


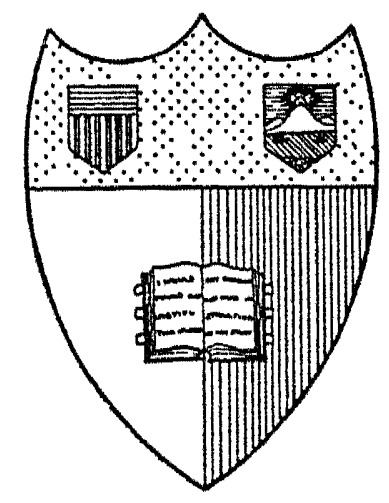

A̛ne 貼axk

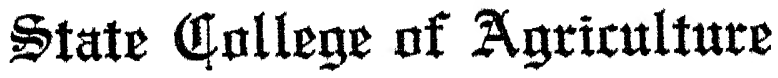

At (T)arnell 引itniuergatty

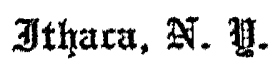

\section{Withrary}




\section{Date Due}
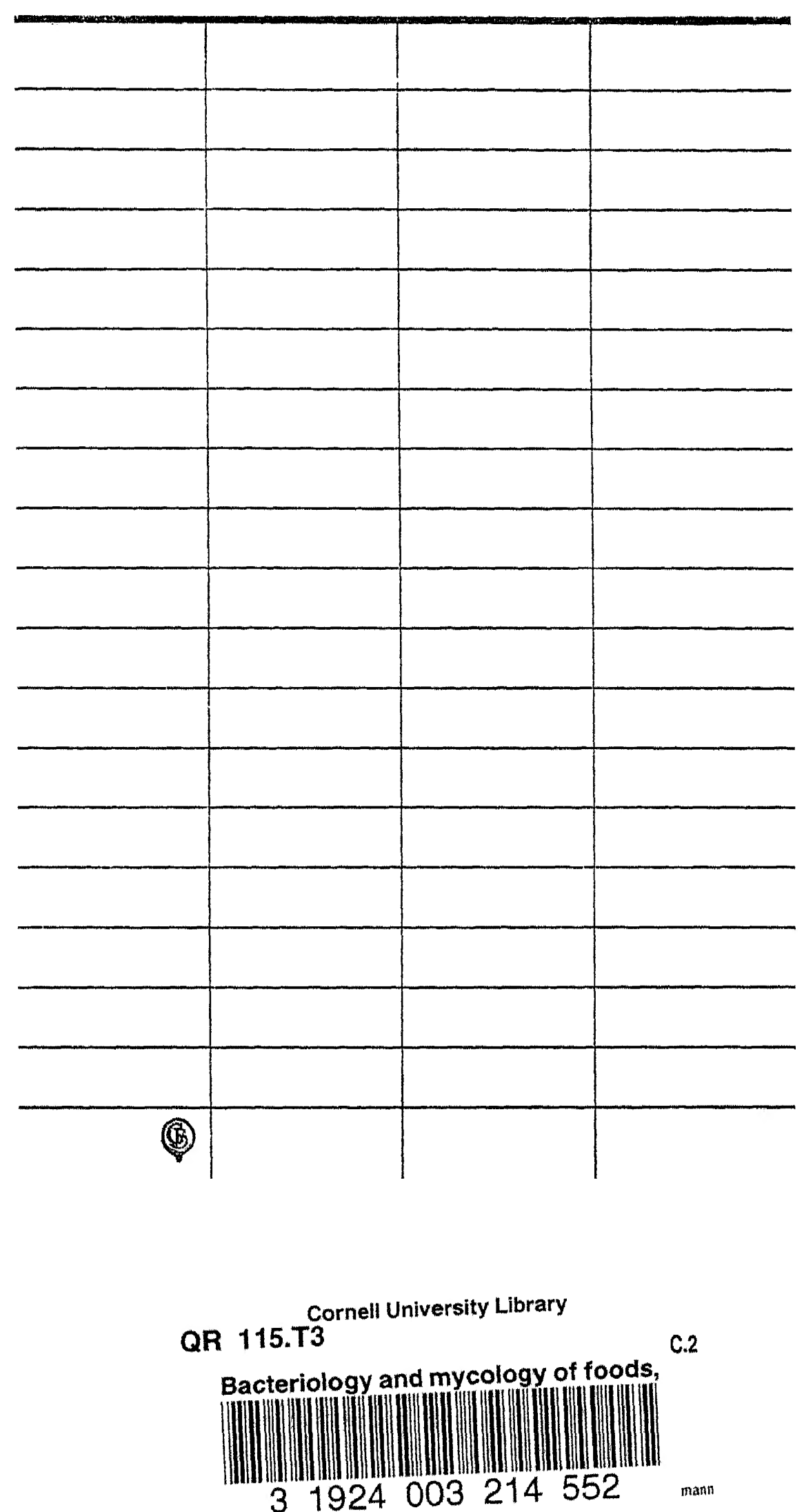


\section{BACTERIOLOGY}

\section{AND \\ MYCOLOGY OF FOODS}

BY

FRED WILBUR TANNER, M.S., Ph.D.

Associate in Bacteriology, University of Illinous

FITST EIITION

NEW YORK

JOHN WILEY \& SONS, INC.

LONDON: CHAPMAN \& HALL, LIMTTED

1919 
CoPYRIGHT, 1919

$B Y$

FRED WILBUR TANNER

PREs OF
BRAUNWORTH O CO.

OOK MANUFACTURERE

BROOKLYN. N, Y. 


\section{PREFACE}

THe present volume is an outgrowth of a course in food microbiology at the University of Illinois. This course has been developed for those who wish to fit themselves for food control, food chemists and for those students in household science who possess a sufficient fundamental training in chemistry. A thorough training in chemistry is essential for intelligent work in microbiology.

The aim of the volume is to present the methods of analysis along with sufficient discussion based on the literature of the subject, to show the history and "make-up" of these methods. An intelligent use of them would be difficult without this. The field of food microbiology is a broad one and the author has drawn freely from the reported investigations of others. Many of these several investigators are experts in their fields, having devoted a lifetime to the study of certain subjects. Some of the methods of analysis are given in the language of their originators, since, by doing this, the personal touch of the author may be retained. No apology is made for the rather liberal quotations from the different "Standard Methods" of the American Public Health Association. These have been worked out by committees composed of men who are experts and their opinions may be given considerable weight.

Numerous references are given at the end of each chapter. Much of the data presented in the chapter has been taken from them; in most instances direct reference is made to them. It is believed that such a feature will render the book more useful to both the practitioner and the student. No serious attempt has been made to list all of the literature on the different subjects which have been treated in this book for this must obviously come from the abstract literature. As the data in this field are accumulating very rapidly, a working reference library is essential.

The author will appreciate helpful suggestions and criticisms from those who use this book. Only by the help of them may the errors and crudities in the first edition of any book be corrected for later editions. 
It is a pleasure to acknowledge the kindness of various individuals and organizations for assistance. To the Cornell Agricultural Experiment station the author is indebted for permission to use the colored plates in the Chapter on Eggs, which were prepared by Professor E. D. Benjamin; to the various supply houses for permission to use illustrations of apparatus; to Bausch \& Lomb Optical Company and the Spencer Lens Company for permission to use parts of their manuals on the "Use and Care of the Microscope"; to the American Public Health Association for permission to use parts of the Standard Methods for the Examination of Sewage and Water, Milk, and Air, finally, to all those various instructors and friends with whom the author has come in contact and who have contributed some of the ideas reflected in the pages of this book.

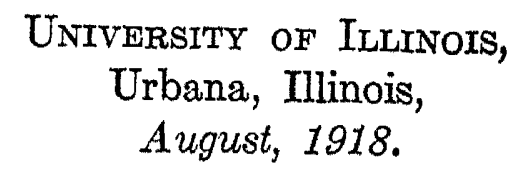




\section{TABLE OF CONTENTS}

\section{CHAPTER}

Bacteriological Apparatus, General Technique, Enumeration of Bacteria, Anaerobic Methods, The Microscope and Microscopic Methods...........

\section{CHAPTER II}

Media and Their Preparation, Bouillon Media, Agar Media, Gelatin Media, Special Media, Potato and Starch Media, Synthetic Media, Blood and Blood Serum Media, Milk Media

\section{CHAPTER III}

Staining Technique. Microscopic Methods, Theory and Methods of Staining. . 76

\section{CHAPTER IV}

Classification and Description of Bacteria. Classification of Bacteria, Historical Life Cycles, Descriptive Chart of the Society of American Bac-

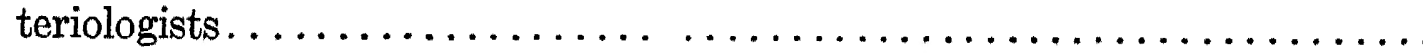

\section{CHAPTER V}

Sterilization and Disinfection. Dry Heat, Moist Heat, Filtration, Light Disinfection, Inorganic Compounds, Organic Compounds, Standardization of Disinfectants.

\section{CHAPTER VI}

Proteins and Carbohydrates. Classification of Carbohydrates, Fats........ 182

\section{CHAPTER VII}

Yeasts and Molds. Fungi, Morphology of Molds, Classification of Molds, Classification of Yeasts, Methods of Study of Yeasts, Compressed Yeast, Yeasts in Bread Making, Methods for the Examination of Bread and Yeasts, 


\section{CHAPTER VIII}

Intestinal Bacteria. The Stomach, Bacteria in Intestines and Feces, The Intestinal Flora, Methods for Determining Bacteria in Feces, Examination of Feces for Cholera Vibrio, Isolation of B. Dysenterio from Feces, Examination of Feces for Tubercle Bacilli, Isolation of Yeasts from Feces.......

\section{CHAPTER IX}

Bacterial Examination of Air. Standard Methods

\section{CHAPTER X}

Water Hygiene. The Chemical Analysis, Bacteriological Analysis, Classification of Water Bacteria, Indicators of Pollution, Test for B. Coli Group, Presumptive and Confirmed Tests, Treasury Department Standard Detection of Pathogenic Bacteria in Water, Self Improvement of Streams, Treatment of Water, Bacteriology of Ice.....................

\section{CHAPTER XI}

Milk and Millk Products. Composition of Milk, Chemical Examination of Milk, Bacterial Examination of Milk, Factors Influencing Bacterial Content of Milk, Sanitary Significance of Bacterial Count, Milk Standards, Standard Plate and Microscopic Methods for Determining Bacteria in Milk, Leucocytes in Milk, Pathogenic Bacteria in Milk, Pasteurization, Bacteriology of Butter, Pathogenic Bacteria in Butter, Cheese, Methods of Examination, Pathogenic Bacteria in Cheese, Condensed Milk, Ice Cream, Pathogenic Bacteria in Ice Cream............................... 363

\section{CHAPTER XII}

Bacteriology of Eggs. Composition of Eggs, Commercial Grading of Eggs, Factors Governing Bacterial Content of Eggs, Methods for the Bacterial Examination of Eggs.

\section{CHAPTER XIII}

Meat and Meat Products. Origin of Bacteria in Meat, Methods of Examination of Meat, Bacteriology and Examination of Oysters............ 479

CHAPTER XIV

Food Preservation. Methods, Examination of Canned Foods, Examination of Tomato Products, Food Poisoning...................... 494

\section{CHAPTER XV}

Epidemiology. Characteristics of Epidemics, Methods for Investigation, Case Cards ........................................ 540

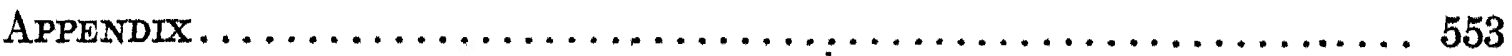

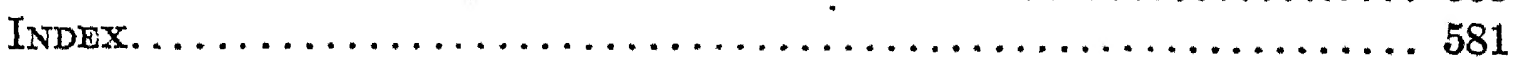




\title{
BACTERIOLOGY AND MYCOLOGY OF FOODS
}

\author{
CHAPTER I \\ BACTERIOLOGICAL APPARATUS
}

The apparatus which is required in a bacteriological laboratory is similar in many respects to that used in a chemical laboratory. Since chemical methods are often used for studying bacteria, it is evident that much the same apparatus may be used. There are several types, however, which may receive special consideration, since a proper construction and type is essential for the best results in bacteriology.

Fermentation Tubes. There are several types of fermentation tubes any of which may be used provided they hold a sufficient excess of the medium over the sample under examination. Standard Methods for the Examination of Water and Sewage, 1917, recommends that the fermentation tubes used in water analysis should hold four times as much medium as sample. All fermentation tubes are constructed with a closed arm which retains the gases that are given off in bacterial metabolism. There are three general types now in use by bacteriologists, Brown's, Smith's (1890), and Durham's (1898).

The Brown fermentation tube was devised for use in connection with water analysis and is quite similar to Smith's. The Smith tube is made both with and without glass feet. Those without the glass feet require a special rack but have the advantage over those with feet that they do not fall over so easily. A convenient rack for holding Smith tubes has been devised by Bain (Ill. State Water Survey Bull. 6, 31, 1908). The Durham tubes are the most satisfactory of all since they are made from ordinary test tubes. The inner tube, according to Lee, should be cut off at an angle to allow the ingress more readily of motil bacteria. Hall (1914) also found this to be true. The efficiency of the Smith and Durham tubes was compared by Browne (1913) and Lee and Fegely (1914). These investigators found that in low dilutions the 
Durham tube was more efficient but with the higher dilutions the Smith tube gave more consistent results. Browne and Ackman (1917) and Browne (1917) have stated that the following factors cause variations in the amounts of gas produced in lactose peptone blle and lactose broth: (1) Temperature; (2) Time of incubation, (3) Initial reaction of the culture medium; (4) Length of inverted vial, (5) Source of bile; (6) Absorption of formed gas.

Graves (1917) inverts the small inner tube over a wooden rod which lifts the tube a distance from the bottom of the outer tube. It is claimed that certain advantages obtain in this procedure.

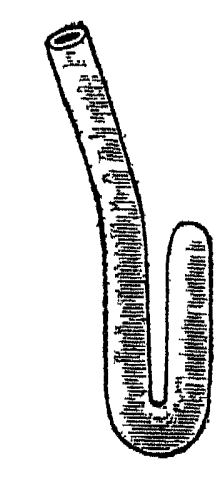

$A$

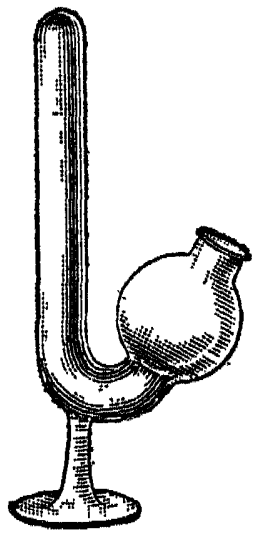

$B$

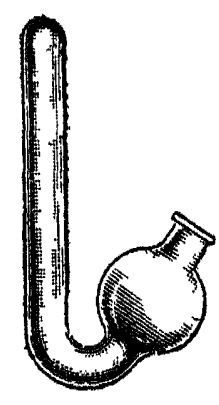

C

Fig 1-Types of Fermentation Tubes. $A$, Brown's, $B$, Smith's $C$, Smith's, without foot, $D$, Graves' Modification of the Durham Fermentation Tube
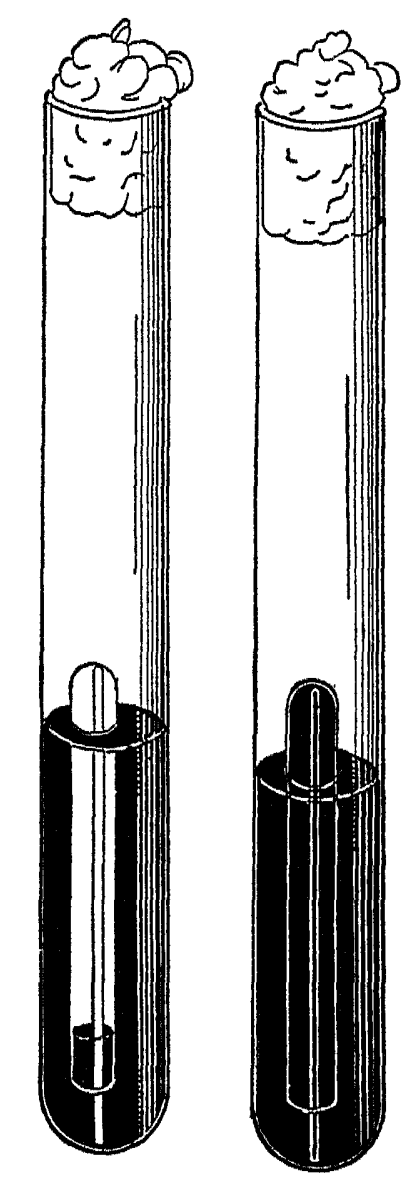

$D$

Petri Dishes. These should be about $10 \mathrm{~cm}$. in diameter, thus having an area of about 65 sq. $\mathrm{cm}$. Either glass or porous tops may be used. The porous tops devised by Hill prevent the spreading colonies which appear so often on Petri dishes in humid weather. Very often it is difficult to secure Petri dishes with flat bottoms. This is essential to an even distribution of bacteria over the plate and a uniform thickness of medium.

Dilution Bottles. These may be of various types and shapes but should contain the amount of dilution water when about half full. This allows ample room for thorough shaking of the diluted sample. Bottles 
with glass stoppers are greatly superıor to those requiring cotton stoppers but these may be used if the cotton is rolled tightly or contains a cork stopper inside of it. Square bottles of the "Blake" type holding
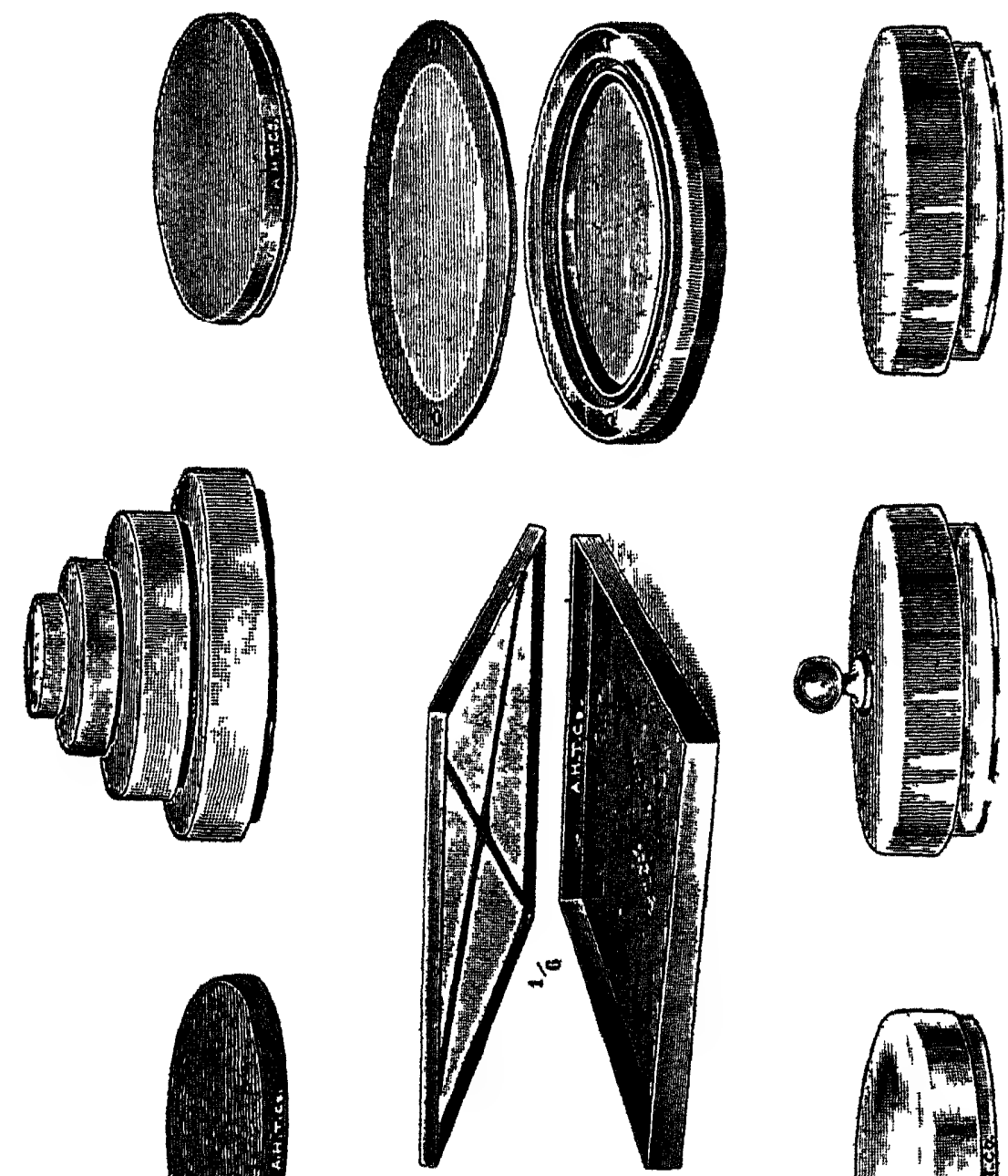

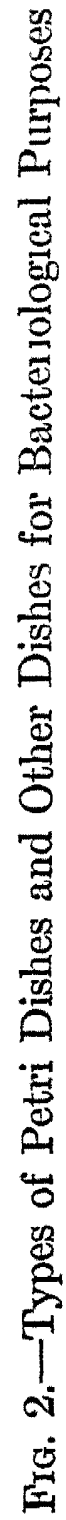
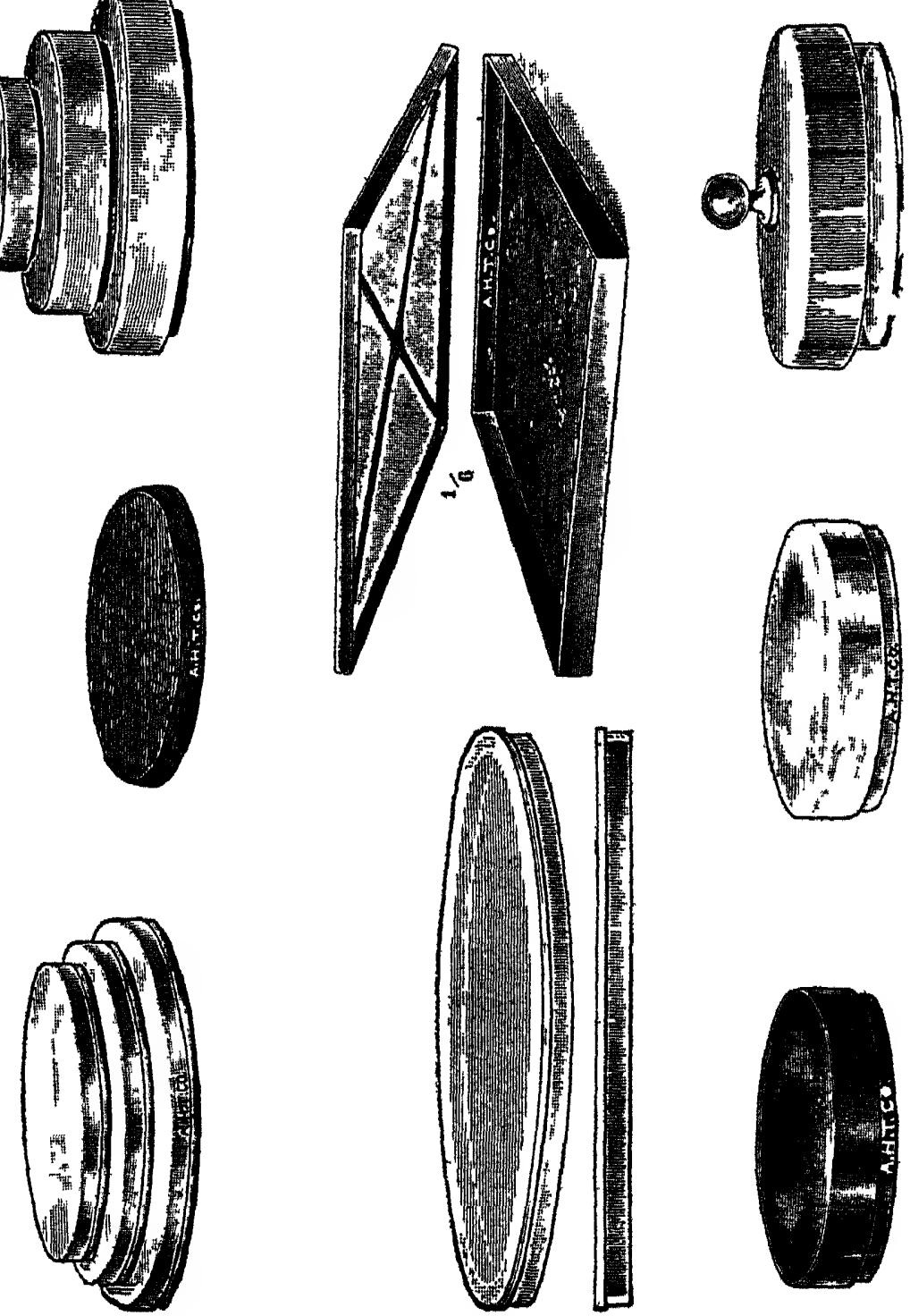

about 200 c.c. are useful and pack together well. If bottles are used for dilutions great care must be exercssed in sterilization to prevent cracking On account of this some laboratories use Florence flasks for holding the dilution water. The dilution bottles should be filled with decimal quantities of water. Where it is desired to obtain exact quantities 

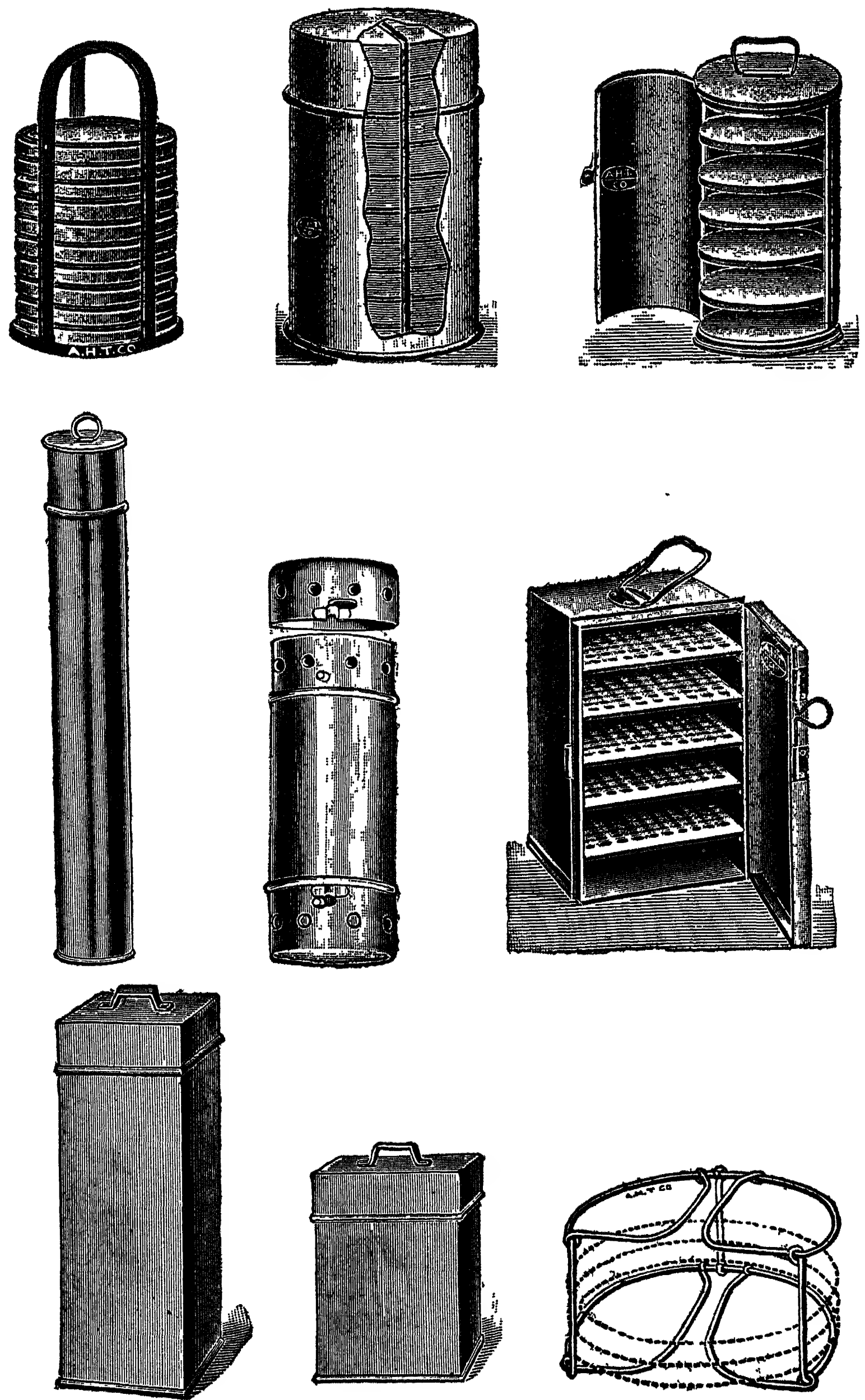

Frg. 3.-Types of Containers and Holders for Sterilizing Petri Dishes, Pipettes and Other Apparatus. 
of dilution water, it may be sterilized in larger quantities and delivered under aseptic conditions in any required amount to the sterile dilution flask. Frost has shown that during sterilization the rolume in dilution bottles may be changed and this might cause considerable error where accuracy is desired.

Sample Bottles. A glass-stoppered bottle of any type may be used. The necks should be wrapped with tin forl before sterlization. Great care should be taken to secure bottles which possess tight stoppers. In general a bottle with a narrow neck is preferable since the possibility of contamination during analysis is greatly decreased.

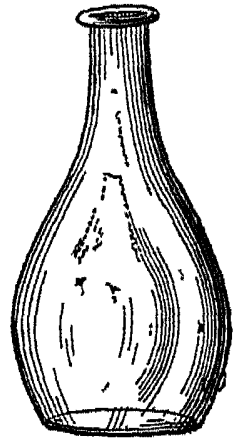

$A$

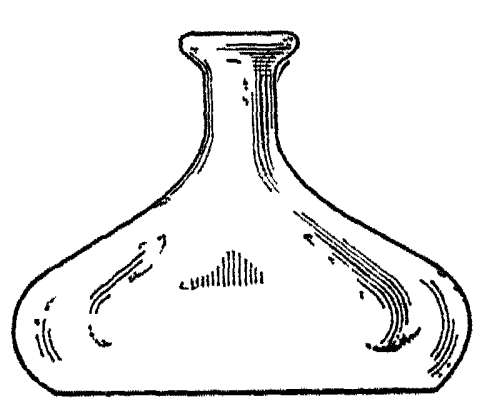

$\boldsymbol{B}$

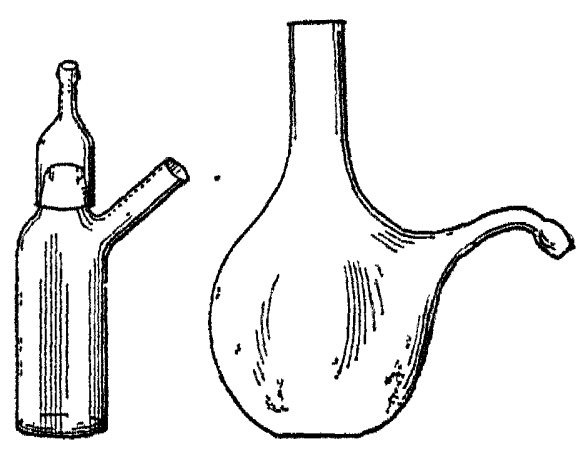

$D$

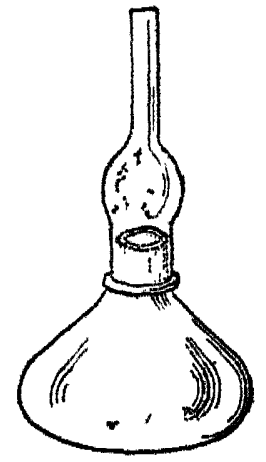

$E$

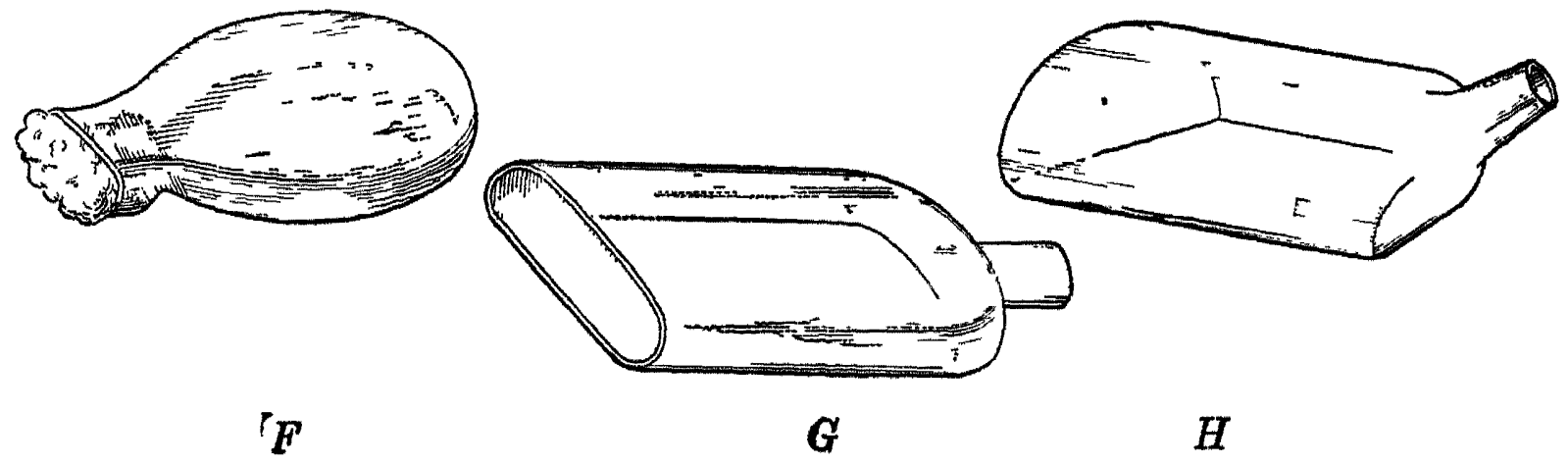

Fig. 4.-Types of Flasks for Bacteriological Work.

- $A$, Koch's Culture Flask, B, Fernbach's Antıtoxan Flask, $C$, Freudenreich's; $D$, Lister Culture Flask, $E$, Miquel Flask; $F$, Kolle Culture for Growing Bacteria in Large Numbers, $G$, Roux Flask, H, Plorowshi's Flask.

Flasks. Many of the flasks which are used for bacterial work are no different from those used in chemistry. Other flasks have been invented for special purposes and usually bear the name of the originator. A few are given in Fig. 4.

Slides and Cover Glasses. Slides should be made of clear white glass and should be free from defects. Different sizes are available but those which are $2.5 \times 7.7 \mathrm{~mm}$. have widest use.

Cover glasses are classified as No. 1, No. 2, and No. 3. The following thicknesses are characteristic of each number. No. $1, \frac{1}{200}$ to $\frac{1}{150}$ in. 
or 0.13 to $0.17 \mathrm{~mm}$. thick. No. $2, \overline{1} \frac{1}{5} \sigma$ to $\frac{1}{10} \overline{0}$ in. or 0.17 to $0.25 \mathrm{~mm}$. thick. No. $3, \frac{1}{100}$ to $\frac{1}{50}$ in. or 0.25 to $0.50 \mathrm{~mm}$. thick.

No. 2 cover glasses are the best for general use since they satisfy the demands of most objectives. No. 1, may be used with oil immersion objectives. The objectives of the different makers are standardized for the following thicknesses of cover glasses:

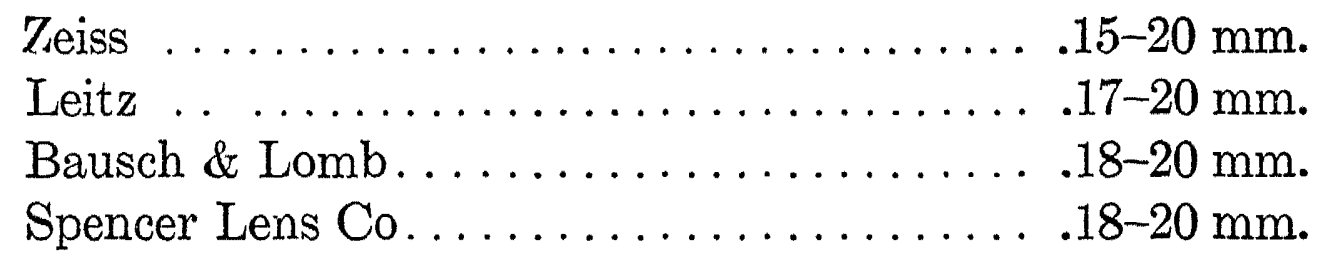

Cleaning of Apparatus. Clean apparatus is the first essential for successful laboratory work in bacteriology. All apparatus whether new or old should be carefully washed and rinsed before using. This removes any free acid or alkali, both of which are very detrimental to the growth of bacteria. Glassware should be washed as clean as possible in tap water with laboratory brushes. These are now constructed in many shapes and sizes which enables one to reach the interior of most flasks. After this is done and if any dirt, etc., remains in the apparatus, it should be taken out by the cleaning solution. This is accomplished by soaking the apparatus, if glassware, in the cleaning solution which is contained in a lead bath. After it has stood for a time, it may be taken out, the cleaning solution allowed to run back into the original container. The clean.ng solution may be used again and should not be thrown away.

The cleaning of slides and cover glasses deserves some special attention. They should be boiled in dilute alkali to saponify any oil or grease which may be on them. After they have been washed in water they should be boiled in cleaning solution for twenty minutes. Then each one should be carefully rinsed in distilled water and stored in ether or dry, as preferred.

Cleaning Solution. This has the following composition:

$$
\begin{aligned}
& \text { Sodium or potassium chromate }\left(\mathrm{K}_{2} \mathrm{Cr}_{2} \mathrm{O}_{7}\right) \ldots .6 \text { gms. } \\
& \text { Concentrated sulphuric acid }\left(\mathrm{H}_{2} \mathrm{SO}_{4}\right) \ldots \ldots \ldots \text { 6. c.c. } \\
& \text { Water........................ 100.c.c. } \\
& \mathrm{K}_{2} \mathrm{Cr}_{2} \mathrm{O}_{7}+4 \mathrm{H}_{2} \mathrm{SO}_{4}=\mathrm{K}_{2} \mathrm{SO}_{4}+\mathrm{Cr}_{2}\left(\mathrm{SO}_{4}\right)_{3}+4 \mathrm{H}_{2} \mathrm{O}+3 \mathrm{O} \text {. }
\end{aligned}
$$

This is a strong oxidizing reagent. Its action may be accelerated by heat. Care should be used in handling it since it is very destructive 
to clothing, etc. The oxygen which is formed is available for the oxidation of any reduced substance.

Platinum Wires. Platinum wires have extended use in bacteriology. Different sizes may be used but gauge No. 26 yields the best results. A heavier wire requires a longer time for cooling after it has been sterilized in the flame. A lighter wire may be too flexible.

In many cases standardized loops are required which deliver the same amount of liquid. Instances are the standardization of disinfectants and determination of bacteria in feces according to the EberleKlien method. St. John (1914) devised a "loop" which gave drops of from 0.019 to $0.021 \mathrm{gm}$. according to the angle at which it was taken from the fluid. Under more unfavorable circumstances the variation

$A$

$B$

c

Frg. 5.-Types of Holders for Inoculating Needles and Loops.

$A$, Special Aluminum and Wooden Handle; $B$, Aluminum Rod; $C$, Glass Rod with Fused Wire.

was from 0.017 to $0.023 \mathrm{gm}$. The size of the drop carlied by this loop is about 0.020 c.c.

Baskets. These should be made of wire with a mesh small enough to retain most of the test tubes used in a laboratory. Any special shape or size may be made at the average tin shop.

\section{General Technique}

Isolation of Microorganisms. This necessitates the plating of bacteria in decimal dilutions on solid media. Agar or gelatin may be used but the latter gives more data. If the organism which is sought in pure culture is known to be mixed with other forms, greater care must be exercised. The plating of decimal dilutions is advisable, especially where little is known with regard to the number of cells which are present. All apparatus must be sterile. Where careful work is 

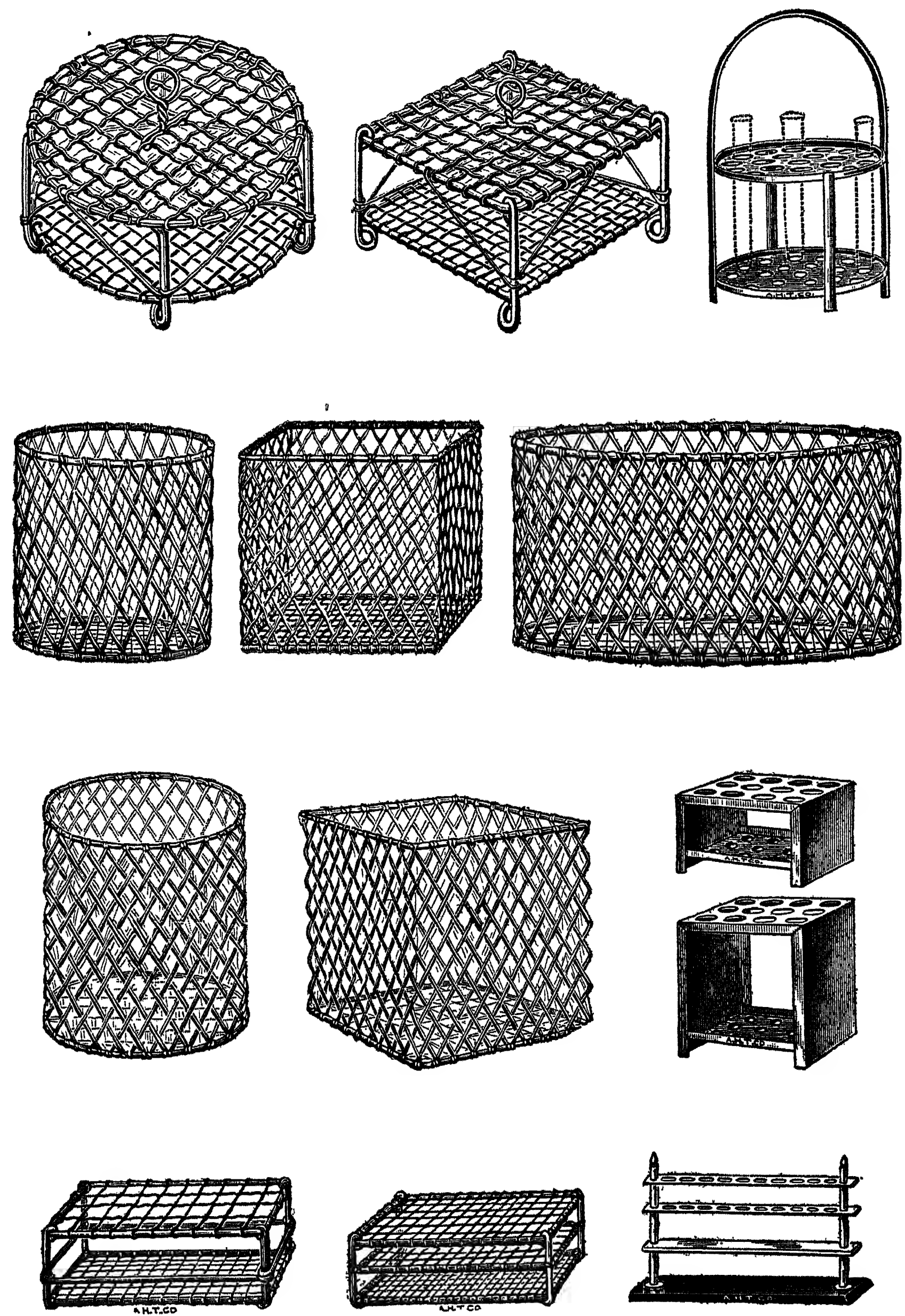

Frg. 6.-Various Types of Wire Baskets for the Bacteriological Laboratory. 
being done, the sample of food should be thoroughly agitated on a shaking machine with glass beads or broken glass.

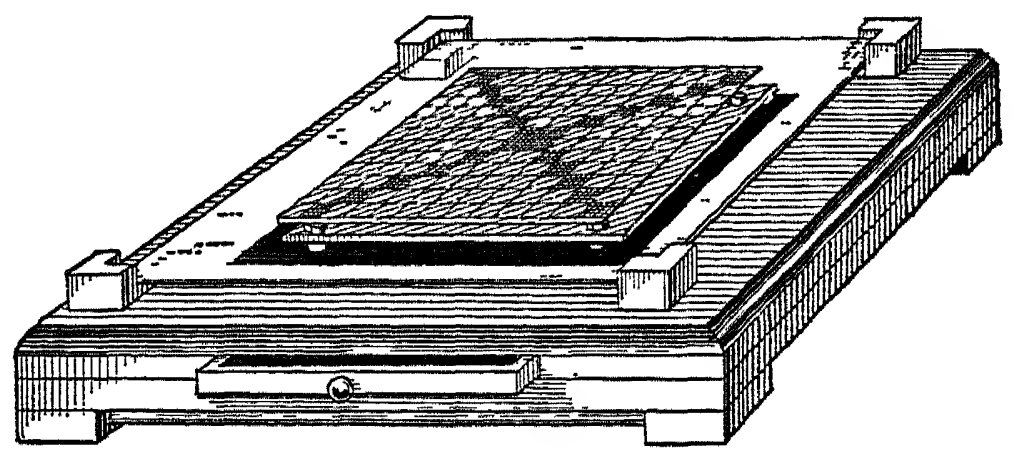

FIG. 7.-Wolfhuegel Counting Apparatus.

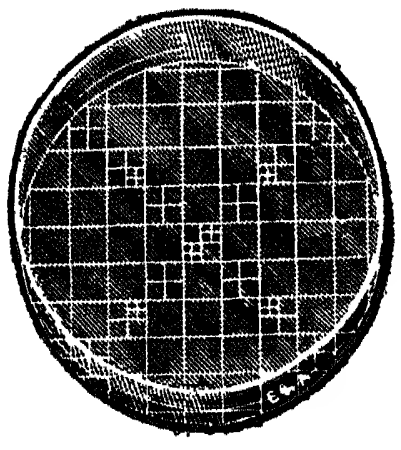

A

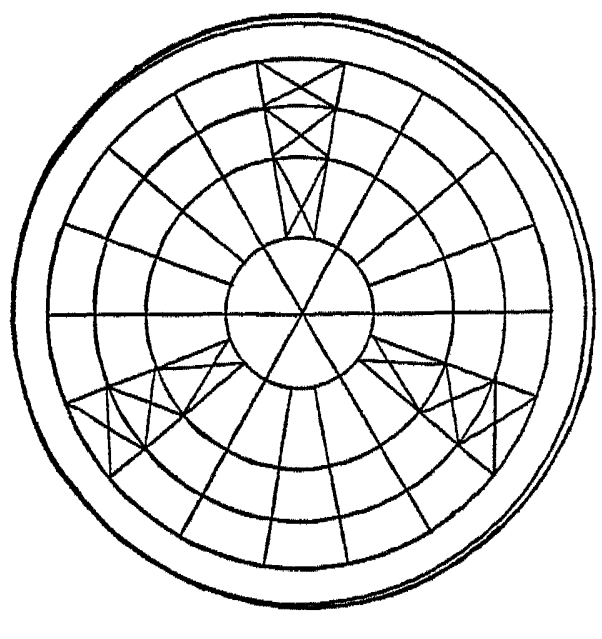

$B$

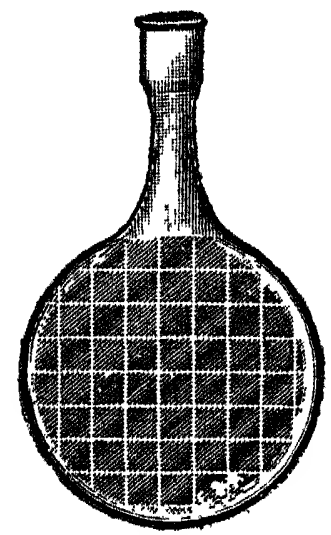

C

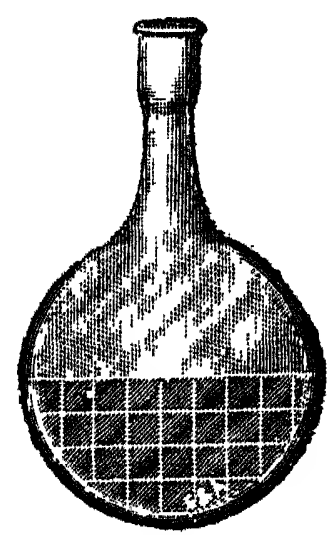

$D$

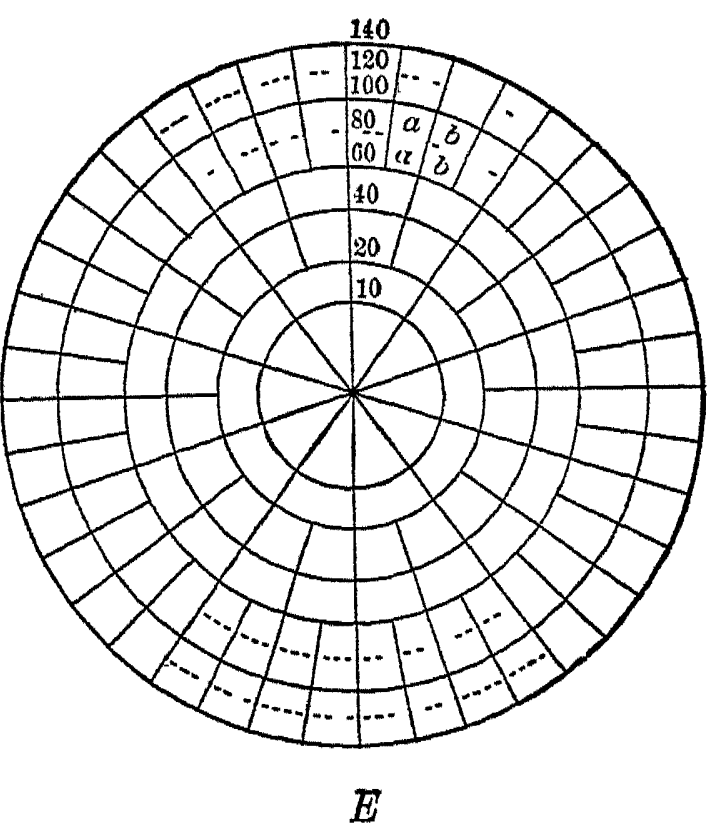

Frg. 8.-Types of Counting Devices.

$A$, Petri Dish with the Bottom Divided into Squares; $B$, Lafar's Counting Plate; $C$ and $D$, Roszahegyi's Counting Flask; $E$, Jeffer's Counting Plate.

Pouring of Plates. A series of these should be poured with different concentrations of the sample. These may be obtained by thoroughly shaking a portion of the sample in sterile water and transferring a loop 
of this suspension to a second tube of stenle water. This procedure is repeated until several concentrations of the sample arè secured. Portions of these are placed in sterile Petri dishes or under these conditions put into tubes of melted medium. These are then put into the Petri dishes.

Isolation of Pure Cultures. A pure culture may be defined in different ways but in this relation it may be regarded as the descendants of a single cell. Each single cell on the Petri dishes poured in the above paragraph will have developed into a colony. In most cases this colony will contain a pure culture if the plate does not contain too many colonies. When there are but a few colonies on the plates, each one that is isolated from the others will be pure. If it is found to be a mixed culture the plating operation must be repeated. In fact in careful work it is essential to replate two or three times. This makes the probability of a pure culture more certain and also serves to "tuneup " or strengthen the organism.

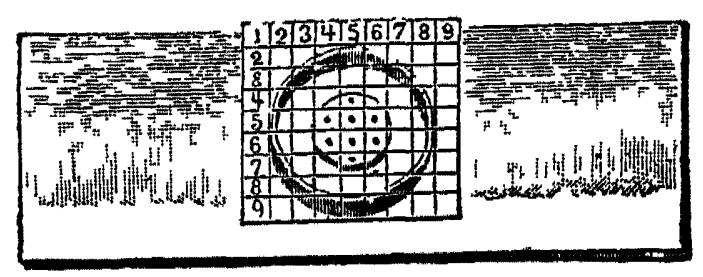

FIG 9-Bottcher's Counting Chamber.

It consists of an ordinary glass slide with a glass ring $20 \mathrm{~mm}$ in diameter and $8 \mathrm{~mm}$ high The cover glass is ruled into 100 squares, 19 of which are numbered

The isolated colonies are picked by means of a sterile platinum wire. A small portion of the colony is removed to either an agar slant or broth tube. Before any extensive investigations are carried out with the organism, it should be studied in hanging drop and stained smear to demonstrate its purity.

Transferring of Cultures. This procedure should be carefully carried out in a room with little dust. Sterile platinum wires should be used which should be sufficiently cooled. If a wire of too great diameter is used, it will retain its heat longer and there is then greater danger that the culture will be killed or altered. Also, if much transferring is done at the same time there will be a tendency for some of the material to cake on the wire. This should be removed. A piece of emery paper or No. 00 sand paper will be found to be a valuable aid.

Dilutions. These are essential in the quantitative enumeration of bacteria. The kind of containers to be used has been described above. 
The dilution water in definite anounts may be put into them before sterilization but the quantity of water has been found to be altered during this procedure. To obviate this difficulty, the containers may be plugged and sterlized empty whlle the water may be sterilized in a larger bottle. This may then be transferred to the dulution bottles with a sterile pipette.

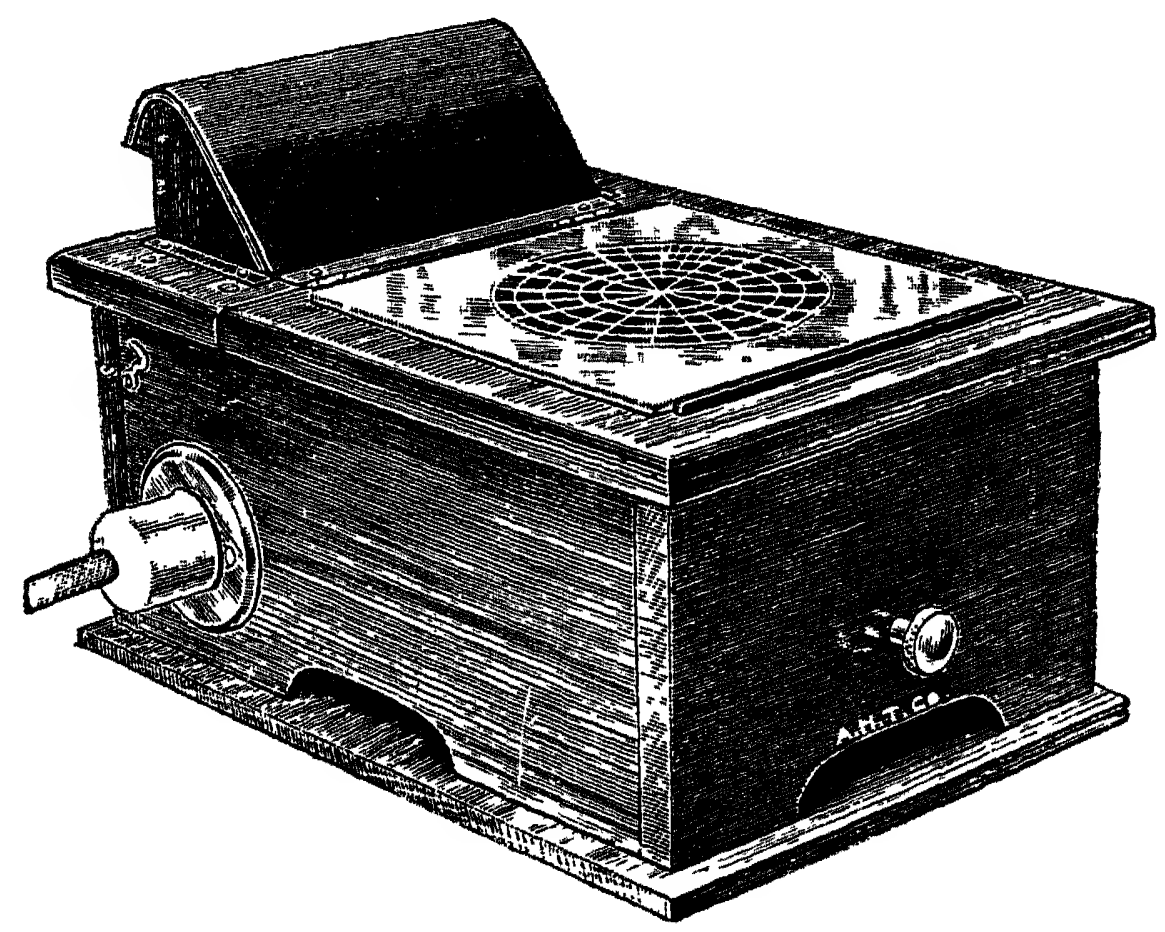

FIG. 10 -Stewart's Counting Apparatus

The Petri dish is Illuminated by oblique rays of light coming from an incandescent light.

\section{EnUmeration of BaCteria}

Several methods have been devised for counting the number of bacteria which are present in any substance. For convenience they may be classified as follows:

A. Plate cultures.

(a) Petri dish cultures.

(b) Frost's microscopic plate method.

B. Direct microscopic method.

(a) Breed's method.

(b) Hæmocytometer methods.

The plate method is probably the oldest and involves the use of the Petri dish. Any transparent solid medium may be used in the Petri dish, but the dilution of sample introduced is important. Hill (1908) and Breed (1916) have both shown that over-crowded plates 
will not give accurate results. Breed, in studying plates made from milk, found that those having more than thirty and less than four hundred colonies gave satisfactory results.

Frost's microscopic plate method and Breed's microscopic method

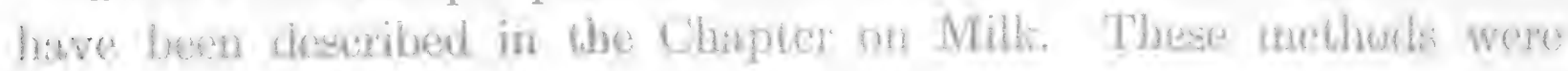

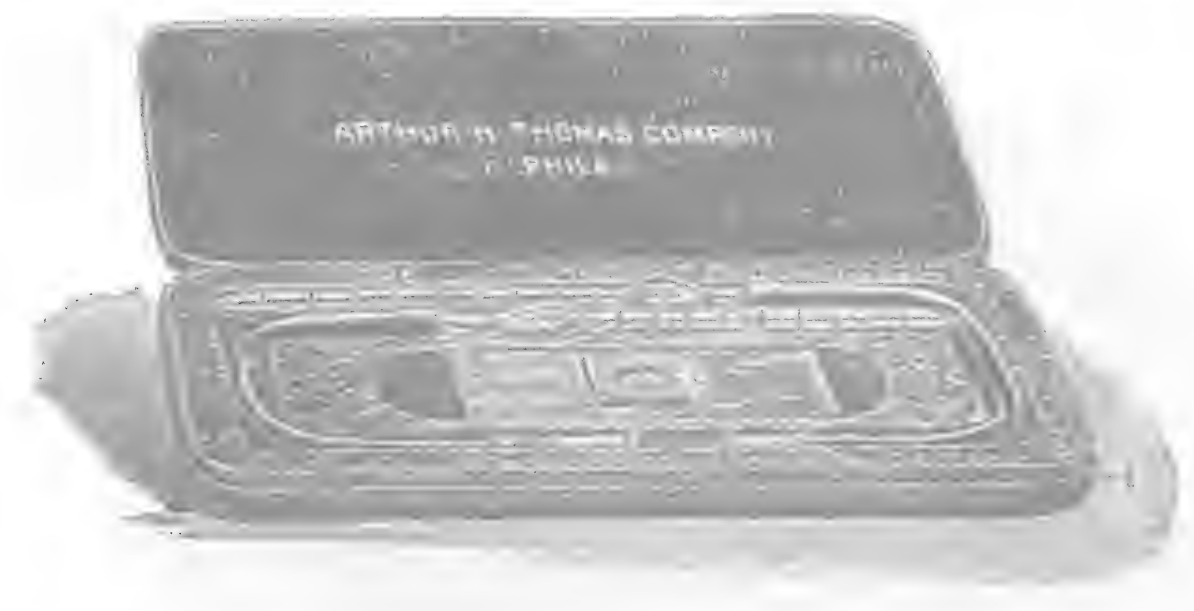

FIG. 11.-Hxmocytometer with Pipettes in Case.

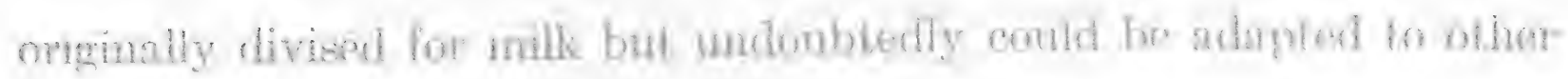
food substances.

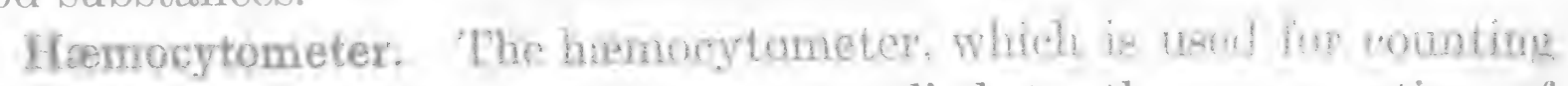
leucocytes and erythrocytes, is now, applied to the enumeration of bacteria in very heavily populated materials.
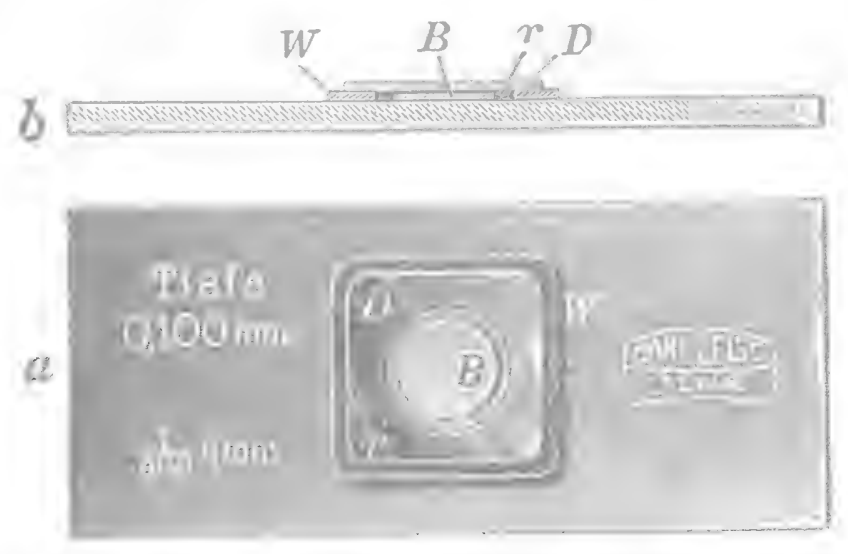

FIG. 12.-Showing Hæmocytometer Chamber.

The instrument consists of a glass slide upon which is fastened pieces of glass in such a way as to form a chamber. The outer glass is square with a round hole in the center. In this is put a round piece of glass of smaller diameter so that there is a shelf, moat and disk formed. The upper surfare of the disk is rulod into snimares. These palinge are

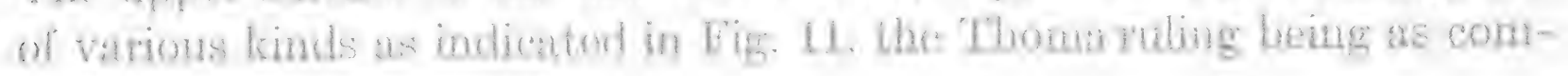


mon as any. This Thoma ruling, however, is more suitable for counting red blood cells.

The pipettes are two in number and are graduated to deliver different amounts. The pipette for erythrocytes delivers dilution of $\frac{1}{10}$ or $\frac{1}{200}$; the pipette for leucocytes delivers a dilution of $\frac{1}{10}$ or $\frac{1}{20}$.

The hæmocytometer was originally devised for counting the blood corpuscles. From this it has been adapted to the enumeration of bacteria in substances containing large numbers. It is best for a person not familiar with the technique to apply it to the counting of blood corpuscles before attempting to count bacteria.

Procedure, Red Corpuscles. Collect a drop of blood on a clean slide and draw part of it up to the mark on the red corpuscle pipette (101). The blood may be secured by cutting the tip of the finger (across the lines) or by a syringe from a vein in the forearm. By drawing the blood to the 0.5 mark a dilution of 200 is secured; when drawn to the 1.0 mark the dilution is 1 to 100 . This first measurement of blood is very important since a slight variation from the mark introduces a larger error in the final results. Should the blood be drawn up too far in the pipette it may be shaken down carefully to the mark. After this the diluting fluid should be drawn into the pipette up to the 101 mark and the pipette thoroughly shaken. The blood is now sufficiently diluted for counting the red blood corpuscles. The contents of the end of the pipette below the bulb should be discarded since it is filled mostly with diluting fluid. A drop of the diluted blood is now placed on the disk of the counting chamber. This should be just sufficient to cover the disk after the cover glass has been put on. The cover glass should be carefully applied with a very slight sliding motion. This should be placed on the chamber as soon as possible in order to secure as even a distribution of corpuscles over the bottom of the chamber as possible. The chamber must be left on a level surface for about a half hour, after which the corpuscles will have settled to the bottom and will be resting on the ruled surface. The number of red corpuscles is now determined by putting the chamber under a microscope fitted with a mechanical stage.

The small squares cover an area $\frac{1}{400}$ sq. $\mathrm{mm}$. since they are $\frac{1}{20} \mathrm{~mm}$. on a slide. The depth of the chamber from the bottom of the cover glass to the top of the disk is $\frac{1}{10} \mathrm{~mm}$., hence the volume of liquid above a square is $\frac{1}{20} \times \frac{1}{20} \times \frac{1}{10}$ equals $\frac{1}{400} \mathrm{~mm}$. $4000 \times$ dilution (200) equals red corpuscles per cubic millimeter. A sufficient number of squares should be counted to give a fairly correct average per square. To accomplish this it is best to count about one hundred squares. By 


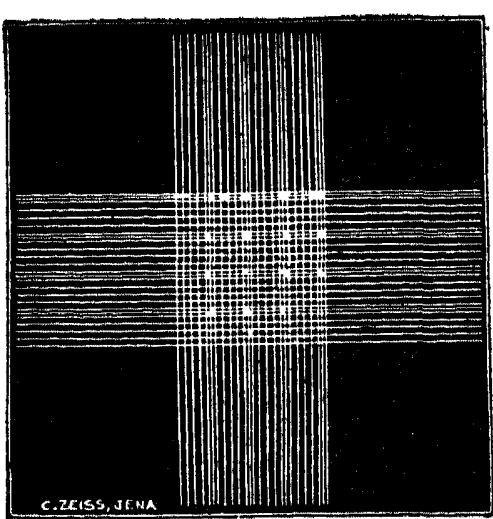

Thoma Ruling

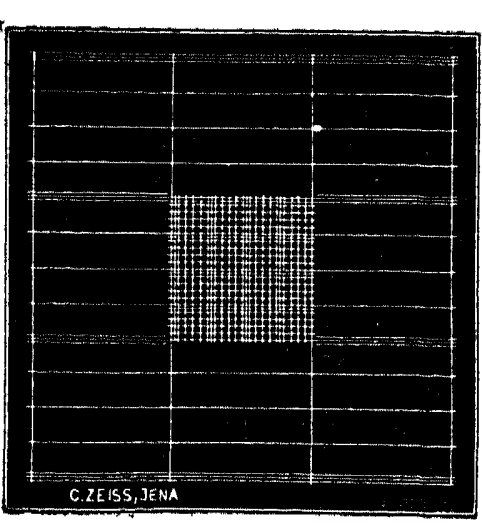

Breuer Ruling

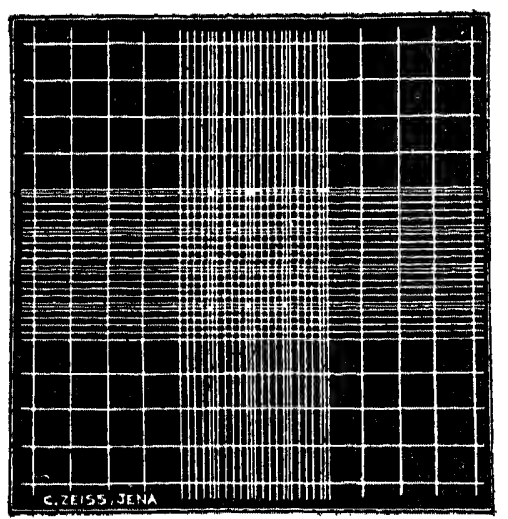

Neubauer Ruling

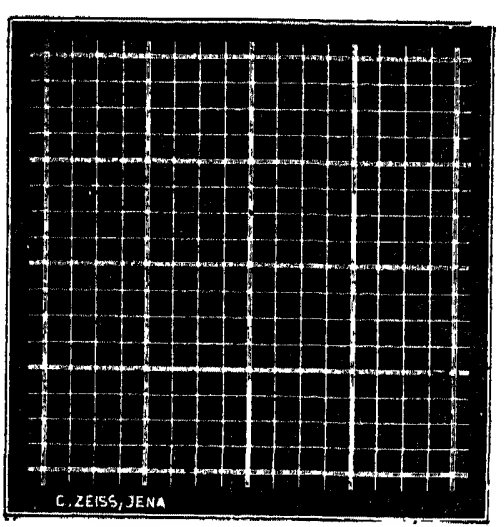

Fuchs and Rosenthal Ruling

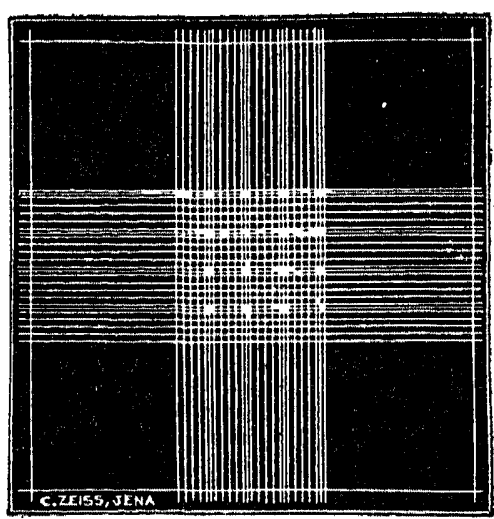

Zappert Ruling

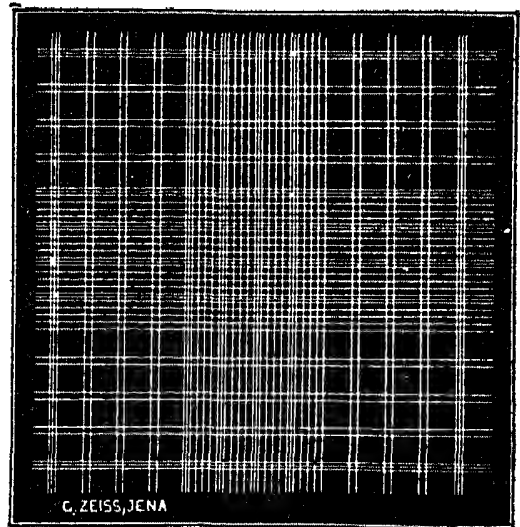

Türk Ruling *

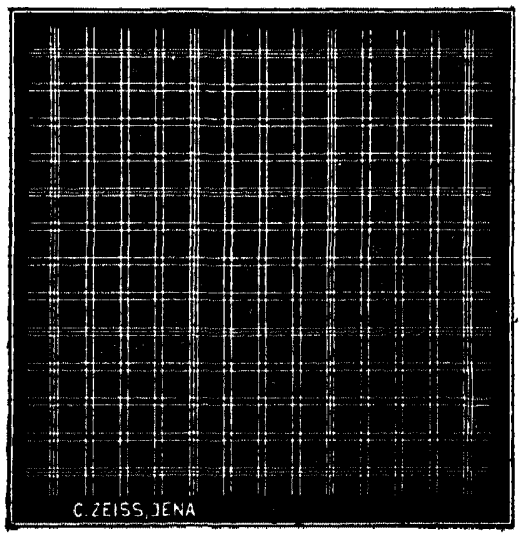

Bürker No. 1 Ruling

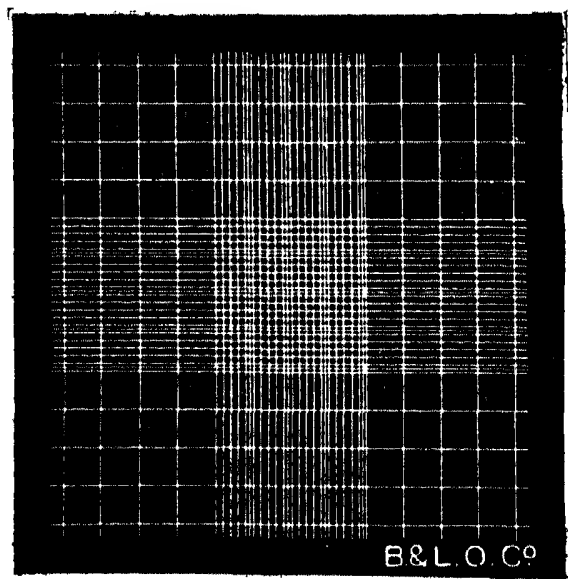

Zappert-Neubauer Ruling

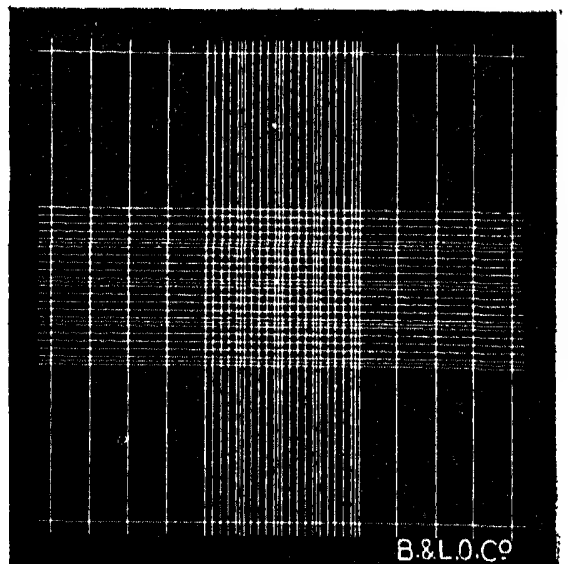

Zappert-Ewing Ruling

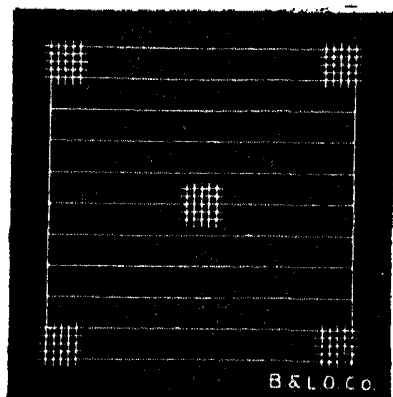

Bass Ruling (15x)

Fig. 13.-Diagrams Showing the Commonest Hxmocytometer Rulings. 
means of the mechanical stage the counting chamber should be moved carefully from square to square. It is useful to always count the cells when they lie on the lines, which are above and to the left. By following some definite system the chance of counting the same corpuscle twice is eliminated.

White Corpuscles. The blood should be drawn into the pipette up to the 0.5 mark after which the special diluting fluid for white corpuscles should be drawn up to the mark. This will give a dilution of $\frac{1}{20}$. A special diluting fluid which will destroy the red and leave the white corpuscles, must be used. The preparation of the disk of the counting chamber is identical with that for counting red corpuscles. A systematic procedure should be followed in going over the slide in order not to count the same square twice. In a well-prepared mount, the number of cells per square should not vary very much from the average for 100 squares.
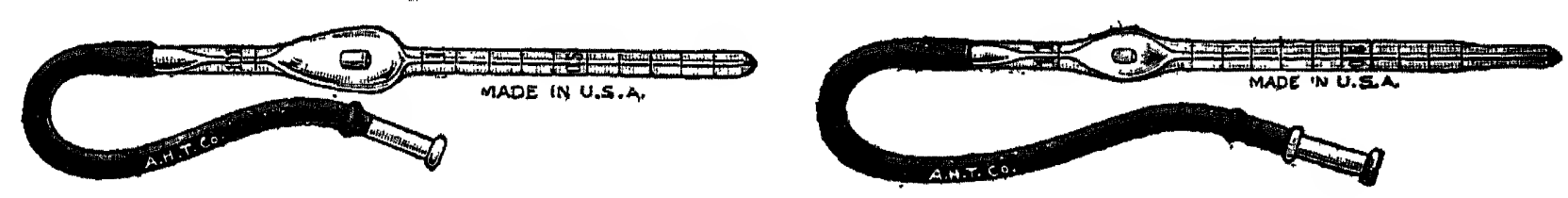

FIG. 14.-Diluting Pipettes for Hæmocytometer.

A counting chamber of more recent construction has been made for the Arthur H. Thomas Co., Philadelphia, known as the ThomaLevy hæmacytometer. This has certain advantages over the types of the older construction and is described as follows:

"In the Levy construction a rectangular depression is cut into the slide itself extending across the entire width. In the middle of this depression is permanently fixed a rectangular strip of glass also extending entirely across the slide, and on this are the rulings. When the cover glass is placed in position on the slide itself the solution over the ruled areas is of the required depth. The Levy method of construction avoids the cemented cell and the attendant danger of its loosening by the drying out of the balsam cement, and the possibility of the loosening of the ruled counting surface is also greatly reduced by this construction. The parallel form of cell greatly facilitates cleaning as compared with the circular type and the method of ruling used in the manufacture of these chambers provides a line with absolutely cleancut edges and of distinctly increased visibility when the chamber is filled with solution for the count. This increase in visibility of the ruling greatly lessens the eye fatigue experienced in making repeated counts."

The manufacturers dlaim for this counting chamber the following advantages:

Increased visibility of the rulings when chamber is filled with solution. 
The new method of construction entrely avoids the cemented cell and the attendant danger of its loosening by the drying out of the balsam cement, and the possibility of the lonsening of the ruled counting surface 15 also greatly reduced by this construction.

The parallel form of cell first suggested by Burker and used in all Levy counting chambers provides a more uniform distribution of corpuscles over the ruled area and entirely removes the effect of atmospheric pressure upon the depth of the solution, a source of considerable error in the circular-form chambers.

The parallel form of cell also greatly faclitates cleaning as compared with the circular type.

Critical tests have shown the Levy rulings to be more accurate, both in the dimensions of the ruling and the depth of the cell, than any counting chambers of best European makes we have tested. All Levy counting chambers are

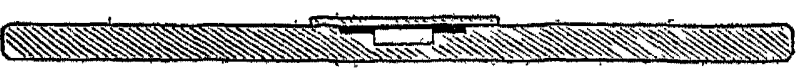

Vertical longitudinal section of slide, with cover glass in position, showing new method of construction

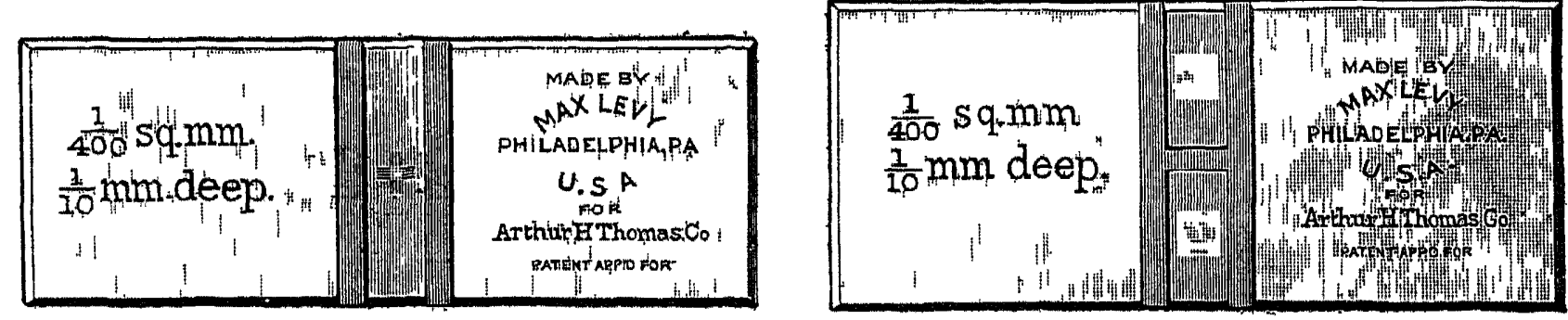

FIG. 15.-Thoma-Levy Counting Chamber.

guaranteed to be well within such limit of error as may be later established by the U. S. Bureau of Standards for instruments of this type.

Application of Hæmocytometer to the Enumeration of Bacteria. Since the samples which are used in the hæmocytometer are small, the bacteria must be present in large numbers. A slight error in making dilutions will cause a large error in the determination of the number of bacteria per cubic centimeter since the factor is large which converts the reading of the microscope into terms of cubic centimeters. It is also an easy matter to confuse organic matter with bacteria. This is especially true in the applications of this apparatus to the enumeration of bacteria in feces and tomato products. These are taken up in other places.

Cleaning of Hæmocytometer Apparatus. This is one of the most important essentials in the use of the apparatus. Pipettes should be cleaned by attaching them to a suction pump and drawing dilute alkali through them. After this alcohol and distilled water may be used. If they become stopped up a small wire may be run through, 
but this should be used with care since the hottom of the pipette may chip off.

The counting chamber should be washed with dilute alkali or soap. It should be thoroughly rinsed in distilled water and wiped dry and should not be sterlized by heating because the cement which holds the disk will be melted.

\section{Anaerobic Mrethods}

For the cultivation of those microorganisms which grow under anaerobic conditions, some special apparatus and methods are necessary to remove free oxygen. Many procedures have been proposed, a few of which will be given here.

Boiling. Air is very soluble in water at room temperature. Winkler has found that 1 liter of water at $20^{\circ} \mathrm{C}$. will dissolve about $9 \mathrm{mg}$. of oxygen. By boiling water for some time this dissolved oxygen may be driven off. Special methods are then necessary to prevent reabsorption of air. In liquid media where heating will cause no change, this may be done by a layer of sterile vaseline or ligroin.

Displacement by an Inert Gas. For this purpose several gases, such as nitrogen, hydrogen, carbon dioxide, coal gas, etc., have been used. Hydrogen has had the widest application on account of its ease of preparation and lack of harmful effects on bacterial development. When these gases are used, they are bubbled through the medium and should be carefully washed by the proper solutions to render them pure. Gas wash bottles of the Bunsen or Friedrich types will allow satisfactory results.

Absorption of Oxygen. For this purpose alkaline pyrogallol which has great affinity for oxygen is usually used. It becomes reduced to compounds which are not definitely known. In applying this chemical to the growth of anaerobes many devices have been used. Any one is quite satisfactory which prevents the ingress of more oxygen after the initial oxygen has been absorbed.

Buchner's Method. In this method the culture tube is placed in a larger one which contains the alkaline pyrogallol. It has the disadvantage that if some special support is not used to keep the culture tube above the surface of the pyrogallol, it is difficult to follow growth during incubation. These tubes may be prepared as follows:

Introduce a few grams of pyrogallol into the bottom of a large test tube and place therein a support for the culture tube. This may be made froin wood, cotton, glass, etc. After the culture tube has been 
placed on its support, introduce the $\mathrm{NaOH}$ by means of a pipette into the bottom of the tube and seal the tube immediately with a rubber stopper.

Wright's Method. This is another absorption method and is different from the others only in method of procedure. It is especially adaptable to the isolation of anaerobic spore-forming bacteria. Decimal dilutions of the sample are introduced into sterile litmus milk. About $1 \mathrm{in}$. above the meniscus of the milk a tight cotton cylinder is forced upon which are put about 2 gms. of pyrogallol. This is treated with strong $\mathrm{NaOH}$ and the original plug replaced in the tubc. This tube should then be heated for fifteen minutes at $80^{\circ}$ to kill all vegetative bacteria. Later incubation will give the characteristic changes.

Lentz's Method. This requires a glass plate $125 \mathrm{~mm}$. square and cellulose absorbent rings. Before using the rings are soaked in pyrogallic acid and immediately before the culture is started in 1 per cent potassium hydroxide. The plate with the culture is inverted over the cellulose ring, being sealed to the glass with plasticine.

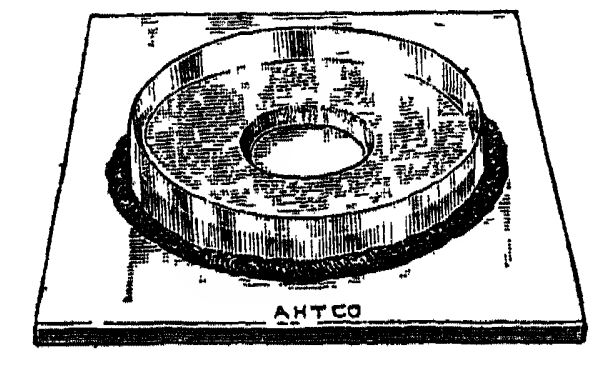

FIG. 16.-Lentz's Anaerobic Apparatus

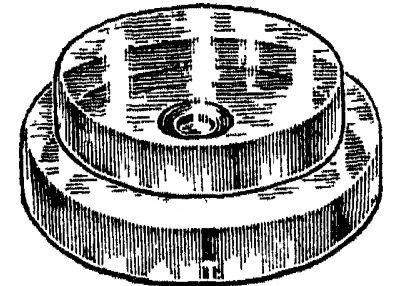

Frg. 17.-Kuster's Anaerobic Culture Apparatus.

Kuster's Method. This depends upon the same principle as McLeod's and Lentz's methods. A glass absorption chamber is used having a small hole in the top through which is introduced the absorption substance. The Petri dish containing the culture is inverted over this and sealed with plasticine.

McLeod's Method. This method requires a porcelain dish consisting of two separate chambers for containing the pyrogallic acid and potassium hydroxide. The special Petri dish has its free edge turned inward and upward. Around the upper cover of the porcelain dish is a groove to contain plasticine which prevents the ingress of air. About 7 c.c. of a 10 to 20 per cent pyrogallic acid is run into the chamber marked $A$ in Fig. 18. Into part $B$ are put 7 c.c. of a 10 per cent potassium hydroxide. The Petri dish is then pressed down into the plas- 
ticine. After this is done air is excluded and the potassium hydroxide and pyrogallic acid are mixed by tilting the dish.

Zinsser's Method. The main apparatus involved in the application of this method is a set of circular glass dishes similar to a Petri dish except that they are much deeper and a larger space is left between the sides. The agar which has been inoculated with the sample is
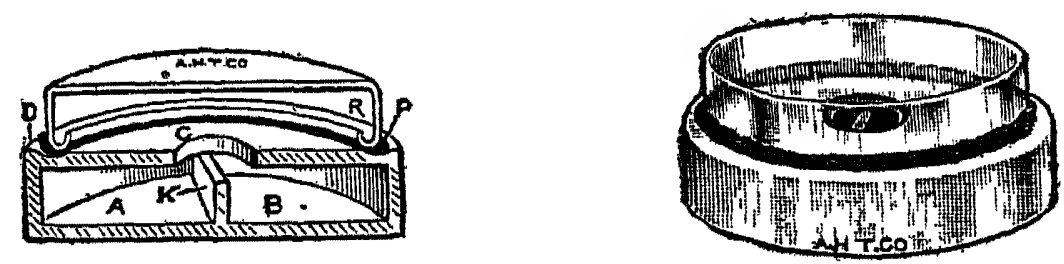

FIG. 18.-McLeod's Anaerobic Culture Apparatus.

poured into the smaller of the dishes. This is allowed to harden and the excess of moisture is evaporated. Into the other dish, is placed a quantity of pyrogallol, over which the smaller dish is inverted. Sodium hydroxide is poured into the larger dish. While this is reacting with pyrogallol, an oil such as abolin is dropped into the same place. This successfully seals the chamber which is formed by the two dishes.

Torrey's Method. This method was especially successful in the hands of Torrey for the isolation of $B$. bifidus and B. acidophilus.

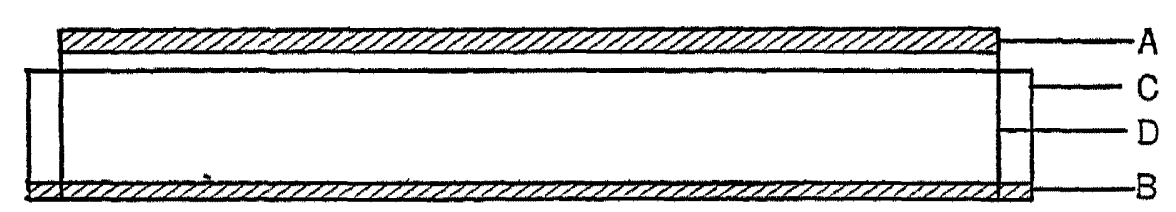

FIG. 19.-Cross-section of Torrey's Anaerobic Chamber.

$A$, Solid Medium Containing the Sample, $B$, Agar which has been Heavily Inoculated with $B$ subtilis or $B$ mesenterncus, $C$ and $D$, Petrl Dishes which Form the Chamber

It is similar to the method devised by Zinsser. A Petri dish about $10 \mathrm{~cm}$. in diameter and about $2 \mathrm{~cm}$. high is poured and inoculated with the sample under investigation. It is placed in an incubator to remove excess moisture and dry the sides of the dish. This is important since too much moisture will allow contamination with the culture which is used to absorb the oxygen. This dish after drying is inverted into another dish, containing agar heavily inoculated with Bacillus cereus, of at least $12 \mathrm{~cm}$. in diameter. The agar in the lower plate forms a seal, and the strict aerobic organism Bacillus cereus will use up the free oxygen. This apparatus is placed in an incubator in a moist chamber to prevent evaporation of the agar in the larger dish. 
Smillie's Method. This method depends upon the catalytic action of platinized asbestos upon oxygen and hydrogen when they are brought into contact. The author claims that the method is well adapted to the study of strict anaerobes such as B. botulinus. The method adaptable to cultures in test tubes is described as follows by the author: "Platinized asbestos is first prepared in the usual way, or it may be purchased from any laboratory supply house. A small mass of the catalyzer is firmly fixed at the end of a platinum wire by coiling the wire about it. The other end of the wire is inserted in the end of a short

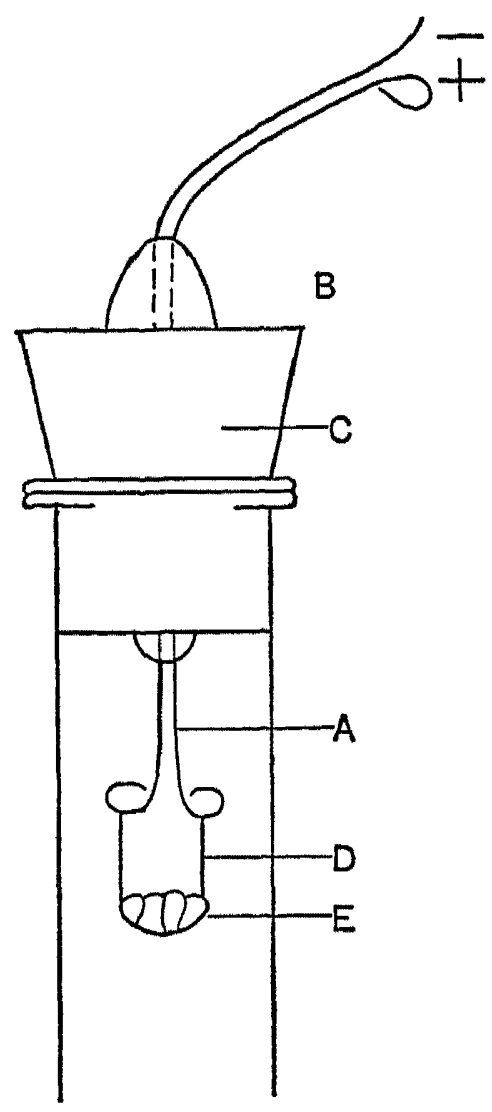

FIg. 20 -Sketch of Anaerobic Culture Apparatus for the Cultivations of Obligate Anaerobes in Test Tubes According to Smillie's Method. glass rod, and the rod is inserted into a No. 1 one-hole rubber stopper. The apparatus is wrapped in a package and autoclaved.

"The water of condensation is removed from a plain agar slant the tube inoculated, inverted, the cotton plug removed and the tube filled with hydrogen by means of a sterile capillary pipette. The hydrogen may be obtained from a Kipp generator, or more satisfactorily from a hydrogen tank. It should be passed-through a series of wash bottles containing silver nitrate, sulphuric acid potassium permanganate, and lead acetate to remove all impurities.

"After allowing the hydrogen to fill the inverted inoculated test tube, the platinized asbestos is heated for a few minutes in a free flame, the rubber stopper is inserted firmly into the inverted tube and the end of the tube dipped into melted paraffin.

"The catalyzer glows for a second or two as the hydrogen and oxygen are actively united, and the water formed is deposited on the surface of the tube. The process is now complete and the tube is ready for incubation." Smillie states that this method is satisfactory for the usual anaerobe but does not remove all traces of oxygen. Another method had to be devised. This is described as follows: "Two lengths of nichrome wire No. $22,6 \mathrm{~cm}$. long, are separately fused into a glass tube so that they are insulated (see Fig. 20a) and the glass tube $B$ closed at each end is passed through a one-hole rubber stopper $C$. To the lower end of the nichrome wire $D$ thus completing 
the circuit. In the coil of the fine nichrome wire is placed a small mass of platinized asbestos $E$. The apparatus is placed in a package and autoclaved." Smillie has applied the same method to the culture of large amounts of bacteria in Blake bottles.

This method applied to the culture of bacteria in anaerobic jars is as follows:

Smillie experienced some trouble in getting jars which would stand up under great vacuum. He finally adopted the ordinary specimen

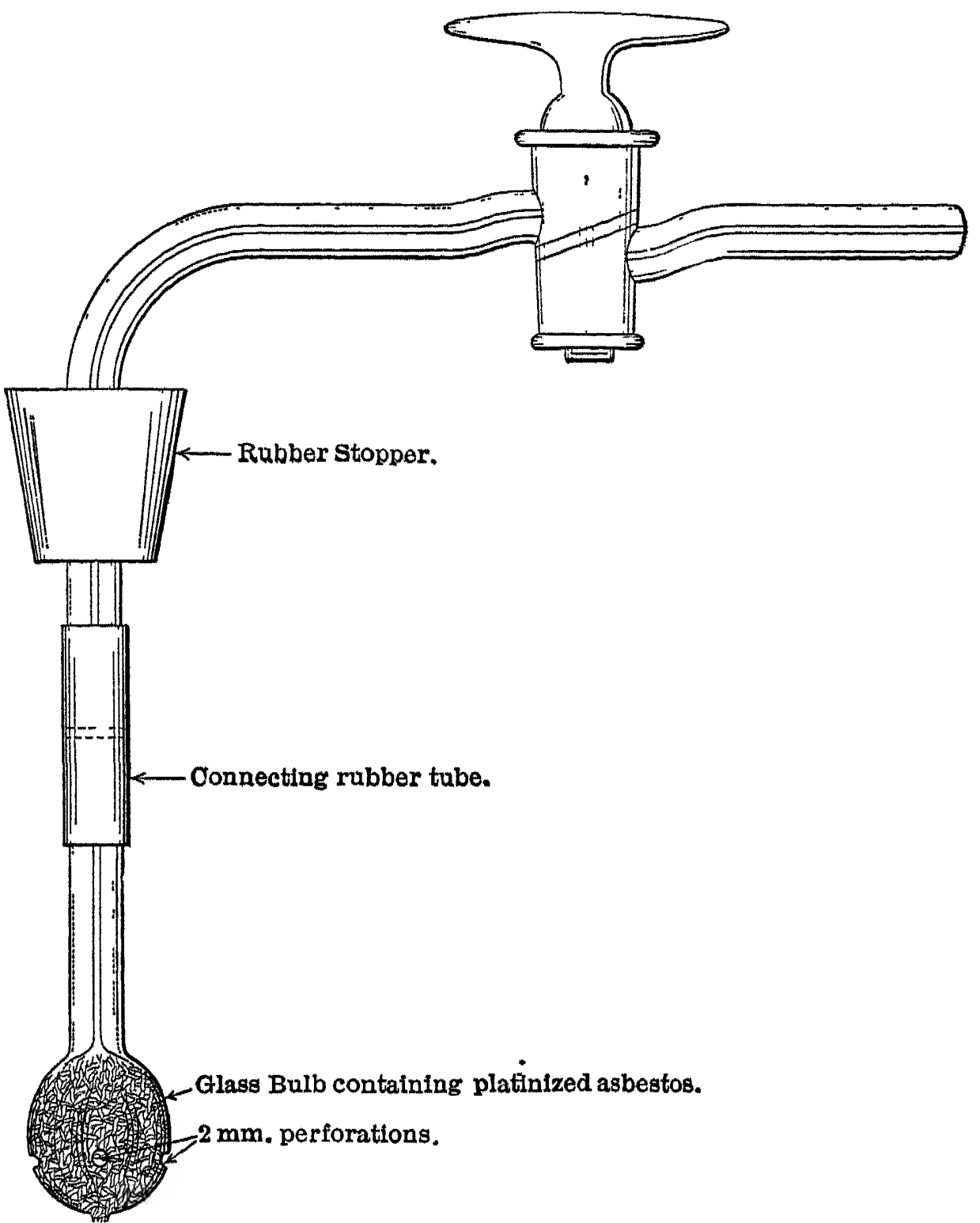

Frg. 21.-Detail of Platinized Asbestos Bulb for Anaerobic Jar According to Smille's Method.

jar about $30 \mathrm{~cm}$. high and an inside diameter of $12.5 \mathrm{~cm}$. In the cover of this were ground two holes into which were fitted No. 4 one-hole rubber stoppers, carrying a glass "angle" stop-cock. A rubber tube extending to the bottom of the jar was attached to one of these. To the other stop-cock, a glass bulb with perforations is attached by means 
of rubber tubing. This glass bulb is filled with platinized asbestos. The cultures are placed in a glass tumbler which is put into the jar. Fig. 21 taken from Smillie's original publication will give the details of the asbestos bulb.

\section{ExCLUSION OF OXYGEN}

Beattie's Method. Ordinary test tubes containing plain or carbohydrate broths are used. Melted sterilized vaseline is poured into the tube until a plug about $\frac{1}{2}$ in. long is formed. The broth and vaseline are then boiled for twenty to thirty minutes in order to expel any dissolved air and allowed to cool. These tubes may be kept for an
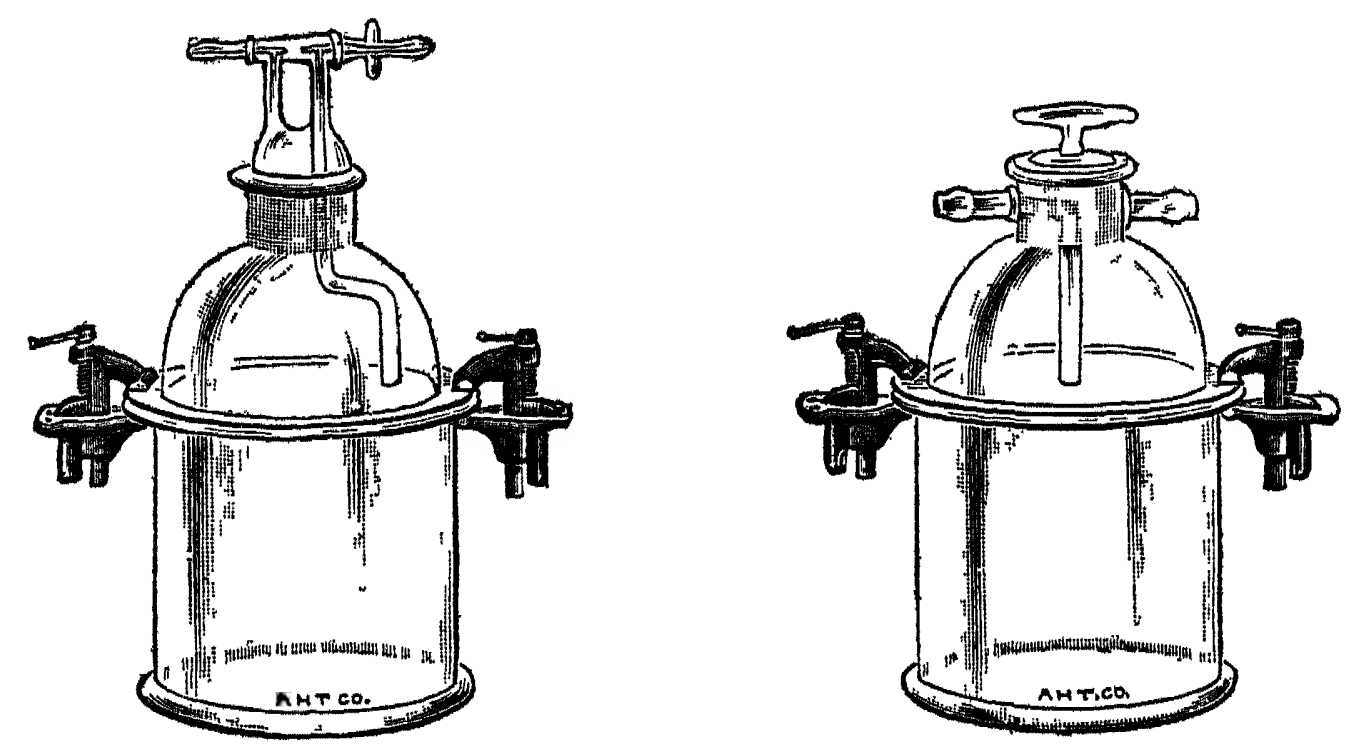

Fia. 22.-Types of Novy Jars.

indefinite time and are air tight. When they are to be inoculated they may be melted in a water bath at $55^{\circ} \mathrm{C}$. The sample may be introduced by means of a sterile pipette. The vaseline may then be heated over a flame to reform the solid plug. The tube may then be placed in the incubator. In order to remove any portion of the culture, a sterile sealed pipette (capillary) is thrust through the plug. This may be broken by forcing the end against the bottom of the tube after which any portion of the culture may be removed. By melting the vaseline plug again the culture tube is sealed for further incubation.

Novy's Method. The apparatus used in the application of this method consists of a large glass jar with a removable top (Fig. 22). The culture may be grown away from oxygen by using an inert gas vacuum or alkalin pyrogallol. The jar is constructed with a glass stopcock which will allow the removal of air with a vacuum pump. This 
may be combined with alkalin pyrogallol and give an atmosphere which is entirely free from oxygen.

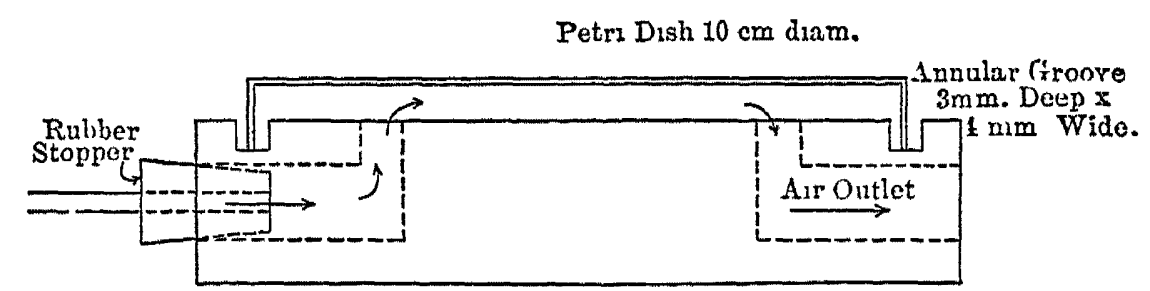

FIg. 23.-Jones Anaerobic Culture Apparatus. Cross-section of the Stone Base with Petri Dish in Position.

Bulloch's Method. This is very similar to Novy's and needs no special attention here. The air in the jar should be replaced by an inert gas.

Jones' Method. This method allows the observation of growth and requires very little gas. It is described by the author as follows: "The apparatus consists of one-half of a Petri dish sealed with paraffin on a square stone or metal base provided for an inlet of the inert gas and outlet of air. The figure gives the general construction of the apparatus.

\section{The Microscope}

A compound microscope is necessary for a study of bacteria. The success which a student has in the pursuit of microbiology is often dependent upon thorough knowledge of the microscope. It is desirable to study the microscope with much care in order to understand its limitations and the significance of each part. It is much better to regard it as a delicate piece of mechanism through whose intelligent use an entire world of living organisms will be made visi-

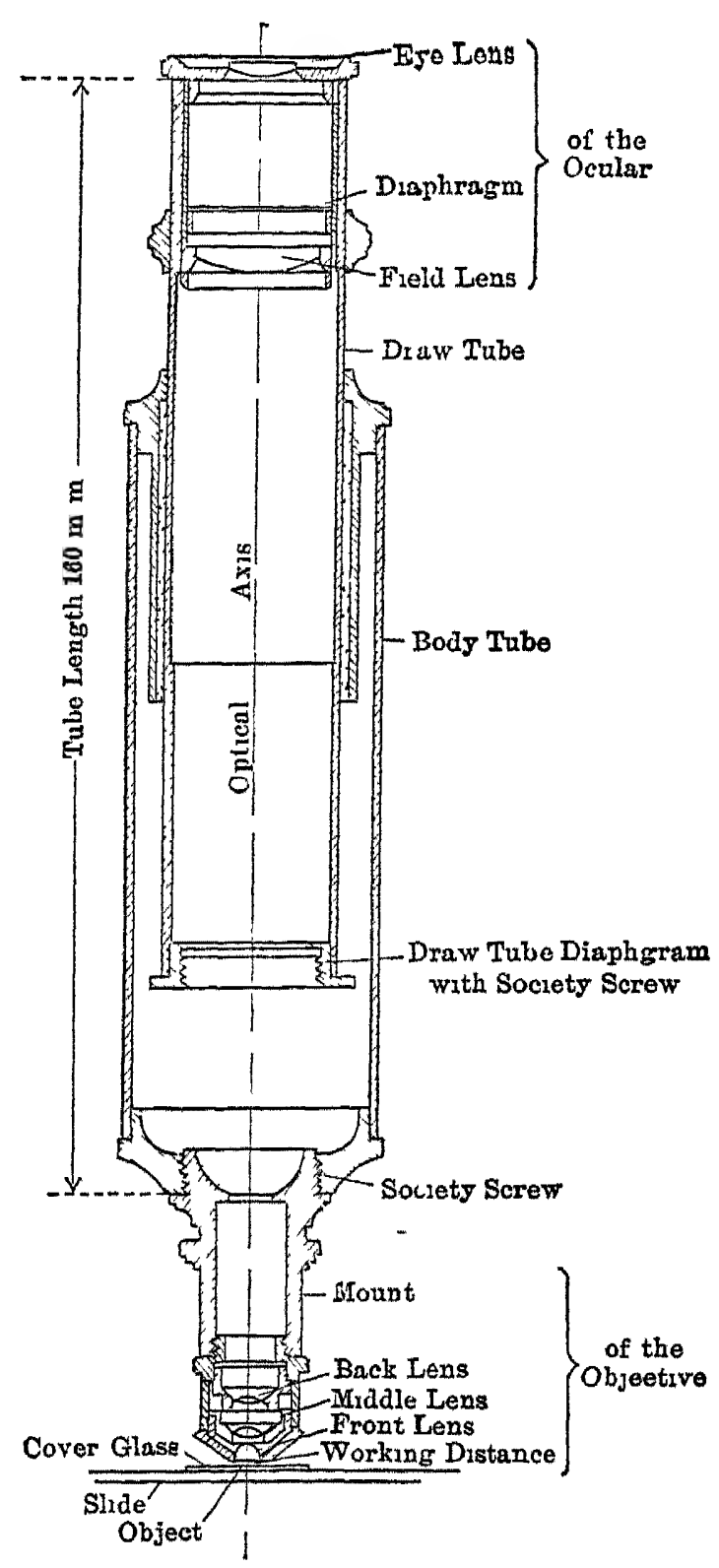

FIG. 24.-Showing Cross-section of a Microscope.

(Courtesy of the Spencer Lens Company) 
ble. The following pages have been prepared from a number of sourccs. Direct reference has been given in a few cases. Most of the data, however, have been taken with permission from microscope manuals which are furnished by the Bausch \& Lomb Optical and Spencer Lens Companies.

\section{DoN'Ts}

Don't allow dust and dirt to settle on the microscope.

Don't carry the microscope by the arm, unless the fine adjustment is protected.

Don't use alcohol on the microscope.

Don't expect toc great a range in the fine adjustment.

Don't take the fine adjustment apart.

Don't bring the objective into contact with the cover glass.

Don't fail to focus up before turning the nosepiece unless you know the objectives are parfocal.

Don't forget that high powers have short working distances.

Don't focus down with the eye at the eyepiece.

Don't fail to secure good even illumination.

Don't drop the objectives and oculars.

Don't try to take an objective apart.

Don't try to clean the lens with a dirty cloth.

Don't fall to clean oil from an immersion lens immediately after using.

Don't try to work with an immersion lens when there are air bubbles in the oil.

Don't use high powers when low ones will do.

Don't use higher oculars than necessary.

Don't expect a lens to work at its best unless used on a cover thickness, and with a tube length, for which it is corrected.

Don't shut one eye.

Don't get discouraged if desired results do not come immediately.

The instrument should always be kept in a case, either the one in which it was received from the factory or one which is specially constructed to hold a series of them in the laboratory. When carrying a microscope or removing it from its case, grasp it by the pillar and not by any other part.

Finger marks and stains should be carefully rubbed with soft cloth or lens paper. If this is not sufficient, a little ether, chloroform or xylol may be used. Alcohol should never be used since this will remove the lacquer. 
Stage. This descrves little attention. It may be cleaned as above described.

Inclination Joint. By means of this joint the body of the microscope may be inclined at an angle. Should the joint become so loose that the microscope will not remain at the desired angle, it may be tightened by tightening a nut.

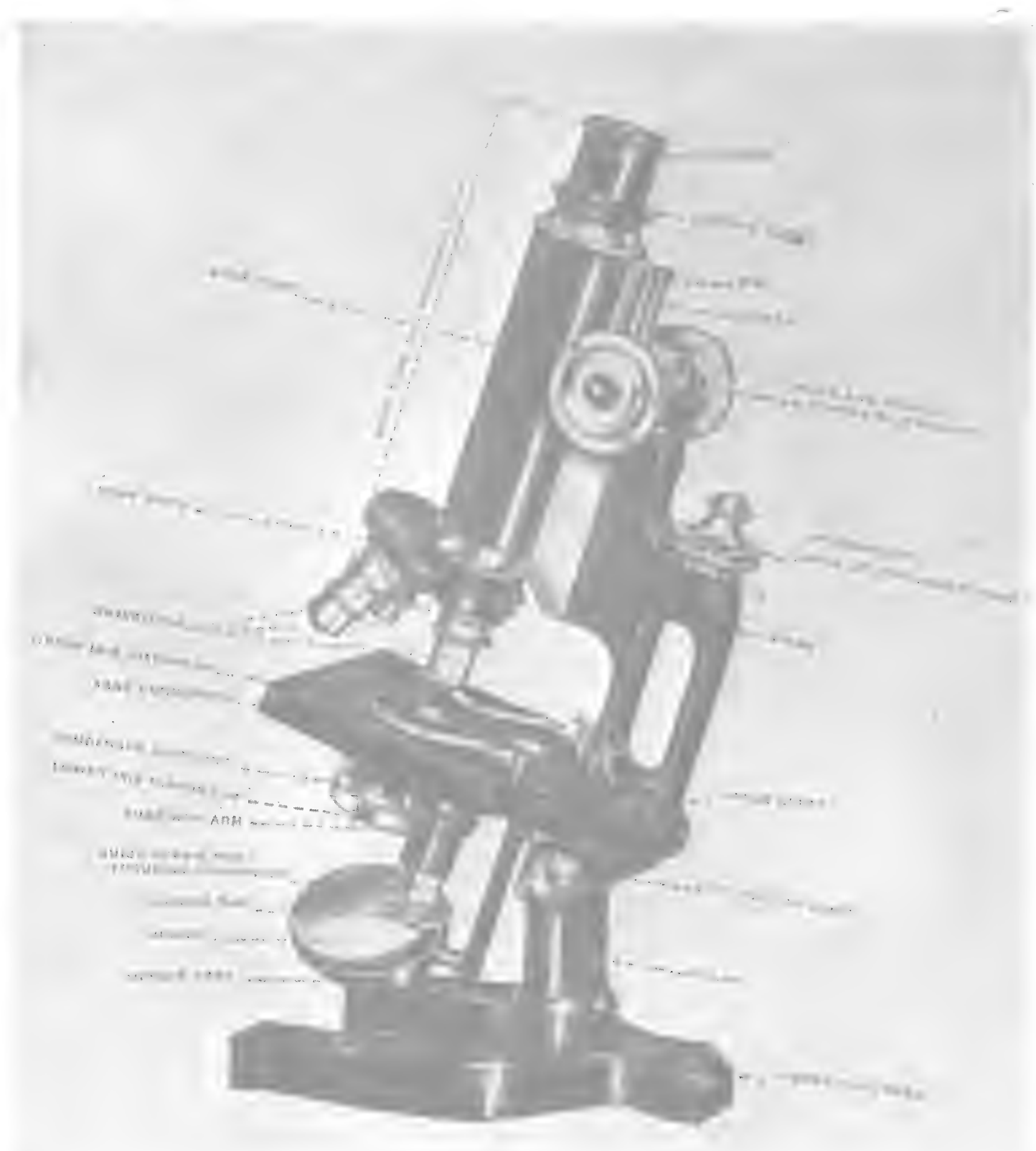

IIIG. 25.-Showing the Different Parts of a Microscope.

Course Adjustment. This is accomplished in one of two ways: The sliding tube and the rack and pinion. The former is found only on the cheaper microscopes, and is practically out of date. In it the body tube is made to slide inside of a sleeve which is fastened to the arm of the microscope. It is very hard to keep in order, because the exposed surfaces of the body tube and the inside of the sleeve 
become gummed and corroded from exposure to the atmosphere and reagents of the laboratory, and particularly from the perspiring or dirty hand of the operator in focusing. In this condition the tube works hard and unevenly, so that one is apt to force an objective into the slide. The tube and sleeve can generally be cleaned by rubbing with a coarse towel after moistening with xylol. Do not grease the tube or sleeve because it gums and corrodes the tube, and is very unsightly. Should the tube be too loose in the sleeve remove the tube and press in the top edge of the sleeve on either side of the one or more slots running down from the top edge.

All of the better microscopes are provided with the rack and pinion coarse adjustment. The bearings must necessarily fit very closely. Any foreign matter on them interferes seriously. Do not strain the teeth of the rack and pinion by forcing the bearings back and forth over one another when they are not clean. A little xylol or chloroform rubbed on the surfaces will clean them. Do not use emery in any form. When the bearings are perfectly clean, oil them slightly with a good acid-free lubricant (paraffin oil or watch oil). If the bearings become so loose that the tube will not stay in place tighten the little screws at the back of the pinion box. All makers have a provision here for taking up lost motion and wear. Do not fill the teeth of the rack with paper paraffin or any other foreign substance. If anything should accumulate in these teeth clean it out.

Fine Adjustment. The fine adjustment is necessarily of limited range and delicate in its mechanism. If, when looking into the eyepiece, no change of focus is noticed by turning the micrometer head or, if the micrometer head ceases to turn, the adjustment has reached its limit. Turn the micrometer back to bring the fine adjustment midway within its range. When the fine adjustment head stops do not force it. All of the better microscopes are made so that the head stops at both ends of the range and so that the micrometer threads cannot be removed from their bearings. Other microscopes do not have this safeguard, and great care should be excrcised not to remove the thread. If by chance it should be removed, exercise great care in replacing it to see that the threads are started properly so that they do not " run." Do not force them if they run at all hard. If they are started to " running" they must go to a machinist. On some microscopes these threads are "left-handed," which should be noted in trying to start the threads.

In some microscopes a little steel pin is fitted loosely into the hollow end of the micrometer thread. Be careful to see that this little pin 
is in its place before starting the micrometer thread. In some cases this pin even drops out of place before the micrometer thread is entirely out of its bearings and the defect is not noticed until the fine adjustment fails to respond. In such a case the top of the fine adjustment must be opened to secure the pin and put it in place. This ought to be done by the maker or experienced mechanic.

In some cases, especially in microscopes (Continental type) where the prism is used in the fine adjustment, the lubricant in the prism becomes gummed so that the adjustment fails to respond promptly and then jumps. On the best microscopes provision is now made against this. The bearings should be thoroughly cleaned and oiled with paraffin oil or watch oil. This ought to be done by the manufacturers, because the mechanism is so delicate that even though safely taken apart it would be put together and adjusted with great difficulty. All the modern microscopes have their fine adjustments so arranged that the fine adjustment ceases to work when the objective rests on the cover glass. This feature should be insisted upon

Draw Tube. This should work easily and smoothly and should be kept clean and dry. In sliding this draw tube, do so with a revolving: motion. The draw tube should always be drawn out to give the proper focal length for which the microscope was standardized. All microscopes give best results if used with cover glasses of definite thicknesses.

Substage. This should be kept dry and clean to prevent cutting and rusting of the delicate bearings. Should the leaves of the iris diaphragm become rusted, they may be cleaned with xylol and oiled. When they become bent, an expert is necessary.

Lenses. Absolute cleanliness is necessary with the lenses. Japanese lens paper of highest quality should be used for cleaning. It is best to use no other material on the lenses. Great care should be used in cleaning them since they are very easily marred and scratched.

Objectives. If these become soiled rub with lens paper using a little chloroform if necessary. Hard rubbing must be avoided. The immersion objective should always be cleaned by wiping with lens paper. If the oil is old and tends to be hard, xylol may have to be used.

All dry objectives are corrected for a definite thickness of the cover glass. (Bausch \& Lomb, $0.18 \mathrm{~mm}$.; Spencer Lens Co., $0.18 \mathrm{~mm}$. Z Zeiss, $15 \mathrm{~mm}$.; Leitz, $0.17 \mathrm{~mm}$.) The objectives are corrected to a certain tube length, which is $160 \mathrm{~mm}$. by most makers and $170 \mathrm{~mm}$. by Leitz. Such tube lengths should always be used for which the objectives have 
been standardized. Variations in the length of this draw tube should be made when cover glasses of a different thickness are used.

Numerical Aperture. N.A. $=n \sin u$.

$n=$ the lowest refractive index that appears between the object and the front lens of the objective;

$u=$ half the angular aperture.

The numerical aperture is important since it determines the resolving power definition and illumination. It is especially important with reference to the resolving power. The resolving power is directly proportional to the numerical aperture. The higher the numerical aperture the greater the resolving power and the finer the detail. Bausch has pointed out the significance of the N.A. (numerical aperture) as follows:

"If a very narrow central pencil is used for illumination, the finest detail that can be shown by a microscope, with high, enough magnification, is equal to $\frac{\lambda}{\text { N.A. }}$, where $\lambda$ is the wave length of the light used for illumination. The wider the pencil used for illumination, the greater the resolving power, until a maximum is reached, when the width of the pencil is sufficient to fill the whole aperture of the objective. In this case the resolving power is twice as great, the finest detail that the objective can show being now equal to 2 N.A. This same limit is reached when a narrow pencil of greatest possible obliquity is used. For example, the wave length of the brightest part of the spectrum may be assumed to equal $0.00053 \mathrm{~mm}$. Consequently an objective of N.A. equal to 1.00 will resolve two lines separated by a distance of $\frac{0.00053}{1.00}$ equal to 0.00053 with a narrow central illumination cone, and $\frac{0.00053}{2 \times 1.00}$ equal 0.000265 , with a cone filling the whole aperture, or with a narrow oblique cone.

"A $4 \mathrm{~mm}$. 0.85 N.A. objective will resolve lines separated by distance ranging between 0.00062 and 0.00031 , dependent upon the aperture employed. For a $4 \mathrm{~mm}$., 0.65 N.A. objective the limiting values are 0.00081 and 0.000405 .

"The N.A. can also be expressed by the equation.

$$
\text { N.A. }=\frac{d}{2 f}=\frac{\text { effective aperture of back lens }}{2 \times \text { equivalent focus }} \text {. }
$$

"Two objectives of the same equivalent focal length (E.F.) and the same N.A. should show the same illuminated area in the back lens, 
when viewed without an eyepiece and illuminated with the widest cone of light they can take in.

"The foregoing explanation shows the importance of the N.A. to the efficiency of an objective.

"It is also evident that an objective cannot show its full efficiency if it is not used with a condenser of a N.A. large enough to fill the back of the objective with light."

The depth of sharpness of an objective is in inverse ratio to the N.A. great penetration goes along with a low N.A. and low penetration but great resolving power with a high N.A.

Illuminating Power. The brilliancy of the objective increases with the square of the numerical aperture of the objective. An objective of 0.40 N.A. will give an image four times as brilliant as one of 0.20 N.A., provided the magnification is the same and the full cone of the illumination is used in both cases.

Magnifying Power. The magnifying power of an objective is in inverse ratio to its focal distance. An objective of $2 \mathrm{~mm}$. focal distance will give, with the same ocular, a magnification eight times greater than one of $16 \mathrm{~mm}$. focal distance. Numerical aperture and magnifying power are of little advantage if the definition is not good.

Chromatic Aberration. This is due to the fact that a ray of white light passing from one medium to another of different refractive index at any angle other than $90^{\circ}$ to the surface between them is refracted and dispersed into its component colors.

Spherical Aberration. This is due to the fact that a spherical surface cannot bring a beam of light which passes through its vertex to the same focus as that of a beam of light passing through any other zone.

Both aberrations are corrected by the use of different kinds of glass (crown and flint) combined as double and triple lenses in the objective. Neither can be corrected absolutely for all colors in an achromatic objective. Apochromatic objectives approach the ideally corrected objective almost to perfection.

An objective can be tested for chromatic correction by using a narrow cone of oblique light and a coarse grating. Abbe's test plate is best. Diatoms are good. No stained object should be used.

If the spherical correction is perfect (see next paragraph) and one side of a line passing through the center of the field shows a clear, narrow greenish yellow border, while the other side is fringed with a violet red (secondary colors) the objective is chromatically corrected. The colors shown in the higher power objectives are of a more primary 
character, i.c., nearer the yellow and blue. Apochromatic objectives show no color borders in this test.

The spherical correction of an objective is perfected for a certain thickness of cover glass and a certain tube length, and is influenced greatly by any variation in either. This is especially true with the high-power dry objectives. The homogeneous immersion objectives are not sensitive to the variation in the cover thickness, because the immersion oil between the cover glass and the lens is of the same refractive index as the glass. They must be used, however, with the proper tube length. In testing an objective for its spherical correction it is therefore very important to supply the proper thickness of cover and tube length. It is manifestly unfair to judge an objective on this point without complying with these conditions. The test for spherical correction can be made on the same object as used for the chromatic test. If the edges of the lines in the center of the field appear equally sharp and clear when illuminated by either a narrow central cone of light or a narrow oblique cone without having to change the fine adjustment the objective is spherically corrected. The color remnants mentioned above will be clear and transparent, while if the lens is poorly corrected spherically, these borders will appear muddy and turbid. Defects in spherical corrections can often be corrected by using cover glasses suitable to them, also by changing the tube length. The fact that the periphery of the field is not in focus at the same time as the center does not bespeak a lack of spherical correction, but a lack of flatness of field with which it is often confused.

Flatness of field depends not only upon the objective itself, but upon the ocular and the cone of light used, whereas the spherical aberration is inherent in the objective itself. No field is absolutely flat. It is a desirable quality in a lens, but spherical and chromatic corrections should never be sacrificed for it. Some lenses appear to be "flatter" than they really are, because their corrections are so poor that little contrast is noticed between objects in the center of the field and at the edge. Narrow cones of light give a flatter field than wide ones. Thin objects are more critical tests for flatness of field than thick ones.

Working distance is the free distance between the cover glass and the objective when the latter is focused. It decreases generally with increasing power and numerical aperture of the objective. Of two lenses with the same focal distance the one with the higher N.A. will have the shorter working distance. The working distance also depends on the mounting of the front lens. If the lens has a prominent mount- 
ing projecting beyond its surface the working distance is lessened thereby.

Oculars. These have a simpler structure than the objectives. They serve as collective lenses, making all of the light constituting the image from the objective enter the eye of the observer.

Illumination. Practically all microscopic studies are carried out by transmitted light, and some special attachment is necessary to direct this light along the optical axis of the microscope. For this purpose substage or Abbe condensers are used. This condenser allows the utilization of all light which enters the object space. Light is sent through the object at an angle great enough to fill the objective.

The condenser is constructed to bring the rays of light to a focus above the upper surface of the uppermost lens. When the concave
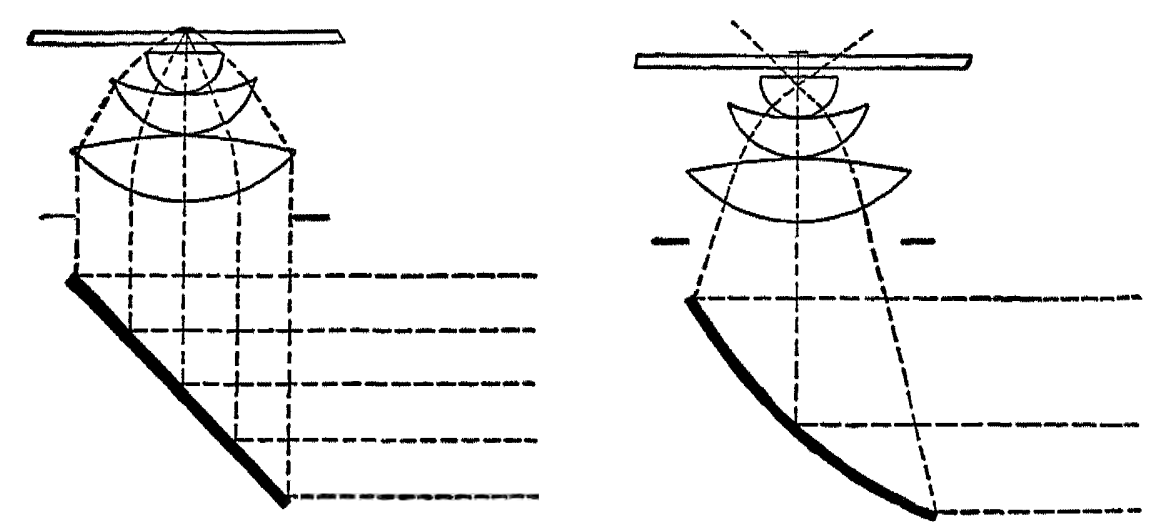

FIG. 26.-Showing Incidence of Light Rays in Condenser. (After Bausch.)

mirror is used the rays of light are brought to a focus within the condenser. The concave mirror acts as a lens and together with the converging effect of the lens in the condenser causes the light to converge more rapidly than that for which the microscope was standardized. Bausch has depicted this in Fig. 26.

Bausch makes the following statement with regard to the use of the condenser: "In the use of the condenser with oil immersion objectives the custom prevails of using the condenser dry. It is well to point out, however, that both the condenser and the objective lose in their efficiency when the former is used dry, and for critical work the condenser should be in immersion contact with the slide.

To make immersion contact between condenser and slide place a drop of oil on the top of condenser, drop the slide upon the stage, first turning the slips to one side.

With immersion objectives the proper focusing of the condenser becomes a matter of nice distinction to obtain best results and can 
only be reliably accomplished by considerable practice and experience. To obtain best position:

Use a $16-\mathrm{mm} .\left(\frac{2}{3} \mathrm{in}\right.$.) objective; focus upon the object; adjust condenser until image of window-sash or flame is in the same plane with object.

Practically all substages are provided with means for focusing the condenser: The rays of light should be focused sharply on the object. A little experience will indicate when the proper aperture is

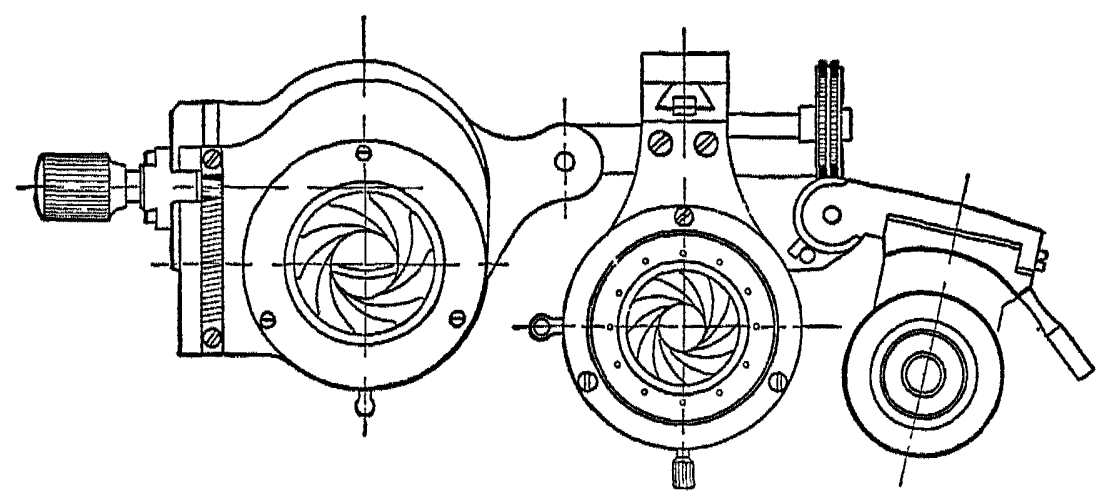

FIG. 27. -Showing Action of Different Parts of the Condenser. (After Bausch.)

being used. The iris-diaphragm when used with dry lenses should not be more than half open. With the oil immersion objective, however, the full aperture should be used.

\section{Final Hints}

"Sometimes the worker may have faithfully carried out all the directions heretofore given and been assured that his lenses possess the above-named qualities as they ought, yet be unable to obtain the desired results. He may be working with a water mount and dry objective become 'immersed' in some water which has worked to the top of the cover glass. His objective may be dirty from a previous 'immersion' or it may have some other dirt upon the front lens. The field may be covered with specks which revolve when the ocular is turned. The field may be dim or hazy, due to dirt on the back of the objective or a film on the inner surfaces of the lenses of the ocular, or because of moisture settling on the lenses because they have just been brought from a cold into a warm room. He may see great streaks on his field, which are due to his own eyelashes, or he may see small slowly moving bodies floating across the field. With the exception of this last, the ailment has only to be mentioned to suggest the remedy. The muscæ volintantes, as these last-named bodies are called, are little specks of 
shreds in the vitreous humor of the eye which cannot be removed, but which can easily be disregarded.

"In water mounts and fresh balsam mounts one is apt to find air bubbles. To be sure that the object is an air bubble, focus up with central light. The bright spot in the center will become clearer while the edge will become darker. With oblique light the bright spot will be thrown to one side. In studying water, blood or any fluid, always cover the drop with a cover glass. The objectives are corrected for rays passing through media with parallel surfaces. If such a mount is not kept horizontal, currents will be set up, due to gravitation, and they will be seen with a magnified velocity seemingly running up hill.

"The fact that the microscope reverses every movement and magnifies it may be mentioned again.

"Beside any movement due to currents there is sometimes a peculiar indefinite to and fro movement of particles from one position to another. This is called Brownian movement.

"In studying sections a true idea of the structure of the tissue can only be obtained by moving the slide about to bring different parts into the optical axis and by focusing with the fine adjustment to bring different levels, or optical planes, successively into view Where serial sections are used each section must be studied in relation to its neighbors.

TABLE I

MAGNIFICATION TABLE

Batsch \& Lomb Optical Co., Rochester, N. Y.

Tube Length $160 \mathrm{~mm}$.

Image Distance $250 \mathrm{~mm}$.

\begin{tabular}{|c|c|c|c|c|c|c|c|}
\hline \multirow{2}{*}{$\begin{array}{l}\text { Objec- } \\
\text { t1ves, } \\
\text { mm }\end{array}$} & \multicolumn{5}{|c|}{ Exepinces. } & \multirow{2}{*}{$\begin{array}{l}\text { Objec- } \\
\text { tives, } \\
\text { Inch }\end{array}$} & \multirow{2}{*}{$\begin{array}{c}\text { Working } \\
\text { Distance } \\
\text { mm. }\end{array}$} \\
\hline & $5 x$ & $64 x$ & $75 x$ & $10 x$ & $12.5 x$ & & \\
\hline 480 & 10 & 13 & 15 & 20 & 25 & 2 & 53 \\
\hline 32.0 & 20 & 26 & 30 & 40 & 50 & $1 \frac{1}{3}$ & 38 \\
\hline 160 & 50 & 64 & 75 & 100 & 125 & $\frac{2}{3}$ & 7.0 \\
\hline 80 & 100 & 130 & 150 & 200 & 260 & $\frac{1}{3}$ & 1.6 \\
\hline 40 & 215 & 275 & 320 & 430 & 560 & $\frac{1}{6}$ & 0.6 \\
\hline $40_{L}$ & 215 & 275 & 320 & 430 & 560 & $\frac{1}{6}$ & 0.3 \\
\hline $30_{S}$ & 285 & 365 & 420 & 570 & 740 & $\frac{1}{8}$ & 0.2 \\
\hline 190 & 475 & 610 & 720 & 950 & 1260 & $\frac{1}{12}$ & 0.15 \\
\hline
\end{tabular}


"Sometimes sections which are freshly mounted in balsam appear cloudy and indistinct. This is because of failure to thoroughly dehydrate the specimen before putting it into the balsam. But this brings us into the realm of laboratory technique, which is beyond the scope of this volume."

TABLE II

SPENCER LENS COMPANY MAGNIFICATION TABLE

Tube Length, $160 \mathrm{~mm}$.

Image Distance, $250 \mathrm{~mm}$.

\begin{tabular}{|c|c|c|c|c|c|c|c|c|c|c|}
\hline \multirow{2}{*}{$\begin{array}{l}\text { Objec- } \\
\text { tives, } \\
\text { mm }\end{array}$} & \multirow{2}{*}{$\begin{array}{c}\text { Inutial } \\
\text { Magnifi- } \\
\text { cation }\end{array}$} & \multicolumn{8}{|c|}{ Oculars } & \multirow{2}{*}{$\begin{array}{l}\text { Objec- } \\
\text { tives, } \\
\text { mm }\end{array}$} \\
\hline & & $4 x$ & $5 x$ & $6 x$ & $8 x$ & $10 x$ & $12:$ & $15 r$ & $20 x$ & \\
\hline 48 & 22 & 8 & 11 & 13 & 18 & 22 & 27 & 33 & 44 & 48 \\
\hline 40 & 28 & 11 & 14 & 17 & 22 & 28 & 33 & 42 & 56 & 40 \\
\hline 32 & 4 & 16 & 20 & 24 & 32 & 40 & $4 \delta$ & 60 & 80 & 32 \\
\hline $30-22$ & $2-45$ & $4-9$ & $5-12$ & $8-19$ & $10-24$ & $15-35$ & $18-43$ & $20-48$ & $30-70$ & $30-22$ \\
\hline 254 & 6 & 24 & 30 & 36 & 48 & 60 & 72 & 90 & 120 & 254 \\
\hline 16 & 10 & 40 & 50 & 60 & 80 & 100 & 120 & 150 & 200 & 16 \\
\hline 12 & 15 & 60 & 75 & 90 & 120 & 150 & 180 & 225 & 300 & 12 \\
\hline 8 & 20 & 80 & 100 & 120 & 160 & 200 & 240 & 300 & 400 & 8 \\
\hline 5 & 36 & 144 & 180 & 216 & 288 & 360 & 432 & 540 & 720 & 5 \\
\hline 4 & 44 & 176 & 220 & 264 & 352 & 440 & 528 & 660 & 880 & 4 \\
\hline 3 & 60 & 240 & 300 & 360 & 480 & 600 & 720 & 900 & 1200 & 3 \\
\hline 18 & 95 & 380 & 475 & 570 & 760 & 950 & 1140 & 1425 & 1900 & 18 \\
\hline 1.5 & 109 & 436 & 545 & 654 & 872 & 1090 & 1308 & 1635 & 2180 & 15 \\
\hline
\end{tabular}

When using the camera lucida with the paper lying on the table add 60 per cent to the above magnifications.

\section{MiCROMETRY}

This has to do with the measurement of objects under the microscope. While there are several ways of doing this the micrometer eyepiece has the widest application. More accurate results are obtained because the real image is measured. Since this image has been magnified by the objective, the " micrometer eyepiece" must be standardized for each object.

The Spencer Micrometer Eyepiece No. 425 is described as follows:

"This micrometer eyepiece represents the highest type of precision in construction and guarantees the greatest possible accuracy of measurement. Instead of the usual cross hairs, a finely ruled glass scale is used in its construction, each interval in the scale being exactly 
equivalent to one revolution of the screw which moves it. This system has important advantages, especially in the measurements of large objects. Unlike the filar micrometer, it does not require the moving of the index over the entire length of the object, as a fraction of one rotation of the screw is all that is necessary.

"The scale is $5 \mathrm{~mm}$. long and is divided into twenty spaces; each millimeter is marked by a line of double length. As the screw is made with a pitch of $\frac{1}{4} \mathrm{~mm}$., one revolution of the screw moves the scale $\frac{1}{4} \mathrm{~mm}$., or one of the intervals.

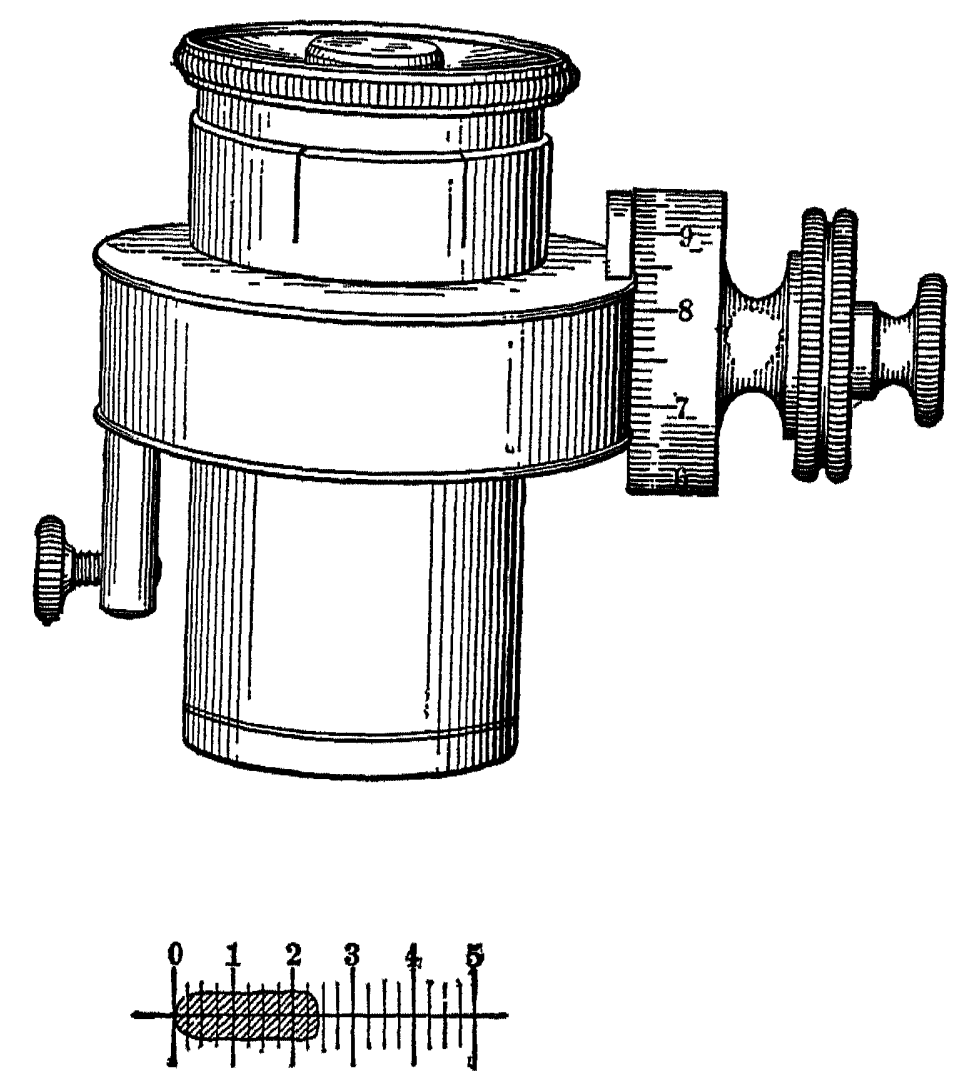

Frg. 28. - Spencer Lens Company's Micrometer Eyepiece.

"Upon the axis of the screw is placed an adjustable drum which may be set to any desired position. This is graduated with one hundred divisions, for reading of which an index pointer is fastened on top of the micrometer case.

"In measuring the length of an object, the scale is moved until one of the millimeter lines coincides with the margin of the object under examination; as for example, line No. 0 in the sketch, then, holding the milled head of the screw fast, the drum is turned until the index stands at zero. Now turning the screw until the line which the other edge of the drum added to the number of full $\frac{1}{2} \mathrm{~mm}$. divisions covered (nine in the sketch) gives the apparent length of the object -its real length depending on magnification used. 
"With an ordinary filar micrometer it would have been necessary to move the cross hairs over the whole length of the object, which in the above instance would have required nine full revolutions besides the fractional turning of the drum, while with our screw micrometer it was only necessary to move the scale over a part of one space.

"For measurements of less accuracy, this micrometer can also be used without turning the screw, simply using the subdivisions of the scale as in the ordinary micrometer."

Zeiss Micrometer Ocular. This consists of a Ramsden ocular of about $20 \mathrm{~mm}$. focal length. The glass plate, with a crossed as well
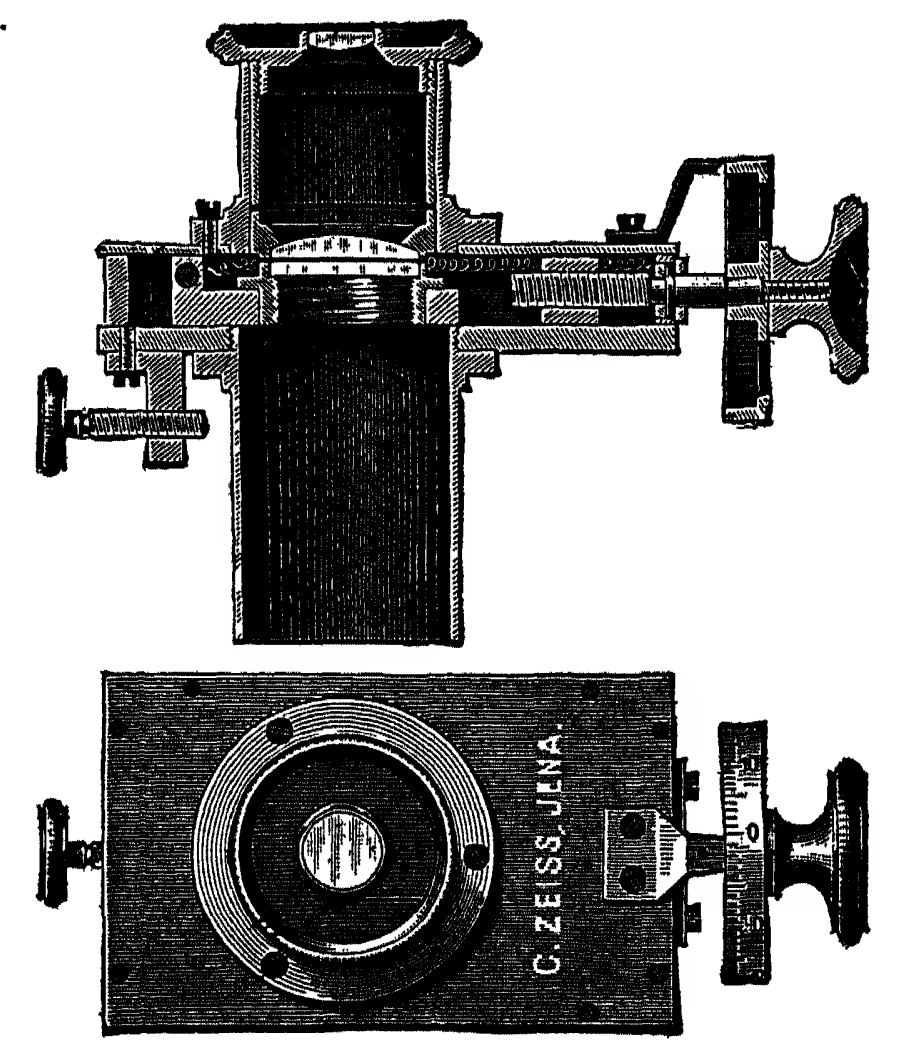

Frg. 29-Zeiss Micrometer Ocular.

as a double line, is moved over the field by turning the micrometer screw. Each unit on the revolving drum corresponds to a movement of $0.01 \mathrm{~mm}$. of the point marked. The absolute values of the units on the drum must be determined from a stage micrometer.

Calibration of Micrometer Eyepiece. For this pull the draw tube to the proper length and focus the lines of an object micrometer sharply. This may be done with an ordinary ocular. Replace this with the micrometer eyepiece, and focus again so that the lines on both micrometers are sharp.

The object micrometer is made from a cover glass bearing a scale which is divided into a certain number of parts. These data are usually 
given on one end of the object micrometer. By focusing the object micrometer with a definite combmation of an objective and micrometer eyepiece at a definitely fixed tube length and comparing the two micrometer scales, it is easy to find the actual length in the plane of the object which corresponds to one scale division of the eyepiece micrometer.

\section{BIBLIOGRAPHY}

BAUSCH, E. Use and Care of the Microscope. Bausch \& Lomb Optical Co., Rochester, N. Y.

BeAtTie, J. M. 1916. Simple and Inexpensive Methods for Fermentation Tests and for Obtaining Culture of Anaerobes. Brit. Med. Jour., 1916-I, 756-7.

Breed, R. S. and Dotterer, W. D. 1916. The Number of Colonies Allowable on Satisfactory Agar Plates. Jour. Bact., 1, 321-31.

Browne, W. W. 1913. A Comparative Study of the Smith Fermentation Tube and the Inverted Vial in the Determination of Sugar Fermentation. Am. Jour. Pub. Health., 3, 701-4.

Browne, W. W. 1917. The Fallacy of Reading Accurately Gas Percentages in the Fermentation of Lactose Peptone Blle and Lactose Broth. Amer. Jour. Pub. Health, 7, 663; Jour. Bact., 2 (1917), 249-267.

BuchNer, H. 1888. Eine neue Methode zur Kultur anaeröber Mikroorganismen. Cent. Bakt., 4, 149-151.

Carpenter, W. B. 1901. The Microscope and Its Revelations. 8th Edit., by W. H. Dallinger.

Durham. 1898-1. A New Method for Demonstrating the Production of Gas by Bacteria. Brit. Med. Jour., 1387.

Graves, K. D. 1917. An Improved Modification of the Durham Fermentation Tube. Jour. Amer. Med. Assn., 69, 2102.

Hall, I. C. 1914. An Improved Durham Fermentation Tube. Amer. Jour. Public Health, 4, 1173-1178.

HiLl, H. W. 1904. Porous Tops for Fetri Dishes. Jour. Med. Res., 18, 93.

Hill, H. W. 1908. The Mathematics of the Bacterial Count. Amer. Jour. Pub. Hyg., 18, 300-310.

Jones, H. M. 1916. A Method of Anaerobic Plating Permitting Observation of Growth. Jour. Bact., 1, 339-341.

Kuster. 1913. Ein einfacher Apparat zur anaeroben Züchtung und eine Verrichtung zur einwandfreien. Entnahme von Untersuchungsmaterial aus der Tiefe von Korperhohlen. Cént. f. Bakt. Ref., 57, 269-271.

LEe, R. E. and Fegeur, W. H. An Experimental Study of the Relative Efficiencies of Certain Fermentation Tubes. Am. Jour. Pub. Health, 4 (1914), 999-1005.

Lentz, O. 1910. Ein neues Verfahren fur die Anaeroben. Cent. f. Bakt. abt., I, 58, 358-365. 
McLeod, J. E. 1913. A Method for Plate Culture of Anaerobic Bacteria. Jour. Path. and Bact., 4, 454-57.

Novy, F. G. 1893. Die Kultur anaerober Bakterien. Cent. Bakt., 14, $581-600$.

Nowak. 1908. Le Bacille de Bang et sa Biologie. Am. Inst. Past., 22, 541.

Smillie, W. G. 1917. New Anaerobic Methods. Jour. Exper. Med., 26, $59-66$.

Sмiтн, T. 1890. Das Gährungskolbchen in der Bakteriologie. Cent. f. Bakt., 7, 50, 2-506.

Spencer Lens Company. How to Use and Care for the Microscope. Buffalo, N. Y.

Spitta, E. J. 1909. Microscopy. 2d Edit.

Stewart, A. H. 1906. A New Colony Counter and Dissecting Microscope. Jour. Med. Res., 14, 423.

Torrex, J. C. 1917. New Differential Plating Methods for B. bifidus (Lissier) and B. acidophilus (Moro). Jour. Bact., 2 (1917), 435-439.

Wrighr. 1900. A Simple Method for Cultivating Anaerobic Bacteria. Jour. Boston Soc. Med. Sci., 5, 114.

Zinsser, H. 1906. A Simple Method for the Plating of Anaerobic Organisms. Jour. Exper. Med., 8 (1906) 542. 


\section{CHAPTER II}

\section{MEDIA AND THEIR PREPARATION}

BACTERIA are such minute organisms that their environment exerts great influence on their development. In the laboratory where it is desired to get as accurate information with regard to their life histories as possible, every attempt should be made to create an environment which simulates the natural one for the variety under examination. The bacteriologist calls this a medium. The characteristics of a good medium are: 1. It must approach the isotonic solution for the bacterial cells. 2. Must contain proper kinds and amounts of the essential food substances such as: (a) Water concentration. (b) Nitrogen requirement, organic and inorganic. (c) Inorganic salts. 3. Should be used at optimum temperature for organism under study.

All media should be made up according to standard methods. In America the Standard Methods for the Examination of Water and Sewage by a Committee of the American Public Health Association are taken as the fundamental basis upon which most official media are made. These may not be the ideal procedures but doubtless embody most of the essential points according to our present information. By following these standard methods the analyst is more certain of securing results which may be compared with those obtained in other laboratories, than if he adopted his own formulæ. Some objections have been raised with regard to parts of these standard methods, but constructive criticism is necessary if they are to be brought to a correct stage.

\section{Constituents of Media}

While there are many different substances which may be used for cultering bacteria, a certain few yield the best results and are therefore used at the present time in most of our common media. These substances furnish the optimum conditions for the average bacterium. For these bacteria, certain pathogenic forms for instance, which do not grow on the ordinary media, special media must be pre- 
pared which embody the essential food substance. Some of the pathogenic bacteria grow better if a little blood serum or hemoglobin is added to our common media. The following substances are in common use by bacteriologists for preparing media.

Peptone. This is added to common culture media to satisfy the organic nitrogen requirement of the bacteria. The peptones which are used in media are mixtures of the various cleavage products of the protein molecule. Important among these are the amino acids, which are precursors for many of the characteristic products of bacterial activity. These must be hydrolyzed from the protein molecule.

Many analyses of peptones are available in the literature, but as Banzhaf and Hirshleifer (1914) have pointed out, they may mean very little because in many cases the methods of analysis are not known. Generally, peptones consist of salts, among which are the phosphates, amino acids and proteoses.

Witte's peptone has formerly been specified for all bacteriological media. Berry (1914-1915) and Conn (1915) have found that equally good results are secured with the domestic peptones as with Witte's. Berry secured her data by comparing colonies of B. typhi, B. coli and streptococcus on agar containing 2 per cent of the different peptones. Conn compared two samples of American-made peptone to Witte's when he studied the methods of milk analysis in New York City. The three peptones showed such slight difference in average that Conn regarded one as good as the other.

Meat Extract. This is an important constituent of laboratory media. For bacteriological purposes it is usually secured in jars. Meat infusion formerly was used in place of meat extract.

This is made by soaking 500 gms. of lean chopped beef in 500 c.c. of distilled water for twenty-four hours. By keeping this in the refrigerator all bacterial changes are inhibited. At the end of this time it is filtered through cheese cloth or flannel and brought back to the original volume by adding distilled water. This meat infusion is used as the base to which the other constituents are added. Its chemical composition is not far different from that of the commercial meat extract. Gage and Adams (1904), however, have shown that the chemical composition is variable. The reaction and the organic solids are variable. This variation is probably due to the different grades of meat which are used. High amounts of fat and connective tissue will tend to give a product weaker in strength. The kind of beef might also explain the variation in chemical content. The variation is illustrated by the following data which are taken from Bigelow and Cook (1908). 
TABLE III

COMPOSITION OF MEAT JUICES PREPARED IN THE LABORATORY

(After Bigclow and Cook, 1908)

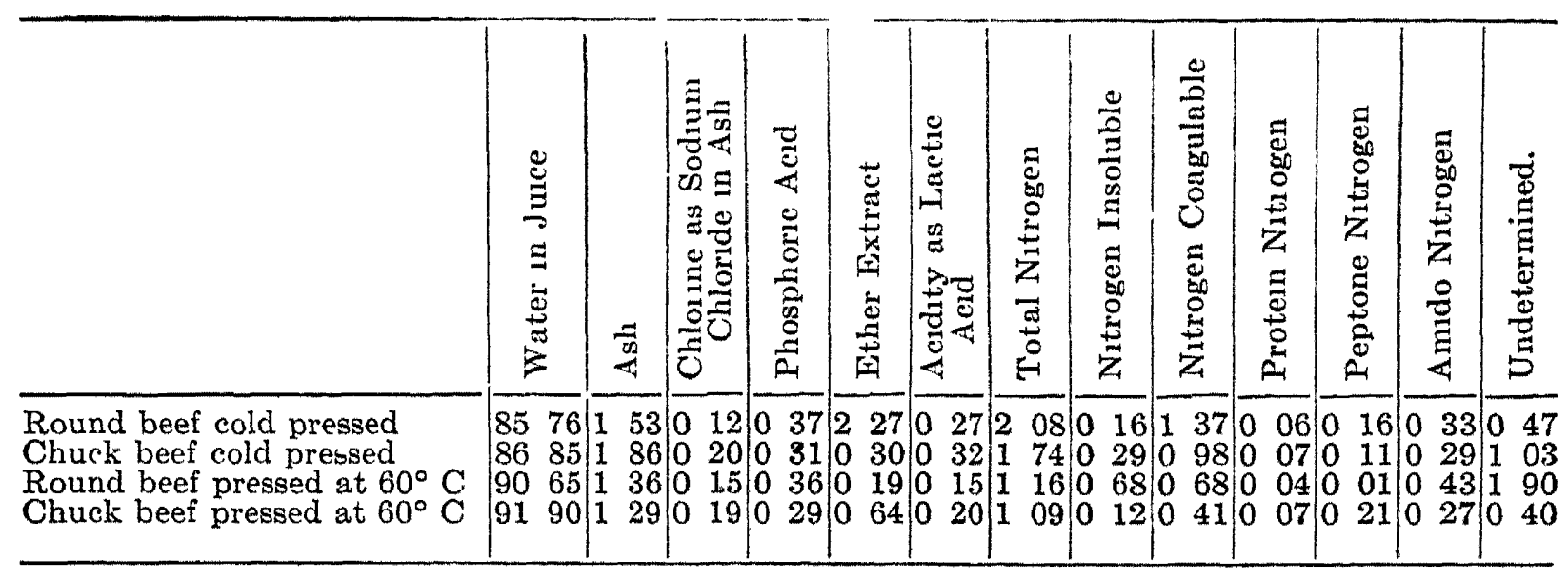

Many different grades of commercial meat extracts are on the market. Bacteriologists have seen fit to specify Liebig's as the "standard" for all bacteriological media. Bigelow and Cook (1908) describe its manufacture as follows:

A first grade extract is prepared from beef alone and is usually sold in jars. An extract of the trimmed bones to which considerable meat adheres is also made. The trimmings include odds and ends of meats, muscles, tissue, bone, etc., and the product is a second-grade article. In preparing this extract, the bones are heated, not boiled, for thirty minutes to forty minutes and the liquor evaporated to the consistency of extract. The extract prepared from cornedbeef liquor constitutes another second-grade product. This extract has a high content of nitrates and sodium chloride. In addition there is an extract prepared from pork and other meats sold under the general term of meat extract. Mixtures of various meat and bone extracts are often made. A fluid meat extract is usually a 50 per cent solution of solid meat extract.

Horse meat is now used to some extent in the preparation of meat extracts. It has the special advantage of possessing less muscle sugar.

The following analysis of Liebig's meat extract is given by Bigelow and Cook (1908).

Moisture.................... 21.14

Mineral constituents:

Total ash . . ............... 21.03

Chlorine as sodium chloride.......... 3.11

Total phosphoric acid.............. 2.40

Organic phosphoric acid.............. 061

Inorganic phosphoric acid............. 1.79 
Acidity:

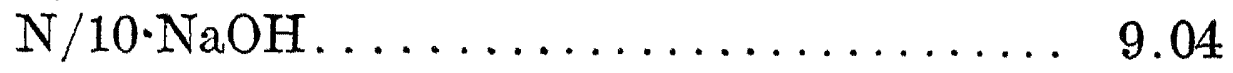

As lactic acid................... 8.13

Nitrogen as:

Total. .......................... 9.07

Insoluble and coagulable............... 0.19

Proteoses........................ 2.01

Peptones...................... 2.68

Total meat bases. . . . . . . . . . . . . . 3.82

Creatin and creatinin................. 1.14

Xanthin bases..................... 0.03

Meat bases other than ............... 2.65

Ammonia . . . . . . . . . . . . . . . . . 0.37

Ether extract..................... 0.94

Undetermined . . . . . . . . . . . . . . . . 5.89

Net weight..................... 57.80

TABLE IV

PARTITION OF THE NITROGEN OF MEAT EXTRACTS

(Cook, 1908)

\begin{tabular}{|c|c|c|c|c|c|c|c|c|c|}
\hline \multirow{3}{*}{$\begin{array}{c}\text { Varicty } \\
\text { and } \\
\text { Sample } \\
\text { Num- } \\
\text { ber. }\end{array}$} & \multirow{3}{*}{$\begin{array}{c}\text { Total } \\
\text { Nitrogen } \\
\text { Per } \\
\text { Cent. }\end{array}$} & \multicolumn{8}{|c|}{ Percentage of Total Nitrogen. } \\
\hline & & \multirow[b]{2}{*}{$\begin{array}{l}\text { Ammonia } \\
\text { Nitrogen. }\end{array}$} & \multirow{2}{*}{$\begin{array}{c}\text { Total } \\
\text { Creati- } \\
\text { nine } \\
\text { Nitrogen. }\end{array}$} & \multirow[b]{2}{*}{$\begin{array}{c}\text { Purine } \\
\text { Nitrogen. }\end{array}$} & \multirow{2}{*}{$\begin{array}{l}\text { Nitrogen } \\
\text { in Phos- } \\
\text { photung- } \\
\text { stic Acid } \\
\text { Filtrate. }\end{array}$} & \multirow{2}{*}{$\begin{array}{c}\text { Nitrogen } \\
\text { in Acid } \\
\text { Alcohol } \\
\text { Filtrate. }\end{array}$} & \multirow{2}{*}{$\begin{array}{c}\text { Nitrogen } \\
\text { in Tannin } \\
\text { Salt } \\
\text { Filtrate. }\end{array}$} & \multicolumn{2}{|c|}{ Amino Nitrogen. } \\
\hline & & & & & & & & $\begin{array}{l}\text { Formol } \\
\text { Method. }\end{array}$ & $\begin{array}{c}\text { Van } \\
\text { Slyke } \\
\text { Method. }\end{array}$ \\
\hline 1 & 9.56 & 2.62 & 22.49 & 3.35 & 7.64 & 78.04 & 54.91 & 10.94 & 18.50 \\
\hline 2 & 9.65 & 2.49 & 22.59 & 3.52 & 6.84 & 78.45 & 55.85 & 10.94 & 18.23 \\
\hline 3 & 7.68 & 1.56 & 32.42 & 2.86 & 71.35 & 100.00 & 73.04 & 10.63 & 15.63 \\
\hline 4 & 9.65 & $\ldots \ldots$ & 29.01 & 4.92 & 49.74 & 76.73 & 59.37 & $10.27^{*}$ & 15.54 \\
\hline 5 & 7.49 & $\cdots$ & 27.50 & 0.13 & $\ldots$. & 89.19 & 64.21 & 9.53 & 17.89 \\
\hline
\end{tabular}

* No correction for ammonia; no determination.

Gelatin. This was first used by Koch as a solid medium. Since that time it has become firmly established in the bacteriological laboratory. It has the advantage over some other substances used in solid media of allowing a larger number of colonies but the great disadvantage that it is a bacterial food and may be liquefied. This often causes a loss of plates.

Gelatin is made from collagen, a constituent of white fibrous tissue. Emmett and Gies (1907) explain the formation of gelatin from collagen 
as being a rearrangement of the molecule. The composition of gelatin is indicated in Table V, which is taken from Mathews' Physiological Chemistry.

TABLE $V$

SHOWING THE AMINO ACID CONTENT OF GELATIN

\begin{tabular}{|c|c|c|c|}
\hline Glycocoll. & 16.50 & Serine. & 0.40 \\
\hline Alanine & 0.80 & Cystine.. & 0.00 \\
\hline Valine & 1.00 & Tyrosine. & 0.00 \\
\hline Leucine. . . & 2.10 & Arginine. . & 7.62 \\
\hline Pro & 5.20 & Hist & 0.40 \\
\hline alanine & 0.40 & Lysine. & 2.75 \\
\hline Asps & 0.56 & Ammon & \\
\hline Glutamic acid. & 1.88 & Tryptophane. & 0.00 \\
\hline
\end{tabular}

The manufacture of gelatin has been described by Thiele (1914). $\mathrm{He}$ states that all varieties, whether used for bacteriological, photographical or food purposes are made in about the same way.

Agar. This according to Whittaker (1911) is derived from many plants, but principally from the genus "Gelidium" which grows on rocks along the coast of Japan. It is carefully cleaned by beating and washing. Finally it is dissolved and filtered to be late ${ }_{1}$ dried in different shapes. Whittaker reports the composition of agar as shown in Table VI. Fellers (1916) found a remarkable uniformity upon analysis of sixteen agars from very different sources.

TABLE VI

CHEMICAL COMPOSITION OF AGAR-AGAR

(After Whittaker, 1911)

\begin{tabular}{|c|c|c|c|c|c|c|}
\hline Form. & Quality. & Moisture. & $\begin{array}{l}\text { Crude } \\
\text { Fiber. }\end{array}$ & $\begin{array}{c}\text { Crude } \\
\text { Protein } \\
N \times 625 .\end{array}$ & $\begin{array}{c}\text { Carbo- } \\
\text { hydrate. }\end{array}$ & Ash. \\
\hline Slender. & No. 1 & 13.10 & 0.47 & 2.21 & 80.34 & 3.88 \\
\hline Best. & $\ldots \ldots$ & 14.39 & 0.35 & 1.49 & 80.17 & 3.60 \\
\hline Powdered......... & $\ldots \ldots$ & 11.39 & 2.62 & 3.58 & 74.91 & 7.50 \\
\hline 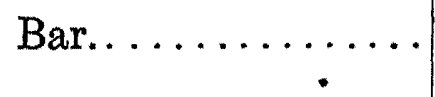 & $\ldots \ldots$ & 15.13 & 0.72 & 3.17 & 77.11 & 3.88 \\
\hline
\end{tabular}

Chemically agar is a carbohydrate, a hexosan of the cellulose group of the polysaccharides. It is not used by bacteria. Very few instances are on record where agar was decomposed by a bacterial enzyme (see Smith's Bacteria in Relation to Plant Diseases, 1905, p. 32).

Reaction of Culture Media. Bacteria require an alkalin or neutral medium. This is an important factor in the growth of all microorgan- 
isms. The reaction should be determined with phenolphthalein since this has becn adopted as the standard indicator. Five cubic centimeters of the media should be titrated and brought to the desired reaction means of standard $\mathrm{NaOH}$ or $\mathrm{HCl}$.

Recent work, however, indicates that this method does not yield satisfactory results. Clark and Lubs (1917) have pointed out that the hydrogen ion concentration must be determined more accurately either by means of the hydrogen electrode or special indicators. The former technique is too long and complicated to be applied to the preparation of ordinary media and it is probable that indicators will have to be used. These are mentioned in the appendix under indicators.

\section{Bouillon Media}

Nutrient Broth. This medium is used as a base to which the other constituents of media may be added. The following procedure for its preparation is recommended by the Laboratory Committee of the American Public Health Association in Standard Methods for the Examination of Water and Sewage, 1917, p. 95.

1. Add 3 gms. of meat extract and 5 gms. of peptone to a liter of distilled water.

2. Heat slowly on the steam bath to at least $65^{\circ} \mathrm{C}$.

3. Make up lost weight, titrate, and if the reaction is not already between +0.5 and +1 adjust to +1 .

4. Cool to $25^{\circ} \mathrm{C}$. and filter * through paper until clear.

5. Distribute in test tubes about 10 c.c. in each tube.

6. Sterilize in the autoclave at $15 \mathrm{lbs}$. pressure $\left(120^{\circ} \mathrm{C}\right.$.) for fifteen minutes after the pressure reaches $15 \mathrm{lbs}$.

Carbohydrate Broths. These are made by adding to plain broth, as prepared above, 1 per cent of the sugar desired just before sterilization. No attempts are necessary to remove muscle sugar since the constituents of broth are free from fermentable sugar. The reaction of carbohydrate broth may be neutral to phenolphthalein or adjusted to neutrality according to Standard Methods, 1917. Mudge (1917) has shown that during sterilization maltose and lactose are broken down and that sucrose and raffinose are not. The Arnold steam sterilizer was found to cause more hydrolysis than the autoclave.

* The Sharples laboratory centrifuge is in use at the University of Illinois for the clearing of solid media. It gives satisfactory results and obviates the use of cotton and egg albumin. It is considered a valuable addition to the laboratory equipment. 
Hasseltine (1917) has confirmed these results and has emphasized the importance of this question in the differentiation of members of the $B$. coti group. The work of these investigators emphasizes the necessity of using some other method then heating for sterilizing media containing disaccharides and trisaccharides.

Trypsin Broth (Gordon et al). Take some fresh bullock's heart free from fat and vessels, mince the meat very finely and weigh. To each $\frac{1}{2}$ kilo add 1 liter of water and make faintly alkaline to litmus with 20 per cent $\mathrm{KOH}$ solution. Heat this slowly to $75^{\circ}$ to $80^{\circ} \mathrm{C}$. for five minutes. Cool to $37^{\circ} \mathrm{C}$., and add 1 per cent of liquor trypsine comp., and keep it at $37^{\circ} \mathrm{C}$. for $2 \frac{1}{2}$ to 3 hours. When trypsinizing is finished, test for peptone with copper sulphate and $\mathrm{KOH}$, then render slightly acid with glacial acetic acid, and bring slowly to the boil for one-fourth hour. Leave over night in a cool place, and siphon off the clear liquid in the morning. Make faintly alkaline to litmus, and sterilize in an autoclave at $118^{\circ} \mathrm{C}$. for one hour on each of two days (if not to be used at once).

Levene (1918) and Burling and Levene (1918) have stated that 0.5 per cent lactose broth should be used in the presumptive test for coli bacteria in water analysis. With the higher carbohydrate content of the broth, the limiting hydrogen ion concentration is reached more quickly.

Nitrate Broth. This is a special broth containing nitrite-free nitrate. It may be prepared in different ways. Standard methods for the Examination of Water and Sewage, 1912, recommended a medium of the following composition:

Tap water....................... 1000 c.c.

Witte's peptone................. $1 \mathrm{gm}$.

Potassium nitrate (nitrite free)......... $0.2 \mathrm{gm}$.

Another formula is as follows:

Distilled water.................... 1000 c.c.

Leibig's meat extract.............. 3 gms.

Potassium nitrate c.p. $\mathrm{KNO}_{3} \ldots \ldots \ldots \ldots \ldots, 10$ gms.

The following has been used with some success:

Distilled water.................. 1000 c.c.

Leibig's meat extract. ............. 3 gms.

Witte's peptone................ 10 gms.

Potassium nitrate, $\mathrm{KNO}_{3} \ldots \ldots \ldots, \ldots \ldots \ldots . \quad 3$ gms. 
Breed (1915) has shown that by increasing the peptone content of nitrate broth to $5 \mathrm{gms}$. per liter more uniform results were obtained when fifty cultures of $B$. coli were tested for nitrate reduction. With soil bacteria 1 per cent peptone scemed to allow more constant results. He concludes that nitrate destruction must be tested for in media which allows a vigorous growth of the organisms. Suitable nitrate broths may have to be devised to fit each group.

Neutral Red Broth (Houston, 1914).

Peptone...................... 10 gms.

Salt, $\mathrm{NaCl} \ldots \ldots \ldots \ldots \ldots \ldots \ldots \ldots \ldots \ldots . \ldots \ldots$ gms.

Plain broth........................ 1000 c.c.

Add 2 c.c sterile aqueous 1 per cent solution of neutral red per liter. Make slightly alkaline with 20 per cent $\mathrm{KOH}$. Tube and sterilize.

Bile Salt Broth (MacConkey, 1900).

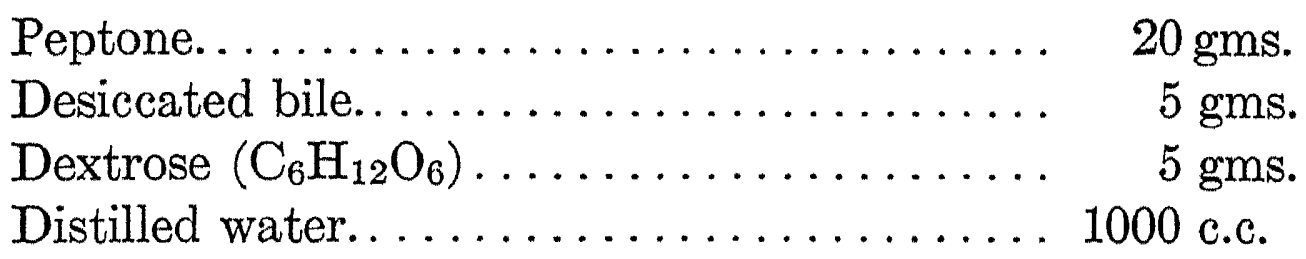

Boil for twenty minutes and add sterile litmus solution to color. Filter and put in fermentation tubes.

Vegetable Bouillon (Berthelot, 1917). This medium may be used as a substitute for meat infusion and is prepared as follows:

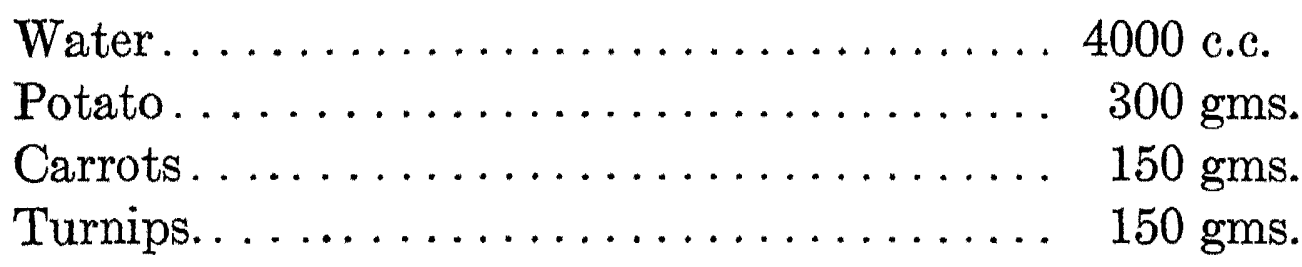

Pcel the potatoes and wash the carrots and turnips. Cut them into small pieces and put in cold water. Boil for four hours, strain and make feebly alkalin. Heat in an autoclave at $120^{\circ} \mathrm{C}$. for one-half hour and allow to stand over night in a refrigerator. Filter through paper.

Chicken Broth (Besson, 1913). Five hundred grams of lean chicken meat are soaked in water and filtered. The filtrate is used in the same manner as beef broth.

Giblet Broth (Besson, 1913). Liver, spleen, etc., may be used for making broth. Five hundred grams of the solid organs are soaked and filtered. The filtrate is, used as in other media of this sort. 
Glucose Formate Broth (Kitasato).

Nutrient broth..................... 1000 c.c.

Glucose $\left(\mathrm{C}_{6} \mathrm{H}_{12} \mathrm{O}_{6}\right) \ldots \ldots \ldots \ldots \ldots \ldots \ldots \ldots \ldots \ldots \ldots \ldots$ gms.

$\mathrm{O}$

Sodium formate $(\mathrm{H}-\mathrm{C}-\mathrm{O}-\mathrm{Na}) \ldots \ldots \ldots \ldots .4 \mathrm{gms}$.

Tube and sterilize.

\section{Peptone Solution (Dunham).}

Witte's peptone................ 10 gms.

Distilled water.................. 1000 c.c.

Reaction not adjusted.

Carbonated Broth (Besson, 1913). Add $\mathrm{CaCO}_{3}, 2$ per cent to lactose, mannite glucose, etc., broth before distributing into test tubes.

$\mathrm{CaCO}_{3}$ is most frequently added to lactose broth. When an organism which ferments lactose is grown in a $\mathrm{CaCO}_{3}$ lactose broth, the acids formed act on the $\mathrm{CaCO}_{3}$ giving off $\mathrm{CO}_{2}$.

Serum Broth (Besson, 1913). This medium is made by adding to tubes of ordinary sterile broth one-half, one-third, or one-quarter their volume of blood serum or blood collected under aseptic conditions. It is best to incubate the tubes before using in order to be certain that they are sterile.

\section{Dextrose Potassium Phosphate Broth.}

Witte's peptone................. 5 gms.

Dextrose $\left(\mathrm{C}_{6} \mathrm{H}_{12} \mathrm{O}_{6}\right) \ldots \ldots \ldots \ldots \ldots \ldots \ldots \ldots \ldots \ldots \ldots$ gms.

Di-potassium hydrogen phosphate $\left(\mathrm{K}_{2} \mathrm{HPO}_{4}\right) . \quad 5 \mathrm{gms}$.

Heat with occasional stirring over steam for twenty minutes. Filter and cool to $20^{\circ}$ C. Dilute to 1000 c.c. with distilled water. Tube and sterilize. (Standard Methods of Water Analysis, 1917, p. 210:)

Tryptophane Broth. To 1000 c.c. distilled water add 5 gms. dipotassium phosphate, $0.3 \mathrm{gm}$. tryptophane, and $1 \mathrm{gm}$. peptone. Heat until the ingredients are thoroughly dissolved, tube and sterilize at $15 \mathrm{lbs}$. for fifteen minutes. Some American peptones are standardized to contain a certain amount of tryptophane. If such peptone is used the tryptophane in the above formula may be omitted and the peptone increased to $5 \mathrm{gms}$. 


\section{Agar Media}

Nutrient Agar. 1. Add $3 \mathrm{gms}$. of beef extract, $5 \mathrm{gms}$. of peptone and 12 gms. of agar dried for one-half hour at $105^{\circ} \mathrm{C}$. before weighing to 1000 c.c. of distilled water. Boil over a water bath or cook in an autoclave until all the constituents are thoroughly dissolved. Make up the loss of evaporation.

2. Cool to about $45^{\circ}$ to $50^{\circ} \mathrm{C}$. and add $10 \mathrm{gms}$. of desiccated egg albumin per liter. Heat in the autoclave for thirty minutes. Make up the loss on evaporation and adjust reaction to $1+$.

3. Filter through cotton or cloth until clear.* Tube and sterilize in the autoclave at $120^{\circ} \mathrm{C}$.

Carbohydrate Agar. Any carbohydrate agar which is desired may be made by adding 1 per cent of the carbohydrate to plain agar. For careful work, carbohydrate media prepared from di- or tri-saccharides should not be sterilized in the autoclave as has been shown by Mudge (1917) and Hasseltine (1917).

Litmus Lactose Agar (Bendick, 1913). Bendick has devised the following for litmus lactose agar.

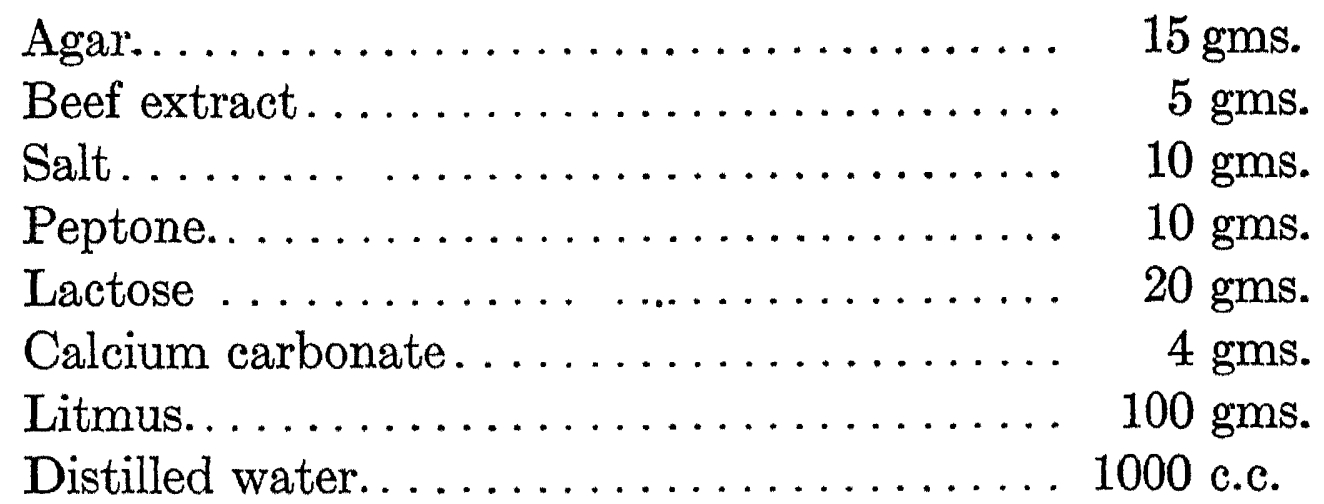

The agar, beef extract, salt and peptone are dissolved in 1000 c.c. of water. This is cleared with egg and filtered. No adjusting of the reactions is necessary. This is put into flasks (250 c.c. in a 500-c.c. flask). One gram of $\mathrm{CaCO}_{3}$ is added and the whole sterilized. Finally to each flask are added 25 c.c. of Kahlbaum's aqueous litmus and 5 gms. of lactose. Tube by pouring direct to the tubes from the flasks keeping the contents well mixed.

Litmus Lactose Agar (Meyer). Meyer (1917) has shown that a litmus lactose agar prepared from 3 per cent agar gives plates which

* The Sharples laboratory centrifuge will give a clear centrifugate in a short time with none of the usual trouble and expense connected with filtration through cotton or paper. 
are free from many of the objections incident to plates made from a lesser per cent of agar. The results obtained were approximately equivalent to Endo's medium agar.

Trypagar (Gordon et al). Take a measured quantity of the trypsinized broth, add 2 per cent of agar fiber, and $0.125 \mathrm{gm}$. of calcium chloride per liter. Autoclave at $118^{\circ} \mathrm{C}$. for three-fourths hour to dissolve the agar. Mix together in a saucepan; titrate with $\mathrm{N} / 10 \mathrm{KOH}$ to give an absolutely neutral reaction. Cool to $60^{\circ} \mathrm{C}$., add white of two eggs beaten up with the crushed shells, autoclave again at $118^{\circ} \mathrm{C}$. for seventyfive minutes (or in the steamer for two hours). Filter, add to the filtrate 5 per cent of the sterile pea extract, and sterilize in the ordinary way.

Neutral Red Bile Salt Agar (Savage, 1901).

Sodium taurocholate.............. 5 gms.

Witte's peptone................. 20 gms.

Distilled water.................... 1000 c.c.

Agar........................ 20 gms.

After filtration 10 gms. lactose and 5 c.c. of a 1 per cent solution of neutral red are added.

Glucose Formate Agar (Kitasato).

Nutrient agar.................... 1000 c.c.

Glucose..................... 20 gms.

Sodium formate.............. 4 gms.

Tube and sterilize.

Soil Extract Agar (Conn, 1914).

Agar........................ 15 gms.

Dextrose........................ $1 \mathrm{gm}$.

Soil extract. . . . . . . . . . . . . . . . . . 100 c.c.

Water........................ 900 c.c.

\section{Synthetic Agar.}

Dextrose....................... 10 gms.

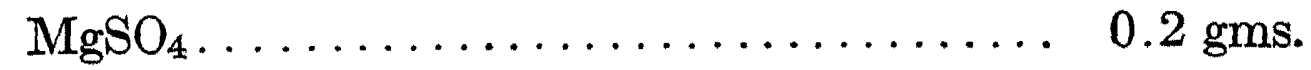

$\mathrm{K}_{2} \mathrm{HPO}_{4} \ldots \ldots \ldots \ldots \ldots \ldots \ldots \ldots \ldots, 0.5 \mathrm{gm}$.

Peptone....................... 0.05 gm.

Agar......................... 20 gms.

Distilled water................... 1000 c.c. 
Hesse and Niedner's (1898) Agar.

Agar-agar........................ 12.5

Nahrstoff Heyden. . . . . . . . . . . . . . . . 7.5

Distilled water.................... 980.0

Claimed to be a suitable medium for the bacterial examination of water.

Dolt's Agar (1908).

Purified agar (3 per cent solution) . . . . . . 250 c.c.

Asparagin..................... 25 gms.

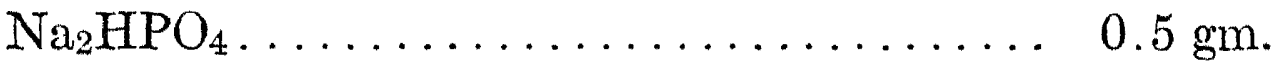

Distilled water................. 100 c.c.

This medium was found to support good growth of B. coli in twentyfour hours.

Neutral Red Agar. Add enough 0.5 per cent noutral red solution to agar containing 1 per cent dextrose to produce a clear red color.

Corn Agar (After Barlow, 1912).

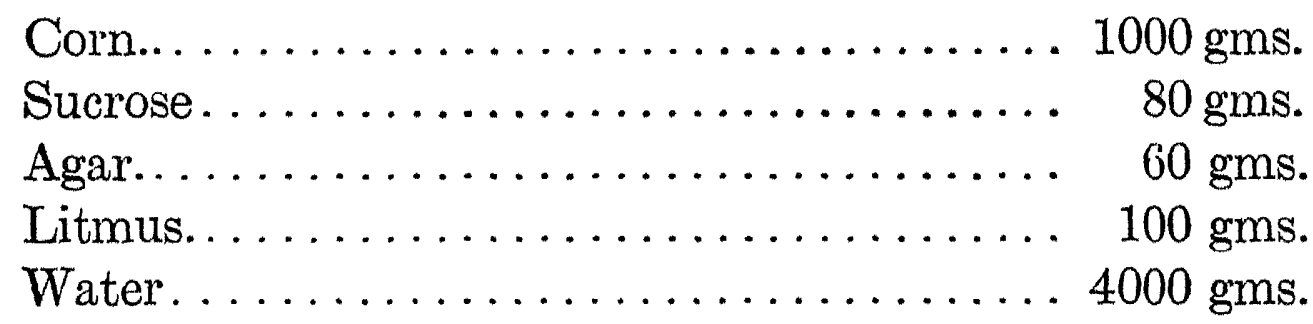

Stir the corn into the boiling water add to other materials, boil five minutes, heat in an autoclave and cool slowly, the heavy matter settles off. The clear supcrnatant agar may be drawn off and tubed.

Agar for Ps. Radicicola (Moore).

Di-potassium phosphate ............ $1 \mathrm{gm}$.

Magnesium sulphate.............. $0.5 \mathrm{gm}$.

Sucrose. . . . . . . .

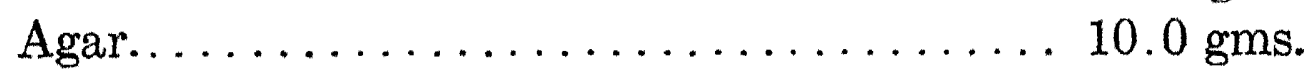

Distilled water..................... 1000 c.c.

Bean Agar (Thom, 1910). Common white beans are heated in five volumes of water. Boiling is stopped just before the swelling of the cotyledons would rupture the seed coats. This gives a clear yellow solution which filters easily and contains sufficient nutrients to grow many species normally. Agar may be as desired. Since this medium is poor in available carbon it is often desirable to add carbohydrate for certain species. 
Sabouraud's Agar for Yeasts (after Anderson, 1917).

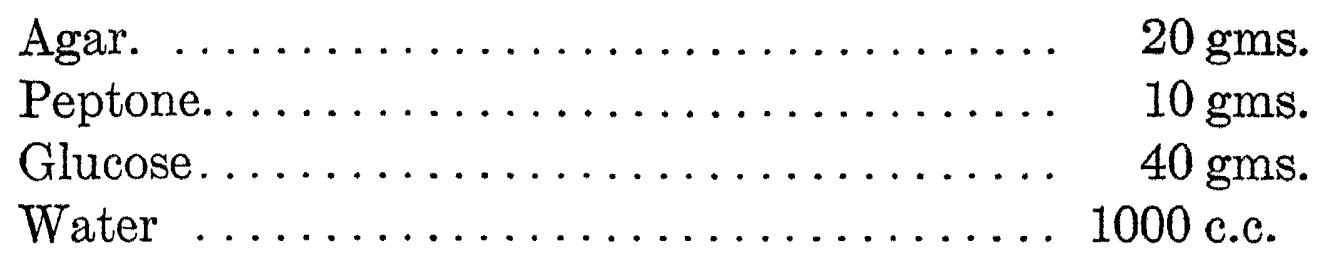

Mix these ingredients in the usual way and adjust the reaction to +2 per cent acid. Without further heating the sugar is added and the medium tubed in previously sterilized tubes. Anderson advises care in sterilizing since the high acidity of this medium may prevent solidification of the agar.

Agar Gelatin Medium (North 1909).

Lean chopped beef or veal............ 500 gms.

Agar....................... 10 gms.

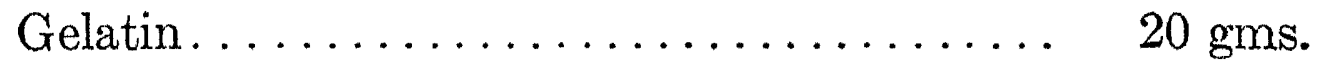

Peptone...................... 20 gms.

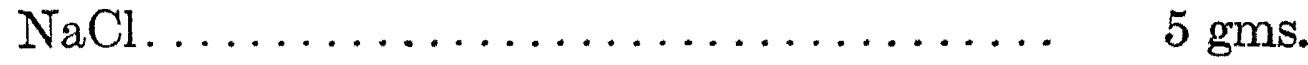

Distilled water.................. 1000 c.c.

Adjust neutral to phenolphthalein. North states that this medium is excellent for streptococci, pneumococci and diphtheria bacilli because it is soft and moist and can be used at $37^{\circ} \mathrm{C}$. It is claimed to be of special value in keeping stock cultures.

\section{Hesse Medium.}

Agar..................... 5 gms.

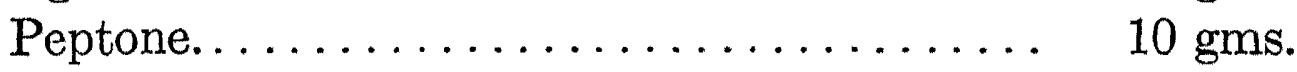

Liebig's beef extract............... 5 gms.

Sodium chloride................ 8.5 gms.

Distilled water................... 1000 c.c.

Agar is dissolved in 500 c.c. $\mathrm{H}_{2} \mathrm{O}$. Other ingredients are dissolved in 500 c.c. $\mathrm{H}_{2} \mathrm{O}$. Solutions are mixed, filtered and tubed.

Hiss Plating Medium (1897).

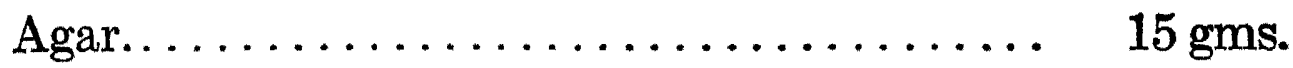

Gelatin........................ 15 gms.

Liebig's meat extract. .............. 5 gms.

$\mathbf{N a C l} \ldots \ldots \ldots \ldots \ldots \ldots \ldots \ldots .5 . \ldots \ldots$ gms.

Dextrose................... 10 gms.

Distilled water.................... 1000 c.c. 
The agar is dissolved in 1000 c.c. $\mathrm{H}_{2} \mathrm{O}$. When melted the extract, gelatin and salts are added. It is cleared and filtered. Dextrose is then added and it is tubed.

Malachite Green Media (1906). 1. Three gms. Liebig's meat extract.

2. Acidify this with 7.5 c.c. $\mathrm{NHCl}$.

3. Dissolve 30 gms. agar by boiling.

4. Neutralize with 7 c.c. $\mathrm{NNaOH}$ until neutral to litmus.

5. Add 5 c.c. $\mathrm{NNa}_{2} \mathrm{CO}_{3}$ and heat in an Arnold for several hours.

6. Add 100 c.c. of 10 per cent sucrose solution. This may be stored in 100 e.c. quantities.

7. Before use redissolve and to 100 c.c. add 2 to 2.9 c.c. of 2 per cent malachite green (trade mark Höchst 120). This should be made in sterile $\mathrm{H}_{2} \mathrm{O}$ and not boiled.

8. Medium is smeared in Petri dishes.

Russell's Medium (1911). "Enough 5 per cent aqueous solution of litmus ( 3 to 5 per cent) is added to plain agar ( 2 or 3 per cent), which usually has a reaction of about 0.8 per cent acid to phenolphthalein, to give it a distinct purple violet color, the amount of litmus depending on the original color of the agar; dark requiring more than light, and the reaction is then adjusted by adding sodium hydrate until the mixture is neutral to litmus. Next, and last, 1 per cent of lactose and one-tenth of 1 per cent of glucose dissolved in a small amount of hot water is added and the medium tubed for slants. The sterilization is done in the Arnold and because of the danger of breaking down the lactose must not be carried too far; if the tubes are packed loosely in the sterilizer basket to allow good circulation of the steam, ten minutes on the first and fifteen on the second day has been time enough. The tubes are then slanted and stored in small quantities in a dark place."

Krumwiede has substituted Andrade for the litmus and secured good results.

Endo's Medium (after Kendall, 1911-12).

I. Preparation of agar.

(a) Prepare plain sugar free agar using 15 gms. agar per liter.

(b) Adjust to a point just alkaline to litmus.

(c) Place in flasks in 100 c.c. quantities and sterilize. 
II. Preparation of the indicator.

(a) Prepare a 10 per cent basic fuchsin solution in 96 per cent alcohol. This solution is fairly stable if kept away from the light.

(b) Prepare a 10 per cent solution of chemically pure anhydrous sodium sulphite. ( $1 \mathrm{gm}$. in 10 c.c. water.) This solution does not keep.

(c) Add 1 c.c. of $\operatorname{II}(a)$ to 10 c.c. of $\operatorname{II}(b)$ and heat in the Arnold sterilizer for twenty minutes. The color of the solution should be nearly discharged. This solution must be prepared each day. It does not keep.

IIT. Preparation of medium.

(a) Add $1 \mathrm{gm}$. of c.c. lactose to 100 c.c. of the agar and place in the autoclave until melted and lactose is dissolved.

(b) Add enough of II (c) to impart a pink color (about 1 c.c.).

(c) Pour into sterile Petri dishes and allow to harden. In the above medium Kendall has reduced the agar content to 1.5 per cent. The original formula of Endo called for a 3 per cent solution which was supposed to prevent diffusion of the acid and prodistinct colonies.

Endo's Medium (Hygienic Laboratory Method). Hasseltine (1918) has described the preparation of this medium as follows: It consists of a 3 per cent agar which is titrated and corrected to +0.5 to phenolphthalein, to which is added 3.7 c.c. of a 10 per cent solution of anhydrous sodium carbonate. For convenience it is flasked, sterilized and stored in 200 c.c. quantities. When ready to use the following ingredients are added to 200 c.c. of agar as follows:

(a) Dissolve 2 gms. chemically pure lactose in 25 to 30 c.c. of distilled water, with the aid of gentle heat.

(b) Dissolve $0.5 \mathrm{gm}$. of anhydrous sodium sulphite in 10 to 15 c.c. of distilled water.

(c) To the sulphite solution add 1 c.c. of saturated solution of basic fuchsin in 95 per cent alcohol.

Add the fuchsin sulphite solution to the lactose solution and then add the whole to the agar. Pour plates at once, and after hardening dry for fifteen minutes in the incubator.

Teague (1918) has shown that a 10 per cent solution of sodium sulphite may be heated for twenty minutes at $15 \mathrm{lbs}$. with practically 
no change. If this is then kept covered with a layer of petrolatum, it may be kept for three weeks at room temperature.

Endo's Medium (After Levine, 1918). Levine states the method of preparation as follows:

Distilled water..................... 1000 c.c.

Peptone (Difco).................. 10 gms.

Dipotassium phosphate............. 2-5 gms.

Agar........................ 15-30 gms.

These ingredients should be boiled until dissolved and the loss by evaporation made up with distilled water. No adjustment of the reaction is necessary; neither is filtration necessary if the medium is to be used for streaked cultures. Store in quantities of 100 c.c. in Erlenmeyer flasks and when used add the following materials:

20 per cent lactose solution ....... 1 gm. or 5.0 c.c.

10 per cent alcoholic solution of basic fuchsin. 0.5 c.c.

Freshly prepared sodium sulphite solution... 2.5 c.c.

Eosin Brilliant-Green Agar (Teague and Clurman, 1916). This medium was devised for the rapid isolation of $B$. typhi from stools and was prepared by these workers after the following method: 500 gms. of chopped beef are placed in 1 liter of distilled water and kept in the icebox over night. The infusion is squeezed through cheese cloth, heated in the Arnold sterilizer and passed through filter paper. Witte's peptone ( 1 per cent), chemically pure sodium chloride ( 0.5 per cent) and agar (1.5 per cent) are added to the warm infusion, the peptone being rubbed into a paste in a little warm water. Then heat the flask of medium in the autoclave for thirty minutes at $120^{\circ} \mathrm{C}$. Adjust the reaction to $1+$ by adding $2 \mathrm{~N}$ sodium hydroxide, cool to $55^{\circ} \mathrm{C}$., clear and filter through cotton. Put into flasks in 100 c.c. quantities and sterilize at $120^{\circ} \mathrm{C}$. for twenty minutes.

When ready to use, melt the agar and add 1 per cent each of sucrose and lactose. To every 50 c.c. of agar add 1 c.c. of a 3 per cent solution of yellowish eosin. From the stock solution of brilliant green in 50 per cent alcohol, a $\frac{1}{6}$ per cent solution is prepared in distilled water and 1 c.c. of this is added to each 50 c.c. of the agar. After the dyes are well distributed, pour into plates.

Eosin-Methylene Blue Agar (Levine, 1918). This is a modification of Harris and Teague's medium and was found by Levine to allow 
a close separation between $B$. coli and $B$. aerogenes. Levine describes its preparation as follows:

Distilled water................... 1000 c.c.

Peptone (Difco)................... 10 gms.

Dipotassium phosphate............ 2 gms.

Agar. .................... 15 gms.

Boil the ingredients until dissolved and make up any loss to evaporation. Place measured quantities in flasks and sterilize at $15 \mathrm{lbs}$. for fifteen minutes. Just prior to use add to each 100 c.c. of the melted agar prepared as above, the following constituents:

Sterile (20 per cent lactose) ........... 1 gm. or 5 c.c.

Aqueous (2 per cent) eosin (yellowish) solution. 2 c.c.

Aqueous ( 2 per cent) methylene blue solution. 2 c.c.

Pour the medium into dishes allow them to harden in the incubator and inoculate in the usual way. There should be no adjustment of the reaction and filtration of the medium is not necessary.

\section{Gelatin Media}

Nutrient Gelatin. 1. Add 3 gms. of beef extract and 5 gms. of peptone to 1000 c.c. of distilled water and add 100 gms. of gelatin dried for one-half hour at $105^{\circ} \mathrm{C}$. before weighing.

2. Heat slowly on a steam bath to $60^{\circ} \mathrm{C}$. until the gelatin is dissolved.

3. Make up lost weight, titrate, and if the reaction is not already between +0.5 and +1 , adjust to +1 .

4. Filter through cloth and cotton until clear.

5. Distribute in test tubes, 10 c.c. to each tube, or in larger containers if desired.

6. Sterilize in the autoclave at $15 \mathrm{lbs} .\left(120^{\circ} \mathrm{C}\right.$.) for fifteen minutes after the pressure reaches $15 \mathrm{lbs}$.

Whey Gelatin. Add 10 per cent to clarified whey. This medium is of value in studying certain members of the lactic acid bacteria.

Soil Extract Gelatin (Conn, 1914).

Gelatin 100-150 gms.

Dextrose

$1 \mathrm{gm}$.

Soil extract...................... 100 c.c.

Water........................ 900 c.c. 
Litmus Gelatin. This may be prepared from lactose gelatin the reaction of which must be neutral. Deposit 1 c.c. of sterile litmus solution in the sterile Petri dish with the sample and pour in the melted gelatin. Shake the dish until the litmus is evenly distributed throughout. Incubate at $20^{\circ} \mathrm{C}$. This medium is of much value in studying the bacteria in milk.

Hiss Medium (1902).

Agar. 15 gms.

Gelatin 15 gms.

Liebig's meat extract............. 5 gms.

Sodium chloride. . . . . . . . . . . 5 gms.

Dextrose................... 10 gms.

Distilled water.................. 1 liter

Adjust the reaction to $1+$.

Raisin Gelatin (Besson). Make a decoction of raisins by grinding and stewing 50 to 100 gms. of raisins in 1000 gms. of $\mathrm{H}_{2} \mathrm{O}$ for one hour. Filter and add $100 \mathrm{gms}$. of gelatin and a pinch of sodium phosphate. Boil for two or three minutes. Neutralize and finish as usual.

Wort Gelatin. 1. Measure out 900 c.c. of beer wort in sterile flask.

2. Weigh out 100 gms. gelatin (10 per cent) and add it to the wort in the flask.

3. Bubble live steam through for ten minutes to dissolve the gelatin.

4. Cool to $60^{\circ} \mathrm{C}$., clarify with egg.

5. Filter and tube.

Gelatin Agar (Besson). By mixing agar and gelatin a medium is obtained with a melting-point between that of either plain agar or plain gelatin.

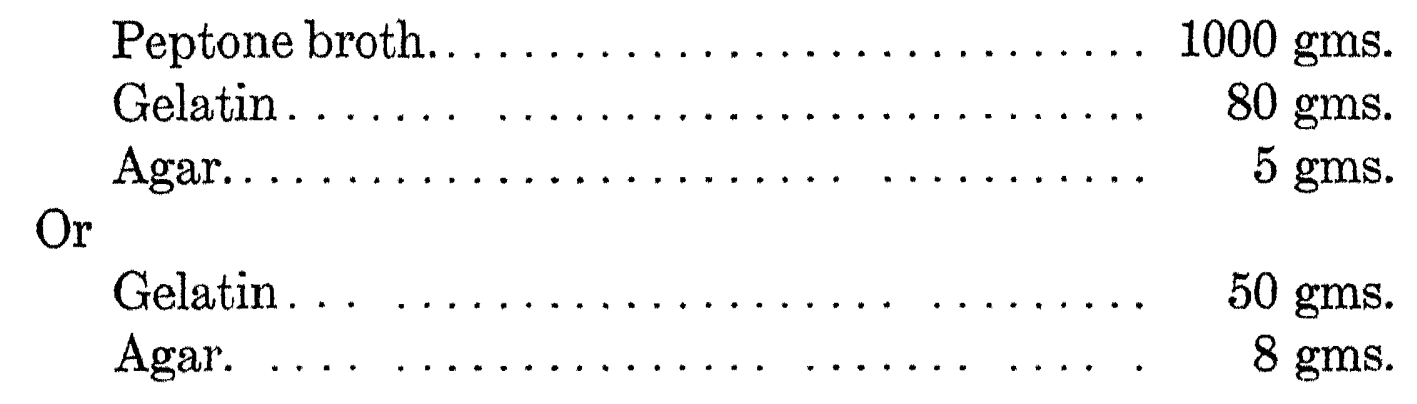

Dissolve the gelatin in the broth, neutralize and then add the agar. Sterilization must not be accomplished above $115^{\circ} \mathrm{C}$. (See North's Agar Gelatin Medium.) 


\section{Special Media:}

Peptone Sucrose Solution (Buchanan, 1909).

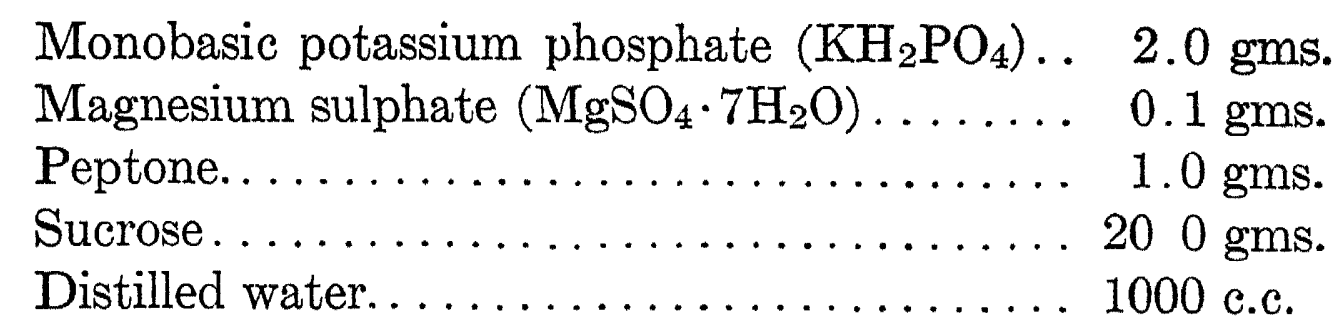

Hay Infusion. Macerate 15-20 gms. of finely chopped hay or in 1000 gms. of $\mathrm{H}_{2} \mathrm{O}$ for one or two hours. Boil for a few minutes, filter, tube and sterilize at $115^{\circ} \mathrm{C}$. The infusion which is sometimes a little acid may be neutralized in the usual way.

Beer Wort (Eyre, 1903). 1. Weigh out 250 gms. malt and put into a flask.

2. Add 1000 c.c. distilled water heated to $70^{\circ}$ C. and close flask with rubber stopper.

3. Place the mixture in a water bath at $60^{\circ} \mathrm{C}$. and allow the mixture to stand for one hour.

4. Strain through cheese cloth into clean flask and heat in an Arnold for thirty minutes.

5. Filter through paper.

6. Tube in 10 c.c. quantities and sterilize in an Arnold.

Spronck's Peptone Yeast Extract. To 5 liters of $\mathrm{H}_{2} \mathrm{O}$ add 1000 gms. commercial yeast, not brewers' yeast. Boil for twenty minutes with stirring, pour into cylindrical vessels and leave for twenty-four hours. Decant the cloudy liquid and add 5 gms. of $\mathrm{NaCl}$ and 10 gms. of peptone per liter. Neutralize exactly and then make alkaline to 7 per cent with $\mathrm{NNaOH}$. Filter through Chardin paper and pour into flasks. Sterilize at $115^{\circ} \mathrm{C}$.

Wine. Before sterilizing make neutral in the ordinary way.

Prune Decoction. Macerate 50 gms. of prunes in 1 liter of distilled water and allow to stand in the ice chest until the next morning. Filter and tube for sterilization.

Pea-flour Extract (Gorden et al., 1916). Take 100 gms. of pea flour and add 1 liter of distilled water with 100 gms. of salt. Mix and steam for one-half hour, stirring constantly. Allow to settle and filter, then sterilize and label "saline pea extract." This pea-flour extract should preferably be freshly made for each batch of agar. This medium was especially designed to replace Witte's peptone. 
Haricot Decoction. Macerate 50 to 60 gms. of white haricot beans in a liter of water for several hours in the cold. Boil for one-half hour. Pour on a coarse sieve, collect the liquid, and add to it 1 per cent of salt, 2 per cent of sugar and a pinch of sodium bicarbonate. Boil and filter through paper. Tube and sterilize at $115^{\circ} \mathrm{C}$.

This medium is used by Maze to cultivate the bacteria in nodules of leguminous plants.

Malt Extract (Besson). 1. Grind up 100 gms. malt and add 1000 gms. of $\mathrm{H}_{2} \mathrm{O}$.

2. Heat to $56^{\circ} \mathrm{C}$. for one hour; the starch is converted into maltose by the diastase and a true beer wort obtained

3. Boil, filter through Chardin paper.

4. Tube, sterilize at $115^{\circ} \mathrm{C}$.

Urine (Besson). 1. Boil some recently passed urine.

2. If the reaction is markedly alkaline after boiling, add a little tartaric acid solution, testing the reaction with litmus paper. -

3. Filter, tube and sterilize at $155^{\circ} \mathrm{C}$. The composition of urine is altcred by this proceeding, the urea in solution being decomposed at boiling temperature. It is better to sterilize through a Chamberland bougie.

Capaldi's Egg Medium. A few loopfuls of egg yolk are added to a tube of liquefied agar, previously cooled to 45 to $47^{\circ} \mathrm{C}$.

Dorset's Egg Medium. Eggs are broken into a flask and the yolks broken with a needle or glass rod. The flask is shaken until the yolks and whites are thoroughly mixed. Foam formation should be avoided. Distribute in culture tubes and sterilize in a Koch inspissator or autoclave in the same manner as blood serum.

Egg-Meat Mixture (Rettger, 1906). A. One-half pound of lean chopped beef is stirred up in 250 c.c. of water, and after neutralizing the meat acids with sodium carbonate, the mixture is heated in an Arnold sterilizer for thirty minutes, with occasional stirring. It is then set away in a cold place for several hours after which the fatty scum is removed.

$B$. The whites of three eggs are mixed with 250 c.c. of water, and after neutralizing, the albumen is coagulated, with occasional stirring, by heating in the Arnold sterilizer for thirty minutes.

$A$ and $B$ are then mixed and introduced into a liter flask, along with 2.5 gms. ( 0.5 per cent) of powdered calcium carbonate. The flask is plugged with cotton and sterilized for thirty minutes, at 110 to $112^{\circ} \mathrm{C}$. (autoclave). 
Redfield's (1912) Medium.

Distilled water.................. 1000 c.c.

Peptone...................... 300 gms.

Potassium chloride $(\mathrm{KCl}) \ldots \ldots \ldots \ldots \ldots, 74 \mathrm{gms}$.

Add the peptone to 700 c.c. of hot water. Stir until it is in solution and cool. Allow to stand over night in the icebox. Filter and put in test tubes.

Ox-bile Medium. Dissolve 1 per cent lactose and 1 per cent peptone in fresh ox-bile, filter and place in fermentation tubes. Sterilize in an Arnold on three successive days.

Decoction of Dried Fruits. 1. Macerate 50 to $100 \mathrm{gms}$. of dried fruit in a liter of water for several hours. Then stew them in the water.

2. Pass through a coarse sieve.

3. Boil. Filter.

4. Tube and sterilize at $115^{\circ} \mathrm{C}$.

The liquid is slightly acid and is useful for cultivating molds. Otherwise make neutral.

Barsiekow's Medium. Prepare and sterilize separately the two following solutions:

A. Sodium chloride, $\mathrm{NaCl} . \ldots \ldots \ldots \ldots \ldots, 0.5 \mathrm{gm}$.

Nutrose.................... $1.0 \mathrm{gm}$.

Water .................... 75.0 gms.

B. Water. . . . . . . 25.0 gms.

Litmus. ................... To give blue color

Sugar (the one desirad)............ $1.0 \mathrm{gm}$.

Add sufficient litmus to give an amethyst color. After cooling mix and distribute in tubes.

Bread. Soak some slices of white bread in distilled water and sterilize at $115^{\circ} \mathrm{C}$.

Crumble some dry bread and allow to dry. Grind when dry and put in Petri disher or Erlenmeyer flask and add water to moisten (about $2 \frac{1}{2}$ pts. of $\mathrm{H}_{2} \mathrm{O}$ to 1 pt. of bread by weight).

Sterilize at $115^{\circ} \mathrm{C}$. for twenty minutes. 


\section{Potato and Starch Media}

Potato Gelatin (Goadby). Add 100 gms. of gelatin to 1 liter of glycerol potato broth. Boil, adjust the reaction, filter, and sterilize.

Glycerol Potato Broth. Wash, peel and grind $1000 \mathrm{gms} \cdot$. of potato. Soak this in a liter of water for twenty-four hours. After filtering add 3 to 5 per cent of glycerol and tube for sterilization.

Potato Agar (McBeth, 1913). Pare, steam, and mash a quantity of potatoes. To 100 gms. of mashed potato add 800 c.c. of tap water and steam for one-half hour, filter through cotton.

Potato solution. . ............. 500 c.c.

Agar...................... 15 gms.

Nutrient solution (same as for cellulose agar). 500 c.c.

Starch Agar. Make 1 liter of ordinary peptone agar. Add to this a 2 per cent paste of potato starch. This should be added at as low a temperature as possible to the neutral agar.

To use, pour plates with the sterile tubed starch agar and streak with the culture. After incubation for 5 days, pour over weak solution of Lugol's iodine.

Starch Agar (Vedder, 1915). Prepare a liter of beef infusion in the usual way. Meat extract does not give as good results. To this add 1.5 per cent of agar. No peptone or salt should be added. Cook. and clarify the medium and add 10 gms. of cornstarch to each liter. The starch is best rubbed up in a mortar in a little agar. One per cent of starch gives the best results. Tube and sterilize in the usual manner. This medium is especially valuable in the growth of gonococci.

Elsner's Potato Medium. 1. Peel and grate 500 grams of potato.

2. Grind up and soak in distilled water for twelve hours.

3. Strain and allow to stand. Filter to clear.

4. Make up to 1 liter and dissolve 15 per cent of gelatin.

5. Adjust reaction to acid and sterilize.

Potato Infusion. Wash and grind up a few potatoes. To each $30 \mathrm{gms}$. of potato, add a liter of distilled water and allow to stand in the icebox over night. In the morning, filter and boil the filtrate. Filter again if necessary and tube for sterilization. If the infusion is too acid adjust the reaction as usual.

Carrot Infusion. This medium may be prepared as above for potato infusion.

Potato Mash. Clean and cut a large potato into slices. Boil until soft and grate or press through a ricer. Distribute this in layers in 
Petri dishes and sterilize at $120^{\circ} \mathrm{C}$. for twenty minutes. The slices may be placed in the Petri dishes without mashing if desired.

Starch Jelly (Smith, 1898). To 10 c.c. of Uschinsky's solution add $1 \mathrm{gm}$. of clean aseptic starch. Rub this up in the slanted fluid. Plug the tubes tightly and place in an inspissator or Arnold in the slanted condition. Heat for two hours on five successive days at 85 to $93^{\circ} \mathrm{C}$. If water is lost, this must be made up by the addition of distilled water.

Potato Agar (Thomas, 1906). The potatoes are carefully washed, pared, sliced, then slowly heated for two hours in approximately two volumes of water. At the close of the heating the water is allowed to boil. The whole is then filtered through cloth, water being added to make up the loss of any evaporation. After filtering, 1 per cent shred agar is added to this fil rate. It may then be heated in an autoclave for thirty minutes at $15 \mathrm{lbs}$. after which it may be tubed or filtered, if desired, before tubing.

Cellulose Agar (McBeth, 1913). "Prepare 1 liter of a dilute ammonium-hydroxide solution by adding 3 parts water to 10 parts ammonia hydroxide, sp.gr. 0.99. Add a slight excess of copper carbonate and shake vigorously, allow to stand overnight, and then siphon off the supernatant solution. Add $15 \mathrm{gms}$. of unwashed sheet filter paper and shake occasionally until the paper is dissolved. Dilute to 10 liters and add slowly a one to five solution of hydrochloric acid, with vigorous shaking until the precipitation of the cellulose is complete. Dilute to 20 liters, allow the cellulose to settle, and decant the supernatant liquid. Wash by repeated changes of water, adding hydrochloric acid each time until the copper color disappears; then wash with water alone until the solution is free from chlorine. Allow it to settle several days and decant off as much of the clear solution as possible. If the percentage of cellulose is still too low, a portion of the solution is centrifugalized to bring the cellulose content up to 1 per cent."

\begin{tabular}{|c|c|}
\hline $\begin{array}{l}\text { Cellulose solution } . \ldots \ldots \ldots \\
\text { Agar. } \ldots \ldots \ldots \ldots \ldots\end{array}$ & $\begin{array}{l}500 \text { c.c. } \\
10 \text { gms }\end{array}$ \\
\hline Nutrient solution, composed of: & \\
\hline 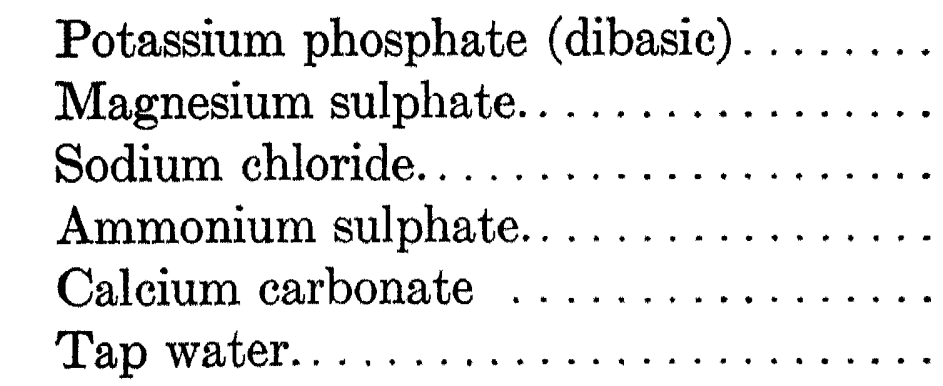 & $\begin{array}{l}1 \mathrm{gm} . \\
1 \mathrm{gm} . \\
1 \mathrm{gm} . \\
2 \text { gms. } \\
2 \text { gms. } \\
1000 \text { c.c. }\end{array}$ \\
\hline
\end{tabular}


Starch Agar (McBeth, 1913). To 800 c.c. of boiling water add 10 gms. of potato starch suspended in a little cold water. Concentrate by boiling to 500 c.c. This breaks up the starch grains and should give a nearly transparent starch solution.

Starch solution................... 500 c.c.

Agar....................... 10 gms.

Nutrient solution (same as for cellulose).. . . 500 c.c.

\section{Synthetic Media}

Under the term synthetic the bacteriologist includes all media which may be prepared from pure chemical compounds. These may always be secured or brought to the same state of purity by the various methods well known to the chemist. The advantages of synthetic media are quite evident.

1. They may be prepared in different lots which have a constant chemical composition.

2. They may be prepared in different laboratories and render the data thus secured, more reliable.

3. They lend themselves to the study of some one compound or element. For instance sulphur free media may be prepared to which certain sulphur compounds may be added.

\section{Parietti's Solution.}

Carbolic acid, $\mathrm{C}_{6} \mathrm{H}_{5} \mathrm{OH} \ldots \ldots \ldots \ldots \ldots \ldots \ldots$ c.c.

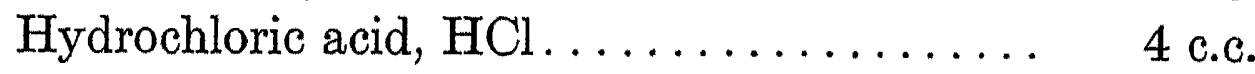

Water......................... 100 c.c.

\section{Drigalski and Conradi (1902) Medium.}

Dextrose (free broth) . . . . . . . . . . . 2000 c.c.

Nutrose. ................... 20 gms.

Agar.................... 40 gms.

Boil, dissolve, neutralize to phenolphthalein, autoclave at $120^{\circ} \mathrm{C}$. for five minutes.

Mayer's Culture-Fluid (from Smith, 1905).

Magnesium sulphate, $\mathrm{MgSO}_{4} \cdot 7 \mathrm{H}_{2} \mathrm{O} \ldots \ldots \ldots \quad 10$ gms.

Ammonium nitrate, $\mathrm{NH}_{4} \mathrm{NO}_{3} \ldots \ldots \ldots \ldots \ldots \quad 15$ gms.

Tribasic calcium phosphate, $\mathrm{Ca}_{3}\left(\mathrm{PO}_{4}\right)_{2} \ldots \ldots 0.01 \mathrm{gms}$.

Monobasic potassium phosphate, $\mathrm{KH}_{2} \mathrm{PO}_{4} \ldots .10 \mathrm{gms}$.

Distilled water.................. 1000 c.c. 
Dissolve cold and add sugar. Add $\mathrm{NaCl}$ ( 3 per cent) if it is to be used for luminous bacteria, and an excess of pure $\mathrm{CaCO}_{3}$ if acid forming bacteria are to be grown.

\section{Prazmowski's Culture-Fluid.}

Dipotassium phosphate, $\mathrm{K}_{2} \mathrm{HPO}_{4} \ldots \ldots \ldots \ldots \quad 5$ gms.

Magnesium sulphate, $\mathrm{MgSO}_{4} \cdot 7 \mathrm{H}_{2} \mathrm{O} \ldots \ldots \ldots \quad 5 \mathrm{gms}$.

Ammonium carbonate, $\left(\mathrm{NH}_{4}\right)_{2} \mathrm{CO}_{3} \ldots \ldots \ldots .5 \mathrm{gms}$.

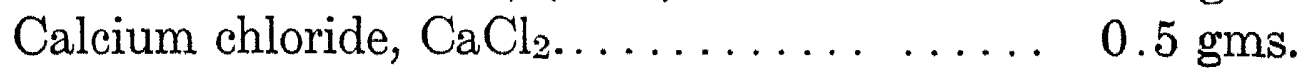

Distilled water.................. 1000 c.c.

Naegeli's Medium (after Besson, 1913).

Water...................... 1000 gms.

Ammonium tartrate.............. 10 gms.

Dipotassium phosphate, $\mathrm{K}_{2} \mathrm{HPO}_{4} \ldots \ldots \ldots \ldots \quad 1 \mathrm{gm}$.

Magnesium sulphate, $\mathrm{MgSO}_{4} \cdot 7 \mathrm{H}_{2} \mathrm{O} \ldots \ldots \ldots, 0.2 \mathrm{gm}$.

Calcium chloride, $\mathrm{CaCl}_{2} \ldots \ldots \ldots \ldots \ldots \ldots .12 \mathrm{gm}$.

\section{Cohn's Solution.}

Distilled water.................. 1000 c.c.

Dipotassium phosphate, $\mathrm{K}_{2} \mathrm{HPO}_{4} \ldots \ldots \ldots \ldots \quad 5$ gms.

Magnesium sulphate, $\mathrm{MgSO}_{4} \cdot 7 \mathrm{H}_{2} \mathrm{O} \ldots \ldots \ldots .5$ gms.

Ammonium tartrate................ 10 gms.

Potassium chloride, $\mathrm{KCl} \ldots \ldots \ldots \ldots \ldots .0 .5 \mathrm{gm}$.

Hydrolyzed Casein Medium (Cannon, 1916). Zipfel proposed the addition of tryptophane to a synthetic medium for the determination of indol formation. Cannon has realized the high cost of tryptophane and suggested the use of a hydrolyzed casein which is prepared as follows: Ten grams of casein are hydrolyzed by 200 c.c. of 10 per cent sulphuric acid. The mixture is kept on the water bath for twentyfour hours after which it is neutralized by saturated barium hydroxide. This will precipitate the sulphate. Evaporate the resulting solution until the amino acids crystallize. Half of the preparation is then dissolved in 500 c.c. of Zipfel's synthetic medium, which has the following composition:

Asparagin

$5.0 \mathrm{gms}$.

Ammonium lactate. .

5.0 gms.

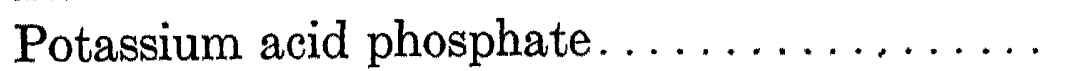

$2.0 \mathrm{gms}$.

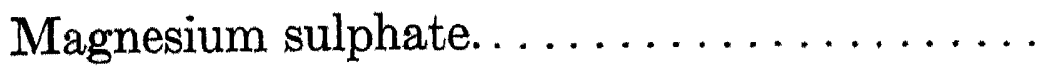

$0.2 \mathrm{gm}$.

Distilled water. $1000.0 \mathrm{gms}$. 


\section{Capaldi's Medium.}

Peptonc..................... 20 gms.

Gelatin.................... 10 gms.

Agar....................... 20 gms.

Dextrose or mannit................ 10 gms.

Sodium chloride, $\mathrm{NaCl} \ldots \ldots \ldots \ldots \ldots \ldots \ldots . \ldots$ gms.

Potassium chloride, $\mathrm{KCl} \ldots \ldots \ldots \ldots \ldots \ldots$ gms.

Distilled water..................... 1000 c.c.

\section{Frankel's Solution.}

Sodium chloride, $\mathrm{NaCl} \ldots \ldots \ldots \ldots \ldots \ldots . \quad 5$ gms.

Monocalcium phosphate, $\mathrm{Ca}\left(\mathrm{H}_{2} \mathrm{PO}_{4}\right)_{2} \ldots \ldots .2$ gms.

Ammonium lactate, $\mathrm{CH}_{3}-\mathrm{CHOH}-\mathrm{CONH}_{2}$. 6 gms.

Asparagin, $\mathrm{C}_{2} \mathrm{H}_{3}\left(\mathrm{NH}_{2}\right) \mathrm{CONH}_{2} \ldots \ldots \ldots \ldots \quad 4$ gms.

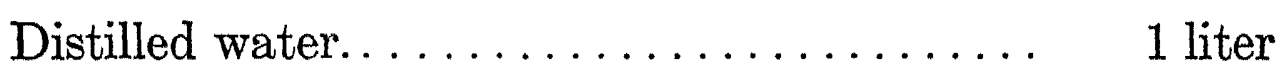

Sodium hydroxide, $\mathrm{NaOH} \ldots \ldots \ldots \ldots \ldots .20$ c.c.

\section{Sullivan's (1905) Medium.}

Asparagin, $\mathrm{C}_{2} \mathrm{H}_{3}\left(\mathrm{NH}_{2}\right){ }_{\mathrm{COOH}}^{\mathrm{CONH}_{2}} \ldots \ldots \ldots \ldots \quad 10$ gms.

Magnesium sulphate, $\mathrm{MgSO}_{4} \ldots \ldots \ldots \ldots \ldots .2$ gms.

Dibasic potassium phosphate, $\mathrm{K}_{2} \mathrm{HPO}_{4} \ldots \ldots . \quad 1 \mathrm{gm}$.

This medium supports good pigment formation by Ps. pyocyaneus and Ps. fluoresceous liquefaciens.

Van Delden's (1904) Solution. For $\mathrm{SO}_{4}$ reducing bacteria.

Dibasic potassium phosphate, $\mathrm{K}_{2} \mathrm{HPO}_{4} \ldots \ldots \quad 0.5 \mathrm{gm}$.

Sodium lactate, $\mathrm{CH}_{3}-\mathrm{CHOH}-\mathrm{COONa} \ldots \ldots .5 .0 \mathrm{gms}$.

Magnesium sulphate $\mathrm{MgSO}_{4} \ldots \ldots \ldots \ldots \ldots 1.0 \mathrm{gm}$.

Asparagin, $\mathrm{C}_{2} \mathrm{H}_{3}\left(\mathrm{NH}_{2}\right)<{ }_{\mathrm{COOH}}^{\mathrm{CONH}_{2}} \ldots \ldots \ldots \ldots \quad 1.0 \mathrm{gm}$.

Ferrous sulphate, $\mathrm{FeSO}_{4} \ldots \ldots \ldots \ldots$ Trace

Tap water.................... 1000 c.c.

This medium is of special value for the determination of sulphate reduction. 
Dolt's Medium (1908). The following media were found to grow B. coli well in twenty-four hours.

Medium I.

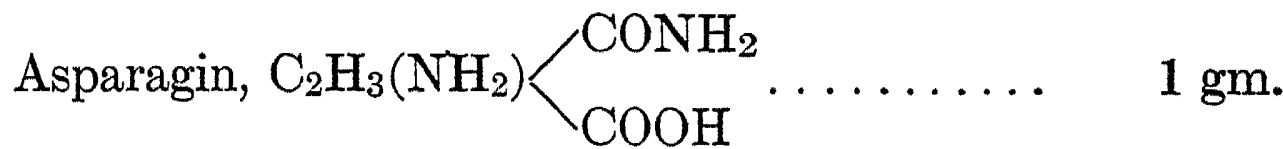

Dibasic sodium phosphate, $\mathrm{Na}_{2} \mathrm{HPO}_{4} \ldots \ldots \ldots \quad 0.2 \mathrm{gm}$.

Distilled water................... 100 c.c.

Medium II.

Asparagin. .................. $1 \mathrm{gm}$.

Dibasic ammonium phosphate $\left(\mathrm{NH}_{4}\right)_{2} \mathrm{HPO}_{4} \ldots \quad 0.2 \mathrm{gm}$.

Distilled water.................. 100 c.c.

The following solid media were prepared:

Purified agar, 3 per cent solution... . . . . 250 c.c.

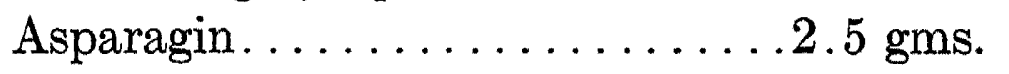

$\mathrm{Na}_{2} \mathrm{HPO}_{4} \ldots \ldots \ldots \ldots \ldots \ldots \ldots$ gm. $\quad 250$ c.c.

Distilled water... .........100 c.c.

Nitrogen-free Medium for Bacteria (Smith, 1905).

Triple-distilled water. . . . . . . . . 1000 c.c.

Cane sugar..................... 5 c.c.

Monopotassium phosphate, $\mathrm{KH}_{2} \mathrm{PO}_{4} \ldots \ldots \ldots \quad 2$ c.c.

Magnesium sulphate, $\mathrm{MgSO}_{4} \cdot 7 \mathrm{H}_{2} \mathrm{O} \ldots \ldots \ldots \quad 0.1$ c.c.

Sodium chloride, $\mathrm{NaCl} \ldots \ldots \ldots \ldots \ldots \ldots . \quad 0.5$ c.c.

All apparatus should be scrupuously clean and oũly chemicals of known purity should be used.

Dox's (1910) Solution for Fungi. Modified Czapek's Medium. (See Thom, 1910).

Distilled water................ 3000 c.c.

Magnesium sulphate, MgSO........... 1.5 gms.

Dibasic potassium phosphate, $\mathrm{K}_{2} \mathrm{HPO}_{4} \ldots \ldots 3.0$ gms.

Potassium chloride, $\mathrm{KCl} \ldots \ldots \ldots \ldots \ldots \ldots$. $1.5 \mathrm{gms}$.

Ferrous sulphate, $\mathrm{FeSO}_{4} \ldots \ldots \ldots \ldots \ldots . .03 \mathrm{gm}$.

Solidified media may be obtained by the addition of agar. 
Czapek's Solution. Cultivation of the fungi. (From Smith, 1905.

Distilled water...................... 1000 c.c.

Magnesium sulphate, $\mathrm{MgSO}_{4} \cdot 7 \mathrm{H}_{2} \mathrm{O} \ldots \ldots \ldots .50 \mathrm{gm}$.

Dipotassium phosphate, $\mathrm{K}_{2} \mathrm{HPO}_{4} \ldots \ldots \ldots \ldots 1.00 \mathrm{gm}$.

Potassium chloride, $\mathrm{KCl} \ldots \ldots \ldots \ldots \ldots \ldots .6 .50 \mathrm{gm}$.

Ferrous sulphate, $\mathrm{FeSO}_{4} \ldots \ldots \ldots \ldots \ldots \ldots .01 \mathrm{gm}$.

Sodium nitrate, $\mathrm{NaNO}_{3} \ldots$. . . . . . . . . . 2.00 gms.

Cane sugar....................

Pasteur's Medium (after Besson).

Water....................... 100 gms.

Candied sugar............... 10 gms.

Ammonium tartrate.............. . . $0.1 \mathrm{gm}$.

Ash of yeast. . . . . . . . . . . . . $0.075 \mathrm{gm}$.

Raulin's Solution for Molds.

Distilled water.................... 1500 c.c.

Cane sugar, $\mathrm{C}_{12} \mathrm{H}_{22} \mathrm{O}_{11} \ldots \ldots \ldots \ldots \ldots \ldots \ldots$ gms.

Tartaric acid, $(\mathrm{CHOH})_{2}(\mathrm{COOH})_{2} \ldots \ldots \ldots \ldots \quad 4$ gms.

Ammonium nitrate, $\mathrm{NH}_{4} \mathrm{NO}_{3} \ldots \ldots \ldots \ldots .4 \mathrm{gms}$.

Ammonium phosphate, $\left(\mathrm{NH}_{4}\right)_{2} \mathrm{HPO}_{4} \ldots \ldots, 0.6$ gms.

Magnesium carbonate, $\mathrm{MgCO}_{3} \ldots \ldots \ldots \ldots .0 .4 \mathrm{gm}$.

Ammonium sulphate, $\left(\mathrm{NH}_{4}\right)_{2} \mathrm{SO}_{4} \ldots \ldots \ldots \ldots 0.25 \mathrm{gm}$.

Zinc sulphate, $\mathrm{ZnSO}_{4} \cdot 7 \mathrm{H}_{2} \mathrm{O} \ldots \ldots \ldots \ldots \ldots 0.07 \mathrm{gm}$.

Iron sulphate, $\mathrm{FeSO}_{4} \cdot 7 \mathrm{H}_{2} \mathrm{O} \ldots \ldots \ldots \ldots \ldots .0 .07 \mathrm{gm}$.

Potassium silicate, $\mathrm{KSiO} \ldots \ldots \ldots \ldots \ldots .0 .07 \mathrm{gm}$.

Lipman-Brown Medium (1910).

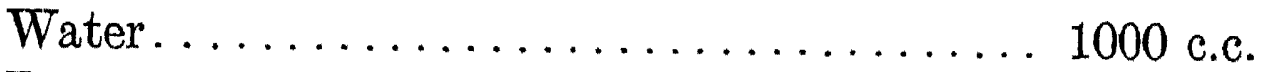

Dextrose, $\mathrm{C}_{6} \mathrm{H}_{12} \mathrm{O}_{6} \ldots \ldots \ldots \ldots \ldots \ldots \ldots \ldots$ gms.

Dipotassium hydrogen phosphate, $\mathrm{K}_{2} \mathrm{HPO}_{4} \ldots 0.50 \mathrm{gm}$.

Magnesium sulphate, $\mathrm{MgSO}_{4} \cdot 7 \mathrm{H}_{2} \mathrm{O} \ldots \ldots \ldots 0.20 \mathrm{gms}$.

Peptone......................... 0.05 gm.

Agar.......................20.00 gms.

Jordan's Non-protein Medium.

Redistilled water................... 1000 c.c.

Asparagin, $\mathrm{C}_{2} \mathrm{H}_{3}\left(\mathrm{NH}_{2}\right){ }_{\mathrm{COOH}}^{\mathrm{CONH}_{2}} \ldots \ldots \ldots \ldots \quad 2$ gms

Magnesium sulphate, $\mathrm{MgSO}_{4} \cdot 7 \mathrm{H}_{2} \mathrm{O} \ldots \ldots \ldots \quad 1 \mathrm{gm}$.

Dipotassium hydrogen phosphate, $\mathrm{K}_{2} \mathrm{HPO}_{4}$... $1 \mathrm{gm}$. 
Beijerinck's Medium for Nitrate Reduction (after Fred).

Dibasic potassium phosphate, $\mathrm{K}_{2} \mathrm{HPO}_{4} \cdot \ldots \ldots \quad 0.5 \mathrm{gm}$.

Potassium nitrate, $\mathrm{KNO}_{3} \ldots \ldots \ldots \ldots \ldots \ldots$ gms.

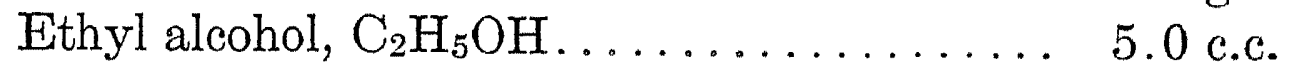

Tap water........................... 1000 c.c.

Winogradsky's Medium for Nitrite Formation (from Ferd., I9I6).

Ammonium sulphate, $\left(\mathrm{NH}_{4}\right)_{2} \mathrm{SO}_{4} \ldots \ldots \ldots \ldots 1.0 \mathrm{gm}$.

Dibasic potassium phosphate, $\left(\mathrm{K}_{2} \mathrm{HPO}_{4}\right) \ldots . \quad 1.0 \mathrm{gm}$.

Magnesium sulphate, $\mathrm{MgSO}_{4} \cdot 7 \mathrm{H}_{2} \mathrm{O} \ldots \ldots \ldots \quad 0.5 \mathrm{gm}$.

Sodium chloride, $\mathrm{NaCl}_{0} \ldots \ldots \ldots \ldots \ldots . \ldots 2.0$ gms.

Ferrous sulphate, $\mathrm{FeSO}_{4} \cdot 7 \mathrm{H}_{2} \mathrm{O} \ldots \ldots \ldots \ldots .0 .4 \mathrm{gm}$.

Magnesium carbonate, $\mathrm{MgCO}_{3}$ in excess. . . $5.0 \mathrm{gm}$.

Distilled water................... 1000 c.c.

\section{Giltay and Aberson's (1891) Solution. For Denitrifying Bacteria.}

Distilled water.................... 1000 c.c.

Potassium nitrate, $\mathrm{KNO}_{3} \ldots \ldots \ldots \ldots \ldots \ldots .2$ gms.

Magnesium sulphate, $\mathrm{MgSO}_{4} \ldots \ldots \ldots \ldots \ldots 2$ gms.

Citric acid................... 5 gms.

Dipotassium hydrogen phosphate, $\mathrm{K}_{2} \mathrm{HPO}_{4} \ldots \quad 2$ gms.

Calcium chloride, $\mathrm{CaCl}_{2} \ldots \ldots \ldots \ldots \ldots \ldots .0 .2 \mathrm{gm}$.

Sodium carbonate, $\mathrm{Na}_{2} \mathrm{CO}_{3} \ldots \ldots \ldots \ldots \ldots+.25$ gms.

Asparagin, $\mathrm{C}_{2} \mathrm{H}_{3}\left(\mathrm{NH}_{2}\right) 〈_{\mathrm{COOH}}^{\mathrm{CONH}_{2}} \ldots \ldots \ldots \ldots 1.00 \mathrm{gm}$.

This medium should be placed in fermentation tubes before sterilization. To prevent any decomposition, the potassium nitrate and asparagin should be dissolved together. The other salts should be dissolved in another portion of water and the whole brought up to a liter.

\section{Omelianski's and Winogradski's Medium.}

Ammonium sulphate, $(\mathrm{NH}) \mathrm{SO} \ldots \ldots \ldots \ldots \ldots \quad 1 \mathrm{gm}$.

Potassium bi-phosphate, $\mathrm{KH}_{2} \mathrm{PO}_{4} \ldots \ldots \ldots \ldots 1 \mathrm{gm}$.

Magnesium sulphate, $\mathrm{MgSO}_{4} \cdot 7 \mathrm{H}_{2} \mathrm{O} \ldots \ldots \ldots, 0.5 \mathrm{gm}$.

Sodium chloride, $\mathrm{NaCl}$. ............ 2.0 gms.

Ferrous sulphate, $\mathrm{FeSO}_{4} \cdot 7 \mathrm{H}_{2} \mathrm{O} \ldots \ldots \ldots \ldots . .4 \mathrm{gm}$.

Basic magnesium carbonate, $4 \mathrm{MgCO}_{3} \cdot \mathrm{Mg}(\mathrm{OH})_{2}$ Excess

Distilled water................... 1000 c.c. 
MacKensie's Culture Fluid (from Smith, I905).

Acid ammonium tartrate........... $1.5 \mathrm{gms}$.

Bipotassium phosphate, $\mathrm{KH}_{2} \mathrm{PO}_{4} \ldots \ldots \ldots \ldots 2.5$ gms.

Potassium sulphate, $\mathrm{K}_{2} \mathrm{SO}_{4} \ldots \ldots \ldots \ldots \ldots$. 1.5 gms.

Sodium chloride, $\mathrm{NaCl} \ldots \ldots \ldots \ldots \ldots \ldots .0 .5 \mathrm{gm}$.

Glucose, $\mathrm{C}_{6} \mathrm{H}_{12} \mathrm{O}_{6} \ldots \ldots \ldots \ldots \ldots \ldots \ldots \ldots$. $5.0 \ldots \ldots$ gms.

Lactose, $\mathrm{C}_{12} \mathrm{H}_{22} \mathrm{O}_{11} . \ldots \ldots \ldots \ldots \ldots \ldots \ldots .5 .0$ gms.

Glycerol, $\mathrm{CH}_{2} \mathrm{OHCHOHCH}{ }_{2} \mathrm{OH} \ldots \ldots \ldots \ldots 15.0$ gms.

Distilled water.................... 1000 c.c.

Alkaline to phenolphthalein.

Proskauer and Beck's Culture Fluid (from Smith, I905).

Commercial ammonium carbonate $\left(\mathrm{NH}_{4}\right)_{2} \mathrm{CO}_{3} \quad 3.5$ gms.

Potassium phosphate, $\mathrm{K}_{3} \mathrm{PO}_{4} \ldots \ldots \ldots \ldots \ldots .1 .5$ gms.

Magnesium sulphate, $\mathrm{MgSO}_{4} \cdot 7 \mathrm{H}_{2} \mathrm{O} \ldots \ldots \ldots 2.5$ gms.

Glycerol, $\mathrm{CH}_{2} \mathrm{OHCHOHCH}{ }_{2} \mathrm{OH} \ldots \ldots \ldots \ldots 15.0$ gms.

Distilled water.................... 1000 c.c.

\section{Fermi's Culture Fluid.}

Distilled water.................. 1000 c.c.

Magnesium sulphate, $\mathrm{MgSO}_{4} \cdot 7 \mathrm{H}_{2} \mathrm{O} \ldots \ldots \ldots \quad 0.2 \mathrm{gm}$.

Dipotassium hydrogen phosphate, $\mathrm{HK}_{2} \mathrm{PO}_{4} \ldots 1.0 \mathrm{gm}$.

Ammonium phosphate, $\left(\mathrm{NH}_{4}\right)_{2} \mathrm{HPO}_{4} \ldots \ldots$. $10 \mathrm{gms}$.

Glycerol, $\mathrm{CH}_{2} \mathrm{OHCHOHCH}{ }_{2} \mathrm{OH} \ldots \ldots \ldots .45$ gms.

This may be added to agar in place of peptone broth or to silicate jelly in which case the volume of $\mathrm{H}_{2} \mathrm{O}$ must be reduced. (Smith.)

Fraenkel and Voges' Solution (from Smith, I905).

Water.......................... 1000 c.c.

Sodium chloride, $\mathrm{NaCl} . \ldots \ldots \ldots \ldots \ldots \ldots . \quad 5$ gms.

Dipotassium phosphate, $\mathrm{K}_{2} \mathrm{HPO}_{4} \ldots \ldots \ldots \ldots \quad 2$ gms.

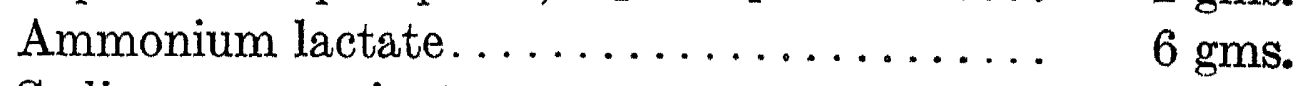

Sodium asparaginate.............. 4 gms.

\section{Uschinsky's Protein Free Medium.}

Asparagin . . . . .

Ammonium lactate.............. 10.0 gms.

Sodium chloride, $\mathrm{NaCl} \ldots \ldots \ldots \ldots \ldots \ldots . \quad 5.0$ gms.

Magnesium sulphate, $\mathrm{MgSO}_{4} \cdot 7 \mathrm{H}_{2} \mathrm{O} \ldots \ldots \ldots \quad 0.2 \mathrm{gm}$.

Calcium chloride, $\mathrm{CaCl}_{2} \ldots \ldots \ldots \ldots \ldots \ldots . \quad 0.1 \mathrm{gm}$.

Dipotassium phosphate, $\mathrm{K}_{2} \mathrm{HPO}_{4} \ldots \ldots \ldots \ldots \quad 1.0 \mathrm{gm}$.

Distilled water................ 1000 c.c. 
When these substances are thoroughly dissolved, add 40 c.c. of glycerol. Tube and sterilize.

Uschinsky's Medium (Smith's 1905 Modification).

Distilled wate

1000 c.c.

Ammonium lactate............... 5 gms.

Sodium asparaginate $\ldots \ldots \ldots \ldots \ldots \ldots .2 .5 \mathrm{gms}$.

Sodium sulphate, $\mathrm{NaSO}_{4} \cdot 10 \mathrm{H}_{2} \mathrm{O} \ldots \ldots \ldots \ldots .2 .5 \mathrm{gms}$.

Sodium chloride, $\mathrm{NaCl} \ldots \ldots \ldots \ldots \ldots \ldots .2 .5$ gms.

Dipotassium phosphate, $\mathrm{K}_{2} \mathrm{HI} \mathrm{J}_{4} \ldots \ldots \ldots 2.5 \mathrm{gms}$.

Calcium chloride, $\mathrm{CaCl}_{2} \ldots \ldots \ldots \ldots \ldots \ldots .010 \mathrm{gm}$.

Magnesium sulphate, $\mathrm{MgSO}_{4} \ldots \ldots \ldots \ldots \ldots .010 \mathrm{gm}$.

Recommended for use with starch jelly.

\section{Blood and Blood Serum Media}

Blood Serum. Beef blood should be collected at the abattoir in clean containers and placed in the refrigerator until a clot has formed. The clear serum should then be decanted or better siphoned off into a sterile flask. For the preparation of Loeffler's blood serum, 1 part of glucose broth is mixed with 3 parts of the serum. This is then distributed into test tubes (or other containers) and placed in the inspissator in a slanted position. Small tin ointment boxes make good containers when the blood serum is to be used for routine diphtheria examinations. After coagulation has been accomplished, the boiled serum should be sterilized in the autoclave at a temperature of about $112^{\circ} \mathrm{C}$. If desired, intermittent heating in the Arnold may be used.

Blood Agar (Besson). 1. Melt tubes of glycerol agar and cool to $40^{\circ} \mathrm{C}$.

2. Add to each tube a small quantity (1 c.c.) of blood from a rabbit's artery. Mix without shaking the tubes and cool in sloping position.

A solution of hemoglobin may be used instead of blood.

Blood Agar for Streptococci (Becker, 1916). Standard agar used in water analysis is placed in quantities of 40 to $100^{\circ}$ c.c. in flasks. These are heated until the agar is melted, and then cooled to $45^{\circ}$ or $50^{\circ} \mathrm{C}$. One cubic centimeter defibrinated human blood is added for each 6 c.c. of the agar base and the whole is thoroughly shaken. Approximately 7 c.c. are used for each plate. Surface streaks are made and observations made after twenty-four hours at $27^{\circ} \mathrm{C}$. Isolated colonies only should be studied. 
Serum Agar. 1. Dissolve 1.5 grams of agar in 100 c.c. distilled water. Filter, tube (5 c.c. $\mathrm{m}$ each tube) and sterilize.

2. Cool to $40^{\circ} \mathrm{C}$. To each tube add an equal volume of sterile serum. Mix gently by rolling the tube and cool in a slanting position.

Alkaline Blood Agar (Dieudonne). Mix equal volumes of bovine blood and a normal solution of potassium hydroxide. Steam for onehalf hour; mix three volumes of this alkalin blood with seven volumes of neutral (to litmus) meat broth agar and pour plates. Dry the plates at $60^{\circ} \mathrm{C}$. for half a hour and keep at room temperature for at least twenty-four hours before using in order to free them from ammonia, the evolution of which would prevent the growth of Microspira cholerae. (Quoted from Goldberger, 1913.)

Alkalin Hemoglobin Agar for Microspira Cholerae (Esch, 1910). Dissolve 5 gms. of horse hemoglobin (Merck) in 30 c.c. of a half normal sodium hydroxide solution. Steam for one-half hour. Mix 15 c.c. of this alkalin hemoglobin solution with 85 c.c. of neutral (to litmus) meat broth agar, pour plates and dry at room temperature with covers removed. The plates are ready for use as soon as dry-a matter of about a half a hour. (Quoted from Goldberger.)

\section{MrLK Media}

Casein Agar (Ayres, 1913). Preparation of 1 liter.

Casein solution: 300 c.c. distilled water.

10 gms. casein (Eimer \& Amend C.P. casein prepared according to Hammarsten).

7 c.c. normal sodium hydroxide.

Agar solution:

500 c.c. distilled water.

10 gms. agar.

After dissolving casein make up to 500 cubic centimeters.

To 300 c.c. of water (distilled) add:

10 gms. casein (Emmer \& Amend C.P. casein prepared according to Hammarsten).

7 c.c. normal sodium hydroxide.

Dissolve casein by heating to boiling. It is desirable to let this solution stand for several hours to get a perfect solution. This is not necessary, however. Make up volume to 500 c.c. and bring the 
reaction of the solution to between +0.1 and +0.2 Fuller's scale. Do not allow solution to become alkaline to phenolphthalein or over +0.2 . If the casein is wcighed accurately and the normal solution accurate the reaction will be about +0.2 .

The agar solution is prepared by dissolving $10 \mathrm{gms}$. agar in 500 c.c. of water. Both casein and agar solutions should be filtered, then mixed. Tube and sterilize in autoclave under pressure for twenty minutes; then cool the tubes quickly in cold water or ice water. The final reaction of the medium will be about +0.1 Fuller's scale. If the medium is alkaline the bacterial growth will be restricted. If the medium is more than +0.1 some of the casein may be precipitated during sterilization. The casein agar should be clear and almost colorless when poured in a Petri dish. Sometimes the casein will be slightly precipitated during sterilization or the cooling, but it is of no consequence since on pouring into plates the precipitate on account of its finely divided condition becomes invisible.

Whey Agar. Add a few drops of acetic acid to boiling milk until the casein is precipitated. Neutralize, or bring to 1 per cent t. Dissolve 1 per cent peptone, 2 per cent dextrose and 1.5 per cent agar. Filter, tube and sterilize.

Plain Milk. This may be made from fresh skimmed milk. It may be used in many of the different laboratory apparatus. Very often it will be found convenient to make it from desiccated milk.

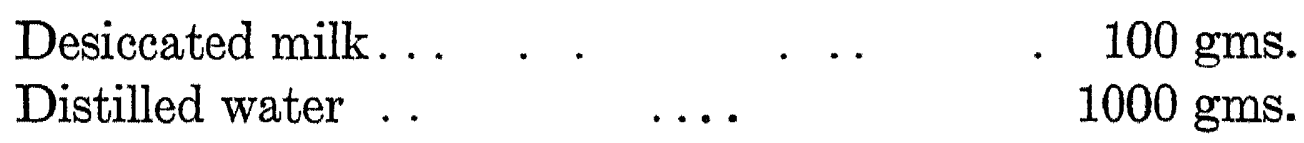

The milk powder should be added to about 200 c.c. of the distilled water and thoroughly mixed. Beating with an egg beater often serves this purpose. It should then be made up to a liter. Sterilize in the Arnold.

Litmus Milk. Add sterile litmus or azolitmin to plain skimmed milk until the color is a distinct lavender. Tube in any of the laboratory apparatus and sterilize.

Litmus Whey (Petruschky). Fresh milk is slightly warmed and treated with dilute hydrochloric acid to precipitate the casein. This is filtered off and dilute sodium carbonate added to the filtrate to neutrality. This is then steamed for two hours to precipitate any casein which is changed to acid albumin by the dilute hydrochloric acid. After filtration a clear neutral solution should be the result. This is colored with litmus, tubed and sterilized. After growth the amount of acid may be determined by standard reagents. 
Rice Milk (Besson).

Milk...................... 150 gms.

Peptone broth................ 50 gms.

Powdered rice................. 100 gms.

Distribute the mixture in Petri dishes 1-2 com. deep. Heat to $115^{\circ} \mathrm{C}$. for twenty minutes. The mixture solidifies and forms an opaque layer.

Dialyzed Milk. Dialyzed milk may be prepared by the addition of .10 c.c. of hydrogen peroxide to 500 c.c. of milk. Two hours later hydrogen peroxide should be added again and after four to five hours the whole is dialyzed. The sugar content is reduced 50 per cent and the normal quantities of fat and proteins are preserved. The milk is diluted one-fifth.

Milk Agar (Hastings, 1904). This medium is prepared by adding to ordinary nutrient agar, which has been melted and cooled to $50^{\circ} \mathrm{C}$. 10 per cent sterile skimmed milk. If the milk is added to the agar while hot, the casein will be precipitated in course flocculent masses. The medium is poured into sterile Petri dishes and after hardening is streaked with the cultures. After incubation, if proteolytic action is present, there will be a clear zone about the streak which will not change when treated with dilute acids. This distinguishes the acid formers from the proteolytic bacteria.

\section{BIBLIOGRAPHY}

Ayers, S. H. 1913. Casein Media Adapted to the Bacterial Examination of Milk. Ann. Rept. Bureau of Anim. Ind., 1913, 225-235.

Banzhaf, E. J. and Hrrschleifer, L. J. 1914. Studies in Preparation of Diphtheria Toxin Media (Analysis of Nutrient Materials) Collected Studies, Bureau of Laboratories, City of New York, 8, 223-225.

BARLow, B. 1912. A Spoilage of Canned Corn Due to a Thermophilic Bacteria. Thesis for Master's Degree, University of Illinois, June, 1913.

Becker, W. C. 1916. The Necessity of a Standard Blood Agar Plate for the Determination of Hemolysis by Streptococci. Jour. Inf. Dis., 19, 754-759.

Bendrck, A. J. 1913. The Use of Calcium Carbonate in Solid Media for the Differentiation of Sugar Fermenting Bacteria, Especially of the Colontyphoid Group. Trans. 15th Intern. Cong. Hygiene and Demography, Vol. II, 40.

Berry Jane. 1914-15. Studies on Laboratory Media. Collected Studies, Bureau of Laboratories, City of New York, 8, 288-293. 
Berthelot, A. 1917. Sur l'emploi du Bouillon de Légumes comme milieu de Culture. Comp. Rend. soc. biol., 80, 131-132.

Besson, A. 1913. Practical Bacteriology, Microbiology, and Serum Therapy. Translated by Hutchens, H. J. Longmans, Green \& Co.

Bigelow, W. D. and Cook, F. C. 1908. Meat Extracts and Similar Preparations. U. S. Depart. of Agriculture, Bureau of Chemistry, Bulletin 114.

Breed, R. S. 1915. Standard Method for Determining Nitrate Reduction. Science N. S., 41, 661.

Buchanan, R. E. 1909. The Gum Produced by B. radicicola. Cent. Bakt. Abt., 2, 22, 392-396.

Burling, H. A. and Levene, M. 1918. Concentration of Glucose and Lactose and Viability of Coli-like Bacteria. Amer. Jour. Pub. Health, 8, 306-307.

Cannon, P. R. 1916. A Rapid and Simple Indol Test. Jour. of Bact., $1,535-536$.

Cook, F. C. 1914. The Partition of Nitrogen of Plant, Yeast, and Meat Extracts. Jour. Amer. Chem. Soc., 36, 1551-1556.

Cons, H. J. 1914. Culture Media for Use in the Plate Method of Counting Soil Bacteria. New York Agric. Exp. Sta., Bulletin 38.

Conn, H. W. 1915. Standard Methods for Determining the Purity of Milk. Public Health Reports, 30, 3-48. Reprint 295.

Dolt, M. L. 1908. Simple Synthetic Media for the Growth of B. coli and for its Isolation from Water. Jour. Inf. Diseases, 5, 616-626.

DrIGalski, V. and Conradi, H. 1902. Ueber ein Verfahren zum Nachweis der Typhusbacillen. Zeit. Hygiene, 39, 283.

Emmetт, A. D. and Girs, W. G. 1907. On the Chemical Relation between Collagen and Gelatin. Jour. Biol. Chem., 3, 33.

Esch. 1910. Zum Bakteriologischen Cholera nachweise mittels der Blutalkali-nahrboden. Deutsch. med. Wochenschr., 36, 559-561.

Eyre, J. W. H. 1903. The Elements of Bacteriological Technique. W. B. Saunders \& Co., Philadelphia.

Fraenkel, C. 1894. Beitrage zur Kenntniss des Bakterienwachstums auf eiweissfreien Nährlosungen. Hyg. Rundschau. 1894. 769.

Fellers, C. F. 1916. The Analysis, Purification, and Some Chemical Properties of Agar Agar. Jour. Ind. Eng. Chem., 8, 1128-1133.

Fred, E. B. 1916. A Laboratory Manual of Soil Bacteriology. W. B. Saunders \& Co., Philadelphia.

GAGE, S. H. and Adams, G. O. 1904. Studies on Media for the Quantitative Estimation of Bacteria in Water and Sewage. Jour. Inf. Diseases, $1,358$.

Girtax, E. and Aberson, J. H. 1891. Recherches sur un mode de Denitrification et sur le Schizomycetes qui et Produit. Archives Neerlandaises des Sciences ex. et nat., 25, 341-361.

GoAdBy, K. W. 1903. The Mycology of the Mouth. Longmans, Green \& Co. 
Gondon, M. H., Hine, T. G. M. and Flack, M. 1916. Cultural Requirements of Meningococcus Brit. Med. Jour., 1916, 678.

Goldberger, J. 1913. Some New Cholera Selective Media. Bulletin 91, Hyg. Lab., Washington.

Hastings, E. G. 1904. The Action of Various Classes of Bacteria on Casein as Shown by Milk Agar Plates. Cent. Bakt. Abt., 2, 12, 590-592.

Hasseltine, H. E. 1917. The Bacteriological Examination of Water. Comparative Studies of Media Used. Public Health Reports, 32, 1878-1887.

Hesse, W. and Nredner. 1898. Die Methodik der Bakteriologischen Wasseruntersuchungen. Zeit. Hygiene, 29, 454-462.

Hiss, P. H. 1897. On a Method of Identifying and Isolating Bacillus Typhosus Based on a Study of Bacillus Typhosus and Members of the Colon Group in Semi-solid Culture Media. Jour. Exper. 'Med., 2, 701-710.

Hiss, P. H. 1902. New and Simple Media for the Differentiation of the Colonies of Typhoid, Colon, and Allied Bacteria. Jour. Medical Research, $8,148$.

Houston, A. C. 1914. Eighth Annual Report Metropolitan Water Board, 41.

Kendali, A. I. and Day, A. 1911. The Rapid Isolation of Typhoid Paratyphoid and Dysentery Bacilli. Jour. Medical Research, 25, 95-99.

Krumwiede, C., Pratt, C. S., and McWildiams, H. I. 1916. The Use of Brilliant Green for the Isolation of Typhoid and Paratyphoid Bacilli from Feces. Jour. Infectious Diseases, 18, 1-13.

Levene, M. 1918. A Simplified Fuchsin-sulphite (Endo) Agar. Abstracts of Bacteriology, 2, 13.

Levene, M. 1918. Presumptive Test for Bacillus Coli. Eng. Contr., 49, 34. Jour. Inf. Dis, 23, 43-47.

Lipman, J. G. and Brown, P. E. 1910. Media for the Quantitative Estimation of Soil Bacteria. Cent. Bakt. Abt., 2, 25, 447-454.

MacConkex, A. 1900. Experiments on the Differentiation and Isolation from Mixtures of Bacillus Coli Communis and Bacillus Typhosus by the Use of Sugars and the Salts of Bile. The Thompson Yates Laboratory, Reports III, 41.

McBeth, I. G. and Scales, F. M. 1913. The Destruction of Cellulose by Bacteria and Filamentious Fungi. U. S. Department of Agriculture, Bureau of Animal Industry, Bull. 266.

Myer, E. M. 1917. The Use of a Three Per Cent.Lactose Litmus Agar Plate for Demonstration of B. coli in Water Examination. Jour. Bact., $2,237-240$.

Mudge, C. S. 1917. The Effect of Sterilization upon Sugars in Culture Media. Jour. Bact., 2, 403-415.

North, C. E. 1909. An Agar Gelatin Medium. Jour. Med. Research, 20, 359-363.

Petruschky. Quoted from Moir and Ritchie's Manual of Bacteriology. Macmillan Company. 6th Edition. New York. 
Redfield, H. W. 1912. A Study of Hydrogen Sulfid Production by Bacteria and its Significance in the Sanitary Examination of Water. Thesis, Cornell University, 1912.

Rettger; L. F. 1906. Studies on Putrefaction. Jour. Biol. Chem., 2, 71-86.

Russeld, F. F. 1911. Isolation of B. typhosus from Feces and Urine. Jour. Med. Research, 25, 217.

SAvige, W. G. 1901. Neutral Red in the Routine Bacteriological Examination of Water. Jour. Hyg., 1, 437.

Smith, E. F. 1898. Potato as a Culture Medium. Proc. Am. Assoc. Adv. 'Science. 47, 411. Cent. Bakt. Abt., 2, 5, 102.

Smith, E. F. 1905. Bacteria in Relation to Plant Diseazes. Carnegie Institution of Washington.

Sullrvan, M. X. 1905. Synthetic Culture Media and the Blochemistry of Bacterial Pigments. Jour. Med. Research, 14, 109-160.

Thom, C. A. 1906. Fungi in Cheese Ripening; Camembert and Roquefort. U. S. Department of Agriculture, Bureau of Animal Industry. Bulletin 82.

Thom, C. A. 1910. Cultural Studies of Species Penicillium. U. S. Dept. of Agr., Bureau of Animal Industry. Bulletin 118.

Teague, O. 1918. Stock Sodium Sulfite Solution for Endo Agar. Jour. Amer. Med. Assn., 70, 454.

Teague, O. and Clurman, A. W. 1916. An Improved Brilliant-green Culture Medium for the Isolation of Typhoid Bacilli from Stools. Jour. Inf. Diseases, 18, 647-652.

Thiele, L. 1912. The Manufacture of Gelatin. Jour. Ind. Eng. Chem., 4, 446-451.

Uschinsky. 1893. Ueber eine eiweissfreie Nahrlosung für pathogene Bakterien nebst einigen Bemerkungen über Tetanusgift. Cent. Bakt. Abt., I, $14,316-319$.

Van Delden, A. 1904. Beitrag zur Kenntniss der Sulfatreduktion durch Bakterien. Cent. Bakt. Abt., II, 11, 88-119.

Vedder, E. B. 1915. Starch Agar as a Useful Culture Medium. Jour. Infectious Diseases, 16, 385-388.

WhITTAKeR, H. A. 1911. The Source, Manufacture and Composition of Commercial Agar-agar. Jour. American Public Health Assn., 1, 632-639. 


\section{CHAPTER III}

\section{STAINING TECHNIQUE}

Since bacteria are such small organisms, they must be exammed collectively and only in rare instances as individuals. Barber (1914) devised a method whereby single cells may be isolated, but this procedure is better adapted to advanced work where very accurate results are desired. For ordınary work the bacteria may be grown either in liquid or solid media. From these media they may be put on slides according to instructions which are given later. After these slides or cover glasses have been examined they should be dropped into a disinfectant since Rettger (1913) has pointed out that the staining procedure may not kill the organism.

Hanging Drop. This preparation enables the bacteriologist to observe bacteria under the microscope in the free condition and not fastened to the slide as in the staining methods. There are several adaptations of this method all of which lead to the same results, i.e., suspending the organisms in a drop of fluid in a well of some sort. Some of the more important of these are mentioned below.

Koch's Concave Slide. This consists of a plain glass slide with a hollow well ground in the middle. The cover glass with the culture is placed over this. Such a slide is quite satisfactory for the examination of bacteria, but may be too shallow for the study and continued observation of molds since the mycelium will soon.fill the cell. For this purpose other slides of a similar nature will be found to give better results.

Böttcher's Moist Chamber. This is essentially for the same purpose as the concave slide, but is constructed in such a way that a deeper well is secured. These may be made by fastening to an ordinary glass slide a glass or rubber ring about $16 \mathrm{~mm}$. in diameter and 2 to $3 \mathrm{~mm}$. high. These are inexpensive and may be easily glued to the slide. A preparation made with one of these may be securely sealed with vaseline and thereby prevent evaporation which is an important factor if continued observations are. to be made. Other forms of apparatus which may be used in this relation are shown in Fig. 30. 
Hanging Block. This preparation allows the bacteria to develop but prevents their movement and thus holds them in one place for observation. In this way the relation of cell to cell may be studied. They may be prepared in the following manner. A sterle agar plate is poured and allowed to harden. From this by means of a sterle scalpel or spatula is cut a block of the agar which is picked up on the spatula. On its surface is rubbed a small amount of the culture which
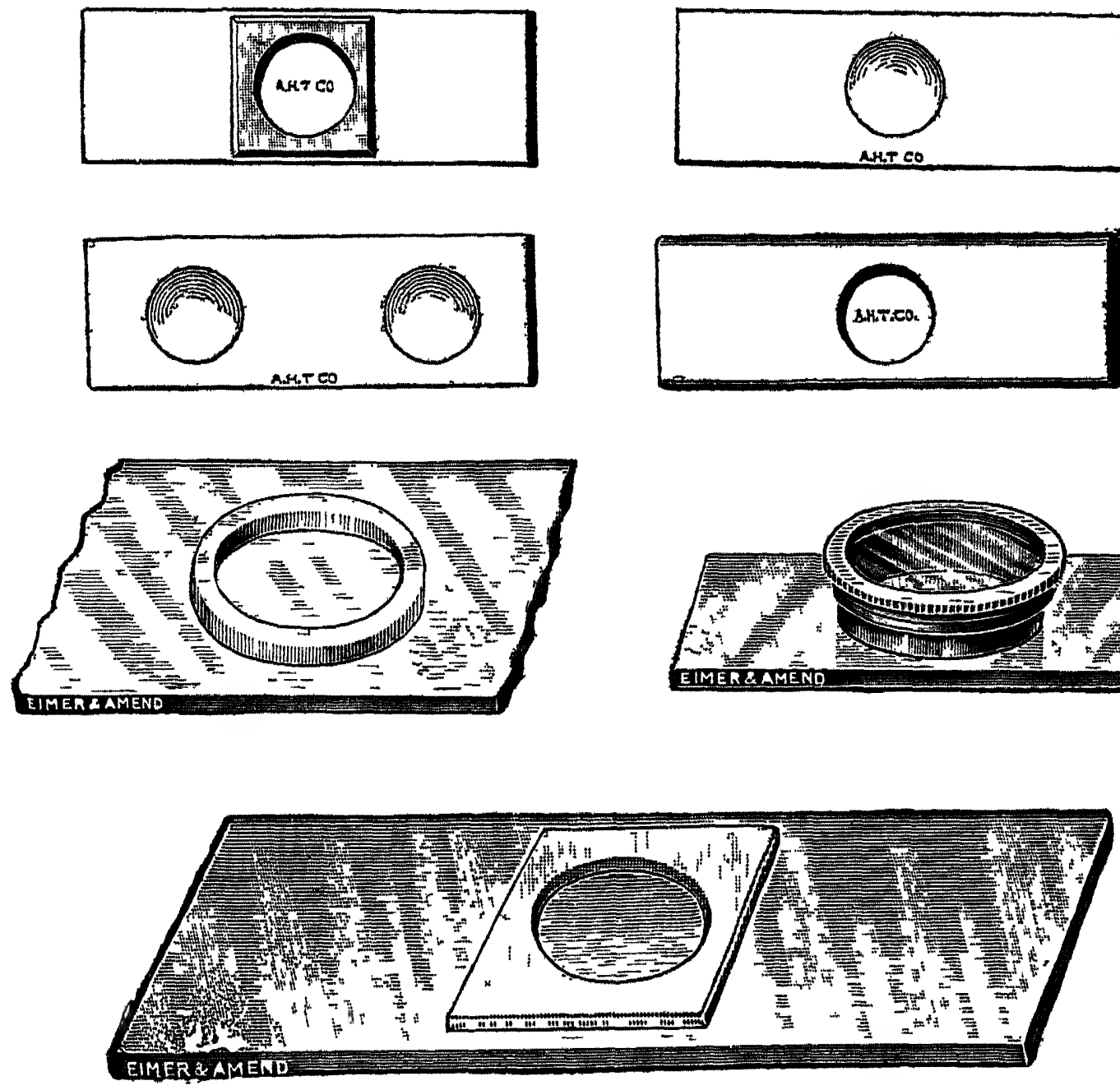

Frg. 30.-Types of Concave Slides and Moist Chambers for Making Hanging Drop and Hanging Block Mounts.

is under study. The agar block is then put up on a sterile cover glass in such a way that the cells of the organism will be between agar block and the cover glass, cover slip. About the edge of the cover slip should be placed a small amount of vaseline to hold the slip in place and also to prevent evaporation if the mount is to be incubated. These preparations may be kept for some time if desired. Hill (1902) has discussed the application of this preparation to the observation of developing bacteria. 
Chinese Ink Preparations. The organisms are mounted in Chinese ink and are unstained. Therein lies the special advantage of such a preparation. Under the microscope the organisms appear as transpurent spots in a dark field. Before using, the ink should be sterilized
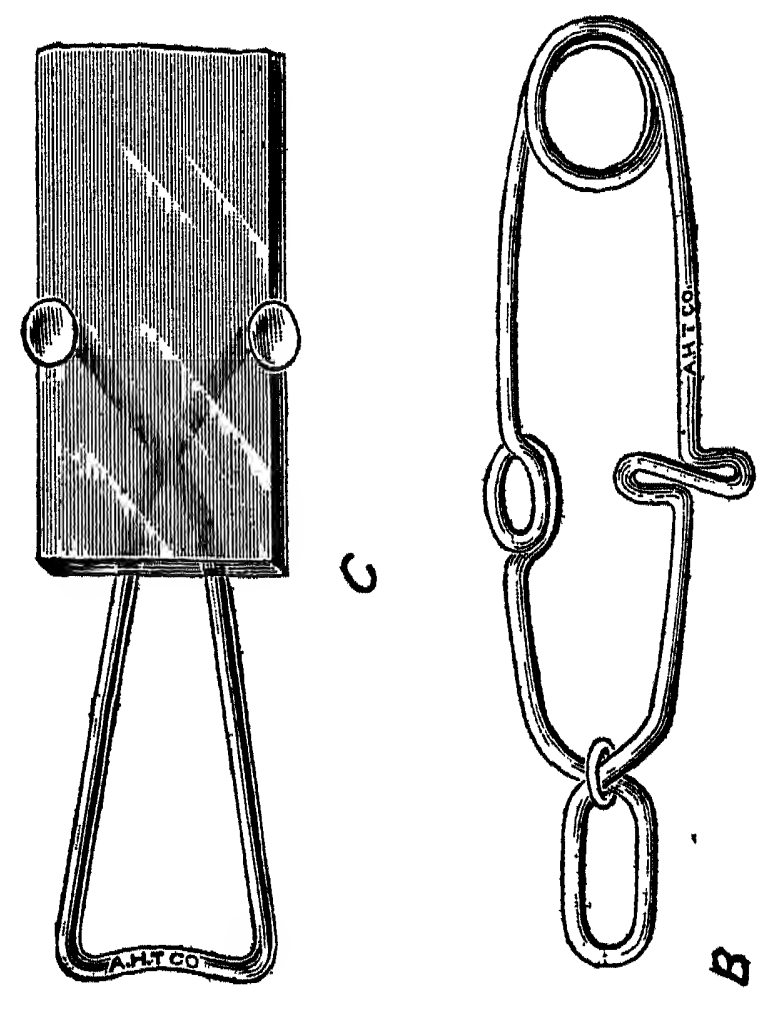

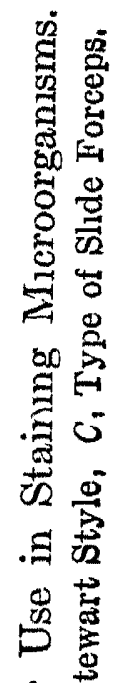
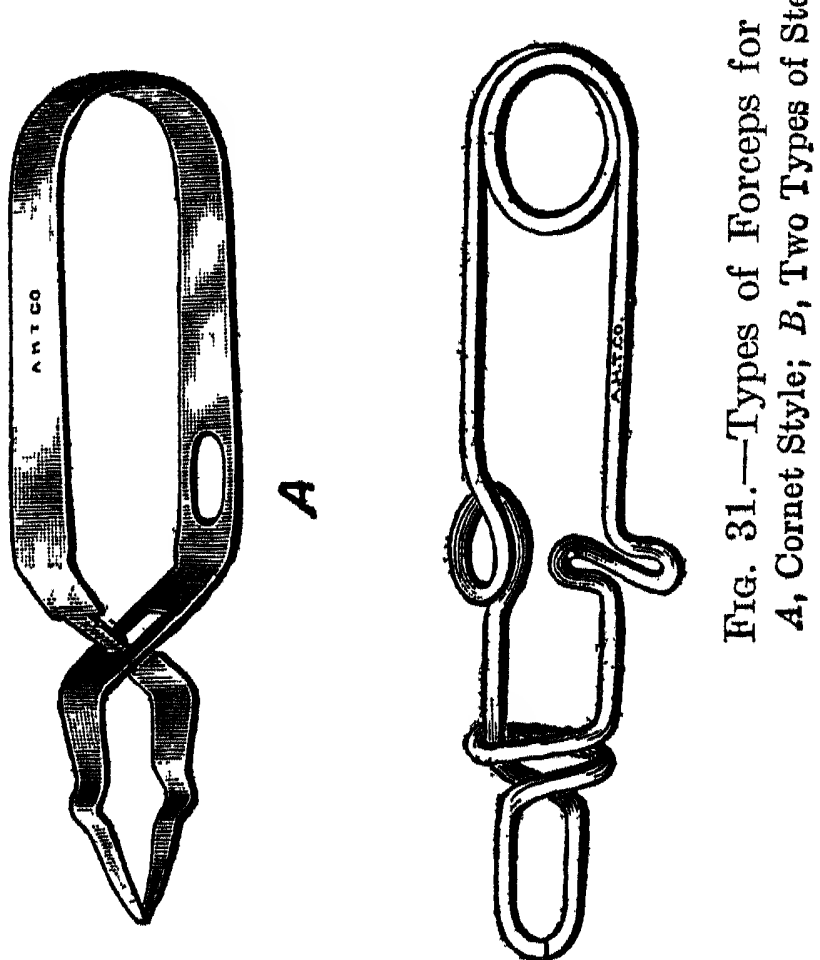

because it may contain many bacteria. After the ink has dried examination may be made either with or without a cover glass.

Dark Ground Illumination. By this method the bacteria are examined in the unstained condition. In order to be observable, the 
objects must be of low refractive index. This method of observing bacteria has been especially valuable for the study of Treponema pallidum and flagella of ordinary bacteria.

\section{Theory and Methods of Staining}

The staining of bacteria should be regarded as a delicate procedure and one requiring much care. It should not be considered-although it probably is in many cases-as a saturation of the cell with the dye. Rather should it be regarded as a chemical reaction involving the same laws which govern chemical changes. It doubtless follows the law of mass action.

Koch (1877) was one of the first to recognize the value of aniline dyes for staining. For convenience the aniline dyes have been divided into two classes-the basic and acid dyes. The basic aniline dyes contain amido groups while the acid dyes contain the hydroxyl groups. The basic dyes are usually used as the salts of hydrochloric acid while the acid dyes are secured as sodium or potassium salts. Unna (1888) has mentioned that these dyes are not always basic but may be salts neutral in character. They are called basic simply because the colored component is basic. The aniline dye does not separate into two parts, the basic or colored part attacking the cell protoplasm, but rather the entire molecule plays its part in the staining process. That the staining ability is determined by the solvent condition of the dye is shown by the following observations.

1. Pure alcoholic water-free solutions of the dyes do not stain.

2. Pure absolute alcohol will not decolorize while alcohol diluted with water decolorizes quickly.

3. The more perfect the solution of the dye in the solvent the lesser does its staining ability become. This helps to explain why absolute alcohol does not decolorize or why pure alcoholic solutions do not stain.

One of the most important essentials of the staining procedure is clean apparatus. All grease and foreign matter must be removed from the slides and cover glasses according to the procedure outlined under cleaning apparatus in the first chapter. After the slides have been thoroughly cleaned they may be kept in a salt-mouth bottle in alcohol. The cover slips may be kept in a short wide-mouth bottle containing alcohol or ether. In this way both the slides and cover glasses will be free from grease provided they are thoroughly cleaned and rinsed in distilled water. 
Preparation of the Smear. The smear on the slide or cover glass should be even and not too thick. For making this, a clcan cover glass is removed from the bottle and allowed to diy in the air or by flaming By means of a sterile platinum loop place a small diop of sterile water on the slide and rub a little of the culture in it. It is best not to stir too much but by gentle tappings add enough of the growth to the drop of water. Spread the drop of water over the glass

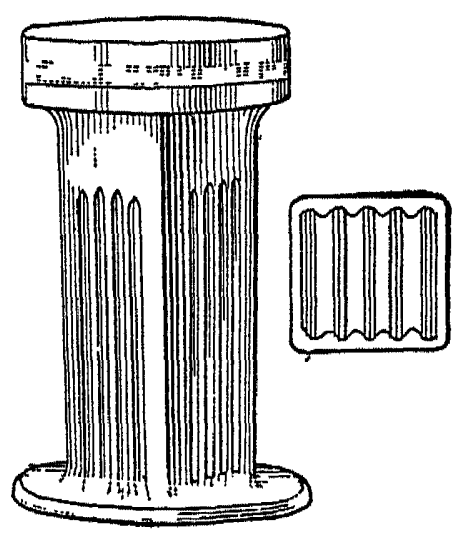

$A$

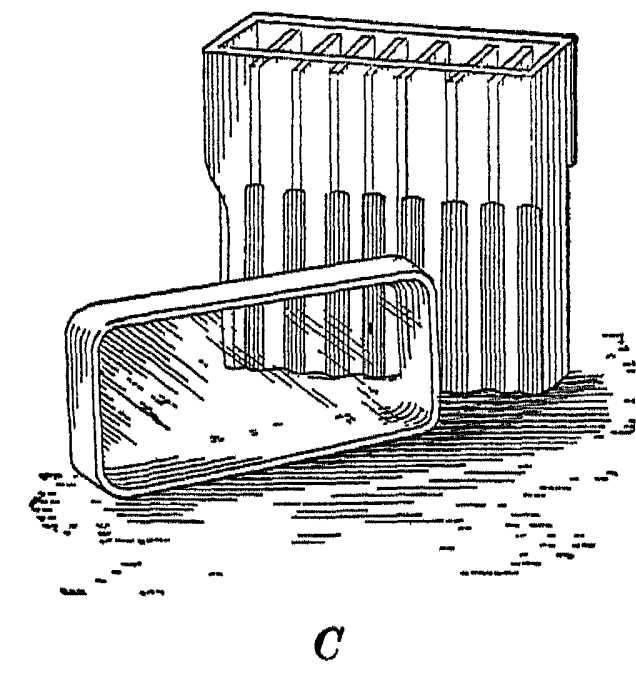

Frg 32 - Types of Staining Jars and Dishes

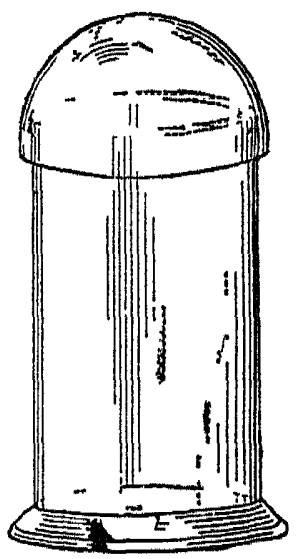

D

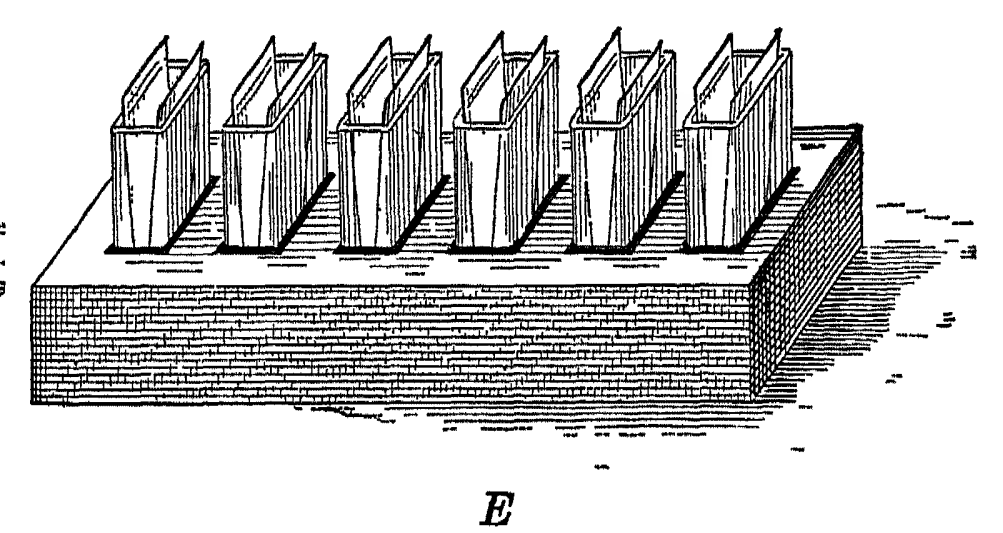

$A$, Coplin's; $B$, Moore's, $C$, Lellendahl's, $D$, Naples Jar, $E$, Series of Jars for Differential Staining

and allow to dry in the air or by gentle heating. After it has evaporated fix by holding above the flame at a point which is not uncomfortable for the hand. The procedure will give the smear. A good preparation should have the organisms evenly distributed over it and should not collect in droplets due to grease. The smear may now be subjected to any of the many staining processes.

Staining Apparatus. While any method may be used to stain bacteria certain pieces of apparatus are more convenient than others. When the organisms are mounted on a slide they may be covered 
with the stain or immersed in it. For immersion, many different types of staining jars are available. When the staining procedure is carried out by the putting the dye on the bacteria on a slide or cover glass, it may be kept in ordinary dropping bottles.

Stock Solutions of Dyes. These may be made up and kept on hand in glass-stoppered bottles. It is best to keep them in the dark Such solutions may be made etther in alcohol or water.

Alcoholic Stains. Such stams are made by dissolving a little of the dye in alcohol. They are not much used in bacteriology and are usually diluted with distilled water.

Aqueous Alcoholic Stains. These are prepared by adding to distilled water a portion of the filtered stock solution of the dye in absolute alcohol. These are the stains which have the greatest use in the laboratory. From 1 to 5 c.c. of the saturated alcoholic solution of the dye are diluted with 100 c.c. of the water.

Aqueous Stains. Pure aqueous solutions are weak stains and have limited use. They are prepared by adding to the distrlled water enough of the dye to give a saturated solution. They have the great advantage that over staining with them is improbable.

Mordants. They have the same purpose in staining that they have in dyeing. They are substances which unite with the aniline dye and with the cell protoplasm. They increase the rapidity of the staining procedure and render the color of the stained protoplasm more intense. The following substances are used as mordants:

Iodine in Lugol's solution.

Bromine in solution with potassium bromide.

Tannin.

Different acids.

Alkalis.

Gram Stain. Gram (1884) devised a differential staining procedure which has been of much service in bacteriology. The explanation of this stain is not definitely settled. Gram in his original work did not attempt to explain it. Unna (1887) put the explanation on a chemical basis. The Gram stain is an arbitrary procedure and a variation in the details of it are very important. It probably depends upon the fact that the pararosaniline (methyl violet, gentian violet) compounds form substances with iodine which are insoluble in alcohol. The iodine salts of rosaniline (fuchsin and methylene blue), however, are decomposed by alcohol. The Gram negative bacteria are stained only with the gentian violet. The alcohol washes out the iodine. The iodine in 
Gram positive bacteria forms a compound between the protoplasm of the cell and the dye which is insoluble in alcohol. In the Gram negative bacteria this compound is soluble in the alcohol and broken up. Kruse (1910) attempted to cxplain this stain by stating that the Gram positive bacteria are more resistant to autolysis, solution in strong alkali, etc., than the Gram negative bacteria. Eisenberg (1909) assumed that the differential Gram staining rested upon the presence of some special compound such as unsaturated fatty acids or phosphatids in the cell membrane. These formed a compound with iodine which renders the cell wall impermeable to alcohol.

Dreter, Kriegler and Walker (1911) in seeking an explanation for this stain proposed the presence of lipoidal substances in the Gram positive bacteria which bind the pararosaniline compounds. This, however, has little foundation according to our present knowledge. Brudny (1908) regarded differences in the permeability of the cell membrane as the important factor. With the Gram positive bacteria the membrane is more easily penetrated by the iodine solution. The structure of thése bacteria is probably "looser" and the stain gains an easy entrance. The Gram negative bacteria, however, are not penetrated and no deposit of the dye takes place on the interior of the cell. Benians (1912) places the explanation of this stain on a definite cell membrane. This prevents the removal of the dye by the alcohol while Hottinger (1916) explains it from the standpoint of colloidal chemistry. He states that the relation of the bacteria to this stain depends upon the degree of dispersion of the nucleoproteins. In the Gram negative bacteria, the stained nucleoproteins are so widely dispersed that they are not seen. In the Gram positive bacteria the stained nucleoproteins form a coarse emulsoid. Gram positive bacteria are said to become Gram negative when the degree of dispersion is increased by the action of proteolytic enzymes. Gram positive bacteria resist ferment action longer than Gram negative. Weinkopff (1911) in studying tryptic digestion of bacteria concluded that the difference between the Gram positive and Gram negative bacteria rested in the penetrability of cell protoplasm. Dreter et al. (1912) extracted a substance from Gram positive bacteria (staphylococci) which when applied to Gram negative $B$. coli made them Gram positive in many cases. When this ether extract was dried on a slide, the residue took the Gram stain. This investigator also treated B. coli with lecithin which made them Gram positive. That the Gram negative bacteria are structurally different from the Gram positive bacteria seems to be indicated by the work of Larson, Hartzell and Diehl (1918). 
These investigators stated that the Gram negative bacteria could be broken up by the sudden release of the pressure when the bacteria were in an atmosphere of carbon dioxide while the Gram positive bacteria resisted such treatment. The latter, although killed, underwent no morphological change.

Procedure for the Gram Stain. Prepare a smear as outlined above.

1. Flood with aniline gentian violet and heat gently for five minutes.

2. Wash in water and immerse in Lugol's iodine solution for five minutes.

3. Wash in water and place in strong alcohol until most of the dye is removed. It may be necessary to repeat this procedure from 2 if any dye remains.

4. Wash in water and examine or counterstain if desired.

5. Counterstain with 10 per cent solution of eosin in water.

There have been several modifications of the Gram method of staining all of which give good results in the hands of those who proposed them.

Nicolles Modification of the Gram Stain. This is similar to the above except that carbol gentian violet is substituted for the aniline gentian violet in the regular Gram's procedure. Decolorization is also accomplished with acetone alcohol (1 part acetone and 2 parts of alcohol).

GRAM POSITIVE BACTERIA

Staphylococcus pyogenes aureus Staphylococcus pyogenes albus

Staphylococcus pyogenes

Pneumococcus

Micrococcus tetragenus

Bacillus Xerosis

Bacillus hoffmanii

Oidium albicans

Bacillus tetanus

Bacterium diphtheriæ

Bacillus ærogenes capsulatus

Bacillus lepræ

Bacillus anthracis
GRAM NEGATIVE BACTERIA

Gonococcus

Micrococcus catarrhalis

Bacillus typhosus

Bacillis coli communis

Bacillus dysenteriæ

Micrococcus melitensis

Pseudomonas pysocyaneus

Bacillus pneumoniæ Fried

Bacillus proteus

Bacillus mallei

Bacillus influenzæ

Koch-Weeks bacillus

\section{Staining of Capsulees}

\section{Muir's Method.}

I. Mordant.

Mercuric chloride (saturated aq. sol.)..... 2 c.c.

Tannin (20 per cent aq. sol.) . . . . . . . 2 c.c.

Potassium alum (Sat. aq. sol.) . . . . . . . 5 c.c. 
II. Stain.

Carbol fuchsin.

III. Counter stain.

Methylene blue.

The smear is mordanted two minutes, washed in alcohol and water and then stained two to three minutes with gentle heat. It is then washed with water and remordanted again for two or three minutes. Counterstain with methylene blue.

Welch's Method. Fix the smear in glacial acetic acid. After a few seconds pour off the acetic acid and flood with aniline gentian violet. Repeat until all acid is removed. Wash and examine.

\section{HunTOON's METHOD}

Preparation of Reagents. Solution 1. To be used as diluent. Three grams of nutrose are sifted into 100 c.c. of distilled water and heated to $100^{\circ} \mathrm{C}$. in the Arnold sterilizer for an hour. Add 5 c.c. of a 2 per cent phenol solution to act as a preservative. Decant into a test tube and allow to settle. Employ the supernatant liquid as the diluent. (Since the supernatant liquid tends to become thinner by constant precipitation of the nutrose, the solution should occasionally be reboiled.)

Solution 2. Fixing and staining solution.

2 per cent aq. phenol solution . . . . 100 c.c.

Concentrated lactic acid . ........ 0.25 to 0.5 c.c.

1 per cent acetic acid solution.......... 1 c.c.

Saturated fuchsin in alcohol. ............ 1 c.c.

Carbol fuchsin, old solution. . . . . . . . 1 c.c.

This staining solution must be kept tightly corked.

\section{Technique of Staining}

1. Employ the solution (No. 1) as a diluent emulsifying the bacteria in 1 or 2 loopfuls and then spreading in as thin a film as possible with the loop. The use of the edge of a slide in spreading the film is not to be recommended.

2. Allow to dry in the air. .

3. Cover the film with the fixative and the staining solution (No. 2) and allow to act for from 30 to 45 seconds.

4. Wash quickly in water, dry and examine. 


\section{StAINING OF SPORES}

Neisser's Method. Flood the smear with hot aniline fuchsin for about an hour. Wash in water and decolorize with acid alcohol (1 part $\mathrm{HCl}$ and 3 parts alcohol). Too long decolorization will remove the stain from the spores also. Counterstain with methylene blue if desired.

Chromic Acid Method. Place the smear in 1 to 20 aqueous chromic acid for five minutes. Wash in water and stain with carbol fuchsin for thirty minutes. Wash and examine.

\section{Staining of Flagella}

This has always been a difficult procedure for many bacteriologists. It may be due to lack of care in some of the preparatory procedures. Smith (1905) has mentioned some of the common errors.

1. Oily or otherwise dirty cover glasses.

2. Unsuitable cultures.

3. Breaking off the flagella.

4. Uneven or too copious distribution of the bacteria.

5. Imperfect mordanting.

6. Excessive mordanting.

7. Understaining.

8. Overstaining.

9. Precipitates on the cover glass during some stage of the process. The greatest error probably lies in dirty slides and cover glasses. These should be cleaned in strong alkali in order to saponify any animal grease which may be on them. Otherwise, the smear will "roll" and not spread evenly. The culture which is stained must be young and vigorous. A small amount of this growth should be transferred to a test tube of sterile water and incubated at $37^{\circ} \mathrm{C}$. After several hours, some of this suspension should be put on slides and allowed to dry by placing the slides in the $37^{\circ} \mathrm{C}$. incubator. The smear should then be fixed and stained by any of the many processes.

The author has secured the best results with Loeffler's method. A double boiler is prepared from two beakers. The inner is supported by pieces of cork. The staining solution should be put into the inner one and the temperature raised to about $65^{\circ} \mathrm{C}$. or until the stain steams. The slides are immersed in this solution and stained as long as required. 


\section{LOEFFLER'S MORDAN'T}

Tannic acid, 20 per cent aqueous. . . . ... 10 c.c.

Ferrous sulphate, saturated aqueous....... 5 c.c.

Basic fuchsin, saturated alcoholic........ 1 c.c.

Mix and filter after a short time.

\section{LOEFFLER'S STAIN}

Basic fuchsin, saturated alcoholic....... 2.5 c.c.

Carbolic acid................... 20.0 c.c.

Van Ermengen's Flagella Stain. I. Van Ermengen's Mordant.

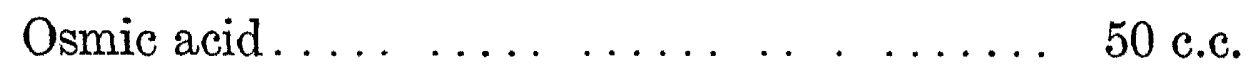

Tannic acid aqueous $10-25$ per cent. . ..... 100

Four drops of glacial acetic acid should be added to the above.

II. Silver Bath. A 0.25 to 0.5 per cent solution of silver nitrate in distilled water.

\section{Reducing Bath.}

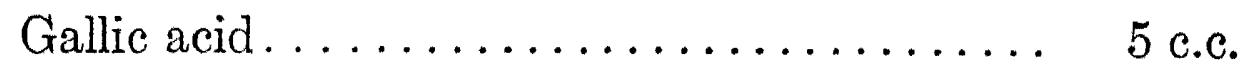

Tannin............................ 3 c.c.

Sodium acetate fused $\ldots \ldots \ldots \ldots \ldots \ldots$ c.c.

Distilled water. . . . . . . . . . . 350 c.c.

The smear should be covered with the mordant for fifteen minutes at $50^{\circ} \mathrm{C}$. The mordant is then washed off in distilled water and alcohol. The smear is then placed in a small amount of the silver bath and gently heated. Without washing, it is then put into the reducing bath and agitated until the solution begins to blacken. It is removed dried and mounted for examination. The apparatus used in this procedure must be absolutely free from animal grease. Kuntze (1902) has suggested some improvements in Van Ermengen's procedure for staining flagella.

\section{AcId Fast Staining}

This involves the formation of a permanent compound between the stain and the cell protoplasm-one which may not be removed by comparatively strong solutions of mineral acids. These bacteria 
require a strong stain with a mordant and once the cell is stained it is decolorized with great difficulty. Different reasons such as a thick membrane about the cell or peculiar structure of the cell contents, have been given to explain the acid fast staining procedure. Klebs (1896) has shown that the acid fast properties are lost if the tubercle bacillus is extracted with ether. Others (Bienstock, 1886) have stated that by increasing the fat content of the cell it may be made to take on acid fast properties. Miller (1916) grew tubercle bacilli in sperm oil and attributed the variations in the staining properties with carbol fuchsin to the production of free oleic acid in the interior of the rod. This acid was believed to be formed by the spores or round granules in the cell. Bulloch (1904) when studying the acid fast properties of bacteria isolated a wax and other substances in the nature of fats from acid fast bacteria. The other constituents of the cells were not acid fast. Baumgarten (1911) thought that unsaturated fatty acids were present in the acid fast bacteria which united with the dye. Benians (1912) claimed that the cell wall was the important factor in retaining the dye. He states "for the present the evidence points to the existence of a waxy layer enclosing a protoplasmic and fatty cell substance and conferring on the organism the property of resisting the penetration of acid and alcohol." Aronson (1910) claims that the peculiar staining properties of the tubercle bacillus are due to the fat content and not to any" peculiar kind of protoplasm. This waxy substance may be extracted by trichlorethylen.

Ziehl-Nielsen Method of Acid Fast Staining. Flood the smear with the stain and heat for ten minutes. Decolorize in 25 per cent mineral acid and wash. Counterstain if desired with eosin or Bismarck brown. The staining solution is prepared as follows:

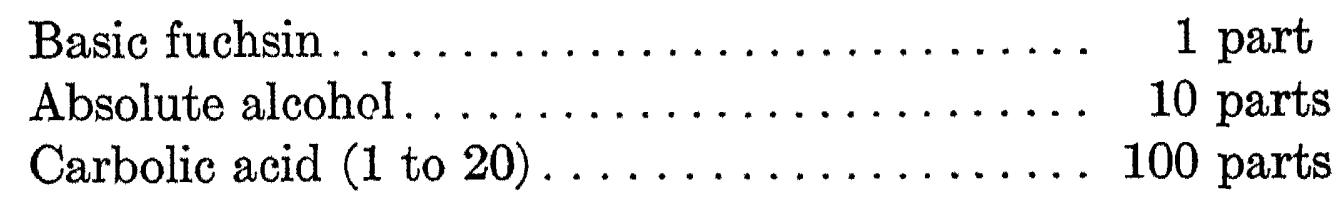

Wright's Method for the Differential Staining of Blood Films and Malarial Parasites. The method of preparing the stain and applying it is outlined by Wright (1902) as follows:

I. Preparation of the Staining Fluid. Make a 0.5 per cent solution of sodium bicarbonate in an Erlenmeyer flask and add to it 1.0 c.c. of methylene blue. Grubler's BX or Koch's or Erlich's rectified methylene blue may be used. The sodium bicarbonate should be entirely dissolved before the methylene blue is added. The mixture should be 
steamed in an Arnold steam sterilizer for one hour. The steaming of the alkalin solution of methylene blue effects certain changes in the methylene blue whereby a polychromatic property is given to it so that the compound with eosin, which is later to be formed with it, has not only the property of differentially staining the chromatine of the malarial parasite but also of differentiating and bringing out more sharply the nuclei and granules of the white blood corpuscles.

When the steaming is completed the mixture is removed from the sterilizer and allowed to cool. When it is cold, without filtering, pour it into a large dish or flask and add to it shaking or stirring meanwhile a 1 to 1000 solution of eosin (Grubler, yellowish, soluble in water sufficient in quantity, until the mixture losing its blue color becomes purple in color, and a scum with yellowish metallic luster form on the surface, while on close inspection a finely granular black precipitate appears in suspension. This will require about 500 c.c. of the eosin solution for 100 c.c. of the alkalin methylene blue solution.

The precipitate is collected on a filter and allowed to dry thereon without washing. When thoroughly dry a saturated solution in pure methyl alcohol is made. Three-tenths of a gram of the dry substance will thoroughly saturate 100 c.c. of the methyl alcohol in a fow minutes.

The saturated alcoholic solution of the precipitate is next filtered and to the filtrate is then added 25 per cent of methyl alcohol; e.g., to 80 c.c. of the saturated alcoholic solution 20 c.c. of methyl alcohol are added.

This somewhat diluted solution of the precipitate is the staining fluid. It is permanent and may be kept on hand. Care should be taken to prevent evaporation of the alcohol so that the fluid may not become too saturated. The object of the dilution is to prevent precipitations on the blood film in the process of staining.

II. The Staining of the Blood Films. The films of blood which are spread thinly are allowed to dry in the air. As much of the staining fluid is poured upon the film as the cover glass or slide will readily hold without draining off. Allow the staining fluid to remain in contact with the film for one minute. Next, add to the staining fluid on the cover glass or slide, sufficient water drop by drop, until it becomes semi-translucent and a reddish tint becomes visible at its margin, while a metallic scum forms on its surface. The amount of water required will vary with the amount of staining fluid on the preparation. Generally 8 or 10 drops will be required if a seven-eighth square cover glass is used. The staining fluid thus diluted should be allowed to remain on the preparation for 2 or 3 minutes, during which time the 
real staining of the preparation takes place, and is then washed off with water.

The differential staining of the preparation is accomplished by washing the preparation with distilled water. The washing is continued until the better spread portions of the film appear yellowish or reddish in color. This method of differentiation of decolorization may require from one to three minutes according to the intensity of the staining and the tint sought in the red blood corpuscles. When the desired color is obtained in the red blood corpuscles the preparation is quickly dried between filter papers and mounted in balsam. It is important to stop the decolorization by drying the preparation as soon as the desired tint in the red blood corpuscles is obtained. A little experience will show the operator how far to carry the decolorization and the time required for to obtain the desired tint in the red blood corpuscles.

\section{Summary for the Method of Staining}

1. Make films of the blood, spread thinly and allow them to dry in the air.

2. Cover the preparation with alcoholic solution of the dye for one minute.

3. Add to the alcoholic solution of the dye on the preparation sufficient water, drop by drop, until the mixture becomes semi-translucent and a yellowish metallic scum forms on the surface. Allow the mixture to remain on the preparation for two or three minutes.

4. Wash in distilled water until the film has a yellowish or pinkish tint in its thinner or better spread portions.

5. Dry between filter paper and mount in balsam.

III. Microscopical Appearance in Blood Films Stained by this Method. The red cells are orange or pink in color. Polychromeatophilia and punctate basophilia (the granular degeneration of Grawitz) are well brought out. The nucleated red cells have deep blue nuclei and the cytoplasm is usually of a bluish tint. The lymphocytes have a dark purplish-blue nucleus and a robin's egg blue cytoplasm in which a few dark blue or purplish granules are sometimes present. The polynuclear neutrophilic leucocytes have a dark blue or dark lilac-colored nucleus and the granules are usually of a reddish color. The eosinophilic leucocytes have blue or dark-colored lilac nuclei. The granules have the color of eosin, while the cytoplasm in which they are imbedded has a blue color. The large mononuclear leucocytes appear in at least 
two forms. Each form has a dark or lilac-colored nucleus. The cytoplasm of one form is pale blue and of the other form is blue with dark lilac or deep purplc-colored granules, which are usually not so numerous as are the granules in the polynuclear neutrophilic leucocytes. The mast cells appear as cells of about the size of polynuclear leucocytes with purplish or dark-blue stained irregular-shaped nuclei and the cytoplasm sometimes bluish in which coarse spherical granules of variable size are imbedded. These granules are of a dark-blue or purple color and may appear almost black. The myelocytes have a dark blue or dark lilac-colored nuclei ard blue cytoplasm in which numerous dark lilac or reddish lilac-colored granules are imbedded. The blood plates are deeply stained and are a prominent feature of nearly all blood preparations. . They appear as blue or purple rounded or oval bodies, usually of a diameter of one-third to one-half of that of a red blood corpuscle. They have ragged margins and present fine bluish or purplish dots or mottlings in their substance. In some instances the blood plate appears to be within the red blood corpuscle or partially within and partially without. The plates found within the red cells must not be mistaken for young malarial parasites.

Preparation of Common Stains. Alkalin Methylene Blue (Loeffler's):

Saturated alcholic methylene blue........ 30 c.c.

Aqueous $\mathrm{KOH}$ solution (0.1 per cent). . . . . 100 c.c.

Carbol Fuchsin (Ziehl):

Saturated alcoholic fuchsin solution....... 10 c.c.

Carbolic acid crystals........... 5 gms.

Distilled water................ 100 c.c.

\section{Anilin rentian. Violet:}

Saturated alcoholic gentian violet........ 11 c.c.

Absolute alcohol. ............... 10 c.c.

Anilin water................... 100 s.c.

The anilin water may be made by shaking 5-6 c.c. of anilin oil in distilled water and filtering.

Lugol's Iodine Solution:

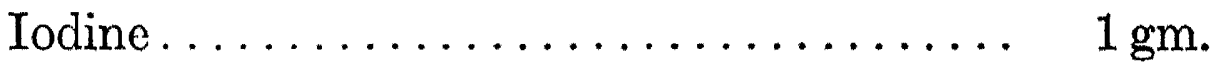

Potassium iodide.............. 2 gms.

Distilled water................ 300 gms. 
Stabilized Gentian Violet (Stovall and Nicholls, 1916). To prevent the usual deterioration of gentian violet, the following stain is proposed:

Aniline.

28 c.c.

Gentian violet. ............... 8 gms.

95 per cent alcohol ................ 100 c.c.

Normal hydrochloric acid........... . 5 c.c.

Distilled water................... 1000 c.c.

The gentian violet should be dissolved in the alcohol. The aniline and hydrochloric acid may be mixed and diluted to 900 c.c. Filter these solutions and add each other with a subsequent filtration.

Another formula for gentian violet for use in the Gram stain has been given by Kilduffe (1909). This has been used in the author's laboratory and has given good results.

Solvent

5 c.c. commercial formalin.

95 c.c. distilled water.

Stain

25 c.c. saturated alcoholic gentian violet,

75 c.c. of the solvent.

or

16 c.c. saturated gentian violet.

84 c.c. solvent.

\section{BIBLIOGRAPHY}

Aronson, H. 1910. In Regard to the Biology of the Tubercle Bacillus. Berlin. klin. Wochenschr., 47, 1917-1620.

Barber, M. A. 1914. The Pipette Method in the Isolation of Single Microorganisms and in the Inoculation of Substances into Living Cells. Philippine Jour. Science, 9B, 307-360.

BaUmgarten. 1911. Lehrbuch der pathogenen Mikroorganismen, p. 641. Original not seen.

BeCHTold. 1886. Fortschritt der Medizin, 1886, 4, 193. Original not seen.

Bienstock, B. 1886. Zur Frage der sogenannten Syphilis Bacillen und der Tuberkelfärbung. Fortschr. der Med., 1886. 193-195.

BRundny. 1908. Ueber die Beziehung der Farbbarkeit der Bakterien nach der Gram und ihrer Permeabilität. Cent. Bakt. Abt., II., 21, 62.

BULloch, W. and M'Leod, J. J. R. 1904. The Chemical Constitution of the Tubercle Bacillus. Jour. Hyg., 4, 1-10.

Dreter, C., Kriegler, S. G., and Walker, E. W. 1911. The Effect of Certain Dyes on Bacteria. Jour. Path. and Bact., 15, 133-136. 
Dreter, C., Scotr, S. A., and Whlker, E. W. 1912. The Nature of Gram Staining. Jour. Path. and Bact., 16, 146.

Eisenberg. 1909. II. L'eber das Lktoplasms und seine Veranderungen im infizierten Tier. Cent. Bakt. Abt., 1, 49, 465-493.

Gram, C. 1884. Ueber die isolierte Farbung der Schizomyceten in Schnitt und Trokenpraparate. Fort. der. Med., 2, 185-190.

Hil, H. W. 1902. "Hanging Block" Preparations for the Microscopic Observation of Developing Bacteria. Journal Medical Research, 7, 202-212.

Hotringer, R. 1916. Contributions to the Theory of Gram's Stain from a Colloidal Chemical Standpoint. Cent. Bakt., 76, 367-383.

Huntoon, F. M. 1917. A Simple Method for Staining the Capsules of Bacteria. Jour. Bact., 2, 241-244.

KILDUFFe, R. 1909. A New and Stable Solution of Gentian Violet for the Gram Stain. Jour. Amer. Med. Assn., 53, 2002.

Klebs, E. 1896. Ueber heilende und immunisierende Substanzen aus Tuberkelbacillen. Cent. Bakt. Abt. I., 20, 488-508.

Kосн. 1877. Cohn's Beitrage Biol. Pflanzen. 2, Original not seen.

KRust, O. 1910. Beziehungen zwischen plasmolyse Verdaulichkeit, Löslichkeit und Färbarkeit von Bakterien. Munchen. med. Wochenschr., 1910, 685, No. 13.

KunTzE. 1902. Einige Bemerkungen ủber die Färbung der Giesseln besonders über das Verfahren von van Ermengen. Cent. Bakt. Orig., 32, $555-560$.

Larson, W. P., Hartzell, T. B., and Diehl, H. S. 1918. The Effect of High Pressures on Bacteria. Journal of Infectious Diseases, 22, 271-279.

Loeffler. 1889. Eine neue Methode zum Farben der Mikroorganismen, im besonderer ihrer Wimperhaare und Giesseln. Cent. Bakt., 6, 209-224.

Miller, A. H. 1916. On the Biochemistry of the Loss of Power of the Tubercle Bacillus to Stain with Carbol Fuchsin. Jour. Path. and Bact., $21,41-46$.

RETTGer, L. F. 1913. Hygiene of the Bacteriological Laboratory. Amer. Jour. Pub. Health, 3, 697-700.

Smith, I. F. 1905. Bacteria in Relation to Plant Diseases. Smithsonian Institution. Washington.

Stovali, W. D., and Nrcholls, M. S. 1916. Stabilized Gentian Violet. Jour. Amer. Med. Assn., 66, 1620-1631.

UnNa, P. G. 1881. Die Rosanilin und Pararosanilin. Eine bacteriologische Farbenstudie. Cent. Bakt. Abt., I, 2, 135-136.

van Ermengen, E. 1894. Nouvelle méthode de coloration des cils des bakteries. Reviewed in Cent. Bakt., 15, 969-970.

Weinkopfr, P. 1911. Tryptic Digestion of Gram Positive and Gram Negative Bacteria. Zeit. Immunitat., 11, 1-17. Chemical Abstracts, 5, 3857.

Wright, J. H. 1902. A Rapid Method for the Differential Staining of Blood Films and Malarial Parasites. Jour. Med. Research, 7, 139-143.

ZneHL. 1882. Deutsch. med. Wochenschr. Original not seen. 


\section{CHAPTER IV}

\section{CLASSIFICATION AND DESCRIPTION OF BACTERIA}

THE early investigators in bacteriology were impressed with the newness of the field and devoted more time to the isolation of new forms. At this time classification received less attention. They examined all sorts of substances and described many shapes and sizes. The condition for working also made it difficult to secure accurate data and this is quite essential in all systematic work. Pure cultures were difficult to secure and the microscope approached in no way the perfection of the modern instrument. Under such conditions it remained for them as for the pioneers in any field to roproduce the facts upon which the later 'workers could build. Consequently the arrangement of bacteria into groups or related masses has been left for the bacteriologists of a later day.

One of the most important ideas which deviated attention from classification was the conception of pleomorphism. The early conception of this theory has been well stated by Fischer (1900). "The pleomorphists maintained that a coccus did not necessarily remain a coccus all its life long, but it could under certain conditions stretch itself and assume the shape of a bacillus, that this again could become curved and change into a vibrio, to return again later on to the coccus form that it commenced with. Words like Micrococcus, Bacillus, Vibrio, Spirillum, which we know now to have a definite taxonomic value, were in the eyes of the pleomorphists worthless designations of transient changes of shape." Such a conception of bacterial forms easily prevented or inhibited attempts at the arrangement of the described forms into a classification. This idea had its origin in the beginnings of biology and botany. In the early days of chemistry it was' one of the earliest objects of the science to change copper into gold.

In bacteriology this theory has constantly received some attention. Buchner revived it when he reported that he had changed Bacillus subtilis into the bacterium specific for anthrax. More recently Rosenow (1914) has reported that he has changed the streptococcus into the pneumococcus. He was able to repeat this several times. The serum 
reactions for the new strain possessed all of those of the true pneumococcus. In turn, he was able to change these serum reactions. This raises the question with regard to what characters may be depended upon for constancy. This work of Rosenow together with the recent reports on life cycles may make it necessary to introduce new methods of classification. In the study of the higher forms of life the term cvolution form conveniently lends itself to abnormal shapes.

Anton van Leewenhoek (1683) was probably one of the first to report different kinds of bacteria. From our present knowledge, it is doubtless true that he had a mixture of the various forms of microscopic life. At this time the methods of securing different dilutions were not perfected. In fact, no real attempt had bcen made up to this time to secure pure cultures since their necessity for accurate bacteriological work had not been realized. Van Leewenhoek recognized curved and straight forms and presented drawings with his paper. This contribution may be considered as a classification only in the most general sense and then only to the extent that different shapes were observed. It is not certain that he saw many bacteria through his crude apparatus.

Müller (1786) studied bacteria from a zoological standpoint and proposed a classification which never had very extended use. Probably he also observed protozoa with perhaps a few bacteria.

Ehrenberg (1828) began his contributions to systematic bacteriology by founding the genus bacterium. A résumé of his work is mentioned by Smith. This genus he later divided into several parts. In 1838 he published his work "Die Infusionsthierchen" and therein describes the genus bacterium as being "Die quergetheilten gehören zu den Zitterheirchen (Vibrionen) die langsgehteilten zu den Stabtheirchen (Bacillen)." Ehrenberg gave us several terms which have been retained up to the present time but to which different meanings are attached.

Cohn (1872) published a classification which was used for some time. He divided the bacteria into the following four groups:

I. Sphaerobacteria Species 1. Micrococcus.

II. Microbacteria: Species 2. Bacterium.

III. Desmobacteria: Species 3 . Bacillus. Species 4. Vibrio. 
IV. Spirobacteria:

Species 5. Spirillum (Ehrenberg).

Species 6. Spirochæte (Ehrenberg).

Cohn's classification is not used at the present time. He did not make any provision for pleomorphism and thus immediately opposed himself to Klebs, Buchner and Lister. It was about this time that Buchner reported the changing of $B$. subtilis into $B$. anthracis. The publications of Cohn and Koch proved the constancy of this characteristic and struck a forceful blow at pleomorphism. The principal objectors to Cohn's classification were the medical men, but after the articles of Cohn and Koch they were more careful.

Lister (1873) was one of the bitterest opponents of Cohn's system. He transferred a drop of sour milk in which he had observed cocci to broth and observed, after a short time, long chains. He used such data to support his views of pleomorphism.

De Dary (1884) divided all bacteria into two classes, those forming endospores and those forming arthrospores. He used spore formation in a different sense than we now use it. Whenever he saw a cell which differed from the normal, he called it an arthrospore. His discussion on the formation and germination of spores is quite complete and interesting.

Eisenberg (1891) for the first time attempted to use physiological characters. Bacteria were divided into pathogenic and non-pathogenic varieties. The pathogens were again divided with regard to their effect on man and animals. No attention was given to spore formation, or flagellation.

Migula (1897) published a classification which has been generally used by bacteriologists. There are objections to it, but it is probably as good a working basis as any which is available. This is given as follows by Jordan (1910):

\section{Classification of Bacteria-(Migula)}

I. Cells globose in a free state, not elongating in any direction before division into one, two, or three planes................... 1. Coccacex.

II. Cells cylindrical, longer or shorter, and only dividing in one plane, and elongating to about twice the normal length before the division. 
a. Cells straight, rod-shaped, without sheath, non-motile, or motile by means of fiagella... ............... . 2 Bacteriacex.

b. Cells crooked, without sheath. ........ 3. Spirillaceæ.

c. Cells inclosed in a sheath..........4. Chlamydobacteriaceæ.

\section{Coccaceæ}

Cells without organs of motion:

a. Division in one plane . ............. Streptococcus.

b. Division in two planes. . ........... Micrococcus.

c. Division in three planes ........... Sarcina.

Cells with organs of motion:

a. Division in two planes. ............ 4. Planococcus.

b. Division in three planes.$\ldots \ldots \ldots \ldots \ldots \ldots$. Planosarcina.

2. Bacteriacese

Cells without organs of motion........... Bacterium.

Cells with organs of motion (flagella):

a. Flagella distributed over the whole body.....2. Bacillus.

b. Flagella polar.................. 3. Pseudomonas.

\section{Spirillaceæ}

Cells rigid, not snake-like or flexuous:

a. Cells without organs of motion.

1. Spirosoma.

b. Cells with organs of motion (flagella):

1. Cells with one, very rarely i,wo to three polar flagella................. 2. Microspira.

2. Cells with polar flagella-tufts. . . . . . 3. Spirillum.

Cells flexuous.

4. Spirochæta.

\section{Chlamydobacteriaceæ (Higher Bacteria)}

Cell contents without granules of sulphur:

a. Cell threads unbranched.

I. Cell division always only in one plane... 1. Streptothrix.

II. Cell division in three planes previous to the formation of gonidia:

1. Cells surrounded by a very delicate, scarcely visible sheath (marine).. 2. Phragmidiothrix.

2. Sheath clearly visible (in fresh water $\ldots . \ldots \ldots \ldots \ldots \ldots$. . . Crenothrix.

b. Cell threads branched .............. 4. Cladothrix.

Cell contents containing sulphur granules....... 5. Thiothrix. 
Fischer (1897) proposed a classification and system of nomenclature in which the root of the generic name expressed the shape of the cell and the ending the arrangement of the flagella as follows:

$$
\begin{array}{ll}
\text { Baktron }=\text { rod } & \text { inium }=\text { monotrichous } \\
\text { Kloster }=\text { spindle } & \text { illum }=\text { lopotrichous } \\
\text { Plectron }=\text { drum stick } & \text { idium }=\text { peritrichous }
\end{array}
$$

Using this system a peritrichous rod would be a "bactronidium." Fischer's system met with little approval and is not used by bacteriologists.

Lehmann and Neumann (1899) published their classification which has had rather extended use in the science. It has been used to a great extent by the medical men. They proposed the following system:

\section{COCCACE}

1. Streptococcus. Dividing in one plane.

2. Sarcina. Dividing in three planes.

3. Micrococcus. Irregular division, including all but clearly marked packets and chains.

\section{Bacteriacem}

1. Bacterium. With no endospores, rods usually $0.8-1.0$ microns in diameter.

2. Bacillus. With endospores, rods, may be more than 1.0 micron in diameter.

\section{SPIRILLACEA}

1. Vibrio. Short, rigid, slightly curved cells, with 1 to 2 polar flagella.

2. Spirillum. Long rigid spiral cells, with lophotrichic flagella.

3. Spirochæte. Long flexible spiral cells, flagella not observed; motility accomplished by undulating membrane.

This is essentially a morphological classification and has many advantages. It was produced at about the same time that Migula proposed his system.

Jensen (1909) made an attempt to group bacteria according to their natural development. He made three divisions as follows:

1. Those bacteria which do not require organic nitrogen or organic carbon. 
2. Those bacteria which require organic carbon but are able to dispense with organic nitrogen. These forms utilize sugars, nitrogen, ammonia or nitrates.

3. Those forms which require both organic nitrogen and carbon. Inorganic substances are of little use.

Jensen attempted to devise a system which would show the relationship of forms. Reasoning from the synthetic processes, he constructed a family tree for bacteria. His methanomonas is proposed as the first bacterium on earth and he considered it the beginning of all life. The method of nomenclature in this system is cumbersome and so far his proposals have been of little more than theoretical interest.

The present-day system and those which are developed in the future must rest upon the past work in this field. Fischer, in his book previously referred to, has stated that the very factor contributing to the progress of bacteriology, the number and variety of its students, has been a great hindrance. There are pathologists, chemists, brewers, botanists, all of whom are making species or varieties. Fischer does not claim that to any one class of investigators should be given the privilege of systematizing the science, but that established principles should be followed.

A student reviewing the systems which have been proposed for the classification of bacteria realizes that the problem is a difficult one. The number of systems which have been proposed supports this statement. Smith has said that no harm will come to anyone if all of these perplexing questions are not settled definitely within his own generation.

The Society of American Bacteriologists has been wrestling with the classification of bacteria for some little time. The Committee (Winslow, 1917) has made an interesting report in which the more important classifications of the past have been discussed. This committee has proposed the following eight families:

Family 1. Nitrobacteriaceæ.

Family 2. Mycobacteriaceæ.

Family 3. Pseudomonadaceæ.

Family 4. Spirillaceæ.

Family 5. Coccaceæ.

Family 6. Bacteriaceæ.

Family 7. Lactobacillaceæ.

Family 8. Bacillaceæ.

The classification will probably demand much more study before a 
satisfactory one has been developed. Much more data may have to be secured with regard to the life histories of the bacteria.

Bacteriological Groups. The arrangement of bacteria into physiological groups have been carried out merely for convenience. In some instances probably these groups are made up of very closely related organisms; in other cases a too insignificant characteristic has been taken as the standard. The first five groups of Fuller and Johnson's classification of water bacteria have been made on the basis of chromogenesis. Jordan also used this same characteristic when he systematized the bacteria which he isolated from the Illinois river. Rahn states, in Marshall's Microbiology, that this is a convenient and helpful method for handling the bacteria. As mentioned above many of the groups are arbitrary and often depend upon characteristics which are not fixed. Many of the groups need to be subjected to careful study. This will tell whether the groups approach natural units and, if they do, the relation and characters of the members. The groups of pathogenic bacteria and those which have sanitary significance have received careful study.

Life Cycles of Bacteria. It has been the custom in bacteriology to regard bacteria as monomorphic. So firmly rooted has this idea become that few bacteriologists have given thought to the possibility of there being life cycles through which the bacteria pass. Involution form has been the term which has been applied to many of the forms which were regarded as "abnormal." In many cases these were explained as contaminations and the culture discarded. That bacteria may possess more or less intricate life cycles has been proposed by different workers. When the data from these different sources are compared, one is impressed with the possibility that bacteriologists have been too hasty in excluding all of these abnormal forms. Löhnis (1916) has given this subject most intensive study. Some of the conclusions which this investigator has reached are as follows:

"A comparative study of 42 strains of bacteria has shown that life cycles of these organisms are not less complicated than those of other microorganisms. As representatives of all groups of bacteria have been tested and all, without exception, behaved essentially in the same manner, in all probability analogous results may be expected with all species of bacteria. All bacteria live alternately in an organized and in an amorphous stage. The latter has been called the "symplastic" stage because at this time the living matter previously inclosed in the separate cell undergoes a thorough mixing, either by a complete disintegration of cell wall, as well as cell content, or by a "melting 
together," of the content of many cells which leave their empty cell walls behind them. In the first case a readily stainable, in the latter case an unstainable "symplasm" is produced. Lohnis later states that all bacteria multiply not only by fission but by gonidia. These may become exospores, grow to full-sized cells, or enter the symplastic stage. Some of the gonidia are said to be filterable. Hort (1917) working independently in England studied B. typh simple binary fission was not the only method of reproduction. $\mathrm{He}$ had previously received similar evidence in a study of typhus fever. - Rosenow (1917) in studying acute anterior poliomyelitis believed that he has observed a transmutation. All of these data from so many different sources point out the fact that bacteria may pass through different stages and that we are not justified in excluding all aberrant forms as contaminations. Should these data be further confirmed, the bacteriologist may find it necessary to alter his methods of analysis.

The Descriptive Chart of the Society of American Bacteriologists. Bacteriologists, for some time, have been working out a system for classifying bacteria. The Descriptive Chart of the Society of American Bacteriologists represents a stage in the development of this effort. A history of the development of the card and the numerical system for recording the characters of bacteria has been prepared by Harding (1910), a very brief outline of which will be given here.

Johnston (1895) first called attention to the possibility of using some such method as the Dewey decimal system for recording the important characteristics of bacteria. A committee which in its report suggested the beginnings of such a procedure was appointed. Conn adopted their suggestion and used such a system in his classification of dairy bacteria. A real attempt to use a group number such as our present one was made by Kendall (1903.) at the Lawrence Experiment Station. This is rèported by Gage and Phelps (1903). The genus classification of bacteria proposed by Migula was used as the basis for their report.

The present chart has a group number depending on the determination of ten characteristics. It has been recently described by Rehn and Harding (1916). This system of recording the characteristics of bacteria has not met with entire approval on the part of many of the workers. One of those who was instrumental in introducing this numerical system has said that it was a thing to be regretted. Whether it is or will be of any value may only be determined after it has been used to study certain groups. This, however, has been done by a very few bacteriologists. Harding (1910) reports the study of the Ps. 
campestris group. For each strain the same group number was obtained which would indicated that it was well fitted to these bacteria. This author believes that it may not " carry the separation to a group synonymous with the ordinary conception of species." This statement seems to have been borne out by later investigations. Harding and Prucha (1908) used the Descriptive Chart in the study of bacteria from cheddar cheese. In their conclusions, they state that this method of recording the reaction of cultures is a marked advance in technique, and that shifts in the checse flora may be thus traced more accurately. The sum and substance of their opinion of the Chart in its application to the study of cheese bacteria is that it is a valuable means to an end. Harding, Morse and Jones (1909) in their study of soft rot organisms used the group number. By examining their data it is apparent that the group number is a valuable aid. Harding reached essentially the same conclusion in 1910 when he studied the same group of bacteria. Conn (1906) when classifying dairy bacteria drew no conclusions with regard to the value of the rudimentary group number which he used. More recently H. J. Conn (1915) reported the study of 130 strains of $B$. subtzlis by means of the Descriptive Chart. In selecting these strains one-half of the determinations represented in the group number are fulfilled because they were implied in the definition of $B$. subtilis. He stated that different group numbers did not always represent different species, and that better methods for making these ten determinations should be devised. This worker later used the Descriptive Chart in another investigation involving the study of about 1000 pure strains isolated from soil (Conn, 1917). The Chart was found to be quite well adapted to the study of spore forms and was of value in the "preliminary study of other forms before learning what special tests were best adapted to them." The following tests were reported to give sufficiently consistent results to be of diagnostic value: shape of vegetative forms, size of vegetative forms, arrangement of flagella when present, presence of spores, size and shape of spores, growth in the absence of oxygen liquefaction of gelatin, nitrate reduction, chromogenesis and form of growth in liquid and solid media. At times some of these gave inconsistent results. Edson and Carpenter (1912) made no statement in their study on sap bacteria about the value of the Descriptive Chart. Tanner (1918) used the Chart in the study of green fluorescent bacteria from water and found the Chart a very convenient method for carrying out such a study.

Description of Microorganisms. The Descriptive Chart of the Society of American Bacteriologists has been given rather extended 


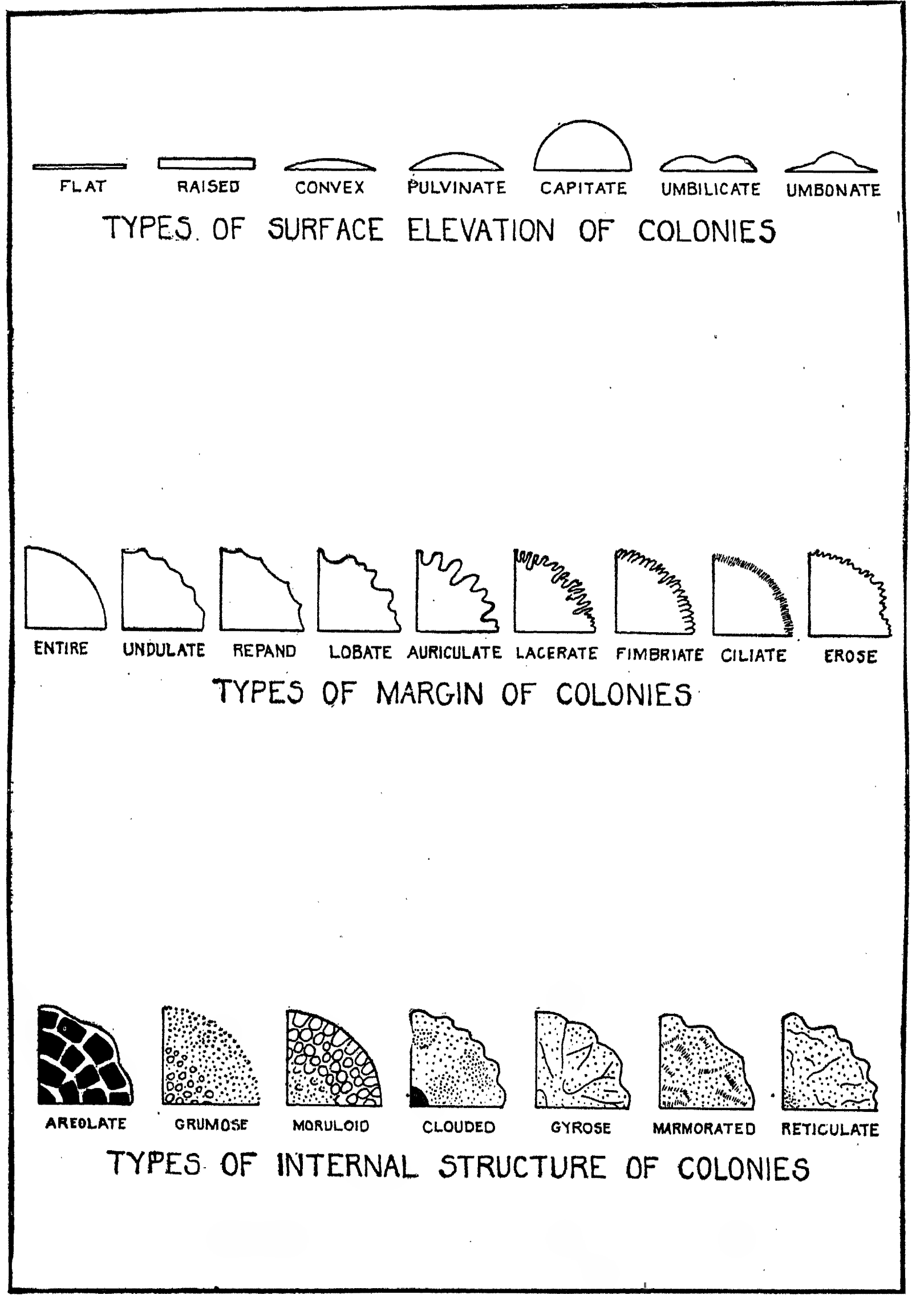

Frg. 33.-Showing the Various Types of Elevation, Margins, and Internal Structure of Colonies. 


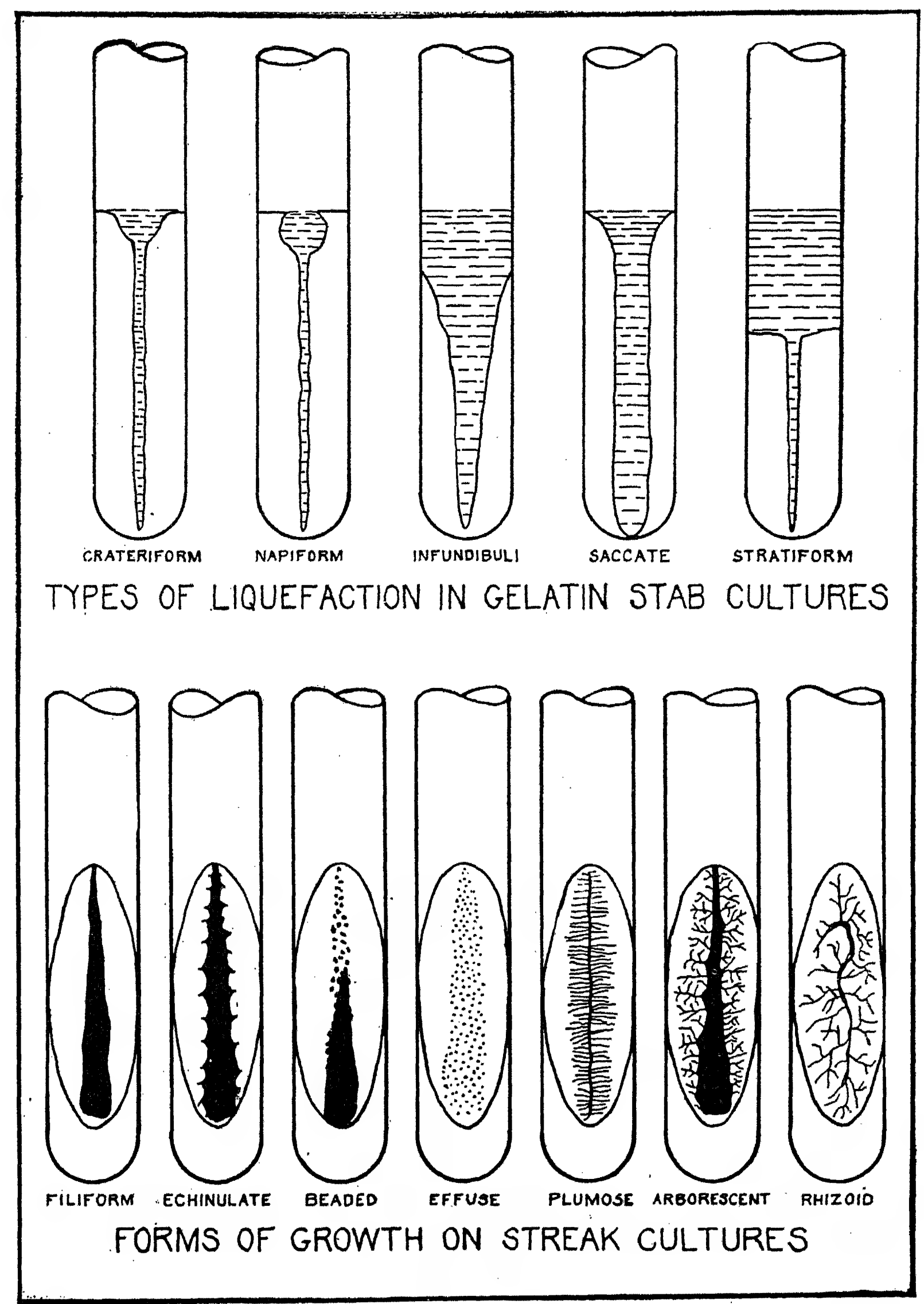

FIG. 34. 
discussion in the previous paragraph. From this it will be seen that those who have really used the Chart regard it as an aid in the study of bacteria. Barring certain recognized limitations it is a convenient
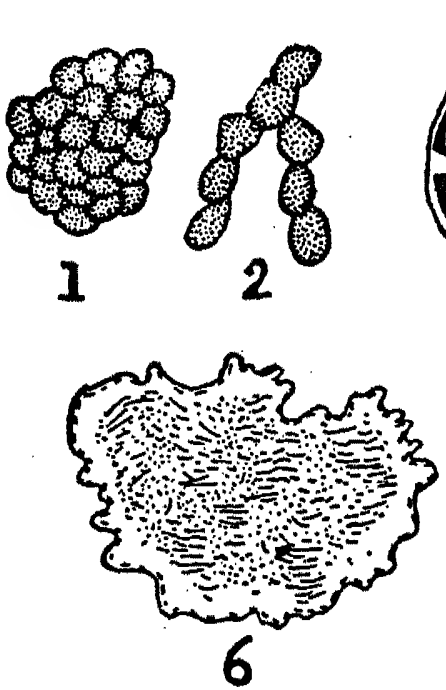

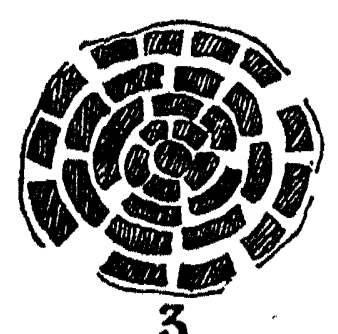

3

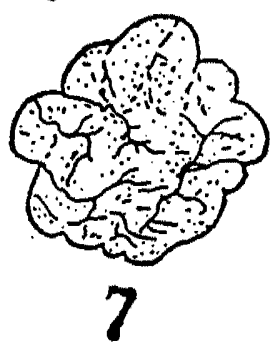

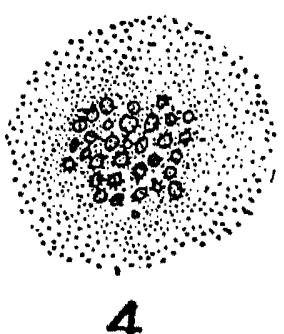

4

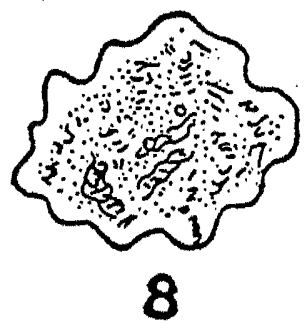

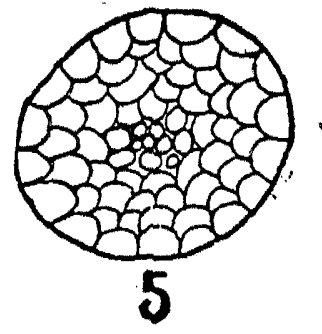

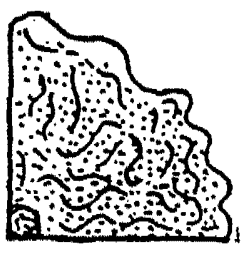

9

Structure of colonies: 1 , conglomerate colony; 2, toruloid colony; 3, alveolate structure; 4 , grumose in center; 5, moruloid; 6, clouded; 7, reticulate; 8, marmorated; 9, gyrose.

(From Moore's Laboratory Directions for Beginners in Bacteriology, Ginn \& Co., 1905.)
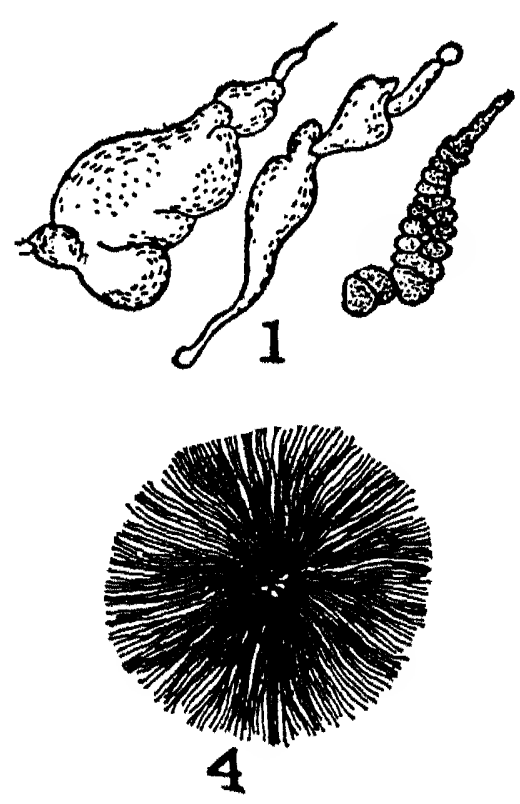

Types of colo curled structure.

(From Moore's Laboratory Directions for Beginners in Bacteriology, Ginn \& Co., 1905.)

FIG. 35.-Showing Characteristics of Colonies. These should not be regarded as absolutely constant. Other terms may be used which will more correctly describe a colony growth.

method for recording the salient characteristics of bacteria. Many of those who criticize it most severely have never used it in any extended research. The student will find the Chart a convenient factor in the 
study of bacteria from foods. The following glossary of terms has been taken from the back of the 1912 Chart:

\section{Glossary of Terms}

Adherent, applied to sporangium wall, indicates that remnants of sporangium remain attached to endospore for some time.

Aerobic, growing in the presence of free oxygen; strictly aerobic, growing only in the presence of free oxygen.

Ameboid, assuming various shapes like an ameba.

Amorphous, without visible differentiation in structure.

Arborescent, a branched, tree-like growth.

Anaerobic, growing in the absence of free oxygen; strictly anaerobic growing only in the absence of free oxygen; facultative anaerobic, growing both in presence and absence of free oxygen.

Beaded, in slat or stroke culture, disjointed or semi-confluent colonies along the line of inoculation.

Bipolar, at both poles or ends of the bacterial cell.

Brief, a few days, a week.

Brittle, growth, dry, friable under the platinum needle.

Bullate, growth rising in convex prominences, like a blistered surface.

Butyrous, growth of a butter-like consistence.

Chains, four or more bacterial cells attached end to end.

Chromogenesis, the production of color.

Ciliate, having fine hair-like extensions, sometimes not visible to the naked eye, resembling cilia.

Clavate, club shaped.

Cloudy, said of fluid cultures which might indicate growth.

Coagulation, the separation of casein from whey in milk.

Contoured, an irregular, smoothly undulating surface, like that of a relief map.

Convex, surface the segment of a sphere.

Coprophyl, dung bacteria.

Coriaceous, growth tough, leathery, not yielding to the platinum needle.

Crateriform, a saucer shaped liquefaction of the medium.

Crecateous, growth opaque and white, chalky.

Cuneate, wedge-shaped.

Curled, composed of parallel chains in wavy strands, as in anthrax colonies.

Diastatic action, conversion of starch into simpler carbohydrates, such as dextrins or sugars, by means of diatase.

Echinulate, a growth along line of inoculation with toothed or pointed margins.

Effuse, growth thin, veily, unusually spreading.

Endospores, thick-walled spores formed within the bacterial cell; i.e., typical bacterial spores like those of $B$. anthracis or $B$. subtilis.

Entire, with an even margin.

Erose, border irregularly toothed. 
Filaments, applied to morphology of bacteria, refers to threacl-like furms, generally unsegmented; if segmented, to be distinguished from chains (q.v.) by the absence of constrictions between the segments.

Filamentous, growth composed of long, irregularly placed or interwoven threads. Filiform, in stroke or stab cultures, a uniform growth along line of inoculation. Fimbriate, border fringed with slender processes, larger than filaments.

Floccose, growth composed of short curved chains, rariously oriented.

Flocculent, containing small adherent masses of bacteria of various shapes floating in the culture fluid.

Fluorescent, having one color by transmitted light and another by reflected light.

Gram's stain, a method of differential bleaching after gentian violet, methyl violet, etc. The + mark is to be given only when the bacteria are deep blue or remain blue after counter-staining with Bismarck brown.

Grumose, clotted.

Granular, composed of small granules.

Infundibuliform, form of a funnel or inverted cone.

Iridescent, exhibiting changing rainbow colors in reflected light.

Lacerate, having the margin cut into irregular segments as if torn.

Lobate, having the margin deeply undulate, producing lobes (see undulate).

Long, many weeks or months.

Luminous, glowing in the dark, phosphorescent.

Maximum temperature, temperature above which growth does not take place.

Medium, several weeks.

Membranous, growth thin, coherent, like a membrane.

Minimum temperature, temperature below which growth does not take place.

Mycelioid, colonies having the radiately filamentous appearance of mold colonies.

Napiform, liquefaction in form of a turnip.

Nitrogen requirements, the necessary nitrogenous food. This is determined by adding to nitrogen-free media the nitrogen compound to be tonted.

Opalescent, resembling the color of an opal.

Optimum temperature, temperature at which growth i" most rapid.

Papillate, growth beset with small nipple-like processes.

Pellicle, bacterial growth forming either a continuous or an interrupted shect over the culture fluid.

Peptonization, rendering curdled milk soluble by the action of trypsin.

Peritrichiate, covered with flagella over the entire surface.

Persistent, lasting many weeks or months.

Plumose, a fleecy or feathery growth.

Polar, at the end or pole of the bacterial cell.

Pseudozoogloex, clumps of bacteria, not dissolving readily in water, arising from imperfect separation, or more or less fusion of the components but not having the degree of compactness and gelatinization seen in zoogloeæ. Pulvinate, decidedly convex, in the form of a cushion.

Punctiform, very small, but visible to naked eye; under $1 \mathrm{~mm}$. in diameter. 
Radiate, showing ray-structure.

Raised. growth thick, with abrupt or terraced edges.

Reduction, removing oxygen from a chemical compound. Refers to the conversion of nitrate to nitrite, ammonia, or free nitrogen, and to the decolorization of litmus.

Rhizoid, growth of an irregular branched or root-like character, as in B. mycoides.

Ring, growth at the upper margin of a liquid culture, adhering to the glass.

Repand, wrinkled.

Rapid, developing in twenty-four to forty-eight.

Rugose, wrinkled.

Saccate, liquefaction in form of an elongated sac, tubular, cylindrical.

Scum, floating islands of bacteria, an interrupted pellicle or bacterial membrane.

Slow, requiring five or six days for development.

Short, applied to time, a few days, a week.

Spindled, larger at the middle than at the ends. Applied to sporangia, refers to the forms frequently called clostridea.

Sporangia, cells containing endospores.

Spreading, growth extending much beyond the line of inoculation, i.e., several millimeters or more.

Stratiform, liquefying to the walls of the tube at the top and then proceeding downwards horizontally.

Thermal Death-point, the degree of heat required to kill young fluid cultures of an organism exposed for ten minutes (in thin-walled test tubes of a diameter not exceeding $20 \mathrm{~mm}$.) in the thermal water-bath. The water must be kept agitated so that the temperature shall be uniform during the exposure.

Transient, lasting a few days.

Truncate, ends abrupt, square.

Turbid, cloudy with flocculent particles, i.c., cloudy plus floceulence.

Umbonate, having a button-like, raised center.

Undulate, border wavy, with shallow sinuses.

Verrucose, growth wart-like, with wart-like prominences.

Vermiform-contoured, growth like a mass of worms, or intestinal coils.

Villous, growth beset with hair-like extensions.

Viscid, growth follows the needle when touched and withdrawn; sediment on shaking rises as a coherent swirl.

Zoogloeæ, firm gelatinous masses of bacteria, one of the most typical examples of which is the streptococcus mesenterioides of sugar vats (leuconostoc mesenterioides), the bacterial chains being surrounded by an enormously thickened firm covering inside of which there may be one or many groups of the bacteria.

Suggested Procedure for Studying Bacteria According to the Descriptive Chart of the Society of American Bacteriologists. The following procedures are suggested for use with the Descriptive Chart. 
( )ne of the factors which ham inhibiled a more extended use of the Chart has been the lack of standard method to une with it. Several of the test's need more study. The Committee on the Chart for the Identification of Bacterial Species of the Society of Amcrican Bacteriologists $h$ us made a preliminary report on methods to be used with the Chart (Conn, H. J., 1918). Since their report represents the attempt on the part of an organized society to standardize tcchnique, parts of their report will be reproduced here.

Media. The importance of using carefully prepared media does not need emphasis. Greatest care should be used in adjusting the reaction to true ncutrality by means of brom thymol blue. All media should be prepared after the Standard Methods of the American Public Hcalth Association.

Invigoration of Cultures. The Committee* has made the following recommendation with regard to this important factor:

Provided a medium can be found upon which the organism to be studied grows vigorously, it should be invigorated before study, even though freshly isolated from its natural habitat. The procedure to be employed is as follows: Prepare duplicate subcultures in standard gluclose broth, and on standard agar slopes, placing cultures of each at $37^{\circ}$ and $25^{\circ}$. On the basis of resulting growth the organism falls into one of the following series:

Sernes I. Organisms which produce good growth (surface growth, distinct turbidity, or heavy precipitate) in twenty-four hours at $37^{\circ}$ in glucose broth.

Series II. Organisms which do not produce good growth in twenty-four hours as above, but do in forty-eight hours at $25^{\circ}$ in glucose broth.

Series III. Organisms which do not grow well in glucose broth but do produce good growth on the surface of agar in twenty-four hours at $37^{\circ}$.

Series IV. Organisms excluded from the above groups but which produce good growth on the surface of agar in forty-eight hours at $25^{\circ} \mathrm{C}$.

Record the series number on the chart at the proper place and proceed with the invigoration by inoculating into another tube of glucose broth for organisms of Series III and IV. Incubate this tube at the temperature, and for the time, called for in the series which it belongs; then transfer from this tube to a third tube and incubate as before. From this third culture make a gelatin or agar plate and incubate at the temperature previously used until colonies of sufficient size for isolation are obtained.

Transfer from a typical colony to one or more agar slants and incubate one day at $37^{\circ}$ or for two days at $25^{\circ}$ according to the temperature relation of the organism studied.

* The term "Committee" when used in this chapter will refer to the Committee on the Chart for Identification of Bacterial Species of the Society of American Bacteriologists. 
In case the organism does not produce growth on either of these media at either temperature, it should be invigorated with any medium and at any temperature known to be adapted to its growth. Under such circumstances, invigorate by the procedure just outlined but using the medium and temperature found most favorable for the organism in question, recording on the chart the method of invigoration adapted. If no conditions are known under which the organism in question produces vgorous growth, it should be studied without preliminary cultiration as soon as possible after isolation from its natural habitat. Such an organism is not likely to give good growth on any ordinary media, and the results of the study called for by the chart will have little significanse.

Motility. Some care is necessary in interpreting the results of motility determinations. Positive results are satisfactory but negative results are convincing oniy after a number of attempts. The safest method to follow is to make flagella stains for if the organism possesses flagella, it is reasonable to assume that it is motile. The Committee states that even negative results do not absolutely prove that the organism is immotile.

Vegetative Cells. Some bacteria go into the spore stage so quickly that in order to secure vegetative cells, as young a culture as possible should be used. For making the various determinations it is necessary to have a young and vigorous culture. This may be secured by passing it through a process of rejuvenation which should consist of continued transferring to a medium which is best adapted for growth. It may be necessary to determine the optimum temperature and incubate all cultures at this temperature.

Endospores. These may usually be observed on the smears which have been stained with the ordinary alcoholic-aqueous stains. On account of a different type of protcplasm, possibly, they resist the staining process and appear on the smear as colorless bodies usually round or oval in shape. In some cells they will be observed in the ends or middle. In this case the cell has not decomposed to set them free. Special stains may be used for indicating the presence of spores, the procedure for which has been given in the Chapter on Staining. Very often these special methods are not attended with much success.

Another method which is often reliable is to kill the vegetative cells by heating in broth culture for thirty minutes at $80^{\circ} \mathrm{C}$. If after this procedure growth is secured by subculturing into other media, it is good indication that the culture was a spore former.

Capsules. The presence of capsules may be indicated either by special staining procedures or the growth in the common media. The 
special staining procedures have been described in the Chapter on Staining. Capsulated bacteria usually grow with a sticky, slimy growth which when touched with a needle, will pull out into a thread. In liquid media the sediment in the bottom of the culture tube will often show a slimy appearance. On the ordinary smears stained with aqueous alcoholic solutions of the aniline dyes, the capsulated bacteria often show a halo about each cell. This seems to resist the staining procedure and may often be made more visible by slightly moving the fine adjustment of the microscope.

Staining Reactions. The theory of staining and the preparation of smears has been fully described elsewhere. To make a complete study of the staining properties of an organism, all of the stains mentioned on the Descriptive Chart should be used. The common special stains, such as the acid-fast, should also be tried.

Oxygen Relations. The relation to oxygen may be determined from the closed arm of the fermentation tubes. Before using, however, it is advisable to reduce the dissolved oxygen to a minimum. This may be done either by a vacuum pump or boiling.

The incubation of streak plates by means of Torrey's method will give good results. Also agar streaks of the organism may be incubated in a Novy jar.

Nutrient Broth. After this medium has been inoculated from a young culture of the organism, it should be incubated at the optimum temperature for the organism. The characteristics of growth should be recorded on the Chart and the culture saved for other tests as hereafter described. Before these observations are made the tube should be shaken as little as possible in order not to destroy any ring or pellicle formation.

Agar Stroke. Streak the agar slant in a straight line from the bottom of the slant to the top. Care should be exercised not to cut through the surface of the slant. . If the inoculum is deposited within the agar when it is intended for the surface anaerobic conditions may be established. With freshly prepared agar streaks, in the bottom of which is water of condensation, care must be used not to lay them flat on the desk since a spreading growth will usually result.

Agar Colonies. Pour agar plates with a dilution which will allow few colonies on the plate. Ten to twenty are sufficient when cultural characteristics are to be determined. Spreading colonies should be avoided either by inverting the plates during incubation or the use of Hill's porous covers. 
Gelatin Colonies. See the directions given for agar colonies. If the culture is a liquefier, observations should be made at frequent intervals before the colonies have spoiled the plate.

Gelatin Stab. A straight needle should be used and the tube stabbed to the bottom. It should be incubated at $20^{\circ} \mathrm{C}$. and frequent observations made to determine the amount of liquefaction. The amount of liquefaction may be determined by means of a millimeter rule. The Descriptive Chart adopted at the 1907 meeting of the Society of American Bacteriologists recommended that gelatin tubes should be held for six weeks to determine liquefaction. This is a test upon which some work must be carried out in order to secure better methods for determining gelatin liquefaction in the shortest time. There is little trouble or error involved in reporting positive results but the time is an important element with negative results. Some bacteria will liquefy gelatin only after several months. This indicates that another method, by which to more sharply distinguish between the liquefiers and the non-liquefiers, is needed.

The Committee has proposed to make the method of Rothberg (1917) provisional until it may be tried out. Rothberg gives the organism a preliminary cultivation in a 1 per cent gelatin solution at $25^{\circ}$ or $37^{\circ}$; then inoculate the surface of gelatin in a test tube and incubate for 15 days at $20^{\circ} \mathrm{C}$.

Potato. The inoculation of potato slants should be made in the same way as for agar slants. After the observations called for on the Descriptive Chart have been made, the potato may be tested for diastasic action according to the directions given under "Potato Starch Jelly."

Potato Starch Jelly. Iodine should be used to determine the presence of diastasic action. Pour the contents of the culture tube into a beaker and dilute with distilled water. If the dilution is sufficient and if the organism possesses a diastase, the various colors of the starch decomposition products with iodine will be obtained. Some of these are as follows:

Starch . ................ blue color with iodine

Soluble starch ............. blue color with iodine

Erythordextrin ............ red color with iodine

Achroodextrin............. no color with iodine

Maltose................. no color with iodine

Dextrose................ no color with iodine 
To secure sucess' with this method both the iodme and starch solutions must be very dilute The same method has been applied to potato. slants. In this procedure the slants are thoroughly mashed and after dilution with water are treated as above.

Allen (1918) has described another convenient method which has had rather extended use. This involves the use of a 0.2 per cent thymol starch agar which is poured into a sterle Petri dish and allowed to harden. Streaks are made on this and after incubation the dish may be flooded with rodine solution. A clear halo about the growth indicates diastasic action on starch. In order to satisfy the requirements of the Descriptive Chart Allen has stated that a clear zone of more than $2 \mathrm{~mm}$. width should be regarded as strong while a feeble action is denoted by a width of less than $2 \mathrm{~mm}$. This differentiation called for on Descriptive Chart is rather ambiguous since to distinguish between feeble and strong diastasic action is probably unnecessary.

Edson and Carpenter (1912) to determine the presence or absence of diastasic action added a 2 per cent thymol starch paste to a 10-dayold broth culture. After incubation for about eight hours, the culture was tested for reducing sugars by means of Fehling's solution.

Temperature Relations. The determination of this characteristic has been usually limited to $37^{\circ} \mathrm{C}$. and $20^{\circ} \mathrm{C}$. Other temperatures may be used as desired. The organism should be inoculated into a medium in which it will grow well. This culture should then be incubated at different temperatures. The possibility of other factors inhibiting growth should be carefully guarded against. It mar be necessary to study this characteristic before starting the other culture work in order to determine the optimum temperature.

Cohn's and Uschinsky's Media. After inoculation they should be incubated at the optimum temperature. The observations which should be made are mentioned on the Chart. These media may also be used as a base to which other compounds, such as fats, proteins, etc., may be added.

Loeffler's Blood Serum. Treat in the same way as described for "Agar Stroke." Make the necessary records on the Chart.

Milk. Freshly skimmed milk should be sterilized either in test tubes or Erlenmeyer flasks. The various observations should be recorded on the Chart. The proteolysis of casein may be determined either by the appearance of the tube or by means of the Hastings (1904) milk agar plate. For preparing this milk agar plate, sterile skimmed milk is added to melted plain agar which has been cooled to $50^{\circ} \mathrm{C}$. and poured into a sterile Petri dish to harden. The strain which is 


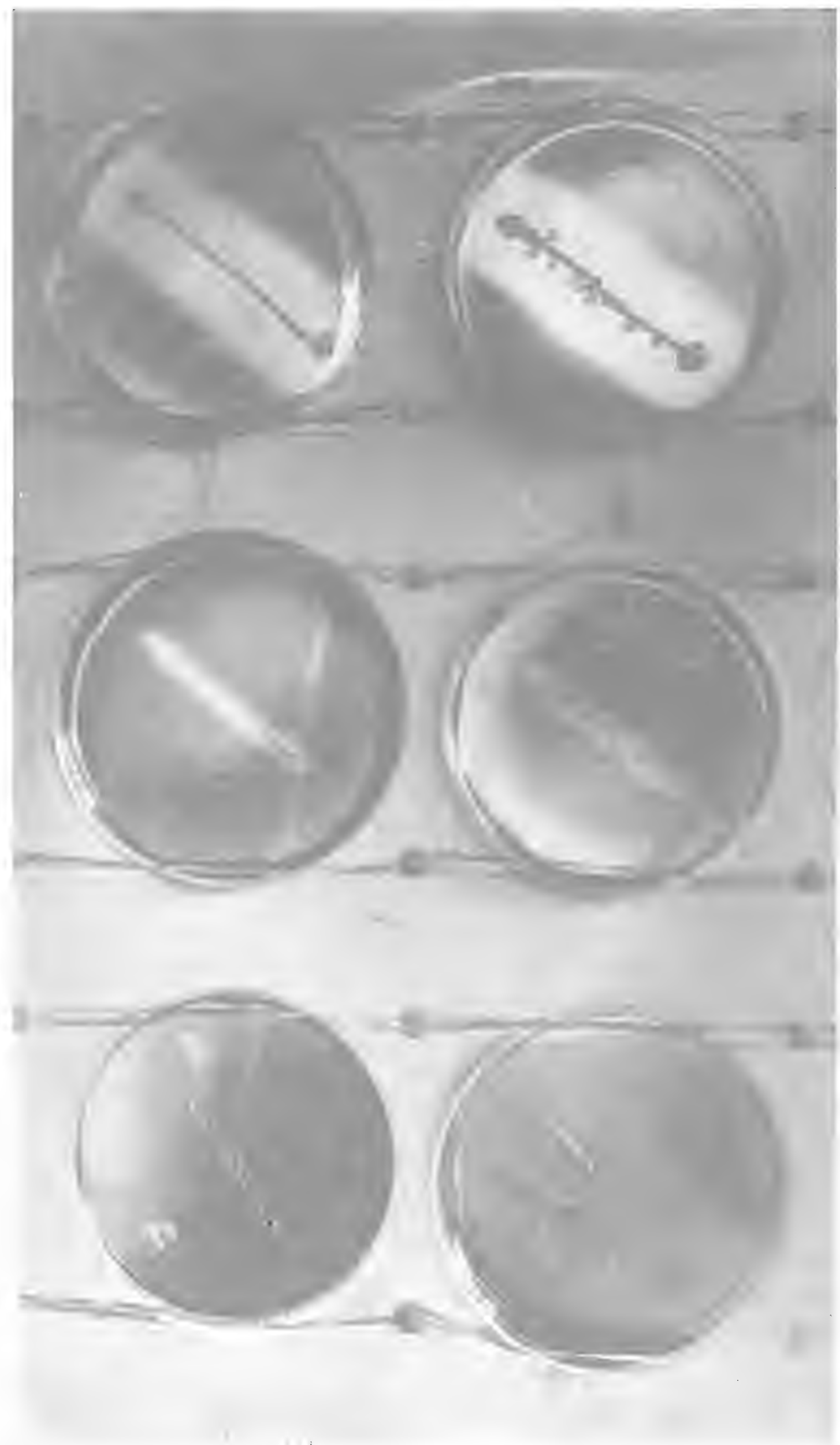

Fia. 36.-Showing Strong, Feeble and Weak Diastasic Action by Bacteria. (After Allen, 1.)18). 
being studied should be streaked across the surface of the agar. After incubation a clear zone will be visible, which, if due to true proteolysis, will not be rendered opaque if treated with weak acid. If a clear zone is present after incubation, it may be due to either proteolysis or the acid formed in the metabolism of the organism. To prove that proteolytic enzymes produced it, flood the plate with dilute acetic acid and if the clear zone remains, the presence of a protease is indicated.

The Descriptive Chart calls for the determination of the reaction on certain days. In the past, this has been determined by titrating 5 c.c. of the culture with $\mathrm{N} / 20 \mathrm{NaOH}$ or $\mathrm{HCl}$. That this method is not accurate has been shown by Clark and Lubs. In order to take advantage of their work the Committec has advised the following procedure:

Acid production in milk can be detected by adding brom cresol purple to the culture and comparing with the color obtained by adding the same proportionate quantity of the indicator to sterile milk. (Brom thymol blue does not give satisfactory results in milk.) Four degrees of acidity that can be recognized in milk are listed in Table II. They correspond closely to those listed in Table I, differing only on that brom cresol purple is used instead of brom thymol blue to show "neutrality" and that the curdling point $\left(P_{H}=4.7\right)$ is used to separate between "moderate" and "strong" acidity instead of the less definite point of maximum red to methyl red. The same method of expression used in recording acidity in clear media should be used in recording that of milk.

\section{TABLE VI}

\section{DEGRLES OF ACIDITY EASILY RECOGNIZED IN MILK}

(After Conn et al., 1918)

\begin{tabular}{|c|c|c|}
\hline Acidity. & Indicator Reaction, etc. & $\begin{array}{l}\text { Approximate } \\
P_{H} \text { Value. }\end{array}$ \\
\hline Neutral & $\begin{array}{l}\text { Same color with brom cresol purple * as sterile milk; } \\
\text { i.e., blue to gray green. }\end{array}$ & $6.2-6.8$ \\
\hline Weak. & $\begin{array}{l}\text { Color with brom cresol purple lighter than in sterile } \\
\text { milk; i.e., gray green to greenish yellow. }\end{array}$ & $5.2-6.0$ \\
\hline Moderate & Yellow with brom cresol purple. Not curdled.... & $4.7-5.0$ \\
\hline Strong. & Curdled. Blue or green to brom phenol blue.... & $3.2-4.6$ \\
\hline Very strong. & Yellow to brom phenol blue $\ldots \ldots \ldots \ldots \ldots \ldots \ldots$ & Under 3.0 \\
\hline
\end{tabular}

* Use a 0.04 per cent solution. 
TABLE VII

SHOWING DEGREES OF ACIDITY EASILY RECOGNIZED IN CLEAR

(1fter Conn ct al., 1918)

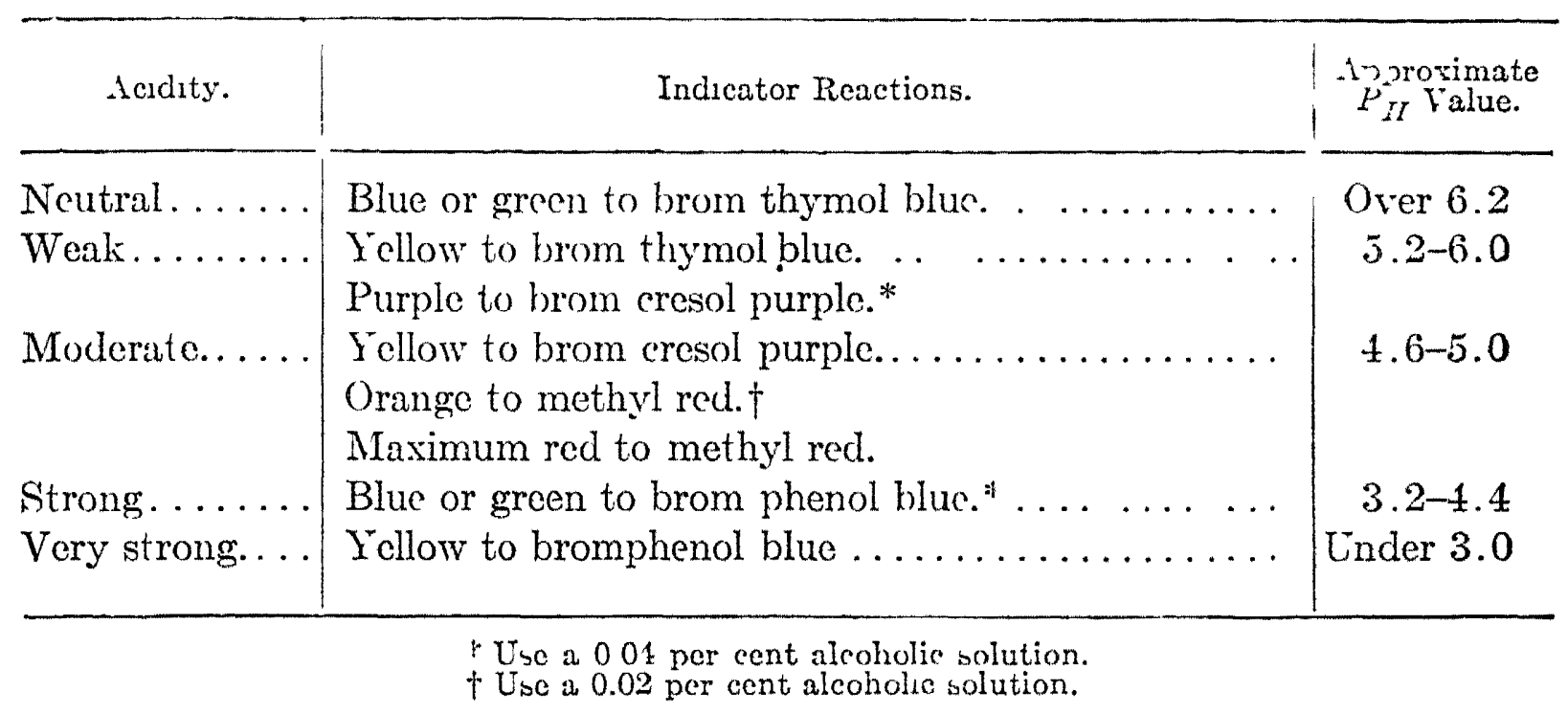

Litmus Milk. Growth in this medium is not much different from that in plain milk. If the tube turns to a white with a pink layer at the surface which is in contact with air, the presence of a reductase for litmus is indicated. Clark and Lubs (1917) have stated that brom eresol purple may be used as a substitute for litmus in milk. The committee regards this as not always to be recommended since this dye does not show the reduction phenomena.

Indol Production. Indol is formed from tryptophane and is produced in media which contains proteins or their split products containing this amino acid. Under routine conditions the production of indol may be determined in Dunham's medium or in the plain broth culture. Zipfol (1912) has shown that trytophane in an inorganic medium will show indol formation in twenty-four hours. Since tryptophane is so expensive Cannon (1916) has prepared a hydrolyzed casein medium with which it is claimed that good indol formation is sccured. This is prepared according to directions which are given in the Chapter on Media. The following tests for indol may be used:

Nitroso-Indol Nitrate Test: Add 0.5 c.c. of concentrated sulphuric acid and 0.5 c.c. of dilute sodium nitrite to the culture. If a red color is obtained the presence of indol is indicated.

Ehrlich's Test: Add 0.5 c.c. of paradimethylamidobenzaldehyde to the culture and if indol is present, a deep crimson color will be formed. 
Hydrogen Sulphide. Hydrogen may be formed from many of the sulphur-containing compounds. When formed from proteins it probably comes from cystine or some other sulphur linkage. It may be detected by suspending a strip of bibulous paper saturated with lead acetate to which a little glycerol has been added. This method has been found to yield satisfactory results by the author.

Ammonia Production. Dilute the culture with ammonia-free water and add 1 c.c. of Nessler's reagent. The presence of a yellowish-green color indicates the formation of ammonia.

Nitrate in Nitrate Broth. Ammonıa, see above. Nitrate. The amount of nitrate decomposed by an organism may be determined quantitatively by the aluminum-reduction method which has been outlined in the Chapter on Water Analysis. For qualitative results the presence of nitrites or ammonia in a medium which was free from these substances before inoculation may be taken as sufficient evidence of nitrate reduction.

Test for Nitrites. The same method may be used for the qualitative estimation of the presence of nitrites that is used in the quantitative determination. The Griess method has had much application and depends on the formation of azobenzolnaphthylamin whenever naphthylamin and sulphanilic acid are present in an acid solution of nitrites. Mason (1912) gives the reaction as follows:
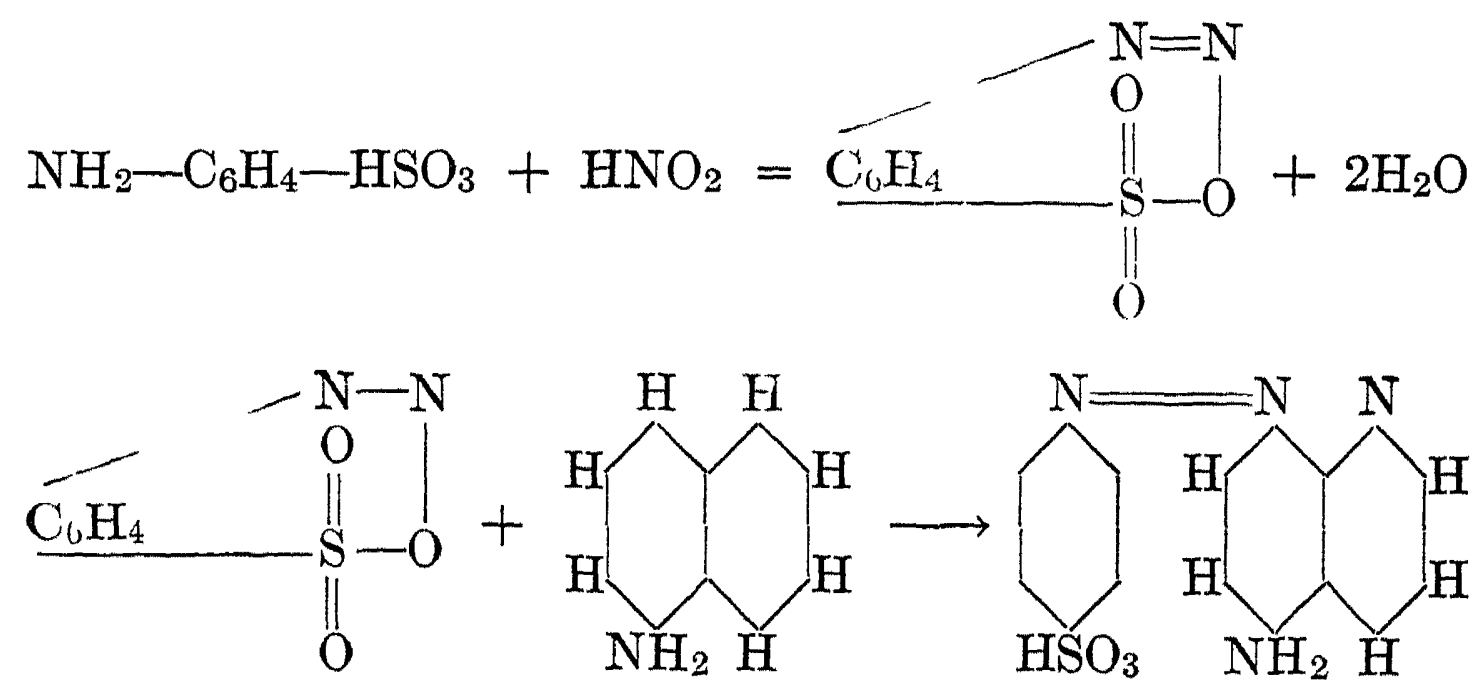

This is a very delicate test and is regarded by a few bacteriologists as too delicate for bacteriological work. If a control tube is made accurate results should be secured by its use.

To determine nitrites, dilute 2 or 3 c.c. of the culture with ammoniafree water and add $\frac{1}{2}$ c.c. each of sulphanilic acid and naphthylamin hydrochloride. The presence of a red color when viewed the long way of the Nessler tube indicates the presence of nitrites. 
Smith (1905) regards the iodine starch test as most satisfactory for microbiologists. He gives the procedure.as follows: "Twenty-five c.c. of distilled water are added to $\frac{1}{2} \mathrm{gm}$. (more or less) of pure potato starch and the fluid boiled. One cubic centimeter of this starch water and 1 c.c. of freshly prepared potassium iodide water $(1: 250)$ are now put into the culture fluid, to which is then added a few drops of strong sulphuric acid water $(2: 1)$. If any appreciable quantity of nitrite is present the culture immediately becomes blue black from the liberation of free iodine which acts upon the starch. Old potassium iodide should never be used without first testing carefully as it usually contains some free iodine." Blank determination should be made.

Silicate Jelly. Make the required observations and record on the Chart.

Fermentation Reactions. The various compounds upon which it is desired to study the action of microorganisms, are added to plain broth. Great care should be used in the sterilization of these media since Mudge (1917) and Hasseltine (1917) have shown that polysaccharides are easily hydrolyzed to monosaccharides. The latter of these investigators found that serious errors were introduced in the study of $B$. proteus, for instance, on common carbohydrates. For investigations requiring accurate data other methods of sterilization than moist heat should be used. The broth may be sterilized by filtration after the carbohydrates have been added, or the carbohydrate solutions may be sterilized by filtration and added to the broth by means of a sterile pipette just before the tubes are inoculated. In determinating the reaction of the fermentation tubes, it has been the custom to titrate 5 c.c. of the medium with $\mathrm{N} / 20 \mathrm{NaOH}$ or $\mathrm{HCl}$ with phenolphthalein as the indicator. The work of Clark and Lubs has shown that this method does not allow accurate results. They have advised the determination of $\mathrm{H}$-ion concentration, but such a procedure is not adapted to routine work on account of the time consumed. In place of this, these workers have prepared a series of indicators with a sufficient range to meet the requirements of bacteriological media. These should be used where it is possible. They are given in the appendix.

Pathogenicity to Animals. The suspected organism should be fed to different laboratory animals and also injected into different tissues. The animal should be kept under observation for a short period before the experiment begins. After injection, it should be constantly watched and weighed.

The Committee recommends the use of these indicators and makes the following statement: 
In careful research work the exact shade of the indicator should be compared with that obtained in standard "buffer" solutions, and the results recorded in terms of $P_{H}$. In laboratories where these solutions cannot be obtained, it is better to record the results as simply + or - according to the reaction of the culture to litmus, than to use the titration method. Under such conditions it is possible, however, to obtain a rough idea of the hydrogen-1on concentration by the use of Clark and Lubs' series of indicators without making accurate determinations of $P_{H}$. Four different degrees of acidity can be easily distinguished by this simple method in sugar broth with initial reaction of neutrality. The indicator reactions for these different degrees of acidity are listed in table II together with the approximate range of $P_{H}$ to which each corresponds. In the absence of accurate determinations, these degrees of acidity may be recorded by the indefinite terms, "weak," " moderate," "strong," and "very strong," or by the symbols,,++++++ , and ++++ .

If this procedure is used in order to secure data, it is possible that a more satisfactory method may be developed whereby the actual acidities of the culture tubes may be defined.

Pathogenicity to Plants. Apply a broth culture of the organism to that part of the plant for which it is supposed to be specific.

Loss of Virulence on Culture Media. The loss of any characteristic may be determined by comparing it before and after prolonged cultivation on artificial media. Attempts should also be made to bring it back after it has been demonstrated to have been lost.

Ferments. The presence of enzymes may be determined by the decompositions which the organisms accomplish. In searching for the presence of any special enzyme, a substrate containing the special compound should be used. Frankel's medium or any other such medium may be used as a base.

Toleration to Acids or Alkalis. This characteristic may be determined by adding different amounts of normal acid to alkali to plain broth before inoculating with the organism. Quite often a large amount of acid or alkali may precipitate the proteins which are in the media. This precipitation must not be confused with growth.

Thermal Death-point. The Committee on Identification of Species of the Society of American Bacteriologists has recommended the exposure of the organism in nutrient broth for ten minutes. The medium should be prepared according to standard methods and the time very carefully limited to ten minutes. 


\section{BIBLIOGRAPHY}

Allen, P. W. 1918. A Simple Method for the Classification of Bacteria as to Diastase Production. Jour. Bact., 3, 15-17.

Cannon, P. R. 1916. A Rapid and Simple Indol Test. Journal of Bacteriology, 1, 535-536.

Chester. 1901. Manual of Determinative Bacteriology. New York. 1901.

Clark, W. M. and Lubs, H. A. 1917. A Substitute for Litmus for Use in Milk Cultures. Jour. Agr. Research, 10, 105-111.

CoHn, F. 1872 Untersuchungen uber Bakterıen. Cohn's Beitrage zur Biol. der Pflanzen. Bd. I. Hefte, 2, 127-224.

Conn, H. W. 1906. Classification of Dairy Bacteria. Ann. Rept. Storrs Agr. Exp. Sta., 107.

Conn, H. J. 1915. A Study of B. Subtilis by Means of the Classification Card. Science N. S., 41, 618.

Conn, H. J. 1917. Soil Flora Studies. Part I. Jour. Bact., 2, 35-45.

DeBary. 1888. Comparative Morphology Biology of the Fungi, Mycetozoa, and Bacteria.

Edson, H. A. and Carpenter, C. W. 1912. Microorganisms in Maple Sap. Vermont Agr. Exp. Sta , Bulletin 167.

Edson, H. A. and CARpenter, C. W. 1912. The Green Fluorescent Bacteria Occurring in Maple Sap. Vermont Agr. Exp. Sta., Bulletin 167, 521-599.

EISENBERG. 1891. Bakteriologische Diagnostik III.

Ellis, H. 1909. Outlines of Bacteriology。 Longmans, Green \& Co., New York.

FISHER. 1900. The Structure and Functions of Bacteria. Oxford.

FlUGGE. 1886. Die Mikroorganismen. II Auf., 1886.

GAGE, S. M., and Phelps, E. B. 1903. On the Classification and Identification of Bacteria with a Description of the Card System in Use at the Lawrence Experiment Station with Record of Species. Proceedings, American Public Health Association, 28, 494-505.

HARDING, H. A. 1910. The Constancy of Certain Physiological Characters in the Classification of Bacteria. Tech. Bull. 13., New York Agr. Exp. Sta., Geneva.

Harding, H. A., Morse, W. J. and Jones, L. R. 1909. The Bacterial Soft Rot of Certain Vegetables. Tech. Bull., N. Y. Agr. Exp. Sta., Geneva.

Harding, H. A. and Prucha, M. J. 1908. The Bacterial Flora of Cheddar Cheese. Tech. Bull., N. Y. Agr. Exp. Sta., Geneva.

Hasseltine, H. E. 1917. The Bacteriological Examination of Water. Reprint No. 43 from Public Health Reports, Nov. 9, 1917.

Hastings, E. G. 1904. The Action of Various Classes of Bacteria on Casein as Shown by Milk Agar Plates. Cent. Bakt. Abt. II, 12, 590-592.

Hort, E. C. 1917. Morphological Studies in the Life-Histories of Bacteria. Proc. Royal Soc. B., Vol. 89, 468-480. 
Jensen, O. 1909. Die Hauptlinien des naturlichen Bakterien systems. Cent. Bakt. II, Abt., 22, 305.

Jensen, O. The Main Lines of the Natural Bacteria System and the Bacteriological Nomenclature. VII Intern Cong. Applied Chem., Part IV, 176-181.

Johnston, W. 1894. On the Grouping of Water Bacteria. Amer. Pub. Health Assn. Proc., 20, 445-449.

Jordan, E. O. 1912. General Bacteriology. 107-109.

Kendald, A. I. 1903. Reported by Gage and Phelps. On the Classification and Identification of Bacteria with a Description of the Card System in Use at the Lawrence Experiment Station for Records of Species. Amer. Pub Health Assn. Proc., 28, 494-505.

Lemmann and Heumann. 1901. Atlas and Principles of Bacterology Philadelphia.

Lister, J. 1872. On the Germ Theory of Fermentation and Other Fermentative Changes. Nature, July 10, and 17, 1872.

Lister, J. 1873. A Further Contribution to the Natural History of Bacteria and the Germ Theory of Fermentation Changes. Quarterly Jour. Microscop. Soc., 380.

LoHnis, F. 1916. Life Cycles of Bacteria. Jour. Agr. Research, 6, 675-702.

Mason, W. P. 1912. Examination of Water. New York, John Wiley \& Sons.

Mrgula, Die Bakterien.

MudGe, C. S. 1917. The Effect of Sterilization upon Sugars in Cultures Media. Jour. Bact. 2, 403-415.

RAHN, O. 1911. Marshall's Microbiology. P. Blakiston's Son \& Co, Philadelphia.

RAHN, O and HARDING, H. A. 1916. Die Bemuhumgen zur einheltlichen Beschreibung der Bakterien im Amerika. Cent. Bakt. Abt. II, 42, 385-393.

Rosenow, E. C. 1914. Transmutation within the Streptococcus-pneumococcus Group. Jour. Inf. Diseases, 14, 1.

Rosenow, E. C. and Towne, E B. 1917. Bacteriological Observations in Experimental Poliomyelitis. Jour. Med. Research, 36.

Rothberg, W. 1917. Observations on Some Methods for the Study of Gelatin Liquefaction. Paper read before the Socipty of American Bacteriologists, December, 1917.

SмrтH, I. F. 1905. Bacteria in Relation to Plant Diseases.

TANner, F. W. 1918. A Study of the Green Fluorescent Bacteria from Water. Jour. Bact, 3, 63-101

Winslow, C. E. A. 1917. The Families and Genera of Bacteria. Jour. Bact., 2, 505-566.

ZrpFet. Cent. Bakt. Orig. Abt., I, 64, 65.

Zopf. 1884. Die Bakterien I. Aufl., 1885. 


\section{CHAPTER V \\ STERILIZATION AND DISINFECTION}

BACTERIA do not exist in nature as pure cultures. Since there are so many varieties, the bacteriologist must use media and apparatus which are free from not only bacteria but all forms of life. This is accomplished by sterlization, which is to be distinguished from disinfectionthe removal of pathogenic bacteria only. The methods for sterilization, may be classified as follows:

I. Dry heat:

a. Flaming and incineration.

b. Hot air oven.

II. Moist heat:

a. Inspissator.

b. Streaming steam in the Arnold.

c. High pressure steam in the autoclave.

d. Boiling.

III. Filtration:

a. Through liquids.

1. Sodium hydroxide, sulphuric acid, etc.

$b$. Through solids.

1. Sand, cotton, glass wool, porcelain, etc.

IV. Light:

a. Sunlight.

b. Ultraviolet.

\section{Dry Heat}

Death by drying may be the result of two processes. The cell protoplasm may decompose or oxidation may take place. This has been studied by Paul (1909) and his co-workers who found, in general, that the rate of death in dry heat is proportional to the oxygen concentration. This would seem to indicate that death in dry heat was an oxidation process, although other reactions may enter. With regard to moist 
bacteria, the oxidation processes would be limited by the solubility of the oxygen in water. According to Winkler (1889) this is 10.14 parts per million at $15^{\circ} \mathrm{C}$. and $760 \mathrm{inm}$.

Incineration. As a means of sterilization, this method needs no discussion. It is plainly a process of oxidation and very efficient. Small incinerators may be purchased which will handle practically all ordinary material.

Flaming. This simple method is a scrviceable one for the bacteriologist. Platinum wires may be sterilized by this method. Watch glasses, slides, etc., may be completely sterilized if a little care is used during the flaming process. The Bunsen burner or alcohol lamp may furnish the heat.

Hot-air Oven. The apparatus for this method is constructed much like the ordinary baking oven. Quite often an attempt is made to insulate it either by means of a double wall or some special material. The temperature is usually maintained by means of a gas flame or electricity. Some hot-air sterilizers are so well insulated that after the temperature has been raised to $180^{\circ} \mathrm{C}$., this temperature is maintained for a long time even though the source of heat is removed. The hot-air oven is used for sterilizing all dry glassware. This should be put into the sterilizer when it is cool and gradually raised to the sterilization temperature. A temperature of $180^{\circ} \mathrm{C}$. for from one hour to one hour and a half will suffice for all ordinary apparatus. This method has definite limitations and should not be applied to the sterilization of media, thick glassware, etc.

\section{Moist Heat}

The theory of sterilization by moist heat has been studied by Chick (1910). Here three essentially different processes take place:

1. Direct effect of heat on bacterial protein.

2. Effect of water possibly hydrolytic on these proteins at high temperature.

3. Desiccation of bacteria.

It was pointed out by Miss Chick that an analogy exists between the disinfection of bacteria by hot water and the "heat coagulation" of proteins. This may help to account for the difficulty with which spores are disinfected by hot water when compared with the vegetative cells. The protein in the spores may be more resistant to hydrolysis than that in vegetative cells. Both changes follow the monomolecular law and both are greatly increased by the presence of minute amounts 
of acid. Chick (1910) states, "The striking similarity between the effects of temperature (dry) on the one hand and hot water on the other indicate that disinfection by the latter is due to the action of water (coagulation and alteration) upon some one protein which is essential for life of the bacterium and that the reaction is conditioned by the chemical action of water upon its constituent proteins." The follow-

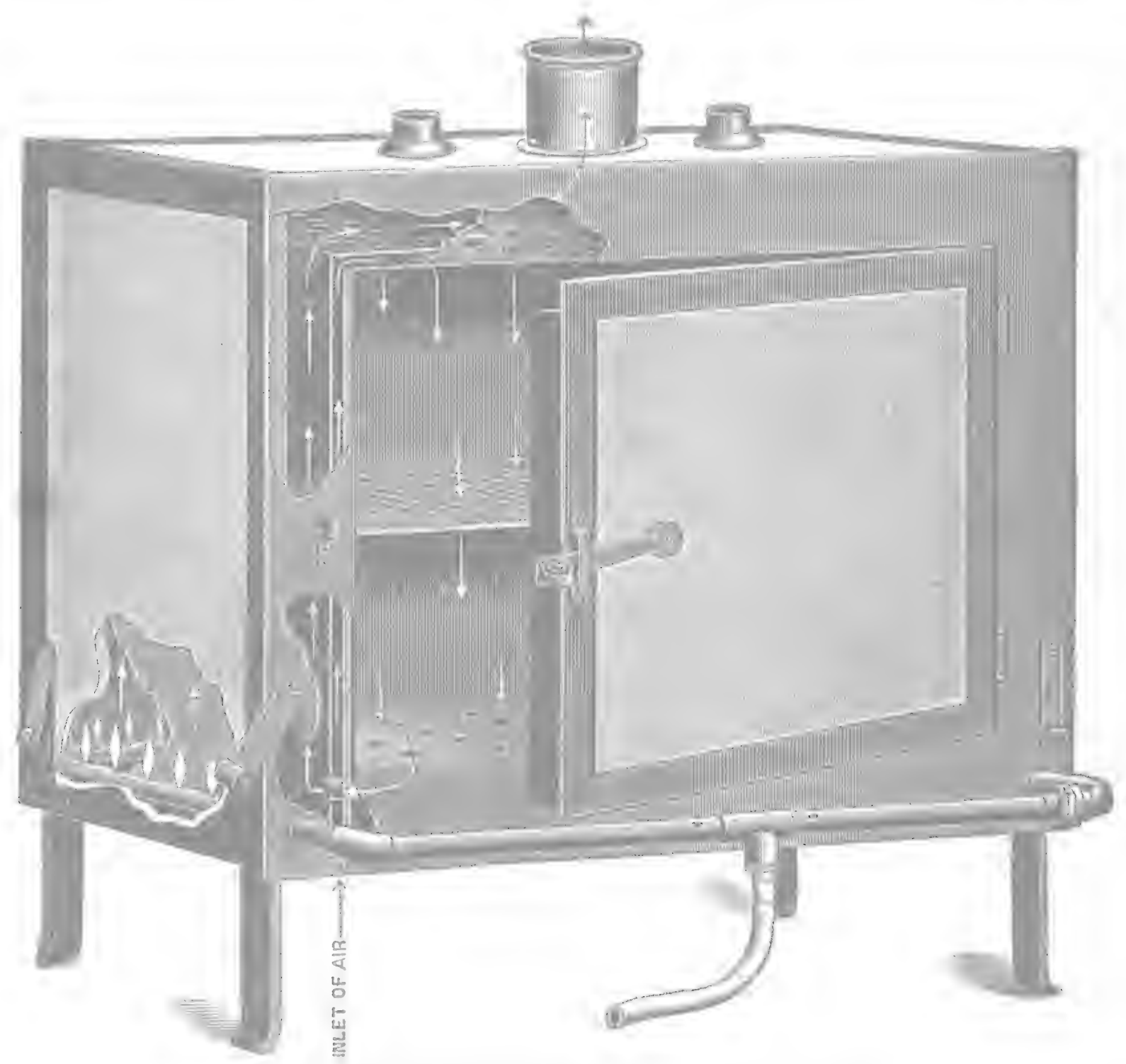

FIG. 37.-Hot-air Sterilizer, Lautenschlager Type.

ing facts with regard to disinfection by moist heat were established by Chick:

1. Disinfection proceeds according to the logarithmic law the rate of disinfection being proportional, at any moment, to the concentration of surviving bacteria.

2. The presence of minute quantities of acid or alkali too small to produce any direct disinfectant action increases the reaction greatly. The acid, however, gave the greatest increase. This presents close analogy to the "heat coagulation" of proteins. 
Rubner (1913) has reviewed the application of steam to sterilization. He points out that vegetative cells contain much more water than spores and thus succumb more quickly to the action of heat. Spores contain hygroscopic water which soon evaporates leaving dry bacterial protein. This will resist heating for some time. Steam at $100^{\circ} \mathrm{C}$. is an important chemical agent since hydrogen sulphide, ammonia and carbon dioxide are liberated from keratin, casein, and dried bacteria when they are subjected to its influence. Rubner found the saturation of the steam to be about as important as the temperature. Steam at $100^{\circ} \mathrm{C}$. with a saturation of 80 per cent requires five times as long to kill bacteria as does saturated steam and steam with a saturation of 70 per cent requires twenty-two times as long as saturated steam. If steam at $100^{\circ} \mathrm{C}$. is superheated, it is altered in two ways. The temperature is raised which makes it a more powerful disinfectant and the saturation is lowered which makes it a weaker disinfectant. In superheated steam at $110^{\circ} \mathrm{C}$. made from steam at $100^{\circ} \mathrm{C}$. spores lived twice as long and at $127^{\circ} \mathrm{C}$. ten times as long. This suggests the correlation between the temperature and saturation which are important factors in sterilization. According to the laws of disinfection, time is the other factor and it cannot be separated from the first two.

High-pressure Steam. This method has extensive use in bacteriology. The apparatus is called an autoclave or dressing sterilizer. Several types are used, the most convenient of which are connected to a steam main from a power plant. Where this is impossible, it may be necessary to generate the steam under the sterilizer. This usually requires more time since it takes some time to get up the pressure. Often it is necessary to keep water in the bottom of the sterilizer in order to prevent the superheating of the steam. Under practical conditions this is usually not necessary since the steam will be saturated. The following table will show the relation between the usual pressures and temperatures required for sterilization in the autoclave:

\begin{tabular}{c|c|c|c}
\hline Gage Pressure. & $\begin{array}{c}\text { Temperature, } \\
\text { Centigrade. }\end{array}$ & Gage Pressure. & $\begin{array}{c}\text { Temperature, } \\
\text { Centagrade. }\end{array}$ \\
\hline 0 & 100 & 15 & 1215 \\
5 & 109 & 20 & 126 \\
10 & 1155 & 40 & 141 \\
\hline
\end{tabular}

In the presence of water, Chick has shown that the temperature is an important factor in sterilization. The temperature coefficient 
was determined for B. Lyphosus and was reported to be 1.635 per $1^{\circ} \mathrm{C}$. or 136 per $10^{\circ} \mathrm{C}$. Here again the killing of bacteria in the presence of water was found to be analogous to the "heat coagulation " of their constituent proteins.

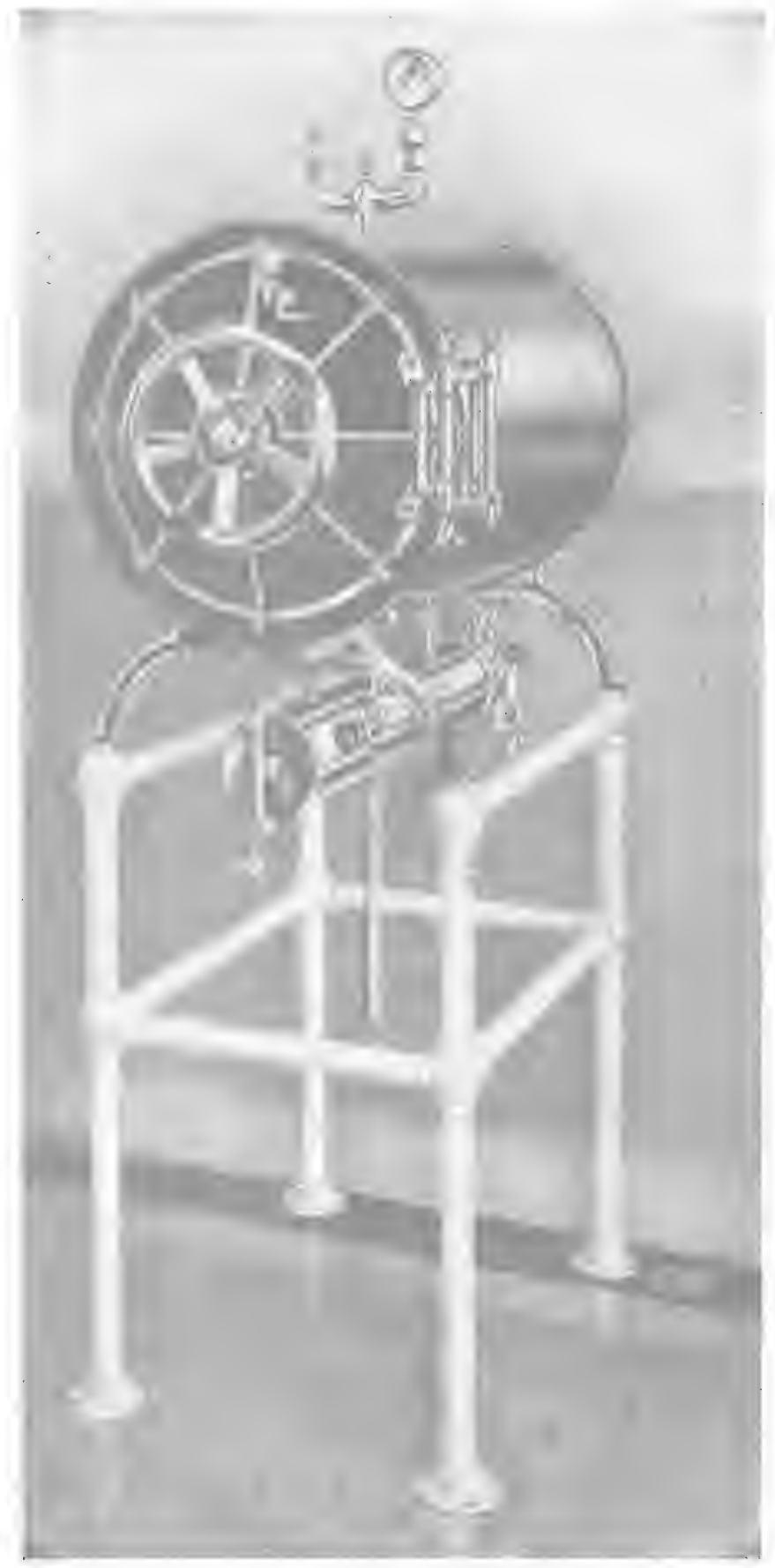

Fig. 38.-Autoclave or Dressing Sterilizer, Kny-Schereer Type.

The autoclave may be used for sterilizing many pieces of apparatus and many media. It has the advantage over other methods of taking less time. It has been recently shown that less hydrolysis of polysaccharides is secured in the autoclave than in the Arnold. This is contrary to what was believed, for it is stated in many places that 
the Arnold steam sterilizer too should be used on such substances as igar's to prevent hydrolysis.

Boiling. Practically no special apparatus is required by this method and any that is demanded may be quickly secured. Probably fifteen minutes is sufficient, for vegetative cells by spores are too resistant to be quickly killed. Surgical instruments may be sterilized by boiling. The water should be boiled for ten minutes before they are put in in order to prevent the possibility of rusting.

Streaming Steam. The apparatus which is used in this method is much like the ordinary steamer which is available in the kitchen.

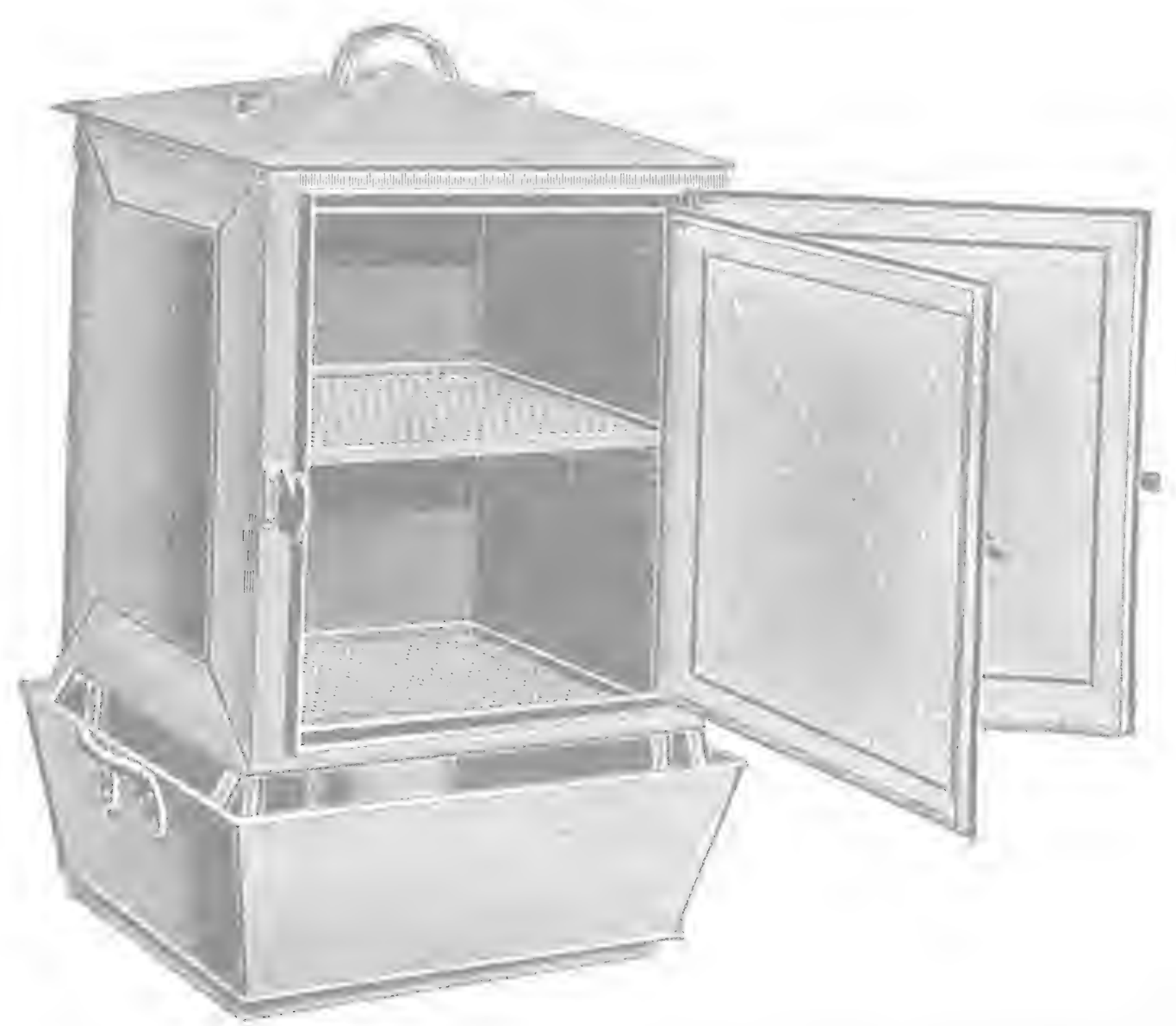

FIG. 39.--Arnold Steam Sterilizer. (Boston Board of Health Pattern.)

In bacteriology the apparatus is known as the Arnold steam sterilizer. It was devised by Tyndall, and improved by Koch and Arnold. It consists of essentially a copper box with a false bottom through which the steam rises to escape at the top. 'There is no superheating in the Arnold as with the autoclave and it requires less attention. It may be used in two ways-continuous or intermittently:

With the continuous method the material to be sterilized is heated for from thirty minutes to a hour and a half. This method has the disadvantage that such prolonged heating may cause changes in the materials. Di-, tri-, and poly-saccharides may be hydrolyzed proteins 
caogulated, etc. Such has been found to be the case as mentioned elsewhere.

With the intermittent method the material is heated for twenty to thirty minutes on three successive days. On the first day the vegetative cells are killed. The second day's heating will destroy those vegetative cells which have come from spores which passed through the first day's heating. On the third day the process is simply checked up.

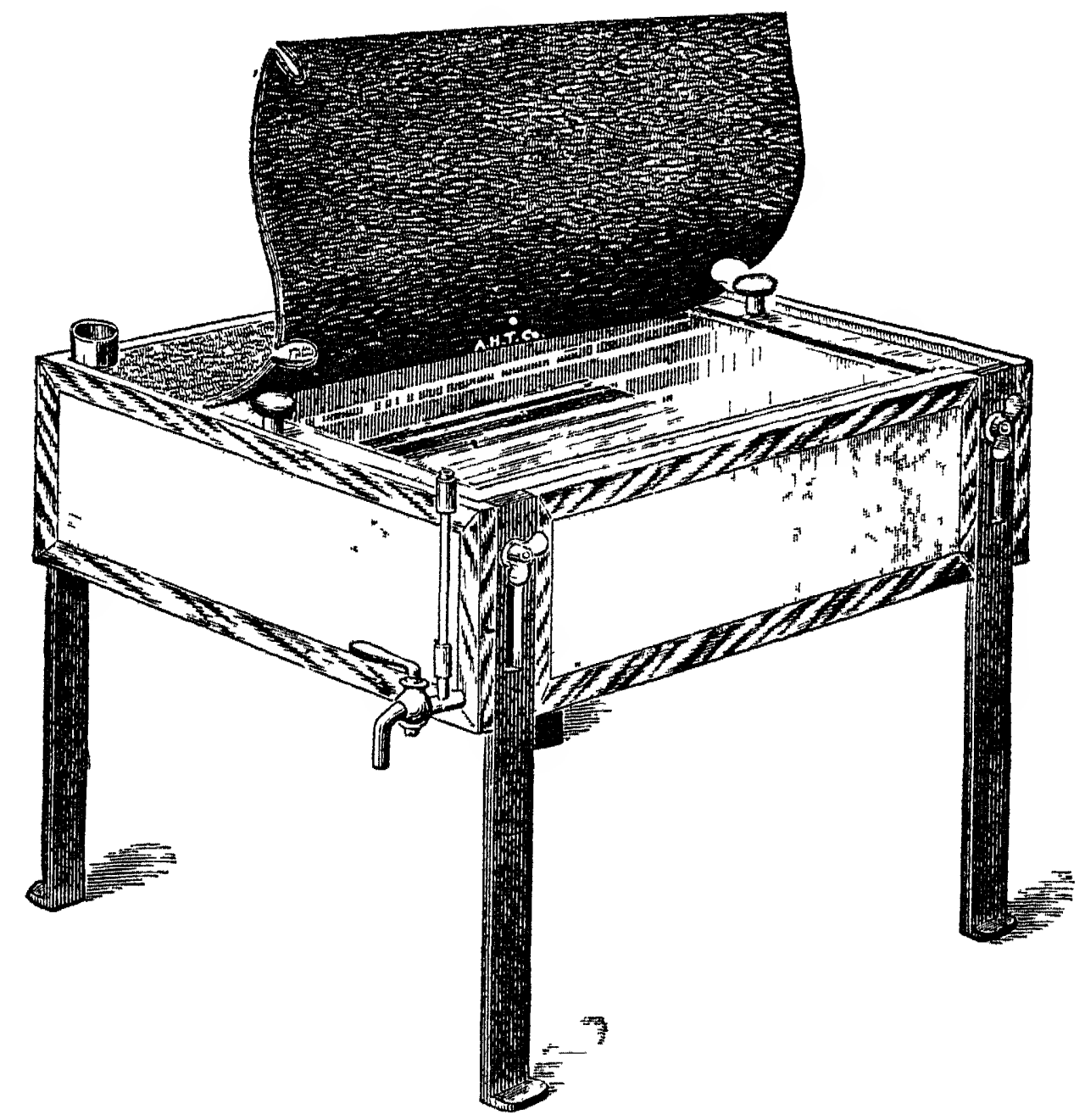

FIg 40 -Bath for Sterlization at Low Temperatures (Inspissator).

Inspissator. Some media which are used by , bacteriologists must be sterilized at low temperatures. For this purpose a serum coagulator or inspissator is used. The media is heated at from $57^{\circ}$ to $60^{\circ} \mathrm{C}$. for different lengths of time. Blood serum may be heated for periods of one hour or for a longer period at one time.

\section{Sterilization by Filtration}

Filtration is an efficient method of sterilization which has certain distinct advantages over other methods. It leaves the filtrate sterile 
and unchanged. Body extracts and scra may be thus treated to render them sterile.

Filtration through Liquids. The action is entirely mechanical unless some strong liquid such as sodium hydroxide or sulphurıc acid are used. In these cases the bacterial protein is decomposed by the chemical. Rettger applied this method to the bacterial examination of air.

Filtration through Solids. The same principle is involved here that is involved in the use of the cotton plug in test tubes and culture flasks. Different substances have been used but most attention has

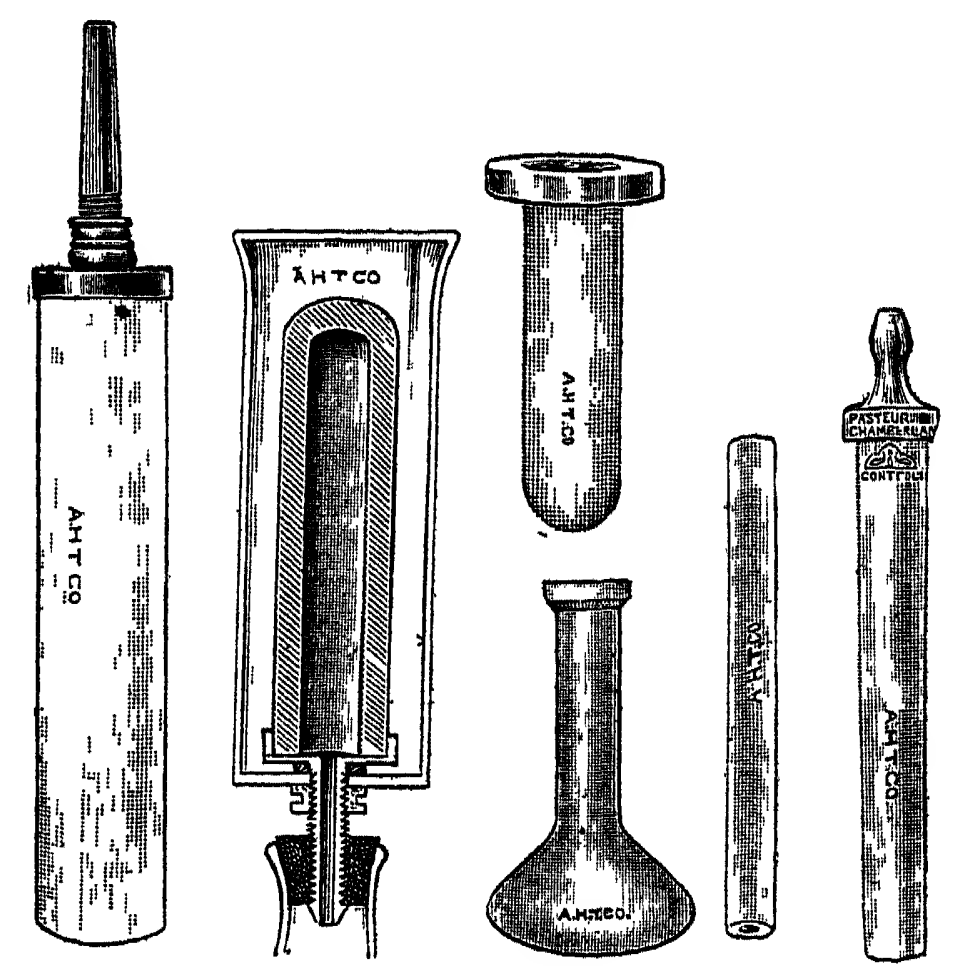

FIG. 41.-Diatomaceous Filters for the Sterilization of Barterial Extracts and Body Fluids.

been given to cotton, glass, wool and sand. Sand is used in the standard method for the bacterial examination of air and also in the purification of water. Stone or porcelain filters are the best substances for laboratory use. They may be secured in many sizes and are easily sterilized and cleaned.

Dialysis. In this medium the semi-permeable membrane is used as the filtering medium. Collodion sacs have had quite a little use in bacterial work.

\section{LIGHT}

Although light does exert an antiseptic action on bacteria and may even totally destroy them after a long period, it is scarcely used 
by the bacteriologist to sterilize media or apparatus. The work on the germicidal action of light is extensive and those wishing bibliographies on the subject should consult the papers of Dieudonne (1894), Ward (1895) and Weinzirl (1914).

Ward (1895) using anthrax bacilli and spores found that exposures of one-half to one hour were necessary for inhibition of growth and that one and a half hours were necessary for sterlization.

Dieudonne (1894) worked with pathogenic bacteria. With $B$. fuorescens pudrtus and $B$. prodigrosus he found that one and a half hours were required in March, July and August and nearly twice as long in November.

Kruse found that an atmosphere of oxygen shortened the length of life by one-half over that of hydrogen when bacteria were exposed to sunlight.

Weinzirl (1914) devised a new technique which involved exposure of the bacteria on paper directly to the sunlight. He stated that nonspore-forming bacteria were killed in from two to ten minutes. Such conditions are rarely secured in nature where the organisms are often accompanied with organic matter. For spores from two to eight hours were necessary.

The various colors of the spectrum exert a different action on bacteria. The intensity of this action increases as one goes from the red toward the violet. Apparent destruction is noticed only after the yellow is passed. From the yellow through the violet and ultraviolet, the germicidal action is at its maximum. In fact it is now well established that the shorter the wave length the greater is the bactericidal action. Newcomer (1917) has reported experiments which indicate that X-ray fluorescence is very bactericidal. X-rays alone exerted a partial bactericidal effect on suspensions of typhoid bacilli. Kempster (1917) exposed tubercle bacilli to X-rays and secured a reduction in their multiplication. Inoculations of tubercle bacilli, which have been exposed to X-rays, into guinea pigs caused no tubercular lesions to develop. He stated that the phagocytes were able to overcome the bacteria if their multiplication was reduced.

Ultra Violet Iight. This light on account of its short wave length possesses decided bactericidal effects. Ward (1892) in studying the effect of arc spectra on bacteria found that the infected plates were sterilized whenever exposed to the violet or ultra violet rays. He successively treated Thames River water by submitting it the effects of ultra violet rays. Houghton and Davis (1913) give the following conclusions to their work: 
A. The ultra violet rays produced by the Cooper Hewitt mercury

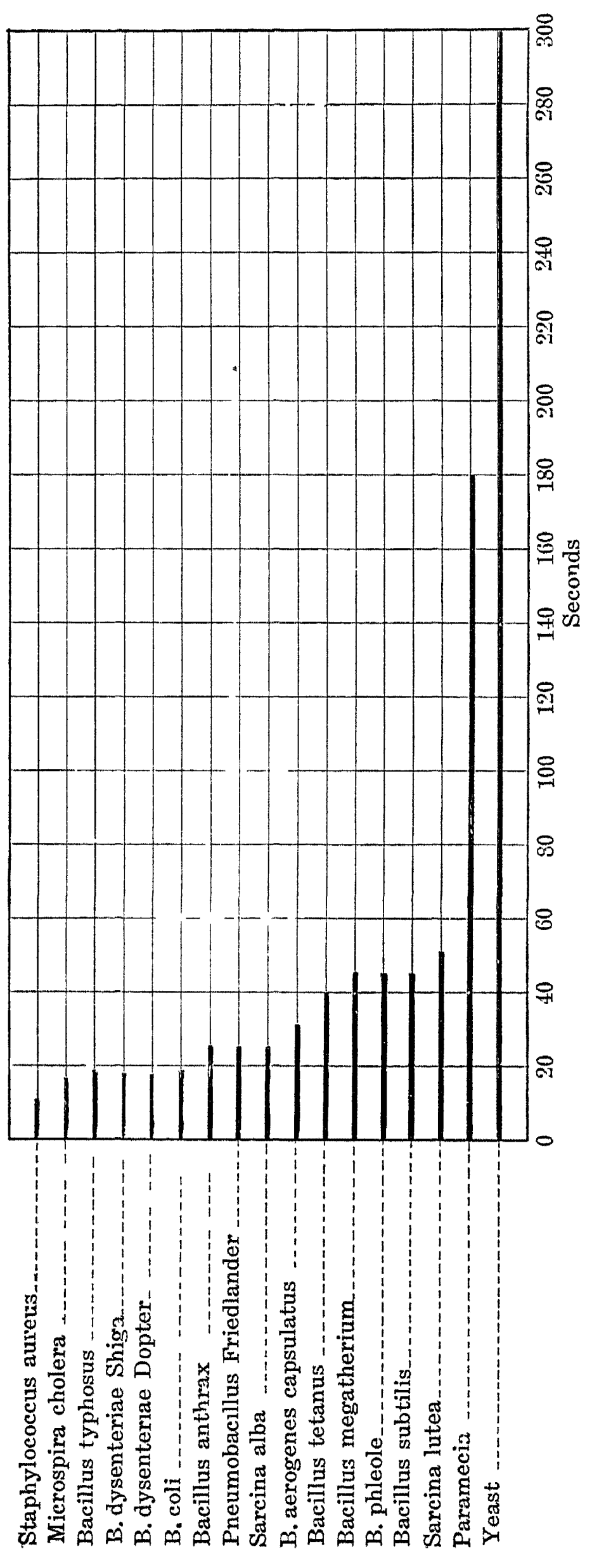

\pm arc have a strong bactericidal power.

B. Certain varieties of bacteria in aqueous suspension, including spore-forming organisms, are killed by exposure to the rays. Molds. however, are only partially destroyed by the ultra violet light.

C. The action seems to be photo-mechanical and is in all probability due to absorption of ultra violet rays by bacterial protoplasm.

D. Protcins and other bodies of high molecular weight interfere with the action 응 of the rays. Turbidity, both organic and inorganic, has a similar action. Color within certain limits seems to have no action.

Ultra violet light was first used for the sterilization of milk and since this time its use has been extended to other materials. Von Recklinghausen studied the action of ultra violet light on bacteria in water. Spores are said to be 
1.5 and five times as resistant as vegetative cells. The resistivity of some of the common bacteria is shown in Fig. 42. This author does not believe that hydrogen peroxide is formed during the action of these rays. Burge has demonstrated that these radiations exert a strong coagulation of the protein and probably the same change is secured on bacterial proteins. He declares (Burge, 1917) that the theory of the destruction of the intracellular enzymes to explain death, is not true. He killed some bacteria by ultra violet radiation and after grinding found that gelatin was liquefied. In this case the intracellular enzymes were not destroyed when the cells had. been killed. This same opinion was advanced by Oker-Blom (1913). He argues that the rays act directly on living protoplasm and do not act through ozone, hydrogen peroxide or nitrous acid. In this connection the greater resistance of the fluorescent bacteria to ultra violet rays found by Burge and Neill (1915) is interesting. They found the fluorescent bacteria to be much more resistant than the non-fluorescent. Harris and Hoyt (1917) state that visible light rays are not as a rule absorbed by protoplasm. Ultra violet rays are generally toxic and they attempted to determine what constituent of the protoplasm was responsible for absorption of these rays. While their experiments were carried out with protozoa, the results are probably applicable to bacteria. The paramecia were suspended in different solutions such as urea, cane sugar, alanin, leucine, and gelatin. They state the susceptibility of protoplasm to ultra violet light is conditioned by the absorption of the toxic rays by amino acid radicles in the proteins. Newcomer (1917) reported that the typhoid bacilli are about $1 / 200$ as sensitive to ultra violet radiations of wave lengths 2100-2800 ̊ngström units as a photographic plate. This decreases to almost zero at 2970 units. Browning and Russ (1917) working the Staphylococcus pyogenes aureus, $B$. coli and $B$. typhi reported that ultra violet radiations with wave lengths between 3800 and 2960 A possessed no marked bactericidal action. Wave lengths between 2960 and 2100 A were markedly bactericidal.

\section{DisINFECTION}

The distinction between sterilization and disinfection is quite artificial in many cases. In both processes the death of bacteria is concerned. Disinfection is generally regarded as the removal of pathogenic bacteria from materials while sterilization is applied to those processes which remove all kinds of life. Obviously all methods of 
sterilization are disinfection processes. Even with this close relation of the process there are certain procedures and reagents which are used more as disinfectants than as sterilizing agents. Many of these could not be applied to media and some apparatus since some of the disinfectant remains after the treatment.

Disinfection is a chemical process and therefore follows chemical laws. Chick (1908) has given the best work on this subject and much of her data form the basis upon which our knowledge with regard to disinfection rests. The chemical reaction in this case would take place between the disinfectant and the bacterium protoplasm. The disinfection process follows the well-known law of physical chemistry-the monomolecular law. The same equation may be used provided legitimate substitutions are made in it to make it fit disinfection.

or

$$
\frac{-d c}{d t}=K C
$$

This may be changed to

$$
\frac{1}{t_{2}-t_{1}} \log \frac{c_{1}}{c_{2}}=K
$$

$$
\frac{1}{t_{2}-t_{1}} \log \frac{n_{1}}{n_{2}}=K
$$

In this equation $c_{1}$ and $c_{2}$ have been substituted for $n_{1}$ and $n_{2}$ the numbers of surviving bacteria. For such a discussion, we may define the monomolecular law as one governing a reaction between two substances where the change in concentration of one may be measured. This law seems to be followed in all cases of disinfection when any agent is used to destroy bacteria. It is apparent that the rate of death is always proportional to the number of cells which are living at any given time. This simply means that where there are larger amounts of reacting substances, more reaction is secured. Consequently a greater d:op is secured in the curve during the first units of time. Some objections may be raised against regarding disinfection in this light. The bacterial cell must be regarded as a molecule. It is quite different since it possesses "life" and is surrounded by a membrane. It is also necessary to neglect the change in concentration of the disinfectant. At present the disinfectant is usually added in such excess that any change which might occur is neglected.

Different opinions exist with regard to how the disinfection process should be explained. For disinfection purposes, a bacterial culture is regarded as made up of a uniform population. Hewlett (1909) and 
Reichel (1909) explain the time clement in disinfection by assuming that the different colls in a bacterial suspension possess different resistances-the weakest or less resistant cells dying first. If such were the case it is reasonable that most of the cells would possess the same resistance and consequently would succumb at about the same time and that at each end of the process we would be dcaling with the weakest (less resistant) and the strongest (most resistant) cells. Such a unimodal curve is usually secured where the element of chance or the law of probability enter. This same monomolecular law is followed among human beings where similar individuals and a multitude of causes of death obtain. The disinfectant may act as a catalyzer since the process demands the presence of water. Where water is absent disinfection is inhibited.

Factors which Influence the Value and Use of Disinfectants. The ideal disinfectant does not exist. If one could be found, it would conform to the following characteristics:

1. Low cost.

2. Non-toxicity to man and animals.

3. Solubility in ordinary reagents in sufficient amounts to destroy bacteria.

4. Minimum time for destruction of bacteria.

5. Great penetrating power.

6. Should be a stable compound under ordinary conditions.

7. Should not react with cloth dyes or wood finishes.

\section{INORGANIC COMPOUNDS}

Lusini (1912) has pointed out with regard to the cations that although the disinfecting property is not a function of the atomic weight the heavy metals exert the greatest action. With a few exceptions, the disinfecting action increases with the chemical affinity of the combined elements. The alkali group is less powerful than the alkalin earth group. The last mentioned group does not fit in with the generalizations above since its disinfecting power is in inverse ratio to the atomic weight. Among the other elements of the periodic system iron has a lower activity corresponding to its low atomic weight; tin has a higher and lead still higher activity. This has been somewhat confirmed by other investigations. Bitter (1912) found that the following metals exerted an antagonistic action toward bacteria drying on them. The intensity of action is in the following order: Copper, brass, silver, gold, platinum, lead, cast iron, steel, aluminum, nickel. 
zine and tin. The action was found to be the same for both polished and corroded metal. Such data are interesting when it is remembered that some of these clements are used for the manufacture of toilet articles and mouth pieces for public drinking fountains. Under these conditions a disinfecting action could hardly be expected. Bacteria will exhibit great development in cultures containing the heavy metals in large pieces.

Halogens. The halogens have rather important positions as disinfectants. Chlorine has had much use as an internal disinfectant in diphtheria, scarlet fever, etc. It is contained in water in a concentration of about 0.4 per cent. The action of chlorine is generally admitted to be an oxidation reaction. Recently it has replaced bleaching powder in the treatment of public water supplies. It is distributed in the liquid form and has many advantages over calcium hypochlorite.

Bromina. This is used as a saturated aqueous solution containing about 3 per cent of the element. When used internally the strength of the solution must be greatly reduced to contain about 0.3 per cent It is a strong oxidizing agent and readily attacks metals. This places some limitations on its use as a disinfectant.

Iodine. A 5 per cent solution with 10 per cent of potassium iodide is a reliable disinfectant. It is available as a tincture which is an alcoholic solution of the element. It is soluble in alcohol to the extent of about 1 part in 10 . Free iodine exerts a powerful toxic action on bacteria since it unites directly with the bacterial protoplasm. Iodoform is an iodine compound $\left(\mathrm{CHI}_{3}\right)$ used in disinfection. It is applied directly to wounds and unites with the organic matter. Free iodine results and iodoform owes its action so the iodine which is thus given off. Often it is made into an ointment by mixing with some carrier. Other forms of iodine which may be used as antiseptics are:

Iodoformogen-iodoiorm albuminate.

Iodo-Hemol Merck-Iodized hemol.

Iodol- $\left(\mathrm{C}_{4} \mathrm{H}_{4} \mathrm{NH}\right)$ iodocarbamid.

Iodomuth-Bismuth iodine compound.

Mahon and White (1915) report that iodine in alcohol is about four times as powerful as a disinfectant as phenol. Their work was done on naked organisms with B. typhi as the test organism. Eugling (1912) in studying the germicidal action of iodoform could not detect a reducing action of bacteria resulting in the liberation of free iodine.

Silver Salts. Silver precipitates proteins from solution as insoluble salts. It probably acts in the same way on bacterial protoplasm. 
Biasiotti (1910) reported that colloidal silver electrically prepared would kill Staphylococcus pyogenes aureus, B. typhi, and Bacternum diphtherice in a few hours. The silver is probably united to the protoplasm in some way because Gram positive bacteria are made Gram negative. Simpson and Hewlett (1914) experimented with colloidal silver in the form of "collosol" on the typhoid bacillus and found it an active bactericide. The "collasols" are said to be non-poisonous but slow of action and very expensive.

Silver is combined with several substances which have wide application in certain phases of the disinfection problem. Argyrol is the silver salt of vitellin and is used in solutions of from 1 to 4 per thousand. Protargol is a compound of silver with protein. It contains 8 per cent of silver nitrate. Albargin is a silver salt of galactose. It may be used on mucous membranes, which explains its use in the venereal diseases. It contains about 10 to 15 per cent of silver. Often it is applied as 10 to 20 per cent solutions in 20 per cent glycerol. Argentamin, a 10 per cent solution of silver nitrate in 10 per cent ethylene-diamin, has much use in gonorrheal infections. It is usually diluted to about 1 part in 300. Argentose is a silver compound of nucleoprotein and is used like silver nitrate. Argonin, a silver caseinate, is used in a 3 per cent solution for treatment of gonorrhea and ophthalmia neonatorum.

Silver Nitrate, $\mathrm{AgNO}_{3}$. This is a common silver disinfectant. It is not as powerful as mercuric chloride but does not precipitate proteins as does mercuric chloride.

Silver Citrate, $\mathrm{Ag}_{3} \mathrm{C}_{6} \mathrm{H}_{5} \mathrm{O}_{7}$. This is used as a wash for wounds and for gonorrhea in dilutions of about 0.25 per cent. Great precautions must be used to obtain fresh solutions. It is also known as itrol.

Silver Lactate. This compound of silver is used as a mild disin- fectant. It has about the same applications as has silver citrate.

Bismuth Compounds. Bismuth subiodide, bismuth subsalicylate, bismuth nitrate are all used as weak disinfectants. They are used in solutions of 1 to 1000 to 1 to 4000 and are probably not very reliable.

Calcium Compounds. Chloride of Lime. This is also known under the names of bleach, hypo and bleaching powder. It has attained an important position in sanitation probably for two reasons; it possesses great bactericidal properties and may be secured for a low price. Thus it satisfies two of the characteristics of a good disinfectant. In the dry condition chloride of lime is calcium oxychloride which, in the presence of water takes on another atom of oxygen.

$$
2 \mathrm{CaOCl}_{2}=\mathrm{Ca}(\mathrm{OCl})_{2}
$$


Chloride of lime is soluble according to Hooker (1913) in about 20 times its own weight of water. It leaves an insoluble residue composed mostly of calcium hydroxide. It is prepared from $\mathrm{Ca}(\mathrm{OH})_{2}$ as follows:

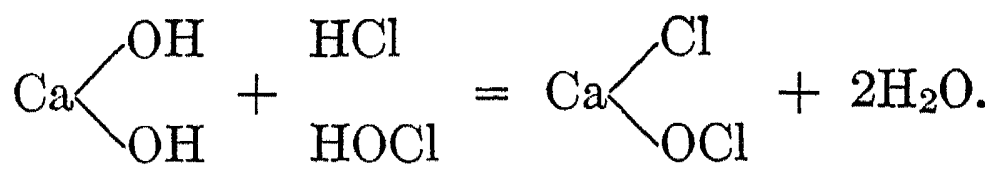

The $\mathrm{HOCl}$ is made by treating water with chlorine. Some care should be used to secure clear solutions for use in disinfection. This is especially true in water treatment. The potential part of thi; compound is very soluble in water while the part with no sterilizing properties quickly settles out. Hooker (1913) gives the following rules for the use of chloride of lime:

First, do not mix too stiff a paste, otherwise a gelatinizing action takes place and greater difficulty in settling out is encountered. Never mix a paste with less than $\frac{1}{2}$ gal. of water for $1 \mathrm{lb}$. of chloride of lime. Second, it is not necessary nor desirable to grind nor break up the lumps too thoroughly; the available chlorine nearly all dissolves readily and too much agitation is detrimental to prompt settling.

The above precautions should be borne in mind in any application of chloride of lime to sanitation.

The strength of chloride of lime is measured in terms of " available chlorine." This is an unfortunate term since it implies that the disinfecting properties of the compound rest in the chlorine. The work in the past indicates that it is an oxidation and not a chlorination. The oxygen is formed in the following manner:

$$
\begin{gathered}
\mathrm{Ca}(\mathrm{OCl})_{2}+\mathrm{H}_{2} \mathrm{CO}_{3}=\mathrm{CaCO}_{3}+2 \mathrm{HClO} . \\
2 \mathrm{HClO}=2 \mathrm{HCl}+\mathrm{O}_{2}
\end{gathered}
$$

It has been suggested by some sanitarians that the term " available chlorine " be substituted by the term " potential oxygen." Lager (1916) has made some interesting statements in regard to the action of calcium hypochlorite. He states that the disinfecting action does not depend upon the available chlorine nor the length of time the chlorine acts. He states that the time for killing depends upon the resistance of the organism.

The United States Pharmacopoia specifies that chloride of lime shall contain 35 per cent of available chlorine. As it is purchased in small cans on the open market it probably falls far short of this and the use 
of such a product allows a false sense of security. Hooker, in the book mentioned above, presents a table which contains much useful information. It appears here as Table VIII and is based on 30 per cent bleach.

TABLE VIII

SHOWING STRENGTH OF SOLUTIONS OF CALCIUM HYPOCHLORITE

(After Hooker, 1913)

\begin{tabular}{c|c|c|c|c|c}
\hline $\begin{array}{c}\text { Pounds } \\
\text { Bleach per } \\
\text { 1,000,000 } \\
\text { Gallons } \\
\text { Water }\end{array}$ & $\begin{array}{c}\text { Parts Bleach } \\
\text { per 1,000,000 } \\
\text { Parts Water }\end{array}$ & $\begin{array}{c}\text { Parts Chlorine } \\
\text { per 1,000,000 } \\
\text { Parts Water }\end{array}$ & $\begin{array}{c}\text { Grains Bleach } \\
\text { per Gallon } \\
\text { Water }\end{array}$ & $\begin{array}{c}\text { Grains A vall- } \\
\text { able Chlorne } \\
\text { per Gallon } \\
\text { Water }\end{array}$ & $\begin{array}{c}\text { Drops Bleach } \\
\text { Solution, 2 Per } \\
\text { Cent or 1 Lb } \\
\text { in Gallon Used } \\
\text { per Gallon } \\
\text { Water. }\end{array}$ \\
\hline 2 & 24 & .08 & .104 & .005 & .25 \\
4 & .48 & .16 & .028 & .009 & .50 \\
6 & .72 & .24 & .042 & .014 & .75 \\
8 & .96 & 32 & .056 & .019 & 1.00 \\
10 & 120 & .40 & .070 & .023 & 125 \\
12 & 144 & .48 & .084 & .028 & 1.50 \\
14 & 168 & .56 & 098 & .033 & 1.75 \\
16 & 192 & .64 & .112 & .037 & 2.00 \\
18 & 216 & .72 & .126 & .042 & 2.25 \\
20 & 240 & .80 & .140 & .047 & 2.50 \\
22 & 264 & .88 & .154 & .051 & 2.75 \\
24 & 288 & .96 & .168 & .056 & 3.00 \\
26 & 312 & 1.04 & .182 & .061 & 3.25 \\
28 & 336 & 1.12 & .196 & .065 & 350 \\
30 & 360 & 1.20 & .210 & .070 & 375 \\
\hline
\end{tabular}

The objections to chloride of lime are important. It takes the color out and exerts great reactivity with organic matter. When it is used in the presence of large amounts of organic matter, a sufficient amount should be used to satisfy the requirements of it and leave enough left over for destroying the bacteria. This is difficult to determine.

Labarraque's Solution. This is a solution of chloride of lime containing 2.4 per cent of available chlorine.

Dakin-Carrel Solution. This solution has been found to be of great value in the treatment of wounds. Different methods may be used in the preparation of this solution. Below are two formulæ:

Formula No. 1. Dissolve 140 gms. of sodium carbonate in 10 liters of distilled water and add 200 gms. of calcium hypochlorite. Shake this solution thoroughly and allow the sediment to settle. After sedi- 
mentation has taken place decant the clear liquid and add 40 gms. of boric acid. The solution is then ready to use.

Formula No. 2. Dissolve 100 gms. of sodium carbonate, 80 gms. of calcium hypochlorite in 10 liters of water. This should give a solution of about $\frac{1}{2}$ per cent of hypochlorite.

In the use of this solution the wound should be kept constantly moist. Doctor Carrel applies the solution every hour or two hours, the amount used generally depending upon the character of the wound. Generally 5 to 10 c.c. were applied every two hours. The use of this solution is being extended to other infections such as puerperal sepsis, etc.

The absolute germicidal power of the chemical was studied by Nissen (1890). He used pure cultures and secured the following results which are quoted from Hooker:

\begin{tabular}{|c|c|c|}
\hline Organisms Killed. & Minutes & $\begin{array}{l}\text { By Dilutions of } \\
\text { Chloride of Lime. }\end{array}$ \\
\hline Bacillus typhosus. ........ & 1 & $1-800$ \\
\hline Bacillus typhosus......... & 10 & $1-1600$ \\
\hline Bacillus choleræ...... $\ldots \ldots \ldots \ldots \ldots$ & 1 & $1-800$ \\
\hline 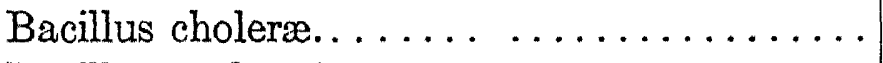 & 10 & $1-600$ \\
\hline Bacillus anthracis. . . . ..... & 1 & $1-1000$ \\
\hline Staph. pyogenes aureus . . . . . . . & 5 & $1-800$ \\
\hline Streptococcus erysipelatus ..... . . & 1 & $1-200$ \\
\hline$\cdot \quad \cdot \ldots \ldots \ldots \ldots \ldots \ldots$ & 15 & $1-20$ \\
\hline Anthrax spores... . . . . . . . . . . & 20 & $1-100$ \\
\hline
\end{tabular}

Hooker has collected an excellent résumé of the literature up to 1913.

Calcium Oxide, $\mathrm{CaO}$. This is known as quick lime and is caustic in the presence of organic matter. It may be used in two forms as slaked lime and milk of lime. Slaked lime is prepared by adding a pint or pound of water in two pounds of lime. The reaction proceeds as follows:

$$
\mathrm{CaO}+\mathrm{H}_{2} \mathrm{O}=\mathrm{Ca}(\mathrm{OH})_{2} .
$$

This will react with carbon dioxide to give calcium carbonate as follows:

$$
\mathrm{Ca}(\mathrm{OH})_{2}+\mathrm{CO}_{2}=\mathrm{CaCO}_{3}+\mathrm{H}_{2} \mathrm{O} \text {. }
$$

Milk of lime is a thick mixture containing 4 to 6 parts of slaked lime. This milk of lime reacts with carbon dioxide to form the carbonate as does the slaked lime. Therefore these mixtures should be used as soon after preparation as possible. 
Chloramine. Toluenc parasulfondichloramin is formed when nascent chlorine formed in aqueous solutions of hypochlorite is brought in contact with suppurating wounds. Dakin $(1916,1917)$ has proposed the name dichloramine- $T$ for this compound. He has shown that most of the substances containing a $\mathrm{NCl}$ group are strongly germicidal. The presence of more than one such groups does not seem to increase the germicidal properties to any marked degree. It may be used in stronger concentrations than with some of the other disinfectants. It is claimed that its use is as simple as the use of tincture of iodine.

Zinc Chloride. This compound had quite a reputation as a disinfectant at one time, but recent knowledge has caused the compound to fall into disuse. McClintic (1905) found that B. coli was not killed in one hour's exposure to a 5 per cent solution and that it required ten minutes for a 25 per , ent solution to kill the same organism. The spores of $B$. subtilis and $B$. anthracis were found to resist 100 per cent and 50 per cent solutions for 30 and 40 days respectively.

Copper Salts. These have been used in the treatment of public water supplies but not generally in ordinary disinfection. Kellerman and Beckwith (1906) have given a résumé of the literature up to the time when their paper was published. Moore and Kellerman (1905) reviewed the application of copper sulphate to water sterilization and the removal of algæ. About 10 lbs. per million gallons of water were sufficient. Its toxic properties are said to be due to the copper salts which are formed with proteins. Embrey (1912) reported that copper sulphate would destroy algæ in a dilution of 1 to 3 million. DeWitt and Sherman (1916) studied the effects of both copper sulphate and cupric chloride on bacteria. Their results showed that copper was not a reliable disinfectant. They state that 2.5 parts of cupric chloride and 4 parts of copper sulphate per million would kill $B$. coli and B. typhi.

Ferrous Sulphate, $\mathrm{FeSO}_{4}$. This has little germicidal value unless in solutions of over 2 per cent. When it is present above 5 per cent, it will restrain development of putrefactive changes. McLaughlin (1903) secured no disinfection in applying it in saturated solution to feces.

Boric Acid, $\mathrm{H}_{3} \mathrm{BO}_{3}$. Boric acid is a mild disinfectant and is used in 5 to 100 per cent solution. It is of especial value for use on mucous membranes such as those of the eye and the nose. Ochsner (1916) stated that boric acid does not destroy pathogenic bacteria but does diminish their virulence. Wet dressings of boric acid were much more effective on the pyogenic cocci than on other infections. 
Carbon. Renon (1914) produced colloidal carbon electrolytically so that the size of the particles was 4 to $6 \mu \mu$. He studied the antiseptic properties by measuring the acidification of milk. The antiseptic action increased with the amount of carbon.

\section{ORganic Compounds}

The infinite number of possibilities for securing compounds destructive to bacterial life renders a discussion of the organic compounds, in this connection, rather difficult. Kligler (1918) has made a study of the action of aniline and some of its derivatives and of the triphenylmethane dyes on bacteria. He stated from this investigation that the action on bacteria might be a function of the benzine nucleus and in the case of the dyes a function of the quinoid structure of the nucleus. Increasing the number of alkyl radicals increased the antiseptic properties. "The antiseptic power is enhanced to a greater extent by an ethyl than by a methyl group and the second alkyl produces a proportionately greater increase than the first. It appears that the relative position of the introduced group may be a factor in determining the relative improvement in the effectiveness of the compound." An interesting fact is mentioned by Kligler that the Gram positive bacteria are more susceptible to the aniline dyes than are the Gram negative bacteria.

Alcohol. Ethyl alcohol is usually used. It precipitates proteins unaltered and if they are allowed to remain in contact with the alcohol they are changed. The precipitation is probably due to dehydration. The greatest disinfective power is secured in strengths of between 50 and 70 per cent. These solutions contain sufficient water for the coagulation of the protein. Beyer (1912) found that concentrations of alcohol under 60 per cent and over 80 per cent were practically worthless as disinfectants. Absolute alcohol, on account of its drying action exhibited a drying and preserving action. Frey (1912) found that treatment with various concentrations of alcohol influenced the swelling and solubility of protein in water. Alcohol diluted to 10 per cent dissolved a little of the protein; above 20 per cent of alcohol solution of the protein stops and swelling decreases; above 90 per cent the protein dissolves. The greatest action of alcohol on protein is at 60 to 70 per cent while the greatest disinfecting power is 70 per cent. Frey explains the use of alcohol as a disinfectant in the irreversibility of the precipitation of protein after treatment in alcohol. 


\section{Phenol and Cresol:}<smiles>CC1CCCCC1</smiles>

o-cresol<smiles>CC1CCCCC1</smiles>

$m$-cresol<smiles>C[C@H]1CC[C@H](O)CC1</smiles>

$p$-cresol<smiles>OC1CCCCC1</smiles>

phenol

The action of these substances as disinfectants has been studied by Cooper (1912), who found that the entrance of the methyl and nitro groups increased the bactericidal and protein penetrating powers of phenol while the entrance of the hydroxyl group decreased these properties. Solutions of phenol in alcohol and in fat had "no bactericidal action on spores and did not penetrate solutions of gelatin. The precipitating action of phenol is increased by adding acid. Cooper points out that the selective action of phenol on different bacteria is connected with the different susceptibilities of proteins to its precipitating action. The absorption of phenol by bacteria is only the beginning of disinfection. No chemical union follows between the phenol and the bacterial protoplasm but it is associated with de-emulsification of the collodial suspension as shown by the precipitation of proteins when a certain phenol concentration is reached.

The superiority of $m$-cresol over phenol as a bactericide is due to the fact that cresol precipitates proteins in a lower concentration than phenol. Steenhauer (1916) showed that $m$-cresol is the strongest and $p$-cresol the next strongest disinfectant. The cresol solutions secured on the market are mixtures of the $0-, m$-, and $p$-cresols. The introduction of the methyl group has decreased the solubility. On account of this the cresols are often suspended in or mixed with some carrier. Both hard and soft soaps, for instance may be used. Lysol, a highly bactericidal substance, is prepared by saponifying linseed oil with commercial cresol in alcoholic solution. This is readily soluble in water and is used in from 2 to 5 per cent solutions. Weaker solutions must be used for disinfection of mucous membranes as the lining of the mouth and vagina. Steenhauer reported that $m$-cresol is the strongest bactericide with $p$-cresol next. He recommended the use of 5 per cent solutions.

Thymol. This compound is probably of little value as a disinfectant. Schmidt (1910) stated that thymol does not affect certain putrefactive bacteria and is not entirely efficient as an antiseptic in 
enzymological work. It is used in many pharmaceutical preparations but probably does no harm.<smiles>CC1CCC(O)C(C)C1</smiles>

Formaldehyde. This is a powerful disinfectant sold in 35 per cent solutions under the name of formalin. Formaldehyde changes to paraformaldehyde by polymerization when kept at room temperatures. It unites easily with many substances especially the organic compounds. For instance when added to gelatin it forms a compound which is insoluble in many of the common reagents. Fermi attempted to secure a gelatin medium in this way which would not melt at room temperature. It may act in the same way on bacterial protein. It is important to have plenty of moisture present when using formaldehyde as a disinfectant. Koch (1901) reported that a 0.05 per cent solution of this compound would kill growing yeast while a 0.005 per cent solution would not. Since carbon dioxide was formed after the cell had been killed, the zymase was not destroyed.

Glycerol. Rosenau has made an interesting study of this compound in its relation to dinsinfection. He found that a 50 per cent solution of glycerol would restrain all bacterial growth and that lower precentages would allow better growth. Bacteria would not grow in media containing 32 per cent of glycerol but molds grew in stronger solutions of 40 and 49 per cent. Glycerol was found to have a distinct but slight germicidal action and probably acted by virtue of its abstracting water from the bacterial cell. Spores were not killed and anthrax spores lived 200 days in strong glycerol solutions. The compound may be used by many bacteria as a food. It is then probably oxidized to glyceric aldehyde and glyceric acid. Glycerol may be used, however, as a carrier for many other disinfectants such as phenol, cresol, etc. Goodrich (1917), however, has shown that glycerol more or less completely destroys the antiseptic power of thymol, phenol, boric acid, and mercuric chloride in aqueous solution. Ruediger (1914) found a distinct but weak bactericidal action of glycerol which was stronger at $30^{\circ}$. than at $15^{\circ} \mathrm{C}$. The action is limited by the diluent. Glycerol diluted with normal salt solution killed bacteria more quickly than when diluted with bouillon. In dilutions of 50 per cent anthrax 
organisms were not killed in fifteen days. A selective action was noticed.

Quinone. Bactericidal activity of quinone has been found to be due to its chemical action on certain constituent proteins of the bacterial cell. According to Cooper quinone is a stronger bactericide than phenol, quinol, or acetone on account of its reactivity towards proteins in much lower eoncentrations.

Dyes as Disinfectants. Recently it has been observed that dyestuffs exert a distinct retarding action on bacteria. Churchman (1912) made one of the most important contributions to this question. $\mathrm{He}$ reported that gentian violet in dilutions of 1 to 100,000 when added to media would inhibit the development of certain bacteria. The Gram negative bacteria grew fairly well on the gentian violet media while generally the Gram positive would not. The subject has been greatly enriched by much recent work especially that of Krumwiede and his co-workers. Some of these researches are mentioned in other places.

\section{TABLE IX \\ SHOWING ANTISEPTIC VALUES OF SOME COMMON SUBSTANCES}

(After Park and Williams)

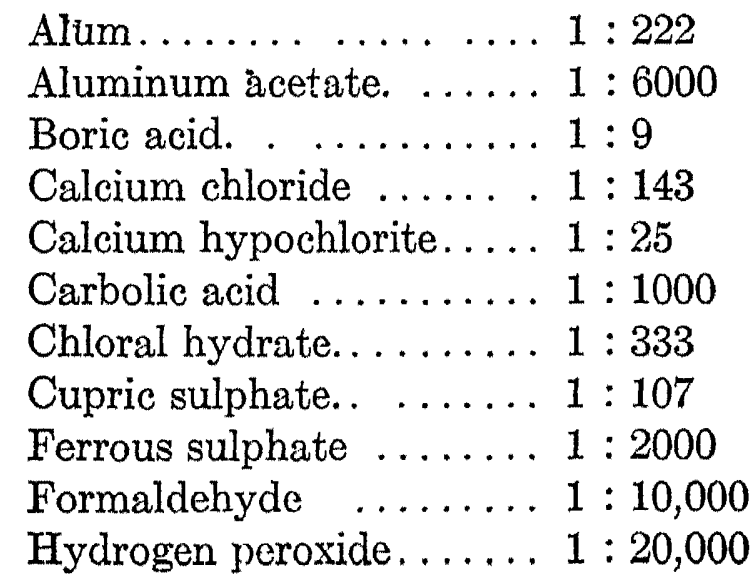

\author{
Mercuric chloride ...... 1:14,300 \\ Mercuric iodide ....... $1: 40,000$ \\ Potassium bromide...... $1: 10$ \\ Potassium iodide. $\quad 1: 10$ \\ Potassium permanganate. . $1: 300$ \\ Pure formaldehyde. . ... $1: 25,000$ \\ Quinine sulphate....... 1:800 \\ Silver nitrate. . ...... $1: 12,500$ \\ Sodium borate ........ 1:14 \\ Sodium chloride. ...... 1:6 \\ Zinc sulphate......... $1: 20$
}

\section{Standardization of Disinfectants}

It is essential to know as far as possible just how toxic to the bacterial cell certain chemicals are. This knowledge is important in the purchase of large amounts of disinfectants and in their application to disinfection problems. That the chemical analysis would not furnish this information is apparent. Furthermore, many of the disinfecting compounds are of such complicated nature that a chemical analysis would be impossible. In view of these facts, many investigators have attempted to devise a purely bacteriological method since 
in disinfection it is the aim to destroy bacteria and the reaction must take place between the bacterial cell and the disinfectant.

The bactericidal activity of chemicals has been studied by many investigators. Robert Koch in the early days of the science gave some attention to the question. He devised a method wherein the bacteria were dried on silk threads. These were then suspended in known dilutions of the disinfectant. After the process had been completed these threads were then transferred to media in order to determine whether the bacteria had been killed. He used Bacillus anthracis for the test organism. Sternberg mixed 5 c.c. of a young culture with equal quantities of the germicide. At regular intervals, loops of this mixture were removed and put in broth. Kronig and Paul (1897) dipped garnets into a culture of Bacillus anthracis containing spores and allowed them to dry. These garnets were then put into the disinfecting solutions and a few taken out at intervals. They were shaken in a definite amount of water and plates made from this. In this way they determined the rate of death. They realized that the value of any disinfectant varied with the conditions under which it acted.

Upon such data as these our present methods have been devised. These represent the present stage in the effort to secure satisfactory methods with which to measure strength of disinfectants. Walters (1917) has pointed out that disinfectants possess a certain specificity for certain bacteria. He further argues that the strength of a disinfectant may only be stated in terms of the organism on which it is to be used in actual practice.

Rideal-Walker Method. Anderson and McClintic (1912) have summed up this method as follows:

Briefly stated, the carbolic coefficient in the Rideal-Walker method is arrived at by dividing the figure indicating the degree of dilution of the disinfectant that kills an organism in a given time by that expressing the degree of dilution of the carbolic acid that kills the same organism in the same time under exactly similar conditions. Leaving out details, the determination of the Rideal-Walker coefficient is substantially as follows:

Certain standard conditions are considered essential to the proper performance of the test. Phenol solutions of known strength are used; cultures are grown in a standard medium, transplants being made every twenty-four hours; the loops used for all inoculations are of a standard size (about $4 \mathrm{~mm}$. in diameter). Usually four dilutions of suitable strengths of the disinfectant to be used are made. Phenol 
controls of a suitable strength are also prepared. Five c.c. of each of these dilutions are placed in sterile test tubes, to which are added at intervals of one-half minute a twenty-four-hour broth culture of $B$. typhosus in the proportion of 1 drop of culture to each cubic centimeter of disinfectant used (according to Partridge, 1 drop of culture equals about 0.1 c.c.).

At the end of two and a half minutes a loopful of each of the mixtures is inoculated into a test tube containing 5 c.c. of standard broth, an interval of half a minute being thus allowed between taking the samples from the different dilutions. This is repeated at five, seven and a half, ten, twelve and a half, and fifteen minutes. The broth tubes, after being incubated at $37^{\circ} \mathrm{C}$. for forty-eight hours, are examined for growth.

The results of the examination are then noted, and if suitable comparative strengths of the disinfectant and carbolic acid have been selected the carbolic acid coefficient is determined as above stated.

The following table (Table 10) illustrates the manner of determining the carbolic acid coefficient of a disinfectant according to the RidealWalker method:

\section{TABLE $\mathrm{X}$}

Name, "A."

Temperature of medication, $20^{\circ} \mathrm{C}$.

Culture used, $B$ typhosus, twenty-four-hour, extract broth, filtered.

Proportion of culture and disinfectant, 01 c.c. +5 c c.

\begin{tabular}{|c|c|c|c|c|c|c|c|c|}
\hline \multirow{2}{*}{ Sample } & \multirow{2}{*}{ Dilution } & \multicolumn{6}{|c|}{$\begin{array}{l}\text { Time Culture Exposed to Action } \\
\text { of Disinfectant for Minutes }\end{array}$} & \multirow{2}{*}{$\begin{array}{l}\text { Phenol Coeffi- } \\
\text { cient. }\end{array}$} \\
\hline & & $2 \frac{1}{2}$ & 15 & $7 \frac{1}{2}$ & 10 & $12 \frac{1}{2}$ & 15 & \\
\hline \multirow[t]{2}{*}{ Phenol.............. } & $1: 90$ & + & - & - & - & & & \multirow{5}{*}{$\begin{array}{l}\frac{100 \lcm{550}}{5.5} \\
\text { coefficient. }\end{array}$} \\
\hline & $1: 100$ & + & + & + & - & - & - & \\
\hline \multirow[t]{3}{*}{ Disinfectant "A" } & $1: 500$ & + & + & - & - & - & - & \\
\hline & $1: 550$ & + & + & + & - & - & - & \\
\hline & $1: 600$ & + & + & + & + & - & - & \\
\hline
\end{tabular}

This method has had to be greatly modified. There are certain things taken for granted which may materially alter the results. It is assumed that the rate of death is proportional to the concentration of the disinfectant. Bacteria are regarded as molecules in their reaction with the disinfectant. Chick has recommended thirty minutes as the standard time in place of fifteen minutes. She pointed out the error 
in the method by working with anthrax spores. The Rideal-Walker method does not consider the presence of organic matter.

Chick and Martin's Modification of the Rideal-Walker Method. These authors have pointed out that an arbitrary time must be selected during which the disinfectant shall be allowed to act. The RidcalWalker method is modified with this in view. The procedure is as follows:

\section{Without Organic Matter}

Everything used in the experiment, tubes, pipettes, etc., being previously sterilized, a series of tubes containing 5 c.c. of disinfectant in different concentrations are placed in a water bath at $20^{\circ} \mathrm{C}$. When the tubes have taken the temperature of the bath, they are one after another inoculated with five drops of a twenty-four hour culture of $B$. typhosus from a standard pipette, the time being registcred by a chronograph. Exactly one minute is allowed to pass between each inoculation. When thirty minutes have elapsed since the first tube was inoculated, samples in duplicate are taken from it with a platinum loop, and sown in 10 c.c. glucose broth containing litmus. One minute later the second tube is sampled and so on. These test cultures are incubated at $37^{\circ} \mathrm{C}$. and always kept four days under observation.

Supposing the value of the disinfectant to be tested is totally unknown, the first series of observations must be scattered over a wide range, e.g., concentrations from 1 in 10 to 1 in 10,000. Having ascertained that the concentration necessary to kill in thirty minutes is betwcen, say, 10 in 1000 and 1 in 1000 the second serics is arranged to narrow it down to between, say, 4 and 5 per 1000, and a third series may determine the necessary concentration as between 4.2 and 4.5 per 1000. At this last trial a series of tubes, containing various strengths of pure phenol, are simultaneously tested.

\section{With Organic Matter}

In order to determine the effect of the presence of organic matter, Chick and Martin use dried, pulverized feces as follows:

The feces used were dried first in a water bath and subsequently at $105^{\circ} \mathrm{C}$., ground to a fine powder, and passed through a fine sieve with a mesh of 130 to the inch. Quantities of 0.15 germs were weighed out and placed in test tubes. To each test tube 2.5 c.c. distilled water was added and the tube sterilized in the autoclave (ten minutes at 
$120^{\circ} \mathrm{C}$.) The tubes were covered with india-rubber caps and kept in jars with greased lids to prevent evaporation.

At the time of the experiment different amounts of a suitable dilution of the disinfectant were added to each tube together with enough distilled water to make the total volume up to 5 c.c. The tubes then contained different concentrations of the disinfectant in question in the presence of 3 per cent feces. The tubes were inoculated and sampled in exactly the same way as when the test was made in distilled water without organic matter.

Lancet Method. This is a modification of the Rideal-Walker method. The following method was advised:

Bacillus coli communis from a twenty-four hour broth culture which had been incubated at $37^{\circ} \mathrm{C}$. was used as the test organism. The disinfectants were diluted with distilled water and placed in special containers $2 \frac{1}{2}$ by $\frac{7}{8}$ ins. Platinum spoons were advised instead of loops for the seeding. By this technique more fluid could be carried than on any ordinary loop. These spoons carried about 0.08 c.c. of water. MacConkey's lactose bile broth was advised for the secondary culture tubes. Samples were to be removed every two and a half minutes from each container up to fifteen minutes. After this the time interval was to be fifteen, twenty, twenty-five and thirty minutes. All tests were carried out between $62^{\circ}$ and $67^{\circ} \mathrm{F}$. The carbolic acid coefficient was deduced as follows: "The figure representing the percentage strength of the weakest lethal dilution of the carbolic acid control was divided by the figure representing the percentage strength of the weakest lethal dilution of the disinfectant being tested. This was done both at the two-and-a-half-minute line and at a thirty-minite line and a mean of the resulting figures was taken as the carbolic acid coefficient."

Determinations of the phenol coefficient have not yielded consistent results in the hands of the various investigators. This may not be due to the method itself, although it is not perfect in its present technique, but to the media used in the various laboratories. Phelps has pointed out some of the limitations and Supfle and Dlugeler, Norton, and have emphasized the necessity of great care in the preparation of the media. The H-ion concentration appears to be very important. Despite these objections, the phenol coefficient is probably the best method available at present, for the comparison of the disinfecting properties of the coal-tar products. 


\section{Hygienic Laboratory Phenol Coefficient}

\section{A. WITHOUT ORGANIC MATTER}

Having discussed the necessity for a satisfactory method of standardizing disinfectants and the factors involved in the examination of disinfectants, we present below the method we* have devised.

When this method is used for the standardization of disinfectants we recommend that it be referred to as the "Hygienic Laboratory phenol coefficient."

We prefer to use the word "phenol" instead of "carbolic acid" when speaking of the coefficient, especially since certain dealers advertise for sale carbolic acids which vary greatly in the proportion of phenol present.

Media. Standard extract broth is used, both for the culture to be tested and for the subcultures made after exposure to the disinfectant. The broth is made from Liebig's extract of beef and is in exact accordance with the standard methods adopted by the American Public Health Association for water analysis. Ten c.c. of the broth are put into each test tube. This amount of broth has been found sufficient to avoid any antiseptic action of the disinfectant carried over. It is important that the reaction of the media is just +1.5 .

Organism. For the test organism a twenty-four-hour-old broth culture in extract broth of the B. typhosus (Hopkins) is used. Before beginning a test the culture should be carried over every twenty-four hours on at least three successive days. For carrying over the culture one loopful of a $4 \mathrm{~mm}$. platinum loop is used.

Before being added to the disinfectant the culture is well shaken, filtered through sterile filter paper, and placed in the water bath in order that it may reach a temperature of $20^{\circ} \mathrm{C}$. before being added to the disinfectant.

Temperature. A standard temperature of $20^{\circ} \mathrm{C}$. has been adopted for all experiments. This temperature is obtained by the use of a specially devised water bath. The cultures and dilutions of the disinfectant are brought to this temperature before the beginning of the test.

Proportion of Culture to Disinfectant. One-tenth c.c. of the culture is used, added to 5 c.c. of the disinfectant dilution. The amount of culture is measured with a pipette graduated in tenths of a cubic centimeter.

* This method is quoted from Anderson and McClintic's original publication. 
Inoculation Loops. For making the transfer of the culture after exposure to the disinfectant a platinum loop $4 \mathrm{~mm}$. in diameter of 23 United States standard gauge wire is used. We.have found it is of advantage to have at least 4 , and preferably 6 , loops. In order to save time in flaming the following method was devised:

A block about 3 ins. wide, 10 ins. high, and 12 ins. long, containing 4 or 6 grooves, spaced 2 ins. apart, is used. Into each of the grooves the platinum loop is laid so that the end of the loops extend about 5 ins. beyond the side of the block. The first step in the operation is to sterilize each loop by flaming with a fantail Bunsen burner before beginning the experiment.

When ready to begin the operation the loop farthest from the operator is taken in the right hand and the inoculation made. It is then replaced in the groove with the right hand and the Bunsen burner (fantail) placed under it with the left hand. The next loop is then used replaced in its groove, and the Bunsen burner placed under it with the left hand, the first loop having been heated to redness while the second loop was in use. This procedure is then continued until all the inoculations have been made. The time required in making the inoculations and in replacing the loop is short, it being found that fifteen seconds is ample.

Incubation. The subcultures are incubated forty-eight hours at $37^{\circ} \mathrm{C}$., and the results then read off and tabulated.

Dilutions. Capacity pipettes for the original dilutions are invariably used. For the phenol controls a standard dilution of pure phenol ("Merck's Silver Label") is made and standardized by the U. S. P. method (Koppeschaar) to contain exactly 5 per cent of pure phenol by weight. From this stock solution the higher dilutions are made fresh each day for that day's test.

For the dilutions of the disinfectant a 5 per cent solution is made by adding 5 c.c. of the disinfectant to 95 c.c. of sterile distilled water. A standardized 5 c.c. capacity pipette is used for this, and after filling the pipette all excess of the disinfectant on the outside of the pipette is wiped off with sterile gauze. The contents of the pipette are then delivered into a cylinder containing 95 c.c. of sterile distilled water and the pipette washed out as clean as possible by aspiration and blowing out the contents of the pipette into the cylinder. The contents of the cylinder are then thoroughly shaken and the dilutions up to $1: 500$ made from it, using delivery pipettes for measuring. For those disinfectants which do not readily form a 5 per cent solution we make a 1 per cent stock solution, and from this make the dilutions greater than $1: 100$ in accordance with the second table of dilutions. If greater 
dilutions than $1: 500$ are to be made a 1 per cent solution is made from the 5 per cent solution and the higher dilutions made from this.

We have adopted the following scale for making dilutions:

For dilutions up to $1: 70$, increase or decrease by a difference of 5 (i.e., 5 parts of water).

From $1: 70$ to $1: 60$ by a difference of 10 .

From 1: 160 to $1: 200$ by a difference of 20 .

From $1: 200$ to $1: 400$ by a difference of 25 .

From 1:400 to $1: 900$ by a difference of 50 .

From 1: 900 to $1: 1800$ by a difference of 100 .

From $1: 1800$ to $1: 3200$ by a difference of 200 .

And so on if higher dilutions are necessary.

It is important that the cylinders used for making the dilutions be correctly graduated, as we have found disregard of this factor an important source of error. It is preferable to use standardized cylinders and pipettes, and we recommend that they be used whenever possible. They, of course, should be perfectly clean. For making the dilutions in accordance with the above scheme we have found the following tables of much service:

Seeding Tubes. The seeding tubes are glass test tubes 1 in. in diameter and about 3 ins. long, with round bottoms. In order to measure the disinfectant into them they are placed in a suitable wooden stand to receive them. We found it convenient to use a wooden block containing 6 rows of 15 holes each for the disinfectant to be tested and a separate stand for the phenol controls. The tubes are placed in the stand and each marked with the strength of dilution it is to contain.

Starting with the lowest dilution (i.e., the strongest), the cylinder is shaken, then 5 c.c. are measured into the tubes marked to receive that strength, using a 5-c.c. delivery pipette. In order to economize glassware, the same pipette is used for measuring out the next dilution, first blowing out as much of the remaining liquid as possible, then drawing a pipetteful of the next dilution to be used and discarding that; then filling the pipette a second time which is emptied into the seeding tube.

The measuring out being completed, the tubes are placed in the water bath and allowed to stand a few minutes in order that the disinfectant solution may reach the standard temperature. We have 
not found it necessary to use cotton plugs in the seeding tubes. They are sterilized in paper-lined wire baskets, with the closed end of the tubes up.

\section{TABLE XI}

\section{(FOR DILUTIONS)-STOCK 5 PER CENT SOLUTION}

[5 c.c. Disinfectant +95 c.c. Distilled Water $=$ Solution $\mathrm{A}]$

\begin{tabular}{|c|c|c|c|c|c|c|c|}
\hline $\begin{array}{l}c c \\
\text { of } \\
A .\end{array}$ & & $\begin{array}{cc}\text { c.c } & c c \\
\text { drst } & \text { of } \\
\text { water } & A .\end{array}$ & & $\begin{array}{c}\text { c c } \\
\text { dist } \\
\text { water }\end{array}$ & $\begin{array}{c}\mathrm{c} c \\
\text { of } \\
\mathrm{A}\end{array}$ & & $\begin{array}{c}c c c \\
\text { dist } \\
\text { water. }\end{array}$ \\
\hline $1: 20=20$ & + & 0 or 10 & - & 0 & or 4 & + & 0 \\
\hline $1: 25=20$ & + & 5 or 10 & + & $2 \frac{1}{2}$ & or 4 & + & 1 \\
\hline $1: 30=20$ & + & 10 or 10 & + & 5 & or 4 & + & 2 \\
\hline $1: 35=20$ & + & 15 or 10 & + & $7 \frac{1}{2}$ & or 4 & + & 3 \\
\hline $1: 40=20$ & + & 20 or 10 & + & 10 & or 4 & + & 4 \\
\hline $1: 45=20$ & + & 25 or 10 & + & $12_{2}^{1}$ & or 4 & + & 5 \\
\hline $1: 50=20$ & + & 30 or 10 & + & 15 & or 4 & + & 6 \\
\hline $1: 55=20$ & + & 35 or 10 & + & $17_{2}^{1}$ & or 4 & + & 7 \\
\hline $1: 60=20$ & + & 40 or 10 & + & 20 & or 4 & + & 8 \\
\hline $1: 65=20$ & + & 45 or 10 & + & $22 \frac{1}{2}$ & or 4 & + & 9 \\
\hline $1: 70=20$ & + & 50 or 10 & + & 25 & or 4 & + & 10 \\
\hline $1: 70=20$ & + & 50 or 10 & + & 25 & or 4 & + & 10 \\
\hline $1: 80=20$ & + & 60 or 10 & + & 30 & or 4 & + & 12 \\
\hline $1: 90=20$ & + & 70 or 10 & + & 35 & or 4 & + & 14 \\
\hline $1: 100=20$ & + & 80 or 10 & + & 40 & or 4 & + & 16 \\
\hline $1: 110=20$ & + & 90 or 10 & + & 45 & or 4 & + & 18 \\
\hline $1: 120=20$ & + & 100 or 10 & + & 50 & or 4 & + & 20 \\
\hline $1: 130=20$ & + & 110 or 10 & + & 55 & or 4 & + & 22 \\
\hline $1: 140=20$ & + & 120 or 10 & + & 60 & or 4 & + & 24 \\
\hline $1: 150=20$ & + & 130 or 10 & + & 65 & or 4 & + & 26 \\
\hline $1: 160=20$ & + & 140 or 10 & + & 70 & or 4 & + & 28 \\
\hline $1: 160=20$ & + & 140 or 10 & + & 70 & or 4 & + & 28 \\
\hline $1: 180=20$ & + & 160 or 10 & + & 80 & or 4 & + & 32 \\
\hline $1: 200=20$ & + & 180 or 10 & + & 90 & or 4 & + & $30 ̀$ \\
\hline $1: 200=20$ & + & or 4 & + & 36 & or 2 & + & 18 \\
\hline $1: 225=20$ & + & 205 or 4 & + & 41 & or 2 & + & $20 \frac{1}{2}$ \\
\hline $1: 250=20$ & + & 230 or 4 & + & 46 & or 2 & - & 23 \\
\hline $1: 275=20$ & + & 255 or 4 & + & 51 & or 2 & + & $25 \frac{1}{2}$ \\
\hline $1: 300=20$ & + & 280 or 4 & + & 56 & or 2 & + & 28 \\
\hline $1: 325=20$ & + & 305 or 4 & + & 61 & or 2 & + & $30 \frac{1}{2}$ \\
\hline $1: 350=20$ & + & 330 or 4 & + & 66 & or 2 & + & 33 \\
\hline $1: 375=20$ & + & 355 or 4 & + & 71 & or 2 & + & $35 \frac{1}{2}$ \\
\hline $1: 400=20$ & + & 380 or 4 & + & 76 & or 2 & + & 38 \\
\hline $1: 450=20$ & + & 430 or 4 & + & 86 & or 2 & + & 43 \\
\hline $1: 500=20$ & + & 480 or & + & 96 & or 2 & + & 48 \\
\hline
\end{tabular}


TABLE XII

(FOR DILUTIONS)-STOCK 1 PER CENT SOLUTION

[1 c c Disinfectant +99 c.c. Distlled Water =Solution B]

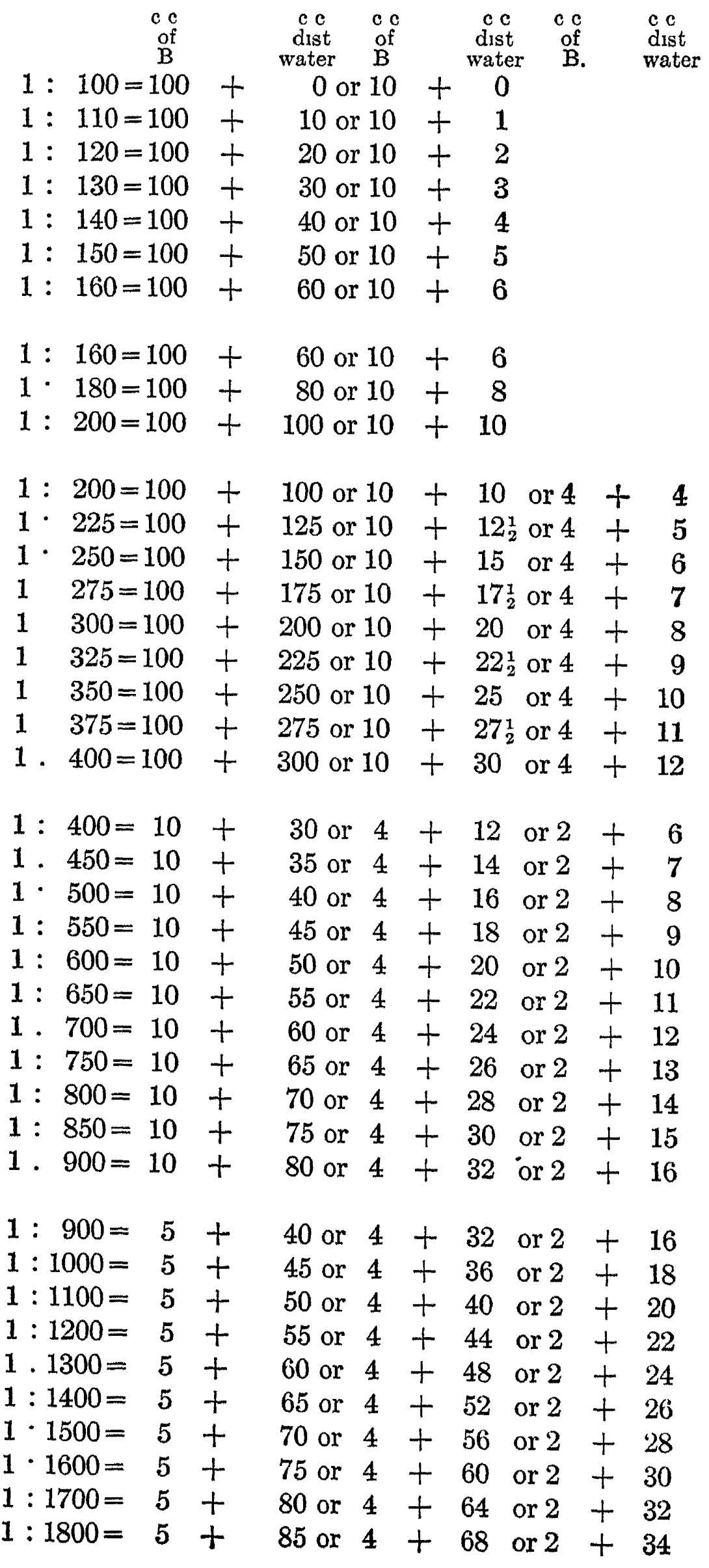


TABLE XII-Continued

(FOR DILUTIONS)-STOCK 1 PER CENT SOLUTION

[1 c c Disinfectant +99 ce Distilled Water =Solution B]

\begin{tabular}{|c|c|c|c|c|c|c|c|c|c|}
\hline & $\stackrel{\text { of }}{\mathrm{B}}$ & & $\begin{array}{c}\text { cc } \\
\text { dist } \\
\text { water }\end{array}$ & $\begin{array}{l}c c \\
\text { of } \\
B\end{array}$ & & $\begin{array}{c}\text { c c } \\
\text { dist } \\
\text { water }\end{array}$ & $\begin{array}{c}\text { c c } \\
\text { of } \\
B\end{array}$ & & $\begin{array}{c}\text { c c } \\
\text { dist } \\
\text { water }\end{array}$ \\
\hline $1: 1800=$ & 5 & + & 85 or & 4 & & 68 & or? & + & 34 \\
\hline & 5 & 1 & 5 or & 4 & - & 7 & 2 & 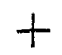 & \\
\hline $0=$ & 5 & + & or & 4 & 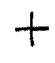 & 8 & o. & 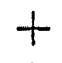 & 42 \\
\hline$\lambda$ & 5 & + & or & 4 & - & 92 & or & + & 46 \\
\hline & 5 & + & 12 & 4 & & 100 & & + & 50 \\
\hline & 5 & + & 13 & 4 & & 10 & or & 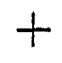 & 54 \\
\hline & 5 & + & & 4 & + & 116 & 01 & + & 58 \\
\hline & 5 & + & 155 or & 4 & & 124 & or: & + & 62 \\
\hline
\end{tabular}

Subculture Tube Racks. Wooden racks, with 5 rows of 14 holes each, are used for holding the subculture tubes, and as plants are made from each mixture of culture and disinfectant every $2 \frac{1}{2}$ minutes up to fifteen minutes, 6 tubes are required for each dilution. Thus, in each rack we have 10 rows of 6 tubes each with 2 empty cross rows of

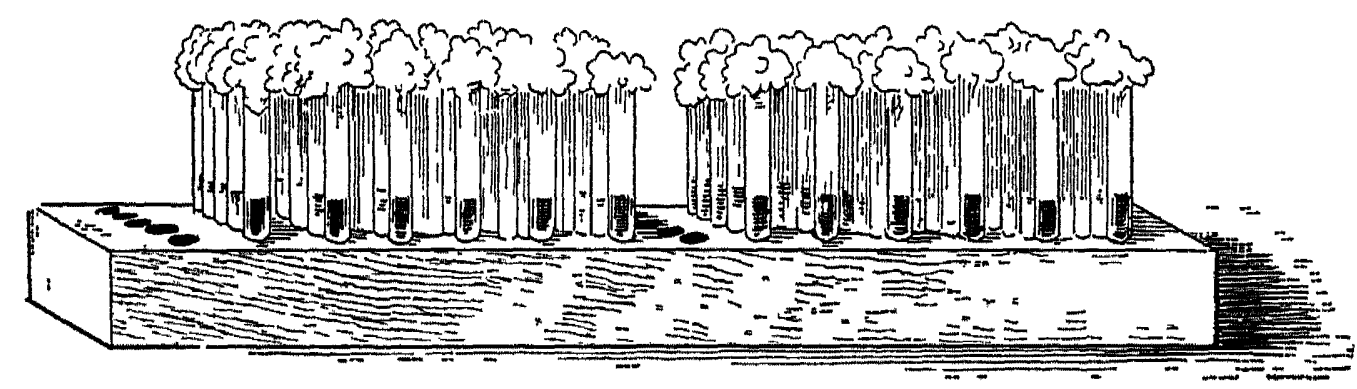

FIG. 43.-Block for Subculture Tubes.

holes remaining, which are utilized by placing over in the next row each tube as it is planted. This makes it easy to keep run of the tubes that are planted. It is well also always to plant from the seeding tube in a certain hole in the water bath into a certain row of tubes in the rack. This, after a little practice, will help to avoid errors in planting.

Method of Conducting the Test. If there are in one experiment more than 10 dilutions of the disinfectant, including the phenol controls, the stronger solutions of the disinfectant and phenol are tested first, as it will not be necessary to plant them after seven and a half minutes. The weaker solutions are then immediately done and are then planted every two and a half minutes for fifteen minutes.

For keeping the time a stop watch can be used, but an ordinary watch will serve the same purpose by simply starting on the two-andone-half- or five-minute periods. 
When everything is in readiness the culture is added to the disinfectant solutions with a sterile pipette in quantities of a tenth of a cubic centimeter to each dilution.

To add the culture, the seeding tube containing the disinfectant is removed from the water bath with the left hand and slanted at an angle of about $45^{\circ}$, and with the right hand the end of the pipette containing the culture is introduced and lightly touched against the side of the tube where the liquid has run away on account of the slanting. At the proper time the culture is allowed to run into the disinfectant solution, the pipette removed, the tube straightened up,

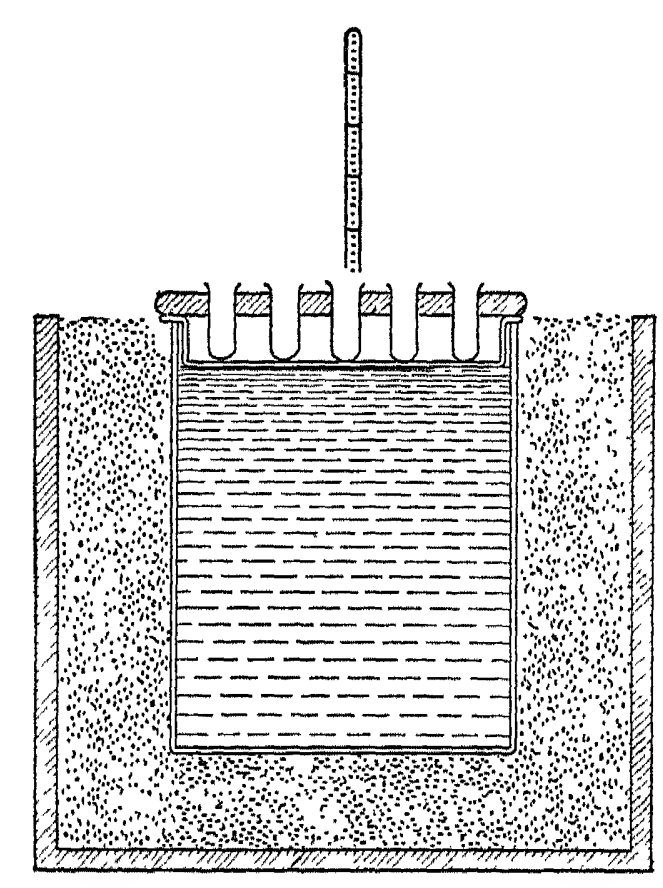

Frg. 44.-Cross-section of Water Bath Showing Seeding Tubes in Place. gently shaken three times, and replaced in the water bath. The other tubes are done the same way in succession, and it will be found that fifteen seconds is ample time for each tube. By adding the culture to the disinfectant with a pipette touched against the side of the seeding tube, accurate measurements can be made and each tube receive exactly the same amount of "seeding," which is not the case when the culture is added by the "drop."

If ten tubes are to be inoculated, only a few seconds will remain after inoculating the last tube before a plant from the first tube will have to be made.

The mixing tubes are not removed or disturbed in making the planting except to insert the loop or spoon into them, touch the botton, withdraw, and then make the plant in broth. Every effort is made to insert and withdraw the loops or spoons in a uniform manner. The loops and spoons are bent to an angle of about $45^{\circ}$ where they are jointed to the shank, and therefore are always filled with the mixture when withdrawn from the seeding tubes. After making the plants the loops or spoons are flamed as already described.

After an experiment is finished the date and any necessary details can be marked on one of the broth tubes. and the rack placed in the incubator at $37^{\circ} \mathrm{C}$. for forty-eight hours. At the end of this time the results are recorded on a chart specially devised for the purpose. 
Determining the Coefficient. After a large number of experiments we have concluded that the method employed by the Lancet commission, with certain modifications, is the best one for determining the coefficient, i.e., the mean between the strength and time coefficients.

In performing the test, plants are made every two and a half minutes up to and including fifteen minutes. To determine the coefficient, the figure representing the degree of dilution of the weakest strength of the disinfectant that kills within two and a half minutes is divided by the igure representing the degree of dilution of the weakest strength of

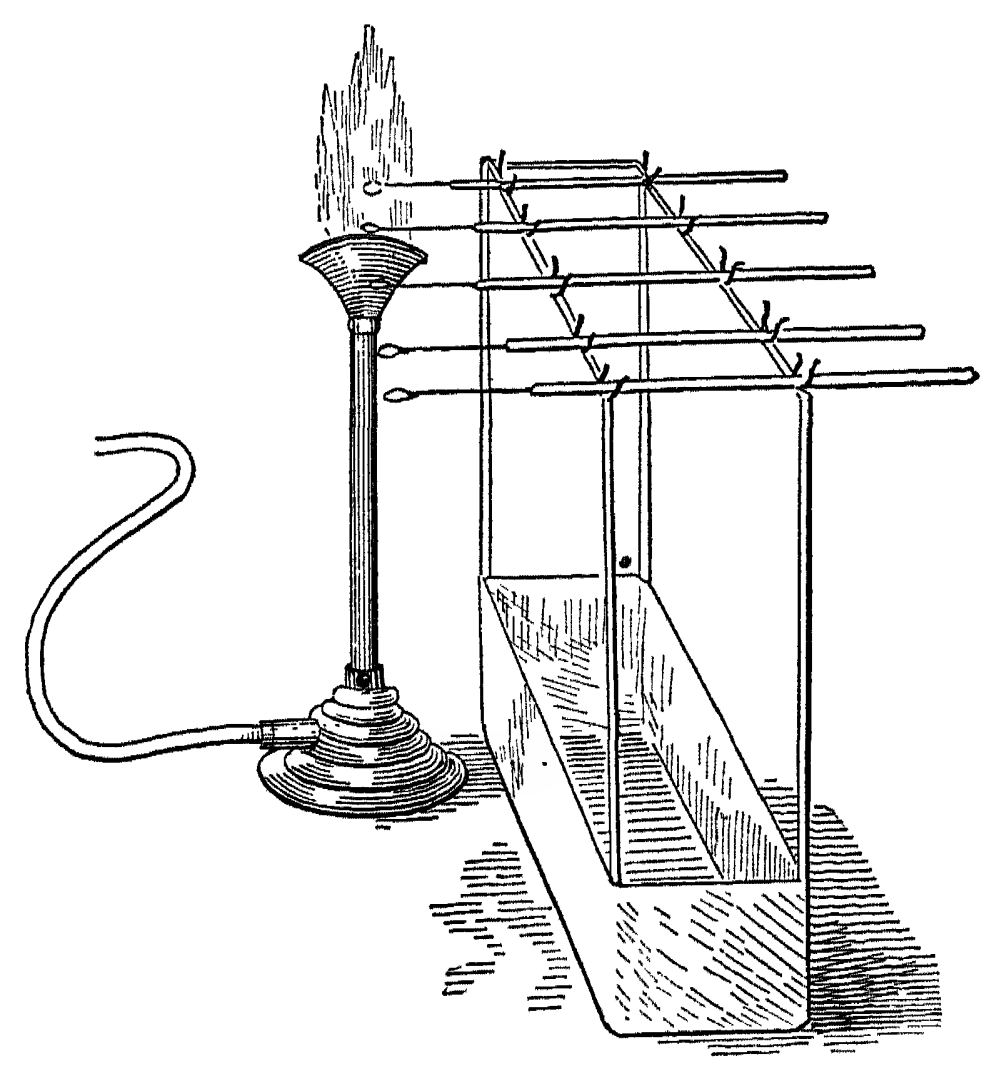

Frg. 45.-Device for Flaming Inoculating Loops.

the phenol control that kills within the same time. The same is done for the weakest strength that kills in fifteen minutes. The mean of the two is the coefficient. The method of determining the coefficient will be seen in Table XIII.

\section{B. THE DETERMINATION OF THE COEFFICIENT WITH THE ADDITION OF ORGANIC MATTER}

It may be well to briefly discuss here the meaning or significance of the term "phenol coefficient," particularly when it is determined in the presence of organic matter. In general terms the coefficient 
of a disinfectant inay, for practical purposes, be defined as the figure that represents the ratio of the germicidal power of the disinfectant to the germicidal power of carbolic acid, both having been tested under the same conditions.

\section{TABLE XIII}

Name "A."

Temperature of medication, $20^{\circ} \mathrm{C}$.

Culture used, B. typhosus, 24-hour, extract, broth, filtered.

Proportion of culture and disinfectant, 0.1 c.c. +5 c.c.

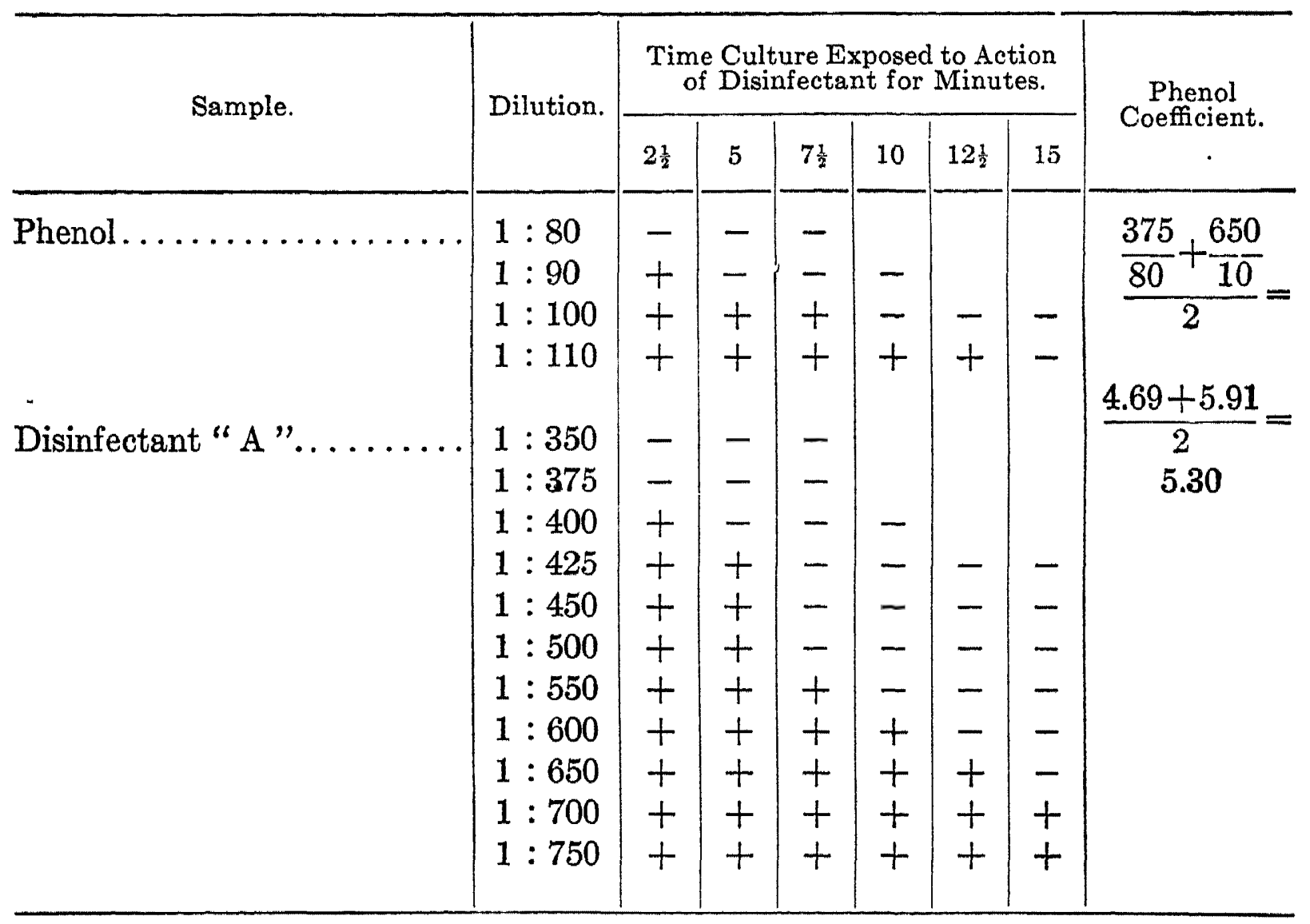

Although the germicidal power of carbolic acid is taken as the unit of comparison, it is influenced to a certain extent by the conditions, particularly the addition of organic matter, or, in other words, it is not a constant unit. This has to be borne in mind when making a comparison of the relative values of the phenol coefficients of a disinfectant determined with and without the addition of organic matter, respectively. It will readily be seen, for instance, that if the germicidal powers of a disinfectant and of carbolic acid were proportionately reduced by the addition of organic matter the coefficient of the disinfectant would remain unchanged regardless of whether or not organic matter was used. However, the germicidal power of carbolic acid, like the other pure phenols, is, as compared with most other disinfectants, only slightly affected by the addition of organic 
matter, and therefore serves as a fairly accurate means of estimating, in the presence of organic matter, the germicidal values of disinfectants in general.

The method of determining the coefficient of disinfectants with the addition of organic matter is identical in many respects with the method in which no organic matter is used, the former differing from the latter principally in the strength of the disinfectant dilutions that have to be prepared and in the preparation and addition of the organic matter to the disinfectant dilutions when performing the test. As the method in which no organic matter is added has already been given in detail, further description here will consist only of the differences between the two methods.

Dilutions. In making the dilutions of a disinfectant for determining its coefficient in the presence of organic matter, allowance must be made for the further dilution of the disinfectant when the volume of organic matter is added thereto. For instance, if 1 c.c. of organic matter is added to 5 c.c. of a 1 per cent dilution of a disinfectant the percentage strength of the disinfectant is proportionately reduced, it then being about 0.83 per cent.

The volume of organic matter that can be added to the disinfectant dilution is, of course, variable, as

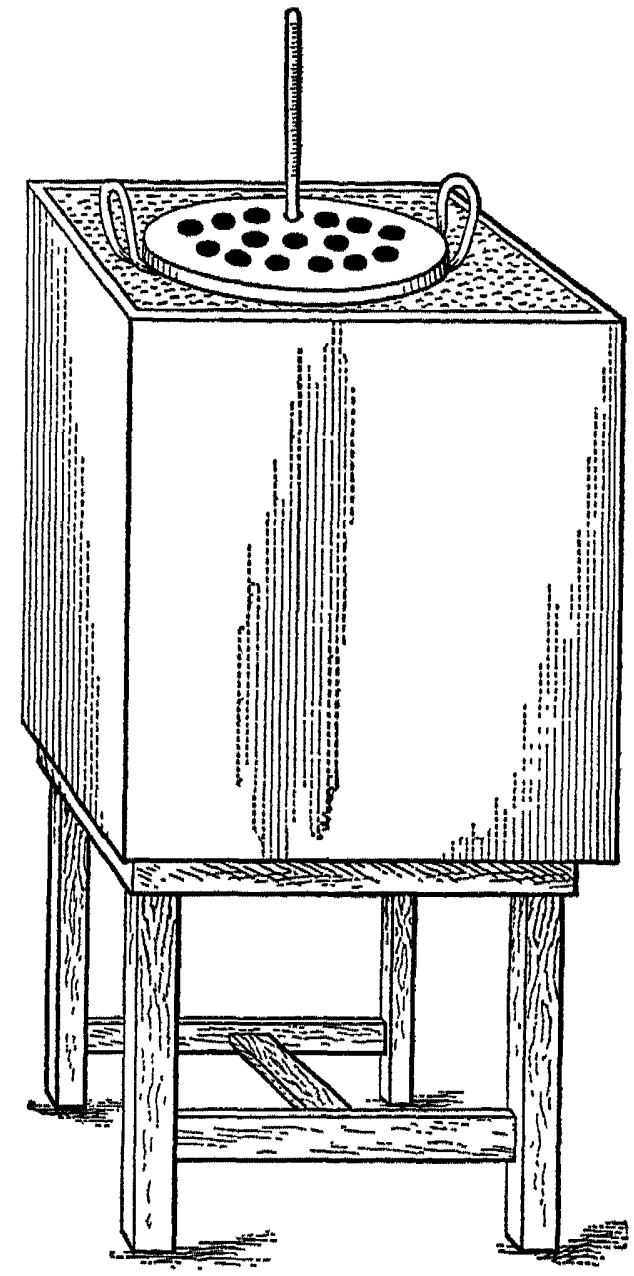

FJg. 46 -Water Bath Showing Position of Holes for Seeding Tubes and Thermometer in Place. is also the volume of disinfectant dilution to which it can be added. Consequently, we have decided rather arbitrarily to use the organic matter by adding 1 c.c. of it to 4 c.c. of the disinfectant dilution contained in a seeding tube. It will be seen that the strength of the disinfectant is reduced 20 per cent by the addition of the organic matter and that the dilutions of the disinfectant must be made accordingly. For example, if it is desired to test a 1 per cent strength of a disinfectant it is necessary to prepare a strength of 1.25 per cent, 4 c.c. of which becomes. a 1 per cent strength when 1 c.c. of the organic matter is added to it. 
We also tried adding 2.5 c.c. of the organic matter to 2.5 c.c. of the disinfectant dilution, but found it rather difficult and cumbersome to do, particularly when the experiment has to be performed with a number of different strengths of the disinfectant. When using the proportions as just stated, the dilutions of the disinfectant are made double the strengths it is desired to test, thus allowing for the further dilution when an equal volume of organic matter is added thereto.

In using the proportions of 1 c.c, of organic matter to 4 c.c. of the disinfectant dilution we have found the following tables (XIV and XV) of service in preparing the dilutions of the disinfectant:

It will be seen that by preparing the dilutions of the disinfectant according to the strengths shown in the second column of Table XIV, and then adding 1 c.c. of organic matter to 4 c.c. of the dilutions, the final dilutions of the disinfectant become as represented in the first columu of the table given abóve.

Preparation and Use of the Peptone-gelatine Organic Matter. As already stated, we prefer to use a mixture of peptone and gelatine dissolve' in distilled water. It is prepared from Witte's peptone (siccum) and "Best French Gold Label" gelatine. The stock preparation is made to contain 10 per cent peptone and 5 per cent gelatine. Proportionate amounts of peptone and gelatine, respectively, are weighed out and liquefied separately in small quantities of water by means of heat. They are then mixed in a graduate and sufficient water added thereto to make a mixture containing 10 per cent of peptone and 5 per cent of gelatine. It is then placed in bottles of appropriate size and sterilized on three successive days. When the mixture has become cold it will be observed that some of the peptone settles to the bottom of the bottles as a flocculent deposit. Consequently, the bottle should be shaken before using it. The stock preparation containing 5 per cent gelatin becomes semi-solid if it is kept in the cold room. at a temperature of $16^{\circ} \mathrm{C}$. However, by warming it until it becomes perfectly liquefied and then not allowing it to go below the temperature of $20^{\circ} \mathrm{C}$. we found that it remains liquid and is easily measured in the pipette.

By adding 1 c.c. of the stock preparation of 10 per cent peptone and 5 per cent gelatin to 4 c.c. of the disinfectant dilutions a resulting mixture is obtained containing 2 per cent of peptone and 1 per cent of gelatin, or a total of 3 per cent organic matter.

The Method of Conducting the Test. Four c.c. of each disinfectant dilution, including the phenol controls, are accurately measured into 
TABLE XIV

STOCK 5 PER CENT SOLUTION

[5 c.c. Disinfectant +95 c.c. Distilled Water $=$ Solution A]

\begin{tabular}{|c|c|c|c|c|c|c|c|c|c|}
\hline $\begin{array}{l}\text { Strength to } \\
\text { be tested }\end{array}$ & $\begin{array}{l}\text { Strength to } \\
\text { be made. }\end{array}$ & & $\begin{array}{c}c c \\
\text { A. }\end{array}$ & & $\begin{array}{l}\text { c c dis } \\
\text { wate }\end{array}$ & & $\begin{array}{l}c c \text { of } \\
A,\end{array}$ & & $\begin{array}{l}\text { c. dist } \\
\text { water }\end{array}$ \\
\hline $1: 30$ & $1: 24$ & $=$ & 20 & + & 4 & or & 10 & + & 2 \\
\hline $1: 35$ & $1: 28$ & $=$ & 20 & + & 8 & or & 10 & + & 4 \\
\hline $1: 40$ & $1: 32$ & $=$ & 20 & + & 12 & or & 10 & + & 6 \\
\hline $1: 45$ & $1: 36$ & $=$ & 20 & + & 16 & or & 10 & + & 8 \\
\hline $1: 50$ & $1: 40$ & $=$ & 20 & + & 20 & or & 10 & + & 10 \\
\hline $1: 55$ & $1: 44$ & $=$ & 20 & + & 24 & or & 10 & + & 12 \\
\hline $1: 60$ & $1: 48$ & $=$ & 20 & + & 28 & or & 10 & + & 14 \\
\hline $1: 65$ & $1: 52$ & $=$ & 20 & + & 32 & or & 10 & + & 16 \\
\hline $1: 70$ & $1: 56$ & $=$ & 20 & + & 36 & or & 10 & + & 18 \\
\hline $1: 70$ & $1: 56$ & $=$ & 10 & + & 18 & or & 5 & + & 9 \\
\hline $1: 80$ & $1: 34$ & $=$ & 10 & + & 22 & or & 5 & + & 11 \\
\hline $1: 90$ & $1: 72$ & $=$ & 10 & + & 26 & or & 5 & + & 13 \\
\hline $1: 100$ & $1: 80$ & $=$ & 10 & + & 30 & or & 5 & + & 15 \\
\hline $1: 110$ & $1: 88$ & $=$ & 10 & + & 34 & or & 5 & + & 17 \\
\hline $1: 120$ & $1: 96$ & $=$ & 10 & + & 38 & or & 5 & + & 19 \\
\hline $1: 130$ & $1: 104$ & $=$ & 10 & + & 42 & or & 5 & + & 21 \\
\hline $1: 140$ & $1: 112$ & $=$ & 10 & + & 46 & or & 5 & + & 23 \\
\hline $1: 150$ & $1: 120$ & $=$ & 10 & $=$ & 50 & or & 5 & + & 25 \\
\hline $1: 160$ & $1: 128$ & $=$ & 10 & + & 54 & or & 5 & + & 27 \\
\hline $1: 160$ & $1: 128$ & $=$ & 10 & + & 54 & or & 5 & + & 27 \\
\hline $1: 180$ & $1: 144$ & $=$ & 10 & + & 62 & or & 5 & + & 31 \\
\hline $1: 200$ & $1: 160$ & $=$ & 10 & + & 70 & or & 5 & + & 35 \\
\hline $1: 200$ & $1: 160$ & $=$ & 5 & + & 35 & or & 2 & + & 14 \\
\hline $1: 225$ & $1: 180$ & $=$ & 5 & + & 40 & or & 2 & + & 16 \\
\hline $1: 250$ & $1: 200$ & $=$ & 5 & + & 45 & or & 2 & + & 18 \\
\hline $1: 275$ & $1: 220$ & $=$ & 5 & + & 50 & or & 2 & + & 20 \\
\hline $1: 300$ & $1: 240$ & $=$ & 5 & + & 55 & or & 2 & + & 22 \\
\hline $1: 325$ & $1: 260$ & $=$ & 5 & + & 60 & or & 2 & + & 24 \\
\hline $1: 350$ & $1: 280$ & $=$ & 5 & + & 65 & or & 2 & + & 26 \\
\hline $1: 375$ & $1: 300$ & $=$ & 5 & + & 70 & or & 2 & + & 28 \\
\hline $1: 400$ & $1: 320$ & $=$ & 5 & + & 75 & or & 2 & + & 30 \\
\hline $1: 400$ & $1: 320$ & 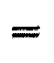 & 5 & + & 75 & or & 2 & + & 30 \\
\hline $1: 450$ & $1: 360$ & $=$ & 5 & + & 80 & or & 2 & + & 32 \\
\hline $1: 500$ & $1: 400$ & $=$ & 5 & + & 85 & or & 2 & + & 34 \\
\hline $1: 500$ & $1: 440$ & 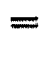 & 5 & + & 90 & or & 2 & + & 36 \\
\hline $1: 600$ & $1: 480$ & $=$ & 5 & + & 95 & or & 2 & + & 38 \\
\hline $1: 650$ & $1: 520$ & - & 5 & + & 100 & or & 2 & + & 40 \\
\hline
\end{tabular}




\section{TABLE XV}

STUCK 1 PER CENT SOLUTION

[1 c.c. Disinfectant +99 c.c. Distilled Water $=$ Solution B]

\begin{tabular}{|c|c|c|c|c|}
\hline $\begin{array}{l}\text { Strength to } \\
\text { be tested }\end{array}$ & $\begin{array}{l}\text { Strength to } \\
\text { be made. }\end{array}$ & ${ }_{\mathrm{B}}^{\mathrm{c}}$. & & $\begin{array}{l}\text { c.c dist } \\
\text { water }\end{array}$ \\
\hline $1: 500$ & $1: 400$ & 5 & + & 15 \\
\hline $1: 550$ & $1: 440$ & 5 & + & 17 \\
\hline $1: 600$ & $1: 480$ & 5 & + & 19 \\
\hline $1: 650$ & $1: 520$ & 5 & + & 21 \\
\hline $1: 700$ & $1: 560$ & 5 & $t$ & 23 \\
\hline $1: 750$ & $1: 600$ & 5 & + & 25 \\
\hline $1: 800$ & $1: 640$ & 5 & + & 27 \\
\hline $1: 850$ & $1: 680$ & 5 & + & 29 \\
\hline $1: 900$ & $1: 720$ & 5 & + & 31 \\
\hline $1: 900$ & $1: 720$ & 5 & + & 31 \\
\hline $1: 1000$ & $1: 800$ & 5 & + & 35 \\
\hline $1: 1100$ & $1: 880$ & 5 & + & 39 \\
\hline $1: 1200$ & $1: 960$ & 5 & + & 43 \\
\hline $1: 1300$ & $1: 1040$ & 5 & + & 47 \\
\hline $1: 1400$ & $1: 1120$ & 5 & + & 51 \\
\hline $1: 1500$ & $1: 1200$ & 5 & + & 55 \\
\hline $1: 1600$ & $1: 1280$ & 5 & + & 59 \\
\hline $1: 1700$ & $1: 1360$ & 5 & + & 63 \\
\hline $1: 1800$ & $1: 1440$ & 5 & + & 67 \\
\hline
\end{tabular}

the seeding tubes and placed in the water bath. For reasons that will be obvious later, it is difficult to handle more than nine dilutions at one time in making a test. The broth culture of $B$. typhosus is filtered and placed in the water bath. The desired quantity of stock peptonegelatine mixture is measured into a large test tube or flask and also placed in the water bath. It is necessary to have a slight excess of the organic matter, so that there will be no trouble in getting the 10 c.c. pipette full when adding the organic matter and culture to the disinfectant. Thus, for nine tubes we usually measure out 15 c.c. of the organic matter. When the disinfectant dilutions, the typhoid culture, and the organic matter have reached the temperature of the water bath $\left(20^{\circ}\right.$ C. $) 1.5$ c.c. of the typhoid culture is added to the 15 c.c. of organic matter and thoroughly mixed by means of a 10-c.c. pipette. With the same pipette the seeding tubes containing the disinfectant dilutions then have added to them, successively every fifteen seconds, 1.1. cc. of the mixture of the organic matter and typhoid culture. The technique of shaking, planting, etc., is then as has already been described.

From the above it will he seen that each seeding tube, as the test is conducted, contains 4 c.c. of the disinfectant dilution plus 1 c.c. 
of organic matter, plus 0.1 c.c. of typhoid culture, and that the quantity of organic matter represented is 3 per cent.

In computing the strengths of the disinfectant and the percentage of organic matter present in the seeding tubes when the test is made, we have disregarded the slight change resulting from the addition of the 0.1 c.c. of typhoid culture to each tube. By so doing the experiment is very much simplified, as it would be very difficult to prepare the dilutions of the disinfectant on the basis of allowing for so slight a change as is caused by the addition of 0.1 c.c. of liquid.

Many of the details of conducting the experiment and the method of determining the coefficient will be seen in Table No. 16:

Name, "A."

\section{TABLE XVI}

Temperature of medication, $20^{\circ} \mathrm{C}$.

Culture used, B. typhosus, twenty-four-hour, extract broth, filtered.

Proportion of culture, organic matter and disinfectant, 0.1 c.c. +1 c.c. +4 c.c. Percentage of organic matter, 2 per cent peptone and 1 per cent gelatin.

Total percentage of organic matter, 3 per cent.

\begin{tabular}{|c|c|c|c|c|c|c|c|c|}
\hline \multirow{2}{*}{ Sample. } & \multirow{2}{*}{ Dilution } & \multicolumn{6}{|c|}{$\begin{array}{l}\text { Time Culture Exposed to Action } \\
\text { of Dismfectant for Minutes }\end{array}$} & \multirow{2}{*}{$\begin{array}{l}\text { Phenol coeffi- } \\
\text { cient. }\end{array}$} \\
\hline & & $2 \frac{1}{3}$ & 5 & $7 \frac{1}{2}$ & 10 & $12 \frac{1}{2}$ & 15 & \\
\hline Phenol.............. & $\begin{array}{l}1: 80 \\
1: 90 \\
1: 100 \\
1: 110\end{array}$ & $\begin{array}{l}- \\
+ \\
+ \\
+\end{array}$ & $\begin{array}{l}- \\
+ \\
+ \\
+\end{array}$ & $\begin{array}{l}- \\
- \\
+ \\
+\end{array}$ & $\begin{array}{l}- \\
+ \\
+\end{array}$ & $\begin{array}{l}- \\
+ \\
+\end{array}$ & $\begin{array}{l}- \\
+ \\
+\end{array}$ & $\begin{array}{l}\frac{\frac{180}{80}+\frac{375}{90}}{2}= \\
2.25+4.17\end{array}$ \\
\hline Disinfectant " A "....... & $\begin{array}{l}1: 180 \\
1: 200 \\
1: 225 \\
1: 250 \\
1: 300 \\
1: 325 \\
1: 350 \\
1: 375 \\
1: 400 \\
1: 450\end{array}$ & $\begin{array}{l}- \\
+ \\
+ \\
+ \\
+ \\
+ \\
+ \\
+ \\
+ \\
+\end{array}$ & $\begin{array}{l}- \\
- \\
- \\
- \\
+ \\
+ \\
+ \\
+ \\
+ \\
+\end{array}$ & $\begin{array}{l}- \\
- \\
- \\
- \\
- \\
- \\
+ \\
+ \\
+ \\
+\end{array}$ & $\begin{array}{l}- \\
- \\
- \\
- \\
- \\
- \\
+ \\
+ \\
+\end{array}$ & $\begin{array}{l}- \\
- \\
- \\
- \\
- \\
- \\
- \\
+ \\
+\end{array}$ & $\begin{array}{l}- \\
- \\
- \\
- \\
- \\
- \\
- \\
+ \\
+\end{array}$ & 2 \\
\hline
\end{tabular}

To Determine the Cost of a Disinfectant per Unit of Disinfecting Efficiency as Compared with Pure Phenol. It is manifestly cheaper to purchase a disinfectant for 60 cents a gallon than it is to purchase one for 30 cents a gallon, provided the former has four times the efficiency of the latter. The true cost of a disinfectant can only be determined by 
taking into consideration the phenol coefficient and the cost of the disinfectant per gallon.

The cost of a disinfectant per 100 units of efficiency as compared with pure phenol is obtained by first dividing the cost per gallon of the disinfectant by the cost per gallon of pure phenol; the efficiency ratio of course is obtained by dividing the coefficient of the disinfectant by the coefficient of phenol, but as the coefficient is always 1 the effieiency ratio is represented by the phenol coefficient of the disinfectant. The cost ratio divided by the efficiency ratio (the coefficient of the disinfectant) gives the cost of the disinfectant per unit of efficiency as compared with the cost per unit of efficiency of pure phenol=1. By multiplying by 100 the relative cost per 100 units is obtained. Thus, $\frac{\text { Cost of disinfectant per gallon }}{\text { Cost of phenol per gallon }}(=$ cost ratio $) \div \frac{\text { Coefficient of disinfectant }}{\text { Coefficient of phenol }(=1)}$ (=efficiency ratio) $=$ the cost of the disinfectant per unit of efficiency as compared with phenol $=1$. And by multiplying by 100 the cost per 100 units is obtained.

For instance, the cost of disinfectant "Car" is $\$ 0.30$ per gallon, and it has a coefficient of 2.12 ; the cost of phenol is $\$ 2.67$ and has a coefficient of 1 . Then.

$$
\frac{.20}{2.67} \div \frac{2.12}{1}=.052
$$

Therefore, the comparative cost per unit of efficiency of "Car" and phenol, respectively, is as 0.052 is to 1 ; or, by multiplying by 100 , the relative cost per 100 units-5.2 to 100 -is obtained.

The following table illustrates the value of the determination of the cost per 100 units of efficiency of a disinfectant as compared with the cost of 100 units of pure phenol:

\begin{tabular}{|c|c|c|c|c|}
\hline Disinfectant. & $\begin{array}{l}\text { Price per } \\
\text { Gallon. }\end{array}$ & Cost, Ratio. & $\begin{array}{l}\text { Efficiency } \\
\text { Ratio, which } \\
\text { is the Phenol } \\
\text { Coefficient. }\end{array}$ & $\begin{array}{l}\text { Relative cost } \\
\text { per } 100 \text { units } \\
\text { of Efficiency } \\
\text { as Compared } \\
\text { with Pure } \\
\text { Phenol }=100\end{array}$ \\
\hline Car.. & $\$ 0.30$ & 0.112 & 2.12 & 5.2 \\
\hline Chl.. & 1.00 & .374 & 4.44 & 8.4 \\
\hline Phi.. & .37 & .138 & 1.40 & 9.9 \\
\hline Cre.. & .44 & .164 & 1.13 & 14.5 \\
\hline Nap... & .41 & .153 & .44 & 34.8 \\
\hline Zod.. & .40 & .150 & .25 & 60.0 \\
\hline Pure phenol. & 2.67 & 1.00 & 1.00 & 100.0 \\
\hline
\end{tabular}


It will be seen that the disinfectant Chl has a higher coefficient than any of the others in the table, but its higher cost per gallon results in its being placed second in cost per 100 units of efficiency.

When bids are solicited for supplying disinfectants they should be required to be made so as to show the cost per 100 units of efficiency of the disinfectant as compared with the cost of 100 units of efficiency of pure phenol. In that way only can a contract for supplying disinfectants be awarded in the combined interests of economy and efficiency.

It may be proper to state here that in the specifications for bids for supplies for the fiscal year beginning July 1, 1912, prepared by the General Supply Committee, is the following specification for bids on disinfectants:

Item 4136 (d), noncorrosive, cresol, phenol, or analogous compound; must be uniform material at $43^{\circ} \mathrm{F}$. and "dilute with water and form a practically perfect emulsion or solution and have a phenol coefficient according to present method of Hygienic Laboratory of not less than $2 ; 1$ gal. sample required.

Note.-The phenol coefficient of disinfectants on which bids are made must be stated.

The General Supply Committee prepares specifications on which bids are solicited for supplies for all the departments in Washington.

McClintic (1912) determined the phenol coefficient of some common disinfectants. The results are indicated in Table XVIII.

Kendall and Edward's Method for Determining Penetrating Power. The authors have attempted to broaden the standardization of disinfectants by determining the relative penetrating power of these agents. The important features of the Rideal-Walker method are retained.

(1) Prepare a twenty-four-hour culture of $B$. Coli in plain broth of standard composition and reaction; (2) add 10 c.c. of this standard culture to 1 liter of agar (1.5 per cent agar) and mix thoroughly; (3) pour the infected agar into sterile tubes of convenient length ( 1 meter) and of exactly $1.5 \mathrm{~cm}$. diameter and allow to harden, after closing the ends with sterile rubber stoppers; (4) allow to harden at $20^{\circ}$ C.; (5) prepare dilutions of the desired disinfectants and a standard 5 per cent carbolic acid solution-the latter is the standard to which the other disinfectants are referred; (6) place the disinfectants so prepared in sterile beakers, allowing 50 c.c. for each agar cylinder; (7) remove stoppers from the infected agar tubes, and permit the contents to run out slowly as a long cylinder; with a sterile knife cut off portions by transverse cuts of $2 \mathrm{~cm}$. length, and allow these smaller cylinders to 


\section{TABle XVIII \\ SHOWING THE PHENOL COEFFICIENTS OF SOME COMMON DISINFECTANTS}

(McClintic, 1912)

\begin{tabular}{|c|c|c|}
\hline Name of Disinfectant. & $\begin{array}{l}\text { With Organic } \\
\text { Matter. }\end{array}$ & $\begin{array}{l}\text { Without Organic } \\
\text { Matter. }\end{array}$ \\
\hline Bacterol. . & 1.34 & 1.58 \\
\hline Benetol $\ldots \ldots \ldots \ldots \ldots \ldots \ldots \ldots \ldots \ldots$ & 0.92 & 1.23 \\
\hline Cabot's silpho-naphthol. ........ & 2.33 & 3.87 \\
\hline Carbolene..................... & 0.65 & 1.36 \\
\hline Carbolozone............. & 0.48 & 1.48 \\
\hline Car-sul. $\ldots \ldots \ldots \ldots \ldots \ldots \ldots$ & 1.75 & 2.00 \\
\hline Chloro-naphtholeum $\ldots \ldots \ldots \ldots \ldots \ldots \ldots \ldots$ & 3.21 & 6.06 \\
\hline Cremoline $\ldots \ldots \ldots \ldots \ldots \ldots \ldots \ldots \ldots \ldots$ & 0.69 & 1.26 \\
\hline Creo-carboline . . . . . . . . . . . . . . & 2.26 & 4.03 \\
\hline Creolin-Pearson $\ldots \ldots \ldots \ldots \ldots \ldots \ldots$ & 2.52 & 3.25 \\
\hline Cresoleum.................... & 1.75 & 2.90 \\
\hline Crude carbolic acid $\ldots \ldots \ldots \ldots \ldots \ldots \ldots$ & 2.63 & 2.75 \\
\hline Dusenberry's liquid creoleum . . . & 0.40 & 1.00 \\
\hline Germol..................... & 1.79 & 2.12 \\
\hline Hycol................... & 9.37 & 12.30 \\
\hline 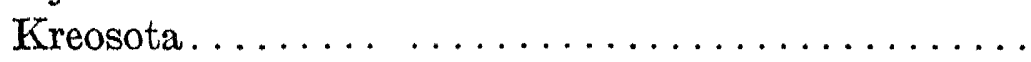 & 0.65 & 1.26 \\
\hline Kreotas................. & 0.30 & 1.10 \\
\hline Kreso........................ & 2.32 & 3.92 \\
\hline Kresolig. . . . . . . . $\ldots \ldots \ldots \ldots$ & 1.48 & 2.13 \\
\hline Li(quor cresolis compositus (U. S. P.). . . . . . . & 1.87 & 3.00 \\
\hline Lysol $\ldots \ldots \ldots \ldots \ldots \ldots$ & 1.57 & 2.12 \\
\hline Phenoco disinfecting fluid. . & 9.86 & 5.00 \\
\hline Saponified cresol. . . . . . . . . . . . & 0.57 & 1.03 \\
\hline 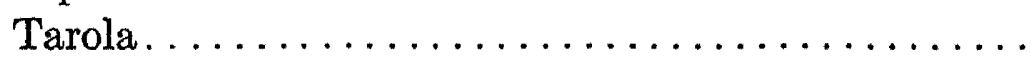 & 1.93 & 3.12 \\
\hline Trikresol........... & 2.50 & 2.62 \\
\hline Zonol............ & 1.57 & 2.37 \\
\hline Creola disinfectant. . . & $\ldots$ & .52 \\
\hline
\end{tabular}

fall directly into the disinfectant solutions, one cylinder for each time interval selected; (8) note the temperature of the solutions (they should be kept at exactly $20^{\circ} \mathrm{C}$. during the experiment); (9) note the time at which the cylinders were dropped into the disinfectant solutions; (10) at the end of stated intervals (usually at hourly intervals for preliminary tests) remove one cylinder from each solution with one of the sterile holders mentioned above, wash it thoroughly with sterile water, and remove a core from the center with a sterile piece of quill tubing (3-mm. bore); (11) place these cores in lactose fermentation tubes, after they are properly labeled, and incubate at $37^{\circ} \mathrm{C}$. for several days, 
making daily obscrvations; (12) compare the killing times of the various solutions tested with that obtained from carbolic acid, and determine the carbolic coefficient of germicidal and penetrating powers combined.

In tests with some common disinfectants and a seventy-two-hour incubation, there was no growth after one hour's exposure to 4 per cent formalin; no growth after three hours' exposure to 1 per cent formalin, or 1 per cent corrosive sublimate solution; and no growth after five hours' exposure to 5 per cent carbolic acid, 0.25 per cent formalin, and 10 per cent chloride of lime. Even after five hours' exposure, however, there was growth with 1 per cent carbolic acid, 0.1 per cent corrosive sublimate, 4 per cent chloride of lime, 2 per cent hyco, 1 per cent cresol, and 2 per cent sulphonaphthol.

TABLE XIX

SHOWING COMBINED GERMICIDAL AND PENETRATING POWERS OF SOME 'COMMON DISINFECTANTS

(After Kendall and Edwards.)

\begin{tabular}{|c|c|c|c|c|}
\hline \multirow{2}{*}{ Disinfectants } & \multirow{2}{*}{$\begin{array}{c}\text { Dilution, } \\
\text { Per Cent }\end{array}$} & \multicolumn{3}{|c|}{ Time of Exposure } \\
\hline & & 1 Hour. & 3 Hours & 5 Hours. \\
\hline Carbolic acid & 5 & + & + & - \\
\hline Carbolic acid . & 1 & + & + & + \\
\hline Formalin.... & 4 & - & - & - \\
\hline Formalin $\ldots \ldots \ldots \ldots \ldots \ldots \ldots$ & 1 & + & - & - \\
\hline Formalin . .............. & 0.25 & + & + & - \\
\hline Mercuric chloride......... & 0.1 & + & + & - \\
\hline Mercuric chloride........ & 1.0 & + & - & - \\
\hline Chloride of lime $\ldots \ldots \ldots$ & 10.0 & + & + & - \\
\hline Chloride of lime......... & 4.0 & + & + & + \\
\hline Hyco $\ldots \ldots \ldots \ldots \ldots$ & 2.0 & + & + & + \\
\hline Cresol................ & 1.0 & + & + & + \\
\hline Sulphonaphthol . ....... & 2.0 & + & + & + \\
\hline
\end{tabular}

The American Public Health Association Standard Phenol Coefficient. This association appointed a committee to report on the standardization of disinfectants and the material given below is taken from that report as published in the American Journal of Public Health for July, 1918.

Apparatus: Subculture Tubes. A test-tube rack of eight rows of ten holes each may be used for the subcultures. If the test be of 
twenty dilutions, eighty tubes are numbered serially and placed in the rack which is placed conveniently at the worker's left hand.

Bunsen Burner. A Bunsen burner with a fantail top is led from the left and back of the bench with a little spare tubing.

Loop Rack. This is made of four pieces of $\frac{1}{2}$-in. wood, each 4 ins. by 9 ins. joined in a frame so that the top is about 10 ins. high. The top is provided with about six grooves for the loops. The latter are placed in their grooves and the rack is placed in front of the worker's right shoulder, close to the front of the bench and almost at right angles to the front edge but with a slight slant to the right and rear. The loops point to the left and the fantail burner is so adjusted as to properly envelop the loop which is over it. The loops are used in succession and, after each one is returned to its place, the burner is moved under it thus providing at all times for a sterile and cool loop.

Loops. The loops used for transferring the culture after exposure to the disinfectant are made as follows: A close cylindrical spiral is made by winding nichrome wire No. 23 B. \& S., as tightly as possible about a piece of steel or other hard wire having a diameter of $.072 \mathrm{in}$. (B. \& S., No. 13). Wind about five full turns, bend the remainder of the wire sharply at a right angle to the wound portion and parallel to the axis of the cylinder. Remove from the core and cut off the lower end to leave exactly four complete turns. When completed the successive turns of the spiral must touch one another continuously.

Seeding Tubes. The characters of the seeding tubes to be used depends entirely upon local conditions. In places in which there is any doubt as to air contamination cotton plugged tubes are to be preferred. However, under conditions as exist in most laboratories the open seeding tubes recommended in the present standard methods are most convenient and quite satisfactory.

Seeding Tube Holder. Cut down an oblong wire test-tube basket to about 2.5 ins. in depth and to the top fit a piece of $\frac{1}{2}$-in. wood through which three rows of seven holes each have been bored, the holes being of proper size to accommodate the tubes loosely. An ordinary tripod is used to support this rack in the water bath. The legs of the tripod are cut so that the tops of the sceding tubes are a little above the surface of the water.

Inoculator. The inoculations of the diluted disinfectant with the culture are made with an apparatus described by Rosenau (Hyg. Lab. Bull. 21, p. 60). A capillary pipette graduated in tenths c.c. is fitted with a rubber bulb and clamped to a ring stand. The bulb is actuated by a second spring clamp the jaws of which engage it. This apparatus 
is placed at the right of the worker so that he can push the loop rack away from him and draw the inoculator toward him and when through inoculating reverse the action.

Water Bath. The water bath consists of a wooden box about 18 ins. square and deep, containing a three- or four-gallon pail packed around with sawdust which is lightly onled so as not to become dusty. The bath is raised from the floor about 6 ins. by cleats and castors, and when not in use is rolled under the bench. During the test it is on the floor at the right of the worker.

Timepiece. A watch or small clock of fair accuracy, provided .with a second hand, is so mounted as to be conveniently and comfortably read by the worker from his place at the bench.

Culture Media, Broth. Ten grams of peptone (Witte's), 3 gms. of extract of meat (Liebig's), 5 gms. of sodium chloride, C. P. Distilled water 1000 c.c. Boil for fifteen minutes, make up to weight, filter tube, sterilize at $10 \mathrm{lbs}$. pressure for twenty minutes. No adjustment whatever of the acidity should be attempted. Determine and record the $P_{h}$ value. This should be between 6 and 7 .

Agar. To 1000 c.c. of the standard broth add 15 gms. of best grade agar, boil thirty minutes, make up to weight, filter and sterilize at $1 \mathrm{lb}$. for twenty minutes. Do not use egg albumen to clarify.

Organism. For the standard test organism in determining the coefficient against the typhoid organism a twenty-four-hour-old broth culture of $B$. typhosus (Hopkins) is used. Before beginning a test the culture is carried over in broth at $37^{\circ}$ every twenty-four hours for at least five successive days, and the last culture well shaken and filtered through sterile filter paper. For carrying over the culture one standard loop is.used. It is important that the transfers be made as nearly as possible as exactly on the twenty-four-hour interval, although a variation of not more than two hours is allowable. The stock culture of $B$. typhosus (Hopkins) is kept on agar slants. These cultures are incubated at $37^{\circ} \mathrm{C}$. for twenty-four hours and then placed in the refrigerator until ready for use. In no case should they be kept more than one month.

Phenol. While pure phenol crystals are undoubtedly the best standard which we have at present, they are far f $10 \mathrm{~m}$ being entirely satisfactory. Commercial phenol crystals are often contaminated with cresols and other phenol homologues. It is important that nothing but the highest grade of pure white synthetic phenol crystals be used of a solidifying point not less than $40^{\circ} \mathrm{C}$. as determined by the method of Weiss and Downs. 
Phenol crystals themselves as well as solutions change in germicidal strength upon standing, particularly when exposed to direct sunlight and to high temperature. Phenol changes more readily when melted or in the presence of moisture than when in crystalline form. It is important, therefore, that the crystals of phenol be kept in a tightly stoppered bottle, in a cool, dry place and exposed to the light and moisture as little as possible. Phenol which has been exposed to the air and allowed to absorb enough water to alter its melting-point or which has become at all red should be discarded for the purpose of this test. The 5 per cent stock solution is prepared as follows: Melt the phenol in the original container and pour out approximately $50 \mathrm{gms}$. into a closed weighing bottle; weigh and dissolve in the proper amount of water to give a 5 per cent solution. Do not attempt any further standardization. This stock solution is kept in the refrigerator in ambered-colored bottles of not more than 500-c.c. capacity, and for a period of not longer than three months.

Dilutions, Distilled Water. Sterile distilled water is recommended for all dilutions. Attention is directed to the danger of metallic copper in the distilled water prepared from the common type of laboratory still in which the condensation surface is of tin-coated copper or brass. If the tin-plate is worn away sufficient copper may be dissolved to materially influence the coefficient.

Five Per Cent Stock Solution. Accurately graduated capacity pipettes are to be used in preparing the stock dilution of disinfectant. Five c.c. of disinfectant are measured into a flask containing 95 c.c. of sterile distilled water the interior of the pipette being rinsed several times with the contents of the flask. From this solution or emulsion, and from the stock 5 per cent phenol solution the required dilutions are prepared.

Initial Dilution from 5 Per Cent Dilution. Take 50 c.c. of the 5 per cent stock solution, place in a 100-c.c. graduated flask and make up to the mark with sterile distilled water. Mix by pouring. This gives a dilution of $1: 40$. With 50 c.c. of this dilution repeat the process to obtain a dilution of $1: 80$ and so on by successive dilutions until the following initial dilutions or such as these as are necessary for the test, are prepared:
$1: 20$
$1: 160$
$1: 40$
$1: 320$
$1: 80$
$1: 640$

Final Dilutions from Initial Dilutions. With sterile delivery pipettes measure the quantities of initial dilutions and sterile distilled water 
required in accordance with the following table to obtain the desired final dilutions:

TABLE XX

\begin{tabular}{|c|c|c|c|c|c|c|}
\hline \multirow{3}{*}{$\begin{array}{l}\text { Initial } \\
\text { Dilution } \\
+ \text { Water (c c) }\end{array}$} & \multicolumn{6}{|c|}{ Initial Dilution } \\
\hline & $1 \quad 20$ & 140 & 180 & $1 \cdot 160$ & $1: 320$ & $1: 64$ \\
\hline & \multicolumn{6}{|c|}{ Final Dilution } \\
\hline $4+4$ & $1: 40$ & $1: 80$ & $1: 160$ & $1: 320$ & $1: 640$ & $1: 1280$ \\
\hline $4+5$ & $1: 45$ & $1: 90$ & $1: 180$ & $1: 360$ & $1: 720$ & $1: 1440$ \\
\hline $4+6$ & $1: 50$ & $1: 100$ & $1: 200$ & $1: 400$ & $1: 800$ & $1: 1600$ \\
\hline $4+7$ & $1: 55$ & $1: 110$ & $1: 220$ & $1: 440$ & $1: 880$ & $1: 1760$ \\
\hline $4+8$ & $1: 60$ & $1: 120$ & $1: 240$ & $1: 480$ & $1: 960$ & $1: 1920$ \\
\hline $4+9$ & $1: 65$ & $1: 130$ & $1: 260$ & $1: 520$ & $1: 1040$ & $1: 2080$ \\
\hline $4+10$ & $1: 70$ & $1: 140$ & $1: 280$ & $1: 560$ & $1: 1120$ & $1: 2240$ \\
\hline
\end{tabular}

Temperature. The standard temperature at which the organism is exposed to the action of the disinfectant is $20^{\circ} \mathrm{C}$., but a variation of not more than $0.5^{\circ}$ on either side of this figure is allowable. The temperature should be maintained by the use of a water bath, the design of which may be left to the individual operator. It is important, however, that the bath be so arranged that the water will rise to a height greater than that of the dilution of disinfectant contained in these seeding tubes. The cultures and dilutions of disinfectants should be brought to a temperature of $20^{\circ} \mathrm{C}$. before starting the test. In very warm weather, it will be found that there is a material increase in the temperature of the water bath during the twenty-minute period. If, however, the bath is brought at the start to a temperature of $19.5^{\circ} \mathrm{C}$. it will not generally exceed $20.5^{\circ} \mathrm{C}$. during the test. For the determination of the temperature coefficient a temperature of $30^{\circ} \mathrm{C}$. is also employed.

All subcultures are incubated at $37^{\circ} \mathrm{C}$. for forty-eight hours. The temperature of the incubator should be maintained between $36^{\circ}$ and $38^{\circ}$.

Technique. In the test of a new preparation the coefficient of which is unknown it will be necessary to make a set of range-finding tests. For this purpose use the series $1: 40,1: 80$, etc., at the head of the colurnns in the table of final dilutions and select for the actual test with phenol a series of final dilutions ranging from the highest dilution which killed in five minutes to the lowest dilution which failed to kill in twenty minutes. If some approximate idea of the coefficient is available, or if the preliminary test indicates a too extensive range of dilutions for the 
final test, a closer range may be obtained by restricting the test to fewer columns and using the middle row of dilutions together with the top row.

The seeding tubes having been properly sterilized are brought to the bench and placed in the seeding racks to the number required. Five c.c. of each of the dilutions of phenol and disinfectant to be tested are placed in order in these seeding tubes, which are appropriately numbered, a separate 5-c.c. delivery tube being used for each dilution. The tube containing the filtered culture is next placed in one of the holes of the seeding-tube holder which is then placed in the water bath and allowed to stand there for sufficient time to bring the contents to temperature. The inoculator is then filled with culture and, at the beginning of an even five-minute period the first seeding tube is inoculated with 0.1 c.c. of culture and the succeeding tubes are then inoculated at appropriate intervals. If the manipulator is sufficiently skilled to make inoculations and transfers at fifteen-second intervals the total number of dilutions including the phenol dilutions that can be carried through a test simultaneously is 20 . Five of these are necessary for the phenol, $1: 80$ to $1: 120$, inclusive, leaving a maximum of 15 for the disinfectant. This will permit the employment of two of the columns of the table of final dilutions, if necessary. In practice, however, it will seldom be found necessary to extend the range over more than one complete column or eight dilutions which with five phenol dilutions gives thirteen in all. This will permit the making of inoculations and transfers at twenty-second intervals, with one minute leeway at the end of each round, a safer and more comfortable schedule. For the beginner thirtysecond intervals are recommended which permit the carrying of ten dilutions simultaneously. Whatever the interval decided upon it is quite desirable that a plan of the test showing the dilutions to be used, intervals between inoculations, and times of making subcultures be carefully laid out on paper so that there shall be no confusion in these matters during the actual test.

When the tubes have been inoculated, the inoculator is pushed away diagonally and the loop holder is drawn up to the edge of the bench and just before the expiration of the time interval, subculture tube No. 1 is taken in the hand, the plug withdrawn, and a loop dipped perpendicularly into the first seeding tube. At the expiration of the time interval, the loop is withdrawn, and the transfer consummated. The loop is returned to its groove on the loop holder, the burner moved in place under it and the subculture tube given a rapid whirling movement to mix its contents and replaced. Another tube and another loop are then taken in hand for the next transfer. At the completion of the test 
the subculture tubes are placed in the incubator and forty-eight hours later they are removed, read and recorded.

Determining the Coefficient. The concentrations of the highest dilutions which gave negative results in five, ten, fifteen and twenty minutes, respectively, are divided by the corresponding concentrations of phenol to obtain a series of four coefficients. The arithmetical mean of the four is taken as the "Mean Phenol Coefficient against Typhoid $20^{\circ} \mathrm{C}$." five to twenty minutes, A. P. H. A." It is recommended that a final report be based upon the results of not less than three separate tests. The coefficient will be known for brevity as the "A. P. H. A. Standard Phenol Coefficient," but for any other organism temperature, time ranges that may be employed the word "Standard" will be omitted and the full expression given. The determination of these special coefficient for special uses is recommended.

Determination of Coagulation Coefficient. (After Schneider, 1912). Certain chemicals which are used as disinfectants precipitate proteins and may be used up in that way. Mercuric chloride, for instance, is such a disinfectant and does not do its maximum work under such conditions. Many of the disinfectants do not coagulate albumin in the dilutions which are used for disinfecting. There seems to be no relation between the "phenol" coefficient and the "coagulating coefficient" but it is often desirable to know just how active a certain substance is in the precipitation of proteins. For this purpose Schneider (1912) devised the following procedure:

\section{Albumen Test Solution}

Based upon the results of the preliminary experiments a 1 per cent aqueous (distilled water) solution of pure dried egg albumen is recommended as the substance upon which the different strength solutions of the various disinfectants is to act.

The following methods for making the albumen solution are submitted for consideration, hoping that other investigators may try them out comparatively:

(a) Gravimetric Method $A$. Place 2 gms. of pure powdered egg albumen in 100 c.c. of boiled distilled water, shake and set aside for six to twelve hours, shaking frequently. Filter through a tared filter paper which has been dried (at $100^{\circ} \mathrm{C}$.) to constant weight. Filtering is slow, requiring perhaps one-hour's time. When the last drop has filtered through, dry the filter paper with the unfiltered albumen residue upon it to constant weight and weigh. Deduct from this weight the 
weight of the albumin residue. From $1 \mathrm{gm}$. of egg albumen dried to constant weight determine the percentage of moisture. From the data thus obtained it is easy to determine the amount of boiled distilled water which must be added to the filtrate (100 c.c.) to make 1 per cent dried albumen solution.

We will suppose that the dried filter paper to be used in filtering the albumen solution weighs 1.570 gms. and this same paper with the undissolved albumen residue (also dried at $100^{\circ} \mathrm{C}$. to constant weight) weighs 1.965 gms., then the weight of the undissolved dried albumen residue equals $0.395 \mathrm{gm}$. We will suppose that $1 \mathrm{gm}$. of albumen loses $0.126 \mathrm{gm}$. on drying, or 12.6 per cent moisture. $0.395 \mathrm{gm}$. raised to its normal air moisture $(0.395 \mathrm{gm} .+12.6$ per cent of $0.395 \mathrm{gm} .=0.444 \mathrm{gm}$.) and subtracted from $2.00 \mathrm{gms}$. leaves $1.556 \mathrm{gms}$., the amount of albumen that passed through the filter paper. 12.6 per cent of $1.556 \mathrm{gms} .=0.196$ gm. and $1.446 \mathrm{gms}$. less $0.196 \mathrm{gm} .=1.360 \mathrm{gms}$. which represents the amount of albumen, dried to constant weight, that passed into solution. Therefore to make a 1 per cent solution it is necessary to add enough boiled distilled water to the filtrate to make $1 / 100$, in this case add water up to the 136 c.c. mark. We now have a 1 per cent solution sufficiently accurate for all practical purposes.

This albumen test solution is now ready for use but it must be kept in mind that it is readily attacked by microbes. However, if carefully prepared with pure albumen, boiled distilled water, in sterile vessels, and put on ice or in a cool place, it will keep for perhaps four days.

Any quantity of albumen solution may be made, it merely being advised not to prepare more than may be required for the tests contemplated.

(b) Gravimetric Method B. In a dried and tarred platinum dish place 5 c.c. of the albumen filtrate $(2$ gms. in 100 c.c. of boiled distilled water), evaporate over water bath and dry to constant weight, and from this determine the percentage of albumen in the solution and the amount of water that must be added to the albumen filtrate to make 1 per cent.

(c) Nitrogen Determination. By means of the Kjeldahl apparatus determine the percentage of nitrogen representing the albumen in solution.

Having prepared the egg albumen solution the next step is to make the phenol control solution, the primary stock solutions of the disinfectants to be tested for coagulating powers, and, from these, the secondary or sub-stock solutions, from which the final test dilutions are made as the experiment progresses. 
The standard of comparison is the opacity produced in 5 c.c. of the 1 per cent egg albumen solution when 5 c.c. of 5 per cent phenol solution is added (in a standard test tube of about 15 c.c. capacity). This phenol tube is placed against a black background. In making a test, varying dilutions of the disinfectant are added to the egg albumen solution until the opacity produced is the same as that in the phenol tube. In each test 5 c.c. of the dilution is added to 5 c.c. of the egg albumen in a standard test tube and the two tubes compared, placed against the black background. The following tentative procedure for making dilutions is suggested:

\section{Dilutions of Disinfectants to Be Used}

The phenol control solution ( 5 per cent) is made as for the AndersonMcClintic method of standardizing disinfectants, using only pure phenol crystals.

Of the disinfectants to be tested 10 per cent and 1 per cent primary stock solutions are made; 10 per cent solutions of liquid disinfectants as alcohol, formalin, and acids, and 1 per cent solutions of the salts of heavy metals and of soluble substances generally. From these primary stock solutions the following secondary dilutions or sub-stock solutions are made, always in those amounts which will serve the purpose, that is, in amounts for perhaps ten sub-dilutions for each and every disinfectant to be tested:

$$
\begin{aligned}
& 1 / 10 \text { (of liquids only) } \\
& 1 / 100 \\
& 1 / 1000 \\
& 1 / 10,000 \\
& 1 / 100,000 \text {. }
\end{aligned}
$$

\section{Method of Testing}

(a) Phenol Standard. Pour 5 c.c. of the egg albumen solution in a standard test tube, using a 5-c.c. pipette having a free outflow. Add to this 5 c.c. of the phenol stock solution ( 5 per cent). Set the tube in the standard test rack (with black background made of cardboard covered with black tissue paper). The degree of opacity developed is to serve as the standard of comparison.

(b) Preliminary Testing. The albumen coagulating power of the disinfectant being unknown, much time and labor can be saved by testing with the four or five sub-stock solutions, adding 5 c.c. to 5 c.c. of 
the egg albumen test solution, in order to find that dilution of the disinfectant which fails to show any opacity. We will suppose that the $1 / 1000$ sub-stock solution shows very marked opacity or precipitation, then the $1 / 10,000$ solution might be tried, which may also show quite marked opacity, then the $1 / 100,000$ may be tried. If this gives negative results then we know that the phenol standard lies between $1 / 10,000$ and $1 / 100,000$, with the probabilities that it is nearer $1 / 10,000$.

\section{TABLe XXI}

\section{PLAN FOR TEN DILUTIONS AT THIRTY-SECOND INTERVALS}

\begin{tabular}{|c|c|c|c|c|c|c|c|c|c|c|c|}
\hline \multirow{2}{*}{ Sube } & \multirow{2}{*}{ Dilution. } & \multicolumn{2}{|c|}{ INOCULATE } & \multicolumn{2}{|c|}{ 5-MrN Test } & \multicolumn{2}{|c|}{ 10-MIN Test } & \multicolumn{2}{|c|}{ 15-MIn Tast } & \multicolumn{2}{|c|}{ 20-MIN TIST } \\
\hline & & Min & $\mathrm{Sec}$ & Min & $\mathrm{Sec}$ & Min & Sec & Min & $\mathrm{Sec}$ & Min & $\mathrm{Sec}$ \\
\hline 1 & $1: 80$ & 00 & 00 & 5 & 00 & 10 & 00 & 15 & 00 & 20 & 00 \\
\hline 2 & $1: 90$ & 00 & 30 & 5 & 30 & 10 & 30 & 15 & 30 & 20 & 30 \\
\hline 3 & $1: 100$ & 1 & 00 & 6 & 00 & 11 & 00 & 16 & 00 & 21 & 00 \\
\hline 4 & $1: 110$ & 1 & 30 & 6 & 30 & 11 & 30 & 16 & 30 & 21 & 30 \\
\hline 5 & $1: 120$ & 2 & 00 & 7 & 00 & 12 & 00 & 17 & 00 & 22 & 00 \\
\hline 6 & $1: 360$ & 2 & 30 & 7 & 30 & 12 & 30 & 17 & 30 & 22 & 30 \\
\hline 7 & $1: 400$ & 3 & 00 & 8 & 00 & 13 & 00 & 18 & 00 & 23 & 00 \\
\hline 8 & $1: 440$ & 3 & 30 & 8 & 30 & 13 & 30 & 18 & 30 & 23 & 30 \\
\hline 9 & $1: 480$ & 4 & 00 & 9 & 00 & 14 & 00 & 19 & 00 & 24 & 00 \\
\hline 10 & $1: 520$ & 4 & 30 & 9 & 30 & 14 & 30 & 19 & 30 & 24 & 30 \\
\hline
\end{tabular}

(c) Concluding Testıng. Going back to the 1/10,000 dilution make ten sub-dilutions, increasing the dilutions by a difference of 1000 by simply adding the required parts of distilled water, using small quantities, thus:

10 parts of $1 / 10,000+1$ part water $=1 / 11,000$.

10 parts of $1 / 10,000+2$ parts water $=1 / 12,000$.

10 parts of $1 / 10,000+3$ parts water $=1 / 13,000$, etc.

Any other quantity proportions may be used, however, as 10, 15 20 , etc., parts of the sub-stock solution with the required parts of distilled water. If the $1 / 1000$ sub-stock solution is to be used then the dilutions should be increased by 100 as follows:

$$
\begin{aligned}
& 10+1=1 / 1100 \\
& 10+2=1 / 1200 \\
& 10+3=1 / 1300 \\
& 10+4=1 / 1400
\end{aligned}
$$


or any other equal proportion of stock solution and distilled water may be used, as $5+0.5,100+10$, or $1000+100$, etc.

If the highest stock dilution $(1 / 100,000)$ is to be used, then the increase should be by 10,000 , thus:

$$
\begin{aligned}
& 10+1=1 / 110,000 \\
& 10+2=1 / 120,000 \\
& 10+3=1 / 130,000, \text { etc. }
\end{aligned}
$$

\section{Derermination of the Phenol Coefficient}

Having determined that dilution which gives the same coagulation opacity as the 5 per cent carbolic acid, it is a very simple matter to determine the phenol albumen cọagulating coefficient by simply dividing the strength of the dilution of the disinfectant tested by the phenol dilution $(1 / 20)$.

\begin{tabular}{|c|c|c|}
\hline 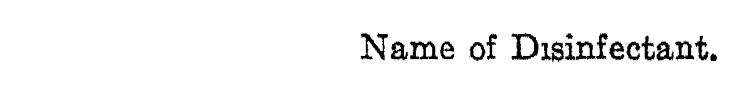 & $\begin{array}{l}\text { Reaction } \\
\text { Limit }\end{array}$ & $\begin{array}{c}\text { Phenol } \\
\text { Coefficient }\end{array}$ \\
\hline Phenol & $1 / 20$ & 1.00 \\
\hline Copper sulphate. & $1 / 15,000$ & 750.00 \\
\hline Mercuric chloride.. & $1 / 10,000$ & 500.00 \\
\hline Silver nitrate & $1 / 9,500$ & 475.00 \\
\hline Alcohol & $1 / 3$ & 0.15 \\
\hline
\end{tabular}

We conclude by giving the coagulating coefficients of a few disinfectants:

The Hale Toxicity Coefficient of Disinfectants. Hale has attempted in this procedure to establish the toxicity of different substances and to give them a definite numerical relation to other toxic agents. $\mathrm{He}$ further proposed that this toxicity coefficient be established for groups of substances having, in general, a similar pharmacological action. The following procedure was proposed for determining the toxicity coefficient of phenol-like compounds:

The animal upon which the substance in question is to be tested shall be the white mouse of not less than 15 nor more than 30 gms. weight. The dose is to be calculated per gram of body weight and should, when diluted, equal between 0.03 and 0.04 c.c. per gram weight; that is, 0.06 to 0.08 c.c. for a 20 -gm. mouse. The diluent is to be dis- 
tilled water and primary dilutions are to be made of such strength that the dose is easily measured with a 1-c.c. pipette graduated in hundredths. This is most easily accomplished by the use of the substance in greater concentration than that required to kill in the above volume dose. After the required dose of the diluted disinfectant has been estimated it is measured into a suitable dish and is then diluted further to the required volume by adding water in sufficient quantity. A series of mice are then to be injected with varying amounts of the substance until the least fatal dose is determined, the mice being kept under observation for a period of twenty-four hours unless death results in a shorter time.

Mice of the same lot are similarly injected with pure phenol properly diluted to make the measurement of the dose easy and then further diluted in a small dish to equal a volume dose of 0.03 to 0.04 c.c. per gram of body weight and the least fatal dose determined as above. The dose thus obtained is considered unity and the least fatal dose of the substance in question is estimated in per cent of this, as is illustrated in the table following:

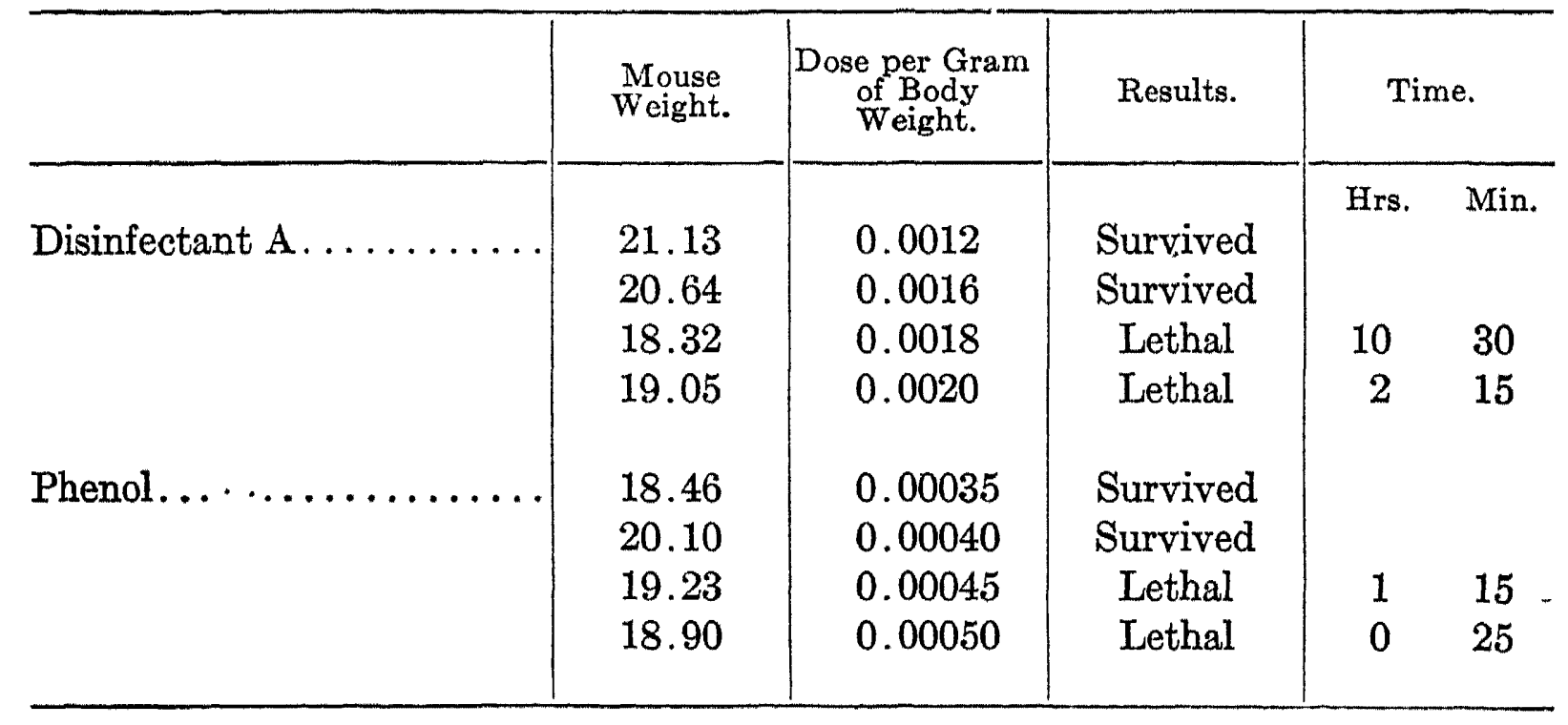

The least fatal dose of disinfectant A was estimated to be 0.0018 ; that of phenol 0.00045. The phenol toxicity coefficient of disinfectant A therefore is, according to the proportion $4.5: 18:: \times: 100,25$ per cent.

The following are some toxicity coefficients secured by Hale in the application of his procedure to some commercial disinfectants. For the sake of comparison, the "phenol coefficients" as determined by McClintic have been included: 


\begin{tabular}{|c|c|c|c|}
\hline & \multirow{2}{*}{$\begin{array}{l}\text { Toxicity } \\
\text { Coefficient. }\end{array}$} & \multicolumn{2}{|c|}{ Phenol Coefficient. } \\
\hline & & $\begin{array}{l}\text { Without Or- } \\
\text { ganic Matter. }\end{array}$ & $\begin{array}{l}\text { With Or- } \\
\text { ganic Matter. }\end{array}$ \\
\hline Bacteriol................ & 45 & 1.58 & 1.34 \\
\hline Benetal. . . . . . . . . . . . . & 33 & 1.23 & 0.92 \\
\hline Creola $\ldots \ldots \ldots \ldots \ldots \ldots \ldots \ldots$ & 12.8 & & \\
\hline Creolin-Pearson . . . . . . . . . . . . & 180 & 3.25 & 2.52 \\
\hline Cresoleum................. & 11 & 2.90 & 1.75 \\
\hline 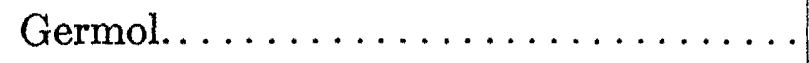 & 16 & 2.12 & 179 \\
\hline Hycol. . . . . . . . . & 32 & 12.30 & 9.37 \\
\hline Kreotas........... & 5.6 & 110 & 0.30 \\
\hline Kreso..................... & 22.5 & 3.92 & 2.32 \\
\hline Lysol . . . . . . . . . . . & 45 & 2.12 & 1.57 \\
\hline Phenol sodique . . . . . . . . & 4.5 & & \\
\hline Trikresol................. & 90 & 2.62 & 2.50 \\
\hline
\end{tabular}

\section{BIBLIOGRAPHY}

Anderson, J. F. and McClintic, T. B. 1912. I. Method of Standardizing Disinfectants with or without Organic Matter. Bull. 82, Hygienic Laboratory U. S. Pub. Health Service, Wash.

Bazzoni, C. B. 1914. The Destruction of Bacteria through Light. Amer. Jour. Pub. Health, 4, 975-992.

Beyer, A. 1912. Alcohol as a Disinfectant. Zeit. Hyg., 70, 225.

Biassioti, A. 1910. The Action of Colloidal Metals on Pathogenic Bacteria. Cent. Bakt. Abt. I., Ref. 45, 680.

Brtter, L. 1912. The Destruction of Bacteria by the Heavy Metals and Building Materials. Zeit. Hyg., 69, 483-512.

Brownivg, C. H. and Russ, S. 1917. Germicidal Action of the Ultra-violet Radiation and its Correlation with Selective Absorption. Proc. Roy. Soc., London, (B) 90, 33-38.

Burge, W. E. 1917. The Action of Ultra-tiolet Light in Killing Living Cells such as Bacteria. American Journal Physiology, 43, 429-432.

Burge, W. E. and NeIld, A. 1915. The Comparative Rate at which Fluorescent and Non-fluorescent Bacteria are Killed on Exposure to Ultra-violet Light. Amer. Jour. Physiology, 38, 399-403.

CHrck, H. 1908. An Investigation of the Laws of Disinfection. Jour. Hygiene, 8, 92.

Chick, H. 1910. The Process of Disinfection by Chemical Agencies and Hot Water. Journal of Hygiene, 10, 237.

Curck, H. 1912. The Factors Influencing the Velocity of Disinfection. Eighth Intern. Cong. Appl. Chem., 26, 167-197. 
Chick, H. and Martin, C. F. 1908. The Principles Involved in the Standardization of Disinfectants and the Influence of Organic Matter on the Germicidal Value. Journal Hygiene, 8, 654 .

Chick, H. and Martin, C. J. 1910. On the Heat Coagulation of Proteins. Journal of Physiology, 40, 404.

Chick, $\mathrm{H}$ and Martin, C. F. 1908. A Comparison of the Power of a Germicide Emulsified or Dissolved with an Interpretation of their Superiority of the Emulsified Form. Journal of Hygiene, 8, 698.

Churchman, J. W. 1912. The Selective Bactericidal Action of Gentian Vılet. Journal Expermental Medicine, 16, 221-247.

Cooper, E. A. 1912. Bactericidal Action of Cresols and Allied Substances and the Best Means of Employing Them. Brit. Med. Journal, 1912, I. 1234-1240.

Cooper, E. A. 1912. Relations of Phenols and their Derivatives to Proteins. A Contribution to Our Knowledge of the Mechanism of Disinfection. Biochem Jour., 6, 362-387; 7 (1913), 175-96.

Dakin, H. D., Cohen, J. B., Daufresne, M., and KenYon, J. 1916. Proceedings Royal Society, London, Series B, 89, 232-251.

Dakin, H. D., Cohen, J. B., and Kenyon, J. 1916. Chloramin, Its Preparation, Properties, and Use. Brit. Med. Journal No. 2874, 160-162.

Dakin, H. D., Lee, W. E., Sweet, J. E., and Hendrix, B. M. 1917. A Report of the Use of Dichloramin-T (Toluene-parasulphondichloramin) in the Treatment of Infected Wounds. Jour Amer. Med. Assn., 69, 27-31.

DeWitt, L. M. and Sherman, H. 1916. The Bactericidal and Fungicidal Action of Copper. Journal Infectious Diseases, 18, 368-382.

Dieudonne, A. 1894. Beitrage zur Beurtheilung der Einwirkung des Lichtes auf Bakterien. Art. a. d. kais. Gesundheitsamte, 9, 405.

DuYser, C. A. and Lewis, W. K. 1914. A New Method for Determining the Value of Disinfectants. Chem. Eng., 19, 113; Jour. Ind. Eng. Chem., 6, 198-200.

Eiskman, C. 1908. Die Ueberlebungskurve bei Abtodtung von Bakterien durch Hitze. Biochem. Jour., 11, 12.

Embrey, G. 1912. The Use of Copper Sulfate in Purifying Water Supplies. Canadian Engineer, 23, 245-6.

Eugling, M. 1912. The Disinfective Action of Iodoform and Novoidin. Cent. Bakt. Abt., I, 60, 397-416.

Findlay, L. and Martin, W. B. M. 1915. The Effect of Daylight and Drying on the Human and Bovine Type of Tubercle Bacilli. Brit. Med. J., 110-111.

Frex, E. 1912. Why is Seventy Per Cent Alcohol so Strongly Bactericidal. Deut. Med. Wochenschr., 38, 1633-5. Chem. Absts., 6: 1912, 3843-4.

Goodrich, H. P. 1917. Glycerol and Antiseptics. British Med. Jour., 1917, $647-648$.

Gosst and Herzog. Theory of Disinfection. II. Disinfectants that Dissolve Lipooid. Zeit. Physiol. Chem., 88 (1), 103-8. 
Hamilton, H. C. 1917. Facts and Fallacies in Disinfection. Am. J. Pub. Health, 7, 282-95.

Hamiton, H. C. and OHno, T. 1913. Standardization of Disinfectants. Am. J. of Pub. Health, 3, 583.

Hamilton, H. C. and OHNo, T. 1914. Bacteriological Standardization of Disinfectants. Amer. Jour. Pub. Health, 4, 486.

Harris, F. J. and Hoyt, H. S. Possible Origin of the Toxicity of Ultra Violet Light. Science, 46, 318-20.

Herzog and Betzed. 1911. Zur Theorie der Desinfection. Zeit. Physiol. Chemie, 67 (1910), 303, 74, 221.

Hewlets. 1909. Milroy Lectures on Disinfection and Disinfectants. Lancet, March 13, 20-27.

Hewlets and Hall. 1911. The Influence of Culture Media on the Germination of Anthrax Spores with Special Reference to Disinfection.Experiments. Jour. Hyg., 11, 473.

Hooker, A. H. 1913. Chloride of Lime in Sanitation. John Wiley \& Sons, New York.

Houghton, E., Madd, Davis L. 1913. A Study of the Germicidal Action of the Ultra Violet Rays. Amer. Jour. Pub. Health, 4, 224-240.

Kendall, A. I. and Edwards, M. R. 1911. A Method for Determining the Germicidal Value and Penetrating Power of Liquid Disinfectants. Jour. Infectious Diseases, 8, 250-57.

Kempster, C. 1917. The Effect of X-Rays upon Diseases of Bacterial Origin. Lancet, 1917, II, 423-424.

Kingzett, C. T. and Woodcock, R. C. The Bacterial Testing of Disinfectants: A Practical Criticism. Analyst, 38, 190-200.

KingzeTt, C. T. and Woodcock, R. C. 1910. Bacteriological Testing of Certain Disinfectants and the Results as Effected by Varying Conditions. Pharm. Jour., 85, 157-8.

KLIgLeR, I. J. 1918. A Study of the Antiseptic Properties of Certain Organic Compounds. Journal of Experimental Medicine, 27, 463-478.

Kocrs. 1901. The Physiological Action of Formaldehyde. Amer. Jour. Physiology, 6, 325-329.

KRöNIG and PAUL. 1897. Die chemischen Grundlagen der Lehre von der Giftwirkung und Disinfection. Zeit. f. Hyg., 25, 1.

LAGER, H. 1916. Disinfection of Water with Calcium Hypochlorite. Zeit. Hyg., 81, 296-310. Chem. Abts., 12 (1918), 394.

Lusini. 1912. Disinfecting Action of Cations According to the Law of the Periodic System. R. Acad. fisicrit. March 20, 1910. Chem. Abts., 6, 1912. 240.

Mahon, T. and White, J. S. 1915. Germicidal Value of Iodine. J. Soc. Chem. Ind, 34 (1915), 244. Chem. and Druggists, 1915, 144.

McCunstic, T. B. 1905. Chloride of Zinc as a Deodorant. Antiseptic and Germicide. Bull. No. 22, Hyg. Lab. U. S. Pub. Health Service, Washington. 
McLaughuin, A. J. 1903. Inefficiency of Ferrous Sulfate as an Antiseptic and Germicide. Bull. 15, Hyg. Lab. U. S. Pub. Health Service, Wash.

Moore, G. T. and Kellerman, K. F. 1905. Copper as a Disinfectant and Algicide in Water Supplies. U. S. Dept. Agr. Bureau of Plant Industry Bull., 76.

Madsen and Nyman. 1907. Zur Theorie der Disinfection. Zeit. Hyg., 57, 388.

Newcomer, H. S. 1917. Bactericidal Fluorescence Excited by X-Rays. Jour. Exper. Med., 26, 657-668.

Newcomer, H. S. 1917. The Abiotic Action of Ultra Violet Light. Jour. Exper. Med., 26, 841-848.

Nissen, F. 1890. Ueber die Desinficirende Eigenschaft des Chlorkalks. Zeit. Hyg., 8, 62-77.

Nonton, J. F. The Coefficients of Germicides. Jour. Ind. Eng. Chem., 8, 197.

Norton, J. F. and Hsu, P. H. 1916. Physical Chemistry of Disinfection. Jour. Inf. Diseases, 18, 180-195.

OChsner, E. H. 1916. The Biochemistry of Topical Applications with Special Reference to the Use of Boric Acid in Septic Infections. Chemical Abstracts, 12 (1918), 191.

Oxer-Blom, M. 1913. The Nature of the Action of Ultra Violet Rays on Bacteria. Zeit. Hyg., 74, 242-247.

Paul Birstein and Reuss. 1910. Beiträge zur Kinetik der Giftwirkung von Geloeteten Stoffen. I. Einfluss der Konzentration. II. Einfluss der Neutralsalze. Einfluss und der Temperature. Biochem. Zeit., 29, 202 and 249.

Paul Brrstein and Reuss. 1910. Beitrag zur Kinetik des Absterben trokener Bakterien in Sauerstoff verschiedener Konzentration und verschiedenen Temperaturen. Biochem. Zeit., 25, 367.

Pheups, E. B. 1911. On the Application of Certain Laws of Physical Chemistry in the Standardization of Disinfectants. Jour. Inf. Dis., 8, 27.

Phelps, E. B. 1913. The Limitations of the Phenol Coefficient in the Standardization of Disinfectants. Amer. Jour. Pub. Health, 3, 53-57.

Reichel, H. 1909. Zur Theorie der Disinfection. Biochem Zeit., 22, 149; 177 and 201.

- Renob, L. (et $a l$ ). 1914. Antiseptic Role of Insoluble Substances. Comp. Rend. Soc. Biol., 76, 64-66.

Reudiger, E. H. 1914. Germicidal Power of Glycerol on Various Microorganisms under Various Conditions. Philippine Journal of Science., 9, B, 564-477.

RIdeal, S. 1912. Suggested International Tests for Disinfectants. Eighth Intern. Cong. Applied Chemistry, 26, 261.

RIDEAL, S. and WALKER, A. 1903. The Standardization of Disinfectants. Jour. Roy. San. Institute, 24, 424.

Rrdeal, (et al). 1915. A Note on Testing Disinfectants. Amer. Jour. Pub. Health, 5, 1175-1176. 
Robertson, T. B. 1914. Disinfection. Jour. Hyg., 14, 143-148.

Robertson, T. B. 1914. On the Conditions under which Disenntinuous Events may be Employed as a Measure of Continuous Processes with Special Reference to the Killing of Bacteria by Disinfectants. Journal of Hygiene, 14, 143-148.

Rosenau, M. J. The Antiseptic and Germicidal Properties of Glycerine. Bull., 16, Hyg. Lab. U. S. Pub. Health Service, Washington.

Rubner, M. 1913. Modern Steam Sterilization. Jour. Am. Med. Assoc., $60,1344$.

Schmid, E. W. 1910. Bactericidal Value of Thymol. Zeit. Physiol. Chem., $67,412-432$.

SchneIDeR, F. 1912. Albumin Coagulation Coefficient for Disinfectants. Pacific Pharmacist, 5, 335-339.

Simpson, W. J. and Hewlett, R. T.. 1914. Germicidal Action of Colloidal Silver. Lancet, Dec. 12, 1914, 1359. Chemical Absts., 8 (1915), 640, 641.

Sommerville, D. 1913. Antiseptics and Germicides. Jour. Roy. Soc. Arts, 61, 927-937; 945-952.

StemnhaUer, A. J. 1916. The Disinfecting Power of o-, m-, and para-Cresol and Basic Mercuric Cyanide. Pharm. Weekbladt, 53, 680-685.

Straus. 1917. Disinfecting Properties of Alcohol. Schweiz. apoth. Ztg., 55, 36.

Supfle, K. and Deugeler, A. 1916. Culture Media for Use after Disinfection Experiments. Arch. Hyg., 85, 189-197.

Von Recklinghausen, M. 1914. Purification of Water by the Ultra Violet Rays. Jour. Amer. Water Works Assn., 1, 565-588.

WALKER, A. 1916. The Standardization of Disinfectants. New York Med. Journal, 1916, 500-505.

Walters, A. L. 1917. The Specificity of Disinfectants and its Bearing on their Standardization. Amer. Jour. Pub. Health, 7, 1030-1036.

WARD, H. M. 1895. The Action of Light on Bacteria. Phil. Trans. Roy. Soc. $1895,961$.

Watson, H. E. 1908. A Note on the Variation of the Rate of Disinfection with Change in Concentration of the Disinfectant. Jour. Hygiene, 8, 536.

WeInzirL, J. 1914. The Germicidal Action of Sunlight upon Bacterial Spores, Amer. Jour. Pub. Health, 4, 969-974.

WiNkLER, L. W. 1888. Die Bestimmung des im Wasser gelösten Sauerstoffs. Berichte, 21, 2, 2843-2854.

Wrighi, J. H. 1917. The Importance of Uniform Culture Media on the Bacteriological Examination of Disinfectants. Jour. Bact., 2, 315-346. 


\section{CHAPTER VI \\ PROTEINS AND CARBOHYDRATES}

Classification of Proteins. Committees for the American Society of Biological Chemists and the American Physiological Society have made the following recommendations concerning the proteins:

Frrst. The word proterd should be abandoned.

SECOND. The word protein should designate that group of substances which consists, so far as at present is known, essentially of combinations of the $a$-amino acids and their derivatives, c.g., $a$-amino acetic acid or glycocoll; $a$-amino propionic acid or alanin; phenyl-a-amino propionic acid or phenylalanin; guanidin-amino valerianic acid or arginin, etc., and are, therefore, essentially polypeptids.

THIRD. That the following terms be used to designate the various groups of proteins:

I. Simple Proteins. Protein substances which yield only $a$-amino acids or their derivatives on hydrolysis.

Although no means are at present available whereby the chemical individuality of any protein can be established, a number of simple proteins have been isolated from animal and vegetable tissues which have been so well characterized by constance of ultimate composition and uniformity of physical properties that they may be treated as chemical individuals until further knowledge makes it possible to characterize them more definitely.

The various groups of simple proteins may be designated as follows:

(a) Albumins. Simple proteins soluble in pure water and coagulable by heat.

(b) Globulins. Simple proteins insoluble in pure water but soluble in neutral solutions of salts of strong bases with strong acids.

(c) Glutelins. Simple proteins insoluble in all neutral solvents but readily soluble in very dilute acids and alkalies.

(d) Alcohol Soluble Proteins. Simple proteins soluble in relatively strong alcohol (70 to 80 per cent) but insoluble in water, absolute alcohol, and other neutral solvents.

(e) Albuminoids. Simple proteins which possess essentially the 
same chemical structure as the other proteins, but are characterized by a great insolubility in all neutral solvents.

$(f)$ Histones. Soluble in water and insoluble in very dilute ammonia and, in the absence of ammonium salts, insoluble even in an excess of ammonia; yield precipitates with solutions of other proteins and a coagulum on heating, which is easily soluble in very dilute acids. On hydrolysis they yield a large number of amino acids, among which the basic ones predominate.

(g) Protamins. Simpler polypeptids than the proteins including the preceding groups. They are soluble in water uncoagulable by heat, have the property of precipitating aqueous solutions of other proteins, possess strong basic properties and form stable salts with strong mineral acids, among which the basic amino acids predominate.

II Conjugated Proteins. Substances which contain the protein molecule with nucleic acid.

(a) Nucleoproterns. Compounds of one or more protein molecules with nucleic acid.

(b) Glycoproteins. Compounds of the protein molecule with a substance or substances containing a carbohydrate group other than a nucleic acid.

(c) Phosphoproteins. Compounds of the protein molecule with some, as yet undefined, phosphorus containing substance other than a nucleic acid or lecithin.

(d) Hemoglobins. Compounds of the protein molecule with hematin or some similar substance.

(e) Lecitho-proteins. Compounds of the protein molecule with lecithins (lecithans, phosphatids).

III. Derived Proteins.

1. Primary Protein Derivatives. Derivatives of the protein molecule apparently formed through hydrolytic changes which involve only slight alterations of the protein molecule.

(a) Proteins. Insoluble products which apparently result from the incipient action of water, very dilute acids or enzymes.

(b) Metaproteins. Products of the further action of acids and alkalies whereby the molecule is so far altered as to form products soluble in very weak acids and alkalies but insoluble in neutral fluids.

This group will include the familiar " acid proteins" and "alkali proteins" not the salts of proteins with acids.

(c) Coagulated Proteins. Insoluble products which result from (1) the action of heat on their solutions, (2) the action of alcohols on the protein. 
2. Secondary Protein Derivatives. Products of the further hydrolytic cleavage of the protein molecule.

(a) Proteoses. Soluble in water, uncoagulable by heat and precipitated by saturating their solutions with ammonium sulphate or zinc sulphate.

(b) Peptones. Soluble in water, uncoagulable by heat, but not precipitated by saturating their solutions with ammonium sulphate.

(c) Peptids. Definitely characterized combinations of two or more amino acids, the carboxyl group of one being united with the amino group of the other, with the elimination of a molecule of water.

Structure of Proteins. Proteins are combinations of amino acids the unions being between the carboxyl group of one acid and the amino group of the other. The following will serve to illustrate the linkage.<smiles>[2H]C(=O)O</smiles>

The free amino and carboxyl groups on the glycyl-alanin molecule are free to react in the same way. This yields infinite possibilities with regard to the size of the protein molecule. Fischer has done much toward synthesizing proteins from these smaller units the amino acids.

The amino acids which have been isolated from proteins may be classified as follows:

\section{Monoamino Monobasic Acids.}

Glycocoll, $\alpha$-amino acetic acid.<smiles>NCC(=O)O</smiles>

Alanin, $\alpha$-amino propionic acid.<smiles>CC(N)C(=O)O</smiles> 
Phenylalanin, $\alpha$-amino, $\beta$-phenyl propionic acid.<smiles>NC(C(=O)O)C1CCCCC1</smiles>

Serine, $\alpha$-amino, $\beta$-hydroxy propionic acid.<smiles>NC(CO)C(=O)O</smiles>

Tyrosine, $\alpha$-amino, $\beta$-para hydroxy phenyl propionic acid.<smiles>NC(C(=O)O)C1CCCOC1</smiles>

Cystine, $\alpha$-diamino, $\beta$-dithiolactylic acid.<smiles>NC(CSS)C(N)C(=O)O</smiles>

Leucine, isobutyl $\alpha$-amino acetic acid.<smiles>CC(C)CC(N)C(=O)O</smiles>

Isoleucine ethyl methyl, $\alpha$-amino propionic acid.<smiles>CCC(N)C(N)C(=O)O</smiles>

Valine, $\alpha$-amino, isovalerianic acid.<smiles>CC(C)C(N)C(=O)O</smiles> 
II. Dicarboxylic acids.

Aspartic acids, $\alpha$-amino succinic acid.<smiles>NCC(C(=O)O)C(=O)O</smiles>

Glutamic acid, $\alpha$-amino, $n$-glutaric acid.<smiles>N[C@@H](CC(=O)O)C(=O)O</smiles>

III. Diamino acids.

Lysine, $\alpha$ - $\epsilon$-diamino caproic acids.<smiles>CCCCC(N)C(=O)O</smiles>

Arginine, $d$-guanidine, amino valerianic acid.<smiles>CC(CCC(=O)O)C(=N)N</smiles>

IV. Heterocyclic amino acids.

Tryptophane, $\beta$-indole, $\alpha$-amino propionic acid.<smiles>NC(C(=O)O)C1CC2CCCC2N1</smiles> 
Histidine, $\alpha$-amino imidazol propionic acid.<smiles>NC(Cc1c[nH]cn1)C(=O)O</smiles>

Proline, $\alpha$-pyrrolidine carboxylic acid.<smiles>O=C(O)C1CCCN1</smiles>

That amino acids are utilized by bacteria has been pointed out in other places. The commercial peptones which are used in the preparation of media are, among other things, mixtures of amino acids. The changes induced by bacteria on certain amino acids in the intestinal tract are discussed in that chapter. Raistrick (1917) has shown that the bacteria of the coli-typhi group are able to change histidine into urocanic acid (iminazolylacrylic acid). Most of the other amino acids may be changed by bacterial enzymes to smaller molecules. Bacterial changes induced in other amino acids have been discussed elsewhere in this book.

Qualitative Analysis for the Elements in Organic Compounds. The inorganic elements may be detected by heating some of the sample on a piece of platinum foil or porcelain. During this ignition the organic clements are driven off. The organic elements are determined by ignition with sodium. It often happens that an element is present in both organic and inorganic forms.

Inorganic Elements. The substance is ignited on a piece of platinum foil or porcelain until all of the organic portion is destroyed. If the sample chars during this procedure, the presence of an organic substance is shown. If an inorganic substance is shown in the charring it may further be determined by the procedures of qualitative analysis.

Organic Elements. Place a small piece of sodium in an ignition tube about 4 ins. long. Incline the tube in a slanting position and 
heat the closed end very gently at first and later heat the bottom to a dull red. As soon as the sodium has begun to volatilize and condense on the sides of the tube, add a few drops of the sample or a few pieces of a solid, being careful to keep off the sides of the tube. As soon as the tube has cooled any excess of sodium may be removed by adding a little alcohol or if the sodium excess is not large the tube may be plunged into warm water. This solution must be filtered free of free carbon. The filtrate may then be tested for the halogens, nitrogen and sulphur. Hydrogen and oxygen are assumed to be present.

Test for Organic Sulphur. Treat about 1 c.c. of the solution secured from the sodium ignition with lead acetate solution. If sulphur is present a black precipitate will be formed according to the following equation. The solution must be strongly alkaline.

$$
\mathrm{Na} 2 \mathrm{~S}+\mathrm{Pb}\left(\mathrm{C}_{2} \mathrm{H}_{3} \mathrm{O}_{2}\right)_{2}=\mathrm{PbS}+2 \mathrm{NaC}_{2} \mathrm{H}_{3} \mathrm{O}_{2} \text {. }
$$

Sodium nitro-prusside may also be used to test for organic sulphur. Add a few drops to about 1 c.c. of the filtrate secured from the sodium ignition and if a violet color results, the presence of sulphur is indicated.

Test for Organic Nitrogen. Add to about 3 c.c. of the filtrate four drops of $\mathrm{NaOH}$ solution, four drops of $\mathrm{FeSO}_{4}$ solution and one drop of $\mathrm{FeCl}_{3}$. Add a little concentrated $\mathrm{HCl}$ to dissolve the precipitated ferrous and ferric hydroxides and boil. If $\mathrm{N}$ is present in the sample a precipitate of Prussian blue will come down. It may be necessary to heat the tube gently and leave it for a time. The following reactions take place:

$$
\begin{aligned}
6 \mathrm{NaCN}+\mathrm{FeSO}_{4} & =\mathrm{Na}_{4} \mathrm{Fe}(\mathrm{CN})_{6}+\mathrm{Na}_{2} \mathrm{SO}_{4}, \\
\mathrm{FeCl}_{3}+\mathrm{NaOH} & =\mathrm{Fe}(\mathrm{OH})_{3}+3 \mathrm{NaCl} \\
4 \mathrm{Fe}(\mathrm{OH})_{3}+3 \mathrm{Na}_{4} \mathrm{Fe}(\mathrm{CN})_{6}+12 \mathrm{HCl} & =\mathrm{Fe}_{4}\left(\mathrm{Fe}(\mathrm{CN})_{6}\right)_{3}+12 \mathrm{H}_{2} \mathrm{O}+12 \mathrm{NaCl} .
\end{aligned}
$$

Test for Sulphur and Nitrogen when Present Together. Acidify about 1 c.c. of the solution with $\mathrm{HCl}$ and add three drops of ferric chloride solution. A dark red indicates the presence of sulphur and nitrogen.

$$
3 \mathrm{NaSCN}+\mathrm{FeCl}_{3}=\mathrm{Fe}(\mathrm{SCN})_{3}+3 \mathrm{NaCl} \text {. }
$$

To be certain of results the above tests for sulphur and nitrogen should be made along with this test.

Test for Organic Halogens: Group Test. This is carried out by removing $\mathrm{H}_{2} \mathrm{~S}$, and cyanide. To do this the solution is acidified with dilute nitric acid and boiling. The addition of silver nitrate at this 
point will precipitate the halids of silver which have the characteristic appearance.

Tests for Bromine and Iodine. Acidify a few cubic centimeters of the sample and boil to remove $\mathrm{H}_{2} \mathrm{~S}$ and the cyanids. Add a few drops of carbon bisulphide and a little chlorine water. If iodine is present, it is taken up by the heavier bisulphide which settle to the bottom of the tube with a distinct violet color.

$$
\begin{aligned}
\mathrm{NaI}+\mathrm{H}_{3} \mathrm{SO}_{4} & =\mathrm{HI}+\mathrm{NaHSO}_{4} . \\
2 \mathrm{HI}+\mathrm{Cl}_{3} & =\mathrm{H}_{4} \mathrm{O}+\mathrm{I}_{2} .
\end{aligned}
$$

Qualitative Tests for Elements in Proteins. Carbon. Heat the protein on a piece of platinum foil and if charring takes place the presence of carbon is indicated.

Hydrogen. Test for water in the top of the test tube.

Nitrogen. Heat with soda lime and test for ammonia by smell and moist litmus paper.

Sulphur. Heat with concentrated potassium hydroxide which will set free potassium sulphide. Cool and add lead acetate solution, which will precipitate black lead sulphide. After adding the lead acetate solution acidify, boil and suspend lead acetate paper above the liquid.

Detection of Proteins. (1) The coagulation of proteins by heat especially in slightly acid solutions may be used in their detection. This precipitate does not disappear on continued addition of acid. (2) Alcohol in excess will form a precipitate which is soluble in water at first; if the precipitate is left in contact with the alcohol, it is changed. The proteins come down unchanged but are probably dehydrated when left in contact with the alcohol. The fixing of tissue in histological work rests upon this fact. (3) Heller's Ring Test rests upon the fact that strong mineral acids will coagulate proteins. A small amount of concentrated nitric acid is put into a test tube and some of the test solution is floated upon it. If proteins are present a whitish layer will be formed at the point of contact between the two liquids.

Action of Bacteria on Proteins. The hydrolysis of proteins by bacteria has been studied by different investigators with the production of data which is not in agreement. Bainbridge (1911) found that bacteria could not decompose " purified native proteins." This was later verified by Sperry and Rettger (1915) who worked with purified serum albumin, egg albumin and edestin and the common proteolytic bacteria. They reported that when all other forms of nitrogen were removed, 
bacteria could not start the decomposition of the proteins. Rettger, Berman and Sturges (1916) have continued this work and reported that proteoses and peptones follow essentially the same law of resistance to bacterial action as do the native proteins. They found that if there was sufficient nitrogen in other combinations to allow growth of the bacteria in order that proteoses could be secreted, the proteins were hydrolyzed. When no growth took place due to the lack of avallable nitrogen, no hydrolysis occurred. The bacteria used in the above experiment might be regarded as "starved" and thus not in a normal condition. Robinson and Tartar (1917) when studying this question made no attempts to use purified proteins: blood fibrin, egg albumin, casein, gliadin and peptone were split by bacteria to ammonia. The chief source of ammonia seemed to be the monoamino and diamino nitrogen. The work of these investigators simulates more closely the conditions in our common media and in nature.

Action of Bacteria on Polypeptids. Sasaki (1912) reported the $B$. coli split glycyl-l-tyrosin and glycylglycine into their component acids and from this they conclude that bacteria probably plays a rôle in digestion. The same author in two other papers reports investigations wherein liquefying and non-liquefying bacteria were used. With nonliquefying types such as typhi, dysentery, etc., glycyl-l-tyrosine and glycylglycine were decomposed to their component acids. Sasaki regards this change as due to an ereptase-like enzyme, excluding peptase because synthetic polypeptids were used. The various liquefying bacteria which he used were also able to split the polypeptids. In 1914 Sasaki killed B. coli with toluene and, after action on polypeptids, demonstrated that they were split into their amino acids. Somewhat similar results have been reported by Mito (1918). He tested the enzymes of $B$. coli communis and Staph. aureus on di-leucylyblycine. Asymetric cleavages were secured. The copper salt of $l$-leucine was isolated. The mother liquor exhibited an optical activity compatible with $d$-leucylglycine. Sasaki (1917) grew $B$. coli communis and $B$. proteus vulgaris in tyrosine containing media of two kinds. One of these media contained lactose to allow acid formation while the other contained a mixture of phosphates to maintain neutrality. In the lactose media both bacteria formed $p$-hydroxyphenyl-ethylamin. In the lactose free media no amine could be isolated but rather large amounts of $d$-p-hydroxyphenyllactic acid were secured.

Bacterial Action on Other Bodies Related to Proteins. There exist a class of compounds which stand between the carbohydrates and proteins-glucosamin, 
<smiles>NCC(O)C(O)C(O)CO</smiles>

This compound possesses an amino group in place of a hydroxyl group and this amino group, to a certain extent, connects it with the proteins although it retains many of its carbohydrate properties. In light of the controversy concerning the action of bacteria on proteins their action on this compound is interesting.

Meyer, in attempting to explain the action of bacteria on $d$-glucosamin, studied the action on acetyl derivatives. ' $\mathrm{He}$ believes that the $\mathrm{NH}_{2}$ group is probably split off after which a further degradation takes place.

Color Reactions of Proteins. By means of these much information may be secured concerning the amino-acids in a protein.

Biuret Reaction. The protein solution is warmed and a little strong sodium hydroxide and dilute copper sulphate is added. Care should be exercised to use dilute copper sulphate two or three drops in a test tube of water. A violet color is obtained. The color is due to the formation of biuret,<smiles>[NH2+]C(=O)N[NH2+]C=O</smiles>

The test is given by those substances which have two $\mathrm{CONH}_{2}$ groups.

Xantho-proteic Reaction. The protein is treated with concentrated nitric acid. The solution or protein will turn yellow. The presence of a $\mathrm{C}_{6} \mathrm{H}_{5}$ group is necessary. This reacts with the $\mathrm{HNO}_{3}$ to yield nitro derivatives of benzine. Nitro-benzine is formed.

Millon's Reaction. The protein or protein solution is heated with Millon's reagent. The protein is turned red or, if a solution is, used a reddish precipitate is formed. The reaction is given by those proteins which possess a hydroxy-phenyl group. Since tyrosine is the only amino acid having this group which has been isolated from proteins, the presence of this amino acid is indicated by a positive test with Millon's reagent. Care must be exercised in the use of Millon's reagent since it may be decomposed by some of the inorganic salts which precipitate mercury. 
Liebermann's Reaction. Boil the protein solution with about 4 c.c. of concentrated hydrochloric acid. A violet-lavender color will result if tryptophane is present.

Bromine Reaction for Tryptophane. Uncombined tryptophane when treated with bromine water will give a violet color.

Test for Tyrosine. Add a few drops of formol solution to concentrated sulphuric acid. On warming with tyrosine, a brown red color is obtained, which, on addition of acetic acid becomes green. Neither proteins nor peptones give the reaction. (Nasse's modification of Denigé's test. Quoted from Smith, 1905.)

Schmidt Test for Tyrosine. Dissolve by boiling in water and add a solution of mercuric nitrate. The red reaction is sharper if a little fuming nitric acid diluted in water is added. Try also the violet reaction with neutral iron chloride. (Quoted from Smith, 1905.)

Precipitation Reactions. The precipitation reactions of proteins are important in removing them from solution and also for detecting them in solution. Most of the metals will precipitate them. Mercury will throw down a heavy white precipitate of mercury proteinate. Copper, when added to a protein solution as the sulphate, will throw down a bluish precipitate. Iron and lead also give heavy precipitates, the one with iron being dissolved by an excess of that compound. Picric acid, trichloracetic acid, phosphotungstic acid, tannic acid and bromine also form precipitates in protein solutions.

\section{CARBOHYDRATES}

The carbohydrates may be classified in different ways. The following is a convenient method:

I. Monosaccharides
A. Pentoses, $\mathrm{C}_{5} \mathrm{H}_{10} \mathrm{O}_{5}$
1. Xylose
2. Arabinose
B. Hexoses, $\mathrm{C}_{6} \mathrm{H}_{12} \mathrm{O}_{6}$
1. Dextrose
2. Levulose
3. Galactose

II. Disaccharides
1. Sucrose
2. Maltose
3. Lactose 
III. Trisaccharides

1. Raffinose

IV. Polysaccharides

A. Starches

1. Starch

2. Glycogen

3. Inulin

$B$. Celluloses

1. Cellulose

2. Hemicellulose

a. Pentosans

Gum arabic

b. Hexosans

Galactans

Agar-agar

Action of Bacteria on Carbohydrates. This class of substances serves bacteria for both energy and building purposes. The action of microorganisms on carbohydrates is a subject too large for extensive treatment here.* The enzymes which will attack carbohydrates are widely distributed among microorganisms. Foods containing carbohydrates are especially susceptible to attack by bacteria unless the sugar is too concentrated. If there is too little moisture present the organisms are unable to carry on their activities.

The changes induced in sugar by bacteria may be discussed in different ways. It is quite natural to discuss them on a physiological basis.

Lactic Acid Fermentation. This is brought about by bacteria by first splitting the lactose into two molecules of a monosaccharide, each of which is further decomposed to lactic acid according to the following equation:

$$
\begin{aligned}
\mathrm{C}_{12} \mathrm{H}_{22} \mathrm{O}_{11}+\mathrm{HOH} & =2 \mathrm{C}_{6} \mathrm{H}_{12} \mathrm{O}_{6}, \\
\mathrm{C}_{6} \mathrm{H}_{12} \mathrm{O}_{6} & =\mathrm{C}_{3} \mathrm{H}_{6} \mathrm{O}_{3} .
\end{aligned}
$$

Since the lactic acid which is thus formed possesses so much latent energy, it may be further decomposed to other compounds such as butyric acid.

The kind of lactic acid formed has been the subject of many investigations. Four lactic acids are known, three of which have the same formula,

* Those wishing an exhaustive treatment of the subject are referred to Kruse's Allgemeine Mikrobiologie, or Lafar's Handbuch der technischen Mycologie. 
$\mathrm{CH}_{3} \mathrm{CH}_{2} \mathrm{OHCOOH}$. The other has the formula $\mathrm{CH}_{2} \mathrm{OHCH}_{2} \mathrm{COOH}$. The last-mentioned lactic acid since it does not possess an asymetric carbon atom is not optically active. The ethylidene lactic acids possess asymetric carbon atoms and are therefore optically active.

Thomas (1916) has shown that the lactic acid produced by Matzoon in synthetic culture media is inactive but that it may be resolved into two active components. According to Heinemann (1907) milk which sours naturally at room temperature contains chiefly $d$-acid; at $37^{\circ} \mathrm{C}$. chiefly $r$-acid with $l$-acid in excess if allowed to stand a few days. He also found that the kind of organism present determined the kind of lactic acid which was formed. The temperature was also believed to have an effect.

Alcoholic Fermentation. These are those changes in sugar and decomposition products of sugar which result in the formation of large amounts of alcohol. Historically it has been known for a very long time and was not thoroughly analyzed until Pasteur proved that it was a biological phenomenon. The chemistry of alcoholic fermentation is now fairly well understood and there is much data available in the literature to review at this time. Kruse believes that dextrose breaks up in a series of reactions whlle Harden maintains that this takes place in one reaction. Kruse's reactions will add up to equal that of Harden's:

$$
\begin{aligned}
3 \mathrm{C}_{6} \mathrm{H}_{12} \mathrm{O}_{6} & =2 \mathrm{C}_{2} \mathrm{H}_{5} \mathrm{OH}+2 \mathrm{CO}_{2} ; \\
2 \mathrm{C}_{6} \mathrm{H}_{12} \mathrm{O}_{6} & =3 \mathrm{CH}_{3} \mathrm{COOH} ; \\
6 \mathrm{C}_{6} \mathrm{H}_{12} \mathrm{O}_{6} & =2 \mathrm{C}_{3} \mathrm{H}_{6} \mathrm{O}_{3} \\
\mathrm{C}_{6} \mathrm{H}_{12} \mathrm{O}_{6}+6 \mathrm{H}_{2} \mathrm{O} & =6 \mathrm{CO}_{2}+6 \mathrm{H}_{2} \mathrm{O} ;
\end{aligned}
$$$$
12 \mathrm{C}_{6} \mathrm{H}_{12} \mathrm{O}_{6}+\mathrm{H}_{2} \mathrm{O}=12 \mathrm{C}_{3} \mathrm{H}_{6} \mathrm{O}_{3}+6 \mathrm{C}_{2} \mathrm{H}_{5} \mathrm{OH}+6 \mathrm{CH}_{3}-\mathrm{COOH}
$$$$
+12 \mathrm{CO}_{2}+12 \mathrm{H}_{2} \mathrm{O} \text {. }
$$

Ethyl Alcohol. Iodoform Reaction. Add about an equal amount of iodine solution to the sample and treat with sodium hydroxide until decolorized. Warm the mixture very carefully and try to detect the odor of iodoform. A yellow precipitate of iodoform may result. This test is not specific as it is given by acetone, aldehyde, acetic ester, and other similar substances.

Acetaldehyde Reaction. Add a few drops of potassium dichromate and dilute sulphuric acid to the sample and heat. An odor of aldehyde will be observed if ethyl alcohol is present. 
Ethyl Acetate Reaction. Add a little sodium acetate and concentrated sulphuric acid to the sample and heat. The fruity odor of ethyl acetate will be detected if alcohol was present in the sample. Quantitative methods for this may be obtained from chemical texts.

ACETONE. Iodoform Reaction. This is the same test as is used to detect ethyl alcohol except that it is carried out in the cold. Add to the same a few drops of sodium hydroxide and very slowly iodinepotassium-iodide solution until the solution is colored yellow. If acetone is present iodoform will settle out.

Nitroprusside Test. Add a few drops of a freshly prepared solution of sodium nitroprusside and about 1 c.c. of sodium hydroxide. A red color is produced which will fade to a yellow on standing.

Molisch Reaction. Add a few drops of Molisch reagent to the sugar solution in a test tube. Incline the tube and pour a few cubic centimeters of concentrated $\mathrm{H}_{2} \mathrm{SO}_{4}$ down the side of the test tube. A lavender color will be formed if sugars are present. If the $\mathrm{H}_{2} \mathrm{SO}_{4}$ is put into the test tube first, and the sugar-Molisch reagent mixture run in on top, a ring test may be observed.

Seliwanoff's Reaction for Ketoses. Add a small amount of the sugar or unknown solution to a few cubic centimeters of Seliwanoff's reagent and heat to boiling. A reddish coloration indicates the presence of ketose sugars.

Fehling's Test. This depends upon the action of reducing sugars. This action is generally attributed to the presence of an aldehyde or ketone group. Some regard other parts of the sugar molecule as involved in this reduction.

Two solutions are used in the test. These are kept apart until used. If they are mixed and allowed to stand, the copper will be slowly reduced by the tartrate. The solutions have the following composition:

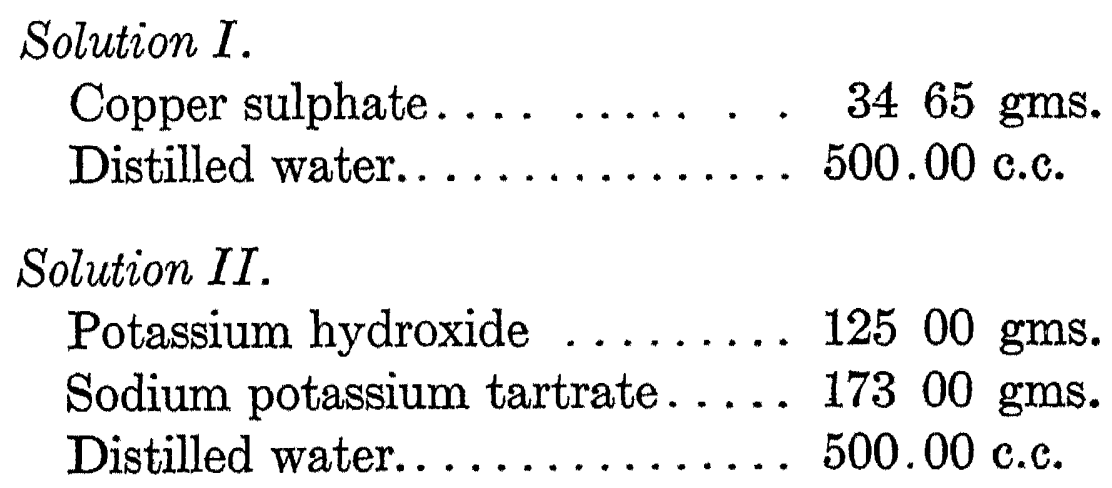

In testing for reducing sugars, mix equal parts of these two solutions and heat to boiling. This will determine whether the solutions are good. If any copper is precipitated by this heating, the solutions must be dis- 
carded. If the solutions are satisfactory, the sugar solution should be added very slowly to the hot Fehling's solution with boiling after each addition. The presence of reducing sugars will be indicated by a yellowbrown precipitate.

Benedict's Method for Reducing Sugars. This is a modification of Fehling's test for reducing sugars. Instead of using the strong $\mathrm{NaOH}$, Benedict has substituted $\mathrm{Na}_{2} \mathrm{CO}_{3}$. Sodium citrate is used to keep the $\mathrm{Cu}(\mathrm{OH})_{2}$ in solution. The method for conducting the test is much like that for Fehling's test. The solution should be boiled before using and allowed to cool. If reducing sugar is present the solution will turn first a green and later a yellow color. Benedict's test is more delicate than the original Fehling's method. The solutions are prepared as follows:

Solution I.

Copper sulphate.......... 34.65 gms.

Distilled water............ 500.00 c.c.

Solution II.

Anhydrous sodium carbonate... 100.00 gms.

Rochelle salt............ 173.00 gms.

Distilled water........... 500.00 c.c.

Nylander's Test for Reducing Sugars. Heat a few cubic centimeters of the solution under examination with $\frac{1}{2}$ c.c. of Nylander's reagent. A black color is formed if a reducing sugar is formed which is due to a precipitation of the bismuth in the reagent.

Phenyl-Hydrazine Reaction. With phenyl hydrazine osazones are formed by action with certain sugars. These are yellow crystalline substances which are characterized by a definite form typical for each different sugar. The test is carried out as follows: Put about 5 c.c. of the sugar solution into a small ieaker or test tube and add about 1.5 c.c. of phenyl-hydrazine acetate solution. Heat on a water bath for threequarters of an hour. Cool and examine the crystals under the microscope.

Quantitative Determination of Dextrose. (Allihn's Method.)

Reagents.

(a) Copper sulphate solution:

Copper sulphate $\mathrm{CuSO}_{4} \cdot 5 \mathrm{H}_{2} \mathrm{O} \ldots \ldots .34 .639$ gms.

Distilled water.............. 500.000 c.c.

(b) Alkalin tartrate solution:

Rochelle salts............ 173 gms.

Potassium hydroxide......... 125 gms.

Distilled water............. 500 c.c. 
Procedure. Place 30 c.c. of the copper sulphate solution, 30 s.c. of the alkaline tartrate solution and 60 c.c. of water in a beaker and heat to boiling. Add 25 c.c. of the solution of the material to be examined, which must be so prepared as not to contain more than $0.250 \mathrm{gm}$. of dextrose, and boil for two minutes. Filter immediately through asbestos without diluting. Wash the precipitate with hot water. Dry, ignite and weigh as cupric oxide. To calculate the amount of copper and its equivalent in dextrose consult Allihn's table in any chemical hand-book.

Benedict's Method for Determination of Dextrose. Twenty-five c.c. of Benedict's special reagent (see Appendix) are measured into a porcelain evaporating dish, 25-30 cm. in diameter, 10-12 gms. of crystalline sodium carbonate, and a small amount of pumice, are added. The solution is boiled vigorously over a free flame and the sugar solution is run in until a white precipitate is formed and the blue color is diminished. After this the sugar solution is run in a few drops at a time until the blue color has entirely disappeared. Water may be added to replace that driven off by evaporation. Twenty-five c.c. of the reagent $=0.05$ $\mathrm{gm}$. glucose or $.053 \mathrm{gm}$. levulose.

\section{FATS}

The fats or lipins are esters of glycerol and certain organic acids which have come to be known as fatty acids. When three molecules of palmitic acid unite with glycerol, the fat plamitin is secured, as follows:

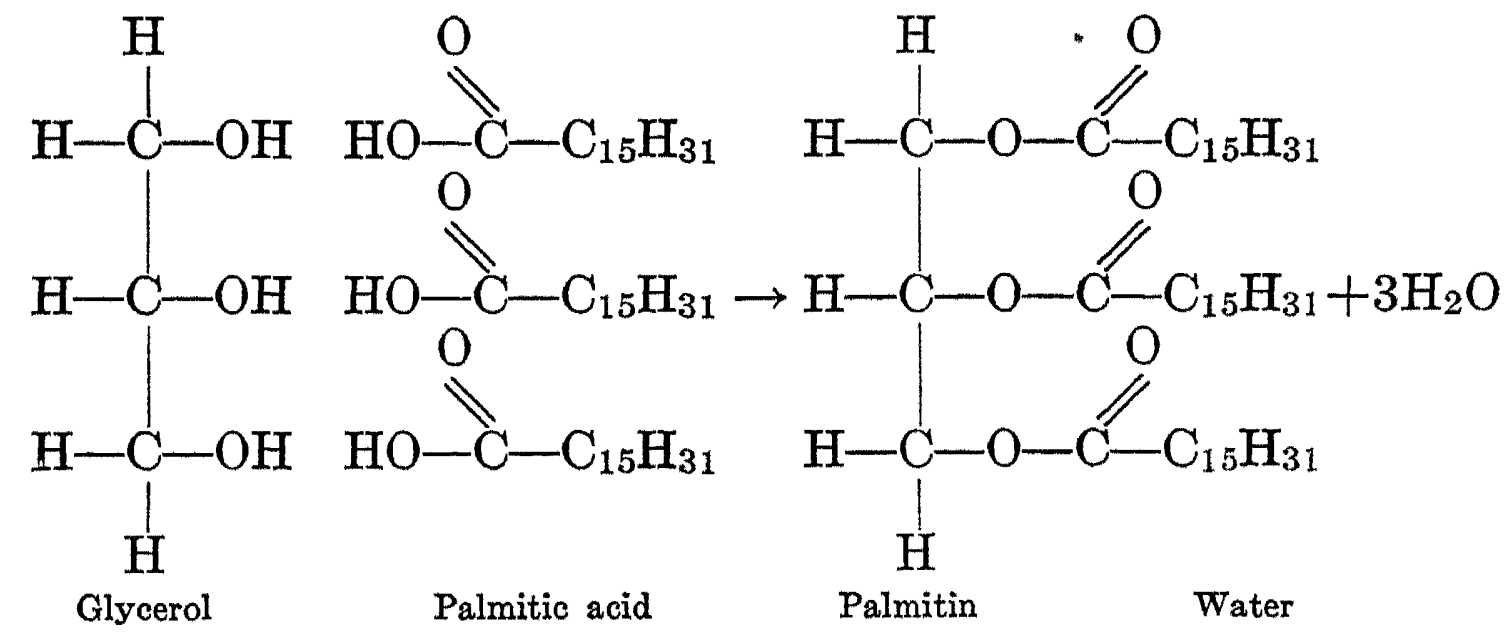

When fats are broken up either by bacterial enzyme or other action, they are resolved into glycerol and fatty acids. This process is known as saponification.

Action of Bacteria on Fats. There is much evidence that fats are split to fatty acids and glycerol by bacteria, although many text books 
state that lipase is not widcly distributed in the bactcrial world or, at least, this subject is little or almost not at all treated. Rancidity in butter has been attributed by some to the formation of butyric acid (Duclaux, 1887). Others maintain that the fat in butter is not attacked, Reinmann (1900) tried the effect of pure cultures on sterile butter and found that most bacteria did not attack fat. In cheese Laxa (1902) demonstrated that $P s$. fluorescens decomposed fat quite extensively. Two other peptonizing bacteria and a yeast were also found which decomposed fat. Rahn (1905) stated that Penicillium glaucum and a few bacteria possessed strong fat-splitting properties. Kendall and Simonds (1914) found that sterile filtrates of plain and dextrose broth cultures of $B$. typhi liberated acid from ethyl butyrate. This was also demonstrated in connection with acid-fast bacteria.

Determination of the Iodine Absorption Number (Hanus Method.) The unsaturated fatty acids will react with the halogens. The amount of iodine differs according to the fat, and consequently this iodine absorption number is of some value for identification of fats. It (Iodine number) may be defined as the number of grams of iodine absorbed by $100 \mathrm{gms}$. of fat.

Reagents. Hanus Iodine Solution. Dissolve 13.2 gms. of iodine in 1000 c.c. of glacial acetic acid (99.5 per cent) showing no reduction with bichromate and sulphuric acid. Add 3 c.c. of bromine to the cold solution.

Starch Paste. Boil a gram of starch in 200 c.c. of distilled water for ten minutes and cool.

Sodium Thiosulphate. Standardize a N/10 solution.

Procedure. Weigh about $0.5 \mathrm{gm}$. of fat into a 250-c.c. glass-stoppered bottle and dissolve by adding 10 c.c. of chloroform. After solution add 30 c.c. of the iodine monobromide solution. Place the bottle in the dark and allow to stand for thirty minutes. The time factor is important. The excess of iodine should be at least as much as is absorbed. One or two blanks should be made under identical conditions. If the first addition of iodine is used up, another addition should be made. Add 10 c.c. of potassium iodide solution and shake after which add 100 c.c. of distilled water washing any iodine on the stopper of the bottle and sides back into the bottle. Titrate the iodine with the $\mathrm{N} / 10$ sodium thiosulphate to a yellow color. Then add a few drops of the starch solution and continue the addition of sodium thiosulphate until the blue color has disappeared. Toward the end of the reaction stopper the bottle and shake violently so that any iodine remaining in the chloroform may be taken up by the potassium iodide solution. The 
number of cubic centimeters of iodine used in the blank minus the number used for the sample gives the thiosulphate value of the sample.

Saponification Number or Koettstorfer Number. Since the amount of alkali which will react with a fat is dependent upon the glycerides in it, the saponification number is valuable for identifying fats and oils. The saponification is the number of milligrams of sodium hydroxide necessary to saponify $1 \mathrm{gm}$. fat.

Reagents. Sodium Hydroxide. Use a N/10 solution each cubic centimeter of which contains $0.0040 \mathrm{gm}$. $\mathrm{NaOH}$ and neutralizes 0.0088 gm. of butyric acid.

Alcoholic Potash Solution. Dissolve $40 \mathrm{gms}$. of pure potassium hydroxide in 1000 c.c. 95 per cent alcohol.

Acid Solution. $\mathrm{N} / 2 \mathrm{HCl}$.

Indicator. Phenolphthalein.

Procedure. Conduct the saponification in wide-mouth Erlenmeyer flasks of 250 c.c. capacity. Place about $5 \mathrm{gms}$. of the fat in a tarred flask and weigh. Add exactly 2 cubic centimeters of the alcoholic potash solution, connect with a reflux condenser and boil for thirty minutes or until the fat is completely saponified. Cool and titrate with the $\mathrm{N} / 2$ hydrochloric acid. The Koettstorfer number is determined as follows: Subtract the number of cubic centimeters of hydrochloric acid used to neutralize the excess of alkali after saponification from the number of cubic centimeters necessary to neutralize the 50 c.c. of alkali added; multiply the result by 28.06 and divide by the number of grams of fat used. Conduct two blanks using the same pipettes and conditions. (Official and Provisional Methods, 1912. A. O. A. C.)

Determination of Volatile Fatty Acids (Reichert-Meissl Method). This determination must be carried out under standard conditions. Under these, the Reichert-Meissl number is the number of cubic centimeters of $\mathrm{N} / 10$ sodium hydroxide required by the soluble fatty acids distilled from 5 gms. of fat.

Procedure. Into two clean Erlenmeyer flasks weigh 5 gms. of the fat. Add 10 c.c. of 95 per cent alcohol and 2 c.c. of sodium hydroxide (100 gms. in 100 c.c. $\mathrm{H}_{2} \mathrm{O}$ ). Attach to a reflux condenser and heat on the steam bath until saponification is complete. After the saponification, in case alcohol was used, remove this by dipping the flasks in a steam bath up to their necks. When the alcohol is nearly gone frothing may occur. Dissolve the soap by adding 135 c.c. of boiled water and warming on the water bath until a clear solution results. After the solution has cooled to about $60^{\circ} \mathrm{C}$., free the fatty acids by adding 8 c.c. of dilute sulphuric acid (200 c.c. in 1000 c.c. of water). Connect to a 
condenser and heat slowly on a water bath until the fatty acids form a transparent layer on the surface. Cool to room temperature and add a few pieces of pumice stone and distill at a rate that will allow about 110 c.c. to be driven over in thirty minutes. The pumice should be prepared by heating small pieces to a white heat and dropping them into distilled water. They should be kept under distilled water to keep them free from air. Mix the distillate, filter and titrate 100 c.c. with standard $\mathrm{N} / 10$ sodium hydroxide solution using phenolphthalein. To obtain the Reichert-Meissl number, increase the cubic centimeters of $\mathrm{N} / 10$ sodium hydroxide used by 100 c.c. of filtrate, by one-tenth, divide by the weight of fat taken, and multiply by 5 .

\section{BIBLIOGRAPHY}

Bainbridge, F. A. 1911. The Action of Certain Bacteria on Proteins. Journal of Hygiene, 11, 341.

Ducladx, E. 1887. Le Lait Études Chimique et Biologiques, Paris.

Heinemann, P. G. Kinds of Lactic Acid Produced by Lactic Acid Bacteria. Jour. Biological Chemistry, 2, 612 .

Kendali, A. I. and Simonds, J. P. 1914. The Esterase Activity of Plain and Dextrose Broth Cultures of the Typhoid Bacillus. Jour. Inf. Diseases, 15, 354-356.

LAXa, O. Ueber die Spaltung des Butterfetts durch Mikroorganismen. Arch. Hyg., 41, 119.

Meyer, K. 1914. Bacterial Decomposition of $d$-Glucosamine. Bichem. Zeit., 58, 415-416.

Meyer, K. 1914. The Behavior of Some Bacteria Towards $d$-Glucosamine. Biochem. Zeit., 57, 297-299.

Mito, I. L. 1918. The Asymetric Decomposition of Racemic Polypeptids by Dead Bacteria. Chem. Abstracts, 12, 816.

Raistrick, H. 1917. A New Type of Chemical Change Produced by Bacteria. The conversion of histidine into urocanic acid by the bacteria of the coli-typhosus group. Biochem. Jour., 11, 71-77.

Reinmann, R. 1900. Investigations on the Causes of Rancidity in Butter. Cent. Bakt. Abt., II, 6, 31.

Rettger, L. F., Berman, N. and Sturges, W. T. 1916. The Utilization of Protein and Non-protein Nitrogen. Jour. Bact., 1, 15.

Robinson, R. H. and Tartar, H. V. 1917. The Decomposition of Protein Substances through the Action of Bacteria. Jour. Biol. Chem., 30, 135-144.

SASAKI, T. 1912. Cleavage, of Polypeptids by Bacteria I. Bacterium Coli Commune. Biochem. Zeit., 41, 174-179.

SASAKI, T. 1913. The Decomposition of Certain Polypeptids by Bacteria. III. Investigations with Liquefying Bacteria. Biochem. Zeit., 47, 472-481. 
SASAKI, T. 1913. The Decomposition of Certain Polypeptids by Bacteria. II. Investigatıons with Non-liquefying Bacteria. Biochem. Zeit., 47, $462-471$.

Sasaki, T. 1916. Bacterial Decomposition of Polypeptids. Physiol. Abstracts, 1917, 2, 15.

SASAKI, T. 1917. The Influence of Conditions of Bacterial Cleavage of Proteins on the Cleavage Products. Jour. Biol. Chem., 32, 527-532.

SASAKI, T., and OTSUKA, I. 1917. The Stereochemistry of the Bacterial Decomposition of Albumin. Jour. Biol. Chem., 32, 533-8.

Sperry, J. A., and Rettger, L. F. 1915. The Behavior of Certain Bacteria toward Animal and Vegetable Proteins. Jour. Biol. Chem., 20, 445.

TAYloR, J. W., and HALL, I. W. 1912. Action of Salira, Tissue Fluids, Bacteria and Bacterial Extracts upon Polypeptıds. Jour. Path. and Bact., $17,121-123$.

Thomas, S. J. 1916. A Study of Stereoisomerism of Fermentation Lactic Acid. Jour. Ind. Eng. Chem., 8, 821-823.

Tsudjr, M. 1918. The Asymetric Decomposition of Racemic Tyrosine by Bacillus Proteus Vulgaris and B. Subtilis (also a Biological Preparation of $d$-tyrosine). Chem. Abstracts, 12, 816. 


\section{CHAPTER VII \\ YEASTS AND MOLDS}

The molds may be responsible for many changes in foods. Their action, however, should be considered from two standpoints since they bring about desirable and undesirable changes. A type of the former in the manufacture of Camembert and Roquefort cheese while the spollage of strawberries and citrus fruits may be offered as an example of the latter. Tas $Y u$, the Chinese drink, is also said to be fermented in part by molds.

Fungi. These are plants which possess no chlorophyl and consequently are unable to utilize the energy in sunlight. All of it must come trom analytic processes. They do not construct their own starch but use that which has been built up by other organisms. For convenience, fungi have been divided into the following main groups. To the food microbiologist, these may not be so important but may be helpful in the identification of unknown fungl.

Ascomycetes. This class produces ascospores or spores in an ascus. The yeasts since they possess this char acteristic are put into this group. The aspergillaceæ are ascomycetes. Some of these form perithecia which contain asci.

Basidiomycetes. The spore-bearing bodies are termed basidia and possess a typical morphology. The conidia are produced from this on the sterigmata which may be either branched or unbranched.

Phycomycetes. In this group are placed the algæ. None of the fungi belonging to this group are commonly met with in food microbiology.

Fungi Imperfecti. Into this group are placed many fungi which do not come under any of the above groups. The fungi imperfecti are characterized by no definite fruiting bodies. Ordium lactis is the best example and in this fungus each mycelial thread breaks up to form oidia which may develop into a new plant.

Structure of Molds. The body of the mold is made up of a cottonlike structure called the mycelium. Each individual thread is called a mycelial thread or hypha. The hyphæ are of two kinds-fertile and 
vegetative. Fertile hyphxe are those which bear the fruiting body and produce the spores. The vegetative hyphæ secure the nutriment and get rid of the excess moisture.

The structure of the hyphæ differs among the molds. Some have cross walls or septa. In other molds these have not been observed. The presence or absence of these septa is an important factor in the

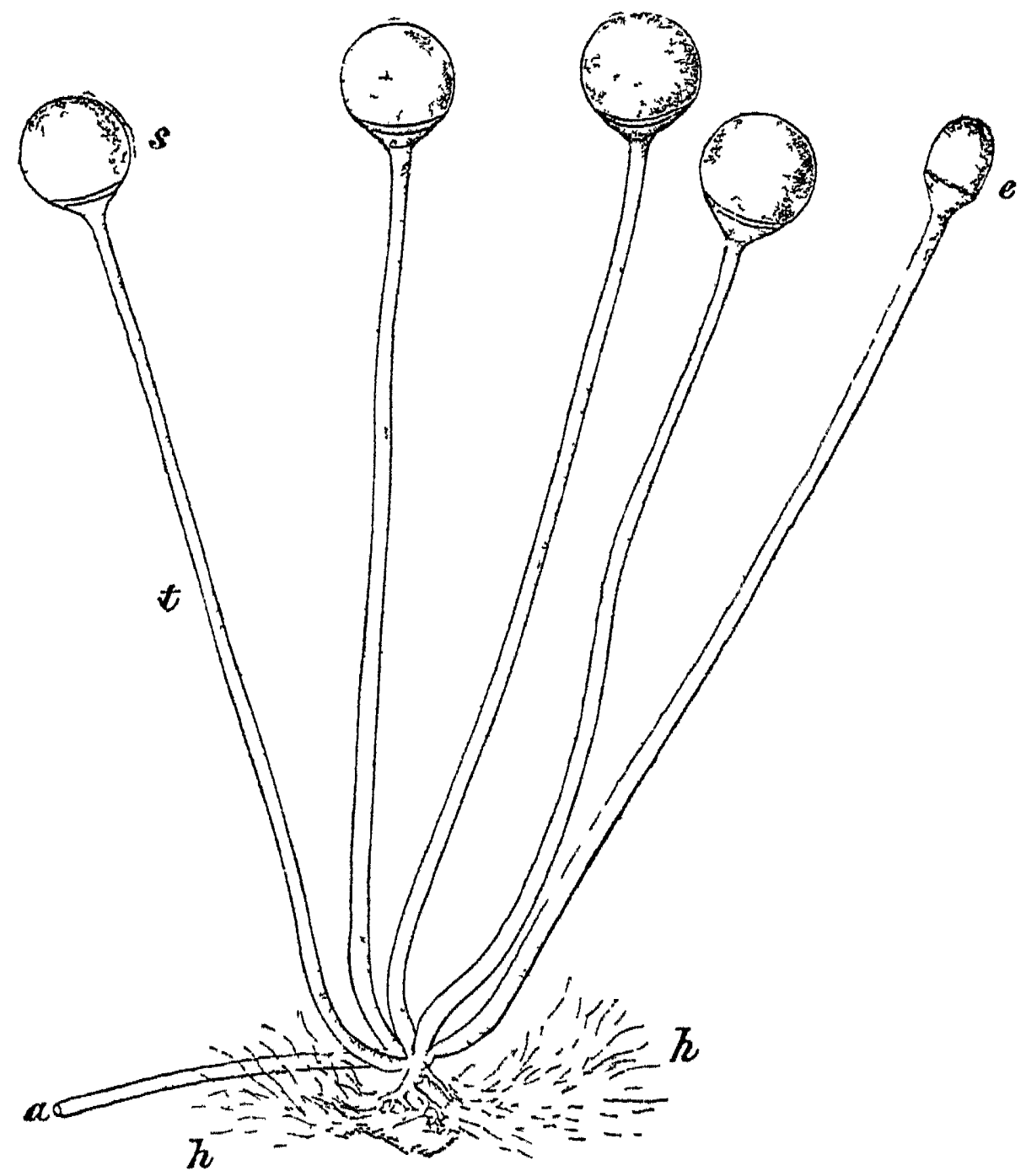

Fig. 47 -Rhızopus nigricans Ehrenberg (After Brefeld)

$(a)$ is the extremity of a stolon, which has developed into the appressorium $(h)$ This latter is the starting point of the sporangiophores $(t)$ four of which are shown with the sporangia $(s)$ unbroken, whilst the columella $(e)$ is all that remains of the fifth Magn 30

identification of molds. The mucoraceæ do not possess these cross walls ordinarily.

Fructification. This takes place on the fertile hyphæ which bear the fruiting bodies. Enormous numbers of spores are formed each of which may develop into a typical mold plant. The spores are of two kinds, sexual and asexual.

The sexual spores are formed by the union of two cells. The resulting cell then possesses the characteristics which are algebraic sums of 
the characteristics inherent in the parent cells. When a sexual spore is formed on a non-septate hyphæ, it is termed a zygospore; if on a septate hyphæ it is an ascospore. Zygospores are formed by the union of two terminal cells on adjacent hyphæ. The ascospore is formed by the

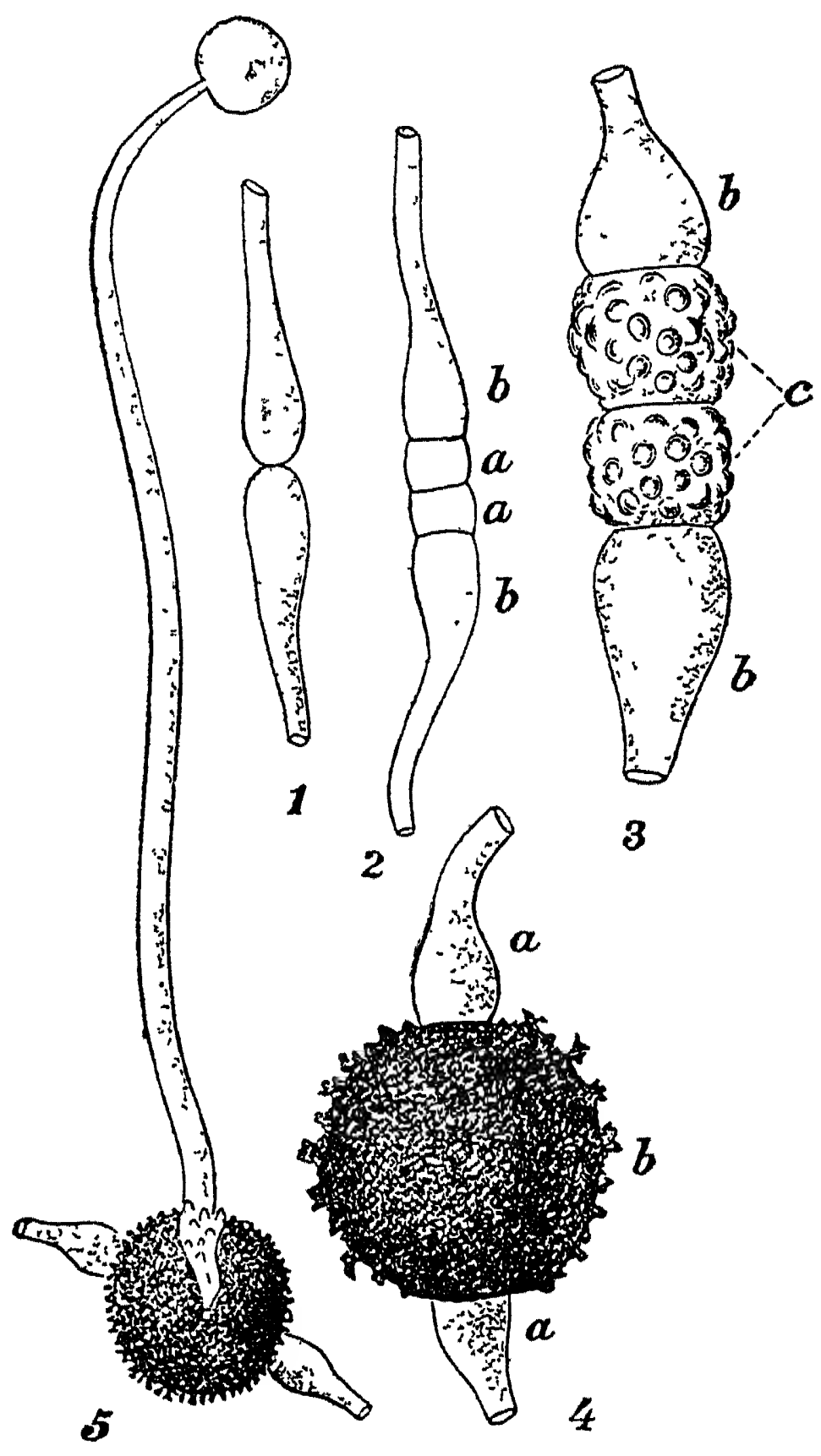

Frg. 48.-Mucor Mucedo. Formation of the Zygospores. (After Brefeld.)

1, two hyphæ in terminal contact; 2, articulation into gamete $a$ and suspensor $b, 3$, fusion of the gametes $a$; the membrane thickens; 4 , ripe zygospore $b$ supported by the suspensors $a a$; 5 , germination of the zygospore to a sporangium stem. Mag. about 60 .

union of adjacent cells. In this mass are formed the asci which hold the spores. Most of the spores formed by molds are asexual being formed mostly at the ends of the fertile hyphæ from typical fruiting bodies.

Classification. The molds are thread fungi and it is often difficult to draw the line between them and some other classes of fungi. Oidium 
lactis (oospora lactis) is often classified with yeasts and has then been given the name Mycoderma lactis. The molds are set apart from bacteria and yeasts in that they are multicellular. The following varieties may be met with in a microbiological examination of foods.

Rhizopus. The most common member of this group is Rhizopus nigricans. It is the common black bread mold and may be responsible for other food spoilage. Stevens (1917) has shown that this fungus causes the rot of strawberries during shipment. This work verifies

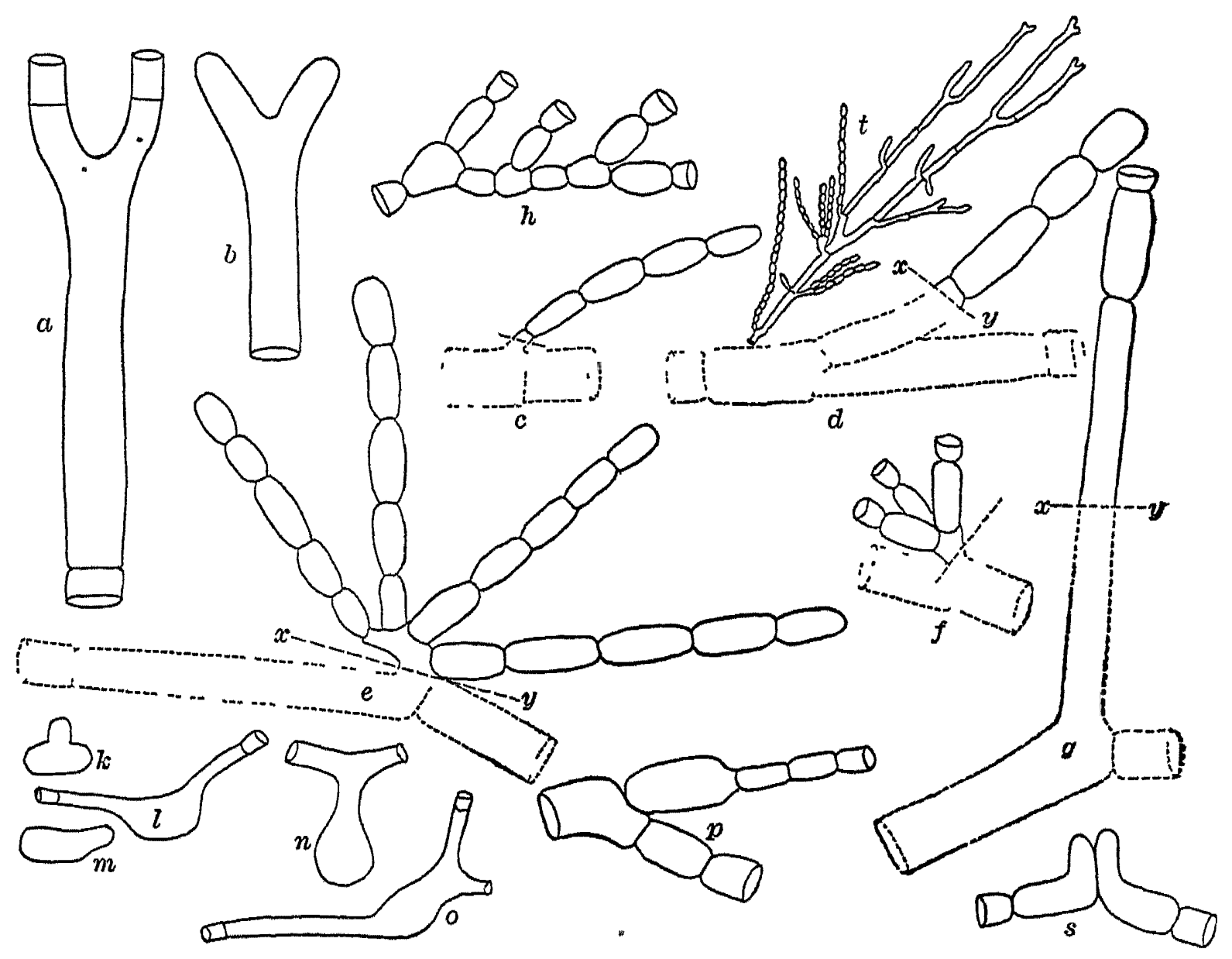

FIG. 49.-Oidium Lactis. (After Thom.)

$a, b$, dichotomous branching of growing hyphæ; $c, d, g$, simple chains of oidia breaking through substratum at dotted line $s-y$, dotted portions submerged; $e, f$, chains of ordia from a branching outgrowth of a submerged cell; $h$, branching chain of oldia, $k, l, m, n, 0, p, s$, types of germination of oldia under varying conditions; $t$, diagram of a portion of a colony showing habit of $O_{2} d i u m$ lactis as seen in culture media.

some done by other investigators on the same subject. SchneiderOrelli (1911) have shown that $R$. nigricans is important in bringing about the decay of over-ripe pears during storage.

Mucor. The mucors have the same structure as Rhizopus molds. They are separated, however, by the fact that the sporangiophores spring from the stolons singly.

Oidium. The sour milk mold Oidium lactis is a common variety of this genus. The most of this mold is below the surface of the medium. 
That part which is not submerged resembles a layer of velvet on the surface of the medium.

Penicillium. Penicillium molds are important in the manufacture of certain cheeses. Thorn $(1906,1905)$ has given much study to this question. Dox (1910) has reported an extended study of the intracellular enzymes in Penicillium camemberti. The following enzymes were demonstrated to be present: erepsin, nuclease, amidase, lipase, emulsin,

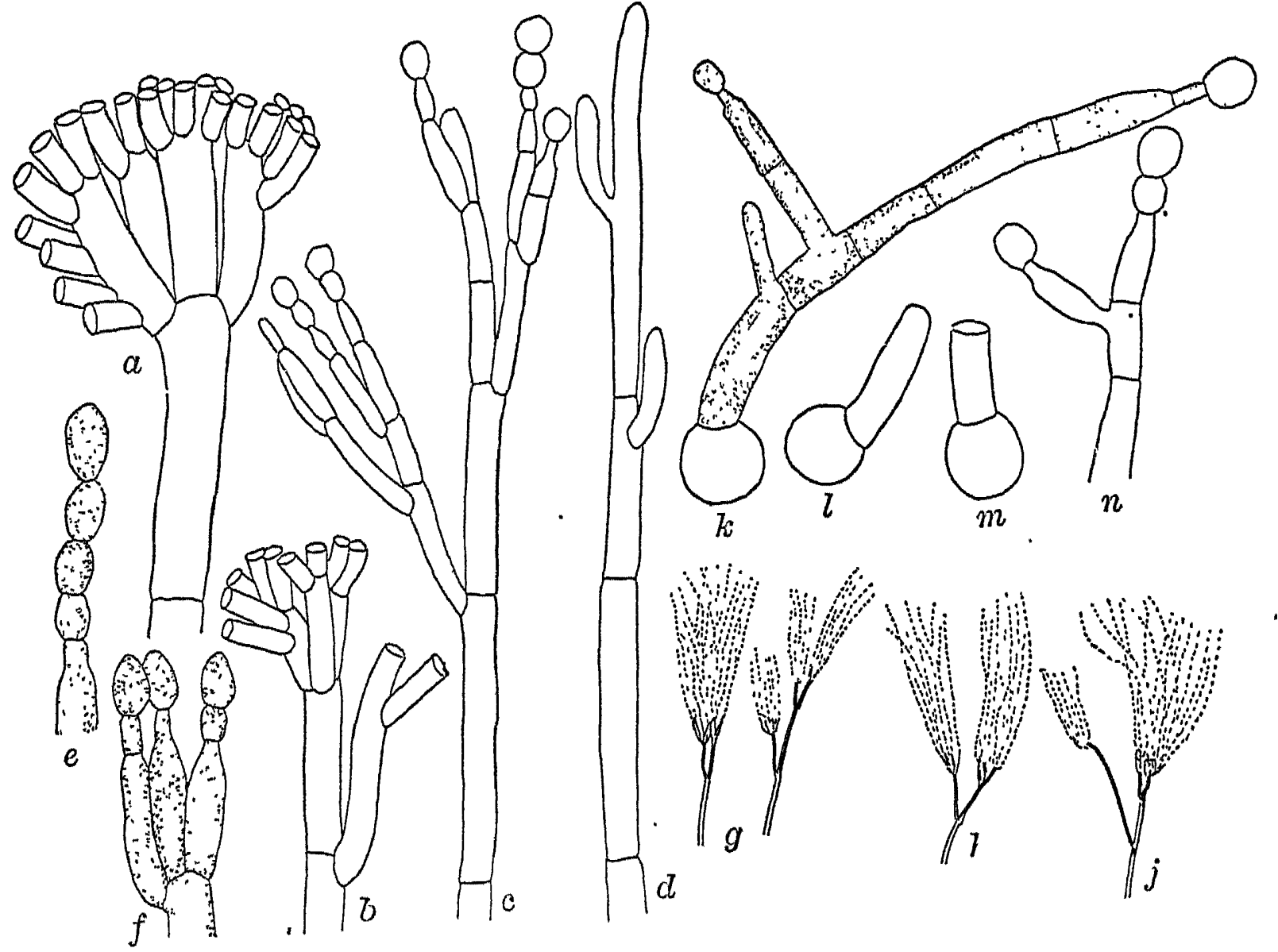

FIG. 50.-Roquefort Penicillium ( $P$. roqueforti). (After Thom, 1906.)

a, part of conidiophore and of bas of fructification, highly magnified showing the production of basidia on the sides as well as at the apex of the basidiophore; $b, c$, other types of branching; $d$, young conidiophore just branching; $e, f$, basidia and the formation of conidia, highly magnified; $g, h, j$, diagrams of types of fructification as seen under low power $(\times 80) ; k, l, m, n$, germination of $g, h, j$, diagrams of types of fructification as seen under low powe

amylase, inulase, raffinase, sucrase, maltase, and lactase. The presence of these enzymes seems to indicate an ability to utilize various food products. Penicillia are widely distributed in nature and many of the molds which contaminate foods belong to this genus. Thorn (1910) has reported cultural studies on the species of penicillium. He has worked out a key which is of much assistance in studying molds and their relation to food spoilage. 


\section{KEY TO PENICILLIUM MOLDS}

(After Thom, 1910)

A. Species fruiting typically by coremia (vertical and definite).

a. Coremia long (3-15 mm.).

1. Conidial masses strictly terminal, olive green,

fragrant. . . . . . .................. claviforme

2. Upper third of coremia fertile, conidia green..P. duclauxii aa. Coremia small.

1. Coremia definite, densely crowded, colony orange below.................... granulatum

2. Coremiform characters indicated incultures by clustering of conidiophores, definite coremia only in old cultures becoming large and definite on apples. ................... expansum

$A A$. Species not (or rarely) producing coremia in culture.

$B$. Species constantly producing sclerotia or ascigerous masses.

b. Producing asciginous masses, yellow or reddish ....................... luteum

$b b$. Sclerotia appearing as white masses in old cultures.................... P. italicum

$b b b$. Sclerotia reddish or pink, globose or elliptical, 500 microns or less in diameter.

c. Conidial fructification in column.

1. Column dense long, sclerotia partiall. buried in substrate............. P. No. 30

2. Column formed of loose chains, sclerotia numerous, exposed........P. No. 29

$c c$. Conidial fructification of divergent chains:

1. Rapid liquefier, spores globose. 2-5 microns................... No. 31

2. Slow liquefier, spores elliptical, $3.5-4$ by $2.5-3$ microns. . . . . . . . . P. No. 32

$B B$. Sclerotia not(or rarely) produced under special conditions.

$C$. Rapid liquefiers (abundant liquid in 5 to 12 days).

$D$. With definite strong ammoniacal odor:

1. Yellowish brown-avellaneous spores rough.. . P. brevicaule

2. White or cream spores rough............ brevicaule

3. White or cream, spores smooth.......... P. brevicaule, ver.

glabrum 
$D D$. Without ammoniacal odor.

$E$. With yellow coloration of liquefied gelatin (not of mycelium in reverse).

1. Colonies small, conidiophores $100-150 \mathrm{mi}-$ crons in length ................ citrinum

2. Colonies broadly spreading conidiophores 250-300 microns................. chrysogenum

$E E$. Without yellow color in liquefied medium (or slight traces only).

e. Colonies white or pink or salmon............ roseum

$e e$. Colonies some shade of green.

$f$. Colonies floccose, margin spreading by stolons....P. stoloniferum

$f f$. Colonies velvety surface growth of fruiting hyphæ only.

g. Conidiophores very short (100-200 microns).

1.............................. No. 12

$2 \ldots \ldots \ldots \ldots \ldots \ldots \ldots \ldots \ldots \ldots$. No. 37

gg. Conidiophores longer (200-400 microns):

1. Conidiophores variously branched, reverse always colorless. ................. P. No. 24

2. Conidiophores each with a verticle of brancheseach branch wearing a columnar fructification -reverse and medium darkening in sugar media......................... atramentosum

CC. Liquefaction of gelatin none or slower than 10-12 days, or only partial.

$h$. Colonies yellowish brown spores elliptical.......P. divaricatum

$h$. Colonies white to lilac, liquefier, 14-16 days.....P. lilacinum

hhh. Colonies floccose or creamy white:

1. Conidiophores long, typical penicillate branching...................... camembertivar.

2. Conidial chains borne upon short branches rogeri of floccose hyphæ................ No. 33

$G G$. Colonies some shade of green.

$H$. Surface with hyphæ definitely in ropes or trailing, bearing numerous conidiophores, as short branches distinctly traceable to their origin in such hyphæ.

$i$. Colonies usually red below and reddening the substratum.

1. Fruiting areas dark green ........... funiculosum

2. Fruiting areas mixed yellow and green.P. pinophilum 
ii. Colonies not producing red color.

1. Colonies gray rarely greenish, very loose floccose .................. intricatum

2. Colonies green, conidial chains in simple compact column ............. P. No. 48

3. Colonies gray to green, hyphæ scattered creeping................. decumbens

$H H$. Surface hyphæ not in well-defined ropes, nor trailing.

$j$. Surface hyphæ woven floccose, course of hyphæ not traceable.

1. Gray green long conidiophores, no odor ...P. camemberti

2. Gray green, shorter conidiophores, strong odor........................ biforme

$j j$. Surface growth at margin simple conidiophores, in older parts both hyphæ and conidiophores.

1. Gray greenish, branching of conidiophores rather loose, odor none or slight. . . ....P. No. 22

2. Green, conidial fructifications rather compact, odor definite "moldy".........P. commune

N. B. There is probably a number of races in this group.

jjj. Fruiting surface velvety-of simple conidiophores or conidiophores borne so close to surface of substratum as to appear simple:

$k$. Conidial mass a dense column of conidial chains.

1. Column from a single verticil of basidia....P. spinulosum

2. Column from a verticil of branchlets with verticillate cells and chains..........P. rubrum

$k k$. Elements of conidial fructification not in column .

$k k$. Elements of fructification not in a column.

l. Conidia smooth.

1. Green, broadly speaking, ripe conidia globose, $4-5$ microns . . . . . . . . . . . . P. ruefort

2. Green, less spreading, conidia elliptical, medium commonly purpled........... P. purpurogenum

3. Gray or olive green, conidia $5-7$ by $3-5$ microns..................... digitatum

13. Conidia delicate rugulose, .P. rugulosum 


\section{SPECIES OF PENICILLIA DETERMINABLE FROM THE SUBSTRATA}

(After Thom, 1910)

(In these species the substratum establishes a presumption of identity.) Cheese (Camembert and Brie):

1. Floccose, white unchangeable, no odor ............ camemberti var. rogeri

2. Floccose, white to gray green, no odor. $\quad \ldots \ldots \ldots$. P. camemberti

3. Powdery, yellowish white, spores smooth, ammoniacal

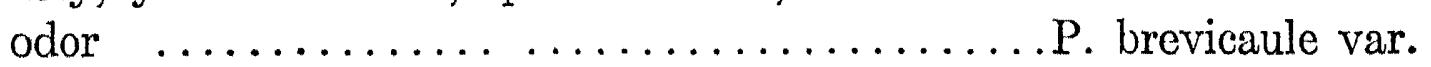
Glabr.

4. Powdery, yellowish white, spores, tuberculate, ammoniacal odor..................... b. brevicaule var. album

5. Forming yellowish brown areas, spores rough, ammoniacal odor... ....................... Previcaule

Cheese Roquefort:

1. Green streaks inside the cheese. Citrus fruits:

1. Colonies of mold blue green.. ................ italicum

2. Colonies of mold olive green $\ldots \ldots \ldots \ldots \ldots \ldots \ldots$. digitatum-olivaceum

Pomaceous fruits:

1. Blue green colonies finally producing coremia ... ...P. expansum Polyporaceæ (Boleti, Polypori, etc.).

1. Colonies green (yellowish green) spreading by stolons...P. stoloniferum Wood (pine):

Producing orange to red stains in pine wood............ piniphilum

Aspergillus. This is a common variety of mold and is responsible for many decomposition processes in nature. The spores are borne from a club-shaped fruiting body called the basidium.

SUMMARY OF THE ASPERGILLUS SPECIES GROUPED ACCORDING TO COLOR OF THE CONIDIAL VEGETATION, THE CHARACTER OF THE STERIGMATA AND THE EXISTENCE OF ASCOSPORES

(After Lafar, 1910)

1. Green (gray, bluish green or yellow green), viz.:

(a) With simple sterigmata. A. GLAUCUS, Link, with ascospores (naked peritheca); A. Clavatus, Desmazieres; A. Fumigatus, Fesenius ascospores (cased perithecia); A. ORYzm (Ahlburg) Cohn; A. varians, Wehmer; A. minimus, Wehmer; A. FLAvus, Link; with sterile sclerotia; A. giganteus, Wehmer; A. ccesiellus, Saito; A. Tokelau, Wehmer; A. penicillopsis (Hennings) Raciborski. 
(b) With branches sterigmata: A. nidulans, Eidam, ascospores (ensheathed sclerotia); A. pseudoclavatus, Puriewitsch, ascospores (naked perithecia); A. variabilis, Gasperini; A. versi color, Vuillemin.

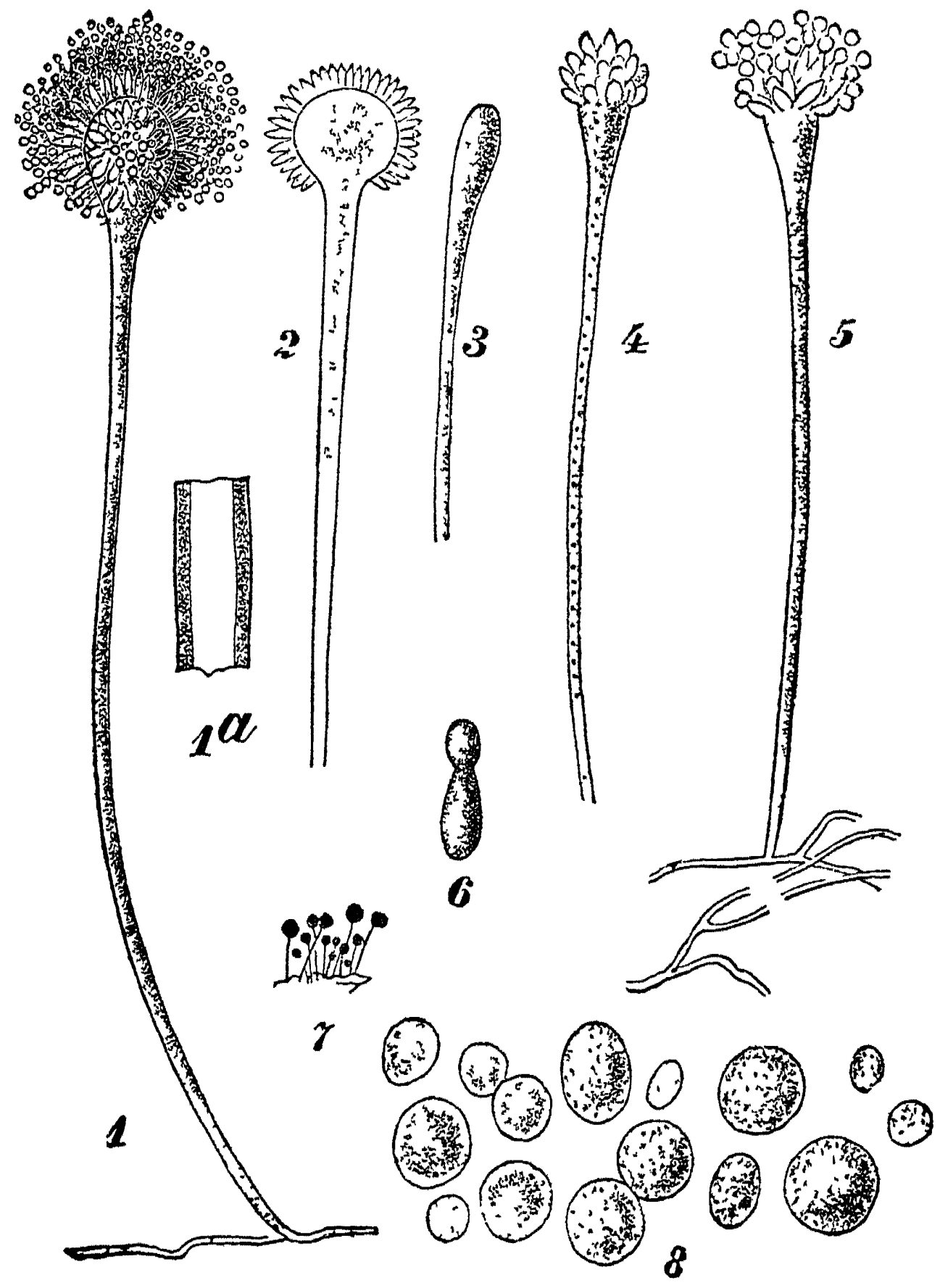

Fig. 51.-Aspergillus Oryzæ. (After Wehmer.)

1-2, conidiophores with clavate and almost spherical globule, 2, in optical section; 3-5, development of a small conidiophore, distension of the hypha, protrusion of sterigmata and incipient formation of conidia, $1 a$, optical section of tough stem, 6, sterigmata; 7, conidal herbage, slightly magnified; 8, conidia. Approximate magnification of $1-5,75$, of 6,400 ; of 8,900 .

2. White, viz.:

(a) With branched sterigmata (associated with simple sterigmata in the case of $A$. candidus, I.; A. candidus, I.; Wehmer, $A$. albus, Wilhelm.

(b) With simple sterigmata: A. candidus (Link) Saccardo. 
3. Blackish brown, viz.:

(a) With branched sterigmata. A. nıger (Cramer), van Tieghm, with sterile sclerotia; A. phcenicis, Fat, and Delacr.; A. strychni, Lindau; A. pulverulenta, MacAlpine; A. atropurpureus, Zimmermann; A. vrolaceo-fuscus, Gasperini.

(b) With simple sterigmata. A. luchuensıs, Inui; A. calyptratus Oudemans.

4. Brownish yellow, yellow brown and reddish, viz:

(a) With simple sterigmata $A$. ostranus, Wehmer, A. WENTII WeHMER; A. perniciosus, Inui; A. giganteo-sulfureus, Saito; A. citrisporus, von Hohnel.

(b) With branched sterigmata (occasionally associated with simple ones). A. sulfureus, Fresenius; A. ochraceus, Wilhelm (with sterile sclerotia); A. rehmin, Zukal (with ensheathed perithecia); A. spurius, Schroter; A. elegans, Gasperini; A. aurcomus, Gueguen (with sterile sclerotia).

Examination of Molds. If molds are present in the foods or material under examination, they will appear on the agar plates after incubation although it may take a longer incubation period than for the bacteria. In the examination of foods or other substances where the entire flora is desired for study, dextrose agar should be used since this will permit the development of bacteria, yeasts and molds. These colonies should then be transferred to dextrose agar slants and, in this way, preserved for further study and identification.

Total Organism. The structure and makeup of the total organism may be secured from a water mount on a plain slide or a hanging drop preparation. The water mount is made by carefully dispersing some of the mold mycelium in a drop of water on a slide. A cover glass is then dropped on the preparation after which it is ready for examination. Such a water mount will yield satisfactory results with objectives with the greater working distances. With the oil-immersion objective, it may be unsatisfactory on account of the very short working distance. From this preparation, the shape and makeup of the entire mold should be determined.

Structure of Hyphæ and Fruiting Body. The examination of this part of the fungus will require higher magnifications. Hanging-drop preparations of the hyphæ should be prepared for examination with the oil-immersion objective. From this, the presence or absence of septa and other facts about the internal structure should be determined. The fruiting body should be carefully gone over in the same manner. The structure of the fruiting head, production of the spores, and other 
data should be carcfully worked out. All of this is necessary when comparing the new fungus to the described varieties. Illustrations of the more common varieties have been included in this chapter.

\section{YEASTS}

The yeasts are fungi which cause many changes in food. Many of them are desirable changes while some are undesirable. For centuries they have played an important part in the domestic life of peoples and are the bases for great industries. Foods which have a high content of carbohydrates are especially susceptible to the attack of yeasts.
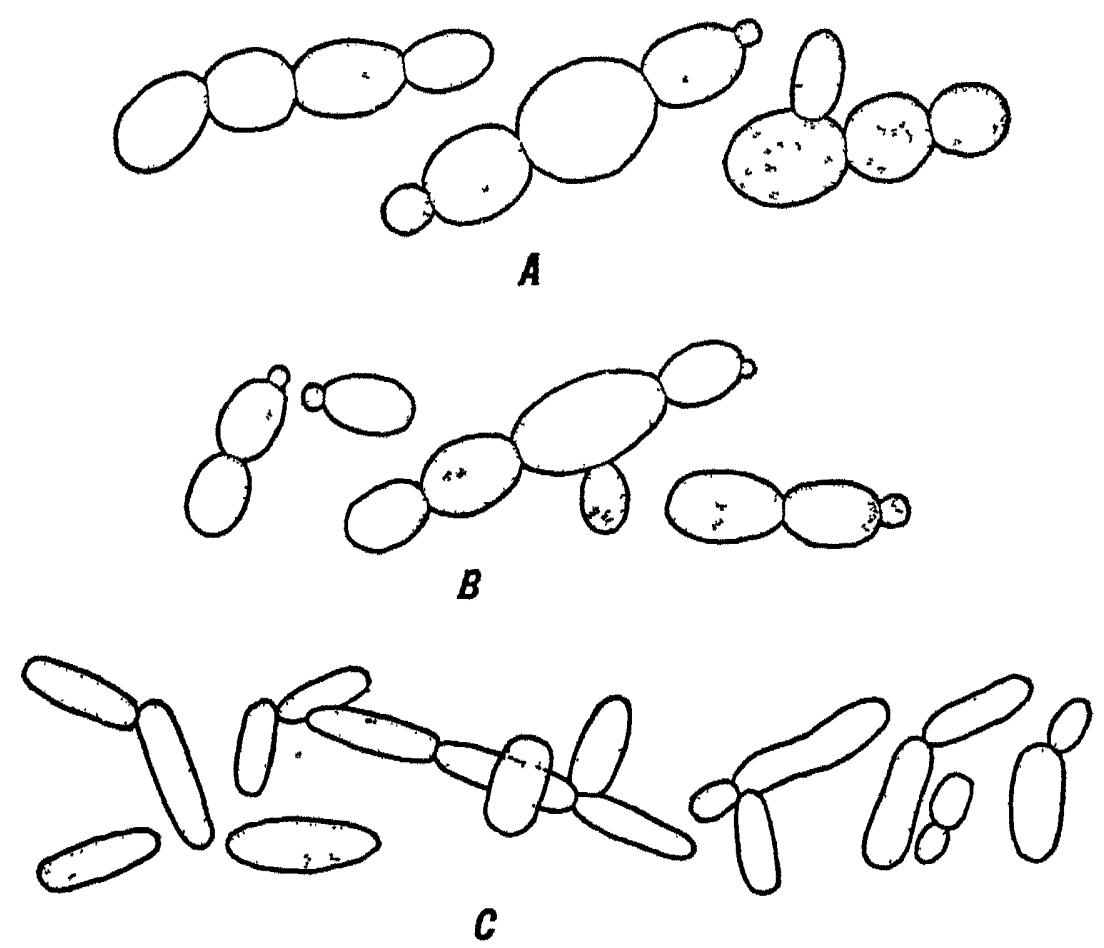

Frg. 52.-Types of Cells in Common Yeasts.

$A$, Saccharomyces cerevısı,$B$, Saccharomyces ellıpsondeus; C, Mycoderma z nnı (Adapted from Gulliermond's Les Levures )

True and Pseudo Yeasts. True yeasts are budding fungi which produce endospores. The false yeasts resemble the true yeasts very closely but differ in the fact that no endospores are formed. These have some of the properties of the molds. The torulæ (Turpin), mycodermæ (Persoona) and cryptococcus (Kützing-Vuillemin) and representatives of the false yeasts.

Classification of Yeasts. The classification of the yeasts is rather confused. This is in part due to the different points from which the subject may be viewed. The fermentologist is concerned with the physiological changes which are brought about by yeasts and does not give much attention to nomenclature. His cultures may be designated 
by such "pseudoscientific" names as "beer yeast," "wine yeast," etc., or they may be even numbered. The taxonomist, however, must be concerned with keeping this group of fungi classified and so far as possible properly named. Many investigators have classified the budding fungi. Anderson (1917) has reviewed the various investigations and a student will find a good summary with discussion of the various classifications in his paper.

Hansen has done most work on the classification of the yeasts. His classification which appeared in 1904 is now accepted as the basis for classification of the true yeasts. He made two families which are divided into nine genera. Not all of these have been accepted by microbiologists. Guillierimond (1912) does not recognize the schizosaccharomycetes which Hansen separated from the saccharomycetes by the fact that they reproduced by fission. He divides the saccharomycetes into five groups. Hansen's classification is as follows:

\section{KTY TO GENERA OF TRUE YEASTS}

(Hansen after Buchanan)

Family I. Vegetative reproduction by budding ...... Saccharomycetaceæ

$A$. Cells do not form a surface membrane at once on sugar media, i.e., do not grow exclusively at the top of the medium.

1. Spores having a single membrane.

a. Cells fusing in pairs before spore formation .......Zygosaccharomycete

b. Cells not fusing in pairs before spore formation.

(1) Spores germinate by ordinary budding..... Saccharomyses

(2) Spores germinate by means of promycelium............ Saccharomycodes

2. Spores having two membranes.... Saccharomycopsis

$B$. Cells forming a surface membrane at once by sugar media.

1. Spores spherical, hemispherical or irregular................. Pichia

2. Spores lemon shaped with pointed ends.................... Willia

Family II. Vegetative reproduction by fission........ Schizosaccharomycetes 


\section{FALSE YEASTS}

(After Guilliermond)

1. Cells generally spherical, often forming a pellicle but only after fermentation; pellicle always viscous without intervention of air................ Torula

2. Cells generally elongated. Pellicle appears at the beginning of development with intervention of air. ..... Mycoderma

3. Yeasts without ascospores, parasitic to animals................... Cryptococcus

Anderson (1917), after a careful study of the field, has proposed the following key, in which the budding phase is predominant. $\mathrm{He}$ has retained many of the names proposed by Hansen:

\section{KEY TO GENERA OF BUDDING FUNGI}

I. Ascospores known.

(Anderson, 1917)

Vegetative cells single or attached in irregular colonies, mycelium not developed, ascospores formed within isolated cells. $\quad \ldots \ldots \ldots \ldots$ (Saccharomycetaceæ)* Spores globose or void:

Spores on germination forming typical yeast cells.

Ascus formation preceded by the conjugation of gametes.................... Zygosaccharomyces

Ascus formation not preceded by the conjugation of gametes:

Spore membrane single .........2. Saccharomyces

Spore membrane double..........3. Sacchromycopsis

Spores on germination forming a poorly developed promycelium ............. Saccharomycodes

Spores pileioform or limoniform, costate....5. Willia

Spores hemispheric, angular or irregular in form on germination forming an extended promycelium .............6. Pichia

Vegetative cells produced predominately by budding but forming a mycelium under some conditions asci terminal or intercalary, differentiated from the mycelium.7. Endomyces

* The genus Schizosaccharomyces, which does not bud, and the relatively unimportant genera, Monospora and Nematospora, are not included in this key. 
II. Ascospores not known, i.e., Fungi Imperfecti.

Heavy dry pellicle formed on liquid media.... 8. Mycoderma

No distinct pellicle formed:

Vegetative cells forming a septate mycelium under exceptional conditions but predominately budding........ . .......9. Parasaccharomyces

Vegetative cells formed only by budding.

Cells apiculate, limoniform. ...........10. Pseudosaccharomyces

Cells frequently elongated into narrow nonseptate hyphal threads $\ldots \ldots \ldots \ldots \ldots$.... Pseudomonilia

Cells typically yeast-like.............12. Cryptococcus

Compressed Yeast. The greater part of the procedure in the manufacture of compressed yeast is concerned with the preparation of the medium or " mash." This is commonly prepared from a mixture of grains, rye, corn and barley being commonly used. After thorough cleaning, the barley is malted in the usual manner. The malt is later put into a kiln to stop germination. The mash is then prepared by mixing the ground grains with the malt and maintaining at a constant temperature of $60^{\circ}$ until all of the starch has been changed to soluble sugars. The mash is then soured for two reasons. It renders the nitrogenous matter available to the yeasts and also prevents the changes induced by the putrefactive bacteria. The souring process is continued until about 1.5 per cent of acid has been produced. The mash is then passed through filter presses which gives a clear filtrate called the wort. This supplies the foods for the yeasts. It is inoculated either by a pure culture or a portion of the previous day's run. An optimum temperature is maintained during which there is a vigorous multiplication of the yeast cells. The cells are carefully filtered out and pressed into cakes with or without starch. The steps in the preparation of compressed yeast are graphically shown in Fig. 53.

Yeast Foods. The use of these substances in bread making has been investigated by Kohman (1916) and his co-workers. The investigation apparently had its origin in the effect of different natural waters on the yeast fermentation in bread as noticed by a large baking company. Kohman showed that by the use of small quantities of ammonium and calcium salts and potassium bromide brought about a saving of from 50 to 65 per cent in the usual amount of yeast. Other distinct advantages connected with the use of these nutriments are also noted. Hoffman (1917) in discussing the same subject has stated:

"Since there is so much evidence in favor of all the ammonium chloride being changed to protein by the yeast, we can calculate how much protein we 


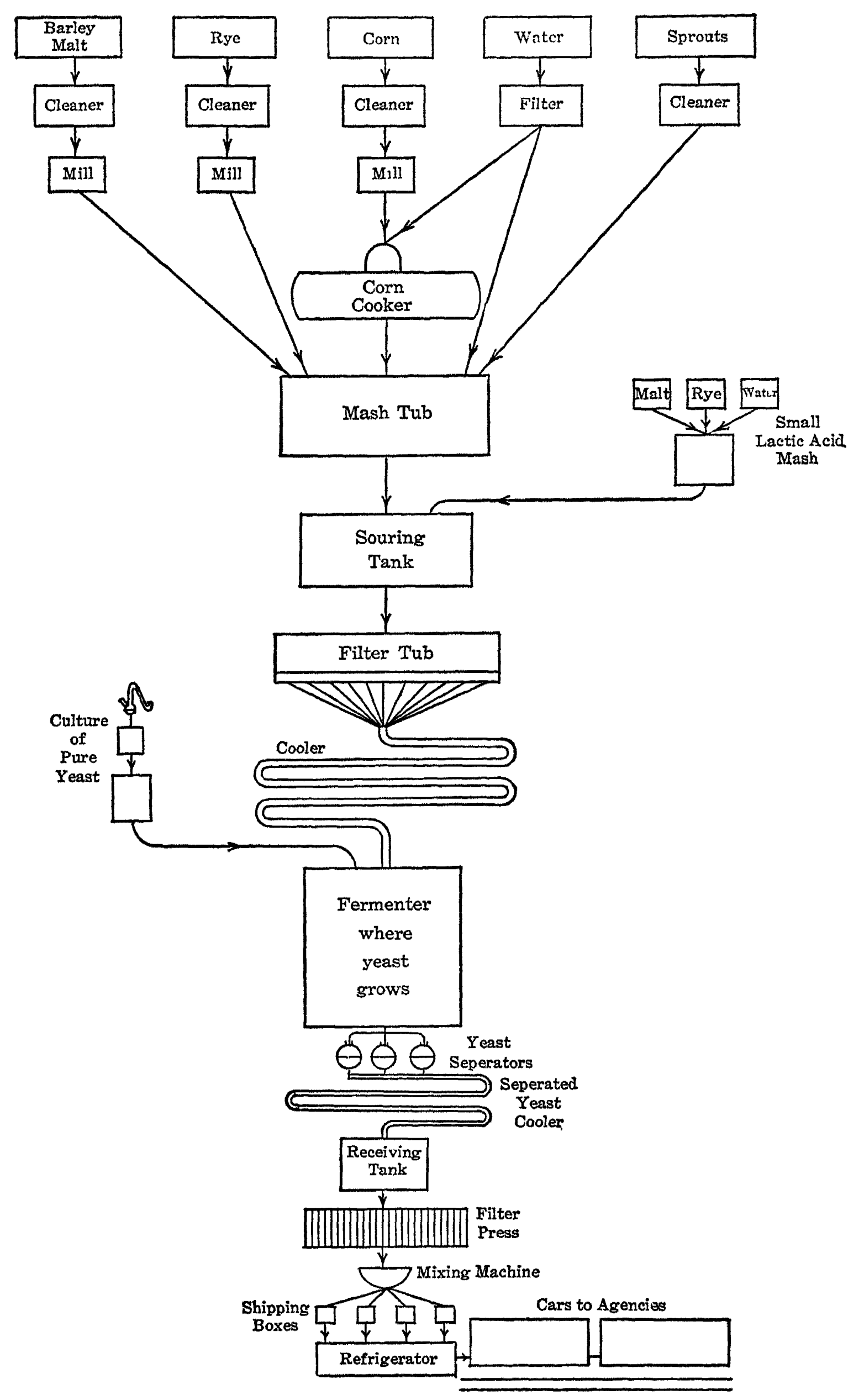

FIg. 53.-Diagram of Yeast Manufacture, (Fleischmann Co.) 
gain in the form of yeast protein, from adding $0.5 \mathrm{lb}$. of ammoniun chloride in a dough batch containing $1000 \mathrm{lbs}$. of flour and how much protem we lose by deducting one-half the quantity of yeast ( $5 \mathrm{lbs}$. would be an average amount) regularly used; $0.5^{\prime} \mathrm{lb}$. ammonium chloride contains $0.131 \mathrm{lb}$. nitrogen; this times 6.25 gives $0.82 \mathrm{lb}$. protein gained by converting the salt into albuminous matter. Compressed bakers' yeast contains 27 per cent dry matter; 5 lbs. of yeast then contains $1.35 \mathrm{lbs}$. of dry matter, 50 per cent, or $0.68 \mathrm{lb}$. of which is albuminous in character. We have not only as much protein produced by the yeast from the ammonium salt but actually more than that lost by deducting half the yeast in the dough batch. The economic value of a process which can utilize such simple substances as sugar and ammonium salts and build therefrom complex but useful food proteins cannot be overestimated."

Table XXII is taken from his work and shows clearly the effect of ammonium chloride on gas formation in bread.

\section{TABLE XXII}

SHOWING THE INCREASE IN GAS PRODUCTION IN DOUGH USING AMMONIUM CHLORIDE

(Hoffman)

\begin{tabular}{|c|c|c|c|c|}
\hline $\begin{array}{l}\text { Fermentation } \\
\text { Hours. }\end{array}$ & $\underset{\mathrm{c} \mathrm{c}}{\text { Control Dough, }}$ & Difference. & $\begin{array}{l}\text { Dough Contain- } \\
\text { ing 0 } 2 \text { Gram } \\
\text { NHCl per } 100 \\
\text { Grams Flour }\end{array}$ & Difference. \\
\hline \begin{tabular}{c}
\multicolumn{1}{c}{0} \\
05 \\
1.0 \\
1.5 \\
20 \\
2.5 \\
3.0 \\
3.5 \\
4.0 \\
4.5 \\
5.0 \\
5.5 \\
6.0 \\
Total gas in six \\
Per cent increas
\end{tabular} & $\begin{array}{l}110 \\
180 \\
285 \\
390 \\
470 \\
570 \\
665 \\
790 \\
880 \\
980 \\
1080 \\
1160 \\
1230 \\
\text { urs } \\
\text { lue to ammoniu }\end{array}$ & $\begin{array}{r}0 \\
80 \\
105 \\
105 \\
80 \\
100 \\
95 \\
125 \\
90 \\
100 \\
100 \\
80 \\
70 \\
1120 \text { c.c. } \\
\text { hloride. }\end{array}$ & $\begin{array}{c}110 \text { c.c. } \\
180 \\
280 \\
390 \\
500 \\
640 \\
785 \\
955 \\
1105 \\
1260 \\
1400 \\
1520 \\
1625 \\
\ldots \ldots \ldots \ldots \\
\ldots \ldots \ldots \ldots\end{array}$ & $\begin{array}{c}0 \\
80 \\
100 \\
110 \\
110 \\
140 \\
145 \\
170 \\
150 \\
155 \\
140 \\
120 \\
105 \\
15153 \\
353\end{array}$ \\
\hline
\end{tabular}

Methods for Study of Yeasts. The study of yeasts demands a pure culture. The securing of this pure culture is not often an easy performance. Two methods have been used and are available to microbiologists at the present time. 
Physiological Method. In this method an environment is created which is favorable to the desired fungus and unfavorable to the others. In applying this method to the yeasts, an acid medium may be used. Bacteria, unless they are acidophiles, are unable to grow in a 2 per cent tartaric acid solution. Pasteur and Hansen grew yeasts in such a medium to free them from bacteria. This method was accompanied with some uncertainty and was replaced by the dilution methods.

Dilution Methods. These have been described before. They consist of diluting or breaking up the yeast's masses in a definite amount of sterile water or physiological salt solution. From these diluted mixtures definite portions are taken for plating on a solid medium. After incubation and growth, cultures should be picked from the colonies and transferred to dextrose agar slants. The yeast is then ready for a cultural and morphological examination.

Moist Chamber Preparations. These are essentially the hanging drop mounts which have been described before. A small portion of the pure culture of yeast should be gently emulsified in a drop of dextrose broth or wort. This should be transferred to a clean cover glass and suspended over a depression in a concave slide or ring slide. The edges of the cover glass should be carefully lined with vaseline in order to make a tight seal and allow no evaporation during incubation. Such a mount will permit observations on a few cells to allow determine budding and cell shape.

Scheme for Identifying a Yeast. Whether the fungus is really a yeast or borderline mold should be determined first of all. The following points may be of assistance:

I. Spore or non-spore former

A. Spore former

1. Shape, morphology

2. Scum or membrane

3. Top or bottom yeast

4. Per cent of alcohol formed

$B$. Non-spore former

1. Non-scum former

a. Color

b. Shape, morphology

2. Scum former

a. Color

b. Morphology 
Microscopic Examination of Compressed Yeasts. Prepare a thin mixture of the yeast in distilled watcr. To this mixture or a small portion of it add a little of a 0.1 per cent solution of methylene blue. Mount a drop of this under a cover glass on a slide and enumerate the dead and living bacteria. The dead cells will stain but the living cells will not.

Test for Adulteration with Beer Yeast. Practically all of the beer yeasts ferment raffinose while the bread yeasts generally do not. One $\mathrm{gm}$. of the yeast is mixed with about 10 c.c. of a 1 per cent solution of raffinose broth and placed into a fermentation tube. Incubate this tube for about twenty-four hours at $30^{\circ} \mathrm{C}$. A blank tube without raffinose should be prepared in order to test for gas produced from stored glycogen. If the difference between the two tubes is decided (about 50 per cent) adulteration with beer yeast is probable. A greater difference renders the test more conclusive.

Efficiency of Bread Yeasts. This may be determined in different ways. A representative sample of the yeast should be obtained and

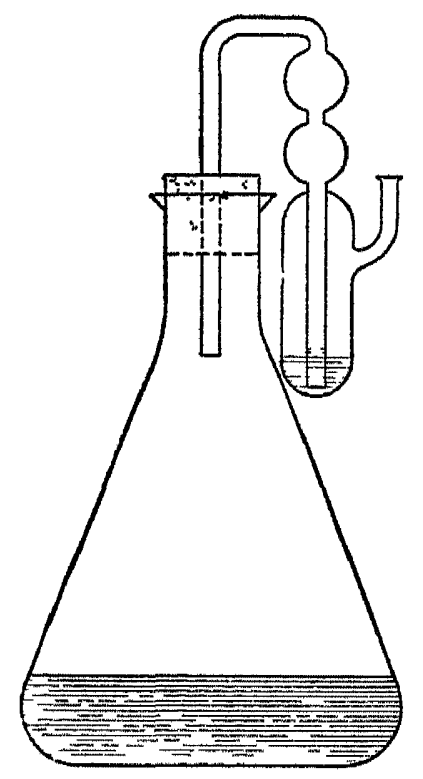

Frg. 54.-Culture Flask for Fermentation Tests. Fitted with Alwood (1908) Ventulation Tube. divided into three parts. Store one part at $20^{\circ}, 30^{\circ}$, and $40^{\circ} \mathrm{C}$. At regular intervals test each part for dead cells, gas formation, and dough-rising power. Test once or twice for adulteration with beer yeast and with mycoderma.

Dough-rising Power of Bread Yeast. Mix 100 gms. of flour, 2 gms. of yeast and 60 c.c. of distilled water by first making a thin batter of the yeast, water and a little of the flour. Then add the rest of the flour and knead for five minutes. About 3 gms. of flour should be kept with which to clean the glass rod. The dough should be formed into a cylinder and dropped into a warmed graduate cylinder which has been greased or powdered with flour. Press the dough down into the cylinder and place at $30^{\circ} \mathrm{C}$. The volume is read every thirty minutes untll the maximum is reached. Compute the maximum volume in terms of the original. Plot a curve which will show the activity of the yeast.

Determination of the Fermenting Power of Yeasts, Meissl Method. Prepare a saccharose phosphate mixture as follows: $400 \mathrm{gms}$. of saccharose, 25 gms. ammonium phosphate, 25 gms. potassium phosphate. Mix this thoroughly and weigh 4.5 gms. into an Erlenmeyer flask which 
carries a fermentation valve. Add 50 c.c. of distilled water and $1 \mathrm{gm}$. of the yeast to be tested and incubate at $30^{\circ} \mathrm{C}$.

Different types of valves are available. Alwood (1908) used a convenient type for studying the fermenting capacity of pure strains of yeasts. The valves are so constructed that the activities of the yeast may be observed and the gases from the fermentation may escape. The moisture is retained by some absorbent such as calcium chloride or sulphuric acid. The apparatus should be weighed before and after the run. The Alwood valve is shown in Figs. 54 and 55.

Determination of the Fermenting Power of Yeasts, Kusserow's Method. Put 400 c.c. of 10 per cent saccharose solution and 10 gms. of FIg. 55.-Detail of Ventilation Tubs the yeast under examination into the for Culture Flask. (Alwood, 1908.) fermentation flask of the Kusserow apparatus. Warm the flask and solution to $30^{\circ} \mathrm{C}$. before starting. After the apparatus has stood for an hour at $30^{\circ} \mathrm{C}$. it is connected and
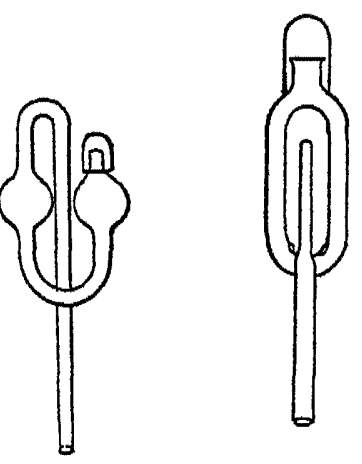

FIG. 56.-Types of Ventilation Valves which may be Used with Erlenmeyer Flasks for Testing the Fermenting Power of Fung1. the amount of gas formed in the next half hour is

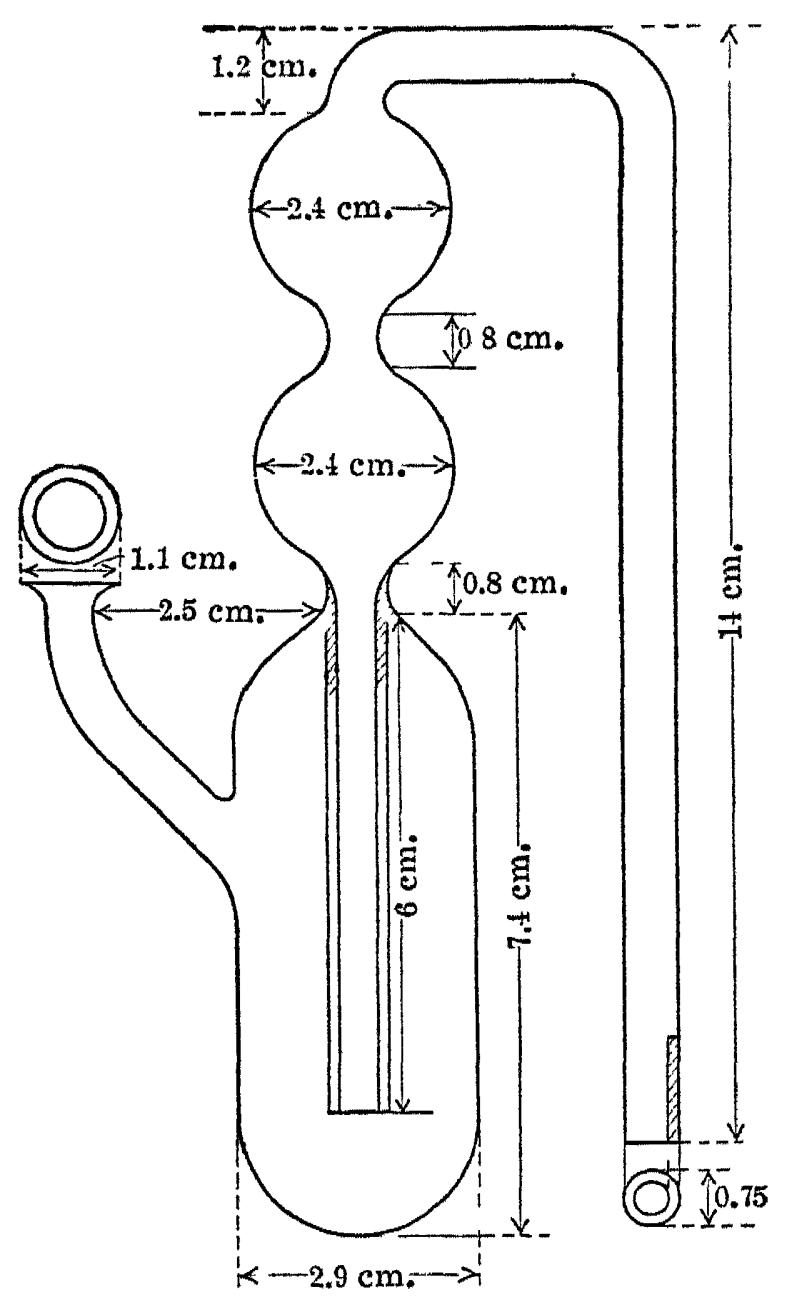
measured; it should be 250 c.c. The amount of gas for the first and second half hours is usually about 50 and 150 c.c., respectively. Five gms. of yeast in 400 c.c. of 10 per cent saccharose solution in a flask fitted with a fermentation valve will cause a loss of about $6.5 \mathrm{gms}$. of carbon dioxide in twentyfour hours if distilled water is used; with tap water the loss should approximate 8 to 12 gms.

Determination of Mycoderma in Bread Yeast. Press the yeast with a sterile spatula into the bottom of a small Petri dish and incubate at $37^{\circ} \mathrm{C}$. for two days. If the yeast contains a mycoderma, pure white characteristic colonies will appear on the yellowish background of the bread yeast. 


\section{Bread Making}

The flour is one of the first things to which the baker pays special attention. Miner (1917), in describing the manufacture of flour, divides it into three general types:
I. Low grade
II. Clear flour
III. Patent flour

The baker demands a uniform flour and to secure it he may resort to blending. Most of them use a baker's "patent" flour which may be superior to the domestic "patent." This is carefully sifted and the other ingredients added such as salt, sugar, lard, malt extract, milk, water, yeasts, etc. In the straight dough method all of these are mixed and allowed to ferment for about five hours in a long trough. The temperature of this room is maintained at $27^{\circ} \mathrm{C}$. with a relative humidity of 75 per cent. From the fermenting room the dough is taken to a divider where it is cut into any desired size. It may be worked a little, allowed to rise and molded for baking. In the larger bakeries this may be entirely a machine process.

Yeast in Bread Making. Bread is one of the oldest foods in the preparation of which fermentative changes are involved. In the early days before commercial yeasts were available, the housewife had to reply on spontaneous fermentations. The organisms which induced these chance fermentations got into the sponge either from the air or along with one of the ingredients. Quite often, a portion of one batch was taken out and saved over to inoculate the next batch of bread. To-day the baker does not have to rely on liquid brewers' yeast nor the housewife on the uncertain fermentations of wild yeasts. The production of compressed and dried yeasts caused a distinct advance to be made in the production of leavened foods. A mixture of yeast and flour will ferment, but the addition of other substances such as potato water, malt water, will greatly accelerate the action of the yeasts. All of the constituents of the flour which are given above are concerned in bread making. The carbohydrates are precursors of the carbon dioxide and in the baked loaf they impart a rich brown color to the crust. According to Wardall (1910) the flavor of the bread is not dependent on the strain of yeast which is used as a leaven. She used thirty-three pure strains of yeasts and could observe no difference in the flavor of bread leavened by them. She regarded the time of incubation as too short for the development of any special flavor. 
Self-rising Bread. This has been known for generations under the name of " salt-rising" bread. As suggested by the name, the fermentation is a spontaneous one. Hunt and Wessling (1917) give the following instructions for making:

1 cup milk

2 tablespoons cornmeal, white 1 teaspoon salt
1 tablespoon sugar

Butter (if used) 1 tablespoon

Flour. "Scald the milk. Allow it to oool until it is lukewarm; then add salt, sugar and cornmeal. Place in a fruit can or heavy crock or pitcher and surround by water at about $120^{\circ} \mathrm{F}$. Allow the mixture to stand six or seven hours or until it shows signs of fermentation. If it has been fermented sufficiently, the gas can be heard as it escapes."

As shown in the above paragraph, the fermentation is a chance one. It is, then, almost an uncontrolled process. The agent inducing this fermentation has received much study. Kohman (1912) has given a review of the literature and some of the data and references mentioned here have been taken from his interesting paper. There seems to be two sides to the question of the organism which is concerned. One side argues that the fermentation is induced by wild yeasts which get in from the air. In support of this are the investigations of Mitchell (1908), Bailey (1914), and others. That it is a bacterial change is supported by the work of Heinemann and Hefferan (1909) and Kohman (1912). The middle ground that both yeasts and bacteria are concerned is taken by Lehman (1894), Peters (1889) and perhaps Harrison (1902). Lehman isolated an organism which he called Bacillus levans. Heinemann and Hefferan isolated a bacillus from corn meal which had many of the characteristics of Bacillus bulgaricus. They proved that the organism came from the corn meal and believed that, in self-rising bread, the high amount of acid formed by this bacillus ( 1.65 per cent) from the milk, united with the inorganic salts to form carbon dioxide. The extensive work of Kohman (1912) indicated that bacteria were the important agents in the fermentation. He believes that members of the colon group are concerned. The gases produced by the salt-rising bacteria consisted of two-thirds hydrogen and about one-third carbon dioxide. These bacteria aerate the bread by decomposing some of the constituents possibly the carbohydrates. The organisms are probably of the type $B$. levans (Lehman). Woodward (1911) believed that the fermentation in salt-rising bread was due to the presence of organisms which are introduced with the corn meal. The organism which was isolated was not a yeast but belonged to another group. It was advised 
that the milk be sterilized hefore the batter was mixed since this allowed more uniform results and decreaned the possibilitios of the bad flavors which are so often inherent in this type of bread.

Examination of Bread. The bacterial examination of bread is important for two reasons: first, the possibility of bread spreading disease bacteria; and secondly, the relation of bacteria to undesirable fermentations and changes which may take place.

Bread is generally regarded as an absolutely safe article in the diet since it is heated before eaten. Roussel (1908) stated that the interior of the loaf reached $101-103^{\circ} \mathrm{C}$; 估e crust reached $125-140.5^{\circ} \mathrm{C}$. Such temperatures were regarded as sufficient to kill the vegetative cells but not the spores except they be present in the crust. Tubercle bacilli in the dough were reported to have retained their vitality. From these data Roussel states that mechanical mixers should be used in order to prevent the spread of discase. Auche $(1909,1910)$ introduced turbercle bacilli into dough before it was made into loaves. The loaves weighed up to $2 \mathrm{~kg}$. The bacilli were killed in the baking process but the author regards it as a possibility that bacteria may pass through the baking process. In the latter paper eited, this same author added such bacteria as the following to bread dough before baking: B. typh , B. paraiyphi, B. dysenterice (Shiga), B. dysenterice (Flexner), B. col, Strept. pyogenes, Staph. aureus and a variety of proteus. Cultures made from the loaves after baking were sterile. Auche states that possibly more resistant bacteria would pass through the baking process. He states that the greatest care must be exercised to prevent contamination after the loaves have left the oven. Fenyvessy and Denies (1911) found that if the temperature of bread reached $94-104^{\circ} \mathrm{C}$. during the baking process, the pathogenic bacteria, if accidentally present, would be destroyed. Gaujoux (1911), discusses some studies of this question. He found the Koch's bacillus (Bacillus tuberculosis) did not survive the temperature of baking bread but assumed that it might pass through the temperatures employed for pastries. Howell (1912) examined bread collected from various districts in Chicago. In general it was found that bread made under clean conditions had a lower bacteria content and fewer streptococci. Jacobs, LeClerc and Mason (1914) studied the effect of wrapping. Wrapped bread had fewer bacteria on the surface. From the above discussion it would seem that bread may carry infection. The evidence is not altogether convincing, however, that it does. Hansen (1913) has reported a typhoid fever epidemic which may have been caused by bread. In this case the bread may have been contaminated after baking.

Slimy or ropy bread has received quite a little attention from the 
bacteriologists. Russell (1903) examined a sample of slimy bread and isolated $B$. mesentericus vulgatus. Temperature studies indicated that the organisms could pass through the baking temperature. Watkins (1906) has studied the question and advised that the flour be subjected to examination when this infection appears. Kornauth (1912) also advised an examination of the flour for the organism. Williams (1912) found a capsulated organism in a case of slimy bread. Kayser.(1911) found that the bacteria causing this infection were introduced with the yeast. He stated that 1 to 2 liters of vinegar added to each $100 \mathrm{~kg}$. of the flour would prevent the infection. Kayser and Delavel (1912) isolated bacteria which were related to the mesentericus group. When a bakery once becomes infected with members of this group, it is disinfected with great difficulty. Kayser suggests the use of acidulated water for washing all of the apparatus and even states that some apparatus may have to be discarded.

Wright (1916), when studying musty bread, found an Aspergillus and Rhrzopus nigricans. Further experimental work indicated that the $R$. nigricans produced a mustiness in bread while Aspergillus produced a sourness. It was believed that the mustiness resulted from proteolytic enzymes from the $R$. nigricans.

Watkins' Method for Detecting in the Flour the Organisms which Cause Ropy Bread. This author (Watkins, 1906) finds the following procedure a delicate one for determining whether a flour contains those bacteria which cause ropy bread. The test is rapid and the author states that there is little possibility of a sound falling to pass the test because they do not yleld appearances of ropiness in the time proposed as a limit. Ten large test tubes ( 6 in. by 1 in ) are thoroughly boiled in water for one hour, washed and drained. When drained sterilize in the oven at $232^{\circ} \mathrm{C}$. for three hours The tubes should be thoroughly sterilized. Cool and then place into each tube a finger of bread $3 \mathrm{~m}$. by $\frac{1}{2} \mathrm{in}$, cut from the center of the same two-day old loaf. The average weight of each loaf should be $5 \mathrm{gms}$. Moisten with distilled water and plug the tubes. Sterilize by boiling in water on three successive days. To test the flour, 2 gms. are well mixed with water and placed in bolling water for thirty minutes. To the series of ten tubes add successively from 1 to $7 \mathrm{c} \mathrm{c}$. of the flour mixture, leaving three tubes to serve as checks. Number the tubes in rotation. Incubate at $28^{\circ} \mathrm{C}$. and at the end of twenty-four hours examine for ropiness.

Kornauth's Method for Detecting Organisms in Flour which Cause Ropy Bread. This is a modification of Kuhl's method and is carried out as follows: 280 gms. of flour, 140 c.c. of salt water, and $0.6 \mathrm{gm}$. of yeast are made into a loaf. After baking, the loaf should be stored at $25^{\circ} \mathrm{C}$. for forty-eight hours. If the organisms causing ropy bread were present in the flour, the loaf will have a characteristic odor. Several loaves should be made at the same time with sound flour as a check. 


\section{BIBLIOGRAPHY}

Alwood, W. B. 1908. The Fermenting Power of Some Pure Yeasts and Some - Associated Fungi. U. S. Dept. Agriculture, Bureau of Chem., Bull. 111.

Anderson, H. W。 1917. Yeast-like Fungi of the Human Intestinal Tract. Jour. Infect. Dis., 21, 341-386.

Auche, B. 1910. Bacteria in Bread. Semaine Méd, 35, Brit. Med. Jour., 1910, No. 2569, 771-772.

Avche, B. 1909. The Destruction of Tubercle Bacilli in Bread by Cooking. Comp. Rend. Soc. Biol., 66, 800-802.

BaILex, E. H. S. 1914. Sanitary and Applied Chemistry. The Macmillan Co., New York.

BALLAND, M. 1894. Sur la temperature intériure du pain sortant du four. Comp. Rend. Acad. Sci., 117, 519-521.

Cadwell, H. V. 1917. Laboratory Control in Yeast Making. Amer. Food Jour., 12, 151.

Fenyvessy, B. von, Denies, L. 1911. Is Baked Bread Sterile? Zeit. Hyg., $69,223-224$.

Gaujoux, E. 1911. Concerning Bread and Pastry. Rev. Hyg. et Pol. Sanit., $33,1176-1180$.

Goutd, R. G. 1917. The Manufacture of Commercial Yeast. American Food Journal, 12, 143-148.

Gound, R. C. The Manufacture of Bread. Amer. Food Jour., 12, 195-201.

Hansen, P. 1913. A Study of Typhoid Fever in Rockford, Illinois, in the Late Summer and Fall of 1913. Illinois State Water Survey Bull., 11, $384-430$.

Harrison, F. C. 1902. Yeast and its Household Use. Ontario Ag. Bull., $118,17 \mathrm{pp}$.

Heinemann, P. G., and Hefferenan, M. 1909. Note on a Lactic Acid-forming Bacillus Closely Resembling $B$. bulgaricus Isolated from Corn Meal. Science, 29, 1011.

Hofrman, C. H. 1917. The Utilization of Ammonium Chloride by Yeast. Jour. Ind. Eng. Chem., 9, 148-153.

HoweLL, K. 1912. The Bacterial Examination of Bread. Amer. Jour. Pub. Health, 2, 321-324.

JACOBS, B. R., LeClerc, J. A., and Mason, MaUd L. 1914. A report on a Chemical and Bacteriological Study of Wrapped Bread. Amer. Jour. Pub. Health, 4, 721-732.

KaYSeR, E. 1912. Bacterial Changes in Bread. Chem. Abstracts, 6, 1067.

Kayser, E., and Delaver, H. 1912. A Study of Stringy Bread. Comp. Rend., 153, 576-578. Chem. Abstracts, 6, 515.

Komman, H. 1912. Salt Rising Bread and Some Comparisons with Bread Made from Yeasts. Jour. Eng. Ind. Chem., 4, 20-30; 100-106.

Komman, H. A., et al. 1916. On the Use of Certain Yeast Nutriments in Bread Making. Jour. Ind. Eng. Chem., 8, 781-789. 
Kohman, H. A. 1917. Feeding the Yeast in Bread Making. American Food Journal, 12, 35-38.

KonNaUth, K. 1912. Ropy Bread. Chem. Abstracts, 7, 847.

LeHMAN, K. B. 1894. Ueber die Sauerteiggärung und Beziehungen des Bacillus levans zum Baclllus coli communis. Cent. Bakt., 15, 350-354

Marchland, H. 1909. The Cooking of Bread. Meun. Franc., 25, 208-10. Exp. Sta. Rec., 22, 64.

Miner, A. 1917. The Manufacture of Flour. Amer. Food Jour., 12, 27-31.

Mitchell, M. 1908. Course in Cereal Foods and their Preparation for Movable Schools of Agriculture. U.S. Dept. Agric. Office Exp. Stations. Bull., 200.

Peters, W. L. 1889. Die Organismen des Sauerteigs und Ihrer Bedeutung fur Brotgahrung. Botan. Zeit., 47, 405, 420 and 435.

Rousseis, J. 1907. The Survival of Pathogenic Bacteria in Bread after Baking. Rev. Intend. Mil., 20, 122-123. Chem. Absts., 2, 1168.

Russeld, H. L. 1903 . Sticky and Slimy Bread and its Causes. Ann. Rept. Wis. Ag. Exp. Sta., 565.

SchNOEDER-ORELLI, O. 1911. Investigations on the Growth and Spread of Decay Fungi in Storage Fruit. Landw. Jahrb. Schweiz., 25, 225-226. Cent. Bakt. Abt. II., 32, 161-169.

Stevens, N. 1917. Rhizopus Rot of Strawberries in Transit. U. S. Dept. Agriculture, Bull., 531.

Thом, C. 1905. Some Investigations from the Study of Dairy Fungi. Journal of Mycology, 2, 117-124.

Thом, C. 1906. Fungi in Cheese Ripening. U. S. Dept. Agriculture, Bureau of Animal Industry Bull., 82.

Thом, C. 1910. Cultural Studies of Species Penicillium. U. S. Dept. Agriculture, Bureau of Animal Industry Bull., 118.

Wardall, Ruth A. 1910. The Relation of Yeast to Flavor in Bread. Jour. Home Economics, 1910, 75-91.

Watkins, E. J. 1906. Ropiness in Flour and Bread and its Detection and Prevention. Jour. Soc. Chem. Ind., 25, 350-357.

Williams, Anna W. 1912. A Study of Ropy Bread. Biochem. Bull., 1, 529-534.

Woodward, W. 1911. The Leavening Agent in Salt Rising Bread. Jour. Home Economics, 3, 100-101.

Wright, A. M. 1916. A Cause of Mustiness in Bread. Jour. Soc. Chem. Ind., 35, 1045-1046. 


\section{CHAPTER VIII}

\section{INTESTINAL BACTERIA}

THE flora of the mouth is dependent upon many factors and is, therefore, continually changing. Excluding the various infections of the mouth which will not be considered here, the bacteria in the mouth are not especially significant. There are, however, several features which, compared with other body fluids, are worthy of mention. The mouth often contains a large number of bacteria and many kinds.

Germicidal Action of Saliva. The work which is available on this subject is neither conclusive nor extensive. Huggenschmidt (1896) studied the various factors which contribute to the protection of the oral cavity and from such factors as bactericidal action of the sputum, mechanical action of the saliva, action of the mucous membrane and bacterial antagonism concluded that the mechanical action of the sputum was the most important factor. Sanarelli (1891) filtered saliva through a Chamberland filter and tested the bactericidal action of the filtrate. He found that saliva did possess a germicidal action quite similar to that exhibited by other body fluids, such as blood, aqueous humor, etc. The speed of the reaction depended upon the initial number of bacteria introduced into the saliva. If a small number of cells (three or four hundred) were introduced, all were killed in a short time but when a few thousand cells were introduced, there was usually a decrease followed by an indefinite increase. With Bacterium diphtheriæ and the pneumococcus no germicidal action was noticed. They were found alive after twenty-eight and forty days. The cause of the germicidal action -was not determined. Gordon (1916) carried out his experiments on this subject in culture media and found a germicidal action against the meningococcus, which he attributed to the antagonistic effect of other bacteria in the saliva. These were supposed to be mixed streptococci.

Effects of Tobacco Smoke on Bacteria in the Mouth. This is a subject of more popular interest and has been of much use to those who wish to justify or decry the habit of smoking. Koerner (1896) found a decided reduction of bacteria in the mouth due to tobacco smoke. 
From the facts secured, this author states that strong smokers (consuming more than one dozen cigars a day) seldom have dental caries. Dunnon (1902) found no bactericidal action on B. tetanus, B. typhi or Leptothrix bucchalis and staphylococcus. Bacillus tuberculosis was inhibited. The action was not due to the nicotine content but to products of combustion. Arnold (1907) compared the bactericidal activity of hay and tobacco smoke. Rideal (1903) reported a slight bactericidal action of tobacco smoke. Ellis (1909) has stated that any beneficial effects resulting from the use of tobacco, is easily overbalanced by the habit of spitting so prevalent among smokers.

\section{The Stomach}

The bacteriological aspects of the stomach have been concerned mostly with its diseases. The bacteria of the stomach gain entrance with the food and drink. The germicidal action of the saliva may probably be neglected as a factor influencing the number of bacteria passing through the mouth.

Germicidal Action of Gastric Juice. The gastric juice is the normal secretion of the stomach. It contains between 2 to 3 per cent solids and about 0.2 per cent of free hydrochloric acid, which is the most powerful of any acid combination in the stomach. A condition of hyperacidity exists when a decidedly greater amount of hydrochloric acid is present than normally, and hyperacidity when the amount of acid is below normal. During conditions of hypoacidity, fermentation may be active with the formation of lactic and butyric acids. Some hold that gastric ulcer and gastric catarrh may be due to a reduced acidity.

That the gastric juice is germicidal was noticed by Spallanzani in 1734. When he moistened meat with gastric juice, it did not putrefy. Meat which was not so treated but which was moistened with other substances, such as water, developed a putrid odor very quickly. $\mathrm{He}$ opened a snake eighteen days after it had swallowed a lizard and while the lizard was partly digested, it had not decomposed. Other experiments by this early scientist demonstrated that putrefaction could be stopped by gastric juice.

Koch (1884) fed cholera vibrios to dogs and after a few hours found that they. had been destroyed. He stated that cholera vibrios were destroyed in the stomach under normal conditions and that if they passed through, it was due to some abnormal condition. Falk (1883) secured no action on $B$. tuberculosis. Kurloff and Wagner (1889) found a selective action of the gastric juice. Cholera vibrio, B. typhi 
and Ps. pyocyaneus were destroyed but B. tuberculosis, B. anthrax, and $B$. tetanus (spores) were not affected. Strauss and Wurtz (1889) showed that anthrax bacilli were killed in fifteen minutes and that the destructive factor in the gastric juice was the hydrochloric acid. The same conclusion was reached by Kabrhel (1890). Hamburger (1890) first called attention to the fact that some of the acid was bound and that the free acid had a stronger germicidal effect. This was proven by testing the action of a solution of $\mathrm{HCl}$ with one of the same strength containing peptone. Kianowsky (1891) showed a direct relation between the number of bacteria in stomachs and the amount of acid. With patients possessing a faulty acid secretion many bacteria were found. Cadeac and Bournay (1893) found that Ps. pyocyaneus was destroyed after six hours and that $B$. anthrax and $B$. tuberculosis were not killed. Pettenkofer (1893) reported some startling work which was carried out in 1893. This was done soon after the great epidemic of cholera in Hamburg and Altoona. He proposed the $X, Y, Z$ theory for cholera where

$$
\begin{aligned}
& X=\text { bacterium; } \\
& Y=\text { host or soil; } \\
& Z=\text { environment. }
\end{aligned}
$$

He stated that $X$ without $Y$ and $Z$ would not cause cholera. So thoroughly did these workers believe in this theory that Pettenkofer and Emmerich drank pure cultures of the cholera vibrio. Each mixed 1 c.c. of a broth culture with 100 c.c. of 1 per cent sodium bicarbonate solution and drank it. Pettenkofer did not change his usual manner of living and, after having a diarrhœa for four days, came back to normal. Emmerich's case ran a longer time. In this case the sodium bicarbonate neutralized the $\mathrm{HCl}$ of the gastric juice. The bacteria thus passed through the stomach and produced the usual results.

Stern (1908) does not regard the gastric juice as such a barrier against bacterial invasion as some of the former workers. He regards persons with hyperacidity as fortunate from this standpoint, but in no way absolutely protected. Bile was found to decrease the bactericidal action of the gastric juice. When proteins were present, cholera vibrios were found to live a long time. Pepsin was found to strengthen the action of the acid. Hansen (1912) infected food with B. coli and secured only a slight decrease of bacteria in the stomach. SchultzSchultzenstein (1908) found the gastric juice to be more germicidal than hydrochloric acid solution of the same strength. Gregersen (1916) reported some of the more recent work on this subject. He studied the action of the acid in the juice and whether any other factors were 
important. Tables XXIII and XXIV are taken from his paper and are quite significant.

\section{TABLE XXIII}

STOMACH CONTENT $3 / 4$ HOUR AFTER EWALD'S TEST BREAIKFAST. TEMPERATURE $37^{\circ} \mathrm{C}$. STAPHYLOCOCCUS PYOGENES AUREUS

(After Gregersen, 1916)

\begin{tabular}{|c|c|c|c|c|c|c|c|}
\hline & Diagnosis & G & $\mathrm{K}$ & $\mathrm{Al}$ & $\mathrm{Ph}$ & Pepsin & Time of Destruction. \\
\hline 1 & Cancer ventriculi & -30 & -14 & 2 & 6 & 0 & No destruction \\
\hline 2 & Neurasthenra & -24 & -10 & 4 & 10 & 0 & No destruction \\
\hline 3 & Alcoholismus chron & -20 & -12 & 0 & 1 & 0 & No destruction \\
\hline 4 & Cancer ventriculi. & -18 & -6 & 1 & 4 & 0 & No destruction \\
\hline 5 & Alcoholismus chron. & -16 & -10 & 1 & 5 & 0 & No destruction \\
\hline 6 & Vicus ventriculi. & -14 & 0 & 24 & 28 & 4 & No destruction \\
\hline 7 & Cancer ventriculi. & -3 & 6 & 14 & 20 & 35 & 90 minutes \\
\hline 8 & Arthroctis chron.. & -3 & 10 & 17 & 22 & 3 & 90 minutes \\
\hline 9 & Anæmia . & 0 & 6 & 10 & 12 & 45 & 90 minutes \\
\hline 10 & Neurasthenia & 0 & 12 & 24 & 32 & 4 & 38 minutes \\
\hline 11 & Ulcus ventriculi & 0 & 23 & 35 & 47 & 5 & 38 minutes \\
\hline 12 & Ulcus ventriculi & 0 & 8 & 12 & 20 & 6 & 45 minutes \\
\hline 13 & Currhosis hepatis. & 0 & 14 & 34 & 44 & 5 & 45 minutes \\
\hline 14 & Neurasthenıa & 3 & 10 & 16 & 24 & 5 & 9 minutes \\
\hline 15 & Colitis & 5 & 14 & 20 & 24 & 4.5 & 6 minutes \\
\hline 16 & Neurasthenia & 5 & 18 & 22 & 30 & 5 & 6 minutes \\
\hline 17 & Ulcus ventriculi. & 5 & 14 & 20 & 24 & 6 & 8 minutes \\
\hline 18 & Colitis & 6 & 12 & 16 & 22 & 4 & 5 minutes \\
\hline 19 & Abstipatic & 8 & 22 & 29 & 34 & 6 & 6 minutes \\
\hline 20 & Colitis & 10 & 22 & 30 & 38 & 6 & 5 minutes \\
\hline 21 & Ulcus ventriculı & 13 & 34 & 50 & 60 & 6 & 3 minutes \\
\hline 22 & Colitis & 20 & 28 & 30 & 34 & 4.5 & 1.5 minutes \\
\hline 23 & Ulcus ventriculi. & 20 & 40 & 54 & 60 & 5.5 & 2 minutes \\
\hline 24 & Ulcus ventriculi. & 26 & 48 & 58 & 66 & 55 & 1.5 minutes \\
\hline 25 & Ulcus ventriculi. & 32 & 42 & 50 & 56 & 6 & 1 minute \\
\hline 26 & Ulcus ventriculi & 46 & 58 & 66 & 74 & 6 & .5 minute \\
\hline 27 & Ulcus ventriculi. . & 50 & 64 & 70 & 78 & 6.5 & .5 minute \\
\hline
\end{tabular}

The letters at the top of the columns refer to the reaction as determined by the following indicators:

$G=$ Gunzberg's reagent. Indicates free hydrochloric acid.

$K=$ Congo red. The blue color indicates free hydrochloric acid. Other mineral acids may also be indicated by this reagent.

$A l=$ Sodium alizarin sulphonate, which indicates all available except that which is combined.

$P h=$ Phenolphthalein, which indicates total acidity. 
It will be noticed from the table that, without free acid, the stomach may not be depended upon to kill bacteria. As the amount of free hydrochloric acid increases, however, the time required for the destruction of bacteria is reduced. The same facts were obtained experimentally by this author and are reported in Table No. XXIV.

\section{TABLE XXIV}

STOMACH CONTENTS WITH OR WITHOUT HCl OR NaOH. STAPH. PYOGENES AUREUS. TEMPERATURE $37^{\circ} \mathrm{C}$.

(After Gregersen, 1916))

\begin{tabular}{|c|c|c|c|c|c|c|}
\hline & G & $\mathrm{K}$ & $\mathrm{Al}$ & $\mathrm{Ph}$ & $\begin{array}{c}\text { Pep- } \\
\text { sin. }\end{array}$ & $\begin{array}{l}\text { Destruction } \\
\text { Time. }\end{array}$ \\
\hline $\begin{array}{l}\text { No. 4. Without addition } \ldots \ldots \ldots \\
\text { After addition of } \mathrm{HCl} \ldots \ldots \\
\text { After addition of } \mathrm{HCl} \ldots \ldots \\
\text { After addition of } \mathrm{HCl} \ldots \ldots \\
\text { After addition of } \mathrm{HCl} \ldots \ldots\end{array}$ & $\begin{array}{r}-18 \\
-12 \\
0 \\
5 \\
10\end{array}$ & $\begin{array}{r}-6 \\
0 \\
0 \\
17 \\
22\end{array}$ & $\begin{array}{r}1 \\
7 \\
19 \\
24 \\
29\end{array}$ & $\begin{array}{r}4 \\
11 \\
23 \\
27 \\
32\end{array}$ & $\begin{array}{l}0 \\
\cdots \\
\cdots \\
\cdots \\
\cdots\end{array}$ & $\begin{array}{l}\text { No destruction } \\
\text { No destruction } \\
45 \text { minutes } \\
6 \text { minutes } \\
3 \text { minutes }\end{array}$ \\
\hline $\begin{array}{l}\text { No. 11. After addition of } \mathrm{NaOH} \ldots \\
\text { Without addition . . . . . } \\
\text { After addition of } \mathrm{HCl} \ldots \\
\text { After addition of } \mathrm{HCl} \ldots \\
\text { After addition of } \mathrm{HCl} \ldots \\
\text { After addition of } \mathrm{HCl} \ldots\end{array}$ & $\begin{array}{r}-23 \\
0 \\
5 \\
10 \\
20 \\
40\end{array}$ & $\begin{array}{r}0 \\
23 \\
28 \\
33 \\
43 \\
63\end{array}$ & $\begin{array}{l}12 \\
35 \\
40 \\
45 \\
55 \\
75\end{array}$ & $\begin{array}{l}24 \\
47 \\
52 \\
57 \\
67 \\
87\end{array}$ & $\begin{array}{l}5 \\
\cdots \\
\cdots \\
\cdots \\
\cdots \\
\ldots\end{array}$ & $\begin{array}{l}\text { No destruction } \\
38 \text { minutes } \\
6 \text { minutes } \\
3 \text { minutes } \\
1.5 \text { minutes } \\
1 \text { minute }\end{array}$ \\
\hline $\begin{array}{l}\text { No. 23. After addition of } \mathrm{NaOH} \ldots \\
\text { After addition of } \mathrm{NaOH} \ldots \\
\text { After addition of } \mathrm{NaOH} \ldots \\
\text { After addition of } \mathrm{NaOH} \ldots \\
\text { Without addition } \ldots\end{array}$ & $\begin{array}{r}-20 \\
0 \\
5 \\
10 \\
20\end{array}$ & $\begin{array}{r}0 \\
20 \\
25 \\
30 \\
40\end{array}$ & $\begin{array}{l}14 \\
34 \\
39 \\
44 \\
54\end{array}$ & $\begin{array}{l}20 \\
40 \\
45 \\
50 \\
60\end{array}$ & $\begin{array}{l}\cdots \\
\ldots \\
\cdots \\
\cdots \\
5.5\end{array}$ & $\begin{array}{l}\text { No destruction } \\
45 \text { minutes } \\
8 \text { minutes } \\
3 \text { minutes } \\
1.5 \text { minutes }\end{array}$ \\
\hline $\begin{array}{l}\text { No. 22. After addition of } \mathrm{NaOH} \ldots \\
\text { After addition of } \mathrm{NaOH} \ldots \\
\text { After addition of } \mathrm{NaOH} \ldots \\
\text { Without addition. } \ldots\end{array}$ & $\begin{array}{r}-8 \\
5 \\
10 \\
20\end{array}$ & $\begin{array}{r}0 \\
13 \\
18 \\
28\end{array}$ & $\begin{array}{r}2 \\
15 \\
20 \\
30\end{array}$ & $\begin{array}{r}6 \\
19 \\
24 \\
34\end{array}$ & $\begin{array}{l}\cdots \\
\cdots \\
\cdots \\
4.5\end{array}$ & $\begin{array}{l}\text { No destruction } \\
9 \text { minutes } \\
4 \text { minutes } \\
1.5 \text { minutes }\end{array}$ \\
\hline $\begin{array}{l}\text { No. 27. After addition of } \mathrm{NaOH} . \\
\text { Without addition. . . . . . . }\end{array}$ & $\begin{array}{l}10 \\
50\end{array}$ & $\begin{array}{l}24 \\
64\end{array}$ & $\begin{array}{l}30 \\
70\end{array}$ & $\begin{array}{l}38 \cdot \\
6.5\end{array}$ & $\begin{array}{l}6 \\
\cdots\end{array}$ & $\begin{array}{l}2 \text { minutes } \\
.5 \text { minute }\end{array}$ \\
\hline
\end{tabular}

The bacterial content of the stomach as shown above is quite dependent upon the acidity of the stomach. In order to standardize these determinations, the acidity of the stomach is usually examined after a test meal. The Ewald test breakfast is much used, which consists of two pieces of toast and 400 c.c. of water. The stomach is pumped out at the end of about one hour. 
The contents should be carefully filtered and the following determinations made.

Total Acidity. Filter 10 c.c. of the gastric contents and add about four drops of phenolphthalein. Titrate to a faint pink color with $\mathrm{N} / 10 \mathrm{NaOH}$. Calculate the number of cubic centimeters of $\mathrm{N} / 10$ $\mathrm{NaOH}$ required for 100 c.c. of stomach contents. This is often termed degrees. Some prefer to express the results in terms of per cent hydrochloric acid. ( 1 c.c. of $\mathrm{N} / 10 \mathrm{HCl}=.00365 \mathrm{gm}$. $\mathrm{HCl}$.)

Free Acidity. This gives both the organic and inorganic free acid. Titrate 10 c.c. of the stomach contents with $\mathrm{N} / 10$ sodium alizarine sulphonate as the indicator. Titrate to a violet color. Determine and report the number of cubic centimeters of $\mathrm{N} / 10 \mathrm{NaOH}$ required by 100 c.c. of the juice.

Combined Acidity. The difference between the total acidity and the free acidity is the combined acidity. This is also reported for 100 c.c. of the stomach contents.

Detection of Lactic Acid. Prepare Uffelmann's reagent by adding ferric chloride to a 1 per cent solution of phenol until a blue color results. Add 3 c.c. of this solution to 3 c.c. of the stomach contents. The presence of lactic acid will be indicated by the formation of a yellow color.

Organic Acids and Acid Salts. Subtract the free hydrochloric acid from the combined acidity and express the results in terms of 100 c.c. of gastric contents.

Microscopical Examination. This may yield much valuable information. The microorganisms to be expected are yeasts, mold spores, rods and sarcinæ. Other bacteria may be present under certain conditions. Other particles, such as cellulose epithelial cells, starch grains, etc., may also be present.

Bacteria in Intestines and Feces. The bacteria which gain entrance to the intestines and feces are those which have passed through the stomach in the chyme or which may have been contributed, in rare cases from the blood stream.

Relation of Bacteria to Life. This has been a much-debated question. Pasteur (1885) believed that bacteria were essential for normal life. Nuttal and Thierfelder (1895, 1896 and 1897) reared guinea pigs under aseptic conditions which had been delivered by Cæsarian section. These increased in weight, but not as regularly as normal pigs. From these data, the authors concluded that bacteria were not necessary. Schotellius $(1899,1902)$ hatched chickens in a sterile environment and from the data which he secured concluded that chicks could not live in absence of bacteria. He stated also that sterilizing the grain may have 
destroyed some essential substance. Lcvin $(1899,1904)$ found the intestines of most Arctic animals completely sterile. He draws no conclusions, but his work is of interest on account of normal growth without bacteria. Metchnikoff (1901) grew tadpoles in sterile bread and water and secured no transformation into frogs in either the sterile or unsterilized tadpoles. The non-sterile grew larger than the sterile. Portier (1905) determined that larvæ of certain lepidoptera lived aseptically. Moro (1905) confirmed the work of Schotellius which has been mentioned above. He used turtles instead of chicks. Bogdanow (1908) concluded that bacteria were necessary for normal development because sterile larvæ of piptera did not do as well as those kept in an unsterile environment. Wollman (1911) criticized the work of Bogdanow, stating that his sterilization was carried out at too high a temperature. He concluded from his work on flies that bacteria were not necessary for normal development. Cohendy (1912) thought that the digestive processes could proceed without bacteria, but that they aided digestion. Life was thought to be possible without bacteria, but bacteria are not indispensable. Kianizine (1915) produced data which he thought weakened the argument of Cohendy. With chickens, Kianizine believed that bacteria were of great aid in digestion. They carried on analytic and synthetic processes which were of much value to the host. In a later paper by the same author (1916) he continues his argument. He made guinea pigs breathe sterile air and eat sterile food and found that even after a few days they were greatly weakened. The bad results from the deprivation of bacteria was thought to be due to reduced oxidation and accumulation of leucomaines in the body. Loeb and Northrop (1916-1917) attempted to determine the ability of flies to synthesize their body proteins without the aid of microorganisms. Larvae grown on sterile media (banana) did not do well, while those placed in a sterile yeast culture developed normally. It was thought that yeasts were essential for this species of fly although the essential element could not be isolated.

This rather extensive presentation of the literature gives the data from which our present conclusions must be drawn. Obviously much of the data which have been used cannot be applied to conditions in the human intestinal tract.

Action of Bile on Bacteria. This juice is poured into the intestine just below the pylorus. It is a thick liquid with a decidedly bitter taste. It is made up of salts of glycocholic and taurocholic acids, pigments (bilirubin, biliverdin, etc.) fats, phosphatids and inorganic salts.

The bile like most of the other body fluids is probably sterile when 
secreted. Toida (1914) found that bile of man and dogs was normally sterile and that it was not a satisfactory medium for all bacteria. It was only slightly suited to Staphylococcus pyogenes aureus and Ps. pyocyaneus. Streptococcus pyogenes and Dip. pneumonioe were better adapted to it. The bactericidal action was found to vary with the origin. Just below the point of entrance into the intestine, the bacterial flora is much simpler. B. coln is often the predominating form.

The regulation of carbohydrate fermentation by bile has been studied by Roger (1912, 1913, 1915). The hydrolysis of starch was increased up to 30-40 per cent but when smaller amounts (10 per cent) were used there was a retardation. Heating the bile did not destroy its activity. Moderate amounts of bile (5-20 parts per 100) retarded the action of intestinal bacteria on glycogen. Larger amounts were erratic, causing sometimes a retardation and sometimes an increase in the action. The same was found to be true with regard to glucose. Pure cultures of Bacillus colon were inhibited in their action on glucose, moderate amounts exerting a more consistent retarding effect than larger amounts.

Bile also exerts a retarding effect on putrefaction. Roger (1913) found that the intensity of the biuret reaction of protein media inoculated with intestinal bacteria in the presence of different amounts of bile, showed that the attack on proteins was retarded. This retardation was evident up to concentration of 20 per cent after which the retardation decreases. Roger (1915) regards the bile as a factor tending to diminish the production of bacterial enzymes and neutralizing toxins. Boudielle (1913) reported that bile as well as bile salts had an inhibiting action on the fermenting power of $B$. coli on glucose. Moderate amounts had more pronounced effects than greater amounts. Lagane (1914) added bile to bouillon cultures and noticed no retardation in growth of intestinal bacteria. He claimed that it favored the development of $B$. coli at the expense of other species.

Whether the bile is a germicide seems hardly to have been settled. However, most of the data which have been accumulated seems to show that bile exerts a selective action. Toida found that under normal conditions the bile to be sterile. In this connection, it is interesting to remember that gall stones may be started by bacteria and that very often typhoid carriers liberate the bacilli in the bile. In these instances the bile seems to exert no bactericidal action, but perhaps a selective action.

Effect of Diet on the Intestinal Flora. It has long been considered that diet was one-of the most important factors determining the intes- 
tinal flora. Much work has been done by various investigators from which facts have been well established with regard to changes induced in the intestines by variations in the diet. The changes in the flora resulting from various diets have been measured in terms of certain large groups and rarely in terms of any one or few organisms. These groups which have thus been used may be enumerated as follows:

I. Putrefactive Group Bacillus colon, B. Welchii

II. Aciduric Group

B. bulgaricus, B. acidophilus, B. bifidus

\section{Amylolytic Group Glycobacter amylolytıcus}

Herter and Kendall (1908), working with monkeys, studied the effect on the intestinal flora of sudden changes in the diet. After the flora from a distinctly protein diet had been established the diet was changed to a milk and sugar diet. From this change they were able to detect a change in the intestinal flora and mental condition of the animal. Also in the urine, putrefactive products were quite evident. In the intestines the acidolphile group of organisms was changed to a proteolytic group. This was one of the first papers from which definite conclusions could be drawn. The authors suggest that in a disease where either the acidophile or putrefactive group tends to become established, a rapid alteration in the diet would tend to prevent either group of bacteria from becoming established. The same conclusions were reached by Herter (1910) in a later paper.

Blatherwick and Hawk (1914) have studied the effect of fasting and of low- and high-protein intake on the bacteria in the intestines. The concentration of bacteria was measured by determining the amount of bacterial nitrogen in the feces according to the method mentioned elsewhere in this chapter and the output of urinary indican. They found that there was no relation between the output of bacteria in the feces and amount of indican in the urine. By means of a seven-day fast (Blatherwick and Hawk, 1914), the bacterial nitrogen excreted by a $76 \mathrm{~kg}$. man was reduced from $1.571 \mathrm{gms}$. to $0.101 \cdot \mathrm{gm}$. The actual weight of excreted bacterial substance was reduced from 14.336 to 0.920 gm. per day. The output of bacterial nitrogen and bacterial substance was about the same during a fast and on a low proten intake. Assumption of a high protein diet caused an increase in the above figures. 
Unfortunately a qualitative study of the bacteria in the feces was not made during this study and to secure such information it is necessary to consult the work of others. Herter and his colleagues, the work of whom has been summarized by Herter (1907), have given information to show that a protein diet induces the proteolytic group. Rettger and his colleagues have studied the effect of carbohydrate feeding. The experiments were made with laboratory animals and man. Hull and Rettger (1915) studied the effect of milk and carbohydrate feeding. They found that lactose when taken by adults along with their usual food, caused a shift in the flora to the acidophilli group of bacteria. $B$. acidophilus and $B$. bifidus appeared with the former more abundant. The most practical diet, however, to produce these results was a combination of milk and lactose. B. bulgaricus was not found which is in accord with the work of others.

Fasting has a decided effect on intestinal bacteria. As quoted above Blatherwick and Hawk (1914), during fasting, noticed a decided reduction in the amount of bacterial nitrogen and bacterial substance. Sisson (1917) has found that, after periods of starvation, there are definite changes in the number of bacteria in contrast to the usually vigorous growth. No change in the kind of organism after starvation was noted. The greatest decrease in the number of bacteria was noticed in the duodenum. Sisson regards the condition produced by starvation as one of relative amicrobism.

In this connection the influence of copious water drinking on fecal bacteria should be mentioned. Fowler and Hawk (1910) noticed a decrease in the total nitrogen appearing as bacterial nitrogen. The following weights of fecal bacteria were excreted per day during the periods mentioned:

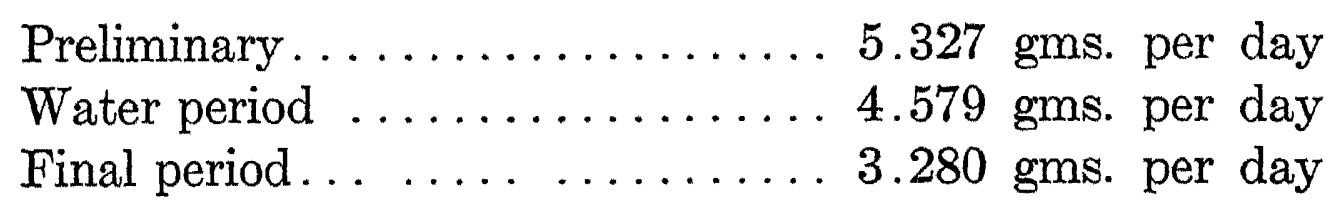

Hattrem and Hawk (1911) by means of copious water drinking obtained a decrease in putrefaction and stated that it was probably due to a decrease in the activities of indol-producing bacteria following accelerated absorption of products of protein digestion. Copious water drinking (1000 c.c.) caused a decrease of indican in the urine which was accompanied by an increase of ethereal sulphates. The drinking of distilled and softened waters were found by Sherwin and Hawk (1914) to cause a decrease in intestinal putrefaction as measured by urinary indican. 
Number of Bacteria in Feces. The different investigators are not in agreement on this question. This may be in part due to different methods of analysis and to the variations in samples. The securing of concordant results is hardly to be expected. The data which are on hand vary from 5 to 50 per cent. Strasburger (1896) stated that onethird of the feces of an individual on a normal diet was bacteria. The quantity of daily bacterial wastes dried in adults was as follows:

a. Eight gms. under normal conditions.

b. In dyspeptic intestinal conditions on an average of $14 \mathrm{gms}$. and even as much as $20 \mathrm{gms}$.

c. In chronic constipation 5.5 gms. and, at times, even as little as 2.6 gms.

The total number of bacteria eliminated by an individual in one day was believed by Strasburger to be 128,000,000,000. Among the determinations which have been made by different investigators are the following collected by Mattill and Hawk (1911):

\begin{tabular}{|c|c|c|}
\hline & $\begin{array}{c}\text { Daily Excretion } \\
\text { of Dry } \\
\text { Bacteria. }\end{array}$ & $\begin{array}{l}\text { Percentage of } \\
\text { Dry Bacteria. }\end{array}$ \\
\hline Strasburger. . & 8.0 gms. & $24.3 \%$ \\
\hline 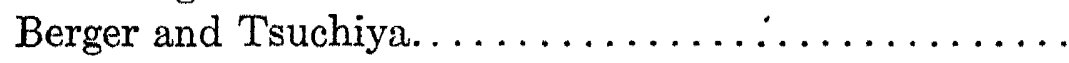 & 3.023 & 12.60 \\
\hline McNeal, Latzer and Kerr. . . . . . . . . . & 5.34 & 26.90 \\
\hline 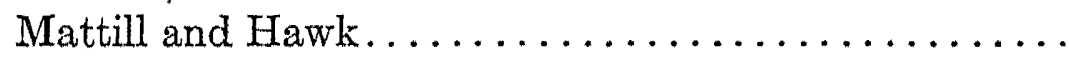 & 8.27 & 27.97 \\
\hline Sato $\ldots \ldots \ldots \ldots \ldots \ldots \ldots \ldots$ & 8.54 & 24.39 \\
\hline Schittenhelm and Tollens.... & $\ldots \ldots$ & 42.00 \\
\hline 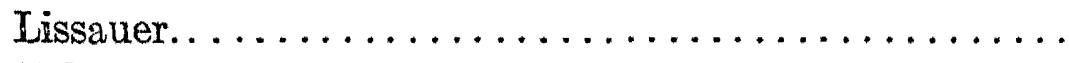 & $\ldots \ldots$ & 8.67 \\
\hline 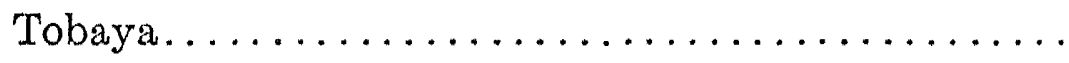 & $\ldots \ldots$ & 11.22 \\
\hline
\end{tabular}

Osborne and Mendel (1914) found that about 70 per cent of the nitrogen in feces of animals was caused by bacteria.

Classification of Intestinal Bacteria. Since the intestinal flora is quite extensive it is not a simple matter to make a classification. The viewpoint and interests of the investigator might influence the kind of classification made. Ford classified the bacteria in the duodenum of infants. The following classification by Distaso (1912) is as satisfactory as any: 


\section{THE INTESTINAL FLORA. BY A. DISTASO \\ Lancet, 182 (1912), 496-98}

A. Gram Negative Bacilli.

1. B. coli group.

2. B. variabilis group. (Anaerobic bacteria with round extremity.)

3. B. thetaiotaomicron group. (Bacilli very polymorphic, elliptical form.)

4. B. rigidis group.

5. B. preacutus group. (Bacillus swollen in the middle and pointed extremities.)

B. Gram Negative Cocci.

1. Sarcina citrea group. (Very common in the mouth and feces, similar in appearance to the gonococcus.)

2. Diplococcus obliculus group. (Strict anaerobes.)

3. Parvulus group. (Strictly anaerobic, small cocci.)

C. Gram-Positive Bacilli.

1. B. Acetogenes group. The greater number of the gram-positive bacilli belong to this group. The bacillus acetogenes $\mathrm{B}$ is its chief representative, since the $B$. bifidus and $B$. acetogenes $\mathrm{A}$ are very rare in the feces examined in London.

2. Streptobacillus group. This microbe always exists in the intestinal flora.

3. Diploc. acuminatus group. An anaerobic bacillus. It produces burytic acid.

4. B. perfrigens group. An anaerobic microbe. It produces enormous quantities of lactic acid.

5. B. cedematic maligni group. With the B. sporogenes (Metchniloff).

6. B. rodella group. Anaerobic group with very long bacilli.

7. A lemon-shaped bacillus, which is stained by iodine and is described by the author as being butyrous acid-producing microbe. It has not yet been obtained pure.

D. Gram-positive Cocci.

1. Enterococci group, in chains, less frequently diplococci.

2. Small cocci group. Are also to be seen corresponding in size to the coccus Banani, to the Staphylococcus pyogenes, and to the Staph. asaccharolyticus.

In addition three kinds of spores are to be found. .

1. Oval spores; rather' large, which may belong to $B$. sporogenes and to other.

2. Round spores, which may belong to B. putrificus (Bienstock-Tissier) or to the bacillus of Rodella and to the $B$. Alkaligines anaerobrcus.

3. Very small spores, strongly refractile, belonging to the $B$. perfrigenes. 
Fermentation in the Intestinal Tract. Putrefaction is used in this case to indicate the bacterial decomposition of proteins. There has been much discussion with regard to the definition of the terms putrefaction and fermentation. Kendall (1911) has given a good discussion of these terms in their relation to the digestive tract. He quotes therein the definitions which have been given to the processes of putrefaction and fermentation by various workers and accepts the definition for fermentation which was given by Alfred Fischer. Fischer defines fermentation as the biochemical decomposition of nitrogen-free, organic compounds, especially the carbohydrates induced by fermenting organisms. If this definition is accepted there may then be different types of the process, depending on the main product: lactic acid, butyric acid, alcoholic fermentations, etc. With regard to putrefaction more confusion exists. The term has been used in so many different ways that it is difficult to correlate them. Kendall states that the phenomena grouped together as putrefaction " represent a scries of symbioses, in which the initial superficial breakdown is brought about by the anaerobes, while the process is brought to its lowest terms by the facultative anaerobes." The Germans have made a distinction between "Faulniss" putrefaction in the popular sense and "Verwesung" eremecausis. This latter is supposed to be an aerobic process carried on by many bacteria. That fermentation should mean decomposition of carbohydrates and putrefaction, decomposition of proteins are the opinions of the biological chemists.

Certain microbiologists, however, have not accepted this definition. They define fermentation as an intracellular process which furnishes energy to the cell and pay no attention to the products which are decomposed. The decomposition of soluble nitrogenous compounds within the cell protoplasm is regarded as fermentation. This seems to be the best definition for the term as has been suggested by Fischer (1902). He gives the following points as characteristic of such a conception of the term:

1. Fermentation is an intracellular process.

2. The products of the fermentation are essentially different from those in the substract and are not mere parts of these original substances.

3. The products are useless to the cell and may be harmful.

4. Vital energy is produced.

According to this definition, then, and as it is stated by Fischer, the hydrolysis of starch to soluble sugars is not a fermentation, because it does not take place within the cell to yield energy to the cell. The burning of the sugar within the cell protoplasm, however, does consti- 
tute fermentation and the energy therefrom is available for cell use. Fermentation may then be regarded as respiration if the latter term is considered to cover those processes which yield energy. There are two kinds of respiration, aerobic respiration and anaerobic respiration.

Aerobic Respiration. This involves the securing of energy from food substances by means of and in the presence of free oxygen. In order to present the facts required in a discussion of this nature, the carbohydrates lend themselves very well. It must be remembered, however, that there are certain well-known objections to the expression of bacterial changes by chemical equations and these should be borne in mind in considering the equations which follow. As a typical example of aerobic respiration the decomposition of dextrose may be taken $\mathrm{C}_{6} \mathrm{H}_{12} \mathrm{O}_{6}+6 \mathrm{O}_{2}=6 \mathrm{CO}_{2}+6 \mathrm{H}_{2} \mathrm{O}+674$ cal. In this change molecular oxygen has been used to burn dextrose completely.*

In the products carbon dioxide and water there is no energy which is available for bacteria. With nitrogenous substances the oxidation of glycocoll takes place as follows:

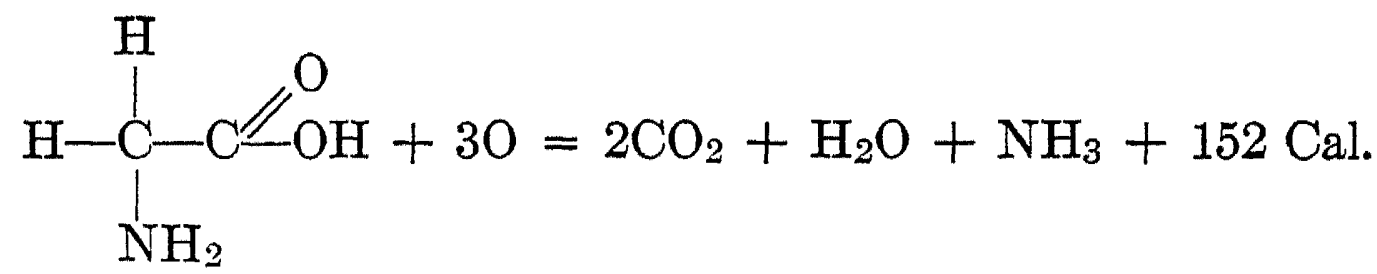

In this equation allowance must be made for the combustion heat of ammonia since this compound may be further oxidized. Leucin is oxidized as follows:

$$
\mathrm{C}_{6} \mathrm{H}_{13} \mathrm{NO}_{2}+15 \mathrm{O}=6 \mathrm{O}_{2}+5 \mathrm{H}_{2} \mathrm{O}+\mathrm{NH}_{3}+755 \text { cal. }
$$

In this equation the same correction must also be made for the combustion heat of ammonia.

Hydrogen sulphide is oxidızed by the sulphur bacteria according to the following equation:

$$
\mathrm{H}_{2} \mathrm{~S}+2 \mathrm{O}_{2}=\mathrm{H}_{2} \mathrm{SO}_{4}+207 \text { cal. }
$$

or to neutral sulphur as follows:

$$
\mathrm{H}_{2} \mathrm{~S}+\mathrm{O}=\mathrm{H}_{2} \mathrm{O}+65 \text { cal. }
$$

* In all considerations of respiration, it must be remembered that the energy secured by complex molecules is latent in them, having been put into them by the organism which formed them. Such synthetic processes are endothermic for the organism which bulds up these complex substances from the simpler compounds. The bacteria secure this energy when they split these complex compounds to simpler ones. 
Anaerobic Respiration. The products of anaerobic respiration are usually left in an incompletely oxidized condition. If dextrose is taken again to show this, any of the following equations might serve.

$$
\begin{aligned}
& \mathrm{CH}_{2} \mathrm{OH}(\mathrm{CHOH})_{4} \mathrm{COH}=\mathrm{CH}_{3} \mathrm{CHOH}-\mathrm{COOH}+15 \mathrm{cal} . \\
& \mathrm{CH}_{2} \mathrm{OH}(\mathrm{CHOH})_{4} \mathrm{COH}=3 \mathrm{CH}_{3} \mathrm{COOH}+34 \mathrm{cal} . \\
& \mathrm{CH}_{2} \mathrm{OH}(\mathrm{CHOH})_{4} \mathrm{COH}=\mathrm{C}_{2} \mathrm{H}_{5} \mathrm{OH}+2 \mathrm{CO}_{2}+22 \mathrm{cal} .
\end{aligned}
$$

The main product in each of these equations may be further oxidized to yleld more energy.

The amount of energy secured by aerobic and anaerobic bacteria from the same amount of food is striking. Take, for instance, the following equation as a typical one for aerobes:

$$
\mathrm{CH}_{2} \mathrm{OH}(\mathrm{CHOH})_{4} \mathrm{COH}+6 \mathrm{O}_{2}=12 \mathrm{CO}_{2} 12 \mathrm{H}_{2} \mathrm{O}+674 \mathrm{cal} \text {, }
$$

and the following as typical for anaerobic bacteria:

$$
\mathrm{CH}_{2} \mathrm{OH}(\mathrm{CHOH})_{4} \mathrm{COH}=\mathrm{CH}_{3} \mathrm{CHOH} \mathrm{COOH}+15 \text { cal. }
$$

The proportion would then exist as follows:

Aerobic : anaerobic $=674.15$

Aerobic : anaerobic $=45.1$

That is, from the same amount of food the aerobes by complete oxidation will get forty-five times as much energy. Looking at it from the standpoint of energy, the anaerobes have to have forty-five times as much food as the aerobes. This explains why the anaerobic bacteria are so destructive in soil, sewage, etc.

Putrefaction in the Intestinal Tract. As stated above the biological chemists have reserved this term for the decomposition of nitrogenous substances. According to the definition of fermentation which has been given above, some decompositions of nitrogenous substances would be regarded as fermentations. The combustion in the protoplasm of those peptones which are diffusible would be regarded as fermentation while the hydrolysis of the proteins and proteoses which goes on outside of the cell wall would not be regarded as fermentation.

The chemistry of the putrefactive changes which take place in the intestines is fairly well known. Straight chain acids are among the first 
compounds which are attacked and the changes may be indicated as follows:

I. Deaminization.<smiles>NCCCCC(=O)O</smiles>

II. Decarboxylation.<smiles>NCCCCCC(=O)OCC([NH3+])=O</smiles>

III. Oxidation.<smiles>CC(=O)OC1OC1CO</smiles>

According to these reactions tyrosin may be putrefied as follows:

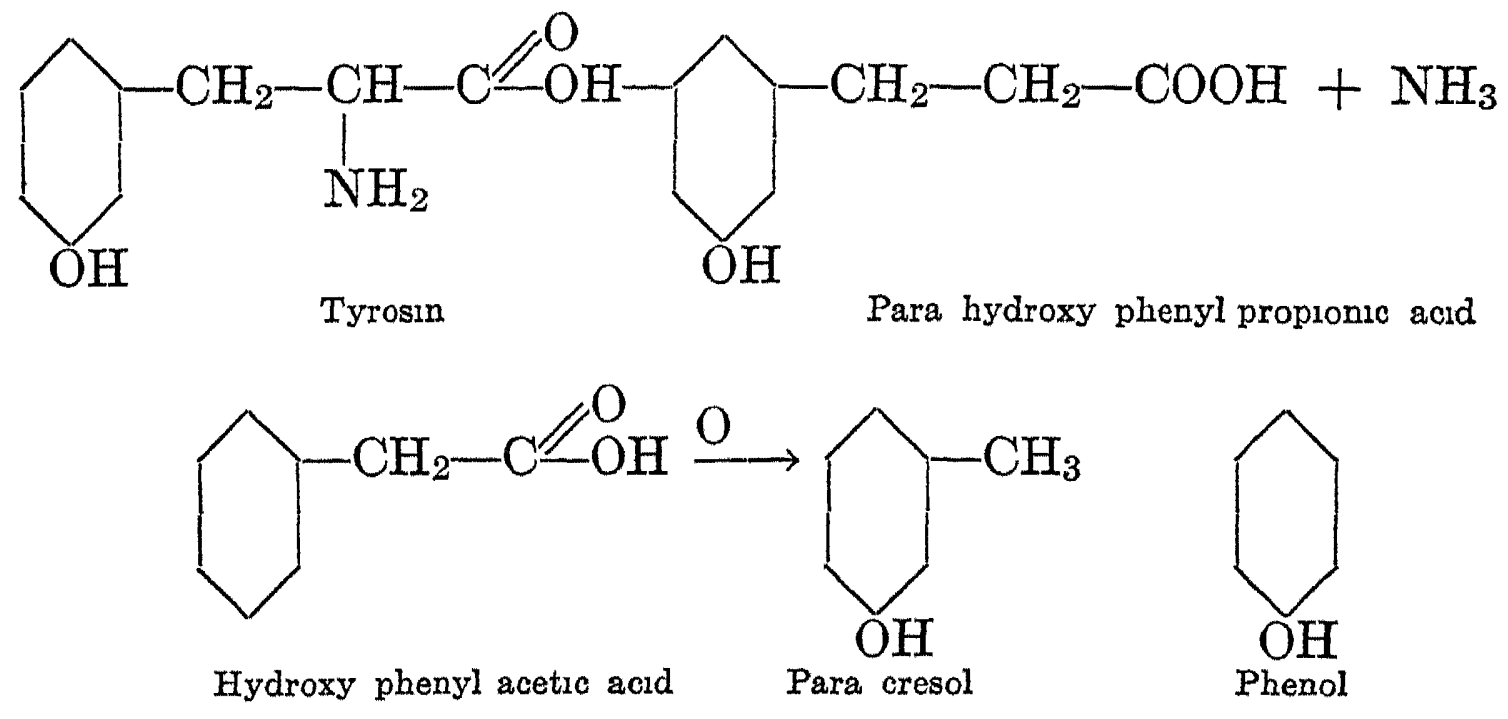

The oxidation stops with phenol. These substances have no wellmarked location in the intestine although it occurs in the large intestine. Their formation is not limited to the intestines for they have been found in other parts of the body. For excretion phenols are detoxicated. 
They are united with sulphuric acid in the liver to form ethereal sulphate.<smiles>O=S(=O)(O[Hg])O[C@H]1CCC[C@@H](OS(=O)(=O)O[TlH])C1</smiles>

This is usually excreted as a sodium or potassium salt.

Putrefaction of Tryptophan. Hopkins and Cole have determined the steps in the decomposition of tryptophan by cells.<smiles>NC(CC1=CNC2CCCC1C2)C(=O)OC1CCC2NC=C(CCC3OC3O)C2C1</smiles>

Tryptophan

Indol propionic acid<smiles>CCC1=CN([N])C2CCCCC12</smiles><smiles>CC1=CNC2CC(C)CCC1C2</smiles><smiles>CC1CCC2C=CNC(C1)C2</smiles>

The final product is methyl indol or skatol. For excretion this may be united with sulphuric acid as follows:<smiles>CC1=CNC2CCCC1C2</smiles><smiles>NC1CCCCC=C1CO</smiles><smiles>[Z17]OS(=O)(=O)OCC1=CCCCC1NC</smiles>

Indol is prepared for excretion in the same way.

Putrefaction of Arginin. Putrecin in the end product and was supposed to be important in certain types of food poisoning. It is formed from arginin as follows: 


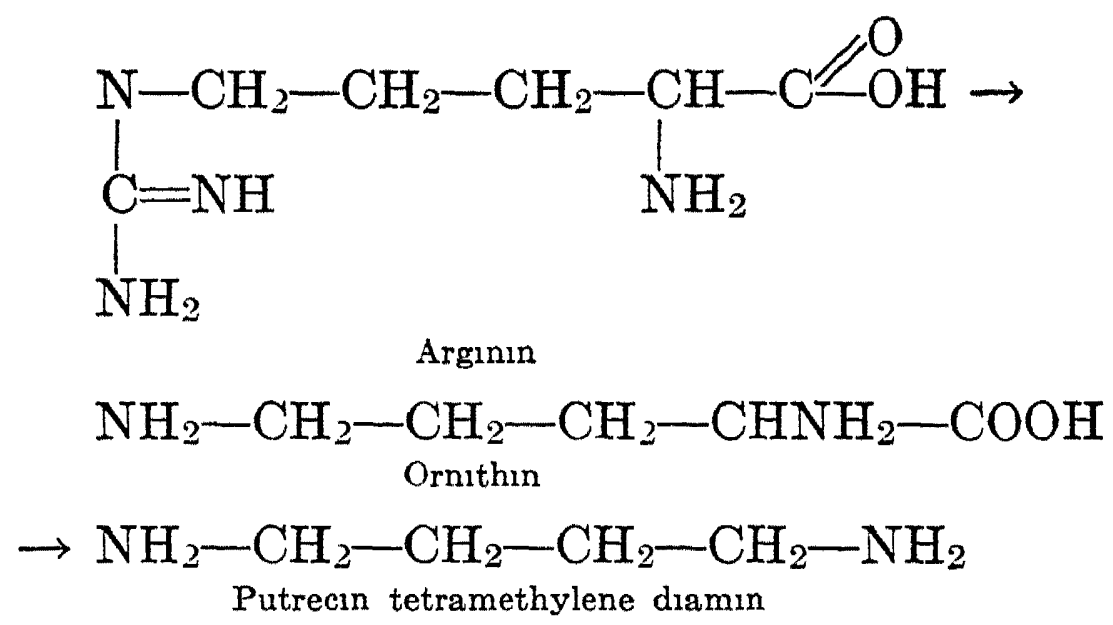

Putrefaction of Lysin. Cadaverin is a common product of putrefaction. It was first observed by Brieger (1885). It is formed from lysin in the following manner:

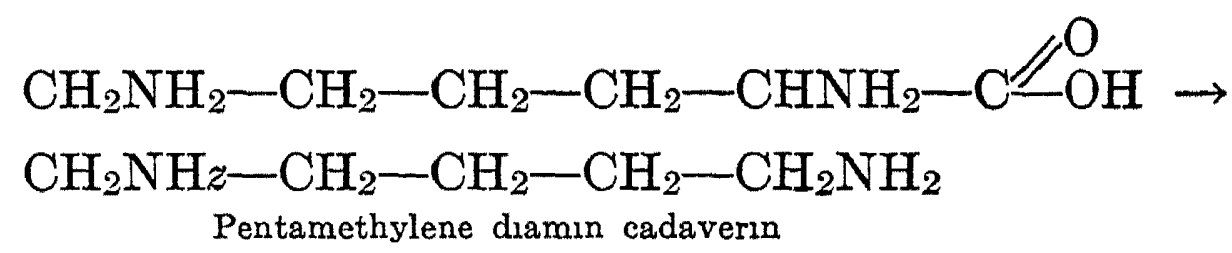

Putrefaction of Cystine.

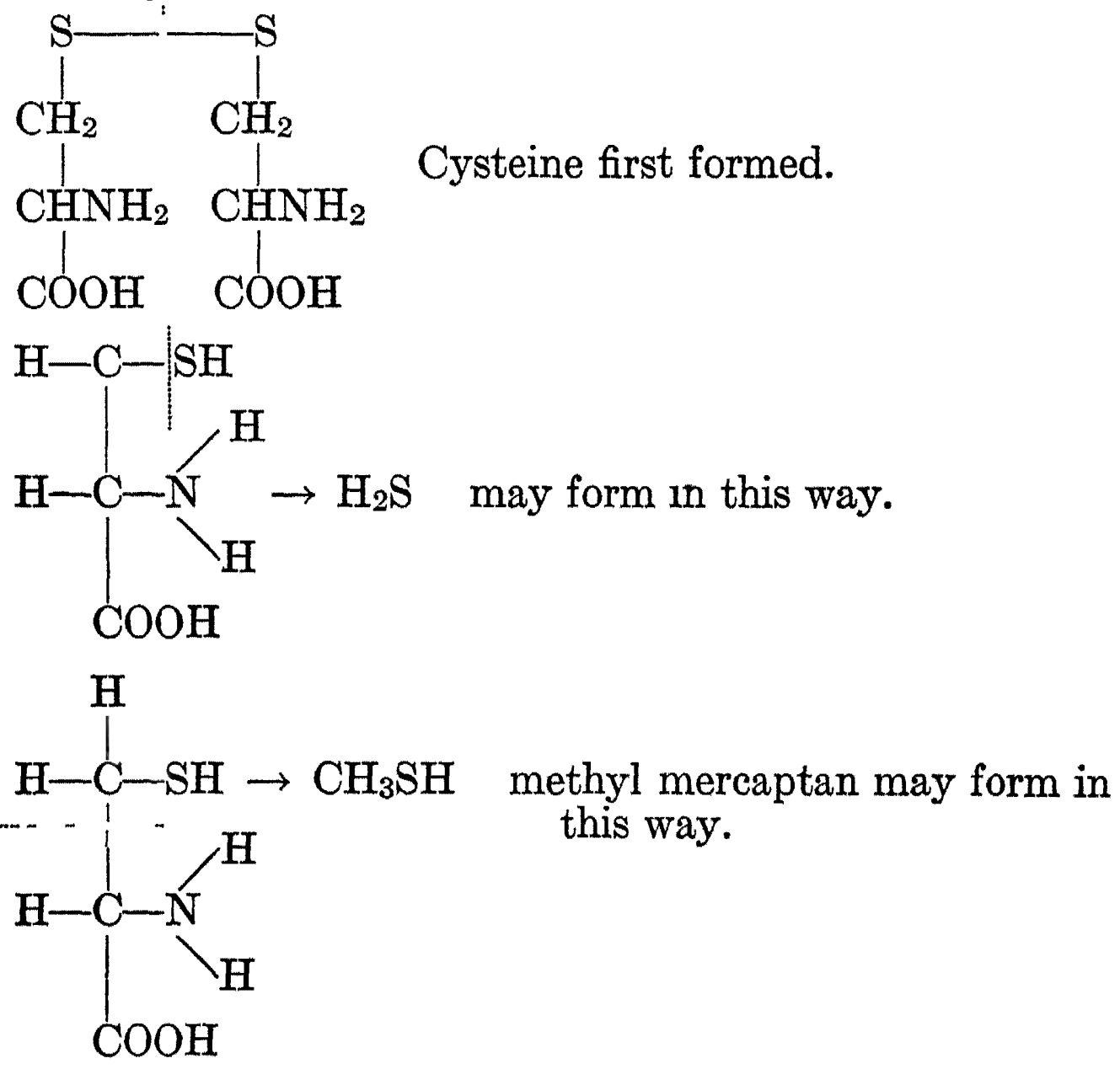


According to Mathews cystine goes to cystcine, and then as:

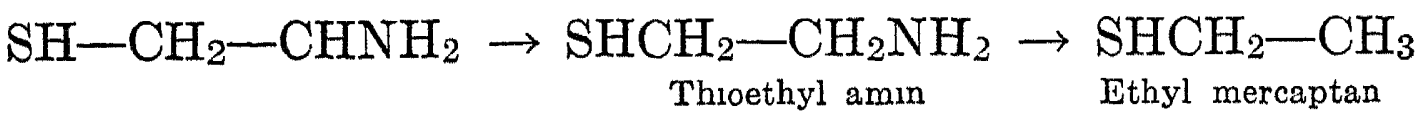

The action of bacteria and yeast-like fungi has been studied by Tanner $(1917,1918)$. These microorganisms were quite able to split hydrogen sulphid from cystine.

Some of these decomposition products of the amino acids and proteins may be detected as follows: The solution to be tested may be either a filtered bacterial culture or a fecal suspension.

Indol Determinations. A number of methods have been devised for the detection of indole. Most of these yield good results when applied to bacteriology.

Nitroso-indol Nitrate Reaction. Acidify a portion of the substance under examination with concentrated nitric acid. The addition of a few drops of potassium nitrate will produce a red coloration if indol is present. The red compound is nitroso-indol nitrate.

Nitro-prusside Reaction. Add a few drops of a freshly prepared solution of sodium nitro-prusside to a filtered sample. Add sodium hydroxide and examine for the production of a violet color. This may be changed to a blue by the addition of a few cubic centimeters of strong acetic acid.

Ehrlich's Para-dimethyl Amino Benzaldehyde Test. Add about one-third the volume of a 2 per cent solution of para-dimethyl amino benzaldehyde in aclohol to the sample under examination. Then slowly add dilute hydrochlorine acid until a red color is evident. This color may be deepened by the addition of a few drops of sodium nitrite solution.

Bergeim's (1917) Method for Determination of Fecal Indol. Rub 30 to 50 gms. of the fresh feces in a mortar with $\mathrm{H}_{2} \mathrm{O}$. Transfer to a 1 liter Kjeldahl flask and add $\mathrm{H}_{2} \mathrm{O}$ to 400 c.c. Add 5 c.c. 10 per cent $\mathrm{KOH}$ and 2 c.c. paraffine and distill with steam until 500 c.c. have been obtained, bringing the volume of the fecal suspension down to about 100 c.c. Re-distill with steam after acidifying slightly with $\mathrm{H}_{2} \mathrm{SO}_{4}$ or else remove the $\mathrm{NH}_{3}$ with permutite. Mix an aliquot of the resulting solution (100 c.c.) in a 150-c.c. separatory funnel with 1 c.c. of a fresh 2 per cent solution of $\mathrm{NaB}$ naphthoquinonesulphonate and 2 c.c. of 10 per cent $\mathrm{KOH}$. After fifteen minutes extract with $\mathrm{CHCl}_{3}$ using 10 and 7 c.c. Dilute extract to 15 c.c. and mix. Compare color in the colorimeter with that of a standard similarly prepared, using $0.1 \mathrm{mg}$. indol. The error appears to be only -1 per cent. 
Skatol Determinations. Skatol is a product of putrefaction which has much in common with indol.

Herter's Test (1907). Add a few cubic centimeters of acid paradimethyl amino benzaldehyde ( 5 gms. to 100 c.c. concentrated $\mathrm{H}_{2} \mathrm{SO}_{4}$ ) to about 6 c.c. of the filtered sample. After heating to boiling a bluishlavender color is obtained. This may be deepened by adding a small amount of hydrochloric acid.

Phenol Determinations. Ferric Chloride Test. By adding a few drops of ferric chloride solution to the solution under examination a blue color will be obtained if phenol is present.

Nitric Acid Test. Add a few drops of nitric acid to the sample and heat. If phenol is present, a yellow color is formed due to the formation of picric acid.

Bromine Water Test. When bromine water is added to a solution containing phenol, mono-, di-, and tribromophenols are formed. The first two possess a very sharp odor. Tribromophenol precipitates as yellow white needles.

\section{Methods for Determining Bacteria in Feces}

The accurate, rapid determination of the bacteria in feces is not a simple matter. Plating on ordinary media is known to give low results. On the other hand there are certain errors in the various microscopic methods. In these, the presence of pieces of organic matter hinder the counting.

MacNeal's (et al.) Modification of the Winterberg Method. The first employment of the Thoma-Zeiss blood-counting chamber for bacteriological technic was made in the one-cell dilution method of obtaining a pure culture. By this method the number of cells per unit volume could be ascertained and the dilution per unit volume of a suspension with one cell in two to five drops required to obtain a suspension with one cell in two to five drops calculated. In this way the first pure bacterial culture was obtained. Henrich Winterberg was the first to use this method of bacterial counting, and to test its accuracy both as regards suspensions of varying dilutions and in comparison with the microscopic plate-counting method. He considers the method more accurate than the plate-counting method, but as a quantitative procedure he would consider it unimportant. He says his determinations were too low. Winterberg's counts were made with suspensions of living bacteria in bacteria-free distilled water.

"The method, as used by MacNeal, Latzer and Kerr, is as follows: 
a portion of the $1: 100$ suspension of feces is diluted ten times, and a portion of this is drawn up to the mark 1 in the capillary of a dilution pipette, ordinarily used in estimating the white blood cells. This is diluted to the mark 11 with a dilute solution of methylene blue in physiological-salt solution. (The stanning solution consists of methylene blue, 1 gm.; glycerine, 25 c.c.; distilled water, 75 c.c. A few drops of this are mixed with 10 c.c. of 0.8 per cent salt solution until the mixture is well colored, but not too opaque. This mixture is used as the diluting flusd. A little practice will show the proper depth of color to be employed.) The suspension is thoroughly mixed in the bulb by shaking and rolling in the usual manner. Several drops are blown out and then a very small drop is placed in the center of the circular elevated portion of the slide, which has been previously thoroughly cleansed by washing in distilled water and alcohol. A clean, thin, ground cover-slip is made slightly moist by breathing upon it and is immediately placed upon the slide. Slight pressure upon it causes the Newton color rings to appear and these remain after the pressure is removed if the preparation has been properly made. The slide is allowed to stand one to two hours to allow the bacteria to settle. Then the bacteria in fifty small squares upon the marked scale are counted microscopically, the No. 7 Leitz objective and No. 3 ocular being used. The calculation is simple as an example will show.

"Example. B252, Subject H, Julv 15, 1908. Fifty squares contain 400 bacteria. Therefore the average per square is 8.0 bacteria. One small square $=1 / 4000$ c.mn. One c.mn. contains $8 \times 4000=32,000$ bacteria. One cubic centimeter contains $32,000 \times 1000=32,000,000$ bacteria. Pipette dilution is $1: 10.32,000,000 \times 10=320,000,000$ bacteria per cubic centimeter of $1 / 1000$ suspension. One cubic centimeter of $1 / 1000$ suspension is equivalent to $1 \mathrm{mg}$. feces. Therefore, there are $320,000,000$ bacteria per milligram feces.

"Counting by this method is an exacting process and much practice is required to see all the bacteria present. Careful adjustment of the light is important and best results have been obtained by illumination with Welsbach light; constant focusing through the different layers is necessary. Perhaps the greatest source of error is the difficulty of distinguishing accurately the bacteria. Skill in this is acquired only by practice. The method has the advantage of simplicity. The results of the authors seem to conform to those of Winterberg in that high dilutions give a relatively high count."

MacNeal's (et al.) Modification of the Eberle-Klein Method for Determining the Number of Bacteria in Feces. Eberle, working in 
Escherich's laboratory, was the first to determine the quantity of fecal bacteria by counting them in stained films. His experiments were carried out with normal infant's stools. Coverglass preparations were made from fecal suspensions of known dilution and after being completely dried were stained with freshly prepared aniline water fuchsin, then washed in water, air-dried, mounted in Canada balsam, and counted. The method was very much improved by Alex, Klein and Hehewerth. Klein had noticed that vegetative bacterla were more sensitive to disinfectants in a moist than a dry state, and this led him to believe that bacteria would also be stained more readily in a moist condition than after drying. Therefore he allowed the dye to act while the bacteria were suspended in a liquid and the coverglass preparation was made, dried, and fixed,. only after the bacteria were stained. Later the same investigator modified the method still further, using gelatin to fix the stained-bacteria to the coverglass. The aqueous solution of gelatin and the stained bacterial emulsion were put upon the coverglass separately, then fixed and spread. When dry, the preparation was immediately mounted in Canada balsam without flaming.

"The procedures as employed by MacNeal, Latzer and Kerr have been considerably modified. In the addition of the gelatin to the bacterial suspension, in spreading the films, in selecting the fields to be counted, and in counting individuals, rather than groups, as units, the technic differs from Klein's method. Of the $1: 100$ suspension of the feces prepared as described above (p. 248), $2 \frac{1}{2}$ c.c. are transferred to a clean, dry bottle, $\frac{1}{2}$ c.c. of melted nutrient gelatin and 2 c.c. of aniline water gentian violet added, and the whole thoroughly mixed and allowed to stand for three to five minutes. Then, by means of a platinum loop, the carrying capacity of which has been determined with great care, a loopful of the mixture, well shaken immediately before, is transferred to a clean, flamed $20 \mathrm{~mm}$. square No. 1 coverglass and deposited near the center of the glass. Immediately another coverglass of the same size is accurately placed on top of the first so that the two glasses are in contact over about three-quarters of their surfaces and the sides evenly fitted together. As soon as the liquid has spread evenly between the two coverglasses, they are quickly slipped apart and allowed to dry. The technic here is the same as ordinarily used in the preparation of coverglass blood films. If the preparation does not appear evenly spread the process is to be repeated with two more coverglasses until a satisfactory result is obtained. The films are next accurately measured by a millimeter rule and then, without further treatment, mounted in Canada balsam upon one slide so that one diagonal of each rectangular 
film is parallel with the long axis of the slide. By laying the slide over coordinate paper these diagonals are readily brought into the same line parallel with the edge of the slide.

"The covers must be so mounted that thece diagonals, now in a straight line, are those which crossed each other as tl.e two coverglasses were originally put together in preparing the films. When properly made each diagonal measures almost exactly $25 \mathrm{~mm}$.

"For counting the bacteria in the preparation, the Leitz 1/12 oil immersion objective and the No. 3 ocular fitted with an Ehrlich ocular square to restrict the field to a convenient size, and a mechanical stage graduated in millimeters are employed. Beginning at the end of the diagonal of one coverglass the bacteria are counted in each of 25 fields $1 \mathrm{~mm}$. apart along this diagonal. In a similar way 25 ficlds are counted on the diagonal of the second coverglass, making a total of 50 fields, the average of which may be considered as representative of both films. The size of the square field is accurately measured by a stage micrometer. From the data then at hand the number of bacteria per milligram feces is calculated.

" Example. B252, Subject H, July 15, 1908. In the preparation made, as described, each film measures $16.5 \times 17.5 \mathrm{~mm}$., total film area therefore, $33 \times 17.5 \mathrm{~mm}$; the field employed measured $0.0445 \mathrm{~mm}$. square; the amount carried by the loop $2.01 \mathrm{mg}$; the number of bacteria counted in 50 fields was 559 and the original $1: 100$ suspension was used (diluted to $1: 200$ by dye and gelatin). From these data, Bacteria per milligram feces $=\frac{559 \times 33 \times 17.5 \times 200}{50 \times 0.0445 \times 0.0445 \times 2.01}=324,000,000$. In this fraction all the members except the size of the films and the number of bacteria counted may be kept constant and the calculation simplified by the use of logarithmic tables.

"With apparatus and reagents ready and the 1:100 suspension prepared, this entire estimation can be completed in about forty minutes. The results cannot be considered very accurate as the platinum loop does not carry an exactly constant quantity. There is also sometimes great difficulty in distinguishing micrococci from other fine particles in the preparations. The concentration of the bacterial suspension also influences the final result. The estimation is in general relatively higher when dilute suspensions are counted as we have observed in applying it to enumeration of bacteria in pure cultures." *

Steele's Modification of the Strassburger Procedure for the Quantitative Enumeration of Fecal Bacteria. Steele describes the method as

* From MacNeal, Latzer and Kerr. J. Inf. Dis. 6 (1909), p. 127. 
follows: "The possibility of separating the bacteria from the rest of the feces depends on the fact that the bacteria are so nearly of the same specific gravity as distilled water that they cannot be centrifugalized out of a watery suspension of the feces, but remain suspended in the supernatant fluid. Taking advantage of this, the bacteria can be removed by washing with the centrifuge. Then, if the specific gravity of the wash-water is lowered by the addition of large amounts of alcohol, the relation of the bacteria to the fluid is changed to such an extent that the microorganisms can be readily centrifugalized out, separated, and weighed. Unless the period of passage of the feces has been ascertained to be normal, it is better to mark the beginning and end of each period of examination by carmine. The use of the Schmidt diet is not necessary. The whole stool is saved. Unless the feces are liquid they are rubbed up with a known amount of distilled water until they are smooth and semi-liquid and as homogeneous as it is possible to make them.

"Two portions of 5 c.c. are measured off with a pipette of large caliber, using for this purpose an ordinary 5 c.c. pipette with the tapering end cut off, and with the necessary correction made at the upper mark. One of these portions of 5 c.c. is put into a porcelain dish and dried over a water bath and later in a drying oven, in order to determine the dricd weight. The addition of a little alcohol and thorough mixing will hasten the process of drying and prevent caking of the feces.

"The second portion is washed free from bacteria. This is done as follows: The wash-watèr is 0.5 of 1 per cent $\mathrm{HCl}$ solution in distilled water. The acid increases the solubility of the salts and soaps of the feces. One hundred c.c. of this solution is employed at the beginning of the washing The feces are thoroughly mixed with the wash solution and then centrifugalized. The use of the water motor or electric centrifuge is almost essential. Each tube is centrifugalized for about $1 \frac{1}{2}$ minutes, then the cloudy supernatant liquid is poured through a layer of gauze. This fluid contains the bacteria in suspension. All of the mixture (the wash-water and the feces) is centrifugalized the same way, and then the residue in the tubes is shaken up with more of the wash-water and centrifugalized again. This is repeated until the supernatant liquid after the centrifugalizing is transparent, showing that approximately all the bacteria have been washed out. If smear is made of the residue at this point, it will be found that the bacteria are not entirely washed away, but are evidently very much reduced. They occur singly, while in the unwashed feces they are in great lumps and masses. The suspension of bacteria is then mixed with a liberal 
portion of alcohol, and evaporated down slowly at a temperature of $40^{\circ}$ to $50^{\circ} \mathrm{C}$. until it amounts to not more than 50 c.c. in all. This takes approximately twenty-four hours. It is then mixed with at least twice its volume of alcohol, preferably absolute alcohol, although this is not absolutely necessary. This lowers the specific gravity of the fluid to such an extent that now the bacteria readily centrifugalize out. The mixture is then centrifugalized until the supernatant liquid is quite clear. This takes thirty minutes or more for each tube. The residue, which consists of the bacteria, is washed with pure alcohol and is shaken up with ether to remove the fat; then it is again washed with alcohol. All of this washing is done by means of the centrifuge. The bacteria are next washed out of the tube with a little alcohol and evaporated to dryness and dried in the oven at moderate heat, dried in the desiccator, and weighed. Smears of the final preparation show that it consists of bacteria with a very few minute particles of other material. These particles are only visible with high power, and are very few in number, perhaps two at each field of the 1/12-inch objective. They stain with methylene blue; Strasburger suggests that they are cellulose, which they may well be. At any rate, the error arising from the inclusion of these small particles in the dried weight of the bacteria must be very small, and is probably balanced by the bacteria that it is not possible to wash out of the residue in the first washing. During the preparation of the bacteria the first portion of 5 c.c. has been dried and weighed. We then know the dried weight of 5 c.c., the weight of the dried bacteria in 5 c.c., the original volume of the stool, and the volume after the addition of a known amount of water. It is then easy to calculate the data that we desire, namely, the volume of the stool, its dried weight, the weight of the dried bacteria, and the percentage of bacteria in the dried weight."

Mattil and Hawk's Method for Quantitative Determination of Fecal Bacteria. "The method is a simplification of MacNeal's adaptation of the Strasburger procedure. About $2 \mathrm{gms}$. of feces are accurately weighed and placed in a 50-c.c. centrifuge tube. To the feces in the tube a few drops of 0.2 per cent hydrochloric acid are added, and the material is mixed to a smooth paste by means of a glass rod. Further amounts of the acid are added with continued crushing and stirring until the material is thoroughly suspended. The tube is then whirled in the centrifuge at high speed for one-half to one minute. The suspension is found sedimented in not more or less definite layers, the uppermost of which is fairly free from larger particles. The upper and more liquid portion of the suspension is now drawn off by means of a pipette 
and transferred to a beaker. The sediment remaining in the tube is again rubbed up with a glass rod with the addition of further amounts of dilute acid, and again centrifugalized for one-half to one minute. The supernatant liquid is pipetted off and added to the first, the same pipette being used for the one determination throughout. A third portion of the dilute acid is then added to the sediment, which is again mixed by stirring and again centrifugalized. All the washings are added to the first one, and, during the process, care is taken to wash the material from the wall and mouth of the centrifuge down into it. Finally, when the sediment is sufficiently free from bacteria, the various remaining particles are visibly clean, and the supernatant liquid, after centrifugalization, remains almost clear. This is removed to the beaker in which are now practically all of the bacteria present in the original portion of feces, together with some solid matter not yet separated. In the centrifuge tubes there is a considerable amount of bacteria-free solid matter.

"The suspension is now transferred to the same centrifuge tube, centrifugalized for a minute, and the supernatant liquid transferred to a clean beaker by means of the same pipette. The tube is then refilled from the first beaker and thus all the suspension centrifugalized a second time. The beaker is finally carefully washed with the aid of a rubbertipped glass rod, the second sediment in the centrifuge tube is washed free of bacteria by means of this wash-water and by successive portions of the dilute acid, and the supernatant liquid after centrifugalization is added to the contents of the second beaker. The second clean sediment is added to the first. The bacterial suspension now in the second beaker is again centrifugalized in the same way and a third portion of bacteriafree sediment is separated. Frequently a fourth serial centrifugalization is performed-always if the third sediment is of appreciable quantity. At all stages of the separation, small portions of the dilute hydrochloric acid are used, so that the final suspension shall not be too voluminous. Ordinarily it amounts to 125 to 200 c.c. At the same time, the final amount of fluid should not be too small, as shown by Ehrenpfordt, because the viscosity accompanying increased concentration prevents proper and complete sedimentation.

"To the final bacterial suspension an equal volume of alcohol is added and the beaker set aside to concentrate. A water bath at $50^{\circ}$ to $60^{\circ}$ is very satisfactory. After two or three days, when the liquid is concentrated to about 50 c.c., the beaker is removed and about 200 c.c. of alcohol are added. The beaker is covered and allowed to stand at room temperature for twenty-four hours. At the end of this time 
the bacterial substance is generally settled, so that most of the clear supernatant liquid, of dark brown color, can be directly siphoned off without loss of solid matter. The remainder is then transferred to contrifuge tubes, centrifugalized, and the remaining clear liquid pipetted off. The sediment consists of the bodies of the bacteria, and is transferred to a Kjeldahl flask for nitrogen determination. This is the bacterial nitrogen. Where a determination of bacterial dry substance is desired, the sediment of bacteria is extracted by absolute alcohol and ether in succession, transferred to a weighed porcelain crucible, and dried at $102^{\circ} \mathrm{C}$. constant weight. This dried sample is then used in the nitrogen determination. Our procedure differs from that of MacNeal in that the bacterial dry matter is not determined. A saving of about seven days' time and of considerable labor is accomplished by this omission.

"Inasmuch as it has been shown by various investigators that such bacteria as are present in the feces contain on the average about 11 per cent of nitrogen, the values for bacterial nitrogen as determined by our method may conveniently serve as a basis for the calculation of the actual output of bacterial substance."

\section{Examination of Feces for Bacillus Typhosus}

Lumsden and Stimson's Method. "Place about 5 gms. of the feces in a conical glass or other suitable vessel, add about 15 or 20 c.c. of sterile physiological salt solution or bouillon and agitate. Let stand one-half to one hour either at room temperature or, preferably, at incubator temperature $\left(37^{\circ} \mathrm{C}\right.$.) in order to permit the heavy particles to settle. Deposit one or two drops of the prenatant fluid in the center of an Endo plate. With a right-angled glass rod distribute the drop over the entirc surface of the plate and then rub the rod over the surface of a second, third, fourth and fifth Endo plate. By carrying the spreader over the surfaces of several plates in this way, one or two of the plates will furnish abundant but sufficiently isolated colonies to permit ' fishing.'

"After inoculation place the plates inverted in the incubator, leave there for twenty to twenty-four hours, and then examine. On the plates colonies of typhoid and paratyphoid bacilli will be transparent, colorless, dew-drop like and usually from 1 to $2 \mathrm{~mm}$. in diameter; while colonies of colon bacilli will be deep red, showing sometimes on the surface a sheen from precipitation of the fuchsin, and measure usually from 
3 to $4 \mathrm{~mm}$. in diameter. If typhoid-like colonies appear on the plates fish five or six of them, and inoculate tubes of Russell's medium in the following manner:

"Touch the colony with a sterilized platinum needle, make two streaks along the slanted surface of the medium in the tube and ther stab the needle down through the center of the block of medium to the bottom of the tube. Incubate the inoculated tubes of Russell's medium for twenty to twenty-four hours and then examine.

Kendall and Day's Method. "The feces are collected preferably in a small rectal tube. A small portion of the feces (about a loopful) is thoroughly emulsified in 10 c.c. of sugar-free broth and preferably incubated one hour at $37^{\circ} \mathrm{C}$. prior to the inoculation of the plates. This preliminary incubation does two things: the clumps of bacteria are thrown down, leaving a more uniform suspension of bacteria in the supernatant solution for inoculation and the bacteria undergo a slight development in a medium particularly suited for their growth. It is believed that better growth is secured if the feces is put through broth than if it is added directly to the solid media. It is essential that sugarfree broth be used.

"The fecal suspension is then rubbed over the surface of the Endo agar plates in the usual manner by means of the sterle glass rods. These plates are then incubated at $37^{\circ} \mathrm{C}$. for eighteen hours. At the end of this time small dewdrops are seen which may be removed entire to broth tubes. (1 c.c. broth which have been held at $37^{\circ}$ C.) These are then incubated for two hours at the end of which time sufficient growth will have taken place, with which to make the agglutination tests."

Holt-Harris-Teague (1916) Method for Isolating B. Typhi from Stools. These authors have devised a medium which they claim will give better results than Endo's medium. They claim that a greater percentage of colorless colonies on this medium turn out to be $B$. typhi than on Endo's medium. It is prepared as follows:

Nutrient agar is made in the usual way, containing 1.5 per cent agar, 1 per cent Witte's peptone, 0.5 per cent sodium chloride, and 0.5 per cent Liebig's meat extract, to the liter of distilled water. It is cleared with egg-white, placed in flasks, and sterilized in the Arnold sterilizer on three successive days. The reaction is brought to +0.8 . The agar is melted and saccharose ( .5 per cent) and lactose ( .5 per cent) are added. The medium is then heated for ten minutes in the Arnold. To every 50 c.c. of the medium are added 1 c.c. of 2 per cent yellowish eosin and 1 c.c. of 0.5 per cent methylene blue. We always add the eosin first 
and then the methylene blue. The mixture is shaken and plates are poured. The surface of the medium is dried in the usual way before the plates are inoculated. We have also obtained excellent results by substituting for Liebig's extract, meat infusion rendered free from sugar by incubation with $B$. coll.

Stock solutions of 2 per cent eosin and 0.5 per cent methylene blue in distılled water are kept in the dark. We have not sterlized these solutions, as we found that they could be kept in the ice-box for weeks without causing contaminations of the medium. Ordinarily we do not heat the agar after the dyes are added, but we have demonstrated that the stained agar can be heated a half hour in the Arnold sterilizer without injury.

Carnot and Halle's Sand Tube Method. This method has had much praise. The method has been described by Gautier (1915) and Levy (1916) somewhat as follows: A pipette tube $33 \mathrm{~cm}$. long by about 5 to 6 $\mathrm{mm}$. in diameter is softened in the middle and the ends brought up to form a U. One of the arms thus formed is filled to a height of $10 \mathrm{~cm}$. with very fine sand (passed through a No. 40 sieve). The other end is filled with hot bouillon and tinted with neutral red, which works up through the sand until the other tube is filled to about the same level. The arm in which there is no sand is inoculated with the sample and incubated for eighteen hours. The most motile bacteris will penetrate the sand and appear in the other arm. Gautier found that B. coli occasionally did this. Agglutination reactions should be made to confirm the results of the sand tube. Other adaptations of the sand-tube method have been reported by Borzone and Carbone (1918) and Piazza (1916).

Isolation of B. typhi from Feces. (Teague and Clurman's Method.) This method is especially adapted to specimens which must be kept for a short time before examination. The specimens are rubbed into a solution of 0.6 per cent sodium chloride containing 30 per cent of glycerol. This environment seems to be very destructive to the colon bacilli and other bacilli in stools but not harmful to the B. typhi. After this, the stool is streaked on plates made from Teague and Clurman's special eosin-brilliant green agar the preparation of which has been mentioned in the chapter on the preparation of media. Beckler (1918) reports that this method has given very satisfactory results at the laboratories of the Massachusetts State Board of Health. One specimen is reported which gave negative results on immediate examination but positive results after holding in the presence of glycerol as advised by Teague and Clurman. This work is also confirmed by Benians (1918). 
He claims that positive results are more likely after preserving the stool in glycerol especially in hot climates or countries.

Isolation of Typhoid Bacilli from Urine. (Morishama and Teague's

Method.) Streak two or three loops of the urine over an Endo or eosinbrilliant green medium plate; add to the urine about one-half its volume of nutrient broth and incubate the mixture over night. If the plate which has been inoculated directly with the urine is negative prepare dilution of the incubated urine next morning and streak on the above plates again. This latter procedure will furnish a higher percentage of positive results.

\section{Examination of Feces for Microspira Cholerm}

Gedding's Method. It would be neither possible nor desirable to fix a technique limited by strict rules for the various operations of bacteriological examinations, but the following general indications may be recommended as permitting in the great majority of cases a positive diagnosis within twenty-four to thirty-five hours:

1. When mucous flakes are available for examination of microscopical investigation of the same, in stained preparations and in the hanging drop.

2. The isolation of the vibrios, employing for the purpose, agar media, at a temperature of $37^{\circ} \mathrm{C}$.

(a) Plant plates of ordinary suitably alkalinized agar and of Dieudonne's medium, using, for the latter, a risiform particle, or an equivalent quantity of feces.

(b) Plant in 50 c.c. of peptone solution 1 c.c. of fecal matter. After a stay of six hours in the incubator (or twelve to eighteen hours, if need be), at $37^{\circ} \mathrm{C}$. take several loopfuls from the surface and plant with them several plates of Dieudonne medium and ordinary agar.

(c) Investigate the agglutination reaction, using drops for the purpose, from the isolated colonies, the properties belonging to cholera vibrios, and secure pure cultures.

3. Demonstrate the character of the vibrios obtained in pure culture, by the reaction of agglutination or that of Pfeiffer.

The conditions are much more favorable to the discovery of vibrios if pathological materials (feces or intestinal contents) are collected as early in the attack as possible, or secured from the cadaver as early as possible after death. Examinations made of the small quantity of material collected by a sound introduced into the rectum, in the living body, or from the cadaver are unreliable. It is sometimes possible to 
recognize that a person even in good health has undergone an attack of cholera by determining whether his blood serum gives with a genuine cholera vibrio the immunity reactions, viz., agglutination or the reaction of Pfeiffer.

Teague and Travis' Method. Two pounds of beef are soaked in 2 liters of water over night. In the morning filter through cloth, heat in the Arnold steam sterilizer and filter through paper. Adjust the reaction to neutrality to litmus by means of sodium hydroxide. Inoculate with $B$. colı and incubate for a few days. Then prepare nutrient agar from it by adding 1 per cent of Witte's peptone, 0.5 per cent of sodium chloride and clear with egg white. Adjust the reaction to 0.5 per cent alkaline, filter and add 0.25 per cent of nutrose. To 50 per cent of this nutrose agar add 1 per cent of sucrose and 1 c.c. of a 3 per cent solution of bluish eosin and 2 c.c. of a 1 per cent solution of Bismarck Brown. Microspira cholerce colonies will show brown centers after twenty-four hours. $B$, coli will show pale pink or yellowish colonies.

\section{Examination of Feces for B. Tuberculosis}

Petrof's Method. In order to remove the food particles, dilute with water and filter through gauze. Saturate the filtrate with sodium chloride and, at the end of a half hour, all of the bacteria will be found in this film. Collect the film and add sodium hydroxide, shake well and incubate at $22^{\circ} \mathrm{C}$. for three hours. Then neutralize to sterile litmus paper, centrifuge and inoculate.

Reh Method. Stir the lump of stool in an Esbach glass with sufficient sterile water to make a soft paste but solid enough not to flow when the glass is tilted. Add a little ether and shake after closing with a rubber stopper. Pour the ether into a centrifuge glass and centrifugalize. The ether is then decanted and the sediment examined after staining with Ziehl's method.

Engleson's Method. Scrape the rectal mucosa with an ordinary sound. Make a smear and stain.

\section{Isolation of Yeasts from Feces}

Anderson's Method. Prepare plates of Sabouraud's agar. By means of a platinum wire which has been dipped into the feces emulsion, touch one of these plates in lines across the plate at distances of about $4 \mathrm{~mm}$. Make as many rows as possible. The yeast colonies which develop in this way should be subjected to further study. 


\section{BIBLIOGRAPHY}

Anderson, H. W. 1917. Yeast-like Fungi of the Human Intestinal Tract. Jour. Inf. Diseases, 21, 341-386.

BAcon, R. F. 1916. The Germicidal Efficiency of Some Copper Cements used in Dental Work. Dental Cosmos, 58, 40-49.

Barker, L. F. 1914. The Diet of Typhoid Fever. Jour. Amer. Med. Assn., 63, 929-931.

Basten, J. 1914. Methods of Determining the Bacterial Flora in Feces of Suckling Infants and our Knowledge of the Bacterial Types. Zeit. Hygiene, $77,282$.

Beckler, E. A. 1918. Use of Glycerine in the Examination of Feces for Typhoid Bacilli. Jour. Amer. Med. Assn., 70, 768.

Benians, T. H. C. 1918. Preservation of Typhoid Bacilli in Stools. Lancet 1, 255, 1918. Jour. A. M. A., 70, 961; Amer. Jour. Pub. Health, 8 (1918), 396.

Bergeim, O. 1917. The Determination of Fecal Indole. Jour. Biol Chem., $32,17-22$.

Blatherwick and Hawk. 1914. Fasting Studies XIII. The Output of Fecal Bacteria as Influenced by Fasting and by Low and High Protein Diet. Jour. Amer. Chem. Soc., 36, 147.

Blatherwick, Sherwin and Hawk. 1912. intestinal Putrefaction and Bacterial Development Accompanying Water Drinking and Fasting. Jour. Biol. Chem., 11, VIII Proceeding.

BOAs and Oppler. 1895. Zur Kenntnis des Mageninhalts beim Carcinoma ventriculi. Deutsch. Med. Wochenschr., 21, 73-75.

Borzone and Carbonne, J. M. 1918. Sand Test of Typhoid Carriers. Jour. Amer. Med. Assn., 70, 895.

Boudielle, T. 1913. Influence of Bile on the Fermenting Action of the Colon Bacillus. Comp. Rend. Soc. Biol., 72, 783-785.

Brugnatelli. 1915. Etiology of Dental Caries. Zent. Biochem. u. Biophys., $18,96-97$.

Cadeac and Bouray. 1893. Rol. microbicide des sucs digestifs et contagion par les matières fécales. Cent. Bakt. Orig., 16, 672.

CAmpo, G. 1902. Micro-organisms of the Mouth of the New Born. Dental Cosmos, 1.00. Cent. Bakt., 31, 496.

Carnot, P. and Halle, B. W. 1915. A New Method of Rapid Bacteriologic Diagnosis of Typhoid Fever. Jour. Amer. Med. Assn., 66, 677.

Collins, K. R. and Grund, M. 1907. Study of the Intestinal Flora. Collected Studies from the Research Laboratories, Department of Public Health, City of New York. 1907, p. 161.

Debono. 1912. On Some Anaerobic Bacteria of the Normal Human Intestine. Cent. Bakt. Orig., 62, 229-234.

Distaso, A. 1913-4. Ueber das Schicksal der per Os eingefuhrten Bacter.en Cent. Bakt. Abt. I. Ref., 60, 255. 
Distaso, A. 1912. The Intestinal Flora. Lancet, 182, 496-498.

Distaso, A. and Schilder, J. 1914. Sur l'acclimation des microbes étrangers à la flore intestinale. Comp. Rend. Soc. Biol., 76, 243-244.

Dunon, E. 1903. Action of Tobacco Smoke upon Certain Microbes of the Mouth. Cent. Bakt, 32, 612.

Ecker, E. E. 1918. The Inhibitive Action of Ox Bile on B. Typhosus. Jour. Inf. Diseases, 22, 95-100.

FORD, W. W. Classification and Distribution of the Intestinal Bacteria in Man. Rockfeller Inst. Med. Res.

Friedenwald, J. and Leitz, L. F. 1909. Experiments Relating to the Bacterial Content of the Feces, with some Researches on the Value of Certain Intestinal Antiseptics. Amer. Jour. Med. Sciences, 138, 653-661.

Fuller, G. W. 1912. Sewage Disposal. McGraw-Hill Book Co., New York. Chapter II. Aerobic and anaerobic Decomposition of Sewage.

Gait ANd Ires. 1914. A Study of the Boas-Oppler Bacillus. Jour. Path. and Bact., 19, 239-244.

GaRHART. 1916. Are there any Laws which Govern the Behavior of Dental Amalgama. Dental Register, 70, Chem. Abstracts, 10, 1379.

GaUtier, P. 1915. Nouveau procédé de recherche du bacille typhique dans les selles. Rev. Médicale de la Suisse Romande, 34, 361-442.

GeDdings, H. D. 1912. The Bacteriological Diagnosis of Cholera. Reprint No. 75, Public Health Reports.

GIEs, W. G. 1915. Chemical Studies of the Relations of Oral Micro-organisms to Dental Caries I. The Importance of a Re-examination and an Extension of the Available Knowledge Pertaining to the Kinds and Chemical Influences of Oral Bacteria in their Relation to the Teeth. Jour. Allied Dental Societies, 10, 137-140.

Gordon. 1916. The Inhibitory Action of Saliva on the Growth of the Meningococcus. Brit. Med. Jour., 1916-I., 849-851.

GREGERSEN. 1916. Untersuchungen uber die antiseptische Wirkung des Magensaftes. Cent. Bakt. Orig., 77, 353-361.

GrINDLEX, H. S. and MAcNeAL, W. J. 1912. Bacteria and Chemistry of Feces of Healthy Men. Vols. 3, 4, and 5. Univ. of Illinois.

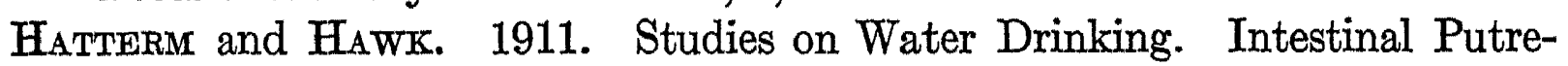
faction During Copious and Moderate Water Drinking with Meals. Arch. Inter. Med., 7, 610 .

HAUSSEN. 1912. Untersuchungen am Hund uber den Einfluss infizierter Milch auf das Bakterienwachstum in Verdauungstraktus speziell im Magen. Cent. Bakt., 62, 89-126.

$H_{A W K}$, P. B. 1916. Quantitative Determination of Fecal Bacteria. Practical Physiological Chemistry, P. Blakiston's Son \& Co.

Heeter, C. A. and Kendall, A. I. 1908. The Use of the Fermentation Tube in Intestinal Bacteriology. Jour. Biol. Chem., 5, 283.

Heniemann and Ecker. 1916. A Study of the Boas-Oppler Bacillus. Jour. Bact, 1, 443 . 
Herter, C. A. 1907. The Common Bacterial Infections of the Intestinal Tract. The Macmillan Co., New York.

Holm-Harris, J. E. and Teague, O. 1916. A New Culture Medium for the Isolation of Bacillus Typhosus from Stools. Jour. Inf. Dis., 18, 596-600.

HopkrNs and CoLe. 1903. Constitution of Tryptophane and the Action of Bacteria on it. Jour. Physiology, 29, 451.

Houston, A. C. 1902-3. Bacteriological Examination of Normal Stools of Healthy Persons. Local Gov. Bd. Med. Officer's Report 1902-3., 511; $1903-4,472$.

Houston, A. C. 1914. Study of Streptococei in Excremental Matter. Mettopolitan Water Board, 10th Rept. on Research Work, 1914, 22-23.

Huld, T. G. and RetTGeR, L. F. 1915. The Influence of Milk and Carbohydrate Feeding on the Character of Intestinal Flora. Cent. Bakt., 75, 365-388. Jour. Bact., 2, 47-71.

Kendali, A. I. 1911-12. The Rapid Isolation of Typhoid, Paratyphoid and Dysentery Bacilli. Jour. Med. Res., 25, 95-99.

Kendall, A. I. 1911. Activity of Bacteria in the Intestinal Tract. Jour. Med. Res., 25, 117-187.

Kranowsky. 1891. Zur Frage uber die antimikrobiellen Eigengeshaft des Magensaftes. Cent. Bakt., 10, 236-237.

KLEIN, A. 1900. Eine neue Mikroskopische Zahlungsmethode der Bakterien. Cent. Bakt. Orig., 27, 834.

KreIN, A. 1906. Ueber den Bakteriengehalt menschlicher und tierischer Feces. Arch. Hyg , 59, 283.

KuIGLER, I. J. 1915. Chemical Studies of the Relations of Oral Microorganisms to Dental Caries. IV. A Biochemical Study and Differentiation of Oral Bacteria with Special Reference to Dental Caries. Jour. Allied Dental Societies, 10, 445-448, 282-330.

KURLOFF and WAGNER. 1889. Ueber die Einwirkung des menschlichen Magensaftes auf Krankheits erregende Keime. Cent. Bakt., 7, 448-450.

Lagane, L. 1914. Action of Bile in Vitro on the Development of Intestinal Bacteria. Comp. Rend. Soc. Biol., 73, 242-243.

Levy, M. D. 1916. The Use of the Sand Tube in the Isolation of the Typhoid Bacillus. Jour. Med. Assn., 66, 1022-1023.

Lumsden, L. L. and Strmson, A. M. 1912. Examination of Excreta for Typhoid Bacilli. Reprint No. 80, Public Health Reports.

MACCoNkeY, A. 1907. Lactose Fermenting Bacteria in the Feces. Jour. Hyg., 7, 331; 5, 333.

MacNeIL, Latzer and KerR. Fecal Bacteria of Healthy Men. Jour. Inf. Dis., $6,123-169 ; 571-609$.

Marshall. 1916. An Acidimetric Study of the Saliva and its Relation to Dental Caries. Dental Items of Interest, 38, 116-127.

MatriLl, H. A. and HawK, P. B. 1911. A Method for the Quantitative Estimation of Fecal Bacteria. Jour. Exper. Med., 14, 433-443.

MatricL and Hawk. 1911. Studies on Water Drinking. IX. Distribution 
of bacterial and other forms of nitrogen and utilization of injected protein under the influence of copious and moderate water drinking with meals. Jour. Amer. Chem. Soc , 33, 1999-2032.

Neucki, M., Sieber, N., and Schoumowa, E. 1898. Die Entgiftung der Toxine durch die Verdauungssafte. Cent. Bakt., 23, 840-847.

NiEdERgESAFS 1915. Anatomical, Bacteriological and Chemical Investigations of the Production of Dental Caries. Arch. Hyg., 84, 220-260.

Osborne, T. B. and Mendel, L. B. 1914. The Contribution of Bacteria to the Feces after Feeding Diets Free from Indigestible Components. Jour. Biol. Chem., 18, 177.

OzaKr. 1912. Zur Kenntniss der Anaeroben Bakterien der Mundhole. Cent. Bakt., 62, 76-98.

Petroff. 1915. A New and Rapid Method for the Isolation and Cultivation of Tubercle Bacilli Directly from the Sputum and Feces with the Aid of Sodium Hydrate and Gentian Violet Egg Meat Juice Medid. Science, $41,661$.

PiazzA. 1916. Sand Tube Method for Isolating Typhoid Bacilli from Stools. Jour. Amer. Med. Assn., 70, 1281-1282.

RaHE. 1914. An Investigation into the Fermentation Activities of Aciduric Bacteria. Jour. Inf. Diseases, 15, 141-150.

RETTGER, L. F. 1915. The Influence of Milk Feeding on Mortality and Growth, and on the Character of the Intestinal Flora. Jour. Exper. Med., $21,365-388$.

Rettger, L. F. and Harton, G. D. A Comparative Study of the Intestinal Flora of White Rats Kept on Experimental and Ordinary Mixed Diet. Cent. Bakt. Abt. I., 73, 362-372.

Rodella. 1908. Magen Carcinoma. Cent. Bakt. Abt. I., 47, 445-447.

ROGER, R. 1912. Influence on Microbic Fermentations of Carbohydrates. Arch. Med. Exp., 24, 461-488.

ROGER, R. 1913. Influence of Bile on the Putrefaction of Nitrogenous Material. Comp. Rend. Soc. Biol., 73, 274-276.

Roger, R. 1913. Influence of Bile on Bacterial Fermentation. II. Fermentation of glycogen. Comp. Rend. Soc. Biol., 72, 544-545.

RoGER, R. 1913. Influence of Bile on Fermentation. III. Fermentation of Glucose. Comp. Rend. Soc. Biol., 72, 603-604.

Roger, R. 1913. Influence of Bile Extracts and Bile Salts on Bacterial Fermentations. Comp. Rend. Soc. Biol., 72, 656-657.

Roger, R. 1915. Antiputrefactive Role of the Bile. Ann. Past. Inst., 29, $545-550$.

SANDBERG. 1904. Ein Beitrag zur Bakteriologie der Milchsauer Garung im Magen mit kesonderer Berucksichtigung der langen Bacillen. Zeit. f. klin. Med., 51, 80-94. Complete bibliography.

Schmmt and Strassburger. 1905. Die Faeces des Menchen, Berlin.

Schmitz, C. 1893. Zur Kenntniss der Darmfaulniss. Zeit. Physiol. Che., 17, 401-403. 
Schulz-Schultzenstein. 1908. Zur Kenntniss der Einwirkung des menschlichen Magensekrete auf Choleravibrionen. Cent. Bakt. Orig., 30, 785.

Surrnow. 1915. Germicidal Action of Dental Cements. Dental Cosmos. $57,1209-1228$.

Steele, J. D. 1907. The Method of Determining the Total Amount of Fecal Bacteria by Weight and its Clinical Significance. Jour. Amer. Med. Assn., August 24, 1907.

Stern. 1908. Ueber das Verhalten der Cholera-vibrionen dem menschlichen Mageninhalt gegenuber. Cent. Bakt., 47, 561, Abt. I.

STR ASSBURGER. 1902. Untersuchungen uber die Bakterienmengen in menschlichen Feces. Zeit. klin. Med., 46, 413.

Strauss. 1909. Ueber die Abhangigkeit der Milch sauer Gahrung vom $\mathrm{HCl}$ Gehalt des Magensaftes. Zeit. klin. Med., 28, 567-578.

Tanner, F. W. 1917. Studies on the Bacterial Metabolism of Sulphur. I. Formation of hydrogen sulphid from certain sulphur compounds under aerobic conditions. Jour. Bact., 3, 565-593.

TANner, F. W. 1918. Studies on the Bacterial Metabolism of Sulphur. II. Formation of hydrogen sulphid from certain sulphur compounds by yeastlike fungi. Jour. Amer. Chem. Soc., 40, 663-669.

Teague, O. and Clurman, A. W. 1916. A Method of Preserving Typhoid Stools for Delayed Examination and a Comparative Study of the Efficacy of Eosin Brilliant Green Agar and Endo Agar for the Isolation of Typhoid Bacilli from Stools. Jour. Inf. Diseases, 18, 653-671.

Teague, O. and Clurman, A. W. 1916. An Improved Brilliant Green Culture Medium for the Isolation of Typhoid Bacilli from Stools. Jour. Inf. Diseases, 18, 674-652.

Teague, O. and Travis, W. C. 1916. A Differential Culture Medium for the Cholera Vibrio. Jour. Inf. Dis., 18, 601-605.

Torda, R. 1914. Sterility of the Bile under Normal Conditions and its Bactericidal Action on Pathogenic Bacteria. Chem. Abstracts, 8, 2558-2559.

Turesson, G. 1916. The Presence and Significance of Molds in the Intestinal Canal of Man and Animals. Exp. Sta. Rec., 35, 559-560.

Washburn and Goadby. 1896. Some Points in Connection with the Bacteria in the Mouth. Trans. Odontological Soc. Great Brit., 1896.

Williams. 1859. A Contribution to tho Bacteriology of the Human Mouth. Dental Cosmos. 1899.

Worman, F. 1912. The Amylolytic Bacteria of the Intestines. Am. Past. Inst., 26, 610-624. 


\section{CHAPTER IX}

\section{BACTERIAL EXAMINATION OF AIR}

THE bacteriological examination of air has not been of much sanitary significance. Many investigations have been carried out and different apparatuses devised to remove the bacteria from a definite quantity of air. A review of these methods has been given by Besson and those wishing a rather complete historical survey should consult that treatise. Ruehle (1915) has devised the most satisfactory technique and probably his method represents the best which has been devised up to the present. Much of the matter presented in this chapter has been taken from his publication. This method involves the use of the aeroscope, which is regarded as an apparatus for gathering bacteria from the air. The other accessories to the procedure are not included in this procedure.

Rettger (1910) made one of the first important advances in the bacterial examination of air. He used 5 c.c. of physiological salt solution as the filtering medium in a special aeroscope described as follows:

The entire apparatus consists of a glass tube with a small round bulb at the end. The bulb has eight or ten small perforations which serve the purpose of allowing the air to pass through at a rapid rate and divide the gas to such an extent that every particle of it is brought into close contact with the filtering fluid. This glass tube or aeroscope is fitted into a small, thick-walled test tube by means of a rubber stopper, which also bears, besides the aeroscope, a short glass tube bent at right angles. The upper end of the aeroscope is at an angle of about $45^{\circ}$, in order to prevent bacteria and particles of dust from falling into the open end of the tube, and still permit of the tube being drawn through the stopper without difficulty.

The standard aeroscope has been described by Ruehle as follows:

A $10 \mathrm{~mm}$. layer of sand which has been passed through a 100-mesh sieve and has been retained by a 200 -mesh sieve is supported within a cylindrical glass tube $70 \mathrm{~mm}$. in length and $15 \mathrm{~mm}$. in diameter upon a layer of bolting cloth folded over the end of a rubber stopper. Through a perforation in the stopper, there passes a tube $6 \mathrm{~mm}$. in diameter and $40 \mathrm{~mm}$. in length. This tube is attached to the aspirator bottle. The upper end of the cylindrical tube is closed by a perforated rubber stopper through which is passed a glass tube $40 \mathrm{~mm}$. in 
length and $6 \mathrm{~mm}$. in diameter bent at an angle of $45^{\circ}$ in order to prevent precipitation of bactoria or dust particles into the aeroscope. In using this aeroscope a measured volume of air is filtered through the tube, the sand shaken out into 10 c.c. of water and aliquot portions of this suspension plated on nutrient agar.

The " modified" form of the aeroscope differs very little. The lower rubber stopper and the bolting cloth is eliminated by fusing a small tube into the large one. A layer of cotton supports the sand.

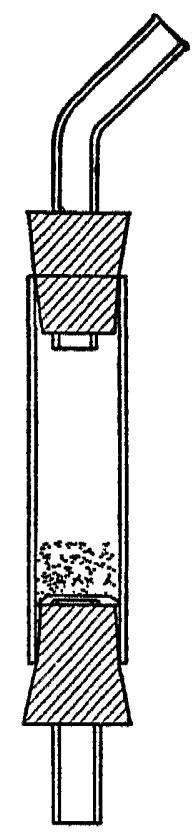

Standard Aeroscope

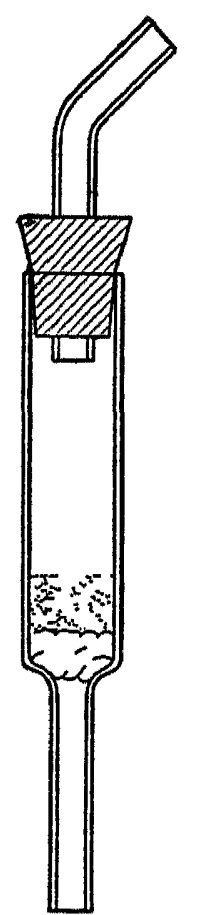

Modified Standard Aeroscope
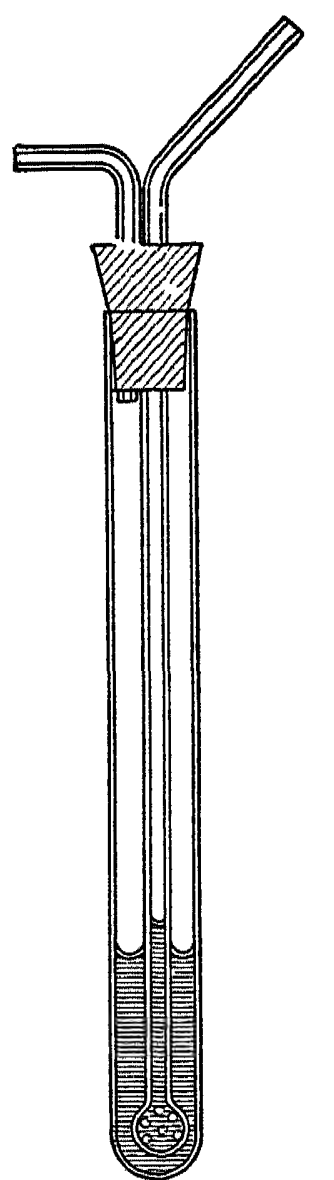

Rettger

Aeroscope

Fig. 57.-Types of Aeroscopes. (After Ruehle.)

In order to render the apparatus capable of being sterilized by hot air, the upper stopper is made of cork.

The Committee on Standard Methods for the Examination of Air of the American Public Health Association recommended the following procedure:

The number of bacteria in the air does not appear to be a factor of any great significance. In special cases, however, as in the study of dairy conditions, it may be of interest.

The sand filter method originally used by Petri has proved essentially sound 
in principle. It was recommended in our 1909 report and again, after careful studies of altertive processes in 1912.

One modification has been made in the apparatus since 1912 which is so clearly an improvement as to warrant immediate adoption. The layer of sand in the filters as previously used was supported on a perforated rubber stopper in a straight section of glass tubing, and the aspiration was applied to a small glass tube passing into the rubber stopper. It was clearly essential that the stopper should fit tightly, which meant that the apparatus must be sterilized in steam. Ruehle (1915) points out that the steam sterilization causes caking of the filter sand and thus introduced appreciable errors in analysis. Ruehle, therefore, made the suggestion that the rubber stopper should be eliminated and the small aspirating tube fused into the large one.

$\mathrm{We}$, therefore, recommend that for the study of bacteria in air there should be used a glass tube of $15 \mathrm{~mm}$. in diameter and $70 \mathrm{~mm}$. long with a smaller tube $6 \mathrm{~mm}$. in diameter and $40 \mathrm{~mm}$. long fused into one end. On the shoulder of the joint between the tubes rests a plug of cotton supporting a layer of sand $10 \mathrm{~mm}$. deep. The sand should be capa ble of passing a 100-mesh sieve but not a 200-mesh sieve. The opposite or inlet end of the larger tube is stoppered by a cork stopper (which need not be exactly tight) perforated by a glass tube $6 \mathrm{~mm}$. in diameter and $40 \mathrm{~mm}$. long bent at an angle of $45^{\circ}$ to prevent direct precipitation of dust particles into the filter tube.

Five cu. ft. of air should be drawn through the filter by the use of an aspirator of known volume, preferably one of the double or continuous type, or by the use of some form of pump or meter. A convenient and ingenious sampling pump was made by Wallace and Tiernan of New York City for the study of Baskerville and Winslow (1913) of the air of New York City schools. After filtration, the sand should be shaken out into 10 c.c. of sterile water, and, after thorough shaking, aliquot portions of the water should be plated on ordinary nutrient agar.

Browne (1917) has proposed a tube which he claims has special advantages over the aeroscope used in standard methods. This consists of the filter tube and dilution tube fused together at right angles. During filtration the sand is placed over the stoppered end. After filtration the sand is shaken into the other end and the filter plug with the bolting cloth is replaced by a sterile stopper. Ten c.c. of sterile water are added and the sand thoroughly shaken. From this decimal dilutions are plated on standard media.

\section{BIBLIOGRAPHY}

Baskervilie, C. and Winstow, C. E. A. 1913. The Air of New York City Schools. Report of the Committee on School Enquiry Board of Estimate and Apportionment, City of New York. III, 611. 
Browne, W. W. 1917. Improved Technique in Bacterial Air Analysis. Amer. Jour. Pub. Health, 7, 52-53.

Gordon, M. H. 1902-3. Report of a Bacterial Test for Estimating Air Pollution. Annual Report of the Local Government Board, containing the Report of the Medical Officer for 1902-3, 421 .

Retrger, L. F. 1910. A New and Improved Method for Enumerating Air Bacteria. Jour. Med. Res., 17, N. S., 161-468.

Ruenle, G. L. A. 1915. Methods of Bacterial Analysis of Air. Jour. Ag. Res., 4, 343-368.

Ruehle, G. L. A. and Kulp, W. L. 1915. Germ Content of Stable Air and its Effect on the Germ Content of Milk. Bull. 409, N. Y. Agric. Exp. Sta., Geneva.

Winslow, C. E. A. and Browne, W. W. 1914. The Microbic Content of Indoor and Outdoor air. Monthly Weather Review, 42, 452. 


\section{CHAPTER X}

\section{WATER HYGIENE}

ThAT water has a close connection with the spread of disease needs no special emphasis. Johnson (1916) has given ample proof of this by collecting statistics from the large registration cities of the United States. (Fig. 59.)

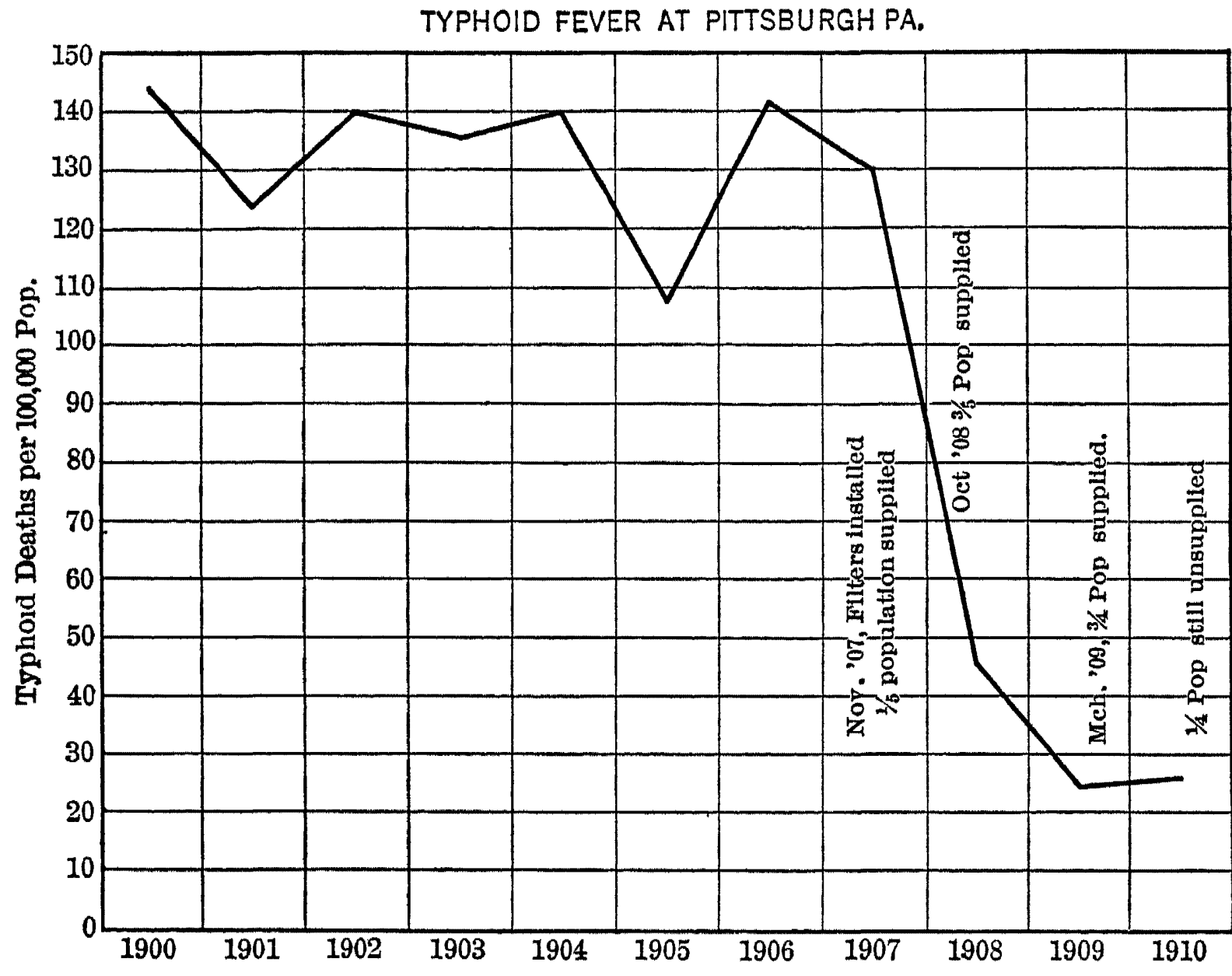

Frg. 58.-Showing the Effect of a Pure Water Supply on the Death Rate from a Typhoid Fever in Pittsburg. (After McLaughlin, 1912.)

The "Mills-Reincke Phenomenon" and the "Hazen Theorem." In 1893, soon after the establishment of the Lawrence, Mass., filtration plant, Mills, the city engineer, noticed a reduction in the general death rate along with a reduction in the typhoid fever death rate. This was also noticed for Hamburg, Germany, by Reincke. When Sedgwick and 
MacNutt (1910) were studying the effect of water treatment on disease they gave the name "Mills-Reincke Phenomenon" to this observation. They stated that infant mortality was closely related to water supply. Also a decrease in tuberculosis was reported for an improvement in the water supply. Hazen (1910) reported somewhat the same fact that "where one death from typhoid fever has been avolded by the use of a pure water, a certain number of deaths, probably two or three, from other causes have been avoided." This has become known as the "Hazen Theorem." These statements have not been enturely borne out by a more careful study. Fink (1916) has pointed out that an error enters in comparing the total mortality with the typhoid death rate. He states that there are two factors which may cause a variation in the ratio between the total and the typhoid fever death rate. " One is the rate at which the general mortality is being lowered and the other is the height of the typhoid fever death rate before filtration and the proportion due to water-borne infection."

The spread of typhoid fever by impure water has been so prevalent in the past that the disease is often referred to as a water-borne disease. Many data are on record to show a decided decrease in the typhoid fever death rate when an improved water supply has been made available to a community. Fig. 58 prepared by McLaughlin shows this reduction for Pittsburg.

Sources of Water Supply. The sources of water supply may be classified as follows:

A. Surface supplies

(a) Lakes

(b) Rivers

(c) Upland gathering grounds

(d) Impounding reservoirs

$B$. Ground water supplies

(a) Shallow walls

(b) Deep wells

1. Artesian

2. Bored

3. Drilled

(c) Springs

Sanitary Inspection. Too little attention is given to sanitary inspection in water analysis: The analyst is quite inclined to regard his results as sufficient data for passing judgment on a water supply. 


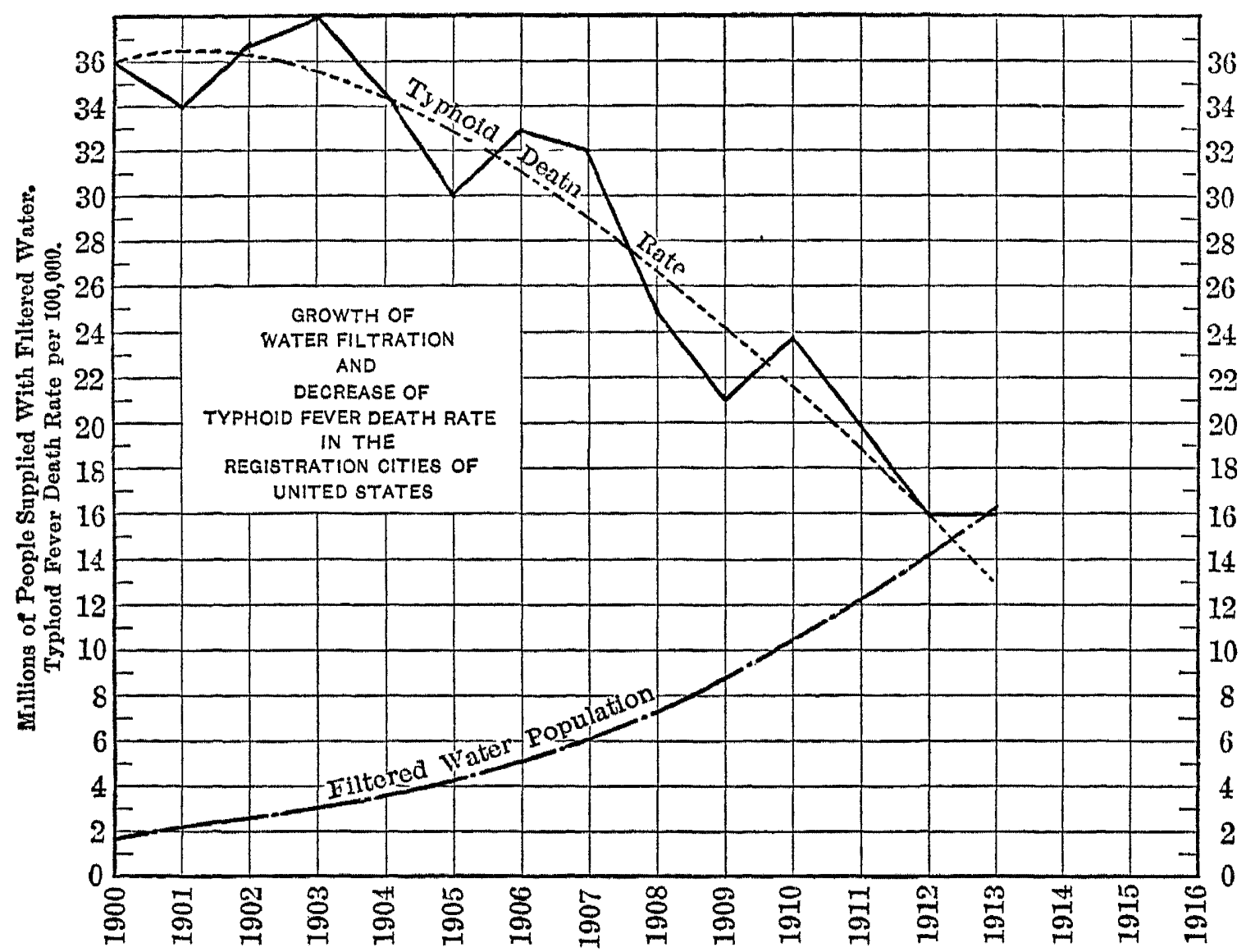

FIG. 59.-Relation of Water Filtration to Typhoid Fever. (After Johnson.)

AVERAGE TYPHOID FEVER DEATH RATE

- FOR -

FIVE YEARS BEFORE AND FIVE YEARS AFTER FILTRATION

$$
\text { - IN- }
$$

ALL CITIES OF U S. OVER 100000 POPULATION IN 1915

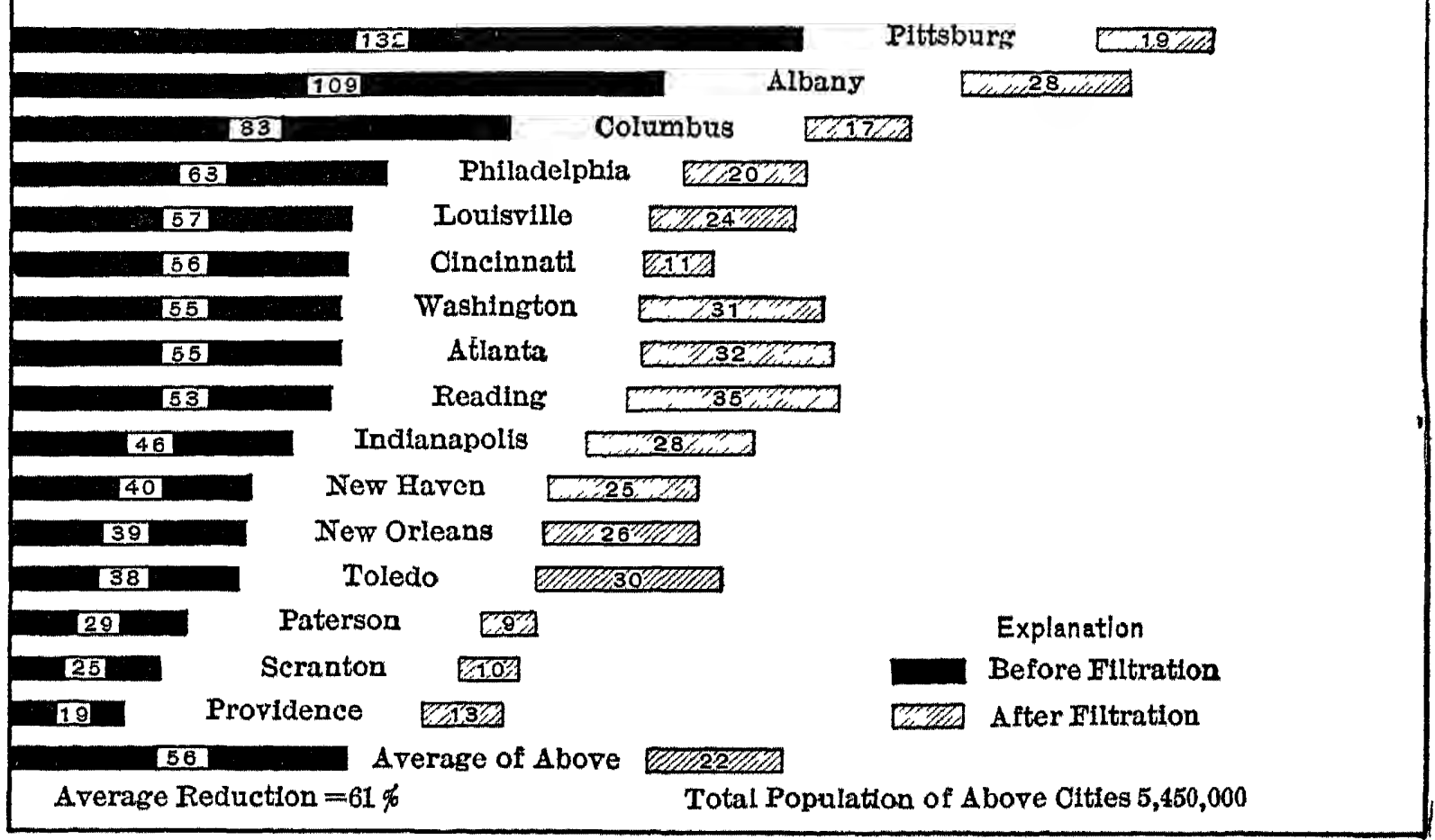

Fig. 60.-Typhoid Fever Death Rate in American Cities. (After Johnson.) 
Sawyer (1915) quotes an instance of a water-borne epidemic which could have been prevented had it been possible to carly out a sanitary inspection. In a certain water supply indications of pollution appeared two months before the epidemic broke out. Could a sanitary inspection have been made to investigate the conditions indicated in the laboratory results, seven deaths and a large number of cases of typhoid fever could have been prevented. In many cases it would not be necessary to make laboratory examinations because the environmental factors would indicate a bad water. In such a case, the laboratory might be used to check up the remedial measures which had been carried out. The field investigations would also yield information with regard to the endemic disease which occurs in a community. Where possible, both field studies and laboratory studies should be made but where one is possible, the sanitary inspection will make it unnecessary, very often to carry out the laboratory examination.

Pure Water. Chemically water is a monoxide of hydrogen and is made chemically pure only with great difficulty. Only the greatest care in technique will yield a water which is free from all traces of metals and gases.

Bacteriologically speaking, a water is pure when it contains no bacteria which may cause disease or sickness. This is a difficult term to define since a water which is pure for one individual may be impure for another. The technique which is available for isolating certain pathogenic bacteria is too crude to give accurate knowledge with regard to purity. Negative results may mean little more than that the certain bacteria sought for, were not found. Generally speaking, a pure water is one which will not cause any disturbance in those who partake of it.

Relation of Chemical and Bacterial Exar ination. In the past there has been much discussion over the superiority of the chemical or bacterial analysis of water. This has probably been quite useless since both are important and necessary for an accurate opinion with regard to the potability of a given water. The more data which an analyst has, the more accurate will his opinion be. Despite all this there are certain outstanding facts with regard to each analysis which might be emphasized.

The chemical analysis may give more data with regard to the history and possibly the age of the water. It gives an idea with regard to the organic matter and the different stages in its decomposition. If most of the nitrogen is in the form of nitrates, it indicates possibly remote pollution and a partial purification. In giving an opinion which must be based on the chemical analysis only, the water analyst has to use the 
organic matter in its different stages of oxidation. The chemical examination of water is important when a municipal supply is treated with alum since in this connection it is important to maintain sufficient alkalinity in the water to decompose all of this coagulant and not allow it to pass into the filtered water. Certain objectionable trade wastes may be detected in a water by chemical analysis.

The bacteriological analysis is more delicate and direct. It is easy to conceive that a water which, according to chemical analysis was of good quality, might contain many pathogenic bacteria. These, unless they were present in enormous numbers could not be detected chemically. In the bacterial examination the analyst is searching for the factors which are most important. In the chemical analysis their presence is inferred when other substances are present. While the isolation of specific pathogenic bacteria from water is not attended with much success, the bacterial examination yields more definite knowledge of their presence. In determining the efficiency of filters, this analysis is important.

The purpose of water examinations are many. Mineral examinations are made by the chemist to determine the effect of any water on boilers, to determine the amount of pollution, presence of any of the heavy metals, etc. The bacteriologist is more interested in the number and kinds of bacteria which may be present. He has to contend with the disadvantage, unlike the chemist, that many of his conclusions are drawn from circumstantial evidence.

For our purposes, a sanitary water analysis may be regarded as made up as follows:

\section{Sanitary chemical examination}

A. Physical (organo leptic) tests

$a$. Turbidity, b. Color, c. Odor

$B$. True chemical determinations

1. Residue

2. Chlorine

3. Nitrogen as

a. Nitrates

b. Nitrites

c. Free ammonia

d. Albuminoid ammonia

4. Alkalinity

5. Oxygen consumed 
Collection of Samples. The collection of the sample of water is an important part of the analysis. If done carelessly, it may determine the results, which are secured in the later analysis. For chemical analysis, the samples should be collected in glass-stoppered bottles which are scrupulously clean. The stoppers should fit tightly and be protected during transportation by tying a piece of cloth over them. For bacteriological analysis, four-, six- or eight-ounce glass-stoppered bottles should be used. They should be cleaned as outlined before (Chapter I) and sterilized. After cooling and before shipment, the top of each bottle should be protected by a piece of cloth or tin foil. Specimens for bacteriological analysis should always be packed in ice during transportation.

Interval before Analysis. The analysis should be started as soon as possible after collection of the sample. The composition of water undoubtedly changes and for this reason certain examinations may be conducted in the field. Any time which may be allowed to elapse between collection and analysis of samples is entirely arbitrary. The committee on "Standard Methods" suggests the following as being fairly reasonable maximum limits.

Physical and Chemical Analysis.

Ground waters.................... 72 hours

Fairly pure surface waters................ 48 hours

Polluted surface water. . . . . . . . . . . . 12 hours

Sewage effluents..................... 6 hours

Raw sewage....................... 6 hours

Microscopical Examination.

Ground waters.................... 72 hours

Fairly pure surface waters. ............... 24 hours

Waters containing fragile organisms........... Immediate examination.

\section{Bacteriological Examination.}

Samples kept at less than $10^{\circ} \mathrm{C} \ldots \ldots \ldots \ldots \ldots 24$ hours

The directions for collecting samples of water are summed up in the following pages which are used by the Illinois State Water Survey. 


\section{STATE WATER SURVEY}

Department of Chemistry, University of Illinois

\section{Instrucrions for ColueCtING WATER for BaCterial Examination \\ THE SAMPLE OF WATER SHOULD BE COL- IECTED IMMEDIATELY BEFORE SHIPPING BY EXPRESS, SO THAT THE SHORTEST POSSIBLE TIME SHALL INTERVENE BETWEEN THE COL- LECTION OF SAMPLE AND ITS EXAMINATION.}

The accompanving "Certificate" must be filled out carefully and enclosed in envelope shipping tag.

The bottle for bacterial sample is packed in cotton in the small tin can. The bottle has been carefully cleaned and sterilized in the laboratory and is in perfect condition for the collection. The rubber cloth cap must not be removed from the bottle until you are actually at the spot and ready to take the sample. Then remove the rubber cap, being exceedingly careful not to touch the inside of the cap or lay it in a place where it will come in contact with anything.

From a Stream, Pond or Reservoir. Immerse the bottle to a depth of about $1 \mathrm{ft}$, then remove the stopper and allow the water to run in until the bottle is about two-thirds full. With the bottle still in the water, replace the stopper and do not again touch it, in or out of the water. Withdraw the bottle, replace the rubber cap, carefully avoiding contact with the inside of the cap, the stopper, or the mouth of the bottle. Next, put the bottle with proper certificate in envelope attached inside the tin can, put the cotton about it, and put the lid in place tightly. In order to keep the sample in good condition until it is actually delivered at the laboratory, it will be necessary to place the small can upright in the large galvanized iron box and fill the galvanized box with ice.

From a Pump or Tap. The water is allowed to flow freely for from five to ten minutes, then diminish the stream. Remove the stopper from the bottle, always careful that the part which is inserted in the mouth of the bottle does not come in contact with anything. Allow the water to flow into the bottle until it is from two-thirds to three-fourths full. Replace the stopper and proceed as directed above.

\section{Important to Note Carefully}

Frrst. Do not wash or rinse the bottle or in any way attempt to clean it.

SECOND. Do not handle or touch the stopper to anything except the mouth of the bottle, only as it is necessary to do so to remove the stopper as directed above.

THIRD. Replace the rubber cap with the same side next to the bottle as when it was removed.

FouRTH. The sample for Bacterial Examination must be accompanied by a gallon sample for chemical analysis. 


\section{STATE WATER SURVEY}

\section{Department of Chemistry, University of Illinois}

Instructions for Collecting Samples of Water for Chemical Analysis

1. From a Well. Water should be pumped out freely for a few minutes before it is collected. The bottle is then to be placed in such a position that the water from the spout may fall directly into it, and rinsed out with the water three times, pouring out the water completely each time. It is then again to be placed under the spout, filled to overflowing, and a small quantity poured out, so that an air space of about an inch shall be left under the stopper. The stopper must be rinsed off with flowing water, and inserted into the bottle while stll wet, and secured by tying over it a clean piece of cotton cloth. The ends of string must be sealed on top of the stopper. Under no circumstances must the inside of the neck of the bottle or the stem of the stopper be touched by the hand or wiped with a cloth.

2. From a Tap. Allow the water to flow freely from the tap for a few minutes and then proceed precisely as directed above.

3. From a Stream, Pond or Reservoir. The bottle and stopper should be rinsed with the water, if this can be done without stirring up the sediment on the bottom. The bottle with the stopper in place should then be entirely submerged in the water and the stopper taken out at a distance of 12 in. or more below the surface. When the bottle is full, the stopper is replaced, below the surface if possible, and finally secured as above. It is important that the sample should be obtained free from the sediment at the bottom of a stream and from the scum on the surface. If a stream should not be deep enough to admit of taking a sample in this way, the water must be dipped with an absolutely clean vessel and poured into the bottle after it has been rinsed.

The sample of water should be collected immediately before shipping by express, so that the shortest possible time shall intervene between the collection of sample and its examination. Shipment on Monday or Tuesday insures prompt delivery and completion of the analysis before the end of the week.

The accompanying "Certuficate" must be filled out carefully and enclosed in the envelope shipping tag.

The sample for chemical analysis must be accompanied by a sample for bacterial examination, unless otherwise directed.

Data Concerning the Sample. This is about as important to the analyst when giving an opinion with regard to the sanitary quality of the water as the data secured in the analysis. Some individuals have tried to "fool " an analyst by furnishing fictitious data for a sample of water and have caused much trouble when a perfectly harmless water was condemned. The following certificates which are used by the Illinois State Water Survey will give an idea with regard to what data should accompany a sample of water for analysis. 


\section{STATE WATER SURVEY}

Department of Chemistry, University of Illinois

Use this Certificate for water whose original source is Well, Spring or Cistern.

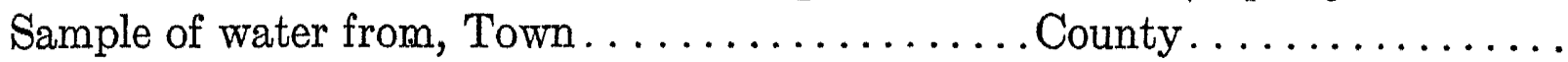
Report to be sent to..

Cellected and sealed by

Date, day and hour of collection

Shipped by...

Express Company

Date and hour of shipment.

Collected from.

Location.

State proximity of privy.

. cesspool.

stable

Feed lot.......... dumping grounds for slops, dish water, wash water, etc. . Is the drainage from all these places toward or from the Well, Spring or Cistern?.

If there is any other possible source of pollution, state it.

Has the water ever been considered unsafe?

Why?

If there have been any cases of Typhoid Fever among users of this water, state number of persons affected.............. Date of illness............... Number of deaths.

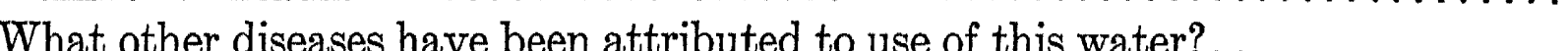

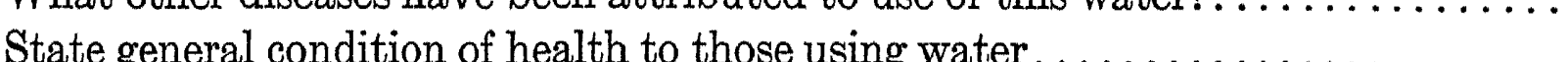
Well-State depth. Is it dug, bored, driven or drilled?.

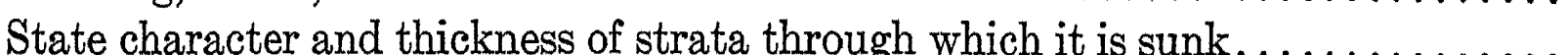

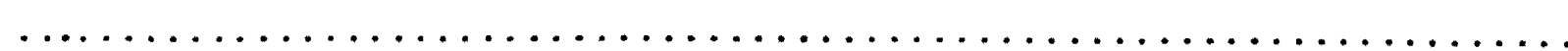

State character of strata from which water is drawn................... Is it a flowing well?

State approximate capacity and effect of dry or wet weather.

With what is it walled or cased?......... How is the well covered?.

Is the cover watertight?.

If cemented or cased with iron pipe, state depth to which cement or casing extends

Spring-What improvements has it?

Character of stratum from which water issues

Character of overlying strata...

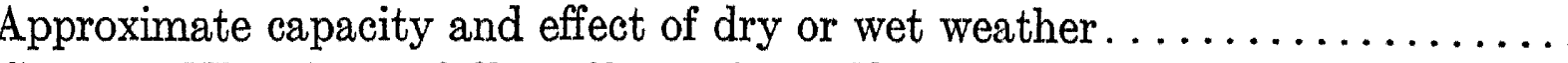
Cistern-What form of filter, if any, is used?.

Does the Cistern leak?.............How long since last cleaned?.

Can small animals get into it at top?.

What care is taken in collecting and storing water?.

Laboratory No........ Keceived. 19. $M$. 


\section{STATE WATER SURVEY}

\section{Department of Chemistry, University of Illinois}

Use this Certificate for water whose original source is Stream, Lake or Pond.

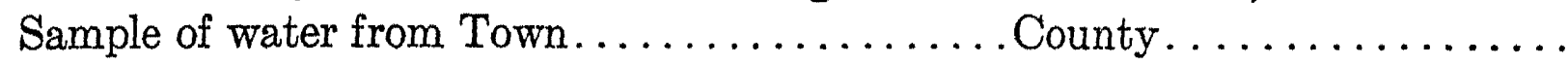

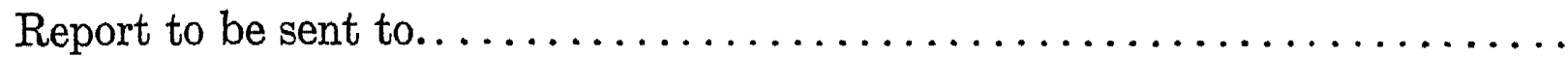

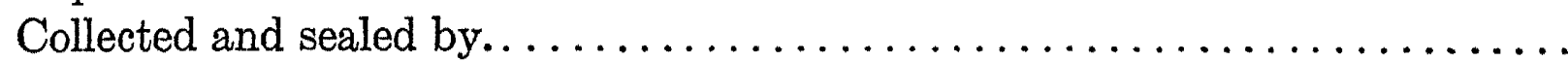
Date, day and hour of collection.

Shipped by....

Express Company.

Date and hour of shipment.

Collected from.

Location.

Has the water ever been considered unsafe?

Why?

If there have ever been any cases of Typhoid Fever among users of this water, state number of prsons an

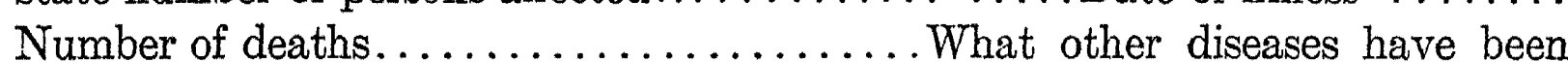
attributed to this water?.

Stream-Name of Stream.

Do sewers discharge into Stream above point of collection?

State distance.................... If the Stream is liable to pollution by drainage from privies, stables, feed lots, slaughter houses, factories or street washings, describe conditions

Have there been recently any cases of Typhoid Fever on territory drained by Stream?....

Condition of Stream at present as affected by drought or recent rains or by temperature.

Lake-Name of Lake.

Do sewers discharge into the Lake at a point liable to pollute the water.

State distance from outfall of sewer to point of collection................ If this Lake is liable to pollution by drainage from privies, stables, feed lots, slaughter houses, factories, or street washings, describe conditions...........

Have there recently been any cases of Typhoid Fever in territory drained into Lake?... Condition of Lake at present as affected by drought or recent rains or by temperature. Pond-Do tile drains discharge into it?....... Do washings from the vicinity

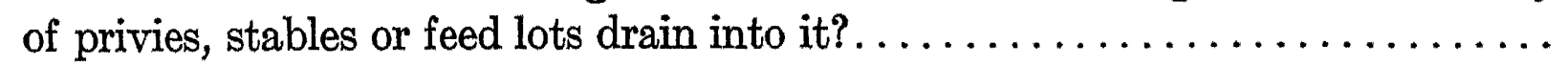
Have there recently been any cases of Typhoid Fever in region draining into it? Condition of Pond at present as affected by drought or recent rains or by temperature. $M$ 


\section{TURBIDITY}

By turbidity is meant the suspended matter which is usually present in water from surface sources. This is gathered from the water shed and, as long as sufficient velocity is maintained, is carried along. Few people care for a turbid water although they may become educaived to the appearance and use of a water having much matter in suspension. There seems to be little sanitary significance connected with the turbidity. This, of course, depends upon the kind of matter which makes up the turbidity.

Determination. Several methods are available for the determination of turbidity. A convenient method is the comparison of the sample of water to a "silica standard." This " silica standard" is a 0.1 per cent suspension of diatomaceous earth in a liter of distilled water. This suspension has a turbidity of 1000 or contains 1000 parts per million of silica. This solution may be diluted with distilled water to give standards of 5, 10, 15, 20,30,40,50, etc. These standard solutions should be stored in clear glass bottles, preferably of the same type in which the samples are taken and should be thoroughly shaken before using. The sample and the standard should be compared by viewing any dark object and noting the details of each.

\section{Color}

Color is of little sanitary significance. It usually comes from organic matter and often is removed from water with difficulty. Very often other substances such as iron may color a water.

Determination. The platinum-cobalt method for determining color is the standard. The standard solution is made by dissolving 1.246 gms. of potassium platinic chloride $\left(\mathrm{PtCl}_{4} \cdot 2 \mathrm{KCl}\right)$ containing $0.5 \mathrm{gm}$. platinum and $1.00 \mathrm{gm}$. crystallized cobalt chloride $\left(\mathrm{CaCl}_{2} \cdot 6 \mathrm{H}_{2} \mathrm{O}\right)$ containing $0.25 \mathrm{gm}$. cobalt, with 100 c.c. of concentrated hydrochloric acid in distilled water and diluting it to a liter. Such a solution is the standard having a color of 500 . It should be diluted to give standards of from 5 to 80 . These may be kept in 50 c.c. Nessler tubes which should be carefully protected when not in use.

The sample of water should be shaken and 50 c.c. poured into a clean 50 c.c. Nessler iube. Compare this with the standards in a colorimeter. Standard methods prescribes the recording of results as follows:

Color between 1 and 50 recorded to nearest unit.

Color between 51 and 100 recorded to nearest 5 .

Color between 101 and 250 recorded to nearest 10 .

Color between 251 and 500 recorded to nearest 20 . 


\section{ODOR AND TASTE}

Odor and taste are probably the most important of any of the organo-leptic tests. A chemically pure water is odorless and tasteless. It also has a decidedly insipid flavor as may be determined by tasting distilled water. Most people demand a water with some taste.

Determination of Odor. Whipple's (1899) method is the standard. The quality of the odor may be expressed in the following terms:
a. aromatic
m. moldy
C. free chlorine
M. musty
d. disagreeable
P. peaty
f. fishy
s. swectish
g. grassy
S. hydrogen sulphic̈e
v. vegetable

The intensity of the odor may be expressed in numerical terms as follows:

\begin{tabular}{c|c|c}
\hline $\begin{array}{c}\text { Numerical } \\
\text { Value }\end{array}$ & Term & Approxumate Definition. \\
\hline 0 & $\begin{array}{c}\text { None } \\
\text { Very faint }\end{array}$ & $\begin{array}{l}\text { No odor perceptible. } \\
\text { An odor that would not be ordinarly detected by the } \\
\text { average consumer, but that could be detected in } \\
\text { the laboratory by an experienced observer. } \\
\text { An odor that the consumer might detect if his atten- } \\
\text { tion were called to it, but that would not otherwise } \\
\text { attract attention. } \\
\text { An odor that would be readily detected and that } \\
\text { might cause the water to be regarded with disfavor. } \\
\text { An odor that would force itself upon the attention } \\
\text { and that might make the water unpalatable. } \\
\text { An odor of such intensity that the water would be } \\
\text { absolutely unfit to drink. (A term to be used only } \\
\text { in extreme cases.) }\end{array}$ \\
\hline 5 & Distinct & Decided \\
\hline
\end{tabular}

\section{RESIDUE}

The residue is obtained by evaporating a known amount of the water to dryness. The amount of the residue depends upon the character of the water. One having a high content of carbon dioxide will dissolve much inorganic matter. The relation of the residue to health depends upon their character. Certain salts cause decided physiological disturbances in some individuals which accounts for the therapeutic value of certain natural waters. 
Determination. Place 100 c.c. of the sample in a tared platinum dish and evaporate to dryness over a water bath. Then heat the dish in an oven at $103^{\circ} \mathrm{C}$. or $180^{\circ} \mathrm{C}$. for one hour. The weight of the residue multiplied by 10 gives the parts per million in the sample.

\section{ChLoRINe}

Chlorine in a water is often a good index of pollution since it is usually high in sewage. A normal urine may contain in a day from 10-15 gms. of chloride calculated to sodium chloride. The interpretation of chlorine results demands a knowledge of the local conditions about the source of the sample. The amount of chlorine which is normal for the district from which the sample was taken must be known.

Determination. Chlorine may be determined by titrating the water with silver nitrate in the presence of potassium chromate as an indicator. Add 1 c.c. of the indicator to 50 c.c. of the sample and titrate with the standard solution of silver nitrate. A standard of 1 c.c. of indicator in 50 c.c. of distilled water should be kept for comparison. The number of cubic centimeters of silver nitrate solution required multiplied by 10 gives the titrated chloride $(\mathrm{Cl})$ in parts per million.

The solutions which are required in the determination of chlorine in water may be prepared as follows:

1. Potassium Chromate. Dissolve 50 gms. of potassium chromate in a small amount of distilled water. Add sufficient silver nitrate to produce a slight red precipitate, filter and dilute to 1 liter with distilled water.

2. Standard Sodium Chlorde Solution. Dissolve 16.48 gms. of pure fused sodium chloride in distilled water. One hundred c.c. of this stock solution should be diluted to a liter in order to secure a solution which will contain $0.001 \mathrm{gm}$. of chloride.

3. Standard Silver Nitrate Solution. Dissolve $2.40 \mathrm{gms}$. of silver nitrate in a liter of distilled water. Standardize this with the above standard sodium chloride solution and adjust the volume so that each cubic centimeter is equivalent to $.0005 \mathrm{gm}$. of chloride.

\section{NITROGEN}

Nitrogen furnishes the chemist a convenient index of bacterial activities in water. There were times in sanitary work when waters were condemned for, what was considered then, excessive amounts of the various nitrogen compounds. At one time nitrites were regarded as an infallible index of pollution. These different determinations of 
nitrogen in water trace the changes through which the organic matter is made to pass by the bacteria. The free and albuminoid ammonias indicate the organic matter while the nitrites and nitrates indicate the amount of oxidation which has taken place.

Nesslerization. This may be done directly or after distillation depending upon the amount which is present in the sample. The following solutions are required:

1. Standard Ammonium Chloride Solution. Dissolve 3.82 gms. of ammonium chloride in 1 liter of ammonium free water. Dilute 10 c.c. of this to 1 liter with ammonia free water. One c.c. of this latter solution equals $0.00001 \mathrm{gm} . \mathrm{N}$.

2. Nessler Solution. Dissolve $50 \mathrm{gms}$. of potassium iodide in a little cold water. To this is added a saturated solution of mercuric chloride until a slight precipitate persists permanently. Add 400 c.c. of a 50 per cent solution of potassium hydroxide. This should be a clear solution made by allowing it to settle. Dilute to a liter and, after allowing it to settle, decant. This should give the required color with ammonia within five minutes and should not give a precipitate with small amounts in two hours.

Folin and Dennis' Modified Nessler Reagent. Double iodide solution is made by dissolving 75 gms. of potassium iodide in 500 c.c. of warm water, adding $100 \mathrm{gms}$. of mercuric iodide and stirring. The solution will be complete in a few minutes but possibly not quite clear on account of impurities. Dilute with 400 to 500 c.c. of water and make the filtrate up to a liter.

To 300 c.c. of the double iodide solution add 200 c.c. of 10 per cent sodium hydroxide, 500 c.c. of water, and mix. Fifteen c.c. of this modified Nessler reagent rapidly added from a measuring cylinder will yield crystal clear mixtures with as large amounts of ammonia nitrogen as are usually involved (0.7 to $1.6 \mathrm{mg}$. ammonia nitrogen).

This has the advantage over the standard Nessler reagent that it may be used very soon after preparation and will not give a precipitate.

For "standards" a series of Nessler tubes is prepared by adding increasing amounts of the standard ammonium chloride solution and diluting with ammonia free water to the 50 c.c. mark. Standard methods recommends the following standards, $0.0,0.1,0.3,0.5,0.7,1.0$, 1.4, 1.7, 2.0, 2.5, 3.0, 3.5, 4.0, 4.5, 5.0, and 6.0 c.c. These standards should be Nesslerized by adding 1 c.c. of Nessler solution to the tubes and allowing them to stand for 10-20 minutes. The sample whether Nesslerized directly or after distillation is treated the same way and compared with the standards. If the amount of ammonia is known to 
be high the distillate may be caught in a flask (200 c.c.) and an aliquot portion Nesslerized. Usually four Nessler tubes are sufficient. The last one should contain no nitrogen. When Nessler tubes are used the following example will serve to illustrate the computation.

First tube $=3.0$ c.c. of standard $\mathrm{NH}_{4} \mathrm{Cl}$ solution.

Second tube $=2.6$ c.c. of standard $\mathrm{NH}_{4} \mathrm{Cl}$ solution.

Third tube $=1.0$ c.c. of standard $\mathrm{NH}_{4} \mathrm{Cl}$ solution.

Fourth tube $=0.0$ c.c. of standard $\mathrm{NH}_{4} \mathrm{Cl}$ solution.

Total 6.6 c.c. of standard $\mathrm{NH}_{4} \mathrm{Cl}$ solution.

$6.6 \times 0.00001=0.000066$ gm. $\mathrm{N}$.

If 500 c.c. of the water were taken this amount must be multiplied by 2 to get the amount of $\mathrm{N}$ in a liter of the water.

$0.000066 \times 2=0.000132 \mathrm{gm} . \mathrm{N}$. in a liter $=.132$ part per million.

If an aliquot portion from a flask is Nesslerized the calculation is about the same.

Free Ammonia. Five hundred c.c. of the sample should be introduced into a 800 c.c. Kjeldahl flask fitted to a condenser so that the distillate may be received in four Nessler tubes. Heat so that not more than 10 c.c. nor less than 6 c.c. comes over in a minute. If the nitrogen is known to be high, catch the distillate in a flask. Nesslerize, as described above.

Albuminoid Nitrogen. This is determined on the same sample after the free ammonia has been distilled off. Alkaline potassium permanganate, prepared as follows is required for this determination. Pour 1200 c.c. of distilled water into a large evaporating dish and boil for ten minutes. Remove the flame and add 16 gms. of potassium permanganate. Dissolve and add 800 c.c. of a 50 per cent solution of potassium hydroxide. Add a little distilled water and boil down to 2000 c.c.

After the distillate for free ammonia nitrogen has been received, remove the flame and add 50 c.c. or more of alkaline potassium permanganate to the flask and collect 200 c.c. of distillate in four Nessler tubes. Nesslerize as described above.

The free ammonia determined above probably represents nitrogen which is present as some inorganic salts. These may be split by boiling. It may represent a stage in denitrification resulting from deaminization of amino acids. As such, free ammonia has little significance but it does represent chenging organic matter. The albuminoid ammonia is of more sanitary significance. It indicates recent organic pollution. 
Nitrites. This determination is carried out on a 50 c.c. sample of the water in a Nessler tube. A series of standards is also prepared by putting the following amounts of the standard solution of sodium nitrite in 50 c.c. Nessler tubes and diluting to the mark with nitrogen-free water: $0.0,0.1,0.2,0.4,0.7,1.0,1.4,1.7,2.0$, and 2.5 c.c. add 1 c.c. of sulphanilic acid and 1 c.c. of the naphthalamin acetate -or hydrochloride solutions to each of the tubes including the sample. Mix and after standing for fifteen minutes compare with the standards. When a 50 c.c. sample is used the standard matched times 0.01 equals the parts per million of nitrites.

The following reagents are required:

1. Sulphanilic Acrd Solution. Dissolve 8 gms. of the purest sulphanilic acid in 1 liter of $5 \mathrm{~N}$ acetic acid (sp. gr. 1.04) or in 1000 c.c. of water containing 50 c.c. of concentrated hydrochloric acid. This will be practically a saturated solution.

2. $\alpha$-naphthylamin Acetaie or Hydrochloride. Dissolve $5 \mathrm{gms}$. of solid and naphthylamin in 1000 c.c. of $5 \mathrm{~N}$ acetic acid or in 1000 c.c. of water containing 8 c.c. of concentrated hydrochloric acid. Filter through alundum or washed absorbent cotton.

3. Stock Sodium Nitrite Solution. Dissolve $1.1 \mathrm{gms}$. silver nitrite in nitrite free water; precipitate the silver with sodium chloride solution and dilute the whole to a liter.

4. Standard Sodium Nitrite Solution. Dilute 100 c.c. of solution 3 to 1 liter; then dilute 50 c.c. of this solution to a liter with nitrite free water, add 1 c.c. chloroform, and preserve in a sterilized bottle. One c.c $=0.0005 \mathrm{mg}$. nitrogen.

Nitrates. (Reduction method.) Place 100 c.c. of the sample in a casserole and, after adding 2 c.c. of the sodium hydroxide solution, reduce the volume by boiling to 20 c.c. Pour this into a 100 c.c. test tube $(16 \times 3 \mathrm{~cm}$.) along with two or three rinsings with nitrogen free water. Put a strip of aluminum foil and close the tuibe by means of a rubber stopper carrying a $5 \mathrm{~m} . \mathrm{m}$. glass tubing which will extend below the surface of distilled water in another tube. The hydrogen is thus allowed to escape. The nitrogen which is lost in this procedure may be neglected. Allow this to stand over night (at least four hours). At the end of this time pour the contents of the tube into a distilling flask and dilute to 200-300 c.c. with nitrogen free water. Distill, collecting the distillate in Nessler tubes or in 200 c.c. flasks if the amount of nitrates is known to be high. If flasks are used to receive the distillate, an aliquot portion must be Nesslerized. Nesslerization may be done directly when the supernatant liquid in the reduction tube is clear. 
The following reagents are required:

1. Sodium or Potassium Hydroxide Solution. Dissolve 250 gms. of hydroxide in 1.25 liters of distilled water. Add several strips of aluminum foil and allow the evolution of hydrogen to continue over night. Concentrate to a liter by boiling.

2. Aluminum Fonl. Strips of pure aluminum about $10 \mathrm{~cm}$. long, $6 \mathrm{~mm}$. wide and weighing about $0.5 \mathrm{gm}$. should be used.

Nitrates represent the end products of nitrification. They represent stable forms of nitrogen and are desired in the various methods of sewage treatment. They indicate that there was originally in the water a relative amount of organic matter which has been changed by ammonification and nitrification to completely oxidized forms.

Nitrites give indication that nitrogen is being oxidized. This in turn represents great bacterial activity on organic matter. All determinations of nitrogen represent the various stages in the oxidation and reduction of nitrogenous compounds.

Oxygen Consumed. Place 100 c.c. of the sample in a flask. Add 10 c.c. of the sulphuric acid solution and 10 c.c. of the standard permanganate solution and digest the solution for thirty minutes on a water bath. The flasks should be placed in the water bath so that the level of the water is kept above the level of the water in the flask. If the amount of permanganate is not sufficient for the oxidation of the sample, repeat the digestion with a larger sample. At least 5 c.c. excess of permanganate should be present when the oxalate is added. Remove the flask and add 10 c.c. ammonium oxalate solution, and titrate until a faint pink color appears and remains with the permanganate solution. If 100 c.c. of water is used the number of cubic centimeters of potassium permanganate solution in excess of the number of cubic centimeters of ammonium oxalate solution is equal to the parts per million of oxygen consumed.

Reagents. 1. Dilute Sulphuric Acid. Dilute 1 part of concentrated sulphuric acid with three parts of distilled water and free the solution of oxidizable matter by adding potassium permanganate until a faint pink color persists after the solution has stood for several hours.

2. Standard Ammonium Oxalate. Dissolve $0.888 \mathrm{gm}$. of the pure salt in a liter of distilled water. One c.c. is equivalent to $0.1 \mathrm{mg}$. of oxygen. An equivalent quantity of oxalic acid may be used.

3. Standard Potassium Permanganate. Dissolve $0.4 \mathrm{gm}$. of crystallized salt in a liter of distilled water. Add 10 c.c. of the dilute sulphuric acid and 10 c.c. of this solution of potassium permanganate to 100 c.c. of distilled water, and digest thirty minutes. Add 10 c.c. of 
the ammonium oxalate solution and the potassium permanganate till a pink coloration appears. This destroys the oxygen consuming capacity of the water used. Now add another 10 c.c. of ammonium oxalate solution and titrate with potassium permanganate. Adjust the potassium permanganate solution so that 1 c.c. is equivalent to 1 c.c. of the ammonium oxalate solution or $0.1 \mathrm{mg}$. of available oxygen.

\section{ALRALINITY}

Determination. Add 4 drops of phenolphthalein to 50 c.c. of water and titrate with $\mathrm{N} / 50$ sulphuric acid solution until the coloration disappears. The number of cubic centimeters of $\mathrm{N} / 50$ sulphuric acid times 20 will indicate the phenolphthalein alkalinity in parts per million. This same sample may be used for the methyl orange alkalinity by adding 2 drops of methyl orange and continuing the titration to a faint pink appearance. The methyl orange alkalinity is equal to the total number of cubic ecntimeters of N/50 sulphuric used multiplied by 20 .

The alkalinity of water is due to the content of inorganic salts such as, carbonates, bicarbonates, hydroxides, silicates, etc. The relations between the phenolphthalein and methyl orange alkalinities are expressed in the following table:

TABLE XXV

\section{RELATIONS BETWEEN ALKALINITY TO PHENOLPHTHALEIN AND METHYL ORANGE IN PRESENCE OF CARBONATE, BICAR- BONATE AND HYDROXIDE}

(Standard Methods, 1917)

\begin{tabular}{l|l|l|l}
\hline \multirow{2}{*}{ Result of Titration. } & \multicolumn{2}{|c|}{ Value of Radial Expressed in Terms of Calcium Carbonate. } \\
\cline { 2 - 3 } & Bicarbonate. & Carbonate. & Hydroxide. \\
\hline & & & \\
$P=0$ & $T$ & 0 & 0 \\
$T=\frac{1}{2} T$ & $T-2 P$ & $2 P$ & 0 \\
$P=\frac{1}{2} T$ & 0 & $2 P$ & 0 \\
$P=\frac{1}{2} T$ & 0 & $2(T-P)$ & $2 P-T$ \\
$P=T$ & 0 & 0 & $T$ \\
& & & \\
\hline
\end{tabular}




\section{Classification of Water Bacteria}

The water bacteriologists have been primarily interested in the presence of certain bacteria which are of sanitary significance. They have done very little with the other bacteria that may be in water with which they are dealing but have confined their attention to some ten organisms which indicate pollution. Only after the other branches of the science had become well advanced did water bacteriologists give any attention to classification. This may have been due to the fact that the flora of a water is heterogeneous and that a few bacteria are probably secured from each material with which the water comes in contact. The environment of a water determines its bacterial flora. One coming from an unpolluted water shed will have quite a different flora than a water flowing from a river which is made to carry the wastes of industrial and domestic life. Each of these examples would have a constant flora but one characteristic for the case mentioned. With the exception of possibly ten bacteria or groups whose presence is supposed to indicate pollution, very little is known about the large number of other bacteria which may be present. More accurate deductions might be made with regard to the sanitary character of a sample of water could more be known concerning its bacterial flora. This question has been discussed in Standard Methods for the Examination of Water and Sewage for 1912, page 106. " In certain cases the determination of species may play directly a useful part in water analysis. It sometimes happens that the bacteria present in a filtered water are different in character from those found in the raw water and unless the facts are known, erroneous inforences may be drawn as to the efficiency of the filters. The determination of particular species is sometimes of importance in proving the identity of a water from a particular source. At times also, the presence of certain species in water may be indicative of pollution."

Bacterial control of filter operations is very important when the health of the public depends upon this factor. A knowledge of the general flora of the raw water might, at certain times, be a valuable factor in determining the efficiency of the filters. 'This would tell whether the bacteria in the effluent were passing through the filters from the raw water or whether they were growing in the under drains. Certain bacterial standards have been formulated that place the limit of 100 per cubic centimeter for bacteria in the filtered water. The character of the bacteria in those effluents which contain more than 100 per cubic centimeter would be very important. 
In order to make some definite bases for describing cultures the committee of the American Public Health Association suggested the following scheme which has been outlined by Savage (1906) as follows:

I. Source and habitat.

II. Morphological characters.

Form. Manner of grouping, dimensions, staining reactions motility, spores, capsules, involution forms.

III. Cultural characteristics.

Mode of growth in and upon nutrient broth, gelatin plates, gelatin tubes, agar plates, agar tubes.

IV. .Biochemical reactions.

Action upon milk, carbohydrates, nitrates; production of indol; relation to free oxygen; temperature relations; pigment formation; liquefaction of gelatin.

Such a scheme as outlined above tends toward greater uniformity in the examination of bacteria in water. It seems to be the general tendency at the present time to arrange bacteria into groups and this is the best method for handling the flora of a substance like water. Tanner (1918) has shown that the fluorescent bacteria in water compose a closely related group. Wyatt Johnston (1894) early called attention to the grouping of water bacteria. In his paper, he discussed the tests which should be applied to separate bacteria into these groups. He analyzed 235 described organisms and reached the following conclusions: That points of difference are given more attention than resemblances. In the grouping of bacteria a single strongly marked characteristic peculiar to a few species is of more value than a number of minor details but the members of any group should not differ unduly in regard to minor points. About this time the American Public Health Association realized the necessity of uniform procedures for the study of bacteria and appointed a committee to study methods for that purpose. This committec reported in 1897 and recommended the following procedures: Necessary Information:

\section{Source and habitat.}

II. Morphological characters.

Form, dimensions, grouping, staining, capsule, flagella spores, pleomorphism, involution forms. 
III. Biological characters.

A. Cultural growth in or on nutrient broth, gelatin plates, gelatin tubes, agar plates, agar tubes, potato, milk and blood serum.

B. Biochemical.

Temperature relations, relation to oxygen, acidity of medium action on gelatin, protein, carbohydrates,

C. Pathogenesis. nitrates, indol, pigments and odor.

Optional Tests:

I. Morphological.

Staining reactions, study of flagella by special stains, permanency of characters.

II. Physiological.

A. Cultural.

Growth-litmus gelatin, blood serum, synthetic media, photograph.

B. Biochemical.

Maximum, minimum and optimum temperature of growth, growth in special gases, limit of acıd and alkali, chemical properties of pigments.

C. Pathogenesis.

Inoculation, toxins, etc.

At the end of the report are given charts for recording the characters of bacteria. This is one of the first attempts in which the organized efforts of a society were brought to bear in systematizing methods for the study of the bacterial" cell.

Marshall Ward (1897) in studying the bacterial flora of the Thames river arranged his strains into the following groups:

I. Forms identical with Bacterium urece.

II. Violet bacteria.

III. B. fluorescens liquefaciens group.

IV. B. fluorescens non-liquefaciens group.

V. Typical B. coll communis.

VI. Series of forms centering around B. proteus.

VII. Like VI except that a yellow pigment is formed.

VIII. Bacteria between VII and IX. Characters changed.

IX. Golden yellow liquefying forms. 
X. Yellow non-liquefying forms. Probably related to IX.

XI. Colorless capsulated forms.

XII. Yellow capsulated forms.

XIII. B. prodrgrosus types.

XIV. Rapid liquefiers, colorless, pronounced putrefactive tendencies.

XV. B. subtılıs like group.

XVI. Yellow sarcina-like bacteria.

XVII. Rose-colored micrococci.

XVIII. Fungus forms.

XIX. White micrococci ( $M$. candicans).

$\mathrm{XX}$. Lemon yellow gelatin liquefiers.

XXI. Short oval cocci forming a red pigment.

That this classification of water bacteria is artificial was realized by Ward for he made the following statement: "My work goes to show that species cannot be made out but that the limits of the species are, in most cases, far wider than is assumed in descriptions-in other words that many so-called species in books are merely variated forms, whose characters, as given, are not constant but depend on treatment. How far this is true for any given case will have to be tested on the particular form in question."

Fuller and Johnson (1899) studied water bacteria from a river in America and arranged their strams into thirteen groups. They realized weaknesses in their system and state that too much importance was given to morphological data and too little to cultural studies. Difficulty was also experienced in separating short rods from cocci. This classification has formed the basis for much systematic work in bacteriology. Their groups are given below:

I. Fluorescent forms.

II. All red chromogenic forms.

III. All orange chromogenic forms.

IV. All yellow chromogenic forms.

V. All violet chromogenic forms.

VI. All non-fluorescent, non-chromogenic, gelatin liquefying bacteria, forming proteus-like colonies on gelatin.

VII. All non-fluorescent, non-chromogenic, gelatin liquefying bacteria, forming subtilis-like colonies on gelatin.

VIII. All non-fluorescent, non-chromogenic, non-proteus like nonsubtilis-like bacteria which liquefy gelatin and ferment carbohydrates with production of gas. 
IX. All bacteria conforming to specified characteristics of VIII, except that fermentation of carbohydrates takes place without formation of gas.

X. All bacteria conforming to group VIII except that no fermentation of carbohydrates occurs.

XI. All non-fluorescent, non-chromogenic, non-liquefying bacteria which ferment carbohydrates with the production of gas.

XII. All bacteria conforming to specified characteristics of XI except that carbohydrates are fermented without gas.

XIII. All bacteria conforming to specified characteristics of group XI except that no fermentation of carbohydrates occurs.

Jordan (1901) studies 543 strains from the Illinois, Mississippi and Missouri rivers and developed the following classification:

I. B. coli communis.

II. B. lactis aerogenes.

III. B. porteus.

IV. B. enteritidzs.

V. B. fuorescens liquefaciens.

VI. B. fluorescens non-liquefaciens.

VII. B. subtzlis.

VIII. Non-gas formers, non-fluorescent, non-spore forming bacilli which liquefy gelatin and acidify milk.

IX. Similar to group VIII save that milk is rendered alkaline.

X. Similar to group VIII save that gelatin is liquefied.

XI. Similar to group IX, save that gelatin is not liquefied.

XII. Similar to group XI, save that the reaction of milk is not altered.

XIII. Chromogenic bacteria not included above.

XIV. Chromogenic staphylococci.

XV. Non-chromogenic staphylococci.

XVI. Sarcinæ.

XVII. Streptococci.

The Bacterial Count. In early sanitary history, this was supposed to be a reliable index of pollution. Many standards were proposed in which it was stated that a water should not contain over a certain number of bacteria per cubic centimeter to be regarded a good drinking water. One of the most elaborate of these was proposed by Miquel (1891).

Less than 10 cells per cubic centimeter........... excessively pure 10-100 cells per cubic centimeter.............. very pure 
100-1000 cells per cubic centimeter........... pure

1000-10,000 cells per cubic centimeter... . . . . . mediocre

10,000-100,000 cells per cubic centimeter.. . . . . . . impure

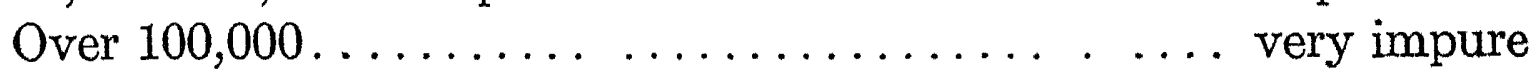

Such a standard would be followed with difficulty to-day. Obviously, according to our present information, such a scheme for grading waters is untenable. Probably Koch's old standard of not over 100 bacteria per cubic centimeter on gelatin is as accurate as any. It is evident that a very few cells of a certain variety would be far more significant than thousands of another variety. In natural waters, however, it has become established that a high content of organic matter usually means a high content of bacteria. High organic content usually means pollution and, in this way, the count itself may mean something. Possibly a water with a large number of bacteria, according to the law of probability, might contain some objectionable forms.

Relation of the $37^{\circ} \mathrm{C}$. Count to the $20^{\circ} \mathrm{C}$. Count. This has received the attention of a number of bacteriologists and their opinions sum up our knowledge with regard to the two counts.

Whipple (1913) made the following comment:

" That the $37^{\circ}$ count more nearly represents the sanitary quality of water than the $20^{\circ}$ count is probably true in a general way, but it is not necessarily so." He cites counts made at the Watertown, N. Y., filtration plant and states:

"The difference between the two counts is striking. The $20^{\circ}$ counts are very high in the winter and spring but are low during the summer, whereas the $37^{\circ}$ counts are low during the winter and spring and high during the summer, at which season the sewage which enters the stream is less diluted." He calls attention to the fact that this seasonable difference gives a varying ratio, and suggests that it would seem advisable to procure more comparative data before making the recommended change. Caird (1913) thinks it advisable to continue the use of gelatin to determine the efficiency of water-treatment plants, and he appends to his paper discussions by various waterworks men, whose opinions seem about evenly divided. Baton (1914) also protests against the recommended change. He concludes that if only one medium is to be used it should be gelatin as it is more reliable and serviceable.

Gaub (1913) at the Washington, D. C., filtration plant found a variable ratio between counts on agar and on gelatin plated from the unfiltered water of the Potomac River. He also found a seasonal ratio which seemed to remain constant for several months at a time and then 
changed. With the filtered water there was a less constant seasonal ration, which was much lower than that with the unfiltered water. This was not due to treatment of the water with calcium hypochlorite. The efficiencies in removal of $B$. coli correspond better to the counts on gelatin, but Gaub does not state how these efficiencies were computed. Gaub forms no conclusion and asks, "Which is the correct medium to "Ise in order to show the efficiency of a plant?" Prescott and Winslow (1915) state that the decomposition of organic matter may be measured either by the material decomposed or by the number of organisms engaged in carrying out the process of decomposition; and that the latter method has the advantage of far greater delicacy since bacteria respond by enormous multiplication to very slight increases in their food supply; thus the count on standard gelatin at $20^{\circ} \mathrm{C}$. is roughly proportionate in not too heavily polluted waters to the free ammonia and oxygen consumed by chemical analysis. Savage asserts that many water bacteria are unable to grow at $37^{\circ} \mathrm{C}$. though most organisms associated with excreta grow readily at $37^{\circ} \mathrm{C}$; ; unfortunately, many harmless soil organisms also grow readily at $37^{\circ} \mathrm{C}$. An experiment by Savage illustrates the limitations of this enumeration. He plated on agar and gelatin a pure tap water collected in a sterile bottle; he then added $1 \mathrm{gm}$. of soil to 500 c.c. of the sample and plated on agar and gelatin and emulsion thus obtained. The agar plates were incubated at $37^{\circ} \mathrm{C}$. for forty hours and the gelatin plates at $21^{\circ} \mathrm{C}$. for three days. The soil contained no $B$. coli in $3 \mathrm{gms}$. The water contained 3 bacteria per cubic centimeter by the agar count and 76 'by the gelatin count. The emulsion contained 1360 bacteria per cubic centimeter by the agar count and 1970 by the gelatin count. Savage concludes that the count at $37^{\circ} \mathrm{C}$. is an index of the presence of bacteria other than those natural to pure water, but not necessarily harmful ones.

Harrison expresses the following opinion: "The number of organisms which develop on beef peptone agar ineubated at blood heat, commonly termed the 'agar' or 'blood-heat' count, is perhaps more important than the gelatin count, as many water bacteria do not grow at blood heat, whereas sewage and soil organisms grow readily at this temperature. The agar count eliminates the water flora, but obscures the sanitary results by reason of the presence of soil bacteria." Muir and Ritchie state that the count on gelatin plates incubated at $20^{\circ} \mathrm{C}$. gives an idea of the number of bacteria which grow at summer heat and that the count on agar plates "incubated at $37^{\circ} \mathrm{C}$. gives an idea of the number of those which grow at blood heat." The following comments are added: "As the pathogenic and intestinal bacteria grow at this 
temperature $\left(37^{\circ} \mathrm{C}.\right)$, the determination of the numbers of blood-heat bacteria is important. The counts on the two media usually differ as each is favorable to the growth of its own group of organisms." The same authors note that the presence of liquefying bacteria on the gelatin plates may make it necessary to count the plates at the end of twentyfour hours, or at the end of a shorter time than is directed in the commonly accepted procedure. Most bacteriologists apparently believe that the agar count at $37^{\circ} \mathrm{C}$. indicates more nearly the bacteria of sewage origin and that it consequently is more important in ascertaining the potability of water. The results of Savage's experiment show, however, that erroneous conclusions will be drawn if the count at that temperature is considered to furnish indication of possible sewage pollution, because soil may contribute large numbers of harmless bacieria that grow at $37^{\circ} \mathrm{C}$.

The advantages and disadvantages of each medium are well known to all bacteriologists. Agar is better for field work in warm weather because it can be transported with less trouble either in test tubes or in Petri dishes; great care must be used to prevent liquefaction if gelatin is used. As gelatin has to be shipped in sooled containers in warm weather it can seldom be used in the field. A less elaborate incubator is required for agar because no special precaution has to be taken to keep the temperature below a certain maximum. In some filter plants in which agar is used to control the operation agar plates are left at room temperature for two days before counting. This could not be done with gelatin plates, for which constant low temperature must be maintained. The count on agar is made at the end of twenty-four hours whereas the count on gelatin is made at the end of forty-eight hours. Spreaders often develop on agar plates but any inconvenience from these is offset by liquefiers on gelatin plates. The two media themselves differ in their properties; gelatin is good for bacteria, but no bacteria have been described that can metabolize agar. Both media are subject to wide variations in chemical content, but agar is more easily purified.

Recent work by Cummings (1916) indicates that in the summer the agar and gelatin counts are nearly equal. In February the gelatin count, on Potomac River water, became sixteen times as great as the agar count. The gelatin count seems to follow the turbidity readings. Where possible as Tanner (1916) has pointed out, both media should be used where it is convenient. Race (1916) has stated that in water analysis no one medium will give a count that will possess any constant ration to the excremental bacteria as shown by $B$. coli content. The $37^{\circ} \mathrm{C}$. count is said to be nearest to the $B$. coli content. The highest 
count for the shortest incubation period is secured at $27^{\circ} \mathrm{C}$. Gelatin was found to yield the highest count. Hill (1908) and Breed and Dotterer (1916) have given some interesting data to show that a good agar plate should not contain over 200 bacteria when counts are to be made. These investigators show that a plate which has less than about 20 and over about 200 bacterial colonies will give a count which is not accurate.

\section{INDICATORS OF POLLUTION}

As soon as sanitary science had demonstrated that diseases were spread by polluted water and foods, it became necessary to find some method of detecting the presence of fecal matter. With this effort originated the Bacillus colon test which has passed through many stages to reach its present status. Other "indicator" organisms have been used in bacteriology such as the streptococci and Bacillus enteridıtzs Gaertner. Indicators of pollution in bacteriology have certain definite characteristics.

1. They should be present in water and on foods which have received sewage pollution.

2. They should be absent from foods which have not been in contact with sewage.

3. They should be easily detected in the shortest time consistent with accurate results.

4. They should possess constant characteristics and not be subject to either morphological or physiological changes.

From the above, it is apparent that an ideal indicator does not exist. Admitting this, the analyst has to resort to the next best thing and take an indicator which best fulfills the necessary requirements. The relative amount of the indicator has come to be the important factor and not the mere presence of the indicator.

The Streptococci. The examination of water for streptococci is not used in America for the routine examination. It would probably be of much value were it used in this country. Houston (1898-9) originally emphasized its value. Possibly one reason why the strep. tococci are not used in routine analysis is the lack of methods for differentiating fecal and non-fecal streptococci. Winslow (1902) and Fuller and Armstrong (1913) pointed out that the amounts of acid formed in carbohydrate media might be used as bases for differentiation. Armstrong and Fuller found that human fecal streptococci were characterized by high acidities in glucose, lactose, and mannite broths and a low acidity in raffinose broth. On the other hand equine and bovine 
types were characterized by a low acidity in glucose, lactose and mannite and a higher acidity in raffinose. The following tables are quoted from Houston's work:

\section{TABLE XXVI}

PERCENTAGE OF STREPTOCOCCI FERMENTING THE SUGARS STATED

F. A. $=$ Fuller and Armstrong. W. $=$ Winslow

\begin{tabular}{|c|c|c|c|c|c|c|}
\hline & \multicolumn{2}{|c|}{ Human Feces } & \multicolumn{2}{|c|}{ Horse Feces } & \multicolumn{2}{|c|}{ Cow Feces } \\
\hline & $\mathrm{FA}$ & $w$ & F A & w. & $\mathrm{FA}$ & W. \\
\hline Glucose .... . & 95 & 89 & 75 & 65 & 81 & 84 \\
\hline Lactose $\quad . . . . .$. & 94 & 62 & 24 & 8 & 77 & $\cdot 52$ \\
\hline Saccharose ............ & 90 & $\ldots$ & 46 & & 75 & \\
\hline Mannite.............. & 65 & 28 & 2 & 2 & 3 & 6 \\
\hline Raffinose.............. & 0 & 6 & 12 & 4 & 73 & 28 \\
\hline
\end{tabular}

TABLE XXVII

PERCENTATE NUMBER OF STREPTOCOCCI FERMENTING SUGARS MENTIONED

Houston's Data. 672 Specimens

\begin{tabular}{|c|c|c|c|c|c|c|c|}
\hline & \multicolumn{5}{|c|}{ Homan Feces (400 Spectmens). } & \multirow[b]{2}{*}{$\begin{array}{c}\text { Milk, } \\
172 \\
\text { Specimens }\end{array}$} & \multirow[b]{2}{*}{$\begin{array}{c}\text { Cow } \\
\text { Dung } \\
100 \\
\text { Specimens. }\end{array}$} \\
\hline & $\begin{array}{l}\text { Series I } \\
100 \text { Specl- } \\
\text { mens }\end{array}$ & $\underset{\text { II }}{\text { Serres }}$ & $\begin{array}{c}\text { Serres } \\
\text { III }\end{array}$ & $\begin{array}{l}\text { Average } \\
\text { of Serres } \\
\text { I, II and } \\
\text { III. }\end{array}$ & \begin{tabular}{|c|} 
Series IV \\
In this \\
series only \\
lactose + \\
specimens \\
were \\
accepted \\
100 speci- \\
mens
\end{tabular} & & \\
\hline Glucose .... & Not tested & & & & & & \\
\hline Lactose. . . . . . & 66 & 78 & 85 & 7634 & 100 & 97 & 85 \\
\hline Saccharose.... & 89 & 84 & 86 & 8634 & 49 & 90 & 89 \\
\hline Mannite....... & 7 & 27 & 39 & 2434 & 4 & 20 & None \\
\hline Raffinose...... & 62 & 8 & 26 & 3200 & 100 & 19 & 74 \\
\hline Salicin ...... & 96 & 91 & 91 & 2967 & 97 & 60 & 93 \\
\hline Inulin........ & 12 & None & 2 & 467 & Not tested & 21 & 13 \\
\hline
\end{tabular}

It is well known that streptococci are present in the intestinal tract of man and animals. They are essentially parasitic in their characteristics and, generally speaking, indicate recent pollution when found 
in water. The evidence, however, is less convincing than that of $B$. coli since it is probable that the methods which exist for differentiating between fecal and non-fecal streptococci are unreliable. Their short life in water points out that a negative result for their examination may not indicate the absence of pollution. More data with regard to the streptococci as indicators of pollution are needed. Savage and Reed (1916) in some work on this subject found that neutral red broth was the best medium. They regard the estimation of the streptococci as of undoubted value but that a positive result has much more meaning than a negative result. The same author with the collaboration of Wood (1917) compared the viability of $B$. colr and streptococci in natural waters. The streptococci were not able to live longer than two weeks while $B$. coli showed a distinct multiplication. In one instance the bacilli increased to fifty times the original number in eleven weeks. The presence of the streptococci in a water would indicate recent pollution and, therefore, of a more dangerous nature. The greatest objection to the use of the streptococci as indicators of pollution is the lack of a rapid method for detecting them in a sample of water. Savage has recommended the use of neutral red media but this does not exert a sufficiently selective action to render the test easily and quickly carried out. The author has found that it was necessary to verify the suspected streptococci with hanging drop determinations in many of the cases where neutral red was used.

Houston's Method for Streptococci in the Feces. Drigalski and Conradi plates are inoculated from an emulsion of the feces, made in sterile water. After incubation at $37^{\circ} \mathrm{C}$., the minute colonies are subcultured in "litmus lemco, lactose peptone" medium. After twentyfour hours the tubes showing no acidity are discarded; those showing acidity are examined microscopically and if results were satisfactory further subcultures were made into a series of lemco peptone sugar media untinted with litmus. After three days incubation at $37^{\circ} \mathrm{C}$. the acidity produced is estimated with $\mathrm{N} / 20 \mathrm{NaOH}$, using phenolphthalein as the indicator. Control tubes are also inoculated. (Mtr. Water Bd. 10th Am. Rept., p. 25, 1914.)

The streptococcus colonies on Drigalski and Conradi plates are small colonies with a tinge of violet. They are to be distinguished from colonies of $B$. typhosus which possess larger colonies.

Detection of Sewage Streptococci. "For this purpose many processes have been devised, but the simplest and best appears to be the examination of the bile-salt glucose broth used for the 'presumptive test' for colon bacilli. The incubation should, however, be prolonged 
to forty-eight hours, and, at the end of that period a loopful from the surface of each tube should be examined as a hanging drop under the microscope. The broth should then be carcfully poured off so as to leave at the bottom any precipitate which has been produced. Add a few drops of dilute sodium carbonate solution, to dissolve the bile salts and examine again in the hanging drop. If distinct chains are not found, streptococci may be regarded as absent." This method has been found by Thresh to yield the best results.

Savage's Method for Isolation of Streptococcus from Water, etc. Add 0.1 and 1 c.c. of the sample (water) to tubes of glucose neutral red broth and 10 c.c of the sample to a tube of double strength neutral red broth. Incubate the tubes at $37^{\circ} \mathrm{C}$. for forty-eight hours and then examine microscopically in hanging drop for chains of cocci. The broth tube containing the quantity of water next below the one giving a positive result is examined several times in fresh preparations. Where it is doubtful whether chains are present, a little of the fluid is centrifugalized and stained with methylene blue. The streptococci are best isolated from glucose broth tubes by adding a platinum loopful to a litmus lactose plate and distributing over its surface. All suspicious strains must be subcultured and worked ont.

Method for Separation of B. coli and Streptococci in Water. "Inoculate the desired quantity of water, prefel ably 1 c.c. into dextrose broth fermentation tube. Incubate at $37^{\circ} \mathrm{C}$. Examine after six to twelve hours. Within two to three hours after gas formation has appeared, plate the broth into litmus lactose agar, incubating for twelve to eighteen hours at $37^{\circ} \mathrm{C}$. If, at the end of this time, no acid-producing colonies are present, it is probably safe to assume that there were no colon bacilii present."

After the first plating from dextrose broth, replace the fermentation tube for thirty-six hours and plate in litmus lactose agar. This culture should give a nearly pure culture of streptococci, if these organisms were originally present in the water.

Bacillus Enteritidis Sporogenes. This organism was discovered by Klein in 1895 in diarrhœa stools. Its use as an indicator of pollution rests on the fact that it is present in feces and, therefore, in sewage. This organism should be regarded as one of a group of bacteria the members of which are very closely related. Table XXVIII quoted from Thresh will indicate the essential differences between certain members of this group. Bacillus welchii (Bacillus aerogenes capsulatus) is probably identical with Klein's Bacillus enteritidis sporogenes. Welch describes his bacillus as non-motile but otherwise the descriptions and the changes 
produced in milk are identical. The nomenclature of this group apparently is in a chaotic state.

TABLE XXVIII

SHOWING THE ESSENTIAL CHARACTERISTICS OF SOME MEMBERS OF THE ANAEROBIC GAS FORMING GROUP OF BACTERIA

(After Thresh, 1913)

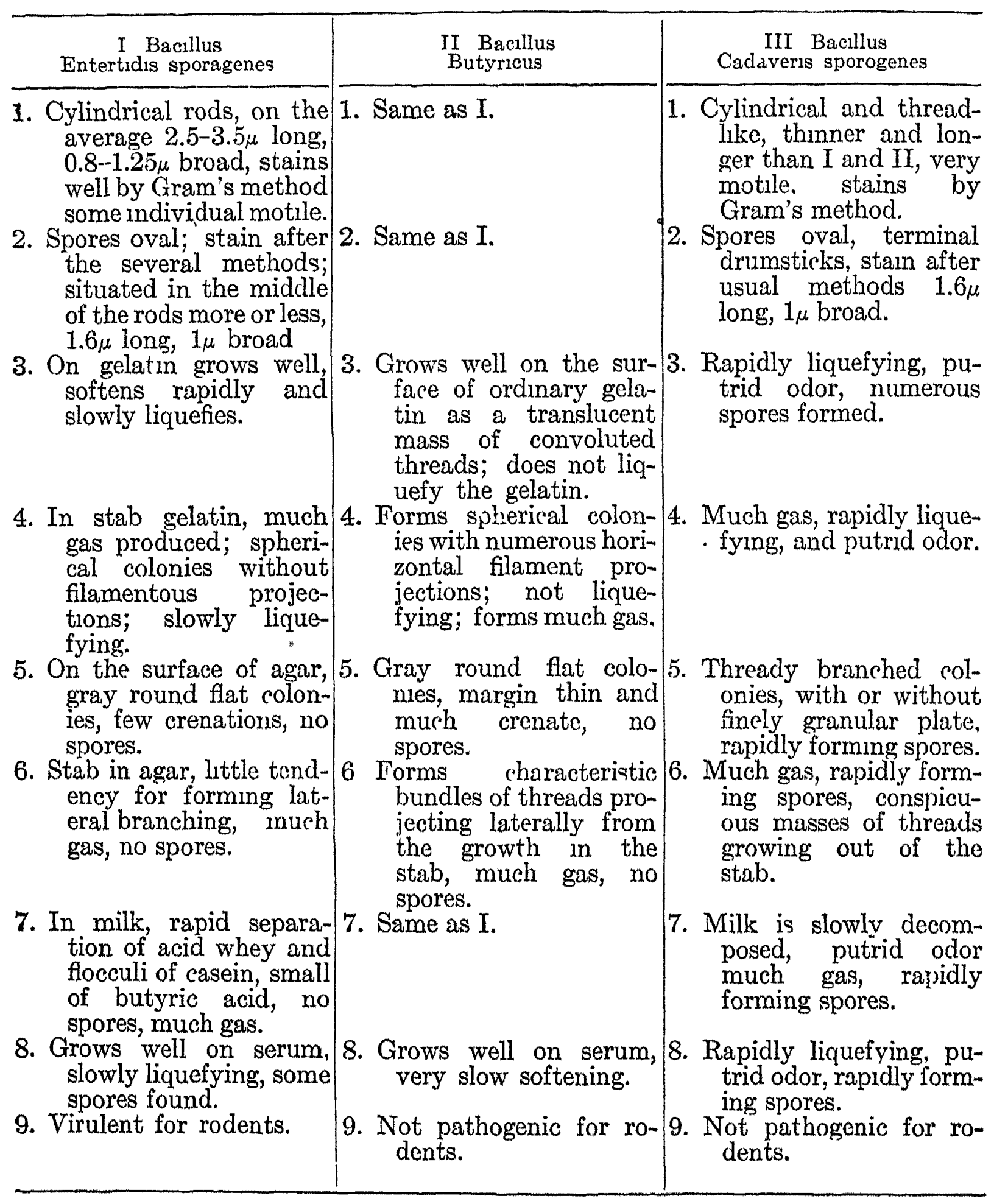

Isolation of Bacillus Enteritidis Sporogenes. In examining water for this organism, it may be necessary to concentrate it either by fil- 
tration or evaporation under reduced pressure at low temperatures. Decimal dilutions of this concentrated sample should be made and 1 c.c. quantities added to sterile litinus milk. It is a g.and plan to boil the tubes of milk for about five or ten minutes before inoculating in order to expel as much air as possible. After the tubes have been inoculated they should be heated on a water bath at $80^{\circ} \mathrm{C}$. for twenty minutes which will kill all vegetative forms. They are then incubated under anaerobic conditions either in a Novy jar or made anaerobic by the Wright method.

The Bacillus Colon Test. Escherich isolated Bacillus colon soon after the discovery of solid media by Koch. Escherich was attempting to find the causal organism of cholera. At first it was thought that he had found the specific bacterium which caused cholera because Bacillus colon was found to be present in the intestinal tract of each patient. This, however, was soon found to be untrue for the bacillus was found in the intestinal tract of normal individuals. Later investigations indicated that it was always present in the feces and from these facts bacteriologists attempted to use it as the indicator of the presence of fecal matter in foods even though the amount was very small. For some time it was accepted as a reliable factor in bacteriology but as knowledge was supplemented with more data it became apparent that some limitations had to be placed in interpreting this test. Much of our work since this time has been concerned with studies to find out the limits in the value of Bacillus colon in this connection.

Different definitions have been given for the Bacillus colon group. Standard Methods (1917) recommended that the $B$. coli group be considered as including all non-spore-forming bacilli which ferment lactose with gas formation and grow aerobically on standard solid media.

The value of the $B$. coli test has been discredited by some of the practical waterworks men. They argue from the several researches which have shown that the organism may not at all times be restricted to sources which have received sewage pollution. Rogers, Clark and Evans (1915), Prescott (1906), Metcalf (1905), Smith (1905), and others have reported the organism or $B$. coli like organisms from grain fields and grains which had not received sewage pollution. Their work was of such import that it became necessary to distinguish between fecal and non-fecal $B$. coli. The English and American bacteriologists have regarded the $B$. coli test with favor while some German bacteriologists have fought its use. Kruse (1894), for instance, believes that B. coli may be found in any water no matter from what source, provided a large enough sample is taken. Chick, representing the English opinion, believes 
that $B$. coli is not present in a watcr except it has received sewage pollution. These, however, may be extreme opinions and may not represent the opinions of the sanitarians in cach country. Race $(1914,1916)$ argues that $B$. coll is a reliable indicator of pollution and states that those cities which have many $B$. coli in their water usually have a high typhoid fever death rate. He warns that $B$. coli should be regarded as merely an inferential indicator. The fact that the presence of $B$. coll in a sample of water may not be sufficient evidence of serious pollution, it is, however, a danger signal which must not be overlooked. Houston (1915) representing the English practice regards $B$. coli as the best index for the control of purification processes. Winslow (1916), in tracing the history of this organism as an indicator has advised the accumulation of as much data as possible to more definitely determine whether $B$. coli is purely a fecal form and $B$. aerogenes a saprophytic form.

The testing of water for this organism has taken some recent advances. Thanks to the research of Rogers and his co-workers in the laboratories of the Bureau of Animal Industry, we have now fairly reliable methods for differentiating between fecal and non-fecal $B$. coli. The methods of analysis which are given in the following pages have been taken with permission from the 1917 report of the committee on water and sewage analysis of the American Public Health Association.

American Public Health Association Method for Determining the Presence of Members of the B. coli Group. It is recommended that the $B$. coli group be considered as including all non-spore-forming bacilli which ferment lactose with gas formation and grow aerobically on standard solid media.

The formation of 10 per cent or more of gas in a standard lactose broth fermentation tube within twenty-four hours at $37^{\circ} \mathrm{C}$. is presumptive evidence of the presence of members of the $B$. coli group, since the majority of the bacteria which give such a reaction belong to this group.

The appearance of aerobic lactose-splitting colonies on lactose-litmusagar or Endo's medium plates made from a lactose-broth fermentation tube in which the gas has formed confirms to a considerable extent the presumption that gas-formation in the fermentation tube was due to the presence of members of the $B$. coli group.

To complete the demonstration of the presence of $B$. coli, as above defined, it is necessary to show that one or more of these aërobic plate colonies consists of non-spore-forming bacilli which, when inoculated into a lactose-broth fermentation tube, forms gas.

It is recommended that the standard tests for the $B$. coli group be 
either $(A)$ the Presumptive, $(B)$ the Partially Confirmed, or $(C)$ the Completed test, as hereafter defined, each test being applicable under the circumstances specified.

\section{A. Presumptive Test}

1. Inoculate a series of fermentation tubes with appropriate graduated quantities of the water to be tested.

2. Incubate these tubes at $37^{\circ} \mathrm{C}$. for forty-eight hours. Examine tube at twenty-four and forty-eight hours, and record gas-formation. The records should be such as to distinguish between:

(a) Absence of gas-formation.

(b) Formation of gas occupying less than 10 per cent $(10 \%)$ of the closed arm.

More detailed records of the amount of gas formed, though desirable for purposes of study, are not necessary for carrying out the standard tests prescribed.

3. The formation within twenty-four hours of gas occupying more than 10 per cent $(10 \%)$ of the closed arm of fermentation tube constitutes a positive presumptive test.

4. If no gas is formed in twenty-four hours, or if the gas formed is less than 10 per cent (10\%), the incubation shall be continued to fortyeight hours. The presence of a gas in any amount in such a tube at forty-eight hours constitutes a doubtful test, which, in all cases, requires confirmation.

5. The absence of gas-formation after forty-eight hours' incubation constitutes a negative test. (An arbitrary limit of forty-eight hours' observation doubtless excludes from consideration occasional members of the B. coli group which forms gas very slowly, but for the purposes of a standard test the exclusion of these occasional slow gas-forming organisms is considered immaterial.)

\section{B. Partially Confirmed Test}

1. Make one or more Endo's medium or lactose-litmus-agar plates from the tube which, after forty-eight hours' incubation, shows gasformation from the smallest amount of water tested. For example, if the water has been tested in amounts of 10 c.c., 1 c.c., and 0.1 c.c., and gas is formed in 10 c.c., and 1 c.c., and not in 0.1 c.c., the test need be confirmed only in the 1 c.c. amount.)

2. Incubate the plates at $37^{\circ} \mathrm{C}$., eighteen to twenty-four hours. 
3. If typical colon-like red colonies have developed upon the plate within this period, the confirmed test may be considered positive.

4. If, however, no typical colonies have developed within twentyfour hours, the test cannot yet be considered definitely negative, since it not infrequently happens that members of the $B$. colv group fall to form typical colonies on Endo's medium or lactose-litmus-agar plates, or that the colonies develop slowly. In such cases, it is always necessary to complete the test as directed under $C 2$ and 3 .

\section{Completed Test}

1. From the Endo's medium or lactose-litmus-agar plate, made as prescribed under $B$, fish at least two typical colonies, transferring each to an agar slant and a lactose broth fermentation tube.

2. If no typical colonies appear upon the plate within twenty-four hours, the plate should be re-incubated another twenty-four hours, after which at least two of the colonies considered to be most likely $B$. coll, whether typical or not, shall be transferred to agar slants and lactose broth fermentation tubes.

3. The lactose broth fermentation tubes thus inoculated shall be incubated until gas formation is noted; the incubation not to exceed forty-eight hours. The agar slants shall be incubated at $37^{\circ} \mathrm{C}$. for forty-eight hours, when a microscopic examination shall be made of at least one culture, selecting one which corresponds to one of the lactose broth fermentation tubes which has shown gas-formation.

The formation of gas in lactose broth and the demonstration of non-spore-forming bacilli in the agar culture shall be considered a satisfactory completed test, demonstrating the presence of a member of the $B$. coll group.

The absence of gas-formation in lactose broth or failure to demonstrate non-spore-forming bacilli in a gas-forming culture constitutes a negative test.

\section{Tests.}

Application of Presumptive, Partially Confirmed, and Completed

A. The Presumptive Test.

1. When definitely positive, that is showing more than 10 per cent $(10 \%)$ of gas in twenty-four hours, is sufficient.

(a) As applied to all except the smallest gas-forming portion of each sample in all examinations.

(b) As applied to the smallest gas-forming portion in the examination of sewage or of water showing relatively 
high pollution, such that its fitness for use as drinking water does not come into consideration. This applies to the routine examinations of raw water in connection with the control of the operation of purification plants.

2. When definitely negative, that is, showing no gas in forty-eight hours, is final and, therefore, sufficient in all cases.

3. When doubtful, that is, showing gas less than 10 per cent (10\%) (or none) in twenty-four hours, with gas either more or less than 10 per cent in forty-eight hours, must always be confirmed.

B. The Partially Confirmed Test.

1. When definitely positive, that is, showing typical plate colonies within twenty-four hours, is sufficient.

(a) When applied to confirm a doubtful presumptive test in cases where the latter, if definitely positive, would have been sufficient.

(b) In the routine examination of water-supplies where a sufficient number of prior examinations have established a satisfactory index of the accuracy and significance of this test in terms of the completed test.

2. When doubtful, that is, showing colonies of doubtful or negative appearance in twenty-four hours, must always be completed.

C. The Completed Test.

The completed test is required as applied to the smallest gasforming portion of each sample in all cases other than those noted as exceptions under the "presumptive" and the "partially confirmed" tests.

The completed test is required in all cases where the confirmed test has been doubtful.

In order that tests for $B$. coli may have a quantitative significance, the following general principles and rules should be observed:

Ordinarily not less than three portions of each sample should be tested, the portions being even decimal multiples of fractions of a cubic centimeter; for example, 10 c.c., 1 c.c., 0.1 c.c., .01 c.c., etc. It is essential that the dilutions should be such that the largest amount gives a positive test (unless the water is such as to give negative tests in 10 c.c.), and the smallest dilution, a negative result. To insure this result, it is often necessary to plant four or five dilutions, especially in 
the examination of a sample of entrrely unknown quantity. The quantitative value of a series of tests is lost, unless all or, at least, a large portion of the smallest dilutions tested have given negative results.

In reporting a single test, it is preferable merely to record results as observed, indicating the amounts tested and the result in each, rather than to attempt expression of the result in numbers of $B$. colz per cubic centimeter. In summarizing the results of a series of tests, however, it is desirable for the sake of simplicity to express the results in terms of the numbers of B. coli per cubic centimeter or per 100 c.c. To convert the result of fermentation tests to this form, the result of each test is recorded as indicating a number of $B$. coli per cubic centimeter equal to the reciprocal of the smallest decimal or multiple fraction of a cubic centimeter giving a positive result. For example the result: 10 c.c. plus; 1 c.c. plus; 0.1 c.c. minus would be recorded as indicating one $B$. coll per subic centimeter. An exception should be made in the case where a negative result is obtained in an amount larger than the smallest portion giving a positive result; for example, in a result such as 10 c.c. plus; 1 c.c. minus; 0.1 c.c. plus. In such a case, the result should be recorded as indicating a number of $B$. colv per cubic centimeter equal to the reciprocal of the dilution next larger than the smallest one giving a positive test, this being a more probable result.

Where tests are made in amounts larger than 1 c.c. giving an average of results less than one $B$. coll per cubic centimeter, it is convenient to express results in terms of the number of $B$. coli per 100 c.c.

The following table illustrates the method of recording and averaging results of $B$. coln tests

\begin{tabular}{l|l|l|l|r|r}
\hline \multicolumn{3}{c|}{ Result of Tests in Amounts Designated } & \multicolumn{2}{|c}{ Indicated No of $B$ coll } \\
\hline 10 c.c. & 1 c.c. & 01 c c & 01 c.c. & per c c. & per 100 c.c. \\
plus & minus & minus & minus & 0 & 10 \\
plus & plus & minus & minus & 10 & 100 \\
plus & plus & plus & minus & 100 & 1000. \\
plus & plus & plus & plus & 1000 & 10,000 \\
plus & plus & minus & plus & 100 & $1,000$. \\
& & & & 121 & 12,110 \\
& & & & 240 & 2,422 \\
\hline
\end{tabular}

The above method of expressing results is not mathematically altogether correct. The average number of $B$. col per cubic centimeter, as thus estimated is not precisely the most probable number calculated by 
application of the theory of probability. To apply this theory to a correct mathematical solution of any considerable series of results involves, however, mathematical calculations so complex as to be impracticable of application in general practice. The simpler method given is, therefore, considered preferable, since it is easily applied and the results so expressed are readily comprehensible.

In order that the results as reported may be checked and carefully valuated, it is necessary that the report should show not only the average number of $B$. coll per cubic centimeter, but also the number of samples examined; and for each dilution, the total number of tests made, and the number (or per cent) positive.

\section{Routine Procedures for Examination of Samples of Water}

First Day.

1. Prepare dilutions as required.

2. Make two (2) gelatin plates from each dilution, and incubate at $20^{\circ} \mathrm{C}$.

3. Make two (2) agar plates from each dilution, and incubate at $37^{\circ} \mathrm{C}$.

4. Inoculate lactose broth fermentation tubes with appropriate amounts for $B$. coli tests, inoculating two (2) tubes with each amount.

Note. Where repeated tests are made of water from the same source as is customary in the control of public supplies, it is not necessary to make duplicate plates or fermentation tubes in each dilution. It is sufficient, in such circumstances, to make duplicate plates only from the dilution which will most probably give from 25 to 250 colonies per plate.

Second Day.

1. Count the agar plates made on the first day.

2. Record the number of additional fermentation tubes which show 10 per cent $(10 \%)$ or more of gas.

Note. In cases only the presumptive test for $B$. coli is required fermentation tubes showing more than 10 per cent (10\%) of gas at this time may be discarded.

Third Day.

1. Count gelatin plates made on the first day.

2. Record the additional number of fermentation tubes which show 10 per cent or more of gas. 
3. Make a lactose-litmus-agar of Endo's medium plate from the smallest portion of each sample showing gas. Incubate plate at $37^{\circ} \mathrm{C}$.

Note. In case the smallest portion in which gas has been formed shows less than 10 per cent (10\%) of gas it is well to make a plate also from the next largest portion so that in case the smallest portion gives a negative end result, it may still be possible to demonstrate $B$. coll in the next larger dilution.

Fourth Day.

1. Examine Endo's medium on lactose-litmus-agar plates If typical colonies have developed, select two and transfer each to a lactosc-broth-fermentation tube and an agar slant, both of which are to be incubated at $37^{\circ} \mathrm{C}$.

2. If no typical $B$. colr colonies are found, incubate the plates another twenty-four hours.

Fifth Day.

1. Select at least two colonies, whether typical or not, from the Endo's medium or lactose-litmus-agar plates which have been incubated an additional twenty-four hours; transfer each to a lactosc-broth-fermentation tube and an agar slant, and completc the test as for typical colonies.

2. Examine-lactose-broth formentation tubes inoculated from plates on the previous day. Tubes in which gas has been formed may be discarded after the result has been recorded. Those in which no gas has formed should be incubated an additional twenty-four hours.

Sixth.Day.

1. Examine lactose-broth-fermentation tubes reincubated the previous day.

2. Examine microscopically agar slants corresponding to lactose-fermentation tubes inoculated from plate colonies and showing gas formation.

Methyl Red Test. As sanitary knowledge increased and it became apparent that $B$. coli or $B$. colz-like bacteria were present on substances which had not received fecal pollution, studies were made to detect those B. coll which came from fecal sources. To this end Rogers (1915) and his co-workers found that the gas ratio separated $B$. coli of fecal origin from $B$. coll of non-fecal origin. $B$. coli from bovine feces fermented dextrose with the formation of equal volumes of carbon dioxide 
and hydrogen while those from grains formed two or three times as much carbon dioxide. The next step was to find a simple procedure for determining this ratio or finding some procedure which was correlated with it since the determination of the gas ratio is too exacting for routine analyses. Clark and Lubs (1915) working in the same laboratory discovered a constant correlation between the gas ratio and the hydrogen ion concentration. The $B$. coll from bovine feces were found to possess a higher hydrogen ion concentration than those from grains or nonfecal origins. A study of indicators yielded the information that methyl red was yellow with the high ratio group and red or acid with the low ratio group. Levine (1916) correlated this test with the Voges-Proskauer reaction.

Voges-Proskauer Reaction. This has been studied by Levine who correlated it with the methyl red test. He found the bacteria which give the Voges-Proskauer reaction were rarely found in feces and that the Voges-Proskauer reaction, like the high gas ratio and the alkalinity to methyl red, is characteristic of non-fecal strains and, therefore, of much sanitary significance. Harden (1901), in his various publications, has reported his studies on the chemistry of this reaction which are well reviewed by Levine. The red color which results is due to a definite end product in the fermentation of glucose. Voges and Proskauer (1898), in their original publication, describe the test as follows:

"On addition of caustic potash, we observed a new and interesting color reaction. If the tube be allowed to stand twenty-four hours and longer at room temperature, after the addition of the potash, a beautiful fluorescent color somewhat similar to that of a dilute alcoholic solution of eosin forms in the culture fluid particularly at the open end of the tube exposed to the air. We have investigated a few of the properties of this coloring substance which is not produced by the action of the alkali on the sugar and have found that it is fairly resistant to the action of the external air. After a time, however, it becomes paler, and finally gives place to a dirty greenish brown."

Harden and Walpole (1905-6) could not account for all of the carbon in the dextrose by the ordinary products (organic acids, alcohol, etc.) along with these substances was secured a glycol which was made up of $2: 3$ butylene glycol.<smiles>CCCO</smiles> 
This was oxidized to acetyl-methyl-carbinol.<smiles>CC(C)C=O</smiles>

When this was mixed with potassium hydroxide, in the presence of peptone, an eosin-like color was obtained after a period. Walpole (1910) found that under aerobic conditions Bacillus aerogenes gave a greater amount of acetyl-methyl-carbinol. The coloration was not secured when butylene glycol or acetyl-methyl-carbinol were mixed with potassium hyrdoxide. Harden (1905) later explained this by finding that the acetyl-methyl-carbinol was oxidized to diacetyl $\mathrm{CH}_{3} \mathrm{CO}-\mathrm{CO}-\mathrm{CH}_{3}$. This, in some way, acted with the pepton to give a pink color. Harden and Norris (1911) later secured the same pink color between diacetyl and arginine, dicyanamide, creatin and gaunidine acetic. They state that the reaction depends upon the group $\mathrm{NH}=\mathrm{C}-\mathrm{N}=\mathrm{HR}, \mathrm{R}$ has not<smiles>N</smiles>

been determined.

Levine demonstrated that the Voges-Proskauer reaction had much sanitary significance and that it would distinguish between fecal and non-fecal strains of Bacillus colon. He states that the natural habitat of those strains which form the acetyl-methyl-carbinol is probably the soil. Bacillus coli from non-fecal origins would then be methyl red and V-P plus.

Standard Methods gives the following procedure for the differentiation of fecal from non-fecal members of the $B$. coli group.

American Public Health Associaiion Method for the Differentiation of Fecal and Non-fecal B. coli. (1) At least ten cultures should be used. If possible these should be sub-cultured from plates made direct from the water since all the cultures obtained by plating from fermentation tubes may be descendants of a single cell in the water. If cultures from water plates are not available those obtained from the plates made as prescribed under $B$ may be used.

(2) Inoculate each culture into dextrose potassium phosphate broth,*

* The preparation of this medium has been described in the chapter on media. Since devising this medium, these investigators (Clark and Lubs, 1917) have described the preparation of a substitute. The new medium is a synthetic solution without Witte's peptone. Aspartic acid is used in its place, thus satisfying the nitrogen requirements and acting as a buffer. The preparation of this new medium is also given in the chapter on media. 
adonite broth and gelatin. For additional confirmatory evidence inoculation may be made into tryptophane broth and saccharose broth. The dextrose broth must be incubated at $30^{\circ}$. Other sugar broths may be incubated at $30^{\circ}$ or $37^{\circ}$ as convenient. Gelatin should be incubated at $20^{\circ}$.

(3) After forty-eight hours record gas formation in adonite and saccharose broths. Determine indol formation in tryptophane broth by adding, drop by drop, to avoid mixing with the medium, about 1 c.c. of a 2 per cent alcoholic solution of $p$-dimethyl amido-benzaldehyde, then a few drops of concentrated hydrochloric acid. The presence of indol is indicated by a violet color.

(4) After five days apply methyl red test and Voges-Proskauer test to dextrose broth.

\section{Methyl Red Test}

Indicator Solution. Dissolve 0.1 gm. methyl red in 300 c.c. alcohol and dilute to 500 c.c. with distilled water.

Procedure in Test. 1. To 5 c.c. of each culture add five drops of methyl red solution.

2. Record distinct red color as methyl red+, distinct yellow color as methyl red-, and intermediate colors as?.

\section{Voges-Proskauer Test}

To the remaining 5 c.c. of medium add 5 c.c. of a 10 per cent solution of potassium hydroxide. Allow to stand over night. A positive test is indicated by an eosin pink color.

(5) Gelatin tubes should not be pronounced negative until they have been incubated at least fifteen days.

The following group reactions indicate the source of the culture with a high degree of probability.

Methyl red+ Voges-ProskauerGelatin -

Adonite-

B. coli of fecal origin

Indol, usually -

Saccharose, usually - 
WATER HYGIENE

Meuhyl red-

Voges-Proskauer +

Gelatin -

Adonite+

$B$. aerogenes of fecal origin

Indol, usually +

Saccharose+

Methyl red-

Voges-Proskauer+

Gelatin -

Adonite-

Indol, usually -

Saccharose +

Methyl red-

Voges-Proskauer+

Gelatin+

Adonite +

Indol, usually -

Saccharoset

The Treasury Department Standard for the Examination of Water on Interstate Common Carriers. The following method for the examination of water on Interstate common carriers has been formulated by a committee of prominent sanitarians. The permissible limits of bacteriological impurity are stated as follows:

1. The total number of bacteria developing on standard agar plates, incubated twenty-four hours at $37^{\circ} \mathrm{C}$., shall not exceed 100 per cubic centimeter; provided, that the estimate shall be made from not less than two plates, showing such numbers and distribution of colonies as to indicate that the estimate is reliable and accurate.

2. Not more than one out of five 10 c.c. portions of any sample examined shall show the presence of organisms of the Bacillus coln group when tested as follows:

(a) Five 10 c.c. portions of each sample tested shall be planted, each in a fermentation tube containing not less than 30 c.c. of lactose peptone broth. These shall be incubated forty-eight hours at $37^{\circ} \mathrm{C}$. and observed to note gas formation.

(b) From each tube showing gas, occupying more than 5 per cent of the closed arm of fermentation tube, plates shall be made after forty-eight hours' incubation, upon lactose litmus agar or Endo's medium.

(c) When plate colonies resembling $B$. coli develop upon either of these plate media within twenty-four hours, a well-isolated characteristic colony shall be 
fished and transplanted into a lactose-broth fermentation tube, which shall be incubated at $37^{\circ} \mathrm{C}$. for forty-eight hours.

For the purposes of enforcing any regulations which may be based upon these recommendations the following may be considered sufficient evidence of the presence of organisms of the Bacillus coli group.

Formation of gas in fermentation tube containing original sample of water $(a)$.

Development of acid-forming colonies on lactose-litmus-agar plates or bright red colonies on Endo's medium plates, when plates are prepared as directed above under $(b)$.

The formation of gas, occupying 10 per cent or more of closed arm of fermentation tube, in lactose peptone broth fermentation tube, inoculated with colony fished from twenty-four-hour lactose litmus agar or Endo's medium plate.

These steps are selected with reference to demonstrating the presence in the samples examined of aerobic lactose-fermenting organisms.

3. It is recommended, as a routine procedure, that, in addition to five 10 c.c. portions, one 1 c.c. portion, and one 0.1 c.c. portion of each sample examined be planted in a lactose peptone broth fermentation tube in order to demonstrate more fully the extent of pollution in grossly polluted samples.

4. It is recommended that in the above-designated tests the culture media and methods used shall be in accordance with the specifications of the'Committee on Standard Methods of Water Analysis of the American Public Health Assoclation, as set forth in Standard Methods of Water Analysis (A. P. H. A., 1912).

This standard has been applied to the examination of water from trains by Bartow (1916), Creel (1914), Hanford (1916), and to water from boats by Cobb, Williams and Letton (1916). Bartow confirmed the presence of $B$. coli in 83 per cent of the samples examined. Creel found that an anaerobic bacillus which formed gas was responsible for gas in the presumptive test in 91 out of 421 samples. Such data indicate the significance of the confirmatory test. More data is required before it may be determined whether this method is the best. Letton (1917) has later stated that the requirement of " not more than 100 colonies per cubic centimeter on agar" of the Treasury Department standard is very lenient. He regards the limit of permissible $B$. coli (not more than two per 100 c.c.) as not too low.

Lactose Bile in Water Analysis. Lactose bile was advocated for some time for use in the presumptive test for $B$. coli. It was supposed to inhibit other bacteria and a few of the weaker types of $B$. coli. For that reason it was deemed superior for the presumptive test than the ordinary carbohydrate broths. Jordan (1915) found that the typical as well as the typical $B$. coli were inhibited. The work of Cummings (1917) for the United States Public Health Service is interesting in this connection. He found, in his stream pollution studies on the Potomac 
river that lactose broth was the better medium. In 1851 parallel sets of lactose broth and lactose bile tubes, lactose broth gave higher results, confirmed as well as presumptive, than lactose bile. It was granted, however, by Cummings that, in the lower reaches of the river, remote from pollution, the bile tubes were more reliable. The opinion of other bacteriologists has been that for highly polluted waters the lactose broth was the better medium, and for slightly polluted waters lactose bile gives the better results. This is attributed to the presence of sporeforming lactose fermenting anaerobic bacteria which are not manifest when the number of $B$. coli are large. Obst (1916) also found that the bile inhibited about one-half of the typical $B$. coli. The general use, then of lactose broth in place of lactose bile seems to be justified.

Hauser (1917) has described the method used for isolating and identifying $B$. coli at the Cincinnati filtration plant. This procedure is interesting and valuable because it is representative of the practice in one of the largest water-treatment plants.

The B.coli group may be defined as aerobic non-spore forming Gram negative bacilli, fermenting lactose with production of gas, and not liquefying gelatin. At the Cincinnati filtration plant the attempt is made to carry out the examinations so that the above definition is fulfilled as completely as possible, consistent with the speed and facility necessitated by routine water analysis.

The particular water samples under examination are planted into lactose broth. Lactose broth is used rather than lactose bile, which is the medium recommended in the 1912 edition of the Standard Methods of Water Analyses of the A. P.H.A., for the reason that, not only at this laboratory, but at the laboratory of the Ohio River Investigation of the United States Public Health Service and other laboratories, it has given consistently a higher percentage of confirmed end-results from original inoculations. In planting the water sample decimal dilutions are used to facilitate computations of the $B$. coli index. In routine examinations, at least three dilutions are used in order to obtain, if possible, a negative and a positive test. The formstion of gas in any amount after forty-eight hours' incubation at $37^{\circ} \mathrm{C}$. is recorded as positive and considered as presumptive positive. The smallest quantity of water giving gas is then confirmed.

A loopful of culture from the tube furnishing the presumptive test is stroked upon Endo's medium previously poured in plates. The plates are incubated at $37^{\circ} \mathrm{C}$. for twenty-four hours. If no aerobic colonies (sterile piate) develop, the respective gas-formation is considered due to anaerobes, and B.coli is recorded as not present. If aerobic colonies develop, the most typical of $B$. coli is transplanted into lactose broth and gelatin and a microscopical examination made directly. As there are several varieties of typical $B$. coli colonies, experience alone will determine the choice.

If in the lactose broth transplant, no gas is formed after the forty-eight 
hours at $37^{\circ} \mathrm{C}$., the result is considered negative, and $B$. coli is recorded as not present. If gas is formed the result is considered positive for $B$. coli, subject to the results of the gelatin transplant and a microscopical examination. If after forty-eight hours' incubation at $37^{\circ} \mathrm{C}$. the gelatin transplant will solidify on cooling, the culture is considered as non-liquefying. If the gelatin does not solidify on cooling, and gas is formed in the lactose broth transplant and the microscopical examination demonstrates a bacillus, the organism is called $B$. cloacce and included in the $B$. coli numerical estimation as it has the same sanitary significance.

The microscopical examination consists of a smear made according to the method of Gram from the Endo plate colony selected for transplanting. A

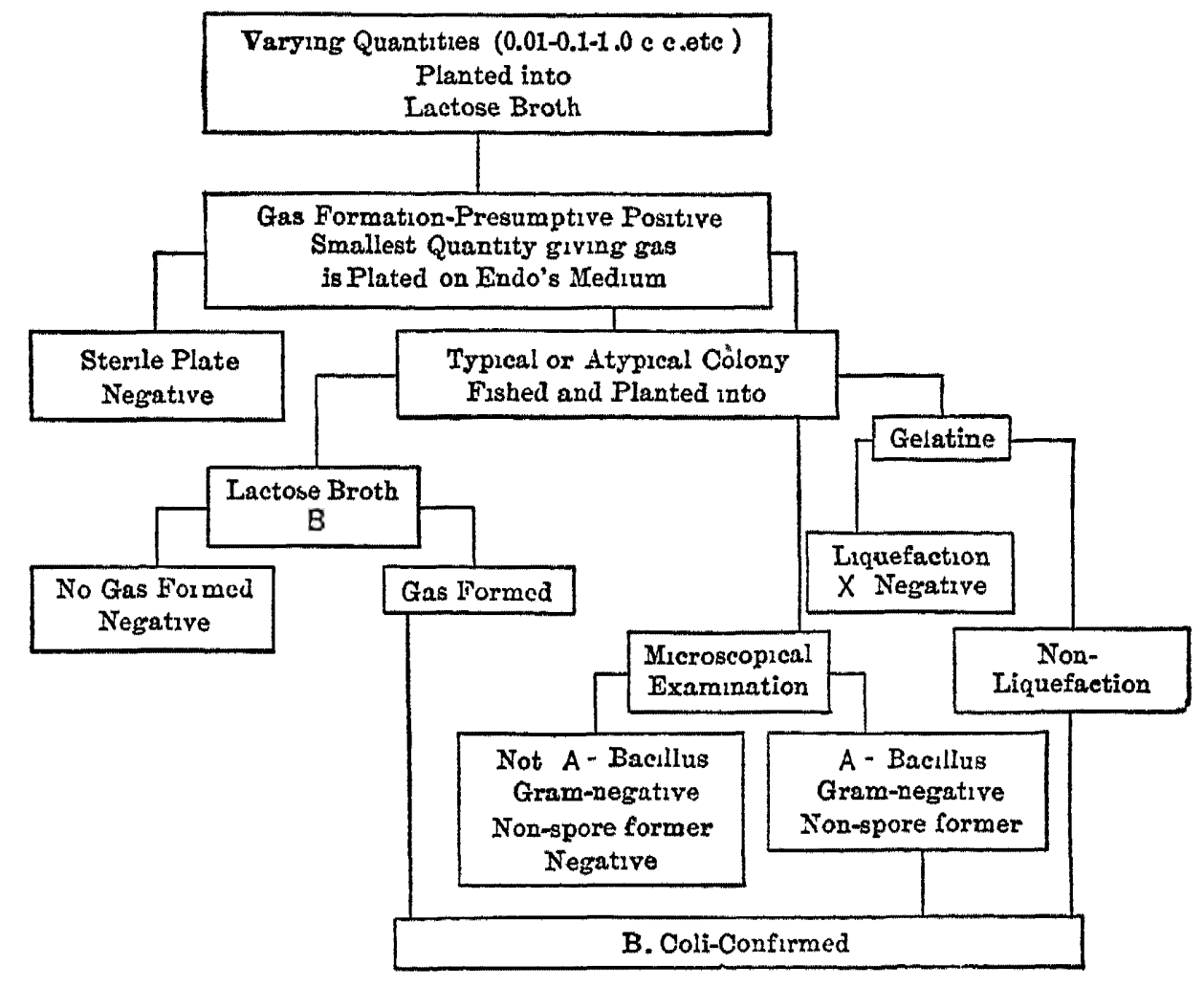

Method Followed at Cincinnati Plant for Identification of B. coli.

Gram negative non-spore-forming bacillus obtained following the above procedure fulfills in every particular the definition for the Bacillus coli. If other than a gram-negative non-spore-forming bacillus is obtained, it is not considered $B$. coli and is so recorded, but such a result is unlikely and has not been enc)untered in experiences at Cincinnati. The liquefaction of gelatin and discordant results in the microscopical examination accompanying gas production in the lactose broth transplant are likely to be due to mixed cultures, in which case'separation should be made by replating from the lactose broth transplant.

" Estimation of the B. coli index is made according to Phelps' method -that is, the reciprocal of the smallest volume of water which gave a positive test is taken as the approximate number of $B$. coli per cubic centimeter and is recorded as such. In case of an anomaly it is assumed that the positive result should have been negative and the negative result positive and is so considered 
in obtaining the $B$. coli index. If a negative confirmation test is obtained, $B$. coli is considered present in the next larger quantity without confirmation. If a negative result is obtained from the largest quantity planted, either by no gas formation in the original planting or by a negative confirmation test, $B$. coli is considered present in the next larger amount that would have been planted, except that a negative result in 100 c.c. is recorded at zero. The assumption is value only in the case of waters such as are encountered in water purification plants where the approximate coli content of the water is known. Such assumptions are rarely necessary as the quantities of water chosen for examination can ordinarily be changed in sufficient time to always obtain a positive test. It is probable that this assumption causes less error than would be introduced by either omitting the figure from the averages or calling it zero.

"The laboratory work above described with the numerical estimations of $B$. coli derived, are recorded for the month under headings of waters in the consecutive stages of purification. The form used is convenient and compact and lends itself readily to a grasp of conditions throughout the plant at any time. Five days from the collection of the water sample, B. coli may, following the above scheme, be satisfactorily and conclusively isolated and identified to conform to the definition.

The following table will show the number of samples examined, according to this method, from January to October, 1916, and the percentage of samples confirmed as B. coli.

\begin{tabular}{|c|c|c|c|c|c|}
\hline & \multirow[b]{2}{*}{$\begin{array}{l}\text { Ruver } \\
\text { Water. }\end{array}$} & \multirow[b]{2}{*}{$\begin{array}{l}\text { Settled } \\
\text { Water. }\end{array}$} & \multirow[b]{2}{*}{$\begin{array}{l}\text { Applied } \\
\text { Water }\end{array}$} & \multicolumn{2}{|c|}{ Fit TERED W } \\
\hline & & & & $\begin{array}{l}\text { Effluent from } \\
\text { Filters. }\end{array}$ & $\begin{array}{l}\text { Outlet Clear } \\
\text { Water } \\
\text { Reservoir. }\end{array}$ \\
\hline Samples tested & 271 & 257 & 266 & 258 & 194 \\
\hline Percentage confirmed & 974 & 91.0 & 966 & $* 94.9$ & $* 86.1$ \\
\hline
\end{tabular}

The B. coli Index. This was proposed by Phelps and was incorporated in a report of the Committee of Water Purification Plants. The following is taken from that report. The B. coli index is the approximate number of $B$. coli per cubic centimeter as determined from qualitative tests made on different quantities of water. For any individual sample, it may be taken as the reciprocal of the smallest volume of water used in the test which gave a positive result. Thus, if a sample gave a negative test with 0.1 c.c. and a positive test with 1.0 c.c. and 10.0 c.c., the $B$. coli index would be $1-0.1=10$. The $B$. coli index for a single sample is not very accurate. The index becomes more accurate as the square root of the number of tests increases. 
The following is an example: Write down the percentage of plus $(+)$ tests for the quantities examined expressed as decimals of 100 . Take the differences between these percentages. Multiply each of these differences by the reciprocal of the quantity corresponding to the larger of the two percentages from which such difference is taken. The sum of these products will be the $B$. coli index. In the following example, the $B$. coli index may be taken as 0.019 -equivalent to $19 B$. coli per cubic centimeter

\begin{tabular}{|c|c|c|c|c|c|}
\hline $\begin{array}{l}\text { Quantity of } \\
\text { Water } \\
\text { Examined }\end{array}$ & $\begin{array}{l}\text { Per Cent } \\
\text { Positive } \\
\text { Tests }\end{array}$ & $\begin{array}{l}\text { Expressed as } \\
\text { Decimals } \\
\text { of } 100\end{array}$ & Differences. & $\begin{array}{c}\text { Reciprocals of } \\
\text { Quantities. }\end{array}$ & Product \\
\hline 100 & 138 & 01380 & .1090 & .01 & 00109 \\
\hline 10 & 29 & 00290 & .0260 & .10 & .00260 \\
\hline 1 & 03 & 00030 & .0026 & 1.00 & .00260 \\
\hline 01 & 004 & 00004 & .0003 & 10.00 & .00300 \\
\hline 001 & 001 & 00001 & .0001 & 100.00 & .01000 \\
\hline
\end{tabular}

In this $B$. coli index the committee has made no provision for percentage reduction. It recommends that it be omitted for various reasons. The bacteria in an effluent from a filter are not derived from the raw water. It required quite a little time for a water to pass through a filter. The percentage removal is, to a certain extent, a function of the number in the raw water and does not vary with the number left in the filtered water. Other objections are also mentioned by the committee.

Interpretation of Results. The accurate interpretation of results of analysis is a part of sanitary work which is often taken too lightly. This problem is rendered more difficult by the lack of an ideal indicator of pollution. No organism has been described which is always and only present in water and on foods which have received sewage pollution. Thresh (1913) gives an interesting discussion with regard to what should be regarded as polluted water. He sent questions to Houston and Winslow seeking their opinions on the subject. He submitted the following questions to Houston of the Metropolitan Water Board: What bacteriological proof would you consider conclusivae as to the pollution of a water with sewage or manurial matter? Houston did not give a definite answer and stated that one was not possible. He advises that the final verdict should rest on data secured from all analyses. His experience seems to indicate that $B$. coli should be absent from 100 c.c. quantities of the water in a majority of representative samples, preferably not less 
than 10. What bacteriological proof would you consider conclusive that a water is free from such pollution or so free that it is safe for drinking purposes? Houston regards the absence of $B$. coli from 100 c.c. of the deep well water as reasonably sufficiont evidence of safety and the greater the number of these negative results, the more satisfactory is the water. A water which never contains B. colz in 100 c.c. quantities is to be regarded as a safe drinking water. A water which contains $B$. coli in less than half of the samples examined occupies an intermediate position but is probably reasonably safe. One which contains $B$. coli in more than half should be regarded with some preliminary disfavor.

Winslow replied to these questions and stated that the presence of colon bacilli in all of ten duplicate 10 c.c. samples, is reasonable proof of the presence of sewage or manurial matter while the absence of colon bacilli from such samples would be reasonable proof of the absence of such sewage. Winslow would carry out a confirmative test to show that the fermentations were due to true $B$. coli.

McLaughlin (1914) regards a safe water as one which contains no pathogenic bacteria. According to his opinion a water containing no more than 2 B. coli per 100 c.c. is satisfactory. A filter plant that cannot furnish such an effluent is either inefficiently operated or is handling a raw water which imposes an unreasonable load on the filters.

In light of the recent contributions of Rogers, Clark and Lubs on the differentiation of the members of the colon group, in which they have shown that $B$. coli of fecal origin is methyl red plus $(+)$ careful extensive work must be done in the future to work out the relation of the methyl red test to the analysis and control of water supplies. Clark (1918) has advised this. Orchard (1918), in view of the lack of uniformity concerning standards has recommended that the societies concerned with water analysis appoint committees to study this problem. This would probably be the quickest and most reliable method of handling the question.

Wording of Reports on the Results of Bacteriological Examination of Water. This is often a difficult procedure for the analyst especially if the certificate accompanying the sample is not filled in. An investigation of this question reveals the lack of uniformity in reports from different laboratories on the same sample of water. This was realized among the English analysts to such an extent that a committee of the Royal Institute of Public Health was appointed to go over the subject. They reported as follows: When the source and surroundings are unknown, it is undesirable to report as follows:

"This water is grossly polluted and is dangerous for drinking" or 
"This water is of superlative quality and is absolutely safe for consumption."

This committee recommended the use of guarded terms as follows:

"This particular sample of water as judged by the above tests yields unsatisfactory results (this may be amplified) and suggests the necessity of inquiring into the local conditions with regard to the source of contamination together with consideration of the desirability of further samples being examined."

Satisfactory results may be reported as follows:

"This particular sample of water, as judged by the above tests yielded satisfactory results" (may be amplified) " but in the absence of knowledge of local conditions, it is impossible to say that the supply will always attain the same standard of purity indicated by the present results."

From the above report some valuable suggestions may be secured with regard to the proper wording of reports. The layman usually must depend on the analyst for a correct interpretation of his results. Too strong opinions should be avoided since they may cause the layman to secure the wrong impression. Some laboratories simply use the words "good " and "bad." It is the case in some laboratories that this part of the work is handled by a person who has had too little experience or who has not informed himself with regard to the prevailing standards. No part of the analysis should have more attention than the reporting of the results and their interpretation to the clients of the laboratory.

\section{Detection of Pathogentc Bacteria in Water}

There are a few diseases that have been spread so much by water that they have become known as water-borne diseases. In some of these the isolation of the causal organism has been atended with little success even if it is present.

Bacillus typhosus. The isolation of Bacillus typhosus from water has not been attended with much success. When attempts have been made on polluted water known to contain the bacilli, there has been no certainty in the results. Those instances in the literature where it is claimed that this has been done, are lacking in sufficient confirmatory evidence. It is possible that by means of recent advances in technique, the isolation of $B$. typh $i$ will be attended with more success than in the past. Most of the methods which are available for isolating the organism from water, have been devised for isolating the organism from other 
material such as feces and urine. The reader is also referred to the methods developed for the isolation of $B$. typhr from feces.

Drigalski and Conradi Method (1902). The preparation of the medium for this method has been described in the chapter on the media. The medium is solldified in Petri dishes which are allowed to become thoroughly dry before use. This may be accomplished by allowing the plates to stand at room temperature with the covers suspended above or in the $37^{\circ} \mathrm{C}$. incubator. The surface is then streaked with the sample by means of a sterile bent glass rod. It is often a good plan to concentrate the water, either by reduced pressure of filtration. Bacillus colon forms red opaque colonies on this medium while Bacillus typhosus forms a blue and often transparent colony. Suspicious colonies must be subcultured into other media and a complete cultural and morphological study made. Identification must be confirmed by agglutination reactions.

Endo's Medium. The preparation of this medium has been described in another chapter. The sample of water is streaked over the surface and the plates incubated at $37^{\circ} \mathrm{C}$. for about fifteen hours. At the end of this time the Bacullus colon colonies will be red with possibly a metallic appearance, while the Bacillus typhosus colonies will be colorless. The same will be true with regard to the paratyphoid colonies. These suspicious colonies should be subcultured into other media and a confirmatory test made by means of the agglutination reactions.

Kligler (1917) has shown that a differentiation in the typhoidparatyphoid group may be made by means of hydrogen sulphid formation and gas formation in the following medium which is semi-solid.

1. Meat infusion agar containing $\frac{3}{4}$ or 1 per cent of agar (depending on the moisture content of the agar shreds).

2. 0.1 per cent of glucose.

3. 0.05 per cent lead acetate.

The agar is prepared in the usual way except that the amount of agar is reduced. The sugar and lead acetate are dissolved in water separately and added to the sterile agar. The agar should be cooled to $60^{\circ} \mathrm{C}$. in order not to precipitate the peptones. Stab inoculations are made. Strains of $B$. typhi brown the medium along the line of inoculation. $B$. paratyphi $B$. causes browning and gas formation; $B$. paratyphi $A$. causes gas but no browning. This has been confirmed by Jordan and Victorson (1917) who used a medium containing 1.5 per cent of agar. Morishima (1918) also described a similar method. The differentiation is secured in about the same way as with Kligler's method.

Russell's medium may be used to good advantage with any of the 
above methods for identifying members of the colon-typhoid group. Russell has described the preparation and use of this medium in the following manner:

" Enough 5 per cent aqueous solution of litmus ( 3 to 5 per cent) is added to plain agar ( 2 to 3 per cent) which usually has a reaction of about .8 per cent to phenolphthalein, to give it a distinct purple violet color, the amount of the litmus depending on the original ' color of the agar; dark requiring more than light, and the reaction then adjusted by adding sodium hydrate until the mixture is neutral to litmus. Next, and last, 1 per cent of lactose and $\frac{1}{10}$ of 1 per cent of glucose dissolved in a small amount of hot water is added and the medium tubed for slants. The sterilization is done in the Arnold and, because of the danger of breaking down the lactose, must not be carried too far; if the tubes are packed loosely in the sterilizer basket to allow good circulation of steam, ten minutes on the first and fifteen on the second day has been time enough. The tubes are then slanted and stored in small quantities in a dark place.

"On this double sugar tube, the typhoid bacillus gives, after an incubation period of from eight to eighteen hours, an extremely characteristic appearance; the surface growth is filiform and colorless, the upper part of the tube is unchanged in color but the lower part, the butt, is a brilliant uniform red. The entire point of the medium rests upon the difference in the changes produced by the growth of the typhoid bacillus under aerobic and under the imperfect anaerobic conditions found in the butt of the tube, where the bacillus obtains its oxygen by breaking down the glucose with the liberation of considerable acid; on the surface, however, in the presence of free oxygen, no acid is formed.

"The colon bacillus, which is often slow in producing acid on the Endo plate, shows abundant gas and acid formation on this medium. The tube is reddened throughout, both above and below, and since the abundant lactose is attacked equally with the glucose there is exuberant gas formation. . . .

"The bacillus fecalis alkaligenes and other alkali formers leave the medium unchanged or slightly bluer. The staphylococcus reddens the tube above but leaves it blue below; the streptococcus intestinalis, when it grows well, gives a beaded growth and reddens the tube slightly throughout. B. subtzlis, which is commonly found in feces, usually leaves the medium unchanged but may redden it below without producing gas, yet the heavy, rough surface growth suffices for its differentiation. B. pyocyaneus gives a greenish blue surface growth and leaves the color of the medium unchanged. B. proteus produces small gas bub- 
bles in the depth and reddens and then decolorizes the butt very early, while the upper part of the tube is unchanged except for the spreading surface growth.

"All dysentery bacilli alter the medium in the same manner as typhoid, yet the quantity of acid produced is small and the reddening is usually confined to the line of inoculation. This reaction is so characteristic that we use this medium regularly in isolating dysentery bacilli; in fact, the same media and technique are used for both typhoid and dysentery.

"The paratyphoids leave the upper part of the medium unchanged, the surface growth is like typhoid but in the butt of the tube in addition to the reddening are found a few small gas bubbles. The only organisms which may simulate the paratyphoids are slow colons and these must be thrown out by agglutination tests and further observation of the sugar tube."

Kligler (1917) has stated that by adding 0.5 per cent of basic lead carbonate to Russell's medium, a good differentiation between $B$. paratyphi $A$ and $B$. paratyhpi $B$ may be secured. Krumwiede and Kohn (1917), by adding 1 per cent of sucrose to Russell's medium found that a large percentage of "intermediates" giving a typical paratyphoid reaction could be excluded.

Bacillus Dysenteriæ. This organism may be isolated by means of Endo's medium plates. It grows as colorless colonies which distinguishes it from other bacteria. After the use of Russell's medium, agglutination reactions should be employed to confirm the results.

Agglutination. The serum of a person who has had typhoid fever or has been immunized against this disease will cause the agglutination of the typhoid bacillus. This reaction is not specific, however, unless high enough dilutions are used to prevent the agglutination of other members of the colon-typhoid group. In low dilutions of serum the reaction is not specific for closely related bacteria. Citron (1914) reports the following data with regard to a strongly agglutinating typhoid and strongly agglutinating cholera serum.

\begin{tabular}{|c|c|c|}
\hline Agglutination Titre. & $\begin{array}{l}\text { Of Typhoid } \\
\text { Serum. }\end{array}$ & $\begin{array}{l}\text { Of Cholera } \\
\text { Serum. }\end{array}$ \\
\hline Against typhoid. & $1-2000$ & $1-10$ \\
\hline Against paratyphoid. & $1-100$ & $1-10$ \\
\hline Against $B$. coli ........ & $1-25$ & $1-10$ \\
\hline Against cholera... & $1-10$ & $1-3000$ \\
\hline
\end{tabular}


Macroscopic Agglutination. By this procedure the presence of agglutinins is demonstrated without the use of a microscope. To carry out this procedure, a series of small tubes are necessary. Mixtures of physiological salt solution, a twenty-four-hour broth culture of $B$. typhi and serum are used. Dilutions of 1-20, 1-40, 1-80 and 1-160 should be prepared. These may be secured as follows: The dilution of serum 1-10 may be secured by drawing the serum up to 1.0 mark on the white blood corpuscle pipette and then filling the bulb to the mark with sterile salt solution or bouillon. Transfer 1 c.c. of this 1-10 dilution to the second test tube and add 1 c.c. of the twenty-four-hour broth culture. This will give the 1-20 dilution. The other dilutions may be prepared in the same way. These tubes should be incubated at $37^{\circ} \mathrm{C}$. and examined at frequent intervals for the presence of a precipitate.

Microscopic Agglutination. This requires the use of a microscope but has the advantage of requiring less time. The same dilutions should be prepared as were mentioned above under macroscopic agglutination. Instead of putting them into small test tubes hanging drop mounts are made which are examined for agglutination after thirty minutes. Agglutination in a dilution of 1 to 80 after thirty minutes constitutes a positive reaction.

Identification of Bacteria by Agglutination. The technique is essentially the same as for the detection of the presence of agglutinins. In this case the bacteria are the unknown factors while the serum is known to be specific for a certain organism. The bacterium should be grown in broth for twenty-four hours and tested against dilutions of the serum.

Coles' Method for the Microscopic Agglutination of Bacteria. The author claims that the method is easy, rapid and requires less blood than some of the other methods.

1. Draw a line across the middle of two slides at right angles to their long axes.

2. Spread a film of blood to be examined on one-half of each slide, and, when dry, spread film of blood from a person who has not had typhoid fever or received protective inoculation as a control over the other half. Dry the smears carefully over a flame.

3. By means of a platinum needle place a small drop of an emulsion of killed typhoid bacilli on the center of each half of both slides. Run the drop well over the smears taking care not to pass from one-half to the other without sterilizing the needle.

4. On one slide carefully place a cover glass on each half of the slide taking care that they are well separated by a wax pencil mark.

5. Place the other slide on a wet.piece of blotting paper and cover 
with a Petri dish to prevent evaporation. Allow to stand for fifteen to twenty minutes. At the end of this time, stain and examine for clumping of the bacteria.

Examine both slides for agglutination of the bacteria.

Isolation of Microspira Choleræ from Water; Metschnokoff's Method after Besson. Prepare a series of flasks and add 200 c.c. of the water under examination. To each flask add the following solution:

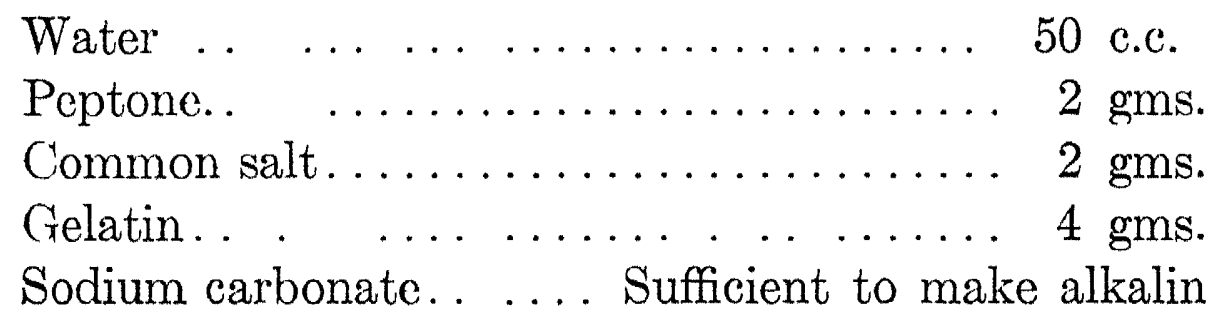

Sterilize this in the autoclave. Then add 150 c.c. of the water under examination and incubate at $37^{\circ} \mathrm{C}$. If the water contains microspira a thin film will form on the surface in about eight hours. This film should then be examined under the microscope and if microspira are present they must be proven to be the pathogenic Microspira cholerce. Besson gives the following " classical characteristics of the M. cholerce."

1. Characteristic appearance on gelatin plates and in gelatin stab.

2. The presence of and the number of flagella.

3. The nitroso-indol reaction in pepton water.

4. Virulence for guinea pigs.

5. The immunity reaction (Pfeiffer's reaction).

6. Agglutination by anti-cholera serum.

The agglutination reaction is probably the most reliable since this is quite specific. Besson mentions that the other characteristics of Microspira cholerce are not fixed and are thus of little use in identifying a suspected organism.

The Cholera-red Reaction. This is a reliable test for the diagnosis of Microspira cholerce. Not'many other organisms. The test is carried out by adding a little sulphuric acid to a broth culture of the organism. A red color results due to the presence of both indol and nitrites which are formed by the organism. Some care must be used in controlling the factors involved in the test. The pepton must be proven to yield the test when a known organism is used. The cholera-red test is of much value in identifying this organism in water.

Detection and Isolation of Bacillus Anthracis from Water. Pasteur used the resistance of the spores as a means of isolating the bacillus from different substances. Heat a small quantity of the witnr in n rinter bath for twenty minutes at $80-85^{\circ} \mathrm{C}$. At the $\mathrm{c:s}$ " , make 
agar plates using one or two decimal dilutions and incubate at $20^{\circ} \mathrm{C}$. for several days. All suspicious colonies must be studied morphologically, culturally and microscopically. The cultures which pass through this treatment should be injected into healthy guinea pigs. Koch's postulates must be satisfied before the suspicious colonies are accepted as anthrax.

Bacillus Tuberculosis. The search for this organism in water has not been carried out to any great extent. If acid-fast bacteria are isolated from water, it is necessary to confirm the findings by animal inoculation.

Brown's et al. Method. Brown and his collaborators used this method to detect the tubercle bacillus in river water. Near the source of pollution (sewer outlet) large bottles of water were collected. Further down the stream different methods were used. Cheesecloth bags were stretched across the surface of the stream and allowed to remain in position from one to two hours, after which they were put into pails and carried to the laboratory. According to this method, they should then be carefully washed with sterile water and the wash water put into wide-mouth bottles, saturated with sodium chloride and allowed to stand in the dark for several hours; at the end of this time, the scum should be collected and transferred to 50 c.c. centrifuge tubes. Ten c.c. of $\mathrm{NaOH}$ are added to each tube and the tube incubated for one-half hour. Neutralize the contents of the tube with $\mathrm{NHCl}$, centrifugalize, and study the sediment microscopically, inoculate part into egg media and inject part into guinea pigs.

Tracing Pollution in Water Courses. Different methods have been used and also different materials such as, sodium chloride, lithium salts fluorescin, and cultures of $B$. prodigiosus.

Salt was used by McCallie (1904), in Georgia, for the purpose of demonstrating the effects of discharging sewage into deep wells. A large amount of salt was put into a 124-ft. drilled well, and the content of chloride in the water of the surrounding wells was determined. Increased content of chloride showed that there was underground connection between the wells and springs in the vicinity.

Dole (1906) summarized his investigations on the use of fluorescein in the following statements:

1. In determining the sanitary value of a well or spring it is more important to study the underground flow than to analyze the water itself. 2. Foreign substances put into the aquifer and traced from point to point are of great value in this study. 3. With the fluorescope one part of fluorescein can be detected in 10 billion parts of water. 4. Fluorescein is a particularly valuable flow indi-. 
cator for fissured or cavernized rocks. 5. It is also available in gravels, where it has been used with success. 6 . It progresses at a slightly lower rate than the water in which it is suspended. 7. It is not decolorized by passage through sand, gravel, or manure; it is slightly decomposed by calcareous soils. 8. It is entirely decolorized by peaty formations and by free acids, except carbonic acid.

Trillat (1899) states and claims that fluorescein can be detected in dilutions of 1 part in 2,000,000,000, but that before the dye is used a study of the soils should be made with regard to the presence of matters which decompose it. Marboutin (1901) comes to somewhat similar conclusions. Martel (1903) shows that fluorescein even in very concentrated solutions decolorizes rather quickly after being kept in the sunlight. When it is kept in complete darkness, which would be the case in the earth, it did not change even after long periods.

Gehrmann (1913) reports that Alba Orlandi and Roudelli, who used a suspension of $B$. prodigiosus, found that cultures of this organism poured on the ground passed through soil 200 meters, and Pfulil found that it took the same organism a short time to pass through $24 \mathrm{ft}$. of - gravel. Gehrmann also reports a place at which wells 200 to $300 \mathrm{ft}$. deep too near an old canal were subject to entrance of contaminated water; no experimental data are given.

\section{Methods of Treatment}

After attempting to use a great many different compounds and methods the use of calcium hypochlorite and liquid chlorine is well established.

Traube (1894) proposed the addition of bleaching powder to water. Since that time the use of hypochlorite has greatly extended in sanitation. Water to which this compound is added in the usual amounts is quite harmless. Professor Hulett, testifying in the Jersey City case, that in the Jersey City water to which had been added $10 \mathrm{lbs}$. of bleach per million gallons of water he was unable to detect the presence of free chlorine. Theoretically, it was possible for free chlorine to be present to the extent of 6.4 parts per trillion. It was also revealed that for a person to get a medicinal dose such as given in typhoid fever it would be necessary to drink $2,500,000$ gals. of water so treated. This, with other data, indicates that there can be little objection to using a water which has received the usual amount of calcium hypochlorite. The waterworks officials have received all kinds of objections against the use of this chemical for water treatment. Some even claim that they cannot raise flowers of a certain tint on account of the chlorine in the water. 
Others have claimed that it was impossible to make tea with such water.

Advantages and Disadvantages of "Bleach" in Water Treatment. These have been given as follows by Johnson (1910, i913):

\section{AdVANTAGes}

1. Destruction of bacteria, especially intestinal forms.

2. Reliability of chemical and ease of application.

3. Absence of poisonous chemical compounds formed.

4. Cheap cost of chemical.

5. Rapid speed of reaction-no storage basins required.

6. Saves coagulating chemicals.

7. Allows much higher rate of filtration.

8. Reduces clogging of filters and lengthens the runs.

\section{Disadvantages}

1. Does not destroy all spore forms.

2. Does not get bacteria inside of suspended matter.

3. Does not remove turbidity.

4. Does not take color out or remove vegetable stain.

5. Inability to remove organic matter.

6. Leaves swampy tastes and odors.

7. Does not soften the water. Increases the hardness slightly. Bleach usually contains a little $\mathrm{CaO}$ which takes out some $\mathrm{CO}_{2}$.

8. Care must be used to allow for reducible compounds such as nitrites or oxidizable iron.

The quantity of "hypo" to add to a water places the sanitarian between two fires-tastes in the filtered or final product and destruction of the pathogenic bacteria. The absolute amount varies greatly. The bacteriological analysis together with turbidity determinations are probably the best indicators to tell whether the amount is sufficient. This must be determined for each separate water supply. Under ordinary conditions when added to the filtered water from 7-12 lbs. per million gallons is sufficient for disinfection. This will give from .28 to .48 p.p.m. of available chlorine (based on 30 per cent bleach). Many factors influence the amount of bleach required by a water but turbidity is one of the most important especially when this is largely organic matter. The amount of bleach added to a water supply must, therefore, vary with the turbidity. Where storage basins are used, less 
attention has to be given to the amount of bleach being added because one important function of storage, as Houston has pointed out, is equalization.

For a time, there was some discussion with regard to whether the bleach should be added to the raw or filtered water. At present it seems to be the consensus of opinion that it should be added to the filtered water. The following facts bear on the consideration:

1. When it is added to the raw water, a greater amount is required.

2. The life in the "schmutzdecke" on the filter may be destroyed.

3. Reactions between the organic matter and chlorine may yield compounds imparting a bad taste.

4. The addition to the filtered water is easier since there would be no cumulative effect.

The method of adding it to the filtered water has been standardized. It is usually mixed to a thick cream and later diluted with water. After settling, it is fed to the water supply through standard orifices by which the amount applied is regulated. This is an important part of the procedure since overdosing must be avoided.

Race (1918) has shown that by using a little ammonia along with the bleach, the amount of the bleach may be materially reduced. $\mathrm{He}$ regards the possibility of chloramine being formed as fairly conclusive. On Ottawa River water at flood period, when the water contained from 500 to $1000 \mathrm{~B}$. coli per cubic contimeter, satisfactory results were obtained with 0.60 p.p.m. of available chlorine, and 0.13 p.p.m. of ammonia. This method of treatment is recommended for those waters where the margin between the dose required for satisfactory treatment and successful purification is small. It is well known among certain practical waterworks men that such is often the case. The character of the raw water may demand an amount of bleach which is very close to the amount which may be tasted in the water.

The question of tastes has caused much trouble to practical waterworks men. Some of the essential oils excreted by microorganisms resemble very much the odor of chlorine. This has caused undue blame to be put on the bleach. Lederer and Bachmann (1912) found that under experimental conditions the average amount of available chlorine that could be detected was around 0.6 p.p.m. while 0.5 p.p.m. could often be detected. Van Brunt placed the amount that could be tasted at 0.6 p.p.m. While the chemical nature of these compounds which cause tastes in water treated with chlorine has not been carefully investigated, it is generally admitted that they may be related to the chloramines. 
Different means may be used to determine the amount of available chlorine which is being added to a water supply. The "bleach" may be analyzed for available chlorine to determine its strength in per cent. Calculations may then be made to find how much should be added to the mixing tanks. Lewis (1912) reports the following scheme used at Evanston, Ill.:

$N=$ No. of gallons pumped per minute. $\frac{1,000,000}{N}=M$, or number of minutes to pump $1,000,000$ gals.;

$Q=$ No. of pounds of " hypo " to be added to each 1,000,000 gals. of water.

$\frac{Q}{M}=P$. or pounds of " hypo" to be added each minute.

$\frac{P}{X}=$ No. of gallons of liquid to be added per minute.

$X=$ pounds of "hypo" in 1 gal. of solution.

Changes in the rate of pumpage demand a change in the amount of chemical which is applied. This is easily accomplished by changing the size of the orifice.

If the strength of the bleach solution is determined from that in the orifice box the following scheme will indicate how much is being applied to the water.

$R \times 70=$ p.p.m. available chlorine;

$R=$ No. of cubic centimeters of $\mathrm{N} / 10 \mathrm{Na}_{2} \mathrm{~S}_{2} \mathrm{O}_{3}$ used per 50 c.c. of bleach solution.

Parts per million of available chlorine in the filtered water equals

$$
1 \text { p.p.m. } \times \frac{\text { Gal. bleach solution bed }}{\text { Gallons of water pumped }} \text {. }
$$

Analysis of the dry bleaching powder which is necessary in the above method, is accompanied by certain errors. To obviate these, the bleach solution may be mixed in the usual way and samples removed directly from the orifice box. This procedure has the advantage that it is more direct-indicating the actual amount of available chlorine which is being applied to the water. The following calculations may be used: 


\section{Evaluation of Bleaching Powder}

Availabie Chlorine. Weigh 7.09 gms. of the sample from a weighing bottle into a mortar. Add water and rub into a smooth cream. Add more water, and after settling, decant the clear liquid into a liter flask. Add more water, grind and decant until all of the powder has been transferred to the flask. Dilute to the mark. Aliquot portions of this may be removed for analysis but in each case the flask should be thoroughly shaken.

Great care must be exercised in the sampling of bleach. It rapidly decomposes in the presence of air. Very often samples taken from the center of a carboy will contain a larger amount of available chlorine than a sample which is removed from the surface.

\section{A. Thiosulphate Method.}

Solution required:

1. $\mathrm{N} / 10$ sodium thiosulphate $\left(\mathrm{Na}_{2} \mathrm{~S}_{2} \mathrm{O}_{3}\right)$.

Dissolve 24.83 gms. of sodium thiosulphate in 1 liter of boiled, cooled distilled water. Boiling the distilled water decreases the error caused by the presence of $\mathrm{CO}_{2}$. The solution may be checked against standard iodine solution. It keeps very well on standing.

2. Starch solution.

Rub a gram of starch to a smooth paste and pour into boiling water with constant stirring. Decant after settling into test tubes putting about 5 c.c. into each tube. Plug and sterilize in the autoclave.

3. Potassium iodide.

Dissolve 25 gms. potassium iodide in 500 c.c. of distilled water.

Procedure. Transfer 50 c.c. of the bleach solution (either that prepared from the powder or that from the orifice box) to an Erlenmeyer flask by means of a pipette. Dilute with $50 \mathrm{c}$ c. distilled water, add 10 c.c. of the potassium iodide solution, a little acetic acid and titrate with the $\mathrm{N} / 10 \mathrm{Na}_{2} \mathrm{~S}_{2} \mathrm{O}_{3}$ until a faint yellow color remains. Add 1 c.c. of the sterile starch solution and continue addition of $\mathrm{N} / 10 \quad \mathrm{Na}_{2} \mathrm{~S}_{2} \mathrm{O}_{3}$ until the blue color is discharged. The number of cubic centimeters of $\mathrm{N} / 10$ sodium thiosulphate used is equivalent to percentage of available chlorine in the sample.

1 c.c. $\mathrm{N} / 10 \mathrm{Na}_{2} \mathrm{~S}_{2} \mathrm{O}_{3}=.003545$ gm. chlorine.

1 c.c. $\mathrm{N} / 10 \mathrm{Na}_{2} \mathrm{~S}_{2} \mathrm{O}_{3}-1$ per cent available chlorine.

B. Arsenious Acid Method.

Solutions required:

1. N/10 arsenious acid. Transfer 4.95 gms. of pure arsenious acid to a beaker, add water and 107 gms. of $\mathrm{Na}_{2} \mathrm{HPO}_{4}-12 \mathrm{H}_{2} \mathrm{O}$. Stir 
and beat until all of the arsenious acid is in solution. Cool and make up to 1 liter with distilled water.

2. Iodized starch paper.

Dip pieces of filter or other bibulous paper into a starch solution containing a very small amount of potassium iodide. Moisten when using.

Procedure. Transfer 50 c.c. of the bleach solution (either that prepared from the powder or that taken from the orifice box) to an Erlenmeyer flask. Add very slowly the $\mathrm{N} / 10$ arsenious acid solution until a drop of the solution causes no dark coloration on the moist iodized starch paper. The number of cubic centimeters of arsenious acid solution used is equivalent to the percentage of available chlorine in the bleach.

1 c.c. $\mathrm{N} / 10$ arsenious acid $=.003545$ gram $\mathrm{cl}$.

The computation to determine how much available chlorine may be made as follows: Take 50 c.c. of bleach solution from the orifice box and titrate with $\mathrm{N} / 10 \mathrm{Na}_{2} \mathrm{~S}_{2} \mathrm{O}_{3}$. One c.c. of this is equivalent to $0.003545 \mathrm{gm}$. chlorine. If the 50 c.c. of bleach solution requires 30 c.c. of $\mathrm{N} / 10 \mathrm{Na}_{2} \mathrm{~S}_{2} \mathrm{O}_{3}$ then $30 \times 0.003545 \times 20=2.127$ gms. chlorine per liter.

One liter $=1000$ gms., then the solution contains .2127 per cent available chlorine. A cubic foot of the solution weighs about 28,320 gms. In this case then $1 \mathrm{cu}$. ft. of the solution will contain 59.094 gms. of available chlorine.

Examination of Water for Available Chlorine. The Committee on the Analysis of Water and Sewage of the American Public Health Association recommended the following procedure. This has not been accepted by the Canadian Public Health Association. They have recommended that it be eliminated from standard methods. (Amer. Jour. Pub. Health 8. (1918) 320.)

In waters that have been treated with calcium hypochlorite or liquid chlorine it is frequently advisable to ascertain the presence or absence of avallable chlorine. As the reagents which have been proposed for its detection are not specific for chlorine but give similar or identical reactions with oxidizing agents or reducible substances, care must be exercised in interpreting the results of such tests; nitrites and ferric salts are of common occurrence, and chlorates also may lead to misinterpretation in waters treated with calcium hypochlorite.

Reagents.-1. Tolidin solution. One gram of o-tolidm, purified by being recrystallized from aclohol, is dissolved in 1 liter of 10 per cent hydrochloric acid.

2. Copper sulphate solution. Dissolve 1.5 gms. of copper sulphate and 1 c.c. of concentrated sulphuric acid in distilled water and dilute the solution to 200 c.c. 
3. Potassium bichromate solution. Dissolve $0.025 \mathrm{gm}$. of potassium bichromate and 0.1 c.c. of concentrated sulphuric acid in distilled water and dilute the solution to 200 c.c.

Procedure--Mix 1 c.c. of the tolidin reagent with 50 c.c. of the sample in a Nessler tube and allow the solution to stand at least five minutes. Small amounts of free chlorine give a yellow and larger amounts a yellow-green color.

For quantitative determination, comparison of the color is made with that of standards in similar tubes prepared from the solutions of copper sulphate and potassium bichromate. The amounts of solution for various standards are indicated in Table XXVIII.

Concentrations greater than 0.10 part per million of chlorine require use of a stronger bichromate solution, containing $0.25 \mathrm{gm}$. of potassium bichromate and 1 c.c. of concentrated sulphuric acid dissolved in distilled water and diluted to 200 c.c. The proper amounts of solution for stronger permanent standards are given in Table XXVIII.

TABLE XXVIII

PREPARATION OF PERMANENT CHLORINE STANDARDS FOR LOW CHLORINE CONTENT

\begin{tabular}{|c|c|c|}
\hline Value in Chlorine & $\begin{array}{c}\text { Copper Sulphate } \\
\text { Solution. }\end{array}$ & $\begin{array}{c}\text { Potassium Bichrom- } \\
\text { ate Solution }\end{array}$ \\
\hline Parts per million & c.c. & c.c. \\
001 & 00 & 0.8 \\
.02 & .0 & 2.1 \\
.03 & .0 & 3.2 \\
.04 & .0 & 4.3 \\
.05 & .4 & 5.5 \\
.06 & .8 & 6.6 \\
.07 & 1.2 & 7.5 \\
.08 & 15 & 8.7 \\
.09 & 1.7 & 9.0 \\
.1 & 1.8 & 10.0 \\
\hline
\end{tabular}

\section{Dissolved Oxygen}

Reagents.-1. Sulphuric acid. Concentrated (sp. gr., 1.83-1.84).

2. Potassium permanganate. Dissolve $6.32 \mathrm{gms}$. of the salt in water and Cilute the solution to 1 liter.

3. Potassium oxalate. A 2 per cent solution.

4. Manganous sulphate. Dissolve $480 \mathrm{gms}$. of the salt in water and dilute the solution to 1 liter.

5. Alkaline potassium iodide. Dissolve 700 gms. of potassium hydroxide and $150 \mathrm{gms}$. of potassium iodide in water and dilute the solution to 1 liter.

6. Hydrochloric acid. Concentrated (sp. gr. 1.18-1.19). 
7. Sodium thiosulphate. A N/40 solution. Dissolve $6.2 \mathrm{gms}$. of chemically pure recrystallized sodium thiosulphate in water and dilute the solution to 1 liter with distilled water. Each cubic centimeter is equivalent to $0.2 \mathrm{mg}$. of oxygen or to 0.1395 c.c. of oxygen at $0^{\circ} \mathrm{C}$. and $760 \mathrm{~mm}$. pressure. Inasmuch as this solution is not permanent it should be standardized occasionally against $\mathrm{N} / 40$ solution of potassium bichromate. The keeping qualities of the thiosulphate solution are improved by adding to each liter 5 c.c. of chloroform and 1.5 gms. of ammonium.

TABLE XXIX

PREPARATION OF PERMANENT STANDARDS FOR HIGH CHLORINE CONTENT

\begin{tabular}{|c|c|c|}
\hline Value in Chlorine. & $\begin{array}{c}\text { Copper Sulphate } \\
\text { Solution. }\end{array}$ & $\begin{array}{c}\text { Stronger Potassium } \\
\text { Bichromate Solution. }\end{array}$ \\
\hline Parts per million & c.c. & c.c. \\
0.10 & 1.8 & 10 \\
.20 & 19 & 20 \\
.30 & 1.9 & 30 \\
40 & 20 & 38 \\
.50 & 20 & 45 \\
.60 & 2.0 & 51 \\
.70 & 20 & 58 \\
.80 & 2.0 & 63 \\
.90 & 20 & 67 \\
1.0 & 20 & 72 \\
2.0 & 2.0 & 120 \\
3.0 & 20 & 21.0 \\
4.0 & 2.0 & 30.0 \\
5.0 & 20 & 39.0 \\
6.0 & 2.0 & 46.0 \\
7.0 & 2.0 & 56.0 \\
8.0 & 2.0 & 63.0 \\
9.0 & 2.0 & 70.0 \\
10.0 & 20 & 75.0 \\
& & \\
\hline
\end{tabular}

Liquid Chlorine. To some extent, this chemical has replaced calcium hypochlorite in water treatment. It has certain distinct advantages over "bleach" as has been outlined by Dutton (1917) and Jennings (1915) as follows:

1. A more accurate means of control.

2. An elimination of dirt and the odor of chlorine.

3. An appreciable decrease in labor.

4. A means of storing the chemical so as to eliminate any decrease in strength. 
5. An elimination of chlorine taste in the treated water which is so often found where hypochlorite is used.

6. A decrease in the cost of the chemical required.

7. The change in the rate of application is quickly and more easily accomplished.

8. Absorption of the gas is more rapid.

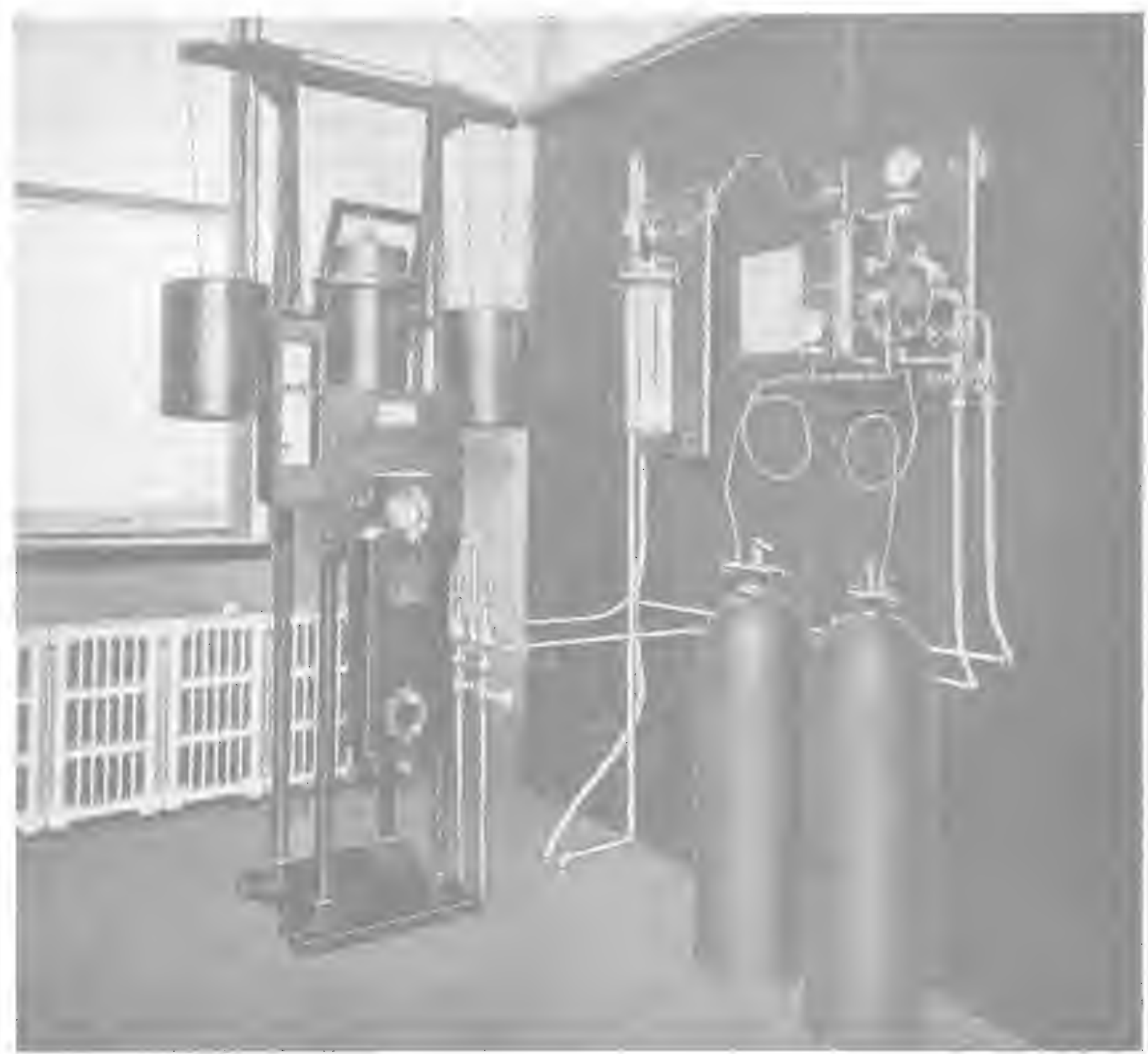

FIG. 61.-Automatic Control Chlorinator. (Wallace-Tiernan Co.)

Dutton (1917) reported that with hypochlorite at 7 cents per pound and liquid chlorine at 20 cents, it would cost $\$ 1.07$ to treat a million gallons of water with bleach and 28 cents to use liquid chlorine. With such data at hand, it is evident that the liquid chlorine is the more efficient chemical to use.

The advantages of softening a water supply with lime are becoming more and more apparent. Houston has shown that lime is a bac-

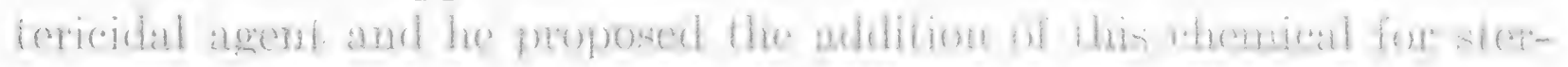


ilization. Experinents on tho ruw Thames river water khowed that B. coli were killed in from 5 to 24 hours in a concentration of 1 to 5000 .

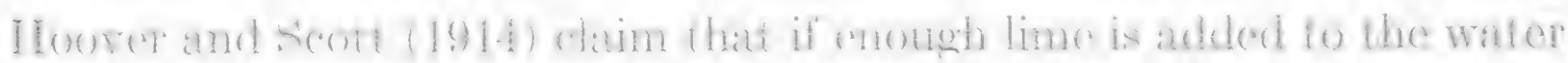

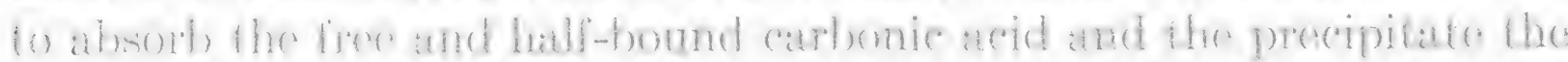
magnesimn. B. colon and B. (yphi are killed in ts hours affer being so

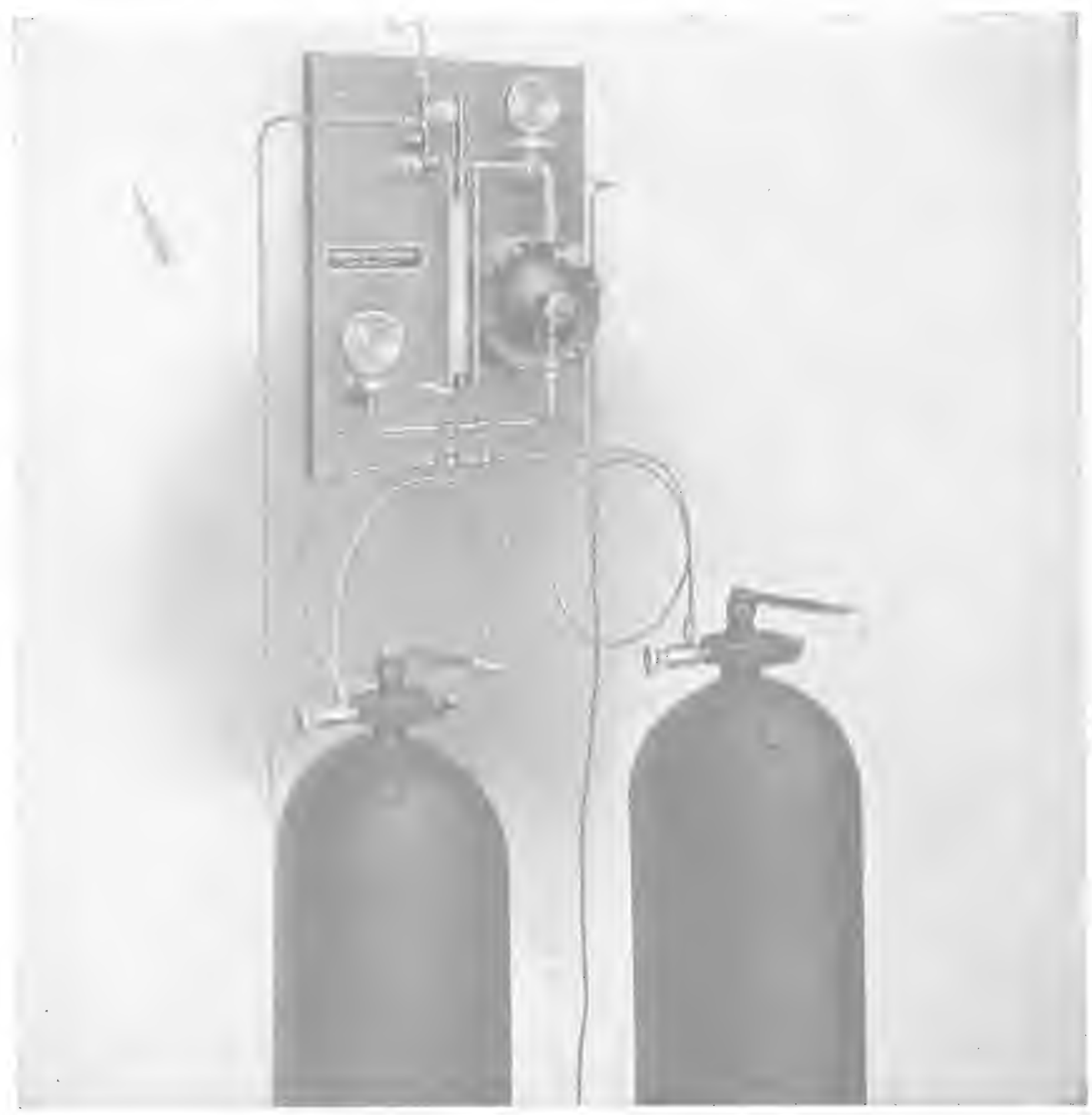

FIG. 62.-Automatic Control Chlorinator Direct Feed. (Wallace-Tiernan Co.)

treated, if the water does not contain large quantities of organic matter. An effective bactericidal action when the lime was added in from $\frac{1}{2}$ to 1 part per million in excess of that which is needed to reduce the temporary hardness. Intestinal bacteria will not live in a water containing no free or half-bound carbonic acid. When a lime-softened water is

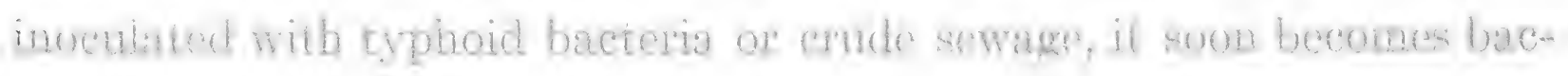


teria free. These authors further state that the action is selective in that certain harmless bacterla grow in this water. Table XXX will show some of the results secured by Hoover and Scott. Haller (1914) con-

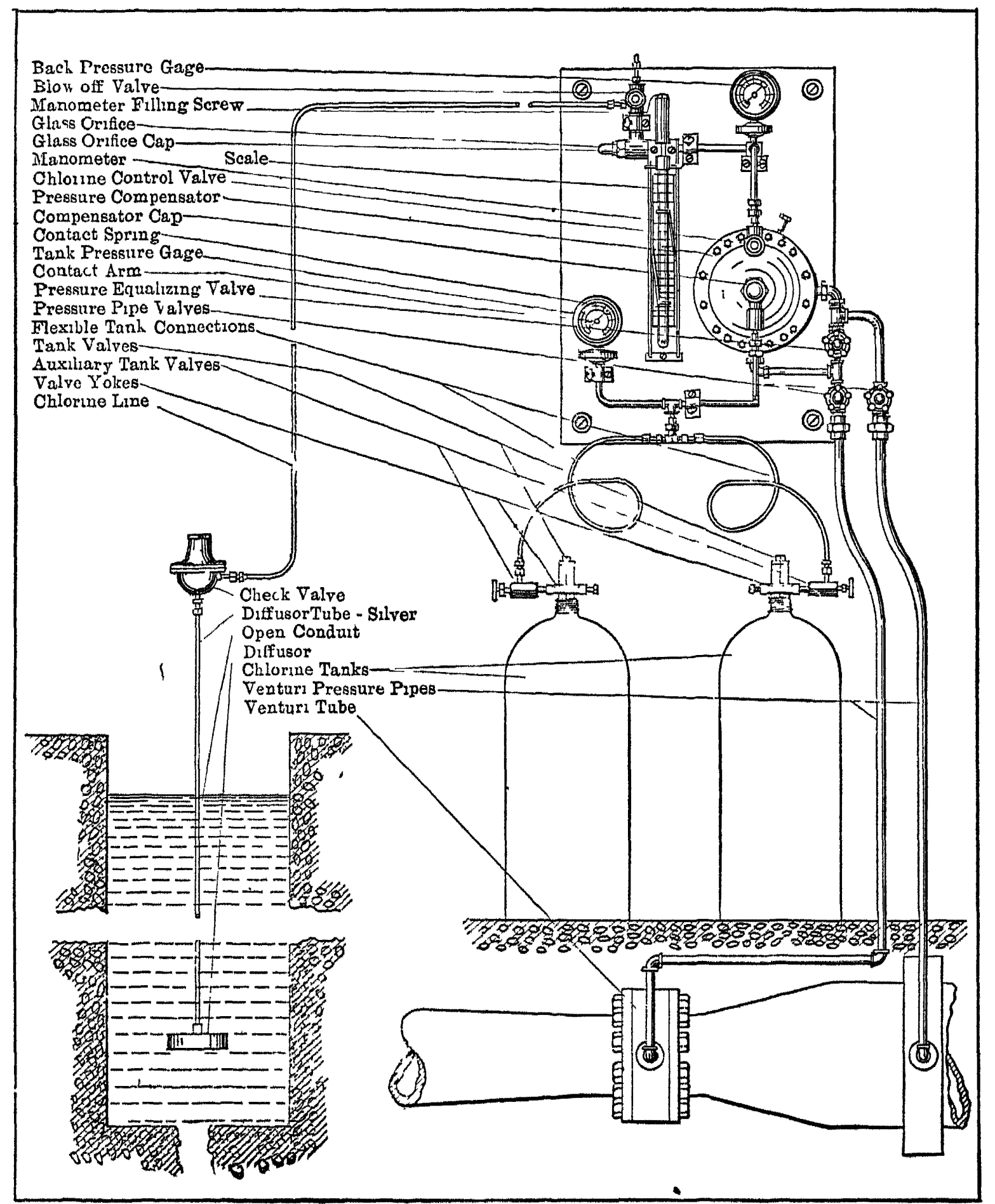

Frg. 63.-Automatic Control Chlorinator, Direct Feed, Type B, Venturi Operated. (Wallace-Tiernan Co.)

firms the statements of Hoover and Scott and also maintains that intestinal bacteria cannot live in a water from which the $\mathrm{CO}_{2}$ has been removed. He regards water which has been treated with calcium oxide safe for consumption very soon even though it was previously contam- 
Inated with typhoid bacill. Watt (1913) reported that three weeks' treatment of river water with three parts of lime per 100,000 reduced the number of $B$. coll and gave generally satisfactory results with the exception of a slight coloring and increased alkalinity. This investigator recommends the "excess lime method" as satisfactory for epidemics of typhoid fever where it is necessary to quickly improve the water supply.

\section{TABLE XXX}

CHEMICAL ANALYSIS. IN PARTS PER MILLION WITH THE ACTION OF LIME-TREATED WATER ON TYPHOID BACTERIA

(Hoover and Scott, 1914)

\begin{tabular}{|c|c|c|c|c|c|c|c|}
\hline \multirow{3}{*}{ Oct, 1912} & \multirow{3}{*}{$\begin{array}{c}\text { Total } \\
\text { Alkalınity }\end{array}$} & \multirow{3}{*}{$\begin{array}{l}\text { Bicarbonate } \\
\text { Alkalinity }\end{array}$} & \multirow{3}{*}{$\begin{array}{l}\text { Normal } \\
\text { Carbonate } \\
\text { Alhalinity }\end{array}$} & \multirow{3}{*}{$\begin{array}{c}\text { Excess } \\
\text { Lime } \\
\text { Caustic } \\
\text { Alkalnity }\end{array}$} & \multicolumn{3}{|c|}{ B LCTERIAL ANALYSIS OF } \\
\hline & & & & & \multirow{2}{*}{$\begin{array}{l}\text { Softened } \\
\text { Water } \\
\text { Control }\end{array}$} & \multicolumn{2}{|c|}{$\begin{array}{c}\text { Softened Water Inocu- } \\
\text { lated With Ty phold } \\
\text { Bacilli }\end{array}$} \\
\hline & & & & & & Initially & $\begin{array}{l}\text { After } \\
24 \text { Hours. }\end{array}$ \\
\hline 20 & 47 & 5 & 42 & 0 & 0 & 180 & 0 \\
\hline 21 & 52 & 8 & 44 & 0 & 0 & 20 & 0 \\
\hline 23 & 59 & 7 & 52 & 0 & 0 & 50 & 6 \\
\hline 24 & 58 & 12 & 46 & 0 & 0 & 165 & 2 \\
\hline 25 & 64 & 8 & 56 & 0 & 0 & 390 & 0 \\
\hline 25 & 58 & 2 & 56 & 0 & 0 & 360 & 1 \\
\hline 26 & 50 & 2 & 48 & 0 & 14 & 3600 & 1 \\
\hline 28 & 46 & 0 & 42 & 4 & 0 & 300 & 0 \\
\hline 29 & 48 & 0 & 48 & 0 & 1 & 3600 & 0 \\
\hline
\end{tabular}

\section{BACTERIOLOGY OF ICE}

The harvesting of natural ice and the manufacture of artificial ice have become well-established industries. The growth of large metropolitan centers which are well removed from the source of food supplies and rural districts, have made these industries important ones.

Kinds of Ice. There are two kinds of ice, artificial and natural. Natural ice is secured, in the northern climates, from natural bodies of water. It is cut into blocks which are convenient for handling and delivered to the consumer after storage. Such ice may be contaminated either from being taken from too badly polluted bodies of water or by being handled in an unsanitary manner.

Artificial ice is produced mostly by the can method. These are usually about the size of an ordinary cake and, before freezing are 
filled with distilled water; if ordinary water with a high mineral content is used, the cake of ice, after it has been frozen, will have a white core through the center. This is formed from the dissolved and suspended matter and will also contain the bacteria which were present in the original water.

Properties of Ice. Ice is crystallized water which is made to form by the removal of heat. The temperature at which this change from the liquid to the solid phase takes place is dependent upon a number of different factors. The presence of dissolved air, inorganic salts pressure, etc., are all concerned. It is well known that salt water freezes at a lower temperature than pure water. During the freezing process, the water contracts until it reaches $4^{\circ} \mathrm{C}$. when it begins to expand and becomes lighter than water. Since the formation of ice is a process of crystallization, it naturally follows the laws of crystallization. Substances which crystallize from solution tend to come out in the pure state. The chemist uses this method for purifying his reagents. The foreign matter is excluded unless a small amount may be occluded during the process between the crystals. The same thing takes place when. water crystallizes. The bacteria and suspended matter are forced out and do not appear in the ice. Ice made from dirty water by the can method usually shows the dirt in a core. This will be found to contain many bacteria; more are found in this core than in the ice at the surfaces of the can.

Bacteriology of Ice. Ice does not furnish the essentials of a good medium and consequently there is no multiplication of bacteria. The question resolves itself into determining how many bacteria may survive in such an environment. The following causes contribute to the inhibitory action on bacteria:

1. Temperature.

2. Lack of oxygen.

3. Absence of moisture.

The longevity of bacteria in ice has received some attention from bacteriologists and sanitarians. Frankland (1894) reports some data which were secured by Prudden. Culture of Bacillus prodigiosus, Bacillus proteus vulgaris, Staphylococcus pyogenes aureus a fluorescent bacillus and Bacillus typhosus were put into samples of water and exposed to temperatures of from $14^{\circ}$ to $30^{\circ} \mathrm{F}$. for 103 days. Bacillus prodigiosus decreased from 6300 in 4 days to 3000 ; in 37 days the number went to 22 and after 51 days all of the cells had died. Bacillus proteus vulgaris disappeared in 51 days. At the end of 11 days there were 


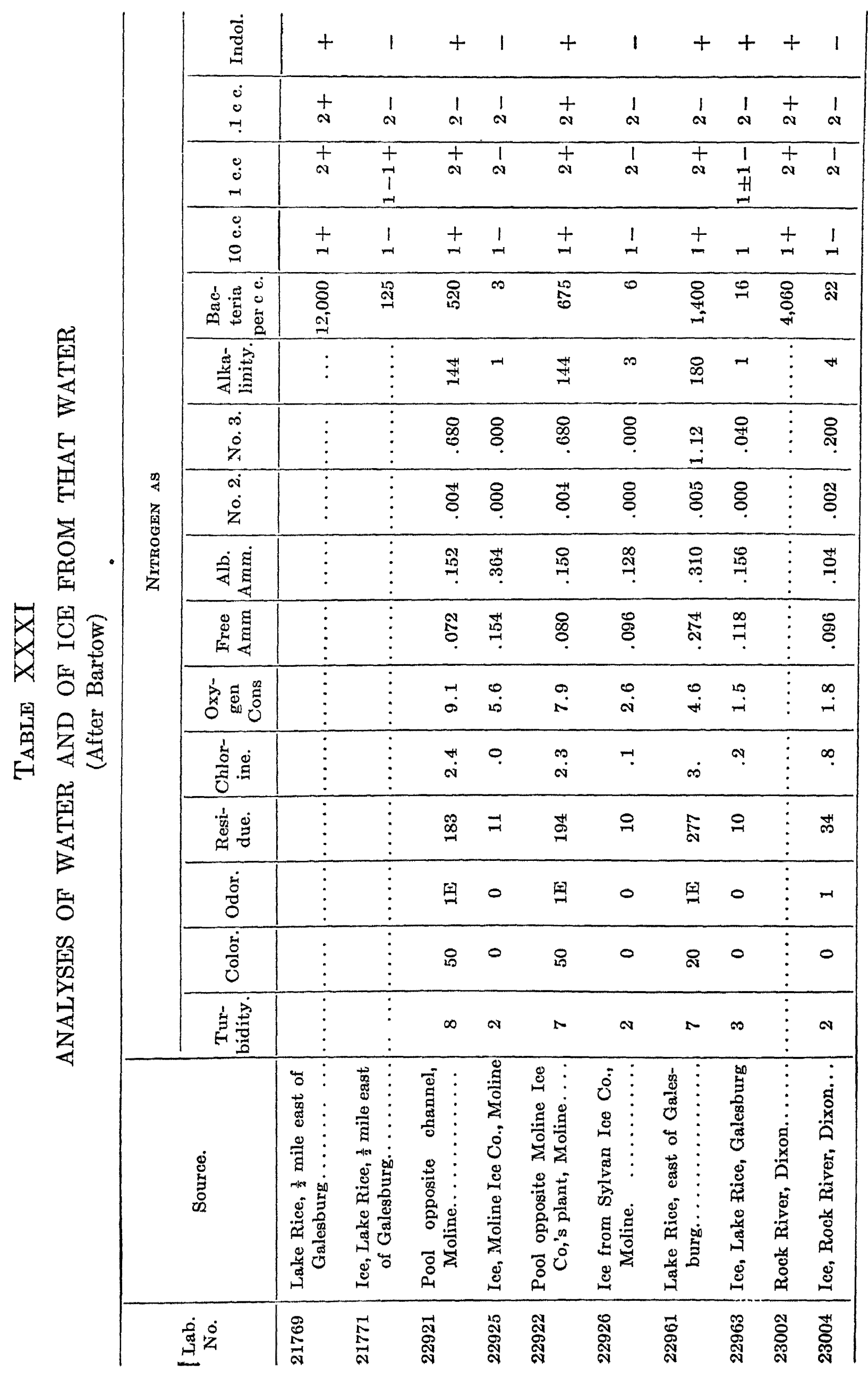


1,000,000 cells of Bacillus typhosus and 7000 after 103 days. Alternate freezing and thawing were very detrimental to bacteria. Experiments with anthrax vegetative and spore cells indicated that the spores resisted for some time while the vegetative cells were rather quickly destroyed. The cholera vibrio is reported to have been destroyed in from 6 to 10 days.

\section{TABLE XXXII}

SHOWING DURATION OF LIFE OF TYPHOID BACILLI AND COLON BACILLI IN ICE KEPT IN A SHED IN WINTER WEATHER

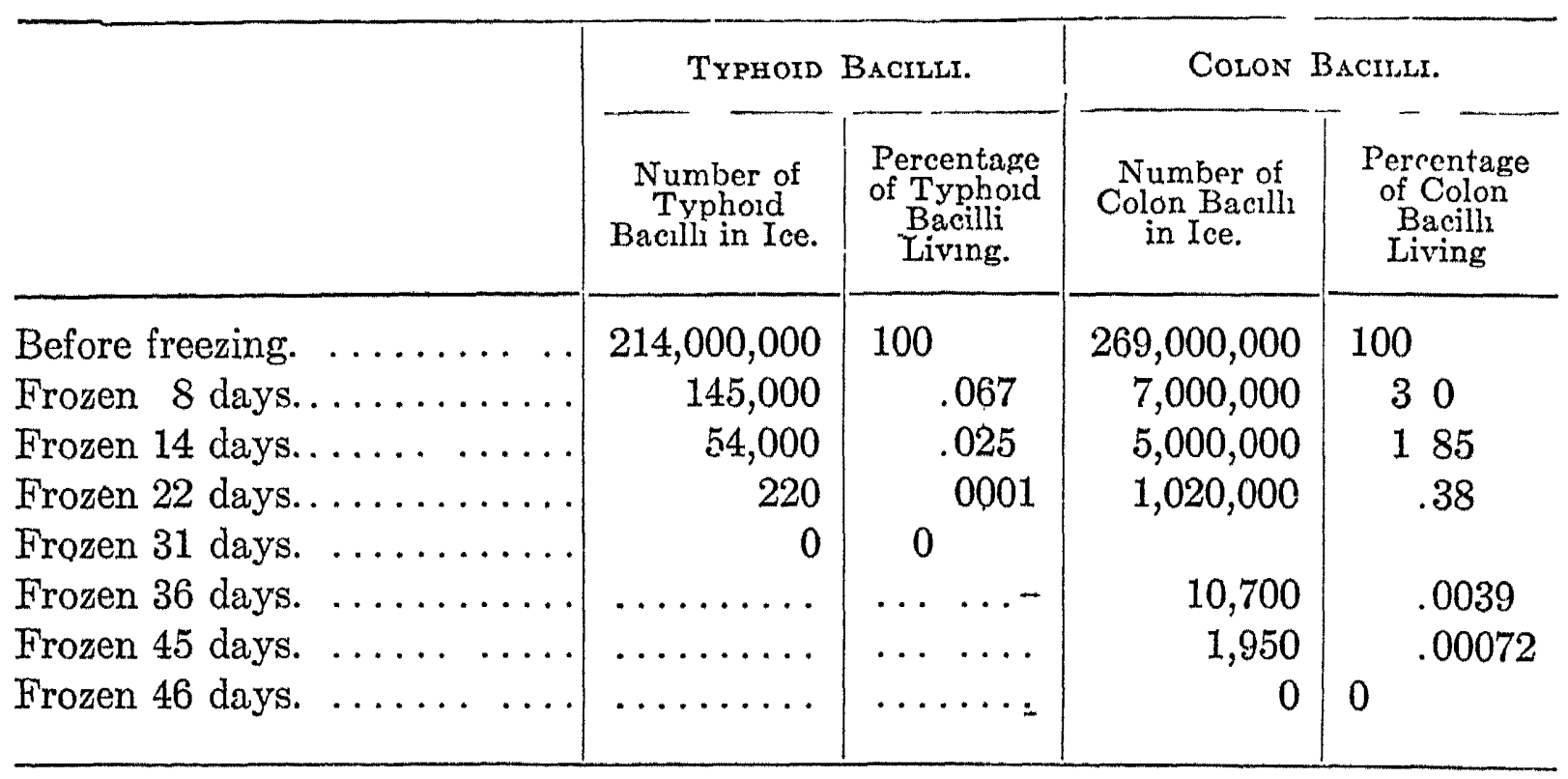

Reudiger (1911) gives in Table XXXII data secured by him on $B$. coli and B. typhosus. From this it will be seen that 99.9 per cent of the $B$. typhi die during the first eight days. Park also found similar data. Jordan, Russell, and Zeit (1904) found that in filtered water B. typhi died rapidly. Two organisms were found after the first day and none later. Hilliard et al. (1915) reported that 99 per cent of $B$. coli were destroyed by freezing in tap water for three hours. B. subtilis showed a less uniform reaction. No apparent difference was noticed by these investigators between alternate freezing and continued freezing. Cream with 30 per cent of milk fat afforded a decided protection to the bacteria.

Ice and Typhoid Fever. The general consensus of opinion among sanitarians is that ice is not an important epidemiological factor in the spread of typhoịd fevęr. , Hutchins and Wheeler (1903) reported an iceborne epidemic of typhoid fever but Hill (1910) believes their evidence to be inconclusive. He mentions the following characteristios of iceborne epidemics' of typhoid fever:

1. They should occur before June.

2. Bulk of the cases should be on one iceman's route, 
3. Ice should have come from grossly polluted sources.

4. It should be young ice-recently frozen.

5. Cases should develop among ice-water drinkers chiefly.

6 . Ice should have been placed in and not around the water. Hill further states: "Ice, were it equally subject to contamination as water and without natural purification processes, could not, on account of its relative small use, constitute a factor accounting for over $\frac{2}{3}$ of 1 per cent of water-borne typhoid (which, of course, would be a much smaller proportion of the total typhoid of the country) even if its use were as general as the use of water.".

Jordan (1911) states, "There has never been much danger from the use of impure ice; there need be none. Certified ice of a purity beyond reproach can be much more readily attained under existing conditions than pure water, and very much more readily than pure milk." About the same opinion has been expressed by other sanitarians. Bartow (1916) has stated, "Nature certainly does her share toward a pure natural ice. If reasonable precautions are taken so that no ice is taken from grossly polluted ponds or rivers and the surface of the ice is protected, there need be no difficulty in placing a pure ice on the market."

Methods for the Bacterial Examination of Ice. After the ice has been sampled and the sample is melted, the methods of analysis, chemical and bacterial are no different than those used in water analysis. The sampling of the ice for the bacteriological analysis does, however, require some special attention. Care must be exercised to secure a sample which is not contaminated. This may be accomplished in different ways. The block of ice should be carefully cleaned and washed with distilled water. Then, it should be copiously rinsed with sterile distilled water. After this it should be split if it is a large block and small pieces chipped off and transferred to sterile containers by means of sterile instruments. If this is carefully carried out no contamination will result. The block may also be bored out with a sterile auger and the shavings collected in a sterile bottle. Greenfield (1916) has described an instrument for sampling ice. It is constructed after the cheese sampler.

\section{Self Purification of Streams}

This is a subject which is becoming more and more important as population increases. It is very closely related to the disposal of sewage by dilution. Directly it may concern the viability of patho- 
genic bacteria in water but indirectly it is concerned with nuisances which almost certainly result when a stream is made to carry too much waste matter. In America the question is being given some official attention by the Federal government and most States have statutes governing the pollution of streams. These have been reviewed by Montgomery and Phelps (1917). The United States Public Health Service has investigated the pollution and sanitary conditions of the Potomac watershed and a very complete report has been prepared by Cumming (1916). In this interesting sanitary survey it was found that the Potomac River " even during the period of lowest stream flow the river in the area of heaviest pollution . . . has, at all times, suffcient oxygen available for the sewage now discharged into the river and enough to take care of that which will probably be added for several years to come." Investigations are also being conducted on the Ohio river but the results are not available.

TABLE XXXIII

SHOWING THE EXTENT OF SELF PURIFICATION IN THE ILLINOIS RIVER

(After Jordan, 1900)

\begin{tabular}{|c|c|c|c|c|}
\hline Collecting Stations. & $\begin{array}{c}\text { Distance from } \\
\text { Bridgeport. }\end{array}$ & $\begin{array}{l}\text { Chlorine, } \\
\text { P.p.m. }\end{array}$ & Bacteria. & $\begin{array}{l}\text { Number of } \\
\text { Analyses. }\end{array}$ \\
\hline Bridgeport, Illinois. . . . . . . . & 0 & 119.2 & $1,245,000$ & 19 \\
\hline Lockport........... & 29 & 117.4 & 650,000 & 30 \\
\hline Desplaines R. at Lockport....... & $\ldots \ldots$ & 7.9 & 9,180 & 28 \\
\hline Joliet. . . . . . . . . . . & 33 & 104.8 & 486,000 & 28 \\
\hline Kankakee River... & $\ldots \ldots \ldots$ & 3.4 & 5,000 & 28 \\
\hline 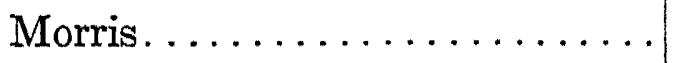 & 57 & 68.1 & 439,000 & 26 \\
\hline Ottawa.............. & 81 & 58.5 & 27,400 & 26 \\
\hline Fox River at Ottawa....... & $\ldots \ldots$ & 5.0 & 6,510 & 29 \\
\hline Big Vermillion at LaSalle... . & $\ldots \ldots \ldots$ & 61.2 & 7,970 & 30 \\
\hline LaSalle............. & 95 & 46.1 & 16,300 & 31 \\
\hline Henry . . . . . . . . . & 123 & 44.2 & 11,200 & 29 \\
\hline Averyville........... & 159 & 40.9 & 3,660 & 30 \\
\hline Wesley City........ & 165 & 40.1 & 758,000 & 22 \\
\hline Pekin .............. & 175 & 38.4 & 492,000 & 29 \\
\hline Havana............ & 199 & 36.2 & 16,800 & 26 \\
\hline Beardstown......... & 231 & 29.3 & 14,000 & 26 \\
\hline Kempsville.......... & 288 & 22.9 & 4,800 & 19 \\
\hline Grafton...$\ldots \ldots \ldots$ & 318 & 18.3 & 10,200 & 28 \\
\hline Mississippi River at Grafton..... & $\ldots \ldots$ & 2.8 & 7,600 & 29 \\
\hline
\end{tabular}

One of the most instructive cases of stream pollution is that of the Illinois River, which is made to carry the sewage of Chicago. The facts 
of the case were brought out when the State of Missouri and the city of St. Louis tried to secure an injunction against the Sanitary District of

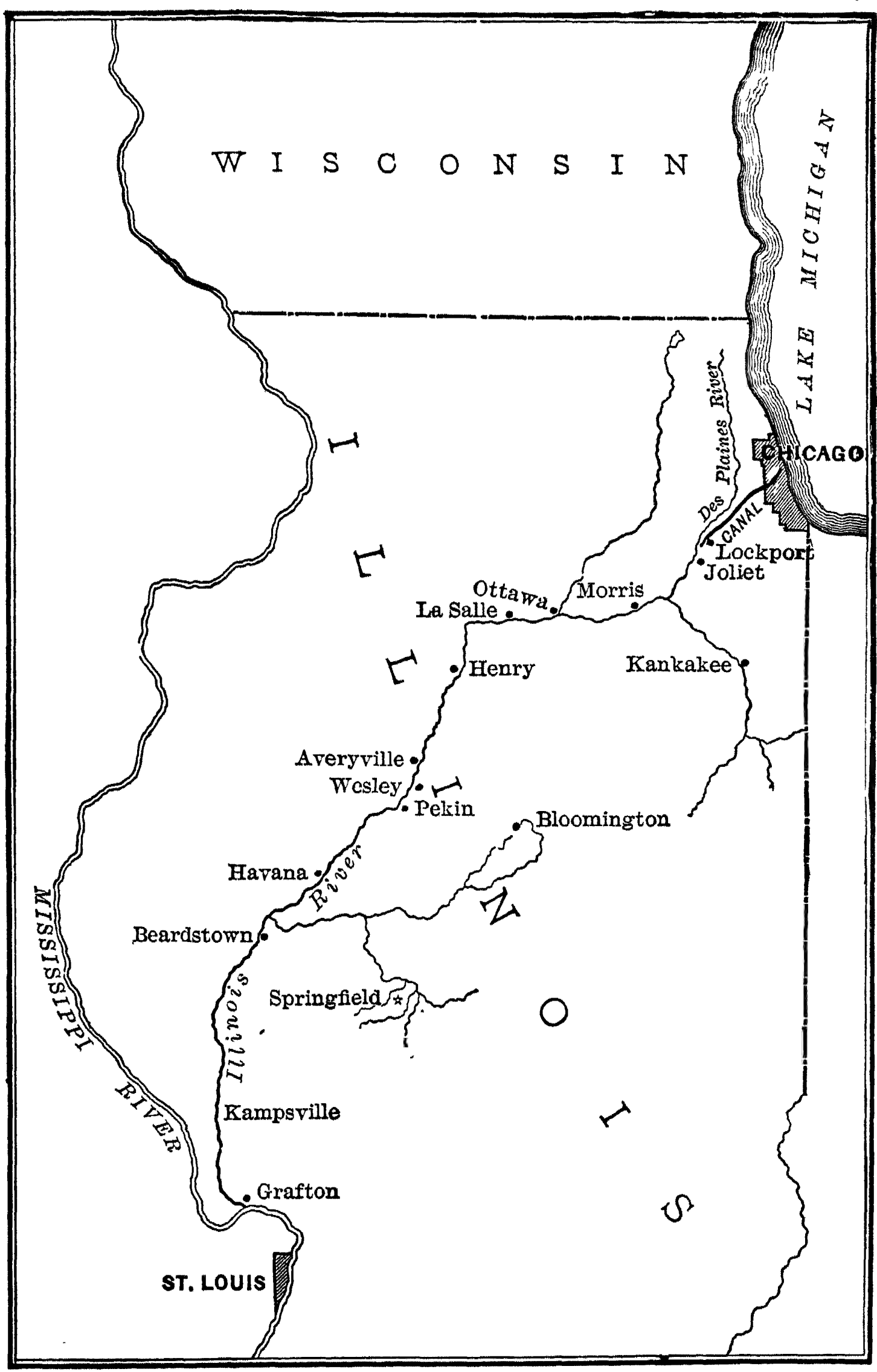

Frg. 64.-Chicago Drainage Canal and the Mlinois River Basin. (From Kinnicut, Winslow and Pratt's Sewage Disposal, John Wiley and Sons, Inc.) 
Chicago to prevent the pollution of this river. It was argued that the water supply of St. Louis was endangered. The testimony heard in
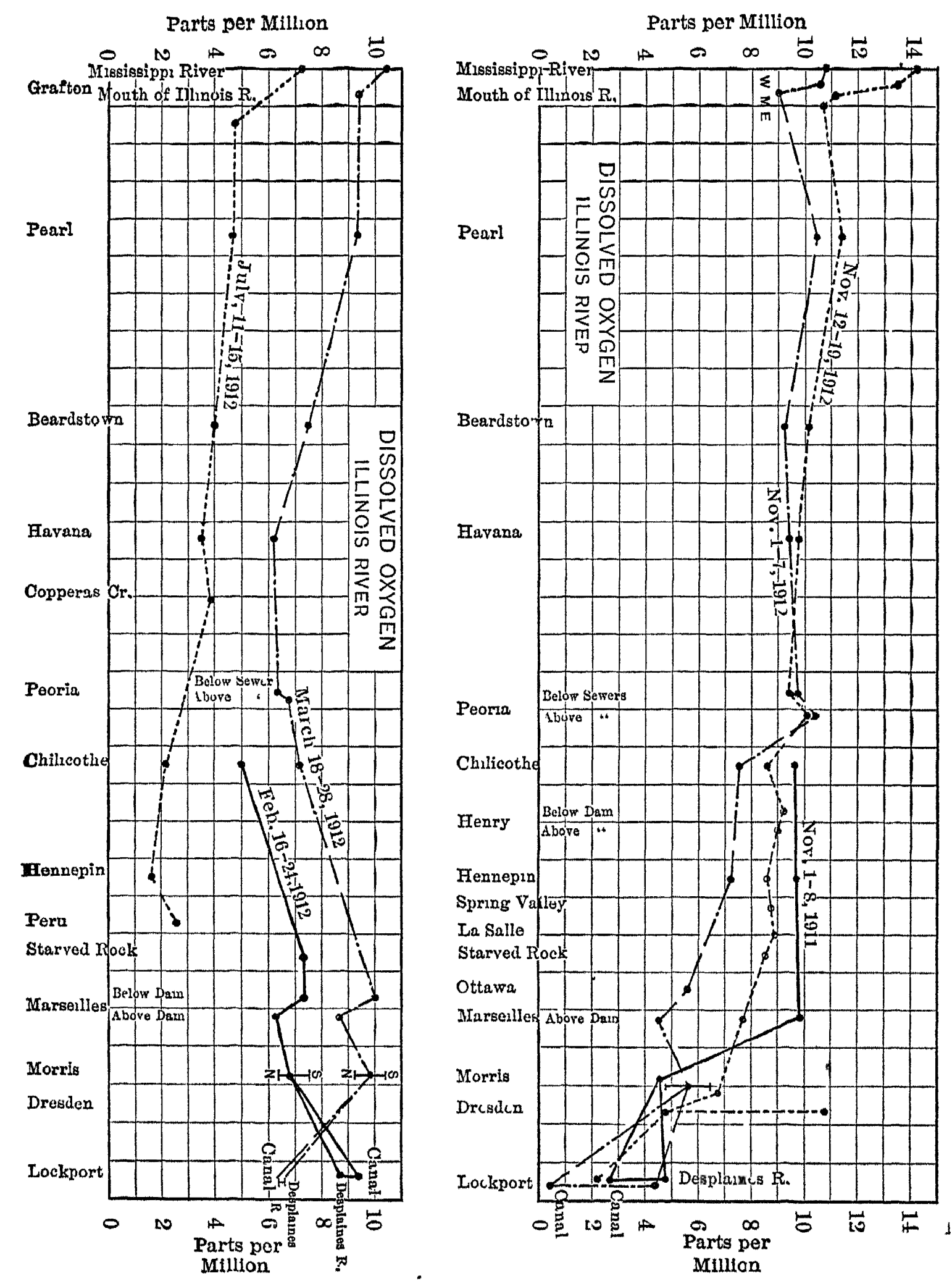

FIc. 65.-Showing Dissolved Oxygen Concentration in Different Parts of the Illinois River. (From Illinois state Water Survey Bulletin.)

this case has been summarized by Leighton (1907). Both sides of the case presented some very interesting opinions and data. Jordan (1900) who did valuable work for the defendants reported some of his data in 
a separate publication. Table XXXIII is taken from that paper. It will be seen from this table that there is a steady decrease in the chlorine content of the water and also in the bacteria content. The rise in the count at Wesley City may be explained by the fact that at Pcoria large amounts of distillery slops and animal refuse were dumped into the river. These would have a greater effect on the bacteria count than on the chlorine. Dissolved oxygen studies have been carried out by the Illinois State Water Survey, some of the data of which are presented. in Fig. 65. From these data, it will be seen that there is evident purification in the Illinois River.

Pathogenic Bacteria in River Water. There are many data available in the literature but in looking it over one is impressed with the diversity of opinion. Much of this disagreement may probably be explained by the different conditions under which each of the investigators worked. It is evident that the factors in the environment influence, to a certain extent, the longevity of bacteria in nature. These factors will not be taken up here, but those who are interested will find discussions in texts on water bacteriology. From the evidence which was presented in the Illinois River case, we know that the typhoid bacilli die rather rapidly in nature. Probably after three or four weeks the water which contained them would be safe. Jordan, Russell and Zeit (1904) studied this question in both pure and polluted waters and their data are taken as the bases upon which our present knowledge rests. They suspended the bacteria in collodion sacs in both pure and polluted waters. In pure Lake Michigan water, the bacilli did not die out until after a week, but three days seemed. to be the limit for the polluted Chicago Drainage Canal water. In 1906 Russell and Fuller confirmed these results using pure Lake Mendota water and sewage as the substrates. In this experiment the bacilli lived for about ten days in Lake Mendota water and three days in sewage. Houston has studied this question in England. He points out that 1 c.c. samples were used by the American workers in most of the cases, and from these small samples conclusions were drawn with regard to the longevity of $B$. typhi. When no organisms were found in 1 c.c. samples, it was stated that the organism had died out. Probably Amcrican bacteriologists have been too much accustomed to use small amounts of water in analytical work. Houston carried on experiments using Thames, Lee and New River waters, using 100 c.c. quantities of the water as the maximum amounts examined. For 100 c.c. quantities in eighteen experiments, it required nine weeks for the total disappearance of $B$. typhi. Ninety-nine per cent of them disappeared in one week under laboratory conditions of the experiment. The above data of 
both American and English bacteriologists point out the value of storage. The rate of death of $B$. typhi and $B$. coli in pure natural water has been reported by Rahn and Hinds (1914) to follow the monomolecular law. Under these conditions the rate increased with the temperature. Oxygen was found to be harmful to $B$. coli but beneficial to B. typhi.

The viability of Microspira cholerce has also been investigated by Houston using the same technique as for B. typhi. Ninety-nine per cent of the cholera organisms perished in three days under the laboratory conditions of the experiment. This is a much shorter period than required for B. typhi. Gelarie (1916) studied the longevity of Microspira cholerce in water of New York Bay. He stated that the survival of these organisms in water depends upon the strain which is used, the number of bacteria seeded and other factors. The organisms are said to live in native bay water from 7 to 45 days and in sterilized bay water up to 285 days. In sterile tap water they lived for from 1 to 18 days and in native tap water from 1 to 3 days. In competition with the other water organisms the life of the cholera organisms was found to be short.

The other phase of the stream pollution question has been concerned with nuisances resulting from overloading the stream with sewage or organic industrial wastes. It is now fairly well established that rivers do purify themselves but it is more often a difficult question to determine the length of time required. Practically every river is able to handle a certain amount of organic matter. Phelps (1914) has stated that three factors are involved-the oxidizing bacteria, organic matter and oxygen. Since the organic matter and bacteria are always present, "the questions of stream purification and prevention of nuisance reduce to one of oxygen supply."

Solution of Atmospheric Oxygen in Water. The amount of oxygen in the water which is available for the oxidizing bacteria is the important question in stream purification. Especially so is the extent to which the oxygen content may be reduced before putrefaction sets in. As long as there is sufficient oxygen, the bacteria will carry to completion the oxidation of organic matter but when this oxygen is reduced to a certain point, putrefaction sets in and this is always accompanied by foul odors. As long as the available oxygen is sufficient, the bacteria will carry it over to the organic matter always driving the reaction in that direction and not allowing an equilibrium to be established. The greater the speed of this reaction, the greater will be the amount of reaeration which will take place and Phelps (1916) has stated that this is the important factor in stream purification and that dams and rapids 
increase this by a mixing action. The balance between the available oxygen and the required oxygen must probably be established for each stream.

Determination of Dissolved Oxygen. Different methods have been in use for determining the oxygen content of a water. The Winkler method has been included in standard methods and has certain distinct advantages over the Levy method which has been advocated by some. It is given in standard methods as follows:

Reagents. 1. Sulphuric acid, concentrated. (Sp. Gr. 1.83-1.84.)

2. Potassium permanganate. Dissolve $6.32 \mathrm{gms}$. of the salt in water and dilute the solution to a liter.

3. Potassium oxalate. A 2 per cent solution.

4. Manganous sulphate. Dissolve $480 \mathrm{gms}$. of the salt in water and dilute the solution to 1 liter.

5. Alkaline potassium iodide. Dissolve $700 \mathrm{gms}$. of potassium hydroxide and $150 \mathrm{gms}$. of potassium iodide in water and dilute to a liter.

6. Hydrochloric acid, concentrated. (Sp. Gr. 1.18-1.19.)

7. Sodium thiosulphate. A N/40 solution. Dissolve $6.2 \mathrm{gms}$. of chemically pure recrystallized sodium thiosulphate in water and dilute the solution to 1 liter with freshly boiled distilled water. Each cubic centimeter is equivalent to $0.2 \mathrm{mg}$. of oxygen or to 0.1395 c.c. of oxygen at $0^{\circ} \mathrm{C}$. and $760 \mathrm{~mm}$. pressure. Inasmuch as this solution is nat permanent it should be standardized occasionally against a $\mathrm{N} / 40$ solution of potassium bichromate. The keeping qualities of the thiosulphate solution are improved by adding to each liter 5 c.c. of chloroform and 1.5 gms. of ammonium carbonate before diluting to the prescribed volume.

8. Starch solution. Mix a small amount of clean starch with cold water until it becomes a thin paste and stir this mass into 150 to 200 times its weight of boiling water. Boil for a few minutes, then sterilize. It may be preserved by adding a few drops of chloroform.

Collection of the Sample. Collect the sample in a narrow-necked glass-stoppered bottle of 250 to 275 c.c. capacity. The following procedure should be followed in order to avoid entrainment or absorption of atmospheric oxygen. In collecting from a tap, fill the bottle through a glass or rubber tube extending well into the tap and to the bottom of the bottle. To avoid air bubbles allow the bottle to overflow for several minutes, and then carefully replace the glass stopper so that no air bubble is entrained. In collecting from the surface of a pond or tank connect the sample bottle to a bottle of 1 liter capacity. Provide each 
bottle with a two-hole stopper having one glass tube extending to the bottom and another glass tube entering, but not projecting, into the bottle. Connect the short tube of the sample bottle with the long tube of the liter bottle. Immerse the sample bottle in the water and apply suction to the outlet of the liter bottle. To collect a sample at any depth arrange the two bottles so that the outlet tube of the liter bottle is at a higher elevation than the outlet of the sample bottle. Lower the two bottles in any convenient form of cage properly weighted, to the desired depth. Water entering during the descent will be flushed through into the liter bottle with a glass stopper in such a manner as to avoid entraining bubbles of air.

Procedure. Remove the stopper from the bottle and add, first, 0.7 c.c. of the concentrated sulphuric acid, and then 1 c.c. of the potassium permanganate solution. These and all other reagents should be introduced by pipette under the surface of the liquid. Insert the stopper and mix by inverting the bottle several times. After twenty minutes have elapsed, destroy the excess of permanganate by adding 1 c.c. of the potassium oxalate solution, the bottle being at once restoppered and its contents mixed. If a noticeable excess of potassium permanganate is not present at the end of twenty minutes again add 1 c.c. of potassium permanganate solution. If this is still insufficient use a stronger potassium permanganate solution. After the liquid has been decolorized by the addition of potassium oxalate add 1 c.c. of manganous sulphate solution and 3 c.c. of the alkaline potassium iodide solution. Allow the precipitate to settle. Add 2 c.c. of the hydrochloric acid and mix by shaking.

The procedure to this point must be carried out in the field, but after the acid has been added and the stopper replaced there is no further change, and the rest of the test may be performed within a few hours as convenient. Transfer 200 c.c. of the contents of the bottle to a flask and titrate with $\mathrm{N} / 40$ sodium thiosulphate, using a few cubic centimeters of the starch solution as an indicator toward the end of the titration. Do not add the starch solution until the color has become a faint yellow and titrate until the blue color disappears.

The use of potassium permanganate is made necessary by high nitrite or organic matter. The procedure outlined must be followed on all work on sewage and partly purified effluents or seriously polluted streams or samples whose nitrite nitrogen exceeds 0.1 part per million. In testing other samples the procedure may be shortened by beginning with the addition of the manganous sulphate solution and proceeding 
from that point as outlined, except that only 1 c.c. of alkaline potassium iodide need be added.

Calculation of Results. Oxygen shall be reported in parts per million by weight. It is sometimes convenient to know the number of cubic centimeters per liter of the gas at $0^{\circ} \mathrm{C}$. temperature and $760 \mathrm{~mm}$. pressure and also to know the percentage which the amount of gas present is of the maximum amount capable of being dissolved by distilled water at the same temperature and pressure. If 200 c.c of the sample is taken the number of cubic centimeters of $N / 40$ thiosulphate used is equal to parts per million of oxygen. Corrections for volume of reagents added amount to less than 3 per cent and are not justified except in work of unusual precision. To obtain the result in cubic centimeters per liter, multiply the number of cubic centimeters of thiosulphate used by 0.698 . To obtain the result in percentage of saturation consult the table on page 68 of the 1917 edition of Standard Methods for the Examination of Water and Sewage, and divide the number of cubic centimeters of thiosulphate used by the figure in this table opposite the temperature of the water and under the proper chlorine figure. The last column of this table permits interpolation for intermediate chlorine values. At elevations differing considerably from mean sea level and for accurate work, attention must be given to barometric pressure, the normal pressure in the region being preferable to the specific pressure at the time of sampling. The term "saturation" refers to a condition of equilibrium between the solution and an oxygen pressure in the atmosphere corresponding to $158.8 \mathrm{~mm}$., or approximately one-fifth atmosphere. The true saturation or equilibrium between the solution and pure oxygen is nearly five times this value and consequently values in excess of 100 per cent saturation frequently occur in the presence of oxygen-forming plants.

When the oxygen concentration becomes too low, the stream becomes putrescible. This same condition may be duplicated in a bottle by allowing it to stand full of sewage tightly stoppered. The following things will be noticed: formation of evident amounts of hydrogen sulphid; heavy deposits of black sediment; disappearance of dissolved oxygen. These are conditions which are not desired in streams and they indicate that the available oxygen is insufficient to care for the organic matter.

Stability and Relative Stability. A stream is stable when its oxygen demand has been satisfied; the products resulting from the decomposition of the organic matter should be completely oxidized. Phelps has stated that this condition obtains when the available oxygen exceeds the 
required oxygen. He stated that available oxygen is used up according to the following order: Dissolved oxygen, nitrates, nitrites, sulphates, phosphates, etc. Since all streams are not stable it is necessary to use other terms to fit their condition. For such conditions the term relative stability is used. Stable streams have a relative stability of 100 since they have all of the oxygen that is demanded by the organic matter. A relative stability of 60 means that a stream has 60 per cent of the oxygen necessary to make it stable. This is determined in the following manner:

Reagent. Methylene blue solution. A 0.05 per cent aqueous solution of methylene blue, preferably the double zinc salt or commercial variety.

Collection of Sample. Collect the sample in a bottle holding approximately 150 c.c. If the dissolved oxygen is low, observe precautions similar to those used in collecting samples for dissolved oxygen (p. 162). Use rubber stoppers or a cork stopper of good grade which has been boiled in water.

Procedure. Add 0.4 c.c. of the methylene blue solution to the sample in the 150 c.c. bottle. As methylene blue has a slightly antiseptic property be careful to add exactly 0.4 c.c. Add the methylene blue solution preferably below the surface of the liquid after filling the bottle with the sample. If the methylene blue is added first do not allow the liquid to overflow as coloring matter will thus be lost. Incubate the sample at $20^{\circ} \mathrm{C}$. for ten days. Four days' incubation may be considered as sufficient for all practical purposes in routine plant-control work. If quick results are desired incubate the sample at $37^{\circ} \mathrm{C}$. for five days using suitable stoppers to prevent the loss and reabsorption of dissolved oxygen. The bacterial flora at $37^{\circ} \mathrm{C}$. is different from the flora at $20^{\circ} \mathrm{C}$. The lower temperature is more nearly the average temperature of surface waters and, therefore, the higher temperature should be used only when quick approximate results are essential. Observe the sample at least twice a day during incubation. Give a sample in which the methylene blue becomes decolorized a relative stability corresponding to the time required for reduction (see Table XXXIV). For routine filter control ordinary room or cellar temperature will give fairly satisfactory results. For accurate studies, room temperature incubation is very undesirable, as the fluctuations in temperature which are ordinarily not noticed are responsible for appreciable deviations from the true values of relative stability. If the samples are incubated less than ten days at $20^{\circ} \mathrm{C}$. and are not decolorized, place a plus sign after the stability value in order to indicate that the stability might have been higher if more time had been allowed. In applying this test to river 
waters it often happens that the blue coloring matter is precipitated either partly or completely through absorption by the clay which many rivers carry in suspension. True relative stabilities cannot be obtained for such waters except by determining the initial available oxygen at the start and the biochemical oxygen demand on incubation at $20^{\circ} \mathrm{C}$. for ten days. Germicides, such as calcium hypochlorite, if present in sufficient quantity vitiate the results. If a sample contains free chlorine, therefore, store it about two hours, or until the chlorine is gone, and then add methylene blue.

Table XXXIV gives the relation between the time of reduction in days at $20^{\circ} \mathrm{C}$. and the relative stability number.

\section{TABLE XXXIV}

RELATIVE STABILITY NUMBERS

\begin{tabular}{|c|c|c|c|}
\hline$t_{20}$ & $S$ & 120 & $S$ \\
\hline 05 & 11 & 80 & 81 \\
\hline 10 & 21 & 90 & 87 \\
\hline 15 & 30 & 100 & 90 \\
\hline 20 & 37 & 110 & 92 \\
\hline 25 & 44 & 120 & 94 \\
\hline 30 & 50 & 130 & 95 \\
\hline 40 & 60 & 140 & 96 \\
\hline 50 & 68 & 160 & 97 \\
\hline 60 & 75 & 180 & 98 \\
\hline 70 & 80 & 200 & 99 \\
\hline
\end{tabular}

$S=$ Relative stability or ratio of available oxygen to oxygen required for equilibrium. Expressed in per cent.

$t_{20}=$ Time in days to decolorize methylene blue at $20^{\circ} \mathrm{C}$.

The theoretical relation is,

$S=100(1-0.794) t_{20}$.

The relation between the time of reduction at $20^{\circ} \mathrm{C}$. and that at $37^{\circ}$ C. is approximately two to one. It is desirable that each observer work out his own comparative $37^{\circ} \mathrm{C}$. table or factor, but results should be reported in terms of $20^{\circ} \mathrm{C}$. stability numbers.

A relative stability of 75 signifies that the sample examined contains a supply of available oxygen equal to 75 per cent of the amount of oxygen which it requires in order to become perfectly stable. The available oxygen is approximately equivalent to the dissolved oxygen plus the available oxygen of nitrates and nitrites. The nitrites in sewage are usually so low as to be negligible. 
The theory of the test is unique. Methylene blue is very sensitive to products of putrefaction. Such compounds as hydrogen sulphide mercaptans, ctc., and other reducing substances quickly reduce it to its colorless leuco base. Consequently when this is mixed with a polluted water, it will change from blue to white as soon as any of these compounds are formed. They are present when putrefaction sets in and, therefore, cause the change in the color of the dye. Phelps has stated that this occurs when the nitrites are used up. This reducing time represents the time " required for the exhaustion of available oxygen."

Lederer (1914, 1915) who, as bacteriologist and chemist for the Sanitary District of Chicago has had ample opportunity to study this subject, has pointed out that great care must be used in the test to secure comparable results. He has devised some unique methods for the study of required oxygen. They are given in Standard Methods as follows:

Biochemical Oxygen Demand of Sewage and Effluents (Lederer, 1914). Relative Stability Method. The relative stability method may be employed to obtain a measure of the putrescible material in sewages and cffluents in terms of oxygen demand.

Procedure for Effluents. Divide the total available oxygen, including the oxygen of nitrites and nitrates, by the relative stability expressed as a decimal.

Procedure for Sewages. Make one or two dilutions with fully aerated distilled water of known dissolved oxygen content. Tap water may be cmployed if it is free from nitrates. Vary the relative proportions of sewage and water to be employed to give a relative stability of from 50 to 75 . Unless proper seals are employed bring the water as well as the sewage to the temperature at which the mixtures are to be incubated before preparing the dilutions. During the manipulation avoid aeration. Having made the proper dilutions, determine the relative stability of each.

Calculate the oxygen demand in parts per million by the following formula:

$$
\text { Oxygen demand is } \frac{O(1-p)}{R p} \text {. }
$$

In this formula $O$ is the initial dissolved oxygen of the diluting water; $p$ is the proportion of sewage; and $R$ is the relative stability of the mixture. Ordinarily the available oxygen in crude sewages, septic tank effluents, settling tank effluents, and tradewastes can be neglected.

Sodium Nitrate Method. For the determination of the bicchemical oxygen demand the sodium nitrate method may be used. The method 
is based on the biochemical consumption of oxygen from sodium nitrate by a sewage or polluted water during an incubation period of ten days at $20^{\circ} \mathrm{C}$. A reasonable excess of sodium nitrate does not give a higher oxygen demand, as do higher dilutions with aerated water. The oxygen absorbed from the air in applying the method to sewages is negligible.

Reagent. Sodium nitrate solution. Dissolve $26.56 \mathrm{gms}$. of pure sodium nitrate in 1 liter of distilled water. One c.c. of this solution in 250 c.c. of sewage represents fifty parts per million of available oxygen. The strength of the sodium nitrate solution may be varied to suit conditions.

Procedure for Sewages. Ordinarily disregard the initial available oxygen as it is very small compared with the total biochemical oxygen demand. Add measured amounts of the sodium nitrate solution to the sewage in bottles holding approximately 250 c.c which have been completely filled and stoppered. Incubate for ten days at $20^{\circ} \mathrm{C}$. A seal is not required during incubation. The appearance of a black sediment and the development of a putrid odor during incubation indicates that too little sodium nitrate has been added. Methylene blue solution in proper proportion may be added at the start to serve as an indicator during the incubation. Domestic sewage usually varies in its oxygen demand from 100 to 300 parts per million, approximately 30 per cent of which is used up at $20^{\circ} \mathrm{C}$. in the first twenty-four hours. At the end of the incubation period determine the residual nitrite and nitrate. Determine the nitrate by the aluminium reduction method, followed by direct Nesslerization. To convert the nitrogen into oxygen equivalents, multiply the nitrite nitrogen by 1.7 and the nitrate nitrogen by 2.9 . The difference between the available oxygen added as sodium nitrate and that found as nitrite and nitrate at the end of the incubation period is the biochemical oxygen demand.

Procedure for Tradewastes. Employ the same procedure using larger quantities of the sodium nitrate solution. Make the reaction alkaline to methyl orange and acid to phenolphthalein. Adjust an acid reaction with sodium bicarbonate, and a caustic alkaline reaction with weak hydrochloric acid. If the liquid is devoid of sewage bacteria seed it with sewage after adjusting the reaction.

Procedure for Polluted River Waters. Determine the initial available oxygen. Unless the river water is badly polluted add ten parts per million of sodium nitrate oxygen. Collect carefully to avoid aeration, three samples in 250 cc. bottles. To one sample add a definite quan- tity of sodium nitrate solution and incubate. Incubate the other two samples for the determination of the residual free oxygen, nitrite and 
nitrate. If there is free oxygen left, the bottle containing the sodium nitrate solution may be discarded. If there is no free oxygen determine residual nitrite and nitrate as drected under the procedure for sewage, and calculate the oxygen demand. (Standard Methods of Water Analysis.)

Examination of Sewage. The chemical examination of sewage and sewage effluents is probably more valuable than the bacterial examination. The problem involved in controlling a sewage treatment plant is quite different than that involved in the control of a water treatment plant. As Lederer and Bachmann (1911) have stated the effluent from a water treatment plant is subjected to different requirements than the effluent from a sewage treatment plant. In the first case the bacteriologist is concerned in the presence of ccrtain types of bacteria and their concentration. Little attention is given to the daily variations in the chemical content of the effluent. With the sewage effluent, however, the question is a different one. It is necessary to watch carefully the variations in the chemical content and keep the organic matter reduced to a minimum. The question of stream pollution is involved if the effluent is to be emptied into a stream. It must not be putrescible and thus cause the nuisances which result from too heavily polluting a stream. Lederer and Bachmann have stated some of the fallacies connected with the bacterial control of sewage plants. They have stated that "to try to obtain reliable information on the number of bacteria in effluents from various devices in a large plant would require a staff of trained men entirely out of proportion to the value of the results obtained." The difficulties in securing careful sampling and the necessity of immediate plating since bacteria in sewage die very rapidly when stored at icebox temperatures, are also mentioned. For these reasons and others more attention is given to the determination of the organic matter content and the biochemical oxygen demand according to the methods which have been given before.

The deposits from rivers and sewage sludges are examined in the following manner according to Standard Methods for the Examination of Water and Sewage, 1917.

\section{Analysis of Sewage Sludge and Mud Deposits}

Collection of the Sample. Collect a representative sample. In general, more than one sample should be taken from a spot and a large number of samples should be collected rather than a few large samples. If the surface layer is darker and a lower layer consists of pure clay 
sample only the surface layer. Samples may be analyzed either separately or as composites of careful mixtures. After the sample has settled a few minutes roughly drain or siphon the excess water. Allow sewage sludge to stand for one hour before draining it free from excess water unless it is essential to determine the moisture content of the sample originally collected. If sludge cannot be analyzed within twenty-four hours it is better not to use air-tight bottles and to add small quantities of chloroform to retard decomposition. At the time of collection carefully examine mud from the bottom of surface water for evidence of sewage pollution and macroscopic and microscopic animal and plant organisms. Record the predominant species. Note the physical appearance of the material, particularly its color, odor, and consistency. Express all analytical results in percentage on a dry basis.

Specific Gravnty. Weigh to the nearest tenth of a gram a widemouthed flask of 100 to 300 c.c. capacity, according to the quantity of material available. Then completely fill the flask with distilled water to the brim, and weigh it again. Empty and fill the flask completely with fresh sewage sludge or mud. If the material is of such consistency that it flows readily, fill the flask to the brim and weigh. The specific gravity is equal to the weight of the sludge or mud divided by the weight of an equal volume of distilled water.

If the material does not flow readily, fill the weighed flask as completely as possible without exerting pressure during the procedure. Weigh and then fill the flask to the brim with distilled water. Let it stand for a few minutes, until trapped air has escaped, then add more water if necessary and weigh. The specific gravity is equal to the weight of the material divided by the weight of the distilled water less the weight of the water added. Record the specific gravity only to the second decimal place.

Morsture. Heat approximately $25 \mathrm{gms}$. of sludge or mud in a weighed nickel dish on the water bath until it is fairly dry. Dry the residue in an oven at $100^{\circ} \mathrm{C}$, cool, and weigh. Repeat to approximate constant weight. The loss in weight is moisture.

Volatile and Fixed Matter. Ignite, in a hood, the residue from the determination of moisture until all the carbon has disappeared. Cool the residue in a desiccator and weigh it. The residue is the fixed matter. The volatile matter is the difference in weight between the original dried sludge and the ignited sludge.

Total Organic Nitrogen. Preparation of Sample. For the determination of organic nitrogen and fat dry approximately 50 to $75 \mathrm{gms}$. of the sludge or mud in a porcelain dish first on the water bath and 
finally in the hot-water oven until all the moisture has disappeared. Crind the dry material to a fine powder and keep it in a glass-stoppered bottle.

Reagents. 1. Sulphuric acid. Concentrated, nitrogen free.

2. Copper sulphate solution. Ten per cent.

3. Potassium permanganate. Crystals.

Procedure. Weigh accurately $0.5 \mathrm{gm}$. of dried sludge or $5.0 \mathrm{gms}$ of dried mud and put it in a 500 c.c. Kjeldahl flask. Digest it with 20 c.c. of sulphuric acid, or more if necessary, and 1 c.c. of copper sulphate solution to assist the oxidation. Boil for several hours until the liquid becomes colorless or slightly yellow. Oxidize the residue with $0.5 \mathrm{gm}$. of potassium permanganate, and follow the "Procedure for Scwage" (p. 165).

The following method is convenient for routine work at sewage disposal plants. Digest $0.5 \mathrm{gm}$. of dried sludge or $5.0 \mathrm{gms}$. of dried mud with 20 c.c. of strong sulphuric acid and 1 c.c. of the copper sulphate solution in a 300 c.c. Kjeldahl flask. After digestion for several hours, cool, transfer to a glass-stoppered 100 c.c. flask, dilute with distilled water to 100 c.c., and mix well. Transfer 50 c.c. with a pipette into another 100 c.c. volumetric flask, and make this portion alkaline with 50 per cent sodium hydroxide, testing a drop of the liquid on a porcelain plate with phenolphthalein to insure neutralization. The formation of a floc usually indicates that the neutralization is complete. Pour the solution into a small glass-stoppered bottle and permit it to stand until the next day. Nesslerize an aliquot portion of the clear, supernatant liquid, and calculate the percentage of nitrogen in the material.

Fats. Fats are usually determined only on sewage sludge, but some mud deposits contain small quantities due to the presence of trade wastes.

Procedure. Weigh, according to the quality of the sewage or mud, 0.5 to $25 \mathrm{gms}$. of dry material. Add water to the weighed portion in a porcelain dish and acidify the mixture with $\mathrm{N} / 50$ sulphuric acid in the presence of litmus tincture or azolitmin solution indicator. Avoid adding too much acid as an excess gives too high results on account of fatty acid residues. Evaporate the acidified mixtures to dryness on the water bath, and heat it in the hot-air oven at $100^{\circ} \mathrm{C}$. for two to three hours. Extract the dry residue with boiling ether, rubbing the sides and bottom of the dish to insure complete solution of the fat. Three extractions with ether are usually sufficient. Filter the ether solution through a $5 \mathrm{~cm}$. filter paper into a small flask. Evaporate the ether slowly, dry the fatty extract for half an hour at $100^{\circ} \mathrm{C}$., cool in a des- 
iccator and weigh. If it is desirable, particularly with certain trade wastes, to determine the quantity of soap fat, determine the fats with and without the addition of acid. The difference between the amounts found by the two determinations is the amount of soap fat present.

Ferrous Sulphide. The liberation of hydrogen sulphide on adding dilute hydrochloric acid to a sludge indicates the presence of ferrous sulphide. As ferrous sulphide quickly oxidizes on exposure to air, a quantitative determination of this constituent must be made immediately after collection of the sample.

Procedure. Heat a definite portion of the sludge with hydrochloride acid in a flask. Pass the liberated gas through bromine water or hydrogen peroxide. Determine gravimetrically the sulphate in the oxidizing solution, and calculate the equivalent of ferrous sulphide by multiplying the weight of barium sulphate by 0.376 .

Biochemical Oxygen Demand. The quantity of river mud most suitable for the determination of the biochemical oxygen demand ranges within certain limits, largely according to the amount of deoxygenating matter present. For examinations of river mud prepare a 1 per cent stock solution in distilled water or tap water saturated with oxygen and free from nitrate; use in the test a dilution of this stock solution equivalent to a concentration of 1 to $10 \mathrm{gms}$. per liter of mud. For examinations of fresh sewage sludge prepare a 1 per cent stock solution in a similar manner, but use in the test a dilution equivalent to only 0.1 to $1.0 \mathrm{gm}$. per liter of wet material. For examinations of dried sludges, which have undergone more or less mineralization, higher concentrations may be required.

Procedure. Place a measured portion of the sample, or the proper amount of the 1 per cent stock solution of the sample, in a 300 c.c. narrow-mouth, glass-stoppered bottle, and dilute it to the desired dilution with water saturated with oxygen. Determine the oxygen content at $20^{\circ} \mathrm{C}$. of the waters that are used for dilution. This determination must be made before the mud or sludge is added, because iron sulphide in the mud or sludge rapidly consumes part of the dissolved oxygen. Incubate at $20^{\circ} \mathrm{C}$. for five days.

Shortly before the determination of the oxygen remaining in solution at the end of five days rotate the bottle once or twice to mix its contents and allow sedimentation for about thirty minutes. Siphon the greater part of the liquid through a narrow-bore siphon into a 150 c.c. bottle, which has been filled with carbon dioxide. Reject the first 25 c.c. of the siphoned liquid and allow a little to overflow at the end of siphoning. Determine the oxygen content of the solution in the bottle in the usual 
way. Report the oxygen demand in grams of oxygen per $100 \mathrm{gms}$. of dried mud or sludge.

\section{BIBLIOGRAPHY}

Bartow, E. 1916. Examination of Drinking Water on Railway Trains. Univ. of Ill. Bull. No. 13. Water Survey Series 12 (1916), 173-182; Jour. Am. Water Works Assn.. 2, 74-82.

Baton, W. U. C. 1914. Investigation into the Advisability of Substituting Agar for Gelatin as a Medium for the Determination of Bacteria Counts in Water Analysis. Jour. Am. Water Works Assn., 1, 11-23.

Brevnig. 1894. Bakteriologische Untersuchungen des Trinkwassers der Stadt Kiel im August und September, 1887. Kiel (1888); quoted from Frankland, in Micro-organisms in Water, London.

BurLING, H. A. 1918. Concentration of Glucose and Lactose and Viability of Coli-like Bacteria. Amer. Jour. Pub. Health, 8, 306-307.

Carrd, J. M. 1913. The Bacterial Count on Gelatin and Agar Media and its Value in Controlling the Operation of Water Purification Plants. Proceedings American Water Works Association, 1913, 325-351.

Crick, H. The Distribution of Bacterium Coli Commune. ThompsonYates Lab. Repts. No. 3, 1.

Clark, W. M. 1918. The Differentiation of Bacteria of the Colon-ærogenes Family. Jour. Amer. Water Works Assn., 5, 26-35.

ClaRk, W. M. and LUBS, H. A. 1917. Improved Chemical Methods for Differentiating Bacteria of the Coli-ærogenes Family. Jour. Biol. Chem., $30,209-234$.

Cobb, Wildiams and Letton. 1916. Drinking Water on Interstate Carriers. Reprint No. 368, Public Health Reports, 31, 2845-67.

Coles, A. C. 1916. An Easy and Rapid Method of Doing Widal's Reaction for Typhoid Fever. Brit. Med. Journal, 1916-I, 684.

Commitree. 1898. Procedures Recommended for the Study of Bacteria with Special Reference to Greater Uniformity in the Description and Differentiation of Species. Being the report of a committee of bacteriologists to the committee on the pollution of water supplies of the American Public Health Association. Amer. Pub. Health Assn. Proceedings, $1898,60-100$.

Crees, R. H. 1914. Examination of Drinking Water on Railway Trains. Bull. 100, Hyg. Lab., Wash.

Cumming, H. S. 1914. Safe Ice. Public Health Reports, $29,3$.

Cumming, H. S. 1916. Investigations of the Pollution and Sanitary Conditions of the Potomac River. Bull. 104, Hyg. Lab., Wash.

DoLe, R. B. 1906. The Use of Fluorescein in the Study of Underground Waters. U. S. Geological Survey, Water Supply Paper, 160.

Dorange. 1898. Epidemie de fièrre typhoide du a l'ingestion de glace

- impure. Rev. d'Hygiéne, 20, 295. 
Dutron, M. S. 1917. Sterilization by Liquid Chlorine and Hypochlorite of Lime. Jour. Amer. W. W. Assn., 4, 228-230.

Dyer, H. G. and KeIth, JR., S. C. 1893. Notes on Normal Intestinal Bacilli of the Horse and of Certain other Domesticated Animals. Tech. Quarterly, $6,256-257$.

Eijkmann. 1904. Die Garungsprobe bei $46^{\circ}$ als Hilfsmittel bei Trinkwasseruntersuchung. Cent. Bakt., Abt. I. Orig., 37, 74.

Eyre, J. W. H. 1904. On the Distribution of Bacillus Coli in Nature. Lancet, 1, 648-649.

Fink, E. B. 1917. American Mortality Statistics and the Mills-Reincke Phenomenon. Jour. Inf. Diseases, 21, 62-94.

Flint, J. M. 1896. Notes on the Distribution of Bacillus Coli Communis. Jour. Amer. Med. Assn., 26, 410-411.

Frankland, Percy and Frankland, G. C. 1894. Micro-organisms in Water, 105-6, London.

Frost, W. H. 1916. Confirmation Tests for B. coli in Routine Water Examinations. Amer. Jour. Pub. Health, 6, 585-8.

Frost, W. H. 1914. The Functions and Value of Epidemiological Studies of Infectious Diseases in Sanitary Administration. Proceedings Indiana Sanitary and Water Supply Assoc., 1914, 95-102.

Fuller and Johnson. 1899. On the Differentiation and Classification of Water Bacteria. Jour. Exp. Med., 4, 609-626. American Pub. Fealth Assn. Proceedings, 25, 580-586.

GAUB, J. 1913. Some Results from the Use of Gelatin and Agar. Proc. Ill. Water Supply Assn. 1913, 155-159.

Greenfield, M. 1916. A New Ice Sampler. Journal Bacteriology, 1, 623.

HALLER, C. 1914. The Sterilization of Water with Lime. Statisch Tiefblau, 4, 299-303. Chem. Abstracts. 8, 2206.

HANFORD, W. W. and BarTow, E. 1916. Quality of the Drinking Water Obtained in Illinois by Common Carriers. Illinois State Water Survey Bulletin, 13, 256-271.

Harden, A. 1901. Action of B. communis on Dextrose. Trans. of the Chemical Society, 1901, 604.

HARDEN, A. 1905. Action on Glucose of Lactose Fermenting Bacteria of Feces. Jour. Hygiene, 5, 488.

HARDEN, A. 1905-1906. On the Voges-Proskauer Reaction for Certain Bacteria. Proc. Roy. Soc., 77, 424.

Harden, A. and Norris, D. 1911-1912. The Bacterial Production of Acetylmethyl-carbinol and 2, 3, Butylene-glycol from Various Substances. Proc. Royal Soc. Series B, 84, 492; 85, 73.

HARDEN, A. and Norris, D. 1911. The Diacetyl Reaction for Proteins. Journal of Physiology, 47, 332.

Harden, A. and Walpole, S. G. 1905-1906. Chemical Action of B. lactis Aerogenes (Escherich) on Glucose. Production of 2, 3, Butylene, Glycol and Acetyl Methyl Carbinol. Proc. Royal Soc. Series B, 77, 399. 
HARRison, F. C. 1912. Microbiology of Water and Sewage, (in Microbiology, edited by C. E. Marshall), 204.

Hauser, S. J. 1917. Methods Used at Cincinnati Filtration Plant for Isolating and Identifying Bacillus Coli. Eng. and Contractor, 48, 296-297. Also published in Amer. Jour. Pub. Health, 7, 1050-1051.

Hazen, A. 1904. The Hazen Theorem. Trans. Amer. Soc. Civil Eng., 54, Pt. D., 131.

HILL, H. W. 1910. Non-relation of the Natural Ice Supply to Typhoid Fever and Dysentery. Cold Storage and Ice Trade Jour., 40, 33-35.

Hilliard, C. M., Torissian, C., and Stone, R. P. 1915. Notes on the Factors Involved in the Germicidal Effect of Freezing and Low Temperatures. Science, 42, 770-1.

Hoover, C. P. and Scott, R. D. 1914. Advantage of the Use of Lime in Water Purification. Monthly Bull. Ohio State Board of Health, December, 1914.

Houston, A. C. First Report on Research. The Vitality of the Typhoid Bacillus in Artificially Infected Samples of raw Thames, Lee and New River Waters, with Special Reference to the Question of Storage. Metropolitan Water Board, London.

Houston, A. C. Fourth Report on Research Work. The Vitality of the Cholera Vibrio in Artificially Infected Samples of raw Thames, Lee and New River Waters, with Special Reference to the Question of Storage. Metropolitan Water Board.

Hutchins and Wheeler. 1903. An Epidemic of Typhoid Fever Due to Impure Ice. Amer. Jour. Science, 126, 680.

Johnson, G. A. 1904. Isolation of B. coli communis from the Alimentary Tract of the Fish and the Significance Thereof. Journal of Infectious Diseases, 1, 348-354.

JoHnson, G. A. 1910. Hypochlorite Treatment of Water Supplies: Its Significance and Limitations. Eng. Record, 62, 321.

Johnson, G. A. 1913. The Purification of Public Water Supplies. U. S. Geological Survey, Water Supply Paper No. 315.

Johnson, G. A. 1916. The Typhoid Toll. Jour. Amer. Water Works Assn., $3,249-326$.

Johnston, W. 1894. On the Grouping of Water Bacteria. Proc. Amer. Pub. Health Assn., 20, 445-449.

Jordan, E. O. 1915. The Bacterial Examination of Water. Trans. XV. Intern. Cong. Hyg. and Demog., 2, 47-50.

Jordan, E. O. 1900. The Self-purification of Streams. Jour.Exper. Med., 5, 302.

JoRdan, E. O. 1903. The Kinds of Bacteria Found in River Water. Journal Hygiene, 3, 1-27.

Jordan, E. O., Russell and Zeit. 1904. The Longevity of Typhoid Bacillus in Water. Jour. Inf. Diseases, $1,641$.

Jordan, E. O. and Victorson, Ruth. 1917. Differentiation of the Paratyphoid Enteritidis Group II. Jour. Inf. Diseases, 21, 554-555. 
KLEIN, E. 1895. Ueber einen pathogenen anaeroben Darmbacillus, Bacillus enteritidis sporogenes. Cent. Bakt., 18, 737.

KLeIN, E. 1897-8. Morphology and Biology of Bacillus Enteritidis Sporogenes; on Association of this Microbe with Infantile Diarrhoa and with Cholera Nostras; on its Relation with Milk and with Manure. Local Government Board; Medical Officer's Report. p. 210.

KLEIN, E. 1898-9. Further Report on Bacillus Enteritidis Sporogenes. Local Government Board, Medical Officer's Report, p. 312.

KLEIN, E. 1899. Ein Beitrag zur Bakteriologie der Leichenverwesung. Cent. Bakt., 25, 278.

KueIn, E. 1901-2. Differentiation of the Several Anaerobic Microbes Commonly Present in the Intestinal Contents of Man and Other Animals. Local Government Board; Medical Officer's Report, p. 404.

KLIGLER, I. J. 1917. A Simple Medium for the Differentiation of Members of the Typhoid Paratyphoid Group. Amer. Jour. Pub. Health, 7, 1042-1044.

KRUmwiede, C. and KoHN, L. A. 1917. A Triple Sugar Modification of the Russell Double Sugar Medium. Jour. Med. Research, 37, 225-227.

KRUSE, W. 1894. Kritische und Experimentale Beitrage zur Hygienischen Beurtheilung des Wassers. Zeit. Hyg., 17, 1894.

LeDerer, A. and Bachmann, F. 1913. Some Interesting Observations on the Disinfection of Lake Water with Calcium Hypochlorite. Proceedings Illinois Water Supply Assn., 1913, 184-201.

LEDerer, A. 1914. A Serious Fallacy of the "Standard" Methylene Blue Putrescibility Test. Amer. Jour. Pub. Health, 4, 241-248.

LEDERER, A. 1914. A New Method for Determining the Relative Stability of Sewage Effluent or Polluted River Water. Jour. Inf. Diseases, 14, 482-497.

LEDeRer, A. 1915. Notes on the Practical Application of the "Saltpeter" Method for Determining Strengths of Sewage. Amer. Jour. Pub. Health, $5,354-361$.

Lederer, A. 1915. Determination of the Biochemical Oxygen Demand by the Saltpeter Method in Stockyards, Tannery and Corn Products Wastes. Jour. Ind. Eng. Chem., 7, 514, 6.

Leighton. 1907. Pollution of the Illinois and Mississippi Rivers by Chicago Sewage. A digest of the testimony taken in the case of the State of Missouri vs. the State of Illinois and Sanitary District of Chicago. U. S. Geological Survey Water Supply Paper No. 194.

Letton, H. P. 1917. The Treasury Department Standard for Drinking Water: Its Value and Enforcement. Jour. Amer. Water Works Assn., 4, 478-481.

Levine, M. 1916. The Significance of the Voges-Proskauer Reaction. Jour. Bact., 1, 153-164.

Levine, M. 1916. The Correlation of the Voges-Proskauer Reaction and Methyl Red Reactions in the Colon-ærogenes Group of Bacteria. Jour. Inf. Diseases, 18, 358-367.

Levine, M. 1918. Presumptive Test for Bacillus Coli. Eng. Contractor, 49, 34. Also see Jour. Inf. Dis., 23, 43-47. 
Lewis, W. L. 1912. Evanston's Experience with Hypochlorite. Proc. Illinois Water Supply Association, 1912, 124-139.

McCallie, S. W. 1904. Experiment Relating to Problems of Well Contamination at Quitman, Ga. U. S. Geol. Survey, Water Supply Paper, 110, $45-54$.

McLaughrin, A. J. 1912. The Necessity for Safe Water Supplies in the Control of Typhoid Fever. Proceeding Illinois Water Supply Association, 1912, 64-84.

McLaughern, A. J. 1914. What is a Safe Drinking Water? Public Health Reports, 29, June 26, 1914.

MacNear, W. J., Latzer, I. and KerR, J. 1909. The Fecal Bacteria of Healthy Men. Jour. Inf. Diseases, 6, 571.

Marboutin, F. 1901. Contribution à l'Etude des Eaux Souterraines. Comp. Rend., 132, 365-368.

Marons. 1910. Bakterium Coli Commune. Arch. Exper. Med., 22, 261.

Martel, E. A. 1903. Sur l'application de la fluorescein à l'hydrologie souterraine. Compt. Rend., 137, 225-227.

Metcalf, H. 1905. Organisms on the Surface of Grains with Special Reference to Bacillus coli. Science, 22, 434-441.

Montgomery, S. D. and Phelps, E. B. 1917. Stream Pollution; a Digest of Judicial Decisions and a Compilation of Legislation Relating to the Subject. U. S. Public Health Service, Public Health Bulletin, 87.

Moore, V. A. and Wright, F. R. A. 1900. A Comparison of B. Coli Communis from Different Species of Animals. Jour. Bost. Med. Soc., 4, 175.

Morishima, K. A Simple and Practical Medium for Difterentiating B. Typhosus, B. Paratyphosus, A. B. Paratyphosus B and B. Enteriditis. Journal of Bacteriology, 3, 19-22.

MurR, R. and RITchIE, J. 1914. Manual of Bacteriology, New York, 5th Edit.

Nankrveld, A. T. 1911. The Sand Filtration and Purification of Chalk Waters. Journal of Hygiene, 11, 235-258.

Nichols, A. H. 1876. Report of an Outbreak of Intestinal Disorder Attributable to the Contamination of Drinking Water by Means of Impure Ice. 7 th Annual Report State Board of Health, Massachusetts, 467.

Obst, M. M. Bile Compared with Lactose Broth for Determining the Presence of B.'Coli in Water. Jour. Bact., 1, 73-79.

ORCHARD, W. J. 1918. Water Supply Standards and their Improvement. Jour. Am. Water Works Assn., 5, 49-58.

PARK, W. H. 1907. The Importance of Ice in the Production of Typhoid Fever. Jour. Amer. Med. Assn., 49, 731-732.

Prescom?, S. C. 1906. The Occurrence of Organisms of Sanitary Significance on Grains. Biol. Studies of Pupils of W. T. Sedgwick, 208-222, Boston.

Prescotr, S. C. and Winsuow, C. E. A. 1915. Elements of Water Bacteriology. New York. 3d Edit., .217. 
Phelps, E. B. 1909. The Disinfection of Sewage and Sewage Filter Effluents, with a Chapter on the Putrescibility and Stability of Sewage Effluents. U. S. Geol. Survey, Water Supply Paper 229.

Phelps, E. B. 1914. Studies on the Self-purification of Streams. Public Health Reports, 29, August 14, 1914.

Phelps, E. B. 1916. Re-aeration Held to be Important in the Self-purification of Streams. Eng. Record, 74, 617-618.

RACE, J. 1914. The Significance of B. Coli in Water Examination. Amer. Jour. Pub. Health, 26, 758-759.

RACE, J. 1914. Bacillus Coli in Water Supply. Municipal Eng., 47, $12-14$.

RACE, J. 1916. Bacterial Counts in Water Examination. Amer. Jour. Pub. Health, 6, 488-496.

RACE, J. 1918. Chlorination and Chloramine. Jour. Amer. Water Wks. Assn., 5, 63-68.

Reynolds. 1902. Report of Stream Examination, Chemic and Bacteriologic, of the Water Between Lake Michigan and the Mississippi River at St. Louis, Chicago, 1902.

Rogers, L. A., Clark, W. M. and Evans, A. 1915. Science, 41, 624.

Royal Institute of Public Health. Committee Report. Jour. State Med., $22,559-567$.

SAVAGE, W. G. 1906. The Bacteriological Examination of Water. Chap. 9, Philadelphia.

SaVAGe, W. G. and Read, W. J. 1916. Significance of Streptococci in Water Supplies. Jour. Hyg.. 5, 334-351.

Savage, W. G. and Wood, D. R. 1917. The Vitality and Viability of Streptococci in Water. Jour. Hygiene, 16, 227, 239.

Sebgwrck, W. T. and Winslow, C.-E. A. 1902. Experiments on the Effects of Freezing and Other Low Temperatures upon the Vitality of the Bacillus of Typhoid Fever with Consideration Regarding Ice as a Vehicle of Infectious Disease. Mem. Amer. Acad. Arts and Sciences, 12.

Sedgwick, W. T. and MAcNutr, J. S. 1910. Study on the Hazen Theorem Jour. Inf. Diseases, 7, 489.

Smith, E. G. B. 1905. Notes on the Occurrence on Grain of Organisms Resembling Bacillus Coli Communis. Science, 21, 710-711.

Tanner, F. W. 1916. Comparison of the Number of Water Bacteria Growing on Agar at $37^{\circ} \mathrm{C}$. and on Gelatin at $20^{\circ} \mathrm{C}$. University of Illinois, Bulletin No. 13. Water Survey Series, No. 12, 242-8.

Thresh, J. C. 1913. Examination of Water and Water Supplies. Blakistons, Philadelphia.

Traube, M. 1894. Einfaches Verfahren Wasser in grossen Mengen Keimfrei zu machen. Zeit. Hyg., 149-188.

Treasury Department. Bacteriological Standards for Drinking Water. Pub. Health Reports, 29, 2959-2966. Jour. Amer. Water Works Assn., 4, 927-946. 
Trillat, A. 1899. Sur l'emploi matières polourantes pour la recherche de l'origine des sources et des caux d'infiltration. Comp. Rend., 128, 698700 .

Tuller, C. A. and Armstrong, V. A. 1913. The Differentiation of Fecal Streptococci by their Fermentative Reactions in Carbohydrate Media. Jour. Inf. Diseases, 13, 442-462.

Van Brunt, G. A. 1911. The Action of Bleaching Powder in Water Purification. University of Illinois Bulletin, Water Survey Series No 8, 53.

WARD, M. 1897. Fifth Report to the Royal Society Water Research Committee. Proc. Royal Soc., London, 61, 415-423.

Watr, J. 1913. Purification of Water Supplies by the Excess Lime Method. Jour. State Medicine, 21, 489-499.

Weissenfeld, J. 1900. Der Befund des Bakterium Coli im Wasser und das Thiere Experiment sind keine brauchbaren Hulfmittels fur die hygienische Beurtheilung des Wassers. Zeit. Hyg., 35, 78-86.

Whrpple, G. C. 1913. The $37^{\circ}$ Bacterial Count. American Journal of Public Health; 3, 36-43.

Winslow, C.-E. A. and Hunnewell, M. P. 1902. Streptococci Characteristics of Sewage and Sewage Polluted Waters. Science, 15, 827.

Winslow, C.-E. A. and Hunnewell, M. P. 1902. A Study of the Distribution of the Colon Bacillus of Escherich and of the Sewage Streptococci of Houston in Polluted and Unpolluted Waters. Journal of Medical Research, 8, 502.

Winslow, C.-E. A. 1916. Tests for Bacillus Coli as an Indicator of Water Pollution. Jour. Amer. Water Works Assoc., 3, 927-946.

Wolman, A. 1917. The Quality of Water and Confirmatory Tests for B. Coli. Jour. Amer. Works Association, 4, 200-205. 
CHAPTER XI

\section{MILK AND MILK PRODUCTS}

Mruk is the special secretion of the mammary glands and is generally used for food by man. Fresh milk is amphoteric to litmus with a specific gravity heavier than water-1.030. It has a yellowish color and a characteristic odor. On account of its chemical composition, which is mentioned later, it is susceptible to many undesirable changes. Many of these are brought about by microorganisms and, therefore, may be controlled by holding the milk in an environment, the factors of which are detrimental to bacterial development.

\section{Chemical Constituents of Milk}

The constituents of milk have been classified by Van Slyke and Bosworth (1914) in the following manner:

1. Milk constituents in true solution in milk serum.
a. Sugar
b. Citric acid
c. Potassium
d. Sodium
e. Chlorine

2. Milk constituents partly in solution and partly in suspension or colloidal solution.
a. Albumin
b. Inorganic phos- phate
c. Calcium
d. Magnesium

3. Milk constituents entirely in suspension or colloidal solution.

a. Fat

b. Casein

Bosworth and Van Slyke (1915) report the composition of cow's, goat's and human milk, shown in Table XXXVI. From this presentation of the chemical composition of milk, it will be seen that it is an excellent medium for bacterial growth.

Van Slyke and Bosworth (1914) studied the composition of milk serum after the other constituents had been removed by filtration through a Pasteur-Chamberland filter. The analysis of the serum is given in Table XXXVII. 


\section{TABLE XXXVI}

COMPOUNDS IN COW'S, GOAT'S, AND HUMAN MILK

(After Bosworth and Van Slyke, 1915)

\begin{tabular}{|c|c|c|c|}
\hline Compounds. & Cow's Milk. & Goat's Milk. & Human Milk. \\
\hline Fat. & $\begin{array}{c}\text { Per cent } \\
3.90\end{array}$ & $\begin{array}{c}\text { Per cent } \\
3.80\end{array}$ & $\begin{array}{c}\text { Per cent } \\
3.30\end{array}$ \\
\hline Milk sugar. . & 4.90 & 4.50 & 6.50 \\
\hline Proteins combined with calcium. & 3.20 & 3.10 & 1.50 \\
\hline Salts. . . & 0.901 & 0.951 & 0.313 \\
\hline Di calcium phosphate... & 0175 & 0092 & 0.000 \\
\hline Tri calcium phosphate . & 0000 & 0.062 & 0.000 \\
\hline Mono magnesium phosphate...... & 0103 & 0.000 & 0.027 \\
\hline Di magnesiuin phosphate..... & 0000 & 0068 & 0.000 \\
\hline Tri magnesium phosphate......... & 0.000 & 0.024 & 0.000 \\
\hline Mono potassium phosphate........... & 0.000 & 0.073 & 0.069 \\
\hline Di potassium phosphate... & 0.230 & 0.000 & $0.00 n$ \\
\hline Potassium citrate....... & 0052 & 0.250 & $0.10 \Omega$ \\
\hline Sodium citrate......... & 0.222 & 0.000 & 0.05 .5 \\
\hline Potassium chloride. . & 0.000 & 0.160 & $0.00 n$ \\
\hline Sodium chloride... . & 0.000 & 0.095 & $0.00 n$ \\
\hline Calcium chloride. . & 0.119 & 0.115 & 0.059 \\
\hline
\end{tabular}

TABLE XXXVII

COMPOSITION OF MILK SERUM

(After Van Slyke and Bosworth, 1914)

\begin{tabular}{|c|c|c|c|c|c|c|}
\hline \multirow[b]{2}{*}{ Constituents } & \multicolumn{3}{|c|}{ SAMPLE No. 1.} & \multicolumn{3}{|c|}{ SAMPLI No. 2.} \\
\hline & $\begin{array}{l}\text { Original } \\
\text { Milk, } \\
100 \text { c.c. }\end{array}$ & $\begin{array}{l}\text { Milk } \\
\text { Serum, } \\
100 \text { c.c. }\end{array}$ & $\begin{array}{l}\text { Prrcentage } \\
\text { of Milk } \\
\text { Constitu- } \\
\text { ents in } \\
\text { Serum. }\end{array}$ & $\begin{array}{l}\text { Original } \\
\text { Milk, } \\
100 \mathrm{cc} .\end{array}$ & $\begin{array}{l}\text { Milk } \\
\text { Serum, } \\
100 \text { c.c. }\end{array}$ & $\begin{array}{l}\text { Percentage } \\
\text { of Milk } \\
\text { Constitu- } \\
\text { ents in } \\
\text { Serum. }\end{array}$ \\
\hline Sugar. & $\begin{array}{l}\text { Grams } \\
\ldots \ldots\end{array}$ & $\begin{array}{l}\text { Grams } \\
\ldots \ldots\end{array}$ & $\begin{array}{c}\text { Per cent } \\
\ldots \ldots\end{array}$ & $\begin{array}{l}\text { Grams } \\
5.75\end{array}$ & $\begin{array}{l}\text { Grams } \\
5.75\end{array}$ & $\begin{array}{l}\text { Per rent } \\
100.00\end{array}$ \\
\hline Casein. & 3.35 & 0.00 & 0.00 & 3.07 & 0.00 & 0.00 \\
\hline Albumin. . . . . & 0.525 & 0.369 & 70.29 & 0.506 & 0.188 & 37.15 \\
\hline Nitrogen in other compounds & & & & 0.049 & 0.049 & 100.00 \\
\hline Citric acid . . . . . . . & & & 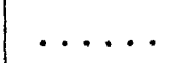 & 0.237 & 0.237 & 100.00 \\
\hline $\begin{array}{l}\text { Phosphorus (organic and in- } \\
\text { organic) } \ldots \ldots \ldots \ldots \ldots\end{array}$ & 0.125 & 0.067 & 53.60 & & & $\ldots \ldots$ \\
\hline Phosphorus (inorganic).. & 0.096 & 0.067 & 70.00 & 0.087 & 0.056 & 64.40 \\
\hline Calcium............ & 0.128 & 0.045 & 35.16 & 0.144 & 0.048 & 33.33 \\
\hline Magnesium... & 0.012 & 0.009 & 75.00 & 0.013 & 0.007 & 53.85 \\
\hline Potassium. . & $* 0354$ & $*_{0} \quad 352$ & $99 \quad 44$ & 0.120 & 0.124 & 100.00 \\
\hline Sodium..... & 0304 & 03.02 & & 0055 & 0.057 & 100.00 \\
\hline Chlorine.... & 0.081 & 0.082 & 100.00 & 0.076 & 0.081 & 100.00 \\
\hline Ash........ & $\ldots \ldots$ & $\ldots \ldots$ & $\ldots \ldots$ & 0.725 & 0.400 & 55.17 \\
\hline
\end{tabular}

* As chlorides. 
Milk Proteins. According to the table from Bosworth and Van Slyke cow's milk has a larger content of protein than goat's or human milk. Data from other sources confirm this fact. The following proteins are found in milk:

Casein

Lact-albumin

Lact-globulin

\section{Changes in Milk}

Milk is subject to many types of changes. One of the most common and one which occupied the attention of chemists for a long time is the coagulation or clotting of the casein. The natural state of this milk constituent may be changed either by acid or the presence of a specific enzyme. Other proteins may be coagulated in the same way by enzymes. This change in the state of the casein is the foundation of the whole cheese industry.

Souring of Milk. This usually refers to the normal changes in milk which are brought about by the continued growth of lactic acid bacteria. The lactic acid which is thus formed removes the calcium from the calcium caseinate. The casein thus formed is insoluble in the milk serum and is precipitated.

The chemical changes which take place in the soluble and insoluble constituents of milk have been studied by Van Slyke and Bosworth (1916). The milk was inoculated with a culture containing Bacterium lactis acidi and $B$. lactis aerogenes and allowed to stand at room temperature for sixty hours. At the end of this time it was filtered through a porcelain filter and subjected to analysis. A blank was made by preserving milk with chloroform and separating the serum in the same way. The changes which took place in sixty hours are given in Table XXXVIII.

Coagulation by Rennin. It was believed for a long time that the coagulation of milk was due only to acid. Fremy, in 1839, one of the earliest workers on the subject, supposed that a special enzyme was present in the lining of calves' stomachs which formed acid from the lactose. Hammarsten (1872) proved that the production of acid from the lactose was not the cause of coagulation by rennin but that it was brought about by a special enzyme, which acted directly on the casein. The phenomenon involved in the action of rennin is the change of casein in milk into soluble paracasein along with another compound. However, before actual clotting takes place an insoluble calcium paracaseinate must be formed from calcium salts in the milk. Van Slyke 
and Bosworth (1913) have shown that the molccular weight of casein is 8888 and that of paracascin 4444 . The valence of the molecule in basic cascinates is 8 and in basic paracaseinates 4. Bosworth (1913), in another paper states that these facts indicate three things:

\section{TABLE XXXVIII}

CHANGES IN CONDITION OF MILK CONSTITUENTS AS A RESULT OF SOURING

(After Van Slyke and Bosworth)

\begin{tabular}{|c|c|c|c|c|c|}
\hline \multirow[b]{2}{*}{ Constituents. } & \multirow[b]{2}{*}{$\begin{array}{l}\text { Original } \\
\text { MIllk, } \\
100 \text { c.c. }\end{array}$} & \multicolumn{2}{|c|}{$\begin{array}{l}\text { SERUM FroM } \\
\text { FrESH MILK. }\end{array}$} & \multicolumn{2}{|c|}{$\begin{array}{l}\text { SFRUM FROM } \\
\text { SOUR MILK. }\end{array}$} \\
\hline & & $\begin{array}{l}\text { Serum } \\
100 \text { c.c. }\end{array}$ & $\begin{array}{l}\text { Percent- } \\
\text { age of } \\
\text { Milk Con- } \\
\text { stituents } \\
\text { in the } \\
\text { Serum. }\end{array}$ & $\begin{array}{l}\text { Serum, } \\
100 \text { c.c. }\end{array}$ & $\begin{array}{l}\text { Percent- } \\
\text { age of } \\
\text { NIlk Con- } \\
\text { stituents } \\
\text { in the } \\
\text { Serum. }\end{array}$ \\
\hline Sugar. & $\begin{array}{l}\text { Grams } \\
5.75\end{array}$ & $\begin{array}{l}\text { Grams. } \\
5.75\end{array}$ & $\begin{array}{c}\text { Per Cent. } \\
100.00\end{array}$ & $\begin{array}{l}\text { Grams. } \\
4.48\end{array}$ & $\begin{array}{c}\text { Per Cent. } \\
\ldots \ldots\end{array}$ \\
\hline Casein. & 3.07 & 0.00 & 0.00 & 0.00 & 0.00 \\
\hline Albumin. & 0.506 & 0.188 & 37.15 & 0.506 & 100.00 \\
\hline Nitrogen in other compounds. . & 0.049 & 0.049 & 100.00 & 0.049 & 100.00 \\
\hline Citric acid........ & 0.237 & 0.237 & 100.00 & 0.000 & 0.00 \\
\hline Phosphorus (inorganic).... & 0.087 & 0.056 & 64.40 & 0.090 & 100.00 \\
\hline 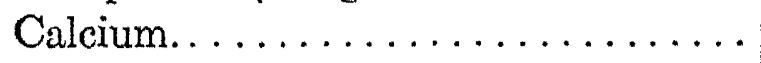 & 0.144 & 0.048 & 33.33 & 0.148 & 100.00 \\
\hline Magnesium. . . . . . . . . & 0.013 & 0.007 & 53.85 & 0.014 & 100.00 \\
\hline Potassium..... & 0.120 & 0.124 & 100.00 & 0.120 & 100.00 \\
\hline Sodium & 0.055 & 0.057 & 100.00 & 0.058 & 100.00 \\
\hline Chlorine... & 0.076 & 0.081 & 100.00 & 0.079 & 100.00 \\
\hline Ash....... & 0.725 & 0.400 & 55.17 & 0.690 & 95.17 , \\
\hline Lactic acid. & 0.000 & 0.000 & 0.00 & 1.124 & $\ldots \ldots$ \\
\hline
\end{tabular}

First, that rennin action consists of the hydrolytic splitting of the casein molecule into two similar molecules of paracasein; perhaps in somewhat the same manner that maltose is split into two molecules of dextrose.

Second, that, as a consequence of this cleavage, it would seem to be doubtful if Hammarsten's whey-protein could be one of the products of rennin action.

Third, that rennin is not, strictly speaking, a coagulating ferment, the coagulation of paracasein being due to the fact that calcium paracaseinates are less soluble than the calcium caseinates, especially in the presence of soluble salts of calcium barium or strontium. 
The action of rennin is then proteolytic since it destroys casein. It might be regarded as a preliminary process in digestion, Bosworth (1913) states that probably the action attributed to rennin may be produced by any proteolytic enzyme. In the bacterial world this has been known for some time that an organism which will split casein will also probably split gelatin and other proteins. This is especially well borne out in the following table compiled by Frost and McCampbell and taken from their text.

From this table one might infer that there was a specificity even for the different proteins.

TABLE XXXIX

ACTION OF CERTAIN BACTERIA ON CERTAIN PROTEINS

(Frost and McCampbell)

\begin{tabular}{c|c|c|c|c|c|c}
\hline \multirow{2}{*}{ Name of Organism. } & \multicolumn{2}{|c|}{ Mrr. } & Gelatin. & Serum. & $\begin{array}{c}\text { Egg } \\
\text { Albumin. }\end{array}$ & Fibrin. \\
\cline { 2 - 5 } & Coag. & Digest. & & & & \\
\hline Bact. anthracis......... & + & + & + & - & + & + \\
Microspira comma...... & + & + & + & + & + & + \\
M. pyogenes aureu...... & + & + & + & - & - & - \\
Pseudomonas pyocyaneus. & + & + & + & + & + & + \\
Pseudomonas violacea.... & - & - & + & - & - & - \\
Bacillus mycoides....... & + & + & + & + & - & - \\
Bacillus prodigiosus...... & - & + & + & + & + & + \\
Aspergillus niger........ & + & + & - & - & - & - \\
Aspergillus oryzæ........ & - & + & + & + & + & - \\
\hline
\end{tabular}

\section{Chemical Examination}

The functions of the chemical and bacteriological examinations may be entirely different although, in many cases, they must go along together. A sanitarian must have all the knowledge which it is possible to secure and should not exclude either data if he would come to an accurate conclusion. In the chemical examinations that are made on milk, data may be secured with regard to the normal or abnormal occurrence of any milk constituent. The presence of preservatives may be directly determined while, in the bacteriological examination, they may be indirectly inferred if the milk does not sour when it is placed in a favorable environment for this change.

Sampling of Milk. To secure accurate results from the chemical or bacterial examination of milk requires good sampling. Too much 
attention cannot be paid to this important step. The larger portion from which the sample is taken should be thoroughly stirred. If the sample is collected in a bottle, the contents should be very thoroughly mixed.

Specific Gravity. This may be determined by any of the usual methods. Hydrometers are convenient and, in this form, are known as lactometers. They are of different types. The New York Board of Health has an arbitrary scale. This is divided into 120 parts with zero equal to the specific gravity of water and 120 equal to the specific gravity of 1.029. The length is usually about 12 ins. The Quevenne lactometer is graduated in accordance with the specific gravity scale with the first two figures omitted for sake of convenience (1.029 sp. gr.=29 Que-

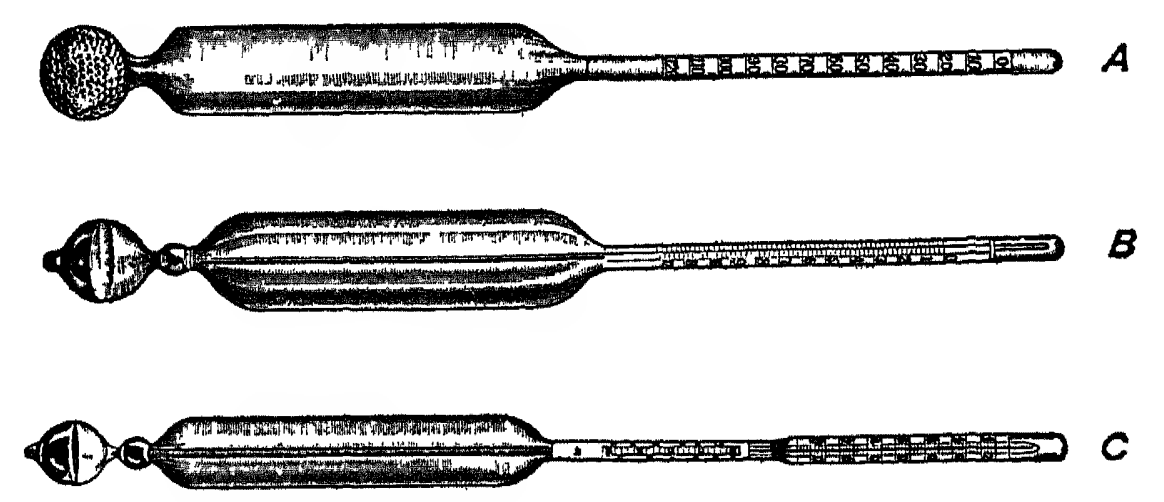

FIG. 66-Types of Lactometers. Type

$A$, New York Board of Health Type, B, Same as $A$ Except with Thermometer, $C$, Quevenne

venne). They are now made with blue and yellow columns. The fractions in the blue column indicate the percentage of water in the skimmed milk, and in the yellow column, in whole milk.

Determination of Ash in Milk. Weigh about $20 \mathrm{gms}$. of milk in a tared dish and, after adding 6 c.c. of nitric acid, evaporate to dryness, ignite just below redness.

Determination of Fat in Milk. Babcock Test. This rests on the fact that concentrated sulphuric acid will dissolve the solids not fat in milk. The fat is unchanged and may be separated in a centrifuge as follows: Special bottles are necessary and into each should be placed 18 gms. (17.6 c.c.) of the well-shaken sample of milk. By means of a special pipette add 17.5 c.c. of commercial sulphuric acid with a specific gravity of about 1.83. During the addition of the sulphuric acid, the contents of the bottle should be constantly shaken. The bottle should now be placed in a centrifuge and whirled for five minutes at about 1000 r.p.m. After this, boiling water should be added to the Babcock bottle, in order to raise the fat column into the neck of the 
bottle. The bottle should be whirled again for about a minute and then the percentage of fat read by means of a pair of calipers. This test is adapted to ice cream and cream with the exception that a larger bottle is used.

Leffmann-Beam Test. The distinctive feature is the use of fusel oil, the effect of which is to produce a greater difference in surface tension between the fat and the liquid in which it is suspended, and thus promote its readier separation. This effect has been found to be heightened by the presence of a small amount of hydrochloric acid.

The test bottles have a capacity of about 30 c.c. and are provided with a graduated neck, each division of which represents 9.1 per cent by weight of butter fat.

Fifteen centimeters of the milk are measured into the bottle, 3 c.c. of a mixture of equal parts of amyl alcohol and strong hydrochloric acid added and mixed. Then 9 c.c. of concentrated sulphuric acid is added in portions of about 1 c.c.; after each addition the liquids are mixed by giving the bottle a gyratory motion. If the fluid has not lost all of its milky color by this treatment, a little more concentrated acid must be added. The neck of the bottle is now immediately filled at about the zero point with one part sulphuric acid and two parts water, well mixed just before using. Both the liquid in the bottle and the diluted acid must be hot. The bottle is then placed at once in the centrifugal machine; after rotation from one to two minutes, the fat will collect in the neck of the bottle and the percentage may be read off.

Gerber's Test. This test is applied as follows: The test bottles are put into the stand with the mouths uppermost; then, with the pipette designed for the purpose, or with an automatic measurer, 10 c.e. of - sulphuric acid are filled into the test bottle, care being taken not to allow any to come in contact with the neck. The few drops remaining in the tip of the pipette should not be blown out. Then 11 c.c. of milk are measured with the proper pipette and allowed to flow slowly onto the acid, so that the two liquids mix as little as possible. Finally, the amyl alcohol is added. (It is important to use the reagents in the proper order, which is-sulphuric acid, milk, amyl alcohol. If the sulphuric acid is followed by amyl alcohol and the milk last, then the result is sometimes incorrect.) A rubber stopper, which must not be damaged, is then fitted into the mouth of the test bottle, and the contents are well shaken, the thumb being kept on the stopper to prevent it coming out. As a considerable amount of heat is generated by the action of the sulphuric acid on the milk, the test bottle should be wrapped in a cloth. 
The shaking of the sample must be done thoroughly and quickly, and the test bottle inverted several times, so that the liquid in the neck becomes thoroughly mixed. By pressing in the rubber stopper the height of the liquid can be brought to about the zero point on the scale.

If only a few samples have to be analyzed and the room is warm, the test bottles can be put into the centrifuge without any preliminary heating, otherwise the test bottles must be warmed for a fow minutes (not longer) in the water bath at a temperature of $60^{\circ}$ to $65^{\circ} \mathrm{C}$. When the tempcrature rises higher than this, say above $70^{\circ} \mathrm{C}$., the rubber stopper is liable to be blown out of the test bottle. After the test bottles have been heated they are arranged symmetrically in the centrifuge and whirled for three to four minutes at a speed of about 1000 r.p.m. When the centrifuge has a heating arrangement attached to it, the preliminary warming is not, of course, neccssary. When the test bottles are taken out of the centrifuge, they are again placed in the water bath at a temperature of $60^{\circ}$ to $65^{\circ} \mathrm{C}$., and left there for several minutes before being read; where the centrifuge is heated, the tubes can be read off as taken from the centrifuge.

By carefully screwing in the rubber stopper, or even by pressing it, the lower limit of the fat column is brought onto one of the main divisions of the scale, and then, by holding the test bottle against the light the height of the column of fat can be accurately ascertained. The lowest point of the meniscus is taken as the level when reading the upper surface of the fat in a sample of whole milk, and the middle of the meniscus for separated milk.

If the column of fat is not clear and sharply defined, the sample must be again whirled in the centrifuge.

Each division on the scale is equivalent to 0.1 per cent, so it is very ' easy to read to 0.05 per cent, or, with a lens, to 0.025 per cent. If the number which is read off is multiplied by 0.1 , then the percentage quantity of fat in the milk is obtained; e.g., if the number on the scale was 36.5, then the percentage of fat is 3.65. (Milk and Dairy Products, Barthel; translated by Goodwin, p. 71.)

Arla-Jensen (1916) advises the use of chemically pure amyl alcohol.

\section{Proteins in Milk}

Procedure. Measure 5 c.c. of the milk into a 500 c.c. Kjeldahl flask delivering it to the bottom of the flask. Add 25 c.c. of concentrated sulphuric acid and $0.7 \mathrm{gm}$. mercuric oxide or the equivalent of pure mercury. Heat the flask directly over a free flame in a hood until the 
contents are yellow or white. After the flask has cooled add about 200 c.c. of ammonia-free water, 20 c.c. of a potassium sulphide solution (40 gms. potassium sulphide per liter of water) and a small piece of zinc. Connect the flask to a condenser and, after adding carefully 50 c.c. of a 60 per cent of sodium hydroxide solution, distill, receiving the distillate in standard acid solution. Titrate the excess of acid with standard alkali, using methyl orange and compute the amount of nitrogen in the milk. This percentage of nitrogen multiplied by 6.25 will roughly give the percentage of protein.

Test for Coloring Matter in Milk. Shake about 10 c.c. of the milk with 5 or 10 c.c. of ether. Fresh milk will give a colorless supernatant ether layer, while, if coloring matter has been added to the milk, this ether layer will be colored.

Detection of Preservatives. The refusal of milk to sour when placed in a warm place is evidence that a preservative has been added. The following preservatives are often found in milk and their detection may be carried out as follows:

Benzoic Acid. Add a few drops of lime water to 300 c.c. of the sample and reduce the volume to about 75 c.c. Mix powdered calcium sulphate with this to a paste and evaporate to dryness. When dry, grind and moisten with dilute sulphuric acid; shake out in 50 per cent alcohol. Repeat this three times and reduce the acid alcohol solution to small volume on the water bath after adding barium hydroxide to neutrality. Acidulate with sulphuric acid and extract with ether. When the ether is evaporated, the benzoic acid is left behind. This precipitate may be subjected to further study.

Boric Acid. Add sodium hydroxide to 75 or 100 c.c. of the milk and evaporate to dryness. Fuse and dissolve the ash in water. Add a little hydrochloric acid and filter. Moisten a strip of turmeric paper with the filtrate and dry on a watch glass. A red color indicates boric acid or its salts.

Salicylic Acid. Add a little sulphuric acid to 15 or 20 c.c. of the milk and shake out with ether. Evaporate to dryness and treat the residue with alcohol and ferric chloride. The presence of salicylic acid is indicated by a violet color.

Formaldehyde. To 5 c.c. of the milk add 5 c.c. of distilled water and 10 c.c. of hydrochloric acid, containing a little ferric chloride. The presence of formaldehyde is indicated by a violet color.

Detection of Heated Milk. Storch's Method. Five c.c. of milk are poured into a test tube; a drop of weak solution of hydrogen dioxide (about 0.2 per cent) which contains about 0.1 per cent of sulphuric acid 
is added, and two drops of a 2 per cent solution of paraphenylendiamin (solution should be renewed quite often), then the fluid is shaken. If the milk or cream becomes, at ance, indigo blue or the whey violet or reddish brown, then this has not been heated, or, at all events, it has not been heated higher than $78^{\circ} \mathrm{C}$.; if the milk becomes a light bluish gray immediately or in the course of half a minute, then it has been heated to $79^{\circ}$ to $80^{\circ} \mathrm{C}$. If the color remains white, the milk has been heated at least to $80^{\circ} \mathrm{C}$. In the examination of sour milk or sour buttermilk, lime water must be added as the color reaction is not shown in acid solution. (Report of the Commission on Milk Standards.)

Arnold's Guaiac Method. A little milk is poured into a test tube and a little tincture of guaiac is added, drop by drop. If the milk has not been heated to $80^{\circ} \mathrm{C}$. $\left(176^{\circ} \mathrm{F}\right.$.) a blue zone is formed between the two fluids; heated milk gives no reaction, but remains white. The guaiac tincture should not be used perfectly fresh, but should have stood a few days and its potency have been determined. Thereafter it can be used indefinitely. These tests for heated milk are only active in the case of milks which have been heated to $176^{\circ} \mathrm{F}$. or $80^{\circ} \mathrm{C}$. (Jensen's Milk Hygiene, Pearson's translation, p. 192.)

Microscopic Test for Heated (Pasteurized) Milk. Frost and Ravenel (1911). About 15 c.c. of milk are centrifuged for five minutes, or long enough to throw down the leucocytes. The cream layer is then completely removed with absorbent cotton and the milk drawn off with a pipette, or a fine-pointed tube attached to a Chapman air pump. Only about $2 \mathrm{~mm}$. of milk are left above the sediment which is in the bottom of the sedimentation tube.

The stain, which is an aqueous solution of safranin 0 , soluble in water, is then added very slowly from an opsonizing pipette. The important thing is to mix stain and milk so slowly that clotting does not take place. The stain is added until a deep opaque rose color is obtained. After standing three minutes, by means of the opsonizing pipette, which has been washed out in hot water, the stained sediment is then transferred to slides. A small drop is placed at the end of each of several slides and spread by means of a glass spreader, as in Wright's method for opsonic index determinations.

In an unheated milk the polymorphonuclear leucocytes have their protoplasm slightly tinged or are unstained.

In heated milk the polymorphonuclear leucocytes have their nuclei stained. In milk heated to $63^{\circ} \mathrm{C}$. or above, practically all of the leucocytes have their nuclei definitely stained. When milk is heated at a 
lower temperature the nuclei are not all stained above $60^{\circ} \mathrm{C}$. The majority, however, are stained.

Frost (1915) has pointed out some of the difficulties of the above method and devised a new one.

Procedure. Add one part of aqueous methylerie blue ( $\bar{\tau}$ gms. of Gruebler's dry dye to 100 c.c. distilled water) to five parts of milk. This should be added slowly to prevent coagulation of the milk. Allow the stain to remain in contact with the milk for from fifteen to thirty minutes. Centrifuge and spread the sediment on a slide. This may be done by using the edge of another shde. Frost reports the following appearance in raw and pasteurized milk. Raw: The entire field is stained a light blue, the depth of the stain depending on the thickness of the film. In this blue background may be clear areas. These may be either leucocytes or fat globules. The leucocytes are practically always colorless. Heated Milk: Smears made from heated milk are not as deeply stained as those made from raw milk. The leucocytes are always more decply stained than the background and appear as dark blue areas in a bluc ficld. The background immediately about the leucocytes frequently shades off into the color of the background, thus forming a halo about them. Under the oil immersion objective the leucocytes are less regular in outline than those in raw milk. The nuclei are distinctly stained.

Balaz's Test for Boiled Millk. To 5 c.c. of the milk; add 2 c.c. of copper sulphate solution which contains $69.26 \mathrm{gms}$. of copper sulphate per liter. Shake and filter. Then, to five drops of the transparent filtrate add Adamkiewicz's reagent (one part of sulphuric acid and two parts of acetic acid). Warm carefully without boiling, shake, and allow to stand for a while. The serum of the boiled milk remains colorless, while the raw milk shows a violet red color. Its greatest intensity occurs within ten or fifteen minutes.

The "Commission on Milk Standards Appointed by the New York Milk Committee" has made a careful study of the production of safe milk. It is composed of the foremost authorities on the relation of mill to the public health. Realizing the lack of uniformity with regard to milk standards, the committee made the following recommendations.

Chemical Standards. The following are proposed and represent those which are enforced in many cities and States.

The Babcock test makes easily practicable the determination of fat and solids-not-fat in milk. Such examinations of milk can be readily adopted and executed by any health board laboratory at a very moderate expense. It is believed that such chemical standards as are suggested 
will help to raise the standards of dairying in this country, and that the provision regarding substandard milk is a liberal one.

Cow's Milk. Standard milk should contain not less than 8.5 per cent of solids-not-fat and not less than 3.25 per cent of milk fat.

Skim Milk. Standard skim milk should contain not less than 8.75 per cent of milk solids.

Cream. Standard cream should contain not less than 18 per cent of milk fat, and should be free from all constituents foreign to normal milk. The percentage of milk fat in cream over or under that standard should be stated on the label.

Adjusted Milks. On the question of milks and creams in which the ratio of the fat to the solids-not-fat has been changed by the addition to or subtraction of cream or milk fat the commission has hesitated to take a position. On the one hand they are in favor of every procedure which will increase the market for good milk and make the most profitable use of every portion of it. On the other, they recognize the sensitiveness of milk, the ease with which it is contaminated, and the difficulty of controlling such processes as standardizing, skimming, homogenizing, souring, adjusting, etc., so as to prevent contamination and the use of inferior materials. On this subject the commission passed a resolution presented by a special committee, as follows:

The committee believes that it is probably necessary to admit standardized and adjusted milk. They believe that such manipulation should be controlled and that such milk should be distinctly labeled as to its modifications.

Milk in which the ratio of the fat to the solids-not-fat has been changed by the addition to or subtraction of cream should be labeled "adjusted milk"; the label should show the minimum guaranteed percentage of fat and should comply with the same sanitary or chemical requirements as for milk not so standardized or modified.

The committee very carefully considered the subject of the agitation which has taken place regarding percentage of solids-not-fat due to the fact that in some large cities much of the milk contains less than 8.5 per cent solids-not-fat. While the commission is disposed to admit that these conditions may exist, yet it believes that these conditions can be remedied, if not immediately at least gradually. On the other hand, experience has shown that to lower the standard would, in a few years, result in the lowering of the general quality of the milk placed on the market, since commerce always tends to approach the minimum standard. The commission, therefore, thinks it is unwise to reduce the standard for solids-not-fat below the percentage of 8.5. In those communities where such a standard can not be rigidly enforced at the 
present time the commission suggests that the standard be gradually applied.

\title{
The Bacterial Examination of Milk
}

The value of the bacterial examination of milk is somewhat dependcnt upon the application of the results and the opinions of those who are interpreting them. The Commission on Milk Standards regards the bacterial content as being due to dirt, temperature and age. The bacterial examination, then is supposed to yield information bearing on these three factors.

Initial Contamination. Milk is such an excellent medium for baco teria that the initial infection determines the number which will be present after a time. The factors which influence this may be mentioned as follows:

\author{
I. Cow \\ a. Udder. \\ b. Coat, etc. \\ II. Barn Conditions \\ III. Milker \\ IV. Utensils \\ a. Pails, cans, etc. \\ b. Special apparatus \\ 1. Separator \\ 2. Clarifier \\ 3. Pasteurizer \\ 4. Cooler \\ 5. Bottler
}

It is fairly well established that milk as it is excreted from the udder is not sterile. Harding and Wilson (1913) and other workers to whom they refer in their paper furnish much information to confirm this state ment. An examination of 1230 samples from the udders of 78 cows showed an average of 428 bacteria per cubic centimeter. Evans (1916) studied 192 samples of milk from 161 cows in dairies which were widely separated. Three types of bacteria were found commonly present in milk from the five dairies which were studied: streptococci, staphylococci and bacilli. Streptococcus lacticus (Kruse) was not found in any of the samples. This author states that "there is a definite udder flora comprising bacteria which belong to parasitic types. It is not 
surprising that the majority of udder bacteria should be of the same type as those common on the skin and mucous membranes of man and animals. The majority of the staphylococci on the skin are of the nonvirulent variety which fails to produce pigment and fails to ferment mannite. But pathogenic varieties are also found on the skin where they ordinarily cause no trouble. . . Whatever the variety may be, conditions in the udder are favorable to multiplication and frequently. large numbers are eliminated in the milk." Rogers and Dahlberg (1914) in studying the origin of streptococci in milk decided that they were 'contributed by the feces; infected udders and the animals' mouths were also mentioned. Licking of the flanks and udders allow the bacteria to reach the milk. Sherman (1915) found 19 out of 142 cows from 6 different herds that gave a milk with over 10,000 bacteria per cubic centimeter. Two of them had a bacterial content of 100,000 bacteria per cubic centimeter in their fresh milk. Sherman states that all of the cows were in a normal healthy condition but he does not state what data were secured to arrive at this conclusion. In 48 cows, in which there were no indications of disease, there were streptococci in the milk of 15. He regards the streptococci of milk as of little significance, which is a very interesting statement in light of the work on scptic sore throat. Colwell (1917) studied the influence of high count and gargety cows on the number of bacteria in milk. Seventy-two per cent of the cows examined produced a milk with less than 10,000 bacteria per cubic centimeters. The other 28 per cent were classed as high-count animals. An interesting fact was established that, where one or more of the quarters were infected with an organism, the same organism could be isolated from the other quarters. Such data have much significance in the question of the relation of milk to septic sore throat; it points out that all of the milk from an animal with an infected udder must be excluded from the milk supply.

From the literature which has beeen presented above, it will be seen that some difference of opinion exists about the udder flora. The predominating organisms seem to be micrococci and, as Evans pointed out, may come from the cow's body. These enter the teat and reproduce since they are in a favorable environment. With regard to high-count cows too little information is available to definitely understand them. They are excluded from certain herds when certified milk is being produced. No exacting study is usually made to determine whether they are really healthy. If nothing is outwardly the matter with them, they are usually considered healthy without any other examination. It is possible that the large numbers of bacteria in the millk of certain cows 
could be explained by a more careful investigation with regard to the condition of the animal.

Barn Conditions. Some difference of opinion still exists with regard to the effect of barn conditions on the bacteria in milk. Harding et al. (1913) found that many procedures about the barn which were supposed to limit the number of bacteria contributed to milk might, under some conditions, increase the count. Ruehle and Kulp (1915) have shown that dusty air in a stable might increase the bacteria in milk but the increase, as such, would be of little sanitary importance. If these added bacteria developed, the increased count, at a later time, would come within sanitary considerations. Savage (1909-10) found a general agreement between the cleanliness of cows' stables and the number of bacteria in milk. This also was borne out by the work of Brainerd (1911).

Utensils. Recent work points to the conclusions that utensils may be one of the most important factors determining the number of bacteria in milk. Pease (1916) claims that, where high counts have been obtained in a dairy which has operated for a long time, they are due to inefticiently cleaned apparatus and by incubation of the bacteria on the moist surfaces of the cans, pails, ctc. Prucha, Harding and Weeter (1915) reported some interesting data to substantiate this contention of Pease. With sterile utensils and sterile bottles, milk leaving the barn was found to contain 2588 bacteria per cubic centimeter and the bottled milk 3875 . Where the utensils were simply washed and with only the bottles sterile, there were increases due to the pails of 57,077 , up to the clarifier of 15,353 , due to the clarifier of 172,763 , due to cooler of 19,841 and due to the bottler of 247,611. More data on the same subject have been since reported by Prucha, Weeter and Chambers (1918). Ayers, Cook and Clemmer (1918) in a careful study of the factors involved in the production of a milk of low bacterial content, state as follows: "Three simple factors were necessary for the production of milk with a low bacterial content, namely, sterilized utensils, clean cows with clean udders and teats, and the small top pail." A fourth factor, holding the milk at a temperature near $10^{\circ} \mathrm{C}$. or lower is necessary in order to keep the bacterial count low. Even cow manure was found to be of less importance in influencing the bacterial count than utensils.

Age. The bacterial count is supposed among other things to yield information with regard to the age of the milk. Up to certain limits this is true, but soon a limit is reached beyond which no further growth may take place unless something happens to disturb the conditions. 
The number of bacteria is probably not proportional to the age of the milk.

Temperature. The effect of temperature on the bacteria in milk has received attention from several standpoints. No single extensive piece of work has been carried out on this subject and consequently our knowledge must come from the various separate pieces of work. Luxwolda (1911) found that milk kept at low temperatures contained an enormous number of bacteria. These did not cause an acid reaction in the milk but the changes were regarded as more dangerous than if the acid bacteria had been present. At $20^{\circ} \mathrm{C}$. lactic acid bacteria appeared and exerted a restraining action on the other forms. This restraining action was steadily diminished as the temperature was lowered. Reed and Reynolds (1916) inoculated sterile flasks of milk with pure cultures and incubated them at four different temperatures for six weeks. The results are shown in Table XL.

TABLE XL

EFFECT OF AGE AND TEMPERATURE ON BACTERIAL GROWTH IN MILK

(After Reed and Reynolds)

\begin{tabular}{|c|c|c|c|c|}
\hline \multirow{2}{*}{ Name of Organism. } & \multicolumn{4}{|c|}{$\begin{array}{l}\text { Age in Days, of MrLk Kept at Various Tempera- } \\
\text { TuRes, Which GaVe Maximum Counts on NuTrient } \\
\text { Agar. }\end{array}$} \\
\hline & $\begin{array}{c}\text { Incubator, } \\
35^{\circ} \mathrm{C} \text {. }\end{array}$ & $\begin{array}{c}\text { Room, } \\
15-28^{\circ} \mathrm{C} .\end{array}$ & $\begin{array}{c}\text { Water Tank, } \\
13^{\circ} \mathrm{C}\end{array}$ & $\begin{array}{l}\text { Cold Stor- } \\
\text { age, }-1^{\circ} \mathrm{C} .\end{array}$ \\
\hline Bacterium lactis acidi.......... & 1 & 3 & 5 & 3 \\
\hline Sarcina lutea................ & 1 & 42 & 42 & 21 \\
\hline Bacillus coli.. $\ldots \ldots \ldots \ldots \ldots \ldots$ & 3 & 5 & 5 & 2 \\
\hline Bacillus cyanogenes............ & 2 & 21 & 4 & 42 \\
\hline Bacillus proteus vulgaris. . . . . . . . & 2 & 2 & 4 & 3 \\
\hline Bacillus aerogenes . . . . . . . . . . & 3 & 5 & 42 & 3 \\
\hline Bacillus fluorescens liquefaciens. . . & 2 & 5 & 42 & 3 \\
\hline Bacillus puditum. . . . . . . . . . & 3 & 4 & 5 & 21 \\
\hline Microspira tyrogena........... & 3 & 21 & 42 & 42 \\
\hline Bacillus subtilis . . . . . . . . . . & 3 & 42 & 42 & 21 \\
\hline Micrococcus citricus. . . . . . . . . . & 21 & 5 & 4 & 4 \\
\hline Oidium lactis $\ldots \ldots \ldots$ & 42 & 42 & 5 & 42 \\
\hline Bacillus prodigiosus. . . . . . . . . . . & 3 & 42 & 42 & 42 \\
\hline
\end{tabular}

Reed, $\mathrm{H}$ S and Reynolds, R. R. Some effects of temperature upon the growth and activity of bacteria in milk. Virginia Ag. Exp. Sta. Bull. (Tech.), 10, 1916.

Conn and his co-worker Esten (1902, 1903, 1904) gave much attention to this subject. Their work was some of the earliest on this subject. In general, they found that at $20^{\circ} \mathrm{C}$. the ordinary lactic acid 
bacteria predominated. At the end of forty hours, when the milk had curdled, Bacillus acidi lactici constituted about 90 per cent of the total bacteria. At $37^{\circ}$ C., B. lactis aerogenes predominated over $B$. acidi lactici as did $B$. coli when it was present. At $10^{\circ} \mathrm{C}$. all types of bacteria developed uniformly. Neutral bacteria grew more rapidly and liquefiers became abundant. Conn and Esten found little difference in the flora at $10^{\circ} \mathrm{C}$. and $1^{\circ} \mathrm{C}$. Growth, however, was more rapid at $10^{\circ} \mathrm{C}$. Ravenel et al. (1910) have studied the changes in milk at $-9^{\circ} \mathrm{C}$. and $0^{\circ} \mathrm{C}$. At $-9^{\circ} \mathrm{C}$. there was no increase in the number of bacteria as determined on agar and gelatin plates. At $0^{\circ} \mathrm{C}$. there was a decided increase. This was accompanied by an increase in acidity and other changes incidont to marked bacterial development. The lactic acid bacteria were inhibited $s \hat{t}$ this temperature but putrefactive bacteria were not.

Pennington et al. $(1908,1913)$ in their studies on colc? storage reported that milk stored at $0^{\circ} \mathrm{C}$. underwent marked proteolysis which was noticeable at the end of two weeks. In a later paper it was stated that proteolysis of casein was due primarily to bacteria while the lactalbumin was destroyed by the enzymes in the milk. The numbers of bacteria greatly increased and this increase was most striking in the raw, untreated milk. The freezing-point was gradually lowered with the decomposition. Market milk held below $0^{\circ} \mathrm{C}$. increased from 15,956 to $376,000,000$ at the end of five weeks. These investigations tend to indicate that milk and other foods may not be held indefinitely even at low temperatures. Changes may eventually take place to render the food unfit for consumption.

"What is Meant by Quality in Milk?" Quality in any food substance may be defined in different ways. The definition often depends entirely on the viewpoint of the definer. The farmer may have quite another conception of quality in milk than the sanitarian; the chemist may define milk quality from the standpoint of food value while the bacteriologist is concerned with disease. It is a far easier propositior to define milk quality than to devise methods for measuring it. One could scarcely find a field in hygiene which is more complicated than that of dairy and milk sanitation. The difficulty of finding practical methods for measuring quality in milk has caused much contention among health authorities. Probably little difficulty would be experienced in reaching a definition for safe milk. A milk to be safe must be clean. In fact cleanliness is the slogan of modern sanitation.

A number of different methods have been used or proposed for measuring quality in milk. Each one has its own special advantages and 
objections. Some have been discussed before in this book and will not be given further attention. The following methods have been proposed at one time or another:

1. Sanitary inspection of the dairies;

2. Bacterial examination of the milk;

3. Dirt or sediment test.

Harding et al. (1917) consider that the elements of quality in city milk to consist of food value, healthfulness, cleanliness and keeping quality. The discussion of these factors leads these authors to the following combination of them to form a basis for grading milk. Park

Gpecial milk $\begin{cases}\text { Element of QUALITy Deod value } & \text { Fat content as stated on package } \\ \text { Healthfulness } & \text { Medical supervision of health of men and } \\ & \text { anmals, or proper pasteurization } \\ \text { Cleanliness } & \text { Sediment, not more than a trace } \\ \text { Keeping quality } & \text { Excellent }\end{cases}$

Table milk $\begin{cases}\text { Food value } & \text { Fat content as stated on package } \\ \text { Healthfulness } & \text { Properly pasteurized } \\ \text { Cleanliness } & \text { Sediment, not more than a small amount } \\ \text { Keeping quality } & \text { Good }\end{cases}$

Cooking milk $\begin{cases}\text { Food value } & \text { Fat content as stated on package } \\ \text { Healthfulness } & \text { Boiled } \\ \text { Cleanliness } & \text { May not be sufficient for table grade } \\ \text { Keeping quality } & \text { May not be sufficient for table grade }\end{cases}$

(1918) does not approve some of the claims in this report. He calls attention to the fact that Harding and his collaborators are interested in the dairy instead of the infant. , He further states, "The experts ... were considering the best methods of grading milk and not the question as to whether excessive numbers of bacteria in market milk were wholesome or not. It would be, to my mind, a step backward to accept the views expressed in the body of the editorial." (From a letter discussing this report.) Park and his colleagues at the Research Laboratories of the New York City Department of Health have given much study to the reiation of milk bacteria to disease in children.

The Dairy Score Card. No attempt will be made to present in this place a complete discussion of the dairy score card. The score card had 
its origin in 1904 when Doctor W. C. Woodward prepared one for use in Washington A year later Pearson at Cornell University devised a similar card. The score card received such approbation by sanitarians that the National Association of Dairy Instructors appointed a committee which had much to do with the present official score card. Much has been written in favor of and against the card. It is regarded by some milk hygienists as one of the most important factors in the production of clean milk. Others, even though they represent large cities do not regard the card as of any great help in milk control. It must be emphasized that the score card was not devised to give information with regard to bacteria in milk but merely to rate the production plants. It has been established, generally speaking, that a high scoring dairy will have a low number of bacteria in its milk. This is to be expected. Some of the recent work in dairy hygiene has emphasized the fact that the present score card lays too much stress on factors which are not directly concerned with the production of milk with few bacteria. Brew (1915) made a comparative study of the bacteria in the milk and the scores of thirty-four commercial dairies. He found no correlation between these two factors. The apparent reason for this was that a large number of items called for on the score card have little or no effect on the bacteria in the milk. Too much emphasis is placed on procedures which do not affect the milk. This study indicates that the score card needs revision if it is to be an index of the bacteria in milk. As indicated by recent studies, within certain limits, the environment of the cow does not seem to exert much influence on the milk. Prucha, Harding and Weeter (1915) also confirm the contention of Brew that the score card needs revision. They found that utensils were important in relation to the number of bacteria in milk. These are not given an important place on the card. Ayers, Cook and Clemmer (1918) reach the same conclusion. Brainerd and Mallory (1914) in studying the Richmond, Virginia, milk supply by means of the bacterial count and score card found the count to be cumbersome-a high count not always being characteristic of a dangerous milk. For some reason which was not determined, they found that the highest scoring dairy showed a larger count than the lowest scoring dairy. North (1917) believes that the life of the present score card is threatened if it is not changed to give a reasonably close indication of the character of the product of dairies. North has presented a revised score card on which he has tried to place emphasis on factors which are concerned in keeping the number of bacteria in milk low. This to a certain extent may change the purpose of the card. 


\section{BOARD OF HEALTH}

RACINE, WIS.

\section{Sanitary Inspection of Dairies}

Dairy Score Card

Owner or lessee or farm.

P. O. Address. State.

Total Number of cows Number milking.

Gallons of milk produced daily.

Product is retailed by producer in.

Sold at wholesale to.

For milk supply of

Permit No

Date of Inspection.

, 191.

REMARKS.

(Signed).

Inspector. 
DAIRY SCORE CARD

DETAILED SCORE

\begin{tabular}{|c|c|c|c|c|c|}
\hline \multirow{2}{*}{ Equipment. } & \multicolumn{2}{|c|}{ SCORE. } & \multirow{2}{*}{ Methods. } & \multicolumn{2}{|c|}{ Score. } \\
\hline & Perfect. & Allowed. & & Perfect. & Allowed. \\
\hline 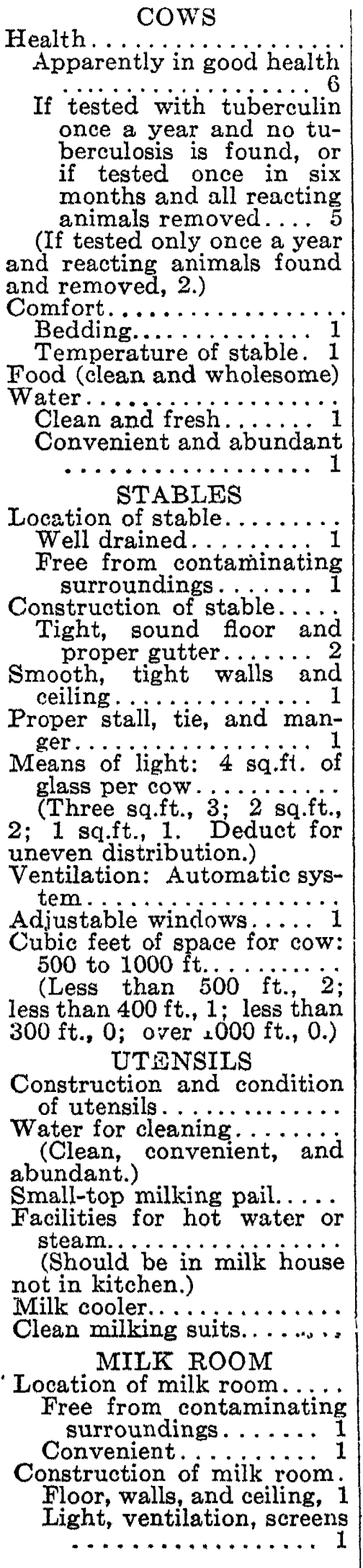 & $\begin{array}{l}3 \\
1\end{array}$ & $\ldots \ldots$ & 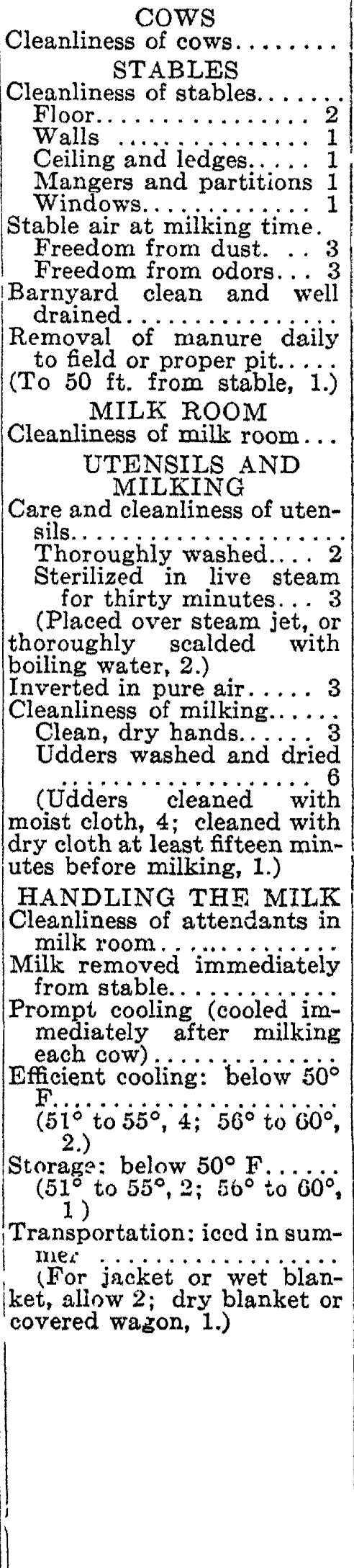 & $\begin{array}{l}1 \\
2 \\
2 \\
5 \\
3\end{array}$ & {$\left[\begin{array}{l}\ldots \ldots \\
\ldots \ldots \\
\ldots \ldots \\
\ldots \ldots \\
\ldots \ldots\end{array}\right.$} \\
\hline Total.......... & 40 & $\ldots \ldots \ldots$ & Total........... & 60 & \\
\hline
\end{tabular}

Equipment. .............. Methods $\ldots \ldots \ldots \ldots \ldots=\ldots \ldots \ldots \ldots \ldots$ Final score. Nore 1. If any filthy condition is found, particularly dirty utensils, the total score shall be limited to 49 .

- Noxe. 2.- If the water is exposed to dangerous contamination, or there is evidence of the presence of a dangerous disease in animals or attendants, the score shall be 0 . 
Dirt or Sediment in Milk. The estimation of dirt in milk has been regarded by many as a reliable index of milk quality. Most attention has been given to insoluble dirt or that which may be removed from the milk by some mechanical means. Schroeder (1914) has given a résumé of the different methods of determining dirt in milk. He stated that the amount of dirt in fresh milk approximates the number of bacteria. This was borne out by the work of Reed and Reynolds whose data bearing on this question have been presented in Table XLI. Campbell (1916) carried out an interesting study on the relation of the number of bacteria to the amount of dirt and stated that the amount of dirt which appeared on the disks was no criterion of the number or kind of bacteria in the milk. Ayers, Cook and Clemmer (1918) found that"the "sediment test bore a somewhat close relation to the number of bacteria in fresh, unstrained milk handled in sterilized utensils." The kind of dirt is, of course, important in this connection. Cow dung would probably be an objectionable type. Ayers and his colleagues, however, state that cow dung (fresh) "though an important source of contamination in general, is not so great a factor as unsterilized utensils in causing high bacterial counts." Kinyoun (1914) regards cleanliness in production as the most important factor in milk hygiene. From an examination of 3000 samples of milk he states that in good milks, there is 1 colon bacillus to every 50,000 bacteria while in dirty milk the ratio is 1 to 555 .

TABLE XLI

RELATION BETWEEN THE AMOUNT OF DIRT IN MILK AND THE BACTERIAL CONTENT

(After Reed and Reynolds)

\begin{tabular}{|c|c|}
\hline Amount of Dirt in 100 c.c. of Milk. & $\begin{array}{l}\text { Number of Bacteria per } \\
\text { Cubic Centimeter of Milk. }\end{array}$ \\
\hline 10 milligrams. . . . & 4,833 \\
\hline 20 milligrams .... & 6,750 \\
\hline 30 milligrams...$\ldots$. & 12,000 \\
\hline 40 milligrams $\ldots \ldots \ldots$ & 17,625 \\
\hline 50 milligrams $\ldots \ldots \ldots \ldots \ldots \ldots \ldots \ldots \ldots$ & 18,375 \\
\hline 100 milligrams. . . . . . . . . . . . . . . . . . & 32,875 \\
\hline
\end{tabular}

The available data seem to indicate that milk may be produced under decidedly filthy conditions and yet have a low bacteria content. The utensils seem to be the contributing factor. These data should not be taken to prove that barns may be kept dirty. Milk, which is produced in dirty plants, is more liable to contain dirt; this dirt may be 
very objectionable. It is reasonable that a farmer who is careful about the condition of his cows will be just as careful about the other features which are known to affect milk quality.

Schroeder (1914) has classified the methods of determining dirt in milk as follows:

Group I. (a) Type in which the sediment is obtained by gravity filtration.

Conn's filter paper method.

The Lorenz or Wisconsin tester.

The Stewart tester.

The Gerber tester.

The Schroeder tester, or multiple filter.

(b) Type in which pressure or suction is used.

The Lorenz improved.

The Wizard.

The Gooch crucible.

Group II. Type in which sediment is obtained by means of the centrifuge.

The Babcock.

The Gerber.

The Stewart-Slack.

Conn's centrifugal method.

Significance of the Bacterial Count. There is no agreement on this important question. The Commission on Milk Standards makes the following statement on the subject:

Bacterial Counts and Decency. On this subject the commission passed the following resolutions:

(a) Because high bacterial counts indicate milk is either warm, dirty, or stale, the bacterial count is an indicator of decency in milk character, entirely apart from its significance as an indicator of the safety of milk.

(b) In determining the sanitary character of milk and the grade in which it belongs, decency must be considered as desirable for its own sake, entirely apart from the consideration of safety. Decency is important as a characteristic of foods and drinks, because it gives pleasure to the consumption of food, while the lack of decency means distaste, displeasure, and even disgust.

(c) The bacterial count is a sufficiently accurate measure of decency to justify the health officer in condemning milk with a high bacterial count because it is lacking in this characteristic.

Bacteriological Laboratory Testing of Milk. On the subject of laboratory examinations of milk for bacteria the commission believes that the interest of 
public health demand that the control of milk supplies, both as to production and distribution, should include regular laboratory examinations of milk by bacteriological methods. They stated by resolution that-

Among present available routine laboratory methods for determining the sanitary quality of milk, the bacterial count occupies first place. and that bacterial standards should be a factor in classifying milk of different degrees of excellence.

The adoption and enforcement of bacterial standards will be more effective than any other one thing in improving the sanitary character of public milk supplies. The enforcement of these standards can be carried out only by the regular and frequent laboratory examinations of milk for the numbers of bacteria it may contain.

It is of the utmost importance that standard methods should be adopted by all laboratories for comparing the bacterial character of milks, since by this means only is it possible to grade and classify milks and properly enforce bacterial standards.

Concerning the methods which should be used by milk laboratories for determining the numbers of bacteria the commission unanimously resolved:

That there be adopted as standards for making the bacterial count the standard methods of the American Public Health Association Laboratory Section.

One of the chief objections raised against pasteurization is the claim that it is frequently employed to cover filthy methods, the milk producer using less care in his methods if he knows that the milk is to be subsequently pasteurized. To meet this objection the commission believes there should be bacterial standards for raw milk as well as bacterial standards for pasteurized milk. In the case of pasteurized milk, standards should be required of the milk before pasteurization as well as after pasteurization.

Reliability of Bacterial Tests. The commission has considered the numerous criticisms that have been raised as to the unreliability of bacteriological analyses, and has made extensive inquiry as to the force of these criticisms. An opinion concerning the reliability of laboratory tests for numbers of bacteria has been reached based on voluminous statistics secured for the most part by groups of observers working together, as well as by individuals. One of these researches alone carried out by members of the commission in cooperation with others included the testing of over 20,000 samples of milk. In other instances repeatedly the same sample of milk was tested one hundred times. Some variations in the analysis of duplicate samples are inevitable, due to the fact that the bacteria are not in solution, but are floating in the milk more or less clustered together in clumps, each of which will count only as a single colony. Under such conditions only an approximate agreement can be expected.

The results of extensive study justify the commission in the conclusion that the analysis of duplicate samples of milk made by routine methods in different laboratories may be expected to show an average variation of about 28 per cent, with occasional samples of wider variation. In some good laboratories the variation may not be greater than 10 per cent. Variations in results diminish witb 
the numbers of samples analyzed. If five samples of the same milk are tested the results may be relied upon as fairly accurate, and always sufficiently accurate to place any particular milk supply unhesitatingly in Grade A, B, or C. The object of bacterial tests of milk samples for the numbers of bacteria should be primarily to determine the sanitary character of the milk supply from which the sample is taken, rather than the character of a single sample of milk. It is strongly urged by this commission that no grading of milk should be made upon the analysis of single samples, and that no prosecutions or court cases should be brought upon the bacterial analysis of a single sample of milk.

Interpretation of Bacterial Tests. The commission has put its opinions on this subject in the form of resolutions, as follows:

Whereas milk is one of the most perishable foods, being extremely susceptible to contamination and decomposition; and

Whereas the milk consumer is justified in demanding that milk should be clean, fresh, and cold, in addition to having the element of safety; and

Whereas milk which is from healthy cows and is clean, fresh, and which has been kept cold, will always have a low bacterial count; and

Whereas milk that is dirty, stale, or has been left warm, will have a high bacterial count; therefore it is resolved:

First. That the health officer is justified in using the bacterial count as an indicator of the degree of care exercised by the producer and dealer in securing milk from healthy cows and in keeping the same clean, fresh, and cold; and

Second. "That the health officer is justified in condemning milk with a high bacterial count as being either unhealthy or decomposed, or containing dirt, filth, or the decomposed material as a result of the multiplication of bacteria due to age and temperature.

Third. That the health officer is justified in ruling that large numbers of bacteria are a source of possible danger, and that milk containing large numbers of bacteria is to be classed as unwholesome, unless it can be shown that the bacteria present are of a harmless type, as, for example, the lactic acid bacteria in buttermilk or other especially soured milks.

Grading by the Bacterial Count. Concerning the number of tests which should be made in order to determine the grade of a milk supply, the commission recommends that the grade into which a milk falls shall be determined bacteriologically by at least five consecutive bacterial counts, taken over a period of not less than one week, nor more than one month, and that at least four out of five of these counts ( 80 per cent) must fall below the limit or standard set for the grade for which classification is desired.

The grading of milk has necessarily been based on its sanitary character, primarily as determined by the bacterial test. The enforcement of grading, therefore, requires the application of the bacterial test in a manner sufficiently comprehensive to fairly determine the sanitary character of milk so that it may be assigned to the grade in which it belongs. Such an administrative system greatly modifies the former conception of milk inspection by public health officials. The inspection service under the grading system becomes subordinate 
to the bacterial laboratory, or at least must look to the bacterial laboratory as a guide. If bacterial tests are recognized as an indication of the sanitary character of milk, then the bacterial laboratory tests should precede the dairy inspection since they will point out to the dairy inspector the location of unsanitary milk. In the enforcement of the grading system, therefore, the milk inspection service should be reorganized in such a manner that the bacterial laboratory makes its tests first, in order to determine the sanitary character of the various milks offered for sale on the city market, and the inspection service then takes up the task of discovering the location and causes of the defects which the laboratory has discovered and of remedying them. The laboratory service and inspection service consequently must be centralized under one head and their work thoroughly coordinated in order to give the greatest economy and effciency.

It is quite apparent from the above rather lengthy quotation from the report of the Commission on Milk Standards, that they regard the bacterial count as of much importance. Contrary to this is the opinion of Harding (1917) and a few others who maintain that the number of bacteria in milk has no relation to the conditions under which the milk was produced. In order to back up this stand the following argument has been used. "The most offensive, and, at the same time, a typical form of filth that may get into milk is cow dung. The bacterial plate count resulting under standard methods of milk analysis gives rather less than 5,000,000 per gram as the germ count of fresh cow dung. A gram of such material added to a liter (approximately $1 \mathrm{qt}$.) of milk would, accordingly, increase the germ count but 5000 per cubic centimeter. Hence, otherwise low-content milk might carry approximately 2 gms. of cow dung per quart and still not exceed the ordinary limit of 10,000 per cubic centimeter for certified milk. Likewise a quart of Grade A milk, which is understood to be clean milk, according to the present accepted standards might contain 12 gms. of cow dung per quart and still be legal Grade A milk. . . . In establishing bacterial count standards as an index of cleanliness, we are lending official sanction to conditions which would outrage public decency and are creating a false sense of security and of cleanliness." It is common knowledge that fresh cow dung has a very variable bacteria content. Usually it is much higher than 5,000,000 per gram and may even approach or exceed a billion. Ayers et al. (1918) found an average bacterial content of 50,000,000 in 57 samples of fresh cow dung. This is probably dependent upon what the cow eats, for with human beings the diet has much to do in determining the bacterial flora. Then, again, fresh cow dung does not ordinarily gain entrance to milk. It is the dry dung 
which happens to fall into the milk from the cow's body and this has a bacteria content which is far different from fresh cow dung. In this connection, Taylor (1918) has shown that 85 per cent of fresh cow dung will dissolve in milk. His studies were carried out with the fresh (wet) cow feces and it required a little restriction to apply his results directly to dirt in milk. Fresh cow dung contains about 85 per cent of moisture and this water ought not to be considered as cow feces. Prucha, Weeter and Chambers (1918) carried out a series of investigations on this subject using dried feces. In discussing this question of bacterial counts in milk Pease $(1913,1916)$ claims that "none of the bacteria found on our counting plates are even recognized by bacteriologists as belonging to the groups of those microorganisms which produce the wellknown specific diseases." His general opinion seems to be that the dairy score card representing sanitary inspection of the dairy should come before the bacterial count and that any attempt to make it of secondary importance to the count is a decided step backwards. North $(1913,1916)$ has stated that confusion exists over the question of dirt and disease and that this has caused some misunderstanding. North (1917) has presented a revised score card and has tried to obviate some of the discrepancies inherent in the present one. In his earlier papers North states that there has been an undercurrent of competition between dairy inspection and bacterial testing and suggests that a system of cooperation be worked out between these two factors. One cannot help but be impressed with the fair-minded stand of this investigator.

Rogers (1915) claims that, how much a high bacterial count is due to contamination and how much is due to multiplication is uncertain. He suggests that it may become necessary to distinguish between measures to reduce the bacterial count and measures in the interest of decency and cleanliness. Conn (1917) has well stated the question. "The bacteriological analysis of milk is not to be taken as indicating in itself either a condition of safety or a condition of danger, but only as a warning. Good, clean, fresh milk will have a low bacterial count, and a high bacterial count means dirt, age, disease or temperature. A high bacterial count is, therefore, a danger signal and justifies the health officer in putting a source with a persistently high bacterial count among the class of unwholesome milk." Rosenau (1912) makes the following statement on this subject:

The enumeration of bacteria in milk is, therefore, one of the readiest and cheapest methods at the disposal of the health officer to determine the general sanitary quality of the market milk supply. The laboratory results serve not 
only as a guide to direct the efforts of the health officer, but to confirm the conclusions arrived at from an inspection of the dairie and dairy farms.

A review of the literature with regard to the bacterial analysis of milk indicates a confused condition. There seem to be two distinct views with regard to the significance of the number of bacteria in milk. That side supporting the view that the bacterial content should not be used is made up of those who are interested in the production of milk and possibly the "protection" of the farmer. The other side is supported by well-known physicians and public health officials who have been engaged for some time in protecting the consuming public from dirty foods. Park and Williams (1910) have shown a close relationship between infant death rate and the number of bacteria in milk. These data were secured after careful experimentation and observation. Numerous data have been secured in this connection when comparing the value of raw and pasteurized milk in infant feeding. In essentially all of the cases, the pasteurized milk has been shown to be superior and this is probably not all due to destruction of certain harmful bacteria. A milk or any other food which contains large numbers of bacteria, is more liable to contain undesirable forms than one with a low count. If the streptococci are of any sanitary significance and there are data on record which support the theory that they are, they are more liable to be present in millks with a large number of bacteria. Breed and Brew (1917), in studying the control of market milk by the microscopic examination, found that one-fifth of all the milk which contained bacteria in excess of $1,000,000$ per cubic centimeter contained large numbers of streptococci. They state that, "So far as was determined, all of the milk of this type was originally infected from the udders of some one or more cows in a herd." In this case, then, the number of the bacteria in the milk was closely related to undesirable conditions at the source of production. The greater part of the argument against the bacterial count seems to rest upon data which have been produced at several experiment stations to show that many of the barn conditions which have, in the past, been supposed to be very important, are not related to the number of bacteria in the milk when it reaches the consumer. These investigators argue that the quality of city milk is too complex a problem to be solved by the consideration of any one factor. They, therofore, put down the four essential factors as food value, healthfulness, cleanliness and keeping quality. From a bacteriological viewpoint the element of cleanliness is the most important because the others may depend upon this one. This element is supposed to be measured by the sediment or visible dirt. 
No attention is given to the "invisible dirt" which may be present. The sediment test of Weld (1907) detects only one kind of dirt-the insoluble or visible. It does not show the amount of invisible dirt or insoluble foreign matter, such as urine; this must be regarded as filth in the same way as other matter. Special mention is made of the "extreme sensitiveness of the public in this matter." It is open to question whether the public is sensitive to dirt in milk or any other food product. If it was so the filthy establishments where food is on sale would not be open for business very long. The absence of dirt in milk may not indicate that the milk has been produced under favorable conditions. That no dirt is visible, does not indicate a safe milk. This question, doubtless, needs more consideration from all standpoints.

Grades of Milk. The commission believes that all milk should be classified by dividing it into three grades, which shall be designated by the letters of the alphabet. It is the sense of the commission that the essential part is the lettering and that all other words on the label are explanatory. In addition to the letters of the alphabet used on caps or labels, the use of other terms may be permitted so long as such terms are not the cause of deception. Caps and labels shall state whether milk is raw or pasteurized. The letter designating the grade to which the milk belongs shall be conspicuously displayed on the caps of bottles or the labels of cans.

The requirements for the three grades shall be as follows:

Grade A. Raw Milk. Milk of this class shall come from cows free from disease as determined by tuberculin tests and physical examinations by a qualified veterinarian, and shall be produced and handled by employees free from disease as determined by medical inspection of a qualified physician, under sanitary conditions, such that the bacterial count shall not exceed 10,000 per cubic centimeter at the time of delivery to the consumer. It is recommended that dairies from which this supply is obtained shall score at least eighty on the United States Bureau of Animal Industry score card.

Pasteurized Milk. Milk of this class shall come from cows free from disease as determined by physical examinations by a qualified veterinarian, and shall be produced and handled under sanitary conditions, such that the bacteria count at no time exceeds 200,000 per cubic centimeter. All milk of this class shall be pasteurized under official supervision, and the bacteria count shall not exceed 10,000 per cubic centimeter at the time of delivery to the consumer. It is recommended that dairies from which this supply is obtained shall score at least 65 on the United States Bureau of Animal Industry score card.

GRADE B. Milk of this class shall come from cows free from disease as determined by physical examinations, of which one each year shall be by a qualified veterinarian, and shall be produced and handled under sanitary con- 
ditions, such that the bacteria count at no time exceeds $1,000,000$ per cubic centımeter. All milk of this class shall be pasteurized under official supervision, and the bacterial count shall not exceed 50,000 per cubic centimeter when delivered to the consumer.

It is recommended that dairies producing grade B milk should be scored, and that the health departments or the controlling departments, whatever they may be, strive to bring these sources up as rapidly as possible.

Grade C. Milk of this class shall come from cows free from disease, as determined by physical examinations, and shall include all milk that is produced under conditions such that the bacteria count is in excess of $1,000,000$ per cubic centimeter.

All milk of this class shall be pasteurized, or heated to a higher temperature, and shall contain less than 50,000 bacteria per cubic centimeter when delivered to the consumer.

Whenever any large city or community finds it necessary, on account of the length of haul or other peculiar conditions, to allow the sale of grade $\mathrm{C}$ milk, its sale shall be surrounded by safeguards such as to insure the restriction of its use to cooking and manufacturing purposes.

\section{MrLk Standards}

If it is difficult and intricate to formulate standards for various foods, it is especially true with regard to milk. To a great extent, this is due to the various number of standpoints from which the milk question may be considered. The farmer is interested more in securing a reasonable financial return on the investment in his milk production plant. The sanitarian, however, often gives little attention to the farmer's side of the question and considers only the effect of the milk on the health of the consumer. Undoubtedly, a broader consideration by any one of these specialists would greatly clear up some of the misunderstanding with regard to certain phases of the milk question.

Milk standards are essential but the question with regard to who shall formulate them is still an open one. If a study is made of the milk standards which are being enforced by many of our large cities, the great discrepancy is apparent. There is essentially no agreement whatever in many of these standards. The Commission on Milk Standards in their third report has attempted to establish a simple system for grading milk and to distinguish between milks which are different in their sanitary and other characteristics. The variation in bacterial suandards for milk analysis is emphasized very well in Reprint No. 192, May 15, 1914, from the Public Health Reports 29. The standards 
for many cities in the United States having a population of 10,000 or over are tabulaterl.

The procedure for the bacteriological examination of milk has been standardized in the same way as has the bacteriological examination of water and sewage. Standard Methods of Bacteriological Analysis of Milk prepared by the Laboratory Section of the American Public Health Association are now used by all bacteriologists making routine analyses of milk and wishing their results to be comparable to those secured in other laboratories. For that reason, much of the following is adapted or copied, with permission, from that report:

Routine Milk Analysis. This type of analysis is designed for the control of the public milk supply and for the purpose of grading milk. For routine analysis the work must be capable of being done quickly and cheaply, and in such a way as to give the most speedy results possible and by methods having the smallest possible expenditure of time and money consistent with fair uniformity of result. The demand for rapid methods, with the smallest possible expenditure of time and money has been forced on different laboratories in the past few years, and has brought about various short cuts from the standard methods as previously formulated. The necessity for such rapid and inexpensive methods must be recognized. But it is also evident that they should be uniform in different laboratories.

Research Methods. Collection of Samples. All collecting apparatus, glassware, pipettes, collecting tubes, bottles, etc., shall be sterilized at a temperature of at least $175^{\circ} \mathrm{C}$. for one hour. Each sample shall consist of at least 10 c.c. of milk. Before taking the sample the milk shall be mixed as thoroughly as possible. If the original container can be inverted the mixing of the milk should be done by inverting it several times. If this is impossible, the milk should be stirred with some sterile stirrer. Any stirrer already in the container may be used. If there is none in the container, the sampling pipette (or any other sterile article) may be used; but it should be used for one container only until it is again sterilized.

A sample simply poured from a large can is not a fair sample unless the milk in the can be thoroughly stirred. The sample shall be taken from the cans by means of a tube with straight sides long enough to reach to the bottom of the original container, and inserted not too rapidly, with the bottom of the tube left open. This will result in the tube containing a cylindrical section of the milk from top to bottom of the can. The finger then placed on the top of the tube will make it possible to withdraw the tube full of milk and transfer it to the sampling bottle. The sample bottle shall be large enough to hold the entire contents of the tube, all of which must be reserved as the sample. Each tube shall be used for collecting a single sample only and must be washed and sterilized again before being used again. An aluminum tube of the diameter of $\frac{1}{4}$ in. and 21 ins. long is very convenient. If the sample is to be taken from the bottle, 
the bottle should bo first hhaken to insure thorough mixing and then the milk may be poured into the sample bottle, although it is better here to use a sampling tube.

If the temperature of the milk is desired, it should be taken from a separate sample that should then be discarded. All records shall be made immediately after taking the sample. The milk sample shall be placed in a properly labeled bottle. The most convenient kind of sample bottles are glass stoppered, or those closing with a cork-lined screw cap. Cotton plugs are not a satisfactory method of closure. The sample bottles shall be placed at once in a carrying case containing cracked ice, so that the milk is cooled at once to near the freezing-point.

The samples shall be transferred to the laboratory as quickly as possible, and shall be plated at once. If the samples can be placed in melting ice and water, they may be kept for several hours (12) without an increase in bacteria. If the plates are not made within four hours of the time of collection, the number of hours that have elapsed should be stated on the report. If the milk is kept at $40^{\circ} \mathrm{F}$. a slight increase may be found in twelve to twenty hours. Up to twenty hours this will not be more than 20 per cent.

Medra. This is essentially identical with that used for water analysis and has been fully treated in the chapter on that subject. Sherman (1916) has demonstrated that lactose agar gives a much higher count than plain agar plates for enumerating bacteria in milk. Lactose agar also of some value in differentiating the types of bacteria which are present. Sherman (1915) has recommended that the reaction of agar media used in the bacterial analysis of milk should be 0.5 per cent plus.

Plating. For miscellaneous samples the character of which is not known, three dilutions shall be made 1-100,1-1000 and 1-10000. Where the character of milk is known the number of dilutions may be reduced. If the milk is pasteurized, certified or known to be fresh. and of high grade, the 10,000 and 100 dilutions may be omitted; if the milk is known to be old, the 100 dilution may be omitted. In no case shall less than two plates be made of each sample. Any convenient method of making dilutions may be used always using pipettes and sterile water blanks. The water for dilutions may be placed in dilution bottles (99 c.c. and 9 c.c. are convenient sizes) and sterilized for one hour in an autoclave at 15 lbs. pressure.

These standard methods do not give the maximum number of bacteria. Some research workers in order to secure the maximum number of bacteria incubate their plates at $37^{\circ} \mathrm{C}$. for five days and at $20^{\circ} \mathrm{C}$. for two days. Such an incubation period of seven days' duration is obviously too long for routine work. Slack (1917) has advised the incubation of agar plates made from milk in routine analysis for forty-eight 
hours rather than twenty-four hours because on an average the count is doubled or trebled. In this connection he arlvises meat infusion agar in place of meat extract agar. Sherman (1916) recommended the addition of dextrose or lactose to agar used in the examination of milk. A much higher count was obtained and the colonies grew much larger.

Incubation. Only one period of incubation and one temperature is regarded as standard, forty-eight hours at $37.5^{\circ}$. C. In crowded incubators ventilation shall be provided.

Counting. If among the different dilutions, there are plates containing from 30 to 300 colonies these should be counted, and the number multiplied by the dilution, be reported as the final count. All colonies on such plates should be counted and the numbers averaged. If there are no plates within these limits the one that comes nearest to 300 is to be counted, unless it happens that there are no other plates with a larger number of colonies, or, unless the numbers in the plates check with other dilutions. 'If the number of colonies on the plate is over 300 , a part of the plate may be counted and the whole plate averaged. Counting shall be done with a lens magnifying 2.5 diameters (or what opticians call a $2.5 \times$ lens. Near-sighted persons shall use their glasses in counting while far-sighted persons shall remove them. In case it is doubtful whether certain objects are colonies or dirt specks they should be examined under a compound microscope.

Reports. In making reports it must be borne in mind that with high numbers obtained by routine methods, only an approximation to accuracy can be obtained. Only the left-hand figures of the final numbers are significant. It is best, therefore, to report only the two left-hand figures of the results, in order to avoid an unwarranted impression of accuracy. For example, where the numbers are in the millions no figures smaller than millions have any significance in the routine analysis of milk. In making the report raise the number to the next highest round number, but never lower it. In no case shall the count of a single plate be regarded as sufficient for the purpose of grading milk. If a single sample of milk only is to be tested, there should be at least three plates counted before a report is made.

Microscopic Method of Analysis. This method has been brought to such a stage by Breed and his colleagues that it is now included in the Standard Methods of Bacteriological Milk Analysis of the Laboratory section of the American Public Health Association, 1917. Brew has summed up the advantages and disadvantages of the plate and direct microscopic method as follows: 
ADVANTAGES AND DISADVANTAGES OF THE DIRECT MICROSCOPIC METHOD AS COMPARED WITH THE PLATE METHOD

Disadvantages

Direct Microscopic Method

1. Difficult to measure accurately such a small quantity of milk.

2. The sample measured is too small to be representative.

3. Dead bacteria may be counted.

4. Error of count is great where bacteria are few or many.

5. Cannot be used for quantitative work when bacteria are few in number.

6. Many fields must be counted, because of the uneven distribution, if an accurate count is desired.

7. Large compact clumps cannot be counted.

8. Bacteria may be lost in the process of preparing slides.

Advantages

\section{Direct Microscopic Method}

1. Less apparatus required, therefore less expensive. Technique simple.

2. The results on a given sample may be reported in a few minutes.

3. Shows the cell content, the presence or absence of streptococci and other important things necessary in estimating the sanitary quality of milk.

4. Gives a better idea with regard to the actual number of germs present.

\section{Plate Method}

1. All bacteria do not grow on the plates because of changes in food temperature relations, or other conditions of environment.

2. The difficulty of breaking up the clumps in the milk affects the accuracy of the count.

3. Requires from two to five days' incubation period.

4. Different species require different incubation temperatures.

5. Gives no idea of the morphology of the bacteria present.

6. More apparatus required, therefore more expensive. Technique complicated and difficult for the trained bacteriologists to use in such a way to secure consistent results.

Samples. Milk samples collected, as above described, may be preserved by icing and handled as in the case of the plate method. All samples on which the cream has risen to the surface must be vigorously shaken before preparations are made from them.

Apparatus. In addition to a microscope and ordinary microscopic slides, stains, etc., the only special apparatus required is a pipette 
which measures $1 / 100$ c.c. The most convenient form of pipette is the straight capillary pipette, calibrated to deliver $1 / 100$ c.c., the graduation mark being $1 \frac{1}{2}$ to $2 \frac{1}{2}$ ins. from the tip. Such pipettes are now for sale by manufacturers, and can be easily obtained. The calibration should be tested by weighing with chemical balances the amount of milk discharged from the tube. Only a single pipette is needed in making a series of tests, provided this is kept clean while in use. In this kind of work cleanliness rather than sterilization is required. Clean towels may be used for wiping the exterior of these pipettes, while their bores may be kept clean by rinsing them in clean water between each sample. The small amount of water left in the tube may be rinsed out into the milk sample under examination. This method of procedure, while adding a small number of bacteria to each sample, introduces only a theoretical error, tests showing that such bacteria cannot subsequently be detected, and make no difference in the final result.

Preparation of Smears. One one-hundredth c.c. of milk or cream is deposited upon a clean glass slide by means of a pipette described above. By the use of a clean, stiff needle this drop of milk is spread over an area of $1 \mathrm{sq}$. $\mathrm{cm}$. This may be most conveniently done by placing the slide upon any glass or paper ruled into areas $1 \mathrm{~cm}$. square. These marks showing through the glass serve as guides. After uniform spreading the preparation is dried in a warm place upon a level surface. In order to prevent noticeable growth this drying must be accomplished within five to ten minutes; but excessive heat must be avoided or the dry films may crack and peel from the slides in later handling.

After drying, the slides are to be dipped in xylol (gasoline may be used) for one minute, then drained and the slides dried. They are then immersed in 90 per cent grain or denatured alcohol, for one minute or more, and then trans-

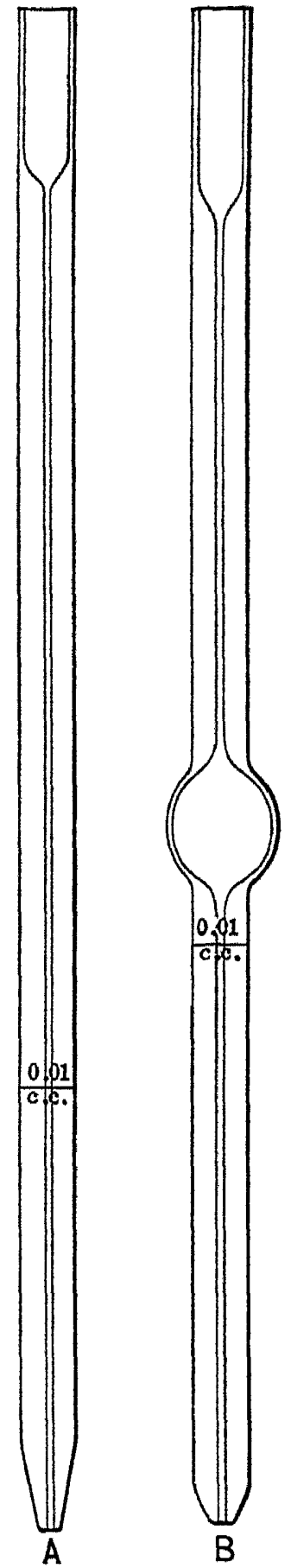

FIG. 67. - Breed's Capillary Pipettes. Several types of pipettes have been devised. Type $A$ gives as much satisfaction as any Prucha at the Ilinois Agricultura Station uses ordinary capillary tubing and standardizes the a mount of mllk deliv ered by weighing it on an analy tical balance.

blue. Old or 
unfiltered stains are to be avoided, as they may contain troublesome precipitates. The slides remain in this solution for five seconds to one minute or longer, depending upon the effect desired, and are then rinsed in water to remove the surplus stain, and decolorized in alcohol. The decolorization takes several seconds to a minuts, during which time the slide must be under observation in order that the decolorization may not proceed too far before they are removed from the alcohol. When properly decolorized the general baskground of the film should show a faint blue tint. Poorly stained slides may be decolorized and restrained as many times as necessary, without any apparent injury. After drying the slides may be examined at once, or they may be filed away and prescrved for further reference.

Standardization of the Microscope. The microscope to be used must be adjusted in such a way that each ficld of the microscope covers a certain known fraction of the total square centimeter's area. This procedure is simple, with the proper materials at hand. The microscope should have a $1.9 \mathrm{~mm}$. (1/12 in.) oil immersion objective, and an ocular giving approximately the field desired, and should preferably be fitted with a mechanical stage. To standardize the microscope, place upon the stage a stage micrometer, and by the selection of oculars or adjusting the draw tube, or both, bring the diameter of the whole microscopic field to $.205 \mathrm{~mm}$. When so adjusted, the microscope field will cover almost exactly $1 / 300,000$ of a cubic centimeter of the milk (actually 1/302,840). This means that if the bacteria in one field only are counted, the number should be multiplied by 30,000 to give the total number for a cubic centimeter. If the bacteria in a hundred fields are to be counted, the total should, of course, be multiplied by 3000 .

Inasmuch as it is difficult to count bacteria lying near the margin of the microscopic field, it is much better to have an eyepiece micrometer, with a circular ruling, $8 \mathrm{~mm}$. in diameter, and divided into quadrants. This will give, in the microscopic field, a smaller area within which the bacteria may be seen most sharply, and which may be more easily counted. Such eyepicce micrometers are now manufactured by laboratory supply houses, and may be casily obtained. In the use of this eyepiece micrometer the inner circle, by the adjustment of the draw tube, should be made to cover a circle with a diameter of $.146 \mathrm{~mm}$. In this case this inner circle will cover $1 / 600,000$ of a cubic centimeter of milk, meaning, of course, that the number of bacteria in a single field should be multiplied by 600,000 , or, if a hundred fields are counted, by 6000 , to obtain the number per cubic centimeter. 


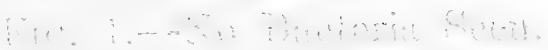

(7)...

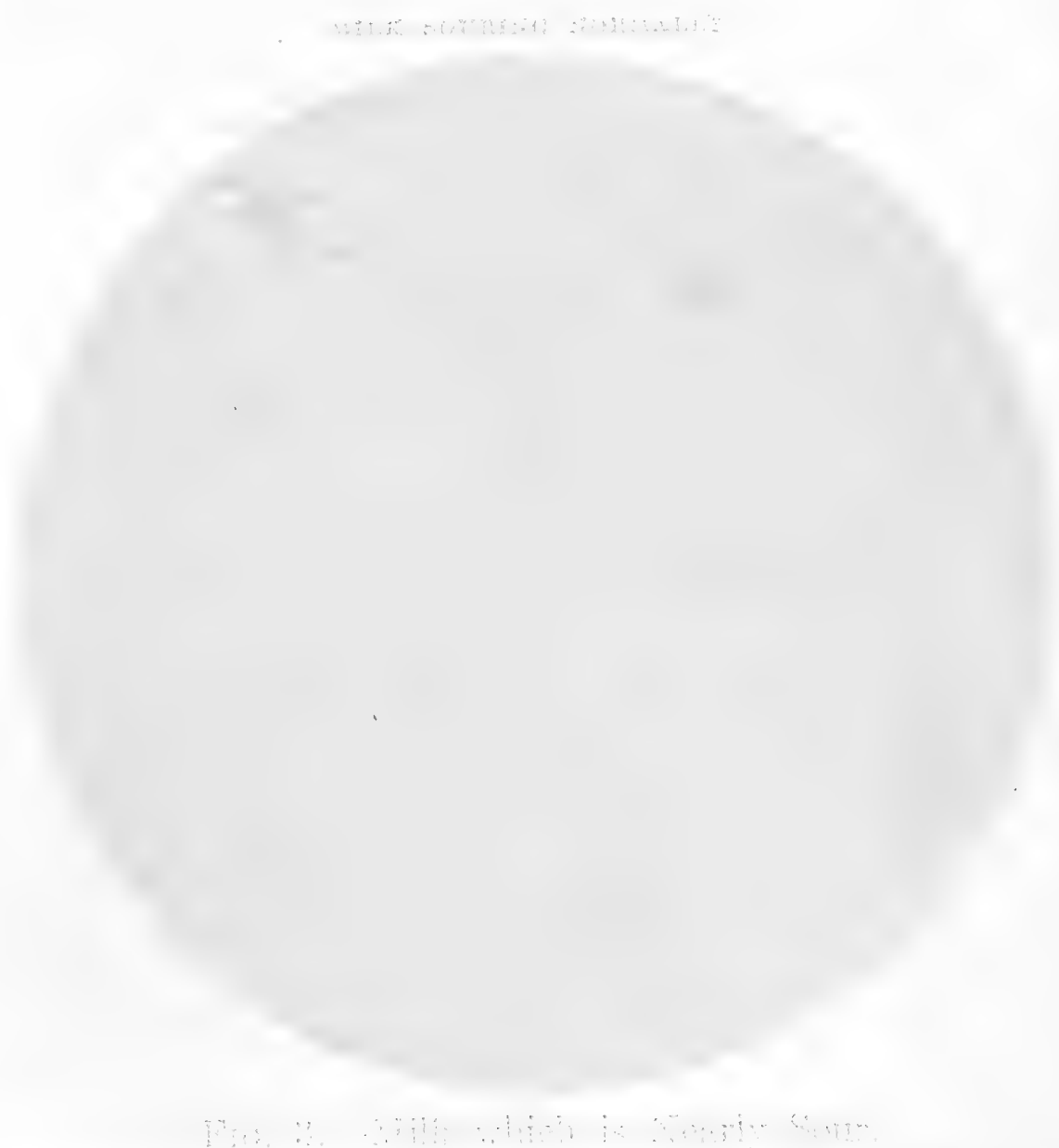

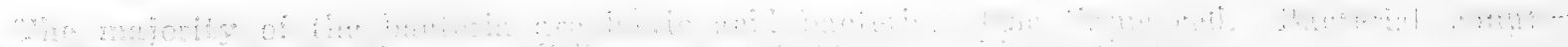
and 

The number of microseopic fields to be counted will depend somewhat upon the kind of data that are desired. If this methorl is to be used simply for the purpose of drviding milk into grades, it will in most cases be unnecessary to do the actual counting, snce a Grade A milk will show field after field without any bacteria at all, while a Crade C milk will show the field crowded with bacteria. In all doubtful cases, however, counting should be done, and there should never be less than thirty fields counted in order to have reliable results. Counting thirty fields is not so tedious a task as would seem to be, since, in ordinary milk, the number of bacteria in each field is small, and the counting may be done very rapidly.

Counting. Counting the bacteria in such a smear may be dore in two ways: 1 . The number of groups of one or more bacteria present. 2. The number of individuals. The second, of course, is really the correct count of the number of bacteria, but the former will give a count much closer to that obtained by the plate count, since the colonies upon the plate represent groups of bacteria rather than individuals, each group growing into a single colony only. Extensive tests have shown that there is a fair correspondence between the number of groups reported by experienced observers and the number of colonies that may grow in plates made from the same milk, although there are occasionally discrepancies of considerable extent. These discrepancies are caused by variations in judgment as to what constitutes a group, variations in the extent to which groups break up in the dilution waters when the smears are made, and the presence of dead bacteria or of bacteria which do not grow on the plates. Some experience is needed by the microscopist in determining just what should be counted. In high-grade milks, an inexperienced person is apt to fail to recognize differences between bacteria and other minute objects. This results, as a rule, in an overcount by inexperienced men. In milk containing many readily recognizable bacteria in each field the inexperienced man is apt to overlook some of them, giving an undercount. These difficulties are overcome, however, by training and experience.

Interpretation of Results. It must be recognized that the results obtained from the microscopic record give a closer approximation to the actual number of bacteria present in the milk than those obtained by the plate method, since the plate method will count as one, either a single bacterium or a group which may sometimes contain a hundred or even more individuals. Inasmuch, however, as the plate count has become a method of analysis that is well known and commonly applied, it becomes desirable to know as closely as possible what relations there 
may be between the plate count and the microscopic count. Experience has shown that the count of individual bacteria is ordinarily 1.5 to 8 times as great as the plate count, the ratio between the two being largely dependent upon the size of the clumps of bacteria present. Where the bacteria are mostly isolated, the ratio of the two counts would be much closer than where there are present long chains of streptococci or masses of cocci. After one has had a little experience in counting clumps it is found that the number of groups shown by the microscope, agrees fairly well with the number of colonies shown by the plate count, though even here there are occasionally discrepancies, due among other things to the appearance in the microscope of kinds of bacteria which fail to grow in the culture media used in making plates. In all cases, however, the direct count of raw milk will give a much closer approximation to the actual numbers of bacteria than the plate count. In view of these facts it is difficult to interpret one count in terms of the other; but a few suggestions will give a fairly satisfactory idea as to how the two may be related.

Grade A raw milk, which should have less than 100,000 bacteria per cubic centimeter, will not show more than three to four small clumps of bacteria for each 30 fields of the microscope where the diameter of the fields is $.205 \mathrm{~mm}$. Such milk also ought not to contain more than 500,000 individual bacteria per cubic centimeter when counted by the microscope. For Grade A pasteurized milk (which should have less than 200,000 per cubic centimeter by the plate count before pasteurization) the microscope should not show more than six to eight clumps per 30 microscopic fields, and not more than 1,000,000 individual bacteria when counted with the microscope.

Grade B milk, which is supposed not to have more than 1,000,000 bacteria before pasteurization, when counted by the plating method, should not show more than 20 individual bacteria per field, where the diameter of the fields is $.205 \mathrm{~mm}$., and not more than three to four groups of bacteria per field.

While the above relation between the plate count and the microscopic counts cannot be relied upon as having a very great amount of accuracy, it will serve to give a general idea of the ratio between the two under ordinary conditions, and may serve as a guide in the use of direct microscopic method.

The direct microscopic method is not as yet recommended by this committee as a method of estimating the numbers of bacteria that are present in samples of milk. For this purpose the plate method, which has long been in use and is fairly well understood, is still recommended as 
the standard method to be employed. For the purpose of rapidly dividing raw milk into a series of grades, in such a way that the results can be obtained in the quickest possible time, the direct microscopic method seems to be extremely useful, and the results which are obtained will, in nearly all cases, agree with those obtained by the plate count, and probably in all cases will give a closer approximation to the fair grading than the plate count can do. For these reasons the use of the direct microscopic method is extremely valuable at the dairy end of the milk route, where the farmer wishes to know the kind of milk he is producing, or the purchaser at the shipping station wishes to know the kind that he is receiving from the farmer. It is of less value at the city end of the milk route, especially if pasteurization of the milk has been introduced anywhere along the route. It is especially useful in the data it gives concerning the kinds of bacteria present in milk, since it sometimes enables the farmer quickly to pick out from his herds such cows as are discharging large numbers of streptococci, thus giving a very efficient means of protecting the milk supply from this type of organisms that are to-day recognized as suspicious and decidedly undesirable.

Goodrich (1914) finds a marked correlation between the microscopic and plate counts. He regards the factor of 20,000 which is used to reduce the microscopic count to terms of the plate count as satisfactory. Breed and Brew (1917) claim that their microscopic method is as satisfactory for controlling city milk supplies as is the agar plate method. Breed and Stocking (1917) found that the plate counts are characterized by greater regularity in the hands of laboratory assistants carrying out routine analyses of milk, than the microscopic method of Breed and Brew. Inexperienced workers are said to secure great errors in the use of the microscopic method. About the same conclusion was reached by Conn (1915). He stated that considerable experience was needed by the analyst to distinguish between bacteria and dirt particles.

The Breed method has been demonstrated by the work of different investigators to be fairly accurate with milks which have large numbers of bacteria. It may scarcely be conceded that where the bacteria are so few that many of the fields of a Breed smear contain no bacteria, the count is very accurate. In this connection the Frost microscopic plate method or Allen's microscopic method may be found to be more satisfactory. Brew has mentioned this last fact as a disadvantage of the microscopic method. To secure the greatest possible accuracy a large number of fields should be counted.

Frost's Microscopic Plate Method. This differs from the Breed Method of securing a microscopic count in that little plates are made 
instead of smears; otherwise the method is somewhat the same. In general, some of the same objections which are applied to Breed's method may be applied to the Frost method. The method is outlined by Frost in the following words:

Preparation of Glass Slides. The little plates are made on the ordinary microscopic glass slides (2.5 by $7.5 \mathrm{~cm}$.). These are carefully cleaned and are then ruled with a grease pencil so that a square surface, over the center of the slide, is surrounded by a grease line. The included area is $4 \mathrm{sq} . \mathrm{cm}$. The ruling is best done by having a wooden or cardboard frame for the slide and some arrangement, as brads, to hold the straight-edge in place. These ruled slides s re then sterilized by passing them through a gas flame, and then they are placed on a warm box $\left(45^{\circ} \mathrm{C} ., 113^{\circ} \mathrm{F}\right.$.) provided with an overhanging top to protect them from dust.

Preparation of the Plates. A ferw tubes of ordinary nutrient agar are then molted and placed in a water bath at $45^{\circ} \mathrm{C}$. to cool. Some sterile plugged test tubes are also put in this water bath to warm up (one for each sample of milk to be tested). The milk sample to be tested is then thoroughly shaken and 1 c.c. is transferred to one of the warm sterile test tubes. To this is added an equal amount (1 c.c.) of the melted agar. The milk and agar are thoroughly shaken, care being taken to prevent the agar from cooling below $40^{\circ} \mathrm{C}$. It is best to warm it up frequently by putting it into the $45^{\circ} \mathrm{C}$. bath.

One-tenth ( 0.1 c.c.) of the milk and agar mixture is then transferred to a warm glass slide and spread as quickly and evenly as possible over the ruled surface. This may be readily done with the help of a pipctte while the slide is on the warm plate. The agar is allowed to set firmly by placing it on a level surface under cover for a few minutes. This makes a little plate culture containing 0.05 c.c. of milk.

When the milk being examined is supposed to contain more than a million bacteria per cubic contimeter, it should be diluted with sterile water or sterile milk before it is mixed with the agar. The dilution may be $1: 10$. This would make the final dilution as much as $1: 200$.

Incubation. This is accomplished by placing the little plates in an incubator in a specially prepared cabinet, or moist chamber with a layer of water in the bottom to maintain a saturated atmosphere. A Petri dish may be used for a few slides. The incubation temperature is $37^{\circ} \mathrm{C}$. for a varying length of time. Five or six hours should be allowed. For samples which contain many bacteria such as market milk which has been kept in a warm place three or four hours will be 
sufficient. For pasteurized milk from ten to twelve hours may bc necessary.

Drying the Plates. When the colonies are sufficiently large the plates are dried. This should be done carefully. Too rapid drying will crack the films. They will dry well in the incubator or over a steam radiator.

Staining. This is accomplished in the following manner:

1. Fix in the flame.

2. Put in a 10 per cent solution of acetic acid in 95 per cent alcohol.

3. Stain with Loeffler's methylene blue $(1: 4)$ for three minutes

4. Decolorize in 95 per cent alcohol for a few seconds or until the background is a pale bluc and the colonies stand out prominently.

5. Dry without washing.

Counting of Colonies. For the purpose of counting the colonies, the slides may be examined with the low-power dry lens. If the structure of the colonies is to be studied it is always best to use a mounting medium and the cover glass. Cedar oil serves quite well and in that casc the cover glass may be removed for use with the oil immersion but Canada balsam may be used in the usual way. A microscopic examination of these plates reveals colonies of considerable size stained a dark blue in a light blue field. The staining process should be so regulated that the colonies will be stained a dark blue in a light blue field. At least twenty fields should be counted on each plate and the plate should be gone over carefully in the selection of these twenty fields.

Calculation. The number of bacteria per cubic centimeter may be determined by multiplying the number of colonies in a microscopic field by the number of times the area of this field is contained in 400 sq. $\mathrm{mm}$. or the area of the little plates times the dilution used. The area of the microscopic field for any definite combination of lenses and tube length is a constant and may be determined by the formula: Area $=R$. The radius may be determined by a stage micrometer. The same tube length must be used that was used in the standardization of the microscope. When the microscope factor is known this formula may be used:

$\frac{\text { Number of colonies counted }}{\text { Number of fields counted }}=$ Reciprocal of dilution of milk.

Microscope factor = number of bacteria per cubic centimeter in the milk. Example: 
$\frac{600}{2 J} \times 20 \times 200=\frac{2,400,000}{20}=120,000$ bacteria per cubic centimeter, where

$600=$ number of colonies counted;

$20=$ number of fields counted;

$20=$ dilution $-1-20$;

$200=$ microscope factor.

Frost $(1915,1917)$ has secured a satisfactory agreement between his method and the standard plate method. In his second communication, this author reports some results secured with his method during a practical test on the Boston milk supply. The table which is presented in this book shows that Frost's microscopic method gives results which are different than those obtained on the standard agar plate.

Allen's Microscopic Method for Counting Bacteria in Milk. Allen (1918) has described his method as based on the fact that a water suspension of aluminum hydroxide readily collects bacteria in milk. This may then be thrown down in one end of a centrifuge tube leaving the normal milk constituents suspended. Allen has described his technique as follows: Preparatıon of Hydroxide Suspension. Mix equal parts of $\mathrm{N} / 20 \mathrm{AlK}\left(\mathrm{SO}_{4}\right)_{2}-12 \mathrm{H}_{2} \mathrm{O}$ and $\mathrm{N} / 20$ sodium hydroxide. After precipitation is complete, wash the precipitate thoroughly by decantation using scrupulously distilled water, keeping in mind" that any dirt in the wash water will be taken up by the aluminum hydroxide and will appear in the film under the microscope. Make up to one-half the original volume and continue to dilute until a proper amount of aluminum hydroxide is obtained after centrifuging. Procedure. 1. Add to the sample of milk to be analyzed enough of the washed aluminum hydroxide precipitate suspension so that it becomes 20 per cent of the mixture. Shake thoroughly for several minutes.

2. Add 2.5 c.c. of the above mixture to a centrifuge tube holding this amount when stoppered. (Use plain centrifuge tube open at both ends for stoppers.)

3. Centrifuge for fifteen minutes at 5000 r.p.m. in a centrifuge of 10-in. diameter or give equivalent centrifugalization.

4. Pull stopper at cream end of the tube and remove the cream with a needle allowing the milk to run out. Then carefully pull the stopper at the other end of the tube and transfer the plug of aluminum hydroxide to a clean glass slide laying over glass or cardboard ruled off in square centimeters. By use of a needle or loop grind up the precipitate on the 
slide and spread it evenly over an arca of 2 sq. cm. using a loopful of sterile water if necesnary for uniform spreading.

5. Place in the incubator until nearly dry, then finish dry ing at room temperature.

6. Heat slightly above the Bunsen flame.

7. Place in a tray of toluene and agitate the tray several minutes to remove butter fat, then take the slides out of the tray and allow the toluene to evaporate from them.

8. Place the slides in 95 per cent alcohol to remove all traces of toluene.

9. Stain in 25 per cent saturated aqueous solution of methylene blue until the film reaches a proper depth.

10. Dry in the warm air above a Bunsen flame and examine under the microscope, using an oil immersion lens of which the number of fields per square centimeter has been determined.

11. Count 20 representative fields on the square centimeter and obtain the average number of bacteria per field.

12. The number of bacteria per cubic centimeter of milk is obtained by multiplying the average number of bacteria per field by the number of microscopic fields in a square centimeter. Usually there are a little over 3000 fields per square centimeter when the ordinary 1.8 oil immersion lens is used but using 3000 as an even number to multiply by is satisfactory in counting.

Allen also gives the following precautions: Care must be exercised not to use a suspension of aluminum hydroxide too strong to give too thick a smear. The thickness of the plug of aluminum hydroxide after centrifugalization should be about $1 \mathrm{~mm}$. No fat or toluene should appear in the field under the microscope. Some samples of pasteurized milk show fine particles of curd due to heating. These may be removed by filtering through a small amount of cotton. The film should be uniformly stained. Rubber stoppers should be used with the centrifuge tubes as cork stoppers will not hold the liquid in the centrifuge. If toe deep a stained film is secured, it may be decolorized sufficiently by dipping in alcohol.

Interpretation of Results. "The interpretation of the bacteriological analysis of market milk must depend upon the history of the milk. It is, therefore, difficult to give any general interpretation. The following are a few significant conclusions:

1. "Where the analysis can be made immediately after the milking the number of bacteria enables conclusions to be drawn as to the cleanliness and care in the dairy and the thoroughness in the cleaning and 
sterilizing of the milk vessels, or sometimes the presence of cows with infected udders. With properly cleaned and sterilized milk vessels and proper care in the farm and dairy the numbers of bacteria should not exceed 10,000, and may easily be brought down to 5000. Numbers beyond these in milk analyzed immediately after the milking may be regarded as an indication of unclean dairy methods, dirty and unsterile millking vessels, or to infected udders. Apart from infected udders the factors in dairying that most noticeably increase the bacteria count are unsterile milk vessels, unsterile strainers, unclean udders, and failure to cool the milk promptly.

2. "If the milk is properly cooled with ice the numbers should rot materially increase in five to seven hours. Communities within five to seven hours of their dairies should, if perfect conditions prevail, be able to obtain milk with nearly as low a count as above indicated. Hence, in such communities bacterial counts above these numbers should not be found in properly guarded milk. A count of 50,000 in such a community is an indication either of unfatisfactory dairy conditions or of failure to properly cool the milk during transportation. Night's milk, if properly cooled, can also easily be brought within thase limits if analyzed the next morning. A count of over 50,000 for a community close to the dairies must be regarded as unsatisfactory, and the number should approach the 10,000 mark for high-grade milk. In hot summer weather the difficulties of kecping low counts are greater, but cven then they need not surpass 50,000 if the milk is properly cooled.

3. "Where milk must be a longer time in transportation from the dairy there will inevitably be an increase in bacteria, depending on the length of time and the temperature. Experience has shown, however, that even in these conditions the excssively high numbers that have frequently been found in city milk are in reality duc to diseased udders, to dirty dairy conditions, to dirty and unsterile milk utensils, or culpable neglect of cooling. Moreover, such high bacterial counts at the shipping station are frequently traceable to a few dirty dairies whose milk with an abnormally high count contaminates the rest of the supply. Dirty shipping cans and warm temperatures in shipping are responsible for most of the high bacterial counts in city milk. Where the milk from healthy cows reaches the city within twenty-four hours, however, the number should not be over 100,000 in winter or 200,000 in summer, and numbers in excess of this may be regarded as due either to improper dairy conditions, dirty milk vessels, insufficient cooling or, perhaps, to diseased udders. In larger cities where much of the milk is forty-eight hours in reaching the city, higher numbers may naturally be expected; 
but even under these conditions there is no good reason why the number of bacteria should reach 1,000,000; and it may mostly be brought down to below 200,000. In such cities, therefore, milk with more than 1,000,000 bacteria must be regarded as improperly guarded either at the dairy or on its transit.

4. "For a Grade A milk higher demands should be made than for the ordinary grade. The standard set by the Milk Commission for Grade A, viz.; of 200,000 for milk to be subsequently pasteurized or for 100,000 to be used raw, is stated by that Commission to be an extreme limit for the most unfavorable conditions. Cities situated near the supplying dairies should demand a much higher standard, which should not allow over 10,000 in bacterial content in Grade A milk in communities favorably situated.

5. "For communities situated where ice is not available it may be necessary to accept a milk with a higher bacterial content; but as rapidly as possible the standard should be made to approach the limits as given above."

Relation of the Microscopic to the Plate Count. The relation of Breed's microscopic to the standard plate count has received some study. Brew (1914), after a rather extensive study of the question pointed out some interesting facts in his summary. He stated that "the relative differences between the two counts are greater where the bacteria are few in number." In such a case, there is probably an error in the microscopic count since it is difficult to see how it is well adapted to milks with few bacteria. Brew finds a greater difference when the individual bacteria on the smear are counted than when only groups of bacteria are counted. This is said to be due to the fact that a colony on a standard plate has developed either from a single bacterium or a group of bacteria. Goodrich (1914) reported a marked correlation between the two counts. He thinks that, for accurate work, more than one slide should be prepared. Brew again studied this relation with the aid of Dotterer (1917) and found that the plate counts were higher than the microscopic counts on milks with a small number of bacteria. Brew has made an attempt to explain this by " unrecognized contamination" on the standard agar plate which would increase this count and by overlooking bacteria under the microscope. This seems a rather feeble attempt to bolster up the Breed microscopic count in one of its apparent deficiencies. More reasonable does it seem that " bacteria where they are very few in number, even though well stained and conspicuous may not occur in the microscopic fields examined." This is probably one of the major objections to Breed's microscopic method. Unlike Allen's 
microscopic method, no concentration of the bacteria in the sample is made. Breed and Brew (1917) compared again, the relation of the two counts when studying the application of the microscopic method to the control of bacteria in market milk. No general agreement was observed.

Frost's method seems to have had little application under commercial conditions. Frost $(1916,1917)$ has carried some studies himself and reported a smaller variation with his "little plate" method than was observed with the standard plate.

The Allen microscopic method may overcome some of the disadvantages of the Breed method. Allen has devised a procedure which is said to be applicable to all grades of milk. With the Breed smear gross errors are involved on milks with low counts. In his original contribution Allen (1916) has shown that on milk with a count of 1000 and slightly over, he was able to check the standard plate count very closely.

Ayers' Milk Tube Method. This is a very satisfactory method for studying the types of bacteria in milk. The milk is plated on agar plates which are incubated at $37^{\circ} \mathrm{C}$. for six days. After counting the plates, each colony should be picked from the plates and transferred to a sterile tube of litmus milk. After fourteen days' incubation these tubes are examined and as a result of the reactions shown, the bacteria picked from the plates are divided into five groups: the acid-forming, acid-forming and coagulating, inert, alkali-forming, and peptonizing. Ayers has used this method in studying the flora of different milk products.

\section{LeUCOCYTES IN MILK}

These cells in milk were first given much attention by Stokes and Wegefarth (1897). It was noticed that they had much resemblance to the white corpuscles and from this it was concluded that they represented "pus" from the cow's udder. Breed (1914) has given a good summary of the literature besides reporting the results of his own investigations. He used the direct microscopical method for counting the cells first suggested by Prescott and Breed (1911) and later applied to the counting of bacteria in milk by Breed (1911). No relationship, if any exists, was established between the number of cells discharged and bacterial infections of the udders. Breed (1913) reports a milk having the enormous number of $54,300,000$ cells per cubic centimeter. This milk when partaken of was found to have a normal taste and the imbiber suffered no evil after effects.

Stokes' Method for Leucocytes in Milk. Centrifugal sediments from 10 c.c. of milk are stained and examined under the one-twelfth oil 
immersion objective. The presence of cells in such a field was regarded by Stokes as justification for excluding an animal from a herd.

Reed's Method for Leucocytes in Milk. 1. Fill 10 c.c. centrifuge tubes with milk and heat for ten minutes at $70-75^{\circ} \mathrm{C}$.

2. Centrifuge the tubes at high speed for ten minutes. Remove the upper layers of cream and milk with a pipette and refill the tubes with distilled water. Centrifuge again for three or four minutes.

3. Draw off all except $\frac{1}{2}$ c.c. of liquid in the point of the centrifuge tube. Wipe out the upper part of the tube with a bit of absorbent cotton fastened to the end of a glass rod. Mix thoroughly the remaining liquid and sediment.

4. Transfer a drop of this mixture to a clean Thoma-Zeiss bloodcounting cell and place the cover glass over it. Count the cells under a one-sixth objective. If the number of leucocytes is low, the entire area of the cell should be counted, using a mechanical stage to move the slide. If their number is large, five or six small squares may be counted and averaged. The average number per small square multiplied by 200,000 will give the number of leucocytes per cubic centimeter in the original milk.

Savage's Method for Leucocytes in Milk. (Savage, 1914.) The ordinary Thoma-Zeiss blood-counting chamber is employed. Direct counting of the cells is impossible owing to the opacity caused by the large amount of fat. One c.c. of the milk is accurately transferred to a centrifugal tube (about 15 c.c. capacity) of the usual pattern, and freshly filtered Toisson's solution is poured in to almost fill the tube. The two fluids are well mixed and then centrifugalized for ten minutes. The cream is well broken up by a clean glass rod, to disentangle leucocytes carried to the surface, and the mixture centrifugalized for an additional five minutes. All the fluid is then removed down to the 1 c.c. mark, great care being taken not to disturb the deposit. This can be conveniently and readily done by means of a fine glass tube connected to an exhaust pump. Theoretically, all the cellular elements present in the original 1 c.c. of milk are now present in the 1 c.c. of fluid. The deposit is thoroughly well mixed (with a wire), and disturbed through the 1 c.c. A sufficient quantity is placed on the ruled squares of the Thoma-Zeiss apparatus, and the cover glass put on. The number of cells is counted in a number of different fields of vision, moving regularly from one field of vision to another. The diameter of the field of vision is ascertained before counting by drawing out the microscope tube until an exact number of sides of the squares spans a diameter of the field of vision. 
The number of cellular clements per cubic millimeter of milk= $\frac{56,000 y}{11 d^{2}}$, where $y=$ the average number per field of vision, $d=$ the number of squares which just spans the diameter. $d$ is determined once for all by marking the nicroscope draw tube so that only 20 fields have to be counted, and the figures substituted in the formula.

Doane-Buckley Quantitative Method for Estimating Leucocytes in Milk. With this method 10 c.c. of milk are centrifuged for four minutes in graduated sedimentation tubes, at an approximate speed of 2000 r.p.m. The cream is lifted out with a cotton swab, care being taken to get as much as possible of the fat. It is then centrifuged one minute more and the cream again removed with a cotton swab. Any fat remaining in the milk interferes seriously with the counting, as, if there are more than a few globules they form a layer on the top of the liqiuid In the counting chamber, and, as the leucocytes settle to the bottom of the chamber, it is difficult to see through the fat. It is only with cows giving milk difficult of separation where this trouble is experienced, and with such animals considerable care is necessary in removing all the cream gathered at the top of the sedimentation tube. The method of removing the fat with cotton is the best one that has occurred to us, and it is the only part of the process that does not operate with entire satisfaction in every instance.

Following the removal of the cream, after the second centrifuging, the bottom of the tube will contain a portion of the sediment which is easily seen. This sediment may, in extreme cases of cows suffering from garget amount to as much as 1 c.c. Ordinarily it will be considerably less than $\frac{1}{2}$ c.c. The amount varies considerably with the number of leucocytes, but not absolutely. The milk above this sediment is removed with a small siphon, which can be easily arranged with bent glass tubes drawn to a fine point and supplied with a small rubber end pinch cock. In using the siphon it is better to keep the point near the surface of the milk in the tube in order not to agitate the precipitated leucocytes and draw a number of them off with the milk. The milk in the tube may be siphoned within an eighth of an inch of the sediment in the tube. This will usually be below the $\frac{1}{2}$-c.c. mark. Two drops of saturated alcoholic solution of methylene blue are then added, thoroughly mixed with the sediment by shaking, and then set in boiling water two or three minutes to assist the leucocytes in taking the color. The contents of the tube can be boiled by holding it directly in the flame, but it has no advantage over the use of the water bath, and it is very likely to break the glass. After heating, some water is added to the tube to 
render the color less dense. Ordnarily filling the tube to the 1 c.c. mark will be sufficient, and this quantity gives an easy factor for calculating the final results.

In putting this liquid containing the leucocytes into the blood counter considerable care is necessary, owing to the tendency of the leucocytes to sink to the bottom. At this place a capillary tube is used, and the cover glass was held in one hand ready to cover the chamber as soon as the drop "was transferred to the counting counter. After placing the glass cover over the chamber, about a minute is allowed the leucocytes to settle to the bottom of the chamber. There are very few foreign bodies likely to be mistaken in counting for leucocytes. Ordinarily the polynuclear leucocytes predominate and the stained nuclei with the unstanned surrounding cell show up very distinctly. A few small leucocytes with large nuclei may be found and these may be confounded with yeast cells untll the worker becomes familiar with the distinction.

As regards counting we have taken a standard with a cubic centimeter as a basis quantity of milk, though we are, of course, aware that the corpuscles in the blood are enumerated with a cubic millimeter basis. We adopted the centimeter largely for two reasons. In counting bacteria in the milk the cubic centimeter is always the basis employed. Simply because the leucocytes were derived from the blood seemed to be no reason why the same basis for counting should be employed as was used with the blood, while to the ordinary bacteriological worker to whom this work will fall, if ever adopted to any extent, the cubic centimeter standard would be a little more easily comprehended because more frequently used. The blood counter holds $\frac{1}{10} \mathrm{c} . \mathrm{mm}$. and $1 / 10,000$ c.c. If 10 c.c. of milk are used and the 1 c.c. of fluid is in the tube after siphoning, and the coloring matter and the water used to dilute has been added, then the resulting number of leucocytes in the counting multiplied by 1000 will be the total number of leucocytes per cubic centimeter in the milk. If a total of 75 leucocytes was counted in the chamber there would be 75,000 leucocytes per cubic centimeter in the milk:

In the actual counting under the microscope a square millimeter of the counting chamber will be found to be ruled off into 400 smaller equal squares. This facilitates an accurate and rapid count. Where the number of leucocytes is not great the entire field can be counted in a short time. Where there is a great number of leucocytes a few squares or sets of squares in different parts of the ruled surface will give approximately the number. 
There are occasionally a few variations desirable from these rules, but it may be well to state that the details have been pretty carefully and thoroughly worked over and compared, and it is seldom that short cuts can be made if correct results are desired. The time and speed of centrifuging are placed as low as possible for accurate work. When there is $\frac{1}{2}$ c.c. or more of sediment, it is necessary to use more of the methylene blue for staining, as there will be too great a number of leucocytes to make a satisfactory count in the counting chamber, it is better to add water until there are 2 c.c., or sometimes even more in the sedimentation tube.

This method of counting, while long in explaining is in reality short and simple in application. Moreover, it is based on accurate measurements in every detail, and the results are correspondingly reliable. (From Report of Committees of the Laboratory Section, American Public Health Assn. Am. J. Pub. Hyg., 6 (1910).

\section{Pathogenic Bacteria in Milk}

Bacillus tuberculosis. The tubercle bacillus is recognized as one of the most important pathogens in relation to the milk question. While it may gain entrance from a tubercular person handling the milk, it is generally admitted that a tubercular cow is usually the source. Human tuberculosis is probably transmitted directly from human to human but may be transmitted by bovines, especially those with udder infections. Griffith (1913) reports the discharge of virulent tubercle bacilli in the milk of a heifer which had been vaccinated when four days old with human tubercle bacilli. Other experiments by Smit (1908), Coquot (1908), Hessler (1909), indicate that there is slight possibility of the tubercle bacilli being discharged into milk unless there are open lesions. Smit found few tubercle bacilli in milk from tuberculous animals with sound udders but where open tuberculosis existed the bacilli could gain entrance to the milk from all channels which communicate with the exterior. This subject has been well summed up by Schroeder (1907).

Delepine's Method for Determining Tubercle Bacilli in Milk (from Savage, 1914). Collect two tubes of milk containing 40 c.c. and centrifugalize for fifteen minutes at 3000 r.p.m. Decant or draw off the cream and milk by means of a pipette leaving about 2 c.c. of milk and residue in each tube. Examine microscopically and inject into guinea pigs. 
Campbell's Method for the Detection of Tubercle Bacilli in Milk. Ten c.c. of a thoroughly mixed sample were placed in each of three centrifuge tubes by means of a sterile pipette. These should be centrifuged for thirty minutes at about 1200 r.p.m. The tubes are then removed from the centrifuge and the cream removed by means of a large sterile platinum loop. Three c.c. of sterile water should be added to this cream to bring the volume up for inoculation. The milk remaining in the centrifuge should be drawn down to 1 c.c. These 1 c.c. portions should be transferred to a sterile test tube. Slides may be made from these samples. Three c.c. of the cream emulsion and 3 c.c. of the milk emulsion should be injected subcutaneously into guinea pigs. The pigs should be observed closely for from six to ten weeks and, if not dead before, should be autopsied.

Anderson's Method. Mix 50 c.c. of the milk with 50 c.c. of sterile water and centrifuge for one hour at 2000 r.p.m. Inject 4 c.c. of the sediment into a guinea pig. If the guinea pig dies, a careful autopsy should be made. If death does not occur by the end of two months, test with tuberculin, chloroform and perform a careful autopsy. All suspicious lesions should be cultured and cultural and morphological studies made.

Besson's Method for Isolation of Bacterium tuberculosis from Milk. Allow the fresh milk to stand for twenty-four hours and examine the deposit. Centrifuge and use the precipitate for making a microscopical examination. Coagulate 200 c.c. of the milk with a little citric acid and filter. Dissolve the precipitate on the filter in a solution of sodium phosphate $\left(\mathrm{Na}_{2} \mathrm{HPO}_{4}+12 \mathrm{H}_{2} \mathrm{O}\right)$ and pour the liquid into a large test tube. Add a few cubic centimeters of ether and, after shaking for about ten minutes, pour off the ether which will carry the fat with it. Centrifuge the aqueous fluid and examine microscopically the sediment.

Beattie and Lewis (1913) have isolated acid fast bacilli from milk which were totally different from tubercle bacilli. They emphasize the futility of using microscopic methods instead of animal inoculation.

The prevalence of tubercle bacilli in market milk has received the attention of investigators in practically all countries. There is an element of error in comparing closely data from the different investigations since a different number of samples were used. This may explain the high incidence of tubercle bacilli which has been reported by some investigators. Table XLIII gives the data which have been secured by some of the investigators on market milk.

Microspira Choleræ. Basenai (1895) reported that there was no germicidal action of milk on Microspira cholerce and that the organisms 
could retain their vitality in milk for thirty-cight days. Some of the cells even retained their vitality after the milk had curdled. These statements are interesting when compared to the results secured by Laser (1891) in butter.

\section{TABLE XLIII}

INCIDENCE OF B. TUBERCULOSIS IN MARKET MILK

(Quoted from Parker 1917)

\begin{tabular}{|c|c|c|c|c|c|}
\hline Date & Place. & Investigator. & $\begin{array}{l}\text { Samples } \\
\text { Sidammed. }\end{array}$ & $\begin{array}{l}\text { Number } \\
\text { Positive. }\end{array}$ & $\begin{array}{l}\text { Percentage } \\
\text { Positive. }\end{array}$ \\
\hline 1899 & England...... & Macfayden. . & 77 & 17 & 221 \\
\hline 1904 & Germany. . & Muller...... & 1596 & 97 & 62 \\
\hline 1904 & Germany. . & Beatty..... & 272 & 27 & 10.0 \\
\hline 1898 & Liverpool. . & Delepine... & 12 & 22 & 17.6 \\
\hline 1897 & Liverpool. . & Hope. . . . . . . & $2 \cdot 28$ & 12 & 5.2 \\
\hline 1900 & London & Klein & 100 & 7 & 7.0 \\
\hline 1893 & Petrograd. & Scharbekow. & 80 & 4 & 5.0 \\
\hline 1900 & Kiew & Pawlowsky. . & 51 & 1 & 20 \\
\hline 1900 & Krakow... & Bunwid. . . . . & 60 & 2 & 3.3 \\
\hline 1900 & Naples... & Marconi.... & 14 & 7 & 500 \\
\hline 1898 & Berlin.... & Petri....... & 64 & 9 & 14.0 \\
\hline 1900 & Berlin.... . & Beik. . . . . . . & 56 & 17 & 30.3 \\
\hline 1898 & Schev. Gueund. & ott $\ldots$ & 27 & 27 & 11.1 \\
\hline 1898 & Königsburg. . . . & Jaeger... & 100 & 7 & 7.0 \\
\hline 1908 & Leipsic. . . . . . . & Eber....... & 210 & 22 & 10.5 \\
\hline 1905 & Rotterdam. & Smit...... & 567 & 14 & 2.7 \\
\hline 1906 & Rotterdam. . & Śmit....... & 1584 & 45 & 2.8 \\
\hline 1908 & Washington.... & Anderson.... & 223 & 15 & 6.7 \\
\hline 1909 & Louisville.. . & Ficld....... & 119 & 46 & 295 \\
\hline 1909 & New York.. & Hess. . . . . . . & 10.5 & 17 & 16.2 \\
\hline 1909 & Philadclphia. & Campbell.. & 130 & 18 & 13.8 \\
\hline 1910 & Chicago .. & Tonney..... & 144 & 15 & 10.5 \\
\hline 1910 & Rochester. . & Goler....... & 237 & 30 & 12.6 \\
\hline
\end{tabular}

Bacillus Diphtheriæ. Diphtheria is a typical milk-borne disease. Marshall (1907) has reported the isolation of the organisms from milk. Trask (1912) has given a good résumé of such epidemics.

\section{DASTEURIZATION}

Pasteurization is the heating of a food substance for a time below the boiling-point. It is now almost exclusively applied to the heating of milk in order to make it a safer food.

It is an old idea having been used by Scheele (McIntosh, 1901), in 1782, to preserve vinegar. In 1831, Appert preserved various animal 
and regetalite products by heating in the closed containers. Pasterur (1879) applied heat to bottled beer to make it keep. In recognition of Pasteur's researches, which revealed the causes of deterioration in fermented liquors and the means of preserving them, the term "pasteurization is now used. Soxhlet (1886) proposed pasteurization in the home for baty feeding. There has been much confusion between the terms "pasteurized" and "sterilized" as applied to mills. Losenau,

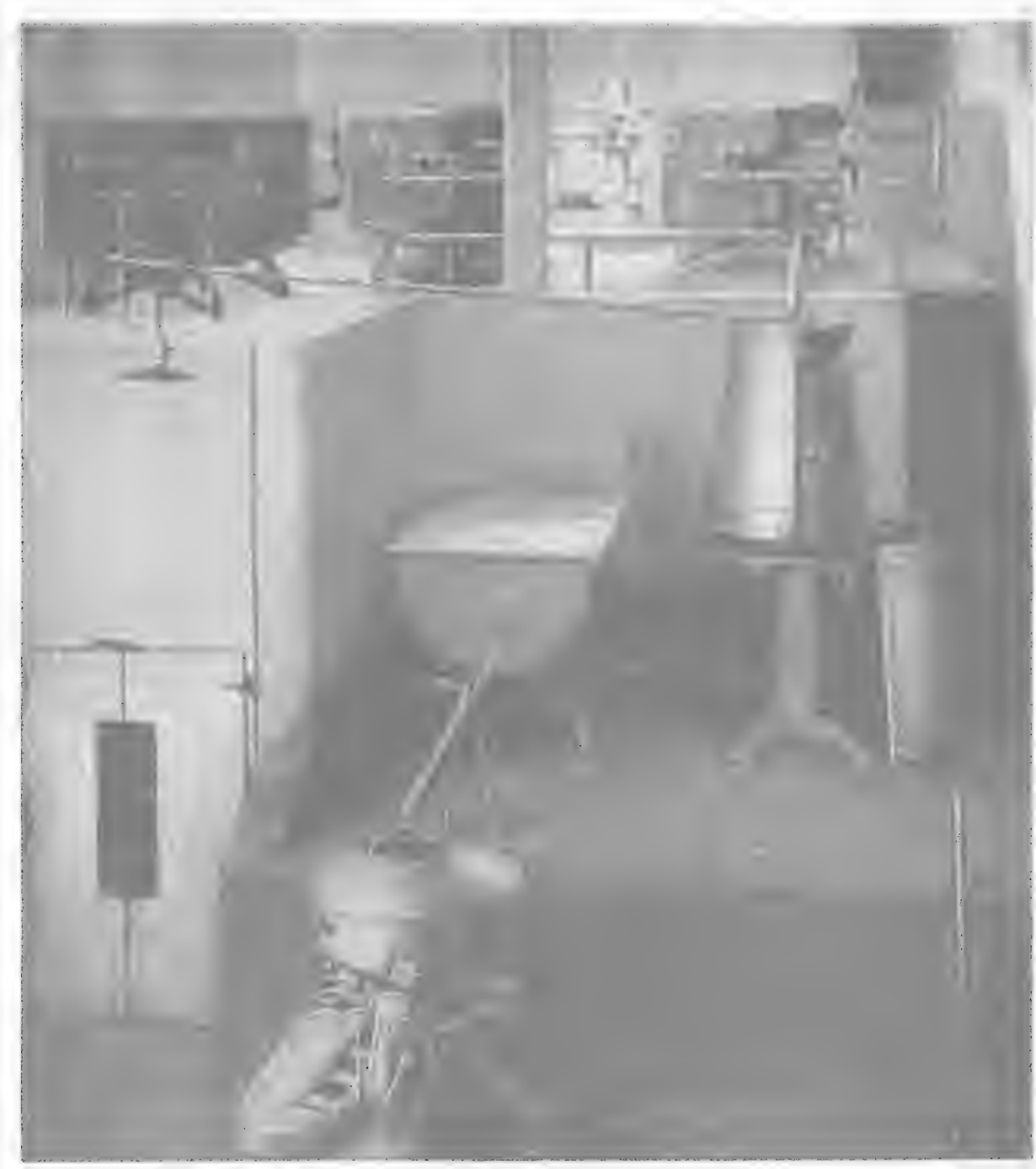

FIG. 68.-Complete Milk Plant. (New York Milk Committce.)

(a) Milk Clarifier; (b) Pasteurizing and Holding. Plants; (c) Milk Cooler; (d) Storagc Tank for Cold Milk; (e) Bottle Filling and Capping Machine.

to avoid this hàs suggested that each bottle indicate the time and temperature of heating.

Theory of Pasteurization. This involves the lus of moist heat as a disinfectant. As discussed in the chapter on sterilization and disinfection, bacteria die according to the monomolecular law and this indicates that the killing is a time process. The bacteria do not die at once as soon as the heat is applied. The same law must obtain in the killing of bacterial by pasteurization. From this it would secm 
that the "continuous" process where heat is applied over a longer time would be inore satisfactory.

Gable (1915) has summed the advantages which might be raised against pasteurization as follows: In the light of recent knowledge some of these are now untenable.

OBJECTIONS

1. Increased cost.

a. By additional apparatus required.

$b$. By fuel required for heating.

c. By ice or ammonia required for cooling.

d. By increased labor of handling.

2. Conceals inferiority.

a. By encouraging carelessness.

b. Discouraging proper care.

3. Interferes with cream rising and whipping.

4. Modifies taste.

5. Less wholesome.

a. Acid less as the lactic acid bacteria are destroyed.

$b$. Spores forms of bacteria are not destroyed.

c. Injurious by-products of bacteria are not destroyed.

d. Milk is chemically changed.

$e$. Is less easily digested.

$f$. Produces scurvy and rachitis.

\section{ADVANTAGES}

1. Economic.

a. Makes milk keep sweet longer.

b. By saving milk which otherwise would spoil.

c. By saving bills for sickness and milk-borne epidemics

2. More wholesome.

a. Pathogenic bacteria are destroyed.

b. Infant mortality less.

c. Bacteria are decreased in numbers.

The methods of pasteurization are two in number, the "flash" and "continuous" processes. In the continuous process the milk is heated for thirty minutes at $60^{\circ} \mathrm{C}$.; the flash process requires a higher temperature $\left(80^{\circ} \mathrm{C}\right.$. to $90^{\circ} \mathrm{C}$.) for a shorter time (1-5 minutes), according 
to the theory of disinfection and since disinfection is a time process the continuous process allows a greater reduction of bacteria.

Effect of Pasteurization on Bacteria. This has been given much study in order to refute the argument against pasteurization that pasteurized milk putrifies rather than sours. Ayers and Johnson (1913)

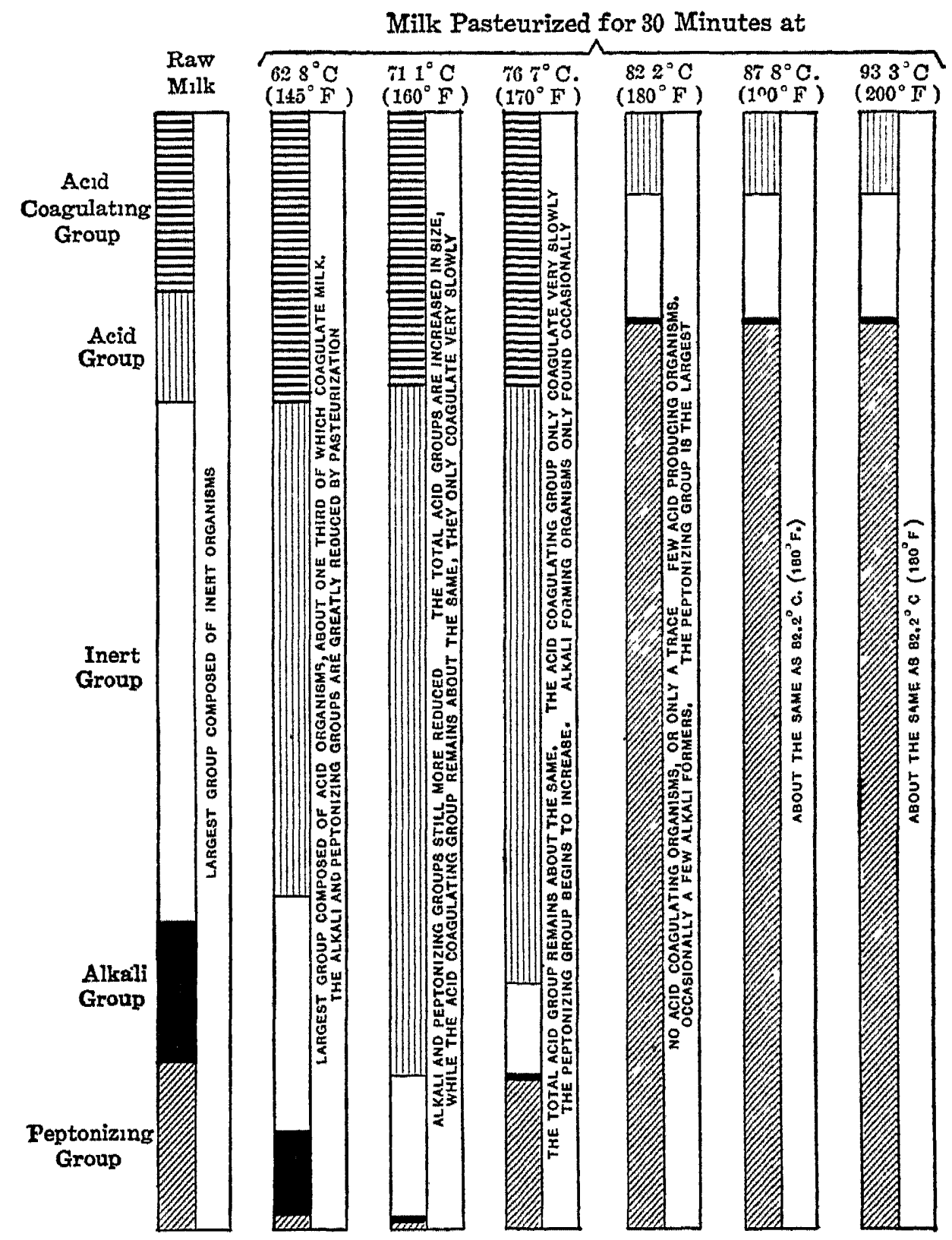

Frg. 69.-The Hypothetical Relation of the Bacterial Groups in Raw and Pasteurized Milk. (After Ayers and Johnson.)

studied the bacteria in pasteurized milk by means of their milk-tube method. They used the holder process with a temperature of $62.8^{\circ} \mathrm{C}$. for thirty minutes in most of their experiments. Their results are graphically summarized in Fig. 69 from which it will be seen that pasteurization increased the percentage of acid-forming bacteria in the milk. Higher temperatures of pasteurization caused a different relation to 
exist between the groups of bacteria in milk. In an earlier paper (1910), these same authors found that pasteurized milk soured about the same as a clean, raw milk. Weigmann et al. (1916) found that, in milk which had been heated from $60^{\circ} \mathrm{C}$. to $63^{\circ} \mathrm{C}$. for thirty minutes, lactic acid bacteria were present in a much larger proportion to remaining organ-

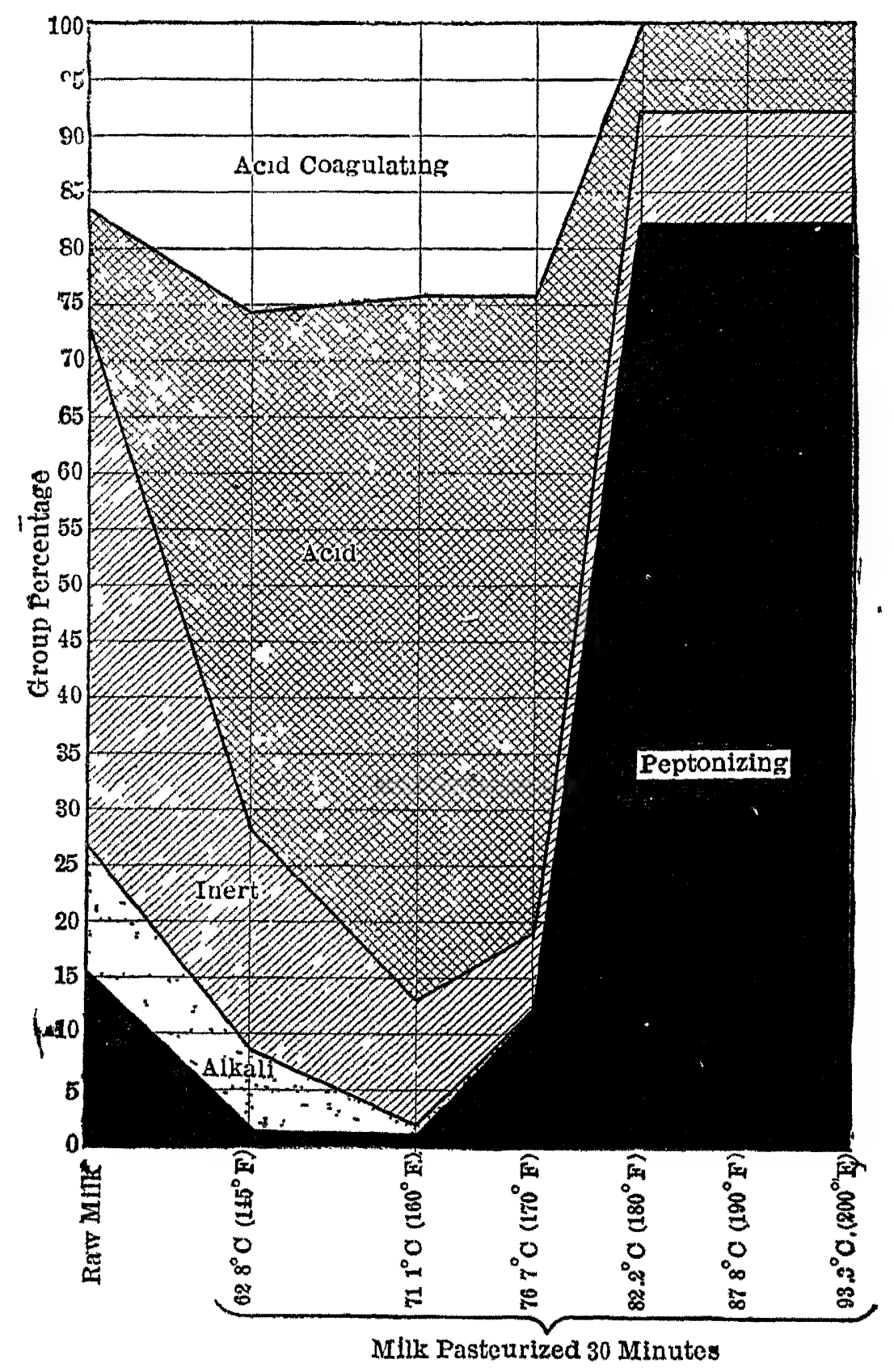

Frg. 70.-The Hypothetical Relation of Bacteria in Raw and Pasteurized Milk (After Ayers and Johnson, 1913.)

isms than in raw mulk. 'The same was also found in milk which had been heated for ten to twenty minutes. Souring, however, was much delayed over that in raw milk, which the authors attribute to attenuation of the lactic acid organusms. With regard to the resistance of Bacillus colon to heat, Ayers and Johnson (1915) state that this organism would 
not be expected, from their results, to survive $65.6^{\circ} \mathrm{C}$. for thirty minutes. Erratic results were secured and it is possible that some strains of Bacillus colon may be found which will survive pasteurization under the above conditions. Gage and Stoughton (1906) found that in 55 per cent of thermal death-point determinations made with $B$. coll, the cultures withstood $80^{\circ} \mathrm{C}$. for five minutes. Russell and Hastings (1902) report the characterıstics of a micrococcus from milk which withstood a temperature of $76^{\circ} \mathrm{C}$. for ten minutes. Different individual resistance, however, was found among the cells. Ford and Pryor (1914) claim that milk heated to any temperature between $65^{\circ} \mathrm{C}$. and $100^{\circ} \mathrm{C}$. and kept between $22^{\circ}$ and $37^{\circ} \mathrm{C}$. will be decomposed by spore-forming bacteria and consequently may be dangerous. They recommend the boilung of milk and storage on ice since heated milk is lable to decompose.

Pathogenic Bacteria. The effect of the pasteunization process on pathogens has been linited almost entirely to Bacillus tuberculosis. Since this organism is especially important in relation to milk hygiene, many attempts have been made to determine its thermal death point in milk Rosenau has prepared table XLIV, which rcviews concisely some of the work which has been done.

The work of Smith (1899) and of Russell and Hastings (1904) has been especially convincing. The former of these investigators found that when tubercle bacilli were suspended in milk they were destroyed in from fifteen to twenty minutes at $60^{\circ} \mathrm{C}$.; the greater portion being klled in 5-10 minutes. The latter tried short periods of pasteurization and found that a temperature of $160^{\circ} \mathrm{F} .\left(71.1^{\circ} \mathrm{C}\right.$.) or above for one minute destroyed the virulence of bovine tubercle bacilli so that guinea pigs were not kllled when inoculated with from 2 to $5 \mathrm{mgs}$. Others have confirmed this work and these temperatures are now accepted in the continuous process. The milkman pasteurizing milk is between two fires. He must avoid a cooked taste in the milk which interests the housewife and must kill all pathogenic bacteria which are the chief interest of the sanitarian.

On the relation of pasteurization to other pathogenic bacteria in milk, our knowledge is very meager. Hesse (1894) stated that raw cows' milk was not a good medium for cholera spirilli and that they died in twelve hours at room temperature and more quickly at higher temperatures. The acidity and other bacteria in the milk kulled them. Fig. 69 prepared by North and taken from the report of the Commission on Milk Standards gives some data concerning $B$. typhi, streptococci and Bact. diphtheria. 
TABLe XLIV

SHOWING THE THERMAL DEATH POINT OF THE TUBERCLE BACILLUS AS FOUND BY VARIOUS INVESTIGATORS

(After Rosenau)

\begin{tabular}{|c|c|c|}
\hline Investigator & Killed at & Not Killed at \\
\hline 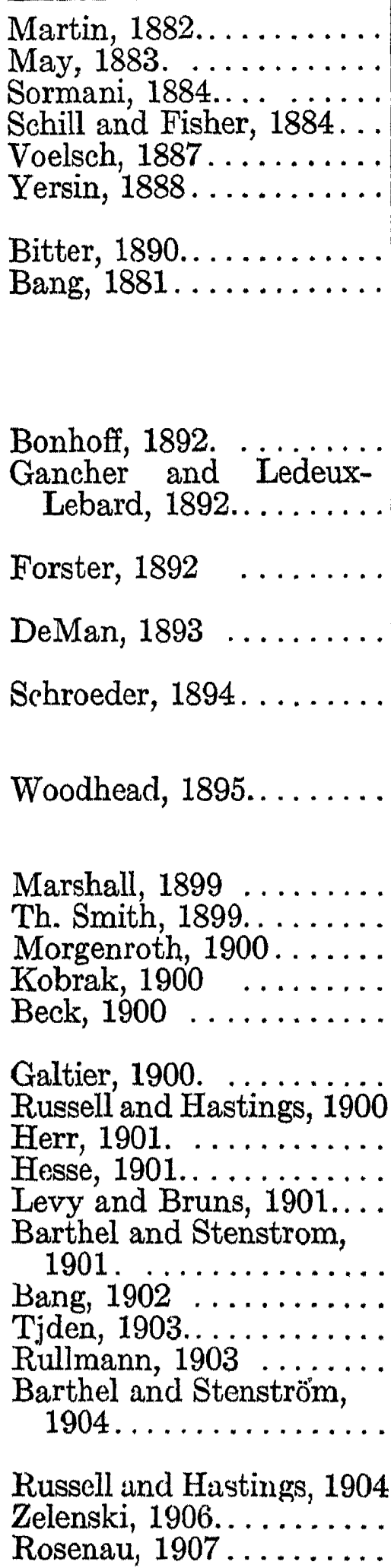 & 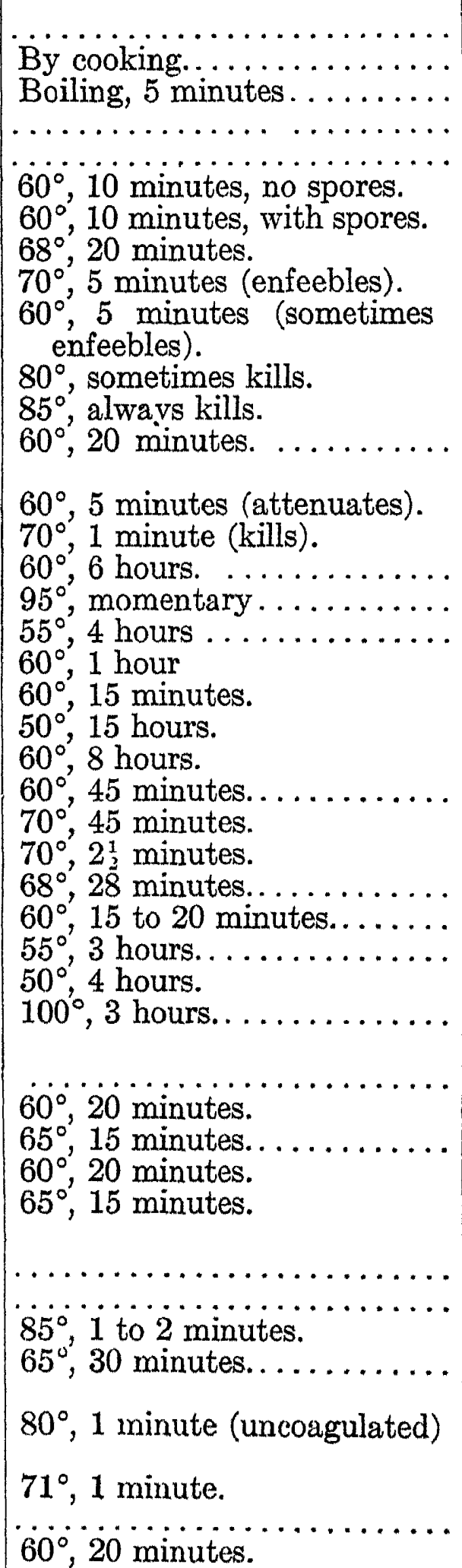 & $\begin{array}{l}90^{\circ}, \text { results contradic- } \\
\text { tory. } \\
60^{\circ}, 10 \text { minutes. } \\
70^{\circ}, 10 \text { minutes. } \\
100^{\circ}, \text { momentary } \\
100^{\circ} . \\
80^{\circ}, 30 \text { minutes. } \\
85^{\circ}, 6 \text { minutes. } \\
80^{\circ}, 5 \text { seconds. } \\
70^{\circ}, 15 \text { minutes. } \\
60^{\circ}, 15 \text { minutes. } \\
60^{\circ}, 30 \text { minuies. } \\
80^{\circ}, 1 \text { minute (coagu- } \\
\text { lated). } \\
76^{\circ}, 20 \text { minutes. }\end{array}$ \\
\hline
\end{tabular}




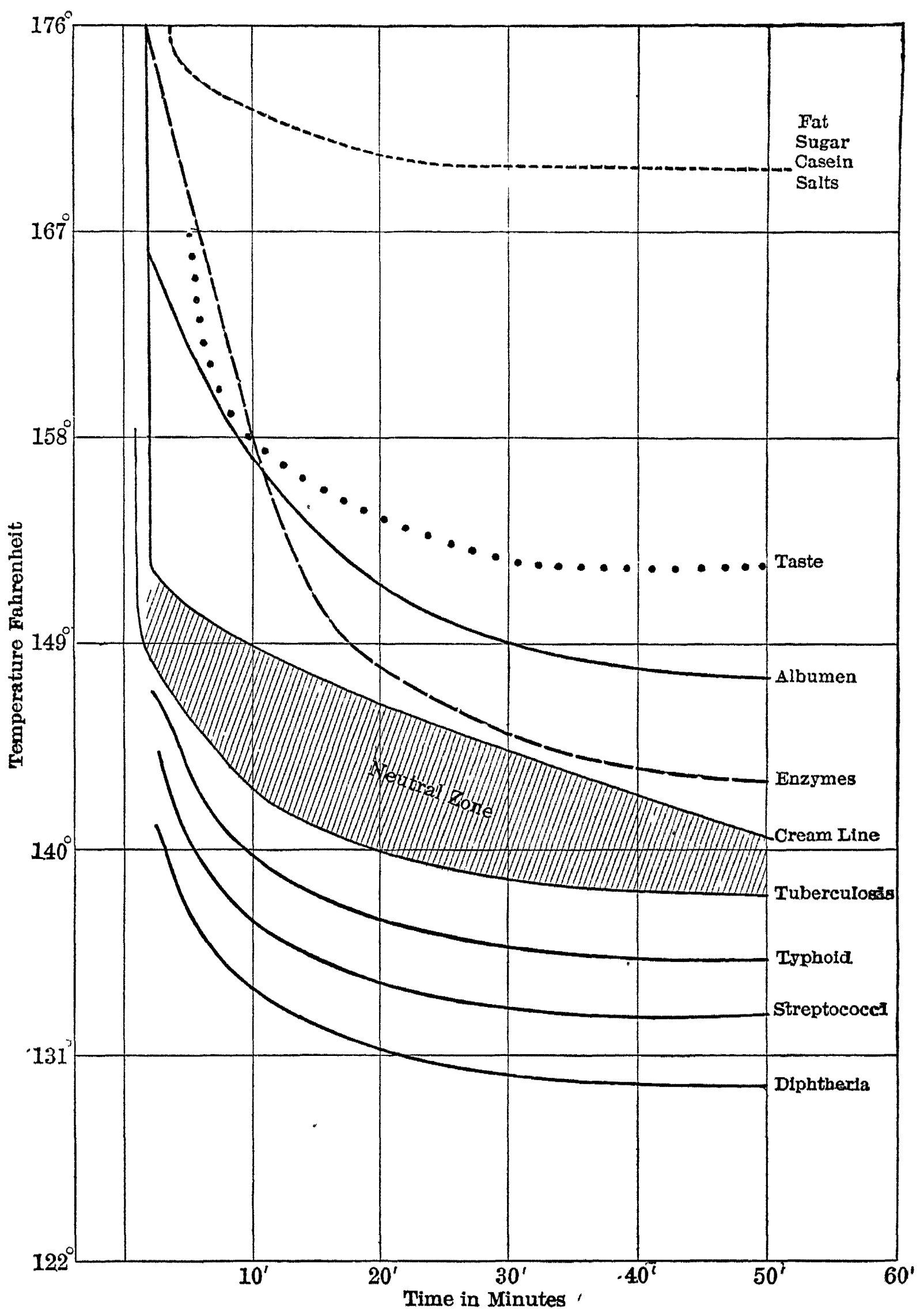

Frg. 71.-Indicating the Relation between the Time and Temperature and Other Factors Involved in the Pasteurization of Milk. (After North.) 
Pathogenic Streptococci. Much evidence has accumulated to show that septic sore throat may be caused by streptococci which originate in milk from cows suffering from mastitis. Cows suffering from garget are regarded as of special importance in this connection. Capps and Downs (1914) found a dairy farm where mastitis was present in the cows and where the milkers had sore throat. The milk from this dairy was delivered to a dairy company which did not pasteurize its milk and consequently an extensive epidemic of septic sore throat resulted. Krumwiede and Valentine (1915) during the investigation of an epidemic regarded the infection as of human rather than of bovine origin. They suggest that "in tracing the source of an epidemic the effort should be toward finding cases of sore throat among those engaged in producing the milk, not mastitis in the cow alone. If human streptococci are found in mastitis, they are most likely secondary agents in an already existing inflammation due to bovine strains. . The streptococci in different epidemics differ culturally and those similar culturally differ in their immunity reactions. Cultural similarity of strains from man and cattle is insufficient to prove their identity. Cultural identity in cvery detail or immunological identity is essential." Rosenow and Moon (1915) studied an cpidemic of septic sore throat and, after tracing it to milk, isolated virulent streptococci from the milk. These showed selective preference for joints, muscles, gall bladder, etc., and resembled certain rheumatic strains morphologically and culturally. These investigators regard virulent streptococci in milk as possible sources of those which cause, rheumatism and other chronic infections. Rosenow and Hess (1917) also traced another epidemic of this disease to the milk from three cows. Winslow and Hubbard (1915) reported a contact epidemic of this infection. Smaller epidemics have been studied by Overman (1914), Henika and Thompson (1917) and others. Smillie (1917) has stated that the streptococci of septic sore throat resemble those of scarlet fever and that discharges from such patients may infect the milk and cause septic sore throat. Ayres and Johnson (1914) studied the possibility of streptococci surviving pasteurization. Out of 139 cultures isolated from cow feces, the mouth of the cow and milk, 64.03 per cent of them survived a pasteurization temperature of $140^{\circ} \mathrm{F}$. Thirty-three and seven hundredths per cent survived at $145^{\circ} \mathrm{F}$. At $165^{\circ} \mathrm{F}$. none were able to survive. These data might indicate that the streptococci which were pathogenic for man could under certain conditions pass through the pasteurization process.

Allen $(1916,1917)$ has shown that raw milk as compared with pasteurized milk exerts a powerful suppressing influence on the multipli- 
cation of certain bacteria. The pasteurized milk was found to be more favorable to the attack of the gas forming colon bacillis and $B$. aerogenes. Allen emphasizes the point that pasteurized milk, although it is safer for human consumption, should be handled with much care since heating has decreased its resistance to many detrimental changes. Ayers and Johnson after a study of this question reported that the bacterial increase in clean raw milk and pasteurized milk was about the same when both were stored under the same conditions.

\section{Bacteriology of Butter}

The bacteriology of butter may be considered from two viewpointsthe use of bacteria to bring about desired changes and the dissemination discase-producing bactcria.

Bacteria in Cream and Cream Ripening. According to present practice cream is pasteurized and ripened before being made into butter. The pasteurization process removes all of the extraneous bacteria and thus prevents spontaneous changes which are liable to produce unfavorable flavors and tastes. After pasteurization, the cream is inoculated with a culture known to produce a desired flavor and allowed to ripen. Certain distinct improvements were introduced into the butter industry by ripening of the cream. (1) A better yield of butter is secured from sour cream. (2) The flavor of the butter is kept constant from batch to batch. (3) Sour cream churns more easily than sweet cream. Peiser (1916) has stated that the greater proportion of bacteria in ripened cream are removed during churning by the buttermilk. Butter contains about one-tenth as many as are in the cream.

Flavor of Butter. This is dependent upon a large number of factors and opinion is still divided with regard to which is the most important. That bacteria are important in changing the flavor of butter after it has been made is improbable. Conn has attempted to make a distinction between "flavor." and "aroma " but such a division is probably quite artificial.

Rancidity is a term which covers a multitude of abnormalities in the taste of butter. Guthrie (1917) in his interesting paper has given a review of the literature. In his experiments, he attempted to determine whether rancidity was due to chemical cow enzyme or biological changes. $\mathrm{He}$ found that none of these factors were important and believes that rancidity as defined by butter judges is rarely found. No marked change in the iodine number was caused by high temperatures, light or air. 
The undesirable flavors in butter may be due to absorbed odors or to products formed in the butter after manufacture. Dyer (1916) showed that the undesirable flavors developing in butter held in cold storage at a temperature of $0^{\circ} \mathrm{C}$. were not due to an oxidation of the fat itself. Gas analyses indicated that the quantity of buttermilk left in

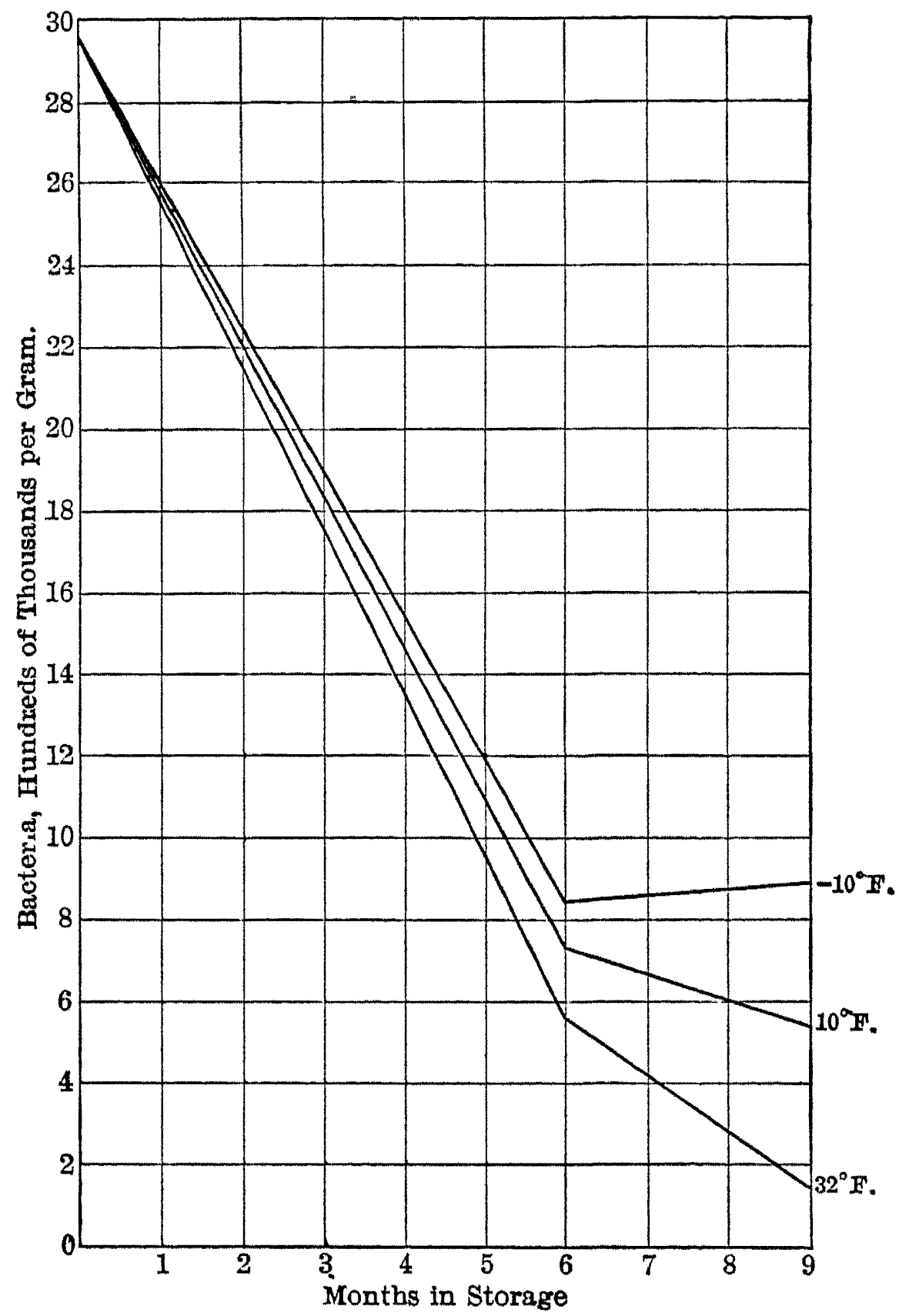

FIG. 72.-Diagram Showing Decrease in Bacterial Content of Butter Stored at Different Temperatures (After Mohler, Washburn and Rogers, 1909.)

the butter has a relation to the quantity of carbon dioxide formed. Rancidity is probably the algebraic sum of a number of factors.

Milk in the fresh condition is a very suitable medium for bacteria. Northrup (1911) studied the longevity of $B$. typhi in sour milk. Before these organisms were destroyed Bacterium lactis acidi had to produce 
$80^{\circ}$ acid in milk and $28^{\circ}$ acid in whey. Bacillus bulgaricus had to produce $208^{\circ}$ acid in milk and $60^{\circ}$ acid in whey. She quotes the work of other investigators as Bassenge, Behla and others that lactic acid is toxic for Baclllus typhosus. Krumwiede and Noble (1915) report that the typhoid bacillus is killed in sour cream by acids and that the destruction is proportional to the amount of acid and number of bacilli. Washburn (1908), in artificially infected milk found that B. typh suffered no diminution in numbers up to twenty days. They had practically disappeared after forty-three days. Potter (1910) concluded that Bacillus bulgaricus and Bacterium lactis acidi exerted no appreciable repressing effect on Bacillus typhi and that these organisms may not be depended upon to make milk, which has been contaminated, safe.

TABLE XLV

VIABILITY OF TYPHOID BACILLI IN SOUR CREAM

(Krumwiede and Noble)

\begin{tabular}{l|c|c|c|c|c|c|c}
\hline Days & 0 & 2 & 7 & 8 & 9 & 10 & 11 \\
\hline $\begin{array}{l}\text { Reaction } * \\
\text { Number of typhord } \\
\text { bacill.. }\end{array}$ & $10 \%$ & & $22 \%$ & $5 \%$ & & & $10 \%$ \\
\hline
\end{tabular}

* Number of cubic centımeters of $\mathrm{N}$ alkali needed to neutralıze $100 \mathrm{c} \mathrm{c}$ of cream

Molds in Butter. Thom and Shaw (1915) found that mold in butter usually took three forms: first, orange yellow spots produced by Oidium lactis; secondly, dirty green spots produced by cladosporium alternaria, thirdly, green spots which are caused by penicillium. A salt content of 2.5 per cent prevented the development of the above fungi and, therefore, their presence indicates a low salting. The presence of much curd was stated to allow a more vigorous growth of mold. Hastings (1916) has recommended that butter tubs and liners be placed in boiling water heated to $150^{\circ}$ to kill the mold spores.

Tubercle Bacilli in Butter. While the literature on the spread of this disease by butter is not extensive, there are several important investigations on the question. Rosenau, Frost and Bryant (1914) examined twenty-five samples of Boston market butter and found tubercle bacilli in two of them, $B$. coli in six and streptococci in fourteen. Hill $(1911,1913)$ has reported the spread of typhoid fever and diphtheria by butter. M. Mohler, Washburn and Rogers (1909) found that, contrary to prevailing opinion, tubercle bacilli were not devitalized by 
cold storage; salt was reported to have no effect on these organisms in butter. Salted butter was found to retain its virulence for six months. Schroeder and Cotton (1908) demonstrated the presence of tubercle bacilli in butter which had been kept for one hundred and sixty days. These data indicate that some butters may contain tubercle bacilli even after comparative long storage periods. Again, the value of pasteurization of dairy products is emphasized.

Savage's Method for Tubercle Bacilli in Butter. To detect tubercle bacilli in butter the inoculation method is the only satisfactory one. The butter is placed in centrifugal tubes which are stood in warm water at $42^{\circ} \mathrm{C}$. until the butter is completely melted. The material is centrifugalized when liquid, and the sediment inoculated into guinea pigs as described under milk. It is difficult to keep the butter liquid during the centrifugalization.

Bacillus typhosus in Butter. Washburn (1908) studied the longevity of $B$. typhi in butter which was artificially infected. In this experiment, the organisms were found 150 days after the preparation of the butter. Boyd (1917) reported an epidemic of typhoid fever from butter. He regards the pasteurization of the products before ripening as a wise procedure to prevent disease and undesirable fermentations. Washburn's data indicate that butter may contain virulent typhoid bacilli for 151 days. Probably the conditions under which the data are obtained greatly influence the results.

Bergey's Method for Typhoid Bacilli in Butter. Transfer 5 gms. of butter by means of a stcrile scalpel to a test tube containing ordinary nutrient bouillon. After incubation for several days, streak plates of Endo's medium, Drigalski-Conradi agar and Loeffler's malachite green agar. Incubate these plates for from 24-48 hours and transfer the suspicious colonies to agar slants and a melted tube of clextrose agar. After incubation only those cultures should be retained for further study which show no fermentation in dextrose agar. Morphological studies should then be made together with cultural studies in lactose, sarbite, raffinose, dextrose, saccharose, dulcite, adonite and inulin broths. Observation has shown that Bacillus typhosus produces a slight acidity in lactose broth and a more definite acidity and coagulation in the sorbite medium.

Determination of Bacteria in Butter, Löhnis Method. For the sterile weighing of butter, cheese and similar substances, put a number of filter papers, $9 \mathrm{~cm}$. and $7 \mathrm{~cm}$. in diameter, into two Petri dishes respectively and sterilize in the air oven. By means of sterilized forceps, place one of the larger papers on the scale pan and then one of the 
TABLE XLVI

INCIDENCE OF TUBERCLE BACILLI IN MARKET BUTTER

(After Briscoe and MacNeal, 1911)

\begin{tabular}{|c|c|c|c|c|c|c|}
\hline Author. & Date & Place. & $\begin{array}{c}\text { Samples } \\
\text { Ex- } \\
\text { amined. }\end{array}$ & $\begin{array}{l}\text { Samples } \\
\text { Posi- } \\
\text { tive. }\end{array}$ & $\begin{array}{l}\text { Per } \\
\text { Cent } \\
\text { Posi- } \\
\text { tive. }\end{array}$ & Remarks. \\
\hline Brusaferro. .. & 1890 & Turin & 9 & 1 & 111 & \multirow{4}{*}{ MLresconic method. } \\
\hline Roth... . . & 1894 & Zurich .. & 20 & 2 & 100 & \\
\hline Obermuller .... & 1895 & Berlin. . .... & 13 & 8 & 610 & \\
\hline Schuchardt ... & 1896 & Marburg $\ldots$ & 42 & 0 & 00 & \\
\hline Obermuller .... & 1897 & Berlin. ..... & 14 & 14 & 1000 & \multirow[t]{7}{*}{16 tested, 2 lost. } \\
\hline Groning. . . . . & 1897 & Hamburg..... & 17 & 8 & 47 & \\
\hline Himesch .... & 1897 & Wien ........ & $?$ & 0 & $\rho$ & \\
\hline Rabinowitch.... & 1897 & Berlin... . . . . & 30 & 0 & 0 & \\
\hline Rabinowitch.... & 1897 & Philadelphia. . & 50 & 0 & 0 & \\
\hline $\begin{array}{l}\text { Petri........... } \\
\text { Hormon and }\end{array}$ & 1897 & Berlin........ & 102 & 33 & 323 & \\
\hline Morgenroth. . & 1897 & Berlin.... & 10 & 3 & 300 & \\
\hline Rabinowitch.... & 1899 & Berlin........ & 15 & 2 & 133 & \multirow{10}{*}{$\begin{array}{l}\text { First series. } \\
\text { Second series. } \\
\text { Third series } \\
\text { Fourth series. }\end{array}$} \\
\hline Rabinowitch... & 1899 & Berlin. . . . . . . & $?$ & $?$ & 872 & \\
\hline Rabinowiteh... & 1899 & Berlin........ & 15 & 15 & 1000 & \\
\hline Rabinowitch... & 1899 & Berlin.... & 19 & 0 & 0 & \\
\hline Obermuller .... & 1899 & Berlin.. & 10 & 4 & 400 & \\
\hline Korn......... & 1899 & Freiburg . . . & 17 & 4 & 235 & \\
\hline Ascher...... & 1899 & Konigsberg .. & 27 & 2 & 74 & \\
\hline Jäger. . . . . . . & 1899 & Kunigsberg .. & 3 & 1 & 333 & \\
\hline Coggi......... & 1899 & Milan. . . . . . . & 100 & 12 & 12 & \\
\hline Weissenfield.... & 1899 & Bonn ...... & 32 & 3 & 91 & \\
\hline Grassberger... & 1899 & Wien....... & 10.0 & 0 & 0 & \multirow[t]{2}{*}{ • } \\
\hline Herbert ...... & 1899 & Tubingen..... & 43 & 0 & 0 & \\
\hline Herbert. ..... & 1899 & Wurttemberg & 58 & 0 & 0 & \multirow{4}{*}{$\begin{array}{l}\text { Pseudo tuberculosis } 5 \text { per. } \\
\text { Pseudo tuberculosis } 8 \text { per. } \\
\text { Pseudo tuberculosis } 4 \text { per. }\end{array}$} \\
\hline Herbert........ & 1899 & Berlin ...... & 20 & 0 & 0 & \\
\hline Herbert. ....... & 1899 & Munchen..... & 5 & 0 & 0 & \\
\hline Abenhausen.... & 1900 & Marburg..... & 39 & 0 & 0 & \\
\hline Hellstróm...... & 1900 & Helsingfors... & 8 & 1 & 125 & \multirow{7}{*}{$\begin{array}{l}12 \text { samples, } 4 \text { lost. } \\
39 \text { samples, } 11 \text { lost. }\end{array}$} \\
\hline Bomhoff........ & 1900 & Marburg..... & 28 & 0 & 0 & \\
\hline Pawlowsky..... & 1900 & Kiew ....... & 23 & $\cdot 1$ & 43 & \\
\hline Tobler........ & 1901 & Zurich...... & 12 & 2 & 16.7 & \\
\hline Lorenz........ & 1901 & Dorpat....... & 30 & 0 & 0 & \\
\hline Markl.. . . . . . . & 1901 & Wien $\ldots . .$. & 43 & 0 & 0 & \\
\hline Herr and & & & & & & \\
\hline Beninde...... & 1901 & Breslau. . . . . . & 52 & 6 & 11.1 & \multirow[t]{4}{*}{ Two wore doubtful. } \\
\hline Aujeszky...... & 1902 & Budapest..... & 17 & 3 & 176 & \\
\hline Thu.......... & 1902 & Christiania... & 16 & 0 & 0 & \\
\hline Teichert........ & 1904 & Rosen........ & 40 & 12 & 30.0 & \\
\hline Reitz........ & 1906 & Stuttgart..... & 94 & 8 & 85 & \multirow[t]{3}{*}{ Butter from 88 dairies. } \\
\hline Eber.......... & 1908 & Leipsic.. . . . . & 150 & 18 & 120 & \\
\hline Briscoe and & & & & & & \\
\hline "MacNeal..... & 1911 & Urbana, Ill .. & 6 & 2 & 332 & \multirow{4}{*}{$\begin{array}{l}\text { Creamery butter. } \\
52 \text { per cent of the samples } \\
\text { contained acid-fast ba- } \\
\text { cilli. }\end{array}$} \\
\hline Eber.......... & 1912 & $\ldots \ldots \ldots \ldots$ & $\ldots$ & $\ldots$ & 15.6 & \\
\hline Rosenau et al... & 1914 & Boston ...... & 21 & 2 & 9.4 & \\
\hline Marchiotti...... & 1917 & $\ldots \ldots \ldots \ldots$ & 25 & $\cdots \cdots$ & 240 & \\
\hline
\end{tabular}


smaller papers on top of it. After taring, with sterilized knife or spoon, place the substance to be weighed on the upper filter paper. Then transfer the material along with the small filter paper, to the first dilution flask. Make the dilutions according to the quantity of butter to be examined and pour plates. The water used for dilutions should be previously warmed to $40^{\circ} \mathrm{C}$. so that the butter $(1 \mathrm{gm}$. in the first tubes or flasks) may be readily emulsified.

Determination of Bacteria in Butter (Schneider's Method). To examine butter directly for the number of bacteria and other contaminations, place $1 \mathrm{gm}$. of the butter into 10 c.c. of ether and shake until all of the butter fat is dissolved. Pour the solution into a special centrifuge tube and centrifuge for five minutes. Wash the contents of the 1 c.c. end into 10 c.c. of ether and again shake and centrifugalize. Pour off the ether and add 2 c.c. of a 2 per cent solution of sodium hydroxide and shake until the casein is dissolved. The sodium hydroxide emulsifies the small amount of fat which is present. Examine the emulsion for bacteria and body cells by means of the hæmocytometer.

Conn's Method for Analysis of Butter. Weigh out upon accurate chemical scales 5 gms. of freshly made butter. Place this in a sterile mortar, with $9 / 5$ c.c. of sterile water. Rub the water and the butter together thoroughly, so as to distribute the bacteria as uniformly as possible through the water. This mixing should be continued for some time, for, at best, many of the bacteria will remain clinging to the fat. Dilute this mixture to 10,000 times and make a series of agar or litmus gelatin plates. Incubate and count as usual.

\section{Cheese}

Cheese may be classified into two general types, rennin curd and acid curd. In the former type, the enzyme rennin is added to the milk which forms calcium paracaseinate from the calcium caseinate. The acid curd cheeses are made from curd secured by the natural or artificial souring of milk.

Cheese Ripening. In this process develops the characteristics which, in part, separate one type of cheese from another. Many theories have been held in the past with regard to this process. Duclaux (1880), Freudenreich (1897), Babcock and Russell (1898), Gorini (1904) and others, have studied the problem and given different theories. The following factors were at times considered the important ones:

1. Enzymes which originated from the cow.

2. Enzymes of bacterial origin.

3. Pepsin which is added with rennin. 
Bacteriology of Cheddar Cheese. Harding and Prucha (1908) studied more than 300 pure cultures of bacteria from cheddar cheese by means of the group number on the classification card of the Society of American Bacteriologists. These finally reduced to 33 groups. Ten of these groups disappeared at once; 9 others were found in but single cheeses; the remaining 14 groups were indicated as the most important members of the cheese flora. Bacterium lactzs acidi included 4 of these 14 groups and was the only species always found and it practically included over 99 per cent of the total bacterial content.

Hastings, Evans and Hart (1912) conducted a similar study. They state that the Bacternum lactıs acıdi groups is an important one and sum up the role of these organisms as follows:

1. They favor the curdling process.

2. They favor the expulsion of the whey.

3. They permit of the fusing of the curd particles.

4. They activate the pepsin in the rennin extract.

5. They have a protective action against the putrefactive bacteria.

They state that the development of $B$. lactis acidi is followed by the Bacillus bulgaricus group.

These same authors in a later paper (Evans, Hastings and Hart, 1914) found the following four groups and assumed that they must function in the ripening process:

1. Bacterium lactis acidi.

2. B. casei.

3. Streptococcus.

4. Micrococcus.

When the Bacterium lactis acidi group was used alone as starters, in pasteurized milk cheese, no cheddar flavor was obtained. Combinations of the Streptococcus and B. lactis acidi groups when added to pasteurized milk improved the character of the cheese. Hart, Hastings, Flint and Evans (1914) isolated coccus forms of bacteria from cheddar cheese and cultured them in sterile milk. Large amounts of volatile acids were found on analysis of the culture. These acids were produced from either citric acid lactose or protein because the milk contained no fat. One strain of streptococcus formed large amounts of alcohols and esters which contribute to the flavor of the cheese. Suzuki, Hastings and Hart (1910) isolated and identified volatile acids during the ripening of cheddar cheese. Alcohol was formed probably from lactose fermentation. The agencies operative in the production of volatile acids and esters are not defined.

Harding and Prucha (1908) analyzed different cheeses and demon- 
strated that the number of bacteria decreases rather rapidly. Fresh cheeses were found to contain large numbers of bacteria. These decreased so that at the end of thirty or forty days there were less than $10,000,000$ bacteria per gram. These authors state that a cheddar cheese commercially ripe usually contains some millions of living bac-

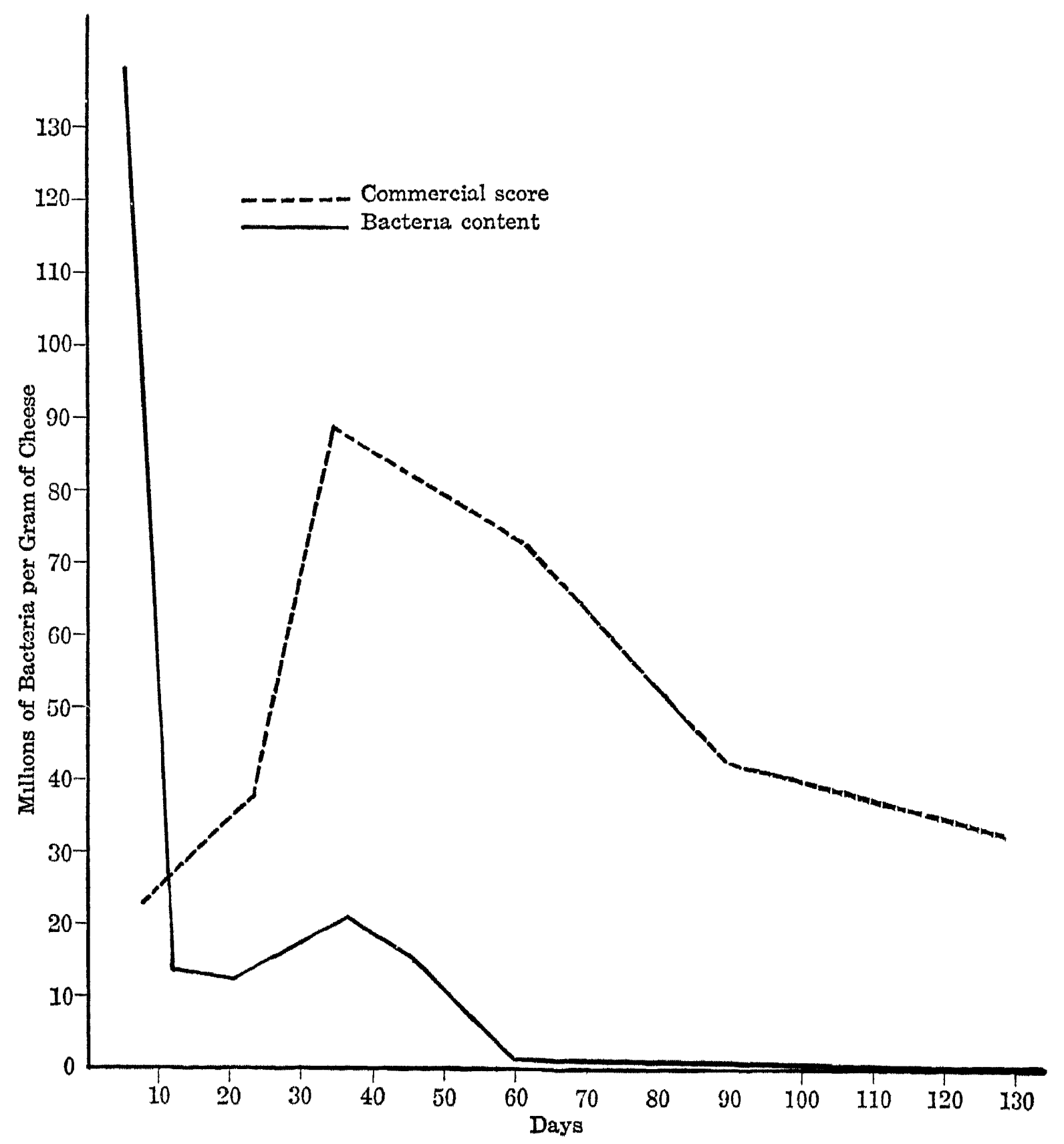

FIG. 73.-Bacteria Content and Commercial Score of Cheddar Chcese. (After Harding and Prucha, 1903.)

teria per gram. Fig. 73 shows the number of bacteria that were found in a cheese at various ages.

The relation of cheese to the spread of disease is an interesting question which has received some attention from investigators. Tuberculosis has received the greater part of the attention. Mohler, Wash- 
burn and Doane (1909) have mentioned and reviewed some of the work which was carried on previous to their publication. Hormann and Morgenroth (1898) upon examination of 15 samples of cottage cheese found 3 of which contained tubercle bacilli. Rabinowitch (1907) reported 3 positive samples out of 5 of the same product. Three samples of soft cheese purchased from the Berne markets by Harrison (1900) were found to produce tuberculosis when injected into guinea pigs. Heim (1889) and Galtier (1887) infected milk before making cheese. Heim reported tubercle bacilli in the cheese for fourteen days but none after four weeks. Galtier found tubercle bacilli in cheese which was two months and ten days old. He believes that such milk products may disseminate the disease among human beings, Mohler, Washburn and Doane (1911) prepared a cheese from infected milk and, by inoculation into guinea pigs, produced generalized tuberculosis from samples 220 days old. Injections of emulsions 260 days old caused slight lesions. Schroeder and Brett (1918) carried out an extensive piece of work which has greatly enriched our knowledge. They purchased 256 samples of cheese from the Washington market and subjected them to examinations for the presence of tubercle bacilli by means of guinea-pig inoculation. Nineteen or 7.42 per cent contained tubercle bacilli. Cheddar cheese was examined to the extent of 59 specimens; no tubercle bacilli were found. None were found in 32 specimens of Neufchatel cheese. Eighteen out of 131 samples of cream cheese were infecte l. They regard the danger of eating ripened cheese as very slight since the bacilli would die during the ripening process. According to Mohler's work, this would depend on the length of the ripening period. Rowland (1895) inoculated cheese and butter with $M$. cholerce and $B$. typhi. After a few days no living organisms could be found, which is regarded as reassuring by this author. He used cheddar, Dutch and American cheeses and both fresh and salt butter.

Cheese Poisoning. This type of food poisoning is said to be rather common. Vaughn named the poisonous substance in cheese tyrotoxicon which he regarded as a ptomaine. Newman (1902) and Lepierre (1894) confirmed his work. Spica (1910) recently isolated a poisonous substance from cheese. Levin (1917) studied this subject and found by means of agglutination reactions that a member of the colon group was involved in a case of cheese poisoning, which he investigated. No tyrotoxicon could be found.

Determination of the Number of Bacteria in Cheese. (Harding and Prucha's Method.) Remove a square inch of the rind by means of a sterile knife. Draw out a plug about 4 ins. long with a carefully flamed 
cheese trycr. Cut a number of thin slices from different parts of this plug and weigh out $1 \mathrm{gm}$. of these slices on a flamed copper foil using an analytical balance. This sample should be triturated in a flamed mortar with $10 \mathrm{gms}$. of sterile granulated sugar or finely ground sterilized quartz. Care should be taken to have the room free from dust and the instruments which come in contact with the cheese, sterile. Dilute this freshly ground cheese to 30 c.c. with sterile water. An aliquot portion should be transferred to a water blank. Plate out in litmus whey gelatin in dilutions of from 1000 to $1,000,000$ or thereabouts. Incubate for ten days at room temperature.

Lohnis' Method. One gm. of cheese taken under aseptic conditions and weighed according to the method described in another place is ground in a sterile mortar with 10 c.c. of sterile tap water, and some sterile quartz sand or glass powder added if necessary. The mixture is put into a dry, stcrile 1000 c.c. flask, which is then filled to the mark with sterile water. After continuous shaking for several minutes, further dilutions with 9 c.c. of water are made in test tubes. Usually dilutions of 1 to 10,000 to 1 to 100,000 are sufficient. This is plated out in plain agar and gelatin. Cultures for anaerobic incubation should also be made.

Harrison's Method. Remove a plug of cheese with a sterile cork barer and transfer to a sterile mortar. Triturate this with sterile water of bouillon. This is injected to a $500 \mathrm{gm}$. guinea pig. A hole is made in the skin with a large needle. Through this by means of a pipette the cheese emulsion is blown in. Animals should be kept under observation and immediately after death should be examined. Smears should be made from the diseased organs and stained from the Ziel Nielson method.

Determination of Tubercle Bacilli in Cheese. (Mohler's et al. Method.) Rub portions of the cheese in a mortar with physiological salt solution. After grinding strain the liquid through a layer of absorbent cotton and the equivalent of 2 gms. of cheese should be injected beneath the skin of each guinea pig. Also feed portions of the cheese to other pigs and keep for observations.

\section{Fermented MrLKs}

These are milks in which the lactose has been changed to lactic acid, alcohol or carbon dioxide by the action of fungi. An extensive literature of the subject, together with a discussion has been prepared by Rogers (1916). 


\section{Condensed Milk}

Milk has been condensed for a long time. In 1835, Newton took out a patent in England. Later, in 1849, Harsford added lactose to condensed milk.

The nomenclature of concentrated milks is not set. Condensed milk often refers to concentrated milks to which a carbohydrate, sucrose or lactose has been added. The term evaporated milk is often restricted to the plain concentrated milk to which no carbohydrates have been added. Again, these may be referred to as condensed sweetened milk and condensed unsweetened milks. The following definition for sweetened condensed milk has been accepted by the Association of American Dairy, Food and Drug Officials, August 7, 1916, and by the Association of Official Agricultural Chemists, November 22, 1916. "Sweetened condensed milk, sweetened evaporated milk, sweetened concentrated milk is the product resulting from the evaporation of a considerable portion of the water from the whole fresh, clean, lacteal secretion obtained by the complete milking of one or more healthy cows, properly fed and kept, excluding that obtained within fifteen days before and ten days after calving, to which sugar (sucrose) has been added. It contains not less than 28.0 per cent of total milk solids and not less than 8.0 per cent of milk fat." (U. S. Agriculture, Food Inspection Decision 158).

Condensed milk is not sterile. The data from many investications are on record in support of this. There seems to be no more bacteria or body cells in condensed milk than in raw market milk. Some of the processes of condensation remove these. The centrifugal separators are, doubtless, responsible for much of this reduction. The bacteria, which do pass through the condensation processes lie dormant on account of insufficient moisture and too concentrated solution. Some are present in the spore stage. This is probably true in those cases where members of the Bacillus subtilis group such as Bacillus subtilis and Bacillus mesentericus have been isolated. Thayer (1912) examined condensed milk which spoiled from twelve to twenty-four hours after condensing and found Bacillus subtilis. To obviate this difficulty, this author recommended a sterilization temperature of $125^{\circ} \mathrm{C}$. for fifteen or twenty minutes. Hammar (1915) isolated an organism which merely coagulatcd the canned milk. The name Bacillus coagulans was given to the organism. The coagulum was firm and had a sweetish taste. There was no indication of putrefaction. Andrews (1913) found 
that there were not enough pus cells in the milk which he examined to cause suspicion. The following sums up his opinion:

The presence, in reasonable number of the bacteria commonly found in fresh milk-Bacillus coli, streptococci, a few staphylococci, and B.enteritidis sporogenes-is comparatively unobjectionable. A large proportion of the bacteria of milk seem to be destroyed in the process of condensation. It was found that condensed milk was almost a differential medium for staphylococci. From his work it would seem that if a few Staphylococcus pyogenes aureus were present in the milk after condensation many more might be found when the can was opened. Efficient pasteurization before condensation should greatly reduce the possibility of the staphylococci getting into the final product.

Park et al. (1915) found that evaporated milk contained fewer bacteria than condensed milk. This may have been due to the higher temperature at which it is prepared. Kossowicz (1908) found that condensed milk was not bacteria free. He found Bacillus fluorescens liquefaciens, Bacillus prodigiosus and Bacillus sinapwagus to be present. Savage (1914) reports several investigations in England, the data from which do not differ materially from those secured in this country. The very interesting experiment of Delepine with regard to the effect of the condensation processes on Bacterium tuberculosis is mentioned. The tubercle bacillus, even though heavily seeded into milks, was killed and could not be demonstrated in the final condensed milk.

Delepine (1915) has studied the effect of preserving and drying milk on the virulence of the tubercle bacillus. In the manufacture of sweetened milk, the mixed raw milk had a bacterial content of $38,000,000$ which was reduced by the treatment to less than fifty in one case. The finished sweetened product failed to produce lesions when inoculated into and fed to rabbits and guinea pigs.

\section{ICE CREAM}

The term is a general one and covers many types of similar substances. Those interested in the history of this food should look up the paper by Washburn (1910).

The bacteriology of ice cream has, of late, received much attention since it became necessary to establish some bacterial standards. Wiley (1912) and his co-workers studied the ice cream manufactured in the District of Columbia and found rather large numbers of bacteria. Many of them contained streptococci. Esten and Mason (1915) studied the effect of storage on the number and kinds of bacteria. The 
cream was frozen in the usual type of freezer and stored in quart bricks. The method of analysis is given in another place. They found that there was little change in the number of bacteria as shown by litmus lactose gelatin plates when cream was kept frozen for a month.

Ayers and Johnson (1915) undertook an investigation on ice cream with the following objects:

1. To determine the number of bacteria in commercial ice cream during the summer and winter seasons.

2. To determine what groups of bacteria are found in commercial ice cream.

3. To determine the relative value of different methods for the determination of Bacillus coli in ice cream.

They found that the average number of bacteria in ice cream is lower during the winter months. A summary of their results is given in the following table:

\begin{tabular}{|c|c|c|}
\hline Item. & $\begin{array}{l}\text { Summer Series } \\
\text { (94 Samples). }\end{array}$ & $\begin{array}{l}\text { W inter Series } \\
\text { (91 Samples). }\end{array}$ \\
\hline $\begin{array}{l}\text { Average number of bacteria per cubic centimeter. } \\
\text { Maximum number.. } \ldots \ldots \ldots \ldots \ldots \ldots \ldots \ldots \ldots \ldots \\
\text { Minimum number. } \ldots \ldots \ldots \ldots \ldots \ldots \ldots \ldots\end{array}$ & $\begin{array}{r}37,859,807 \\
510,000,000 \\
120,000\end{array}$ & $\begin{array}{r}10,388,222 \\
114,000,000 \\
13,000\end{array}$ \\
\hline
\end{tabular}

Studies by means of the milk-tube method showed that the bacterial groups bore about the same relation to each other in the average summer and winter samples. During the summer months the averages based on 71 samples were as follows:

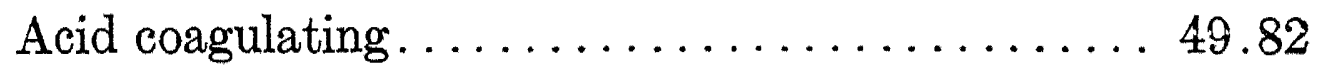

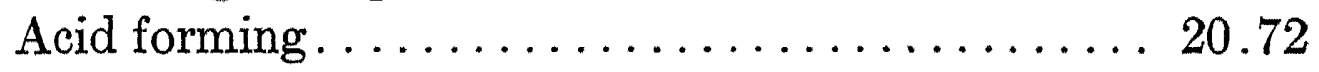

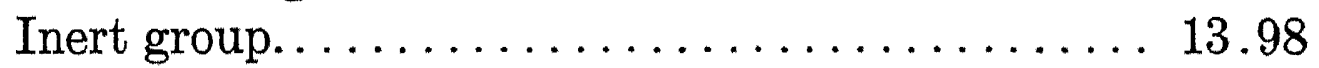

Alkali forming groups............... 1.86

Peptonizing group............... 13.62

The averages for the winter samples are:

Acid coagulating group.............. 30.84

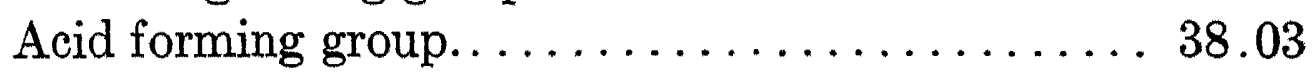

Inert group................... 4.81

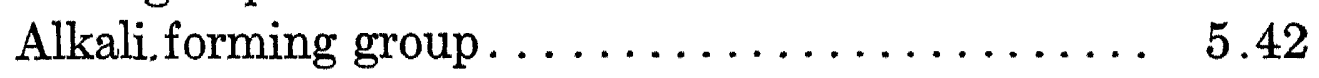

Peptonizing group................ 20.90

The peptonizing group is of most significance since it is these bacteria which cause poisoning from ice cream. 
Ayers and Johnson (1917) in a later paper report the results from a study of some phases of ice-cream analysis. It was found that bacteria are evenly distributed through a can of ice cream and that storage does not tend to cause an uneven distribution. These were factors which had been suspected of causing difficulties in the bacterial examination of ice cream. This was later confirmed by Heinemann and Gardan (1917). As freezing progressed clusters of bacteria were broken up and this tended to increase the count. No marked difference was found in the counts on samples from difierent parts of the same can.

Hammar and Goss (1917) analyzed ice cream and water sherbets. They reported very few organisms in the water sherbets compared with the ice cream. Ice creams, other than vanilla, were found to contain from 130,000 to $40,850,000$ bacteria per cubic centimeter. In general there was no increase in the number of bacteria during storage and generally there was a reduction. With some samples increases were reported. Table XLVII contains a few analyses reported by Hammar and Goss.

Table XLVII

SHOWING THE EFFECT OF STORAGE ON THE NUMBER OF BACTERIA IN ICE CREAM

(Hammer and Goss, 1917)

\begin{tabular}{|c|c|c|c|c|c|c|c|}
\hline \multicolumn{2}{|c|}{ Mix } & 236,000 & $32,800,000$ & 120,000 & $172,500,000$ & $110,000,000$ & $120,000,000$ \\
\hline \multicolumn{2}{|c|}{ Freeze. } & 735,000 & $30,850,000$ & 146,000 & $271,000,000$ & $170,000,000$ & $140,000,000$ \\
\hline \multicolumn{2}{|c|}{1 day old. } & 360,000 & $7,750,000$ & 137,000 & $157,000,000$ & $194,000,000$ & $70,000,000$ \\
\hline \multirow{2}{*}{$\begin{array}{l}2 \\
3\end{array}$} & "، & 310,000 & $4,450,000$ & 216,000 & $128,000,000$ & $216,000,000$ & $71,000,000$ \\
\hline & "، & 260,000 & $2,435,000$ & 10 & $52,000,000$ & $102,000,000$ & \\
\hline 4 & " & & $1,150,000$ & 152,000 & & & \\
\hline \multirow{2}{*}{$\begin{array}{l}5 \\
6\end{array}$} & "“ & 310,000 & & 300,000 & $34,000,000$ & $39,000,000$ & $41,000,000$ \\
\hline & "، & & & 139,000 & & & \\
\hline 7 & “" & & & 156,000 & $31,000,000$ & $54,000,000$ & \\
\hline \multirow{2}{*}{$\begin{array}{l}8 \\
9\end{array}$} & " & & & & & & $61,000,000$ \\
\hline & “" & & & & & $36,000,000$ & \\
\hline \multirow{2}{*}{$\begin{array}{l}10 \\
11\end{array}$} & " & & & & & & \\
\hline & " & & & & & $15,000,000$ & \\
\hline 12 & "6 & & & & & & \\
\hline
\end{tabular}

No special difficulties appear in the bacterial analysis of ice cream. Secure a brick of the cream or a pint or quart container if it is not available in brick form. As soon as it has reached the laboratory unwrap 
and remove samples from the interior of the mass of ice cream. This may be done by scraping off the exterior with a sterile spatula and by means of a sterile scoop transfer portions to sterile flasks. Heat these on a water bath at $40^{\circ} \mathrm{C}$. with occasional stirring until melted. Dilute with sterile pipettes and plate out using the standard media.

Bacillus Typhosus in Ice Cream. Several severe epidemics of typhoid fever have been caused by ice cream. Mitchell (1915) found that these bacteria could live for 12 to 39 days in ice cream and that ice cream could thus become an important epidemiological factor in the spread of the disease. Cumming (1917) reported an epidemic of typhoid fever caused by a carrier who participated in the preparation of the cream. Lumsden (1917) has given the characteristics of epidemics caused by ice cream. The difficulty of securing accurate data in such epidemics is mentioned. Waterman (1917) has reported a small outbreak of typhoid fever which probably came from ice cream; the typhoid history of the people involved is rather striking. Bolten (1918) reported experiments on freezing $B$. typhi in cream. At the end of a month the number of bacteria is reported to have been reduced to about one-twentieth of the original number. At the end of about 45 days after freezing in brine, some of the bacilli were found to be alive. As the author points out, ice cream may be a potent factor in the dissemination of typhoid fever.

Determination of the Number of Bacteria in Ice Cream (Esten and Mason). Samples are taken with sterilized knives, the outer layer being cut off before the sample is taken out. Allow the cream to melt at room temperature in the same manner as milk samples. Plate out on litmus lactose gelatin; incubate for 7 days at $21^{\circ} \mathrm{C}$. and count. Make four plates from each sample and report the results as an average of the four plates. By using litmus lactose gelatin the number of acidforming bacteria and the number of liquefying bacteria may be determined.

Savage's Method for Bacterial Analysis of Ice Cream. The ice cream should be collected in a sterile vessel, e.g., a wide-mouthed sterile bottle with glass stopper-and packed in ice if it cannot be examined at once.

To examine, melt the ice cream by placing for fifteen to twenty minutes in the $22^{\circ} \mathrm{C}$. incubator then treat as a milk sample.

The degree of dilution and the methods of examination are similar to those used in the examination of milk.

Determination of Streptococci in Ice Cream (Wiley et al.). The method for the detection of streptococci in ice cream has been used by 
Wiley and his co-workers as follows: The molted sample should be centrifuged for half an hour in a Stewart lactocrite with a speed of approximately 3000 r.p.m. This apparatus, which consists of a flat aluminum pan holding twenty tubes of 1 c.c. capacity and stoppered at the outer end with a specially constructed rubber plug, causes the sediment not only to be thrown to the end of the tube but drives it against the rubber plug with such a force that it is almost quantitatively adherent to the plug. Accordingly, if one removes the rubber stopper and by rubbing on a glass slide and over an area of known surface attaches the sediment, one can obtain, on staining and examining the film microscopically, an approximation of the number of organisms and leucocytes in 1 c.c. of the liquid. Because of the débris in the ice cream, which ordinarily renders the usual method of centrifuging milk and cream samples quite impracticable, the above method was resorted to so far as the detection of the presence of streptococci was concerned, it was found eminently satisfactory.

Davis (1914), in connection with investigations of septic sore throat decided that all of the streptococci concerned were of the hemolytic variety. These were found to remain alive in ice cream for eighteen days with little diminution in numbers. He concludes that ice cream is a suitable medium for the growth and dissemination of dangerous streptococci.

The Food and Drugs Act of the United States Department of Agriculture attempts to promote the production of pure.clean foods. With certain foods, as with ice cream, a satisfactory method of laboratory control has not been worked out, and to this end more attention must be given to the conditions of manufacture. The Commission on Milk Standards has proposed the following score card by which the ice cream plants may be rated. It is then assumed that there is some direct relation to the quality of ice cream and the conditions under which it is produced. 
SCORE CARD FOR ICE-CREAM MANUFACTURING PLANTS

\begin{tabular}{|c|c|c|}
\hline & Perfect. & Allow. \\
\hline 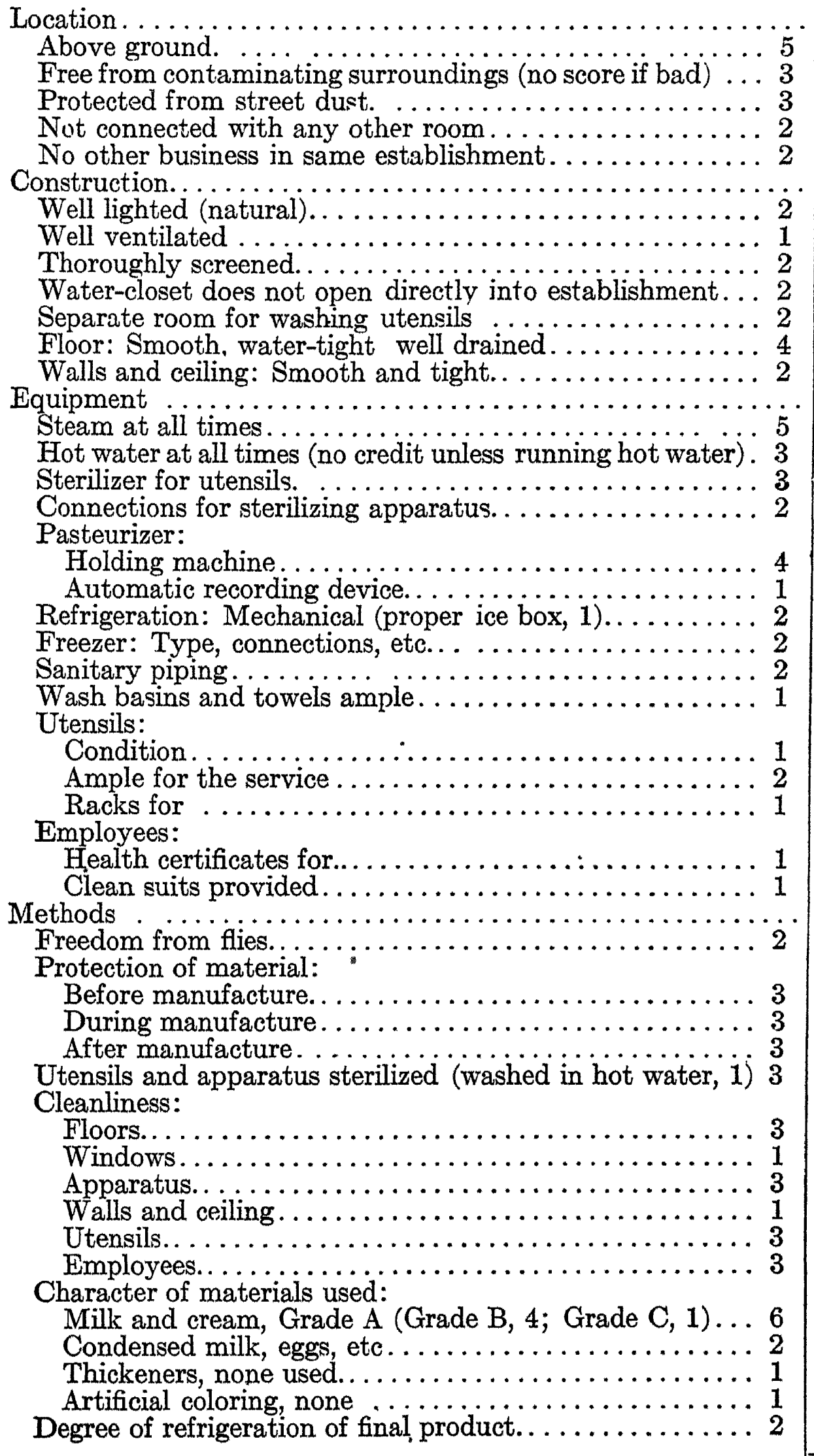 & 30 & \\
\hline 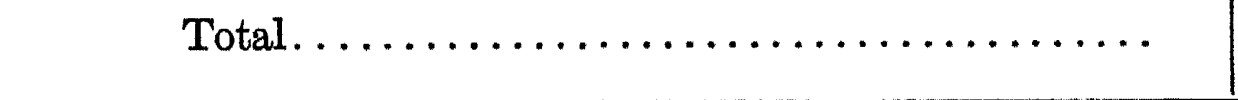 & 100 & \\
\hline
\end{tabular}




\section{BIBLIOGRAPHY.}

Abenhausen. 1900. Investigation on the Presence of Tubercle Bacilli in Marburg Butter and Margarine. Dissertation, Marburg, 1900.

Allen, P. W. 1916. Comparison of the Rate of Multiplication of Bacteria in Raw Milk with the Rate in Pasteurized Milk. Jour. of Infectious Diseases, 19 (1916), 721-728.

Allen, P. W. 1917. Comparisons of the Rate of Gas Production by Certain Bacteria in Raw and Pasteurized Milk. Jour. Infectious Diseases, 21 (1917), 219-225.

ANDrews, F. W. 1913. The Cytology and Bacteriology of Condensed Milk. Jour. Path. and Bact., 18, 169-178. Exp. Sta. Rec., 1913.

Anon. 1912. Tubercle Bacilli and.Uncooked Food. Vt. Bd. Health Bull. 12, $58-60$.

Ascher, N. 1899. Untersuchungen von Butter und Milch auf Tuberkelbaclllen. Zeit. f. Hyg., 32, 329.

Ayers, S H. and Jornson, W. T. 1910. The Bacteriology of Commercially Pasteurized and Raw Market Milk. U. S. Dept. of Ag., Bureau of Animal Industry, Bull. 126.

Ayers, S. H. and Johnson, W. T. 1913. A Study of the Bacteria which Survive Pasteurization. U. S. Dept. of Ag., Bureau of Animal Industry, Bull. 161.

Ayers, S. H. and Johnson, W. T. 1915. A Bacteriological Study of Retail Ice Cream. U. S. Dept. of Agr. Bull. 303.

Ayers, S. H. and Johnson, W. T. 1915. Ability of Colon Bacilli to Survive Pasteurization. Jour. Agr. Research, 3, 401-410.

Axers, S. H. and Johnson, W. T. 1917. The Determination of Bacteria in Ice Cream. U. S. Dept. Agr. Bull. 563.

Ayers, S. H., Cook, L. B. and Clemmer, P. B. 1918. The Four Essential Factors in the Production of Milk of Low Bacterial Content. U.S. Department of Agriculture Bulletin 642.

BABCOCK, S. M. and Russell, H. L. 1897. Unorganized Ferments of Milk; a New Factor in the Ripening of Cheese. Wisc. Ag. Exp. Sta. 14th Annual Report, 1897, 161-193.

Batr, A. C 1916. Ice Cream Making. Agr. Exp. Station, Univ. of Wis. Bull. 262.

Balazs, A. 1913. About the Detection of Boiled and Raw Milk. Abst. in Chem. Ztg., 37, 554.

BANG, B. 1891. Experimentelle Untersuchungen über Tuberculose Milch. Deut Zeit. f. Thiermed, 17, 1.

Basenar, F. 1895. The Behavior of Cholera Bacilli in Raw Milk. Arch. Hyg., 23 (1895), 170-183. 
Beattie, J. M. and Lewis, F. C. 1913. Acid Fast Bacilli in Milk. Jour. Path. and Bact., 18, 122-123, Abstract.

Bergey, D. H. 1911. The Isolation of Bacillus Typhosus from Butter. Jour. Med. Res., 25 (1911-12), 231-33.

Bolten, J. 1918. Effect of Freezing on the Organisms of Typhoid Ferer and Diphtheria. Public Health Reports, 33, 163-166.

Bosworth, A. W. 1913. The Action of Rennin on Casein. N. Y. Agr. Exp. Station Tech. Bull. 31.

Boyce. 1900. Results Quoted from Aunett Laucet, 1900, p. 160.

Boyd, M. F. 1917. Butter as a Vehicle of Infection in Typhoid. Jour. Amer. Med. Assn., 69, 2030-2032.

Brainerd, W. K. Clean and Sanitary Milk. Virginia Ag. Exp. Sta. Bull. 185.

BREed, R. S. 1911. The Determination of the Number of Bacteria in Milk by Direct Microscopical Examination. Cent. Bakt. Abt. II, 30, 337-340.

BreEd, R. S. 1913. The Significance of Body Cells in Milk. Proceedings, 6th Ann. Convention International Milk Dealers' Assn., 1913, 25-32.

Breed, R. S. 1914. Cells in Milk Derived from the Udders. N. Y. Agr. Exp. Station Bull. 380.

Breed, R. S. and Brew, J. D. 1917. The Control of Bacteria in Market Milk by Direct Microscopic Examination. New York Agr. Exp. Station Bull. 443.

BRew, J. D. 1915. Milk Quality as Determined by Present Dairy Score Cards. N. Y. Agr. Exp. Sta. Bull. 398.

Brew, J. D. and Dotterrer, W. D. 1917. The Number of Bacteria in Milk. New York Agr. Exp. Station (Geneva) Bull. 439.

Briscoe, C. F. and MacNeal, W. J. 1911. Tuberculosis of Farm Animals. Illinois Ag. Exp. Sta. Bull. 149, 317-431.

Browne, Wm. W. 1915. A Comparison of the Acid Production of the Bacillus Coli Group Isolated from Different Sources. Am. J. Pub. Health, 5 (1915), $39-48$.

BRuck, C. Experimentelle Beitrage zur Frage der Typhusverbreitung durch Butter. Cent. Bakt. Abt. I. Referate, 34, 778.

Brussaferro. 1890. Experiments on the Inoculation of Commercial Butter. Gironali di Med. Veter. Prat. Torino. Cited by Reitz.

Campbeld, H. C. 1909. Tubercle Bacilli in Market Milk in Philadelphia. 26th Ann. Rept. Bureau Animal Industry, 163-177.

CAmpbeli, H. C. 1916. Comparison of the Bacterial Count of Milk with the Sediment or Dirt Test. U. S. Department of Agriculture Bulletin 361.

CApPs, J. A. and Davis, D. J. 1914. The Relationship of Septic Sore Throat to Infected Milk. Jour. Inf. Dis., 15, 130-134.

Colwell, R. C. 1917. The Influence of Gargety and High-count Cows on the Number of Bacteria in Milk. Abstracts Bact., 1, 48-9.

Combs, W. B. and Eckers, C. H. 1917. The Relation of Oidium Lactis and Penicillium to the Keeping Qualities of Butter. Jour. Dairy Science, 1, 347-355. 
Commission on Milk Standards, Third Report of (1917), appointed by the New York Milk Committee. Reprint 386. Public Health Reports, 32, 271-296. See also 27, No. 19, May 10, 1912.

Committee Report. 1915. Standard Methods of Bacteriological Analysis of Milk. Am. J. Pub. Health, 5, 1315-25.

Conn, H. W. 1915. Standards for Determining the Purity of Milk. Reprint No. 295. Public Health Reports, 30, No. 33.

Cons, H. W. 1916. Reliability of the Bacteriological Analyses of Milk. Milk Dealer, 6, 50-45.

Conn, H. W. and Esten, W. M. 1902. The Comparative Growth of Different Species of Bacteria in Normal Milk. Ann. Report Storrs Ag. Exp. Sta., $14,13-80$.

Conn, H. W. and Esten, W. M. 1904. The Effect of Different Temperatures in Determining the Species of Bacteria which Grow in Milk. Ann. Rept. Storrs Agr. Exp. Sta., 16, 27-88.

Conn, H. W. and Stocking, W. A. 1903. Aseptic Milk. Ann. Rept. Storrs Ag. Exp. Sta., 15, 52-62.

Conn, H. W. and Stocking, W. A. 1903. Strained and Unstrained Milk Preserved at $70^{\circ}$ and $50^{\circ}$ F. Ann. Rept. Storrs Ag. Exp. Sta., 15, 38-51.

Coquot, A. 1908. The Passage of Tubercle Bacilli into Milk. Bull. Soc. Cent. Med. Vet., 85, 146-152.

Cumming, J. G. 1917. An Epidemic Resulting from the Contamination of Ice Cream by a Typhoid Carrier. Jour. Am. Med. Assn., 68, 1163-1165.

Dauson, C. F. 1898. Vitality and Retention of Virulence by Certain Pathogenic Bacteria in Milk and its Products. U. S. Dept. Agr., Bureau Animal Industry. Ann. Rept., 1898, 224-228.

Davis, D. J. 1914. The Growth and Viability of Streptococci of Bovine and Human Origin in Milk Products. Jour. Inf. Dis., 15, 378-388.

Delepine, S. 1915. Effects of Preserving Processes upon the Virulence of Tubercle Bacilli in Milk. Reviewed in the American Jour. Pub. Health, $5,379-380$.

Doane, C. F. and Lawson, H. W. 1908. Varieties of Cheese. U. S. Dept. of Agr. Bureau of Animal Industry Bulletin 105.

Du Claux, E. 1894. Le Lait, Paris.

Dyer, D. C. 1916. Progressive Oxidation of Cold-storage Butter. Jour. Ag. Res., 6, 927-952.

Eber, A. 1908. Investigations on the Tubercle Bacilli Content of Milk and Milk Products Sold in Liepsic. Zeit. Fleisch u. Milchhyg., 18, 309-319.

EBER, A. 1912. Investigations on the Presence of Tubercle Bacilli in Milk and Milk Products. Zeit. Fleisch u. Milchhyg., 22, 243-349.

Ennst, H. C. 1890. How Far May a Cow Become Tuberculous before her Milk Becomes Dangerous as an Article of Food. Amer. Jour. Med. Sciences, $88,439$.

Esten, W. M. 1909. Bacterium Lactis Acidi and its Sources. Storrs Ag. Exp. Sta. Bull. 59. 
Esten, W. M. and Mason, C. J. 19079 Soures of Bacteria in Milk. Ann. Rept. Storrs Ag. Exp. Sta. 1907-9, 65-107.

Evans, Alice C. 1916. Bacteria of Milk Freshly Drawn from Normal Udders. Jour. Inf. Dis., 18, 437-476.

Evans, Alice Hastings, E. G. and Hart, E. B. 1914. Bacteria Concerned in the Production of the Characteristic Flavor in Cheese of the Cheddar Type. Jour. Agricultural Research, 2, 167-192.

Ford, W. W. and Pryor, J. C. 1914. Observations upon the Bacteria found in Milk Heated to Various Temperatures. Bull. Johns Hopkins Hosp., 25, $270-276$.

Fremy. 1839. Quoted from another author. Ann. de pharm., 31, 188.

Freudenreich, E. 1897. Ueber die Erreger der Reifung der Emmenthaler Käse. Landw. Jahrb. Schweiz. Jahrg., 11, 85-101.

FreudenREICH, E. 1902. Milchsauerefermente und Käsereifung. Landw. Schweiz. Jahrb., 16, 91-104.

Freudenreich, E. and Thoni, J. 1905. Sur l'action de differents lactiques sur la maturation du fromage. Rev. Generale du Lait., 4, 169-181; 200209; 225-232; 247-259. Also see Vol. 2, 241-247; 271-280.

Frost, W. D. 1915. A Microscopic Test for Pasteurized Milk. Jour. Amer. Med. Assn., 64, 821-822.

Frost, W. D. 1916. Confirmation Tests for B. Coli in Routine Water Examination. Am. Jour. Pub. Health, 6, 1916, 585.

Frost, W. D. 1916. Comparison of a Rapid Method of Counting Bacteria in Milk with the Standard Plate Method. Journal Infect. Dis., 19, 273-287.

Fróst, W. H. 1916. Relationship of Milk Supplies to Typhoid Fever. Public Health Reports, 31, 3291-3302.

Frost, W. D. 1917. Counting the Living Bacteria in Milk-A Practical Test. Jour. of Bact., 2, 567-583.

Frosr, W. D. and Ravener, M. 1911. A Microscopic Test for Heated Milk. Proc. Am. Assn. Med. Milk Commissions, 1911, p. 127.

GAGE, S. M. and Stoughton, Grace V. 1906. A Study of the Laws Governing the Resistance of Bacillus Coli to Heat. Technology Quarterly, 19, 41-45.

Galtier, B. 1887. Dangers de l'utilization des produites tel que le petitlait et le fromage, oblenus aves le lait de raches tuberculeuses. Comp. Rend. Acad. Sci., 104, 1333-1335.

GebHaRdr, F. 1890. Experimentelle Untersuchungen über den Einfluss der Verdünnung auf die Wirksamkeit des Tuberkelosen Giftes. Virch. Arch., $109,127$.

Goodrich, G. W. 1914. Comparison of the Plating and Microscopic Methods in the Bacteriological Examination of Milk. Jour. Inf. Dis., 14, 512-519.

Gorins, C. 1904. Sur la presence de bacteries productrices d'acidité et de presure dans les fromages en maturation. Rev. Générale du Lait, 3, 505510.

Grassburger, R. 1899. The Intraperitoneal Injection of Market Butter and the Resulting Changes. Münchener Med. Wochenschr., 46, 341-344. 
Gróning. Cited in Kolle und Wassermann. Handbuch der Pathogenen Mikroorganisms, 2, 140.

GUTHRIE, E. S. 1917. Concerning Rancịdity of Butter. Jour. Dairy Science, $1,218-233$.

Hammar, B. W. 1915. Bacteriological Studies on the Coagulation of Condensed Milk. Iowa Ag. Exp. Sta. Res. Bull. 19.

Hammar, B. W. 1912. Bacteria and Ice Cream. Iowa Agr. Exp. Sta. Ames. Bull. 134.

Hammar, B. W. and Goss, E. F. 1917. Bacteria in Ice Cream. II. Iowa Agric. Exp. Sta. Bull. 174.

Hammansten. Quoted from another author. Maly's Jahresbericht, 1872, p. $118 ; 1874$, p. $135 ; 1877$, p. 158.

Harding, H. A. 1916. Present Status of the Score Card of Milk Quality. Amer. J. Pub. Health, 6 (1916), 562.

Harding, H. A. 1917. Bacterial Count as an Index of Cleanliness in Milk. Abstracts of Bacteriology, 1 (1917), 53-54.

Harding, H. A. and Prucha, M. J. 1908. The Bacterial Flora of Cheddar Cheese. New York Agr. Exp. Station Tech. Bull. 8.

Harding, H. A., Breed, R. S., Stocking, Jr., W. A. and Hastings, E. E. 1917. What is Meant by Milk Quality. University of Illinois Agr. Exp. Sta. Circular No. 205.

Harding, H. A., RueHze, G. L., Wilson, J. K. and Sminh, G. A. 1913. The Effect of Certain Dairy Operations upon the Germ Content of Milk. N. Y. (Geneva) Agr. Exp. Sta. Bull. 365. (Contains a number of references on this subject.)

HarmanN and Morgauroth. 1898. Weitere Mittheilungen uber Tuberkelbacillen-Befunde in Butter und Kàse. Hyg. Rund., 8, 1081-1084.

Harrison, F. C. 1896. Bacterial Contamination of Milk. Ann. Rept. Ontario Ag. Coll., 22, 105-114.

Harrison, F. C. 1902. The Duration of the Life of the Tubercle Bacillus in Cheese. U.S.Dept. Agr., Bureau Animal Industry 19th Ann Rept., 217-233.

Hart, E. B., Hastings, E. G., Flint, E. M. and Evans, Alice C. 1914. Relation of the Action of Certain Bacteria to the Ripening of Cheese of the Cheddar Type. Jour. Agr. Res., 2, 193-216.

Hastings, E. G. 1916. Cause and Prevention of Mold on Butter. Proc. Wis. Buttermakers' Assn., 16, 145-152.

Hastings, E. G., Evans, A. C. and Hart, E. B. 1912. Studies on the Factors Concerned in the Ripening of Cheddar Cheese. Wisconsin Agr. Exp. Station. Research Bulletin 25.

Hastings, E. G., Evans, A. C. and Hart, E. B. 1912. The Bacteriology of Cheddar Cheese. U. S. Dept. of Agriculture. Bureau of Animal Industry Bull. 150.

HeIm. 1889. Ueber das Verhalten der Krankheitserreger der Cholera, des Unterleibtyphus und der Tuberculose in Milch, Butter, Molken, und Käse. Arb. a. d. kaiserl. Gesundh., 5, 294-311. 
Heinemand, P. G. and Gordan, J. E. 1917. Creamery and Milk Plant, 5 $44-52$.

Hellstrom, F. E. 1900. The Estimation of Tubercle Bacilli in Butter and a Few Investigations on Pathogenic Bacteria in Butter from Pasteurized and Unpasteurized Cream. Cent. Bakt. Abt. I. Ref., 28, 542-555.

Henike, G. W. and Thompson, I. F. 1917. Septic Sore Throat: Epidemiological study of a milk-borne epidemic at Galesville, Wis., due to the use of infected milk. Jour. Am. Med. Assn., 68, 1307.

HeRbert. Investigation on the Presence of Tubercle Bacilli in Butter. Cited by Reitz, A. (1906).

HerR, F. and Beninde, M. 1901. Investigation on the Presence of Tubercle Bacilli in Butter. Zeit. Hyg., 38, 152-181.

Hess, A. H. 1908. The Incidence of Tubercle Bacilli in New York City Milk. Jour. Am. Med. Assn., 52, 1011-1016.

Hesse, W. 1894. Die Beziehung zwischen Kuhmilch und Cholera Baketerien. Zeit. f. Hyg., 17, 238.

Hessler, G. 1909. Ein Beitrag zur Frage der Infektiosität der Milch tuberkulöser Kühe. Inaug. Dissert. Univ. Bern., 1909.

Hrwl, H. W. 1911. Typhoid Fever from Butter. Third Biennial Rept. Minn. State Bd. Health, p. 203.

HIrschbergeR, K. 1889. Experimentelle Beiträge zur Infektiosität der Milch tuberculoser Kuhe. Deut. Arch. Klin. Med., 44, 400.

Horman and Morgenroth. 1898. The Presence of Bacteria in Butter. Hygienische Rundschau, 8, 217-230.

Horman and Morgenroth. 1898. Further Contribution on the Presence of Tubercle Bacilli in Butter and Cheese. Hyg. Rund., 8, 1081-1084.

Ice Cream, Report of Hearing on, before Dr. C. A. Alsberg, Chief of the Bureau of Chemistry, U. S. Dept. of Ag., February 10, 1914 and March 7, 1914. Subject: The Use of Colloids as Stabilizers in Ice Cream, the Butter Fat Standards for Ice Cream and the Bacteriology of Ice Cream, with Special Reference to the Cincinnati Ice Cream Cases. Published by the National Association of Ice Cream Manufacturers, June, 1914.

Jensen, OrLA. 1916. The Use of Amyl Alcohol in Gerber's Method for the Determination of Fat in Milk. Maelkeritid, 29, 104-106.

Jordan, E. O. 1917. The Bacterial Examination of Foods. American Food Journal, 12, 251-252. Jour. Am. Med. Assn., 68, April 14, 1917.

JosHr, L. L. 1913. Observations on the Bacteriological and Chemical Examination of the Milk Supply of Bombay. (Calcutta, 1913, 20 pp.)

Josmi, L. L. 1915. Bacteriological Examination of the Bombay Milk Supply. Jour. Dairying (India), 3, 5-36.

KInYoun, J. J. 1914. A Bacteriological Index for Dirt in Milk. U. S. Naval Medical Bulletin 8, 435-442.

KuEIN, A. 1900. Zur Kenntniss der Verbreitung des Bacillus Tuberculosis und Pseudotuberculosis in der Milch Sowie der Biologie des Bacillus Tuberculosis. Cent. Bakt. Abt. I, 28, 111. 
KonN, O. 1899. Tubercle Bacilli in Butter. Arch. Hyg., 36, 57-65.

Kossowicz, A. 1908. Bacterial Content of Condensed Milk. Z. landw. Vers. Wes. Oesterr., 11, 719-24.

Krumwiede, C. and Noble, W. C. 1915. The Viability of the Typhoid Bacillus in Sour Cream. Collected Studies from the Bureau of Laboratories. City of New York, Vol. 8, 311.

Krumwiede, C. and Valentine, E. 1915. A Bacteriological Study of an Epidemic of Septic Sore Throat. Jour. Med. Res., 33, 231-238.

LAPIERRE, C. 1894. Chimie unimale-analyse d'un fromage avarie extraction d'une ptomaine nouvelle. Comp. Rend. Acad. Sci., 118, 476-482.

LASER, H. The Behavior of Bacteria of Typhoid Fever, Tuberculosis and Cholera in Butter. Zeit. Hyg., 10, 513-520.

Levin, W. 1917. Cheese Poisoning. A toxicogenic bacillus isolated from cheese. Jour. Lab. Clinical Medecin, 2, 3-26.

Lumsden, L. L. 1917. The Determination of Ice Cream as a Factor in the Spread of Typhoid Fever Infection. Am. Jour. Pub. Health, 7, 1005-1009.

Luxwolda, W. B. 1911. The Growth of Some Milk Bacteria at Different Temperatures. Cent. Bakt. Abt. II, 31, 129-175.

Marchisotiti, A. C. 1917. The Presence of the Tubercle Bacillus in Butter. Bev. Facult. Agron. y. Vet. La Plata, 2, Series 2, 12. 279-298.

MarkL. 1901. On the Presence of Tubercle Bacilli in Vienna Market Butter and Margarine. Cent. Bakt. Abt. I. Ref., 29, 954.

Marshall, W. E. 1907. Note on the Occurrence of Diphtheria Bacilli in Milk. Jour. Hyg., 7, 32-34.

Mason, C. J. 1915. The Bacteria in Ice Cream. Storrs (Conn.) Agr. Exp. Sta. Bull. 83.

Melvin, A. D. 1912. The Score-card System of Dairy Inspection. U. S. Dept. Agr. Bur. of Anim. Ind. Cir. 199.

Mohler, J. R.. Washburn, H. J. and Doane, C. F. 1909. The Viability of Tubercle Bacilli in Cheese. 26th Ann. Rept. Bureau Animal Industry, 187-191.

Mohler, J. R., Washburn, H. J. and Rogers, L. A. 1909. The Viability of Tubercle Bacilli in Butter. U. S. Department of Agriculture, Bureau of Animal Industry, Annual Report for 1909, 179-185.

Morganroth. 1899. On the Presence of Tubercle Bacilli in Margarine. Hyg. Rundschau, 9, 1121.

North. 1913. Discussion following Article by Pease. (1913).

North, C. E. 1916. Bacterial Testing versus Dairy Inspection. Am. J. Pub. Health, 6 (1916), 569.

North, C. E. 1917. A Survey of Dairy Score Cards. Amer. Jour. Pub. Health, 7, 25-39.

Northrup, Z. 1911. The Influence of the Products of Lactic Organisms upon Bacillus Typhosus. Mich. Ag. Exp. Sta. Tech. Bull. 9.

Obermulemer. 1899. Tubercle Bacilli in Market Butter. Hyg. Rund., 9, $57-79 ; 7,712-714,1897 ; 5,877,1895$. 
Overton, F. 1914. An Epidemic of Septic Sore Throat at Easthampton (N. Y.) and its Control. Health News, N. Y. State Department of Health, 9, 379-371.

Overton, F., Krumwiede, C. and Jaques, A. D. 1914. An Epidemic of Septic Sore Throat in Rockville Center, N. Y. Health News, N. Y. State Department of Health, 9, 230-233.

PaRK, W. H., et al. 1915. A Sanitary Study of Condensed Milk. N. Y. Med. Jour., 102, 1073-1083.

PARK, W. H. 1918. "What Constitutes Good Milk." Jour. Am. Med. Assn., 70, 720.

PARK and Williams. 1917. Pathogenic Ilicroörganisms. Lea \& Febiger.

Parker, H. N. 1917. City Milk Supplies. McGraw-Hill Book Co., New York City.

Pawlowsky. 1900. The Presence of Tubercle Bacilli in Market Butter. Comp. Rend. 10 Intern. Cong. Hyg. et Demog., 187-189.

Pease, H. D. 1913. The Sanitary Significance of Bacteria in Milk. 6th Ann. Convention, International Milk Dealers' Association Proceedings, 1913, 105-116.

Pease, H. D. 1916. Some Observations on Causes of High Bacterial Counts in Market Milk. Amer. Jour. Pub. Health, 6, 563-568.

Peiser, L. 1916. Bacteriology of Cream Ripening. Milk Dealer, 5, 26-27.

Pennington, M. E. et al. Bacterial and Enzyme Changes in Milk and Cream at $0^{\circ}$ C. Jour. Biol. Chem., 16, 331-368.

Pennington, M. E. 1908. Bacterial Growth and Chemical Changes in Milk Kept at Low Temperature. Jour. Biol. Chem., 4, 353.

Petri, R. F. 1898. Zum Nachweis der Tuberkelbacillen im Butter und Milch. Arb. Gesundsheitsamt, 14, 1.

Prescott, S. C. and Breed, R. S. 1911. The Determination of the Number of Body Cells in Milk by a Direct Method. Jour. Inf. Dis., 7, 632-40.

Prucha, M. J., Harding, H. A. and Weeter, H. M. 1915. Utensils as a Source of Bacterial Contamination of Milk. Science, 42. 353.

Prucha, M. J., Weeter, H. M. and Chambers, W. H. 1918. Germ Content of Milk. II. As influenced by the utensils. Univ. of Ill. Ag. Exp. Sta. Bull. 204.

Prucha, M. J., Weeter, H. M. and Chambers, W. H. 1918. Germ Life in Cow Feces. Abstracts of Bacteriology, 2, 6 .

Rabinowitch, L. 1897. On the Question of Tubercle Bacilli in Market Butter. Zeit. Hyg, 26, 90. Exp. Sta. Record, 9, 689.

Rabinowitch, L. 1899. Tubercle Bacilli in Butter. Deut. Med. Wochenschr., 25,5 .

RabiNowITSCH, M. 1907. Verbreitung der Tuberkelbacillen durch Milch und Fleisch. In. G. Cornet's Die Tuberkulose. 2d Edit. Pt. I, 123 Vienna, 1907.

Ravenel, M. P., Hastings, E. G. and Hammar, W. B. 1910. The Bacterial Flora of Milk held at Low Temperatures. Jour. Inf. Dis., 7, 38-46. 
REed, H. S. 1914. Manual of Bacteriology. Ginn \& Co.

ReEd, H. S. and Reynolds, R. R. 1916. Some Effects of Temperature upon the Growth and Activity of Bacteria in Milk. Virginia Ag. Exp. Sta. Tech. Bull. 10.

Reirz, A. 1906. Bacteriological Examination of Butter. Cent. Bakt. Abt II., 16, 193-212.

Rogers, L. A. 1916. Fermented Milks. U. S. Dept. Ag. Bull. 319.

Rogers, L. A., Clark, W. M. and Evans, A. 1914. The Characteristics of Bacteria of the Colon Type found in Bovine Feces. Jour. Inf. Dis., 15, 100-123.

Rogers, L. A. and Dahlberg, A. O. 1914. The Origin of Some Ntreptococci Found in Milk. Jour. Agr. Res., 1, 491.

Rogers, Clark and Evans. Colon Bacteria and Streptococci and their Significance in Milk. Am. J. Pub. Health, 6 (1916), 381.

Rosenar, M. J. 1912. The Number of Bacteria in Milk and the Value of Bacterial Counts. In milk and its relation to the public health. Hyg. Lab. Bull. 56, 427-453.

Rosenar, M. J., Frost, W. D. and Bryant, R. 1914. A Study of Market Butter of Boston. Jour. Med. Res., 30, 69.

Rosenow, E. C. and Hess, C. L. 1917. An Epidemic of Septic Sore Throat Due to Milk. Jour. Am. Med. Assn., 68, 1305.

Rosenow, E. C. and Moon, V. H. 1915. On an Epidemic of Sore Throat and the Virulence of Streptococci Isolated from the Milk. Jour. Inf. Dis., 17, 69-71.

Rowland, S. D. 1895. Cheese and Butter as Possible Carriers of Typhoid and Cholera. Brit. Med. Jour., 1895, No. 1799.

Ruehle, G. L. A. and KuLP, W. L. 1915. Germ Content of Stable Air and its Effect upon the Germ Content of Milk. N. Y. (Geneva) Agr. Exp. Sta. Bull. 409.

Russeli, H. L. 1893-4. The Source of Bacterial Infection and the Relation of the Same to the Keeping Quality of Milk. Ann. Rept. Wis. Ag. Exp. Sta., 11, 150-165.

Russell, H. L. and Hastrngs, E. G. 1902. A Micrococcus, the Thermal Death Point of which is $76^{\circ}$ C. Cent. f. Bakt. Abt. II., 8, 339-342.

Russeld, H. L. and Hastings, E. G. 1904. Effect of Short Periods of Exposure to Heat on Tubercle Bacilli in Milk. 21st Ann. Rept. Wis. Ag. Exp Sta., 178-192.

Savage, W. G. 1906. Streptococci and Leucocytes in Milk. Jour. Hygiene, $6,123-138$.

Savage, W. G. 1909-10. Report upon the Bacterial Measurement of Milk Pollution. Ann. Rept. Local Govt. Bd. (Gt. Brit.), 39, 474-503.

SAvAGE, W. G. 1914. The Bacteriological Examination of Food and Water. Cambridge Univ. Press, p. 112.

Schrieder, M. C. 1914. Dirt Sediment Testing-a Factor in Obtaining Clean Milk. Amer. Jour. Pub. Health, 4, 50-64. 
Schroeder, E. C. 1907. Milk and its Products as Carriers of Tuberculous Infection. 24th Ann. Rept. Bureau of Animal Industry, 183-199. (Good Bibliography appended.)

Schroeder, E. C. and Brett, G. W. 1918. Public Health Studies Concerning Cheese. Jour. Am. Vet. Assn., 52, 674-685.

Schroeder, E. C. and Cotton, W. E. 1908. Tubercle Bacilli in Butter. Their Occurrence, Vitality and Significance. U. S. Dept. Agric., Bureau of Animal Industry Circular 127.

Schuchardt. 1896. An Investigation of the Presence of Tubercle Bacilli in Butter. Dissertation, Marburg.

Sherman, J. M. 1915. Studies on the Produetion of Sanitary Milk. Penn. Ag. Exp. Sta. Rept., 1915, 299-305.

Sherman, J. M. and Reynolds, F. P. 1915. Bacteriological Methods for Determining the Quality of Milk. I. The reaction of agar for the bacterial analysis of milk. Penn. Ag. Exp. Sta., 1915, 294-299.

Sherman, J. M. 1916. The Advantages of a Carbohydrate Medium in the Routine Bacterial Examination of Milk. Jour. Bact. I., 481-492.

Slack, F. H. 1917. Some Observations on the Bacterial Examination of Milk. Amer. Jour. Pub. Health. 7, 690-697.

SmrLuIE, W. G. 1917. Studies of the Beta Hemolytic Streptococcus (Smith and Brown). Jour. Inf. Dis., 20, 45-48.

Sмiт, H. J. 1908. Ueber das Vorkommen von Tuberkelbacillen in der Milch und den Lymphdrusen des Rindes. Inaug. Diss. Univ. Bern., 1908.

Sмгтн, Tн. 1899. The Thermal Death Point of Tubercle Bacilli in Milk and Some Other Fluids. Jour. Med. Res., 4, 233.

-. 1916. Standard Methods of Bacteriological Milk Analysis. Provisional report of the laboratory section of the American Public Health Association.

SteIN, G. 1884. Experimentelle Beiträge zur Infektion der Milch Perlsuchtiger Kuhe. Inaug. Dissert., Berlin.

Stockrng, W. A. 1906. Quality of Milk Affected by Common Dairy Practices. Storrs. Ag. Exp. Sta. Bull. 42.

Stokes, W. R. and WeGeForth, A. The Microscopic Examination of Milk. Med. News, 71 (1897), 15-48.

Suzuki, S. K., Hastings, E. G. and Hart, E. B. 1910. The Production of Volatile Acids and Esters in Cheddar Cheese and their Relation to the Development of the Flavor. Wisconsin Agr. Exp. Station Research Bull. 11.

Sweer, E. A. 1917. Safe Milk, an Important Food Problem. Public Health Reports Supp. No. 31.

TAYLOR, G. B. 1917. Experiments on Determinations of Cow Manure in Milk; Moisture Content and Solubility of Cow Manüre. Journal of Dairy Science, 1, 303-312.

Tetchert. 1904 Bacteriological and Chemical Studies of the Butter in the Province of Posen with Special Reference to the Tubercle Bacillus. Cent. Bakt. Abt. II., 13, 560-561. 
Thayer, L. A. 1912. A Bacterial Infection of Condensed Milk. Vermont Agricultural Experiment Station Bull. 170, 123-124.

Thom, J. 1914. On the Hygienic Bacteriological Character of Bern Market Milk with Reference to the Presence of Tubercle Bacilli. Cent. Bakt. Orig. Abt I., 74, 11-69.

Thue, H. 1905. Investigations on the Presence of Tubercle Bacilli in Milk, Butter and Margarine in Christiania. Cent Bakt. Abt. I., Ref., 36. 597.

Tobler, M. 1901. Contribution on the Question of the Presence of Tuhercle Bacilli and other Acid-fast Bacteria in Market Butter. Zert. f. Hyg., 36, 120-148.

Trask, J. W. 1912. Milk as a Cause of Epidemics of Typhoid Ferer. Scarlet Fever and Diphtheria. In milk and its relation to the public health. Bull. 56. Hyg. Lab. U. S. Public Health Service.

Van Slyke, L. L. and Bosworth, A. W. 1914. Studies Relating to the Chemistry of Milk and Casein. N. Y. Ag. Exp. Sta. Tech. Bull. 37.

VAN Slyke, L. L. and Bosworth, A. W. 1912. Composition and Properties of Some Casein and Paracasein Compounds and their Relations to Cheese. New York Agricultural Experiment Station, Tech. Bull. 26.

Van Slyke, L. L. and Bosworth, A. W. 1916. Chemical Changes in the Souring of Milk. N. Y. Agr. Exp. Sta. Tech. Bull. 48.

Vaughn, V. C. 1886. A Ptomaine from Poisonous Cheese. Zeit. Physiol. Chem., 10, 146.

Washburn, H. J. 1908. The Viability of Typhoid Bacilli in Milk and Butter. 25th Ann. Rept. Bureau Animal Industry, 297-300.

Washburn, R. M. 1910. Principles and Practice of Ice Cream Making. Vermont Agr. Exp. Sta. Bull. 155.

Washburn, R. M. and Dahlberg, A. C. 1917. The Influence of Salt on the Changes Taking Place in Butter. Journal of Dairy Science, 1, 114-126.

Waterman, E. L. 1917. Typhoid Fever from Ice Cream. Public Health Bulletin of the Michigan State Board of Health, 5, 157-159.

Weigmann, H., Wolff, A., Treusch, Marg., Steffen, M. 1916. The Behavior of Streptococcus Lacticus on Heating Milk at $60^{\circ}$ to $73^{\circ} \mathrm{C}$. (modern pasteurization). Cent. f. Bakt. Abt. II., 45, 63-107.

Weinzire. A Bacteriological Method for Determining Manural Pollution of Milk. Am. J. Pub. Health, 5 (1915), 862-66.

Weissenfald. 1899. Bacteria in Butter and Other Milk Products. Berl. klin. Wchschr., 36, 1053-1055.

WrLeY, H. W. 1912. Ice Cream. In milk and its relation to the public health, pp 249-312.

Winslow, C. E. A. and Hubbard, L. W. 1915. A Contact Outbreak of Septic Sore Throat in Westchester County (N. Y.). Health News, N. Y. State Dept. Health, 9, 266-270. 


\section{CHAPTER XII}

\section{EXAMINATION OF EGGS}

EGGs have always been an important factor in the diet. As the density of population increased, especially in urban centers, the problem of securing fresh eggs became more and more acute. This was rendered more intricate by the fact that the greatest part of the annual lay is secured during the months of March, April, May and June. This makes it necessary to hold over, either by cold storage or preservation, the abundance of these months against the want of the others. It has become of much importance to determine what factors are important in the keeping of this food product.

A knowledge of the physiology of the hen which is concerned with the development of the egg is important in this connection. This has been well described by Benjamin (1915) from whose publication the following has been adapted. The oviduct of the hen is located in the rear part of the body cavity. The yolk is the first part to develop and this takes place in the ovary which may contain a large number of minute yolks. Each is contained in a sac, or follicle through which it sccures its nourishment while developing. After development the yolk enclosed in its vitellin membrane escapes from the yolk sac and descends through the oviduct. If fertilization occurs, it takes place soon after the egg has entered the oviduct and before any albumen is deposited about it. About 40 per cent of the albumen is said to be laid down as the yolk passes through the upper half of the oviduct. After passage through this albumen-forming region, it reaches the isthmus where the shell membranes are added with about 10 to 20 per cent more albumen. The uterus is then reached, where the remainder of the shell is added. During its passage through the vagina, just before its expulsion from the cloaca, it probably receives the outer gelatinous coating on the shell. The structure of the egg and shell is given in Figs. 75 and 76.

The chemical composition of any food substance is important in determining the type of composition which may take place. This is especially true with a substance like eggs. The analysis quoted in Table XLVIII is taken from the paper of Benjamin (1915). 


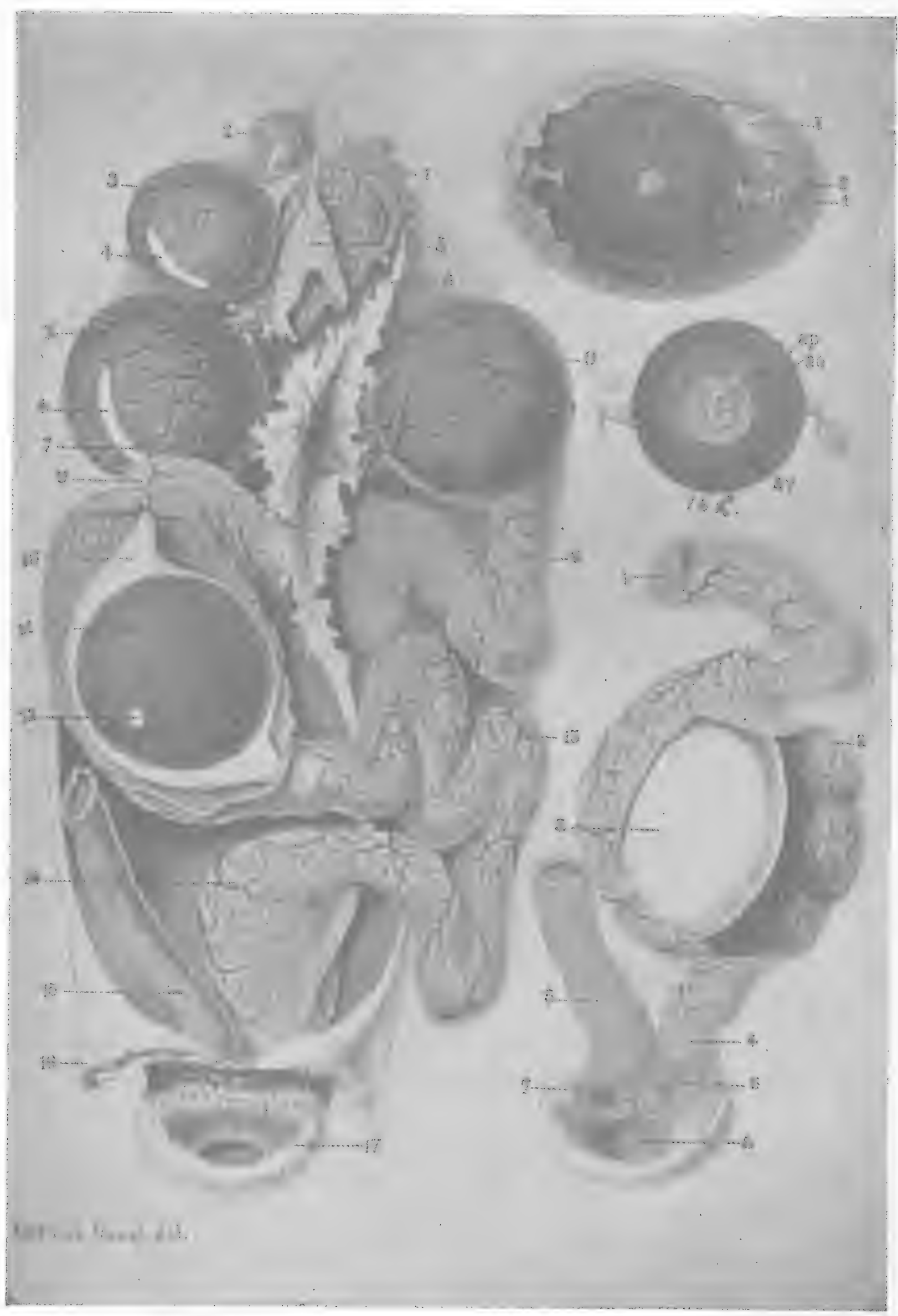

1. ovary, with minute oviles; 2-3, yolk sacs; 4, suture line; 5 , empty yolk sac; 7 , funnel opening into oviduct; 8 , yolk in oviduct; 9 , albumen-secreting region; 10 , al bumen being secreted; 11, yolk passing through oviduct; 12, germinal disk; 13, isthmus; 14, uterus; 15, large intestines; 17, cloaca.

On the right-hand side of the figure are shown, from the top downwrd: Complete egg; yolk of egg incubated for sixteen hours; completed egg in uterus-(1) isthmus, (2) glands of uterus, (3) complete egg, (4) vagina, (8) cloaca, 


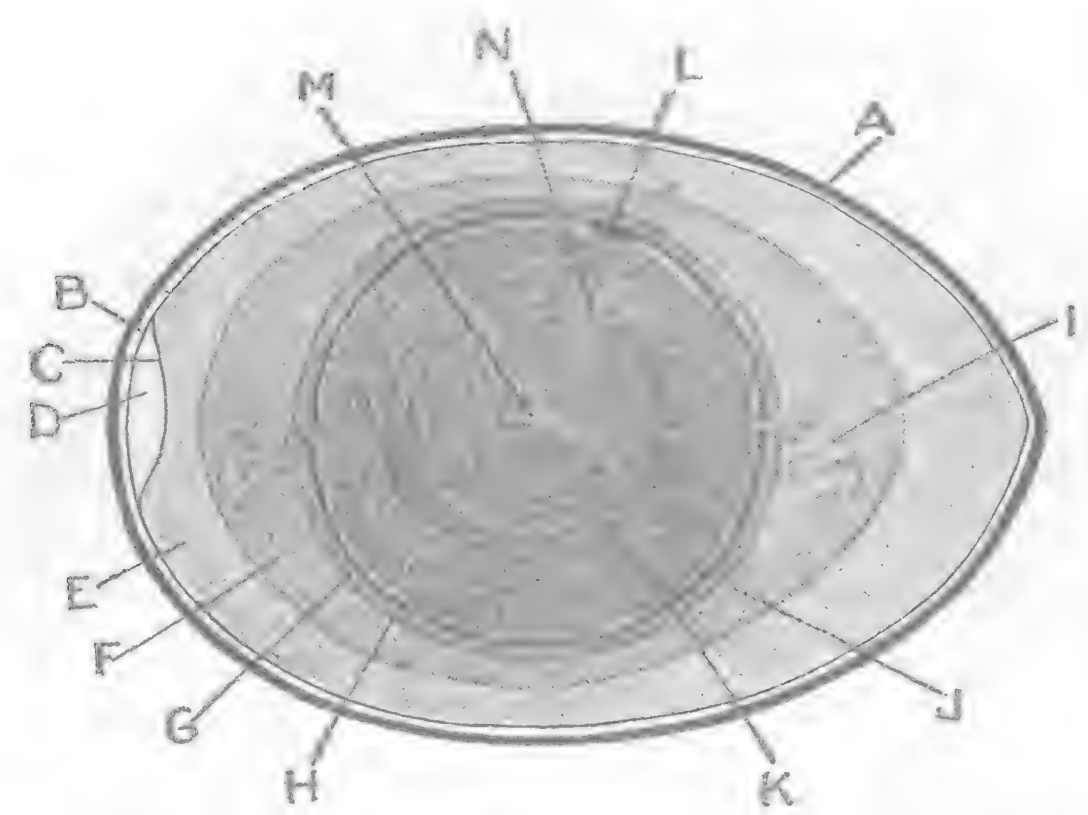

FIG. 75.-Structure of an Egg. (After Benjamin, 1914.)

$A$, outer shell; $B$, outer shell membrane; $C$, inner shell membrane; $D$, air cell; $E$, outer thin portion of albumen; $F, G$, inner dense portion of albumen; $I I$, vitelline membrane; $T$, chalaza; $J$, thin film of white yolk inside of vitelline membrane; $K$, layers of yellow yolk separated by thin lavers of white yolk; $L$, germinal disk: $M$, central part of yolk filled with white yolk; $N$, slender tube connecting center of yolk with region of germinal disk.

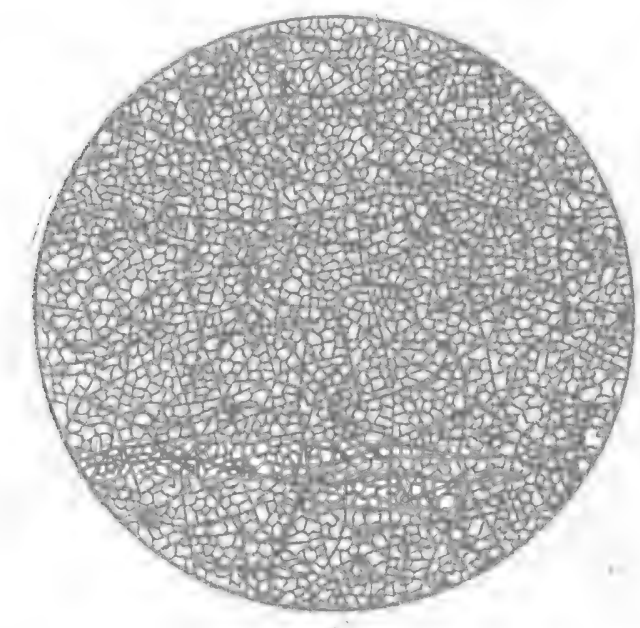

A

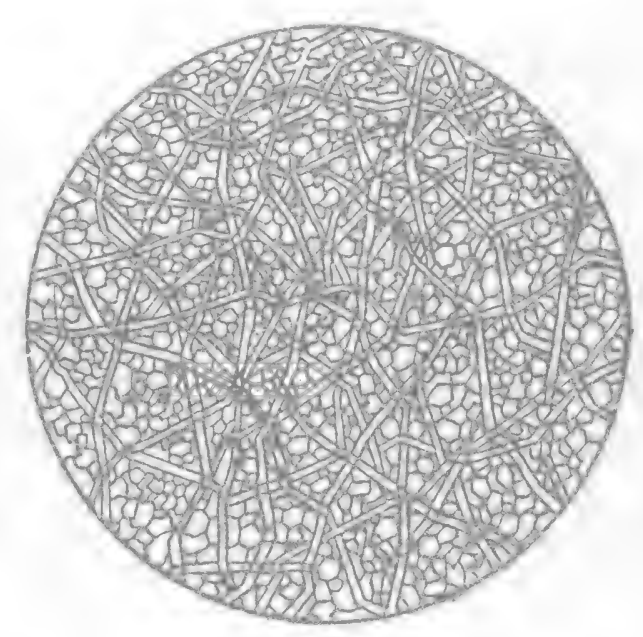

E

Fra. 76.-. Shell Mombranes of an Egg Highly Magnifed. (Lfter Benjamin, 1914.)

$A$, inner shell membrane. Note the fine cellular structure. The cells are bound together by many intertwining fibers. $B$, outer shell membrane. This is much coarser in structure than the inner membrane. The fibers are very transparent.

\section{TABLE XLVIII}

\section{COMPOSITION OF HENS' EGGS}

\begin{tabular}{|c|c|c|c|}
\hline & $\begin{array}{c}\text { Yolk, } \\
\text { Percentage. }\end{array}$ & $\begin{array}{l}\text { White of Egg, } \\
\text { Percentage. }\end{array}$ & $\begin{array}{l}\text { Whole Egg, } \\
\text { Percentage. }\end{array}$ \\
\hline 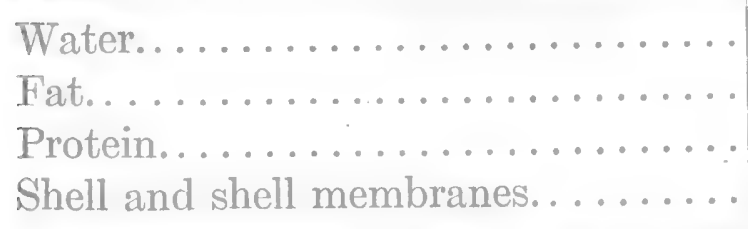 & $\begin{array}{r}46-52 \\
30-35 \\
14-16 \\
\ldots\end{array}$ & $\begin{array}{c}80-88 \\
\text { Traces } \\
10-13 \\
\ldots \ldots\end{array}$ & $\begin{array}{r}70-76 \\
9-14 \\
10-15 \\
9-12\end{array}$ \\
\hline
\end{tabular}


From Table XLVIII it is seen that the whole egg usually contains over 10 per cent of fat. Nearly all of this fat is in the yolk, and obviously the fowl must have a surplus of fat before the yolks can begin to develop. The eggs of ducks, geese, turkeys, guinea fowls, and other birds vary to some extent from the analysis noted above, but the differences are slight.

The detailed analysis of hens' eggs which follows is taken from Simon's textbook of physiological chemistry:

Analysis of shell (9-11 per cent of whole egg).

Calcium carbonate

Percentage. 90.00

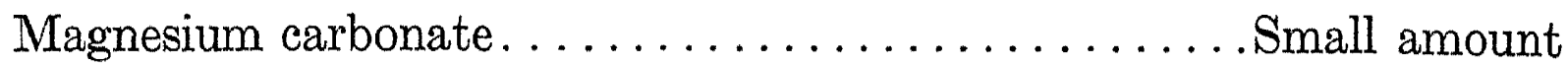

Calcium and magnesium phosphate............ Small amounts

Water............................. 1.00

Analysis of albumen (60.5 per cent of whole egg).

Water Percentage.

Albumins........................... 11.50-12.27

Extractives....................... $0.38-0.77$

Glucose. . ....................... $0.10-0.50$

Fats and soaps...................... Traces

Mineral salts...................... $0.30-0.66$

Lecithins and cholesterin................. Traces

The mineral ash of the albumen has been found by Poleck and Weber to consist of:

Sodium $\left(\mathrm{Na}_{2} \mathrm{O}\right)$.

Percentage.

Potassium $\left(\mathrm{K}_{2} \mathrm{O}\right)$

$32.56-32.93$

Calcium $(\mathrm{CaO})$

$27.66-28.45$

Magnesium $(\mathrm{MgO})$

$1.74-2.290$

Iron $\left(\mathrm{Fe}_{2} \mathrm{O}_{3}\right)$

$1.60-3.17$

Chlorine. (Cl)

$0.44-0.55$

Phosphoric acid $\left(\mathrm{P}_{2} \mathrm{O}_{2}\right) \ldots \ldots \ldots \ldots \ldots \ldots \ldots \ldots \ldots$

$2384-28.56$

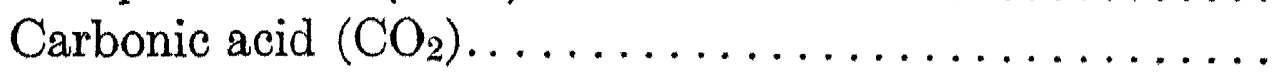

$3.16-4.83$

Sulphuric acid $\left(\mathrm{SO}_{3}\right) \ldots \ldots \ldots \ldots \ldots \ldots \ldots \ldots \ldots \ldots \ldots$

$9.67-11.60$

Silicic acid $\left(\mathrm{SiO}_{2}\right)$

$1.32-2.6 \dot{3}$

Fluorine (Fl).

0.28- 0.49

Analysis of yolk (29 per cent of whole egg). 
According to Gautier we have the following:

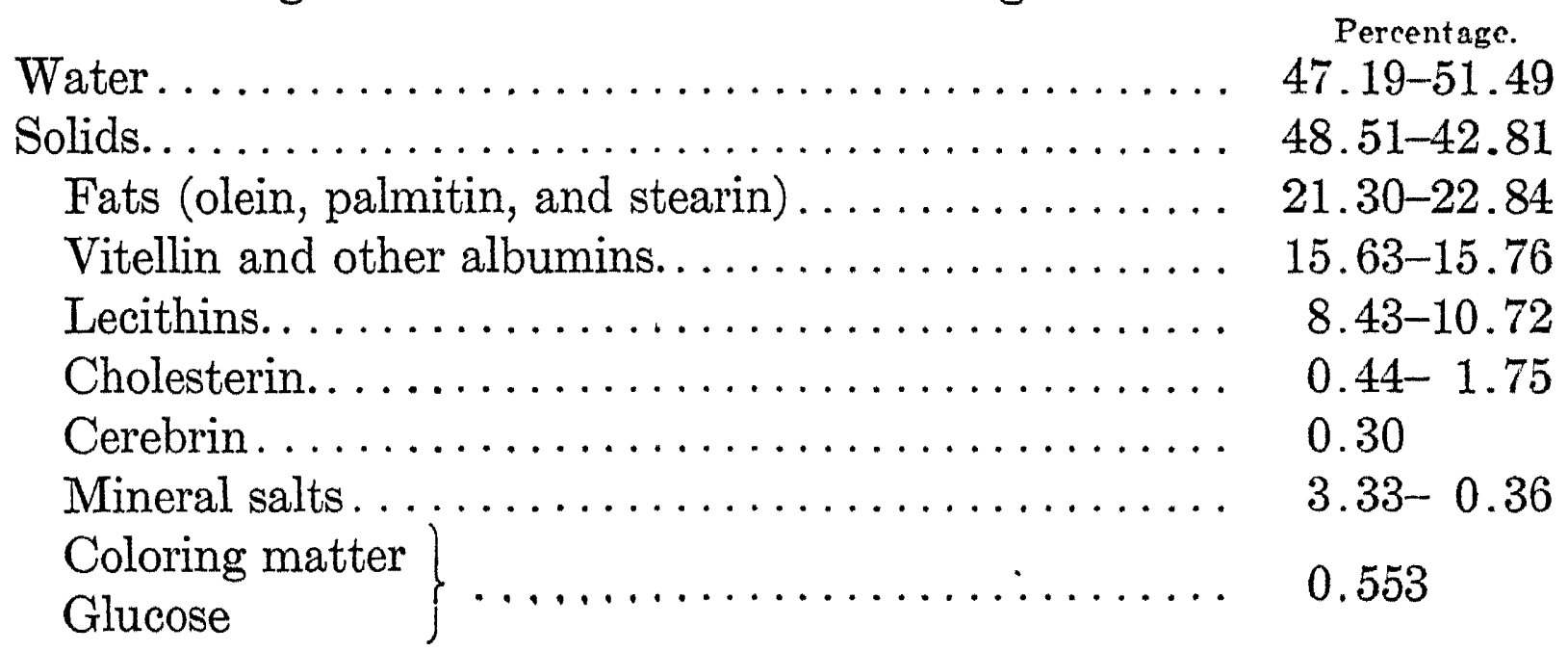

Poleck and Weber also give the following as the analysis of the mineral salts of the yolk:

Percentage

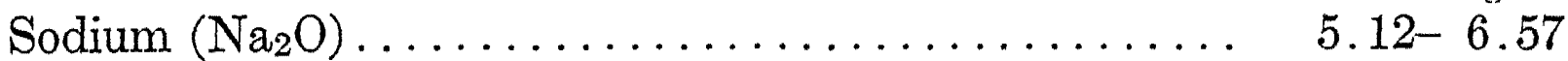

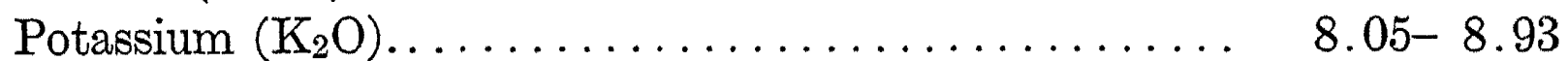

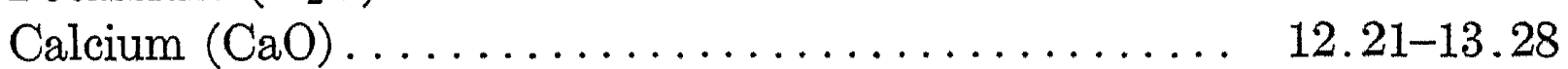

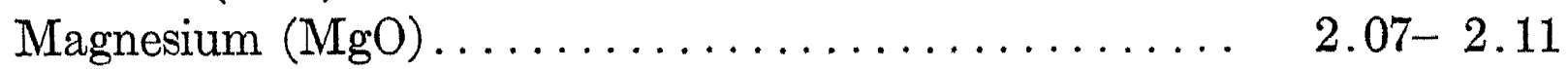

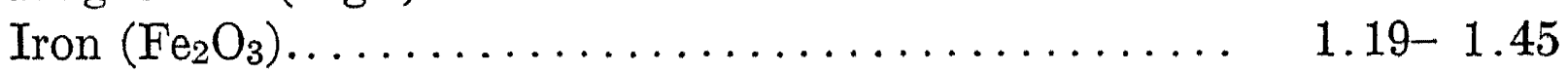

Phosphoric acid, free $\left(\mathrm{P}_{2} \mathrm{O}_{3}\right) \ldots \ldots \ldots \ldots \ldots \ldots \ldots \ldots .6 .72$

Phosphoric acid, combined.............. 63.81-66.70

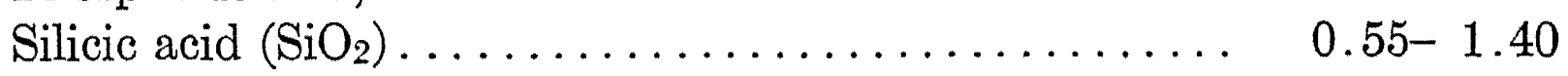

Chlorine............................. Traces

According to Simon, the shell is made up of an organic matrix of the nature of keratin. This matrix is largely impregnated with lime salts. The pigments of the shell are said to be derived from the common pigment of blood. The shell membranes are also composed largely of keratin, with a small amount of mineral salts, principally calcium phosphate. The white of the egg is supposed to consist of compartments which are divided by thin membranes and which contain the liquid albumen. These membranes are continuous with the chalazæ and the shell membranes. The yolk consists largely of spherical cells, most of which are filled with fat.

The bacteriology of eggs has received considerable attention and much interesting and useful data are available. As far as is known eggs have not been important in spreading pathogenic bacteria.

Grading of Eggs. This is essentially a commercial problem. Under some conditions, the grading of eggs is very difficult. However, in practice they are graded by the candling process and observations with 
regard to their internal condition and appearance, are made. Frazier (1916) and Stiles and Bates (1912) describe the dufferent grades of eggs.

Firsts and Extras. This class includes all freshly laid, sound, whole, clean shell, medium and good-sized eggs. The term "fresh" is used to Indicate an egg with practically no age. Before the candle such an egg is characterized by a very small air cell at the large end, absence of defects, transparence and a slow-moving yolk. They appear perfectly homogeneous. Some fresh eggs are laid with defects as "liver spots" "meat spots," foreign material, etc. The appearance of the opened egg gives much information to the microbiologist. The yolk should stand up with a definite shape on account of the strong vitelline membrane about it. The albumen should be transparent and firm. The contents should have no odor and should be practically free from a large number of bacteria.

\section{TABLE XLIX}

\section{COMMERCIAL SAMPLES OF LEAKING EGGS BROKEN IN THE CANDLING ROOM (Pennington)}

\begin{tabular}{|c|c|c|c|c|c|c|c|c|c|c|}
\hline \multirow{2}{*}{$\begin{array}{c}\text { Sample } \\
\text { No. }\end{array}$} & \multirow{2}{*}{ Source } & \multirow{2}{*}{$\begin{array}{l}\text { Date } \\
\text { of Col- } \\
\text { lection }\end{array}$} & \multicolumn{2}{|c|}{$\begin{array}{c}\text { BaCTERIA PER } \\
\text { GRAM ON PLAIN } \\
\text { AGAR INCUBATED }\end{array}$} & \multirow{2}{*}{$\begin{array}{c}\text { Gas- } \\
\text { produc- } \\
\text { ins bac- } \\
\text { teria } \\
\text { per } \\
\text { Gram } \\
\text { in Lac- } \\
\text { tose } \\
\text { Blle }\end{array}$} & \multirow{2}{*}{$\begin{array}{c}\text { Liquefy- } \\
\text { ung or- } \\
\text { ganism } \\
\text { per } \\
\text { Gram }\end{array}$} & \multicolumn{2}{|c|}{$\begin{array}{c}\text { AMMONIAL } \\
\text { NITROGEN } \\
\text { (Folin). }\end{array}$} & \multirow{2}{*}{$\begin{array}{l}\text { Mols- } \\
\text { ture }\end{array}$} & \multirow{2}{*}{$\begin{array}{l}\text { Wght } \\
\text { of } \\
\text { Sam- } \\
\text { ples, } \\
\text { Lbs }\end{array}$} \\
\hline & & & $20^{\circ} \mathrm{C}$. & $37^{\circ} \mathrm{C}$ & & & $\begin{array}{l}\text { Wet } \\
\text { Basis }\end{array}$ & $\begin{array}{c}\text { Dry } \\
\text { Basis }\end{array}$ & & \\
\hline 4214 & $F-1$ & May 1 & $4,300,000$ & $2,100,000$ & 10,000 & $1,400,000$ & 0017 & 0054 & 6974 & 420 \\
\hline 4224 & $F-1$ & May 2 & $3,800,000$ & $1,800,000$ & 100,000 & 430,000 & 0016 & 0054 & 7035 & \\
\hline 4243 & $F-1$ & May 3 & $1,600,000$ & 950,000 & 10,000 & 650,000 & 0017 & 0062 & 7255 & \\
\hline 4370 & $F-1$ & May 23 & $25,000,000$ & $6,300,000$ & 100,000 & $12,000,000$ & 0020 & 0067 & $70 \quad 09$ & 150 \\
\hline
\end{tabular}

Seconds. In this grade are classed all eggs not included under the first, except the third grade mentioned below. Stiles and Bates report the following divisions:

(a) Undersized. If not for their small size these eggs would be classed as firsts. They conform to the standards mentioned above for a first-grade product.

(b) Checks and Cracks. Eggs, the shells of which have become broken by careless handling but which have intact shell membranes. 
(c) Leakers. Eggs whose shells and shell membranes are sufficiently broken to permit a portion of their contents to escape.

(d) Dirties. Eggs, the shells of which have become soiled from unclean nests, etc.

(e) Weak Eggs. In this grade are placed all eggs in which the albumen has become weak or watery, due to high or varying temperature.

Leakers and dirties present the greatest problem to food bacteriologists since they allow the ingress of bacteria. The leakers are either sold for local consumption or are broken for drying. Table XLIX presents some data on commercial samples of leaking eggs secured by Pennington et al. (1914).

Dirties appear during wet weather and sometimes during hot weather when moisture from the hen's body allows more dirt to adhere to the shell. Many of these are used for local consumption or for drying since they do not keep well. In the examination of eggs with dirty shells Pennington (1914) secured widely divergent results. The minimum number of bacteria was 400 and the maximum number $1,600,000$ per gram at $20^{\circ} \mathrm{C}$. The number of $B$. coll varied from 10 to 10,000 in six samples. It has been reported by many investigators that dirty eggs do not keep well. Often these eggs are washed but this process reduces the keeping quality since it removes the delicate membrane or film of mucus on the surface of the shell.

Spots. All eggs which show a spot before the candle are put into this class. These may be due to bacterial, meat, blood or mold spots. The term "spot-rot" is applied to a heated egg caused by decomposition of the dead germ. Molds often develop in eggs and the hyphæ extend toward the air cell. To prevent this eggs must be stored in a dry place. Kossowicz found that old eggs were more susceptible to mold invasion than are fresh eggs; this is somewhat to be expected. Postolka (1916) confirmed this opinion. Marked growth of molds took place after either natural or artificial infection in the testacea but rarely in the yolk. Natural infection was caused by Penicillium glaucum and Cladospornum herbarum. Under experimental conditions almost any mold will attack and penetrate the egg. Postolka states that with even great infection with molds, no putrefaction takes place. Meat spots are caused by broken-down tissue of the hen's oviduct. In the candling process they appear as small floating bodies and are usually near the shell. Blood clots result from injuries to 
the oviduct which causes blood to be laid down with the albumen. Bloody cggs are not used by the average housewife and they cause a great loss.

Rots. Different kinds of rots are known since bacteria develops in eggs in different ways. The fertile egg is much more liable to rot than is the sterile egg. Benjamin (1915) describes the following rots: White rots are common and often called watery rots, sour, or addled eggs. They represent the first stages in bacterial decomposition. Such eggs have an enlarged cell before the candle and a mixed interior. When opened they are usually a light yellow in color and watery. Mixed rots represent a more advanced stage of decomposition and are characterized by thin interiors; in the open condition they give off an odor of hydrogen sulphide and sourness. The yolk is rarely intact. Black rots are eggs in which the contents are very dark and may be easily shaken about the shell. When opened the odor is much like that of hydrogen sulphide. In appearance the contents are mixed and very watery.

Kuhl (1914) from the examination of large numbers of eggs concluded that for trade purposes the following would be a good classification of commercial eggs: fresh eggs, those up to eight or ten days old; eggs, those not over four weeks old; cooking eggs, any offered for sale which are not spoiled.

Factors Influencing Bacterial Content of Eggs. A study of this side of the qucstion involves two points, first the entrance of bacteria before the shell is put down and, secondly the contamination after laying, due to improper handling, etc. While the first point has been studied, the data are not convincing. Mauer (1911), in quoting the work of Pernot (1909) and Conradi states that the infection of the yolks even in the normal ovary is possible. Horowitz (1902), Zimmermann (1878), Abel (1895), Cao (1908), Dräer (1895), McClintock (1894), Poppe (1910) and others, maintain that the oviduct is not sterile. Contrary to this are the data of Horowitz (1902) and Rettger (1913). This stage in our knowledge points out that more work is needed on this subject. Obviously, under abnormal conditions, the oviduct may be infected. This has been found to be true with Bacillus pullorum and the white diarrhœa of chicks. Bushnell and Mauer (1914) point out that there are factors which lower the vitality of the hen and render her unable to resist invading bacteria. Hadley and Caldwell (1916) think that the preponderance of yolk infections indicates that bacteria are present in the ovaries of the hen. 
Permeability of the Shell. The shell about the egg may be regarded as a semi-permeability membrane and, therefore, not resistant to bacteria. Wittich (1851) reported the infection of eggs by molds. Wilm (1895) succeeded in infecting eggs with cholera vibrios. When the eggs were covered with a broth culture, the organisms passed through the shell in from fifteen to sixteen hours, Golokow (1896), Piorowski (1895), Lange (1907), and Poppe (1910) demonstrated the same thing with other bacteria both pathogenic and non-pathogenic. Opposed to this work is that of Mauer (1911), Hoppert (1912), Sachs-Müke (1907), Zorkendorfer (1894) and Schlegel (1904). Kossowicz (1913) showed that $B$. proteus penetrated the shell very easily. The above indicates the ability of bacteria to penetrate the shells of eggs and this emphasizes the necessity of storing eggs under sanitary conditions.

Bacteria in Egg White. Available data on this subject are conflicting. Many of those who have found no bacteria in egg white have assumed that this comp onent of the egg possesses a bactericidal action. Wurtz (1890), Rettger and Sperry (1912), Scholl (1893), Horowitz (1902), Turro (1902), Laschtschenko (1909), and Riezicka (1912) produce data indicating the same result. Sperry (1913) showed that cold storage eggs exhibited the same action. Bainbridge (1911), Poppe (1910), Hoppert (1912), Mauer (1911), Laschtschenko (1909) found no germicidal action. Mauer was especially interested in B. coli. Rettger and Sperry employed B. putrificus and B. edematis maligni as the best organisms. Egg white in test tubes was heavily inoculated with the test organisms and incubated under conditions favorable for the development of the organisms. In the yolk which was treated in the same manner evident putrefaction set in while in the egg white the bacteria were destroyed for microscopic examination failed to demonstrate the presence of the cells. Hadley and Caldwell (1916) in their excellent report on eggs reported data which confirm that of Rettger and Sperry.

Bacteria in the Egg Yolk. Practically all of the investigators are agreed that the yolk contains the greater number of the bacteria. Hadley and Caldwell found 8.7 per cent of 2520 eggs infected in the yolks. They quote from other authors: Mauer is said to have found 18.1 per cent of the yolks to be infected. Bushnell and Mauer raised this to 23.7 per cent. Rettger's data is corrected to show a yolk infection of 9.9 per cent covering examinations over a period of three years.

Qualitative studies of the bacteria in eggs have been reported by several investigators. Rettger (1913) gives the following bacteria: 


\begin{tabular}{|c|c|}
\hline Fresh Ëggs. & Number of Times Found. \\
\hline 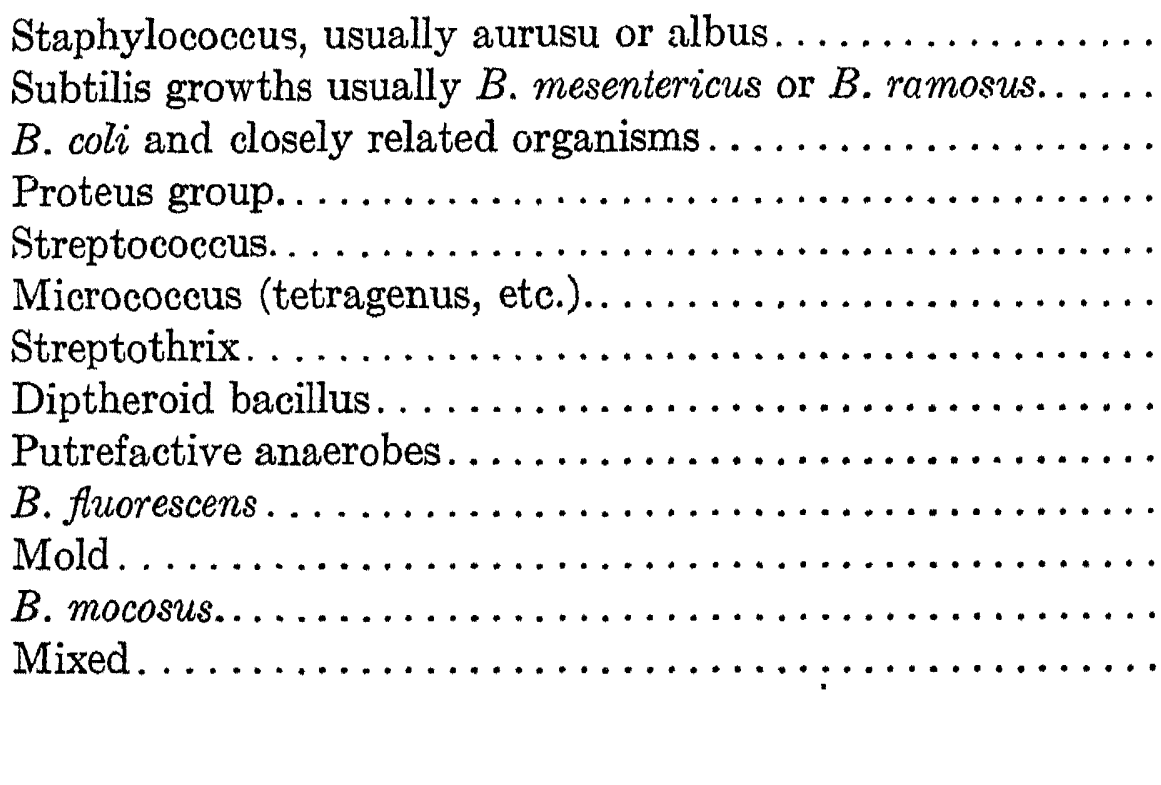 & $\begin{array}{r}74 \\
60 \\
43 \\
30 \\
14 \\
9 \\
6 \\
5 \\
5 \\
2 \\
4 \\
3 \\
2 \\
257\end{array}$ \\
\hline
\end{tabular}

Hadley and Caldwell studied 40 different strains isolated from eggs. Among them were found 11 cocci, 28 rods, and 1 spirillum. No streptococci were reported. They observed no member of the hemorrhagic, septicemia, intestinal, proteus, colon, enteritidis, typhoid dysentery nor diphtheria groups. Bacterium pullorum was not observed in 2520 eggs. No anaerobic bacteria were sought.

That eggs in the fresh condition contain bacteria is still an open one. As pointed out by Hadley, it is difficult to explain the presence of bacteria by contamination during plating. Hadley and Caldwell noticed different types of bacteria on the control plates than on the egg plates. The percentage of infected eggs found by different investigators is given below:

\begin{tabular}{|c|c|c|}
\hline Name. & Examined. & Per cent Infected. \\
\hline Rettger. & 3510 & 9.5 \\
\hline Rettger (10 c.c. samples) ............. & 647 & 3.86 \\
\hline Bushnell and Mauer............... & 2759 & 23.70 \\
\hline Hadley and Caldwell.......... & 2520 & 8.70 \\
\hline Mauer.............. & 600 & 18.10 \\
\hline
\end{tabular}

Enzymes in Eggs. Rullman (1915) found catalase in eggs which were bacteria free. The amount had no relation to the age and was about equal in the yolks and whites. In putrid eggs the amount was so large that the sample had to be diluted. Pennington and Roberston (1912) studied this question using eggs of known history. They experi- 
mented to determine the presence of pepsin, trypsin, lipase, catalase, and reductase. Lipase content increases from a little in a fresh egg to a large amount in a stale egg. The catalase content of a fresh egg was found to be variable.

Dried Eggs. The production of desiccated eggs has become a wellestablished industry. Most of our knowledge with regard to its control rests on the work of Pennington (1916). The methods of manufacture and their bacteriological control are carefully gone over. From their data the following facts were established. For breaking purposes reputable firms use all under-sized or over-sized, dirty, cracked or

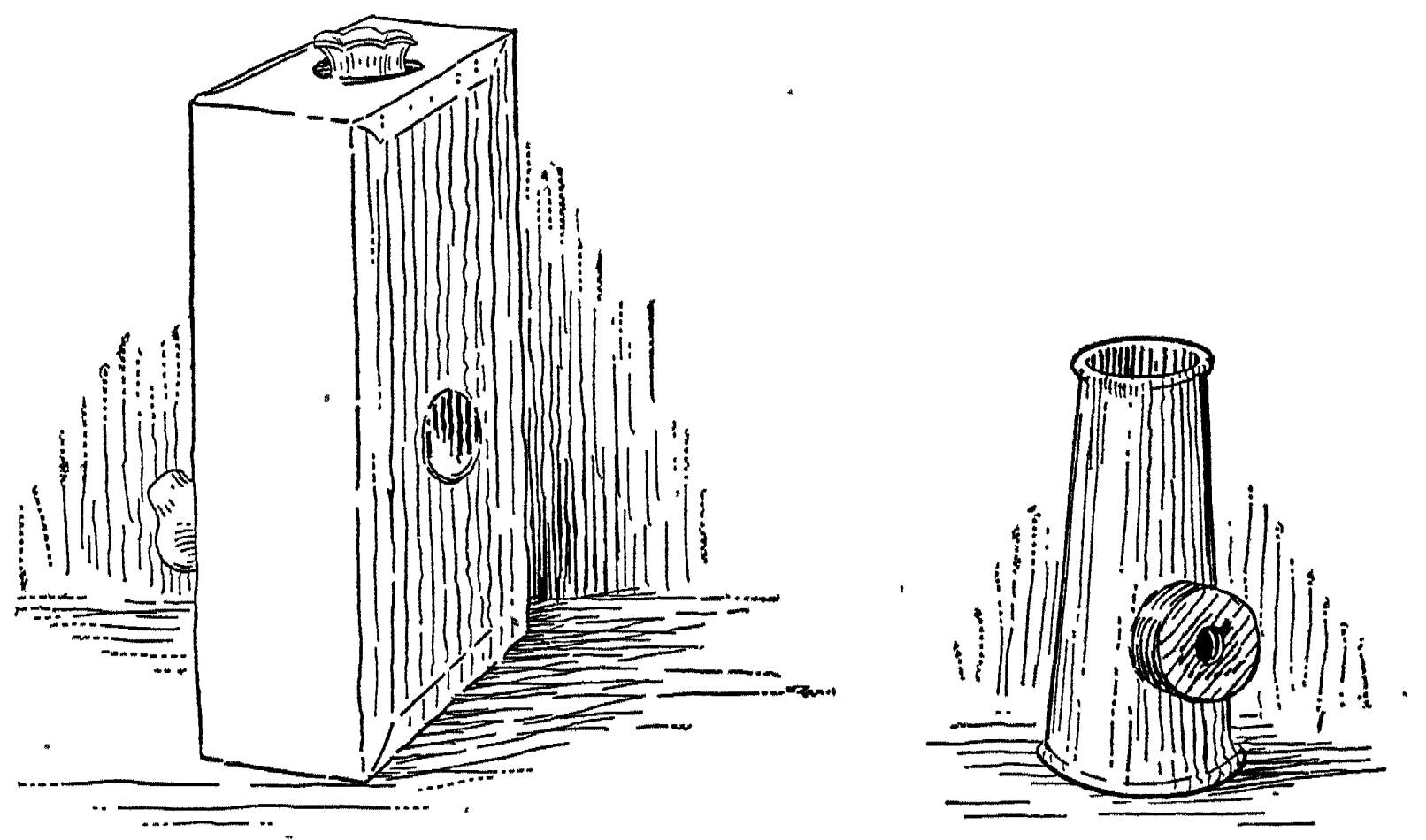

FIG. 77.-Types of Candling Devices. (After Benjamin.)

The one at the left is made by arranging an ordinary oil lamp inside of a pasteboard box. The one at the right is a metal device which is placed on a lamp in place of the chimney.

shrunken eggs. Such eggs should be candled, broken and dried under chilled conditions. The lowest count on flaky dried eggs was 65,000 per gram and the highest count was $20,000,000$ per gram. The average count for 48 samples was $3,600,000$. The number of $B$. coli varied from 0 to $1,000,000$. About three times as many bacteria were found in the dried product as in the liquid egg. Ross (1914) examined 248 samples of dried eggs which had been stored at different temperatures. He gave the following conclusions to his work. "The desiccated egg loses a large percentage of the bacteria originally present if stored for a relatively short period. A more rapid diminution of bacteria results if the storage takes place at higher temperatures. This author states 


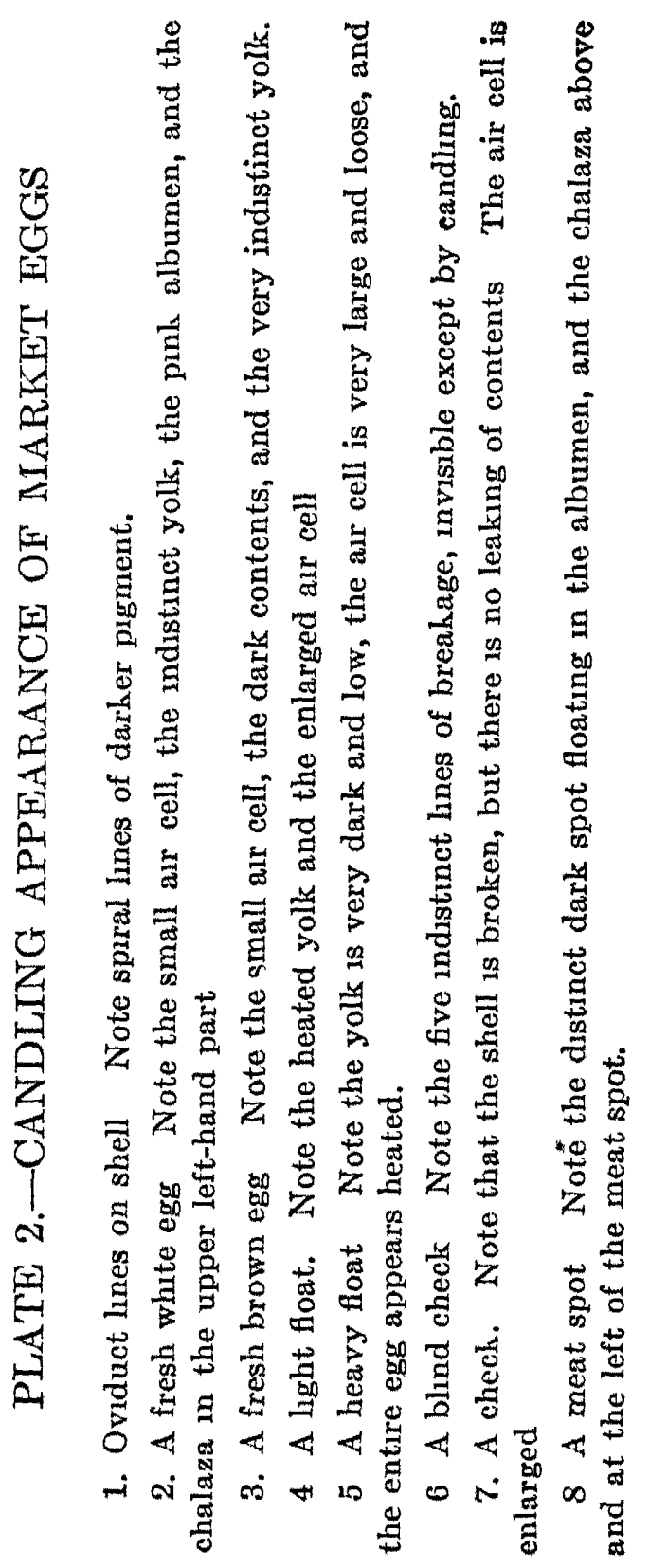




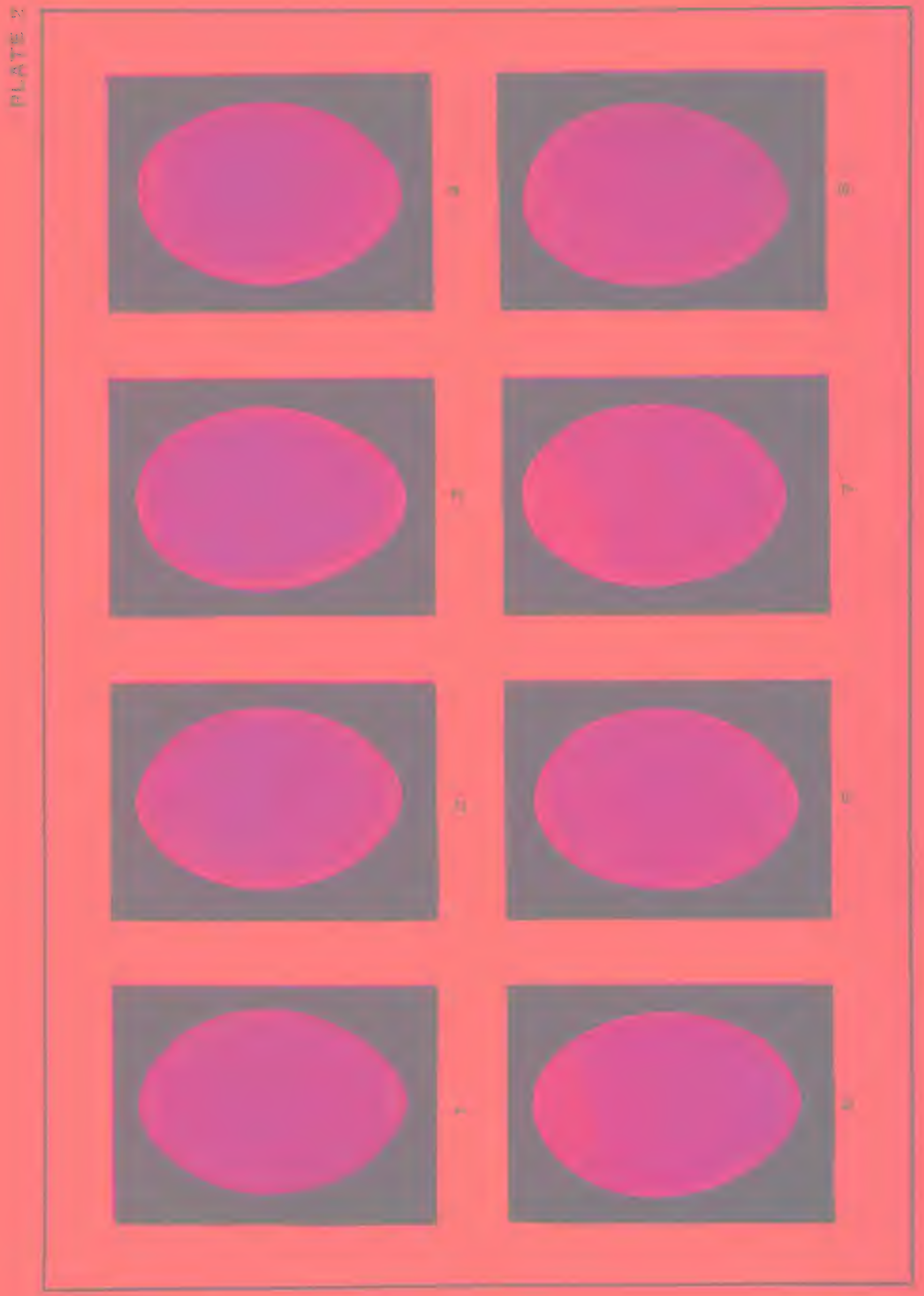


that a product prepared even from spots and worse, might satisfy the ordinary bacterial tests if stored for a period of a few months.

Frozen Eggs. Stiles and Bates (1912) prepared frozen eggs in the laboratory from second-grade eggs. Such a product had generally a bacterial content of 1,000,000 per gram. Frozen eggs prepared from spots, rots and blood rings gave a bacterial content of from $4,000,000$ to $1,000,000,000$ per gram with a relatively high content of $B$. coli and streptococcus. Pennington (1916) reported a careful study of this subject and pointed out many features in the sanitary control of the industry.

Candling of Eggs. The control of the commercial egg industry by chemical or bacterial analyses is very impracticable. Pennington (1909) has pointed out that each egg is a package by itself and an analysis of it would in no way indicate the condition of the lot. This has made it necessary for those engaged in food control to look for other methods for determining the fitness of eggs for consumption. Candling has developed to satisfy this need. Frazier (1917) states that this consists in ascertaining the character of an egg by allowing light to penetrate the contents. Different contrivances are used to do this. An electric light yields the most satisfactory results since it is constant in intensity and quality. Many cheap types of candling devices are used. Benjamin has described two common types. These are shown in Fig. 77. The accurate use of the candling apparatus demands some experience before reliable results may be obtained. The colored plates will be of much assistance. They are taken by permission from the bulletin by Professor Benjamin. Pennington et al. (1918) have recently published instructions for candling eggs. Those wishing a complete classified description of eggs before the candle and outside of the shell will be rewarded by reading this bulletin.

Bacterial Examination of Eggs. Much discussion has passed back and forth over the methods of egg analysis. The early methods were probably faulty as Hadley has said and data from the earlier work must be accepted with some reservation. Hadley has discussed the methods of analysis and any one interested in that subject should consult his work.

Stiles' Method. The eggs should be washed in a solution of bichloride of mercury $(1 / 1000)$ or 5 per cent phenol for a few minutes after which they should be dried with sterile cotton and placed with the large end uppermost in a small beaker. The air space is then scorched with a gas flame for a few seconds. An opening should be made immediately into the cavity with sterile forceps, a sufficient 


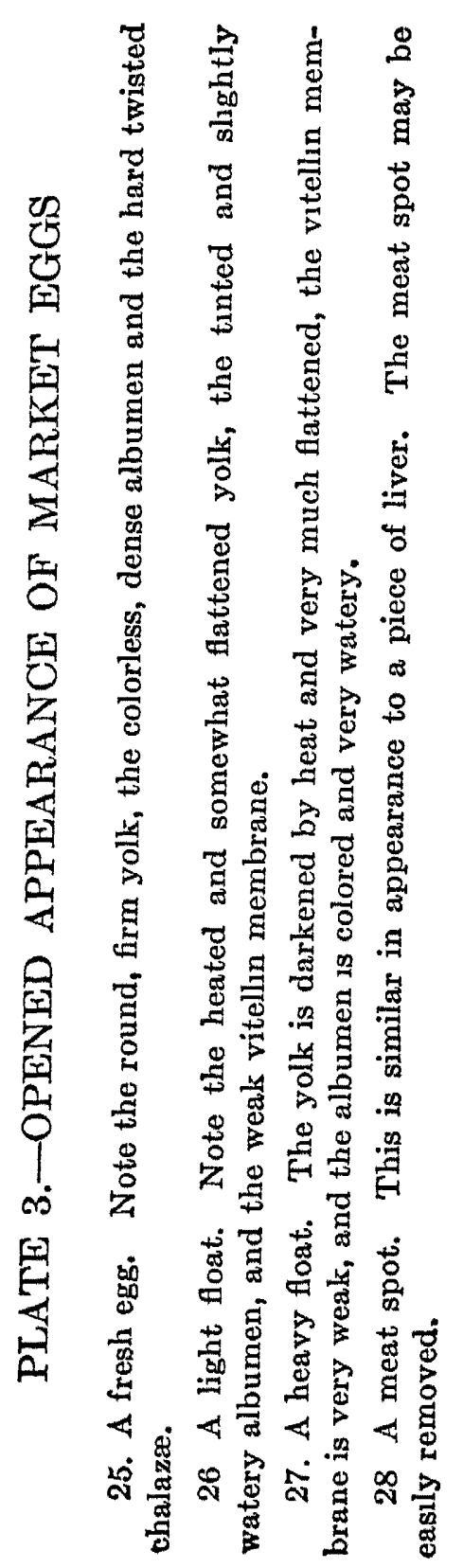




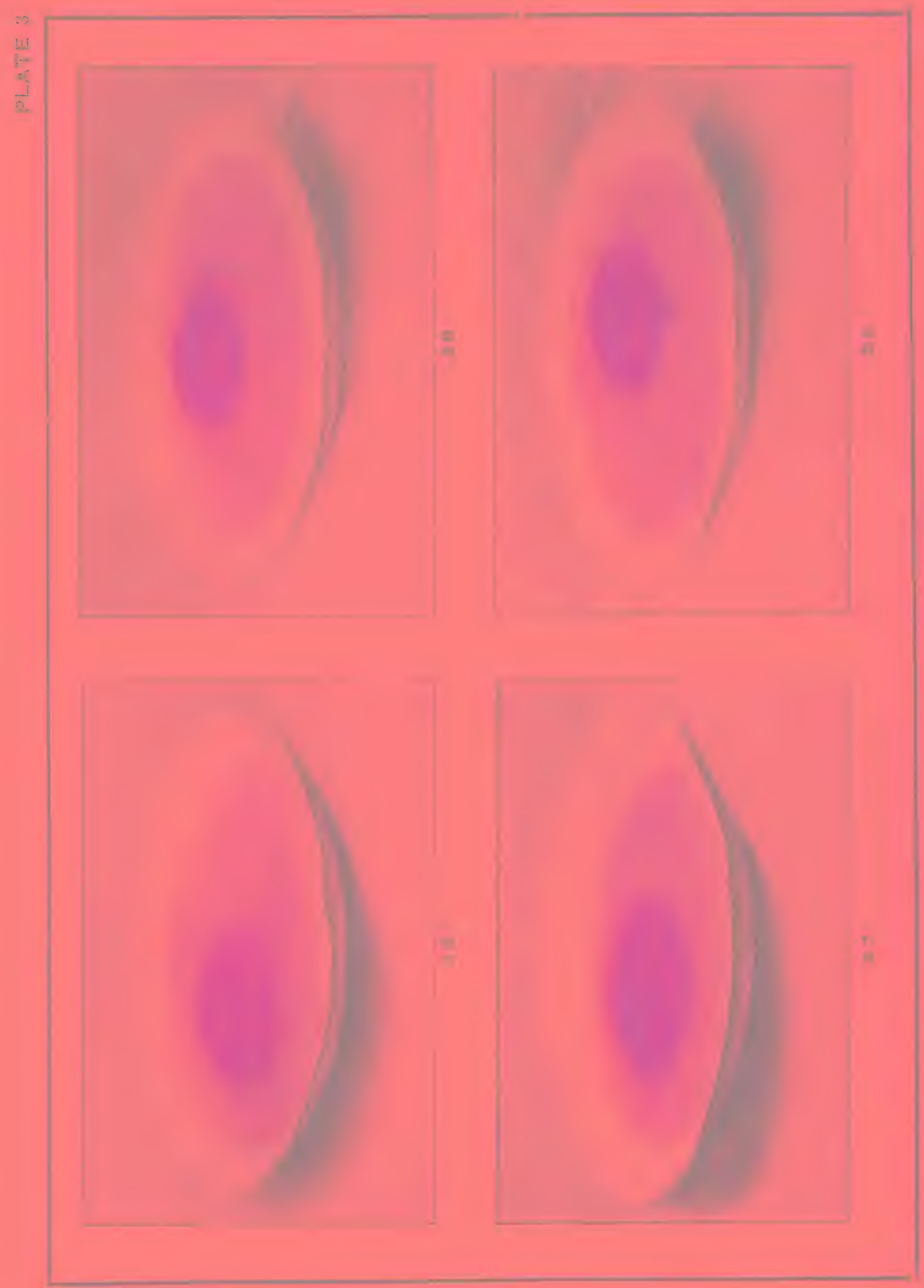


amount of the shell being removed without rupturing the membrane below. When this is accomplished the latter should be broken with a hot platinum spatula and with a sterile pipette 0.5 c.c. of the white of the egg quickly removed and placed in the necessary Petri dishes for cultures. The remaining egg white is then decanted, leaving the unbroken yolk in the shell. With another sterile pipette, the yolk sac is ruptured and suitable portions of its contents removed for study. While this procedure guards against contamination, the breaking up of the respective layers of the egg when out of this shell is difficult and sometimes the inability to do so interferes seriously with the obtaining of quantitative results. With the eggs which have been in cold storage for considerable periods a separation of the whites and the yolks is not possible.

Bushnell and Mauer's Method. The egg should be cleaned with brush and soap and immersed for ten minutes in a 1 to 500 solution of corrosive sublimate. It is transferred with sterile crucible forceps to a small conical graduate, acute pole uppermost. The corrosive sublimate is removed and the egg dried by washing it first with alcohol and then with ether. The acute pole is scorched to kill spores, etc., that might remain. The egg is then immediately removed from the graduate by the operator's holding it by the blunt pole, turning the acute pole down. The hands of the operator should have been thoroughly greased with vaseline to avoid contamination of the flasks by bacteria which might drop off the hands while handling the eggs. With sharp, stout forceps, which have been sterilized in the flame, a hole about $\frac{1}{2} \mathrm{~cm}$. in diameter is made into the acute pole. Holding the egg with the acute end down, and making the stab from below prevents contamination from above. The shell around the hole is flamed briskly and the egg is put with the acute pole upon the neck of a tall 300 c.c. Erlenmeyer flask containing 100 c.c. of sterile bouillon. The blunt end of the egg is now heated with a Bunsen flame, while a close watch is kept on the hole. The heating expands the air in the air space and this expels the contents of the egg. As soon as about half of the albumen has run into the flask the heating is interrupted. The cotton plug is quickly removed from a sterile flask, the neck of the flask is flamed and the egg is transferred from the first to the second flask. Sometimes it is necessary to invert the egg, as soon as the heating is discontinued, to prevent all of the albumin from running into the first flask. In this case it often happens that a little of the egg content runs down the outside of the shell, where it may become contaminated. To prevent such material from getting into the next flask, it is cemented to the 


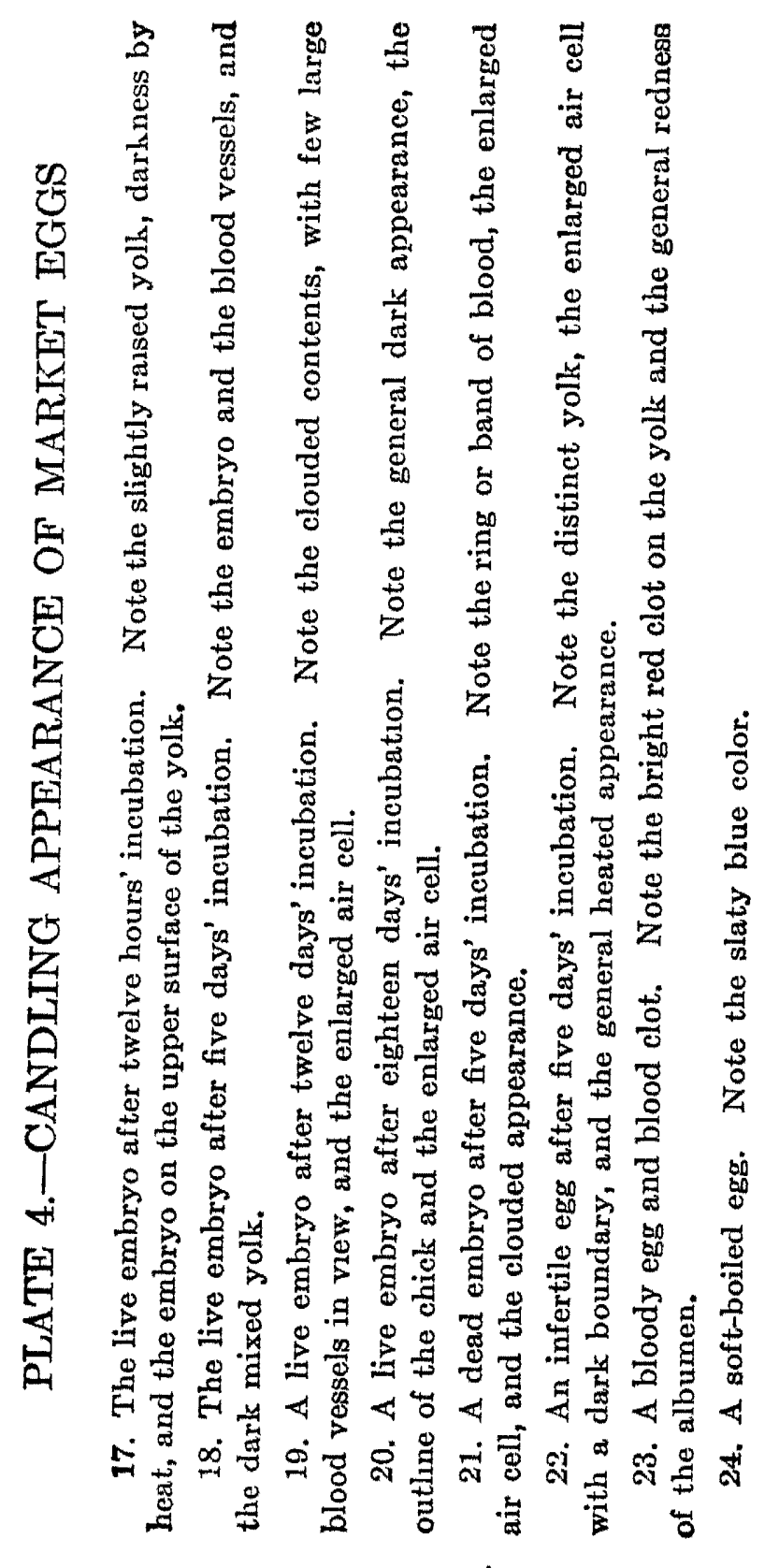




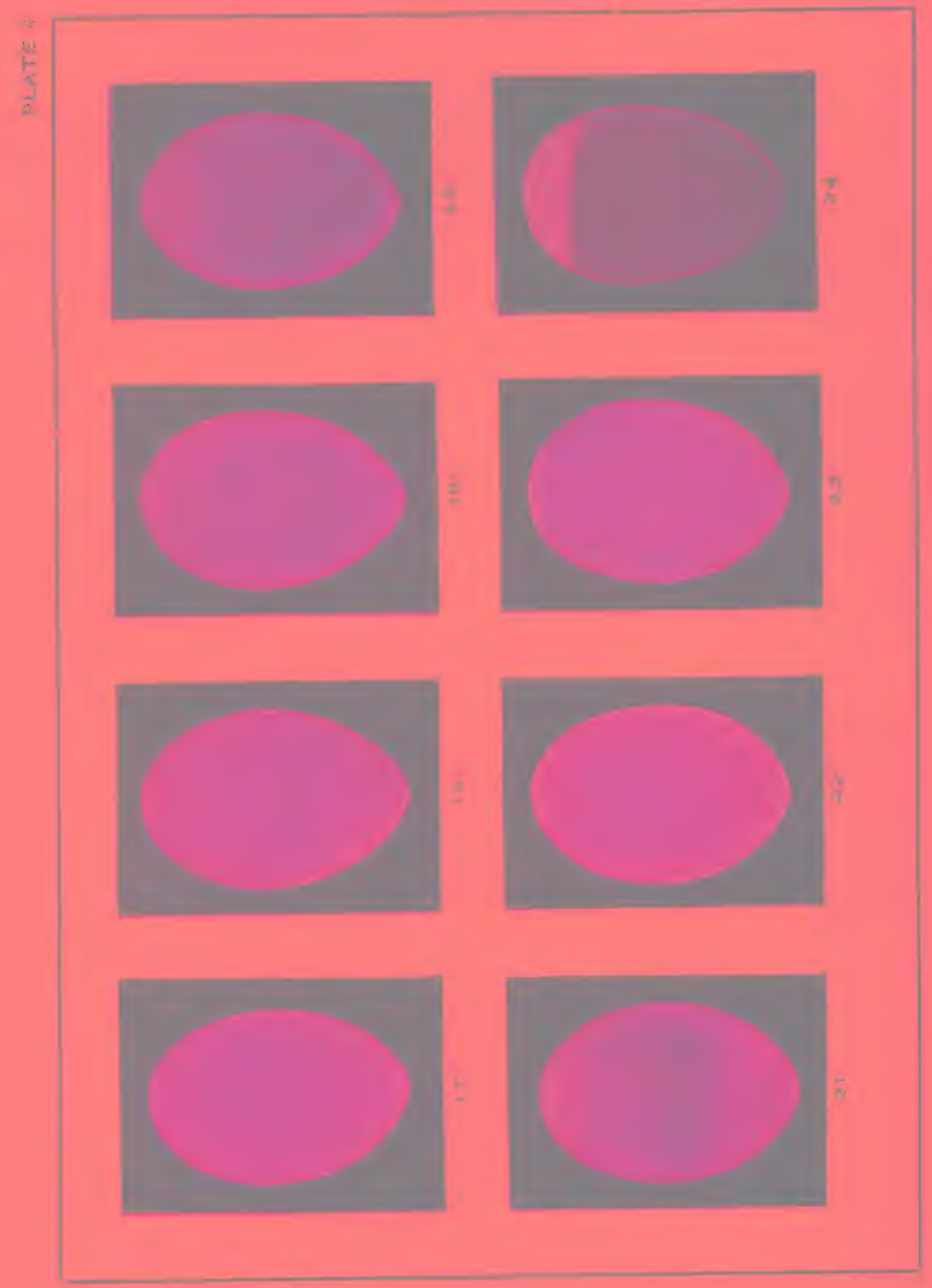


shell by being heated in the flame. The expulsion of the albumin into the second flask should be done slowly and watched closely. As soon as the yolk appears in the hole the heating is interrupted and the egg is tilted from one side to the other to allow the rest of the albumen to run out. In the same manner the yolk is expelled in two portions. The success of this method depends largely on the size of the hole. If this is too small, it is hard to separate the white from the yolk; if it is too large, it is difficult to expel the yolk in two separate portions. Sometimes the yolk obstructs the hole before all of the albumen is obtained. If the yolk does not retract after cooling, the egg is inverted for a moment. Often the vitelline membrane will not rupture, and the yolk will come out in one piece. This can be prevented by puncturing the membrane with a sterile platinum needle. In this manner four flasks are obtained from each egg, two of them containing albumin and two of them containing yolk. The flasks are repeatedly shaken to mix the contents well. It is of advantage to have tall flasks because the contents can be mixed more easily without wetting the cotton plug. Two flasks, one with albumin and one with yolk, are incubated at $38^{\circ} \mathrm{C}$. for forty-eight hours, and the other flasks are incubated at $20^{\circ} \mathrm{C}$. for five days. After this period of development subcultures on agar slants are made to determine if growth has taken place.

Rettger's Method for the Bacterial Examination of Eggs. The egg to be examined is placed small end up, in an egg cup or holder. The upper half of the egg is flamed with a Bunsen burner, the cup being turned constantly so that every part of the upper half of the shell is brought into brief contact with the flame. While the egg is held in one hand the upper end of the shell is removed with sterile scissors, leaving an opening about 1 in. in diameter. The white is poured out, care being taken to prevent it from running down the side of the shell. At this point the edge of the opening is flamed after which the entire yolk is poured out into a wide-mouthed flask, or better still, a large tube especially designed for this work by Rettger. Previous to introducing the yolk, definite amounts of nutrient bouillon are placed in the flasks (25-50 c.c.) or tubes ( 25 c.c.) which are plugged with cotton and sterilized. The yolk and bouillon should be thoroughly mixed. In case the test tube is used this process is greatly facilitated by the presence of a small glass rod about $1 \frac{1}{2}$ in. in length. The tubes should then be placed in the $37^{\circ} \mathrm{C}$. incubator and after seventy-two hours' incubation agar streaks made from them. These should be incubated for from twenty-four to forty-eight hours. 


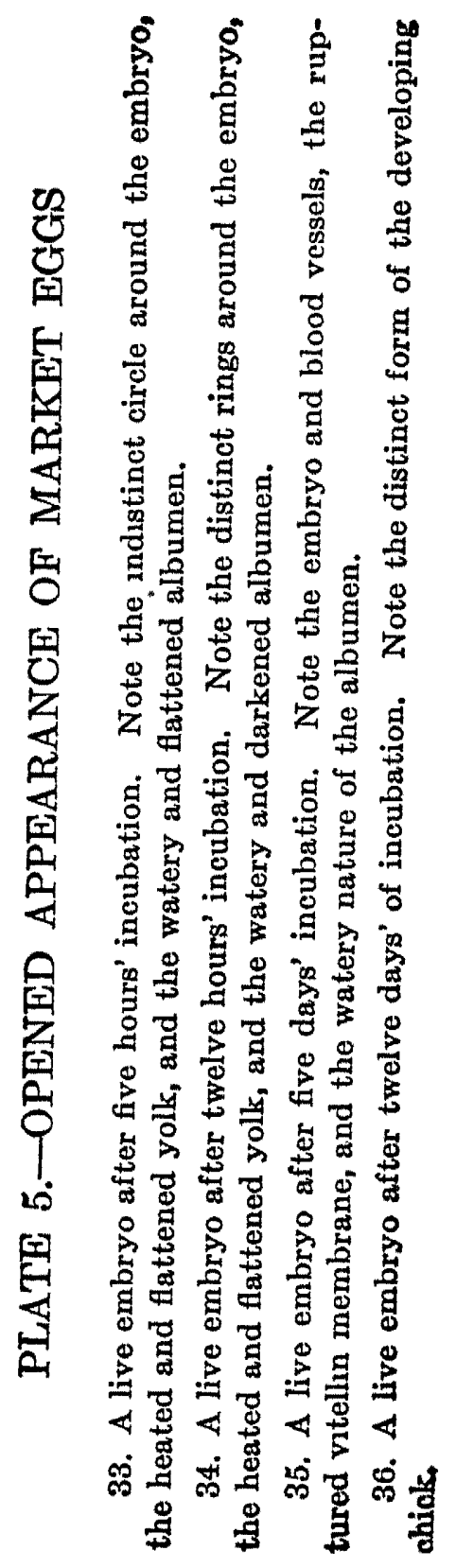




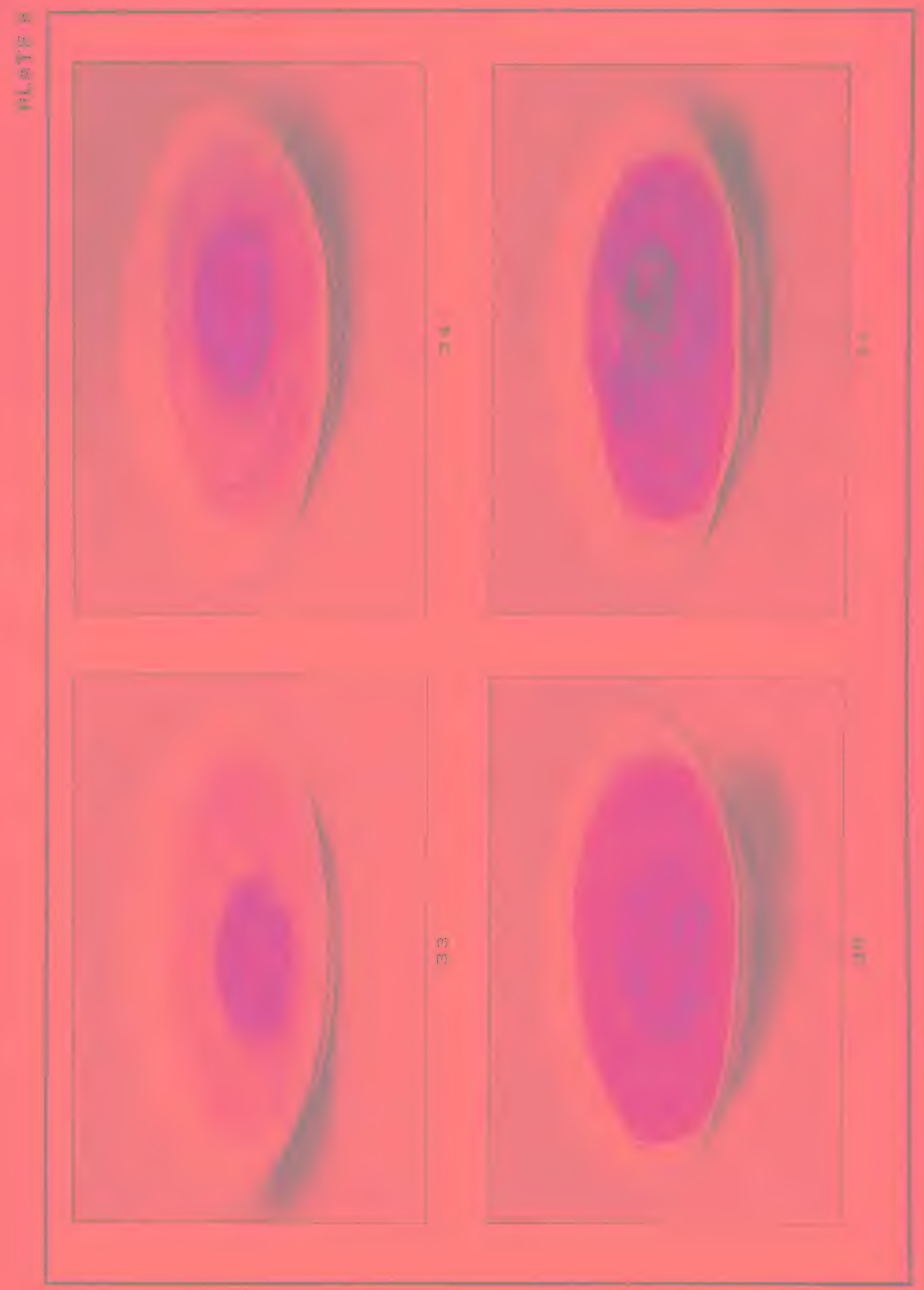


Hadley and Caldwell's Method. The egg shell should be thoroughly disinfected before any opening is made. This may be accomplished by first washing the egg, if soiled, with soap and water and a wad of cotton, then immersing for ten minutes in 1:5000 mercuric chloride solution, containing either citric acid or ammonium chloride to increase the penetrating power. The egg may then be plunged into 95 per cent alcohol, removed, drained and ignited to dry the surface. These operations can be carried on in a suitably constructed wire rack in which the eggs can await examination, being meanwhile protected from air contamination.

Next, as a final precaution, the egg should be well fiamed at the end opposite the air space until a very thin layer $(1-2 \mathrm{~mm}$.) of the albumen lying close to the shell is coagulated. The amount of heating required can be ascertained by experience. After this, by means of sterile forceps, a hole about $2 \mathrm{~cm}$. in diameter is made at the flamed end and the white poured into a tube or flask containing 25 c.c. of the desired medium. Next, the opening in the shell is enlarged to about $3 \mathrm{~cm}$. The yolk is gently allowed to run out of the shell onto a circle of sterile filter paper (kept in a small pile under the bell jar). The paper is then so inclined that the yolk rolls about until the white is entirely removed by the paper, after which the yolk is rolled off the edge of the paper into a tube or flask containing at least 25 c.c. of culture medium, an amount somewhat greater than the volume of the average yolk. Great care must, of course, be taken not to rupture the yolk membrane before the yolk is poured into the tube. The yolk may then be broken by means of a sterile glass rod and mixed with the broth.

The preparation is now ready for incubation which may be carried on for forty-eight hours at $37^{\circ} \mathrm{C}$., followed by forty-eight hours at $20^{\circ} \mathrm{C}$. The white may be mixed with the broth and grown in the same manner. At the end of the period of incubation the tubes should be again mixed by rotation to insure distribution of the bacteria, and a small amount on a straight needle transferred from each to ordinary tubes containing broth. The broth tubes are incubated at $37^{\circ}$ and at $20^{\circ} \mathrm{C}$., as were the original tubes and examined for growth at the end of four days. In. case growth appears plate cultures are made.*

* In addition to the points of technique mentioned above, minor details can perhaps best be worked out by the individual investigator. For instance, cotton plugs in the large tubes are unwieldy. They may be replaced by close-fitting glass covers, coming well down over the tops of the egg tubes. The breaking of the yolk and the mixing with the broth may be accomplished by means of broken glass placed in the tubes with the broth, rather than by means of glass rods, a procedure which may favor contamination from the air. Wire cages or racks of various sizes and 


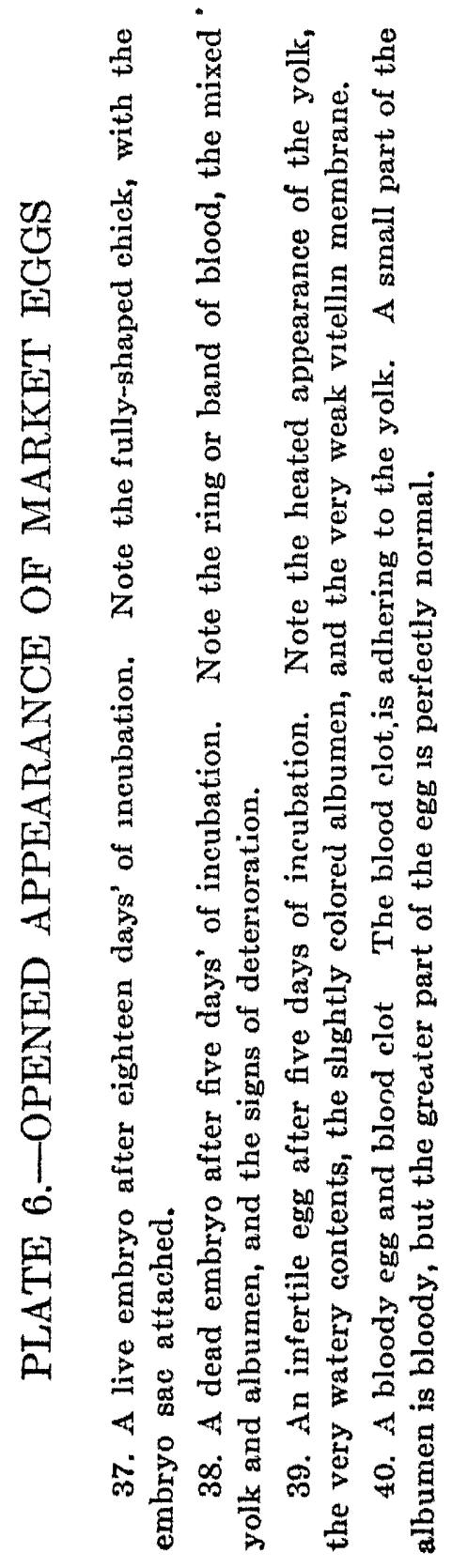




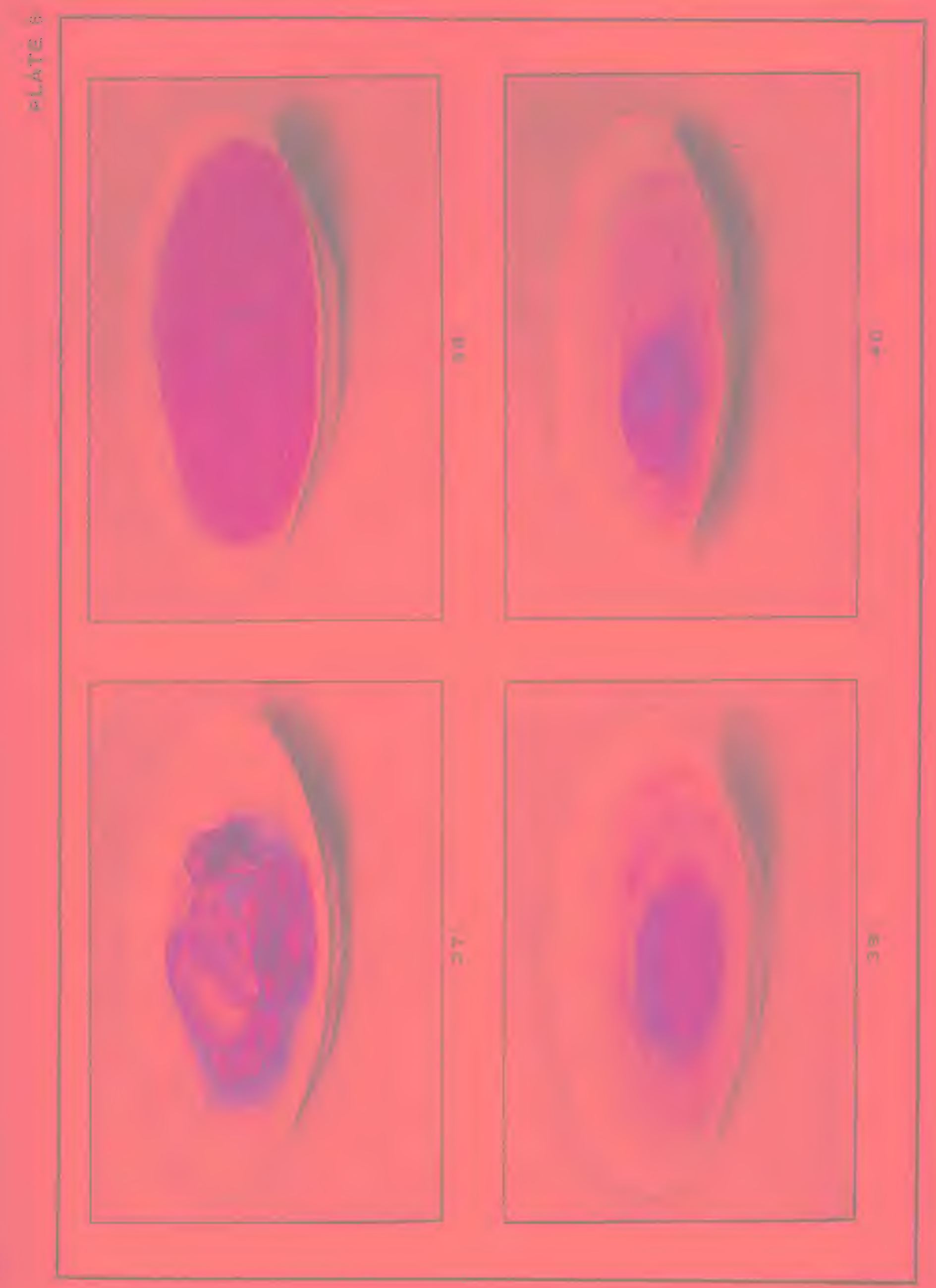


It should be borne in mind that the recommendations made above are suitable only for fresh eggs in which it is desired to examine the yolk and white separately. In old market eggs, or preserved eggs in which the yolk is weakened, and in decomposed eggs in which the yolk and white have become mixed, different methods would be required; and here, as in the bacteriological examination of other egg products, the technique must be evolved in accordance with the particular aim of the investigation.

\section{BIBLIOGRAPHY}

Abel, R. and Draer, A. 1895. Das Huhnerei als Kulturmedium für Cholera Vibrionen. Zeit. f. Hyg., 19, 61.

Artault, T. 1893. Recherches bacteriologiques, mycologiques, zoologiques et médicales sur l'œuf de poule. Thèse Paris. Cent. Bakt., 1894, 16, 461.

Artault, T. 1893. Le bacille pyocyanique dans un œuf de poule. Compt. Rend. soc. biol., series $9,5,78$.

ARtault, T. 1895. Tuberculose provoquée chez lapins par des injections de contenu d'œufs de poule. Comp. rend. soc. biol. ser. 10, 2, 683.

BaINBRidge, F. A. 1911. The Action of Certain Bacteria on Proteins. Journal of Hygiene,. 11, 341.

Barthelmy, M. A. De l'incubation des œufs d'une poule atteinte du cholera des poules. Compt. Rend. Acad. Sciences, 96, 1322.

Bechamp, A. and Eustache, G. 1877. Sur l'alteration des œufs provoquée par des moississures venus de l'extérieur. Comp. Rend. Acad. Sci., 85, 854.

Beckwith, T. D. and Horton, G. D. 1914. Is Poor Hatching of Normal Eggs Due to the Presence of Microorganisms within the Egg? Science N. S., $40,240$.

Benjamin, E. D. 1914. The Interior Quality of Market Eggs. New York Agricultural Exp. Station Bull. 353. Cornell Univ., Ithaca.

BorNaud, M. 1914. Recherches sur les parasites vegetaux des œufs des poules. Cent. Bakt. Ref., 63, 43.

Bushneld, L. D. and Mauer, O. 1914. Some Factors Influencing the Bacterial Content and Keeping Quality of Eggs. Kansas Ag. Ex. Sta. Bull. 201.

CAO, G. 1908. Sur la permeabilita delle uova ai microorganismi. Bull. Inst. Pasteur, 6, 472.

shapes may be used for the immersion of the eggs in the disinfecting solution, in the alcohol and during ignition.

Methods for the detection of anaerobic microorganisms probably do not present unusual difficulties. The method of Hesse, involving the use of a layer of sterile olive or paraffin oll over the surface of the mixture is probably the best. In this case samples may be withdrawn by means of a sterle pipette. 


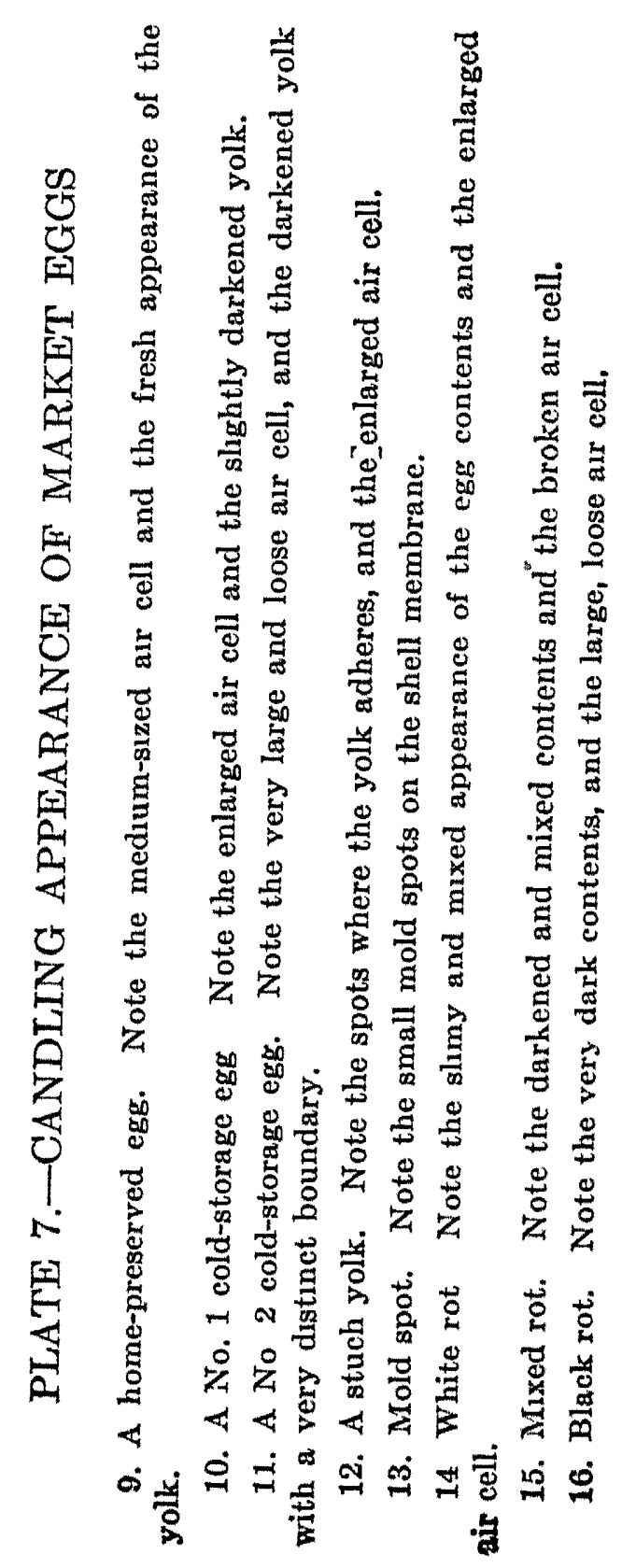




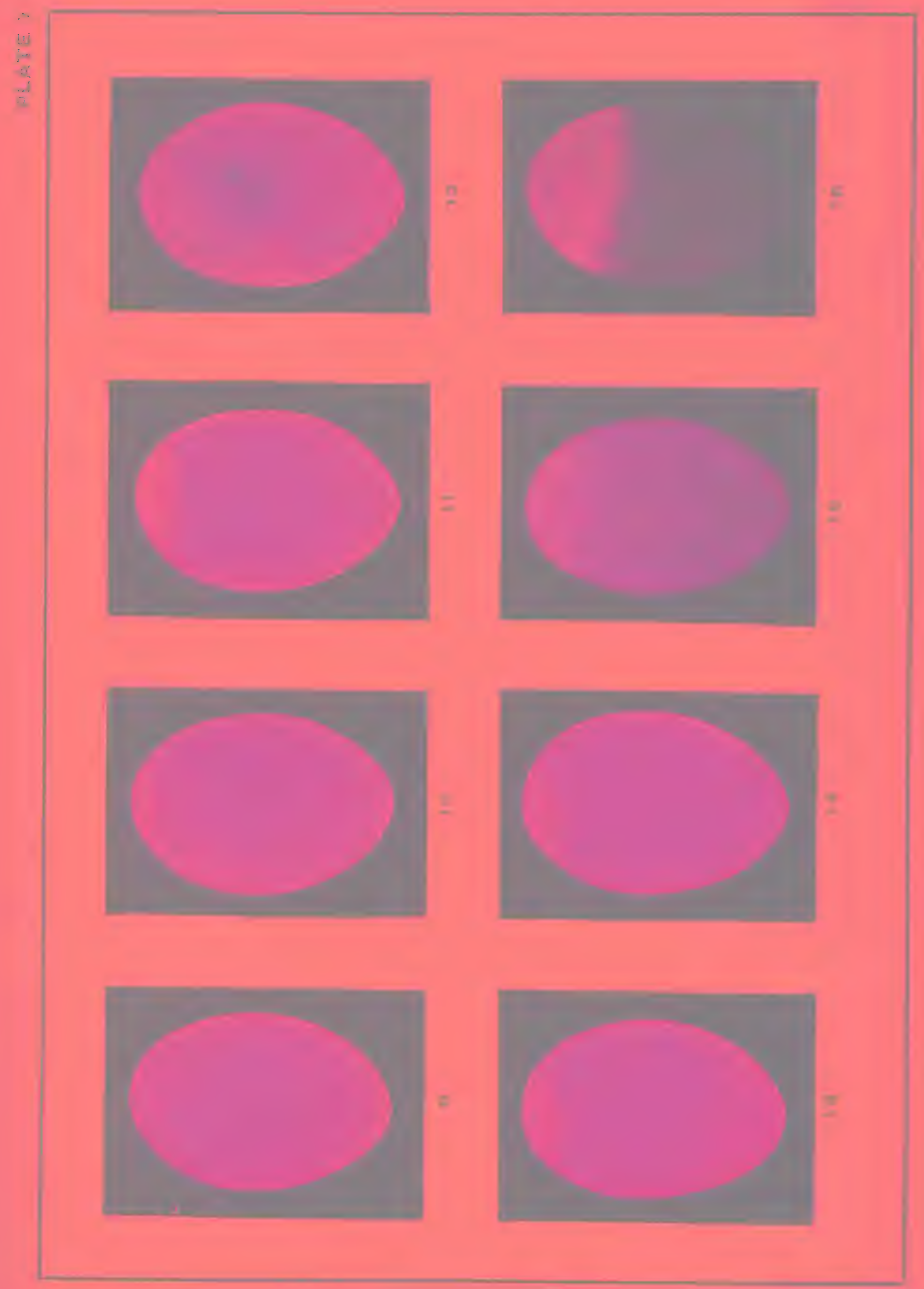


DonItz. 1895. Ueber das Verhalten der Choleravibrionen Hülnerei. Zeit. Hyg., 20, 31.

Frazier, D. J. 1917. Candling Eggs. American Food Journal, 12, 39.

Gayon, U. 1873. Sur l'altération spontanée des œufs. Comp. Rend. Acad. Sci., 76, 232.

GAYON, U. 1875. Recherches sur l'altération spontanée des œufs. Ann. Sci. l'Ecole Norm. Supérieur, Series 2, 4, 205.

GoLokow. 1896. Ueber das Eindringen von Cholera Vibrionen im Hühnerei. Baumgarten's Jahresbericht, 12, 583.

GRIGORIEW, A. W. 1894. Vergleichende Studien über die Zersetzung des Hühnereiweisses durch Vibrionen. Arch. Hyg., 21, 142.

Hadley, P. B. and CacDwell, D. W. 1916. The Bacterial Infection of Eggs. Rhode Island Ag. Exp. Sta. Bull. 164.

HAMMERL, H. 1894. Ueber die im rohen Eiren durch das Wachstum von Cholera Vibrionen hervor gerufen Veränderung. Zeit. Hyg., 18, 153.

Hoppert, M. J. Factors Relating to the Bacteria Content of Eggs. Thesis University of Wisconsin.

Horowitz, A. 1902. Contribution à l'étude des moyens de defense de l'organisms contre l'invasion microbienne; recherche sur l'oviducte de la poule de le blanc d'œuf. Thèse Paris. Baumgarten's Jahresbericht. 19, 984.

Huteppe, F. 1888. Ueber die Verwendung von Eiern zu Kulturwecken. Cent. Bakt., 2, 80 .

Kempner, W. 1894. Ueber Schwefelwasserstoffbildung der Choleravibrionen im Hühnerei. Arch. Hyg., 21, 317.

Kossowicz, A. 1913. Die Zersetzung und Haltbarmachung der Eier. Wiesbaden. J. F. Bergman.

KuHL, H. 1914. The Relation between Spoiling and the Age of Eggs. Hyg. Rundschau, 24, 253-259.

Lamson, G. H. 1909. Infection and Preservation of Eggs. Storrs Ag. Exp. Sta. Bull. 55.

LANGE, R. 1907. Ueber das Eindringen von Bakterien in das Hühnerei durch die Eischale. Arch. Hyg., 62, 201.

LASCHTSCHENKo, P. 1907. Ueber die keimtötende und entwicklungshemmende Wirkung von Hühnereiweiss. Zeit. Hyg., 64, 419.

Linnossier et Lemoine. 1910. Sur la toxicité normale des aliments albuminoides frais. Influence de la conservation. Comp. Rend. soc. biol., 68,671 .

McCuntock. 1894. Bacteria in Eggs. Modern Medicine and Bact. Rev., 3,144 .

Mauer, O. 1911. Bacteriological Studies on Eggs. Kansas Ag. Exp. Sta. Bull. 180.

MeninI, G. 1908. Richerche intorno alla penetrazione dei bacteria nelle uova di gallina. Lo. Sperimentale, $61,711$.

Moster, F. 1864. Mykologische Studien am Hühnerei. Arch. Path. Anat. Physiol. Virchow., 29, 510. 


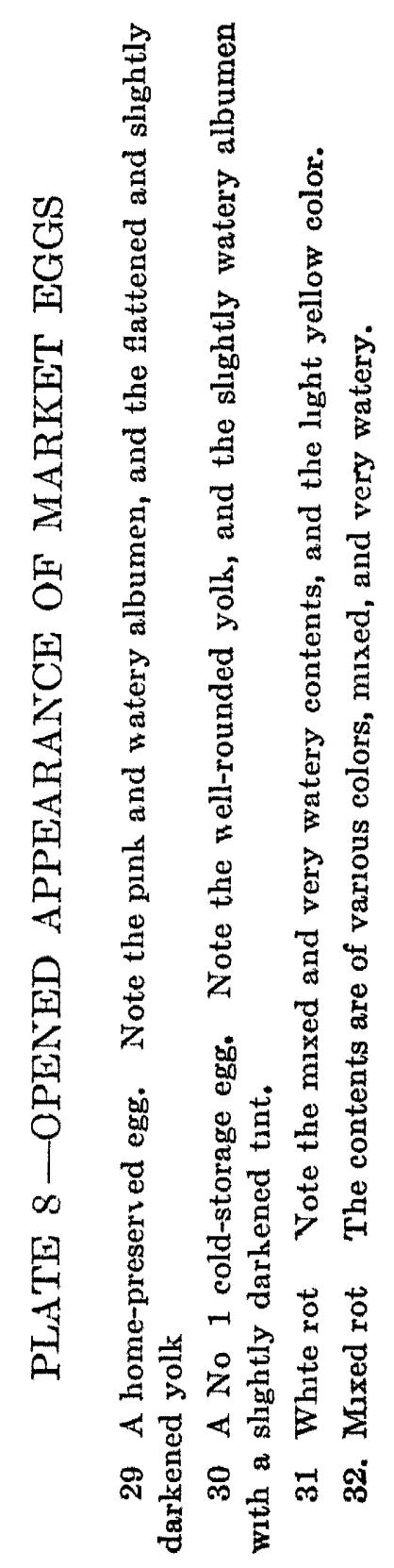




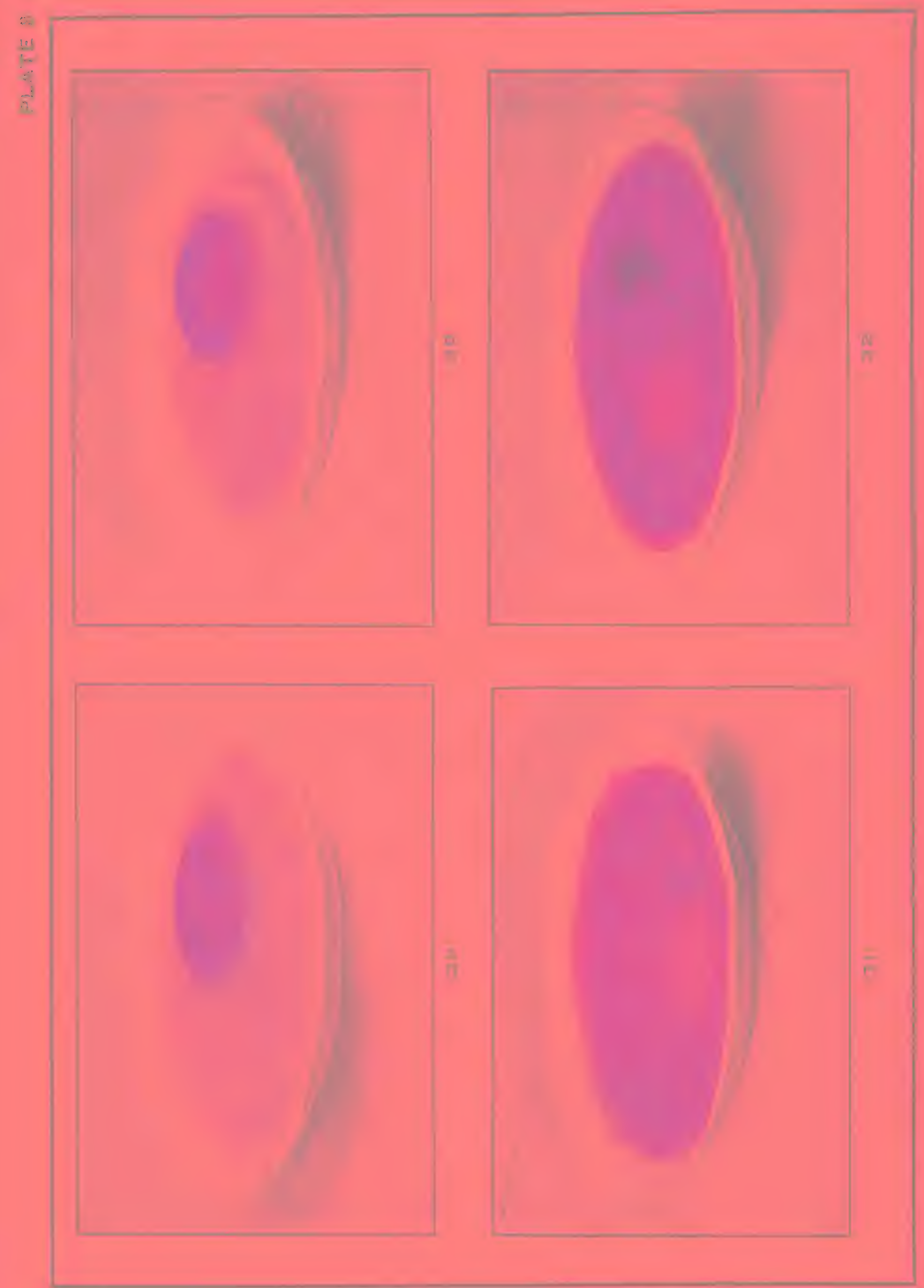


Pennington, M. E. 1909. A Chemical and Biological Study of Fresh Eggs. Jour. Biol. Chem., 7, 109.

Pennington, M. E., et al. 1914. A Bacteriological and Chemical Study of Commercial Eggs in the Producing Districts of the Central West. U. S. Dept. Agric. Bureau Chem. Bull. 51.

Pennington, M. E. and Robertson, H. C. 1912. A Study of the Enzymes of the Egg of the Common Fowl. Circular 104, U. S. Dept. Agric. Bureau of Chemistry.

Pennington, M. E., et al. 1916. A Study of the Preparation of Frozen and Dried Eggs in the Producing Section. U. S. Dept. Agric. Bull. 224.

Pennington, M. E., Jenkins, M. K. and Betrs, H. M. P. 1918. How to Candle Eggs. U. S. Dept. Agriculture Bulletin 565.

Pronkowskr. 1895. Ueber die Einwanderung der Typhus Bacillen in das Hühnerei. Arch. Hyg., 25, 145.

Poppe, K. 1910. Zur Frage der Uebertragung von Krankheitserregern durch das Hühnerei. Zugleich ein Beitrag zur Bakteriologie des normalen Eise. Arb. a. d. Kaiserl. Gesndhsmt., 34, 186.

Postolka, A. 1916. The Growth of Molds in Eggs. Cent. Bakt. Abt. II., 46, 320-330. Chem. Abts., 11 (1917), 3215.

RaEbiger, W. 1900. Ueber die Rotfärbung eines Hühnereis durch B. prodigiosus. Zeit. Fleisch u. Milchhyg., 11, 115.

Retrger, L. F. and Sperry, J. A. 1912. The Antiseptic and Bactericidal Properties of Egg White. Jour. Med. Res., 26, 55.

RetTGer, L. F. 1913. The Bacteriology of the Hen's Egg with Special Reference to its Freedom from Microbic Invasion. Cent. Bakt. Abt. II, 39, 611. Storrs. Ag. Exp. Sta. Bull. 75.

Retrger, L. F. 1911. Bacilliary White Diarrhoea of Chicks. Storrs Ag. Exp. Sta. Bull. 60.

RIEzICKA, V. 1912. Ueber die natürliche Schutzkraft in Entwicklung begriffener Hühnerei. Arch. Hyg., 77, 369.

Ross, L. S. 1914. Bacterial Content of Desiccated Eggs. Proc. Iowa Acad. Sci., 21, 33-49.

Rullman, W. 1915. The Bacteria and Catalase Content of Hen Eggs. Cent. Bakt. Abt. II., 45. 219-230. Chem. Abts., 11, 2509.

SchENK. 1850. Ueber Pilzbildung in Hühnereiern. Verhandl. Phys. Med. Gessell., 1, 73.

ScHoll, H. 1892. Untersuchungen über Giftige Eiweisskörper bei Cholera Asiatica und einigen Fäulnisprocessen. Arch. Hyg., 15, 172.

Scholr, H. 1893. Bakteriologische und chemische Studien über das Hühnereiweiss. Arch. Hyg., 17, 535.

Schrank, J. 1888. Untersuchungen über den im Hühnerei die stinkende Faulniss hervorrufenden Bacillus. Wiener. med. Jahrb., 84, 303.

SchraNCK, J. 1895. Bakteriologische Untersuchungen fauler Kalkeier. Zeit. Allg. Osterr-Apoth. Ver., 33, 295. 
Fig. 78.-Rate of Evaporation of Hens' Eggs.

1 to 9. Eggs that have been held for one day, one week, two weeks, three weeks, four weeks, five weeks, six weeks, seven weeks, and eight weeks, respectively. The eggs were kept at a livingroom temperature of about $70^{\circ} \mathrm{F}$, in an open pasteboard carton. There was no breeze blowing over the eggs, and each egg illustrated is typical of several eggs that were examined at each stage; therefore this series represents the normal results of such holding.

10 to 12 . Eggs held for twelve weeks, one week, and one-half week, respectively, at a temperature of $40^{\circ} \mathrm{F}$. This is a very good temperature at which to hold eggs for short periods. Even at twelve weeks of age (section 10), the egg is less evaporated than at four weeks of age, if held at a living-room temperature of $70^{\circ} \mathrm{F}$. (section 5). Eggs may be held for two weeks at $40^{\circ} \mathrm{F}$. without much change.

13 and 14. Each of these eggs has been held for six weeks at a temperature of about $50^{\circ} \mathrm{F}$ The porous shell of the second egg (14) has caused more rapid evaporation. The porosity of the shell may be easily distinguished in candling.

15 and 16. These eggs have been held for five weeks and for two and one-half weeks, respectively, at a temperature of $50^{\circ} \mathrm{F}$ It should be noted that the evaporation is more rapid than that which took place at $40^{\circ} \mathrm{F}$., but much slower than that at $70^{\circ} \mathrm{F}$. 


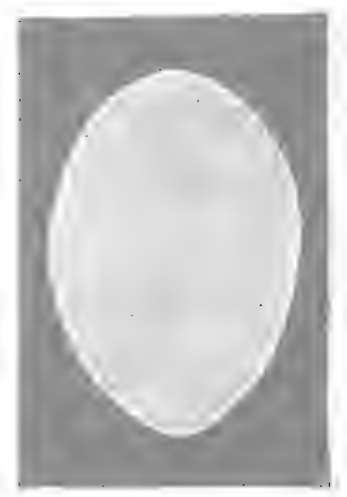

1

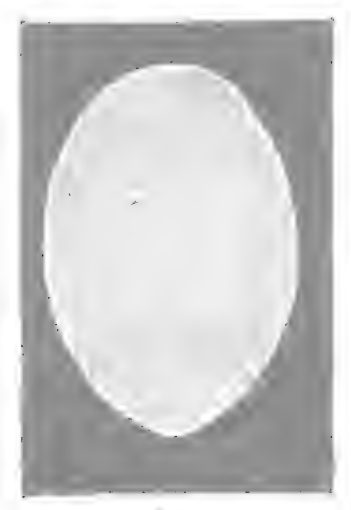

5

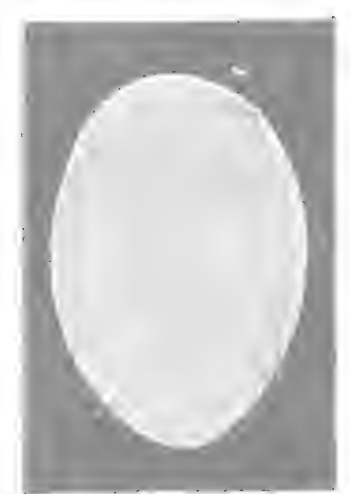

5

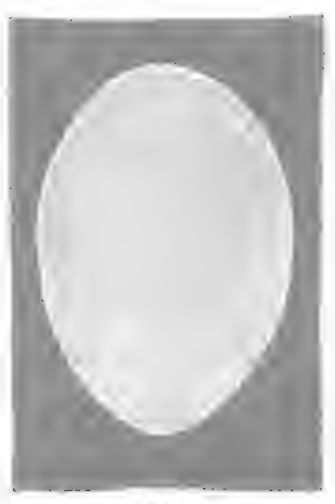

13

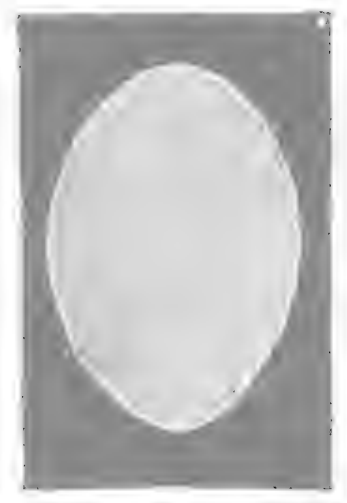

2

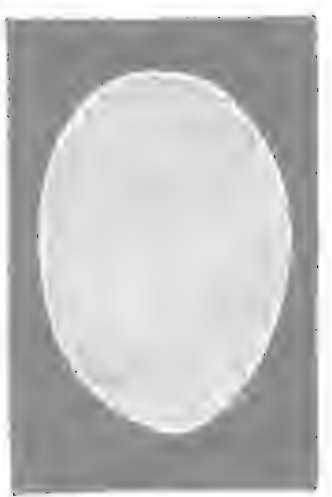

6

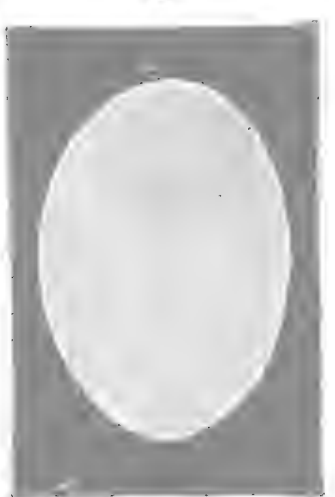

io

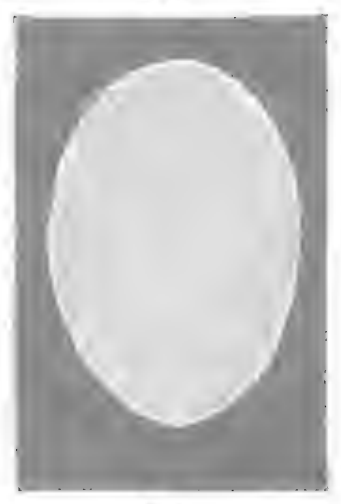

1.2

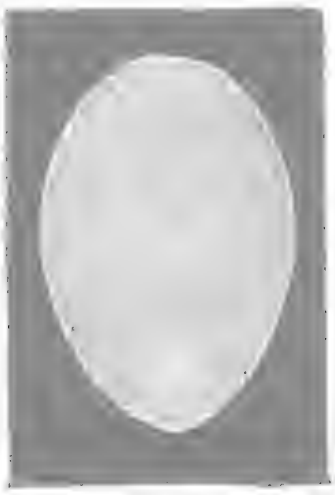

3

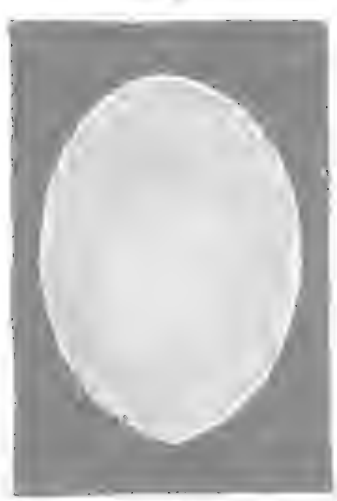

7

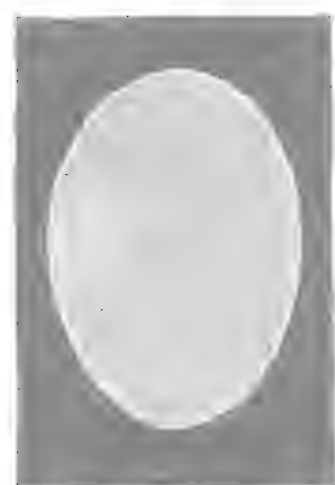

11

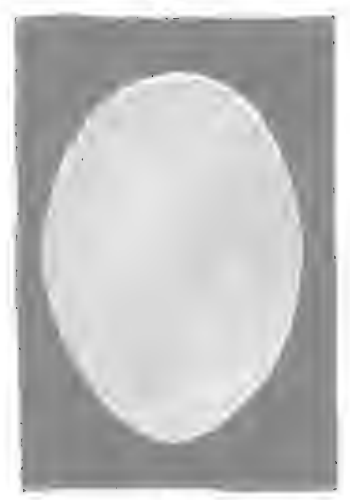

15
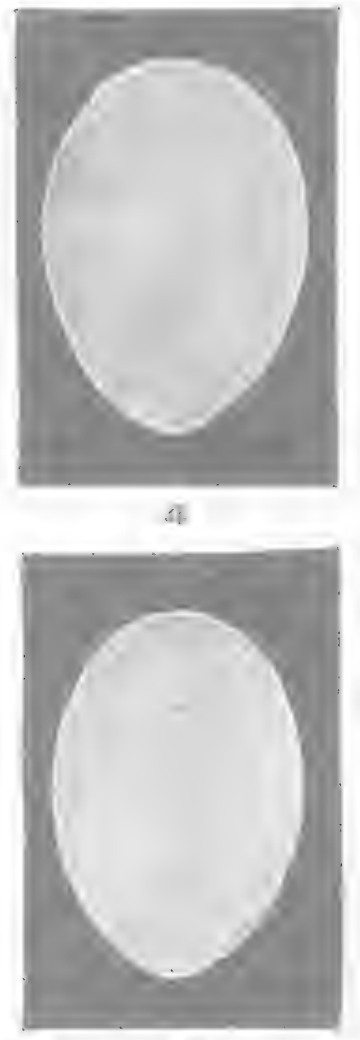

3

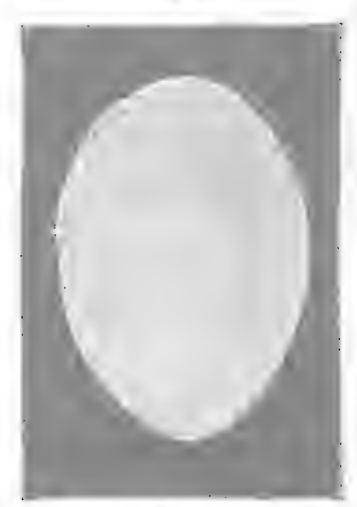

$1 \mathrm{a}$

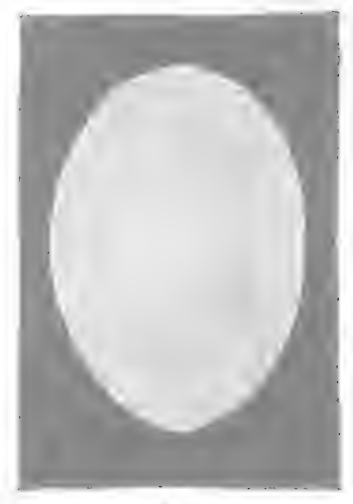

16

Fis. 78. 
Simon, C. E. 1904. A Text Book of Physiological Chemistry for Students of Medicine and Physicians, 455.

SPERry, J. A. 1913. On Antiseptic and Bactericidal Properties of Egg White. Science N. S., 38, 413.

Spring, A. Des champignons qui se developent dans les œufs de poule. Bull. Acad. Roy. Sci. Let. St. Beaux-Arts Belg., 19, 555.

Stiles, G. W. and Bates, C. 1912. A Bacteriological Study of Shelled, Frozen and Desiccated Eggs made under Laboratory Conditions at Washington. U. S. Dept. Agric. Bur. Chem. Bull. 158.

TICE, W. G. A Chemical and Bacteriological Study of Eggs. 35th Ann. Rept. Ncw Jersey Bd. Health, 275.

TURRo, R. 1902. Zur Bakterienverdauung IV. Bakteriologische Wirkung des Huhnerei. Cent. Bakt. Abt. I. Orig., 32, 107.

VoN WirTICH. 1851. Ueber Pilzbildung im Hühnerei. Zcit. Wiss. Zool., $3,213$.

Wiley, H. W., et al. 1908. A Preliminary Study of the Effects of Cold Storage on Eggs, Quail, and Chicken. U. S. Dept. Agric. Bureau of Chem. Bull. 117.

WrLm. 1895. Ueber die Einwanderung von Choleravibrionen im Huhnerei. Arch. Hyg., 23, 145.

WurTz, R. De l'action bactericide du blane d'œuf. Semaine Méd., 3, 21. -

Z a MmermanN, O. E. R. 1878. Ueber dir Mikroorganismen welche die Verderbnis der Eier veranlassen, 6. Ber. d. Natur. Gesell. Chemnitz, p. 3, Landwir. Jahersb., 755.

ZoRGENDORFER. 1893. Ueber die im Hühnerei vorkommenden Bakterienarten nebst Vorschlägen zu rationellen Verfahren der Eiconservierung. Arch. Hyg., 16, 367. 
CHAPTER `XIII

\section{MEAT AND MEAT PRODUCTS}

MEAT is interesting from the bacteriological viewpoint for several reasons. As the activities of man became more complex, it became necessary to keep meat for future consumption. In order to do this, he had to study the action of the bacteria. The meat industry is one in which a large amount of capital is invested. Since meat is very susceptible to the attacks of bacteria and since it may be taken from diseased animals, it has become necessary for the Federal authorities to control the slaughter and disposal of meat. The Federal Meat Inspection Service of the United States Department of Agriculture is a public health agency which is constantly protecting the consumers. Melvin (1906) has outlined the scope and application of the laws governing this product. The opinions regarding what constitutes diseased meat are by no means in agreement. Stiles (1917) has discussed this subject and pointed out that there are several definitions of the term " diseased" as applied to meat. Meat is essentially protein in composition and this chemical constitution determines the type of decomposition to which it is susceptible.

Origin of Bacteria in Meat. The presence of bacteria in the organs and flesh of healthy animals is still a debated question. Many investigators have reported their presence but the health of the animal in these instances may be questioned. Maurel (1911) found a diplococcus in meat. Conradi (1909), using a special enrichment method found aerobic and anaerobic bacteria in muscles and organs. Bierotti and Machida (1910) secured similar results. Zwick and Weichel (1911) found bacteria frequently in the liver but not in the muscles with the exception of one case. Conradi (1909) in examining many organs found bacteria but concluded that they came from the intestines, being absorbed along with the food. Hoagland et al. (1917) in studying the changes in beef during storage above freezing, states as follows with regard to the presence of bacteria:

Certain bacteria (chiefly micrococci) may be normally present in the carcass of healthy animals slaughtered for beef. These bacteria possess no pathological 
significance and do not appear to multiply in the cold-stored carcasses provided the cold-storage room is maintained at the proper temperature.

Chopped Meats. These may contain many bacteria for a number of reasons: (1) The meat that is used for such purposes may be dirty and old and unfit for consumption in other ways. (2) The hashing process probably distributes bacteria throughout the meat This treatment increases the surface area of the meat, allowing a more vigorous development of bacteria. (3) Careless handling of the product may cause high counts in the same way as in other food.

The bacteriology of chopped meat has been studied by a number of investigators. Zweifel (1911) regarded the count as of little sanitary significance. Marxer (1903) made a bacteriological study of Hamburger steak and concluded that 1,000,000 bacteria per gram or a large number of representatives of the protein group indicated a meat that was on the verge of decomposition. Weinzirl and Newton (1914), after developing a satisfactory method for enumerating bacteria in chopped meats studied forty-four samples of market Hamburger steak. To determine the degree of composition comparisons were made between the organoleptic, ammonia tests, and the bacterial content. From their data, they conclude that the Marxer standard given above is too low since nearly all samples have to be condemned without regard to the evidence of putrefaction. There was a good correlation between the ammonia and organoleptic tests but not one between these and the bacterial content. These authors propose a standard of 10,000,000 bacteria per gram which, according to their data, would condemn about 50 per cent of the samples. Le Fevre (1917), using the technique devised by Weinzirl and Newton, made a semi-qualitative study of the bacteriain Hamburger steak. The presence of liquefying bacteria was evident in nearly all of the samples. Acidifiers presented in the table do not agree with statements in the article. Anaerobic bacteria were found in some samples. Le Fevre believes that the Marxer standard is high enough and that the bacterial count should be relied upon for the control of retail supplies of chopped meat.

Whether this may be done is doubtful. At least much more information should be accumulated before any "standard" is established. The kind of bacteria may be more important than the numbers. Weinzirl and Newton (1915) undertook an investigation to determine the effect of cold storage below freezing on the bacterial content. After one year at $-10^{\circ} \mathrm{C}$. six of the ten samples fell within the $10,000,000$ standard. This compared favorably with the organoleptic tests. 
Sausage. The bacteriology of sausage is essentially the same as that of Hamburger steak. One difference is the content of spices in sausage. While most of the work on bacteria in sausage has been concerned with meat poisoning, a little has been done which gives information with regard to the normal bacteria which may be present. V. D. Sloonton (1907) found few bacteria in sausage and in one instance the sausage was sterile. Drying and smoking greatly reduced the number of bacteria. Highly seasoned sausage was very detrimental to certain bacteria. Cary (1916) made a comprehensive study of the sausage with regard to the numbers of bacteria which were present and the factors influencing them. The sausages were purchased upon the market in Chicago. It is interesting to note that Cary regards the bacterial count of sausage as of little importance. Many factors such as precautions in manufacture, handling in shops, and presence of preservatives intluence the count.

The following bacteria which are of special importance were isolated from thirty-four samples:

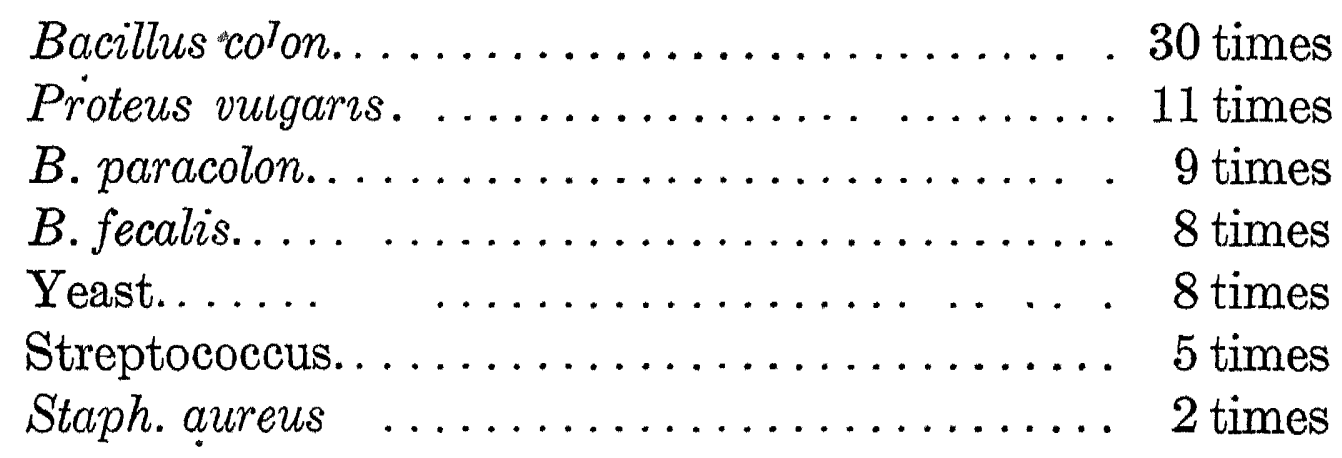

The cultures of $B$. paracolon resembled $B$. paratyphosus morphologically and culturally but were not agglutinated by either paratyphoid or enteritidis sera. The casings were regarded as unimportant in influencing the count of sausage, if they were properly prepared.

Canned Meats. Some aspects of the canning problem have been taken up in another chapter. More attention may be given, however, to the canning of meats at this time. Practically all kinds of meats are canned. The procedure which is followed depends very much on the kind of meat. Canned meat possesses characteristics which are in sharp contrast with some of those of fresh meat. It is trimmed free from bone cartilage and contains a smaller amount of water than fresh meat. The cost, therefore, is not much more than that of fresh meat.

Some investigations have been made on canned meats. Beveridge and Fawcus (1908) reported that when tins of meat of identical size and shape are heated, there is a difference in the length of time required for the interior of the cans to reach the same temperature. The lowest 
temperature that could be used for sterilizing tinned 'meats was $120^{\circ}$ for sixty minutes. These experiments were carried out with $B$. cadaveris sporogenes and B. putrificus coli. Fowler (1909) studied five samples of canned meat, three of which were blown. An organism like B. putrficus coli was isolated. The spores of the organism resisted boiling for two minutes. Beveridge (1909) found that the blackening of the intcrior of the tins was due to products of bacterial action such as $\mathrm{H}_{2} \mathrm{~S}$, etc.

McBryde (1907) reported a study of the spoilage of tinned corned beef. The sound cans were found to be sterile while the leakers revealed the presence of bacteria yeasts and molds. The bacteria were facultative with regard to oxygen. McBryde points out that while the bacterla which he found were non-pathogenic, they may give rise to toxic substances which may serve to cause severe physiological disturbances in those who partake of the meat.

Bushnell and Utt (1917) examined canned salmon purchased on the open market and found it sterile and relatively free from tin salts. Apparently all of the tins which they examined were normal. Swelled canned sardines have been investigated by Sadler (1918). B. vulgarns and members of the colon-aerogenes group were isolated. Apparently no effort was made to determine the presence of anaerobic bacteria. Obst (1918) found a bacillus possibly identical with $B$. Walfishrauschbrand in pure culture in 287 cans of swelled sardines. Koch's postulates were satisfied using this organism and sound cans of sardines. The organism was an anaerobic, gas-producing, non-spore forming bacillus. In a rather extended investigation on spoiled canned mackerel and cod, the author has isolated both aerobic and anaerobic organisms. These were put according to the usual factory methods and processed at the usual temperatures.

\section{BACTERIAL EXAMINATION OF MEAT AND MEAT PRODUCTS}

Hoagland's et al. Method. This is applicable to large pieces of meat and was used by these workers in studying changes in meats under coldstorage conditions. A slice or section from $\frac{3}{4}$ in. thick is cut off (of the round) and from this a chunk measuring $4 \frac{1}{2}$ by 8 ins. is taken for bacterial examination. This is immersed in boiling water for three minutes and next in mercuric chloride ( 0.5 per cent) for five minutes. It is then wrapped in sterile gauze which has been wrung out in the mercuric solution. This is done in order to sterilize the surface of the meat and to prevent the growth and possible penetration of bacteria from the outside. Beginning about 1 in. down from the outer surface take a 
series of cultures at intervals of an inch, proceeding from the outside toward the center. In taking the cultures, use a serus scalpel. Plugs of meat about $1 \mathrm{~cm}$. square should be taken and cultures made in neutral beef broth and glucose agar. When clouding appears, the contents of these tubes should be plated out.

Weinzirl and Newton's Method for the Examination of Hamburger Steak. Three different portions of the sample to be analyzed are taken. The meat is triturated in a mortar with sand by means of a pestle. After grinding with sand for a time sterile salt solution is added and grinding continued. Finally the salt solution is increased to 100 c.c. and a thorough mixture is made. This is sampled and incubated at room temperature. Ordinary media may be used.

Eyre's Method for Examination of Meat. The meat is minced by means of sterile instruments and added to plain broth. The flasks are incubated at $42^{\circ} \mathrm{C}$. for thirty minutes. They are shaken from time to time. Then this is sampled.

Examination of Sausage. Thoroughly sterilize the sausage by passing through a flame. Cut open the casing with a sterile knife and remove 3 or 4 gms. of the interior. This should be added to a sterile tared weighing bottle and carefully weighed. Thoroughly emulsify in sterile water and put on shaking machine for twenty minutes. Plate out on plain agar, plain gelatin and litmus lactose agars.

St. John's Method for Examination of Sausage. The skin is seared and removed; then small fragments are torn from the muscular tissue. These, weighing about $0.3-0.7 \mathrm{gm}$. are shaken for ten minutes, in sterile salt solution with ground glass. Plates were made with neutral glycerol agar and plain agar. Three incubation temperatures were used $37^{\circ}, 20^{\circ}$, and $1.6^{\circ}$. (Effects of cold storage on eggs, quail and chickens. Wiley et al. Bull. $115 \mathrm{Bu}$. of Chem., 1908, p. 75.)

Bacterial Examination of Shellfish. Oysters were regarded by sanitarians for some time as possible agents in the spread of typhoid fever and other intestinal infections. Not until 1894, however, were they definitely connected with this disease. Professor H. W. Conn, of Wesleyan University, in a masterly piece of epidemiological work, proved to the satisfaction of those interested in public health that an outbreak of typhoid fever among those who had attended a fraternity banquet was caused by oysters. The chain of evidence was so complete that the study of oyster grounds, in relation to possible sewage pollution, was greatly stimulated. Since this time many investigations have been carried out. Stiles (1911) has given a rather complete bibliography of the articles up to the date of his paper. Hindman and Goodrich (1917) 
upon examination of Puget Sound oysters found a great variation in the bacterial content. They state that oysters intended for human consumption should be grown in water free from pollution and that after collection they should be handled with great care.

The relation between polluted shellfish and disease is so close that the American Public Health Association appointed a committee to prepare a code of standard methods of shellfish examination. The report of this committee was published in 1912 and included the following recommendations for the bacteriological examination of shellfish.

Many laymen assume and argue that because oysters and other foods are cooked, they are safe for human consumption. In many cases cooking may render a food product sterile. So many experiments have been performed, however, along this line that a student of the subject does not accept such a sweeping statement. Stiles (1911) reported data which were secured in five different experiments to demonstrate the effect of heat for the destruction of bacteria in oysters. The shellfish were exposed to live steam $\left(98^{\circ}\right.$ and $99^{\circ} \mathrm{C}$.) in a steam sterilizer for periods varying from two to thirty minutes. He found that by steaming contaminated oysters and clams in the shell, or cooking them after shucking for fifteen minutes at boiling temperature destroyed practically all organisms of a questionable character, but since in practice shellfish are rarely cooked for this length of time, cooking cannot be depended upon to remove the danger. In this connection it is well to remember the work of Howell (1912), Roussel (1907), and Marchland (1909) on bread and of Sawyer (1914) on cooked spaghetti.

\section{Recommendations for Standard Methods for the Bacteriological Examination of Shellfish}

Oysters in the Shell. Selection of Sample.

Twelve (12) oysters of the average size of the lot under examination, with deep bowls, short lips, and shells tightly closed, shall be picked out by hand and prepared for transportation to the laboratory.

As complete a record of such data as is possible to obtain shall be made covering the following points:

The exact location of the bed from which the sample has been selected.

The depth of the water over the bed at time of collection.

The state of the tide.

The direction and velocity of the wind. 
Other weather conditions.

The day and hour of the removal of the stock from the water.

The conditions under which the stock has been kept since removal from the water and prior to the taking of the sample.

The day and hour of the taking of the sample.

Transportation of the Sample. The oysters so selected shall be packed in suitable metal or pasteboard containers of such size and shape that a number of them can be enclosed in a shipping case capable of satisfactory refrigeration by means of ice. The important points in this connection are:

$A$. The prevention of the mixing of the oyster liquor of different samples, and of the mixing of the ice water with the oysters.

$B$. The icing of the samples, if they are not to arrive at the point of laboratory examination inside of thirty-six hours, or, if the outside temperature is above $50^{\circ} \mathrm{F}$.

It is not necessary to enclose the oysters in an absolutely tight container, providing the above conditions are maintained.

Condition of Samples. Record shall be made of the general condition of the oysters when received, especially whether the shells are open or closed; of the presence of abnormal odors; and of the temperature of the stock.

Technical Procedure. The bacteriological examination shall be started as soon as possible after the receipt of the sample.

The oysters shall be thoroughly cleaned with a stiff brush and clean running water and then dried. The edges of the shell shall be passed through the flame or burned with alcohol.

The opening of the shell shall be accomplished by either of the following methods:

$A$. By the use of a sterile oyster knife in the usual manner.

$B$. By drilling through a flamed portion of the shell near the hinge with a sterile drill. The drill shall be sterilized, and the site of the operation on the shell shall be flamed at least once during the drilling process.

Bacterial Counts. Bacterial counts shall be made of a composite sample of each lot obtained by mixing the shell liquor of five oysters. Agar shall be used for the culture medium and in general the procedure shall be in accordance with the method recommended for the examination of water by the Committee on Standard Methods of Water Analysis of the American Public Health Association.

The water used for dilution purposes shall contain 1 per cent of sodium chloride, in order to approximate the natural salinity of oyster liquor. 
The agar plates shall be incubated at $20^{\circ} \mathrm{C}$. for three days and the colonies then counted.

Determination of Bacteria of the Bacillus coli Group. The quantitative determination of the presence of $B$. coli shall be in accordance with the following procedure:

Measured quantities $(1.0 ; 0.1 ; 0.01$ c.c., etc., or their equivalents in dilutions) of the shell water of each of five oysters selected from the dozen, shall be placed in fermentation tubes containing lactose peptone bile, prepared according to the method recommended by the Committee on Standard Methods of Water Analysis. These shall be incubated for three days at $37^{\circ} \mathrm{C}$., and the presence or absence of gas noted daily. For all ordinary purposes of routine work a development of from 10 to 85 per cent of gas during this time period shall constitute a positive test indicating a presumption of the presence of at least one bacterium of the $B$. coli group in the quantity of the shell water tested. But no final $B$. coli rating based on these results shall be used for official approval or condemnation unless positive confirmatory tests for the presence or organisms of the $B$. coli group shall have been obtained from the tube of highest or next highest dilution from each oyster, showing the presence of gas. These confirmatory tests shall be begun immediately upon noting the formation of gas, and carried out in accordance with the procedure recommended by the Committee on Standard Methods of Water Analysis.

Statement of Results. The results of the bacterial counts shall be expressed as Number of Bacteria per Cubic Centimeter. The results for the tests for $B$. coti shall be expressed either in the form of the following arbitrary numerical system to be known as "The American Public Health Association Method of Rating Oysters for B. coli; or in Estimated Number of Bacteria of the B. coli Group per Cubic Centimeter of the Sample."

The American Public Health Association Method of Rating Oysters for B. coli.* The following values shall be assigned to the presence of bacteria of the $B$. coli group in each of the five oysters examined, these figures being the reciprocals of the greatest dilutions in which the test for B. coli was positive:

If present in 1.0 c.c. but not in 0.1 c.c., a value of 1 .

If present in 0.1 c.c. but not in 0.01 c.c., a value of 10 .

If present in 0.01 c.c. but not in 0.001 c.c., a value of 100 , etc.

* Where the term $B$. coli is used, it refers in all cases to bacteria of the $B$. coli group and not to the specific prototype. 
The sum of these values for the five oysters gives the total value for the sample and this figure shall be taken as the " rating for $B$. coli."

The results shall be expressed in the following tabular form:

RESULTS OF TESTS FOR B. COLI IN DILUTIONS INDICATED

\begin{tabular}{c|c|c|c|c}
\hline Oysters & 10 c c. & 0.1 c.c. & $0.01 \mathrm{cc}$. & Numerical Value. \\
\hline 1 & + & + & 0 & 10 \\
2 & + & + & 0 & 10 \\
3 & + & 0 & 0 & 1 \\
4 & + & 0 & 0 & 1 \\
5 & + & 0 & 0 & 1 \\
\multicolumn{2}{l}{ Total, or rating for B. coli.. } & $\ldots \ldots \ldots \ldots \ldots$ & $\ldots \ldots \ldots \ldots$ & $\ldots \ldots .23$ \\
\hline
\end{tabular}

$+=$ Presence of bacteria of the $B$. coli group in fermentation tube test with lactose bile.

$0=$ Fallure to demonstrate presence of bacteria of the $B$. colv group.

Estimated Number of B. coli per Cubic Centimeter. If the standard $B$. coli rating above described is divided by 5 or, in general, if the rating is divided by the number of oysters tested, the result will be approximately the number of $B$. coli per cubic centimeter of shell water. Partly because it does not do this exactly, but also for simplicity and the avoidance of fractions, the method of stating results as an arbitrary rating is preferred by the committee. Practical experience with the method also has appeared to justify this preference.

Illustrations of the Application of the Method of Rating Oysters for B. coli. Sometimes results similar to the following are obtained, that is, one or more oysters may show positive results in small quantities of shell water, while an equal number may show negative results in larger quantities. In this case the next lower numerical value shall be given to the positive results in the high dilutions, and such positive results shall be considered as being transferred to a lower dilution giving negative results in another oyster. This is done on the theory that inconsistent results, mathematically considered, may follow naturally from an unequal distribution of the bacteria in the shell water. This recession of the assigned values, however, shall not be carried beyond the point where the number of such recessions is greater than the number of instances where other oysters in the series failed to give positive $B$. coli results.

As examples of the method of obtaining the rating for $B$. coli, the following illustrations are given. They represent results that may be met in practice. 
CASE A.-RESULTS OF B. COLI TESTS IN DILUTIONS INDICATED

\begin{tabular}{c|c|c|c|l}
\hline Oysters. & 1.0 c.c. & 0.1 c.c. & 0.01 co.c. & Numerical Value. \\
\hline 1 & + & + & 0 & 10 \\
2 & + & + & 0 & 10 \\
3 & + & + & 0 & 10 \\
4 & + & 0 & 0 & 10 (not 1) \\
5 & + & + & + & 10 (not 100) \\
& & & & 50 Rating \\
\hline
\end{tabular}

CASE B.-RESULTS OF B. COLI TESTS IN DILUTIONS INDICATED

\begin{tabular}{c|c|c|c|c}
\hline Oysters. & 1.0 c.c. & 0.1 c.c. & 0.01 c.c. & Numerical Value. \\
\cline { 1 - 3 } 1 & + & + & + & 10 (not 100) \\
2 & + & + & + & 10 (not 100) \\
3 & + & 0 & 0 & 1 \\
4 & 0 & 0 & 0 & 1 (not 0) \\
5 & 0 & 0 & 0 & 1 (not 0) \\
& & & & 23 Rating \\
\hline
\end{tabular}

CASE C.-RESULTS OF B. COLI TESTS IN DILUTIONS INDICATED'

\begin{tabular}{c|c|c|c|l}
\hline Oysters. & 1.0 c.c. & 0.1 c.c. & 0.01 c.c. & Numerical Value. \\
\hline 1 & + & + & 0 & 10 \\
2 & + & + & 0 & 10 \\
3 & + & + & + & 100 \\
4 & + & + & + & 10 (not 100) \\
5 & + & 0 & 0 & 10 (not 1) \\
& & & & $140=$ Rating \\
\hline
\end{tabular}

Oysters Removed from the Shell (Opened or Shucked Stock). Except as hereinafter stated, all the procedures and requirements for the examination of opened oysters, i.e., shucked stock, shall be those specified for the examination of oysters in the shell.

Seiection and Preparation of Sample. The stock in the container from which the sample is to be taken shall be thoroughly mixed, and one or more wide-mouthed sterile jars of a total capacity of one quart shall be each half filled with the sample by means of a clean ladle or other instrument sterilized by flaming alcohol. The jar or jars shall be so sealed as to exclude all possibility of contamination from without. 
Transportation of Samples. When the time between the collection of the sample and its examination exceeds three hours, or, if the outside temperature is above $50^{\circ} \mathrm{F}$., the sample shall be thoroughly refrigerated by means of ice placed around, but not in, the sample jars.

Technical Procedure. The bacteriological examination shall be begun as soon as possible after taking the sample. The sample shall be thoroughly shaken at least twenty-five times immediately before opening.

Bacterial Counts. The procedure specified for oysters in the shell be followed:

Determination of Bacteria of the Bacillus coli Group. The procedure specified for oysters in the shell shall be followed, but attention is called to the fact that higher dilutions than $1 / 100$ c.c. are usually required. Triplicate fermentation tubes shall be inoculated from each dilution of the sample.

Statement of Results. The results of the bacteriological examination of the opened oysters, or shucked stock, shall be expressed in the same way as that specified for oysters in the shell, except that in the calculation of the $B$. coli rating the values for the results of the positive fermentation tests after confirmation shall be recorded for each of the inoculations of each dilution. In order that the rating from these triplicate tests may be compared with that obtainod from testing five oysters in the shell, the sum of the values for the triplicate tests shall be multiplied by $5 / 3$. If, instead, the sum is divided by 3 , the result will give approximately the number of $B$. coli per cubic centimeter.

\section{Clams and other Shell Fish}

The methods for examining clams and shell fish, other than oysters, shall be those given above. Certain modifications are necessary in the method of handling the samples and the opening of the shells, etc.

Clams are more likely to lose water during transportation than oysters. It is, therefore, necessary to take greater precautions to separate different samples of clams from each other than in the case of oysters.

In opening soft clams it has been found that if two incisions are made through the mantle the shell water may be poured out without opening the shell.

Hard clams are more difficult to open, but if the shell be struck over the dorsal muscie with a small hammer an opening will be formed permitting the insertion of the knife to cut the muscle. 
Sometimes clams and other shell fish contain too little liquor to make all of the tests above described. This is always the case when the shells are very small. Under these conditions the water from two or more shellfish shall be taken together and tested and considered as one.

The English practice for the examination of oysters is somewhat different than that used in America. Savage (1914) has outlined the method which was used by Houston for the Royal Commission on Sewage Disposal, as follows:

1. The outside of the oyster shells are well scrubbed with soap and water, and cleaned as thoroughly as possible in running tap-water, finally with sterile water.

2. The hands of the investigator are thoroughly cleaned, washed in 1 in 1000 corrosive sublimate solution, and finally with sterile water.

3. The oysters are opened by a sterile knife held in position by a sterile cloth, and with the concave shell underneath. Great care must be taken to avoid any loss of the liquor. The liquor in the shell is poured into a 1000 c.c. cylinder, and the oyster and oyster liquor are added after the oyster has been cut into small pieces by sterile scissors.

4. Ten oysters are treated as above in each experiment.

5. The volume of oysters plus oyster liquor is read off, and usually varies between 80 and 120 c.c. For qualitative work 100 c.c. may, therefore, be taken as a fair average of the total shell contents of ten oysters.

Sterile water is then poured into the cylinder up to the 1000 c.c. mark and the whole well stirred with a sterile rod. Each 100 c.c. of this liquor may be considered to contain the bacteria in one oyster.

The Precipitin Test for the Detection of Foreign Protein in Meats. The food microbiologist may be called upon at any time to determine the presence or absence of a suspected protein in certain foods-horse or deer meat in sausage. The chemical methods for doing this are very indirect if not impossible. The bacteriological methods are very sensitive.

Meat Extract. About $30 \mathrm{gms}$. of the meat under examination should be covered with 100 c.c. of sterile physiological salt solution. This is then allowed to stand over night in a refrigerator after which it should be filtered clear by any of the usual methods. Hiss and Zinsser (1914) advise that this should be adjusted to a neutral reaction and diluted with sterile water until only a slight even turbidity is formed by the addition of concentrated nitric acid. The less the amount of fat in the above protein solution, the easier will it filter. Before injection, the 
protein solutions should be sterilized by passage through a stone filter in order not to cause an infection in the animal.

Anti-protein Sera. These should be active in fairly high dilution and each serum must be specific for its protein. As many anti-sera will have to be made as kinds of meat suspected. Usually, however, the problem is simpler and involves the question of horse meat in sausage, etc. Schneider (1915) gives the following for this examination:

Tube 1,2 c.c. unknown extract $(1-300)+0.1$ c.c anti-horse serum.

Tube 2,2 c.c. unknown extract $(1-300)+0.1$ c.c. normal rabbit serum.

Tube 3,2 c.c. horse-flesh extract (1-300) +0.1 c.c. of anti-horse serum.

Tube 4,2 c.c. pork extract $(1-300)+0.1$ c.c. of anti-horse serum.

Tube 5, 2 c.c. beef extract (1-300)+0.1 c.c. of anti-horse serum.

Tube 6,2 c.c. saline solution +0.1 c.c. of anti-horse serum.

Schneider advises stratifying the immune sera in the tube. They should be kept at room temperature. If " tubes 1 and 3 show a clouding within five minutes and if a definite precipitate forms within thirty minutes, the other tubes remaining perfectly clear, the extract is probably one of horse flesh or the flesh of some other single-toed animal."

\section{BIBLIOGRAPHY}

Bates, C. 1916. The Handling of Shucked Oysters. Amer. Jour. Public Health, 6, 987.

Bates, C. 1916. Sanitary Control of Shellfish Industry. Amer. Jour. Pub. Health, 6, 453.

Bates, C. and Round, L. 1916. A Comparison of Bacteriological Methods for the Examination of Oysters. Amer. Jour. Pub. Health, 6, 841.

Beveridge, W. W. O. and Fawcus, H. B. 1908. Experiments on Canning Meat. London. Great Britain War Office, 57-72.

Beveridge, W. W. O. 1908. Report on the Nature and Causes of the Blackening of the Interior of Tins. Third report of the committee on physioological effects of food, training and clothing on the soldier. London. Great Britain War Office, 1908. Jour. Royal Army Medical Corps, $13,326-332$.

Bierotti and Machilda. 1910. Múnchener med. Wochenschr., 57, 636.

Bushnelt, L. D. and Urт, C. A. A. 1917. The Examination of Canned Salmon for Bacteria and Tin. Kansas State Board of Health, 13, 36-38. Jour. Ind. Eng. Chem., 12, 678.

CARY, W. E. 1916. The Bacterial Examination of Sausages and its Sanitary Significance. Amer. Jour. Pub. Health 6, 124-135.

Cons, H. W. 1894. 17th Annual Report of the Connecticut State Board of Health. 
ConRadr. 1909. A New Bacteriological Method for Meat Analysis. Zeit. Fleisch. u. Milchhygiene, 19, 341-345. See also Münch. med. Wochenschr., 56, 1318-1320.

Fowler, C. E. P. 1909. Bacteriological Report. Great Britain War Office, $13,323-325$.

Hindman, E. F. and Goodrich, F. J. 1917. A Study of Puget Sound Oysters. Amer. Food Jour., 12, 611-614.

Hoagland, F., McBryde, C. N. and Powick, W. C. 1917. Changes in Fresh Beef During Cold Storage above Freezing. U. S. Department of Agriculture Bulletin 433.

Howeld, K. 1912. The Bacterial Contamination of Bread. Am. J. Pub. Health, 2, 321-324.

Johnstone, J. 1909. Routine Methods of Shellfish Examination with Reference to Sewage Pollution. Jour. Hyg., 9, 412.

Kossowicz, A. and NASSAU, R. 1916. Contribution to Bacteriology and Technology of the Preservation of Meat. Wiener Tierärtz. Wochenschr., 3, 81-102. Chem. Zentr., 1916, II, 69.

LeFevre, E. 1917. A Bacteriological Study of Hamburger Steak. Amer. Food Journal, 12, 140-142.

MacManus, R. D. 1917. The Manufacture of Meat Food Products. Amer. Food Journal, 12, 559-563.

McBryde, C. N. 1907. A Study of the Methods of Canning Meats with Reference to the Proper Disposal of Defective Cans. 24th Ann. Rep. Bureau of Animal Husbandry, U. S. Dept. Ag., 279-296.

Marcitand, H. 1909. The Cooking of Bread. Meun. Franc., 25, 208-210. Exp. Sta. Rec., 22 (1910), 64.

MarXer. 1903. Beiträge zur Frage des Bakteriengehalts und der Haltbarkeit des Fleisches. Quoted from LeFevre, 1917.

MaUReI, E. 1911. The Occurrence of Microorganisms in the Interior of Meat, Potatoes, and Sausage. Comp. Rend. Soc. Biol., 70, 241-244. Exp. Sta. Rec., 25 (1911), 265.

Melvin, A. D. 1906. The Federal Meat Inspection Service. 23d Ann. Report Bureau of Animal Industry, 65-100.

Pennington, M. E. 1911. The Comparative Rate of Decomposition of Drawn and Undrawn Market Poultry. U. S. Dept. Ag. Bu. Animal Industry. Circular 70.

Round, L. 1916. Comparative Bacteriological Examination of Oysters. Amer. Jour. Pub. Health, 6, 686.

Rousser, J. 1907. The Survival of Pathogenic Bacteria in Bread after Baking. Rev. Intend. Mil., 20, 122-131. Chem. Abts., 2 (1908), 1168.

SADLER, W. 1918. The Bacteriology of Swelled Canned Sardines. Amer. Jour. Pub. Health, 8, 216-220.

Sawyer, W. A. 1914. Ninety-three Persons Infected by a Typhoid Carrier at a Public Dinner. Jour. Amer. Med. Assn., 63, 1537-1542.

SMItH, G. H. 1913. Size of the Sample Necessary for the Accurate Deter- 
mination of the Sanitary Quality of Shell Oysters. Amer. Jour. Pub. Health, 3, 705.

Stiles, G. W. 1911. Shellfish Contamination from Sewage Polluted Waters and from Other Sources. U. S. Department of Agriculture, Bureau of Chemistry Bull. 136.

Stiles, C. W. 1917. What is Diseased Meat? Amer. Food. Jour., 12, 193194. Jour. Amer. Med. Assn., 68, March 3, 1917.

Weinzrre, J. and Newton, E. B. 1914. Bacteriological Analysis of Hamburger Steak with Reference to Sanitary Conditions. Amer. Jour. Public Health, 4, 413-417.

Weinzirl, J. and Newton, E. B. 1915. The Fate of Bacteria in Frozen Meat Meat Held in Cold Storage and its Bearing on a Bacteriological Standard for Condemnation. Amer. Jour. Public Health, 5, 833-835.

ZwICK and WeICHeL. The Presence of Bacteria in the Flesh of Normal Slaughtered Animals and the Technique of the Bacteriological Meat Examination in Forced Slaughter. Arb. kaiserlich. Gesundh., 3, 327-337. 


\section{CHAPTER XIV}

\section{FOOD PRESERVATION}

THE relation of bacteria to foods may be considered from several viewpoints. With regard to the changes which they induce in foods they may be divided into two general classes - the desirable and the undesirable. These may be divided somewhat after the following manner:

I. Desirable microorganisms:

A. Bacteria
(a) Fermented milks
(b) Cheese and butter
(c) Pickles, sauerkraut, etc.
(d) Ensilage
(e) Industries, leather, retting, etc.

B. Yeasts

(a) Leavening agents

(b) Wine and beer

C. Molds

(c) Cheese

II. Undesirable Microorganisms:

$A$. Those which cause spoilage

(a) Fermentation, putrefaction

$B$. Those which cause food poisoning

The question of food spoilage may be discussed from a number of viewpoints. Some have taken up each special food such as milk, meat, etc., and discussed the types of change, organisms, etc., which are involved. More recently, however, Rahn (1913) has pointed out the possibility of discussing the large groups of food substances which undergo decomposition and not mentioning each particular food. For this purpose, he has devised the following groups, which serve very well as a basis for a consideration of food spoilage: 
Group I. Proteins (Acids and carbohydrates in traces).

Example: Meat, fish and eggs.

Group II. Carbohydrates (Acids and protcins in traces).

Example: Starch, sugar, honey.

Group III. Proteins + Carbohydrates (Acid in traces).

Ex:mple: Milk, cereals, flour, greens (cabbage), vegetables.

Group IV. Acids (Proteins and carbohydrates in traces). Example: Vinegar.

Group V. Acid+Proteins (Carbohydrates in traces). Example: Hard cheese, sauerkraut.

Group VI. Acid+Carbohydrate (Protein in traces). Example: Must.

Group VII. Acid+Protein+Carbohydrate.

Example: Sour milk, butter, fruit.

Group VIII. Acids, carbohydrates and proteins in traces.

Example: Water.

As Rahn points out the type of decomposition in each group is quite sharply defined. The decomposition of foods in Group I is a typical putrefaction (Fäulnis). The foods under Group II contain too little water to permit of microbial cleavage. Acid fermentations are the types of change for Group III.

\section{Food Preservation}

Asepsis. According to Rahn's system the following groups of food substances may be preserved by asepsis.

Group I. Meat, fish, eggs.

Group III. Milk, vegetables, nuts.

Group VII. Fruits.

By asepsis is meant the prevention of contamination of food substances by undesirable microorganisms during their preparation. The following factors influence this method of food preservation.

I. Conditions of production.

II. Amount of moisture.

III. Physical structure.

IV. Chemical composition.

V. Temperature.

Few foods are produced absolutely free from microorganisms. If such were the case, they would keep indefinitely. Since this is practically impossible, it has become necessary to reduce to a minimum 
unnecessary contamination during production. In this connection, it should be remembered that many foods contain bacteria in the tissue. This has been shown in other places in this book. Hoagland, McBryde and Powick (1917) found a micrococcus to be normally present within the tissues of fresh beef. Bacteria have also been found in tissues from other sources.

The relation of environmental conditions during production to infection of foods needs no extended discussion. Many cases of food poisoning from meats have been traced back to infection during slaughter. Other factors tend to render the aseptic production of meat impracticable and impossible. Certified milk is the nearest approach to the production of aseptic milk. Water glass is used for preservation of eggs and this is probably a good example of aseptic preservation of foods.

The humidity of the atmosphere in which foods are kept, is an important determining factor. Water is necessary to carry food into the cell and remove the waste products from the cells of microorganisms. Spores will not germinate if there is insufficient moisture present.

The physical structure of the food may determine the type of decomposition. Nature has provided many foods with efficient barriers against the attack of microorganisms. Unless these are broken in some way, the microorganisms are not able to accomplish any damage. Fruits, such as apples, cherries, pears, etc., are provided with a thick cellulose skin which is very resistant to the attack of microorganisms. Few of them possess a cellulase with which to attack this barrier. Eggs are protected by a shell which is covered with a gelatinous layer. If this is removed by washing, the egg will spoil much more quickly.

In general, the conditions under which a food is produced greatly influence any method that may be adopted to preserve it.

Spices. Condiments have probably been a little over-emphasized as food preservatives. Bitting (1909), in studying the preserving action of spices in ketchup found that cinnamon and cloves were the strongest antiseptics. He boiled 20 gms. of the whole spice in 200 c.c. of water for forty-five minutes. This was then filtered and from 0.1 to 5 c.c. of the filtrate added to tomato bouillon. Mustard, paprika and cayenne pepper checked growth, but 5 c.c. of the highest strength did not inhibit growth. Bachmann (1916), using different technique, secured somewhat the same results. Using the oils, she found that cinnamic aldehyde was most effective in preventing the growth of all organisms studied. Eugenol and oil or allspice had a distinct preservative action. Molds were found to be more sensitive than the bacteria or yeasts. 
The investigations carried out at the University of Wisconsin have given us much information on the use of spices as preservatives (Hoffmann and Evans, 1911, Bachmann, 1916). Cinnamic aldehyde and eugenol were found to be especially important in preserving. Both of these are found in cinnamon and cloves and probably accounts for the preserving action of these condiments. In the later paper by Bachmann a new method was used by which the plain and spice agar could be used in the same Petri dish. The results are quite similar to those presented in a former paper. Pepper and nutmeg were not able to retard growth of microorganisms. Cloves and allspice in large amounts were effective. In a later paper Bachmann (1918) reported that bacteria, molds and yeasts show a marked sensitiveness to different brands of spice. The growth of microorganisms on a spiced medium may be taken as a criterion of the preservative value of the spice.

Garlic was found to possess a prophylactic action by Minchin (1917) in diseases of diphtheria, typhus and typhoid. It would possibly possess the same action in food preservation.

Fermentation. This process of preservation is limited to a certain few foods. The acids, principally lactic, are relied upon to suppress the growth of putrefactive bacteria. Brown (1916), in studying the brine pickle fermentation reports that many bacteria may enter the tank, but only those which can tolerate 12 to 20 per cent of salt are concerned in the normal fermentation. He found that the acidity of new brine is practically zero and gradually increases to 50 per cent $\mathrm{N} / 10$ or above. Lactic and acetic acids predominate with a ratio of about $2: 1$. When the scum yeasts reduced the acid from the surface downward, the pickles were liable to decomposition.

From the fact that lactic acid is so important in the keeping of pickles it may be wondered whether the salt is really necessary. Rahn (1913) has answered this:

This question must doubtless be answered with yes. If no salt, or only little salt would be added the keeping quality of brine pickles would be like that of the dill pickles, or even less, since the spices of the dill pickles have a certain, though very small, disinfecting value. Salt retards all life activities of bacteria; it makes the fermentation go slower and it makes the acid destruction by the scum yeast slower, too. The more salt the longer will the pickle keep.

The fermentations involved in the preservation of sauerkraut are little different from those of pickles. According to Round (1916) bacteria are the important organisms concerned in the fermentation 
and when air is allowed to come in contact with the brine or kraut, there is a vigorous growth of scum yeasts. Vats which showed an abnormal fermentation contained a different species of bacteria from the normal which got into the brine before the acid was high enough to prevent their growth. In a later paper (1917) Round states that the respiration of living tissue is important in gas production. Bacillus brassica (Wehmer) and Bacillus cucumeris fermentatce (Henneberg) represented the types which produced the greatest amounts of acid.

Smoking. Smoke has been used for a long time to preserve foods, especially meats. It probably acts by closing the pores of the meat and thus preventing the entrance of bacteria. The smoke contains different volatile products which are deleterious to the organisms causing putrefaction. The creosote compounds are also objectionable to certain insects which might pass their larval stage on it.

Smoke also imparts a flavor to meats which is desired. This is especially true with meats which have passed through a pickle. Resininous woods are never used since these impart an undesirable flavor to the meat. Green hickory is advised for smoking under domestic conditions or corn cobs may serve as fuel. Under commercial conditions, piles of hickory logs are burned under the meat (hams). Toward the end of the smoking period, hickory sawdust is thrown on to give the hams a brown color (MacManus, 1917).

McBryde (1911) has studied an economically important infection of hams known as ham souring. In advanced cases, the ham will possess a distinctly putrefactive odor while in the early stages the odor may be indefinite and a little " off." McBryde isolated an anaerobic bacillus (B. putrefacies) with which he satisfied the postulates of Koch. Since it is an anaerobic bacillus it develops in the deeper portions of the ham. The portions nearest the bone seem to be attacked first.

Concentrated Solutions. The cell membrane should be regarded as a semi-permeable membrane and the passage of liquids through it is governed by the laws of osmosis and osmotic pressure. This is dependent upon the size of the molecule and type of substance in solution. The osmotic pressure is proportional to the number of molecules in solution. In salt and sugar solutions then, of equal concentration by weight, the salt will exert a greater destructive action than the sugar which is present in a fewer number of molecules.

Salt as a Preservative. Much more data are available in regard to the use of salt than carbohydrates. In this case it is used to prevent the growth of certain organisms and favor the growth of others. In early times salt served as currency and was too expensive to be used 
in food preservation. As methods for its manufacture were developed it became too abundant for currency and gradually was used for preservation of foods. Different reasons have been ascribed for the keeping power of salt.

I. Exerted a poisonous action.

II. Made the moisture unavailable for the microorganisms.

III. Destroyed cells by plasmolysis.

Rapin and Weigman (1913) found that salt contained bacteria. Salt which was kept in barrels had a high number of bacteria in the upper layers. Spore-forming varieties were found which would attack milk fat when this was salt used dairying.

Peterson (1900) reported some interesting data from which the change of species of microorganism with salt concentration may be noted. In low concentrations the flora is more heterogeneous. Between 12 and 15 per cent concentration the rods are killed. Wild yeasts develop in 25 per cent of salt. These data were secured from studies on fish. (See Table 48.)

\section{TABLe XLVIII}

INFLUENCE OF SALT UPON THE BACTERIAL FLORA AND DECOMPOSITION OF FISH

(Peterson, 1900)

The figure indicates the day after salting when bacteria or chemical change was first noticed.

\begin{tabular}{|c|c|c|c|c|c|c|c|c|}
\hline & $\underset{\text { Cent. }}{\text { Per }}$ & $\begin{array}{c}\text { Per } \\
\text { Cent. }\end{array}$ & $\begin{array}{c}\text { Per } \\
\text { Cent. }\end{array}$ & $\begin{array}{c}\text { Per } \\
\text { Cent. }\end{array}$ & $\underset{\text { Cent. }}{\text { Per }}$ & $\begin{array}{c}\text { Per } \\
\text { Cent. }\end{array}$ & $\begin{array}{c}\text { Per } \\
\text { Cent. }\end{array}$ & $\underset{\text { Cent. }}{\text { Per }}$ \\
\hline Salt concentration..... & 5 & 8 & 10 & 12 & 15 & 18 & 20 & 23 \\
\hline Rods, appear. . . . . . . & 2 & 14 & 23 & 52 & & & & \\
\hline Rods, plenty.......... & 12 & 29 & 50 & 75 & & & & \\
\hline Cocci, appear....... & 2 & 2 & 2 & 2 & 14 & 15 & 50 & 75 \\
\hline Cocci, plenty . . . . . . . & 2 & 2 & 6 & 6 & 34 & 15 & & \\
\hline Yeast, appear........ & $\ldots \ldots$ & $\ldots \ldots$ & $\ldots \ldots$ & 6 & 14 & 15 & 50 & 75 \\
\hline Yeast, plenty. ......... & $\ldots \ldots$ & $\ldots \ldots$ & $\ldots \ldots$ & $\ldots \ldots$ & 14 & 15 & & \\
\hline Odor. .......... & 6 & 8 & 8 & 12 & 34 & 75 & & \\
\hline Alkaline reaction & 4 & 6 & 10 & 12 & 34 & 69 & & \\
\hline Butyric acid...... & 12 & 23 & 23 & 12 & 34 & 75 & 75 & \\
\hline Peptones......... & 10 & $\ldots$ & 30 & 58 & 75 & 75 & & \\
\hline Ammonia......... & 7 & 6 & 10 & 12 & & & & \\
\hline $\mathrm{H}_{2} \mathrm{~S} . \ldots \ldots \ldots \ldots$ & 6 & 14 & 28 & 58 & & & & \\
\hline Indol $\ldots \ldots \ldots \ldots \ldots$ & 16 & 29 & 68 & 75 & & & & \\
\hline Phenol........... & 55 & 75 & & & & & & \\
\hline
\end{tabular}

The blank spaces indicate that change was not observed in seventy-five days. 
De Freytag (1890) studied the effect of concentrated salt solutions on pathogenic bacteria. The brine used for preserving meat had the following effect on bacteria:

\section{In Salt-Concentrated-The Following Died:}

(After de Freytag)

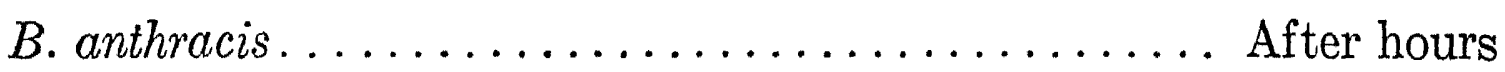

B. anthracis spores.................... Not in 6 months

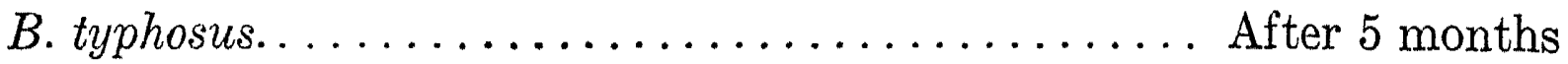

B. of hog erysipelas.................. After 2 months

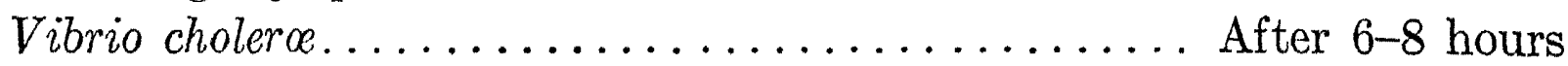

Strep. erysipelatis. .................. Not in 2 months

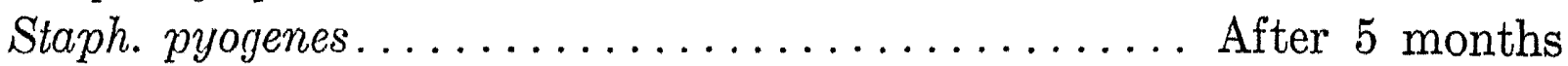

B. tuberculosis....................... Not in 3 months

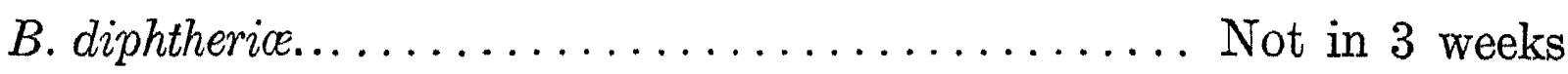

(After Stadler)

B. coli .......................... Not in 6 weeks

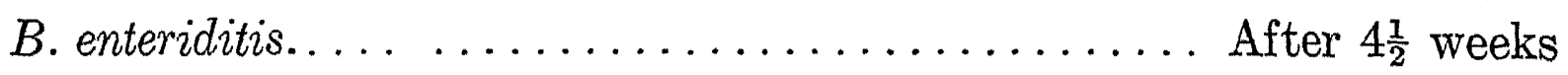

B. morbificans bovis..................... After 3 weeks

B. proteus......................... Not in 3 weeks

B. typhosus...................... Not in 6 weeks

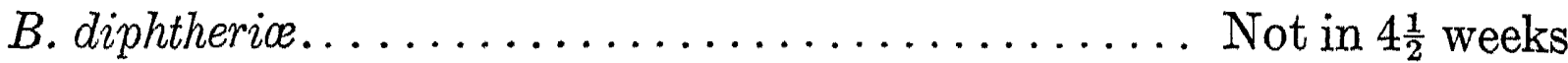

Staph. pyogenes..................... Not in 6 weeks

B. lact. aerogenes...................... Not in 6 weeks

B. pestis........................... Not in 16 weeks

Stadler (1899) found that Bact. coli commune, Bac. morbificans bovis, Bac. enteritidis, Bac. (proteus) vulgaris and Bacillus botulinus were inhibited in salt concentrations of 7-10 per cent. Fettick (1908) studied the relation of salt to butter preservation. His conclusions were drawn from counts. They were about the same on salted and unsalted butter. Inoculating pure cultured into sterile butter, several molds Bacillus fuorescens liquefaciens, and lactic acid bacteria grew at 3 per cent concentration. Bacillus colon, Bacillus subtilis, Bacillus aerogens and some torulæ multiplied at 6 per cent concentration. From these data, Fettick concludes that a concentration of about 3 per cent of salt should be used in butter. This will allow the lactic acid bacteria to grow but prevent the others. In connection with butter the data of Washburn and Dahlberg (1917) is interesting. They conclude that salt hastened the deterioration of the butter. They found little relation 
between the bacteria acidity and score. Rahn, Brown and Smith (1909) found that salted butter kept better than unsalted. Microorganisms were found in slated butter which multiplied slowly at $-6^{\circ} \mathrm{C}$. Thom and Shaw (1915) found that 2.5 to 3 per cent of salt was necessary to keep down mold growth in butter.

Giltner and Baker (1915) in studying the effect of salt on the flora in butter have given some information which might be considered in this place. They found that 12 per cent of salt does not retard growth in all cases and that some bacteria are able to withstand 20 per cent of salt. Streptococci were found to be sensitive to salt while staphylococci and micrococci were not. The greater part of the yeasts and torulæ in butter cannot withstand as much salt as the cocci. Eight per cent of salt was believed to limit the physiological activities of most bacteria. Continued cultivation of some organisms on salt agar increased the salt tolerance.

Weichel (1910) studied the effect of salt on the bacteria which are concerned in food poisoning. He found that by inoculating broth containing various amounts of salt with $B$. enteritidis, B. paratyphi " $B$," $B$. aertryke, that the cultures lived for between 33-95 days. Extending this study to meat inoculated before pickling, he found that inoculated meat showed a complete reduction in bacteria in 12 to 19 per cent of salt only after 75 days. Ptomaines formed by*bacteria were not destroyed by salting. In this connection Serkowski and Tomczak (1911) stated that 15 to 20 per cent of salt was required to be of prophylactic value with respect to bacteria which cause food poisoning.

Lewandowsky (1904) observed a differential action of certain salts. A 25 per cent solution of sodium chloride was about as efficient as a 30 per cent solution of potassium nitrate. The chlorides of potassium and sodium possessed about the same efficiency in solutions of equal molecular concentration.

Bitting (1909) in studying spoilage of tomato ketchup tried the effect of salts. Increasing amounts were put into 100 c.c. portions of bouillon which were later inoculated with molds and yeasts. A 5-gm. solution had no effect on molds. A 30 -gm. solution allowed no development of the mold. The yeast did not develop in a $15-\mathrm{gm}$. solution. These data are interesting in connection with the work of Wehmer and Pettersson (1900). Wehmer isolated a yeast from herring brine which was inhibited by 15 per cent of salt. Pettersson confirmed this during a study of fish brine.

Thom (1914) inoculated Czapek's solution containing differen' amounts of salt with different cultures of penicillia. The results are 
shown in Table XLIX. From this, as Thom has pointed out, it will be seen that the lower percentages of salt delayed growth but did not prevent it completely. At 15 per cent salt concentration the molds are inhibited.

TABLE XLIX

SHOWING THE EFFECTS OF DIFFERENT CONCENTRATIONS OF SALT ON THE DEVELOPMENT OF MOLDS

\begin{tabular}{|c|c|c|c|c|c|c|c|c|}
\hline & \multicolumn{2}{|c|}{ Check. } & \multicolumn{2}{|c|}{$\begin{array}{l}\text { With } 5 \text { Per } \\
\text { Cent Sodium } \\
\text { Chloride. }\end{array}$} & \multicolumn{2}{|c|}{$\begin{array}{l}\text { With } 10 \text { Per } \\
\text { Cent Sodium } \\
\text { Chlor!de. }\end{array}$} & \multicolumn{2}{|c|}{$\begin{array}{l}\text { With } 15 \text { Per } \\
\text { Cent Sodium } \\
\text { Chloride. }\end{array}$} \\
\hline & 7 days & 34 days & 7 days. & 34 days. & 7 days & 34 days & 7 days & 34 days. \\
\hline P. camemberti. & 0.8 & 1.0 & 0.7 & 1.0 & 03 & 0.6 & 0.2 & 0.8 \\
\hline P. italicum...... & 0.8 & 1.0 & 0.3 & $\ldots$ & 0.0 & 0.4 & 0.0 & 0.1 \\
\hline P. expansum.. & 0.9 & 7.0 & 0.8 & 1.0 & 0.5 & 0.8 & 0.1 & 0.4 \\
\hline P. roqueforti.. & 0.6 & 1.0 & 0.4 & 0.7 & 0.3 & 0.6 & 0.1 & \\
\hline P. commune. . & 0.8 & 1.0 & 0.7 & 1.0 & 0.1 & 0.7 & 0.1 & 0.8 \\
\hline P. chrysogenum. & 0.8 & 1.0 & 0.9 & 1.0 & 0.4 & 0.6 & 0.3 & 0.8 \\
\hline P. viridicatum. & 0.8 & 1.0 & 0.9 & 1.0 & 0.4 & 0.8 & & \\
\hline P. cit. glabrum. . & 0.9 & 1.0 & 0.8 & 1.0 & 0.3 & 0.6 & 0.2 & \\
\hline P. cyclopium.... & 0.8 & 1.0 & 0.6 & 1.0 & 0.4 & 1.0 & 0.2 & 0.9 \\
\hline P. solitum.... & 0.9 & 1.0 & 0.9 & 1.0 & 0.5 & 0.9 & 0.2 & 0.8 \\
\hline P. piscarium. & 0.9 & 1.0 & 0.9 & 1.0 & 0.4 & 1.0 & 0.2 & 0.9 \\
\hline P. lanosum. . & 0.7 & 1.0 & 0.7 & 1.0 & 0.3 & 0.6 & 0.2 & 0.8 \\
\hline
\end{tabular}

In recording observations 8 the normal colony on this medium as represented by the check culture at its typical stage of development is recorded as 10 . Less vigorous growths are recorded in tenths upon the observer's judgment. Germination of spores without further development of colony is recorded arbitrarily as 0.1. (After. Thom, 1914.)

Karaffa-Karbutt (1912) found salt to possess weak bactericidal power. Saprophytic bacteria required a higher concentration than pathogenic bacteria. Eight to 9 per cent inhibited the colon groups while the pyogenic bacteria required 10 to 12 per cent. Many yeasts grew in a 25 per cent solution. Concentrated salt solutions kill vegetative bacteria in two to three months but not spores.

The preparation of cod which has been described by Bitting (1911) furnishes a good illustration of the use of salt for preserving meat. He reports that 25,000 long tons of salt are used in a year at Gloucester, Mass., alone. As soon as the fish are caught, they are cleaned, which consists in removing the head, viscera and backlone. They are then piled up in the hold and sprinkled with salt. About $1.5 \mathrm{bu}$. of salt may be used per $100 \mathrm{lbs}$. of fish. The water is drawn out of the fish and this brine runs to the bottom of the hold from which it is pumped out. 
The fishermen often attempt to reduce the amount of water taken from the fish by using lesser amounts of salt so that the fish will weigh more in port. Souring may result. After the fish are on shore they may be further dried by means of salt. The amount of salt taken up by fish is given in the following table from Bitting.

AMIOUNT OF SALT TAKEN UP BY FISH PUT UP IN DIFFERENT FORMS (After Bitting, 1911)

\begin{tabular}{|c|c|c|}
\hline & Salt, Per Cent. & Moisture, Per Cent \\
\hline Fish cakes.. . & 18.9 & 47.6 \\
\hline Slack salted.. . & 145 & 37.2 \\
\hline Export....... & 19.6 & 25.6 \\
\hline 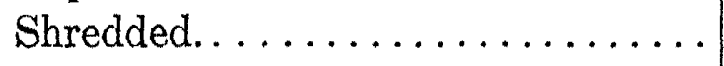 & 20.6 & 46.2 \\
\hline
\end{tabular}

Reddening of Cod Fish. Bitting (1911) has discussed the bibliography of this subject. His own work on this question yielded a coccus and bacillus as the possible causes. He has reported the complete cultural characters of these organisms. Indications point to the infection of the fish during its preparation. Undoubtedly the organism is able to resist the action of salt.

Pickling. This might be discussed under another heading. Pickles are usually combinations of sugar, salt and often molasses. They are usually used in such combinations and concentrations that they inhibit bacterial life but do not blot it out entirely. Salt acts as a dehydrating agent. One result of this is a hardening of the tissue. Sugar does not do this, and is, therefore, often used with salt. It imparts a sweet flavor to the meat. Hoagland (1908) regards the action of saltpeter in influencing the color of meat as due to the formation of no hemoglobin. Saltpeter is reduced within the meat to nitrites which, in turn, are changed to nitric oxide. This unites with the hemoglobin to give the characteristic red color. Saltpeter, which is used in many pickling solutions, probably exerts little effect on bacteria.

Sugar. Carbohydrates lack some of the preserving action of the șalts. Bitting (1909) also tried the effect of sugar in preserving tomato bouillon. No effect of sugar was noticed until the amount of sugar had reached 25 gms. per 100 c.c. of bouillon. With the molds studied, sirups were prepared up to 200 gms. of sugar per 100 c.c. of tomato bouillon. The growth became slower up to $170 \mathrm{gms}$. Above $170 \mathrm{gms}$. it required two months to get growth. Yeasts were not able to survive $80 \mathrm{gm}$. solutions of sugar. 
Drying. All forms of life demand moisture. Especially is this the case with the lower forms whose body structures are made up of about 90 per cent'water. Nature uses this method for preserving the cereals against attack of microorganisms. Crackers, if kept dry, may be preserved indefinitely. Varıous meats are not susceptible to the attack of bacteria in the dry condition.

According to Rahn's classification the following groups may be preserved by drying:

Group I. Protein foods.

Group II. Carbohydrate foods.

Group III. Proteins + carbohydrates.

Group VII. Acid + protein + carbohydrates.

Some of the food products coming under these groups do not lend themselves conveniently to evaporation or drying. Beattie and Gould (1917) gave an excellent treatise on the methods and apparatus used in the commercial application of drying to food preservation.

Cold Storage. All microorganisms are very susceptible to changes in temperature. The great majority have their range of optimum temperature between $20^{\circ} \mathrm{C}$. and $40^{\circ} \mathrm{C}$. In any discussion of cold storage the psychrophilic bacteria are concerned. About all that may be said with regard to freezing is that it inhibits or delays bacterial development and exerts a selective action. Much data has been recorded in the literature to prove this. Pictet and Joung (1884) soon after pure cultures were made possible, subjected many bacteria to low temperatures $\left(-130^{\circ} \mathrm{C}.\right)$. Koch $(1884)$ demonstrated that the cholera vibrio could resist $-10^{\circ} \mathrm{C}$. for ten hours. MacFayden (1900), using liquid air temperatures of $-190^{\circ} \mathrm{C}$., found that bacteria were resistant. Paul and Prall (1907) used the garnet method to study the effects of low temperatures on bacteria and the rate of death. For 100 days the number of bacteria remained constant at liquid air temperatures. Those which were kept at room temperature and icebox temperature decreased rapidly. Sedgwick, Hamilton and Funk (1917) have shown that some bacteria remain alive longer in solutions at low temperatures which, at higher temperatures, are decidedly toxic. They assume "that the mechanical protection given bacteria from shearing ice crystals by solutes is important." Ruata (1918) has secured results in this connection which indicate that cold if applied long enough will destroy bacteria. He worked with dry cold at temperatures of $-4^{\circ} \mathrm{C}$. and $-12^{\circ} \mathrm{C}$. Tubes were withdrawn from the freezing environment and incubated on successive days. On the fourth day the number of colonies which developed had decreased to from 3 to 49 while, on the second 
day, thousands of colonies had appeared. After the fourth day nono developed. This author believes that other investigators on this subject have not allowed a sufficiently long contact with the cold. It is stated that even one cell on the interior could start a culture.

Some difference is made with regard to whether the food is kept above or below freezing. Below freezing, growth and multiplication are probably impossible on account of lack of moisture. Above freezing, the moisture is available and multiplication takes place even though it is very slow. Just above freezing, it is possible for the putrefaction bacteria to grow. These may cause the formation of toxic substances.

Cold storage is relied upon to save many foods. Some of these are produced in greater abundance in one season or are eaten many miles from the source of production. Refrigeration has made this possible. Mutton preserved by cold storage during shipment from Australia is now eaten in England. Beef from Argentina is shipped to many parts of the world. Burrows (1914) described a quarter of beef which had been kept frozen for eighteen years. Microscopic examination of the fibers showed a normal appearance. The meat was consumed with no signs of digestive disturbance. Burrows stated that the meat had been kept in a chamber in and out of which other beef was passing and regarded this as a factor in the preservation of eighteen-year beef.

The bacteriological features concerned with cold storage have been studied by the Bureau of Chemistry of the U. S. Department of Agriculture. Milk stored at freezing temperatures was found to contain many millions of bacteria at the end of a month. The chemical characteristics of the milk also underwent a detectable change. (Pennington, 1908).

Wiley (1908) and his colleagues have secured much data with regard to the cold storage of eggs. They found that the flavor of the eggs began to deteriorate quickly. Certain constituents during continued cold storage were found to develop rosette crystals in the yolks. Eggs which were stored for a year decreased about 10 per cent in weight. With regard to poultry, these investigators found that undrawn poultry kept better than the drawn. It was stated that cleaning of the fowl should be done carefully or there was danger of serious infection of the muscle tissue.

Pennington has studied the changes which take place in poultry during cold storage. Visible changes were found. The cold-stored poultry were found to contain appreciable numbers of bacteria in the tissue while the tissues of fresh fowls were sterile. In a paper published in 1917, Pennington reports the results of chemical, bacteriological 
and histological studies made on poultry stored between temperatures of -9 and $-13^{\circ} \mathrm{C}$. below freezing. These birds were frozen hard. There was no loss in nutritive value but a change in flavor was detected after nine months. Chemical changes which were observed were thought to be due to enzymic rather than bacterial causes. The aerobic bacteria decreased in the muscle during storage as the period lengthened.

Above freezing, different lots were stored at $29.3^{\circ} \mathrm{C}$. at from 7.2 to $12.8^{\circ} \mathrm{C}$. and at $0^{\circ} \mathrm{C}$. Bacteria in muscles and skin increased at all three temperatures but the increase was smaller as the temperature approached $0^{\circ} \mathrm{C}$. The amino and basic nitrogen increased at the expense of protein nitrogen.

Pressure. Hite, Giddings and Weakley (1914) studied the effect of pressure on bacteria in canned fruits and vegetables. The bacteria which caused spoilage of sweet, ripe fruits could be destroyed by pressure. One hundred thousand pounds for ten minutes stopped the fermentation of grape juice. Apple juice which was subjected to 60,000 to $80,000 \mathrm{lbs}$. for thirty minutes remained sweet. These authors kept apple juice which had been subjected to 90,000 to $120,000 \mathrm{lbs}$. for five years. Inconsistent results, however, were secured with blackberries and raspberries. Tomatoes also gave such results. The work indicates that more study is necessary to overcome some of the difficulties of the process in its application to food preservation. Bridgman (1914) found that egg white became somewhat stiffened when it was exposed to $75,000 \mathrm{lbs}$. per square inch for thirty minutes at $20^{\circ} \mathrm{C}$. Larson, Hartzell and Diehl (1918) found that a direct pressure of 6000 atmospheres would kill non-spore forming bacteria when applied for fourteen hours. It required 12,000 atmospheres for the same length of time to kill the spore formers. This has much significance in the sterilization of foods by this method since the spore formers cause much of the trouble in spoilage. In an atmosphere of $\mathrm{CO}_{2} 50$ atmospheres for 1.5 hours killed non-spore formers.

Canning. Appert in 1802 first showed that foods could be preserved if they were sterilized and kept in hermetically tight containers. This discovery stimulated the use of the process and it spread rapidly to various parts of the world. Bitting (1917) has shown the extent of the canning industry. For the year 1914, 103,765,923 cases of canned food products were put up. These were valued at $\$ 258,798,036$. He states that the United States is not only the largest producer but also the largest consumer of canned foods. The future of this industry seems very bright and each day sees a new type of food preserved by this method. 
Process of Canning. The following pages on this topic are taken from the various publications of Bitting (1917, 1915, 1912):

Raw Materials. These must be of first-class quality in order to secure a high-grade canned product. The canning factory should be located near the point of production in order to have the produce delivered in fresh and unharmed condition. Corn and peas lose their fresh appearance soor after harvesting. Beans become tough and stringy. The same may be said with regard to the canned fish industry. A recent type of fish cannery is constructed on a boat which is floated to the fishing grounds.

Preparation. The procedure in this case depends solely on the product for obviously, the operations will depend on the food to be canned. As the produce is brought to the cannery it is roughly graded according to size, quality or maturity. These are handled separately. Some fruits need no more preparation than picking out foreign matter and effective material. Others need peeling, pitting, coring and sizing. Certain vegetables, on the other hand, require more preparation than the fruits. Peas must be threshed from the vines, corn cut from the cob, and string beans snipped and stringed. Most of this work is done by machinery.

Grading. This is done to secure a uniformity of the product. Bitting regards this as having. been carried to excess when it is realized that there are ten or twelve grades of peas, fifteen to eighteen grades of peaches and ten grades of cherries. Grading for size is purely mechanical. The produce, when possible, is sifted through perforated cylinders, screens, vibrating rolls, etc.

Washing. This is a thorough process in industrial canning. The packing of peas requires nearly 1 gal. of water for each can.

Blanching. This is carried out for several reasons, depending upon the product. The object is not to whiten as the term would indicate. It may be to remove the sticky or gelatinous coating from beans, peas, etc., or to make more flexible and of uniform color as with cherries.

Filling the Cans. Whether this is done by hand or machinery depends upon the product. Material like peas, squash, corn, beans, and hominy are put into the cans by machinery while such fruits as pears, peaches, must be put into the cans by hand in order to secure uniform filling of the cans.

Siruping and Brining. Most canned foods have a sirup or brine added. The lowest grade products are packed in water. This brine may be either salt, sugar or a mixture of both depending upon the product. 


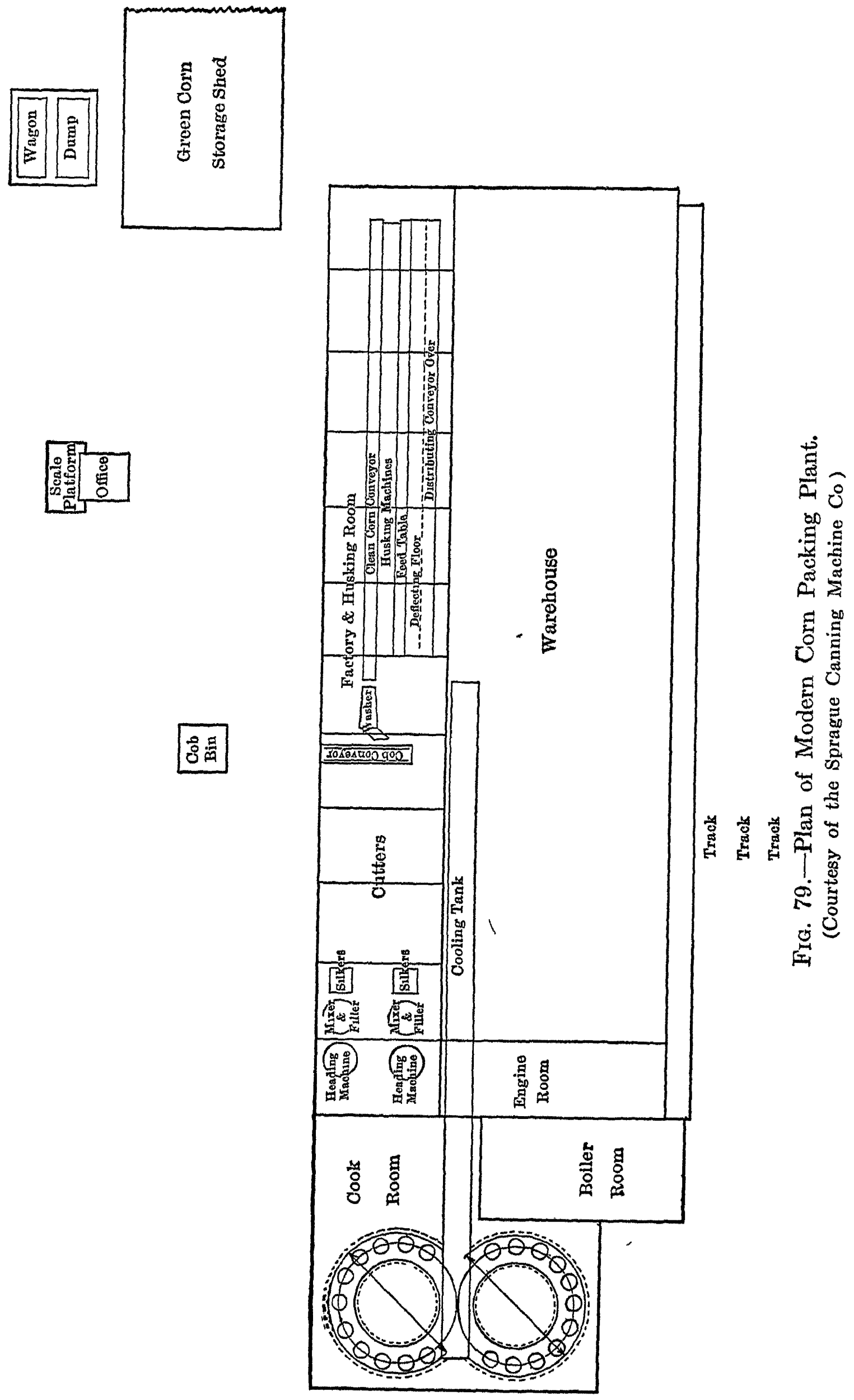


Exhausting. The can is heated until hot in order to drive out as much air as possible. This is not done with produce which is canned hot or which receives a hot brine.

Closing the Cans. This is now done mostly by machinery whether the cans are soldered or crimped.

Processing. This is the final treatment given the cans and is done soon after the cans are capped. This may be done either below or above the boiling-point. The desired temperature is maintained either by hot water or an atmosphere of steam. After the "process" the cans must be cooled else the products will be over cooked. This is probably one of the most important steps in the canning procedure. Much loss is often caused by errors at this point. It is often necessary to make the "process" a subject of careful study because the length must often vary even from day to day during the packing of the same goods.

Furthermore, it is known that the process is dependent upon the character of the contents of the cans. Acid products like strawberries require a lower process than a product like corn or beans. The thermal death point of microorganisms is lowered by the presence of acid. Bitting and Bitting (1917) have studied the heat penetration of cans and found a great variation with the character of the food. The temperature of a No. 3 can filled with water raised from $85^{\circ} \mathrm{F}$. to $210^{\circ} \mathrm{F}$. in four minutes. Sweet potatoes gave different results and were decidedly more sluggish in their change of temperature.

The Container. In commerce tin cans are almost exclusively used although paper cans have a promising outlook. Many shapes and varieties are now used. Bitting makes the following statement with regard to this:

First, the cans were made to utilize a standard sheet of metal with the minimum of waste by the method of can making in vogue at the time. This resulted in cans of arbitrary volume, bearing no definite relation to standards of volume, like the pint, quart or gallon and unfortunately they did not bear a close relationship to the quantity that would be consumed at a single meal by an average family. A size of package was started, however, which has persisted because of a fixture in trade and the expense in changing machinery, cans, shipping cases, etc. Secondly, was the introduction of sizes to fit a given weight of a certain product. This has been particularly true of meat products, as $4,8,12$, and 16-ounce cans. The sizes that will hold these weights of a ground meat will not hold the same quantity if cereal be added or if large cuts or pieces be used in place of the finely ground product. Thirdly, is the attempt to make cans that will hold a quantity of a given article to retail at a popular price, like 
10 cents. This applies to soup, beans, etc. Table $\mathrm{L}$ gives the sizes and volumes of the standard cans.

TABLE L

SHOWING THE SIZES AND CAPACITIES OF STANDARD CANS

(After Bittıng)

\begin{tabular}{c|c|c|c}
\hline Number & Diameter in Inches & Height in Inches & Capacity in Ounces \\
\hline 1 & $2 \frac{11}{16}$ & 4 & 12 \\
2 & $3 \frac{7}{16}$ & $4 \frac{9}{16}$ & 222 \\
$2 \frac{1}{3}$ & $4 \frac{1}{16}$ & $4 \frac{3}{4}$ & 326 \\
3 & $4 \frac{1}{4}$ & $44_{8}^{7}$ & 364 \\
10 & $6 \frac{3}{16}$ & 7 & 1161 \\
\hline
\end{tabular}

Under domestic conditions, glass cans have been much used. Great care, however, has to be used to secure a tight seal, which is often rendered difficult on account of poor rubber gaskets.

Swells. According to Bigelow (1914) a "swell" is a can which has undergone decomposition by microorganisms accompanied by generation of gas, which first releases the vacuum and then causes pressure in the can. This decomposition is often of putrefactive nature and may be rapid or slow, according to the organisms and temperature. They are caused by different factors. Insufficient sterilization or leaky cans are evident causes. One leaky can may infect other cans in a similar condition.

Baker (1912) has given some attention to the composition of gases occurring in commercial canned foods. Some of the analyses given below are taken from his paper:

Can of red raspberries, eighteen months old:

Carbon dioxide..................................

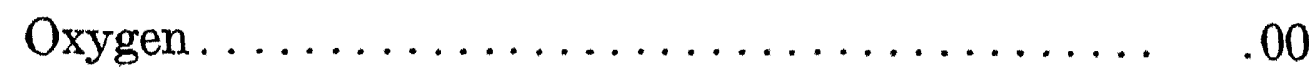

Hydrogen...................... 6550

Nitrogen .................... 26.10

Can of strawberries, eighteen months old:

Per Cent.

Carbon dioxide................... 12.60

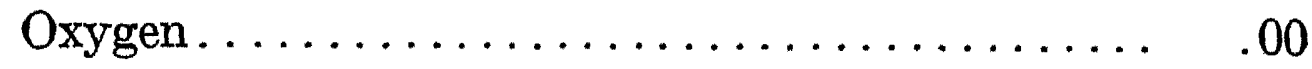

Hydrogen ........................ 72.40

Nitrogen..................... 15.00 
Baker states that the analyses of probably 100 samples of gas from sound cans has never shown any oxygen. He attributes this to the oxidation of tin and iron salts, and combination with nascent hydrogen.

Springers. Bigelow (1914) defines a springer as a " can whose ends are more or less bulged, owing to pressure from hydrogen generated as a result of the chemical action of the contents on the metal of the container or because the can was overfilled or insufficiently exhausted." Cans with bulged ends may be caused by expansion due to a rise in temperature. These will soon return to normal shape when the temperature is lowered. Baker (1912) states that the gases in the head spaces of springers are never more than three; carbon dioxide, nitrogen and hydrogen. Often hydrogen is absent and oxygen is rarely found.

Flat Sour. This is a general term and may be used to cover many abnormalities of canned goods. Flat sours contain no gas and since there is nothing to indicate any abnormal condition when looking at a can, their discovery is delayed until the can is opened.

\section{Examination of Canned Foods}

The examination of canned foods is accompanied with danger unless the examiner has had experience in interpreting his data, both chemical and bacteriological. Bigelow (1917) has pointed out this side of the question very strikingly. The methods which are used are quite similar to those used in other branches of bacteriology. The objects of the examination may be varied. Quite often it is to determine the sterility of certain foods in order to check up the process of manufacture; or the yuality of raw materials may be the object. The examination of canned foods presents the same difficulty that Pennington has mentioned with regard to the examination of eggs: the examination of one can or one egg may not yield information with regard to other cans or eggs. Each must be regarded as a sealed package and when once opened may be brought back to the original condition with a little difficulty.

The bacteriological examination of canned foods has been fully discussed by Bitting and Bitting (1917). Some of the discussion below hàs been taken from that paper.

The first step in the examination is to observe the appearance of the can. Rusty cans suggest the possibility of perforations. The cans should be without dents and with slightly concave or flat ends. In certain cases bulged ends may be due to springers, which will go down 
a little after the can is cooled. Bitting points out that incubation at $37^{\circ} \mathrm{C}$. and at from 50 to $55^{\circ} \mathrm{C}$. for fifteen days should precede the bacterial examination.

The second step, is the opening of the can, which must be carried out under very carefully controlled conditions. The can should be thoroughly sterilized about the place for opening either by flaming or covering with alcohol and allowing it to burn off. The can may be opened by cutting around the top with a sterile can opener or a hole put through the top by a sterile awl. The contents for examination may then be removed by means of a sterile loop or a sterile pipette. When the hole is punched into the can a flame should be played on the spot so that no bacteria will be sucked into the can with the air. The can should be entirely opened, later as soon as possible, in order to determine the appearance of the contents.

The third step, is concerned with the actual microscopical examination. This should be direct as well as cultural. The direct methods of examination have been sufficiently discussed elsewhere in this book. The cultural methods require special media such as corn, agar, tomato bouillon, etc., depending upon the substances which are being examined. Such media create for the organisms which are searched for, an environment as much as possible like the normal. Both aerobic and anaerobic methods of culture should be used. It is often advisable to incubate the cultures in a vacuum, to come more nearly to the conditions inside of the can.

Organisms in Canned Foods. These have not received very extended study. Much of our knowledge must come from analogy and there is some danger in reasoning thus. In relatively few instances has time been taken to identify the various varieties of microorganisms. Burgess (1912) isolated and identified about 12 organisms from "flat sour" corn. Among others, he found B. coli, B. acidi lactici, $P$ s. syncyanea, $B$. megatherium and B. vulgatus. The first work on the bacteriology of canned foods in this country was done by Russell (1895). He examined swelled canned peas. He isolated two organisms, one of which was a copious gas producer. Prescott (and Underwood, 1897, 1898, 1900 ), gave this subject quite a little attention. They did much to establish the close relationship of bacteria to the spoilage of canned foods. The importance of the canned food industry should stimulate the examination of these foods.

The author has found spore-forming facultative anaerobes to be common. In a few cases vegetative cells have been isolated. In one case mushrooms packed in brine of the following composition-5 lbs. 
salt, $1 \mathrm{lb}$. citric acid, $12 \frac{1}{2}$ gals. of water, developed swelled cans. Bacteria with the following group numbers were isolated.

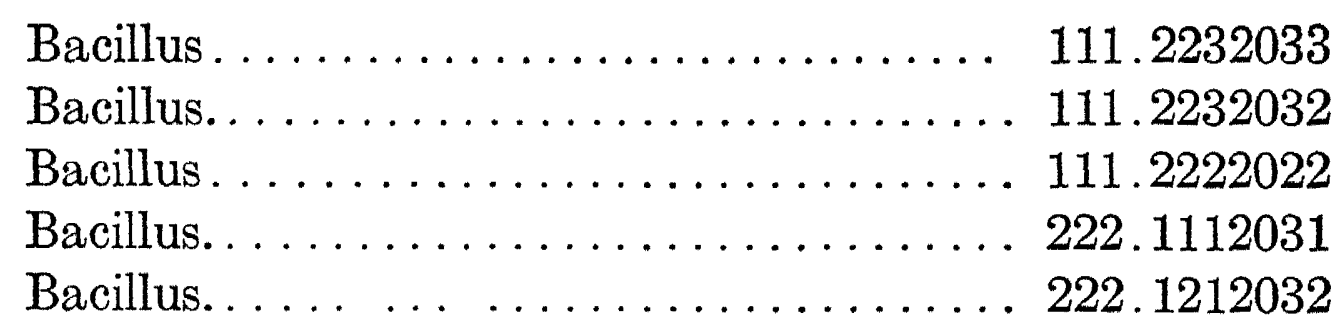

The growth of such organisms in the finished products probably indicates insufficient sterilization. The gas-forming bacteria as indicated by the above group numbers are members of the colon group. These, undoubtedly, got onto the mushrooms during growth, since they are usually grown on heavily manured areas.

Examination of Cans for Leaks. Several ingenious methods have been proposed.

1. One which is widely used is to tap the end of the can. A sound can with a vacuum will give a characteristic ring while one which is a leaker with no vacuum will give a dead sound. The examination of a few cans of both types will show the difference.

2. The suspected cans may also be examined for leaks by plunging them into hot water. If leaks are present, bubbles will be given off.

3. The Meade tester may be used. This subjects the suspected can under water to a vacuum. If the can is a leaker, a stream of bubbles will be given off from it.

Storage of Food in Tin Cans. It has become almost an instinct for people to immediately take food out of a tin can after it has been opened. When the housewife is asked why she does this, various reasons are given. One which has often been given is that "ptomaine" poisoning will result. Bigelow (1918) has given some definite information on this subject. He regards the storage of food in the tin cans after part has been removed as no more dangerous than storage in containers made of other material, since it is becoming more and more known that food "poisoning" is really an infection. Bigelow determined the tin content in progressive samples from the same tin of food and it is interesting to note that after fermentation had set in (pineapple) larger amounts of tin were dissolved. Bigelow considers that the only reasons which make it advisable to remove food from opened cans are appearance and economy of space secured by putting the food into a smaller container. 


\section{Tomato Products}

The canning of tomato products is often beset with many pitfalls for the canner. The chemical constitution of the tomato renders it liable to attack from microorganisms. The following table is prepared from some data reported by Bigelow and Fitzgerald (1914).

TABLE LI

COMPOSITION OF TOMATO PULP

(Bigelow and Fitzgerald)

\begin{tabular}{|c|c|c|c|c|c|c|c|}
\hline \multirow{2}{*}{$\begin{array}{l}\text { Sample } \\
\text { No. }\end{array}$} & \multirow{2}{*}{ Description of Sample. } & \multicolumn{2}{|c|}{ Specific Gravity. } & \multirow{2}{*}{$\begin{array}{c}\text { Total } \\
\text { Solids. } \\
\text { Per Cent. }\end{array}$} & \multirow{2}{*}{$\begin{array}{c}\text { Salt Free } \\
\text { Solids. } \\
\text { Per Cent. }\end{array}$} & \multirow{2}{*}{$\begin{array}{c}\text { Insoluble } \\
\text { Solids } \\
\text { Per Cent }\end{array}$} & \multirow{2}{*}{$\begin{array}{l}\text { Salt Free } \\
\text { Soluble } \\
\text { Solids. } \\
\text { Per Cent. }\end{array}$} \\
\hline & & $\frac{68^{\circ} \mathrm{F}}{68}$ & $\frac{203^{\circ} \mathrm{F}}{203}$ & & & & \\
\hline 893 & $\begin{array}{l}\text { Peeled tomatoes not } \\
\text { concentrated........ }\end{array}$ & & & 4.47 & 4.42 & 0.56 & 3.86 \\
\hline 701 & $\begin{array}{c}\text { Trimming stock not } \\
\text { concentrated........ }\end{array}$ & & & 4.53 & 4.43 & 0.50 & 3.90 \\
\hline 1048 & Whole tomatoes.. & & . & 5.57 & 5.53 & 1.12 & 4.41 \\
\hline 1051 & Whole tomatoes.. & & & 6.55 & 6.43 & 0.75 & 5.68 \\
\hline $\begin{array}{c}\text { Sample } \\
\text { No. }\end{array}$ & Description of Sample. & $\begin{array}{c}\text { Sugar. } \\
\text { Per Cent. }\end{array}$ & $\left\{\begin{array}{c}\text { Acid as } \\
\text { Citric. } \\
\text { Per Cent. }\end{array}\right.$ & $\begin{array}{c}\text { Salt. } \\
\text { Per Cent. }\end{array}$ & $\begin{array}{l}\text { Undeter- } \\
\text { mined. } \\
\text { Per Cent }\end{array}$ & $\begin{array}{c}\text { Salt Per } \\
\text { Cent of } \\
\text { Total } \\
\text { Solids. }\end{array}$ & $\begin{array}{l}\text { Insoluble } \\
\text { Solids. } \\
\text { Per Cent } \\
\text { of Total } \\
\text { Solids. }\end{array}$ \\
\hline 893 & $\begin{array}{l}\text { Peeled tomatoes not } \\
\text { concentrated......... }\end{array}$ & 2.26 & 0.40 & 0.05 & 1.20 & 1.2 & 12.50 \\
\hline 701 & $\begin{array}{l}\text { Trimming stock not } \\
\text { concentrated........ }\end{array}$ & 2.36 & 0.30 & 0.10 & 1.04 & 2.4 & 11.00 \\
\hline 1048 & Whole tomatoes....... & 3.55 & 0.35 & 0.04 & 0.51 & 0.7 & 20.10 \\
\hline 1051 & Whole tomatoes....... & 3.96 & 0.37 & 0.12 & 1.35 & 1.80 & 11.50 \\
\hline
\end{tabular}

Ketchup. This is one of the most important tomato products and one which has received much attention with regard to the enforcement of the Food and Drugs Act. It is made in the following way: Clean, ripe tomatoes are thoroughly washed and pulped. The skins, seeds, etc., are removed after which the pulp is concentrated. To this are then added the condiments to taste. The ketchup is then sieved and bottled. After the bottles are sealed, they may be processed to insure sterility.

Much trouble has been experienced in the control of this industry from the standpoint of the Food and Drugs Act. Undoubtedly, in 


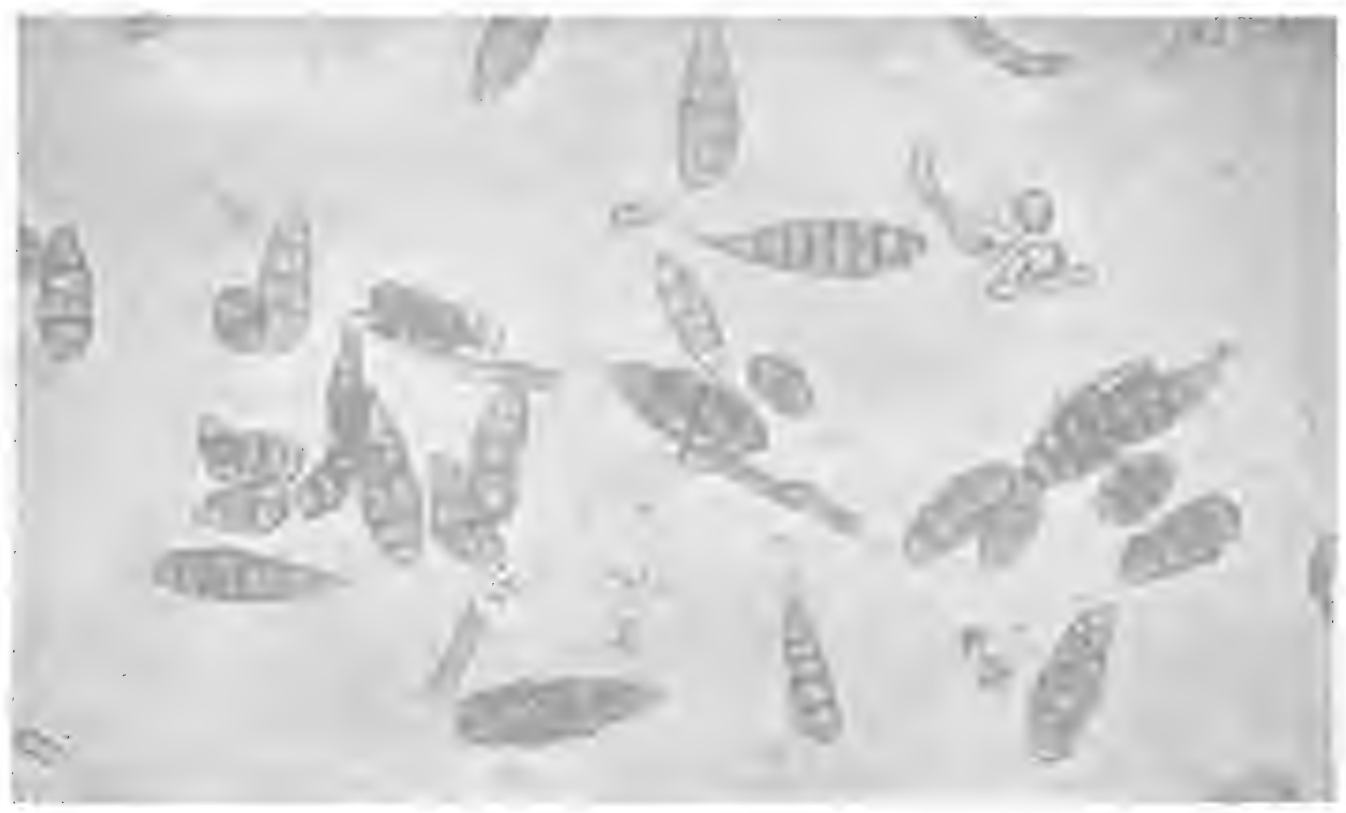

FIG. 1.- Spores and Fragments of Filaments of Mold from Decaying Sweet Pepper $(\times 150)$

The same or an allied species is one cause of "dry rot" in tomatoes.

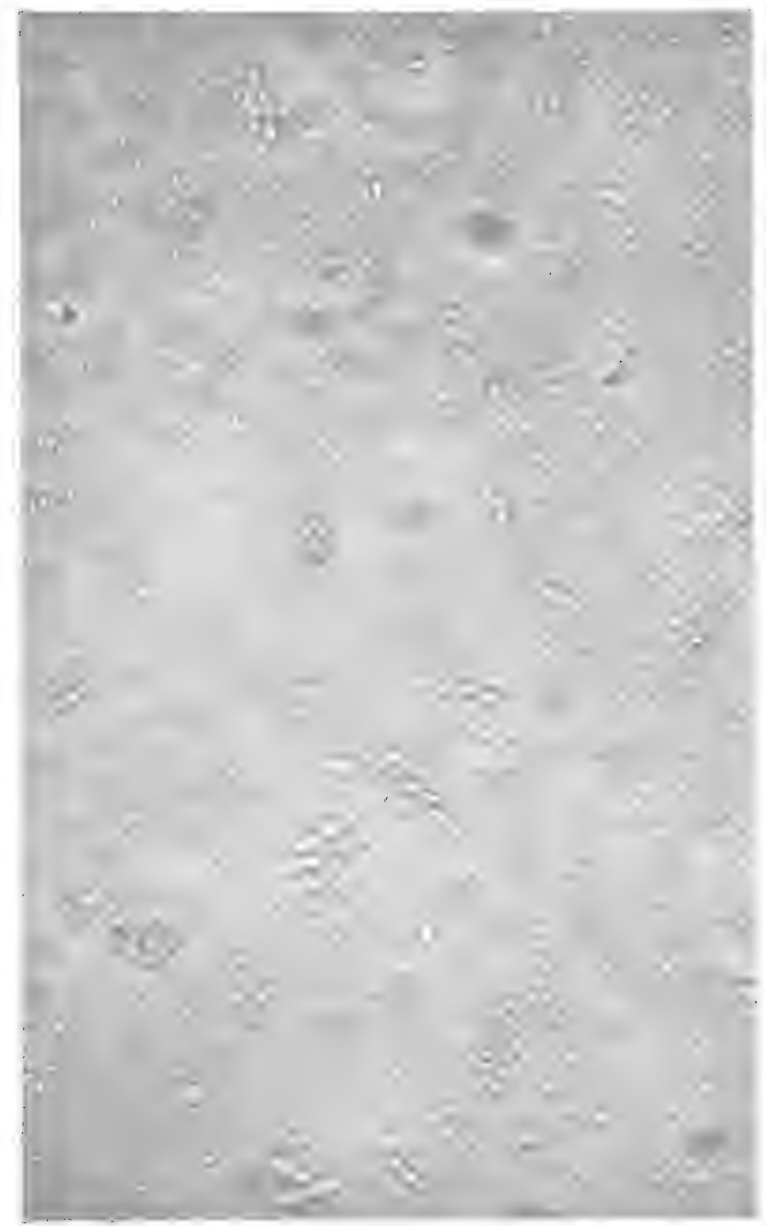

IIG. 2.-Yeasts and Spherical Bacteria frorn Decaying Tomatoes $(\times 500)$.

The oval bodies are the yeasts, some in budding stage; the bacteria appear as small spheres, or pairs of spheres.

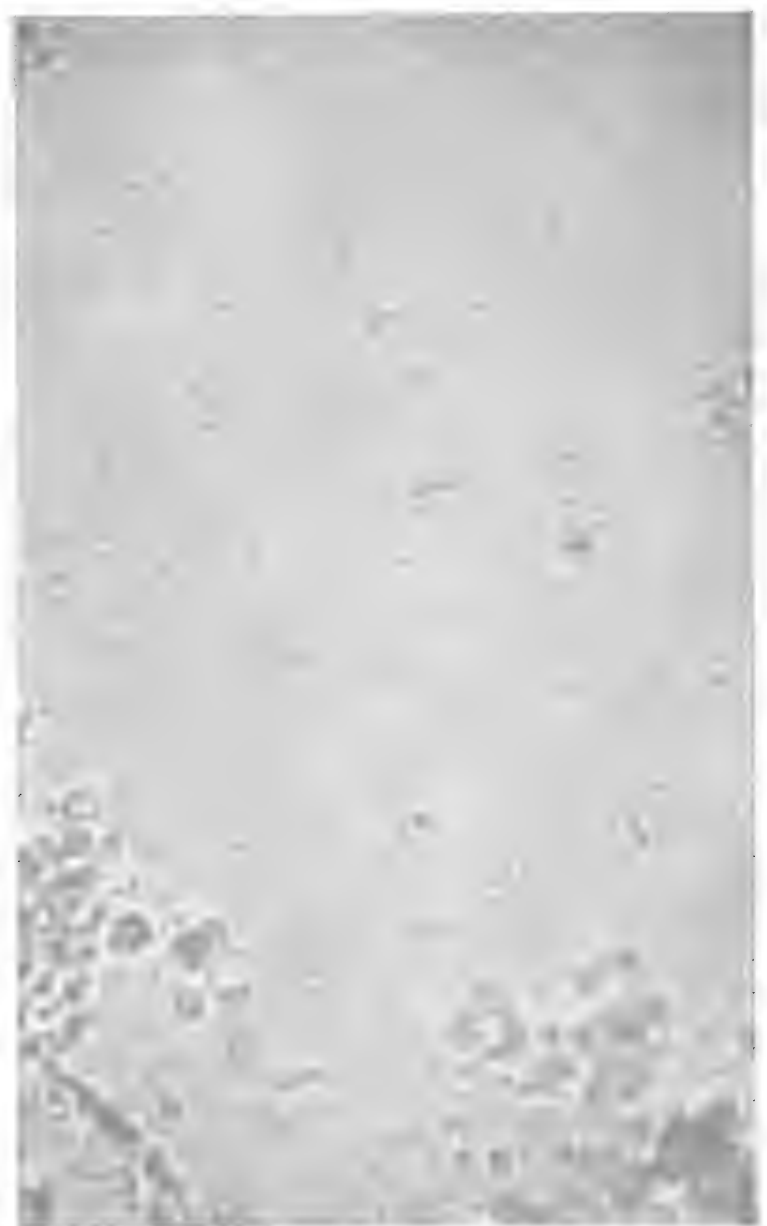

FIG. 3.-Rod-shaped Bacteria from Tomato Pulp, Common in Bad Ketchups $(\times 500)$.

To face page $\mathbf{5 1 5 .}$ 
former times, many unscrupulous manufacturers used spoiled fruit, and by-products from the tomato canning industry for making ketchup Satisfactory methods for separating good and bad ketchups have been hard to find. In general, a bad or low-grade ketchup is one which has been made from decayed or partly decayed fruit. The methods which are now used are microscopical and center around determining the numbers of bacteria, yeasts and molds. Bitting (1909) used superficial methods for studying spollage of tomato ketchup. He found that ketchup prepared from whole ripe stock had a low content of microorganisms and in that prepared from decayed fruit large numbeis of microorganisms were present.

Howard (1911) proposed a method for the examination of ketchup which has been in use since then probably becausc no one has proposed a better one to replace it. This method is based on cxamming the tomato product for yeasts, molds and bacteria, and it is thus assumed that these microorganisms indicate that decayed fruit was used or that the method of manufacture is faulty. This latter possibility was found to be the case in an instance quoted by Howard (1917). A manufacturer was using ripe fresh tomatoes and still secured high counts in the final product.

\section{Howard's Method for the Microanalysis of Tomato Products AND INTERPRETATION OF the Results}

Apparatus Required. The outfit used is as follows:

A good compound microscope giving magnifications of approximately 90, 180, and 500 diameters. This is accomplished by the use of a $16 \mathrm{~mm}$. (two-thirds of an inch) objective and an $8 \mathrm{~mm}$. (one-third of an inch) objective, together with a medium ( $\times 6$ compensating) and also a high-power ocular ( $\times 18$ compensating). A Thoma-Zeiss bloodcounting cell,* a 50-c.c. graduated cylınder, and ordınary slides and cover glasses complete the apparatus required. It is impractical to use objectives of a higher power than those mentioned, because of their short working distance, which makes their use with the counting cell inpossible.

Estimation of Molds. A drop of the product to be examined is placed on a microscope slide and a cover glass is placed over it and

* This is a cell named after the designer of the form of rulings used, and consists of a slide with a disk ruled in $\frac{1}{20} \mathrm{~mm}$ squares, so arranged that when the cover is in place the film of liquid under examination is $\frac{1}{10} \mathrm{~mm}$ deep They were originally intended for counting corpuscles in the blood and are obtainable from practically all manufacturers of microscopic accessories. 
pressed down till a film of the product about $0.1 \mathrm{~mm}$. thick is obtained. After some experience this can be done fanly well. A film much thicker than this is too dense to be examined successfully, while a much thinner film necessitates pressing the liquids out, which gives a very unevenappearıng preparation. When a satisfactory mount has been obtained, it is placed under the microscope and examined. The power used is about 90 diameters, and such that the area of substance actually examined in each field of view is approximately $1.5 \mathrm{sq} . \mathrm{mm}$.

A ficld is cxamined for the presence or absence of mold filaments, the result noted, and the slide moved so as to bring an entirely new field into view. This is repeated till approximately 50 fields have been examined, and the percentage of fields showing molds present is then calculated. Our experience has demonstrated that for homemade ketchups this is practically zero, and with some manufactured ketchup it is as low as from 2 to 5 per cent, whlle for carelessly made products it may be 100 per cent; that is, every field would show the presence of mold. Investigations under factory conditions clearly indicate that with only reasonable care the proportion of ficlds having molds can be kept below 25 per cent. A specimen in which 60 per cent of the fields have molds is in more than twice as bad a condition as one containing 30 per cent.

After the percentage reaches 30 to 40 per cent it will be found that some of the fields frequently have more than one filament or clump of mold, and the number of such fragments might be counted, but in this laboratory this usually is not done. A Thoma-Zeiss counting cell with a center disk of 0.75 in. instead of 0.25 in., as usually furnished, would give a regular depth of liquid and would be more exact than the method described, but this must be specially manufactured, not being listed in any of the catalogues of microscopic supplies, and the method as given is sufficiently accurate for the purpose. When the number of fragments of mold per cubic centimeter is estimated, it has been found to range from virtually zero to over 20,000. There is no excuse for a manufacturer allowing such conditions to prevail that his ketchup shows more than 2000 per cubic centimeter, while some manufacturers by careful handling hold it down to 150.

Estimation of Yeasts and Spores. Though the spores referred to are those coming from molds and correspond to seeds in more highly developed plants, it is frequently very difficult to differentiate some of them with certainty from some yeasts without making cultures, which is obviously impossible in a product that has been sterilized by heat. For this reason the yeasts and spores have been reported together, and 


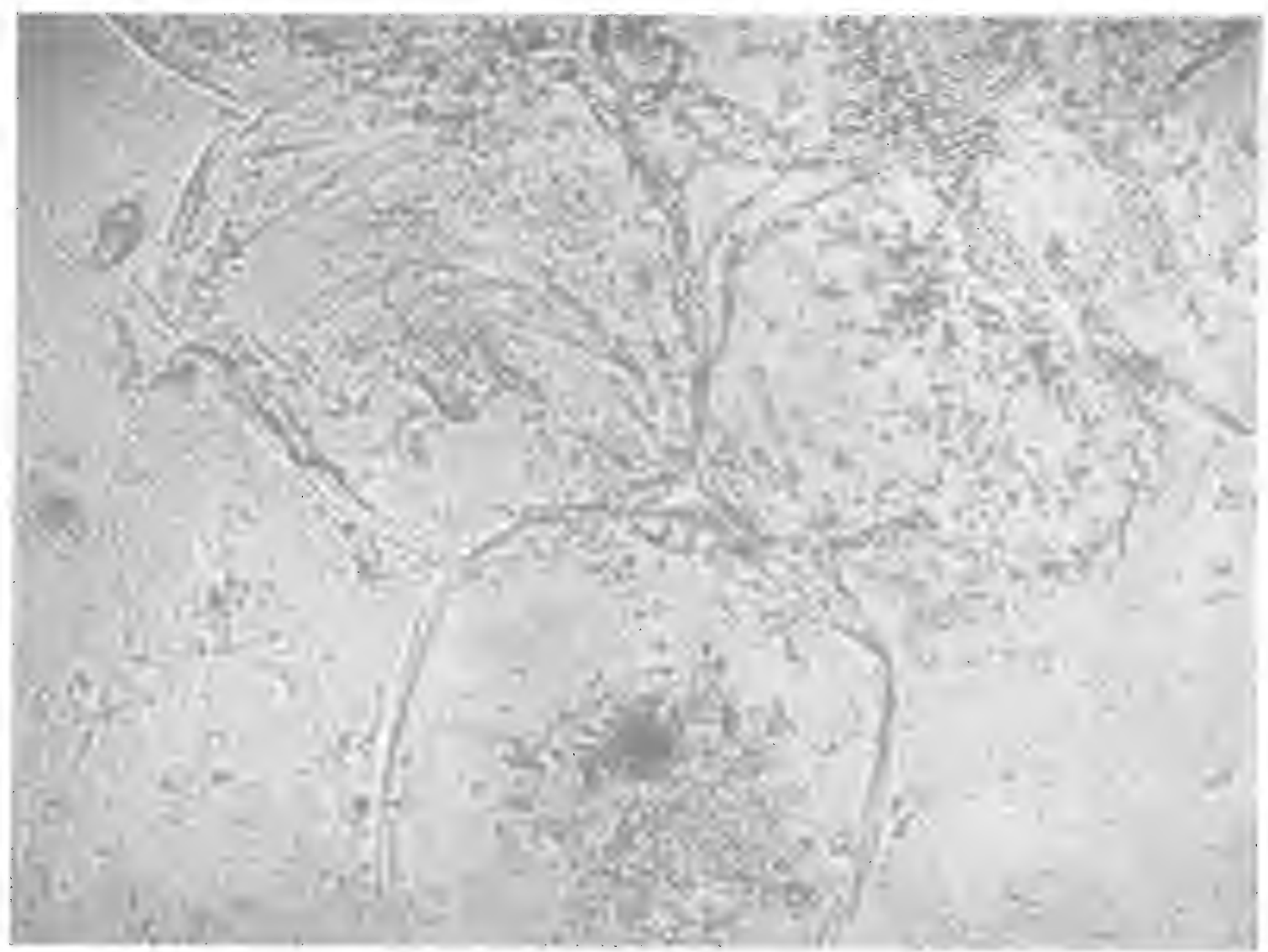

FrG. 1.-Normal Tomato Ketchup, Showing Cells and Granular Contents $(\times 200)$.

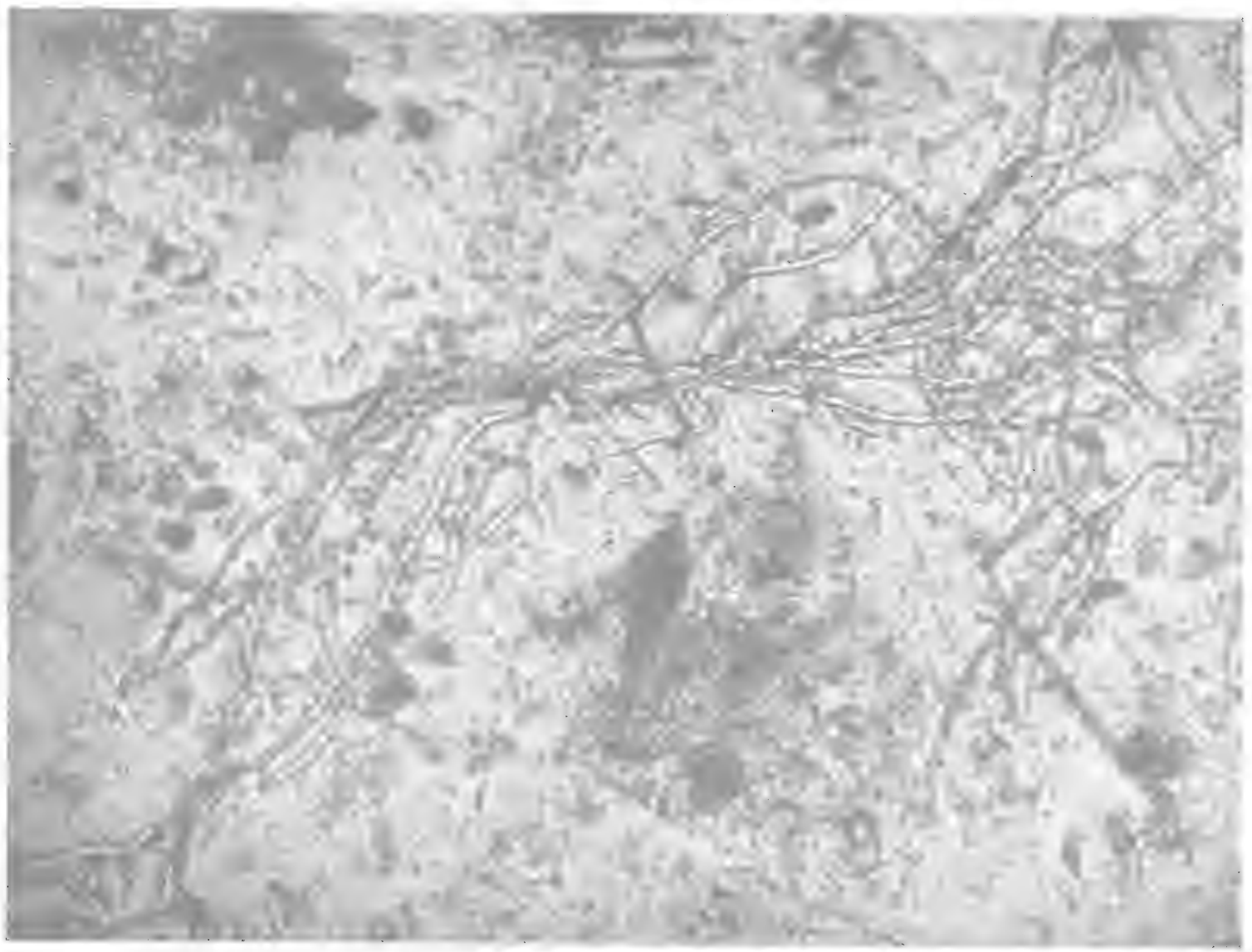

FIG. 2.-Mold Filament from Ketchup Made "rom Partially Decayed Stock $(\times 150)$. 
if there seemed to be a larger percentage of the latter, mention was made of that fact.

To make a count 10 c.c. of the product is thoroughly mixed with 20 c.c. of water, and, after being allowed to rest for a moment to permit the very coarsest particles to settle out, a small drop is placed on the central disk of the Thoma-Zeiss counting cell and then covered with a glass. Care must be exercised to have the slide perfectly clean, so that, when the cover glass is put in place, a series of Newton's rings * results from the perfect contact of the glass surfaces; and furthermore, the drop should be of such size as not to overrun the moat around the central disk and creep in underneath the cover glass, thus interfering with the contact.

With the magnification of 180 , it has been the practice in this laboratory to count the number of yeasts and spores on one-half of the ruled squares on the disk. With the dilution used this calculates back to a volume equal to one-sixtieth of a cubic millimeter in the original sample, and reports are made on that basis rather than on the number in a cubic centimeter, because the former number is more readily grasped by the mind and affords a simpler notation. To obtain the numbers per cubic centimeter the count made is simply multiplied by 60,000 .

It has been found in practice that the number of yeasts and spores varies, for one-sixtieth of a cubic millimeter, from practically none in homemade and first-class commercial ketchups up to 100 or 200 , and in one sample the number was as high as 1200. Laboratory experiments show that, when the number of yeasts in raw pulp reaches from 30 to 35 in one-sixtieth of a cubic millimeter the spoilage may frequently be detectable by an expert by odor or taste, and from experiments made under proper factory conditions, it seems perfectly feasible to keep the number in commercial ketchups below 25 .

Estimation of Bacteria. The bacteria are estimated from the same mounted sample as that used for the yeasts and spores. A power of about 500, obtained by using a high-power ocular, is employed in this case, and because of the greater number present a smaller area is counted over. Usually the number in several areas, each consisting of five of the small-sized squares, is counted and the number of organisms per cubic centimeter is calculated by multiplying the average number in these areas by $2,400,000$. Thus far it has proved impracticable to count the micrococci present, as they are likely to be confused with other bodies

* These are rainbow-colored rings produced at the point of contact when polished plates of glass are pressed against each other. 
frequently present in such products, such as particles of clay, etc. A comparison of this method with the ordinary cultural methods on samples in which the organisms had not been killed has almost invariably shown that the one used gives too low instead of too high results. In some cases it was found to give not more than one-third of the entire number present. The estimates of the laboratory on this point may, therefore, be considered very conservative.

As regards the limits which may be expected in the examination of ketchups for bacteria, it might be stated that some manufactured samples as well as good, clean products made by household methods, have been examined and the count found to be so low when estimated by this method that the numbers present were reported as negligible. In other words, it was found that for the areas counted over the number of bacteria averaged less than one-that is, less than 2,400,000 per cubic centimeter. It is unusual, however, for the final number per cubic centimeter to be less than from $2,000,000$ to $10,000,000$ organisms. Contrasted with this number as a minimum, it has been found that the number has occasionally exceeded $300,000,000$ per cubic centimeter. Such a number as this would indicate extremely bad conditions and carelessness in handling, as the studies of factory conditions have shown that there is little excuse for the number ever exceeding 25,000,000 per cubic centimeter. While experiments have also shown that although the effect produced by the bacteria on the product varies with different species, it is true that their presence can frequently be detected in the raw pulp by odor or taste when the number exceeds $25,000,000$ per cubic centimeter and sometimes when the count is as low as $10,000,000$.

To one who has not been initiated into the mysteries of the microscope the presence of such a number of bacteria in a food product seems inexcusable. It must be remembered in this connection that the most of these are probably nonpathogenic forms, and many occur naturally on the skins of the fruits. It does not seem just to set a standard so high as to virtually prohibit the manufacture of the product under commercial conditions; rather the idea is to set a limit that the manufacturer can attain if due care is exercised and which will insure a cleanly product. It is, however, perfectly possible to make a cleanly, wholesome product commercially even though the number of bacteria exceed that in the homemade article.

The allowable limits for the bacterial content of tomato pulp vary according to the concentration. The number, however, should be low enough so that when the amount of concentrating necessary for its conversion into ketchup has been accomplished the final product 
will still be within permissible linits $(25,000,000$ per cubic centimeter). Thus, for a pulp which must be concentrated one-half the bacterial counts should not exceed about half the limits stated above for the ketchup itself, i.e., it should not be more than 12,500,000 per cubic centimeter. The same general rule should also apply to the content of molds and of yeasts.

To insure a sound product, free from decay or any filthy material, many factors must be carefully watched, for not infrequently oversight in one particular has been found to have undone the good effects of the care exercised in all other ways. Thus it is possible for the washing of the fruit to be ideal and the sorting out or removing of the decayed portions beyond criticism, and yet a delay in making up the pulp into the final product may allow an amount of decomposition to occur which offsets the care previously exercised. It has been a matter of surprise to some manufacturers to find with what rapidity some of these organisms increase. In one factory where this point was tested, the bacterial content in a batch of tomato trimming juice was found to be about 7,000,000 per cubic centimeter when taken from the peeling tables, and after standing at room temperature for five hours it had increased to $84,000,000$. This was a twelvefold increase in a length of time which was less than half the working day for some of the factories visited. At the end of five days the number had increased to nearly $3,000,000,000$ per cubic centimeter. Thus it is seen that delay in manufacture is very liable to result disastrously.

Such facts as these serve to emphasize the great importance of absolute cleanliness in every detail ahout factories of this kind. Dirty floors and ccilings and apparatus left with residues of tomato product clinging to them are most fruitful sources for the contamination of new batches of the product. To clean such an establishment properly it is almost imperative that machinery and woodwork be washed by means of live steam used lavishly at frequent intervals. To leave buckets, tables, conveyors, or any other part of the equipment or floors overnight without cleansing them, as was the practice in some factories, is reprehensible and tends to contaminate the product and lead to spoilage and loss.

There are some objections to this method. Prescott, Burrage and Philbrick (1917) have stated that the method is grossly inadequate. They pointed out the following facts which render the method open to criticism. 1. Low magnification for examining the unstained mounts. 2. No account of coccus forms is taken. 3. Personal equation is given too much importance. 4. Directions are too indefinite. Bitting and 
Bitting (1915) have also stated that the method is unsatisfactory. Indefinite directions are especially mentioned. Vincent (1918) has maintained the above objections. Bitting and Bitting have pointed out the practical impossibility of concentrating tomato pulp to the consistence of tomato paste and having it pass the standard of 25,000 ,000 . The inadequacies of the procedure are soon apparent to anyone who attempted to use the method. The difficulty of distinguishing between particles of organic matter and bacteria makes the results very uncertain. These criticisms caused a restatement of the procedure. More definite details have been given by Howard (1917) as follows:

\section{Howard's Revised Method for the Microanalysis of Tomato Products}

Apparatus. (a) Compound Microscope. Equipped with apochromatic objectives and compensating oculars, giving magnifications of approximately 90, 180 and 500 diameters. These magnifications can be obtained by the use of 16 and $8 \mathrm{~mm}$. Zeiss apochromatic objectives with X6 and X18 Zeiss compensating oculars, or their equivalents. such as the Spencer 16 and $8 \mathrm{~mm}$. apochromatic objectives with Spencer $\mathrm{X} 10$ and X20 compensating oculars, the draw tube of the microscope being adjusted as directed below.

(b) Thoma-Zeiss Blood-counting Cell.*

(c) Howard Mounting Cell. Constructed like a blood-counting cell but with the inner disk (which need not be ruled) about $19 \mathrm{~mm}$. in diameter.

Molds (Tentative). Clean the Howard cell so that the Newton's rings are produced between the slide and the cover glass. Remove the cover and place by means of a knife blade or scalpel, a small drop of the sample of the central disk; spread the drop evenly over the disk and cover with the cover glass so as to give an even spread to the material. It is of the utmost importance that the drop be mixed thoroughly and spread evenly; otherwise the insoluble matter and the molds are most abundant at the center of the drop. Squeezing out of the more liquid portions around the margin must be avoided. In a satisfactory mount, Newton's rings should be apparent when finally mounted and none of the liquid should be drawn across the moat and under the cover glass.

* Comment by the authors. In using these cells the plane parallel cover glasses furnished with them should be used instead of the ordinary microscope cover glasses. Since the latter are subject to curvatures that introduce errors in the thickness of the mounts. 
Place the slide undlor the microscope and examine with a magnification of about 90 diameters and with such adjustment that each field of view represents approximately $1.5 \mathrm{sq} . \mathrm{mm}$. of area on the mount.*

This area is of vital importance and may be obtained by adjusting the draw tube to the proper length as determined by actual measurement of the field, a $16 \mathrm{~mm}$. Zeiss apochromatic objective with a Zeiss $\mathrm{X} 6$ compensating ocular or a Spencer $16 \mathrm{~mm}$. apochromatic objective with a Spencer X10 ocular, or their equivalents, being used to obtain the proper magnification.

Observe each field as to the presence or absence of mold filaments and note the result as positive or negative. Examine at least 50 fields, prepared from two or more mounts. No field should be considered positive unless the aggregate length of the filaments present exceeds approximately one-sixth of the diameter of the field. Calculate the proportion of positive fields from the results of the examination of all the observed fields and report as percentage of fields containing mold filaments.

Yeasts and Spores (Tentative). Fill a graduated cylinder with water to the 20 c.c. mark, and then add the sample till the level of the mixture reaches the 30 cc.. mark. Close the graduate, or pour the contents into an Erlenmeyer flask, and shake the mixture vigorously fifteen to twenty seconds. To facilitate thorough mixing the mixture should not fill more than three-fourths of the container in which the shaking is performed. For tomato sauce or pastes or products running very high in the number of organisms, or of heavy consistency, 80 c.c. of water should be used with 10 c.c. or $10 \mathrm{gms}$. of the sample. In the case of exceptionally thick or dry pastes it may be necessary to make an even greater dilution.

Pour the mixture into a beaker. Thoroughly clean the Thoma-Zeiss counting cell so as to give good Newton's rings. Stir thoroughly the contents of the beaker with a scalpel or knife blade, and then, after allowing to stand three to five seconds, remove a small drop and place upon the central disk of the Thoma-Zeiss counting cell and cover immediately with the cover glass, observing the same precautions in mounting the sample as given above. Allow the slide to stand not less than ten minutes before beginning to make the count. Make the count

* Comment by the authors. In order to have an area of $1.5 \mathrm{sq} . \mathrm{mm}$. the diameter of the microscopic field should be $1.382 \mathrm{~mm}$. This is determined by using a stage micrometer and adjusting the length of the microscope draw tube. Obviously after the proper draw tube length has been secured the adjustment should be noted and always used in making the mold counts. 
with a magnification of about 180 to obtain which the following combinations, or their equivalents, should be employed: $8 \mathrm{~mm}$. Zeiss apochromatic objective with X6 Zeiss compensating ocular, or an $8 \mathrm{~mm}$. Spencer apochromatic objective with X10 Spencer compensating ocular with draw tube not extended.

Count the number of yeasts and spores * on one-half of the ruled squares on the disk (this amounts to counting the number in eight of the blocks, each of which contains twenty-five of the small ruled squares). The total number thus' obtained equals the number of organisms in $1 / 60 \mathrm{c} . \mathrm{mm}$. if a dilution of one part of the sample with two parts of water is used. If a dilution of one part of the sample with eight parts of water is used the number must be multiplied by three. In making the counts the analyst should avoid counting an organism twice when it rests on a boundary line between two adjacent squares.

Bacteria (Tentative). Estimate the bacteria from the mounted sample used above, but allow the sample to stand not less than fifteen minutes after mounting before counting. $\dagger$ Employ a magnification of about 500, which may be obtained by the use of an $8 \mathrm{~mm}$. Zeiss apochromatic objective with an X18 Zeiss compensating ocular with draw tube not extended, or an $8 \mathrm{~mm}$. Spencer apochromatic objective with an X20 Spericer compensating ocular with a tube length of 190, or their equivalents. Count and record the number of bacteria in a small area consisting of five of the small-sized squares. Move the slide to another portion of the field and count the number on another similar area. Count five such areas, preferably one from near each corner of the ruled portion of the slide and one from near the center. Determine the average number of bacteria per area and multiply by $2,400,000$, which gives the number of bacteria per cubic centimeter. If a dilution of one part of the sample with eight parts of water instead of one part of the sample with two parts of water is used in making up the sample, then the total count ol,uained as above must be multiplied by $7,200,000$. Omit the micrococci type of bacteria in making the count.

Explanation of Calculations. Fig. 80 has been prepared to make somewhat clearer the explanation of the areas denoting the yeast and spore and "oacterial counts. The light lines in the figure show the arrangement of rulings on the entire slide. The squares $(A, A$, etc.), and rectangles $(B, B$, etc.) designated in the figure by the heavy lines indicate the portions used for the yeast and spore and for the bacterial

\footnotetext{
* Comment by authors. The organisms counted as "yeasts and spores" are the yeast cells, and yeast and mold spores, not bacteria spores.

$\dagger$ The estimation of yeast, molds and bacteria are made from the same mount.
} 
counts, respectively. The eight large squares, $A, A$, etc., are the squares used for yeast and spore counts. Each of these squares has twenty-five of the small squares. The sum of the organisms counted in the eight squares marked $A, A$, etc., is the number in $1 / 60 \mathrm{c} . \mathrm{mm}$. if a dilution. of one part of product to two parts of water is used.

Yeast and Spore Count. The ruled square on the slide is $1 \mathrm{~mm}$. on each side and the cell is $1 / 10 \mathrm{~mm}$. deep. The volume of the ruled part is, therefore $1 / 10 \mathrm{c.mm}$. The ruled area is divided into sixteen large squares and the number of organisms is counted in eight of these, which is equivalent to $1 / 2$ of $1 / 10 \mathrm{c.mm}$., or $1 / 20 \mathrm{c.mm}$. If a dilution of sne

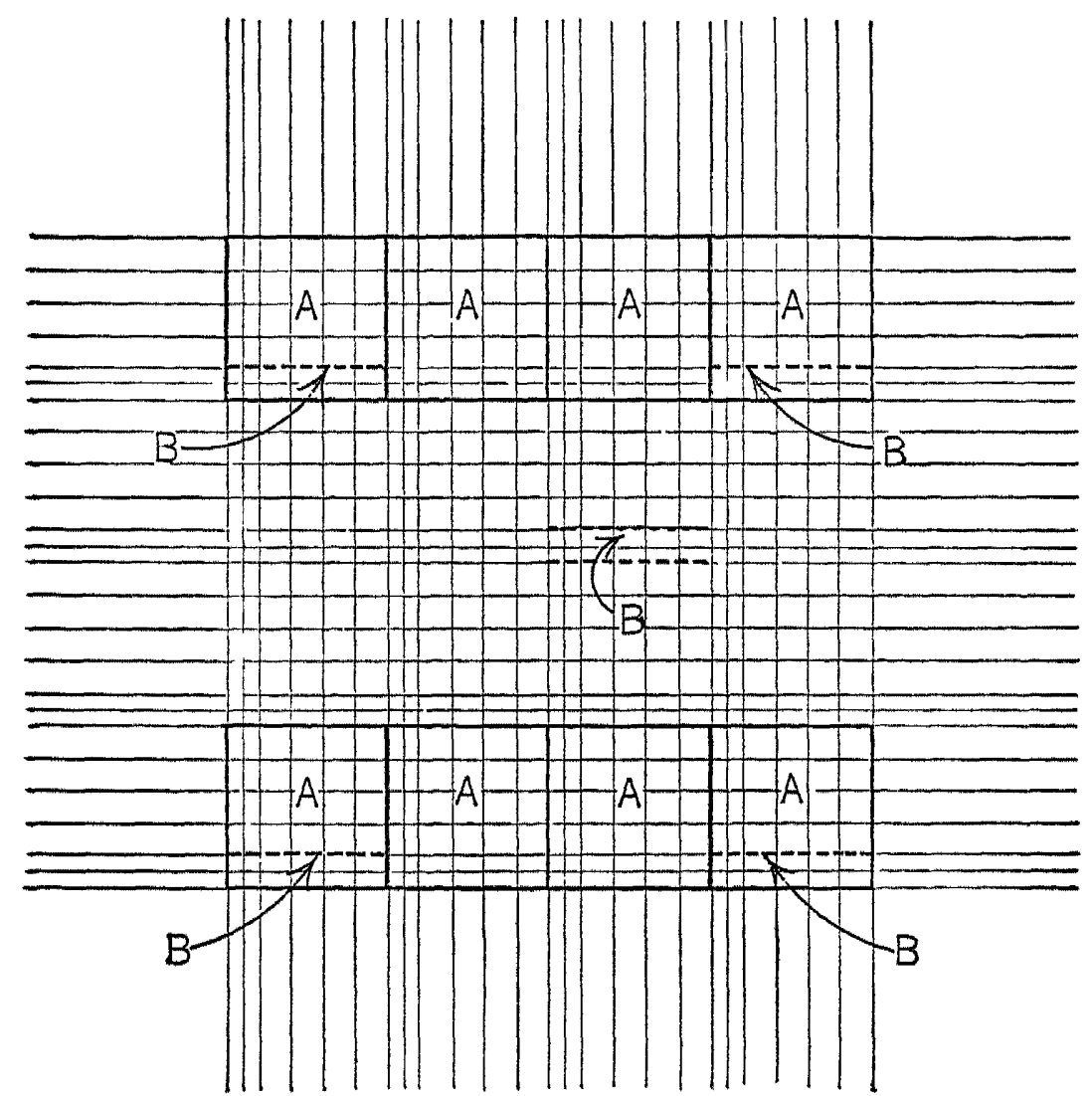

FIG. 80.-Diagram of Thoma Rulings. (After Howard, 1917.)

One millimeter divided by lines into twenty spaces in each direction each space equalling $\frac{1}{20} \mathbf{m m}$. To facilitate counting, every fifth space is subdivided by a line through the nnddle.

part of the product to two parts of water is used $1 / 3$ of $1 / 20 \mathrm{c.mm}$., or $1 / 60 \mathrm{c} . \mathrm{mm}$. as representing the actual amount of original stock in which organisms are counted, is obtained.

Bacterial Count. The rectangles, $B, B$, etc., each including five of the smallest squares, represent the areas used in making the bacterial count. Similar rectangles of equal area might be selected, the object being to count five such areas well distributed over the ruled portion of the slide. The average number of bacteria counted on five rectangles, such as $B, B$, etc., multiplied by 2.4 million, equals the number of 
bacteria per cubic centimeter. In calculating the bacteria, it is observed that there are $400(20 \times 20)$ small squares on the slide. The number of bacteria in rectangles $(B, B$, etc.), each containing five of these small squares, are counted and an average made. This average represents the bacteria in $1 / 80$ of the total ruled area. Since the cell is $1 / 10 \mathrm{~mm}$. deep, the volume represented by the organisms counted is $1 / 80 \times 1 / 10$ or $1 / 800 \mathrm{c.mm}$. With the usual dilution of one part of product to two parts of water the actual volume in which the number of organisms is determined is $1 / 3$ of $1 / 800 \mathrm{~cm}$. or $1 / 2400 \mathrm{c.mm}$. or $1 / 2,400,000$ c.c.

Howard (1917) made a study of the relation between the number of yeasts and spores, mold counts, and bacteria (rods) and the amount of rot in the tomatoes. With regard to yeasts and spores a count of twenty was represented by 1 per cent decay. From this point the rate of increase was lower. A count of thirty-five yeasts and spores indicated, according to Howard about 4 per cent of rot. Fig. 81 devised by Howard shows the "Zone of Possible Yeast and Spore Counts." From this chart it is possible to determine the relation between a count and the per cent of rot which it represents.

The relation between the number of bacteria and the percentage of rot is shown in Fig. 82. Howard thinks that below 15,000,000 little is indicated with regard to the amount of rot. Above this "and up to 20 per cent of rot, the ratio of increase as shown on this chart is about $20,000,000$ for each per cent of rot." Howard states that a low bacterial count does not always indicate a sound stock but a high number of bacteria always indicates bad stock.

Fig. 83, devised by Howard, shows the relation between the mold count and the percentage of rot. From this chart it is possible to calculate the percentage of decay from a mold count. The following is quoted from Howard. "Attention should be called to the fact that beyond 20 per cent of rot the chart is plotted on the basis of the assumption that 100 per cent rot would give a mold count of 100 per cent of the fields. As a matter of fact, the mold count reaches this maximum of 100 per cent of the fields with less than this amount of rot. In the study of the relation between the rot and count under factory conditions, 20 per cent of rot was the highest on which the full data was secured. From 0 to $\frac{1}{2}$ per cent of rot the mold count rises rapidly. Beyond $\frac{1}{2}$ per cent the rate of rise gradually decreases, until after 20 per cent of rot the rate of increase is slow. On the whole the zone was higher on factory than on laboratory samples. The chart shows that a count of 60 per cent molds represents a rot content of not less than about 4 per cent. It is interesting that the mold count of twenty-five, which was 


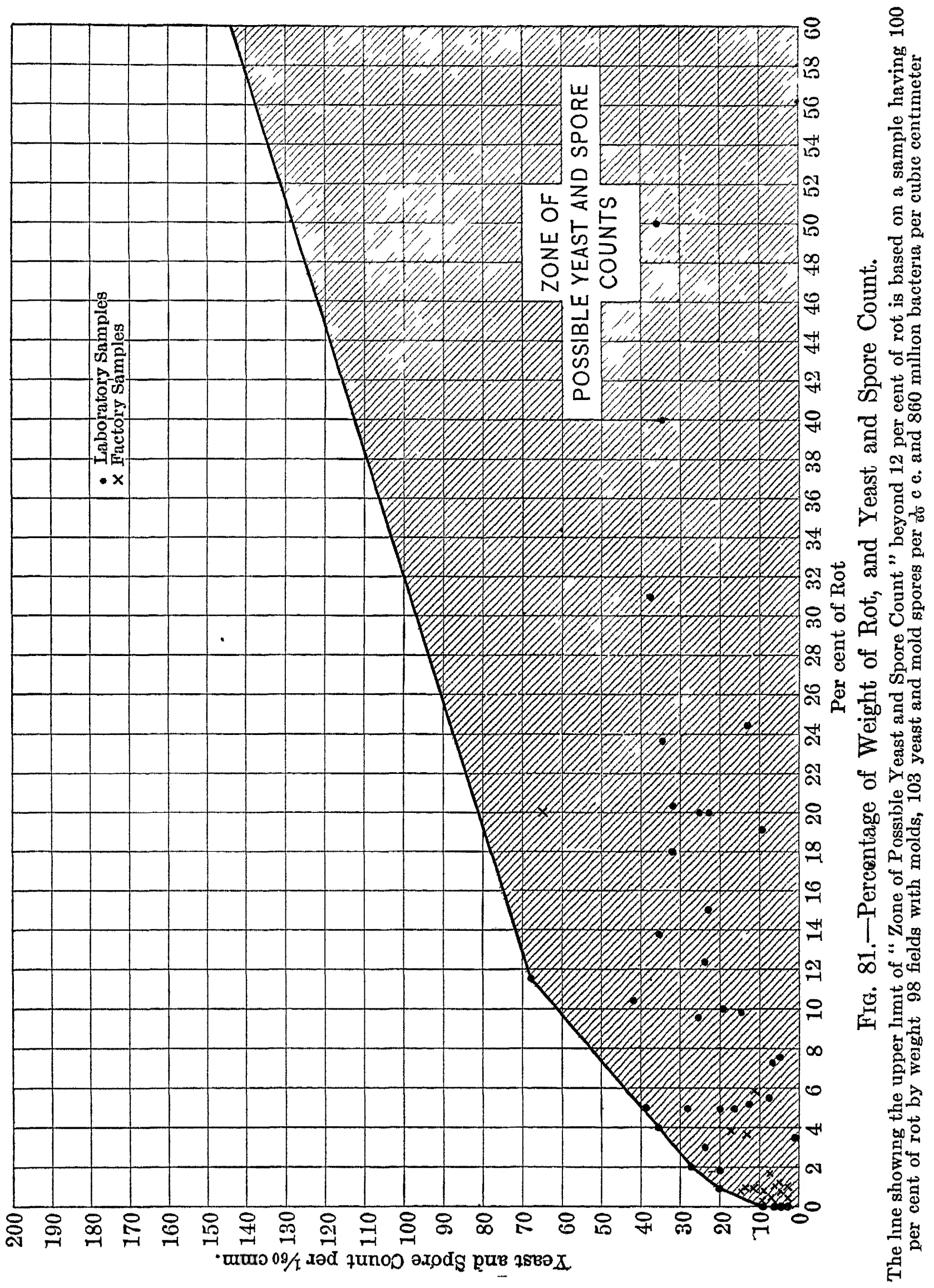




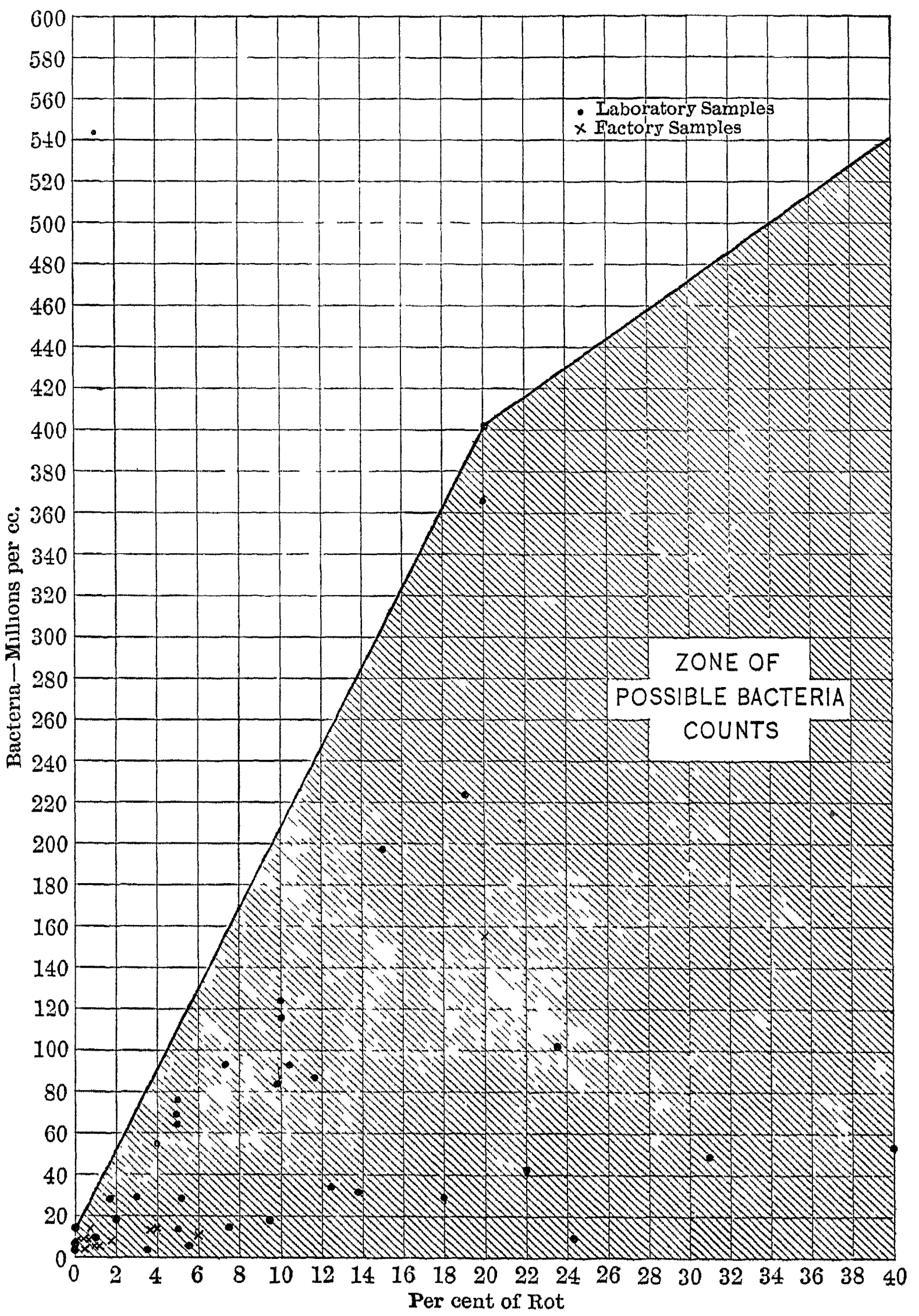

Frg. 82.-Percentage by Weight of Rot and Bacterial Count. (After Howard and Stevenson, 1917.)

The line showing the upper limit of "Zone of Possible Bacteria Counts" beyond 20 per cent of rot is based upon the count of a sample having 100 per cent of rot, 100 fields with molds, 22 yeast and spores per of c.c. and 960 million bacteria per cubic centimeter. 


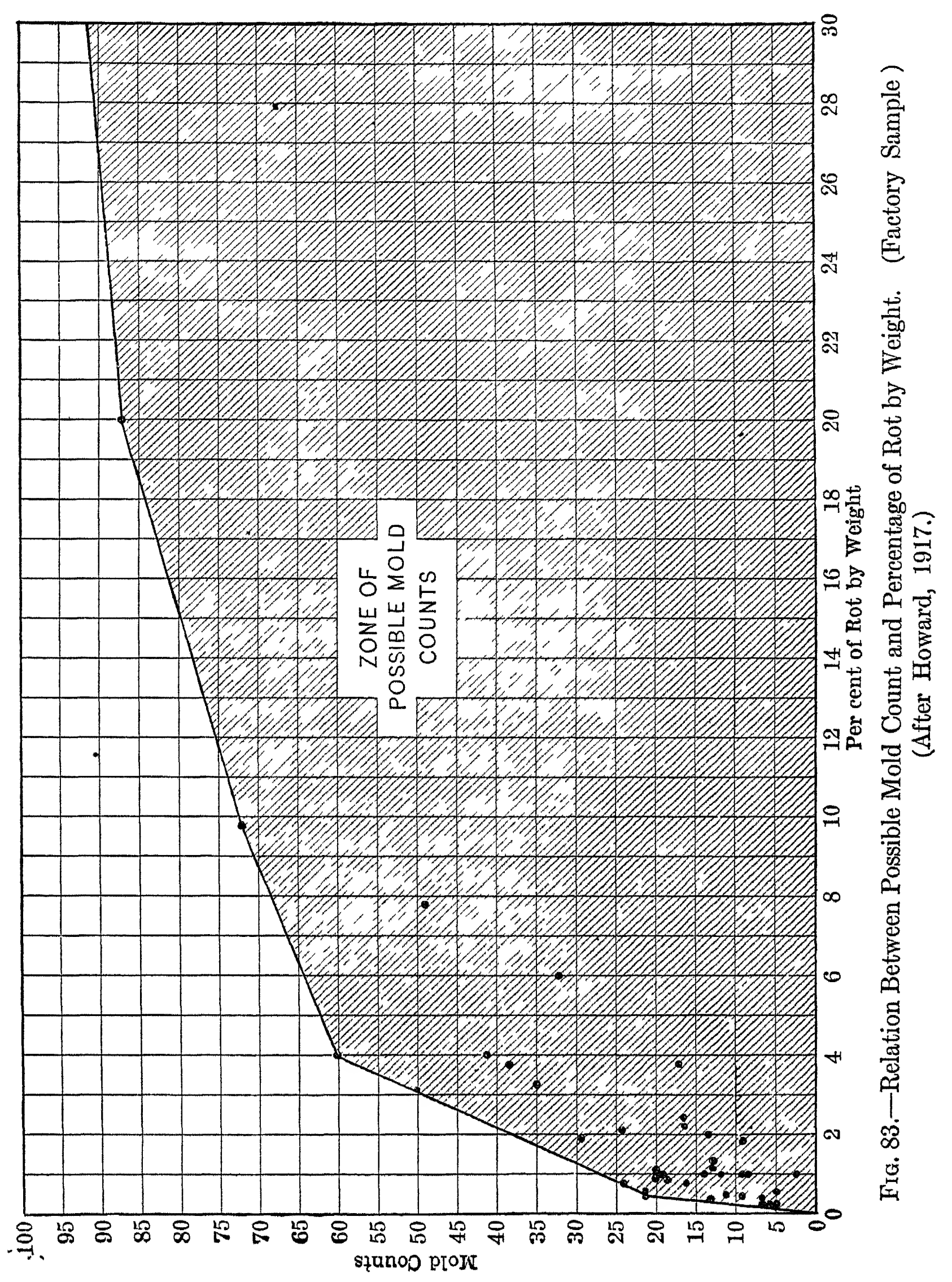


suggested as a factory-working basis in 1911, represents at least 0.8 to 0.9 per cent of rot. There may be more rot than that present, but, on the basis of the data at hand, it is highly improbable that it represents less than that amount. From the chart it is possible to calculate the approximate minimum percentage of decay represented by a given mold count. Thus, for instance, a mold count of forty enters the 'Zone of Permissible Mold Counts' at a point representing 2.2 per cent of decay. Therefore, a count of forty may be obtained in samples having any amount of rot between 2.2 and 100 per cent."

Vincent's Method for the Examination of Tomato Paste. Vincent (1918) has attempted to overcome the errors of the Howard method by adapting the Breed smear to tomato products. The method is described as follows: "The catsup is diluted with two parts of sterile water, since this dilution has proven satisfactory in counting most specimens when the Zeiss counting chamber is used. With a sterile pipette calibrated to deliver 0.01 c.c. the diluted catsup is deposited on a second glass slide and evenly spread over an area of $1 \mathrm{sq} . \mathrm{cm}$. by means of a sterile needle. After drying, the slide is immersed in 95 per cent alcohol for one minute to fix the smear, dried in air, stained with Loeffler's methylene blue for two minutes, washed in water, dried and examined with the 1/12 in. oil immersion lens." The rest of the procedure is identical with that outlined under Breed's microscopic method for counting bacteria in milk. The microscope is standardized to bring the diameter of the field to $0.205 \mathrm{~mm}$. Thirty fields are counted and the average for one field is multiplied by three on account of the dilution of the catsup. This result is then multiplied by 300,000 , which represents the number of bacteria per cubic centimeter.

Vincent compared this microscopic count with the count secured with the Zeiss blood counter. The latter count was always much greater than the former. Vincent believes that the cocci should be counted since they probably take as important a part in the spoilage of tomatoes as the rods.

\section{Food Poisoning}

In the past, this term has covered a multitude of physiological disturbances in the alimentary tract. For some, it has been a very easy matter in the past to diagnose an ailment as food poisoning or ptomaine poisoning when other possibilities have been excluded. The term "ptomaine poisoning" was used by many for a time, but this is gradually being discarded for terms with more meaning. More 
recently the conception that foods may cause infection has crept in and this is being confirmed. Specific bacteria are now being recognized as the principal factors in many cases. In others, the toxins produced by bacteria are ingested and these produce intoxications.

Certain characteristics of food infection or poisoning have prevented rapid advances in our knowledge. The occurrence of a small number of cases in a family is investigated with some difficulty because these individuals often are engaged in quite different activities. Much easier is it to study the problem when the individuals are members of units whose activities are much alike. Consequently some of the most valuable data with regard to food poisoning has been collected from prisons, barracks and similar groups. Probably, in the smaller units, cases of food poisoning have been overlooked and attributed to other causes. Then, in the past, many cases of food poisoning have been reported, the study of which included no bacteriological or chemical examination.

The causes of food poisoning are varied because there is little agreement with regard to a definition for the term "poison," which is a difficult term to define. However, for convenience, the following causes of food poisoning may be mentioned:

I. Ingestion of foods containing pathogenic bacteria.

II. Ingestion of foods containing poisonous products of bacterial katabolism-toxins.

III. Ingestion of foods which are naturally poisonous, such as mushrooms. Certain alkaloids are present.

IV. Ingestion of foods containing poisonous metals.

Ptomaines. These are toxic amines and, as indicated in the chapter on intestinal bacteria, they are the products of bacterial action. They are basic in character and have been called " animal alkaloids." Most of them do not contain oxygen; the following are typical ptomaines:

$\left(\mathrm{CH}_{3}\right) \mathrm{NH}_{2} \ldots \ldots \ldots \ldots \ldots \ldots \ldots \ldots \ldots \ldots \ldots \ldots \ldots \ldots$ methylamin

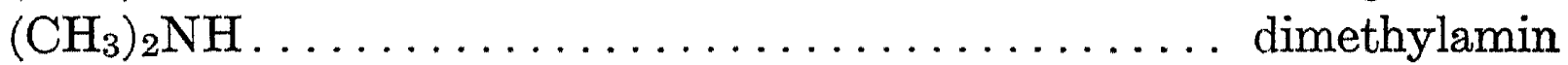

$\left(\mathrm{CH}_{3}\right)_{3} \mathrm{~N} \ldots \ldots \ldots \ldots \ldots \ldots \ldots \ldots \ldots \ldots \ldots \ldots \ldots \ldots \ldots \ldots \ldots$ trimethylamin

$\mathrm{NH}_{2}-\mathrm{CH}_{2}-\mathrm{CH}_{2}-\mathrm{CH}_{2}-\mathrm{CH}_{2}-\mathrm{NH}_{2} \ldots \ldots \ldots \ldots$ putrescine

$\mathrm{NH}_{2}-\mathrm{CH}_{2}-\mathrm{CH}_{2}-\mathrm{CH}_{2}-\mathrm{CH}_{2}-\mathrm{CH}_{2}-\mathrm{NH}_{2} \ldots \ldots$ cadaverine

Not all of the ptomaines are poisonous. The more elementary amino compounds are probably not poisonous. The ptomaines do not hold the position in food poisoning as formerly. Their importance in the past probably originated in their isolation from bacterial cultures and the 
lack of more definite bodies to which to attribute the effects of disease caused them to be cmphasized in this connection. Little data was necessary to prove that they were not important in bacterial toxemia.

In relation to food poisoning, their significance is negligible. It has been very casy in the past for diagnosticians to attribute the various alimentary disturbances to the presence of ptomaines. This was especially true if the patient had been eating of canned foods. It is unfortunate that such a close relation between ptomaine poisoning and canned foods has been established. Probably no food is less liable to be of any importance in this connection than canned foods. A food which has putrefied to the point where ptomaines are present will be excluded by the organoleptic tests; it is also reasonable to assume that the ptomaines themselves are soon destroyed by bacterial action. Vaughan and Novy have given extended attention to the ptomaines and it may be due almost entirely to their investigations that ptomaines have held such an important position in food poisoning. They proposed a rather elaborate nomenclature for the different types of poisonings. Some of them are as follows:

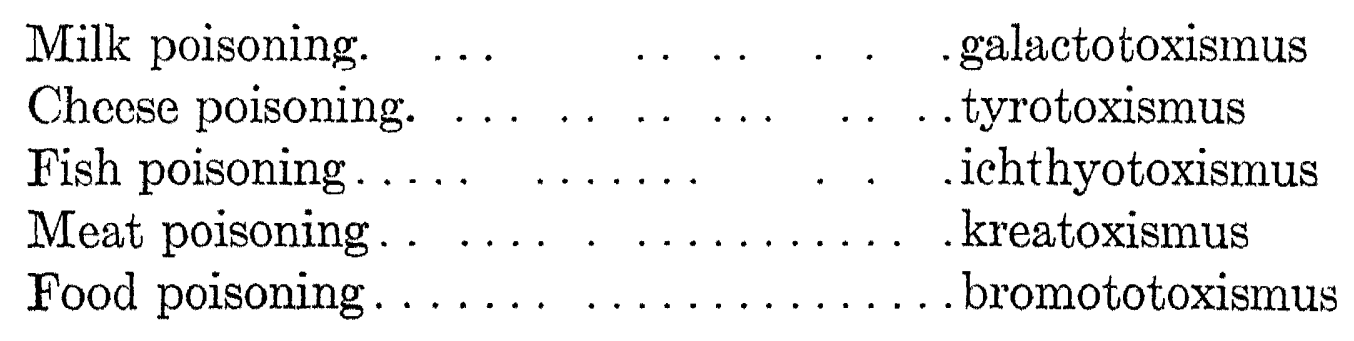

Relation of Carriers to Food Infections. Common infections of foods by carriers are, dysentery, typhoid fever, paratyphoid fever and streptococcus sore throat. Data are being accumulated very rapidly to enlighten this question.

The question is often asked, how long may a person continue to distribute the bacilli in feces and urine? In answer to this question there are several cases which might be mentioned. Bolduan and Noble (1912) report a dairyman who had typhoid fever at fiftcen ycars of age. He was a carrier for forty-six years showing $B$. tuphi in his stools. Martz (1917) reported a carrier of fifty-five years' standing. The case of a woman who was apparently a carrier for fifty-four years is men"tioned in an Editorial of the Jour. Am. Med. Assn., Vol. 52 (1909), 388.

The handling of carriers is not a settled question. Kendall (1916) pointed out the danger of carriers handling foods. Several severe epidemics of typhoid are known to have been caused by food infected from a carrier. The ideal situation will only be reached after all handlers 
of foods in public places arc examined for various diseases which arc transmissible through foods.

In an effort to control and keep in touch with "carriers" the California State Board of Health causes the following agreement to be entered into between the "carrier" and the department. (Cumming, 1917).

I have this day been informed that my excreta contain typhoid bacilli and that, unlcss unusual precautions are taken, persons will contract the infection from me. Realizing this danger I agree to observe the precautions stated below, and request that I be permitted to remain in free communication with other persons.

1. I will take no part in the preparation or handling of food which will be consumed by persons outside of my immediate family, and I will not participate in the management of a boarding house, restaurant, food store, or in any other occupation involving the preparation or handling of food.

2. I will not dispose of my excretions in a toilet to which flies have access without first exposing such excretions to either a 5 per cont dilution of liquor formaldehyde or 5 per cent phenol (carbolic acid).

3. I will notify the local health officer of any cases of typhoid among persons with whom I come in contact.

4. I will inform that local health officer of any contemplated change of residence so that he can notify the State Board of Health and obtain its approval.

5. I will submit specimens for examination when requested by the State Board of Health.

6. I will fill out the following report blank when submitted to me semiannually, and return the same to the California State Board of Health:

"I have, during the last six months, complied to the best of my knowledge with the five separate agreements entered into between mysclf and the California State Board of Health. Precautions involved in these separate agreements are for the purpose of preventing typhoid infection."

That "carriers" may be found among the animals is suggested by much recent data. The work of Mitchell and Bloomer (1914) seems to indicate that chickens may not become carriers although the authors recommend further study.

Paratyphoid Fever. This infection is produced by a bacillus closely related to $B$. typhi but possessing certain distinct differences. Many cases of food poisoning have been traced to the paratyphi bacilli. Bain- 
bridge (1912) has attempted to make a distinction between paratyphoid fever and meat poisoning. He bases his argument on the inadequacies of agglutination reactions for separating members of the group and states that precipitation or complement fixation reactions are necessary to distinguish between the bacteria, to which food poisoning has been attributed. The symptoms of food or meat poisoning are quite different and Bainbridge is probably correct in making this distinction. The true paratyphoid fever resembles typhoid in many of its characteristics but the mortality from it and the mildness of the disease are distinct differences.

Jordan (1917) has pointed out the occurrence of at least two clinical types of paratyphoid infection, one having almost the identical picture of the true infection with $B$. typhi and the other the commoner gastrointestinal type.

Bongartz (1910) studied samples of meat from both condemned animals and apparently normal animals. Many of these were found to contain paratyphoid bacilli. He suggests the education of the public to the eating of cooked meat which, to a certain extent, would reduce the danger of diseased meat or foods. Hoffenreich (1914), on the other hand, examined 249 samples of meat from typical abbatoirs and found no indications of the presence of bacteria of the paratyphoid-Gaertner group. He concludes that their distribution is not as general as has been supposed. Bernhardt (1914) has found Bacillus voldagsen and $B$. typhi suis (Glasser) in a case of meat poisoning which resulted in death. He advises that a polyvalent paratyphoid serum be used for diagnosis.

Fowler (1909) has described an epidemic of paratyphoid fever resulting from eating goose at a Christmas dinner. He stated that the goose may have been infected during slaughter; bacteria may have invaded the flesh and excreted a toxin which would account for the acute onset of the cases. Bernstein and Fish (1916) described an epidemic of sixty cases and four deaths resulting from paratyphoid infection from a pie. This was confirmed by clinical and laboratory investigation. Such epidemics point out that restaurants (Kendall, 1916) may be important factors in the dissemination of infection. Müller (1915) reported that several cases of fish poisoning were caused by bacteria of the paratyphoid-enteriditis group. Seele (1913) in discussing food poisoning in the German army during the years 1912 and 1913 stated that meat and sausage were the foods which caused most trouble. The following organisms were identified: $B$. coli communis, B. proteus, $B$. enteritidis and $B$. paratyphosus. From 
the above epidemics it is apparent that foods may distribute paratyphoid bacilli.

Bacillus enteritidis. Gaertner (1888) first showed the relation of this organism to food poisoning. The infection came from the meat of a cow which was abnormal before it was slaughtered. The bacillus was isolated from the diseased meat and also from the spleen of a fatal case. Later Durham (1898) and De Nobele (1899) also isolated bacilli like $B$. enteritidis from abnormal meat. Van Ermengen (1892) reported the study of an extensive epidemic of food poisoning which was believed to have originated from eating the meat of diseased calves. Bacteria like $B$. enteritidis were isolated. Other investigations have been reported by Holst (1895), Poels and Dhont (1894), Jacobitz and Kayser (1910) and others.

\section{BotULISM}

Botulism or "sausage poisoning" has been known for a long time. The earliest report was by Kerner, who described a series of cases among which were a number of deaths. Dickson, in America (1915), has given the subject quite a little study. Most of the outbreaks in this country have been in California. This is probably due to their being alert to the possibility of botulism which, in other cases, has been diagnosed as ptomaine poisoning.

The organism was discovered by Ermengen (1899) in a ham which had been responsible for a severe attack of meat poisoning. It was found in muscle in the spore stage. $\mathrm{He}$ also isolated this organism from the spleens of the fatal cases. A further study gave more evidence that the organism was distinctly pathogenic.

The organism has the following characteristics: It is gram positive, anaerobic and a spore former. The temperature range is between 18 and $30^{\circ} \mathrm{C}$. The vegetative cells are easily destroyed by heat but the spores are quite resistant. Although Van Ermengen stated that they could be destroyed by heating at $85^{\circ} \mathrm{C}$. for fifteen minutes, Dickson (1915) has reported a greater resistance. The organism does not grow above $30^{\circ} \mathrm{C}$. and, therefore, the toxin is the important factor in this type of food poisoning.

Toxin of Bacillus botulinus. The toxin of Bacillus botulinus is soluble and produces a strict toxemia. It is thermolabile and very toxic not only when administered by injection but when administered by the mouth. This separates it very closely from the toxins of $B$. tetani and $B$. diphtherice. It was formerly thought that the toxin for- 
mation occurred only on certain food substances, as meat, but Dickson has shown that it may take place in such foods as canned beans and peas.

The symptoms have been stated by Dickson (1915).

The symptomology of botulism has been summarized by Van Ermengen as a neuroparalytic symptom complex with disturbances of secretion and symmetrical partial or complete motor paralyses which apparently have their seat in lesions of the central nervous system. The first symptoms do not occur earlier than from twelve to twenty-four hours after ingestion of the infected food, although they may appear much later. In contrast to the usual types of food poisoning there is apt to be little evidence of gastro-intestinal disturbance, and obstinate constipation is more frequent than is diarrhœa. There is usually a decrease, but there may be an increase in the secretion of saliva and of mucus in the mouth and in the throat, when mucus is present it is extremely viscid. There is early disturbance of the external and internal muscles of the eyes which manifests itself by blepharoptosis mydriasis, disturbances of accommodation, diplopia and strabismus. General muscular weakness is common. There may be dysphasia or aphonia and there is usually great difficulty in swallowing. The absence of sensory disturbances is characteristic.

The symptoms gradually increase in severity and when death occurs it is usually from respiratory or cardiac failure, as in bulbar paralysis. When recovery takes place the e is usually a long and tedious convalescence with a slow return of muscular strength. The condition so closely resembles that seen in gelsemium or hyoscyamin poisoning, polyomyelitis, cerebral syphilis and bulbar paralysis that isolated cases may readily escape recognition, but when a series of cases is seen and especially if all have partaken of a common article of food there is usually little difficulty in establishing a diagnosis.

Botulism may be diagnosed microscopically by detecting the Bacillus botulinus in the suspected food. The bouillon cultures should be tested for toxin and inoculated food fed to laboratory animals. In the cultural examination of the food, careful anaerobic technique should be used.

Bacillus botulinus has caused severe poisonings in modern times. Wilbur and Ophüls (1914) reported the investigation of an epidemic which was caused by home-canned string beans. Dickson (1915) has mentioned some other epidemics. He (1917) called attention also to the fact that vegetables put up by the cold-pack method might cause the disease. This was later denied, to a certain extent, by the U. S. Department of Agriculture. Fischer (1906) studied an epidemic of botulism resulting from the eating of bean salad. The symptoms were characteristic and appeared between twenty-four and forty-eight hours. The beans were canned and when opened were not cooked since they 
seemed to be soft. Had they been thoroughly cooked it is improbable that any unusual results would have been noticed. Another epidemic of botulism from canned string beans has been reported by Landmann (1904). In this epidemic the value of boiling canned food is again emphasized. Some of the beans were boiled by accident and when eaten produced no evil results. Those who partook of the uncooked beans suffered the usual symptoms. The presence of $B$. botulinus was indicated by a bacteriological examination.*

Diagonosis of Food Poisoning. Extract some of the suspected food with physiological salt solution. Inject boiled and unboiled decimal dilutions of this extract into guinea pigs. Feed the suspected food to experimental animals. If they will not eat it, sprinkle other foods with a physiological salt solution extract of the suspected food.

\section{BIBLIOGRAPHY}

Bachmann, F. M. 1916. A Study of the Effect of Spices on the Growth of Certain Organisms. Abstracts of Bacteriology, 1, 109.

Bachmann, F. M. 1916. Inhibiting Action of Certain Spices. Jour. Ind. Chem., 8, 620-623.

Bachmann, F. M. 1918. The Use of Different Microorganisms to Determine the Preservative Value of Different Brands of Spices. Jour. Ind. Eng. Chem., 10, 121-123.

Bacon, R. F. and Dunbar, B. P. Changes Taking Place During Spoilage of Tomatoes with Methods for Detecting Spoilage in Tomato Products. U. S. Department of Agriculture, Bureau Chemistry Circular 78.

Bainbridge, F. A. 1912. The Milroy Lectures on Paratyphoid Fever and Meat Poisoning. Lancet, 1912-I, 705, and lectures in two succeeding numbers.

Baker, H. A. 1912. "Springers" in Canned Foods, Causes and Prevention. 8th Intern. Cong. Applied Chem., 18, 39-41.

Baker, H. A. 1912. The Disappearance of Oxygen in Canned Food Containers. 8th Intern. Cong. Applied Chem., 18, 45-49.

Beattie, J. H. and Gould, H. P. 1917. Commercial Evaporation and Drying of Fruits. U. S. Dept. Agriculture, Farmer's Bulletin 903.

Bernhardt, G. 1912. In Regard to the Causes of Meat Poisoning. Paratyphoid B. bacilli Voldagsen Type as a Cause of Meat Poisoning in Man. Zeit. f. Hyg., 73, 64-78.

Bernstern, H. S. and Fish, E. S. 1916. Food Poisoning by the Bacillus paratyphosus B. An epidemic due to the organism isolated from a pie. Jour. Amer. Med. Assn., 66, 167-171.

* Those desiring an extended discussion of botulism should read Monograph No. 8 (1918) of the Rockefeller Institute for Medical Research by Dickson. 
Bigelow, W. D. 1914. Swells and Springers. Research Laboratory, National Canners' Assn., Washington, D. C., Bull. 2.

Bigelow, W. D. 1917. The Chemist in the Canned Food Industry. Jour. Ind. Eng. Chem., 9, 187-189.

Bigelow, W. D. 1918. Problems of Canning Operations. Amer. Jour. Pub. Health, 8, 212-215.

Bigelow, W. D. and Fitzgerald, F. F. 1914. Tomato Pulp. Research Laboratory, National Canners' Assn., Washington, D. C., Bull. 3.

Birting, A. W. 1909. Experiments on the Spoilage of Tomato Ketchup. U. S. Dept. Agriculture, Bureau of Chem. Bull. 119.

Bitting, A. W. 1909. The Canning of Peas. U. S. Dept. Agriculture, Bureau of Chemistry Bull. 125.

Brrming, A. W. 1911. Preparation of Cod and Other Salt Fish for the Market. U. S. Dept. Agriculture, Bureau of Chemistry Bull. 123.

Bitting, A. W. 1915. Methods Followed in the Commercial Canning of - Foods. U. S. Dept. Agriculture, Bull. 196. See also Bull. 151.

Bitring, A. W. 1917. Canned Foods. Modern Processes of Canning in the United States, General System of Grading and Description of Product Available for Export. U. S. Dept. Commerce. Bureau of Foreign and Domestic Commerce. Miscellaneous Series No. 54.

Birming, A. W. and Birting, K. G. 1915. Ketchup. Murphey-Bevins Co., Lafayette, Indiana.

Bitring, A. W. and Bitring, K. G. 1917. Bacteriological Examination of Canned Foods. Research Laboratory, National Canners' Laboratory Bull. 14.

Bolduan, C. F. 1909. Bacterial Food Poisoning by A. Dieudonne. E. B. Treat \& Co.

Bolduan, C. and Noble, W. C. 1912. A Typhoid Bacillus Carrier of Fortysix Years' Standing and a Large Epidemic from this Source. Jour. Amer. Assn., 58, 7 .

Bongartz, C. 1910. Do Paratyphus-like Bacteria Occur in Meat under Normal Conditions and if so is Bacteriological Meat Inspection Necessary? Inaug. Dissert. Univ. Berne.

Bridgman, P. W. 1914. The Coagulation of Albumin by Pressure. Jour. Biol. Chem., 19, 511-512.

Brown, C. W. 1916. Notes on Brine Pickle Fermentation. Abstracts of Bacteriology, 1, 104-105.

Burgess, P. S. 1912. A Bacteriological Study of "Flat Soured" Canned Corn and Pumpkin Together with Length of Time Required for Complete Sterilization. The Canner and Dried Fruit Packer, about 1912.

Cathcart, E. P. 1906. The Bacterial Flora of "Blown" Tins of Preserved Foods. Jour. Hyg., 6, 248-250.

Courmont and Rochoux. 1910. Dogs as Typhoid Carriers. Amer. Jour. Med. Sciences, 140, 899. 
Cumming, J. G. 1917. An Epidemic Resulting from Contamination of Ice Cream by a Typhoid Carrier. Jour. Amer. Med. Assn., 68, 1163-1165.

De NoBle. 1899. Quoted from Bolduan, 1916.

Dickson, E. C. 1915. Botulism, an Experimental Study. Jour. Amer. Med. Assn., 65, 492-496.

Durham, H. E. 1898. On an Epidemic of Gastro-enteritis Associated with the Presence of a Variety of the Bacillus enteritidis (Gaertner). Brit. Med. Jour., 1898-I.

Durham, H. E. 1899. Infection by Unsound Meat. Brit. Med. Jour., $1899-\mathrm{I}, 1216$.

Feтtick, O. 1908. Quantitative und Qualitative Untersuchungen über die Bakterien, Hefen, und Pilze der Butter und uber den Einfluss des Kochsalzgehaltes auf dieselben. Cent. Bakt. Abt. II., 22, 32.

Fischer, A. 1906. Ueber eine Massenerkrankung an Botulismus infolge Genusses "verdorbener." Zeit. f. klin. Med., 59, 58-77.

Fowler, G. E. P. 1909. Outbreak of Food Poisoning Following a Christmas Dinner. Jour. Roy. Army Medical Corps, 13, 271-274.

Freytag, C. L. DE. 1890. Ueber die Einwirkung konzentrierter Kochsalzlosungen auf Bakterien. Arch. Hyg., 11, 60.

Giltner, W. and Baker, J. D. 1915. Effect of Salt on Butter Flora. Mich. Sta. Rept., 1915, 209.

Gould, R. G. 1917. Commercial Dehydration. Amer. Food Journal, $373-375$.

Hilliard, C. M. 1918. The Fish Canning Industry. Amer. Jour. Pub. Health, 8, 202-204.

Hite, B. H., Giddings, N. J. and Weakiy, C. E. 1914. The Effect of Preqsure on Certain Microorganisms Encountered in Preserving Fruits and Vegetables. West Virginia Ag. Exp. Sta. Bulletin 146.

Hoffenreich, E. 1914. Investigations of the Presence of Bacteria in Places where Meat is Slaughtered and Sold with Special Reference to the Paratyphoid-Gaertner Group. Tierarz. Zentrl., 37, 337-346.

Hofrman, C. and Evans, A. 1911. The Use of Spices as Preservatives. Jour. Ind. Eng. Chem., 3, 835-838.

Howard, B. J. 1911. Decomposition and its Microscopical Detection in Some Food Products. Year Book, U. S. Dept. Agriculture, 1911, 297-308.

Howard, B. J. 1911. Tomato Ketchup under the Microscope. U. S. Dept. Agriculture, Bureau of Chemistry Circular 68.

Howard, B. J. and Stevenson, C. H. 1917. Microscopical Studies on Tomato Products. U. S. Dept. Agriculture Bull. 581.

Howard, B. J. and Stevenson, C. H. 1917. The Sanitary Control of Tomato Canning Factories. U. S. Department of Agriculture Bull. 569.

JACOBItz and Kayser, H. 1910. Bacterial Food Poisoning. Cent. Bakt. Abt., 1. Orig., 53, 377-378.

Jordan, E. O. 1917. Food Poisoning. The University of Chicago Press.

Jordan, E. O. 1918. Food-borne Infections. Science, 47, 80-86. 
Inaraffa-Korbutt, K. 1912. The Influence of Common Salt on the Life and Growth of Microorganisms. Zeit. IIyg., 71, 162-170.

Landmann, G. 1904. Ueber die Ursache der Darmstädter Bohnenvergiftung. Hyg. Rund., 14, 449-452.

Larson, W. P., Hartzeld, T. B. and Diehl, H. S. 1918. The Effect of High Pressures on Bacteria. Journal of Infectious Diseases, 22, 271-279.

Lewandowsky, F. 1904. Ueber das Wachstum ron Bakterien in Lablösungen von höher Konzentration. Arch. Hyg., 49, 47.

MacFayden, A. 1900. On the Influence of the Temperature of Liquid Air on Bacteria. Lancet, 1900-I., 849, 1130.

Martz, Hans. 1917. A Typhoid Carrier for Fifty-five Years. Zeit. Hyg., 80, No. 3. Quoted from Amer. Jour. Pub. Health, 7, 431.

Minchin, W. C. 1917. The Germicidal and Therapeutic Action of Garlic. Med. Press and Circular 103, 493-495.

Mrtcheld, O. W. H. and Bloomer, G. T. 1914. Experimental Study of the Chicken as a Possible Typhoid Carrier. Jour. Med. Res., 31, 247-250.

Múlder, M. 1914. The Value and Purpose of Animal Experimentation in Meat Examination. Zeit. Hyg., 16, 11.5-138.

MúlleR, R. 1914. Fish Poisoning by Bacteria of the Paratyphoid-enteriditis Group. Münch. med. Wochenschr., 61, 471-473.

Nelson. 1910. The Pungent Constituent of Pepper. Jour. Ind. Eng. Chem., 2,419 .

Pennington, M. E. 1911. The Comparative Rate of Decomposition in Drawn and Undrawn Market Poultry. U. S. Dept. Agriculture, Bureau of Chemistry, Circular 70.

Pennington, M. E., et al. 1917. The Influence of Temperature above Freezing on the Changes in Composition, Bacterial Content and Histological Structure of the Flesh of the Common Fowl. Jour. Biol. Chem., $29,31,32$.

Pernot, E. F. Experiments on the Canning of Fruits. Oregon Ag. Exp. Sta. Bull. 81.

Petrersson, A. 1900. Experimentelle Untersuchungen über das Conservieren von Fisch und Fleisch mit Salzen. Arch. Hyrg., 37, 171.

PFute, E. 1904. Beitrag zur bakteriologischen Untersuchung der Fleischkonserven. Zeit. Hyg., 48, 121.

Poels and Dhont. 1894. Quoted from Bolduan, 1916.

Prescott, S. C. 1900. The Bacteriology of Canned Foods. Science, 11, 442.

Prescott, S. C. and Underwood, I. 1897. Microorganisms and Sterilizing Processes in the Canning Industry. Tech. Quarterly.

PrescotT, S. C. and Underwood, L. 1898. Contributions to our Knowledge of the Microorganisms and Sterilizing Processes of Canning Industries. Tech. Quarterly.

Prescott, S. C., Burrage, S., and Philbrick, B. G. 1917. Some Sources of Error in the Microscopical Examination of Certain Food Products. Abstracts of Bacteriology, 1, 51 . 
RaHN, O. 1913. Bacteriology of Foods on a Physiological Basis. Cent. Bakt. Abt. II., 37, 492-497.

Rahn, O., Brown, C. W. and Smith, L. M. 1909. Keeping Qualities of Butter II, and III. Mich. Ag. Exp. Sta. Tech. Bull. 2.

Rapin. The Microbic Flora of Salt as a Cause of Butter and Cheese Defects. Milk Zeitung, 20, 433.

Round, L. A. 1916. Normal Fermentation of Sauerkraut. Absts. Bact., 1, 108.

Round, L. A. 1917. The Bacteriology of Sauerkraut, a Further Study. Abstracts of Bacteriology, 1, 50 .

Ruata, A. Q. 1918. Action of Cold on Microorganisms. Annali d'Igiene Rome, 28; Jour. Amer. Med. Assoc., 70, 1125 (1918); Amer. Jour. Pub. Health, 8 (1918), 399.

SADLER, W. 1918. The Bacteriology of Swelled Canned Sardines. Amer. Jour. Pub. Health, 8, 216-220.

Sedowick, W. T., Hamidton, H. W. and Funk, F. J. 1917. Experimental Studies on the Effects of Various Solutions upon the Viability of Bacteria at Low Temperatures. Abstrants of Bact., 1, 49.

Seele, W. 1913. Cases of Food Poisoning Noted in the German Army During the Last Two Years. Inaug. Dissert. Univ. Berlin. Hyg. Rund., $24,531,532$.

Serkowski, S. and Tomczer, P. 1911. The Influence of Common Salt on the Bacteria which Cause Meat Poisoning. Zeit. Untersuch. Nahr. Genussmitel, 21, 211-216.

StADLER, E. 1899. Ueber die Einwirkung von Kochsalz auf Bakterien die bei den sog. Fleischvergiftungen eine Rolle spielen. Arch. Hyg., 35, $40-82$.

Trom, C. 1914. The Salt Factor in the Mold Ripened Cheeses. Storrs Agric. Exp. Sta. Bull. 79, 387-394.

Trom, C. and Shaw, R. H. 1915. Moldiness in Butter. Jour. Ag. Research, $3,301-310$.

Werchel, A. 1910. The Effect of Salt upon Bacteria of the Ptomaine Group. Arb. kaiserl. Gesundh., 34, 247-265. Chem. Abstracts, 4, 3261

Weigmann, H. 1913. Germ Content of Salt. Jahresbericht. Vers. Stat. Molkw. Landw. Kammer. Schleswig-Holstein, 1913, 9, 10.

WrLbur, R. L. and Ophuls, W. 1914. Botulism. A report of food poisoning apparently due to eating of canned string beans with a report of a fatal case. Arch. Intern. Med., 589. 


\section{CHAPTER XV}

\section{EPIDEMIOLOGY}

No attempt will be made here to present a complete discussion of the subject, but merely a few remarks about epidemics and the method of investigating them. Boudreau (1914) has defined an epidemic as an unusual number of cases of a communicable disease, arising within a short period, in a limited area, and traceable to a common source or sources. The food bacteriologist may often find it necessary to investigate the cause of an epidemic either to protect an innocent article of food or to check the spread of infection. It is by no means an easy task in some epidemics to pick out the real cause; in others, by using a logical procedure, the epidemiologist may select with great accuracy the focus of the infection and see the reward of his efforts by the immediate decrease of the number of cases. He must be on his guard for any and all information which may help him towards his goal.

Different methods have been used in the past by epidemiologists. It used to be customary for him to use indirect methods in securing his data. If called to a community where there was an unusual amount of disease, he would go equipped with sample bottles for the collection of specimens of milk and water and would spend much time in the early part of his investigation in inspecting water supplies, methods of sewage treatment, etc. The mode of attack is now quite different and marks one of the distinct advances which preventive medicine has made. The present practice in investigating epidemics has been well stated by Hill (1912). A certain amount of information is always necessary with regard to the characteristics of the disease and some of the different methods of spreading. Certain characteristics have been worked out for epidemics caused by food, water, carriers, etc. These are by no means constant but serve in a general way along with such other general data such as, population of the community, endemicity of the disease in the community, general appearance, etc. If this preliminary, general study is well made the epidemiologist may save much time when he comes to analyze his other data because suggested causes of the infection may be quickly eliminated. 
Milk-borne Epidemics. The character of this article and the type of consumers gives a milk-borne epidemic of infection rather marked characteristics. Newman (1904) has mentioned the following:

(a) There is a special incidence of the disease upon the track of the implicated milk supply. It is localized to such area.

(b) Better class of houses and persons suffer most.

(c) Milk drinkers are chiefly affected and they suffer most who are large consumers of raw milk.

(d) Women and children suffer most and frequently adults suffer proportionately more than children.

(e) Incubation periods are shortened.

(f) There is a sudden onset and rapid decline.

(g) Multiple cases in one house occur simultaneously.

(h) Clinically the attacks of the disease are often mild. Contact infectivity is reduced and the mortality rate is lower than usual.

The above characteristics as outlined by Newman (1904) for milkborne epidemics also fit other food epidemics. Sawyer $(1914,1915)$ reported investigations of two typhoid epıdemics, one caused by water and the other caused by food. Fig. 84 shows the incidence of cases for both epidemics. These curves show very well the types which are secured for food and water-borne epidemics of typhoid fever.

Water-borne Epidemics. Such epidemics of disease have been common in the past and many such epidemics are described in the literature. The important characteristics of such epidemics may be described as follows:

(a) Water-borne epidemics may be preceded by a period of dysentery.

(b) There is usually a slow onset of the epidemic and a rapid decline.

(c) The cases are evenly distributed over the city if the city is served by a municipal supply.

(d) The larger water-borne epidemics have occurred in the spring.

Epidemics caused by other foods will show, in general, other characteristics. If the typhoid fever is caused by oysters, it will be confined to that part of the year when oysters are usually eaten. Furthermore, the great majority of the cases will be primary cases-those who have partaken of the oysters. To detect carriers may require much inferential evidence and the elimination of other suggested causes. After this has been done, the indication that a carrier is concerned must be supported by bacteriological examinations of the urine and feces of the suspected individual. 


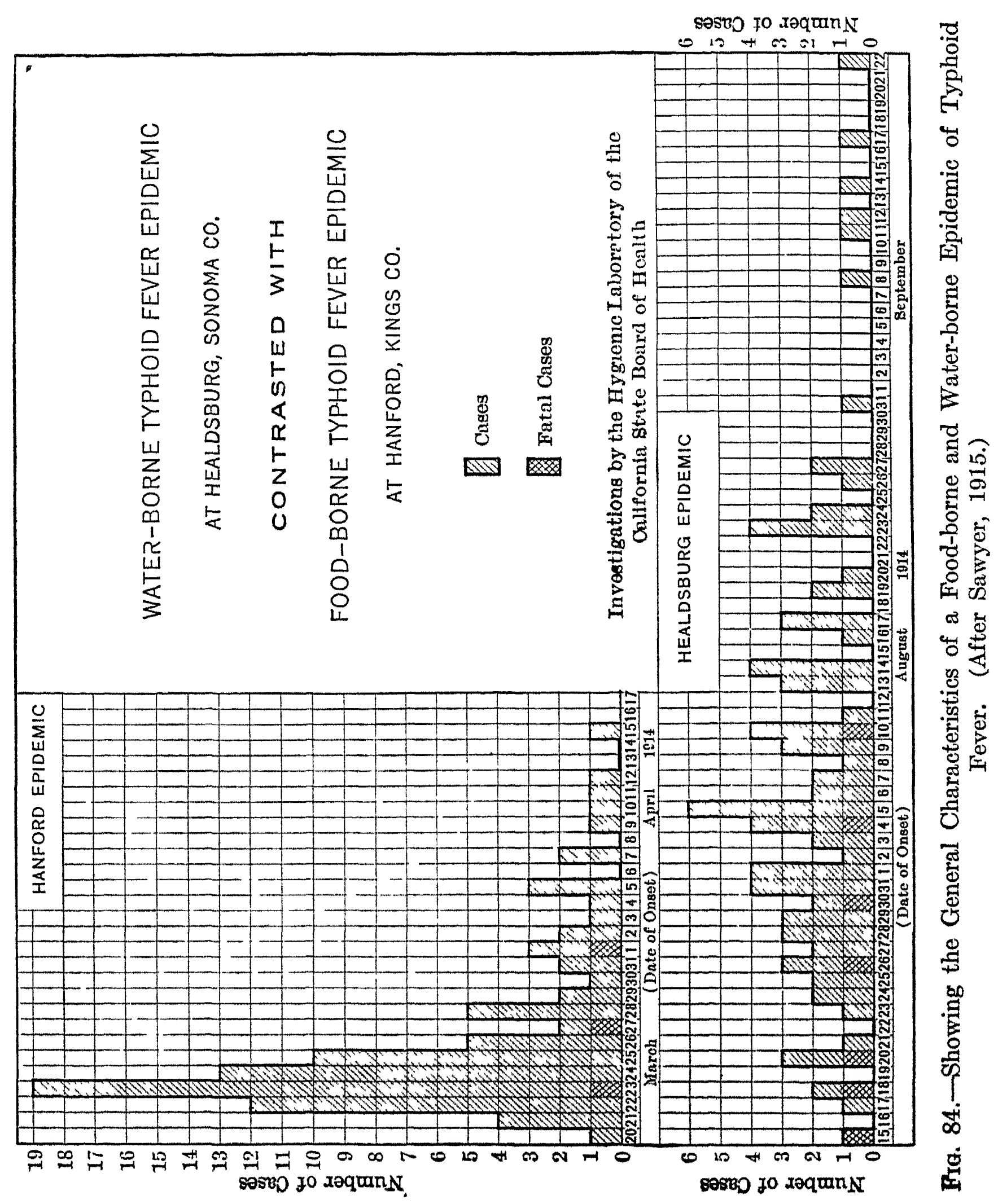




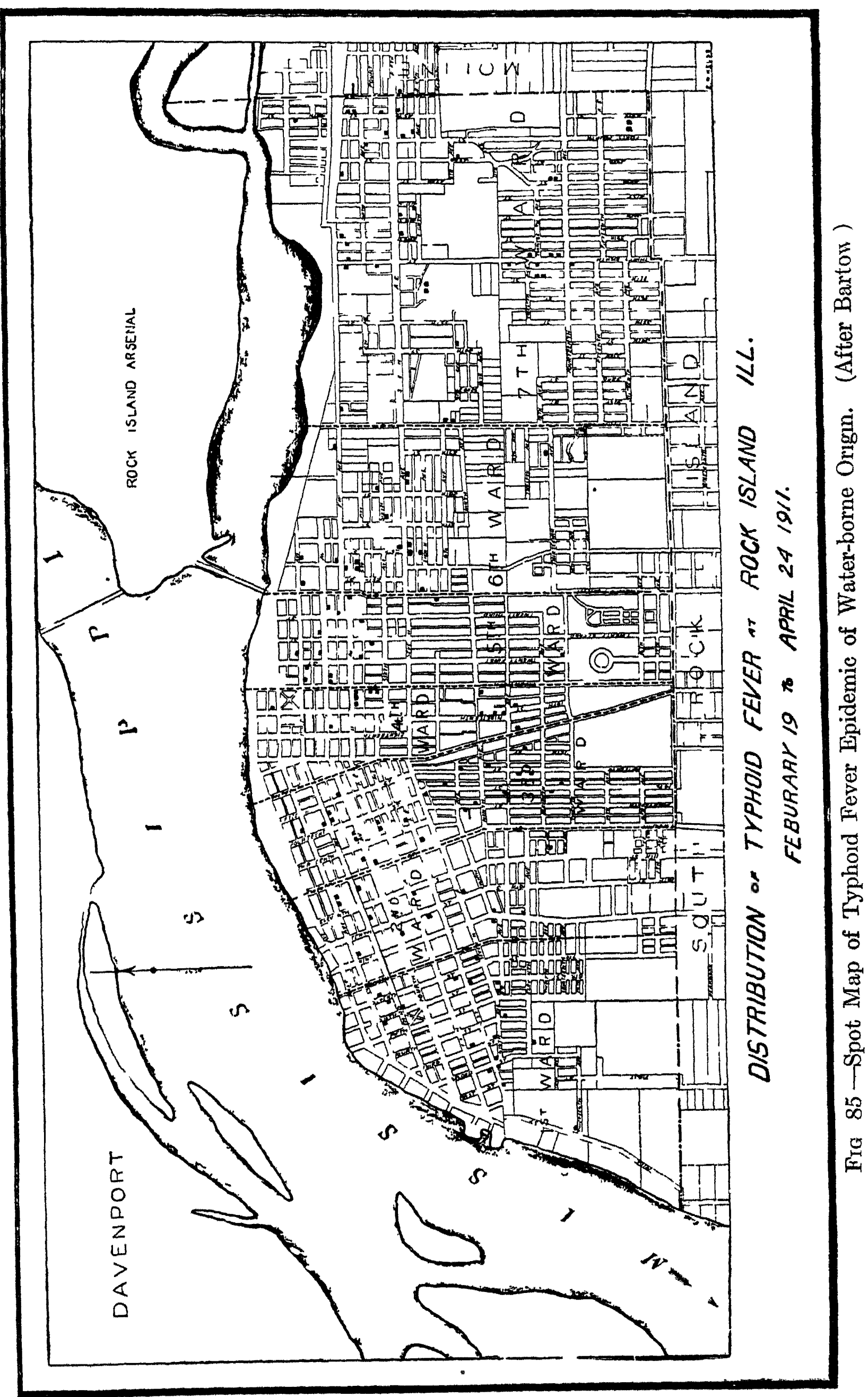


Determination of the Origin of an Epidemic. In the majority of epidemics, the foci may be discovered with a fair degree of accuracy. Hill (1912) has given the detailed procedure and part of his paper is reproduced below. On account of the standing of this authority in this field, the author feels justified in quoting rather extensively from his work. In the last analysis, the epidemiologist must be a many-sided man; he must be somewhat of a bacteriologist, psychologist, and detective. He must have the intuition to separate the essential from the non-essential data. Then, somewhat like the lawyer, he should have studied reported epidemics in order to determine how others have been investigated.

"To illustrate the general principles, let us suppose notification be received that a typhoid fever outbreak exists in a far-off community. The public health detective packs his grip and goes. He knows no details; he has never heard of this particular community before; he has not even any general information about the character of the country; he enters the community with no preconceived ideas. But he does know how typhoid fever originates and how it spreads. Water, milk, food, flies, and fingers are the routes-typhoid cases or typhoid carriers the source. His duties are to find both; and to find them, not as a scientific amusement, or as a matter of record; not to furnish food for speculation-above all not to make a show of doing something-but to stop the outbreak; and then to advise measures to prevent recurrence.

"The public health detective on entering the community affected by typhoid fever does not first examine the water supply, the milk supply, the sewage disposal system, the markets, the back alleys, the dairies or anything else. He goes directly to the bedsides of the patients. Of course he must obtain the names and addresses of the patients from someone-from the local health officer, if he has them; from the attending physicians, if the health officer has no list; from the lay citizens themselves, if no one else is immediately available. The more complete the list, the faster he can work, because then he is not compelled to hunt up the cases personally. But if there be no list, he begins making one himself. His intention is to see just as many patients as he can, for each furnishes evidence and he wants it all. But he knows that it is not always necessary at this stage to see absolutely all the patients, so long as he sees the majority.

"Reaching the patient's * bedside, his investigation begins. Auto-

* If the patient is a child or delirious or not strong enough for an interview, or speaks only some foreign tongue, the relatives, friends or associates must supply the information. 
matically, almost mechanically, he decides whether or not the patient has typhoid fever or not. Satisfied on that point, his first question is not, "Tell me all the different water supplies you have used, or all the sources of milk you have used.' The first question is, 'When did you first show the earliest symptoms of the disease?' Why? Because this date once fixed, the date at which infection entered the patient's mouth is fixed also, i.e., a date between one and three weeks previous to the date of earliest symptoms.* Remember that at this stage the detective may not have even an inkling as to which of the usual factors, water, milk, food, thies or fingers, is involved. Still less can he guess which particular water supply, milk supply, etc., of the many possible ones, may be the guilty one. But the answer to this question reduces possible routes to those used by this patient-not at anytime-but during a specific period, i.e., from one to three weeks preceding his date of earliest symptoms.

"Not yet, however, are the milk and water questions offered. The second question is, 'Where were you during that period?' Why? Because if the patient were not in the community during that period, he could not have contracted his infection within it, and does not belong to the outbreak under examination at all but to some other. $\mathrm{He}$ is, in brief, an 'imported case' and while, of course, he is to be supervised lest he spread his infection to others, he cannot help to locate the source of the main outbreak-unless perchance he be himself that source, i.e., the introducer to the community of the original infection. If he be an imported case he is noted for further reference and the detective goes to another patient. If not, the questions continue. But not yet is water or milk or flies mentioned. The third question is 'Were you associated during your period of infection with any then known typhoid case?' Why? Because such association, especially if intimate, makes it more than probable that the case under examination received his infection from the preceding case, rather than from any general route and that he is, therefore, a 'secondary' case. If he had such associations, this is noted for further reference and the investigator passes on to another bedside. If not, the questions continue and now, at last, take up milk, water, food, etc., but, of course, only so far as to determine those used by the patient during his infection period.

"Then the investigator passes to the next patient. What has he learned so far? Nothing much yet. But he has narrowed the possible

* The occasional exceptions do not affect the validity of this statement as a practical working rule. 
routes of infection to certain water supplies, certain milk supplies, ccrtain food supplies, etc., i.e., those used by the first patient durng a certarn period, and he has done this in thirty minutes-in scarcely the time it takes for the old-style investigator to get his bottles ready to collect his first water sample.

"At the bedside of the second patient, the same inquiries in the same order are made. If this second patient be an imported case, or a secondary case, he also is merely noted for future reference. If he be a primary, however, the origins of his drinking water, milk, food, etc., during his infection pcriod are also ascertained. Perhaps he coincides with the first patient in every detail of alimentary supplies, in history and associations. If so, nothing much has been added to the detective's knowledge. But more than likely, dissimilarities have developed. Since the responsible water supply, milk supply, etc., must be one of those water supplies, milk supplies, etc., used in common by primary cases all those not common to both of these primary cases may be dropped from consideration (except in rare instances of multiple routes). Thus, if both have used the same water, water from that origin remains as a possibility. But if the water supplies have been different, water is eliminated from the question entirely. If the milk supplies are identical, milk remains as a possible route of infection; if not, milk is eliminated from the question eniirely.

"In brief, provided the information obtained be reliable, and it is a part of the public health detcctive's training to distinguish at a glance truth from falsehood, the honestly mistaken, or forgetful, or stupid replies from the reliable ones-and above all never to believe anything (to the extent of recording it) unless it is checked, confirmed and established as a fact, the modern investigator has in one hour narrowed his investigation to a point which the old-style investigator often would not reach for weeks.

"And so from patient to patient the inquiry proceeds. In the course of the day the investigator has seen perhaps thirty patients. The tabulation (probably already made in his own mind) shows, say, three imported cases, five secondaries, two uncertain or indefinite. The remaining primary cases show in common, say, one water supply only, the milk, etc., varying; or one milk supply only, the water, etc., varying; or no connection except attendance at some one social function.

"Going straight to the route thus indicated, the public health detective quickly confirms the indications of his results. $\mathrm{He}$ knows that the route indicated must be the guilty one for only that route can account for all the cases. He concentrates on that route until 
the evidence is complete- when and how that route became infected, when and by what sub-routes the infection was distributed, why it infected the patients found and not others, etc.

"In this illustration I have assumed complete ignorance on the part of the epidemiologist as to everything connected with the community he is investigating, except what he finds by cross-examining the pationts. As a matter of fact, every epidemiologist, however much a stranger to the particular community he enters, begins to learn about it from the moment he enters it.

"Thus almost unconsciously he notes the size of the town and comparcs it with the number of cases reported as existing; if it is summer time he almost automatically notes the presence or absence of open toilets in the back yards, of manure piles and of garbage cans-all bearing upon fly infection. If it is winter time or the community be well sewcred, he does not even consider flies. If the cases are grouped in one quarter of the town, while the public water supply extends all over it, he tentatively eliminates the water supply, before he asks a question. If good surface drainage and a sandy soil exist, or driven wells are chiefly in vogue, he tentatively eliminates well water-even before he registers at the hotel.

"This is not and cannot be a complete synopsis of all the combinations of circumstances which the epidemiologist meets. It is intended to illustrate his methods and to show why they are incredibly rapid and incredibly accurate-how they eliminate speculation and guarantee a correct solution-which means of course the achievement of the great end, the finding of proper measures for suppression.

"As soon as the route is indicated, he must go to that route, and establish beyond peradventure that it was in truth responsible. A water supply cannot convey typhoid if typhoid fever discharges have not entered it. There is no object in attributing an outbreak to fly infection from toilets into which typhoid feces have not been discharged at such a time as to account for the cases. A milk supply, not handled at some point by an infected person, nor adulterated at some time with infected extraneous matter cannot convey typhoid. Whatever his results, they cannot be true unless they are consistentthey should not be accepted unless they are provable-and proved.

"If the public health detective is familiar with the community where the outbreak occurs, including its water supplies, its milk supplies, the sociological relationships of its people, etc., etc., he can often tentatively determine the cause of the outbreak by a mere inspection of the names and addresses of primary cases, especially if plotted on 
a map of the community, taking into account also the time of year, and other general points. But such deductions, while often wonderfully reliable, can never be as conclusive and satisfactory as are the results of an investigation by even a total stranger, if the investigation be conducted as above described."

The data received from each patient should be recorded on a case card. Many different cards have been described in the literature. The one given below has been used by the Illinois State Water Survey. Others have been described by Hill (1912), Boudreau (1914), Ferguson (191) and Hansen (1914).

Suggested Form for Securing Information Relative to Typhoid Fever Cases by the Local Health Authorities.

Record No.

\section{TYPHOID FEVER RECORD}

1. Name of Patient.

2. Residence, Street and Number

3. Age.

4. Sex.

5. Give Occupation and Place of Business.

6. Name of Physician

7. Date when Patient Took to Bed

8. Date of First Symptoms. .

9. Date of Physician's First Visit.

10. Date of Leaving Bed on Recovery

11. Date of Relapse.

12. Date of Death

13. Was Widal Test Made?

Date.

Positive or Negative

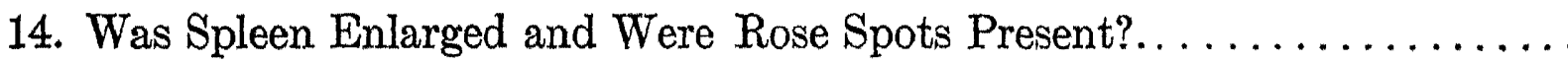

15. Was Patient Absent from City During the Two Months Previous to Illness?............... If so, state where he visited and between what dates.

16. If Patient Changed Residence During Two Months Previous to Illness, Give Former Address and Date of Change.

17. Character of Residence (private house, boarding house, apartment, hotel, etc.).

18. State Source of Drinking Water. At Home, at Place of Business, Elsewhere. 
19. State Source of Milk Supply and Name of Dealer. Was Milk Habitually Drunk? Was it Occasionally Used, as in Tea or Coffee or in Ice Cream?

20. Were Raw Oysters, Lettuce or Celery Eaten Raw within Three Weeks before Illness?

21. State from Whom Bread was Obtained.

22. Did Patient Fish or Bathe in Neighboring Streams?

If so, State at what Point or Points.

23. Did Patient use any Public Baths or Swimming Pools?

If so, give Dates.

24. Give Number of Persons Living in Same House or Apartment. Also Give Number in Whole Building in Case of Hotel, Apartment House, etc..

25. Give Names of Other Cases in Same Building and Dates when they Went to Bed....

26. Give Names and Approximate Dates for Cases among Business Associates

27. Give Names and Approximate Dates for Cases among Friends (Include Schoolmates)

28. Did Patient Attend any Social Gatherings where Food was Served within Two Months before Illness? If so, Gives Dates.

29. Describe Sanitary Condition of Premises. Note Especially Outdoor Privies, Cesspools, Wells and Refuse Piles.

30. Is House Well Screened?

31. Had Patient a Separate Room?...

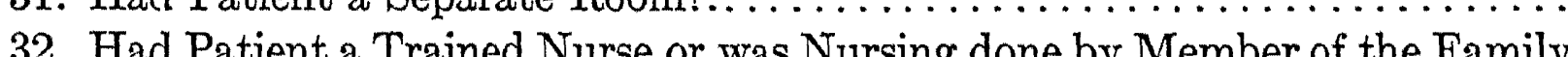
or Household?

33. Where Stools and Urine Disinfected? If so, Describe How this was Carried Out and Note Length of Time Continued.

Remarks. To Include any Facts, Statements or Comments not Included in the Foregoing that May Throw Light on the Source and Mode of Infection

Information Given by

Information Obtained by ...

Date of Obtaining Information. 


\section{APPENDIX I. FORMS FOR TYPHOID INVESTIGATIONS}

Suggested Form for Physicians' Report on Typhoid Fever

TYPHOID FEVER

Name

Street and Number

Sex.

Age. . .

Place of Business.

Occupation.

Physician.

Date of Taking to Bed.

Date of First Symptons.

Date of Physician's First Visit.

Was Widal Test Made?............ Positive or Negative

Was Patient Out of Town within Thirty Days?.

Between what Dates.

Are There Other Cases in House?

If So, How Many.

State Source of Drinking Water.

Give Name of Milk Dealer.

Remarks.

The preparation of a spot map is very often an aid in studying epidemics. If the cases are about evenly distributed over a city it is a fair indication that the water supply is to blame. If the cases are grouped in several localities, it may indicate that a milk supply or a carrier is to blame. Fig. 84 shows such a map which was prepared by Bartow (1912) for a water-borne epidemic.

\section{BIBLIOGRAPHY}

Bartow, E. Discussion to Paper by Hill (1912).

Boudreau, F. G. 1914. The Mode of Procedure in the Study of Epidemics. Ohio State Board of Health Bull. 4, 1277-1286.

Ferguson, H. F. 1916. Epidemic of Typhoid Fever Caused by Polluted Water Supply at Old Salem Chautauqua. Illinois State Water Survey Bulletin No. 13, 272-286.

Hansen, P. 1914. A Study of Typhoid Fever in Rockford, Illinois, in the Late Summer and Fall of 1913. Illinois State Water Survey Bull. No. 11, $384-430$. 
Hrus, H. W. 1912. The Detailed Procedure to be Followed in an Epidemiological Determination of the Origin of a Typhoid Outbreak. Proceedings Illinois Water Supply Assn, 1912, 96-105.

Jordan, E. O. and Irons, E. E. 1912. The Rockford (Ill.) Typhoid Epidemic. Jour. Inf. Diseases, 11, 21-43.

SAwYeR, W. A. 1914. Ninety-three Persons Infected by Typhoid Carrier at a Public Dinner. Jour. Amer. Med. Assn., 63, 1537-1542.

SAwYer, W. A. 1915. A Water-borne Typhoid Fever Epidemic. California State Board of Health, 10, 303-311. 



\section{APPENDIX}

Indicator Solutions. The following table is taken from 'Hawk's Physiological Chemistry and will be found useful in bacterial work. The amounts of the indicator solutions and their strengths to be used in the determination of hydrogen ion concentrations in 10 c.c. portions of unknown solution are indicated below:

INDICATOR SOLUTIONS

\begin{tabular}{|c|c|c|}
\hline & Drops. & Preparation of Solution. \\
\hline 1. Alizarin & & \\
\hline acid) & $10-5$ & $0.1 \mathrm{gm}$. in 1000 c.c. water. \\
\hline 2. Azolitmin (litmus).. & & Aqueous solution. \\
\hline 3. Cochineal... & & Alcoholic solution. \\
\hline 4. 2.5-dinitro-hydrochinone. . & $5-2$ & $1 \mathrm{gm}$. to 1000 c.c. alcohol. \\
\hline 5. Mauvein.. & $8-1$ & $0.5 \mathrm{gm}$. to 1000 c.c. \\
\hline 6. Methyl orange.. & $5-3$ & 0.1 recrystallized salt to 1000 c.c. water. \\
\hline 7. Methyl red.... & $4-2$ & Saturated solution in 50 per cent alcohol. \\
\hline 8. Methyl violet. & $8-1$ & $0.5 \mathrm{gm}$. in 1000 c.c. water. \\
\hline 9. Neutral red... & $20-10$ & $\begin{array}{l}0.1 \mathrm{gm} \text {. in } 500 \text { c.c. alcohol and } 500 \text { c.c. } \\
\text { water. }\end{array}$ \\
\hline 10. $p$-nitr & $20-3$ & 0.4 gm. to 60 c.c. alcohol, 940 c.c. water. \\
\hline 11. Phen & $20-3$ & 0.5 gm. to 500 c.c. water, 500 c.c. alcohol. \\
\hline 12. Roso & $15-6$ & $0.4 \mathrm{gm}$. in 400 c.c. alcohol, 600 c.c. water. \\
\hline 13. Thym & 10-3 & 0.4 gm. to 500 c.c. alcohol, 500 c.c. water. \\
\hline 14. Tropaeolin O... & $10-5$ & $0.1 \mathrm{gm}$. to 1000 c.c. water. \\
\hline 15. Tropaeolin OO ... & $5-3$ & $\begin{array}{l}\text { Of recrystallized salt, } 0.1 \mathrm{gm} \text { to } 1000 \text { c.c. } \\
\text { water. }\end{array}$ \\
\hline 16. Tropaeolin 000. & $10-4$ & $0.1 \mathrm{gm}$. to 1000 c.c. water. \\
\hline
\end{tabular}

In an extensive study of the application of H-ion determination to bacteriological work, .Clark and Lubs (1917) have pointed out the error in determining reaction by titration. They used the electrometric method and by means of it prepared a series of new indicators 
which will be valuable in future bacteriological work. They are given in Table LII.

TABLE LII

IIST OF INDICATORS, PREPARED BY CLARK AND LUBS, 1918

\begin{tabular}{|c|c|c|c|c|}
\hline Chemical Name. & Common Name. & $\begin{array}{l}\text { Concen- } \\
\text { tration, } \\
\text { Per Cent. }\end{array}$ & Color Change. & $\begin{array}{c}\text { Range } \\
P_{H}\end{array}$ \\
\hline 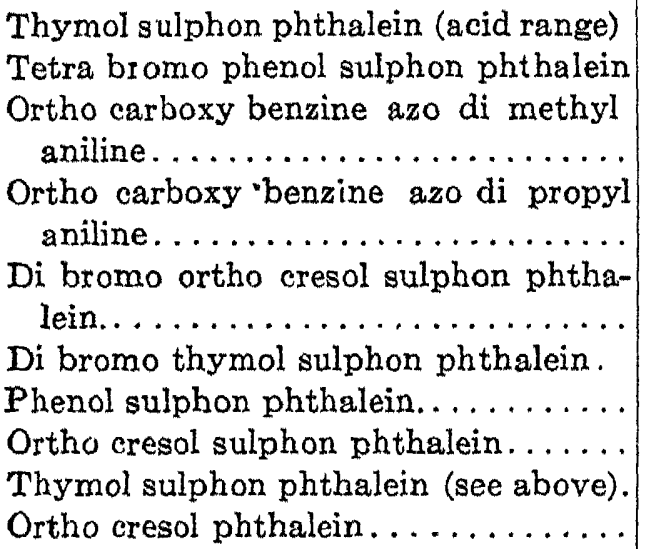 & $\begin{array}{l}\text { Thymol blue..... } \\
\text { Brom phenol blue } \\
\text { Methyl red....... } \\
\text { Propyl red....... } \\
\text { Brom cresol purple } \\
\text { Brom thymol blue. } \\
\text { Phenol red....... } \\
\text { Cresol red........ } \\
\text { Thymol blue...... } \\
\text { Cresol phthalein... }\end{array}$ & $\begin{array}{l}0.04 \\
0.04 \\
0.02 \\
0.02 \\
0.04 \\
0.04 \\
0.02 \\
0.02 \\
0.04 \\
0.02\end{array}$ & $\begin{array}{l}\text { Red-yellow } \\
\text { Yellow-blue } \\
\text { Red-yellow } \\
\text { Red-yellow } \\
\text { Yellow-purple } \\
\text { Yellow-blue } \\
\text { Yellow-red } \\
\text { Yellow-red } \\
\text { Yellow-blue } \\
\text { Colorless-red }\end{array}$ & $\begin{array}{c}1.2-2.8 \\
3.0-4.6 \\
4.4-6.0 \\
4.8-6.4 \\
5.2-6.8 \\
6.0-7.6 \\
6.8-8.4 \\
7.2-8.8 \\
8.0-9.6 \\
8.2-9.8\end{array}$ \\
\hline
\end{tabular}

\section{Preparation of Standard Reagents}

A standard solution contains a known amount of reacting substance and is to be distinguished from a normal solution. A normal solution contains the hydrogen equivalent of the active constituent in grams per liter. This is that amount which brings into the reaction $1.008 \mathrm{gms}$. of hydrogen, 8 gms. of oxygen, etc., or the equivalent. This definition has so many distinct advantages that it is now generally accepted.

The following are some of the standard solutions used in bacteriology:

N Sodium Hydroxide. Weigh out 40.06 or better about $45 \mathrm{gms}$. of sodium hydroxide and dissolve in 1 liter of boiled cooled distilled water. Standardize against normal hydrochloric acid. It is best to allow the two solutions to stand at the same temperature for several hours or overnight. The titration should be repeated several times. Calculation is as follows:

If

$$
50 \text { c.c. } \mathrm{NaOH}=52.6 \text { c.c. } \mathrm{NHCl}
$$

the $\mathrm{NaOH}$ is too strong by 2.6 c.c. per every 50 c.c.

$$
50: 2.6=1000: x \quad x=\frac{2.6 \times 1000}{50}=50 . c . c .
$$




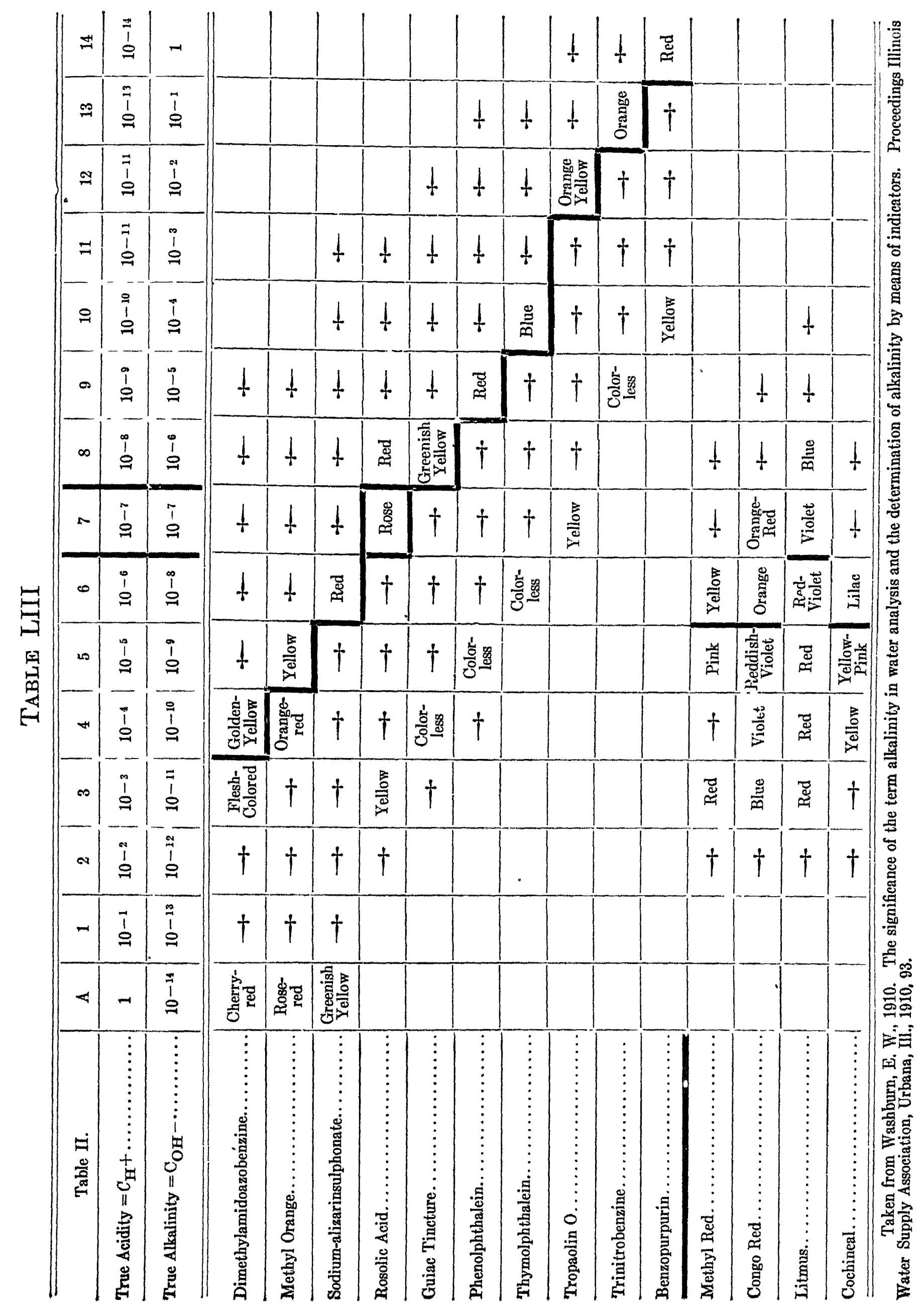


This amount of water should be added, after which the solution will be exactly normal. It should be checked, however, against the $\mathrm{N} \mathrm{HCl}$. From this the $\mathrm{N} / 20 \mathrm{NaOH}$ may be prepared by diluting 50 c.c. of the $\mathrm{N} \mathrm{NaOH}$ to a liter.

N Potassium Hydroxide. Dissolve 56 gms. of $\mathrm{KOH}$ in 1000 c.c. of distilled water and standardize against $\mathrm{N} \mathrm{HCl}$ as was done with $\mathrm{N} \mathrm{NaOH}$.

$\mathbf{N}$ Sodium Carbonate. Ignite the bicarbonate $\mathrm{NaHCO}_{3}$ to redness and dissolve 53 gms. in a liter of water. Standardize against normal acid and dilute accordingly with distilled water.

$\mathbf{N}$ Sulphuric Acid. Follow the procedure which has been given elsewhere for $\mathrm{N} \mathrm{HCl}$. This may be standardized against $\mathrm{N} \mathrm{NaOH}$ or gravimetrically by precipitating with $\mathrm{BaOH}$.

N Hydrochloric Acid. This should contain $36.46 \mathrm{gms}$. of $\mathrm{HCl}$ in a liter of water. The pure concentrated $\mathrm{HCl}$ may be diluted to a specific gravity of 1.020. According to the table on page 559, it will be seen that this solution is too strong, having about $4.13 \mathrm{gms}$. of $\mathrm{HCl}$ in a liter. To be made exactly normal, it should be titrated against pure $\mathrm{Na}_{2} \mathrm{CO}_{3}$. This is obtained by heating the $\mathrm{NaHCO}_{3}$ being careful not to allow it to fuse. This is then put into a weighing bottle from which it is weighed into Erlenmeyer flasks for titrating. Dissolve in boiled distilled water and titrate with the $\mathrm{HCl}$ solution using methyl orange as the indicator. Calculations are made as follows, assuming for example that 1.9864 gms. of $\mathrm{Na}_{2} \mathrm{CO}_{3}$ neutralized 37.28 c.c. of the $\mathrm{HCl}$ solution.

If our solution had been exactly normal 1 liter would have neutralized

$$
\begin{aligned}
\frac{\mathrm{Na}_{2} \mathrm{CO}_{3}}{2} & =\frac{106.00}{2}=53 \text { gms. of } \mathrm{Na}_{2} \mathrm{CO}_{3} \\
53: 1000 & =1.9864: x \\
x & =37.48 .
\end{aligned}
$$

37.28 c.c. of the $\mathrm{HCl}$ solution were required and it is evident that this solution is too strong and that for each 37.28 c.c. of the solution, $37.48-37.28=.20$ c.c. of water must be added. The amount of water to be added to each liter of the $\mathrm{HCl}$ solution to make it exactly normal is computed as follows:

$$
\begin{aligned}
37.28: .20 & =1000: x \\
x & =\text { amount of distilled water to be added. }
\end{aligned}
$$

$\mathrm{N}$ Nitric Acid. See preparation of $\mathrm{N} \mathrm{HCl}$.

N Oxalic Acid. Dissolve 47 gms. of pure dry oxalic acid in 1000 c.c. of distilled water. Standardize against $\mathrm{N} \mathrm{Na}_{2} \mathrm{CO}_{3}$. 
N/10 Potassium Permanganate. Dissolve $3.163 \mathrm{gms}$ in a liter of water and standardize against ferrous ammonium sulphate, oxalic acid or pure iron.

$$
\begin{array}{rlrl}
1 \text { c.c. }= & .0056 \text { gm. } \mathrm{Fe} & 1 \text { c.c. }= & .0008 \text { gm. O. } \\
& .0072 \text { gm. } \mathrm{FeO} & .0063 \mathrm{gm} . \mathrm{H}_{2} \mathrm{C}_{4} \mathrm{O}_{4} .2 \mathrm{H}_{2} \mathrm{O} . \\
& .008 \text { gm. } \mathrm{Fe}_{2} \mathrm{O}_{3} . &
\end{array}
$$

$\mathrm{N} / 10$ Iodine. Dissolve 12.685 or $12.8 \mathrm{gms}$. of pure iodine and 18 gms. of potassium iodide in a liter of distilled water. Standardize against $\mathrm{N} / 10 \mathrm{Na}_{2} \mathrm{~S}_{2} \mathrm{O}_{3}$.

$$
\begin{aligned}
1 \text { c.c. } & =.0158 \text { gm. } \mathrm{Na}_{2} \mathrm{~S}_{2} \mathrm{O}_{3}, \\
& =.00495 \mathrm{gm} . \mathrm{As}_{2} \mathrm{O}_{3}, \\
& =.00575 \mathrm{gm} . \mathrm{As}_{2} \mathrm{O}_{5} .
\end{aligned}
$$

$\mathbf{N} / 10$ Sodium Thiosulphate. Dissolve $24.9 \mathrm{gm}$. of the crystals in 1000 c.c. of water and standardize against $\mathrm{N} / 10$ iodine.

$\mathbf{N} / 10$ Sodium Chloride. Dissolve 5.9 of the salt in 1000 c.c. of water. Standardize gravimetrically by precipitating as $\mathrm{AgCl}$.

$$
\begin{aligned}
1 \text { c.c. } & =.0058 \mathrm{gm} . \mathrm{NaCl}, \\
& =.0035 \mathrm{gm} . \mathrm{Cl}, \\
& =.0108 \mathrm{gm} . \mathrm{Ag} .
\end{aligned}
$$

$\mathrm{N} / 10$ Silver Nitrate. Dissolve 16.966 gms. of $\mathrm{AgNO}_{3}$ in a liter of water and standardize by precipitating as $\mathrm{AgCl}$. The preparation of standard silver nitrate for water analysis is outlined in another place.

Preparation of Antiformin. Dissolve 720 gms. of chloride of lime and $260 \mathrm{gms}$. of sodium carbonate in 2500 c.c. of water. Allow to stand and filter. By means of $\mathrm{N} / 10$ sodium thiosulphate determine the amount of available chlorine and adjust so that it will contain about 5.6 per cent. Add 7.5 per cent of sodium hydroxide, filter and store for future use.

Azolitmin Solution. Add 1 per cent of Kahlbaum's azolitmin to distilled water and boil for five minutes. Adjust the reaction, if necessary, with $\mathrm{NaOH}$. It should give a distinct blue plate when it is diluted in the Petri dish with the medium.

Benedict's Reagent for the Quantitative Estimation of Dextrose.

Copper sulphate (crystallized)........ 18.00 gms.

Sodium carbonate (anhydrous)......... 100.00 gms.

Sodium citrate................ 200.00 gms.

Potassium thiocyanate........... 125.00 gms.

Potassium ferrocyanide (5 per cent solution). $\quad 5.00$ c.c.

Distilled water to make 1 liter. 
Dissolve the carbonate, citrate and thiocyanate in 800 c.c. of water and filter if necessary. Dissolve exactly $18 \mathrm{gms}$. of copper sulphate in 100 c.c. of distilled water. Add this to the first solution with constant stirring after which add the 5 c.c. of potassium ferrocyanide and dilute to a liter.

Diluting Fluid for Leucocytes. Prepare a 0.5 per cent solution of acetic acid and tinge slightly with gentian violet.

Hayem's Diluting Fluid for Erythrocytes.

Sodium sulphate $\left(\mathrm{Na}_{2} \mathrm{SO}_{4}\right) \ldots \ldots \ldots \ldots \ldots$ gms.

Mercuric chloride $\left(\mathrm{HgCl}_{2}\right) \ldots \ldots \ldots \ldots \ldots 0.5 \mathrm{gm}$.

Sodium chloride $(\mathrm{NaCl}) \ldots \ldots \ldots \ldots \ldots 1.0$ gms.

Distilled water................... 200 c.c.

Litmus or Azolitmin Solution. The standard solution of litmus shall be a 2 per cent solution of reagent litmus in water. Crush the litmus in a mortar with a little water and, after diluting to the mark, boil for five minutes. Distribute in flasks or tubes and sterilize. This solution generally needs no correction but may be adjusted with dilute $\mathrm{NaOH}$ or $\mathrm{HCl}$ if necessary.

Millon's Reagent. Dissolve 30 c.c. of mercury in 570 c.c. of concentrated nitric acid and dilute with two parts of water.

Molisch Reagent. Dissolve 15 gms. of $a$-naphthol in 95 per cent alcohol.

Nylander's Reagent. Boil 4 gms. of potassium hydrogen tartrate and 2 gms. of bismuth subnitrate in 100 c.c. of 10 per cent $\mathrm{KOH}$. When cool filter.

Phenylhydrazine Mixture. Thoroughly mix two parts of sodium acetate and one part of phenylhydrazine hydrochloride.

Seliwanoff's Reagent. Dissolve $.05 \mathrm{gm}$. resorcinol in 100 c.c. of dilute $\mathrm{HCl}$.

Toisson's Diluting Fluid for Erythrocytes.

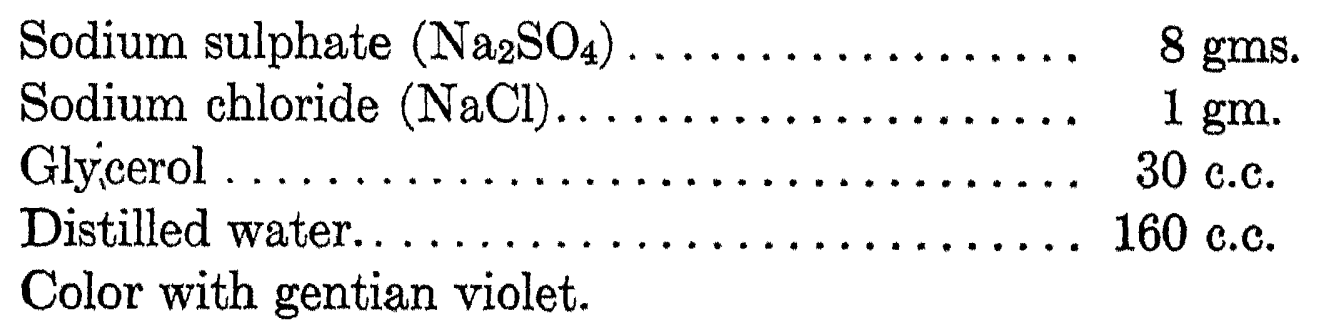

Uffelmann's Reagent. Add ferric chloride to phenol until a bluishviolet color results. 
SPECIFIC GRAVITIES OF HYDROCHLORIC, NITRIC, AND SULPHURIC - ACIDS

(After G. Lunge)

\begin{tabular}{|c|c|c|c|c|c|c|c|}
\hline \multirow{2}{*}{$\begin{array}{l}\text { Specific } \\
\text { Gravity } \\
\text { at } \frac{15^{\circ}}{4^{\circ}} \\
\text { (Vacuo). }\end{array}$} & \multicolumn{3}{|c|}{ Per Cent by Weight. } & \multirow{2}{*}{$\begin{array}{l}\text { Specific } \\
\text { Gravity } \\
\text { at } \frac{15^{\circ}}{4^{\circ}} \text {. } \\
\text { (Vacuo). }\end{array}$} & \multicolumn{3}{|c|}{ Per Cent by Weight. } \\
\hline & $\mathrm{HCl}$. & $\mathrm{HNO}_{3}$ & $\mathrm{H}_{2} \mathrm{SO}_{4}$ & & $\mathrm{HCl}$. & $\mathrm{HNO}_{3}$ & $\mathrm{H}_{2} \mathrm{SO}_{4}$ \\
\hline 1.000 & 0.16 & 0.10 & 0.09 & 1.225 & & 36.03 & 30.48 \\
\hline 1.005 & 1.15 & 1.00 & 0.83 & 1.230 & & 36.78 & 31.11 \\
\hline 1.010 & 2.14 & 1.90 & 1.57 & 1.235 & & 37.53 & 31.70 \\
\hline 1.015 & 3.12 & 2.80 & 2.30 & 1.240 & & $38 \quad 29$ & 32.28 \\
\hline 1.020 & 4.13 & 3.70 & 3.03 & 1.245 & & 39.05 & 32.86 \\
\hline 1.025 & 5.15 & 4.60 & 3.76 & 1.250 & & 39.82 & 33.43 \\
\hline 1.030 & 6.15 & 5.50 & 4.49 & 1255 & & 40.58 & 34.00 \\
\hline 1.035 & 7.15 & 6.38 & 5.23 & 1.260 & & 41.34 & 34.57 \\
\hline 1.040 & 8.16 & 7.26 & 5.96 & 1.265 & & 42.10 & 35.14 \\
\hline 1.045 & 9.16 & 8.13 & 6.67 & 1.270 & & 42.87 & 35.71 \\
\hline 1.050 & 10.17 & 8.99 & 7.37 & 1.275 & & 43.64 & 36.29 \\
\hline 1.055 & 11.18 & 9.84 & 8.07 & 1.280 & & 44.41 & 36.87 \\
\hline 1.060 & 12.19 & 10.68 & 8.77 & 1.285 & & 45.18 & 37.45 \\
\hline 1.065 & 13.19 & 11.51 & 9.47 & 1.290 & & 45.95 & 38.03 \\
\hline 1.070 & 14.17 & 12.33 & 10.19 & 1.295 & & 46.72 & 38.61 \\
\hline 1.075 & 15.16 & 13.15 & 10.90 & 1.300 & & 47.49 & 39.19 \\
\hline 1.080 & 16.15 & 13.95 & 11.60 & 1.305 & & 48.26 & 39.77 \\
\hline 1.085 & 17.13 & 14.74 & 12.30 & 1.310 & & 49.07 & 40.35 \\
\hline 1.090 & 18.11 & 15.43 & 12.99 & 1.315 & & 49.89 & 40.93 \\
\hline 1.095 & 19.06 & 16.32 & 13.67 & 1.320 & & 50.71 & 41.50 \\
\hline 1.100 & 20.01 & 17.11 & 14.35 & 1.325 & & 51.53 & 42.08 \\
\hline 1.105 & 20.97 & 17.89 & 15.03 & 1.330 & & 52.37 & 42.66 \\
\hline 1.110 & 21.92 & 18.67 & 15.71 & 1.335 & & 53.22 & 43.20 \\
\hline 1.115 & 22.86 & 19.45 & 16.36 & 1.340 & & 54.07 & 43.74 \\
\hline 1.120 & 23.82 & 20.23 & 17.01 & 1.345 & & 54.93 & 44.28 \\
\hline 1.125 & 24.78 & 21.00 & 17.66 & 1.350 & & 55.79 & 44.82 \\
\hline 1.130 & 25.75 & 21.77 & 18.31 & 1.355 & & 56.66 & 45.35 \\
\hline 1.135 & 26.70 & 22.54 & 18.96 & 1.360 & & 57.57 & 45.88 \\
\hline 1.140 & 27.66 & 23.31 & 19.61 & 1.365 & & 58.48 & 46.41 \\
\hline 1.145 & 28.61 & 24.08 & 20.26 & 1.370 & & $59 \quad 39$ & 46.94 \\
\hline 1.150 & 29.57 & 24.84 & 20.91 & 1.375 & & 60.30 & 47.47 \\
\hline 1.155 & 30.55 & 25.60 & 21.55 & 1.380 & & 61.27 & 48.00 \\
\hline 1.160 & 31.52 & 26.36 & 22.19 & 1.385 & & 62.24 & 48.53 \\
\hline 1.165 & 32.49 & 27.12 & 22.83 & 1.390 & & 63.23 & 49.06 \\
\hline 1.170 & 33.46 & 27.88 & $23: 47$ & 1.395 & & 64.25 & 49.59 \\
\hline 1.175 & 34.42 & 28.63 & 24.12 & 1.400 & & 65.30 & 50.11 \\
\hline 1.180 & 35.39 & 29.38 & 24.76 & 1.405 & & 66.40 & 50.63 \\
\hline 1.185 & 36.31 & 30.13 & 25.40 & 1.410 & & 67.50 & 51.15 \\
\hline 1.190 & 37.23 & 30.88 & 26.04 & 1.415 & & 68.63 & 51.66 \\
\hline 1.195 & 38.16 & 31.62 & 26.68 & 1.420 & & 69.80 & 52.15 \\
\hline 1.200 & 39.11 & 32.36 & 27.32 & 1.425 & & 70.98 & 52.63 \\
\hline 1.205 & & 33.09 & 27.95 & 1.430 & & 72.17 & 53.11 \\
\hline 1.210 & & 33.82 & 28.58 & 1.435 & & 73.39 & 53.59 \\
\hline 1.215 & & 34.55 & 29.21 & 1.440 & & 74.68 & 54.07 \\
\hline 1.220 & & 35.28 & 29.84 & 1.445 & & 75.98 & 54.55 \\
\hline
\end{tabular}

From Reed's Manual of Bacteriology, Ginn \& Co. 
SPECIFIC GRAVITIES OF HYDROCHLORIC, NITRIC, AND SULPHURIC ACIDS.-Continued

\begin{tabular}{|c|c|c|c|c|c|c|c|}
\hline \multirow{2}{*}{$\begin{array}{l}\text { Specifie } \\
\text { Gravity } \\
\text { at } \frac{15^{\circ}}{4^{\circ}} \\
\text { (Vacuo). }\end{array}$} & \multicolumn{3}{|c|}{ Per Cent by Weight. } & \multirow{2}{*}{$\begin{array}{l}\text { Gravity } \\
\text { at } \frac{15^{\circ}}{4^{\circ}} \\
\text { (Vacuo) }\end{array}$} & \multicolumn{3}{|c|}{ Per Cent by Weygth. } \\
\hline & $\mathrm{HCl}$. & $\mathrm{HNO}_{3}$ & $\mathrm{H}_{2} \mathrm{SO}_{4}$ & & $\mathrm{HCl}$. & $\mathrm{HNO}_{3}$. & $\mathrm{H}_{2} \mathrm{SO}_{4}$. \\
\hline 1.450 & & 77.28 & 55.03 & 1.670 & & & 74.51 \\
\hline 1.455 & & 78.60 & 55.50 & 1.675 & & 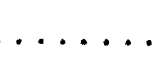 & 74.97 \\
\hline 1.460 & & 79.98 & 55.97 & 1.680 & & $\ldots$ & 75.42 \\
\hline 1.465 & $\ldots$ & 81.42 & 56.43 & 1.685 & $\ldots$ & $\ldots \ldots$ & 75.86 \\
\hline 1.470 & . & 82.90 & 56.90 & 1.690 & $\cdots$ & $\ldots \ldots$ & 76.30 \\
\hline i. 475 & . . & 84.45 & 57.35 & 1.695 & & $\ldots \ldots$ & 76.73 \\
\hline 1.480 & $\ldots$ & 86.05 & 57.83 & 1.700 & 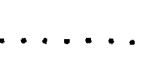 & $\ldots \ldots$ & 77.17 \\
\hline 1.485 & $\ldots$ & 87.70 & 58.28 & 1.705 & & & 77.60 \\
\hline 1.490 & $\ldots$ & 89.60 & 58.74 & 1.710 & & $\ldots$ & 78.04 \\
\hline 1.495 & 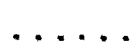 & 91.60 & 59.22 & 1.715 & $\cdots$ & $\cdots$ & 78.48 \\
\hline 1.500 & & 94.09 & 59.70 & 1.720 & $\cdots$ & $\ldots$ & 78.92 \\
\hline 1.505 & . & 96.39 & 60.18 & 1.725 & & $\ldots$ & 79.36 \\
\hline 1.510 & 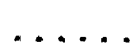 & 98.10 & 60.65 & 1.730 & & $\ldots$ & 79.80 \\
\hline 1.515 & . & 99.07 & 61.12 & 1.735 & & $\cdots$ & 0.24 \\
\hline 1.520 & . & 99.67 & 61.59 & 1.740 & & $\cdots$ & 80.68 \\
\hline 1.525 & .. & $\ldots \ldots$ & 62.06 & 1.745 & & $\cdots$ & 81.12 \\
\hline 1.530 & & & 62.53 & 1.750 & & & 81.56 \\
\hline 1.535 & & & 63.00 & 1.755 & & & 82.00 \\
\hline 1.540 & & $\cdots$ & 63.43 & 1.760 & & $\cdots$ & 82.44 \\
\hline 1.545 & & & 63.85 & 1.765 & & 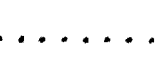 & 82.88 \\
\hline 1.550 & & & 64.26 & 1.770 & & & 83.32 \\
\hline 1.555 & & & 64.67 & 1.775 & & & 83.90 \\
\hline 1.560 & . & . & 65.08 & 1.780 & & $\ldots$ & 84.50 \\
\hline 1.565 & & 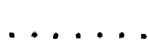 & 65.49 & 1.785 & & $\ldots$ & 85.10 \\
\hline 1.570 & & & 65.90 & 1.790 & & & 85.70 \\
\hline 1.575 & & & 66.30 & 1.795 & & & 86.30 \\
\hline 1.580 & & & 66.71 & 1.800 & & & 86.90 \\
\hline 1.585 & & . & 67.13 & 1.805 & & & 87.60 \\
\hline 1.590 & & .. & 67.59 & 1.810 & & & 88.30 \\
\hline 1.595 & & .. & 68.05 & 1.815 & & & 89.05 \\
\hline 1.600 & & .. & 68.51 & 1.820 & & & 90.05 \\
\hline 1.605 & * & . & 68.97 & 1.825 & & & 91.00 \\
\hline 1.610 & & . & 69.43 & 1.830 & & & 92.10 \\
\hline 1.615 & & & 69.89 & 1.835 & & & 93.43 \\
\hline 1.620 & & . & 70.32 & 1.840 & & & 95.60 \\
\hline 1.625 & & 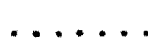 & 70.74 & 1.8405 & & & 95.95 \\
\hline 1.630 & & & 71.16 & 1.8410 & & & 97.00 \\
\hline 1.635 & & & 71.57 & 1.8415 & & & 97.70 \\
\hline 1.640 & & & 71.99 & 1.8410 & & & 98.20 \\
\hline 1.645 & & & 72.40 & 1.8405 & & & 98.70 \\
\hline 1.650 & & & 72.82 & 1.8400 & & & 99.20 \\
\hline 1.655 & & & 73.23 & 1.8395 & & & 99.45 \\
\hline 1.660 & & & 73.64 & 1.8390 & & $\cdots$ & 99.70 \\
\hline 1.665 & & & 74.07 & 1.8385 & & & 99.95 \\
\hline
\end{tabular}


ALCOHOL TABLE MODIFIED FROM WINDISCH

\begin{tabular}{|c|c|c|c|c|c|}
\hline $\begin{array}{l}\text { Specific } \\
\text { Gravity of } \\
\text { Distillate. }\end{array}$ & $\begin{array}{l}\text { Per Cent of } \\
\text { Alcohol by } \\
\text { Weight. }\end{array}$ & $\begin{array}{l}\text { Per Cent of } \\
\text { Alcohol by } \\
\text { Volume. }\end{array}$ & $\begin{array}{l}\text { Specific } \\
\text { Gravity of } \\
\text { Distillate. }\end{array}$ & $\begin{array}{l}\text { Per Cent of } \\
\text { Alcohol by } \\
\text { Weight. }\end{array}$ & $\begin{array}{l}\text { Per Cent of } \\
\text { Alcohol by } \\
\text { Volume. }\end{array}$ \\
\hline 1.0000 & 0.00 & 0.00 & 0.9908 & 5.20 & 6.55 \\
\hline 0.9998 & 0.11 & 0.13 & 6 & 5.32 & 6.71 \\
\hline 6 & 0.21 & 0.27 & 4 & 5.45 & 6.86 \\
\hline 4 & 0.32 & 0.40 & 2 & 5.57 & 7.02 \\
\hline 2 & 0.42 & 0.53 & 0 & 5.70 & 7.18 \\
\hline 0 & 0.53 & 0.67 & 0.9898 & 5.83 & 7.33 \\
\hline 0.9988 & 0.64 & 0.80 & 6 & 5.95 & 7.50 \\
\hline 6 & 0.74 & 0.93 & 4 & 6.08 & 7.66 \\
\hline 4 & 0.85 & 1.07 & 2 & 6.21 & 7.82 \\
\hline 2 & 0.96 & 1.20 & $\hat{0}$ & 6.34 & 7.99 \\
\hline 0 & 1.06 & 1.34 & 0.9888 & 6.47 & 8.15 \\
\hline 0.9978 & 1.17 & 1.48 & 6 & 6.59 & 8.31 \\
\hline 6 & 1.28 & 1. 61 & 4 & 6.73 & 8.48 \\
\hline 4 & 1.39 & 1.75 & 2 & 6.88 & 8.64 \\
\hline 2 & 1.50 & 1.88 & 0 & 6.99 & 8.81 \\
\hline$\overline{0}$ & 1.60 & 2.02 & 0.9878 & 7.12 & 8.98 \\
\hline 0.9968 & 1.71 & 2.16 & 6 & 7.26 & 9.15 \\
\hline 6 & 1.82 & 2.30 & 4 & 7.39 & 9.32 \\
\hline 4 & 1.93 & 2.44 & 2 & 7.53 & 9.48 \\
\hline 2 & 2.04 & 2.58 & 0 & 7.66 & 9.66 \\
\hline 0 & 2.16 & 2.72 & 0.9868 & 7.80 & 9.83 \\
\hline 0.9958 & 2.27 & 2.86 & 6 & 7.94 & 10.00 \\
\hline 6 & 2.38 & 300 & 4 & 8.07 & 10.17 \\
\hline 4 & 2.49 & 3.14 & 2 & 8.21 & 10.35 \\
\hline 2 & 2.60 & 3.28 & 0 & 8.35 & 10.52 \\
\hline 0 & 2.72 & 3.42 & 0.9858 & 8.49 & 10.70 \\
\hline 0.9948 & 2.82 & 3.56 & 6 & 8.63 & 10.88 \\
\hline 6 & 2.94 & 3.71 & 4 & 8.77 & 11.05 \\
\hline 4 & 3.06 & 3.85 & 2 & 8.91 & 11.23 \\
\hline 2 & 3.17 & 4.00 & 0 & 9.06 & 11.41 \\
\hline 0 & 3.29 & 4.14 & 0.9848 & 9.20 & 11.59 \\
\hline 0.9938 & 3.40 & 4.29 & 6 & 9.34 & 11.77 \\
\hline 6 & 3.52 & 4.43 & 4 & 9.49 & 11.95 \\
\hline 4 & 3.64 & 4.58 & 2 & 9.63 & 12.14 \\
\hline 2 & 3.75 . & 4.73 & 0 & 9.79 & 12.32 \\
\hline 0 & 3.87 & 4.88 & 0.9838 & 9.92 & 12.50 \\
\hline 0.9928 & 3.99 & 5.03 & 6 & 10.07 & 12.69 \\
\hline 6 & 4.11 & 5.18 & 4 & 10.22 & 12.88 \\
\hline 4 & 4.23 & 5.33 & 2 & 10.36 & 13.06 \\
\hline 2 & 4.35 & 5.48 & 0 & 10.52 & 13.25 \\
\hline 0 & 4.47 & 5.53 & 0.9828 & 10.66 & 13.44 \\
\hline 0.9918 & 4.59 & 5.78 & 6 & 10.81 & 13.63 \\
\hline 6 & 4.71 & 5.93 & 4 & 10.96 & 13.82 \\
\hline 4 & 4.83 & 6.09 & 2 & 11.12 & 14.01 \\
\hline 2 & 4.95 & 6.24 & 0 & 11.27 & 14.20 \\
\hline 0 & 5.08 & 6.40 & & & \\
\hline
\end{tabular}


ALCOHOL TABLE MODIFIED FROM WINDISCH-Continued

\begin{tabular}{|c|c|c|c|c|c|}
\hline $\begin{array}{l}\text { Specific } \\
\text { Gravity of } \\
\text { Distillate. }\end{array}$ & $\begin{array}{l}\text { Per Cent of } \\
\text { Alcohol by } \\
\text { Weight. }\end{array}$ & $\begin{array}{l}\text { Per Cent of } \\
\text { Alcohol by } \\
\text { Volume. }\end{array}$ & $\begin{array}{l}\text { Specific } \\
\text { Gravity of } \\
\text { Distillate. }\end{array}$ & $\begin{array}{l}\text { Per Cent of } \\
\text { Alcohol by } \\
\text { Weight. }\end{array}$ & $\begin{array}{l}\text { Per Cent of } \\
\text { Alcohol by } \\
\text { Volume. }\end{array}$ \\
\hline 0.9818 & 11.42 & 14.39 & 0.9718 & 19.30 & 24.32 \\
\hline 6 & 11.57 & 14.58 & 6 & 19.45 & 24.51 \\
\hline 4 & 11.72 & 14.77 & 4 & 1960 & 24.70 \\
\hline 2 & 11.88 & 14.97 & 2 & 1976 & 24.89 \\
\hline 0 & 12.03 & 15.16 & 0 & 1991 & 25.08 \\
\hline 0.9808 & 12.19 & 15.36 & 0.9708 & 20.06 & 25.27 \\
\hline 6 & 12.34 & 15.55 & 6 & 20.21 & 25.47 \\
\hline 4 & 12.50 & 15.75 & 4 & 20.36 & 25.66 \\
\hline 2 & 12.65 & 15.95 & 2 & 20.51 & 25.84 \\
\hline 0 & 12.81 & 16.14 & 0 & 20.66 & 26.03 \\
\hline 0.9798 & 12.97 & 16.34 & 0.9698 & 20.81 & 26.22 \\
\hline 6 & 13.13 & 16.54 & 6 & 20.96 & 26.41 \\
\hline 4 & 13.28 & 16.74 & 4 & 21.10 & 26.59 \\
\hline 2 & 13.44 & 16.94 & 2 & 21.25 & 26.78 \\
\hline 0 & 13.60 & 17.14 & 0 & 21.40 & 26.96 \\
\hline 0.9788 & 13.76 & 17.34 & 0.9688 & 21.54 & 27.14 \\
\hline 6 & 13.92 & 17.54 & 6 & 21.69 & 27.33 \\
\hline 4 & 14.08 & 17.74 & 4 & 21.83 & 27.51 \\
\hline 2 & 14.23 & 17.94 & 2 & 21.97 & 27.69 \\
\hline 0 & 14.29 & 18.14 & 0 & 22.12 & 27.87 \\
\hline 0.9778 & 14.55 & 18.34 & 0.9678 & 22.26 & 2805 \\
\hline 6 & 14.71 & 18.54 & 6 & 22.40 & 28.23 \\
\hline 4 & 14.87 & 18.74 & 4 & 22.54 & 28.41 \\
\hline 2 & 15.03 & 18.94 & 2 & 22.68 & 28.59 \\
\hline 0 & 15.19 & 19.14 & 0 & 22.82 & 28.76 \\
\hline 0.9768 & 15.35 & 19.34 & 0.9668 & 22.96 & 28.94 \\
\hline 6 & 15.51 & 19.55 & 6 & 23.10 & 29.11 \\
\hline 4 & 15.67 & 19.75 & 4 & 23.24 & 29.29 \\
\hline 2 & 15.83 & 19.95 & 2 & 23.38 & 29.46 \\
\hline 0 & 15.99 & 20.15 & 0 & 23.52 & 29.64 \\
\hline 0.9758 & 16.15 & 2035 & 0.9658 & 23.65 & 29.81 \\
\hline 6 & 16.31 & 20.55 & 6 & 23.79 & 29.98 \\
\hline 4 & 16.47 & 20.75 & 4 & 23.93 & 30.15 \\
\hline 2 & 16.63 & 20.96 & 2 & 24.06 & 30.32 \\
\hline 0 & 16.79 & 21.16 & 0 & 24.19 & 30.49 \\
\hline 0.9748 & 16.95 & 21.36 & 0.9648 & 24.33 & 30.66 \\
\hline 6 & 17.11 & 21.56 & 6 & 24.46 & 30.82 \\
\hline 4 & 17.27 & 21.76 & 4 & 24.59 & 30.99 \\
\hline 2 & 17.42 & 21.96 & 2 & 24.73 & 31.16 \\
\hline$\cdot 0$ & 17.58 & 22.16 & 0 & 24.85 & 31.32 \\
\hline 0.9738 & 17.74 & 22.35 & 0.9638 & 24.99 & 31.49 \\
\hline 6 & 17.90 & 22.55 & 6 & 25.12 & 31.65 \\
\hline 4 & 18.05 & 22.75 & 4 & 25.25 & 31.81 \\
\hline 2 & 18.21 & 22.95 & 2 & 25.37 & 31.98 \\
\hline 0 & 18.37 & 23.14 & 0 & 25.50 & 32.14 \\
\hline 0.9728 & 18.52 & 23.34 & 0.9628 & 25.63 & 32.30 \\
\hline 6 & 18.68 & 23.54 & 6 & 25.76 & 32.46 \\
\hline 4 & 18.84 & 23.73 & 4 & 25.88 & 32.62 \\
\hline 2 & 18.99 & 23.93 & 2 & 26.01 & 32.78 \\
\hline 0 & 19.14 & 24.12 & 0 & 26.13 & 32.93 \\
\hline
\end{tabular}


INTERNATIONAL ATOMIC WEIGHTS*

$\mathrm{O}=16$

\begin{tabular}{|c|c|c|c|c|c|}
\hline Name. & Symbol. & $\begin{array}{l}\text { Atomic } \\
\text { Weight. }\end{array}$ & Name. & Symbol & $\begin{array}{l}\text { Atomic } \\
\text { Weight. }\end{array}$ \\
\hline Aluminium. & $\mathrm{Al}$ & 27.1 & Molybdenum... & Mo & 96.0 \\
\hline Antimony...... & $\mathrm{Sb}$ & 120.2 & Neodymium..... & $\mathrm{Nd}$ & 144.3 \\
\hline Argon........... & A & 39.88 & Neon........... & $\mathrm{Ne}$ & 20.2 \\
\hline Arsenic........ & As & 7496 & Nickel. . . . . . . . & $\mathrm{Ni}$ & 58.68 \\
\hline Barium........ & $\mathrm{Ba}$ & 137.37 & Niton........... & $\mathrm{Nt}$ & 222.4 \\
\hline Bismuth........ & $\mathrm{Bi}$ & 208.0 & Nitrogen........ & $\mathrm{N}$ & 14.01 \\
\hline Boron........... & $\mathrm{B}$ & 11.0 & Osmium. . . . . . . & Os & 190.9 \\
\hline Bromine. . . . . . . & $\mathrm{Br}$ & 79.92 & Oxygen...... & 0 & 16.00 \\
\hline Cadmium..... & $\mathrm{Cd}$ & 112.40 & Palladium. . . . . & $\mathrm{Pd}$ & 106.7 \\
\hline Caesium......... & $\mathrm{Cs}$ & 132.81 & Phosphorus. . . . . . & $P$ & 31.04 \\
\hline Calcium........ & $\mathrm{Ca}$ & 40.07 & Platinum........ & $\mathrm{Pt}$ & 195.2 \\
\hline Carbon.......... & $\mathrm{C}$ & 12.00 & Potassium....... & $\mathrm{K}$ & 39.10 \\
\hline Cerium....... & $\mathrm{Ce}$ & 140.25 & Praseodymium.... & $\operatorname{Pr}$ & 140.6 \\
\hline Chlorine......... & $\mathrm{Cl}$ & 35.46 & Radium......... & $\mathrm{Ra}$ & 226.4 \\
\hline Chromium....... & $\mathrm{Cr}$ & 52.0 & Rhodium........ & $\mathrm{Rh}$ & 102.9 \\
\hline Cobalt. . . . . . & Co & 58.97 & Rubidium. ...... & $\mathrm{Rb}$ & 85.45 \\
\hline Columbium. . . . . & $\mathrm{Cb}$ & 93.5 & Ruthenium...... & $\mathrm{Ru}$ & 101.7 \\
\hline Copper......... & $\mathrm{Cu}$ & 63.57 & Samarium....... & $\mathrm{Sm}$ & 150.4 \\
\hline Dysprosium... & Dy & 162.5 & Scandium.... & $\mathrm{Sc}$ & 44.1 \\
\hline Erbium..... & $\mathrm{Er}$ & 167.7 & Selenium.... & $\mathrm{Se}$ & 79.2 \\
\hline Europium.... & $\mathrm{Eu}$ & 152.0 & Silicon...... & $\mathrm{Si}$ & 28.3 \\
\hline Fluorine..... . & $\mathrm{F}$ & 19.0 & Silver. . . . . . & $\mathrm{Ag}$ & 107.88 \\
\hline Gadolinium... & Gd & 157.3 & Sodium.... & $\mathrm{Na}$ & 23.00 \\
\hline Gallium....... & $\mathrm{Ga}$ & 69.9 & Strontium... & $\mathrm{Sr}$ & 87.63 \\
\hline Germanium.... & $\mathrm{Ge}$ & 72.5 & Sulphur..... & $\mathbf{S}$ & 32.07 \\
\hline Glucinum... . & $\mathrm{Gl}$ & 9.1 & Tantalum.... & $\mathrm{Ta}$ & 181.5 \\
\hline Gold........ & $\mathrm{Au}$ & 197.2 & Tellurium... & $\mathrm{Te}$ & 127.5 \\
\hline Helium...... & $\mathrm{He}$ & 3.99 & Terbium.... & $\mathrm{Tb}$ & 159.2 \\
\hline Holmium. . . . . . . & Ho & 163.5 & Thallium.... & $\mathrm{Tl}$ & 204.0 \\
\hline Hydrogen.... & $\mathrm{H}$ & 1.008 & Thorium.... & Th & 232.4 \\
\hline Indium....... & In & 114.8 & Thulium..... & $\operatorname{Tm}$ & 168.5 \\
\hline Iodine....... & I & 126.92 & $\operatorname{Tin} . . . \ldots \ldots$ & $\mathrm{Sn}$ & 119.0 \\
\hline Iridium . . . . . & $\mathrm{Ir}$ & 193.1 & Titanium... . & $\mathrm{Ti}$ & 48.1 \\
\hline Iron. . . . & $\mathrm{Fe}$ & 55.84 & Tungsten.... & W & 184.0 \\
\hline Krypton. & $\mathrm{Kr}$ & 82.92 & Uranium.... & $\mathrm{U}$ & 238.5 \\
\hline Lanthanum. . & $\mathrm{La}$ & 139.0 & Vanadium... & $\mathrm{V}$ & 51.0 \\
\hline Lead. . . . . . . . & $\mathrm{Pb}$ & 207.10 & Xenon...... & $\mathrm{Xe}$ & 130.2 \\
\hline Lithium. . & $\hat{\mathrm{Li}}$ & 6.94 & Ytterbium... & $\mathrm{Yb}$ & 172.0 \\
\hline Lutecium. & $\mathrm{Lu}$ & 174.0 & (Neoytterbium) & & \\
\hline Magnesium. . & $\mathrm{Mg}$ & 24.32 & Ytterium........ & $Y t$ & 89.0 \\
\hline Manganese. & $\mathrm{Mn}$ & 54.93 & Zinc......... & $\mathrm{Zn}$ & 65.37 \\
\hline Mercury .. & $\mathrm{Hg}$ & 200.9 & Zirconium....... & $\mathrm{Zr}$ & 90.6 \\
\hline
\end{tabular}

* Compiled by the International Committee on Atomic Weights consisting of F. W. Clarke, W. Ostwald, T. E. Thorpe, and G. Urbain. 
SOLUBILITY OF OXYGEN IN WATER

(Winkler, Berichte, 22, 1772.) (1889)

\begin{tabular}{c|c||c|c||c|c}
\hline $\begin{array}{c}\text { Tempera- } \\
\text { ture. }\end{array}$ & Oxygen. & Temperature. & Oxygen. & Temperature. & Oxygen. \\
\cline { 2 - 3 } 0 & 14.70 & 9 & 11.58 & 18 & 9.56 \\
1 & 14.28 & 10 & 11.31 & 19 & 9.37 \\
2 & 13.88 & 11 & 11.05 & 20 & 9.19 \\
3 & 13.50 & 12 & 10.80 & 21 & 901 \\
4 & 13.14 & 13 & 10.57 & 22 & 8.84 \\
5 & 12.80 & 14 & 10.35 & 23 & 8.67 \\
6 & 12.47 & 15 & 10.14 & 24 & 851 \\
7 & 12.16 & 16 & 9.94 & 25 & 8.35 \\
8 & 11.86 & 17 & 9.75 & & \\
\hline
\end{tabular}

CIRCUMFERENCES AND AREAS OF CIRCLES

Formula for Area

$$
A=\frac{\pi D^{2}}{4}=.785398 D^{2}
$$

Formula for Diameter

$$
D=2 \sqrt{\frac{\bar{A}}{\pi}}=1.128379 \sqrt{A}
$$

\begin{tabular}{c|c|c||c|c|c}
\hline Diameter. & Circumference. & Area. & Diameter. & Circumference. & Area. \\
\hline \multicolumn{1}{|c|}{} & .39270 & .012 & 7 & 21.991 & 38.485 \\
$\frac{1}{8}$ & .78540 & .049 & 8 & 25.133 & 50.265 \\
$\frac{1}{3}$ & 1.1781 & .110 & 9 & 28.274 & 63.617 \\
$\frac{3}{8}$ & 1.5708 & .196 & 10 & 31.416 & 78.54 \\
$\frac{1}{2}$ & 1.9635 & .307 & 20 & 62.832 & 314.16 \\
$\frac{5}{8}$ & 2.3562 & .442 & 30 & 94.248 & 706.86 \\
$\frac{3}{4}$ & 2.7489 & .601 & 40 & 125.664 & 1256.6 \\
$\frac{7}{8}$ & 3.1416 & .785 & 50 & 157.080 & 1963.5 \\
1 & 6.2832 & 3.142 & 60 & 188.496 & 2827.4 \\
2 & 9.4248 & 7.069 & 70 & 219.911 & 3848.5 \\
3 & 12.566 & 12.566 & 80 & 251.328 & 5026.5 \\
4 & 15.708 & 19.635 & 90 & 282.744 & 6361.7 \\
5 & 18.805 & 28.274 & 100 & 314.160 & 7854.0 \\
6 & & & & & \\
\hline
\end{tabular}


TABLE SHOWING EQUIVALENTS OF PRESSURE AND HEAD OF WATER

Head in Feet and Equivalent Pressrue in Pounds

\begin{tabular}{|c|c|c|c|c|c|}
\hline \multicolumn{2}{|c|}{5 TO 60 FEET. } & \multicolumn{2}{|c|}{70 то 180 Fent. } & \multicolumn{2}{|c|}{200 TO 1000 FEET. } \\
\hline Feet Head. & Pounds Press. & Feet Head. & Pounds Press. & Feet Head. & Pounds Press. \\
\hline 5 & 2.17 & 70 & 30.3 & 200 & 86.6 \\
\hline 10 & 433 & 80 & 34.6 & 250 & 108.2 \\
\hline 15 & 6.50 & 90 & 39.0 & 300 & 129.9 \\
\hline 20 & 8.66 & 100 & 433 & 350 & 151.5 \\
\hline 25 & 10.83 & 110 & 47.6 & 400 & 173.2 \\
\hline 30 & 12.99 & 120 & 52.0 & 500 & 216.5 \\
\hline 35 & 15.16 & 130 & 56.3 & 600 & 259.8 \\
\hline 40 & 17.32 & 140 & 60.6 & 700 & 303.1 \\
\hline 45 & 19.49 & 150 & 65.0 & 700 & 346.4 \\
\hline 50 & 21.65 & 160 & 69.2 & 900 & 389.7 \\
\hline 60 & 26.09 & 180 & 78.0 & 1000 & 433.0 \\
\hline
\end{tabular}

Pressure in Pounds and Equivalent Head in Feet

\begin{tabular}{|c|c|c|c|c|c|}
\hline \multicolumn{2}{|c|}{5 то 60 Pounds. } & \multicolumn{2}{|c|}{70 то 170 Pounds. } & \multicolumn{2}{|c|}{180 to 500 Podnds. } \\
\hline Pounds Press. & Feet Head. & Pounds Press. & Feet Head. & Pounds Press. & Feet Head. \\
\hline 5 & 11.5 & 70 & 161.6 & 180 & 415.6 \\
\hline 10 & 23.0 & 80 & 184.7 & 190 & 438.9 \\
\hline 15 & 34.6 & 90 & 207.8 & 200 & 461.7 \\
\hline 20 & 46.2 & 100 & 230.9 & 225 & 519.5 \\
\hline 25 & 57.7 & 110 & 253.9 & 250 & 577.2 \\
\hline 30 & 69.3 & 120 & 277.0 & 275 & 643.0 \\
\hline 35 & 80.8 & 130 & 300.1 & 300 & 692.7 \\
\hline 40 & 92.3 & 140 & 323.2 & 325 & 750.4 \\
\hline 45 & 103.9 & 150 & 346.3 & 350 & 808.1 \\
\hline 50 & 115.4 & 160 & 369.4 & 400 & 922.6 \\
\hline 60 & 138.5 & 170 & 392.5 & 500 & 1154.5 \\
\hline
\end{tabular}


U. S. GALLONS TO LITERS (METRIC)

\begin{tabular}{|c|c|c|c|}
\hline Gallons. & Liters. & Gallons. & Liters. \\
\hline 1 & 3.78544 & 51 & 193.05744 \\
\hline 2 & 7.57088 & 52 & 196.84288 \\
\hline 3 & 11.35632 & 53 & 200.62832 \\
\hline 4 & 15.14176 & 54 & 204.41376 \\
\hline 5 & 18.92720 & 55 & 208.19920 \\
\hline 6 & 22.71264 & 56 & 21198464 \\
\hline 7 & 26.49808 & 57 & 215.77008 \\
\hline 8 & 30.28352 & 58 & 219.55552 \\
\hline 9 & 34.06896 & 59 & 223.34096 \\
\hline 10 & 37.85440 & 60 & 227.12640 \\
\hline 11 & 41.63984 & 61 & 230.91184 \\
\hline 12 & 45.42528 & 62 & 234.69728 \\
\hline 13 & 49.21072 & 63 & 238.48272 \\
\hline 14 & 52.99616 & 64 & 242.26816 \\
\hline 15 & 56.78160 & 65 & 246.05360 \\
\hline 16 & 60.56704 & 66 & 249.83904 \\
\hline 17 & 64.35248 & 67 & 253.62448 \\
\hline 18 & 68.13792 & 68 & 257.40992 \\
\hline 19 & 71.92336 & 69 & 261.19536 \\
\hline 20 & 75.70880 & 70 & 264.98080 \\
\hline 21 & 79.49424 & 71 & 268.76624 \\
\hline 22 & 83.27968 & 72 & 272.55168 \\
\hline 23 & 87.06512 & 73 & 276.33712 \\
\hline 24 & 90.85056 & 74 & 280.12256 \\
\hline 25 & 94.63600 & 75 & 283.90800 \\
\hline 26 & 98.42144 & 76 & 286.69344 \\
\hline 27 & 102.20688 & 77 & 291.47888 \\
\hline 28 & 105.99232 & 78 & 29526432 \\
\hline 29 & 109.77776 & 79 & 29904976 \\
\hline 30 & 11356320 & 80 & 302.83520 \\
\hline 31 & 11734864 & 81 & 306.62064 \\
\hline 32 & 121.13408 & 82 & 310.40608 \\
\hline 33 & 124.91952 & 83 & 314.19152 \\
\hline 34 & 128.70496 & 84 & 317.97696 \\
\hline 35 & 132.49040 & 85 & 321.76240 \\
\hline 36 & 136.27584 & 86 & 325.54784 \\
\hline 37 & 140.06128 & 87 & 329.33328 \\
\hline 38 & 143.84672 & 88 & 333.11872 \\
\hline 39 & 147.63216 & 89 & 336.90416 \\
\hline 40 & 151.41760 & 90 & 340.68960 \\
\hline 41 & 155.20304 & 91 & 344.47504 \\
\hline 42 & 158.98848 & 92 & 348.26048 \\
\hline 43 & 162.77392 & 93 & 352.04592 \\
\hline 44 & 166.55936 & 94 & 355.83136 \\
\hline 45 & 170.34480 & 95 & 359.61680 \\
\hline 46 & 174.13024 & 96 & 363.40224 \\
\hline 47 & 177.91568 & 97 & 367.18768 \\
\hline 48 & 181.70112 & 98 & 370.97312 \\
\hline 49 & 185.48656 & 99 & 374.75856 \\
\hline 50 & 189.27200 & 100 & 378.54400 \\
\hline
\end{tabular}

CUBIC FEET TO LITERS (METRIC)

\begin{tabular}{c|c||c|c}
\hline Cu.ft. & Liters. & Cu. ft. & Liters. \\
\hline 1 & 28.3166 & 51 & 1444.1466 \\
2 & 56.6332 & 52 & 1472.4632 \\
3 & 84.9498 & 53 & 1500.7798 \\
4 & 113.2664 & 54 & 1529.0964 \\
5 & 141.5830 & 55 & 1557.4130 \\
6 & 169.8996 & 56 & 1585.7296 \\
7 & 198.2162 & 57 & 1614.0462 \\
8 & 226.5328 & 58 & 1642.3628 \\
9 & 254.8494 & 59 & 1670.6794 \\
10 & 283.1660 & 60 & 1698.9960 \\
11 & 311.4826 & 61 & 1727.3126 \\
12 & 339.7992 & 62 & 1755.6292 \\
13 & 368.1158 & 63 & 1783.9458 \\
14 & 396.4324 & 64 & 1812.2624 \\
15 & 424.7490 & 65 & 1840.5790 \\
16 & 453.0656 & 66 & 1868.8956 \\
17 & 481.3822 & 67 & 1897.2122 \\
18 & 509.6988 & 68 & 1925.5288 \\
19 & 538.0154 & 69 & 1953.8454 \\
20 & 566.3320 & 70 & 1982.1620 \\
21 & 594.6486 & 71 & 2010.4786 \\
22 & 622.9652 & 72 & 2038.7952 \\
23 & 651.2818 & 73 & 2067.1118 \\
24 & 679.5984 & 74 & 2095.4284 \\
25 & 707.9150 & 75 & 2123.7450 \\
26 & 736.2316 & 76 & 2152.0616 \\
27 & 764.5482 & 77 & 2180.3782 \\
28 & 792.8648 & 78 & 2208.6248 \\
29 & 821.1814 & 79 & 2237.0114 \\
30 & 849.4980 & 80 & 2265.3280 \\
31 & 877.8146 & 81 & 2293.6446 \\
32 & 906.1312 & 82 & 2321.9612 \\
33 & 934.4478 & 83 & 2350.2778 \\
34 & 962.7644 & 84 & 2378.5944 \\
35 & 991.0810 & 85 & 2406.9110 \\
36 & 1019.3976 & 86 & 2435.2276 \\
37 & 1047.7142 & 87 & 2463.5442 \\
38 & 1076.0308 & 88 & 2491.8608 \\
39 & 1104.3474 & 89 & 2520.1774 \\
40 & 1132.6640 & 90 & 2548.4940 \\
41 & 1160.9806 & 91 & 2576.8106 \\
42 & 1189.2972 & 92 & 2605.1272 \\
43 & 1217.6138 & 93 & 2633.4438 \\
44 & 1245.9304 & 94 & 2661.7604 \\
45 & 1274.2470 & 95 & 2690.0770 \\
46 & 1302.5636 & 96 & 2718.3936 \\
47 & 1330.8802 & 97 & 2746.7102 \\
48 & 1359.1968 & 98 & 2775.0268 \\
49 & 1387.5134 & 99 & 2803.3434 \\
50 & 1415.8300 & 100 & 2831.6600 \\
\hline & & &
\end{tabular}


USEFUL FACTORS FOR WATER

Based on Weights at $62^{\circ} \mathrm{F}$. (Standard Temperature)

U. S. gallons $\times \quad 8.3356=$ pounds

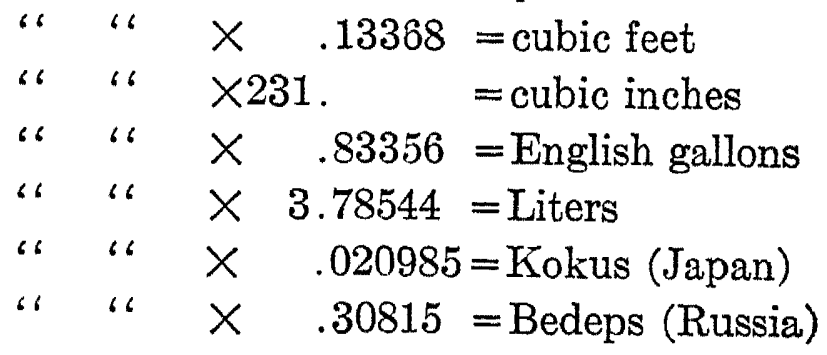

English gallons $\times 10 . \quad=$ pounds

$\begin{array}{lllll}\text { " } & \text { " } & \times & .160372 & =\text { cubic feet } \\ \text { " } & \times 277.12 & =\text { cubic inches } \\ \text { " } & \times & \times & 1.1997 & =\text { U. S. gallons } \\ \text { " } & \times & 4.5413 & =\text { Liters } \\ \text { " } & \times & .025175 & =\text { Kokus (Japan) } \\ & \times & .36969 & =\text { Bedeps (Russia) }\end{array}$

Liters $\times 2.202 \quad=$ pounds

" $\times .035302=$ cubic feet

" $\times 61.023=$ cubic inches

" $\times .26417=$ U. S. gallons

" $\times .2202=$ English gallons

" $\times \quad .0055435=$ Kokus (Japan)

، $\times .081405=$ Bedeps (Russia)

Kokus $\times \quad 397.22028=$ pounds

" $\times 6.3703=$ cubic feet

" $\times 11008.00=$ cubic inches

" $\times 47.6535=$ U. S. gallons

" $\times 39.722028=$ English gallons

" $\times 180.39=$ Liters

" $\times 14.6847$ =Bedeps (Russia)

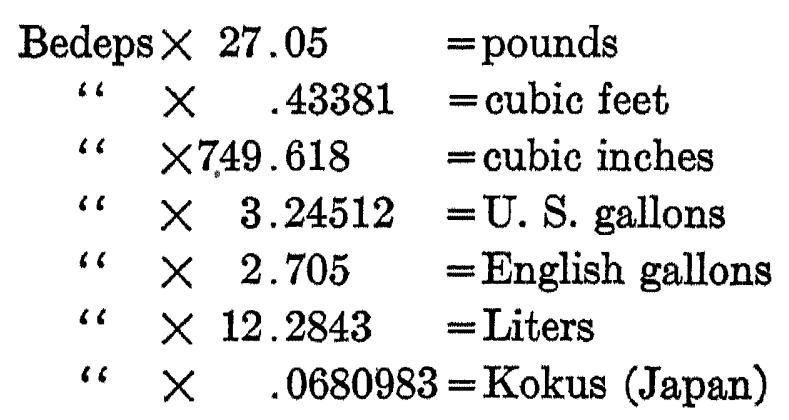


Cubic feet of water $\times 62.355=$ pounds

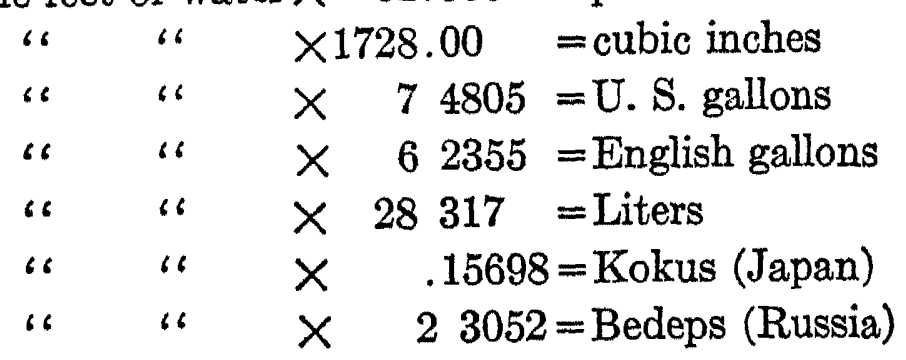

Pounds of water $X \quad .016037=$ cubic feet
" " $\times 27.712=$ cubic inches
" " $\quad$ " $.11997=U$. S. gallons
" " $\times .1 \quad$ =English gallons
" " $\times .45413=$ Liters
" " $\times .0025175=$ Kokus (Japan)
" " $\times .036969=$ Bedeps (Russia)

\section{SURVEYOR'S MEASURE}

7.92 inches

$=1$ link

25 links

$=1 \mathrm{rod}$

4 rods

$=1$ chain

10 square chains or 160 square rods $=1$ acre

640 acres

$=1$ square mile

36 square miles ( 6 miles sq.) $\quad=1$ township

\section{CUBIC MEASURE}
1728 cubic inches $=1$ cubic foot
128 cubic feet $\quad=1$ cord (wood)
27 cubic feet $\quad=1$ cubic yard
40 cubic feet $\quad=1$ ton (shipping)
2150.42 cubic inches $=1$ standard bushel
268.8 cubic inches $=1$ standard gallon
1 cubic foot $\quad=$ about four-fifths of a bushel 


\section{METRIC EQUIVALENTS}

\section{Linear Measure}

\begin{tabular}{|c|c|}
\hline ter $=$ & \\
\hline & \\
\hline$=39$ & 1 yarc \\
\hline $\begin{array}{l}\text { dekameter }=1 \\
\text { kilometer }=0\end{array}$ & $1 \mathrm{rod}=0.5029$ \\
\hline
\end{tabular}

\section{Square Measure}

1 square centimeter $=0.1550$ square inch 1 square inch $=6.452$ sq. centimeters 1 square decimeter $=0.1076$ square foot 1 square foot $=9.2903$ sq. decimeters 1 square meter $=1.96$ square yards 1 square yard $=0.8361$ sq. meter 1 are $\quad=3.954$ square rods 1 square rod $=0.2529$ are 1 hektar $\quad=2.47$ acres 1 acre $=0.4047$ hektar 1 square kilometer $=0.386$ square mile 1 square mile $=2.59$ square kilometers

\section{MeAstre of Vor,dMe}

1 cubic centimeter $=0.061$ cubic inch $\quad 1$ cubic inch $=16.39$ cubic centimeters 1 cubic decimeter $=0.0353$ cubic feet $\quad 1$ cubic foot $=28.317$ cubic decimeters

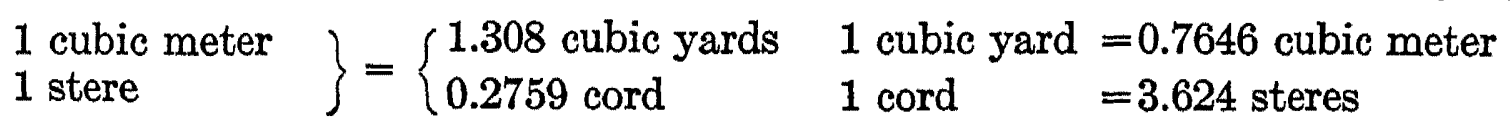

1 liter $\quad= \begin{cases}0.908 \text { quart dry } & 1 \text { quart dry }=1.101 \text { liters } \\ 1.0567 \text { quart liquid } & 1 \text { quart liquid }=0.9463 \text { liter }\end{cases}$

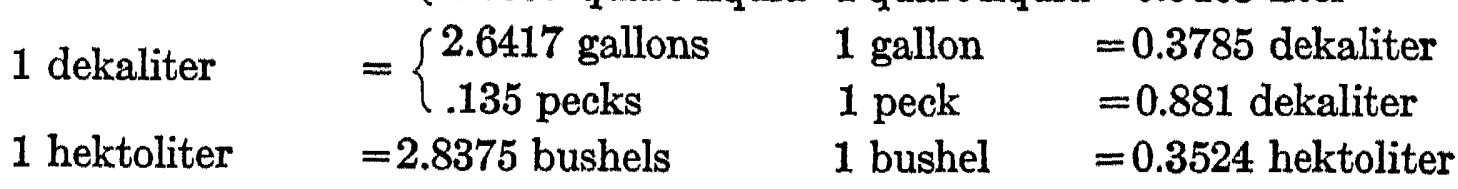
WEIGHTS

1 gram $=0.0527$ ounce

1 kilogram $=2.2046 \mathrm{lbs}$.

1 metric ton=1.1023 English ton
1 ounce $=28.85$ grams

$1 \mathrm{lb} .=0.4536$ kilogram

1 English ton $=0.9072$ metric ton.

\section{Approximate Metric Equivarents}

$\begin{array}{ll}1 \text { decimeter } & =4 \text { inches } \\ 1 \text { meter } & =1.1 \text { yards } \\ 1 \text { kilometer } & =\frac{5}{8} \text { of mile } \\ 1 \text { hektar } & =2 \frac{1}{2} \text { acres } \\ 1 \text { stere, or cubic meter } & =\frac{1}{4} \text { of a cord }\end{array}$

1 liter $\quad=\left\{\begin{array}{l}1.06 \text { quart liquid } \\ 0.9 \text { quart dry }\end{array}\right.$

1 hektoliter $=2 \frac{5}{8}$ bushels

1 kilogram $=2 \frac{1}{5}$ pounds

1 metric ton $=2200$ pounds 
Equivalents of grams or c.c. in apothecary's weight. (From Merck's Materia Medica): The following are approximate equivalents.

$0.001 \mathrm{gm}$. or c.c. $=\frac{1}{60}$ grn. or $\min$.

\begin{tabular}{|c|c|c|c|c|}
\hline 0.003 & ، & 66 & $=\frac{1}{20}$ & 66 \\
\hline 0.004 & 16 & 6 & $=\frac{1}{15}$ & 66 \\
\hline 0.008 & ، & " & $=\frac{1}{8}$ & "6 \\
\hline 0.01 & "6 & " & $=\frac{1}{6}$ & 66 \\
\hline 0.015 & a & "6 & $=\frac{1}{4}$ & " \\
\hline 0.03 & 66 & "6 & & ، \\
\hline 0.05 & "6 & $" 1$ & $=\frac{3}{4}$ & "6 \\
\hline 0.6 & 66 & 66 & $=1$ & 6 \\
\hline 0.1 & " & $" 6$ & $=1 \frac{1}{2}$ & "6 \\
\hline 0.2 & 's & " & $=3$ & 16 \\
\hline 0.25 & 66 & " & $=4$ & " \\
\hline
\end{tabular}

$0.3 \mathrm{gm}$. of c.c. $=5 \mathrm{grn}$. or $\min$.

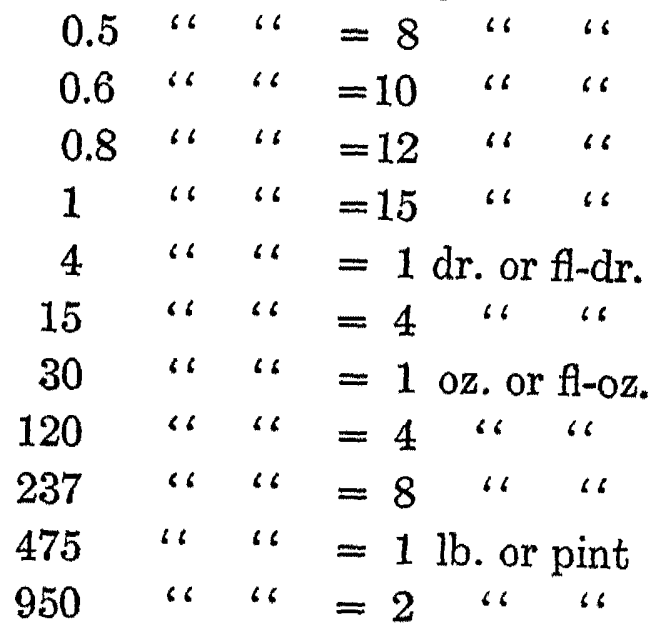

COMPARISON OF THERMOMETER SCALES

\begin{tabular}{r|r|r||c|c|c||c|c|c}
\hline $\begin{array}{c}\text { Centi- } \\
\text { grade. }\end{array}$ & Reaumur. & $\begin{array}{c}\text { Fahren- } \\
\text { heit. }\end{array}$ & $\begin{array}{c}\text { Centi- } \\
\text { grade. }\end{array}$ & Reaumur. & $\begin{array}{c}\text { Fahren- } \\
\text { helt. }\end{array}$ & $\begin{array}{c}\text { Centi- } \\
\text { grade. }\end{array}$ & Reaumur & $\begin{array}{c}\text { Fahren- } \\
\text { heit. }\end{array}$ \\
\hline-30 & -24.0 & -22.0 & 14 & 11.2 & 57.2 & 58 & 46.4 & 136.4 \\
-28 & -22.4 & -18.4 & 16 & 128 & 60.8 & 60 & 48.0 & 1400 \\
-26 & -20.8 & -14.8 & 18 & 14.4 & 64.4 & 62 & 49.6 & 1436 \\
-24 & -19.2 & -11.2 & 20 & 16.0 & 68.0 & 64 & 512 & 147.2 \\
-22 & -17.6 & -7.6 & 22 & 17.6 & 71.6 & 66 & 528 & 1508 \\
-20 & -16.0 & -4.0 & 24 & 19.2 & 75.2 & 68 & 54.4 & 154.4 \\
-18 & -14.4 & -0.4 & 26 & 20.8 & 78.8 & 70 & 56.0 & 1580 \\
-16 & -12.8 & 3.2 & 28 & 22.4 & 82.4 & 72 & 57.6 & 161.6 \\
-14 & -11.2 & 6.8 & 30 & 24.0 & 86.0 & 74 & 59.2 & 165.2 \\
-12 & -9.6 & 10.4 & 32 & 25.6 & 89.6 & 76 & 60.8 & 168.8 \\
-10 & -8.0 & 14.0 & 34 & 27.2 & 932 & 78 & 62.4 & 172.4 \\
-8 & -6.4 & 17.6 & 36 & 28.8 & 96.8 & 80 & 64.0 & 176.0 \\
-6 & -4.8 & 21.2 & 38 & 30.4 & 100.4 & 82 & 65.6 & 179.6 \\
-4 & -3.2 & 24.8 & 40 & 32.0 & 104.0 & 84 & 67.2 & 183.2 \\
-2 & -1.6 & 28.4 & 42 & 33.6 & 107.6 & 86 & 68.8 & 186.8 \\
0 & 0.0 & 32.0 & 44 & 35.2 & 111.2 & 88 & 70.4 & 190.4 \\
2 & 1.6 & 35.6 & 46 & 36.8 & 114.8 & 90 & 72.0 & 194.0 \\
4 & 3.2 & 39.2 & 48 & 38.4 & 118.4 & 92 & 73.6 & 197.6 \\
6 & 4.8 & 42.8 & 50 & 40.0 & 122.0 & 94 & 75.2 & 201.2 \\
8 & 6.4 & 46.4 & 52 & 41.6 & 125.6 & 96 & 76.8 & 204.8 \\
10 & 8.0 & 50.0 & 54 & 43.2 & 129.2 & 98 & 78.4 & 208.4 \\
12 & 9.6 & 53.6 & 56 & 44.8 & 132.8 & 100 & 80.0 & 212.0 \\
\hline & & & & & & & & \\
\hline
\end{tabular}


CONVERSION OF PARTS PER MILLION TO GRAINS PER UNITED STATES AND IMPERIAL GALLONS

\begin{tabular}{c|c|c||c|c|c}
\hline $\begin{array}{c}\text { Parts per } \\
\text { Million. }\end{array}$ & $\begin{array}{c}\text { Grains per } \\
\text { Imperial } \\
\text { Gallon. }\end{array}$ & $\begin{array}{c}\text { Grains per } \\
\text { United States } \\
\text { Gallon. }\end{array}$ & $\begin{array}{c}\text { Parts per } \\
\text { Millon. }\end{array}$ & $\begin{array}{c}\text { Grains per } \\
\text { Imperial } \\
\text { Gallon. }\end{array}$ & $\begin{array}{c}\text { Grains per } \\
\text { United States } \\
\text { Gallon. }\end{array}$ \\
\cline { 1 - 5 } 1 & .0700 & .0583 & 40 & 2.8000 & 2.3327 \\
2 & .1400 & .1166 & 50 & 3.5000 & 2.9129 \\
3 & .2100 & .1749 & 60 & $4.2000^{*}$ & 3.4990 \\
4 & .2800 & .2332 & 70 & 4.9000 & 4.0822 \\
5 & .3500 & .2915 & 80 & 5.6000 & 4.6654 \\
6 & .4200 & .3499 & 90 & 6.3000 & 5.2486 \\
7 & .4900 & .4082 & 100 & 7.0000 & 5.8318 \\
8 & .5600 & .4665 & 200 & 14.0000 & 11.6630 \\
9 & .6300 & .5248 & 300 & 21.0000 & 17.4950 \\
10 & .7000 & .5831 & 400 & 28.0000 & 23.3270 \\
20 & 1.4000 & 1.1663 & 500 & 35.0000 & 29.1290 \\
30 & 2.1000 & 1.7495 & & & \\
\hline
\end{tabular}

* Other values may be computed from the above table.

Sp. Gr. $=\frac{140}{B^{\circ}+130}$ for Baumé lighter than water.

$B^{\circ}=\frac{140}{\text { Sp. Gr. }}-$ for Baumé lighter than water.

Sp. Gr. $=\frac{145}{154-B^{\circ}}$ for Baumé heavier than water.

$B^{\circ}=145 \frac{145}{\text { Sp. Gr. }}$ for Baumé heavier than water. 
SPECIFIC GRAVITIES IN DEGREES BAUMÉ

Liquids Lighter than Water

\begin{tabular}{|c|c|c|c|c|c|}
\hline $\begin{array}{l}\text { Degrees } \\
\text { Baumé. }\end{array}$ & $\begin{array}{l}\text { Specific } \\
\text { Gravity. }\end{array}$ & $\begin{array}{l}\text { Degrees } \\
\text { Baumé. }\end{array}$ & $\begin{array}{l}\text { Specific } \\
\text { Gravity. }\end{array}$ & $\begin{array}{l}\text { Degrees } \\
\text { Baumé. }\end{array}$ & $\begin{array}{l}\text { Specific } \\
\text { Gravity. }\end{array}$ \\
\hline 10 & 1.000 & 15 & 0.966 & 20 & 0.933 \\
\hline 11 & 0.993 & 16 & 0.959 & 21 & 0.927 \\
\hline 12 & 0.986 & 17 & 0.952 & 22 & 0.921 \\
\hline 13 & 0.979 & 18 & 0.946 & 23 & 0.915 \\
\hline 14 & 0.972 & 19 & 0.940 & 24 & 0.909 \\
\hline 25 & 0.903 & 30 & 0.875 & 35 & 0.849 \\
\hline 26 & 0.897 & 31 & 0.870 & 36 & 0.843 \\
\hline 27 & 0.892 & 32 & 0.864 & 37 & 0.838 \\
\hline 28 & 0.886 & 33 & 0.859 & 38 & 0.833 \\
\hline 29 & 0.881 & 34 & 0.854 & 39 & 0.828 \\
\hline 40 & 0.824 & 48 & 0.787 & 58 & 0.745 \\
\hline 41 & 0.819 & 50 & 0.778 & 60 & 0.737 \\
\hline 42 & 0.814 & 52 & 0.769 & 65 & 0.718 \\
\hline 43 & 0.805 & 54 & 0.761 & 70 & 0.700 \\
\hline 46 & 0.796 & 56 & 0.753 & 75 & 0.683 \\
\hline
\end{tabular}


SPECIFIC GRAVITIES IN DEGREES BAUMÉ AND TWADDLE Liquids Hgavier than Water

\begin{tabular}{|c|c|c|c|c|c|}
\hline \multicolumn{2}{|c|}{$\begin{array}{c}\text { HYDROMETER } \\
\text { READING DEGREES. }\end{array}$} & \multirow{2}{*}{$\begin{array}{l}\text { Specific } \\
\text { Gravity. }\end{array}$} & \multicolumn{2}{|c|}{$\begin{array}{c}\text { HYDROMETER } \\
\text { READING DEGREES }\end{array}$} & \multirow{2}{*}{$\begin{array}{l}\text { Specific } \\
\text { Gravity. }\end{array}$} \\
\hline Twaddle. & Baumé. & & Twaddle. & Baumé. & \\
\hline $\begin{array}{l}0 \\
1 \\
2 \\
3 \\
4\end{array}$ & $\begin{array}{r}.0 \\
.7 \\
1.4 \\
2.1 \\
2.7\end{array}$ & $\begin{array}{l}1.000 \\
1.005 \\
1.010 \\
1.015 \\
1.020\end{array}$ & $\begin{array}{r}43 \\
44 \\
45 \\
46 \\
47\end{array}$ & $\begin{array}{l}25.5 \\
26.0 \\
26.4 \\
26.9 \\
27.4\end{array}$ & $\begin{array}{l}1.215 \\
1.220 \\
1.225 \\
1.230 \\
1.235\end{array}$ \\
\hline $\begin{array}{l}5 \\
6 \\
7 \\
8 \\
9\end{array}$ & $\begin{array}{l}3.4 \\
4.1 \\
4.7 \\
5.4 \\
6.0\end{array}$ & $\begin{array}{l}1.025 \\
1.030 \\
1.035 \\
1.040 \\
1.045\end{array}$ & $\begin{array}{l}48 \\
49 \\
50 \\
51 \\
52\end{array}$ & $\begin{array}{l}27.9 \\
28.4 \\
28.8 \\
29.3 \\
29.7\end{array}$ & $\begin{array}{l}1.240 \\
1.245 \\
1.250 \\
1.255 \\
1.260\end{array}$ \\
\hline $\begin{array}{l}10 \\
11 \\
12 \\
13 \\
14\end{array}$ & $\begin{array}{l}6.7 \\
7.4 \\
8.0 \\
8.7 \\
9.4\end{array}$ & $\begin{array}{l}1.050 \\
1.055 \\
1.060 \\
1.065 \\
1.070\end{array}$ & $\begin{array}{l}53 \\
54 \\
55 \\
56 \\
57\end{array}$ & $\begin{array}{l}30.2 \\
30.6 \\
31.1 \\
31.5 \\
32.0\end{array}$ & $\begin{array}{l}1.265 \\
1.270 \\
1.275 \\
1.280 \\
1.285\end{array}$ \\
\hline $\begin{array}{l}15 \\
16 \\
17 \\
18 \\
19\end{array}$ & $\begin{array}{l}10.0 \\
10.6 \\
11.2 \\
11.9 \\
12.4\end{array}$ & $\begin{array}{ll}1 & 075 \\
1.080 \\
1.085 \\
1 & 090 \\
1 & 095\end{array}$ & $\begin{array}{l}58 \\
59 \\
60 \\
61 \\
62\end{array}$ & $\begin{array}{l}32.4 \\
32.8 \\
33.3 \\
33.7 \\
34.2\end{array}$ & $\begin{array}{l}1.290 \\
1.295 \\
1.300 \\
1.305 \\
1.310\end{array}$ \\
\hline $\begin{array}{l}20 \\
21 \\
22 \\
23 \\
24\end{array}$ & $\begin{array}{l}13.0 \\
13.6 \\
14.2 \\
14.9 \\
15.4\end{array}$ & $\begin{array}{l}1.100 \\
1.105 \\
1.110 \\
1.115 \\
1.120\end{array}$ & $\begin{array}{l}63 \\
64 \\
65 \\
66 \\
67\end{array}$ & $\begin{array}{l}34.6 \\
35.0 \\
35.4 \\
35.8 \\
36.2\end{array}$ & $\begin{array}{l}1.315 \\
1.320 \\
1.325 \\
1.330 \\
1.335\end{array}$ \\
\hline $\begin{array}{l}25 \\
26 \\
27 \\
28 \\
29\end{array}$ & $\begin{array}{l}16.0 \\
16.5 \\
17.1 \\
17.7 \\
18.3\end{array}$ & $\begin{array}{l}1.125 \\
1.130 \\
1.135 \\
1.140 \\
1.145\end{array}$ & $\begin{array}{l}68 \\
69 \\
70 \\
71 \\
72\end{array}$ & $\begin{array}{l}36.6 \\
37.0 \\
37.4 \\
37.8 \\
38.2\end{array}$ & $\begin{array}{l}1.340 \\
1.345 \\
1.350 \\
1.355 \\
1.360\end{array}$ \\
\hline $\begin{array}{l}30 \\
31 \\
32 \\
33 \\
34\end{array}$ & $\begin{array}{l}18.8 \\
19.3 \\
19.8 \\
20.3 \\
20.9\end{array}$ & $\begin{array}{l}1.150 \\
1.155 \\
1.160 \\
1.165 \\
1.170\end{array}$ & $\begin{array}{l}73 \\
74 \\
75 \\
76 \\
77\end{array}$ & $\begin{array}{l}38.6 \\
39.0 \\
39.5 \\
39.8 \\
40.1\end{array}$ & $\begin{array}{l}1.365 \\
1.370 \\
1.375 \\
1.380 \\
1.385\end{array}$ \\
\hline $\begin{array}{l}35 \\
36 \\
37 \\
38 \\
39\end{array}$ & $\begin{array}{l}21.4 \\
22.0 \\
22.5 \\
23.0 \\
23.5\end{array}$ & $\begin{array}{l}1.175 \\
1.180 \\
1.185 \\
1.190 \\
1.195\end{array}$ & $\begin{array}{l}78 \\
79 \\
80 \\
81 \\
82\end{array}$ & $\begin{array}{l}40.5 \\
40.8 \\
41.2 \\
41.6 \\
42.0\end{array}$ & $\begin{array}{l}1.390 \\
1.395 \\
1.400 \\
1.405 \\
1.410\end{array}$ \\
\hline $\begin{array}{l}40 \\
41 \\
42\end{array}$ & $\begin{array}{r}24.0 \\
.24 .5 \\
25.0\end{array}$ & $\begin{array}{l}1.200 \\
1.205 \\
1.210\end{array}$ & $\begin{array}{l}83 \\
84 \\
85\end{array}$ & $\begin{array}{l}42.3 \\
42.7 \\
43.1\end{array}$ & $\begin{array}{l}1.415 \\
1.420 \\
1.425\end{array}$ \\
\hline
\end{tabular}


SPECIFIC GRAVITIES IN DEGREES BAUME AND TWADDLE.(Continued)

Liquids Heavier than Water

\begin{tabular}{|c|c|c|c|c|c|}
\hline \multicolumn{2}{|c|}{$\begin{array}{c}\text { HYDROMLTER } \\
\text { READING DEGREES. }\end{array}$} & \multirow{2}{*}{$\begin{array}{l}\text { Specific } \\
\text { Gravity. }\end{array}$} & \multicolumn{2}{|c|}{$\begin{array}{c}\text { HYDROMETER } \\
\text { READING DEGREES. }\end{array}$} & \multirow{2}{*}{$\begin{array}{l}\text { Specific } \\
\text { Gravity }\end{array}$} \\
\hline Twaddle. & Baumé. & & Twaddle. & Baumé. & \\
\hline $\begin{array}{l}86 \\
87 \\
88 \\
89 \\
90\end{array}$ & $\begin{array}{l}43.4 \\
43.8 \\
44.1 \\
44.4 \\
44.8\end{array}$ & $\begin{array}{l}1.430 \\
1.435 \\
1.440 \\
1.445 \\
1.450\end{array}$ & $\begin{array}{l}129 \\
130 \\
131 \\
132 \\
133\end{array}$ & $\begin{array}{l}56.6 \\
56.9 \\
57.1 \\
57.4 \\
57.7\end{array}$ & $\begin{array}{l}1.645 \\
1.650 \\
1.655 \\
1.660 \\
1.665\end{array}$ \\
\hline $\begin{array}{l}91 \\
92 \\
93 \\
94 \\
95\end{array}$ & $\begin{array}{l}45.1 \\
45.4 \\
45.8 \\
46.1 \\
46.4\end{array}$ & $\begin{array}{l}1.455 \\
1.460 \\
1.465 \\
1.470 \\
1.475\end{array}$ & $\begin{array}{l}134 \\
135 \\
136^{\circ} \\
137 \\
138\end{array}$ & $\begin{array}{l}57.9 \\
58.2 \\
58.4 \\
58.7 \\
58.9\end{array}$ & $\begin{array}{l}1.670 \\
1.675 \\
1.680 \\
1.685 \\
1.690\end{array}$ \\
\hline $\begin{array}{r}96 \\
97 \\
98 \\
99 \\
100\end{array}$ & $\begin{array}{l}46.7 \\
47.1 \\
47.4 \\
47.8 \\
48.1\end{array}$ & $\begin{array}{l}1.480 \\
1.485 \\
1.490 \\
1.495 \\
1.500\end{array}$ & $\begin{array}{l}139 \\
140 \\
141 \\
142 \\
143\end{array}$ & $\begin{array}{l}59.2 \\
59.5 \\
59.7 \\
60.0 \\
60.2\end{array}$ & $\begin{array}{l}1.695 \\
1.700 \\
1.705 \\
1.710 \\
1.715\end{array}$ \\
\hline $\begin{array}{l}101 \\
102 \\
103 \\
104 \\
105\end{array}$ & $\begin{array}{l}48.4 \\
48.7 \\
49.0 \\
49.4 \\
49.7\end{array}$ & $\begin{array}{l}1.505 \\
1.510 \\
1.515 \\
1.520 \\
1.525\end{array}$ & $\begin{array}{l}144 \\
145 \\
146 \\
147 \\
148\end{array}$ & $\begin{array}{l}60.4 \\
60.6 \\
60.9 \\
61.1 \\
61.4\end{array}$ & $\begin{array}{l}1.720 \\
1.725 \\
1.730 \\
1.735 \\
1.740\end{array}$ \\
\hline $\begin{array}{l}106 \\
107 \\
108 \\
109 \\
110\end{array}$ & $\begin{array}{l}50.0 \\
50.3 \\
50.6 \\
50.9 \\
51.2\end{array}$ & $\begin{array}{l}1.530 \\
1.535 \\
1.540 \\
1.545 \\
1.550\end{array}$ & $\begin{array}{l}149 \\
150 \\
151 \\
152 \\
153\end{array}$ & $\begin{array}{l}61.6 \\
61.8 \\
62.1 \\
62.3 \\
62.5\end{array}$ & $\begin{array}{l}1.745 \\
1.750 \\
1.755 \\
1.760 \\
1.765\end{array}$ \\
\hline $\begin{array}{l}111 \\
112 \\
113 \\
114 \\
115\end{array}$ & $\begin{array}{l}51.5 \\
51.8 \\
52.1 \\
52.4 \\
52.7\end{array}$ & $\begin{array}{l}1.555 \\
1.560 \\
1.565 \\
1.570 \\
1.575\end{array}$ & $\begin{array}{l}154 \\
155 \\
156 \\
157 \\
158\end{array}$ & $\begin{array}{l}62.8 \\
63.0 \\
63.2 \\
63.5 \\
63.7\end{array}$ & $\begin{array}{l}1.770 \\
1.775 \\
1.780 \\
1.785 \\
1.790\end{array}$ \\
\hline $\begin{array}{l}116 \\
117 \\
118 \\
119 \\
120\end{array}$ & $\begin{array}{l}53.0 \\
53.3 \\
53.6 \\
53.9 \\
54.1\end{array}$ & $\begin{array}{l}1.580 \\
1.585 \\
1.590 \\
1.595 \\
1.600\end{array}$ & $\begin{array}{l}159 \\
160 \\
161 \\
162 \\
163\end{array}$ & $\begin{array}{l}64.0 \\
64.2 \\
64.4 \\
64.6 \\
64.8\end{array}$ & $\begin{array}{l}1.795 \\
1.800 \\
1.805 \\
1.810 \\
1.815\end{array}$ \\
\hline $\begin{array}{l}121 \\
122 \\
123 \\
124 \\
125\end{array}$ & $\begin{array}{l}54.4 \\
54.7 \\
55.0 \\
55.2 \\
55.5\end{array}$ & $\begin{array}{l}1.605 \\
1.610 \\
1.615 \\
1.620 \\
1.625\end{array}$ & $\begin{array}{l}164 \\
165 \\
166 \\
167 \\
\cdot 168\end{array}$ & $\begin{array}{l}65.0 \\
65.2 \\
65.5 \\
65.7 \\
65.9\end{array}$ & $\begin{array}{l}1.820 \\
1.825 \\
1.830 \\
1.835 \\
1.840\end{array}$ \\
\hline $\begin{array}{l}126 \\
127 \\
128\end{array}$ & $\begin{array}{l}55.8 \\
56.0 \\
56.3\end{array}$ & $\begin{array}{l}1.630 \\
1.635 \\
1.640\end{array}$ & $\begin{array}{l}169 \\
170 \\
171\end{array}$ & $\begin{array}{l}66.1 \\
66.3 \\
66.5\end{array}$ & $\begin{array}{l}1.845 \\
1.850 \\
1.855\end{array}$ \\
\hline
\end{tabular}




\section{Chlorine Data}

Chlorine [ $\chi \lambda \omega$ pós $=$ green] a greenish yellow 'gas, easily compressed to a liquid, was discovered in 1774 by Scheele, a Swedish Chemist. Atomic weight 35.45 , molecular weight 70.9 , vapor density 35.8 . Liquefies at $-33.6^{\circ} \mathrm{C} .\left(-28.5^{\circ} \mathrm{F}.\right)$, solidifies at $-102^{\circ} \mathrm{C} .\left(-151.36^{\circ} \mathrm{F}.\right)$. Under pressure of 6 atmospheres liquefies at $0^{\circ} \mathrm{C}$. $\left(32^{\circ} \mathrm{F}\right.$.).

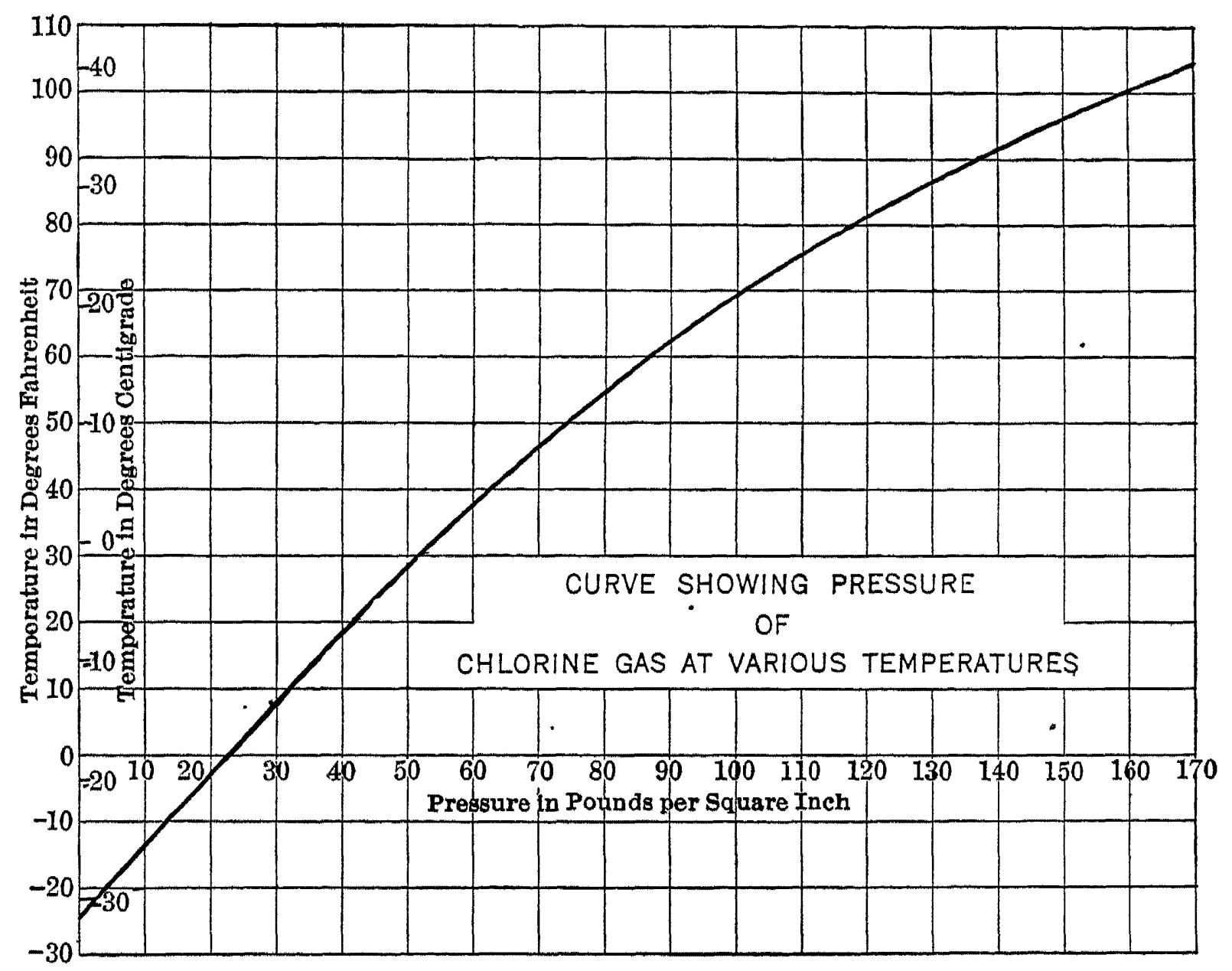

WEIGHTS OF CHLORINE

\begin{tabular}{l|l|l}
\hline \multicolumn{1}{c|}{ Datum. } & Gaseous Chlorine. & Liquid Chlorine. \\
\hline Specific gravity & 2.49 (air $=1)$ & 1.44 (water $=1)$ \\
Weight of 1 liter & $3.167 \mathrm{gms.}$ & 1440 gms. \\
Weight of 1 cu. ft. & $0.198 \mathrm{lb}$. & $89.752 \mathrm{lbs}$. \\
Weight of 1 gal. & $0.026 \mathrm{lb}$. & $11.999 \mathrm{lbs}$. \\
\hline
\end{tabular}

One volume of liquid chlorine is equivalent to 444.4 volumes of chlorine gas. 
SOLUBILITY OF CHLORINE

\begin{tabular}{c|c|c|c}
\hline \multicolumn{2}{c|}{ Temperature } & Solubility Ratio by Volume & $\begin{array}{c}\text { Pounds of Chlorine Soluble } \\
\text { in 1,000,000 Gals of Water. }\end{array}$ \\
\hline $\mathrm{C}^{\circ}$ & $\mathrm{F}^{\circ}$ & 15 & 20,000 \\
\hline 0 & 32 & 30 & 40,000 \\
10 & 50 & 18 & 24,000 \\
\hline 0 & 88 & & \\
\hline
\end{tabular}

One part per million $=834 \mathrm{lbs}$ per million gallons of water $=0058$ grain per gallon One grain per gallon $=1712$ parts per millon $=142.86$ lbs. per million gallons of water

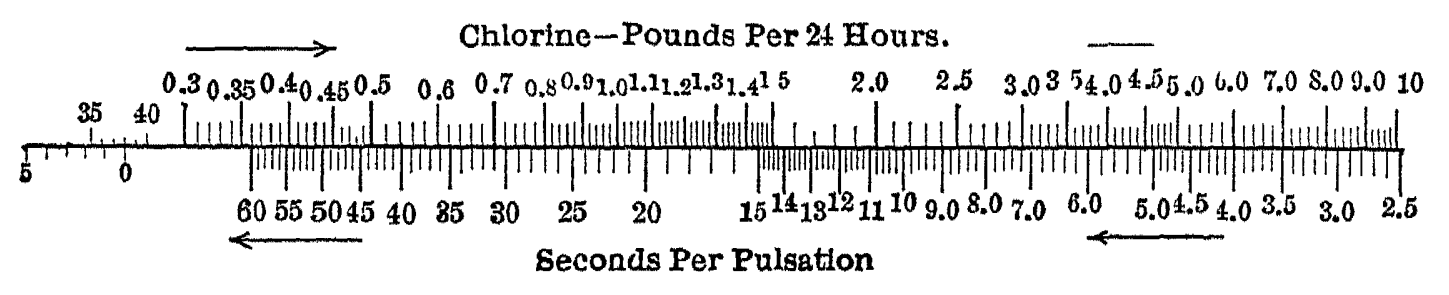

Chlorine - Pounds Per 24 Hours.

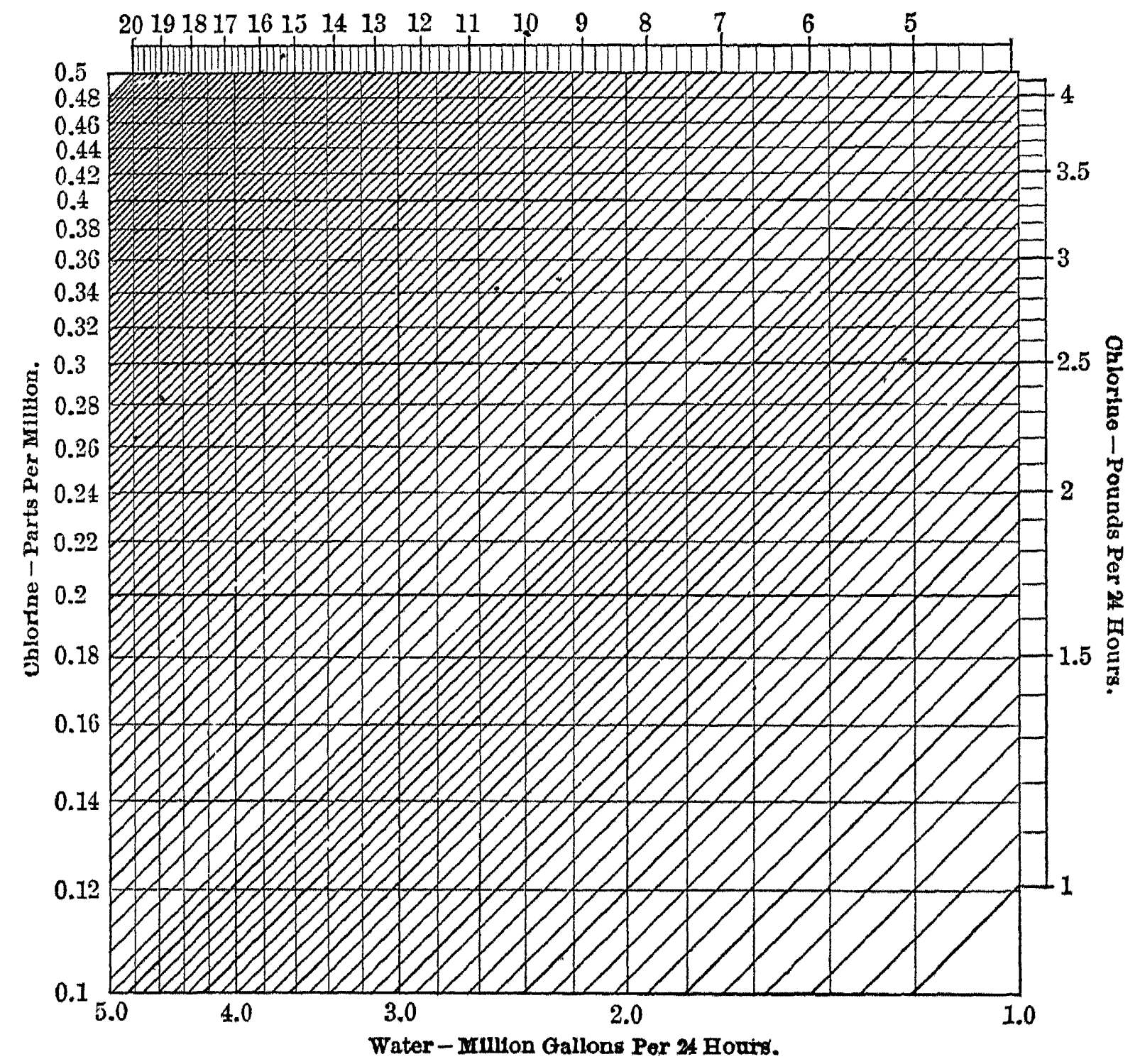

FIg 86.-Chlorine Data for the Treatment of Water Supplies. (Wallace-Tiernan Co.), See also data on another page. 
Transportation of Bacteriological Specimens. The preparation and shipment of specimens for bacteriological examinationis a very important procedure. Unfortunately the bacteriologist and 'chemist may not have much to do with this step in the examination of any substance; usually, he is expected to handle the specimen after it has been delivered to his laboratory. At times, he may be quite justified in refusing to handle certain specimens which are sent because it would be dangerous to himself or to those with whom he comes in contact. Since much of the material for bacteriological examination is sent through the United States mails it is important that he have a knowledge of the postal rules and regulations which concern these shipments. Hasseltine (1918)* has given a good résumé of the subject. The following quotation is cited by this author from the Postal Regulations issued by the Post Office Department. Sections 472 and 473 read as follows:

Sec. 472. All kinds of poison, and all articles and compositions containing poison, and all poisonous animals, insects and reptiles, and explosives of all kinds, and inflammable materials and infernal machines, and mechanical, chemical, or other devices or compositions, which may ignite or explode, and all disease germs or scabs, and all other natural or artificial articles, compositions or materials of whatsoever kind which may kill, or in any wise hurt, harm or injure another, or damage, deface, or otherwise injure the mails or other property, whether sealed as firstclass matter or not, are hereby declared to be non-mailable matter, and shall not be conveyed in the mails or delivered from any post office or station thereof, nor by any letter carrier; but the Postmaster General may permit the transmission in the mails, under such rules and regulations as he shall prescribe as to preparation and packing, of any articles hereinbefore described which are not outwardly or of their own force dangerous or injurious to life, health or property. (The rest of this section is not concerned with shipment of bacteriological materials.)

Sec. 473. 1. Specimens of diseased tissues may be admitted to the mail for transmission to United States, State, municipal, or other laboratories in possession of permits referred to in paragraph 3 of this section only when inclosed in mailing cases constructed in accordance with this regulation: Provided, That bacteriologic or pathologic specimens of plague and cholera shall under no circumstances be admitted to the mails.

2. Liquid cultures, or cultures of microorganisms in media that are

* Hasseltine, H. E. 1918. Public Laboratory Specimens, Their Preparation and Shipment. Public Health Reports 32, 2016-2032. Reprint from the same No. 438-1918. 
fluid at the ordinary temperature (below $45^{\circ}$ C. or $113^{\circ} \mathrm{F}$. ), are unmailable. Such specimens may be sent in media that remain solid at ordinary temperature.

3. No package containing diseased tissue shall be delivered to any representative of any of said laboratories until a permit shall have first been issued by the Postmaster General, certifying that said institution has been found to be entitled, in accordance with the requirements of this regulation, to receive such specimens.

4. (a) Specimens of tubercular sputum (whether disinfected with carbolic acid or not disinfected) shall be transmitted in a solia glass vial with a mouth not less than 1 in. in diameter and capacity of not more than 2 oz., closed by a cork stopper or by metallic serew top protected by a rubber or felt washer. Specimens of diphtheria, typhoid, or other infectious or communicable diseases or diseased tissue shall be placed in a test tube made of tough glass, not over $\frac{3}{4}$ in. in diameter and not over $7 \frac{1}{2}$ ins. in length, closed with a stopper of rubber or cotton and sealed with paraffine or covered with a tightly fitting rubber cap.

(b) The glass vial or test tube shall then be placed in a cylindrical tin box, with soldered joints, closed by a metal screw cover with a rubber or felt washer. The vial or test tube in this tin box shall be completely and evenly surrounded by absorbent cotton, closely packed.

(c) The tin box, with its contents, must then be inclosed in a closely fitting metal, wooden, of papier-mâché block or tube, at least $\frac{3}{16}$ in. thick in its thinnest part, of sufficient strength to resist rough handling and support the weight of the mails piled in bags. This last tube shall be tightly closed with a screw-top cover with sufficient screw threads to require at least one and one-half full turns before it will come off, and fitted with a felt or rubber washer. (See Fig. 8.)

5. Specimens of blood dried on glass microscopic slides for the diagnosis of malaria or typhoid fever by the Widal test may be sent in any strong mailing case which is not liable to breakage or loss of the specimen in transit.

6. Upon the outside of every package of diseased tissues admitted to the mails shall be written or printed the words "Specimen for bacteriological examination. This package to be pouched with letter mail." 


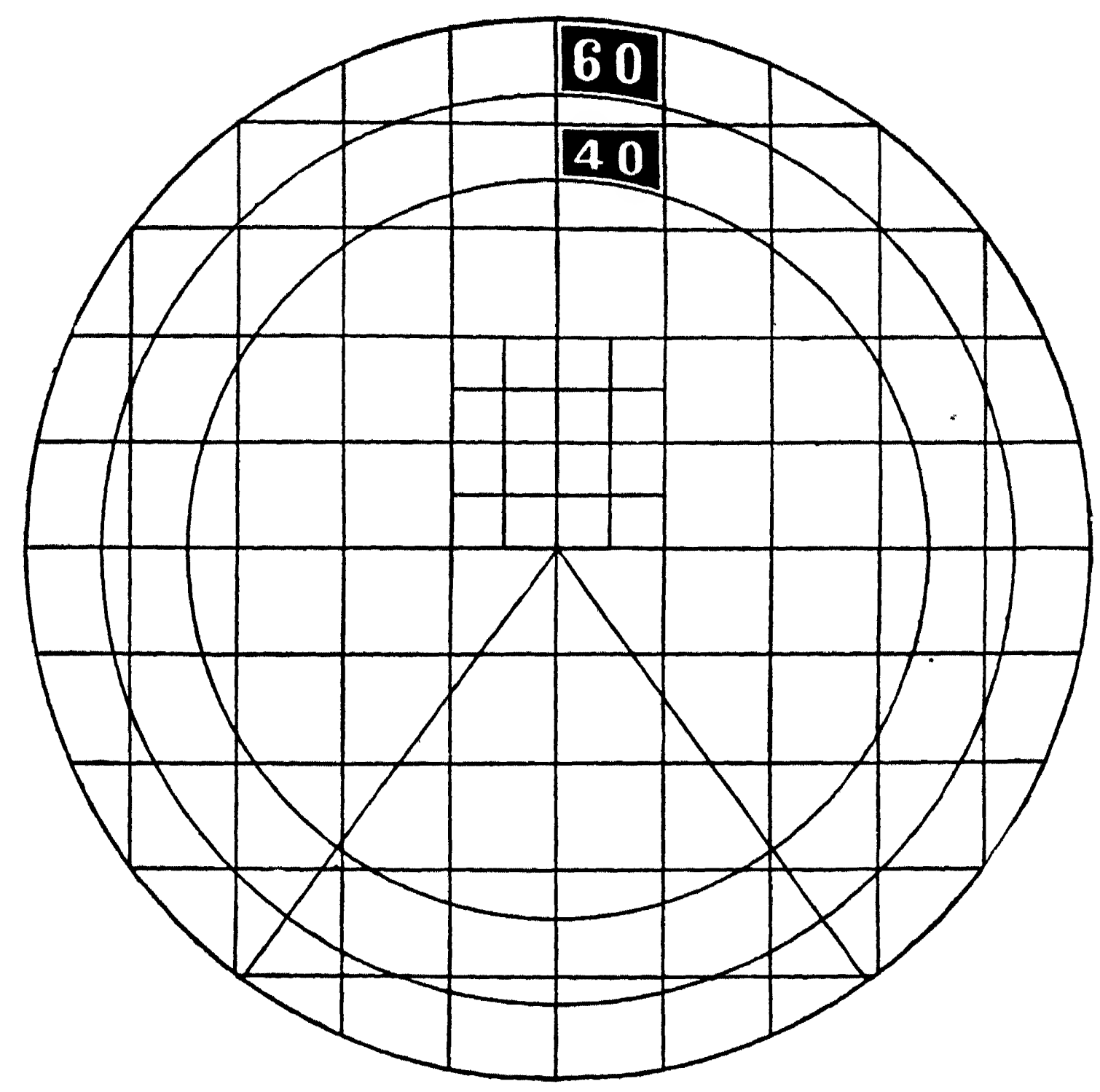

Frost's Plate Counter.

Each of the larger squares represents an area of $1 \mathrm{sq} \mathrm{cm}$. The figures 60 and 40 indicate the number of square centimeters in the circles bounded by the respectivelines Each sector is one-tenth the area of its circle.

Department of Bacteriology, University of Illinois. 


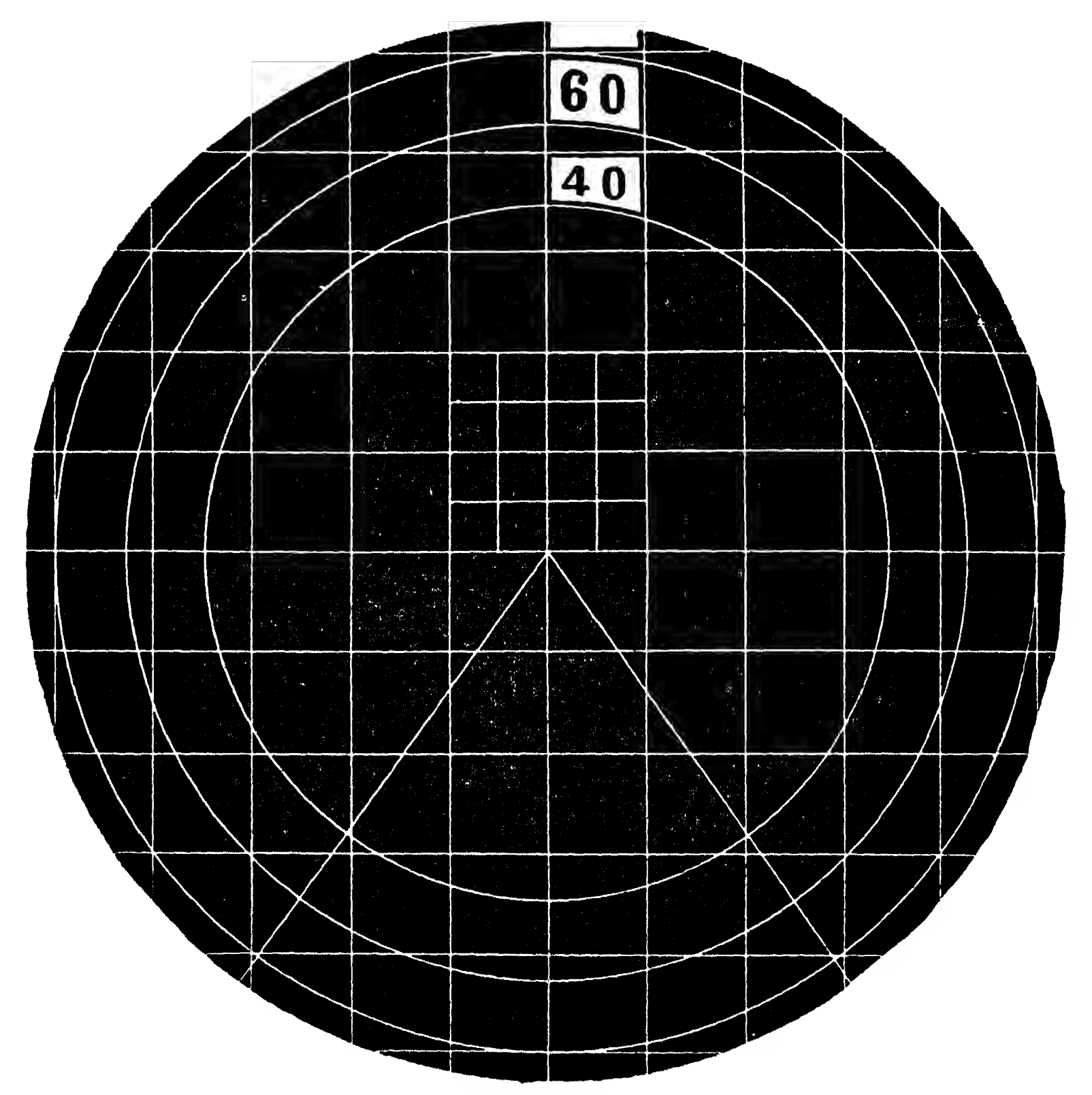

Frost's Plate Counter.

Each of the larger squares represents an area of $1 \mathrm{sq} . \mathrm{cm}$. The figures 60 and 40 indicate the number of square centimeters in the circles bounded by the respective lines. Each sector is onetenth the area of its circle.

Department of Bacteriology, University of Illinois. 


\section{INDEX}

A

Acetaldehyde reaction for ethyl alcohol, 194

Acid fast staining, theory of, 87

- - - Ziehl-Niehlsen method, 86

Acidity, degrees recognized in milk, 114

- degrees recognized in clear media, 115

Aciduric bacteria, 236

Action of bacteria on carbohydrates, 193

- - - glucosamine, 191

- - - polypeptides, 190

- - - proteins, 189

- - bile on bacteria, 234

- - certain bacteria on proteins, 367

Adjusted milks, defined, 374

Adulteration of compressed yeast with brewer's yeast, 220

Advantages of "bleach" in water treatment, 325

- - pasteurization of milk, 416

Aerobic respiration, 241

Aeroscope, 264

Agar, 43

- colonies, study of, 110

- for Ps. radicicola, 50

- gelatin medium (North), 51

- medium, plain, 48

- stroke, study of, 110

Age of milk, 377

Agglutination of bacteria, 320

Alanin, 184

Albuminoid ammonia, determination in water, 282

Alcohol as a disinfectant, 140

- table, 561

Alcoholic fermentation, 194

Alkaline blood agar, 70

- hemoglobin agar, 70

- methylene blue, 90

Alkalinity, determination in water, 285
Allen's microscopic method of milk analysis, 404

Alwood fermentation valve, 220, 221

Analysis of mud deposits, 352

- - sewage sludge, 352

Anderson's method for tubercle bacilli in milk, 413

Amino acids in gelatin, 41

- - - proteins, 184

Ammonia production by pure cultures, 116

Ammonium chloride in bread, 218

Anaerobic methods, 17

- respiration, 242

Analysis of Liebig's meat extract, 41

Aniline gentian violet, 90

Anti-protein sera, 491

Antiseptic values, 143

Albargin, disinfectant, 135

Aperture, numerical, 28

Aqueous alcoholic stains, 81

- stains, preparation, 81

Argentamin, disinfectant, 135

Argentose, 135

Arginine, bacterial decomposition, 244

-, chemical structure, 244

Arnold steam sterilizer, 126

Arnold's test for heated milk, 372

Ascomycetes, 202

Asepsis in food preservation, 495

Ash in milk, 368

Aspartic acid, 186

Atomic weights, 563

Autoclave, 125

Available chlorine, 136

- oxygen in water, 344

Ayer's milk tube method, 408

\section{B}

Basidiomycetes, 202

Babcock test for fat in milk, 369 
Bacillus anthracis, isolation from water, 322

- colon index, 314

— - test in water analysis, 299

- diphtheriæ in milk, 414

- dysenteriæ, isolation from water, 320

- enteritidis in water, 297

- tuberculosis in feces, 258

- - milk, 412

- - water, 323

- typhosus in butter, 426

- - cream, 437

- - feces, 254

- - isolation from water, 317

- - Carnot and Halle's method, 256

- - Holt-Harris-Teague method, 255

- - Kendall and Day's method, 255

- - Lumsden and Stimson's method, 254

- - Morishama and Teague's method, 257

_- - Teague and Clurman's method, 257

- - longevity in water, 343

Bacteria and life, 233

-, Eberle-Klein method, 248

- in baked bread, 224

- cream and cream ripening, 423

-

- - yolk, 459

- - feces, 238

- - fresh eggs, 460

- - self-rising bread, 223

- - the stomach, 230

-, MacNeal's procedure for Winterberg method, 247

-, Matill and Hawk's procedure, 252

-, Steel's modification of the Strassburger method, 250

Bacterial count in milk, significance, 385

- - water analysis, 290

- examination of butter, 423

- - eggs, 463

- - - ice, 337

- - milk, 375

- groups in ice cream, 435

- - raw and pasteurized milk, 417

- vs. chemical examination of water, 271
Bacteriological specimens, transportation, 577

Bacteriology of butter, 423

- - cheddar cheese, 429

- - ice, 336

Balaz's test for bolled milk, 373

Barn conditions in milk production, 377

Barsiekow's medium, 59

Baskets, various types of, 7,8

Bean agar, 50

Beattie's method for anaerobic bacteria, 22

Beer wort, 57

Beijerinck's medium for nitrate reduction, 67

Benedict's method for estimation of dextrose, 197

- - - reducing sugars, 196

Benzoic acid, detection in milk, 371

Bergey's method for typhoid bacilli in butter, 426

Besson's method for tubercle bacilli in milk, 413

Bile, germicidal action, 234

- salt broth, 46

Biochemical oxygen demand of sewage, 350

Bismuth compounds in disinfection, 135

Blanching, 507

Blood agar, 69

- - alkaline, 70

- - for streptococci, 69

- films, staining, 87

- serum, 69

Boiling in sterilization, 126

Boric acid in disinfection, 139

- - milk, determination, 371

Böttcher's counting chamber, 10

- moist chamber, 76

Botulism, 533

Bread making, 222

- medium, 59

Breeu's capillary pipettes, 397

Breed smears in milk analysis, 397

Bromine as a disinfectant, 134

- reaction for tryptophane, 192

- water test for skatol, 247

Brown's fermentation tube, 1

Buchner's anaerobic methods, 17 
Bushnell and Mauer's method for egg analysis, 465

Butter flavor, 423

\section{C}

Cadaverine, 529

Calcium compounds in disinfection, 135

— oxide, 138

Calibration of micrometer oculars, 36

Campbell's method for tubercle bacilli in milk, 413

Candling eggs, 461, 463

Canned meats, 481

Canning, 506

Cannon's hydrolized casein medium, 63

Capaldi's egg medium, 58

Capsules, determination on bacteria, 109

Capsule stain, Huntoon's method, 84

- - Muir's method, 83

- - Welch's method, 84

Carbohydrate agar, 48

- broths, 44

Carbohydrates, action of bacteria on, 193

-, classification, 193

Carbol fuchsin, 90

Carbonated broth, 47

Carbon in disinfection, 140

Carnot and Halle's method for typhoid bacilli in feces, 256

Carriers and food infection, 530

Carrot infusion medium, 60

Case history of botulism, 535

Casein agar after Ayers, 70

- medium, 63

Causes of food poisoning, 533

Cellulose agar, 61

Certificates for chemical examination of water, 276, 277

Characteristics of a good indicator of pollution, 294

- - anaerobic water bacteria, 298

- fermentation, 240

- milk-borne epidemics, 541

- water-borne epidemics, 541

Cheddar cheese, bacteriology, 429

Cheese bacteriology, 428

- poisoning, 431

- ripening, 428
Chemical analyses of ice, 337

- and bacterial examination of water, 271

- changes in milk from souring, 366

- constituents of milk, 363

- examination of milk, 367

- standards for milk, 373

Chicago drainage canal and Illinois River, 341

Chicken broth medium, 46

Chinese ink preparations, 78

Cholera red reaction for $M$. cholerce in water, 322

Chlorine data, 575

Chloramine, 139

- in water treatment, 326

Chloride of lime in disinfection, 135

Chlorine as a disinfectant, 132

-, determination in water, 280

Cholera, $M$. microspira in feces, 257

Chopped meats, 480

Chromatic aberration, 29

Classification of water bacteria, 193, 286

- - proteins, 182

- - yeasts, 213

Cleaning apparatus, 6

- hemocytometers, 16

- solution, 6

Coagulation coefficient of disinfectants, 171

- of milk by rennin, 365

Coarse adjustment on microscope, 25

Cohn's classification of bacteria, 94

- solution, preparation, 63

- - , characteristics of bacteria in, 112

Cold storage, 505

Cole's method for microscopic agglutination, 321

Collection of milk samples, 393

- - water samples, 273

Colloidal silver in disinfection, 135

Color, determination in water, 278

- reactions of proteins, 191

Coloring matter in milk, 371

Combined acidity of the stomach, 233

Comparison of thermometer scales, $\mathbf{5 7 0}$

Composition of hens' eggs, 453

- - milk serum, 365

- tomato pulp, 514 
Completed test for $B$. coli in water, 302

Compounds in goats' and human milk, 364

Compressed yeast, 216

- -, microscopic examination, 220

- - outline of manufacture, 217

Concave slides, types, 77

Concentrated solutions in food preparation, 498

Condensed milk, 433

Conjugated proteins, 183

Conn's method for bacteria in butter, 428

Constituents of media, 39

Coplin's jars for staining, 80

Copper salts in disinfection, 139

Corn agar medium, 50

Cover glasses, sizes and thicknesses, 5

Cresol as a disinfectant, 141

Cystine, decomposition, 245

-, structure, 185

Czapek's solution, 66

\section{D}

Dairy score card, 380

Dakin-Carrel solution in disinfection, 137

Dark ground illumination, 78

Deaminization, 243

De Bary's classification of bacteria, 95

Decarboxylation, 243

Decoction of dried fruits, 59

Delepine's method for tubercle bacilli in milk, 412

Derived proteins, 183

Descriptive chart, 100

Description of microorganisms, 101

Detection of foreign proteins, 490

Determination of the origin of epidemics, 544

Development of the Descriptive Chart, 100

Dextrose potassium phosphate broth, 47

Diagnosis of food poisoning, 535

Dialysis sterilization, 128

Dialyzed milk, 72

Diet and the intestinal bacterial flora, 235

Differential staining of blood films, 87

Dilution bottles, 2

- pipettes for blood counts, 15
Dilutions, 10

Direct vs. plate count on milk, 396

Dirt in milk, 384

Disadvantages of "bleach" in water treatment, 325

Disinfectants, standardization, 144

Disinfection, 131

- vs. periodic system, 133

Dissolved oxygen in water, 344

Doane-Buckley method for leucocytes in milk, 410

Dolt's agar medium, 50

- medium, 65

Dorset's egg medium, 58

Dough raising power of bread yeasts, 220

Dox's solution for fungi, 65

Draw tube on microscope, 27

Dried eggs, 46

Drigalski and Conradi's medium, 62

Dry heat sterilization, 121

Drying in food preservation, 504

Dunham's solution, 47

Durham's fermentation tube, 1

Effect of baking on microorganisms in bread, 224

- - low temperatures on bacteria, 504

- pasteurization on bacteria in milk, 417

- - pressure in food preservation, 506

- - storage on ice cream bacteria, 436

- - yeast on flavor of bread, 222

Efficiencies of peptone, 40

Efficiency of bread yeasts, 220

Egg-meat mixture for putrefaction, 58

Ehrenberg's work on classification of bacteria, 94

Ehrlich's reaction for indol, 115

Elements in inorganic compounds, 187

- of "quality" in milk, 381

Elsner's potato medium, 60

Endo's agar medium, Kendall's method, 52

- - - Hygienic Laboratory method, 53

- - Levine's method, 54

- medium for isolating $B$. typhosus from water, 318 
Endospore, observation of, 109

Enzymes, 118

— in eggs, 460

Eosin-brilliant green agar medium, 54

- -methylene blue agar medium, 54

Epidemics of paratyphoid fever, 532

Errors in staining flagella, 85

Erythrocytes, enumeration, 13

Esten and Mason's method for bacteria in ice cream, 437

Estimation of bacteria in tomato products, 517

- - dextrose (Allihn's method), 196

- molds in tomato products, 515

Ethyl acetate reaction for alcohol, 195

- alcohol, aldehyde reaction, 194

- - iodoform reaction, 194

Evaluation of bleaching powder, 328

Examination of bread, 224

- canned foods, 511

- clams, 489

- Hamburger steak, 483

- - meat, 482

- molds, 212

- oysters, 485

- - sausage, 483

- shell fish, 483

- - tin cans for leaks, 513

- - water for available chlorine, 329

\section{F}

Factors influencing bacteria in milk, 375

- - - - eggs, 458

- - disinfection, 133

Fasting and intestinal bacteria, 236

Fats, 197

— in milk, 368

Fecal and non-fecal B. coli, 308

Fehling's solution, preparation, 195

- test for reducing sugars, 195

Fermentation in food preservation, 497

- - the intestinal tract, 240

— of sugars by streptococci, 295

- reactions of pure cultures, 117

- tubes, types, 1

Fermented milks, 433

Fermenting powers of yeasts, determination of, 220
Fermi's culture fluid, 68

Fernbach's antitoxin flask, 5

Ferric chloride test for phenol, 247

Ferrous sulphate in disinfection, 139

Filtration through liquids, 128

- sterilization, 127

Fine adjustment of the microscope, 26

Flagella staining, 86

Flaming sterilization, 122

Flasks, types of, 5

Flat sours in canning, 511

Flavor of butter, 423

Flours used in bread making, 222

Fluorescein for tracing water pollution, 323

Food-borne epidemics of disease, 542

Food poisoning, 528

— preservation, 494

Formaldehyde in disinfection, 142

Forms of growth, 102

Frankel and Voges' solution, 68

Trankel's solution, 64

Free ammonia in water, determination, 282

Freudenreich's flask, 5

Frost and Ravenel's test for heated milk, 372

Frost's microscopic method for milk analysis, 401

- plate counter, 579,580

Frozen eggs, 463

Fructification in molds, 203

Fuller and Johnson's classification of water bacteria, 289

Fungi, 202

- imperfecti, 202

\section{G}

Gases in canned foods, 510

Gastric analysis, 233

- juice, germicidal action, 229

Gedding's method for $M$. cholerce in feces, 257

Gelatin, preparation and composition, 42,43

- agar, 56

-, colonies, study of, 111

— stab, study of, 111 
Gerber's test for milk fat, 369

Germicidal action of saliva, 228

_ - - gastric juice, 229

Giblet broth, 46

Giltay and Aberson's solution, 67

Glossary of terms, 105

Glucosamines, bacterial action on, 191

Glucose formate broth, 47

- - agar, 49

Glutamic acid, 186

Glycerol in disinfection, 142

- potato broth, 60

Glycocoll, 184

Grades on milk, 391

- milk by bacterial count, 387

Grading eggs, 455

Gram positive and negative bacteria, 83

- stain, Nicoll's modification, 83

- - theory of, 81

Graves' modification of the Durham fermentation tube, 2

Griess method for nitrites, 116

Groups, bacteriological, 99

— of bacteria in intestines, 236

\section{H}

Halogens as disinfectants, 134

Ham souring, 499

Hanging blocks, 77

- drops, 77, 78

Harding and Prucha's method for bacteria in cheese, 431

Haricot decoction, 58

Harrison's method for bacteria in cheese, 432

Hay infusion, 57

Hazen theorem, 269

Heated milk, determination of, 371

Hemocytometer rulings, 14

- chamber, 12

Heese agar medium, 51

- and Niedner's agar, 50

High-pressure steam sterilization, 122

Hiss agar-gelatin medium, 51

- medium, 56

Histidine, 187

Holt-Harris-Teague method for B. typhosus in feces, 255
Hot air oven sterilization, 122

Houston's method for isloating streptococci from feces, 296

Huntoon's method for staining capsules, 84

Hydrogen sulphid formation, 116

Hydrolysis of carbohydrates during sterilization, 44

Hyphæ, structure of, 212

\section{I}

Ice and typhoid fever, 339

- cream, 434

- plant score card, 439

Identification of bacteria by agglutination, 321

Illuminating power of the microscope, 29

Illumination of the microscope, 31

Incidence of tubercle bacilli in market butter, 427

- - - - milk, 414

Incineration in sterilization, 122

Inclination joint on the microscope, 25

Indol from tryptophane, 244

- , test for, 115

Indicator solutions, 553

Indicators of pollution, 294

Infection of food by carriers, 530

Influence of salt on bacteria, 499

Initial contamination of milk, 375

Inorganic disinfectants, 133

- elements in organic compounds, 187

Inspissator, 127

Instructions for collecting water samples, 274

Intermittent sterilization, 127

Interpretation of bacterial tests on milk, 387

- - results in water analysis, 315

Intervals before analysis of water samples, 273

Intestinal bacteria, effect of protein diet, 236

- water drinking, 237

- flora, classification, 238

Invigoration of cultures, 108

Iodine absorption number of fats, 198

- in disinfection, 134 
Iodoformogen, 134

Iodoform reaction for acetone, 195

- - - ethyl alcohol, 194

Iodo-hemol, 134

Iodol, 134

Iodomuth, 134

Isolation of $B$. enteritzdrs sporogenes from water, 299

- B. typhosus from water, 317

- - pure cultures, 10

Isoleucine, 185

\section{$\mathrm{J}$}

Jeffer's counting plate, 9

Jensen's system of classification of bacteria, 97

Jones' anaerobic method, 23

Jordan's classification of water bacteria, 290

- non-protein medium, 66

\section{$\mathbf{K}$}

Kendall and Day's procedure for $B$. typhosus in feces, 255

Ketchup, 514

Key to Aspergillus molds, 210

- - genera of budding fungi, 215

_ - - true yeasts, 214

- penicillia, 207

Koch's concave slide, 76

- culture flask, 5

Koettstörfer number of fats, 199

Kolle culture flask, 5

Kusserow's method for determining fermenting power of yeasts, 221

Kuster's anaerobic method, 18

\section{I}

Labarraque's solution, 137

Lactic acid fermentation, 193

_ — in the stomach, 233

Lactose bile in water analysis, 311

Lafar's counting plate, 9

Leavening agent in self-rising bread, 223

Leeuwenhoeck's work on bacteria, 94

Leffman-Beam test for milk fat, 369
Lehmann and Neumann's work on classification, 97

Lellendahl's staining dish, 80

Lentz's anaerobic apparatus, 18

Leucine, structure, 185

-, enumeration, 15

Leucocytes in milk, 408

Levine's eosin-methylene blue agar, 54

Liebermann's reaction, 192

Liebig's meat extract, 41

Life cycles of bacteria, 99

Light, sterilization, 128

Lime in disinfection, 136

Lipman-Brown medium, 66

Liquid chlorine, addition to water, 331

Lister culture flask, 5

Lister's work on milk, 95

Litmus gelatin, 56

- lactose agar, 48

- milk, preparation, 71

- - cultural characteristies in, 115

- whey, 71

Löhnis' method for bacteria in butter, 426

- - - - cheese, 432

Loss of virulence on culture media, 118

Low temperature, effect on bacteria, 504

Lugol's iodine solution, 90

Lumsden and Stimson's method for B. typhosus in feces, 254

Lymphocytes, staining of, 89

Lysine, decomposition, 245

-, structure, 186

Lysol in disinfection, 141

\section{M}

MacKenzie's culture flask, 68

McLeod's anaerobic method, 18

Magnifying power of microscope, 29

Magnification tables, microscopes, 33, 34

Malachite green medium, 52

Malt extract medium, $\mathbf{5 8}$

Mayer's culture fluid, 62

Meat extract, composition of, 40,41

- - partition of nitrogen in, 42

-infusion, preparation, 40

- juice (infusion), 41

Medium for fungi, 65 
Meissl's method for determining fermenting power of fungi, 220

Metals, action of on bacteria, 133

Methods of adding "bleach" to water, 327

- - water treatment, 324

- for study of yeasts, 218

Methyl red test in water bacteriology, 307

Micro-analysis of tomato products, 515

Micrometer, ocular, 436

Micrometry, 34

Microscope, 23

Microscopic agglutination, 321

- examination of gastric contents, 233

- method for milk analysis, 395

Microspira cholerce, in feces, 257

- - - milk, 413

- - water, 322

Migula's classification of bacteria, 95

Milk agar, preparation of, 72

- borne epidemics, 541

- medium, characters of bacteria in, 112

- , preparation of, 71

- of lime, 138

- proteins, 365

- samples, 396

- standards, 392

Millon's reaction, 191

Mills-Reincke phenomenon, 268

Modified aeroscope, 265

Mohler's method for bacteria in cheese, 432

Moist chamber preparations of yeasts, 219

- heat sterilization, 122

Molds in butter, 425

-, structure, 203

Moore's staining dish, 80

Miquel culture flask, 5

Mordants, 81

Morishama and Teague's method for B. typhosus in feces, 257

Motility, 109

Mucor, 205

- mucedo, 204

Muir's method for staining capsules, 83

Müller's classification of bacteria, 94

Mycoderma in bread yeast, 221

\section{N}

Naegeli's medium, 63

Naples jar for staining, 80

Neisser's method for spore staining, 85

Nesslerization, 281

Neutral red agar, 50

- - bile salt agkr, 49

- - broth, 46

Nicoll's modification of Gram's stain, 83

Nitrate broth, 45

Nitrates, determination in water, 283

Nitrite formation, 116

-, test for, 116

Nitrites, determination in water, 283

Nitrogen-free medium for bacteria, 65

-, significance in water analysis, 280

Nitroprusside reaction for acetone, 195

Nitrosoindol test, 115

North's agar-gelatin medium, 51

Novy's anaerobic method, 23

Number of bacteria in feces, 238

Numerical aperture, 28

Nutrient broth, preparation, 44

- - , characteristics of bacteria in, 110

- gelatin, 55

Nylander's test for reducing sugars, 196

\section{0}

Objections to pasteurization, 416

Objectives on microscope, 27

Oculars on microscope, 31

Odor, determination of, in water, 279

Oidium lactis, 205

Omelianski and Winogradski's medium, 67

Opinions on bacterial count in milk, 389

Organic acids in the stomach, 233

Organic halogens, test for, 189

- nitrogen, test for, 187

- sulphur, test for, 188

Origin of bacteria in meat, 479

- - epidemics, 544

Ox-bile medium, 59

Oxygen consumed, determination of, in water, 284

- relations, study of, 110

- solution in water. 345 


\section{$\mathbf{P}$}

Paratyphoid fever, 531

Parietti's solution, 62

Partially confirmed test for $B$, coli in water, 301

Pasteur's medium, 66

Pasteurization of milk, 414

Pathogenic bacteria in river water, 343

- - and pasteurization of milk, 419

- streptococci in milk, 422

Pathogenicity of pure cultures, to animals, 117

- - , to plants, 118

Pea-flour extract, 57

Penicillium, 207

Peptone, 40

- sucrose solution (Buchanan), 57

Permeability of egg shells, 459

Petri dishes, types of, 2, 3

Pettenkofer's experiment on cholera, 230

Phenol in disinfection, 141

— from tyrosine, 243

Phenylamin, 185

Phenyl-hydrazine reaction, 196

Phycomycetes, 202

Physiological method for dilutions, 219

Pickling in food preservation, 503

Piorowski's culture flask, 5

Plain milk medium, 71

Plan of modern corn packing plant, 508

Plate vs. microscopic count on milk, 396

Platinum wires, 7

Polynuclear neutrophilic leucocytes, 89

Polypeptids, bacterial action on, 190

Potato agar, 61

- gelatin, 60

- infusion, 60

- mash, 60

- slant, study of, 111

- starch jelly, 111

Pouring plates, 9

Prazmowski's culture flask, 63

Precipitin test for proteins, 491

Preparation of common stains, 90

- - standard reagents, 554

Preservation of food by asepsis, 495

- - by concentrated solutions, 498

Preservatives in milk, 371
Pressure and head of water, 565

Presumptive test for $B$. coli, 301

Procedure for $B$. coli identification at Cincinnati, 313

- - studying water bacteria, 287

Process of canning, 507

Processing canned foods, 509

Proline, 187

Properties of ice, 336

Proskauer and Beck's culture medium, 68

Protargol in disinfection, 135

Proteins, bacterial action on, $\mathbf{1 8 9}$

-, color reactions of, 191

—, in milk, 371

-, precipitation reactions, 192

-, structure of, 184

* Prune decoction, 57

Pseudo yeasts, 213

Ptomaines, 529

Pure cultures, invigoration of, 108

- - isolation of, 10

- - methods of study, 108

- - transferring, 10

- water, 271

Putrefaction in the intestines, 243

Putrescine, 529

\section{Q}

Qualitative tests for elements in proteins, 189

- estimation of fecal indol, 246

"Quality" in milk, 379

Quinone in disinfection, 143

\section{$\mathbf{R}$}

Rack for fermentation tubes, 1

Raisin gelatin, 56

Rating of oysters, 487

Raulin's solution for molds, 66

Reaction in milk cultures, 114

Reagents, preparation of, 554

Red corpuscles, 13

Reddening of cod, 503

Redfield's medium, 59

Reed's methods for leucocyte in milk, 409

Reichert-Meissl number of fats, 199 
Relation between bacteria and dirt in milk, 384

- of microscopic to plate count, 407

- pressure to temperature, 122

- - the $37^{\circ}$ to the $20^{\circ} \mathrm{C}$. count, 291

- water filtration to typhoid fever, 270

Relative stability of streams, 347

Reliability of bacteria tests on milk, 386

Reporting milk analysis, 395

Required oxygen in water, 345

Residue, determination of, in water, 279

Rettger's aeroscope, 264

- method for egg analysis, 467

Revised method for tomato products, 520

Rhizopus, 205

Rice milk medium, 72

Ropy bread, 224

Roszahey's counting flask, 9

Routine milk analysis, 393

- steps in bacterial water analysis, 305

Roux culture flask, 5

Russell's medium, preparation of, 52

_-, use in water analysis, 318

\section{$\mathbf{S}$}

Sabourand's agar for yeasts, 51

Salicylic acid in milk, detection, 371

Saliva, germicidal action of, 228

Salt in food preservation, 498

Sample bottles, 5

Sampling of milk, 367

Sanitary inspection in water hygiene, 269

Saponification number of fats, 199

Sausage bacteriology, 481

Savage's method for bacteria in ice cream, 437

_- leucocytes in milk, 409

- - tubercle bacilli in butter, 426

Scheme for identifying yeasts, 219

Schneider's method for bacteria in butter, 428

Score card, 380

Sediment in milk, 384

Self-improvement of streams, 340

Self-rising bread, 223

Seliwanoff's reaction for ketoses, 195
Separation of $B$. coli and streptococci in water, 297

Serine, 185

Serum agar, 70

Serum bouillon, 47

Sewage streptococci, 296

Shell membrane of eggs, 453

Significance of bacterial count in milk, 385

Silicate jelly, 117

Silver citrate, 135

-, colloidal in disinfection, 135

- salts as disinfectants, 135

Sizes and capacities of tin cans, 510

Slaked lime, 138

Slides for mounting bacteria, 5

Slimy bread, 224

Smear, preparation, 80

Smillie's method for anaerobic bacteria, 20

Smith's fermentation tube, 1

Smoking in food preservation, 497

Soil extract agar, medium, 49

- - gelatin medium, 55

Solubility of oxygen in water, 564

Sources of water supply, 269

Souring of milk, 365

Specific gravities of common acids, 559

- gravity of milk, 368

Spherical aberration, 29

Spore staining, 85

Springers in canning, 511

Spronck's peptone yeast extract, 57

Stability in streams, 347

Stabilized gentain violet, 91

Staining, methods, 81

一, theory, 79

Standard aeroscope, 264

- loops, 7

Standardization of the microscope, 398

Starch agar, 60, 62

- jelly, 61

Steam sterilization, theory of, 124

Steel's method for fecal bacteria, 252

Sterilization methods, 121

Stewart's counting apparatus, 11

Stiles' method for egg examination, 463

Stokes' method for leucocytes in milk, 409 
Storage of food in tin cans, 513

Storch's method for heated milk, 371

Strassburger method for fecal bacteria, 250

Streaming steam sterilization, 126

Strength of yeasts, 220

Streptococci in water, 297

Structure of eggs, 453

- - hyphæ, 212

Structure of molds, 202

Substage on the microscope, 27

Sugar in food preservation, 503

Sullivan's culture fluid, 64

Swells in canning, 510

Synthetic agar, 49

\section{$\mathbf{T}$}

Teague and Clurman's eosin-brilliant green agar, 54

- - method for B. typhosus in feces, 257

- Travis' method for $M$. cholere in feces, 258

Temperature of milk, 378

- relations of bacteria, 112

Theory of pasteurization, 415

- - staining, 79

Thermal death point, 118

Thymol, disinfectant, 141

Tin cans, 509

Tobacco smoke, effect on mouth bacteria, 228

Toleration of bacteria to acids and alkalis, 118

Torrey's anaerobic method, 19

Total acidity of the stomach, 233

Toxicity coefficient of disinfectants, 175

Toxin of Bacillus botulinus, 533

Tracing pollution in water, 323

Transporting bacteriological specimens in the U. S. mail, 577

Treasury Department Standard for water examination, 311

True yeasts, 213

Trypsin broth, 45

Tryptagar, 49

Tryptophane, bromine reaction, 192

- broth, 47
Tryptophane, decomposition of, 244

-, structure, 186

Tubercle bacilli in butter, 425

Turbidity, determination in water, 278

Typhoid bacilli in ice, 338

- death rate in American cities, 271

- fever case record, 548

- - from oysters, 487

- - in Pittsburgh, 268

Tyrosine, Decomposition, 243

-, structure, 185

\section{U}

Ultra-violet light, action on microorganisms, 130

Urine medium, 58

Uschinsky's medium, characteristics of bacteria in, 112

Use of the microscope, 24

Utensils influencing milk production, 377

\section{V}

Valine, 185

Van Delden's solution, 64

Van Ermengen's procedure for staining flagella, 86

Vegetable bouillon, 46

Vegetative cells, 109

Ventilation tube, 220

Vincent's method for tomato products, 528

Voges-Proskauer reaction in water analysis, 307

\section{W}

Ward's classification of water bacteria, 288

Water bacteria, classification, 286

Water-borne epidemics of disease, 541

- drinking and bacteria in intestines, 237

Welch's method for staining capsules, 84

Whey agar, 71

- gelatin, 55

White corpuscles, enumeration, 15

Wiley's method for streptococci in ice cream, 437 
Wine medium, 57

Winogradski's medium for nitrite formation, 67

Winterberg method for fecal bacteria, 247

Working distance of the microscope, 30

Wolffhugel's counting apparatus, 9

Wording reports on water analysis, 317

Wort gelatin, 56

Wright's stain, preparation, 88

- anaerobic method, 18

- procedure for staining blood films, 87

$\mathrm{X}$

Xantho-proteic reaction, 191
X-ray fluorescence, bactericidal properties, 129

\section{$\mathbf{Y}$}

Yeast foods, 216

-, genera of true, 214

- in bread making, 222

- in feces, 258

Yeasts, classification of, 213

\section{$Z$}

Zichl-Niehlsen method of acid fast staining, 87

Zinc chloride in disinfection, 139

Zinsser's anaerobic method, 19 


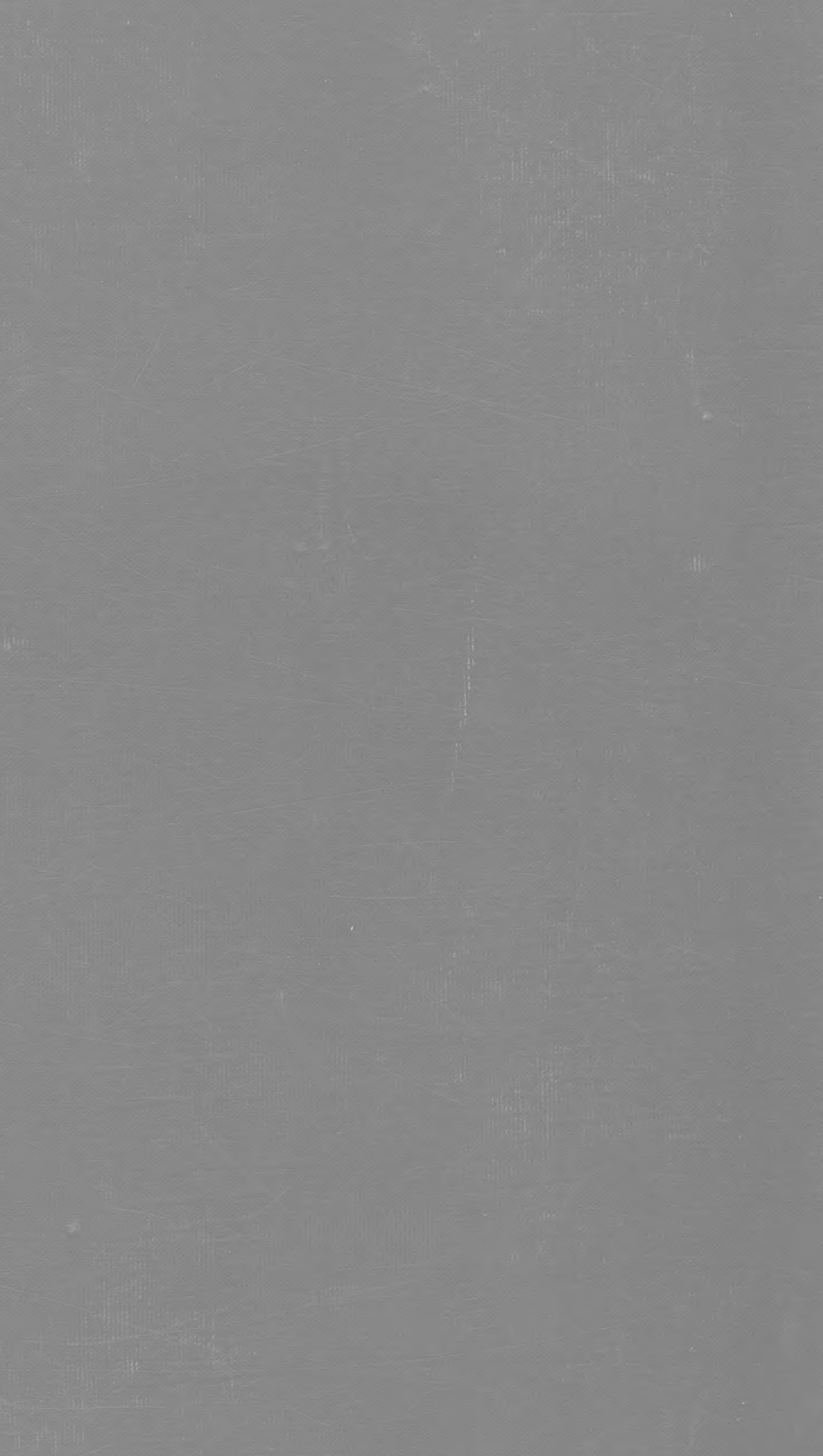

